WHC-SD-W025-RPT-001, Rev. 0

\title{
This Report
}

was Prepared by:

Golder Construction Services, Inc.

Redmond, Washington

for the Westinghouse Hanford Company

Richland, Washington

DISCLAIMER

This report was prepared as an account of work sponsored by an agency of the United States Government. Neither the United States Government nor any agency thereof, nor any of their employees, makes any warranty, express or implied, or assumes any legal liability or responsibility for the accuracy, completeness, or usefulness of any information, apparatus, product, or process disclosed, or represents that its use would not infringe privately owned rights. Reference herein to any specific commercial product, process, or service by trade name, trademark, manufacturer, or otherwise does not necessarily constitute or imply its endorsement, recommendation, or favoring by the United States Government or any agency thereof. The views and opinions of authors expressed herein do not necessarily state or reflect those of the United States Government or any agency thereof.

Prepared by:

Tiant S. Suari for

Scott A. Matthees

$\frac{9-13-94}{\text { Date }}$

Construction Qualify Assurance Engineer

Prepared by:

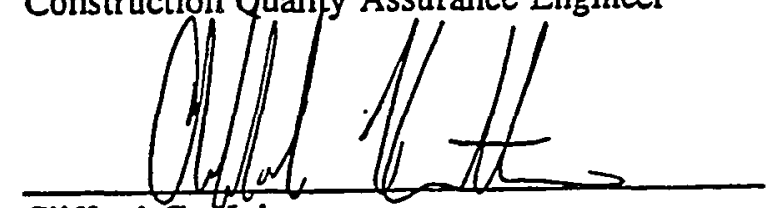

Clifford C. Knitter

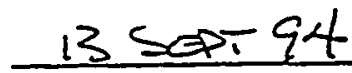

Associate/CQA Manager

Approved by:

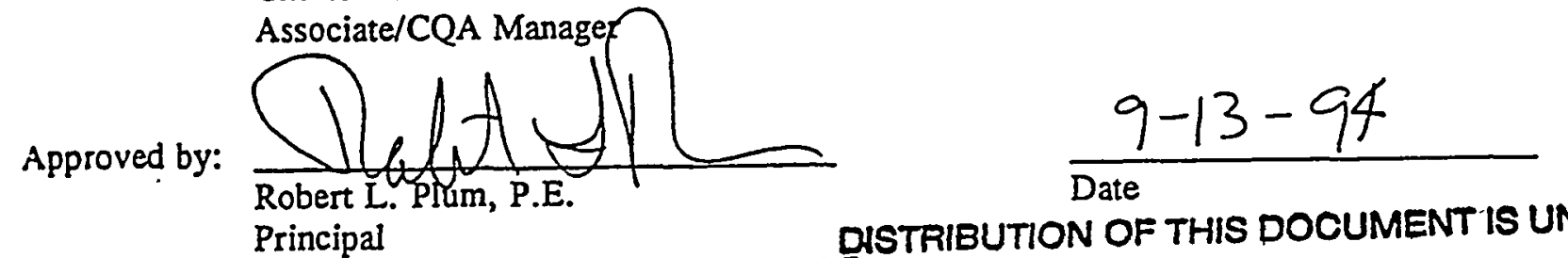

Date

Principal

September 8, 1994
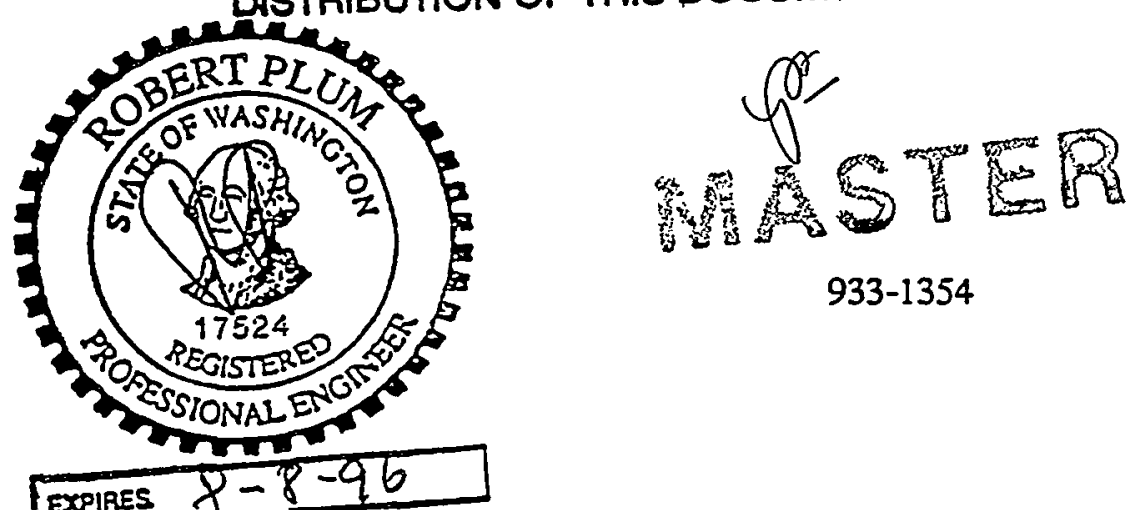

933-1354 


\section{DISCLAIMER}

Portions of this document may be illegible in electronic image products. Images are produced from the best available original document. 
2. To: (Receiving Organization) WRAP 1

\section{Proj./Prog./Dept./Div.:} H025

\section{Originator Remarks:}

For release

11. Receiver Remarks:

3. From: (Originating Organization)
WRAP 1
6. Cog. Engr.:
V. Roscha

4. Related EDT No.:

NA

7. Purchase Order No.:

Nit

9. Equip./Component No.:

IVA

10. System/Bldg./Facility:

NA

12. Major Assm. Dwg. No.:

NA

13. Permit/Permit Application Ho.: NA

14. Required Response Date: ASAP

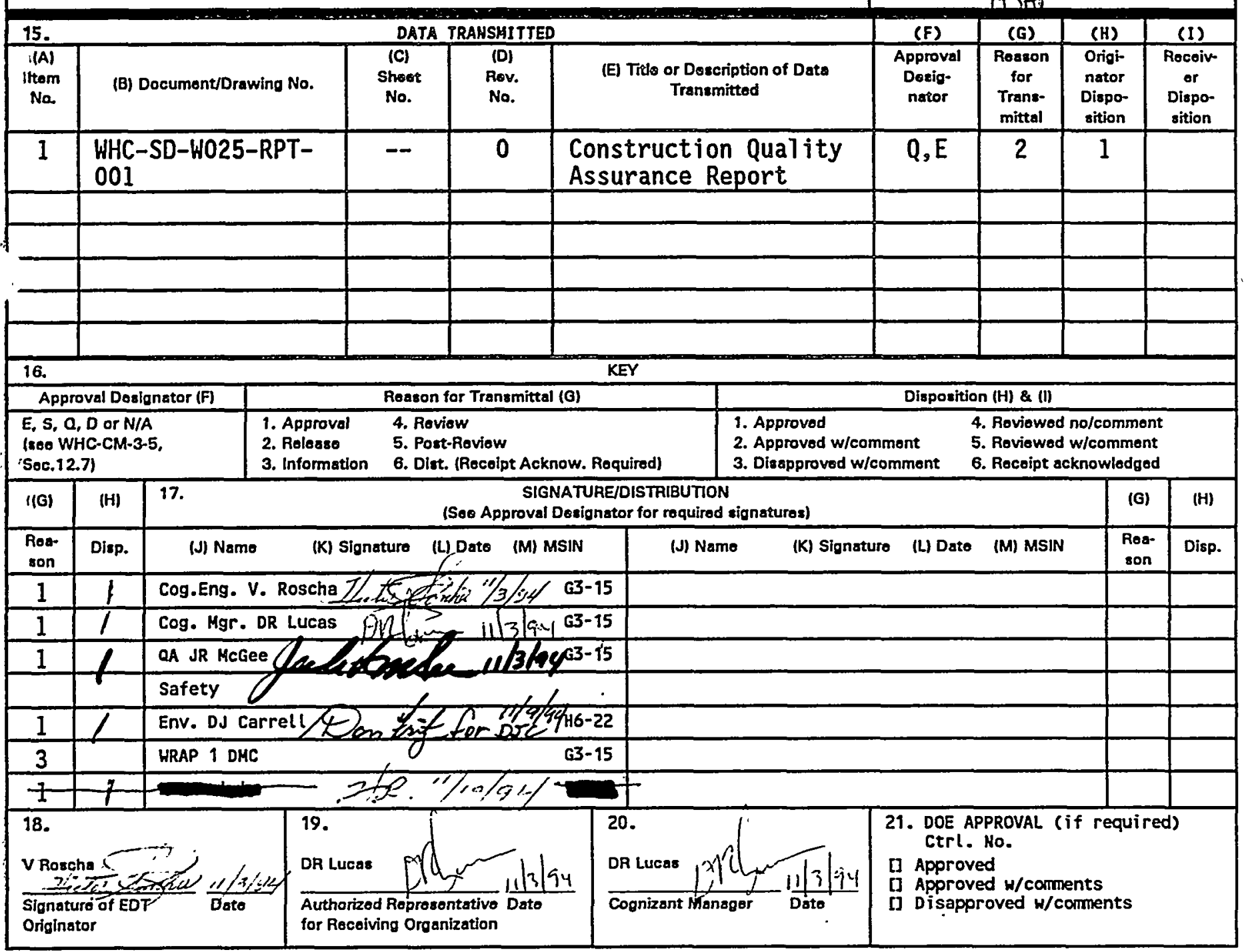




\section{INSTRUCTIONS FOR COMPLETION OF THE ENGINEERING DATA TRANSMITTAL}

(USE BLACK INK OR TYPE)

BLOCK TITLE

(1)* EDT

(2) To: (Receiving Organization)

(3) From: (Originating Organization)

(4) Related EDT No.

(5)* Proj./Prog./Dept./Div.

(6)* Cognizent Engineer

(7) Purchase Order No.

(8) * Originator Remarks

(9) Equipment/Component No.

(10) Systom/Bldg./Facitity

(11) Roceiver Remarks

(12) Major Assm. Dwg. No.

(13) Pormit/Pormit Application No.

(14) Required Response Date

(15) - Data Transmitted
(A). Itom Number
(B)- Document/Drawing No.
(C) * Sheat No.
(D)* Rev. No.
(E) Title or Description of Data Transmitted
(F) * Impact Lovol
(G) Resson for Transmittal
(H) Originator Disposition
(I) Receiver Disposition

(16) Koy

(17) Signature/Distribution

(G) Resson

(H) Disposition

(J) Name

(K) * Signature

(L)* Date

(M) * MSIN

(18) Signature of EDT Originator

(19) Authorized Representativo for Rocoiving Organization

(20)" Cognizant Manager

(21)* DOE Approval
- Pro-assigned EDT number.

- Enter the individual's name, title of the organization, or entity (e.g.. Distribution) that the EDT is being transmitted to.

- Enter the title of the organization originating and transmitting the EDT.

- Enter EDT numbers which relate to the data being transmitted.

- Enter the Project/Program/Depertment/Division titlo or Project/Program acronym or Project Number, Work Order Number or Organization Code.

- Enter the name of the individual identified as being responsible for coordinating disposition of the EDT.

- Entar rolated Purchase Order (P.O.) Number, if availablo.

- Enter special or edditional comments concerning transmittal, or "Key" retrieval words may be ontered.

- Enter equipment/component number of affected item, if appropriate.

- Enter appropriate system, building or facility number, if appropriate.

- Enter special or additional comments concerning transmittal.

- Enter applicable drawing number of major assembly, if appropriate.

- Enter applicable permit or pormit application number, if appropriato.

- Enter the date a response is required from individuals identifiod in 8lock 17 (Signature/Distribution).

- Enter sequential number, beginning with 1, of the information listed on EDT.

- Enter the unique identification number assigned to the document or drawing being transmitted.

- Enter the sheet number of the information being transmitted. If no sheet number, loevo blank.

- Enter the rovision number of the information boing transmitted. If no revision number, leave blank.

- Enter the title of the document or drawing or a brief description of the subject if no title is identified.

- Enter the appropriate Impact Level (Block 15). Also, indicete the appropriate approvals for each item liztod, i.o., SQ, ESQ, otc. Use NA for non-ongineering documents.

- Enter the appropriate code to identify the purpose of the data transmitzal (see Block 16).

- Entor the appropriate disposition codo (seo Block 16).

- Enter the appropriate disposition code (seo Block 16).

- Number codes used in completion of Blocks 15 (G), (H), and (I), and 17 (G), (H) (Signature/Distribution).

- Enter the cade of the roason for trensmittal (Block 16).

- Enter the code for the disposition (Elock 16).

- Enter the signature of the individual completing the Disposition $17(H)$ and the Transmittal.

- Obtain appropriate signature(8).

- Enter date signature is obtained.

- Enter MSIN. Note: If Distribution Sheet is used, show ontire distribution (including shet indicatod on Pago 1 of the 5 Ty on the Distribution Sheot.

- Enter the signature and date of the individual originating the EDT lentered prior to transmittal to Receiving Organization). If the EDT originator is the cognizant ongineer, sign both Blocks 17 and 18.

- Enter the signature and deto of the individual identified by the Recoiving Organization es authorized to approve disposition of the EDT and acceptance of the date transmitted, as epplicable.

- Enter the signature and date of the cognizant manager. (This signature is authorization for relosse.)

- Enter DOE opproval (if roquired) by lottor number and indicate DOE action.

-Asterisk denote the required minimum items check by Configuration Documentation prior to release; these aro she minimum release requirements. 


\section{RELEASE AUTHORIZATION}

Document Number: WHC-SD-W025-RPT-001, Rev. 0

Document Title: Construction Quality Assurance Report

Release Date: $\quad 11 / 17 / 94$

This document was reviewed following the procedures described in WHC-CM-3-4 and is:

APPROVED FOR PUBLIC RELEASE

WHC Information Release Administration Specialist:

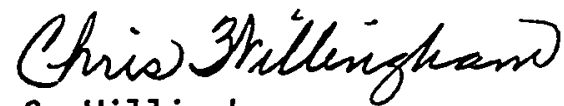

c. Wi1lingham

TRADEMARK DISCLAIMER. Reference herein to any specific commercial product, process, or service by trade name, trademark, manufacturer, or otherwise, does not necessarily constitute or imply its endorsement, recormendation, or favoring by the United States Government or any agency thereof or its contractors or subcontractors.

This report has been reproduced from the best available copy. Available in paper copy and microfiche. Printed in the United States of America. Available to the U.S. Department of Energy and its contractors from:

U.S. Department of Energy

Office of Scientific and Technical Information (OSTI)

P.0. Box 62

Oak Ridge, TN 37831

Telephone: (615) 576-8401

Available to the public from:

U.S. Department of Commerce

National Technical Information Service (NTIS)

5285 Port Royal Road

Springfield, VA 22161

Telephone: (703) 487-4650 
2. Title

Construction Quality Assurance Report

4. Rev No.

5. Key Words

H-025

Radioactive Mixed Waste Land Disposal Facility Non-Drag-Off

3. Number WHC-SD-W025-RPT-001

6. Author

Name: V. Roscha

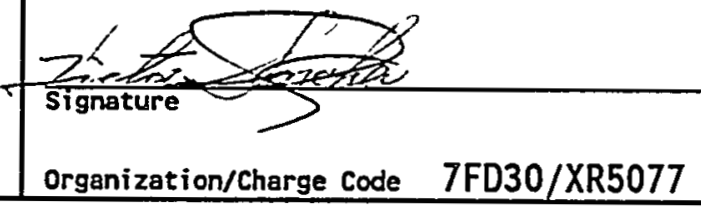

7. Abstract

This report provides a summary of the construction quality assurance (CQA) observation and test results, including:

- The results of the geosynthetic and soil materials conformance testing.

- The observation and testing results associated with the installation of the soil liners.

- The observation and testing results associated with the installation of the HDPE geomembrane 1 iner systems.

- The observation and testing results associated with the installation of the leachate collection and removal systems.

- The observation and testing results associated with the installation of the working surfaces.

- The observation and testing results associated with in-plant manufacturing process.

- Summary of submittal reviews by Golder Construction Services, Inc.

- The submittal and certification of the piping material specifications.

- The observation and verification associated of the Acceptance Test Procedure results of the operational equipment functions.

- Summary of the ECNs which are incorporated into the project. 
WHC-SD-W025-RPT-001, Rev. 0

\section{CONTENTS}

\section{VOLUME I}

$1.0 \quad$ INTRODUCTION $\ldots \ldots \ldots \ldots \ldots \ldots \ldots \ldots \ldots \ldots \ldots \ldots \ldots \ldots \ldots \ldots$

1.1 SCOPE OF SERVICES $\ldots \ldots \ldots \ldots \ldots \ldots \ldots \ldots \ldots \ldots \ldots \ldots \ldots \ldots$

1.2 PROJECT DESCRIPTION $\ldots \ldots \ldots \ldots \ldots \ldots \ldots \ldots \ldots \ldots \ldots \ldots \ldots \ldots \ldots \ldots$

1.3 PROJECT SPECIFICATIONS $\ldots \ldots \ldots \ldots \ldots \ldots \ldots \ldots \ldots \ldots$

2.0 PRECONSTRUCTION TESTING AND CONFORMANCE TESTING $\ldots \ldots \ldots \ldots .8$

2.1 EXCAVATION AND BACKFILL $\ldots \ldots \ldots \ldots \ldots \ldots \ldots \ldots \ldots, 8$

2.2 SOIL LINER $\ldots \ldots \ldots \ldots \ldots \ldots \ldots \ldots \ldots \ldots \ldots \ldots \ldots \ldots \ldots \ldots \ldots$

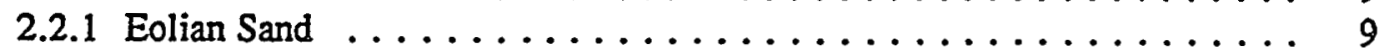

2.2.2 Bentonite ............................ 10

2.2 .3 Admix ....................... 10

2.3 GRANULAR DRAINAGE MATERIAL $\ldots \ldots \ldots \ldots \ldots \ldots \ldots \ldots \ldots$

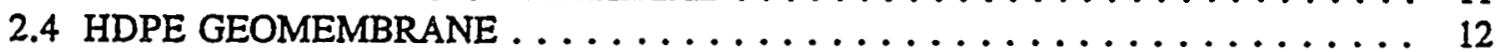

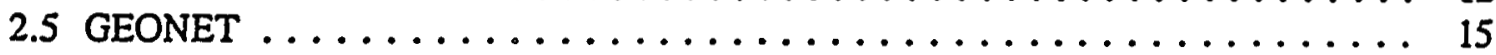

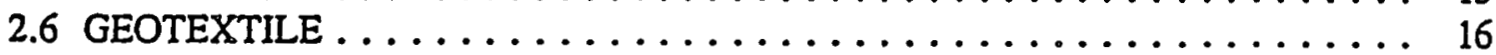

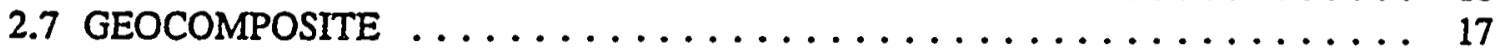

2.8 HDPE PIPE AND STAINLESS STEEL PIPE $\ldots \ldots \ldots \ldots \ldots \ldots \ldots \ldots 19$

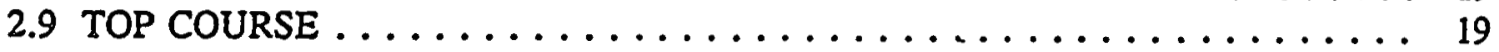

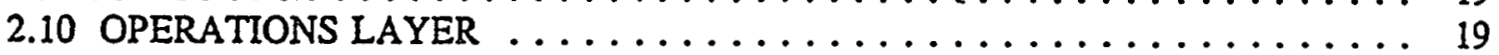

3.0 EXCAVATION/SUBGRADE PREPARATION $\ldots \ldots \ldots \ldots \ldots \ldots \ldots \ldots 21$

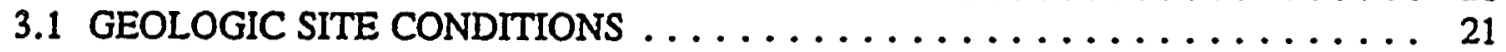

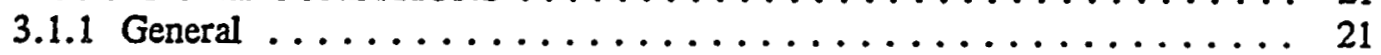

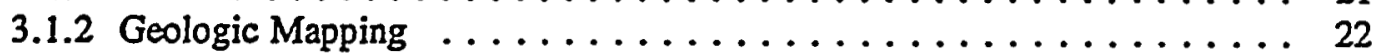

3.1.3 Geologic Units Encountered ................... 22

3.2 SUBGRADE PREPARATION AND BACKFILL PLACEMENT $\ldots \ldots \ldots \ldots 22$

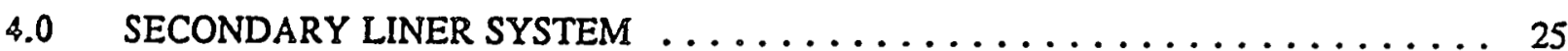

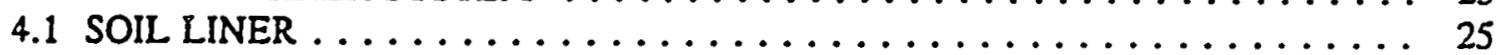

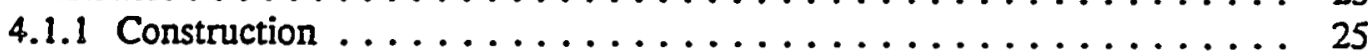

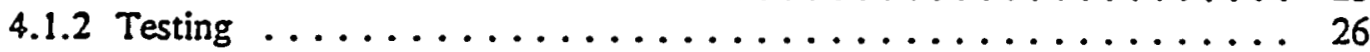

4.2 HDPE GEOMEMBRANE LINER INSTALLATION $\ldots \ldots \ldots \ldots \ldots \ldots \ldots 28$

4.2.1 HDPE Geomembrane Liner Deployment . . . . . . . . . . . . 29

4.2.2 HDPE Geomembrane Liner Seaming . . . . . . . . . . . . . 29

4.2 .3 Destructive Testing ..................... 30

4.2.4 Nondestructive Seam Continuity Testing . . . . . . . . . . 31

4.2.5 HDPE Geomembrane Liner Repairs ................ 32

4.3 LEACHATE COLLECTION AND REMOVAL SYSTEM $\ldots \ldots \ldots \ldots \ldots \ldots, 32$

4.3.1 Geonet Installation .......................... 32

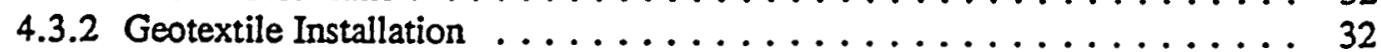

4.3 .3 Geocomposite Installation ................... 33

4.3.4 Granular Drainage Material . . . . . . . . . . . . . . 33

4.3.5 Leachate Removal System ................... 34 
$5.0 \quad$ PRIMARY LINER SYSTEM $\ldots \ldots \ldots \ldots \ldots \ldots \ldots \ldots \ldots \ldots \ldots \ldots \ldots \ldots \ldots \ldots$

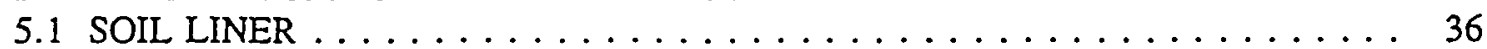

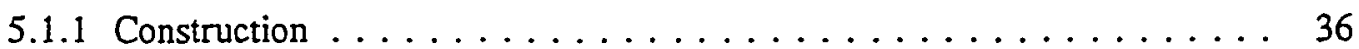

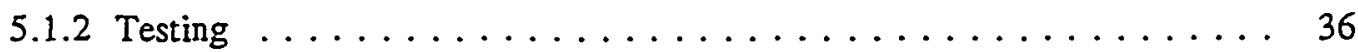

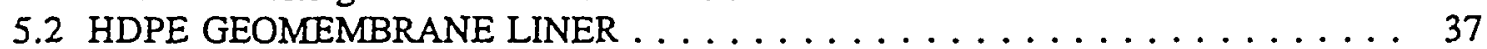

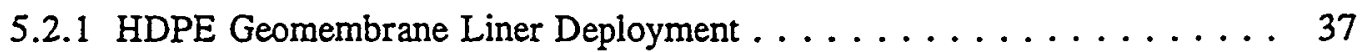

5.2 .2 HDPE Geomembrane Liner Seaming $\ldots \ldots \ldots \ldots \ldots \ldots \ldots \ldots . \quad 37$

5.2 .3 Destructive Testing . . . . . . . . . . . . . . 38

5.2 .4 Nondestructive Seam Continuity Testing $\ldots \ldots \ldots \ldots \ldots \ldots \ldots .38$

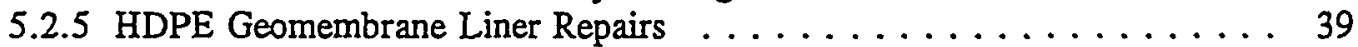

5.3 LEACHATE COLLECTION AND REMOVAL SYSTEM $\ldots \ldots \ldots \ldots \ldots . \ldots 39$

5.3 .1 Geonet Installation . . . . . . . . . . . . . . . . . 39

5.3 .2 Geotextile Installation $\ldots \ldots \ldots \ldots \ldots \ldots \ldots \ldots \ldots \ldots \ldots \ldots$

5.3.3 Geocomposite Installation $\ldots \ldots \ldots \ldots \ldots \ldots \ldots \ldots \ldots, 40$

5.3 .4 Leachate Collection Piping $\ldots \ldots \ldots \ldots \ldots \ldots \ldots \ldots, 40$

5.3 .5 Granular Drainage Material . . . . . . . . . . . . . 40

5.3.6 Leachate Removal System..................... 41

6.0 WORKING SURFACES $\ldots \ldots \ldots \ldots \ldots \ldots \ldots \ldots \ldots \ldots \ldots \ldots \ldots$

6.1 OPERATIONS LAYER $\ldots \ldots \ldots \ldots \ldots \ldots \ldots \ldots \ldots \ldots \ldots \ldots \ldots$

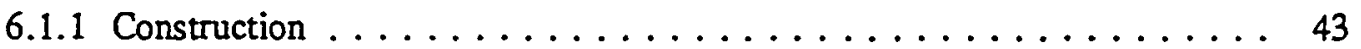

6.1 .2 Testing $\ldots \ldots \ldots \ldots \ldots \ldots \ldots \ldots \ldots \ldots \ldots \ldots \ldots \ldots \ldots \ldots, 44$

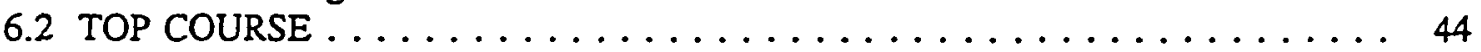

6.2 .1 Construction $\ldots \ldots \ldots \ldots \ldots \ldots \ldots \ldots \ldots \ldots \ldots \ldots \ldots, 44$

6.2 .2 Testing $\ldots \ldots \ldots \ldots \ldots \ldots \ldots \ldots \ldots \ldots \ldots \ldots \ldots \ldots \ldots \ldots \ldots, 44$

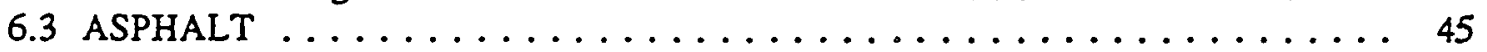

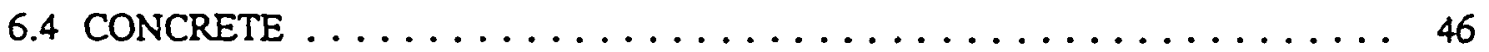

7.0 OPERATIONAL FEATURES $\ldots \ldots \ldots \ldots \ldots \ldots \ldots \ldots \ldots \ldots$

7.1 LEACHATE TEMPORARY STORAGE TANK, PIPING, AND TRANSFER

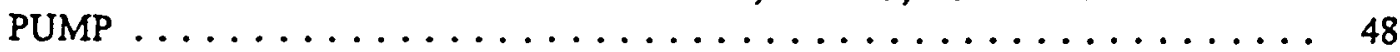

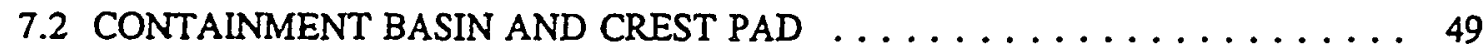

7.3 CONTROL BUILDING AND ELECTRICAL SYSTEM $\ldots \ldots \ldots \ldots \ldots \ldots .49$

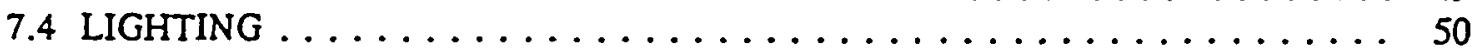

7.5 DRAINAGE FEATURES $\ldots \ldots \ldots \ldots \ldots \ldots \ldots \ldots \ldots \ldots \ldots \ldots \ldots \ldots \ldots \ldots \ldots$

8.0 ACCEPTANCE TEST PROCEDURES $\ldots \ldots \ldots \ldots \ldots \ldots \ldots \ldots \ldots \ldots \ldots$

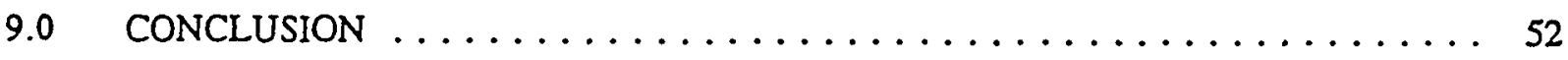


WHC-SD-W025-RPT-001, Rev. 0

CONTENTS (C̣ont.)

\section{TABLES:}

1. Lining and Leachate Collection Systems 3

2. Engineering Change Notice Summary 5

3. Excavation \& Backfill Materials Evaluation - Preconstruction Test Summary 8

4. Eolian Sand Preconstruction Test Summary - 10

5. Bentonite Preconstruction Test Summary 10

6. Admix Preconstruction Test Summary 12

7. Granular Drainage Material Preconstruction Test Summary . 13

8. HDPE Geomembrane Conformance Test Summary 15

9. Geonet Conformance Test Summary 17

10. Geotextile Conformance Test Summary 18

11. Geocomposite Conformance Test Summary 20

12. Top Course Preconstruction Testing Summary 21

13. Operations Layer Preconstruction Testing Summary 22

14. Secondary Soil Liner Field and Laboratory Testing Summary 31

15. Destructive Test Criteria 33

16. Secondary Granular Drainage Material Construction Testing Summary 38

17. Primary Soil Liner Field and Laboratory Testing Summary 40

18. Primary Granular Drainage Material Construction Testing Summary 45

19. Top Course Laboratory Testing Summary 50

20. Summary of Concrete Pours 51

\section{FIGURES:}

1. Hanford 200 West Area Location

2. Project $W-025$ Location in 200 West Area

3. Geologic Map of Project W-025

4. Geologic Cross Sections

5. Subgrade, General Fill, and Top Course Moisture-Density Test Locations

6. Secondary Soil Liner Moisture-Density Test Locations

7. Shelby Tube Sample Locations

8. Extrusion Weld Destructive Test Codes

9. Fusion Weld Destructive Test Codes

10. Toe of Access Ramp Tie-in Detail

11. Primary Soil Liner Moisture-Density Test Locations 
WHC-SD-W025-RPT-001, Rev. 0

CONTENTS (Cont.)

\section{APPENDICES:}

Appendix A Construction Schedule and GCS Onsite CQA Staff

Appendix B Summary of Submittal Reviews by GCS and GAI

Appendix C Specification Clarifications and Engineering Change Notices

Appendix C-1 Preconstruction Meeting Minutes

Appendix C-2 Geosynthetics Preconstruction Meeting Minutes

Appendix C-3 Engineering Change Notices

Appendix D Soil Materials Laboratory Test Results

Appendix D-1 Excavation and Backfill

Appendix D-2 Eolian Sand

Appendix D-3 Bentonite

Appendix D-3.1 CQA Laboratory Test Results

Appendix D-3.2 Viscosity Correlation

Appendix D-3.3 Certificates of Analysis

Appendix D-4 Admix/Soil Liner

Appendix D-5 Granular Drainage Materials

Appendix D-6 Top Course

Appendix D-7 Operations Layer

Appendix E Geomembrane Conformance Tests

Appendix E-1 Surveillance Inspection Reports

Appendix E-2 CQA Conformance Tests

Appendix E-3 Manufacturer's and Installer's QC Documentation

Appendix F Geonet Conformance Tests

Appendix F-1 CQA Conformance Tests

Appendix F-2 Manufacturer's QC Documentation

Appendix G Geotextile Conformance Tests

Appendix G-1 CQA Conformance Tests

Appendix G-2 Manufacturer's QC Documentation

Appendix $\mathrm{H}$ Geocomposite Conformance Tests

Appendix $\mathrm{H}-1$ CQA Conformance Tests

Appendix H-2 Manufacturer's QC Documentation

Appendix I Pipe Manufacturers' QC Documentation

Appendix I-1 HDPE Pipe

Appendix I-2 Stainless Steel Pipe

Appendix J Subgrade and General Fill Moisture-Density Tests

Appendix K Soil Liner Moisture-Density Tests

Appendix K-1 Secondary Soil Liner

Appendix K-2 Primary Soil Liner

Appendix L Subgrade Acceptance Certificates

Appendix $M$ Secondary HDPE Geomembrane Liner Installation

Appendix M-1 Geomembrane Deployment Observation Summary

Appendix M-2 Trial Seam Observation Summary

Appendix M-3 Geomembrane Weld Observation and Non-Destructive Testing

Summary

Appendix M-4 Destructive Test Observation Summary

Appendix M-5 Geomembrane Repair Summary 


\section{CONTENTS (Cont.)}

APPENDICES (cont):

Appendix N Primary HDPE Geomembrane Liner Installation

Appendix N-1 Geomembrane Deployment Observation Summary

Appendix N-2 Trial Seam Observation Summary

Appendix N-3 Geomembrane Weld Observation and Non-Destructive Testing

Summary

Appendix N-4 Destructive Test Observation Summary

Appendix N-5 Geomembrane Repair Summary

Appendix 0 Top Course Moisture-Density Tests

Appendix P Asphalt Paving

Appendix P-1 Laboratory Tests and Field Density Tests

Appendix P-2 Design Engineer's Memo Regarding Tolerances

Appendix Q Concrete

Appendix $\mathrm{R} \quad$ Leachate Temporary Storage Tank

Appendix R-1 Manufacturer's QC Documentation

Appendix R-2 Registered Professional Engineer's Statement

Appendix R-3 Self Priming Pump Testing

Appendix R-4 Leachate Piping Pressure Testing

Appendix R-5 Containment Basin Leak Test

Appendix S Electrical Design Engineers Observations During ATPs

Appendix $T$ CQA Verification Surveys

Appendix T-1 Subgrade

Appendix T-2 Top of Secondary Soil Liner

Appendix T-3 Top of Secondary Gravel

Appendix T-4 Top of Primary Soil Liner

Appendix T-5 Top of Primary Gravel

Appendix T-6 Top of Operations Layer

Appendix $U$ Report: Soil Liner Test Fill and SDRI Test

\section{Drawings}

Sheet S-1 Secondary HDPE Geomembrane Liner As-Built Drawing

Sheet $\mathrm{P}-1$ Primary HDPE Geomembrane Liner As-Built Drawing

Sheet 1 of 6 Subgrade As-Built

Sheet 2 of 6

Sheet 3 of 6

Sheet 4 of 6

Sheet 5 of 6

Sheet 6 of 6

Sheet E1

Sheet E2

Sheet E3

Sheet PG1

Top of Secondary Admix As-Built

Top of Secondary Gravel As-Built

Top of Primary Admix As-Built

Top of Primary Gravel As-Built

Top of Operations Layer As-Built

Pump Control Panel Layout and Schedule

Pump Control Wiring Diagram

Pump Control Wiring Diagram

Schedule of Control Panel Equipment

Sheet PG2

Schedule of Control Panel Equipment 


\section{WHC-SD-W025-RPT-001, Rev. 0}

\subsection{INTRODUCTION}

Golder Construction Services, Inc. (GCS) has performed quality assurance observation and testing for the construction of the Radioactive Mixed Waste Land Disposal Facility, Project W-025, located in the 200 West Property Protected Area of the Hanford Nuclear Reservation, approximately 30 miles north of Richland, Washington (Figures 1 and 2). This work was performed under Contract No. DE-ACOG-85RL11615 between the United States Department of Energy and Golder Associates Inc. (GAI).

This report provides a summary of the construction quality assurance (CQA) observation and test results, including:

- The results of the geosynthetic and soil materials conformance testing;

- The observation and testing resulis associated with the installation of the soil liners;

- The observation and testing results associated with the installation of the HDPE geomembrane liner systems;

- The observation and testing results associated with the installation of the leachate collection and removal systems;

- The observation and testing results associated with the installation of the working surfaces.

The engineering design basis for the Non-Drag-Off Landfill, Project W-025, are not included in this report, but are given in the following reports by Golder Associares Inc.:

- $\quad$ Site Investigation Report, Non-Drag-Off Landfill Site, Low Level Burial Area No. 5 . 200 West Area dated December 4, 1989.

- Soil Liner/Leachate Compatibility Testing in Support of Project W-025, Radioactive Mixed Waste Disposal Facility, not dated.

- Design Report, Project W-025, Radioactive Mixed Waste (RMW) Land Disposal Facility, Non-Drag-Off; Revision 1, dated November 12, 1992.

\subsection{SCOPE OF SERVICES}

The scope of GCS' services included CQA observation and testing of Project W-025 in accordance with the project Construction Quality Assurance Plan (CQAP). The CQA observation and testing services included in this report took place between September 7, 1993 and October 7, 1994. A schedule of CQA onsite personnel and specific construction activities is shown in Appendix A.

Construction documentation included daily field reports which were submitted by all field personnel. The CQA Engineer prepared and submitted summary weekly field reports to the DOE Contract Officer's Technical Representative (COTR), Westinghouse Hanford Company (WHC). 
These reports are not included in this document, but are available for review at the COTR and at GCS's Redmond, Washington, office. In addition, photographs were taken on a regular basis during the construction. Copies of these photographs have been tumed over to WHC.

Delhur Industries, Inc. (Delhur) of Port Angeles, Washington, was the prime contractor for the project. Delhur completed the Project W-025 excavation, subgrade preparation, general fill, soil liners, earthwork for the leachate collection and removal systems, and the protective layer for the Project W025 construction project. Installation of the secondary and primary geosynthetic liner systems, and the geosynthetic materials for the secondary and primary leachate collection systems was performed by GeoPacific Lining Inc. (GeoPacific) of Seattle, Washington. The leachate temporary storage tank was fabricated by Quality Tank \& Construction of Sandy, Oregon. Pumps and piping systems were installed by Pilchuck Mechanical, Inc. of Renton, Washington. Electrical systems were installed by JRT Electric, Inc. of Richland, Washington. Paving was installed by ACME Materials \& Construction Co. of Richland, Washington. Surveying services were provided by Permit Surveying, Inc. of Kennewick, Washington. Permit Surveying, Inc. (Permit) was also the Surveyor of Record for this project.

GCS CQA personnel or GAI Design representatives reviewed contractors' submittals which proposed methods and materials to be used during construction of the Non-Drag-Off facility for compliance with the project specifications. A summary of the submittals reviewed by GCS and GAI is presented in Appendix B.

GCS also reviewed CQA verification surveys performed on GCS's behalf by Rogers Surveying Inc. of Richland, Washington, and record surveys performed by Permit, for the following:

- Subgrade Surface

- Secondary Soil Liner Surface

- Secondary Drainage Gravel Surface

- Primary Soil Liner Surface

- Primary Drainage Gravel Surface

- Operations Layer Surface

Record drawings, prepared by Permit, as well as the geomembrane liner as-built panel layout drawings, prepared by GCS, are included in Volume 2 of this report.

\subsection{PROJECT DESCRIPTION}

The Project W-025 Radioactive Mixed Waste Land Disposal Facility, Non-Drag-Off, is a landifill which was designed to accept Low Level Radioactive Mixed Waste (LL-RMW). LL-RMW is the combination of hazardous constituent and low level radioactive material. The landfill consists of a double-lined, composite, soil liner/geosynthetic liner system, and a leachate collection and removal system. The layers of the lining system and the leachate collection system, from bottom to top, are as shown in Table 1. 
WHC-SD-W025-RPT-001, Rèv. 0

Table 1. Lining and Leachate Collection Systems.

\begin{tabular}{|c|c|c|}
\hline LAYER & FLOOR OF FACIITY & SLOPES OF FACILITY \\
\hline Secondary Liner & $\begin{array}{l}\text { 3.1-ft thick soil liner } \\
\text { 60-mil, smooth HDPE liner }\end{array}$ & $\begin{array}{l}\text { 3.1-ft thick soil liner } \\
\text { 60-mil, textured HDPE liner }\end{array}$ \\
\hline $\begin{array}{l}\text { Secondary Leachate } \\
\text { Collection System }\end{array}$ & $\begin{array}{l}\text { Geonet } \\
16-0 z / \mathrm{yd}^{2} \text { geotextile } \\
1.0 \text {-ft thick layer of drainage } \\
\text { gravel } \\
16-0 z / \mathrm{yd}^{2} \text { geotextile }\end{array}$ & $\begin{array}{l}\text { Geonet-Geotextile } \\
\text { Geocomposite }\end{array}$ \\
\hline Primary Liner & $\begin{array}{l}\text { 1.5-ft thick soil liner } \\
\text { 60-mil, smooth HIDPE liner }\end{array}$ & 60-mil, textured HDPE liner \\
\hline $\begin{array}{l}\text { Primary Leachate } \\
\text { Collection System }\end{array}$ & $\begin{array}{l}\text { Geonet } \\
\text { 16-oz/yd }{ }^{2} \text { geotextile } \\
\text { 1.0-ft thick layer of drainage } \\
\text { gravel } \\
\text { 7-oz/yd }{ }^{2} \text { geotextile }\end{array}$ & $\begin{array}{l}\text { Geonet-Geotextile } \\
\text { Geocomposite }\end{array}$ \\
\hline Operations Layer & 3.0-ft thick gravel/eolian sand & 3.0-ft thick Eolian sand \\
\hline
\end{tabular}

The leachate removal systems included pumps, HDPE piping, stainless steel piping, and a leachate temporary storage tank.

\subsection{PROJECT SPECIFICATIONS}

The work performed for the Project W-025 construction project was completed according to the following documents, plans, and specifications:

- Material Specifications and Construction Requirements for the Radioactive Mixed Waste Land Disposal Facility, Non-Drag-Off, Project W025, Revision 2 , prepared for the U.S. Department of Energy-Richland Operations Office, Contract No. DE-AC0689RL11615, by Golder Associates Inc., dated May 26, 1993.

- Construction Plans for Radioactive Mixed Waste (RMW) Land Disposal Facility, NonDrag-Off, Project W-025, prepared by Golder Associates Inc., dated May 21, 1993. 
- Construction Quality Assurance Plan for Project W-025, Radioactive Mixed Waste (RMW) Land Disposal Facility - Non-Drag-Off, Revision 3 , prepared for the U.S. Department of Energy - Richland Operations Office, Contract No. DE-AC0689RL11615, by Golder Associates Inc., dated May 21, 1993.

- Quality Assurance Program Plan for Project W-025, Radioactive Mixed Waste (RMW) Land Disposal Facility - Non-Drag-Off, Revision 3, prepared for the U.S. Department of Energy - Richland Operations Office, Contract No. DE-AC06-89RL11615, by Golder Associates Inc., dated April, 1991.

Construction issues were discussed and clarified in a preconstruction meeting which was held at the offices of Kaiser Engineers Hanford on August 25, 1993. Minutes of the meeting, recorded by Kaiser Engineers Hanford, are included in Appendix C-1.

A second preconstruction meeting, with GCS and GeoPacific personnel in attendance, was held onsite on November 17, 1993, to discuss and clarify geosynthetics installation issues. Minutes of the meeting, recorded by GCS, are included in Appendix C-2.

During construction of the landfill, several issues regarding design and construction were encountered. These issues were discussed with the Design Engineer of record, Golder Associates Inc. (GAD), and resolutions to the issues were determined. An Engineering Change Notice (ECN) was prepared by the Design Engineer for each construction issue which warranted a design or specification change. These documents are included in Appendix C-3.

Engineering Change Notices (ECN) W-025-006 through W-025-026, W-025-028, W-025-029. W-025-031, and W-025-032 are summarized in Table 2. ECNs W-025-001 through W-025-005 were completed prior to the bidding date for this project, were incorporated to the specification package and Design Drawings prior to the bid date and are not included in Table 2. ECNs W-025-027 and W-025030 involved the second landfill, Project W-025A, and are not included in Table 2.

Table 2. Engineering Change Notice Summary.

\begin{tabular}{|l|l|}
\hline ECN No. & \multicolumn{1}{c|}{ Description of Change } \\
\hline \hline W-025-006 & $\begin{array}{l}\text { Submit qualifications of organization and personnel who will serve as } \\
\text { geosynthetics installer. } \\
\text { Add section to specifications for Dry Type Transformers. } \\
\text { Pole mounted transformers - change from } 75 \mathrm{KV} \mathrm{BIL} \mathrm{to} 95 \mathrm{KV} \mathrm{BIL.} \\
\text { Open fuse cutouts shall be extra heavy duty, } 100 \text { Amp continuous, } 5600 \mathrm{Amp} \\
\text { interrupting duty or better. Cutouts shall be fused with 2T fuses. } \\
\text { Grounding shall be performed in accordance with NESC rules } 96 \text { \& } 97 .\end{array}$ \\
\hline
\end{tabular}


WHC-SD-W025-RPT-001, Rev. 0

\begin{tabular}{|c|c|}
\hline ECN No. & Description of Change \\
\hline$W-025-007$ & $\begin{array}{l}\text { Change Instrumentation/Electrical Acceptance Test Procedure: } \\
\text { Circuit breaker for pump W5P1 to be located on Panel A. } \\
\text { Edit WP51 to W5P1. } \\
\text { Measure phase currents for pumps prior to testing. Verify that overload } \\
\text { devices are sized correctly. }\end{array}$ \\
\hline W-025-008 & Editorial changes to Design Drawings and title blocks of Drawings. \\
\hline W-025-009 & Editorial changes to CQA Plan tables. \\
\hline$W-025-010$ & $\begin{array}{l}\text { Only two friction angle tests are required for each of the following interfaces: } \\
\text { Textured geomembrane/soil liner } \\
\text { Texured geomembrane/geocomposite } \\
\text { Geocomposite/operations layer }\end{array}$ \\
\hline W-025-011 & $\begin{array}{l}\text { Clarified CQA Plan such that only two friction angle tests are required for the } \\
\text { interfaces indicated in ECN \#10 (W-025-010). }\end{array}$ \\
\hline $\mathrm{W}-025-012$ & $\begin{array}{l}\text { Move site access road northward approximately } 10 \mathrm{ft} \text { to avoid an existing } \\
\text { power pole. }\end{array}$ \\
\hline$W-025-013$ & $\begin{array}{l}\text { Nominal Transmissivity of geonet shall be a minimum of } 0.001 \mathrm{~m}^{2} / \mathrm{sec}(4.8 \\
\text { gal/min/ft) at } 10,000 \mathrm{psf} \text { normal load, and the Mass/Unit Area shall be a } \\
\text { minimum of } 22 \mathrm{oz} / \mathrm{yd}^{2} \text {. } \\
\text { Type A geotextile shall have Mass/Unit Area of } 7.0 \mathrm{oz} / \mathrm{yd}^{2} \text { or greater, and } \\
\text { Permittivity shall be } 1.2 \mathrm{sec}^{-1} \text { or greater. } \\
\text { 1.5-in diameter, SDR 11, HDPE pipe is acceptable for submersible pump } \\
\text { discharge piping. }\end{array}$ \\
\hline W-025-014 & $\begin{array}{l}\text { Type B geotextile shall have a minimum thickness of } 170 \text { mils, a minimum } \\
\text { Trap Tear Strength of } 135 \mathrm{lbs} \text {, and a minimum permitivity of } 0.40 \mathrm{sec}^{-1} \text {. }\end{array}$ \\
\hline W-025-015 & The first lift of admix material may be placed in a 12-inch thick loose lift. \\
\hline W-025-016 & $\begin{array}{l}\text { The minimum density of the resin used to manufacture the geomembrane } \\
\text { material shall be } 0.930 \text {. } \\
\text { For Friction Angle tests, combinations of shear strength parameters, including } \\
\text { such parameters as friction angle and cohesion, may be used in stability } \\
\text { analyses to evaluate the static and dynamic factors of safety against slope failure } \\
\text { to determine the acceptability of the materials. }\end{array}$ \\
\hline
\end{tabular}


WHC-SD-W025-RPT-001, Rev. 0

\begin{tabular}{|c|c|}
\hline ECN No. & Description of Change \\
\hline $\mathrm{W}-025-017$ & $\begin{array}{l}\text { If the installer elects to weld geomembrane seams in ambient temperatures } \\
\text { below } 40^{\circ} \mathrm{F} \text { or above } 104^{\circ} \mathrm{F} \text {, the installer must certify that such seams will be } \\
\text { comparable to seams welded in ambient temperatures between } 40^{\circ} \mathrm{F} \text { and } 104^{\circ} \\
\mathrm{F} \text {, and additional coupons (from the ends of such seams) shall be tested to } \\
\text { verify the quality of the welds. } \\
\text { SDR } 13.5 \mathrm{HDPE} \text { pipe or SDR } 15.5 \mathrm{HDPE} \text { pipe may be used for the Collector } \\
\text { Well. } \\
\text { End caps shall be provided for the cleanout access pipes. } \\
\text { "Valve" section of specifications shall be replaced with new section which } \\
\text { includes specifications for a hose bib, and ball valves. Adds six valves to the } \\
\text { valve schedule. } \\
\text { Supply additional butterfly valves to allow recirculation of leachate. } \\
\text { Supply two turbine meters to allow determination of the quantity of discharge } \\
\text { from each the primary and secondary sumps. } \\
\text { Mount a high level limit switch on the liquid level gauge. }\end{array}$ \\
\hline W-025-018 & $\begin{array}{l}\text { Changed Design Drawings to incorporate valves, meters, piping, and switch } \\
\text { indicated by ECN } \# 17 \text { (W-025-017). }\end{array}$ \\
\hline W-025-019 & $\begin{array}{l}\text { Modified Acceptance Test Procedure. Major changes included testing spare } \\
\text { pumps at the ground surface, testing flowmeters, and testing control panel } \\
\text { heaters. }\end{array}$ \\
\hline$W-025-020$ & $\begin{array}{l}\text { Heat trace cable can be either } 120 \text { volt or } 240 \text { volt. A } 30 \text {-Amp, two pole circuit } \\
\text { breaker shall be used for } 240 \text { volt heat trace cable. } \\
\text { Provide control panel heaters to maintain temperature within operating range of } \\
\text { components during cold weather. }\end{array}$ \\
\hline $\mathrm{W}-025-021$ & $\begin{array}{l}\text { Lower subgrade elevations of truck staging area and site entrance road to allow } \\
\text { installation of eight inches of top course and four inches of asphalt. }\end{array}$ \\
\hline$W-025-022$ & $\begin{array}{l}\text { Provide electric pump, accessories, and connections to allow removal of liquid } \\
\text { from containment basin under leachate tank. } \\
\text { Provide stairway and railing to enable access to containment basin. }\end{array}$ \\
\hline
\end{tabular}


WHC-SD-W025-RPT-001, Rev. 0

\begin{tabular}{|c|c|}
\hline ECN No. & Description of Change \\
\hline$W-025-023$ & $\begin{array}{l}\text { On Drawings, add heat trace and insulation to piping system components } \\
\text { between leachate tank and valves near crest wall. } \\
\text { Add vacuum release valve to discharge pipe immediately upstream of leachate } \\
\text { tank. } \\
\text { Terminals shall be installed in junction boxes for transducers. } \\
\text { Relay CR7 shall be a time-delay relay. } \\
\text { Photocell on exterior of control building may be deleted if photocells are } \\
\text { provided for each light pole. }\end{array}$ \\
\hline W-025-024 & $\begin{array}{l}\text { Revise Acceptance Test Procedures to incorporate design changes made during } \\
\text { construction. }\end{array}$ \\
\hline W-025-025 & $\begin{array}{l}\text { Revise piping between headwall and leachate temporary storage tank to } \\
\text { incorporate new meters and values. }\end{array}$ \\
\hline$W-025-026$ & $\begin{array}{l}\text { Revise design drawings: } \\
\text { 1. H-2-131587, Sheet 1, Rev. } 2 \\
\text { 2. H-2-131585, Rev. } 2 \\
\text { 3. H-2-131587, Sheet 2, Rev. } 2\end{array}$ \\
\hline$W-025-028$ & $\begin{array}{l}\text { Revise Acceptance Test Procedures to incorporate changes made as a result of } \\
\text { previous ECNs. }\end{array}$ \\
\hline W-025-029 & $\begin{array}{l}\text { Edit design drawings. } \\
\text { Cross reference design drawings between Project W-025 and Project W-025A. } \\
\text { List contractor's submittals on design drawings. } \\
\text { Cross reference design drawings with as-built drawings contained in CQA } \\
\text { report. }\end{array}$ \\
\hline$W-025-031$ & $\begin{array}{l}\text { Remove and replace flowmeters, and modify the piping configuration near the } \\
\text { crest wall. }\end{array}$ \\
\hline $\mathrm{W}-025-032$ & $\begin{array}{l}\text { Edit Acceptance Test Procedures to account for the changes in the sizes of the } \\
\text { flowmeters. }\end{array}$ \\
\hline
\end{tabular}




\subsection{PRECONSTRUCTION TESTING AND CONFORMANCE TESTING}

The Quality Assurance Program Plan and the CQA Plan required preconstruction testing of the soil materials and conformance testing of the geosynthetic materials proposed for use on this project. Testing of the proposed materials was performed to verify compliance with the project specifications. The frequencies and results of the preconstruction and conformance tests are presented below.

\subsection{EXCAVATION AND BACKFILE}

During the excavation of Project W-025, GCS personnel visually classified the excavated soils at least once per shift, as specified. Two general soil groups: silty fine sand (eolian sand); and gravelly sand to sandy gravel, were segregated and stockpiled as the excavation progressed. The eolian sand was eventually used as a component in the admixture for the soil liner, as a general fill material, and as an operations layer material. The gravelly soil was eventually used as a general fill material and as an operations layer material.

Approximately 4,500 cubic yards of the eolian sand and gravelly sand materials were used for backfilling in and around the landfill excavation. Three samples of these soils were tested by GCS personnel in the onsite soils laboratory to evaluate the materials. The material evaluation testing frequency is summarized in Table 3.

Table 3. Excavation \& Backfill Materials Evaluation - Preconstruction Test Summary.

\begin{tabular}{|c|c|c|c|}
\hline $\begin{array}{c}\text { MINIMUM } \\
\text { FREQUENCY }\end{array}$ & TESTS & $\begin{array}{c}\text { TESTS } \\
\text { REQUIRED }\end{array}$ & $\begin{array}{c}\text { TESTS } \\
\text { PERFORMED }\end{array}$ \\
\hline \hline $\begin{array}{c}\text { Excavation } \\
\begin{array}{c}\text { One Test } \\
\text { Per } \\
\text { Shift }\end{array}\end{array}$ & Visual Classification (ASTM D2487) & 11 & 13 \\
\hline $\begin{array}{c}\text { Backfill } \\
\text { One Test } \\
\text { Per }\end{array}$ & $\begin{array}{l}\text { Modified Proctor (ASTM D1557) } \\
\text { Hydrometer and Sieve (ASTM D422) } \\
\text { Atterberg Limits (ASTM D4318) }\end{array}$ & 3 & $3^{*}$ \\
\hline * ${ }^{3}$ & The tested soils were non-plastic, so Atterberg Limits tests were not possible. \\
\hline
\end{tabular}

The specifications required that the proposed backfill soils conform to any one of the following Unified Soils Classification System classifications:

- GP - Poorly graded gravel

- GM - Silty gravel

- GC - Clayey gravel 


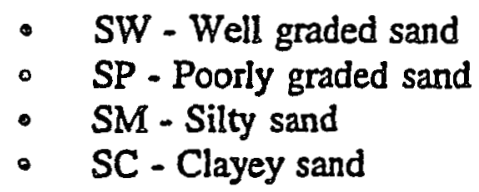

The results of the preconstruction materials evaluations indicated that the tested samples met the requirements of the specifications for both backfill materials and operations layer materials. The results of these tests are presented in Appendix D-1.

\subsection{SOIL LINER}

Approximately 20,000 cubic yards of soil liner material were prepared between October 4, 1993 and November 15, 1993 for use in the Project W-025 test fills, secondary soil liner, and primary soil liner. The soil liner material consisted of an admixture (admix) of eolian sand stockpiled during landfill excavation and imported bentonite. A nominal bentonite content of 12 percent with an allowable range of 11 percent to 14 percent (by weight) was required by the project specifications. American Colloid Company of Upton, Wyoming, supplied the bentonite used in the admix. Prior to mixing, preconstruction testing of the admix components was required.

\subsubsection{Eolian Sand}

Two sets of grain-size determinations were performed on samples of eolian sand before the sand was mixed with bentonite. During the excavation of Project W-025, the eolian sand was sampled and tested as the material was stockpiled. This first set of grain-size determinations was performed to verify that the stockpiled eolian sand was suitable for use as soil liner material, backfill material, or operations layer material. Just prior to mixing the stockpiled eolian sand with bentonite, a second set of grain-size determinations was performed. The second set of tests was performed to determine the percentage of fines (minus \#200-sieve particles) in each sample. This percentage of fines in the sand was used, along with the percentage of fines in the sand-bentonite admix, to determine the bentonite content of the admix. Less than 20,000 cubic yards of eolian sand were admixed with bentonite, and the tests performed on the eolian sand resulted in an approximate frequency of one test for every 500 cubic yards of eolian sand used in the admix, exceeding the specified requirement of one test for every 1,000 cubic yards of eolian sand used. The frequency of the preconstruction tests performed on the eolian sand is summarized in Table 4.

Table 4. Eolian Sand Preconstruction Test Summary.

\begin{tabular}{|c|c|c|c|}
\hline $\begin{array}{c}\text { MINIMUM } \\
\text { EREQUENCY }\end{array}$ & TESTS & $\begin{array}{c}\text { TESTS } \\
\text { REQUIRED }\end{array}$ & $\begin{array}{c}\text { TESTS } \\
\text { PERFORMED }\end{array}$ \\
\hline $\begin{array}{c}\text { One Test } \\
\text { Per } \\
1,000 \mathrm{yd}^{3}\end{array}$ & Particle Size (ASTM D422) & 20 & 40 \\
\hline
\end{tabular}

The tests on the eolian sand were performed by GCS personnel in the onsite soils laboratory. The results of these tests are presented in Appendix D-2 and show that the tested samples met the project specifications. 
WHC-SD-W025-RPT-001, Rev. 0

\subsubsection{Bentonite}

As bentonite was delivered to the site, samples of the bentonite were periodically tested to verify that the bentonite's properties were consistent with the specified properties for 91-barrel yield bentonite. A total of 3,743.3 tons of bentonite were delivered to the jobsite, and nine samples were tested, resulting in an approximate frequency of one preconstruction test for every 416 tons of bentonite delivered to the site. This testing frequency exceeded the required frequency of one test for every 500 tons of bentonite delivered. A summary of tests performed on the bentonite is presented in Table 5.

Table 5. Bentonite Preconstruction Test Summary.

\begin{tabular}{|c|c|c|c|}
\hline $\begin{array}{l}\text { MDNMUM } \\
\text { FREQUENCY }\end{array}$ & TESTS & $\begin{array}{c}\text { TESTS } \\
\text { REQUIRED }\end{array}$ & $\begin{array}{c}\text { TESTS } \\
\text { PERFORMED }\end{array}$ \\
\hline $\begin{array}{l}\text { One Test } \\
\text { Per } \\
500 \text { Tons }\end{array}$ & $\begin{array}{l}\text { Fines Content (API 13A, Sec 4) } \\
\text { Filtration (API 13A, Sec 4) } \\
\text { Viscosity (API RP 13B, Sec 2.3)* } \\
\text { Free Swell (USP-NF-XVI) }\end{array}$ & 8 & 9 \\
\hline \multicolumn{4}{|c|}{$\begin{array}{l}\text { Specifications indicated API } 13 \mathrm{~A}, \mathrm{Sec} 4 \text { (Direct indicating viscometer). American } \\
\text { Colloid established a correlation between the direct indicating viscometer and the Marsh } \\
\text { funnel viscosity. Therefore, API RP 13B, Sec } 2.3 \text { (Marsh funnel) was used to determine } \\
\text { the viscosity. A copy of the correlation between the two methods of viscosity } \\
\text { measurement is included in Appendix D-3.2. }\end{array}$} \\
\hline
\end{tabular}

The bentonite preconstruction tests were performed by GCS personnel in the onsite soils laboratory. The results of the tests indicated that the tested bentonite samples met the requirements of the specifications. A summary of the bentonite test results is presented in Appendix D-3.1.

The specifications required the manufacturer to submit a bentonite yield certificate for every 500 tons of bentonite delivered to the site. Thirty-nine rail cars delivered a total of approximately 3,743 tons of bentonite, and the supplier submitted 31 certificates of analysis, resulting in an approximate frequency of one certificate of analysis for every 121 tons of bentonite delivered. GCS reviewed the certificates and verified that the bentonite conformed to the specifications. Copies of the certificates of analysis are included in Appendix D-3.3.

\subsubsection{Admix}

After the completion of the mixing of bentonite, eolian sand, and water, but prior to the placement of the material, preconstruction tests were performed on the admix. The specifications required an admix moisture content of 1 percent to 5 percent above the optimum moisture content, a bentonite content of 11 percent to 14 percent, and a hydraulic conductivity (permeability) of less than or equal to $1 \times 10^{-7} \mathrm{~cm} / \mathrm{s}$.

Preconstruction moisture content tests were performed at an approximate frequency of one test for every 513 cubic yards of admix produced, exceeding the specification's requirement of one moisture test for every 1,000 cubic yards of admix produced. The admix was tested for bentonite 
content at an approximate frequency of one test for every 833 cubic yards of admix produced, exceeding the specification's requirement of one bentonite content test for every 1,000 cubic yards of admix produced.

A suite of three tests, including a laboratory recompacted hydraulic conductivity test, a Standard Proctor moisture-density test, and an Atterberg Limits test was performed on each of six samples of the admixture, resulting in an approximate testing frequency of one suite of tests for every 3,333 cubic yards of admix produced, and exceeding the specification's requirement of one suite of these tests for every 5,000 cubic yards of admix produced. The recompacted hydraulic conductivity tests were performed by GAI's soils laboratory, located in Redmond, Washington. Standard Proctor and Atterberg Limits preconstruction tests were performed in GCS's onsite soils laboratory. The preconstruction testing frequency for the admix is summarized in Table 6.

Table 6. Admix Preconstruction Test Summary.

\begin{tabular}{|c|c|c|c|}
\hline $\begin{array}{c}\text { MINIMUM } \\
\text { FREQUENCY }\end{array}$ & TESTS & $\begin{array}{c}\text { TESTS } \\
\text { REQUIRED }\end{array}$ & $\begin{array}{c}\text { TESTS } \\
\text { PERFORMED }\end{array}$ \\
\hline \hline $\begin{array}{c}\text { One Test } \\
\text { Per } \\
1,000 \mathrm{yd}^{3}\end{array}$ & Moisture Content (ASTM D2216) & 20 & 39 \\
\hline $\begin{array}{c}\text { One Test } \\
\text { Per } \\
5,000 \mathrm{yd}^{3}\end{array}$ & $\begin{array}{c}\text { Bentonite Content (ASTM D422) } \\
\text { Atterberg Limits (ASTM D498) } \\
\text { Permeability (ASTM D5084) }\end{array}$ & 20 & 24 \\
\hline
\end{tabular}

The preconstruction test results indicated that the tested samples met the requirements of the project specifications. The results of these conformance tests are presented in Appendix D-4.

Preconstruction laboratory testing indicated that the designed admixture; when properly mixed, moisture conditioned, placed, and compacted; would meet the project permeability requirement of less than or equal to $1 \times 10^{7} \mathrm{~cm} / \mathrm{s}$. CQA tests on test fills and a Sealed Double-Ring Infiltrometer (SDR) test demonstrated that the contractor's equipment and methods were capable of preparing, placing, and. compacting the admix such that the specified permeability could be achieved. The results of tests performed on the test fills, and the results of the SDRI test are presented in GCS's report Quality Assurance Observation and Testing, Soil Liner Test Fill and SDRI Test, Radioactive Mixed Waste Land Disposal Facility, Non-Drag-Off, Project W-025, dated May, 1994, and presented in Appendix $U$.

\subsection{GRANULAR DRANAGE MATERIAL}

Two types of granular drainage media were used in the drainage layers for both the secondary and primary leachate collection systems. Drainage gravel, generally a sandy pea-gravel, was used on the floor of each of the systems; and sump gravel, generally a clean, medium-sized gravel, was used in the sump of each system. Prior to the use of these materials, samples were obtained from the supplier, ACME Materials and Construction Co., of Richland, Washington, and preconstruction tests were performed. Preconstruction testing of the drainage gravel and sump gravel included a suite of 
three tests: Sieve analysis; permeability; and slake durability. The suite of preconstruction tests was performed on four samples of the drainage gravel and one sample of the sump gravel, resulting in preconstruction testing frequencies of one suite of tests for every 450 cubic yards of drainage gravel, and one suite of tests for every 250 cubic yards of sump gravel. These preconstruction testing frequencies exceeded the specification's required testing frequency of one suite of tests for every 500 cubic yards of gravel. The sieve analyses were performed in GCS's onsite soils laboratory.

Permeability tests were performed by GAI's soils laboratories located in Redmond, Washington, and Mt. Laurel, New Jersey. A simulated leachate was used during the slake durability tests, which were performed by GAI's soils laboratory located in Redmond, Washington. The preconstruction testing frequencies for the granular drainage materials are summarized in Table 7.

Table 7. Granular Drainage Material Preconstruction Test Summary.

\begin{tabular}{|c|c|c|c|}
\hline $\begin{array}{c}\text { MINIMUM } \\
\text { FREQUENCY }\end{array}$ & TESTS & $\begin{array}{c}\text { TESTS } \\
\text { REQUIRED }\end{array}$ & $\begin{array}{c}\text { TESTS } \\
\text { PERFORMED }\end{array}$ \\
\hline \hline Drainage Gravel & $\begin{array}{c}\text { Particle Size (ASTM C136) } \\
\text { Permeability (ASTM D2434) } \\
\begin{array}{c}\text { One Test } \\
\text { Per } \\
500 \mathrm{yd}^{3}\end{array}\end{array}$ & 4 & 4 \\
\hline $\begin{array}{c}\text { Slake Durability (ASTM D4644) } \\
\text { Sump Gravel } \\
\begin{array}{c}\text { One Test } \\
\text { Per } \\
500 \mathrm{yd}^{3}\end{array}\end{array}$ & $\begin{array}{c}\text { Particle Size (ASTM C136) } \\
\text { Permeability (ASTM D2434) } \\
\text { Slake Durability (ASTM D4644) }\end{array}$ & 1 & 1 \\
\hline
\end{tabular}

The preconstruction test results indicated that the sampled granular drainage materials met the requirements of the specifications. The results of these preconstruction tests are presented in Appendix D-5.

\subsection{HDPE GEOMEMBRANE}

The geomembrane liner delivered to the site was high density polyethylene (HDPE), manufactured by SLT North America, Inc. (SLT), of Conroe, Texas. Three types of this material were delivered between October 29, 1993, and January 12, 1994, and included the following:

- Textured, 60-mil thick, HDPE geomembrane liner (for use on the slopes and in the sump of the landfill)

- Smooth, 60-mil thick, HDPE geomembrane liner (for use on the floor of the landfill)

- Smooth, 80-mil thick, HDPE geomembrane liner (for lining beneath the truck unloading area and lining the concrete containment areas around the leachate tank)

During the manufacture of the above HDPE liner materials for Project W-025, a GCS representative visited SLT's manufacturing plant on four occasions to review the manufacturing 
process, quality control, laboratory facilities, and testing procedures. During one visit, GCS's representative observed an excessive amount of recycled regrind being added to the resin, and brought this to the attention of the manufacturer. The manufacturer opted to prepare a new batch of resin for material production for Project W-025. The manufacturer certified that no more than two percent recycled regrind had been added to the resin for any of the HDPE liner material produced for this project. A Surveillance Inspection Report was written by the GCS representative for each of the four manufacturing plant visits. Copies of these reports are included in Appendix E-1.

Approximately 362,000 square feet of textured, 60-mil thick, HDPE geomembrane liner; 33,500 square feet of smooth, 60-mil thick HDPE liner; and 25,200 square feet of smooth, 80-mil thick HDPE liner were delivered to the site. These materials were sampled and tested by GCS for conformance with the project specifications. Ten rolls of the textured material were sampled and tested, resulting in an approximate frequency of one conformance test for every 36,200 square feet of textured HDPE geomembrane liner delivered to the site. One 60-mil thick roll of the smooth, HDPE liner material was sampled and tested, resulting in an approximate frequency of one conformance test for every 33,500 square feet of material delivered. Three samples, one from each resin lot delivered to the site, of the 80-mil thick, smooth, HDPE liner material were sampled and tested, resulting in an approximate frequency of one conformance test for every 8,400 square feet of material delivered. The conformance testing frequencies met or exceeded the specifications' requirement of a minimum of one conformance test for every 50,000 square feet of material delivered to the site, or a minimum of one conformance sample for each lot of material delivered.

Additional conformance testing of the material properties of the geomembrane included fingerprinting analyses and direct shear testing. Fingerprinting analyses, including tests of the material's specific gravity; melt index, and crystallinity were performed on two samples of the geomembrane. Two direct shear tests were performed on samples of the textured HDPE liner and the soil liner material as required by the specifications. A summary of the HDPE liner conformance testing frequency is presented in Table 8.

Table 8. HDPE Geomembrane Conformance Test Summary.

\begin{tabular}{|c|c|c|c|}
\hline $\begin{array}{l}\text { MINIMUM } \\
\text { FREQUENCY }\end{array}$ & TESTS & $\begin{array}{c}\text { TESTS } \\
\text { REQUIRED }\end{array}$ & $\begin{array}{c}\text { TESTS } \\
\text { PERFORMED }\end{array}$ \\
\hline $\begin{array}{l}\text { Textured } 60-\mathrm{mil} \\
\text { One Test per } \\
50,000 \mathrm{ft}^{2} \text { or } \\
\text { One test/Lot }\end{array}$ & $\begin{array}{l}\text { Sp. Gravity (ASTM D792 or D1505) } \\
\text { Carbon Black Content (ASTM D1603) } \\
\text { Carbon Black Dispersion (ASTM } \\
\text { D1603) } \\
\text { Thickness (ASTM D1593) } \\
\text { Tensile Properties (ASTM D638) }\end{array}$ & 10 & 10 \\
\hline Two Tests Total & $\begin{array}{l}\text { Friction Angle of Interface with Admix } \\
\text { (Direct Shear Method) }\end{array}$ & 2 & 2 \\
\hline
\end{tabular}


WHC-SD-W025-RPT-001, Rev. 0

\begin{tabular}{|c|c|c|c|}
\hline $\begin{array}{l}\text { MINIMUM } \\
\text { FREQUENCY }\end{array}$ & TESTS & $\begin{array}{c}\text { TESTS } \\
\text { REQUIRED }\end{array}$ & $\begin{array}{c}\text { TESTS } \\
\text { PERFORMED }\end{array}$ \\
\hline $\begin{array}{l}\text { Smooth } 60 \text {-mil } \\
\text { One Test per } \\
50,000 \mathrm{ft}^{2} \text { or } \\
\text { One test/Lot }\end{array}$ & $\begin{array}{l}\text { Sp. Gravity (ASTM D792 or D1505) } \\
\text { Carbon Black Content (ASTM D1603) } \\
\text { Carbon Black Dispersion (ASTM } \\
\text { D1603) } \\
\text { Thickness (ASTM D1593) } \\
\text { Tensile Properties (ASTM D638) }\end{array}$ & 1 & 1 \\
\hline $\begin{array}{l}\text { Smooth } 80 \text {-mil } \\
\text { One Test per } \\
50,000 \mathrm{ft}^{2} \text { or } \\
\text { One test } / \text { Lot }\end{array}$ & $\begin{array}{l}\text { Sp. Gravity (ASTM D792 or D1505) } \\
\text { Carbon Black Content (ASTM D1603) } \\
\text { Carbon Black Dispersion (ASTM } \\
\text { D1603) } \\
\text { Thickness (ASTM D1593) } \\
\text { Tensile Properties (ASTM D638) }\end{array}$ & 3 & 3 \\
\hline
\end{tabular}

The conformance samples were tested by GCS's geosynthetics laboratory located in Duluth, Georgia and the direct shear tests were performed at Golder Associates Ltd. soils laboratory located in Calgary, Alberta. The results of the direct shear tests were reviewed by GCS CQA personnel and the GAI Design Engineer. The direct shear test results met the requirements of the specifications. The remainder of the conformance test results were reviewed by GCS for compliance with the project specifications. With only one exception, the tested material met the requirements of the specifications. One roll of smooth, 80-mil thick, HDPE geomembrane liner from a different lot than those used was rejected because the conformance test results indicated that the density and carbon black content were lower than specified. This roll was rejected for use in Project W-025. The results of the conformance tests are presented in Appendix E-2. An inventory of the rolls of HDPE geomembrane liner delivered to the site is also included in Appendix E-2.

The manufacturer of the geomembrane (SLT North America) provided the following documentation for the HDPE liner materials delivered to the site:

- Fingerprinting analytical data

- A report, issued by Geosyntec Consultants, of Atlanta, Georgia, which presents the results of friction angle tests

- Certification that HDPE liner materials meet the requirements of the specifications

- Quality Control (QC) Roll Test Data Report for each roll of HDPE geomembrane liner

- Certificate of Analysis for each resin lot used to produce the delivered rolls

- Certification that the resin contains no reclaimed polymer and less than two percent of recycled trim 
WHC-SD-W025-RPT-001, Rev. 0

- Certification that the materials passed the 2000-hour Environmental Stress Crack Resistance test

- Certification that the materials meet the specification's requirements for Resistance to Soil Burial

- Certification that the welding extrudate (rod and resin) is manufactured from the same base resin type and will exhibit similar physical characteristics as the geomembrane

- Certification that the material contains only virgin polyethylene resin and carbon black

- Certification of the consistency of the material

GCS personnel and/or GAI Design Engineers reviewed the above documents for conformance to the project specifications. Copies of these certificates are included in Appendix E-3.

\subsection{GEONET}

The HDPE geonet delivered to the site was TENAX CE-2, manufactured by TENAX Corporation, of Jessup, Maryland. Approximately 50,700 square feet of geonet were delivered to the site on October 13, 1993. A total of two rolls were sampled and tested for conformance with the project specifications, resulting in an approximate testing frequency of one conformance test for every 25,350 square feet of material delivered to the site, exceeding the specification's requirement of one conformance test for every 50,000 square feet of geonet delivered to the site. The geonet conformance testing frequency is summarized in Table 9.

Table 9. Geonet Conformance Test Summary.

\begin{tabular}{|c|c|c|c|}
\hline $\begin{array}{l}\text { MINIMUM } \\
\text { FREQỤENCY }\end{array}$ & TESTS & $\begin{array}{c}\text { TESTS } \\
\text { REQUIRED }\end{array}$ & $\begin{array}{c}\text { TESTS } \\
\text { PERFORMED }\end{array}$ \\
\hline $\begin{array}{l}\text { One Test per } \\
50,000 \mathrm{ft}^{2}\end{array}$ & $\begin{array}{l}\text { Specific Gravity (ASTM D792 or } \\
\text { D1505) } \\
\text { Thickness (ASTM D1777) } \\
\text { Transmissivity (ASTM D4716) } \\
\text { Mass per Unit Area (ASTM D3776) }\end{array}$ & 2 & 2 \\
\hline
\end{tabular}

The conformance samples were tested by GCS's geosynthetics laboratory in Duluth, Georgia. GCS reviewed the conformance test results for compliance with the project specifications. The conformance tests indicated that the tested samples met the requirements of the project specifications.

- The conformance test results are given in Appendix F-1. An inventory of the rolls of geonet delivered to the site is also included in Appendix F-1.

The manufacturer of the geonet (TENAX Corporation) provided certification and quality control $(\mathrm{QC})$ data for the material delivered to the site. GCS reviewed these documents for compliance with the specifications. With one exception, the documents indicated compliance with the specifications. The manufacturer's QC tests indicated that the specific gravity of the geonet was 
0.946 , whereas GCS's conformance tests indicated a geonet specific gravity of 0.940 to 0.941 . The specifications require a geonet specific gravity less than that of the 60-mil thick HDPE geomembrane upon which the geonet was to be installed. GCS's conformance tests on the 60-mil geomembrane indicated specific gravities ranging from 0.942 to 0.945 , and the geomembrane manufacturer's (SLT's) QC tests on the 60-mil geomembrane indicated specific gravities ranging from 0.942 to 0.947. GCS's conformance tests on the geonet indicated that the specific gravity of the geonet met the requirements of the specifications, whereas TENAX'es QC tests indicated specific gravity of the geonet was greater than the specific gravity of the geomembrane. TENAX wrote a letter which clarified their position that interlaboratory precision and the accuracy of the standard test method were the most probable causes for the discrepancies. TENAX also indicated that valid comparisons of the specific gravities of the geonet and geomembrane can only be made if both materials are tested by the same testing laboratory. GCS personnel and GAI design personnel reviewed the data and the letter from TENAX, and determined that, based on the data from a common laboratory, the geonet's specific gravity met the requirements of the specifications. The manufacturer's quality control documents and the letter regarding the geonets' specific gravity are included in Appendix F-2.

\subsection{GEOTEXTUL}

The geotextile materials delivered to the site were Nicolon $S 700$ (seven-oz/yd fabric) and Nicolon $S 1600$ (16-oz/yd $\mathrm{d}^{2}$ fabric) manufactured by Nicolon Corporation, of Norcross, Georgia. The geotextiles are non-woven, needle-punched, polypropylene. Approximately 72,000 square feet of S700 geotextile were delivered to the site on October 4, 1993, and on January 12, 1994, and 112,500 square feet of $S 1600$ geotextile were delivered to the site on October 4, 1993. Two rolls of S700 fabric, and three rolls of the $S 1600$ fabric were sampled and tested for conformance with the project specifications, resulting in approximate frequencies of one conformance test for every 36,000 square feet of seven-ounce material, and one conformance test for every 37,500 square feet of the 16-ounce material. These conformance testing frequencies exceed the specified minimum frequency of one conformance test for every 50,000 square feet of material. The geotextile conformance testing frequencies are summarized in Table 10.

Table 10. Geotextile Conformance Test Summary.

\begin{tabular}{|l|l|c|c|}
\hline \multicolumn{1}{|c|}{$\begin{array}{c}\text { MINIMUM } \\
\text { FREQUENCY }\end{array}$} & \multicolumn{1}{|c|}{ TESTS } & \multicolumn{1}{c|}{$\begin{array}{c}\text { TESTS } \\
\text { REQUIRED }\end{array}$} & $\begin{array}{c}\text { TESTS } \\
\text { PERFORMED }\end{array}$ \\
\hline \hline Seven-oz/yd & & \\
\hline Fabric & $\begin{array}{l}\text { Mass per Unit Area (ASTM D3776) } \\
\text { Grab Strength (ASTM D4632) } \\
\text { Tear Strength (ASTM D4533) } \\
\text { Burst Strength (ASTM D3786) }\end{array}$ & 2 & 2 \\
$\begin{array}{l}\text { One Test per } \\
\text { 50,000 } \mathrm{ft}^{2}\end{array}$ & $\begin{array}{l}\text { Puncture Strength (ASTM D4833) } \\
\text { Thickness (ASTM D1777) } \\
\text { Permittivity (ASTM D4491) } \\
\text { AOS (ASTM D4751) }\end{array}$ & 2 & \\
\hline
\end{tabular}


WHC-SD-W025-RPT-001, Rev. 0

\begin{tabular}{|l|l|c|c|}
\hline \multicolumn{1}{|c|}{$\begin{array}{c}\text { MINIMUM } \\
\text { FREQUENCX }\end{array}$} & \multicolumn{1}{|c|}{ TESTS } & $\begin{array}{c}\text { TESTS } \\
\text { REQUIRED }\end{array}$ & $\begin{array}{c}\text { TESTS } \\
\text { PERFORMED }\end{array}$ \\
\hline \hline 16-02/yd ${ }^{2}$ Fabric & $\begin{array}{l}\text { Mass per Unit Area (ASTM D3776) } \\
\text { Grab Strength (ASTM D4632) } \\
\text { Tear Strength (ASTM D4533) }\end{array}$ & 3 & 3 \\
One Test per & $\begin{array}{l}\text { Burst Strength (ASTM D3786) } \\
\text { Puncture Strength (ASTM D4833) } \\
\text { Thickness (ASTM D1777) } \\
\text { Permittivity (ASTM D4491) } \\
\text { AOS (ASTM D4751) }\end{array}$ & 3 & \\
\hline
\end{tabular}

The conformance samples were tested by GCS's geosynthetics laboratory in Duluth, Georgia. GCS reviewed the conformance test results for compliance with the specifications. The conformance test results indicated that the tested samples of the materials delivered to the site on October 4, 1993, including 48,000 square feet of $\$ 700$ fabric and 112,500 square feet of $\$ 1600$ fabric, met the requirements of the specifications. These materials were designated for use inside the landfill:

A conformance test result for a sample of the 24,000 square feet of 5700 fabric delivered to the site on January 12,1994, indicated that the sample did not meet the specification's minimum requirement for thickness. This material was rejected for use in Project W-025 where seven-oz/yd ${ }^{2}$

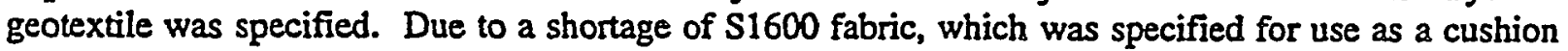
layer on the geomembrane liner in the truck unloading area, three layers of the deficient S700 fabric were designated for use as replacement material for the S1600 fabric in the truck unloading area by the Design Engineer (Appendix C-3). The use of S700 fabric was allowed in the truck unloading area because three layers of the deficient $S 700$ fabric would provide a thicker cushion on the geomembrane than would a single layer of the $\$ 1600$ fabric.

The conformance, test results for the geotextiles are presented in Appendix G-1. An inventory of the rolls of geotextile delivered to the site is also included in Appendix G-1. In addition to reviewing conformance test results, GCS personnel reviewed manufacturer's certifications and QC data for compliance with the specifications for the geotextiles. The manufacturer's certification and quality control documents for the delivered materials are included in Appendix G-2. Additionally, GCS reviewed the manufacturer's certification of the thread used for sewing the geotextiles. This document is also included in Appendix G-2.

\subsection{GEOCOMPOSITE}

The geocomposite delivered to the site was SLT's GS-228. The geocomposite consisted of Nicolon $\$ 700$ geotextile thermally bonded to the top and bottom of TENAX CE-2 geonet. Approximately 355,700 square feet of GS-228 geocomposite were delivered to the site between November 8, 1993 and January 12, 1994. Eight rolls of GS-228 were sampled, and the geotextile component was tested for Apparent Opening Size (AOS) to determine conformance with the project specifications. The resulting testing frequency was approximately one conformance test for every 44,500 square feet of geocomposite delivered to the site, exceeding the specification's requirement of one conformance test for every 50,000 square feet of geocomposite. 
WHC-SD-W025-RPT-001, Rev. 0

In addition to the above conformance testing of the geocomposite, two friction angle tests, using the direct shear method, were performed on the interface between the geocomposite material and the textured HDPE liner. Two friction angle tests were also performed on the interface between the geocomposite and the operations layer soil. The geocomposite conformance testing frequencies are summarized in Table 11.

Table 11. Geocomposite Conformance Test Summary.

\begin{tabular}{|l|l|c|c|}
\hline \multicolumn{1}{|c|}{$\begin{array}{c}\text { MINIMUM } \\
\text { FREQUENCY }\end{array}$} & $\begin{array}{c}\text { TESTS } \\
\text { REQUIRED }\end{array}$ & $\begin{array}{c}\text { TESTS } \\
\text { PERFORMED }\end{array}$ \\
\hline $\begin{array}{l}\text { One Test per } \\
50,000 \mathrm{ft}^{2}\end{array}$ & AOS (ASTM D4751) & 8 & 8 \\
\hline Two Tests Total & $\begin{array}{l}\text { Friction Angle of Interface } \\
\text { with Textured HDPE } \\
\text { (Direct Shear Method) }\end{array}$ & 2 & 2 \\
\hline Two Tests Total & $\begin{array}{l}\text { Friction Angle of Interface } \\
\text { with Operations Layer } \\
\text { (Direct Shear Method) }\end{array}$ & 2 & 2 \\
\hline
\end{tabular}

The AOS conformance tests were performed by GCS's geosynthetics laboratory, located in Duluth, Georgia. The direct shear tests were performed by Golder Associates Ltd. soils laboratory, located in Calgary, Alberta. The test results were reviewed for conformance to the specifications by GCS personnel and the GAI Design Engineer, and are presented in Appendix-H-1. The tested samples met the project specifications. An inventory of the rolls of geocomposite delivered to the site is included in Appendix $\mathrm{H}-1$.

In addition to reviewing conformance test results, GCS reviewed manufacturer's certification and QC test results for the geocomposite. The manufacturer's informion indicated that the first 50 delivered rolls of geocomposite were manufacturered using a geotextile that did not meet the requirements of the project specifications. These 50 rolls of geocomposite were rejected. The manufacturer then produced geocomposite composed of the specified materials, and shipped the new geocomposite to the jobsite. The manufacturer's QC test data for the new material indicated that several rolls failed the manufacturer's QC tests. Assuming that the manufacturer tested samples from the trailing edge of each roll of material, and that the trailing edge of one roll is the same as the leading edge of the next consecutively produced roll of material, deficient material on the trailing edge (outside) of a roll of material would also indicate deficient material on the leading edge (inside) of the next consecutively produced roll. Therefore, GCS determined that the failing tests affected a total of eight rolls of the new material. All eight of these rolls of geocomposite were also rejected by GCS for use in Project W-025, and the contractor removed all of the rejected rolls from the jobsite. The manufacturer's QC test data and certification of the geocomposite are included in Appendix H-2.

Delhur arranged for the performance of two friction angle tests each for the interface between the geocomposite and the textured HDPE liner, and for the interface between the geocomposite and the operations layer material. GCS CQA personnel and GAI Design personnel reviewed the contractor's test results for conformance to the requirements of the specifications. The results of the contractor's friction angle tests for the geocomposite/textured geomembrane and 
WHC-SD-W025-RPT-001, Rev. 0

interfaces are included in Appendix E-3 with the friction angle data for the tests performed on the soil liner/textured geomembrane interface.

\subsection{HDPE PIPE AND STAINLESS STEEL PIPE}

The HDPE pipe delivered to the site was produced by Polaris Pipe Co., of Van Nuys, California, and was supplied by Industrial Plastics, Inc., of Washougal, Washington. The specifications required no QA conformance tests for these materials. However, the contractor was required to submit quality control certificates for the pipe, and qualifications of the pipe joining personnel. GCS reviewed these documents. The documents indicated that the pipe conforms to ASTM D1248, Type III, Class C, Category 5, Grade 34; cell classification 345434C per ASTM D3350; and that the proposed joining personnels' qualifications met the requirements of the specifications. All onsite joining of the HDPE pipe was performed by certified personnel. Copies of the manufacturer's certificates and the qualifications of the pipe joining personnel are included in Appendix I-1.

The stainless steel pipe delivered to the site was Schedule 40 Type 316 , as required by the specifications. No QA conformance tests were required for the stainless steel pipe. However, manufacturers' certifications for the pipe are included in Appendix I-2.

\subsection{TOP COURSE}

Top course material for Project W-025 was supplied by ACME Materials and Construction Co., of Richland, Washington. The top course was used as a road-base material on the access ramp, the perimeter road, the truck staging area, the truck unloading area, and the site entrance. Prior to construction, GCS personnel obtained samples of the top course material from the supplier, and performed the required preconstruction tests. The top course preconstruction tests are summarized in Table 12.

Table 12. Top Course Preconstruction Testing Summary.

\begin{tabular}{|l|l|c|c|}
\hline \multicolumn{1}{|c|}{$\begin{array}{c}\text { MINIMUM } \\
\text { FREQUENCY }\end{array}$} & TESTS & $\begin{array}{c}\text { TESTS } \\
\text { REQUIRED }\end{array}$ & $\begin{array}{c}\text { TESTS } \\
\text { PERFORMED }\end{array}$ \\
\hline $\begin{array}{l}\text { One Test } \\
\text { Prior to } \\
\text { Construction }\end{array}$ & $\begin{array}{l}\text { Particle Size (WSDOT 1991 } \\
\text { Sec. 9-03.9.3) } \\
\text { Mod. Proctor (ASTM D1557) }\end{array}$ & 1 & 1 \\
\hline
\end{tabular}

The results of the preconstruction tests on the top course material indicated that the tested sample met the requirements of the specifications. The test results are presented in Appendix D-6.

\subsection{OPERATIONS LAYER}

The operations layer materials for Project W-025 were segregated and stockpiled as excavation of the facility progressed. Eolian sand was stockpiled, and designated for use as operations layer material on the slopes of the facility, for use in the admix, and for use as general fill material. Sandy gravel to gravelly sand was stockpiled, and designated for use as operations layer material on the floor 
of the facility, and for use as general fill material. GCS personnel performed 21 preconstruction sieve analyses and hydrometer tests on the eolian sand, and two preconstruction sieve analyses and hydrometer tests on the gravelly materials as they were stockpiled. Based on the Design Engineer's estimate of 15,690 cubic yards of soil required for use as operations layer material, the 23 preconstruction tests resulted in a frequency of one preconstruction test for every 682 cubic yards of operations layer material. This testing frequency exceeded the specification's requirement of one preconstruction test for every 2,000 cubic yards of operations layer material. The preconstruction tests are summarized Table 13.

Table 13. Operations Layer Preconstruction Testing Summary.

\begin{tabular}{|c|c|c|c|}
\hline $\begin{array}{c}\text { MINIMUM } \\
\text { FREQUENCY }\end{array}$ & TESTS & $\begin{array}{c}\text { TESTS } \\
\text { REQUTRED }\end{array}$ & $\begin{array}{c}\text { TESTS } \\
\text { PERFORMED }\end{array}$ \\
\hline $\begin{array}{l}\text { One Test per } \\
2,000 \mathrm{yd}^{3}\end{array}$ & $\begin{array}{c}\text { Hydrometer and Sieve } \\
\text { (ASTM D422) }\end{array}$ & 8 & 23 \\
\hline
\end{tabular}

The specifications required that the operations layer material meet the specification's requirements for backfill material, previously discussed in Section 2.1 of this report. The results of the preconstruction tests indicated that the tested samples met these requirements. The preconstruction test results for the operations layer material are presented in Appendix D-7. 
WHC-SD-W025-RPT-001, Rev. 0

\subsection{EXCAVATION/SUBGRADE PREPARATION}

Project W- 025 was excavated by Delhur Industries, Inc., of Port Angeles, Washington, in loose to compact eolian sand, and dense to very dense sand and gravel deposits. Delhur began the excavation on September 13, 1993, and completed the excavation on September 25, 1993. The following equipment was used during the excavation and subgrade preparation phases of construction:

- Hitachi EX 1000 Excavator

- 3 International, 50-ton, Payhauler, End-Dump Trucks

- Caterpillar D-9 Bulldozer

- Caterpillar D-5 Bulldozer

- John Deere (JD) 772A Grader

- 3500-Gallon Water Truck

- Ray Go Rascal 410A, Smooth-Drum, Vibratory Compactor

- Caterpillar 988B Loader

\subsection{GEOLOGIC SITE CONDITIONS}

The excavation of the Project W-025 Landfill was monitored to confirm the geological/geotechnical conditions described in the design report and to confirm that the excavated materials were placed in the proper stockpiles. Upon completion of the excavation, the landfill subgrade was geologically mapped. The results of the geological mapping are described below.

\subsubsection{General}

The Project W-025 Landfill is located within the 200 West area of the Hanford Nuclear Reservation. The Hanford Nuclear Reservation lies within the Columbia Plateau geological province. The Columbia Plateau province is defined by the lateral extent of the Columbia River Basalt Group, a sequence of Miocene basaltic lava flows that cover an estimated 63,000 square miles of the states of Washington, Oregon, and Idaho. Large-scale tectonic deformation in the plateau has resulted in the formation of several broad structural and topographic basins. The Hanford Reservation lies within the Pasco Basin.

The Pasco Basin represents a topographic low within the Columbia Plateau. The confluence of the Columbia, Snake, Yakima, and Walla Walla Rivers occurs in the Pasco Basin. Late Pleistocene catastrophic flooding and Holocene eolian processes have created an extensive system of channel bar deposits, flood plains, sand dunes, and loess deposits within the central portion of the basin.

Significant geomorphic features within the central part of the basin include the Cold Creek Bar on which the 200 West area is located, the Central Hanford Sand Plain, and the Cold Creek-Dry Creek Alluvial Plain.

The geology of the Pasco Basin is dominated by a thick sequence of tholeiitic basalt flows collectively known as the Columbia River Basalt Group. These flows erupted during the Miocene Epoch, between 6 to 17 million years before the present. Interbedded with the basalt flows are a series of epiclastic and volcaniclastic sediments generally referred to as the Ellensburg Formation. Overlying the Columbia River Basalt Group within the Pasco Basin are Miocene to Holocene fluvial, glaciofluvial, and eolian sediments. These sediments make up the Miocene-Pliocene Ringold 
WHC-SD-W025-RPT-001, Rev. 0

Formation and the informally named Pleistocene Hanford formation. Unconsolidated, Holocene fluvial sediments, sand dune, and loess are the predominant surficial deposits.

\subsubsection{Geologic Mapping}

The geologic mapping of the Project W-025 excavation was performed by an engineering geologist with GCS who was familiar with the geology of the Hanford region. The field geologic mapping was performed between October 15 and October 28, 1993. The base map for the geologic mapping was Drawing No. H-2-131589, revision 2, "Subgrade Grid Points, RMW Land Disposal Facility, Non-Drag-Off", prepared by Golder Associates Inc., dated May 21, 1993. Stratigraphic contacts were located in the field by measuring directly from marked survey grid points in the Project W-025 excavation. The survey grid points were spaced every 25 feet and allowed for relatively accurate location of stratigraphic contacts in the excavation.

In general, the geologic units were well exposed in the excavation side walls and floor. Minor cleaning of the exposure using a shovel was required in several instances to accurately locate the stratigraphic contacts or to collect representative samples of the geologic units for description.

The geologic map for the Project W-025 excavation is shown in Figure 3 and the cross-sections for the cell are shown on Figure 4.

\subsubsection{Geologic Units Encountered} follows:

The geologic units exposed in the Project W-025 excavation, from youngest to oldest, are as

- Recent Eolian Sand: Quaternary, wind deposited, loose to compact, olive gray, silty, fine sand was encountered in the upper portion of the excavation. The unit had a basal elevation ranging from approximately 691 on the west end to 683 on the east end. A six-inch thick volcanic ash layer was observed interbedded with the eolian sand in the northeast corner of the excavation. The volcanic ash layer ranged in elevation from approximately 691 to 693.

- Glaciofluvial Flood Deposit: Underlying the Recent Eolian Sand was dense to very dense, olive black, gravelly, fine to coarse sand. This, in turn was underlain by dense, moderate olive brown, fine sandy silt, which was underlain by dense to very dense, fine to coarse sand in which the Project W-025 excavation was terminated. The base of the gravelly sand unit ranged from approximately 677 on the west to 672 on the east. The base of the sandy silt unit ranged from approximately 675 on the west to 670 on the east.

\subsection{SUBGRADE PREPARATION AND BACKFULL PLACEMENT}

The subgrade of the Project W-025 excavation was prepared for installing the secondary lining system by grading and recompacting the excavation surfaces. Delhur used Caterpillar D-9 and D-5 bulldozers, and a JD 272A grader to scarify the subgrade to a depth of approximately eight inches. A water truck was used to moisture condition the scarified soils. The subgrade soils were then 
compacted to at least 90 percent of their Modified Proctor maximum dry density (ASTM D1557) using a Ray Go Rascal 410A, smooth-drum, vibratory roller. In a few areas on the lower sideslopes of the excavation, the contractor experienced difficulty achieving the required compaction of clean, sandy gravels. In these areas, Delhur used relatively fine-grained soils as fill material to provide a firm surface upon which the secondary lining system could be installed. The fill materials were moisture conditioned and compacted in the same manner as the subgrade soils.

Field moisture-density tests were performed by GCS personnel on the compacted subgrade using a Troxler Model 3440 Nuclear moisture-density gauge (ASTM D2922 and ASTM D3017). At least one side-by-side rubber-balloon test (ASTM D2167) was performed each day, or once for every 20 Troxler tests per day, to verify the accuracy of the nuclear densometer tests. At the completion of each nuclear moisture-density test, a sample of the soil at the test location was obtained and sealed in a plastic bag for laboratory water content determination using the oven-dried method (ASTM D2216). In areas where the test results indicated that the compaction did not meet the requirements of the specifications, the contractor was required to rework and recompact the soil until passing test results were achieved. The specifications required moisture-density tests on the subgrade at a minimum frequency of one test per 10,000 square feet of surface area. The surface area of the excavation was approximately 160,000 square feet, and 32 moisture-density tests (excluding retests) were performed on the subgrade soils, resulting in an approximate frequency of one moisture-density test for every 5,000 square feet of subgrade surface area. A summary of the rest results is included in Appendix $J$.

An as-built survey of the excavation was performed by Permit Surveying, Inc. A CQA survey was performed by Rogers Surveying; Inc. to verify Permit's survey. GCS reviewed data from both surveys, and concluded that the CQA survey data reasonably verified Permit's data. Permit's survey indicated that all surveyed grid points were within the tolerances required by the project plans and specifications. Rogers' CQA survey data are included in Appendix T-1. Permit's Record Drawing, Subgrade As-Built, Sheet 1 of 6 , is included in Volume 2 of this report.

On the periphery of the excavation, subgrade preparation was required in the footprint of the perimeter road, the truck unloading area, the truck staging area, and the site entrance road. Delhur used similar equipment and methods to compact these subgrade areas to at least 90 percent of the soil's Modified Proctor maximum dry density (ASTM D1557). The peripheral area of the excavation was approximately 80,000 square feet in size, and GCS personnel performed 14 moisture-density tests. The resulting approximate frequency of one moisture-density test for every 5,714 square feet of peripheral subgrade area exceeded the specification's requirement of one test for every 10,000 square feet of subgrade area. A summary of these test results is included in Appendix J.

The placement and compaction of approximately 4,500 cubic yards of backfill were required to establish the design grade of the site entrance, the truck staging and unloading areas, and segments of the perimeter road. Backfill material was hauled by an International, 50-ton, Payhauler, end-dump truck, or by a Caterpillar 988B front-end loader. The material was spread in maximum loose-lift thicknesses of eight inches by a JD 272A grader. The backfill was moisture conditioned using a 3,500-gallon water truck, and then compacted to 90 percent of the material's Modified Proctor maximum dry density (ASTM D1557) by a Ray Go Rascal 410A smooth-drum, vibratory roller.

Field moisture-density tests were performed on each lift of backfill material. A total of 58 moisture-density tests were performed, resulting in a frequency of one test for every 78 yards of backfill, exceeding the specifications' requirement of one moisture-density test for every 500 cubic yards of backfill placed. A summary of the backfill moisture-density test results is included in 
WHC-SD-W025-RPT-001, Rev. 0

Appendix J. The locations of the field moisture-density tests for the subgrade, general fill, and top course materials are shown on Figure 5. 


\subsection{SECONDARY LINER SYSTEM}

The secondary liner system for the Project W-025 landfill consisted of a soil liner, geomembrane liner, and leachate collection and removal system (LCRS).

\subsection{SOIL LINER}

\subsubsection{Construction}

The soil liner admix was prepared using onsite eolian sand and imported bentonite. The sand for the soil liner was obtained from the eolian sand stockpile located on the west side of the site. The eolian sand had been stockpiled during the excavation of the landfill. Preconstruction testing indicated that the eolian sand and the bentonite met the requirements of the specifications for use as soil liner constituents (See Sec. 2.1). The eolian sand, bentonite, and water were mixed in a pug mill to produce an admixture which contained approximately 11 percent to 14 percent by dry-weight bentonite, and a moisture content of 1 percent to 5 percent above the admixture's optimum moisture content, as determined by ASTM D698. After the soil liner was mixed in the pug mill, the placement of the soil liner was generally completed in the following stages:

- The soil liner was stockpiled around the edge of the excavation and allowed to moisture cure for approximately 24 hours.

- Water was applied to the subgrade just prior to the placement of the soil liner.

- The first lift of soil liner was. spread in a loose-lift thickness of approximately a 12 inches, using a Caterpillar D-6 dozer and a JD 450 dozer.

- The soil liner was compacted to at least 90 percent of the material's Standard Proctor maximum dry density (ASTM D698), using four, one-way passes of a Caterpillar $825 \mathrm{C}$ self-propelled wedgefoot compactor.

- Five additional lifts of soil liner were placed and spread in maximum loose-lift thicknesses of approximately 10 inches. Compaction of each lift was achieved using four passes of the Cat $825 \mathrm{C}$ compactor.

- The top lift of the soil liner was trimmed using a D-6 dozer equipped with low ground pressure (LGP) tracks.

- The surface of the soil liner was prepared for geomembrane deployment by rolling with a Ray Go Rascal smooth-drum vibratory roller.

- The soil liner was watered as necessary during dry weather to prevent excessive drying.

The placement and compaction of the first lift of soil liner commenced in the northwest corner of the landfill, and generally proceeded in a clockwise direction. Delhur completed this circular cycle six times during the placement and compaction of the six lifts of soil liner. To enable large trucks to 
haul admix to the floor of the facility, the access ramp was not covered with soil liner material until just prior to the completion of the soil liner in the rest of the landfill.

A total of approximately 20,000 cubic yards of admix material was produced by the pug mill. Approximately 15,150 cubic yards of this material were placed and compacted on the floor and slopes of the Project W-025 Landfill during the construction of the secondary soil liner.

Following the completion of the placement, compaction, and trimming of the secondary soil liner, an as-built survey of the soil liner surface was performed by Permit Surveying, Inc., and a CQA survey was performed by Rogers Surveying, Inc. GCS reviewed the data from both surveys, and determined that Rogers' survey data reasonably verified Permit's survey data. The specifications required a minimum secondary soil liner thickness of 3.1 feet, measured perpendicular to the subgrade surface. Permit's survey data indicated that three of the surveyed grid points did not meet the tolerance requirements of the specifications, and that four grid points had not been surveyed. Delhur was required to rework the three areas which were initially out of tolerance. Permit then surveyed the four grid points which were missed during the first survey, and resurveyed the three areas which had been reworked. A review of the new survey data indicated that a minimum of 3.1 feet of soil liner had been installed at each of the surveyed grid points. Permit's Record Drawing, Top of Secondary Admix As-Built, Sheet 2 of 6, is included in Volume 2 of this report. Roger's CQA survey data for the secondary soil liner are included in Appendix T-2.

The trench for the secondary slope riser pipe was excavated using a Case 580 backhoe. The trench was trimmed using hand tools. The anchor trench in the access ramp and the anchor trench around the perimeter of the landfill were excavated using the Hitachi EX1000 excavator. The anchor trench in the access ramp was prepared for geomembrane deployment by rolling with a Ray Go Rascal 410A smooth-drum vibratory roller and a Bomag hand operated smooth-drum vibratory roller. GCS observed the excavation of the anchor trenches, and confirmed that the trenches had been constructed per the project plans.

After the surface of the secondary soil liner had been prepared for geomembrane deployment by rolling with a smooth-drum vibratory roller, cold weather caused the top lift of soil liner to freeze. When the soil liner thawed, a sheen of mud remained on the surface. Due to concerns regarding the friction angle of the interface between the geomembrane and the muddy surface of the soil liner, the contractor was required to dry the muddy surface before deployment of the geomembrane liner was allowed to begin. Delhur used propane fueled weed-burners, mounted on a D-6 dozer, to dry the surface of the soil liner.

\subsubsection{Testing}

A total of 162 field moisture-density tests were performed at a frequency of at least one test per 250 cubic yards of soil liner placed. All field moisture-density tests were conducted using a Troxler Model 3440 Nuclear density gauge (ASTM D2922 and D3017). At least one side-by-side rubber balloon test (ASTM D2167) was conducted each day, or once for every 20 nuclear density tests per day, to verify the accuracy of the nuclear densometer tests.

After completion of the nuclear moisture-density test, a sample of the soil from the density test location was obtained and sealed in a plastic bag for laboratory water content determination using the oven-dried method (ASTM D2216) or the microwave oven method (ASTM D4643). Upon completion of the test, the hole was backfilled with soil liner material and compacted by hand. Using 
moisture content data obtained during construction of the test fill and during the admix preparation, a statistical analysis (regression method) was performed to determine the correction factor for correcting the microwave oven moisture content to the equivalent of the conventional oven moisture content. The microwave oven moisture method was used to allow quick determinations as to whether compaction tests were passing or failing. Conventional oven moisture determinations require approximately 24 hours for a result. Microwave oven moisture determinations require only approximately five minutes for a result. For this reason, the correlation was established. At least one side-by-side microwave moisture and conventional oven moisture test were performed each day to continually update the regression analysis.

Four of the 162 moisture-density tests indicated that the moisture content of the soil liner was too dry. Delhur reworked the affected areas of the soil liner, and retests indicated acceptable moisture-density test results. The results of field moisture-density tests are summarized in Appendix K-1. Test locations are shown in Figure 6.

In addition to the field tests, Shelby tubes were used to obtain relatively undisturbed samples of the compacted soil liner. Laboratory tests were performed on the Shelby tube samples. The ends of the Shelby tubes were sealed in the field with plastic caps and were later sealed in the on-site laboratory with wax. The samples were shipped to the GAl soils laborarory located in Redmond, Washington, for permeability testing. Hydraulic conductivity tesis (ASTM D5084) were performed at a frequency of at least one test for every 5,000 cubic yards of soil liner placed. Hydrometer and sieve tests (ASTM D422) and Atterberg Limits tests (ASTM D4318), were performed on samples of the soil taken immediately adjacent to the location of the Shelby tube samples. These index tests were performed at GCS's onsite soils laboratory.

The specifications required a hydraulic conductivity less than or equal to $1 \times 10^{-7} \mathrm{~cm} / \mathrm{s}$. The hydraulic conductivity test results indicated that the permeabilities of the Shelby tube samples ranged $5 \times 10^{-8} \mathrm{~cm} / \mathrm{s}$ to $5 \times 10^{-9} \mathrm{~cm} / \mathrm{s}$, with the exception of one Shelby tube sample taken in the southwest corner of the landfill that had a hydraulic conductivity of $1 \times 10^{-6} \mathrm{~cm} / \mathrm{s}$. To determine the reason for the higher permeability of this sample, a test pit was excavated at the location at which the sample was obtained. The admix in the test pit contained lenses of sandy soil which likely caused the poor permeability test result. To determine the extent of the contaminated admix, four additional test pits were excavated in the soil liner approximately $15 \mathrm{ft}$ to the north, south, east, and west of the original test pit. No sandy lenses were observed in the additional test pits. Having bracketed the extent of the contaminated soil liner, all of the soil liner between the four additional test pits was removed and replaced with new admix material. Shelby tube samples were then taken in the replacement soil liner area, adjacent to the replacement area, and in the northwest corner of the landfill. The results of hydraulic conductivity tests on the three new Shelby tube samples indicated permeabilities that met the requirements of the specifications.

The most likely reason for the contamination of the admix in the southwest corner of the landfill involved trucks hauling soil liner material to the floor of the landfill. At the time of the contamination, approximately three lifis of soil liner had been installed on the slopes and floor of the facility, but the access ramp had not been covered with soil liner. The ramp was left bare to enable easy access to the floor of the facility. Trucks hauling admix to the floor would travel down the bare access ramp and turn sharply into the southwest corner of the landfill. The trucks turned into the corner to enable them to back onto the floor of the facility to dump their loads of admix. The trucks likely tracked sand from the bare access ramp onto the admix in the southwest corner of the landfill. 
Following the completion of the installation of the secondary soil liner, cold weather caused the top lift of the soil liner to freeze. Due to concerns that freezing and thawing could adversely affect the permeability of the soil liner, three Shelby tube samples were taken while the soil was frozen. Hydraulic conductivity tests were performed on the Shelby tube samples. The results of the tests indicated that the permeability of each sample met the requirements of the specifications. The locations of all Shelby tube samples taken in the secondary and primary soil liners are shown on Figure 7. After the soil liner thawed, and the muddy sheen on the surface of the soil liner was mechanically dried by the contractor, the installation of the secondary geomembrane liner was allowed to begin.

With the exceptions noted above, all field and laboratory tests met the requirements of the specifications. Corrective measures were taken to rectify the noncompliances indicated by the failing tests. A summary of the field tests and laboratory tests performed on the secondary soil liner is presented in Table 14. The results of the laboratory tests are presented in Appendix D-4, and the results of the field moisture-density tests are presented in Appendix K-1.

Table 14. Secondary Soil Liner Field and Laboratory Testing Summary.

\begin{tabular}{|l|l|c|c|}
\hline \multicolumn{1}{|c|}{$\begin{array}{c}\text { MINIMUM } \\
\text { FREQUENCY }\end{array}$} & \multicolumn{1}{|c|}{ TESTS } & $\begin{array}{c}\text { TESTS } \\
\text { REQUIRED }\end{array}$ & $\begin{array}{c}\text { TESTS } \\
\text { PERFORMED }\end{array}$ \\
\hline \hline $\begin{array}{l}\text { One Test Per } \\
250 \mathrm{yd}^{3}\end{array}$ & $\begin{array}{l}\text { Moisture-Density (ASTM D2922, } \\
\text { D3017, D2216, \& D4643) }\end{array}$ & 61 & 162 \\
\hline $\begin{array}{l}\text { One Test Per } \\
5,000 \mathrm{yd}^{3}\end{array}$ & $\begin{array}{l}\text { Hydraulic Conductivity } \\
\text { (ASTM D5084) }\end{array}$ & 4 & 11 \\
\hline None required & $\begin{array}{l}\text { Sieve and Hydrometer (ASTM D422) } \\
\text { Atterberg Limits (ASTM D4318) }\end{array}$ & 0 & 12 \\
\hline
\end{tabular}

\subsection{HDPE GEOMEMBRANE LINER INSTALLATION}

Material used in the construction of the Project W-025 Landfill secondary geomembrane liner consisted of 60-mil thick, smooth, HDPE geomembrane, and 60-mil thick, textured, HDPE geomembrane. The smooth geomembrane was used on the floor of the facility, and the textured geomembrane was used on the sideslopes and in the sump area of the facility. The HDPE liner material was manufactured by SLT North America, of Conroe, Texas.

The geomembrane liner installer for this project was GeoPacific Lining Co. (GeoPacific) of Seattle, Washington. Installation of the secondary geosynthetic liner commenced on November 19 , 1993, and was completed on January 20,1994. The installation of the geomembrane started in the northeast corner of the landfill, and generally proceeded around the landfill in a counterclockwise direction. 


\subsubsection{HDPE Geomembrane Liner Deployment}

Prior to deployment of the geomembrane liner, a panel layout drawing was submitted by GeoPacific showing the number and orientation of the various panels comprising the Project W-025 Landfill geosynthetic liner. The HDPE liner was generally installed per the panel layout drawing with slight deviations to allow for correct joining of panels in the field. During deployment, GCS documented the as-built locations of the actual panels deployed. The approximate as-built geomembrane liner panel layouts are shown on Sheet $S-1$.

Prior to deployment of the geomembrane liner, GCS and the installer observed the subgrade for conformance with the project specifications. A subgrade acceptance certificate was issued and signed by the installer for each area where geomembrane was to be deployed. Copies of these subgrade acceptance certificates are presented in Appendix $L$.

The rolls of geomembrane material were mounted on a spreader bar and deployed from the crests of the landfill's sideslopes. The geomembrane liner panels were deployed using either hand labor or a 4-wheel, all terrain vehicle (ATV). Both deployment methods minimized subgrade disturbance. During deployment of the geomembrane liner panels, the following services were provided by GCS personnel:

- Measurement of the panel thickness;

- Confirmation of panel overlap;

- Visual observation of overall sheet quality; and

- Assignment of an unique identification number to each panel deployed.

A nominal overlap of five inches was maintained between adjoining panels. The average panel thickness was determined by averaging five measurements made along each of the leading edge, trailing edge, and one side of the panel. Approximately 187,700 square feet of secondary geomembrane liner were deployed. A summary of GCS' deployment observations is presented in Appendix $M-1$.

\subsubsection{HDPE Geomembrane Liner Seaming}

Trial seams were made by each welding apparatus to be used and by each welding technician who was going to operate a welding apparatus (welder), at the start of each seaming period and at least once every four hours while seaming was being performed. Sample coupons were cut from each end of each trial seam and tested in the peel and shear test modes using a calibrated tensiometer supplied by GeoPacific. If a trial seam failed the field testing, the welder and welding apparatus associated with the failing trial seam were not allowed to weld on the geomembrane liner until a passing trial seam had been made in accordance with the specifications. A summary of the trial seam results is presented in Appendix.M-2.

The geomembrane liner panel seaming process proceeded concurrently with the panel deployment. The majority of the seams were made using a double hot-wedge fusion welding apparatus. Repairs, corner seams, and some of the butt-seams between panels were made using an extrusion welding apparatus. The seaming operations were observed and documented by GCS personnel. The entire length of all seams, patches, or other repairs were observed and documented by GCS. A portion of the seaming was completed when the ambient temperature was less than $40^{\circ}$ Fahrenheit. The specifications required the installer to certify the quality of seams welded in ambient 
temperatures less than $40^{\circ}$ Fahrenheit. GeoPacific certified that such seams were equivalent to seams welded in temperatures ranging from $40^{\circ}$ Fahrenheit to $104^{\circ}$ Fahrenheit. A copy of this certification is included in Appendix E-3. GCS also increased the frequency of destructive testing of seams welded at temperatures below $40^{\circ}$ Fahrenheit.

Approximately 10,103 feet of seams were welded on the secondary geomembrane liner, including 9,366 feet of fusion welded seams and 737 feet of extrusion welded seams. A summary of seaming operations is presented in Appendix M-3. The locations of the secondary geomembrane liner seams are shown on Sheet $S-1$.

\subsubsection{Destructive Testing}

A total of 35 destructive test samples, excluding retests, were obtained from seams on the secondary HDPE liner, resulting in an average frequency of one destructive test sample for each 289 feet of welded seam. This frequency exceeded the project specification's requirement of at least one sample per every 500 feet of seam welded. Of the 35 destructive test samples, 31 were obtained from fusion welded seams, and 4 were obtained from extrusion welded seams and caps. The test locations were selected by GCS personnel based on the completion of approximately 500 feet of welding, on observations of the welded seams, or on the general performance of the seaming crew. Destructive samples were tested onsite by GCS personnel using GCS's calibrated tensiometer.

The destructive testing consisted of cutting ten test coupons from each destructive test sample. Five coupons were tested for adhesion (peel test mode) and five coupons were tested for bonded seam strength (shear test mode). The specified acceptance criteria for destructive tests are summarized in Table 15. Destructive test codes are shown in Figures 8 and 9.

Table 15. Destructive Test Criteria.

\begin{tabular}{|l|l|l|}
\hline Seam Type & \multicolumn{1}{|c|}{ Peel Test Mode } & \multicolumn{1}{c|}{ Shear Test Mode } \\
\hline \hline Fusion Weld & $\begin{array}{l}\text { 1. Each of the five test coupons must } \\
\text { not fail in the weld on either the } \\
\text { inside or outside track of the weld. } \\
\text { (FTB)* }\end{array}$ & $\begin{array}{l}\text { 1. Each of the five test coupons per } \\
\text { track have a minimum yield } \\
\text { strength of 135 pounds per inch } \\
\text { width. } \\
\text { 2. Each of the five test coupons per } \\
\text { track must not fail in the weld. } \\
\text { (FTB)* }\end{array}$ \\
\hline $\begin{array}{l}\text { Extrusion } \\
\text { W'eld }\end{array}$ & $\begin{array}{l}\text { 1. Each of the five test coupons must } \\
\text { not fail in the weld. (FTB)* }\end{array}$ & $\begin{array}{l}\text { 1. Each of the five test coupons have } \\
\text { a minimum yield strength of } 135 \\
\text { pounds per inch width. } \\
\text { FTB }=\text { Film Tear Bond }\end{array}$ \\
\hline
\end{tabular}


In the event a destructive test sample failed, the path of the welder was traced in both directions from the location of the failed test sample and the seam was resampled and retested at intervals that generally ranged from 10 to 50 feet until the deficient portion of the seam was bounded in both directions by passing test samples. A total of 19 additional destructive test samples were obtained to bracket deficient portions of seams. The section of seam between the passing test samples was then either reconstructed or capped. The destructive test locations are shown on Sheet S-1. A summary of the destructive test results is presented in Appendix M-4.

\subsubsection{Nondestructive Seam Continuity Testing}

All nondestructive seam continuity testing was performed by GeoPacific personnel and observed and documented by GCS personnel. Two types of nondestructive testing were used for this project:

- Vacuum box testing on extrusion welds and some short double hot-wedge fusion welds; and

- Air pressure testing on double hot-wedge fusion welds.

A vacuum box is a rigid-wall box with a clear plexiglass top and a neoprene gasket around the bottom of the box that forms a seal between the box and the HDPE geomembrane. Vacuum box testing consisted of:

- Applying a soapy water solution to the seam;

- Placing the vacuum box over the seam;

- Applying a vacuum of at least five-inches of mercury to the inside of the box for at least 10 seconds;

- Observing the seam for bubbles, which would indicate a discontinuity in the seam.

Air pressure testing consisted of:

- Sealing off of the air channel between the inside and outside tracks of the fusion weld;

- Inserting a needle with a pressure gauge attached into the air channel;

- Using an air pump, inflating the air channel to 25 to $30 \mathrm{psi}$; and

- Observing the pressure gauge over a five minute period. A pressure drop of greater than two psi during this period indicated a possible discontinuity in the seam.

Any leaks or discontinuities detected in the seams were marked and subsequently repaired in accordance with the project specifications. Documentation summarizing the nondestructive testing of the seams and repairs is presented in Appendix M-3 and Appendix M-5, respectively. 


\subsubsection{HDPE Geomembrane Liner Repairs}

In accordance with the specifications, defects in the geomembrane liner were assigned a unique identification number by GCS. These defects were located and marked in the field by GCS personnel for repair. The defects were repaired and nondestructively tested by GeoPacific in accordance with the project specifications. A summary of the defects and repairs is presented in Appendix M-5. Defect repair locations are shown on Sheet S-1.

\subsection{LEACHATE COLLECTION AND REMOVAL SYSTEM}

The secondary leachate collection and removal system (LCRS) system consisted of geosynthetic transmission media and granular drainage material to collect leachate, and a submersible pump and HDPE piping to remove leachate.

On the floor of the Non-Drag-Off Landfill, geonet was installed directly on top of the secondary geomembrane liner. The geonet was overlain by $16-0 z / y d^{2}$ geotextile. The geotextile was overlain by a 1.0 foot thick layer of drainage gravel, and the gravel was covered by a layer of $16-0 z / \mathrm{yd}^{2}$ geotextile.

On the sideslopes and in the sump of the landfill, geocomposite material was installed directly on the surface of the secondary geomembrane liner. In the sump, the geocomposite was overlain by $16-0 z / \mathrm{yd}^{2}$ geotextile, the sump was filled with sump gravel, and the sump gravel was covered with a layer of $16-0 z / \mathrm{yd}^{2}$ geotextile, as shown in the project drawings.

\subsubsection{Geonet Installation}

The geonet panels were oriented similar to the geomembrane panels shown on Sheet S-1. All geonet was deployed manually by uncoiling rolls of the geonet which had been positioned on the floor of the landfill. Adjacent geonet panels were overlapped approximately four inches, and the panels were connected with plastic net ties along the length of each seam at the following intervals:

- Longitudinal seams (lengthwise) across the floor - a tie every five feet

- End-to-end seams (butt seams) - two rows of ties, the ties in each row spaced every six-inches, the rows spaced three-inches apart, and the ties staggered between the rows

Repairs to any damaged geonet were patched with geonet extending two feet beyond the edges of the hole or tear, orienting the ribs in the patch parallel to the ribs in the existing geonet, and tying every six inches. The installation and repair of the geonet was observed by GCS personnel.

\subsubsection{Geotextile Installation}

The geotextile installation was completed concurrently with the installation of the geonet, such that the geonet was covered the same day it was deployed. All the geotextile was deployed in an orientation similar to the geomembrane panels shown on Sheet $S-1$. The geotextile was deployed by manually uncoiling rolls of the material which had been positioned on the floor of the landfill. Adjacent geotextile panels were overlapped approximately three inches. All geotextile seams were 
WHC-SD-W025-RPT-001, Rev. 0

sewn with a locking-stitch sewing machine. To enable complete encapsulation of the gravel which was to be installed on top of the geotextile, the geotextile was extended up the sideslopes approximately six feet to eight feet. The installation of the geotextile was observed by GCS personnel. None of the geotextile was damaged, so no repairs were necessary. The installed geotextiles were exposed to sunlight for less than 15 days before being covered by the next consecutive layer of the LCRS.

\subsubsection{Geocomposite Installation}

Geocomposite was installed on the sideslopes of the landfill by manually deploying the material from a roll mounted on a spreader bar. at the crest of the slope. The geocomposite panels were deployed in an orientation similar to the textured geomembrane liner panels. During deployment of the geocomposite, a temporary rub sheet was used between the geocomposite and the textured geomembrane liner to prevent damage to the geotextile component, and to prevent a reduction of the friction at the interface between the geocomposite and the geomembrane. Geocomposite panels were positioned such that the geonet component of adjacent panels overlapped approximately three to four inches. Along end-to-end seams and longitudinal seams, the geonet overlaps were secured by tying in accordance with the guidelines followed for geonet (See Section 4.3.2). In the anchor trenches, the geonet overlaps were secured with ties spaced six inches apart. The geotextile components of adjacent geocomposite panels were overlapped approximately eight inches, and the overlapping top layers of geotextile were carefully heat seamed.

The geocomposite was tied-in to the geonet and geotextile materials on the floor of the landfill. Tie-in seams were located at least three feet from the toe of the slope. The geonet materials were secured with ties in accordance with the guidelines indicated in Section 4.3.2 of this report. Geotextile materials were secured by continuously heat-bonding the bottom of the geotextile on the floor to the top of the geocomposite. The geocomposite was exposed to sunlight for a period of less than 15 days prior to being covered by the next consecutive layer of the lining system.

\subsubsection{Granular Drainage Material}

4.3.4.1 Installation. Two types of granular drainage material were installed in the secondary leachate collection system. Drainage gravel was installed on the floor of the landfill, and sump gravel was installed in the sump. Both materials were supplied by ACME Materials and Construction Co., of Richland, Washington.

Delhur used Payhauler end-dump trucks to haul the drainage gravel to the toe of the access ramp. The access ramp in the landfill had been completed prior to the installation of the granular drainage materials. The drainage gravel was spread toward the sump using a D-6 dozer equipped with LGP tracks. The dozer spread the gravel such that a three feet thick access pad was constructed from the toe of the access ramp to the secondary sump. A Caterpillar 988 loader was then used to transport sump gravel to the sump. A JD 450 dozer then spread the sump gravel in the sump. The D-6 dozer and the JD 450 dozer then spread the drainage gravel from the three feet thick access pad to the remainder of the floor, forming a one-foot thick lift of drainage gravel. Approximately 800 cubic yards of drainage gravel and 100 cubic yards of sump gravel were placed in the secondary leachate collection system. 
The granular drainage materials were track-walked and trimmed using the D-6 and JD 450 dozers. Permit Surveying performed an as-built survey of the gravel surface, and Rogers Surveying performed a CQA survey of the gravel surface. GCS personnel reviewed the data from each of the surveys. Rogers' survey reasonably verified Permit's survey. Permit's survey data indicated that a minimum gravel thickness of 1.0 foot had been installed at each surveyed grid point. Roger's CQA survey data are included in Appendix T-3. Permit's Record Drawing, Top of Secondary Gravel AsBuilt, Sheet 3 of 6 , is included in Volume 2 of this report.

Following the completion of the surveys, the secondary gravel surface was covered with $16-0 z / \mathrm{yd}^{2}$ geotextile. The geotextile was installed and sewn as described in Section 4.3.2 of this report. To completely enshroud the gravel layer, the geotextile which was installed on top of the gravel was sewn, near the toe of each sideslope, to the geotextile which had been previously installed on the secondary geonet.

4.3.4.2 Testing. GCS personnel obtained samples of the granular drainage materials as the gravels were installed. Laboratory tests were performed on four samples of the drainage gravel, resulting in a frequency of one set of laboratory tests for every 200 cubic yards of secondary drainage gravel. One set of laboratory tests was performed on a sample of the sump gravel, resulting in a frequency of one set of laboratory tests for every 100 cubic yards of secondary sump gravel. These testing frequencies exceed the specified construction testing frequency of one set of laboratory tests for every 250 cubic yards of material placed. The laboratory tests completed during construction are summarized in Table 16.

Table 16. Secondary Granular Drainage Material Construction Testing Summary.

\begin{tabular}{|c|c|c|c|}
\hline $\begin{array}{l}\text { MTNDMUM } \\
\text { FREQUENCY }\end{array}$ & TESTS & $\begin{array}{c}\text { TESTS } \\
\text { REQUIRED }\end{array}$ & $\begin{array}{c}\text { TESTS } \\
\text { PERFORMED }\end{array}$ \\
\hline $\begin{array}{c}\text { Drainage Gravel } \\
\text { One Test } \\
\text { Per } 250 \mathrm{yd}^{3}\end{array}$ & $\begin{array}{l}\text { Particle Size (ASTM C136) } \\
\text { Permeability (ASTM D2434) }\end{array}$ & 4 & 4 \\
\hline $\begin{array}{c}\text { Sump Gravel } \\
\text { One Test } \\
\text { Per } 250 y^{3}\end{array}$ & $\begin{array}{l}\text { Particle Size (ASTM C136) } \\
\text { Permeability (ASTM D2434) }\end{array}$ & 1 & 1 \\
\hline
\end{tabular}

The permeability tests were performed by GAI's soils laboratory located in Redmond, Washington. The particle size analyses were performed in GCS's onsite soils laboratory. The results of these tests indicated that the tested samples met the requirements of the specifications. A summary of the test results is presented in Appendix D-5.

\subsubsection{Leachate Removal System}

The secondary leachate removal system consists of HDPE piping and a submersible pump. An eight inch diameter, HDPE slope riser pipe was installed horizontally on the floor of the secondary sump, and extends up the east sideslope of the landfill. Two 2-inch diameter, HDPE pipes were 
installed adjacent to the eight inch diameter pipe in the sump and on the sideslope. HDPE geomembrane liner boots were installed around the HDPE pipes at the crest of east slope where the pipes penetrate the secondary HDPE liner. In the sump, the end of each of the three pipes is perforated. The eight inch diameter pipe houses a 1.5-horsepower submersible pump attached to a 1.5-inch diameter HDPE discharge pipe. The pump discharges through the 1.5-inch diameter pipe to the leachate temporary storage tank, located near the crest of the east sideslope. One of the two inch diameter pipes contains a transducer which monitors the water level in the secondary sump. The second two inch diameter pipe is a spare level sensor pipe. The system was designed such that the submersible pump will automatically begin pumping when the transducer indicates a leachate level 12 inches above the bottom of the secondary sump. The submersible pump will automatically shut-off when the leachate level is drawn-down to six inches above the bottom of the sump. This system was designed to maintain the leachate level (in the secondary sump) between six inches and 12 inches above the bottom of the secondary sump. 


\subsection{PRIMARY LINER SYSTEM}

The primary liner system consisted of a soil liner on the floor of the landfill, geomembrane liner, and a leachate collection and removal system.

\subsection{SOIL LINER}

\subsubsection{Construction}

Construction of the primary soil liner was conducted in a manner similar to that used to install the secondary soil liner. However, the primary soil liner was only placed on the floor of the landfill, and in the anchor trench on the access ramp.

On the floor of the landfill, the minimum thickness of the primary soil liner was 1.5 feet. The first lift of soil liner material was placed in a loose-lift thickness of approximately 18 inches to 20 inches, resulting in a compacted lift thickness of approximately 14 inches to avoid damaging the underlying secondary geosynthetic liner system.

In the anchor trench on the access ramp, a 1.0-foot thick layer of compacted soil liner was installed on the secondary geosynthetic liner system, and a 1.0-foot thick layer of compacted soil liner was installed on the primary geosynthetic liner system. Both layers of soil liner were compacted by track walking with a D-6 dozer (equipped with LGP tracks) and by rolling with a smooth-drum roller. Figure 10 depicts the manner in which the lining system on the access ramp was tied-in to the lining system at the toe of the ramp.

Approximately 1,700 cubic yards of admix were placed in the primary soil liner. After compacting and trimming. The surface of the primary soil liner was surveyed by Permit Surveying. A CQA verification survey was performed by Rogers Surveying. Rogers' survey data reasonably verified Permit's survey data. However, Permit's data indicated that the primary soil liner thickness was out-of-tolerance at three survey grid points. Delhur was required to rework the soil liner in the affected areas, and Permit was required to resurvey the reworked areas. A review of the new survey data indicated that a minimum thickness of 1.5 feet of soil liner had been installed at each survey grid point. Permit's Record Drawing, Top of Primary Admix As-Built, Sheet 4 of 6, is included in Volume 2 of this report. Rogers' CQA survey data are included in Appendix T-4.

\subsubsection{Testing}

Testing of the primary soil liner was also conducted in a manner similar to the secondary soil liner. A total of 16 field moisture-density tests were conducted at a frequency of at least one test per 250 cubic yards of soil liner placed. The field moisture-density test locations are shown in Figure 11 and the test results are given in Appendix K-2.

In addition to the field tests, a laboratory permeability test was performed on a Shelby tube sample of the primary soil liner. The Shelby tube sample location is shown on Figure 7 . The field and the laboratory tests performed on the primary soil liner are summarized in Table 17. 
Table 17. Primary Soil Liner Field and Laboratory Testing Summary.

\begin{tabular}{|l|l|c|c|}
\hline \multicolumn{1}{|c|}{$\begin{array}{c}\text { MINIMUM } \\
\text { FREQUENCY }\end{array}$} & \multicolumn{1}{|c|}{ TESTS } & $\begin{array}{c}\text { TESTS } \\
\text { REQUIRED }\end{array}$ & $\begin{array}{c}\text { TESTS } \\
\text { PERFORMED }\end{array}$ \\
\hline \hline $\begin{array}{l}\text { One Test Per } \\
250 \mathrm{yd}^{3}\end{array}$ & $\begin{array}{l}\text { Moisture-Density (ASTM D2922, } \\
\text { D3017, D2216, \& D4643) }\end{array}$ & 7 & 16 \\
\hline $\begin{array}{l}\text { One Test Per } \\
5,000 \mathrm{yd}^{3}\end{array}$ & $\begin{array}{l}\text { Hydraulic Conductivity } \\
\text { (ASTM D5084) }\end{array}$ & 1 & 1 \\
\hline
\end{tabular}

All test results met the project specifications. A summary of the laboratory test results is included in Appendix D-4.

\subsection{HDPE GEOMEMBRANE LINER}

The materials, methods, and equipment used to install the primary HDPE geomembrane liner were generally the same as those used to install the secondary HDPE geomembrane liner. GeoPacific began installing the primary HDPE liner on December 19,1993, and completed the installation on January 27, 1994. GCS performed the same CQA services for the consiruction of the primary geomembrane liner system as on the secondary system.

Included in the primary geomembrane lining system is the 80-mil thick HDPE geomembrane liner installed in the truck unloading area. The 80-mil thick HDPE geomembrane liner was installed on a prepared subgrade consisting of eolian sand.

\subsubsection{HDPE Geomembrane Liner Deployment}

Deployment of the primary geomembrane in the landfill was completed in a manner similar that used during the installation of the secondary geomembrane liner. Deployment of the HDPE panels started on the south slope and proceeded clockwise around the landfill. Approximately 180,100 square feet of 60-mil thick, HDPE, geomembrane were deployed in the landfill's primary geosynthetic liner system.

Deployment of the 80-mil thick, smooth, HDPE, geomembrane liner in the truck unloading area was completed using similar equipment and methods as those used to install the secondary lining system. Approximately 11,900 square feet of 80 -mil thick HDPE liner were deployed in the truck unloading area. The approximate as-built geomembrane liner panel layouts for the primary HDPE liner in the landfill and in the truck unloading area are shown on Sheet P-1. A summary of the deployment observations is presented in Appendix $\mathrm{N}-1$.

\subsubsection{HDPE Geomembrane Liner Seaming}

Seaming of the primary geomembrane liner took place concurrently with deployment of the primary HDPE panels, and was performed in a manner similar to that used during the installation of 
the secondary lining system. Trial seaming was performed as indicated in Section 4.2 .2 , and a summary of the trial seam results is presented in Appendix $\mathrm{N}-2$.

Approximately 8,853 feet of seam were welded during the installation of the primary geomembrane liner in the landfill, including 8,790 feet of fusion welded seam, and 63 feet of extrusion welded seam. Approximately 494 feet of seam were welded during the installation of the primary geomembrane liner in the truck unloading area, including 446 feet of fusion welded seam, and 48 feet of extrusion welded seam. The 80-mil thick, smooth, HDPE geomembrane liner in the truck unloading area was tied-in to the 60-mil thick, textured, HDPE geomembrane liner in the southeast corner of the landfill, by seaming with an extrusion welder. A summary of the seaming records is presented in Appendix N-3. The locations of the primary geomembrane liner seams are shown on Sheet P-1.

\subsubsection{Destructive Testing}

Destructive testing of the primary HDPE geomembrane seams was performed in a manner similar to that used during the installation of the secondary system. A total of 24 destructive test samples, excluding retests, were initially obtained from seams on the primary HDPE geomembrane liner in the landfill. This resulted in an average frequency of one destructive test sample for each 369 feet of welded seam. This testing frequency exceeded the specification's requirement of one destructive test for every 500 feet of seam. Of the 24 destructive test samples, 19 were obtained from fusion welded seams, and five were obtained from extrusion welded seams and caps. In the event that a destructive test sample failed to meet the requirements of the specifications, additional samples were obtained to bracket the deficient portion of the seam. A total of five samples were obtained to bracket deficient portions of seams.

A total of three destructive test samples were obtained from seams welded on the HDPE geomembrane liner in the truck unloading area, resulting in an average frequency of one destructive test sample for every 165 feet of welded seam. One of the samples was obtained from a fusion welded seam, and the other two samples were obtained from extrusion welded seams. One of the extrusion weld samples failed the destructive test, and all seaming performed by the apparatus which welded the seam was capped. The test locations were selected by GCS personnel based on the completion of approximately 500 feet of welding, on observations of the welded seams, or on the general performance of the seaming crew. Destructive test samples were tested onsite by GCS personnel using GCS's calibrated tensiometer. The destructive test locations are shown on Sheet P-1. A summary of the destructive test results is presented in Appendix N-4.

\subsubsection{Nondestructive Seam Continuity Testing}

Nondestructive testing of the geomembrane seams and repairs was completed in a manner similar to that used during the installation of the secondary liner system. Any leaks or discontinuities detected in the seams were marked and subsequently repaired in accordance with the project specifications. Documentation summarizing the nondestructive testing of the seams and repairs is presented in Appendix N-3 and Appendix N-5, respectively. 


\subsubsection{HDPE Geomembrane Liner Repairs}

In accordance with the specifications, defects in the geomembrane liner were assigned a unique identification number by GCS. These defects were located and marked in the field by GCS personnel for repair. The defects were repaired and nondestructively tested by GeoPacific in accordance with the project specifications. A summary of the defects and repairs is presented in Appendix N-5. Defect repair locations are shown on Sheet P-1.

\subsection{LEACHATE COLLECTION AND REMOVAL SYSTEM}

The primary leachate collection system consists of geosynthetic transmission media, and granular drainage material, a leachate collection piping system, and a leachate collection well. The leachate removal portion of the primary system consisted of a submersible pump, a self priming pump, and HDPE piping.

On the floor of the Project W-025 Landfill, geonet was installed directly on top of the primary geomembrane liner. The geonet was overlain by $16-0 z / y^{2} d^{2}$ geotextile. The geotextile was overlain by a 1.0 foot thick layer of drainage gravel, and the gravel was covered by a layer of seven-oz/yd $\mathrm{d}^{2}$ geotextile.

On the sideslopes and in the sump of the landfill, geocomposite material was installed directly on the surface of the primary geomembrane liner. In the sump, the geocomposite was overlain by $16-0 z / \mathrm{yd}^{2}$ geotextile, a collector well was installed, the sump was filled with sump gravel, and the sump gravel was covered with a layer of seven-oz/yd $\mathrm{d}^{2}$ geotextile.

\subsubsection{Geonet Installation}

The geonet installation was completed in a manner similar to that used to install the geonet in the secondary system. The orientation of the geonet panels is similar to that of the geomembrane panels shown in Sheet P-1. Repairs to any damaged geonet were made with geonet patches and net ties in accordance with the project specifications. The installation and repair of the geonet was observed by GCS personnel.

\subsection{Geotextile Installation}

The geotextile installation was completed concurrently with the installation of the geonet, such that the geonet was covered the same day it was deployed. All geotextile was deployed parallel to the orientation of the geonet panels. The geotextile was deployed by manually uncoiling rolls of the material which had been positioned on the floor of the landfill. Adjacent geotextile panels were overlapped approximately three inches. All geotextile seams were sewn with a locking-stitch sewing machine. To enable complete encapsulation of the gravel which was to be installed on top of the geotextile, the geotextile was extended up the sideslopes approximately six feet to eight feet. Geotextile boots were formed around slope riser pipes which protruded through the material. The geotextile boots were secured to the HDPE pipes using stainless steel banding. The installation of the geotextile was observed by GCS personnel. None of the geotextile was damaged, so no repairs were necessary. The geotextile was exposed to sunlight for a period of less than 15 days before being covered by the next consecutive layer of the lining system. 
In the truck unloading area, three layers of seven-oz/yd $\mathrm{d}^{2}$ geotextile were installed as a cushion layer on top of the 80-mil thick, HDPE, geomembrane liner. Each of the three layers was installed perpendicular to the underlying layer of geosynthetic material. Adjacent panels of geotextile were overlapped approximately six inches, and seamed by heat bonding using a leistering device.

\subsubsection{Geocomposite Installation}

The geocomposite installation was completed in a manner similar to that used to install the secondary geocomposite materials. Geocomposite material was installed on the sideslopes and in the sump area of the primary system. The geocomposite materials were tied-in to the geonet and geotextile on the floor of the landfill as described in Section 4.3.3.

\subsubsection{Leachate Collection Piping}

A leachate collection piping system was installed on the floor of the primary system prior to the installation of granular drainage materials. An HDPE, 30-inch diameter, leachate collection well, mounted on a four-foot square by one-inch thick slab of HDPE flatstock, was installed in the primary system sump. The bottom of the collection well is perforated, and wrapped with two layers of geonet. A perforated, HDPE, four-inch diameter, leachate collection piping system was installed around the edges and in the center of the floor of the primary system. The piping system drains to the leachate collection well, and the piping is connected to the collection well. Non-perforated, HDPE, four-inch diameter, cleanout riser pipes, connected to the four-inch diameter perforated pipes at strategic locations, provide for cleanout access to the leachate collection piping system. During the assembly of the piping system, the HDPE pipes and fittings were joined by butt fusion welding.

In addition to the HDPE piping noted above, a four-inch diameter, perforated, HDPE pipe was installed on the surface of the primary geosynthetic materials in the center of the truck unloading area, and along the south edge of the access ramp. This drainage pipe is continuous from the truck unloading area to the toe of the access ramp, at which point the pipe connects to the primary drainage system piping. The perforated pipe in the truck unloading area and on the access ramp was covered by top course material.

\subsection{Granular Drainage Material}

The installation and testing of the primary granular drainage materials were similar to the installation and testing of the secondary granular drainage materials.

5.3.5.1 Installation. Two types of granular drainage material were installed in the primary leachate collection system. The installation methods were similar to those used during the installation of the secondary granular drainage materials. Approximately 1,000 cubic yards of drainage gravel were installed in the primary system on the floor of the landfill. The gravel was placed by hand around the HDPE piping system. Approximately 150 cubic yards of sump gravel were installed in the primary sump area. The sump gravel was placed by hand around the leachate collection well.

Permit Surveying performed an as-built survey of the gravel surface, and Rogers Surveying performed a CQA survey of the gravel surface. GCS personnel reviewed the data from each of the surveys. Rogers' survey confirmed Permit's survey. Permit's survey data indicated a minimum 
gravel thickness of 1.0 foot had been installed at each surveyed grid point. Roger's CQA survey data are included in Appendix T-5. Permit's Record Drawing, Top of Primary Gravel As-Built, Sheet 5 of 6 , is included in Volume 2 of this report.

Following the completion of the surveys, the primary gravel surface was covered with seven$\mathrm{oz} / \mathrm{yd}^{2}$ geotextile. The geotextile was installed and sewn as described in Section 4.3.2 of this report. To completely enshroud the gravel layer the geotextile which was installed on top of the gravel was sewn near the toe of each sideslope to the geotextile which had been previously installed on the primary geonet.

5.3.5.2 Testing. GCS personnel obtained samples of the granular drainage materials as they were stockpiled or installed. Laboratory tests were performed on four samples of the primary drainage gravel, resulting in a frequency of one set of laboratory tests for every 250 cubic yards of primary drainage gravel. One set of laboratory tests was performed on a sample of the primary sump gravel, resulting in a frequency of one set of laboratory tests for every 150 cubic yards of primary sump gravel. These testing frequencies met or exceeded the specified construction testing frequency of one set of laboratory tests for every 250 cubic yards of material placed. The laboratory tests completed during construction are summarized in Table 18.

Table 18. Primary Granular Drainage Material Construction Testing Summary.

\begin{tabular}{|c|c|c|c|}
\hline $\begin{array}{c}\text { MINIMUM } \\
\text { FREQUENCY }\end{array}$ & TESTS & $\begin{array}{c}\text { TESTS } \\
\text { REQUIRED }\end{array}$ & $\begin{array}{c}\text { TESTS } \\
\text { PERFORMED }\end{array}$ \\
\hline \hline Drainage Gravel & $\begin{array}{c}\text { Particle Size (ASTM C136) } \\
\text { One Test } \\
\text { Per 250 yd }\end{array}$ & $\begin{array}{c}\text { Permeability (ASTM D2434) } \\
\text { Sump Gravel }\end{array}$ & $\begin{array}{c}\text { Particle Size (ASTM C136) } \\
\text { Permeability (ASTM D2434) }\end{array}$ \\
$\begin{array}{c}\text { One Test } \\
\text { Per 250 } \mathrm{yd}^{3}\end{array}$ & 1 & 4 \\
\hline
\end{tabular}

The permeability tests were performed by GAI's soils laboratory located in Redmond, Washington. The particle size analyses were performed in GCS's onsite soils laboratory. The results of these tests indicated that the tested samples met the requirements of the specifications. A summary of the test results is presented. in Appendix D-5.

\subsubsection{Leachate Removal System}

The primary leachate removal system consists of HDPE piping, a submersible pump, and a selfpriming pump. An eight-inch diameter, HDPE pipe was installed on the floor of the primary sump. The eight-inch HDPE pipe is connected to the leachate collection well and extends up the eastern sideslope of the landfill. Two, two-inch diameter, HDPE pipes were installed adjacent to the eightinch diameter pipe in the sump and on the sideslope. The two-inch diameter pipes are also connected to the 30-inch diameter leachate collection well. HDPE geomembrane liner boots were installed 
around the pipes at the crest of the eastern slope, the point at which the pipes penetrate the primary geomembrane liner.

A 1.5-horsepower submersible pump attached to a 1.5-inch diameter HDPE discharge pipe was installed in the sump end of the eight-inch diameter pipe. The pump discharges through the 1.5 -inch diameter pipe to the leachate temporary storage tank, located near the crest of the eastern sideslope. One of the two-inch diameter pipes contains a transducer which monitors the water level in the primary sump. The second two-inch diameter pipe is a spare level sensor pipe. The system was designed such that the submersible pump will automatically begin pumping when the transducer indicates a leachate level 12 inches above the bottom of the primary sump. The submersible pump will automatically shut-off when the leachate level is drawn-down to six inches above the bottom of the sump.

A five-horsepower self-priming pump is mounted near the top of leachate collection well. The suction intake for this pump is located at the bottom of the collection well. The pump discharges through a three-inch diameter, HDPE pipe to the leachate temporary storage tank. The three-inch diameter HDPE pipe was pressure tested at 50 psi for at least one hour. GCS personnel observed the pressure test. See Appendix R-3 for pressure test documentation.

A float switch, located 30 inches above the bottom of the primary sump, is designed to automatically start the self-priming pump if the leachate reaches the level of the float switch. A second float switch, located six inches above the bottom of the primary sump, is designed to automatically shut off the self-priming pump when the leachate level drops below this float switch. 
WHC-SD-W025-RPT-001, Rev. 0

\subsection{WORKING SURFACES}

The working surfaces for the Project W-025 Landfill include the operations layer; the top course surfacing on the perimeter road and access ramp; the asphalt surfacing on the truck staging and unloading areas; and the concrete installed for the containment basin, crest pad and crest, and control building slab.

\subsection{OPERATIONS LAYER}

The operations layer is a protective soil cover installed on the surface of the landfill. The operations layer provides insulation for the soil liner and provides a protective cushion for the lining systems.

\subsubsection{Construction}

Two general soil types were used for the operations layer. Eolian sand was used on the slopes of the landfill, and gravelly sand to sandy gravel was used on the floor of the landfill. Both materials were stockpiled during excavation of the landfill. Delhur used a D-6 dozer, equipped with LGP tracks, to spread the operations layer materials in a lift of three feet. On the sideslopes, the eolian sand was spread up the slopes. Approximately 15,690 cubic yards of operations layer material, including both eolian sand and gravelly soils, were installed on the surface of the landfill.

To provide access through the operations layer to the leachate collection well, a 48-inch diameter, concrete, vertical riser pipe was installed over the top of the collection well. The vertical riser pipe protrudes through the operations layer, and is secured to a six feet square by six inch thick, reinforced concrete base. The base rests on the surface of the primary sump gravel. A 32-inch diameter hole in the base allows the leachate collection well to protrude through the base, and the annulus between the well and the base will allow for future settlement of the vertical riser. Four 4feet long segments of vertical riser pipe have been installed to provide access through future layers of waste. The vertical riser pipe segments and base were coated with Tnemec Series 61 primer and costing. The assembled vertical riser was wrapped with a layer of 60 -mil thick, smooth, HDPE, goomembrane liner material. The HDPE liner was wrapped around the vertical riser to provide a smooth surface which will reduce friction when waste settles around the riser in the future.

Permit Surveying, performed an as-built survey of the operations.layer surface and Rogers Surveying, performed a CQA verification survey. GCS reviewed data from both surveys, and decermined that Rogers' survey data confirmed Permit's survey data. Permit's data indicated that the operations layer was slightly thinner than the specified 3.0 feet thickness at several grid points. Dethur reworked the areas in question, and Permit resurveyed these areas. The new survey data indicated that a minimum thickness of 3.0 feet had been installed at each of the surveyed grid points. Permit's Record Drawing, Top of Operations Layer As-Built, Sheet 6 of 6, is included in Volume 2 of this report. Rogers' CQA survey data are presented in Appendix T-6.

Shortly after the completion of the installation of the operations layer, wind erosion removed a few inches of operations layer material from the sideslopes of the landfill. Delhur used a D-6 dozer to regrade the sideslopes to re-establish the 3.0 feet thickness of the operations layer. A mixture of water and lignosite was then sprayed in the sideslopes to stabilize the surface and inhibit future wind 
WHC-SD-W025-RPT-001, Rev. 0

erosion of the operations layer. GCS personnel observed the regrading and the application of the lignosite mixture.

\subsubsection{Testing}

During the installation of the operations layer, GCS personnel obtained samples of the operations layer soils. Hydrometer and sieve analyses (ASTM D422) were performed on eight samples of the operations layer soil, resulting in a frequency of one sieve analysis for every 1,961 cubic yards of material placed, exceeding the specification's requirement of one sieve analysis for every 2,000 cubic yards of material placed. The tests were performed in GCS's onsite soils laboratory. The specifications required that the operations layer material meet the specification's requirements for backfill materials, previously indicated in Section 2.1, and that the material used on the sideslopes should have a maximum diameter of one inch. The test results indicated that the sampled materials met the requirements of the specifications for operations layer materials. A summary of the laboratory tests performed on the operations layer materials is presented in Appendix D-7.

\subsection{TOP COURSE}

Top course material was used for surfacing the perimeter road and the access ramp in the landfill, and was used as a base course material for the asphalt paving in the truck unloading and staging areas, and the site entrance.

\subsubsection{Construction}

Top course material was supplied by ACME Materials and Construction Co. (ACME). Delhur installed the top course for the perimeter road and the access ramp. ACME installed the top course in the areas requiring asphalt paving. In the truck unloading area, three layers of seven$0 z \mathrm{yd}^{2}$ geotextile were installed on the surface of the HDPE liner prior to the placement of top course. On the access ramp, a layer of $16-0 z / y^{2}$ geotextile was installed on the surface prior to the placement of top course. Both contractors used similar methods of spreading and compacting the top course material. A grader was used to spread the material in the truck staging area and on the perimeter road. A D 4 dozer was used to spread the top course material in the truck unloading area, and a JD 450 dozer was used to initially spread the material on the access ramp. Top course was placed by hand around the four-inch diameter, perforated HDPE pipe in the truck unloading area and on the access ramp. A water truck was used to saturate the material, and a smooth drum, vibratory roller was used to compact the material to at least 95 percent of the material's Modified Proctor (ASTM D1557) maximum dry density. The top course material was installed in a three foot thick layer on the landfill access ramp, and in an eight-inch thick layer elsewhere. Approximately 760 cubic yards of top course were installed on the access ramp, and approximately 1,975 cubic yards of top course material were installed on the perimeter road, the truck staging and unloading area, and the site entrance.

\subsubsection{Testing}

During the installation of the top course material, GCS personnel performed field moisture-density tests (ASTM D2922 and D3017), and obtained samples of the placed material for laboratory testing. 
Three field moisture-density tests were performed on the compacted top course material on the landfill access ramp, resulting in a frequency of one moisture-density test for every 2,274 square feet of top course surface area, exceeding the specification's requirement of one moisture-density test for every 10,000 square feet of surface area. Sixteen moisture-density tests were performed on the top course on the perimeter road, site entrance road, and truck staging and unloading areas, resulting in a frequency of one moisture-density test for every 123 cubic yards of top course placed in these areas, exceeding the specifications' requirement of one test for every 500 cubic yards of top course placed in these areas. The test results indicated that the top course had been compacted to at least 95 percent of the material's Modified Proctor maximum densiry as determined by ASTM D1557. The top course moisture-density test results are presented in Appendix 0.

Laboratory testing included sieve analyses (ASTM C136) and Modified Proctor compaction tests (ASTM D1557). Two sieve analyses and two Modified Proctor tests were performed during the installation of the top course, resulting in a frequency of one sieve analysis and one Modified Proctor test for every 1,367 cubic yards of top course placed, exceeding the specification's requirement of sieve analysis and one Modified Proctor test for every 2,000 cubic yards of top course placed. The laboratory testing was performed in GCS's onsite soils laboratory. The results of the tests indicated that the sampled top course material met the requirements of the specifications. The top course laboratory test results are presented in Appendix D-6. A summary of the construction tests performed during the installation of the top course material is shown in Table 19.

Table 19. Top Course Material Construction Testing Summary.

\begin{tabular}{|c|c|c|c|}
\hline $\begin{array}{c}\text { MNNIMUM } \\
\text { FREQUENCY }\end{array}$ & TESTS & $\begin{array}{c}\text { TESTS } \\
\text { REQUIRED }\end{array}$ & $\begin{array}{c}\text { TESTS } \\
\text { PERFORMED }\end{array}$ \\
\hline \hline Access Ramp \\
$\begin{array}{c}\text { One Test } \\
\text { Per 10,000 } \mathrm{f}^{2}\end{array}$ & $\begin{array}{c}\text { Moistrre-Bensity (ASTM D2922, } \\
\text { D3017, \& D2216) }\end{array}$ & 1 & 3 \\
\hline $\begin{array}{c}\text { Perimeter Road } \\
\text { One Test } \\
\text { Per 500 yd }\end{array}$ & $\begin{array}{c}\text { Moisture-Density (ASTM D2922, } \\
\text { D3017, \& D2216) }\end{array}$ & 4 & 16 \\
\hline $\begin{array}{c}\text { One Test } \\
\text { Per 2,000 yd }\end{array}$ & $\begin{array}{c}\text { Sieve Analysis (ASTM C136) } \\
\text { Modified Proctor (ASTM D1557) }\end{array}$ & 2 & 2 \\
\hline ₹ Includes perimeter road, truck unloading area, truck staging area, and site entrance road. \\
\hline
\end{tabular}

\subsection{ASPHALT}

A liquid asphalt primer, MC-250, was applied by ACME Materials and Construction, to the surface of the top course on the site entrance road, the truck staging area, and the truck unloading area, prior to paving these areas with asphait concrete. After a curing period of five days, ACME installed asphait paving on the treated areas. ACME placed and compacted the asphait in two, 
approximately two-inch thick lifts. The asphalt was compacted to at least 93 percent of the material's theoretical maximum density using a smooth-drum vibratory roller.

CQA laboratory tests and field density tests were performed by Intermountain Materials Testing, Inc. (Intermountain) of Pasco, Washington. Laboratory testing included sieve analysis (ASTM C136 and C117), extraction test (ASTM D2172), maximum density (ASTM D2041), and specific gravity. During compaction of the asphalt, Intermountain performed field density tests on the asphalt. Four field density tests (ASTM D2922) were performed on each lift of compacted asphalt, resulting in an approximate frequency of one field density test per 7,500 square feet per lift of asphalt placed, exceeding the specification's requirement of one density test for every 25,000 square of asphalt placed.

Following the completion of the asphalt installation, Permit Surveying, Inc. performed an as-built survey of the asphalt surface. GCS reviewed the survey data. The surface of the asphait was outof-tolerance at a majority of the surveyed grid points. The survey data indicated that the asphalt had generally been installed approximately 0.1 foot thicker than specified, and that the drainage pattern on the north edge and in the southwest corner of the truck unloading area did not meet the intent of the project design. The Design Engineer determined that the thickness discrepancy would be tolerable, but that the drainage pattern would have to be corrected so that runoff from the truck unloading area will drain into the landfill. A copy of the Design Engineer's memo addressing the drainage issue is included in Appendix P-2. ACME subsequently rectified the drainage problems by placing and compacting additional asphalt in the southwest corner, and by installing a curb on the north edge of the truck unloading area to correct the drainage pattern. Intermountain performed laboratory tests on the additional asphalt, and GCS performed field density tests on the compacted paving. The results of all asphalt laboratory tests and field density tests indicated that the material met the requirements of the specifications, and are included in Appendix P-1.

A performance test was performed to demonstrate that the drainage in the truck unloading area had been corrected. A 2,000-gallon water truck was used to spread water on the asphalt surface. The water drained into the landfill as designed. GCS personnel observed the demonstration. See Appendix P-1 for documentation of all CQA observations related to the construction and testing of the unloading area asphalt paving. The reworked asphalt surface was resurveyed by Permit Surveying, Inc. These survey data are included in the Record Drawing, Top of Operations Layer As-Built, Sheet 6 of 6 , included in Volume 2 of this report.

\subsection{CONCRETE}

Several small concrete pours were completed for structures on the east side of the Project W-025 Landfill. The concrete pours are summarized in Table 20.

Table 20. Summary of Concrete Pours.

\begin{tabular}{|c|l|}
\hline $\begin{array}{c}\text { DATE } \\
\text { OF POUR }\end{array}$ & \multicolumn{1}{|c|}{ STRUCTURE } \\
\hline \hline $10-27-93$ & Base Slab for Leachate Tank Containment Basin \\
\hline $10-29-93$ & Walls for Leachate Tank Containment Basin \\
\hline
\end{tabular}


WHC-SD-W025-RPT-001, Rev. 0

\begin{tabular}{|c|l|}
\hline $\begin{array}{c}\text { DATE } \\
\text { OF POUR }\end{array}$ & \multicolumn{1}{|c|}{ STRUCTURE } \\
\hline \hline $11-09-93$ & Base Slab for Control Building - (Slab was rejected) \\
\hline $12-01-93$ & $\begin{array}{l}\text { Replacement Base Slab for Control Building, Steps and Pump } \\
\text { Platform in Containment Basin }\end{array}$ \\
\hline $02-10-94$ & Base Slab for Crest Wall \\
\hline $02-14-94$ & Crest Wall \\
\hline
\end{tabular}

GCS observed the forming and rebar installation for each of the concrete pours. All rebar was epoxy coated prior to delivery. Intermountain Materials Testing performed CQA testing of samples of the concrete as it was placed. A set of four concrete cylinders was cast for each pour, and laboratory tests were performed by Intermountain to determine the seven-day, 14-day, and 28-day compressive strength of each set of cylinders. The specified 28-day strength of 3,000 psi was exceeded by the test results for each pair of 28-day tests performed for each pour. The results of Intermountain's tests are included in Appendix Q.

The concrete pour which was made on November 9, 1993, was rejected because the pour was completed earlier than the scheduled time, prior to the arrival of Intermouncain personnel onsite to perform CQA sampling and testing. The rejected pour was intended for use as the base slab-for the control building. The Design Engineer determined that a replacement slab could be poured directly on the surface of the rejected slab. The replacement slab was poured on December 1, 1993. 


\subsection{OPERATIONAL FEATURES}

\subsection{LEACHATE TEMPORARY STORAGE TANK, PIPING, AND TRANSFER PUMP}

The leachate temporary storage tank was constructed by Quality Tank and Construction, of Sandy, Oregon. Carbon steel, Type A36 was used to construct the 10,000-gallon tank. The tank was ASME Code stamped, rated for $25 \mathrm{psi}$. A manufacturer's data report/certificate of compliance, required by the provisions of the ASME Code rules, is included in Appendix R-1. A Registered Professional Engineer's statement regarding the integrity of the tank system is included in Appendix R-2.

Coatings were applied to the interior and exterior of the tank, in accordance with the specifications. A GCS representative observed the interior and exterior of the tank prior to coating. After the tank was coated, GCS's representative measured the thickness of the interior and exterior coatings. The thickness of the exterior coating met the requirements of the specifications. The interior coating was thinner than specified, so an additional coating application was required on the interior of the tank. Subsequent measurements indicated that the interior coating thickness met the requirements of the specifications.

The temporary storage tank receives the discharge of leachate from the submersible pumps located in the landfill's secondary and primary sumps, and from the self-priming pump located in the primary sump. A series of valves and turbine meters located between the crest wall and the tank allow the measurement of the quantity of leachate pumped from each the primary sump and the secondary sump.

The leachate temporary storage tank is equipped with a high level, float-acuated shutoff switch that deactivates the pumps to prevent tank overflow. The tank is also equipped with a high, high level shutoff switch to deactivate the pumps in the event that the high level switch fails.

A high capacity transfer pump, mounted inside the containment basin for the leachate tank, enables the iransfer of the leachate from the tank to a tanker truck, and also allows recirculation of the leachate in the tank.

Piping associated with the leachate temporary storage tank includes the HDPE discharge piping from the sump pumps, recirculation HDPE piping, and stainless steel suction and discharge pipes for the high capacity transfer pump. The discharge pipes from the sump pumps direct flow into the tank. Suction and discharge piping for the transfer pump allow the removal of leachate from the tank. Recirculation valves and piping, attached to transfer pump and the tank, enable recirculation of the liquid in the tank. The HDPE piping, valves, and meters located between the valves near the crest wall and the leachate tank were pressure tested at 33 psi for hour. Simultaneously, the recirculation piping and valves, and the valves and stainless steel piping associated with the transfer pump were pressure tested at 33 psi for one hour. GCS observed the pressure testing and documentation can be found in Appendix R-4.

The manufacturer of the stainless steel pipes provided certification of the pipes. The certification is included in Appendix I-2. The tank, associated piping, and transfer pump were wrapped with electrical heat trace cable and insulated per the specifications.

Heat trace cable and insulation were not required for the discharge pipes between the sump pumps and the crest wall, because the piping system was designed such that these pipes contain no water 
when the pumps are not operating. Factory installed check valves have been removed from the submersible pumps to cause liquid in the subject discharge pipes to back-drain to the sumps during non-pumping periods. The discharge pipe for the self-priming pump is equipped with drain-down valves which cause liquid in this pipe to back-drain to the sump during non-pumping periods.

\subsection{CONTAINMENT BASIN AND CREST PAD}

The containment basin for the leachate tank was lined with 80-mil thick, smooth, HDPE geomembrane liner, by GeoPacific. The geomembrane was installed and tested in a similar manner to that described for the secondary and primary liner systems. After the tank was installed in the lined containment basin, a leak test was conducted to verify the performance of the HDPE liner. A quantity of water, slightly greater than the capacity of the tank, was placed in the containment basin, and the water level was monitored by GCS for 48 hours. Measurements of the water level were measured to the nearest 0.004 inch. The measurements indicated no change in the water level during the 48-hour test. See Appendix R-5 for leak testing documentation. After the 48-hour period, the water was removed from the basin, and GCS observed the floor of the HDPE-lined basin for signs of water accumulated beneath the liner. GCS observed no evidence of soft spots beneath the liner that would indicate leaking.

The concrete pad at the crest of the east slope was lined with 80-mil, smooth, HDPE geomembrane liner, by GeoPacific. The geomembrane was installed and tested in a similar manner to that described for the secondary and primary liner systems. The crest pad was lined to contain drips or leaks from the array of pipes, valves, and meters installed immediately above the pad. The lined pad drains into the containment basin for the leachate temporary storage tank. Only a few minor extrusion welds were required to install the HDPE liner on the crest pad. The welds on the floor of the crest pad were nondestructively tested using a vacuum box.

\section{CONTROL BUILDING AND ELECTRICAL SYSTEM}

The control building was supplied by Par Construction, of Kennewick, Washington, and installed by Delhur. The control building is a metal structure which measures approximately 12 feet long by 9 feet wide by 8.5 feet high. The building houses electrical equipment and control panels for the leachate collection and removal system pumps and ancillary equipment.

JRT Electric, Inc. (JRT) installed the electrical system for Project W-025. A Pump Control Panel Lyyout drawing, Sheet E1, and Pump Control Wiring Diagrams, Sheets E2 and E3, were provided by

JRT and are included in Volume 2 of this report. The electrical system installation included overhead service, a transformer, site lighting, and pump controls. The pump control panel was assembled by Technical Systems, Inc. of Lynnwood, Washington. The face of the main electrical control panel door contains displays which indicate the leachate levels in each the primary and the secondary lining system sumps. The digital readings are in inches, and are transmitted through a signal cable from a transducer installed in each sump. Automatic switching equipment activates the submersible pump in the secondary sump when the secondary sump transducer indicates that the leachate level has risen to 12 inches above the floor of the sump. The pump is deactivated when the transducer indicates that the leachate level in the sump has fallen to six inches. Automatic switching equipment operates the submersible pump in the primary sump in the same manner, based on the level of leachate indicated by the primary system transducer. 
The self-priming pump in the primary system is automatically activated by a float switch. For the purpose of the initial calibration, the switch was set to activate the pump when the liquid level reached a depth of 30 inches and shut the pump off when the depth was reduced to six inches (as measured from the floor of the primary sump). During the operation of the landfill, the float switch can be set to activate the pump before the leachate head on the geomembrane liner reaches one foot (as measured from the lip of the sump).

To prevent overfilling of the leachate temporary storage tank, the submersible pumps and the selfpriming pump are automatically shut-off when a float switch in the leachate temporary storage tank indicates that the leachate level in the tank is within 12 inches of the top of the tank. In the event that this float switch fails to deactivate the pumps, a high level limit switch on the tank will shut-off the pumps if the leachate level in the tank rises to within 10 inches of the top of the tank.

A flashing beacon is mounted on the roof of the control building. The beacon is energized when a sump pump fails, or when the leachate temporary storage tank is nearly full as indicated by the high level switches in the tank.

The heat trace cable is controlled by a thermostat mounted on the outside of the control building. The thermostat energizes the heat trace cable when the ambient temperature drops below $40^{\circ}$ Fahrenheit.

The electrical system was reviewed by the Design Engineer's consultant, Sparling, Inc., during the Acceptance Test Procedures. Sparling's representative indicated that the electrical system performed as designed. A copy of Sparling's report is included in Appendix S.

\subsection{LIGHTING}

The indoor and outdoor lighting systems were installed by JRT Electric, Inc. Indoor lighting was provided for the inside of the control building. The outdoor lighting system included lights mounted on four 40 -feet long wooden poles. The poles were set in holes drilled approximately six feet deep. Tro 180-watt, low-pressure sodium (LPS) lamps are mounted on the pole nearest the control building. One 180-watt LPS lamp was installed on each of other three poles. Each of the outdoor lights is equipped with a photocell. The outdoor lighting system was tested by covering the

photocells, and the system performed as required. GCS observed the testing.

\subsection{DRAINAGE FEATURES}

The drainage for Project W-025 was designed and installed such that the paved truck unloading area drains into the landfill, and all other areas beyond the crest of the landfill drain outward, away from the landfill. The pavement in the truck staging area drains away from the landfill. Between the landfill crest and the perimeter road, the area was graded to provide drainage toward the perimeter road. The perimeter road is sloped outward, at a grade of approximately one percent, to provide drainage away from the landfill. On the outside of the perimeter road, on the north and west sides of the landfill, drainage ditches were excavated as required to provide drainage away from the landfill. 
WHC-SD-W025-RPT-001, Rev. 0

\subsection{ACCEPTANCE TEST PROCEDURES}

The Acceptance Test Procedures (ATPs), outlined in the project specifications, were performed to demonstrate that the electrical and instrumentation systems function as intended by the design. The following is a list of the components tested:

- Heat träe system

- Transducers and meter/controllers - primary and secondary sumps

- Submersible pumps and spare - primary and secondary sumps

- Self priming pump - primary sump

- Transfer pump and spare - leachate tank

- Flow meters

- Leachate tank high level switches

- Control building power and lighting

- Outdoor lighting

- Control panel heaters

The ATPs were initially performed on April 14, 1994, and were observed by GCS, the Design Engineer, and the COTR. The results of the ATPs indicated that the electrical and instrumentation systems function as intended by the Design Engineer, with a few minor exceptions. The exceptions included discrepancies in flow meter readings, minor dripping from vacuum release valves, and occasional sticking of the high-level limit switch. The three minor discrepancies were included as punch list items to be reconciled in the near future. Documentation of the results of the initial ATPs were presented in a report prepared by Westinghouse Hanford Company, Acceptance Test Report. WHC-SD-W025-ATR-001, Rev. 0, dated May, 1994.

Engineering Change Notices subsequently required the replacement of the flow meters, the replacement of the vacuum release valves, and modifications to the piping configuration near the flow meters. Acceptance Test Procedures were performed on October 4, 1994 to demonstrate that the new components function as intended by the design. These ATPs were observed by GCS, WHCOperations, and the COTR. The results of these ATPs indicated that the new flow meters, piping, and vacuum/air release valves function properly. Documentation of the results of these ATPs were presented in a report prepared by Westinghouse Hanford Company, Acceptance Test Report, WHCSD-W025-ATR-001, Rev. 1, dated October, 1994. 
WHC-SD-W025-RPT-001, Rev. 0

\subsection{CONCLUSION}

The activities described above associated with the construction of the Radioactive Mixed Waste Land Disposal Facility, Non-Drag-Off, Project W-025, in the 200 West Area of the Department of Energy's Hanford Nuclear Reservation, near Richland, Washington, were observed by GCS personnel. Based on GCS's observations and the results of testing reported herein, it is GCS' opinion that the above mentioned system has been constructed in substantial compliance with the design drawings and project specifications, including the engineering changes documented in this report. 
WHC-SD-W025-RPT-00I，Rer。0"

FIGURES 


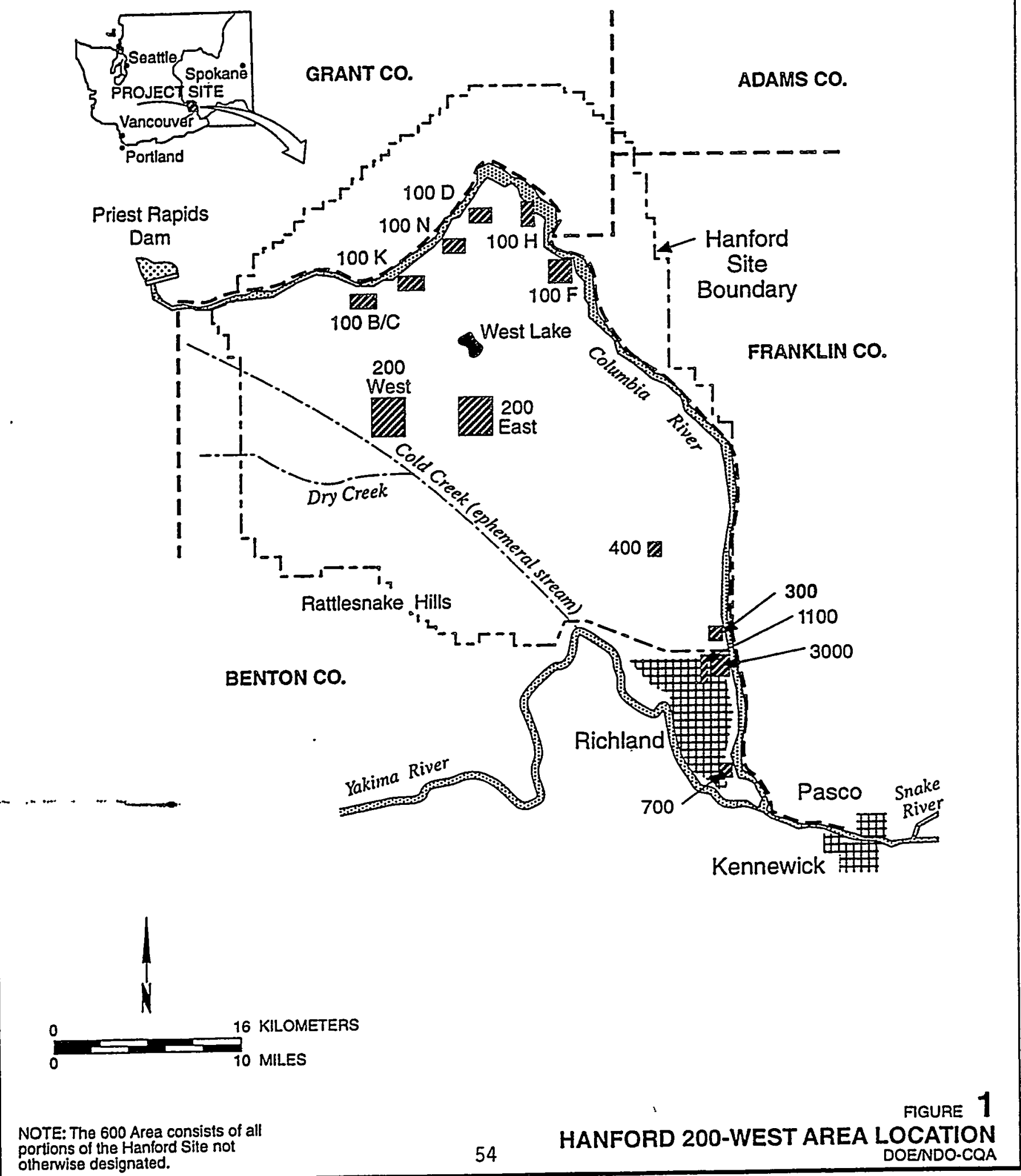




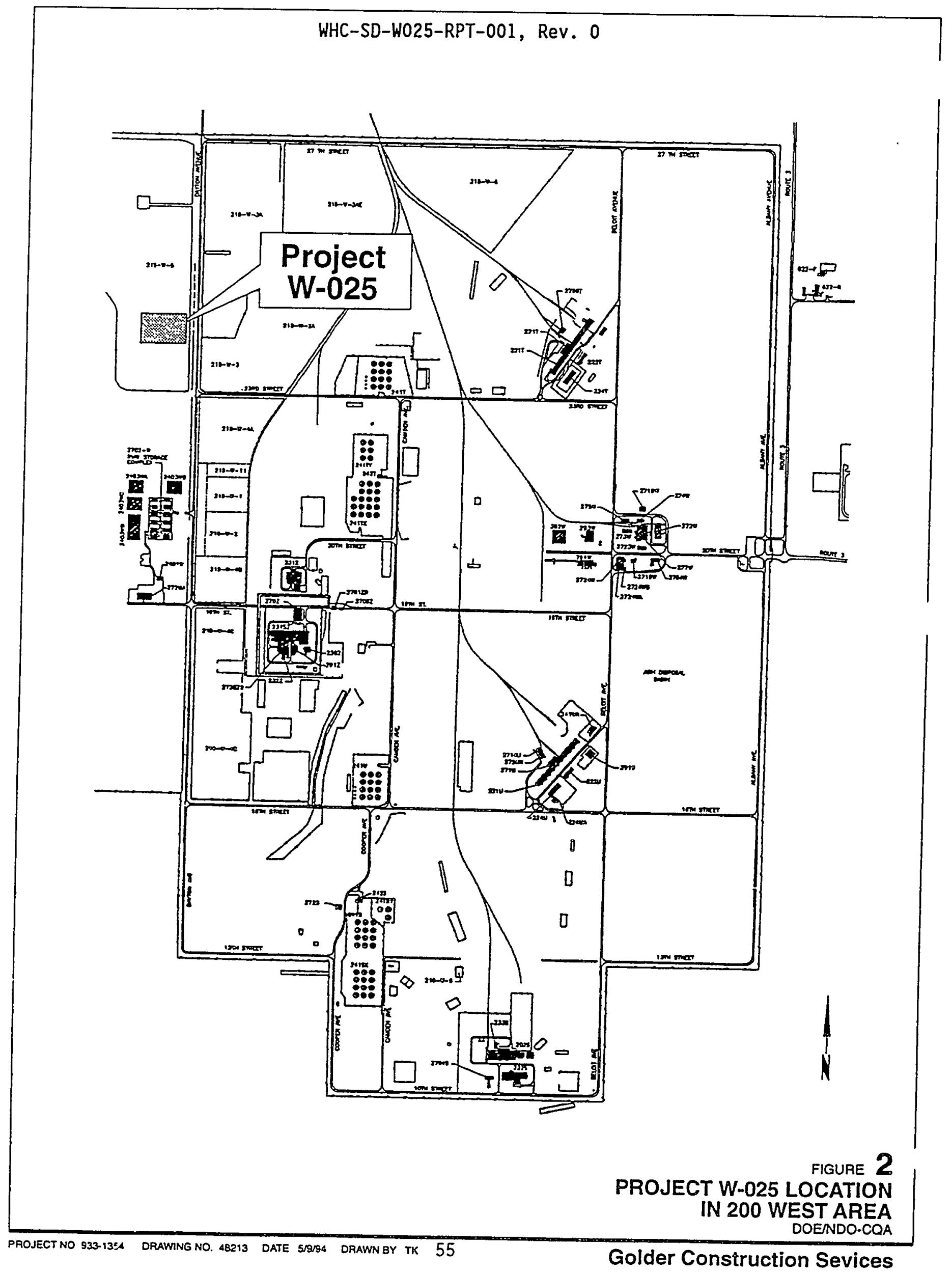


WHC-SD-W025-RPT-001, Rev. 0

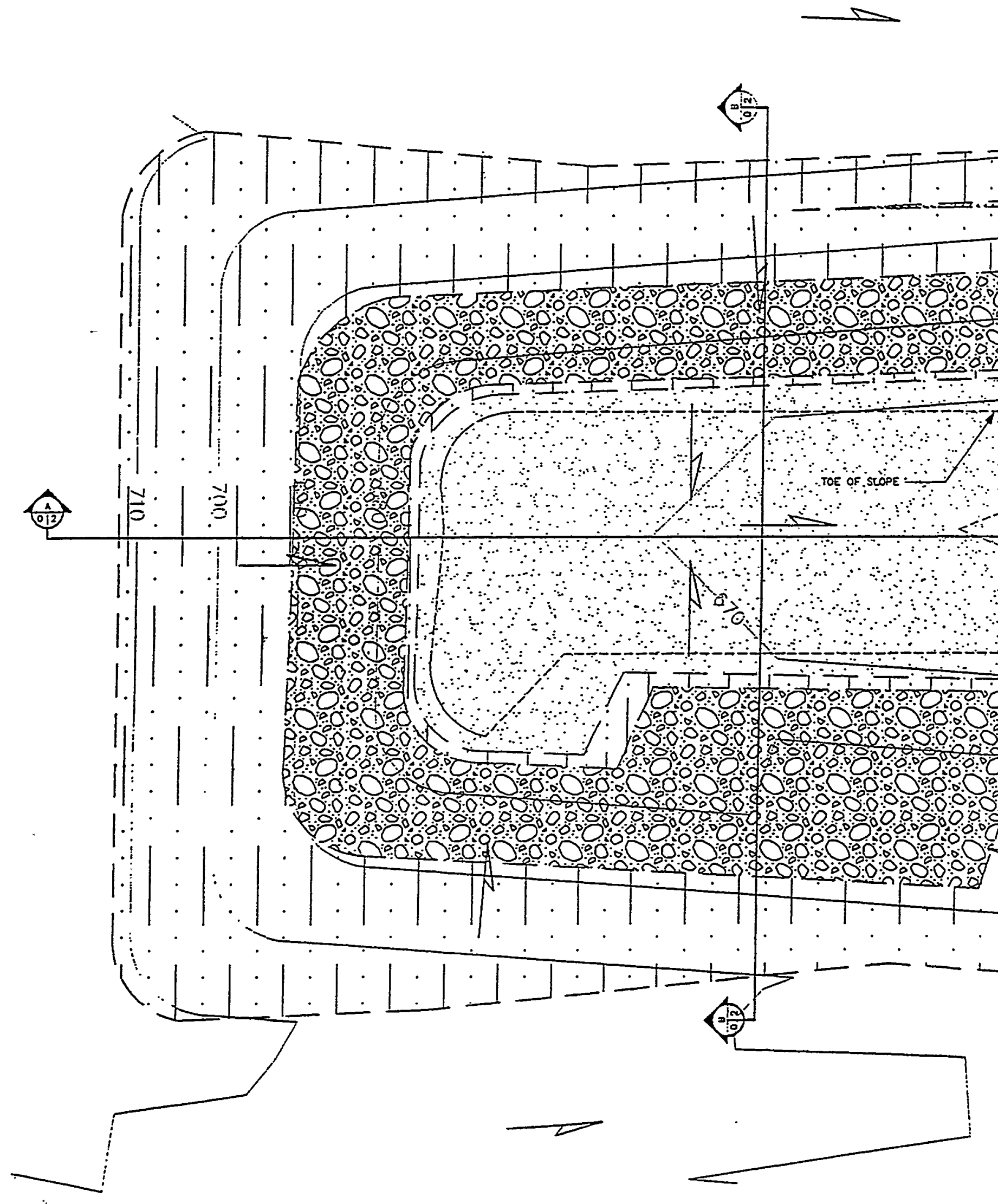


HHC-SD-W025-RPT-001, Rev. 0
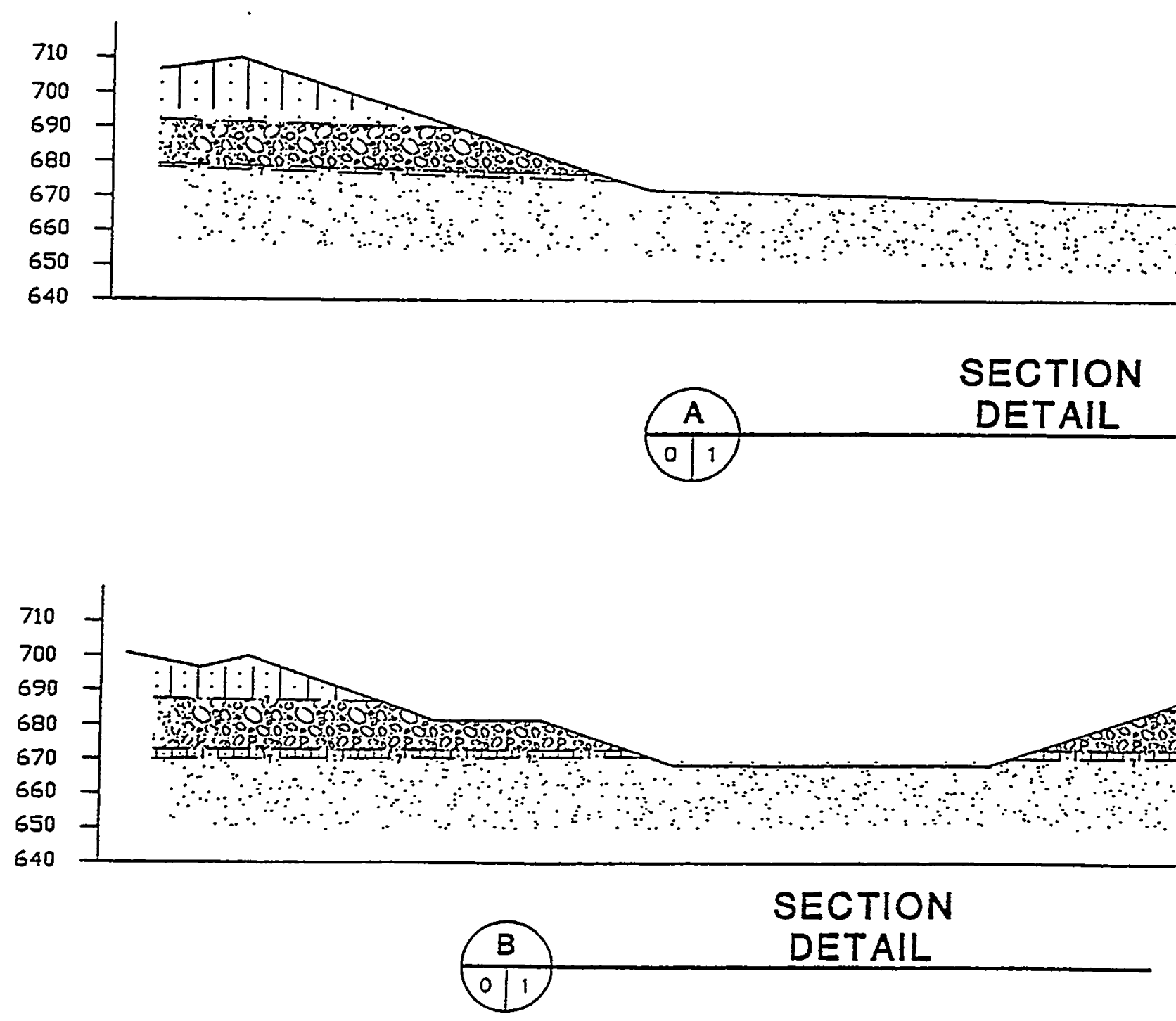
EXPLANATION

\begin{tabular}{|c|c|c|c|}
\hline \multirow{5}{*}{$\frac{}{5}$} & \multirow{2}{*}{ 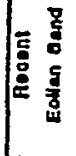 } & $\vdots \vdots \vdots \vdots$ & Eolian Sand - Compoct, dive groy $(5 Y 3 / 2)$. silty fine SAND \\
\hline & & 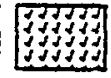 & Loose to compoct, pale yellowish brown (10YR 6/2) ASH \\
\hline & \multirow{3}{*}{ 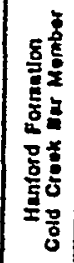 } & 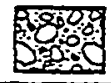 & Dense to very dense, Olive block (5Y 2/1). grovelly fine to coorse SAND \\
\hline & & 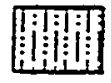 & Dense, moderote olive brown ( $5 Y$ 4/4). fine sondy SILT \\
\hline & & & Dense to very dense. light olive groy $(5 Y 5 / 2)$. fine to coorse SAND \\
\hline
\end{tabular}

- - Approximate stratigraphic unit contact location.
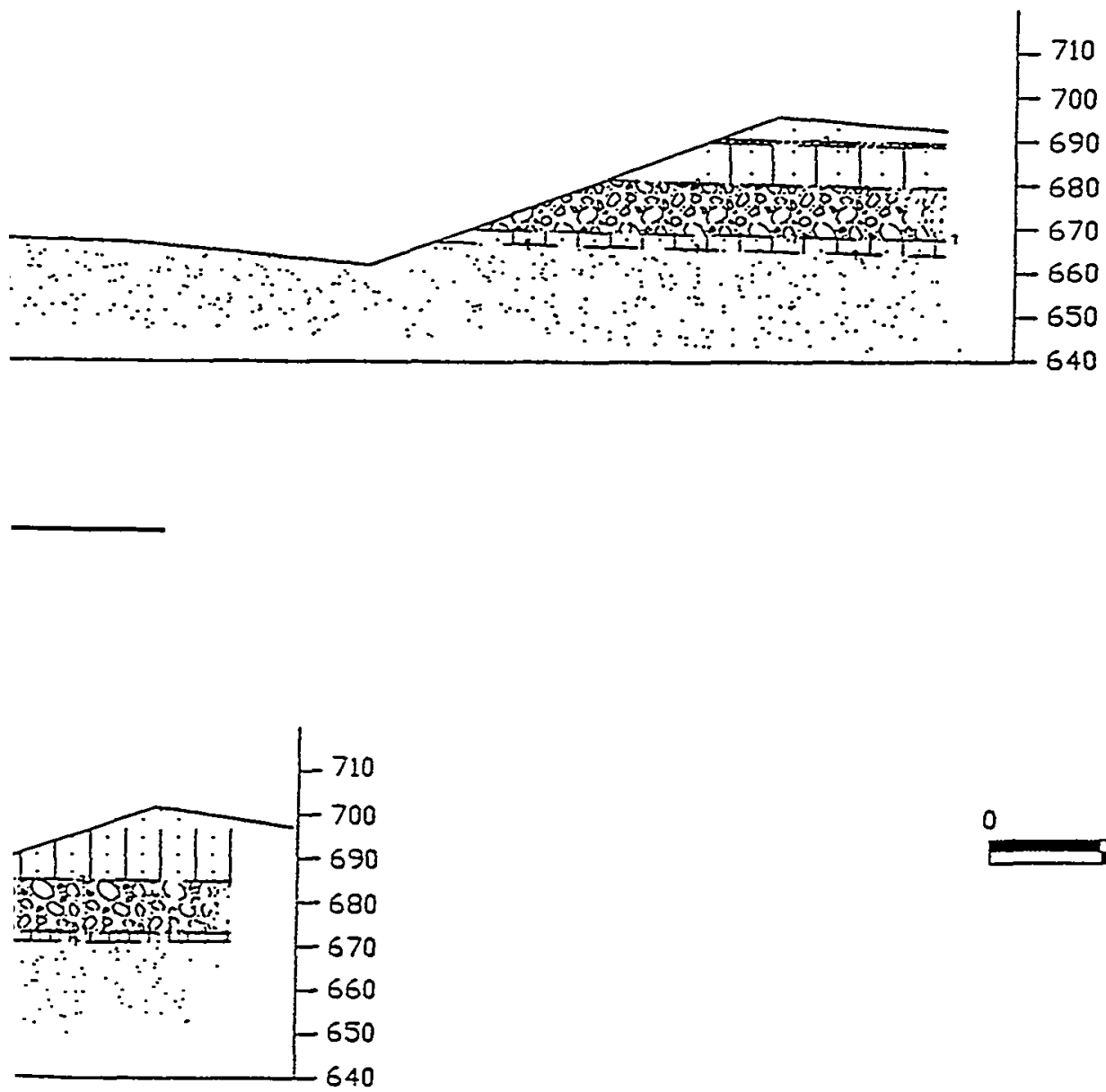

0

FEET 


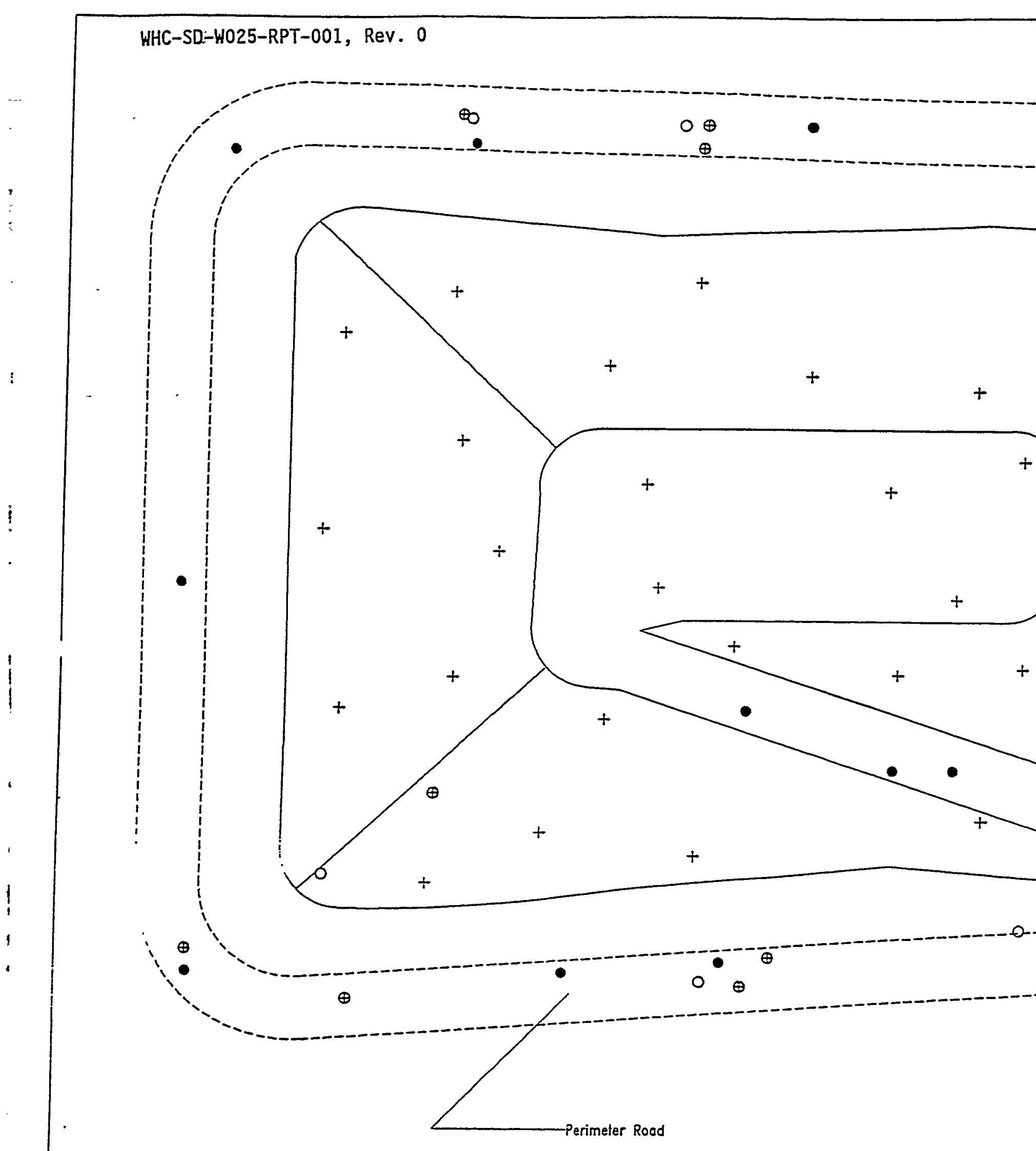




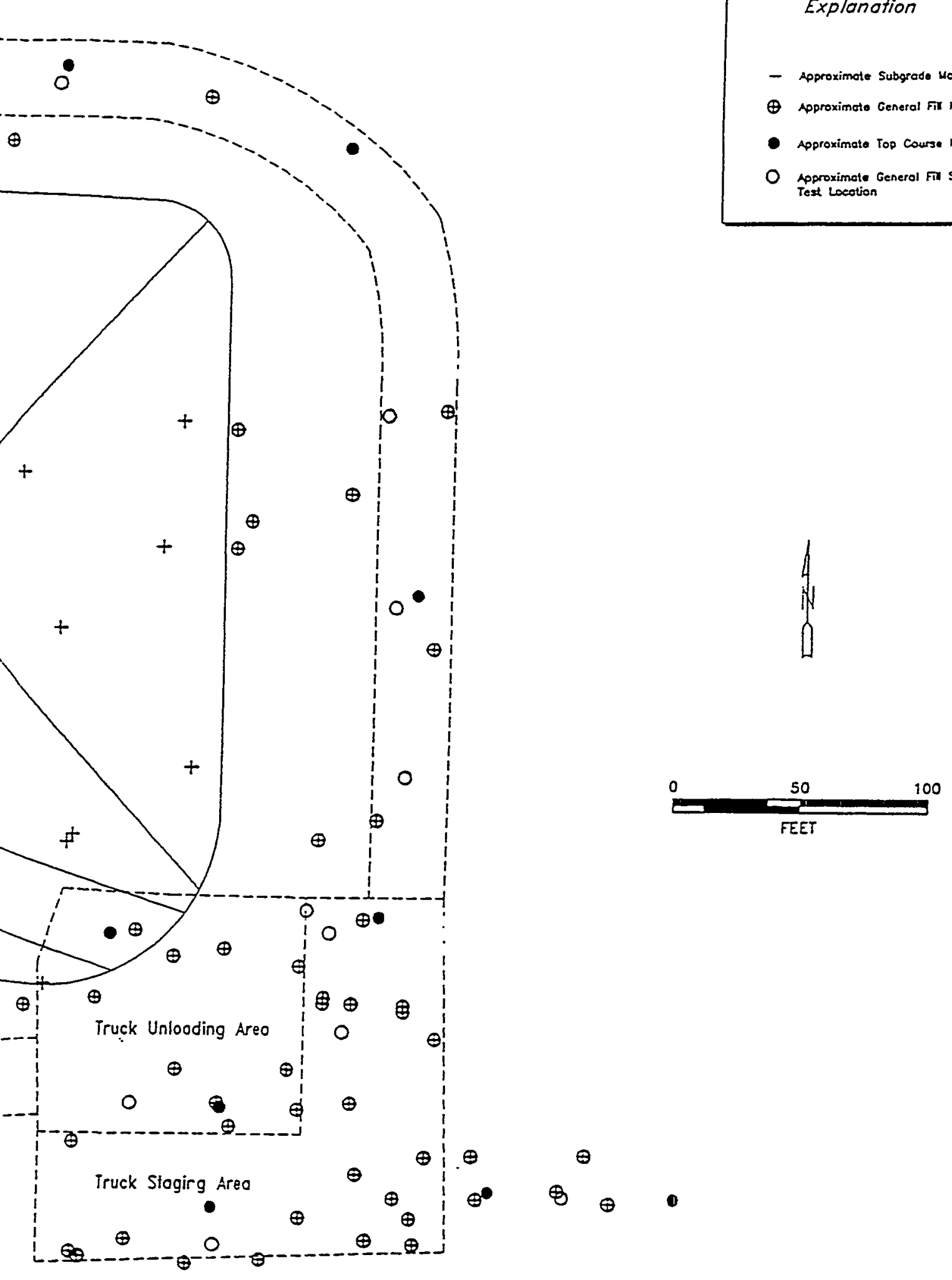


HHC-SD-W025-RPT-001, Rev. 0

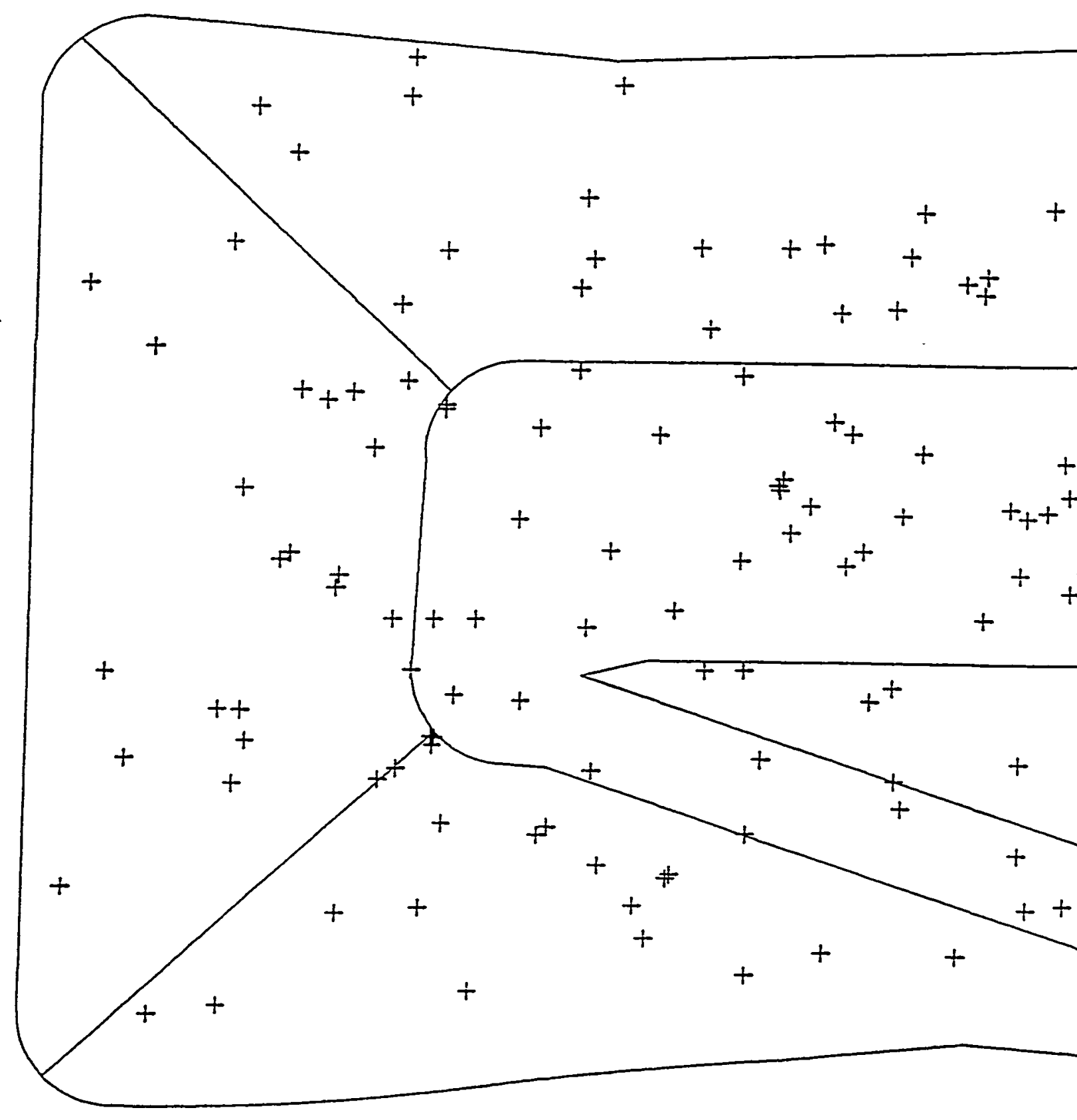




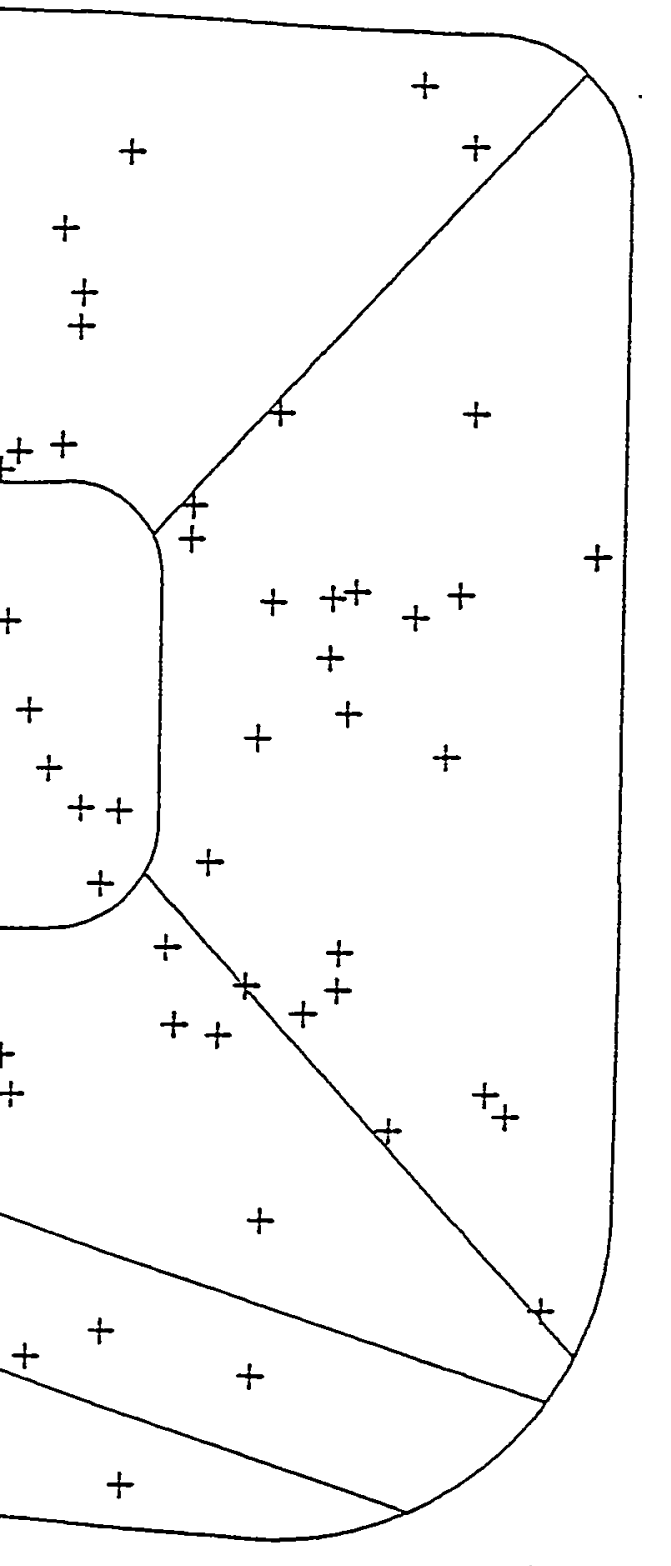

\section{Explanation}

Approximate Moisture-Density Test Locotion

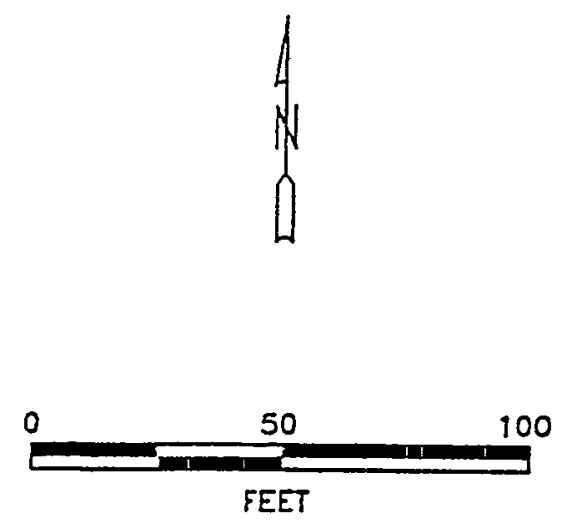

FIGURE 6

Secondary Soil Liner Moisture-Density Test Locations DOE/NDO CQA/WA 
WHC-SD-W025-RPT-001, Rev. 0

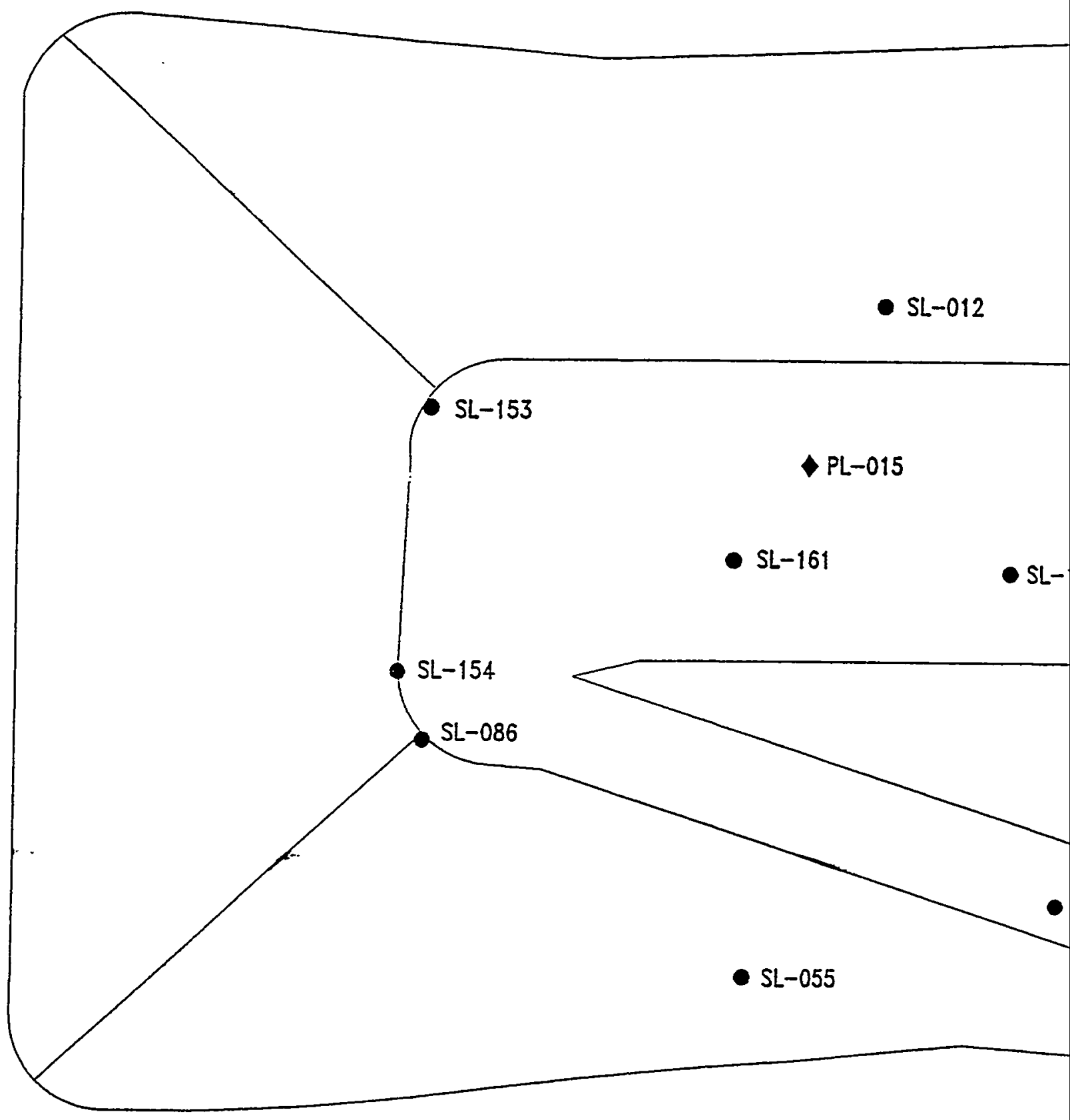




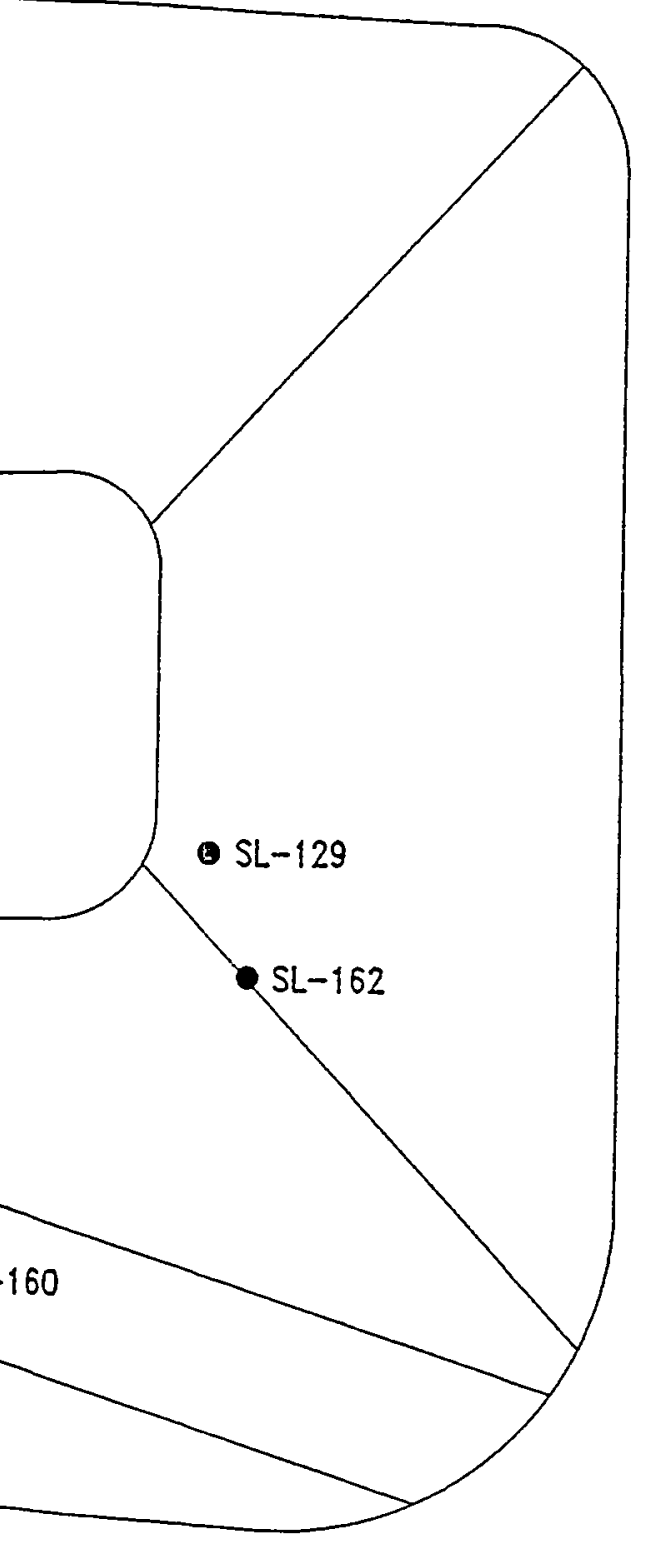

\section{Explanation}

- Secondary Soil Liner Shelby Tube Sample Number and Approximote Sample Location

Primory Soil Liner Shelby Tube Somple Number and Approximate Sample Locotion
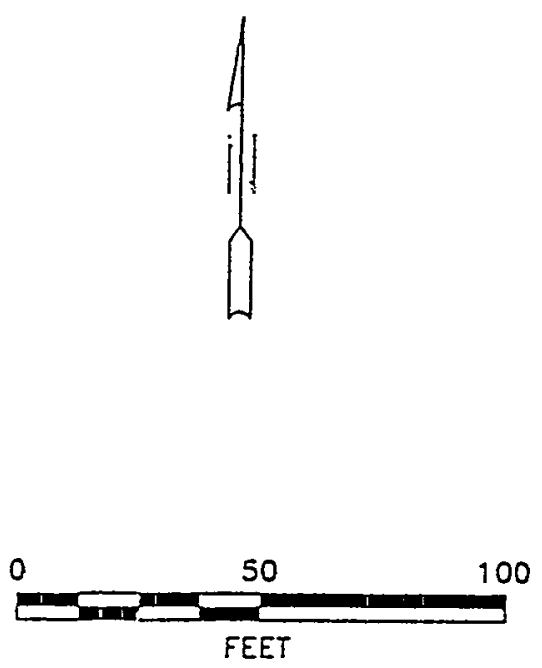

FIGURE 7

SHELBY TUBE SAMPLE LOCATHONS DOE/NDO CQA/WA 


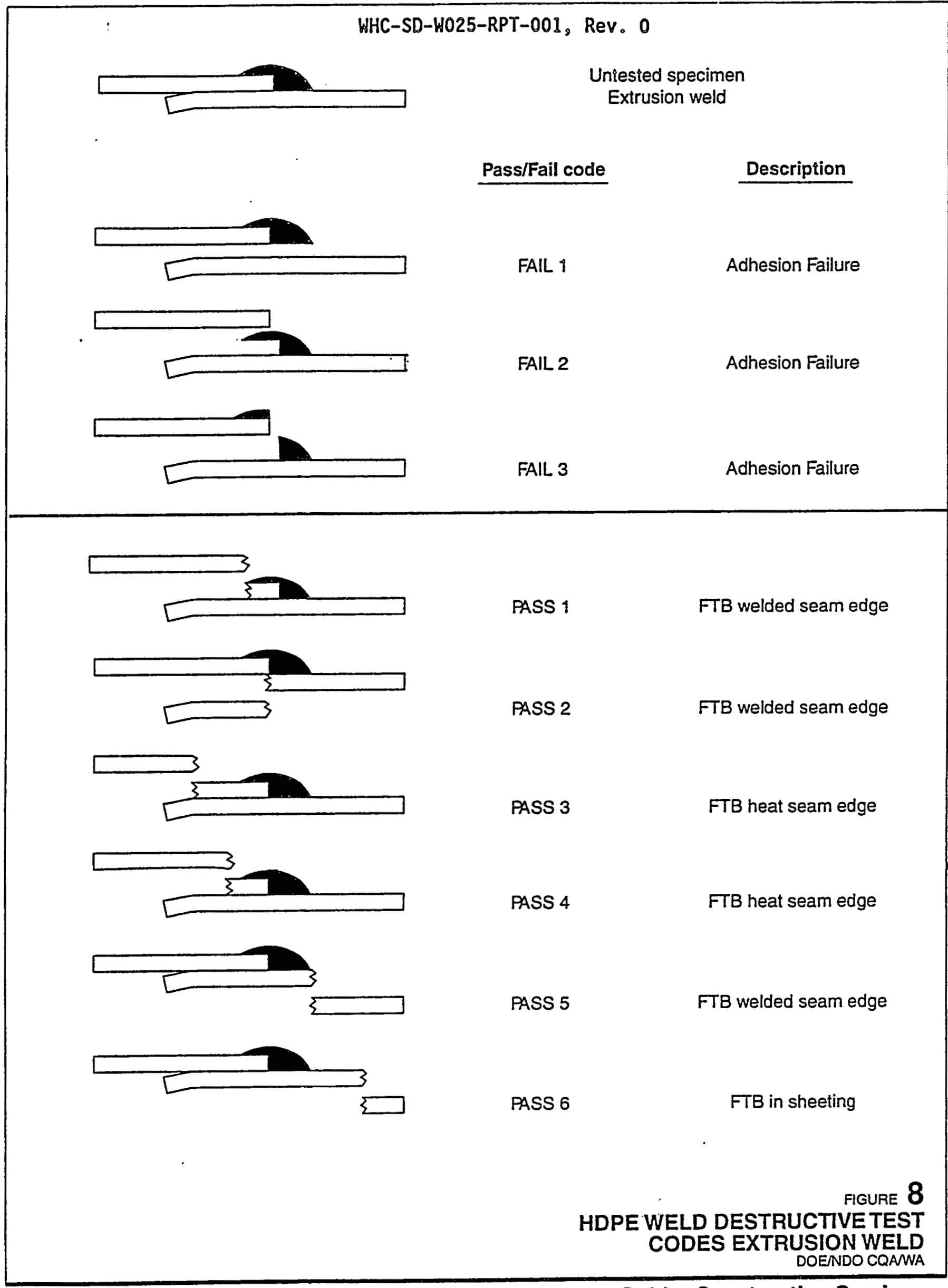




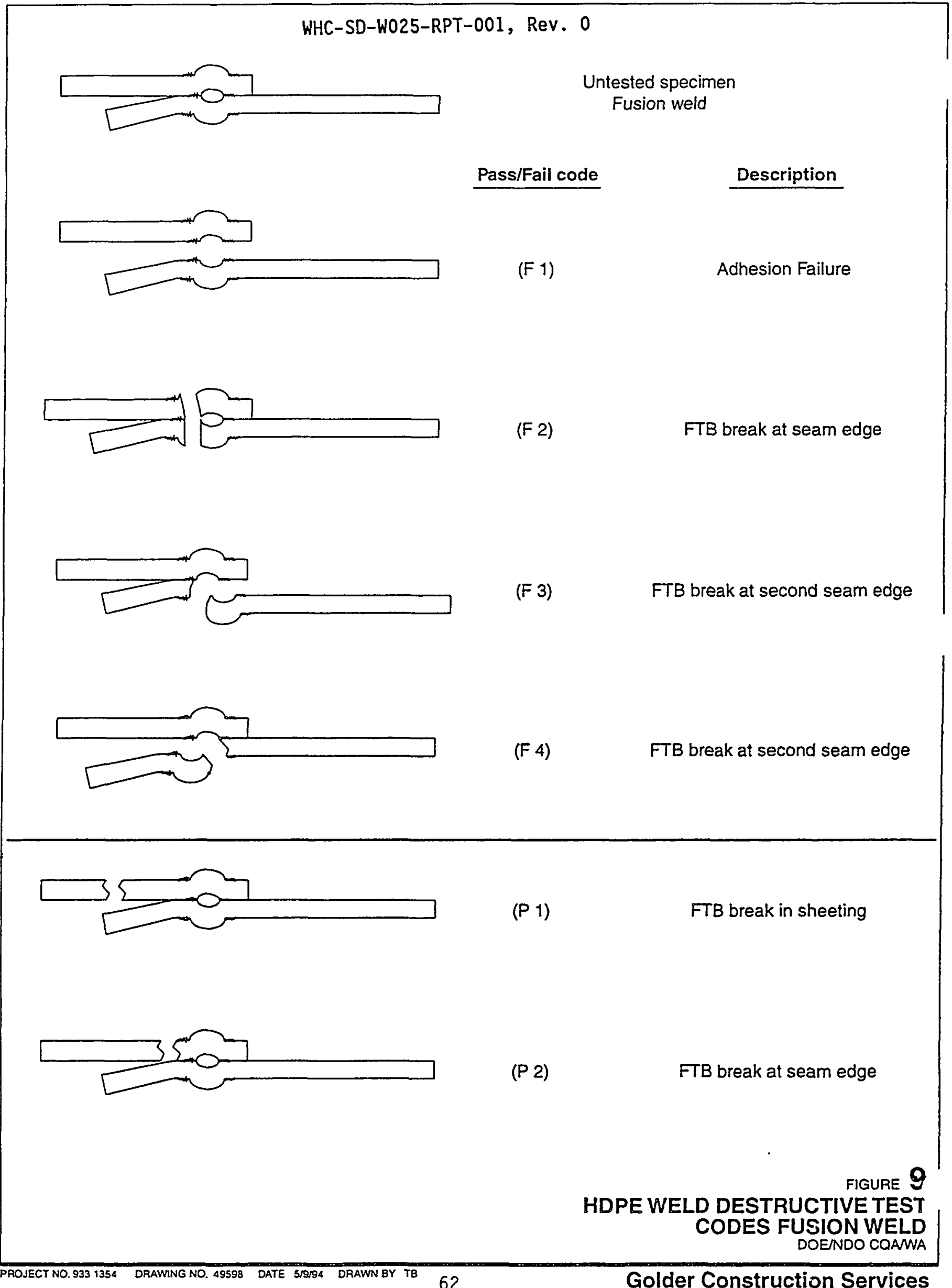


WHC-SD-W025-RPT-001, Rev. 0

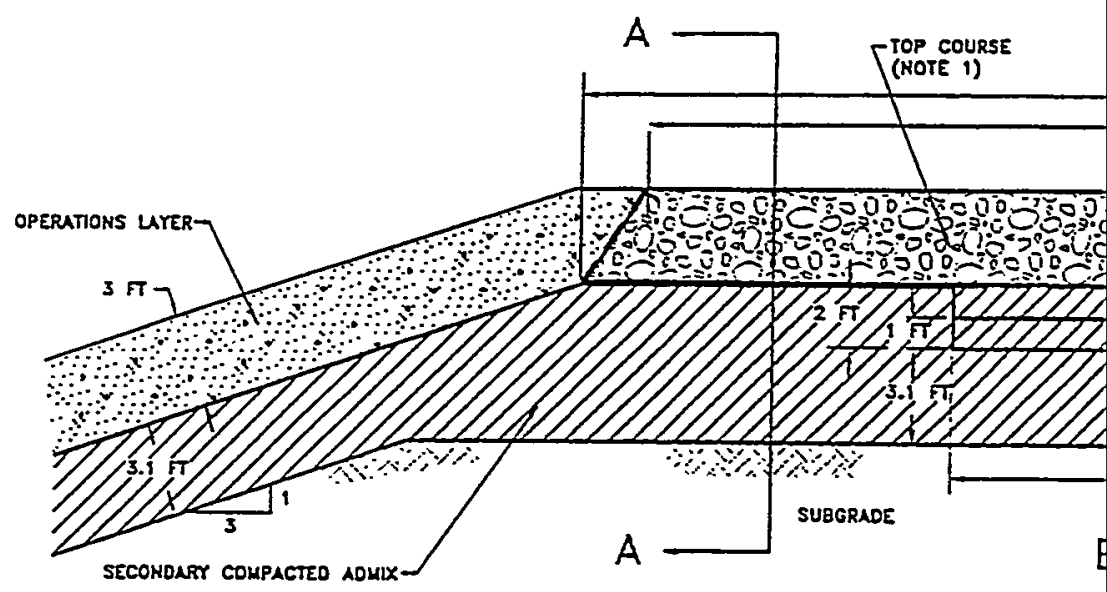

ACCESS RAMP SE

TYPE A GEOTEXTILE GEONET/GEOCOMPOSITE AND PRIMARY HDPE

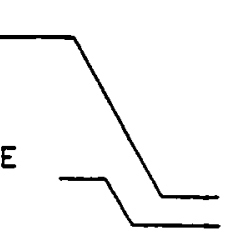

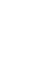

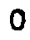

8

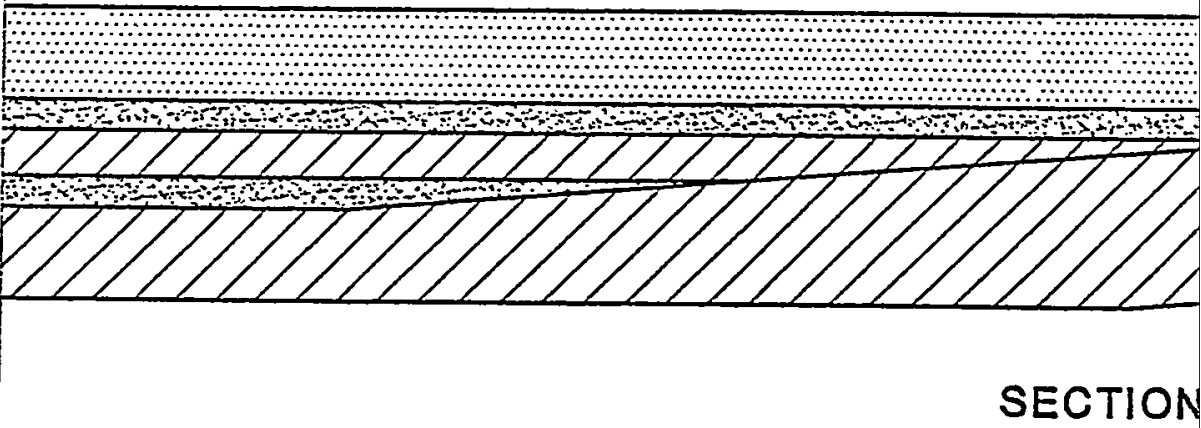
GEONET/GEOCOMPOSITE
AND SECONDARY HDPE GEONET/GEOCOMPOSITE
AND SECONDARY HDPE

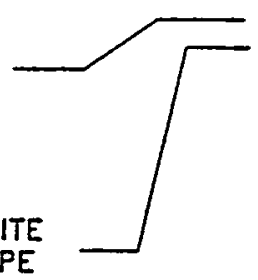

TYPE B GEOTEXTILE

SECTION
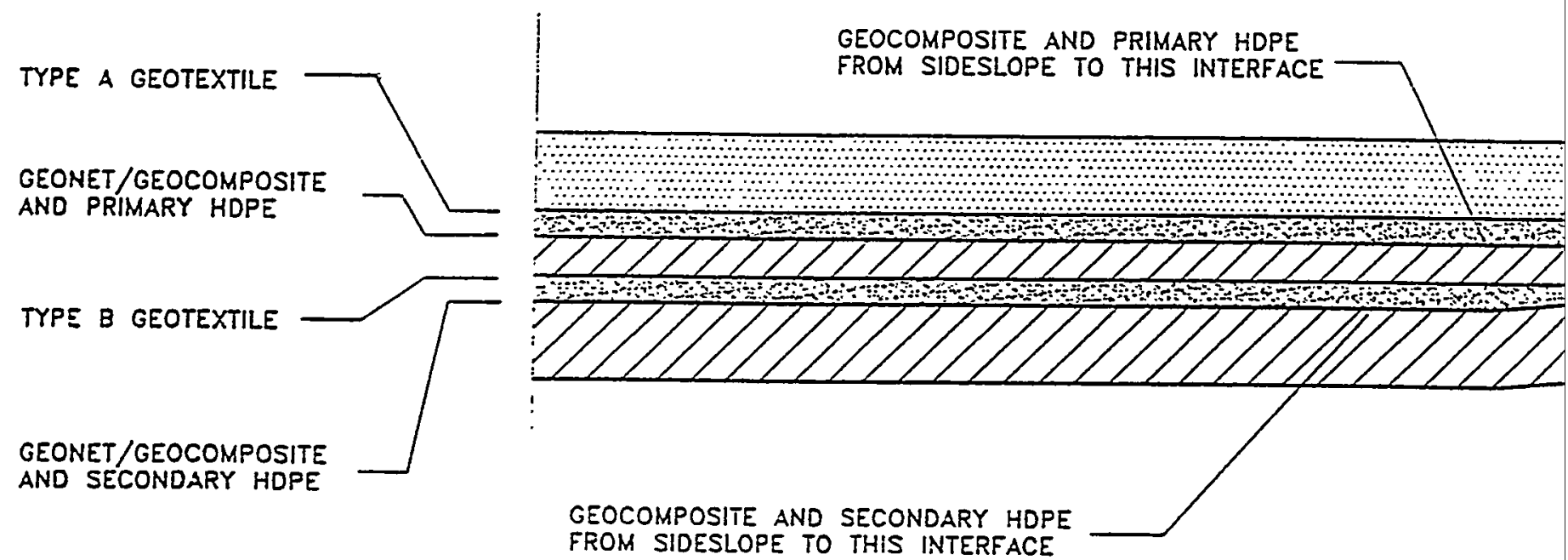
FROM SIDESLOPE TO THIS INTERFACE 


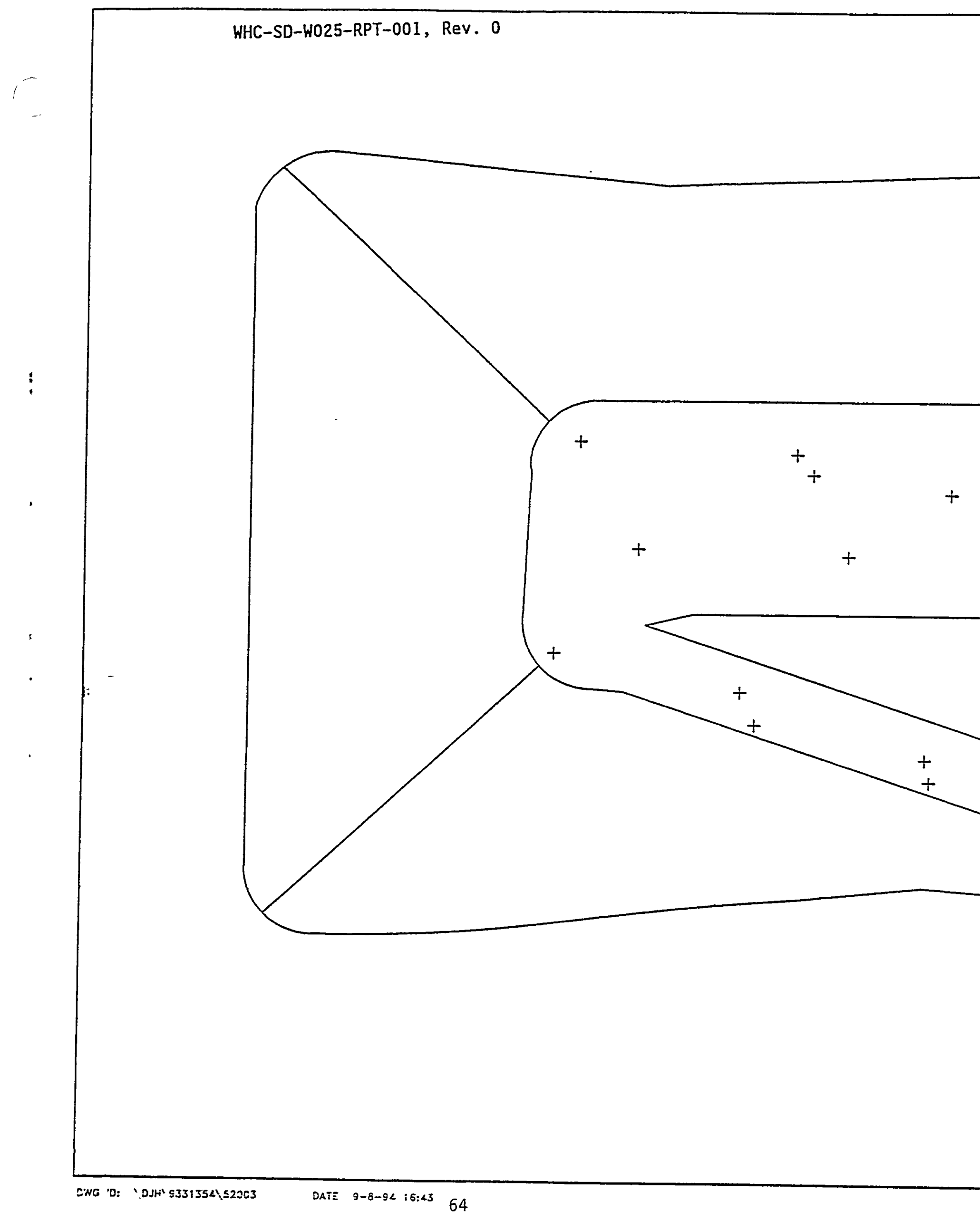




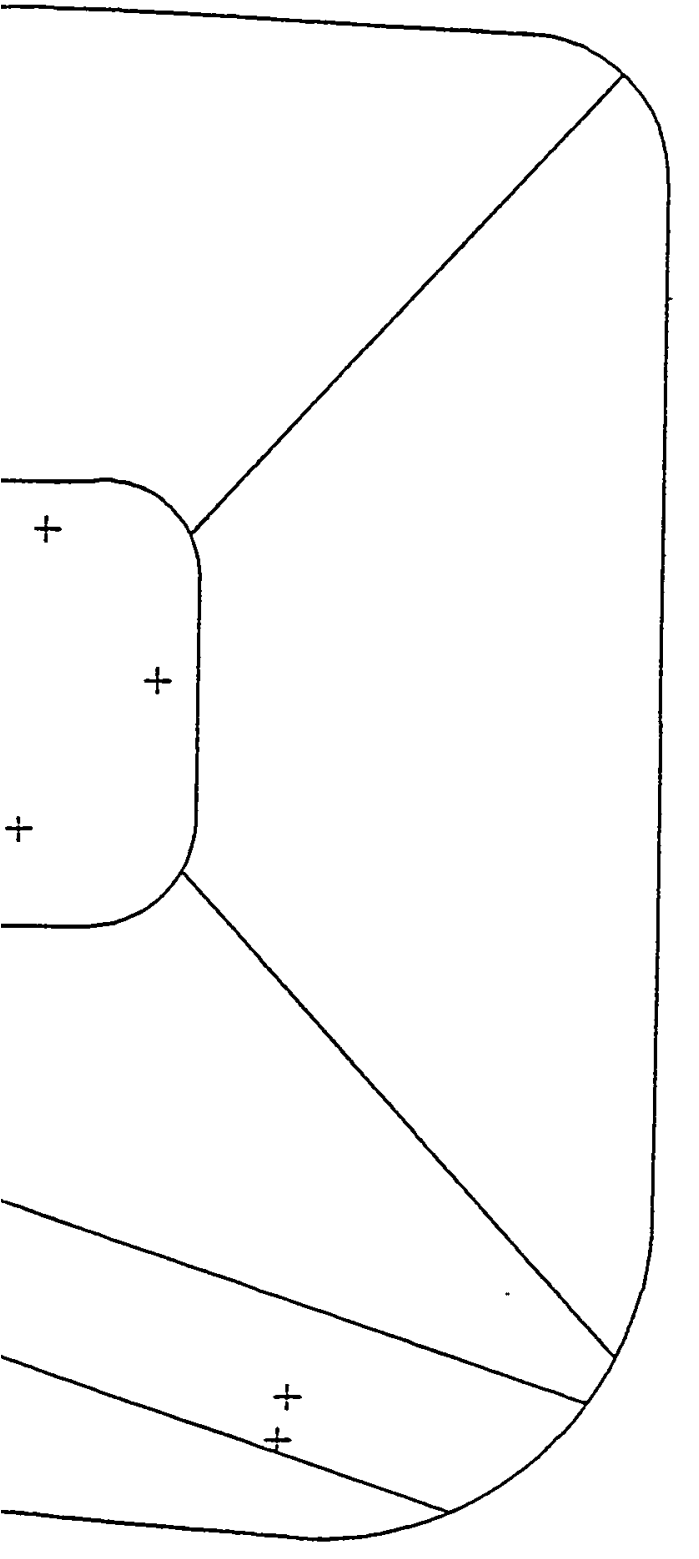

+ Approximate Moisture-Density Test Location

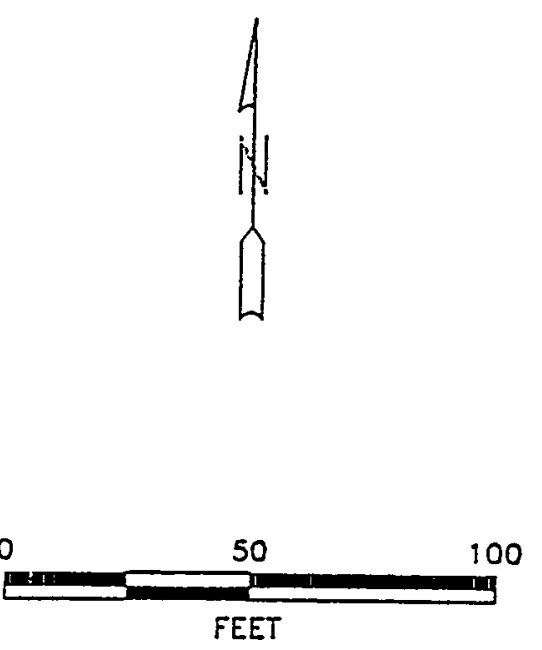

FIGURE 11

PRIMARY SOIL LINER MOISTURE-DENSITY TEST LOCATIONS DOE/NDO CQA/WA 
WHC-SD-W025-RPT-001, Rev。 0

APPENDIX A

CONSTRUCTION SCHEDULE AND GCS ONSITE STAFF 
WHC-SD-W025-RPT-001, Rev. 0

菂

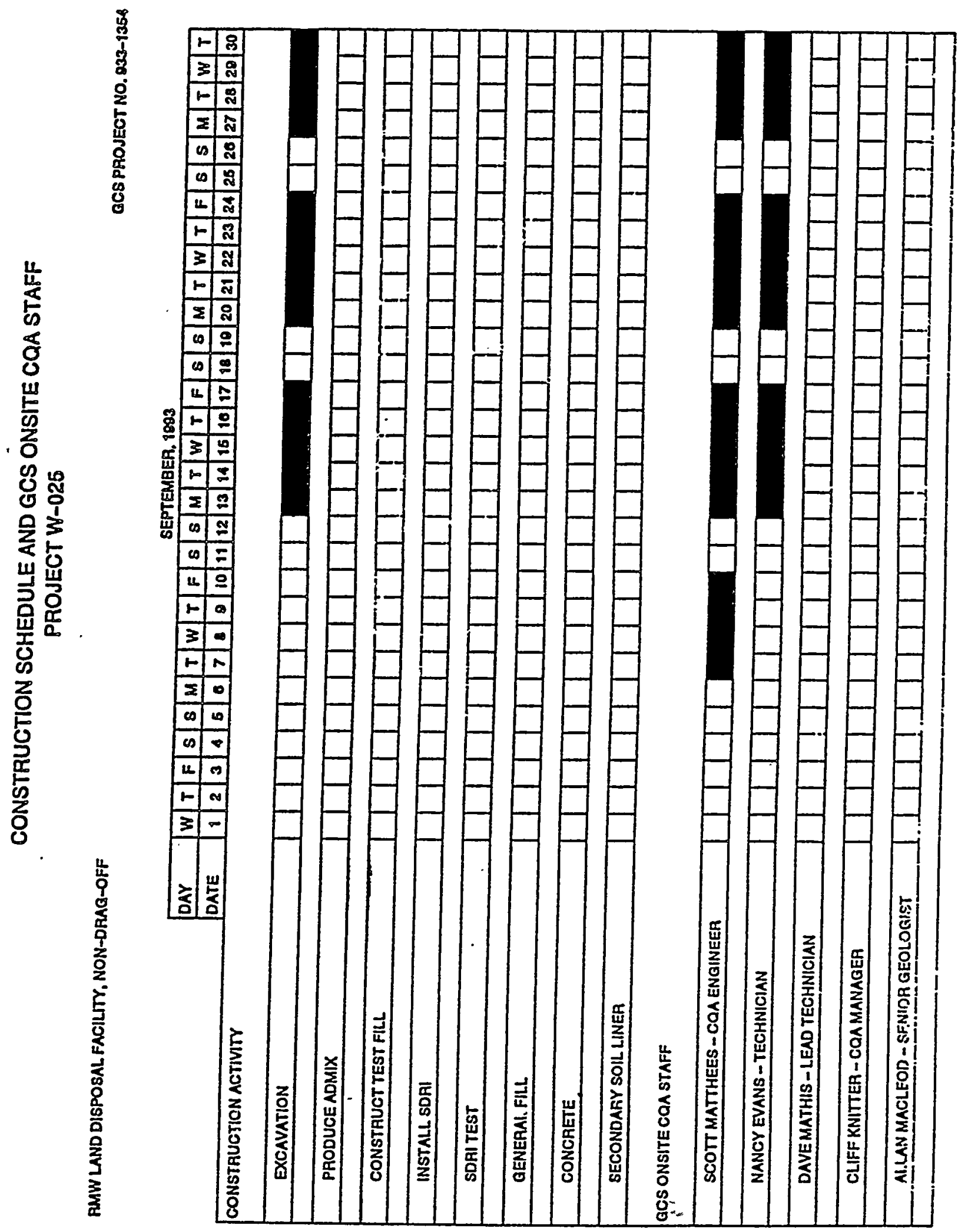


WHC-SD-W025-RPT-001, Rev. 0

$\underset{0}{w}$

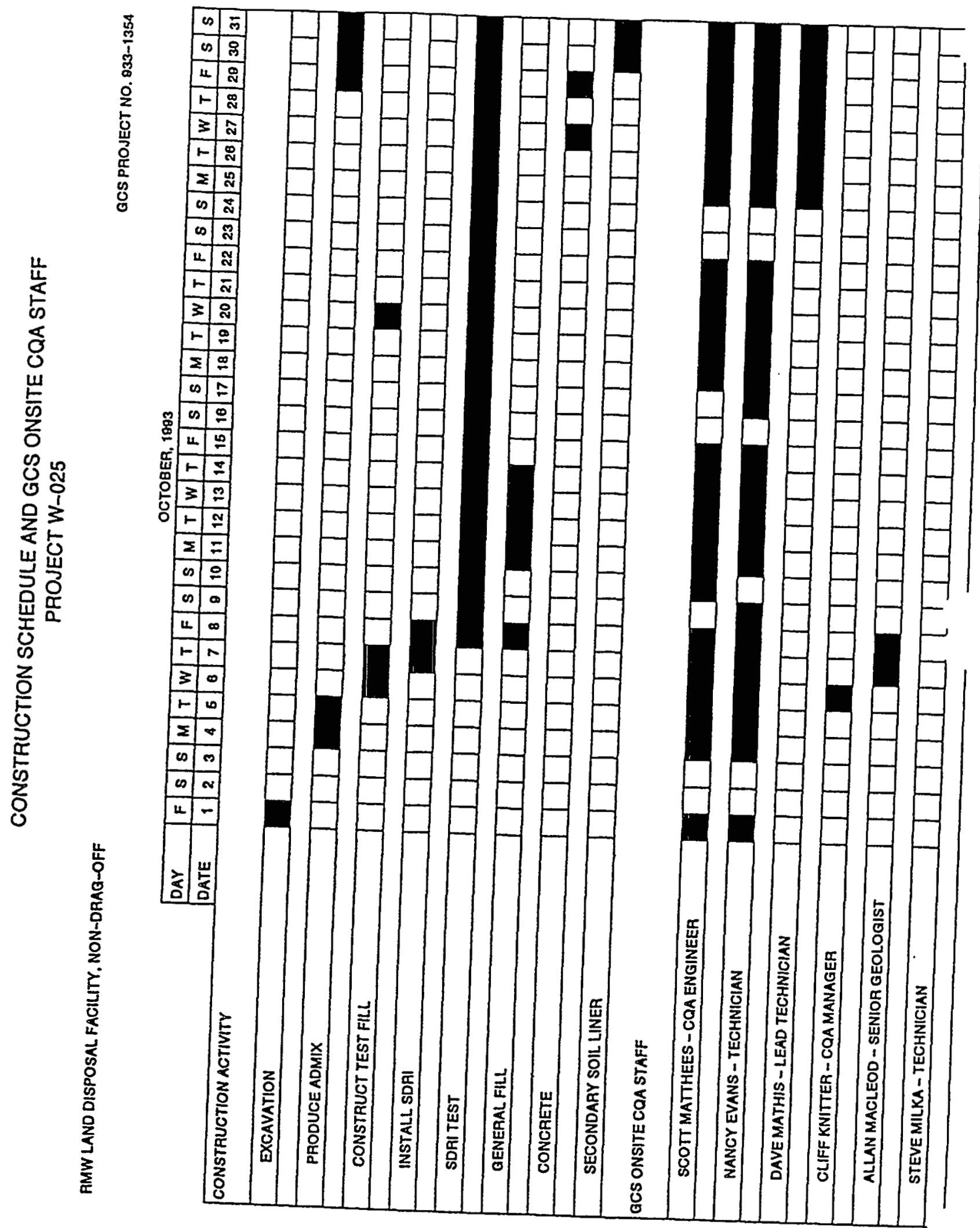


WHC-SD-W025-RPT-001, Rev. 0

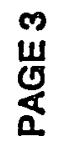

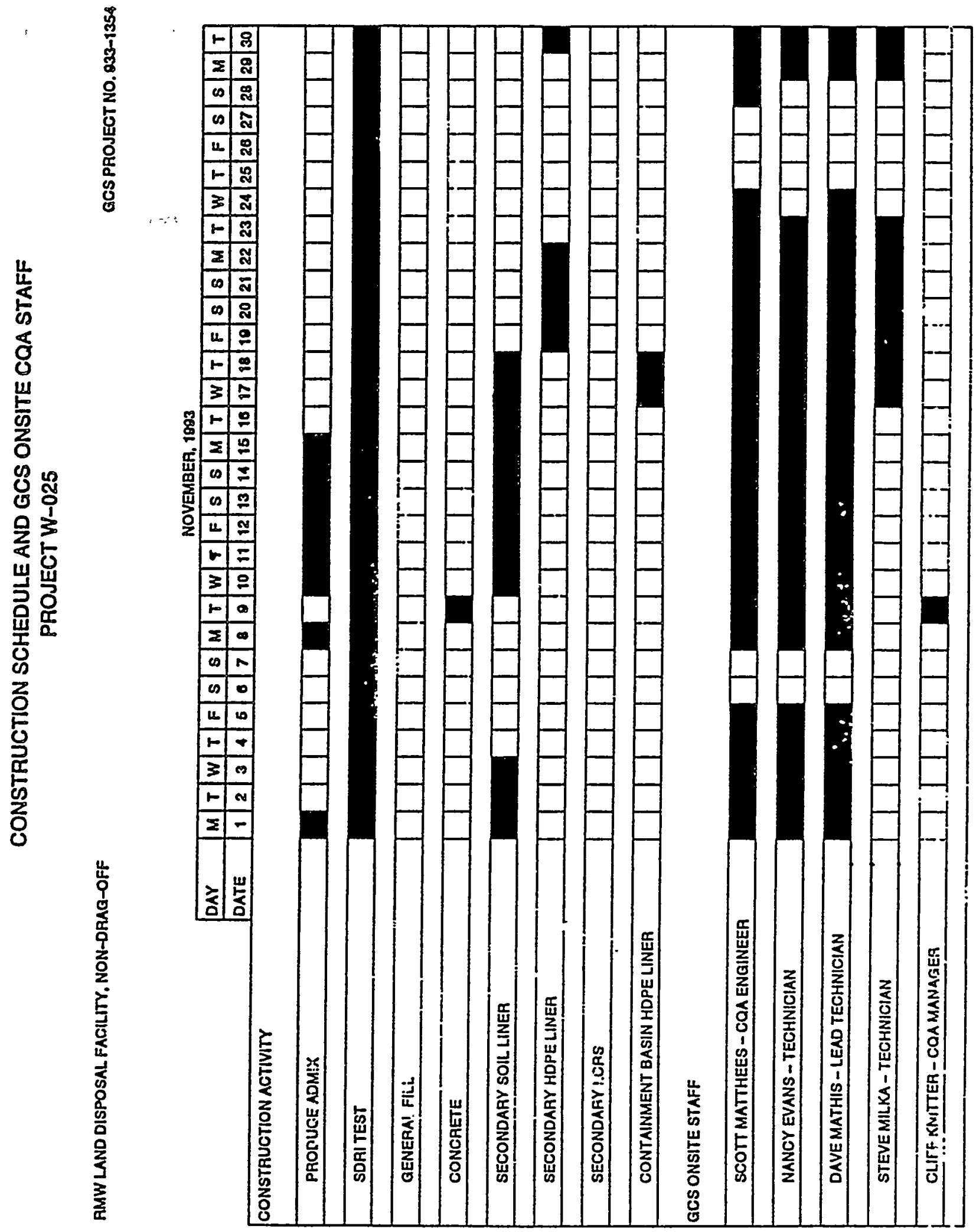


WHC-SD-W025-RPT-001, Rev. 0

$\underset{\substack{u \\ \hdashline}}{\frac{w}{a}}$

$\frac{1}{6}$
$\frac{5}{6}$
$\frac{5}{0}$
$\frac{11}{6}$
$\frac{5}{2}$
0

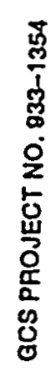

ญ ్ㅇ

只立

荘

운

$\frac{1}{0}$

क

方

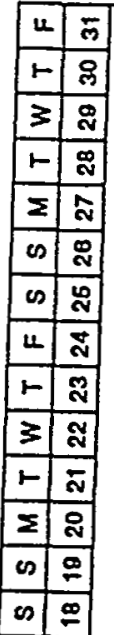

\begin{tabular}{|l|l|}
\hline 4 & $=$ \\
\hline 1 & 9 \\
\hline 3 & 9 \\
\hline 1 & 9 \\
\hline 0
\end{tabular}

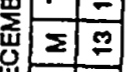

㟧事

$\infty=$

4 우

\begin{tabular}{|l|l|}
\hline-1 & $\infty$ \\
\hline 3 & $\infty$ \\
\hline $1-$ & 0
\end{tabular}

$1-1$

\begin{tabular}{|l|l|}
\hline$\Sigma$ & $\infty$ \\
\hline 6 & 0 \\
\hline 0
\end{tabular}

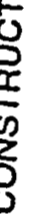

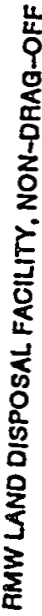

\begin{tabular}{|l|l|}
\hline$\infty$ & \\
\hline 4 & $\infty$ \\
\hline- & $N$ \\
\hline 3 & - \\
\hline
\end{tabular}

$3-$

高㟔 
岂

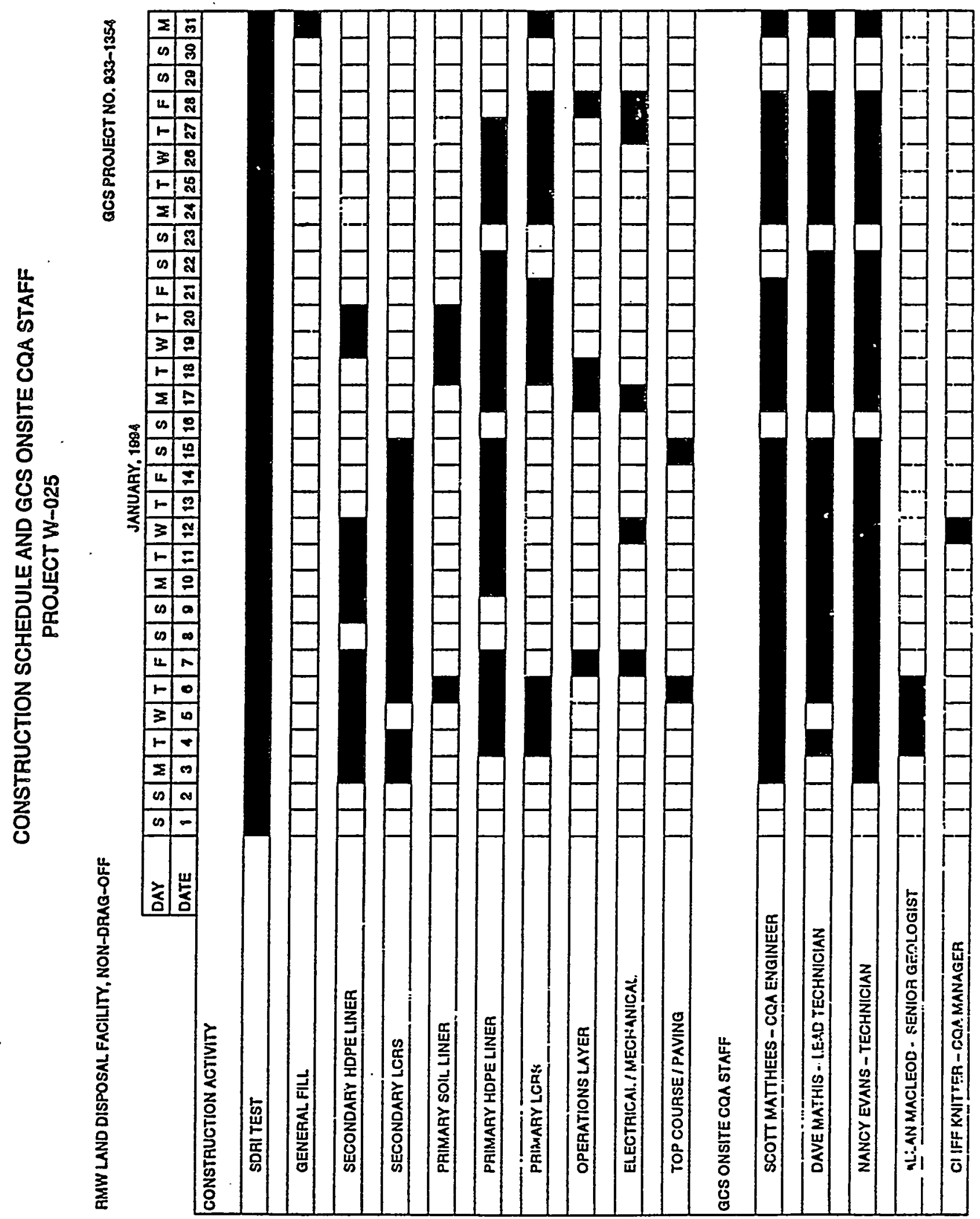


WHC-SD-W025-RPT-001, Rev. 0

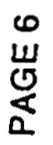

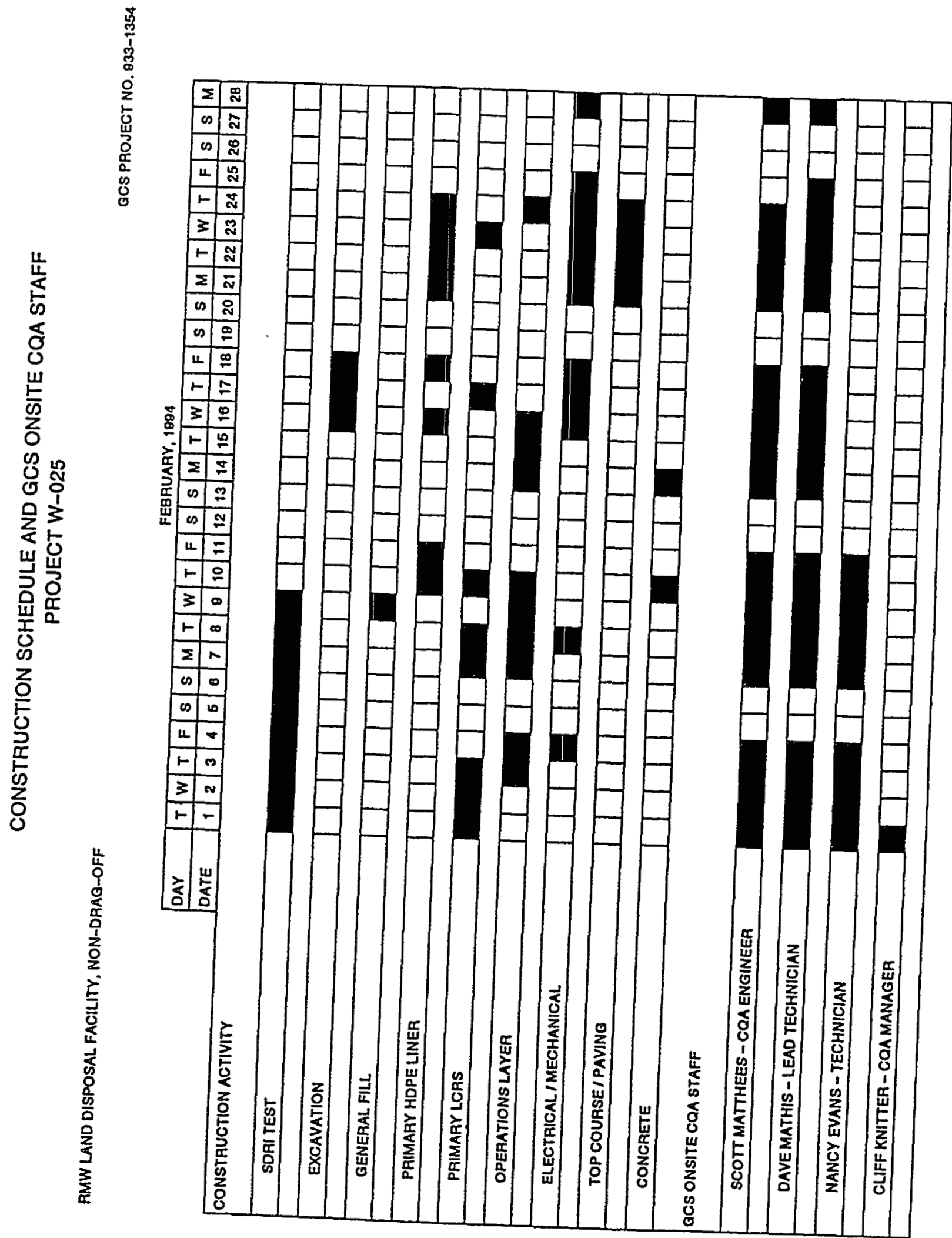


WHC-SD-W025-RPT-001, Rev. 0

U
0
0

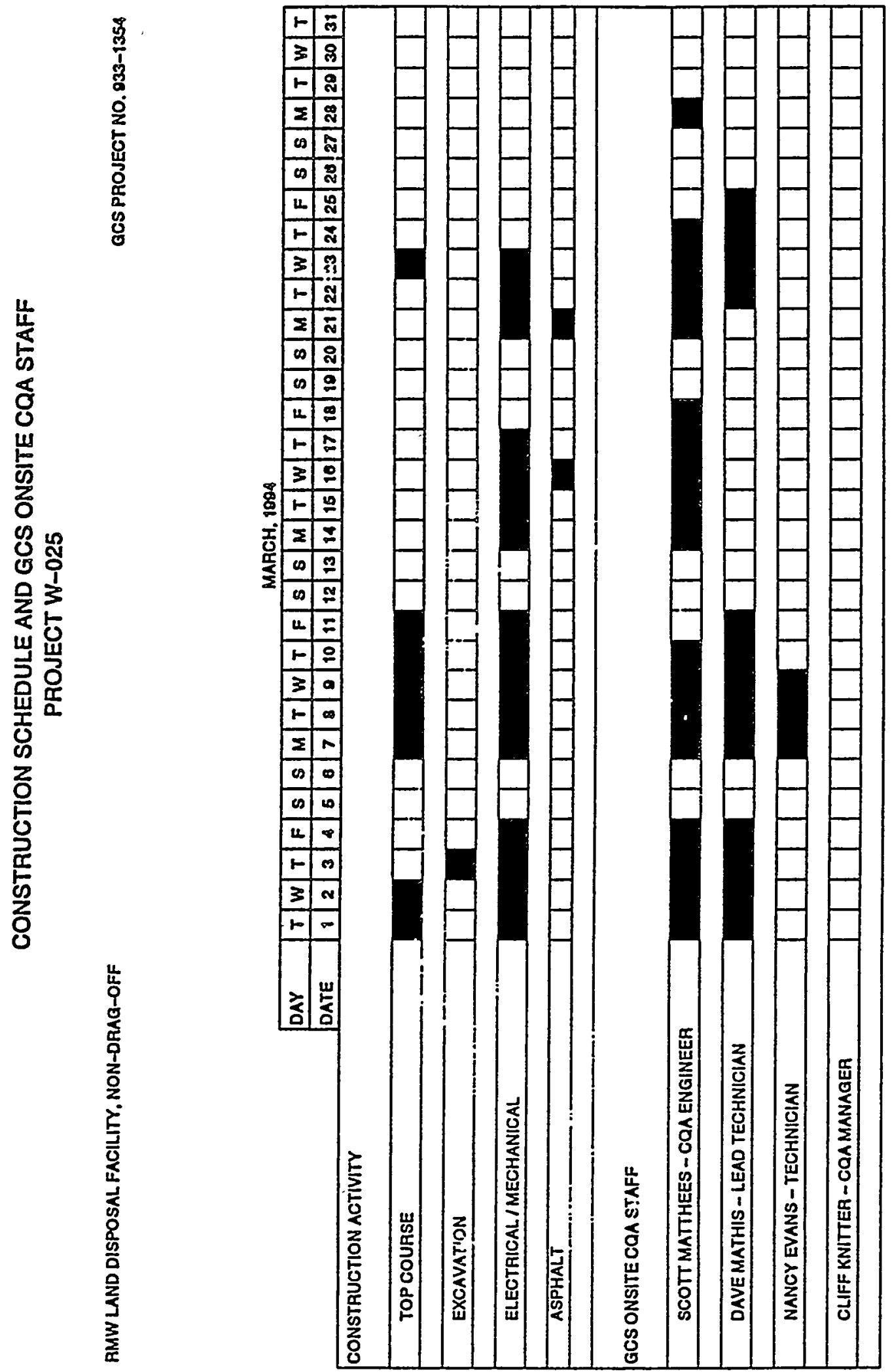


WHC-SD-W025-RPT-001, Rev. 0

$\frac{\infty}{\mathbb{N}}$

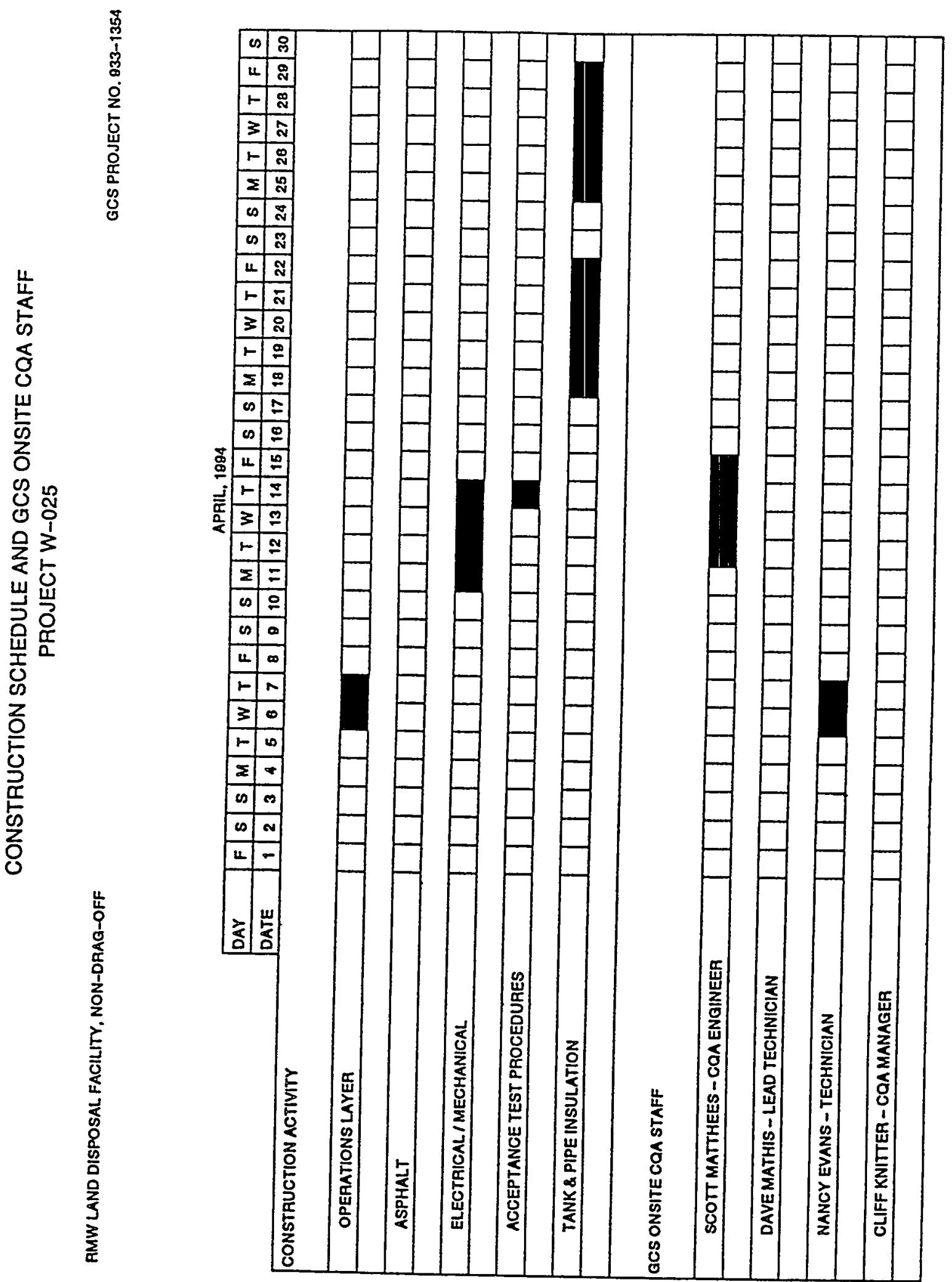


WHC-SD-W025-RPT-001, Rev. 0

党

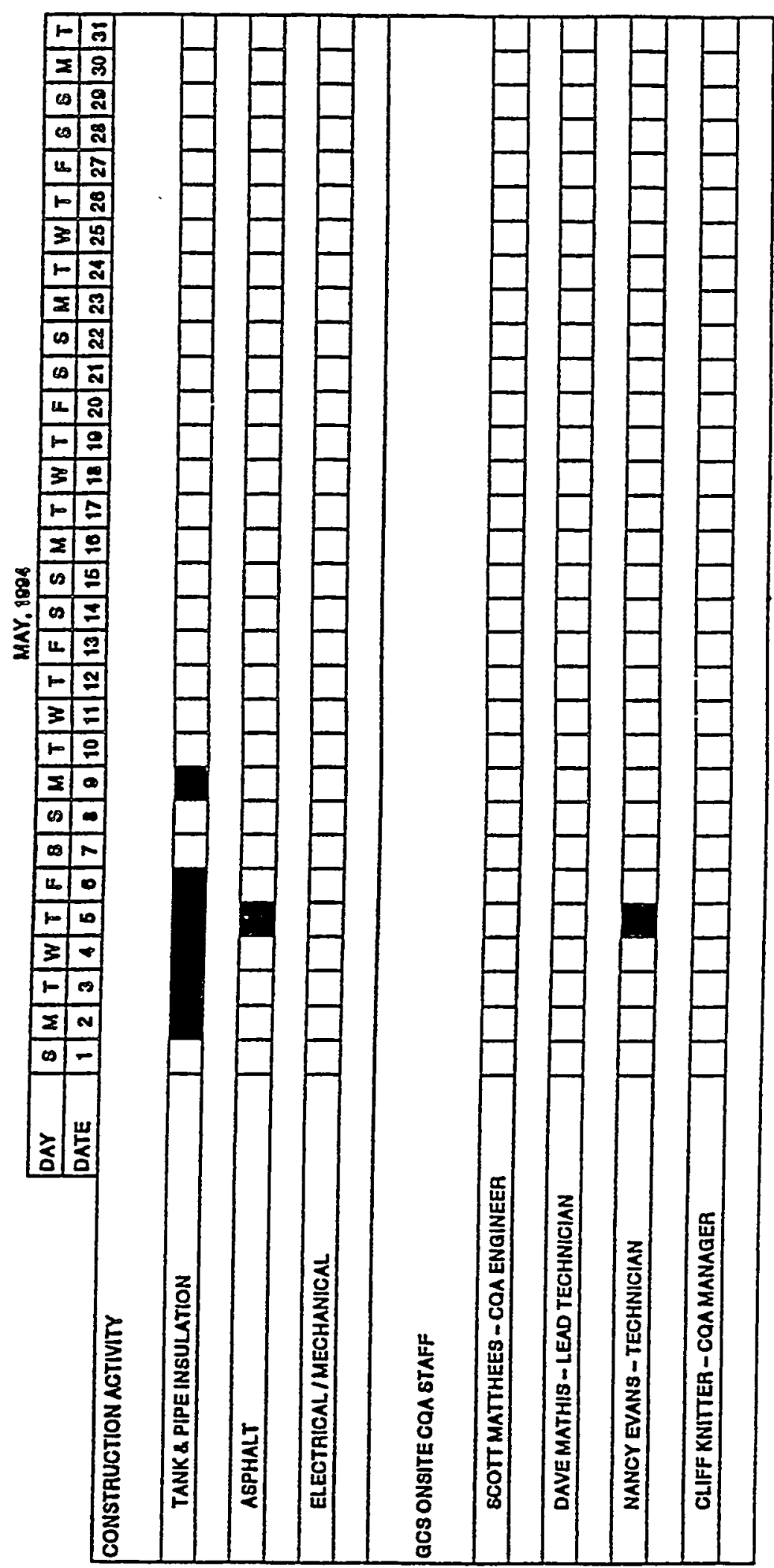


WHC-SD-W025-RPT-001, Rev. 0

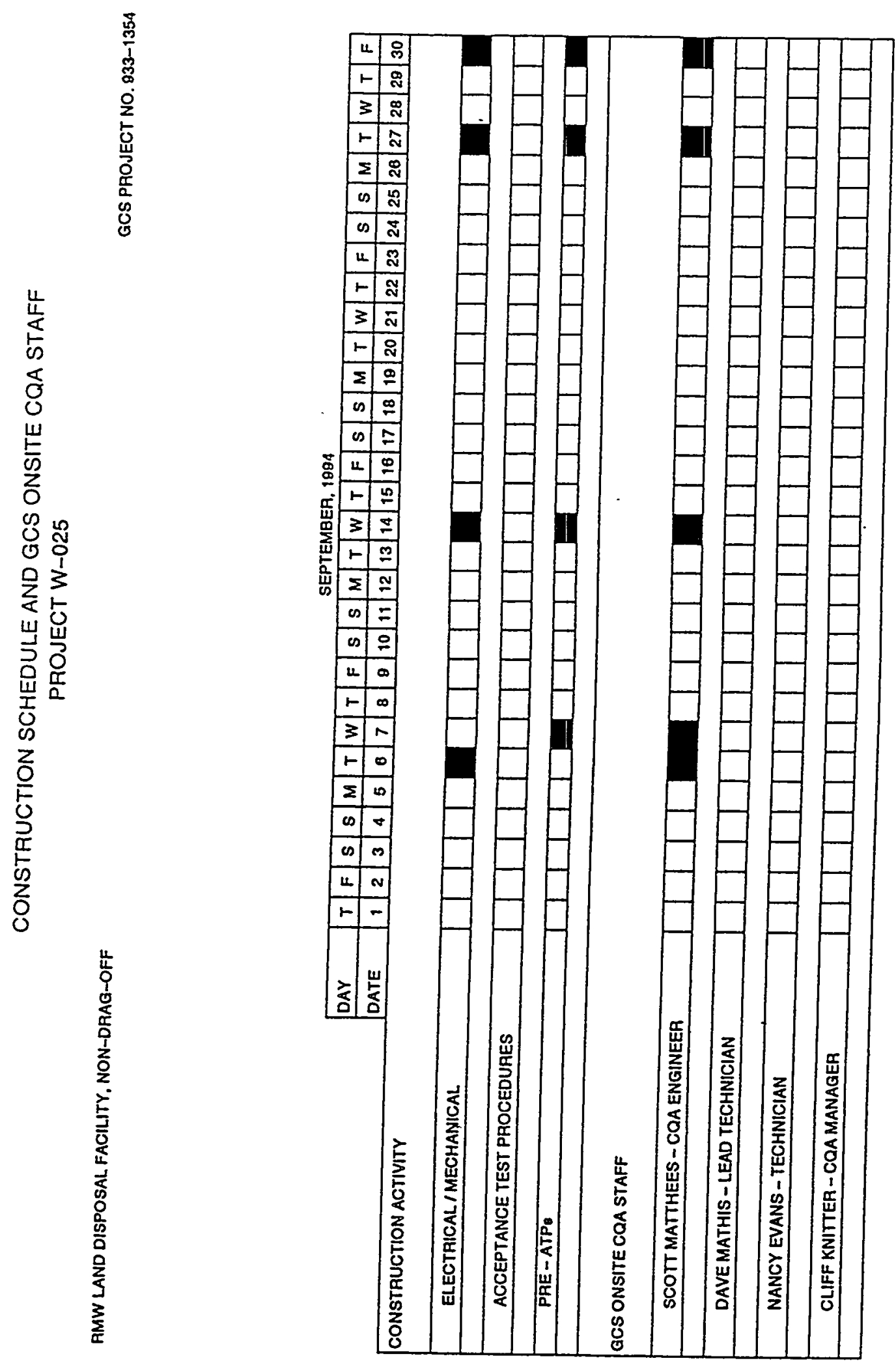


WHC-SD-W025-RPT-001, Rev. 0
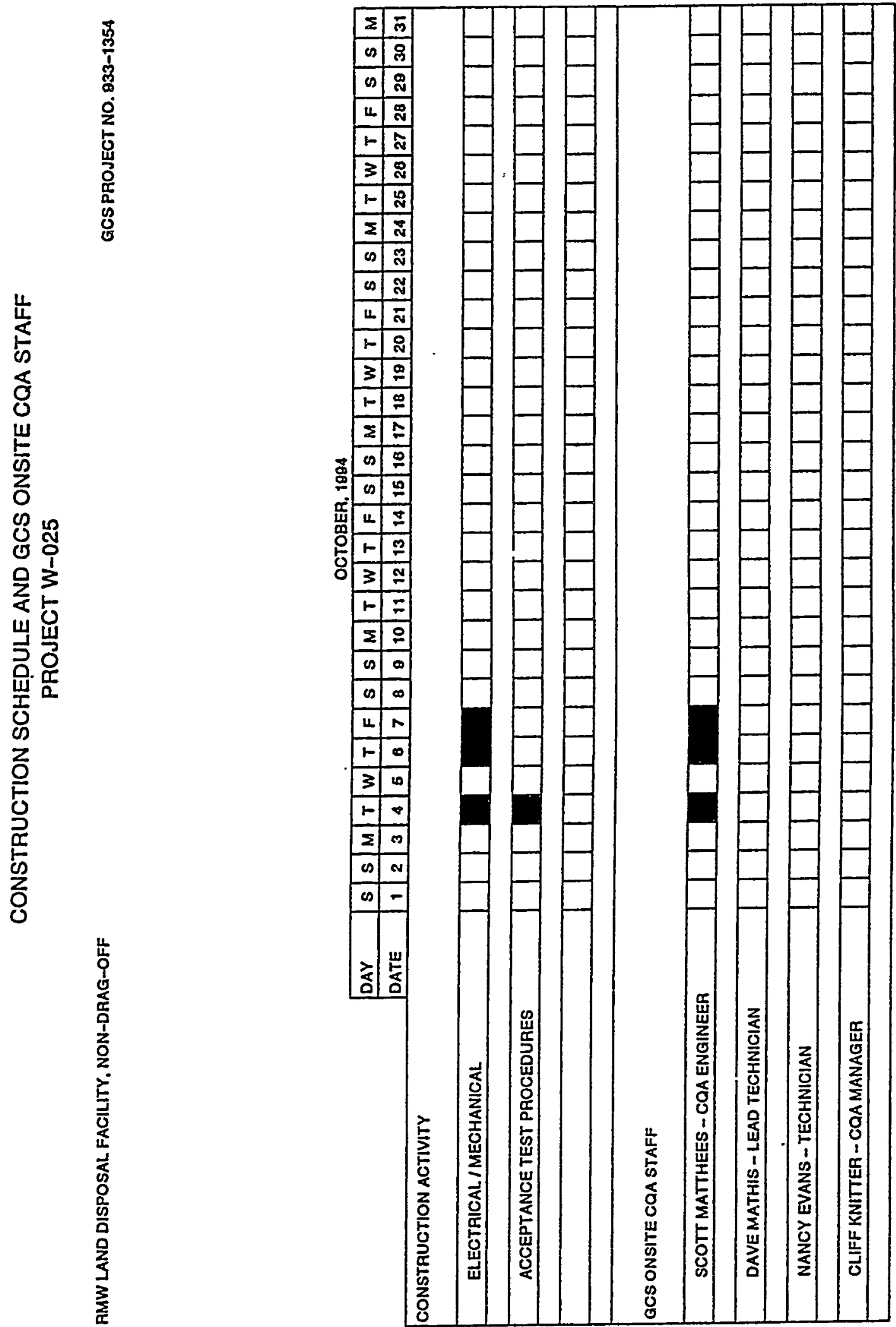
WHC-SD-W025-RPT-001, Rev. 0

APPENDIX B

SUMMARY OF SUBMITTAL REVIEWS BY GCS AND GAI

$B-i$ 
WHC-SD-W025-RPT-001, Rev. 0

fage-y

SUMMARY OF SUBMITTAL REVIEWS BY GCS AND GAI

Project W-025, Non-Drag-Off

GCS Project No. 933-1354

\begin{tabular}{|c|c|c|}
\hline $\begin{array}{l}\text { SUBMITTAL } \\
\text { NOO. }\end{array}$ & 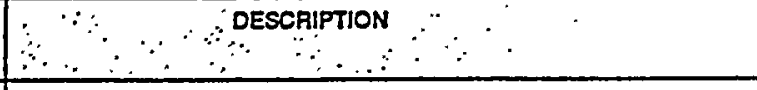 & STATUS \\
\hline 12 & Earthwork operations plan and schedule & Approved by GCS: 09-02-93 \\
\hline 13 & Trenching and Shoring Plans & Information only \\
\hline 18 & $\begin{array}{l}\text { Trenching and Backfilling (Operations Plan and Schedule) } \\
\text { (Soe Submitals 10 \& 12) }\end{array}$ & Approved by GCS: 03-10-94 \\
\hline 15 & $\begin{array}{l}\text { Trenehing and Backrilling (Tronching and Shoring) } \\
\text { (See Submittal 13) }\end{array}$ & Approved by GCS: 03-10-94 \\
\hline 16 & Admix proparation plan (bentanito) & $\begin{array}{l}\text { Approved w/ exception by GCS: 09-17-93 } \\
\text { Resubmit w/ test pad results }\end{array}$ \\
\hline $10 A$ & Admix preparation plan (Pug mill) & $\begin{array}{l}\text { Approved w/ exception by GCS: 09-29-93 } \\
\text { AGsubril: }\end{array}$ \\
\hline 16B & Admix preparation plan (Pug mill) & Approved by GCS: $10-05-93$ \\
\hline 16.1 & Admix Prep. Plen (Belt Scalo Cert.) & Approved by GCS: $10-12-93$ \\
\hline 17 & Soll tinor placement plan & $\begin{array}{l}\text { Not approved by GCS: } 09-27-93 \\
\text { Resubmil }\end{array}$ \\
\hline 17A & Soil liner placement plan & Approved by GCS: $10-25-93$ \\
\hline 18 & $\begin{array}{l}\text { Admix Produetion, Placement, and Trimming (Ops. Plan and Schedule) } \\
\text { (See Submitals 10, 16A, 168, 17, \& 17A) }\end{array}$ & Approved by GCS: 03-10-94 \\
\hline 10 & Trenching and shoring plan & App:ored by GCS: $11-12-93$ \\
\hline 20 & Granulas Drainage Layers/Earthwork Operations Plan and Schedule & Apfroved by GCS: $12-05-83$ \\
\hline 21 & Granular Drainago Layers/Gravel Samples & Approved by GCS: $12-05-93$ \\
\hline 22 & Operations Leyer/Earthwork Operations Plan and Schedulo & Approved by GCS: $12-05-83$ \\
\hline 23 & Operations Layer/Trenching and Shoring Plans & Approved by GCS: $12-05-83$ \\
\hline 24 & Gedextile $Q A$ and $Q C$ documentation & Approved by GAl: 09-20-93 \\
\hline 24.1 & ONOC documentation (thread for geotextile) & Approved by GCS: $10-25-93$ \\
\hline
\end{tabular}


WHC-SD-W025-RPT-001, Rev. 0

Page?

\section{SUMMARY OF SUBMITTAL REVIEWS BY GCS AND GAI}

Project W-025, Non-Drag-Off

GCS Project No. 933-1354

\begin{tabular}{|c|c|c|}
\hline $\begin{array}{l}\text { SUBMITTAL } \\
\text { NO. }\end{array}$ & DESCRIPTION & STATUS \\
\hline 25 & FML QA OC documentation (SLTS OA Manual) & $\begin{array}{l}\text { Approved w/ exception by GAl: } 02-21-93 \\
\text { Resubmit }\end{array}$ \\
\hline $25 \mathrm{~A}$ & FML $Q A$ and $Q C$ documentation (Crystallinity letter) & $\begin{array}{l}\text { Approved w/ exception by GAl: 10-01-93 } \\
\text { Resubmit }\end{array}$ \\
\hline 258 & Friction Angle Test (admix / FML) & Approved by GCS: 11-19-93 \\
\hline 25.9 & Geomembrani OA \& OC Dala & Approved by GCS: $11-19-93$ \\
\hline 25.2 & Extrudate Certification & Approvad by GCS: $11-19-93$ \\
\hline 25.3 & Geomembrano QA \& OC Data & $\begin{array}{l}\text { Approved w/ exception by GCS: } 11-19-93 \\
\text { Resubmit }\end{array}$ \\
\hline 25.3A & $\begin{array}{l}\text { Geomembrane QA \& OC Documentation (Resin lot centification } \\
\text { and Geocomposite OA\& OC dala for } 32 \text { rolls) }\end{array}$ & Approved by GCS: $01-18-24$ \\
\hline 25.4 & Geomembrane QA \& OC Data & Approved w/ exception by GCS: $1 ;-19-83$ \\
\hline $25.4 \mathrm{~A}$ & Same submittal as $25.3 \mathrm{~A}$ & Approved by GCS: 0:-18-94 \\
\hline $25.4 \mathrm{~B}$ & $\begin{array}{l}\text { Goomembrane OA\& QC Deoumentation (Environmental Stress Crack } \\
\text { Resistance Cerification) }\end{array}$ & Approved w/ exceptions by GCS: $02-09-84$ \\
\hline $25.4 \mathrm{C}$ & $\begin{array}{l}\text { Geomembrane QA \& OC Deoumentation (Environmental Stress Crack } \\
\text { Besistance Certification) }\end{array}$ & Approved by GCS: 03-22-84 \\
\hline 25.5 & $\begin{array}{l}\text { Geomembrane OA \& OC Dcoumentation (Centification - welding in } \\
\text { temperatures lets than } 40 \text { degrees) }\end{array}$ & Approved by GCS: $12-08093$ \\
\hline 25.6 & Geomembrane QA \& QC Documentetion (Cert for roll 8388) & Approved w/ exception by GCS: $01-14-84$ \\
\hline $25.6 \mathrm{~A}$ & Geomembrane QA \& OC Documentation (Direct Shear Tea Aeport) & Approved by GAI \& GCS: 02-09-94 \\
\hline 25.7 & Goomembrane QA \& OC Documentation (Resin, and rolls 9604 \& 8505) & Approved by GCS: 01-18-94 \\
\hline 25.8 & Geomembrane QA \& OC Documentation (Resistance lo Soil Burial Data) & Approved by GCS: $03-10-84$ \\
\hline 25.8 & Geomembrane OAOC Documentation (Cert. - Resistance to Soil Burial) & Approved by GCS: 03-22-84 \\
\hline
\end{tabular}




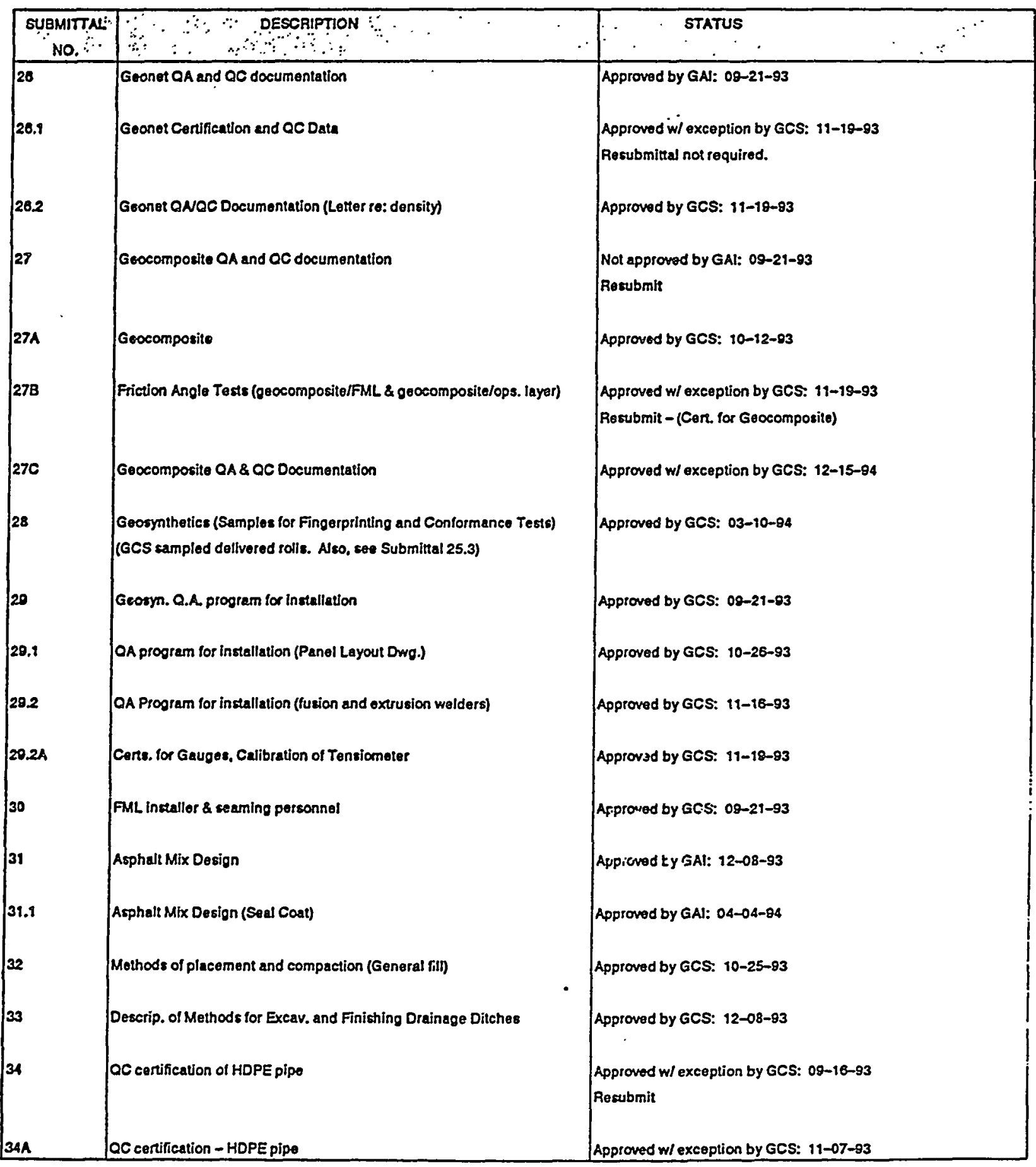


WHC-SD-W025-RPT-001, Rev. 0

\section{SUMMARY OF SUBMITTAL REVIEWS BY GCS AND GAI}

Project W-025, Non-Drag-Off

GCS Project No. 933-1354

\begin{tabular}{|c|c|c|}
\hline $\begin{array}{c}\text { SUBMITTAL" } \\
\text { NO. }\end{array}$ & DESCRIPTION & STATUS \\
\hline 348 & OC Certification - HOPE Pipe & Approved by GCS: $12-08-93$ \\
\hline 35 & Fabrication Drwg. - (HDPE pipe) & Approved by GCS: $11-07-93$ \\
\hline $35 \mathrm{~A}$ & Fabrication Drawings for HDPE Collection Well & Approved by GCS: $12-08-93$ \\
\hline 36 & Oualifications of joining personnel & Approved by GCS: 09-16-93 \\
\hline 37 & Vertical riser pipo & $\begin{array}{l}\text { Approved w/ exception by GAl: } 09-27-83 \\
\text { Resubmit }\end{array}$ \\
\hline $37 \mathrm{~A}$ & Precact concrete pipe & Approved by GAI: 11-04-93 \\
\hline 38 & Rebar and coating & Approved by GCS: $10-26-93$ \\
\hline 39 & Shop drawings - rebar & Approved by GAl: $10-26-93$ \\
\hline 40 & Certificate of Admixture (Air-entraining admix for concrete) & Approved by GA: $04-04-94$ \\
\hline 41 & Conctele mix design & Approved by GAl: 10-26-93 \\
\hline 42 & Anchor bolt type & Approved by GAl: $10-28-93$ \\
\hline 43 & Waterstops & Approved by GA: $10-26-93$ \\
\hline 4 & Metal FabJShop Dwgs (Vertical riser lid end grating) & Approved by GAl: 05-11-94 \\
\hline 46 & Manufacturer's Tech. Info. (Door for Control Building) & Approved by GAl: $04-04-94$ \\
\hline 46.1 & Manufaeturer's Tech. Info. (Frame for Control Building Door) & Approved by GCS: $04-18-94$ \\
\hline 47 & $\begin{array}{l}\text { Paint Coating Systems (Coating Systems Lif) } \\
\text { (See Submittals 48, 48A, and 48.1) }\end{array}$ & Approved by GCS: $03-10-24$ \\
\hline 48 & Manufacturer's Specifications (Manhole coating) & $\begin{array}{l}\text { Nol approved by GAl: } 10-26-93 \\
\text { Recubmit }\end{array}$ \\
\hline $48 \mathrm{~A}$ & Manu's Specifications (manhole coating) & Approved by GAl: 11-16-93 \\
\hline 48.1 & Manu's Specifications (lank coating) & Approved by GAl: $19-09-93$ \\
\hline
\end{tabular}


WHC-SD-W025-RPT-001, Rev. 0

Pages

\section{SUMMARY OF SUBMITTAL REVIEWS BY GCS AND GAI}

Project W-025, Non-Drag-Off

GCS Project No. 933-1354

\begin{tabular}{|c|c|c|}
\hline $\begin{array}{l}\text { SUBMITTAL } \\
\therefore \text { NO,. }:=\end{array}$ & 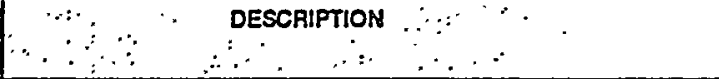 & STẠTUS \\
\hline 49 & Puint Costing Syrtems (Color Semples) & Approved by GCS: 03-16-24 \\
\hline 50 & Pumps, motors, materials, etc. & Approved by GA: 09-17-93 \\
\hline 50.1 & Into. on pumps, molors, etc. & $\begin{array}{l}\text { Approved w/ exception by GAl: } 10-07-93 \\
\text { Resubmit }\end{array}$ \\
\hline 50.18 & Inlo. on pumps, motors, ote. & Approved by GAl: 11-01-83 \\
\hline 51 & Manu. Inlo. - Control bidg., hardware, etc. & $\begin{array}{l}\text { Approved w/ exception by GAl: } 10-26-93 \\
\text { Reeubmit }\end{array}$ \\
\hline 51.2 & Control building foundation & Azproved by GAl: $11-04-93$ \\
\hline 512 & Manu's Information (control building) & Approved by GAl: 11-17-83 \\
\hline 52 & Manu. Into. - Dosign load - bldg. & Approved by GA: 11-12-83 \\
\hline 53 & Leachate tank: fabricator drawings & $\begin{array}{l}\text { Approved w/ exception by GAI: 09-20-93 } \\
\text { Resubmit }\end{array}$ \\
\hline $53 \AA$ & Fabricator drawings & $\begin{array}{l}\text { Approved w/ exception by GAl: } 10-14-93 \text {. } \\
\text { Resubmit }\end{array}$ \\
\hline 538 & Liquid lovel indicator - tank & Approved by GA: 11-04-93 \\
\hline 53.1 & Fabrication dwgz - Vent - leachate tank & Approvod by GAl: 11-04-83 \\
\hline 53.2 & Fabrication dwos. - Ebow for leachate tank & Approved by GAl: $11-05-93$ \\
\hline 53,3 & Fab. Drawings (Leachate Tank) & Approved by GAl: $12-08-93$ \\
\hline 53.4 & Fabricalor Drawings (letter from tank fabricator) & Afproved by GAl: $12-08-93$ \\
\hline 53.5 & Fab. Dwgs. (ASME Cern. for Leachate Tank) & Approved by GAl: $12-08-93$ \\
\hline 53,6 & Fab. Drawings (Leachate Tank) & Approved by GAl: $12-08-93$ \\
\hline 54 & Pump Controls (Detailed Drawings and Manulacturer's Information) & Approved by GAl: 04-04-94 \\
\hline 55 & Electrical System and Pump Controls & Approved W/ comments by GAl: $12-08-93$ \\
\hline
\end{tabular}


WHC-SD-W025-RPT-001, Rev. 0

Paga 6

SUMMARY OF SUBMITTAL REVIEWS BY GCS AND GAI

Project W-025, Non-Drag-Off

GCS Project No. 933-1354

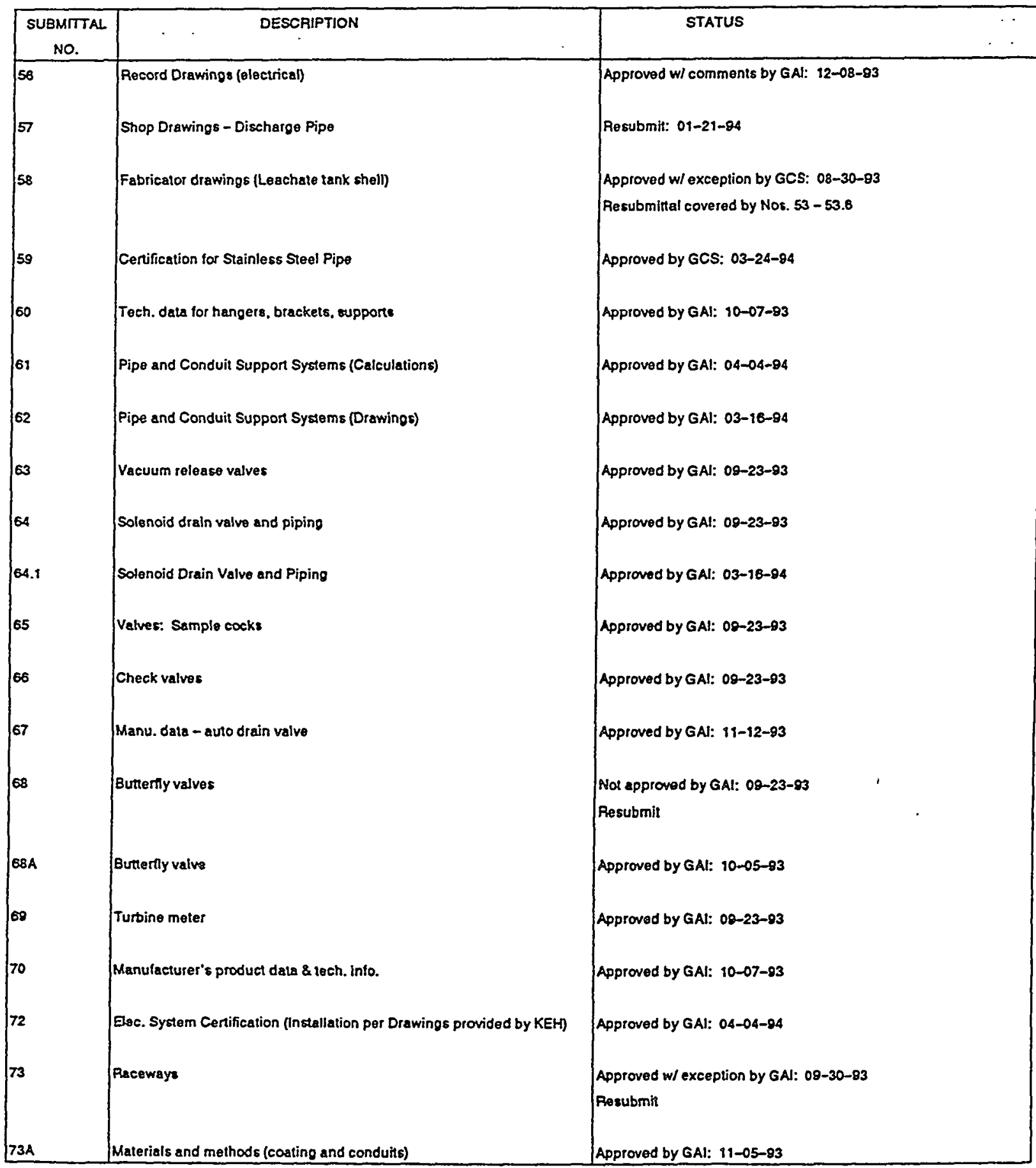


WHC-SD-W025-RPT-001, Rev. 0

Page 7

\section{SUMMARY OF SUBMITTAL REVIEWS BY GCS AND GAI}

Project W-025, Non-Drag-Off

GCS Project No. 933-1354

\begin{tabular}{|c|c|c|}
\hline $\begin{array}{c}\text { SUEMITTAL } \\
\because \text { NO. . : } \\
\end{array}$ & 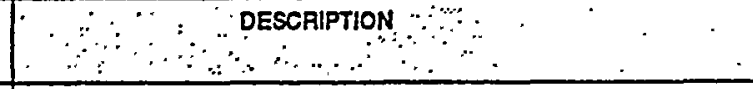 & STATUS \\
\hline 74 & Wire and cables & Approwed by CA: 09-30-83 \\
\hline 75 & Connectors and terminations & Approved by GAI: 09-30-93 \\
\hline 76 & Motor controllere & $\begin{array}{l}\text { Approved w/ exception by GAl: } 10-28-93 \\
\text { Renubmit }\end{array}$ \\
\hline $76 A$ & Motor Controllere & Approved w/ exception by GCS: $12-08-93$ \\
\hline 77 & Power service equipment & Approved by GAl: 10-28-93 \\
\hline 78 & Transformers & Approved by GAl: 11-05-93 \\
\hline 78.1 & Transformers & Approved by GAl: 11-15-93 \\
\hline 79 & Panel boards & $\begin{array}{l}\text { Approved w/ exception by GAl: } 11-05-93 \\
\text { No resubmittal required. }\end{array}$ \\
\hline 80 & Product Data (Ughting Fixtures and Ballacts) & Approved by GAI: 04-08-94 \\
\hline 89 & Wood poles & Approved by GAl: $09-30-93$ \\
\hline 82 & Open fure cutouts & $\begin{array}{l}\text { Approved w/ exception by GAl: 11-07-93 } \\
\text { No resubmittal required. }\end{array}$ \\
\hline 83 & Surge arresters/product data & $\begin{array}{l}\text { Approved w/ exception by GAl: } 10-05-93 \\
\text { Rasubmit }\end{array}$ \\
\hline $83 A$ & Product data - surge arrestor & Approved by GA: $11-09-93$ \\
\hline 84 & Push button stations & $\begin{array}{l}\text { Approvad w/ exception by GAl: } 10-28-93 \\
\text { Resubmit }\end{array}$ \\
\hline $84 A$ & Push Buttor Stations & Approved w/ exception by GCS: $12-08-83$ \\
\hline 85 & Pullbox & Approved by GAL: $09-30-93$ \\
\hline 86 & Heat trece & $\begin{array}{l}\text { Not approved by GAL: 09-30-93 } \\
\text { Resubmit }\end{array}$ \\
\hline
\end{tabular}


WHC-SD-W025-RPT-001, Rev. 0

Rago 8

SUMMARY OF SUBMITTAL REVIEWS BY GCS AND GAI

Project W-025, Non-Drag-Off

GCS Project No. 933-1354

\begin{tabular}{|c|c|c|}
\hline $\begin{array}{l}\text { SUBMTTAL } \\
\text { NO. }\end{array}$ & DESCRIPTION & STATUS \\
\hline $86 \mathrm{~A}$ & Heat trace & $\begin{array}{l}\text { Approved w/ exception by GAl: } 11-05-93 \\
\text { No resubmittal required. }\end{array}$ \\
\hline 87 & Materials and methods (panelboard) & $\begin{array}{l}\text { Approved w/ exception by GAl: } 11-09-93 \\
\text { No resubmittal required. }\end{array}$ \\
\hline 88 & Motor Controllers & $\begin{array}{l}\text { Approved w/ exception by GAI: } 10-28-83 \\
\text { Resubmit }\end{array}$ \\
\hline $88 \mathrm{~A}$ & Motor Controllers & Approved w/ exception by GCS: $12-08-93$ \\
\hline 89 & Shop drawings - transformers & Approved by GAl: $11-05-93$ \\
\hline 89.1 & Dry Type Transformers/Shop Drawings & Approved by GAl: $12-08-83$ \\
\hline 90 & Shop drawing (panelboard) & $\begin{array}{l}\text { Approved w/ exception by GAl: } 11-09-93 \\
\text { No resubmittal required. }\end{array}$ \\
\hline 91 & Certified Laboratory Test Report - Lighting & Approved w/ exception by GAI: $11-05-93$ \\
\hline $81 \mathrm{~A}$ & Certified Laboratory Test Repon - Lighting & Approved by GAl: $12-22-83$ \\
\hline 92 & Lighting & Approved w/ exception by GAl: $11-12-93$ \\
\hline 93 & Electric Heat Trace-Catalog data & $\begin{array}{l}\text { Not approved by GAl: } 09-30-93 \\
\text { Resubmit }\end{array}$ \\
\hline $93 A$ & Eec. heat trace & $\begin{array}{l}\text { Approved w/ exception by GAl: } 11-09-93 \\
\text { No resubmittal required. }\end{array}$ \\
\hline 84 & Manufacturer's QC test results & Resubmit-cannot review until cable has been submitted: $10-12-9$ \\
\hline $94 A$ & Manufacturer's QANQC Tests & Approved by GAl: $12-06-93$ \\
\hline 300 & Info. request: Piggy back pump & $\begin{array}{l}\text { Arrangement approved by GAl: } 09-09-93 \\
\text { Resubmit w/ additional information }\end{array}$ \\
\hline
\end{tabular}


WHC-SD-W025-RPT-001, Rev. 0

APPENDIX C

SPECIFICATION CLARIFICATIONS AND ENGINEERING CHANGE NOTICES 
WHC-SD-W025-RPT-001，Rev。0

APPENDIX C-1

PRECONSTRUCTION MEETING MINUTES

$c-1$ 
WHC-SD-W025-RPT-001, Rev. 0

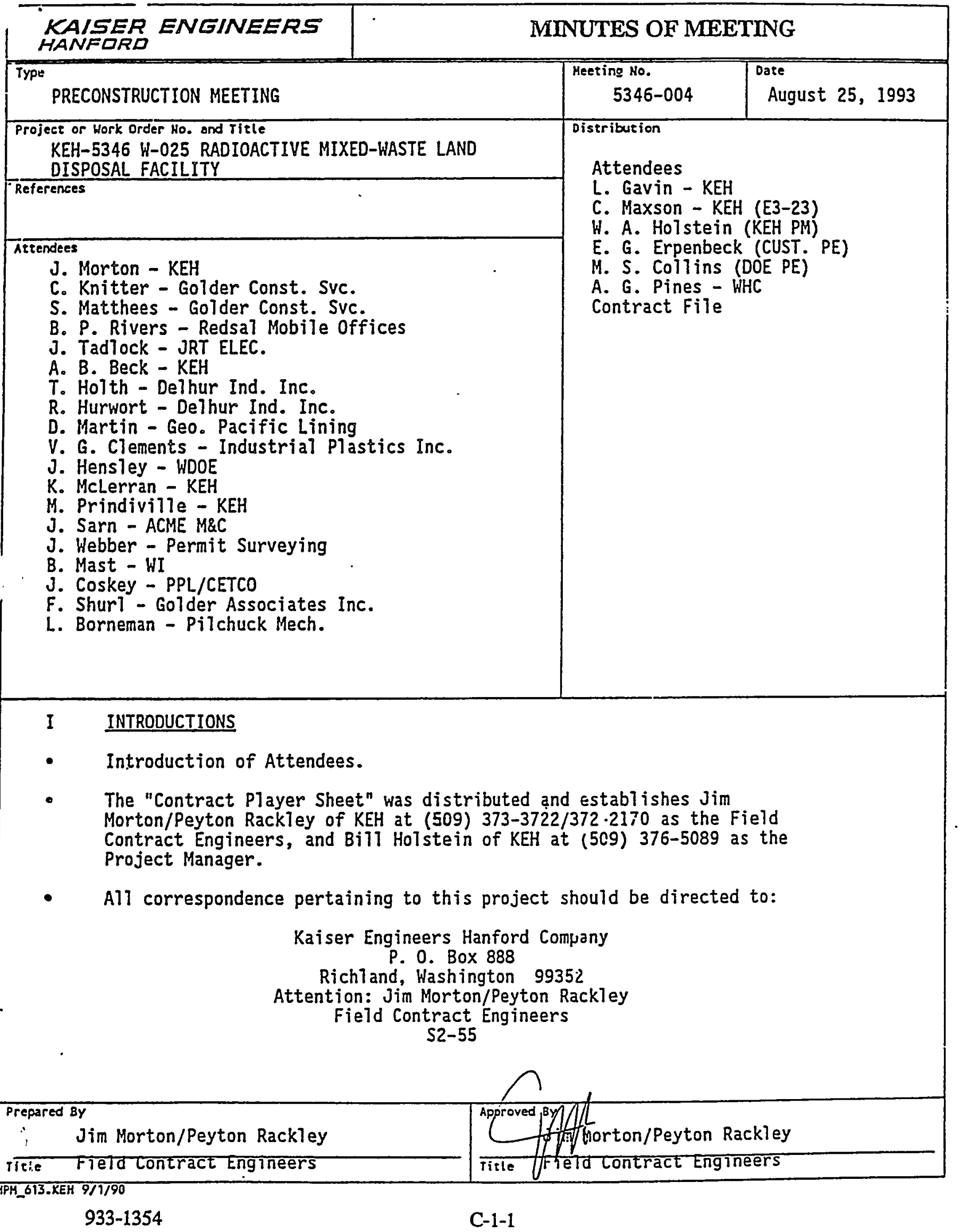


KAISER ENGINEERS HANFORD

MINUTES OF MEETING - CONTINUED

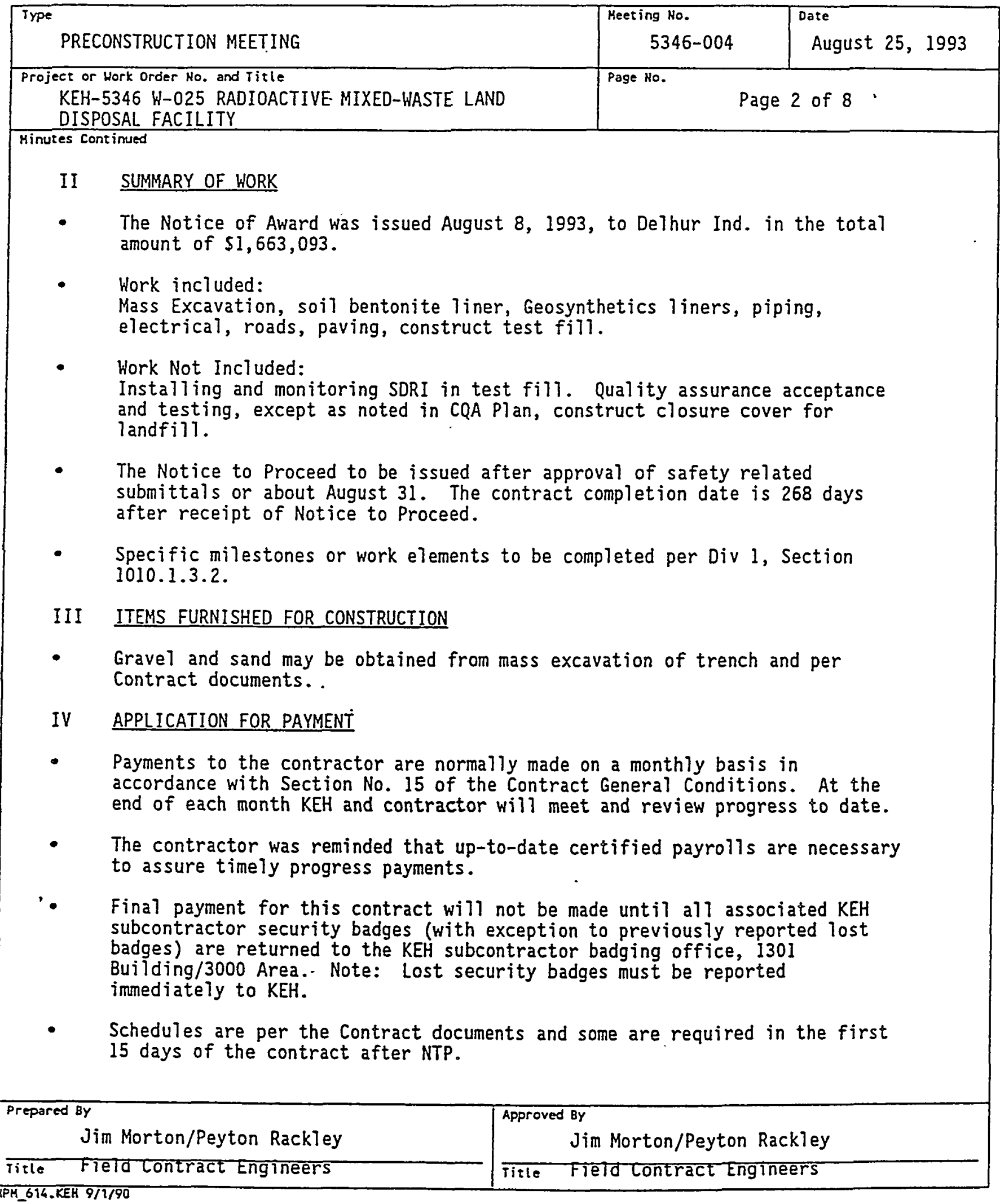


WHC-SD-W025-RPT-001, Rev. 0

KAISER ENGINEERE

HANFORD

MINUTES OF MEETING - CONTINUED

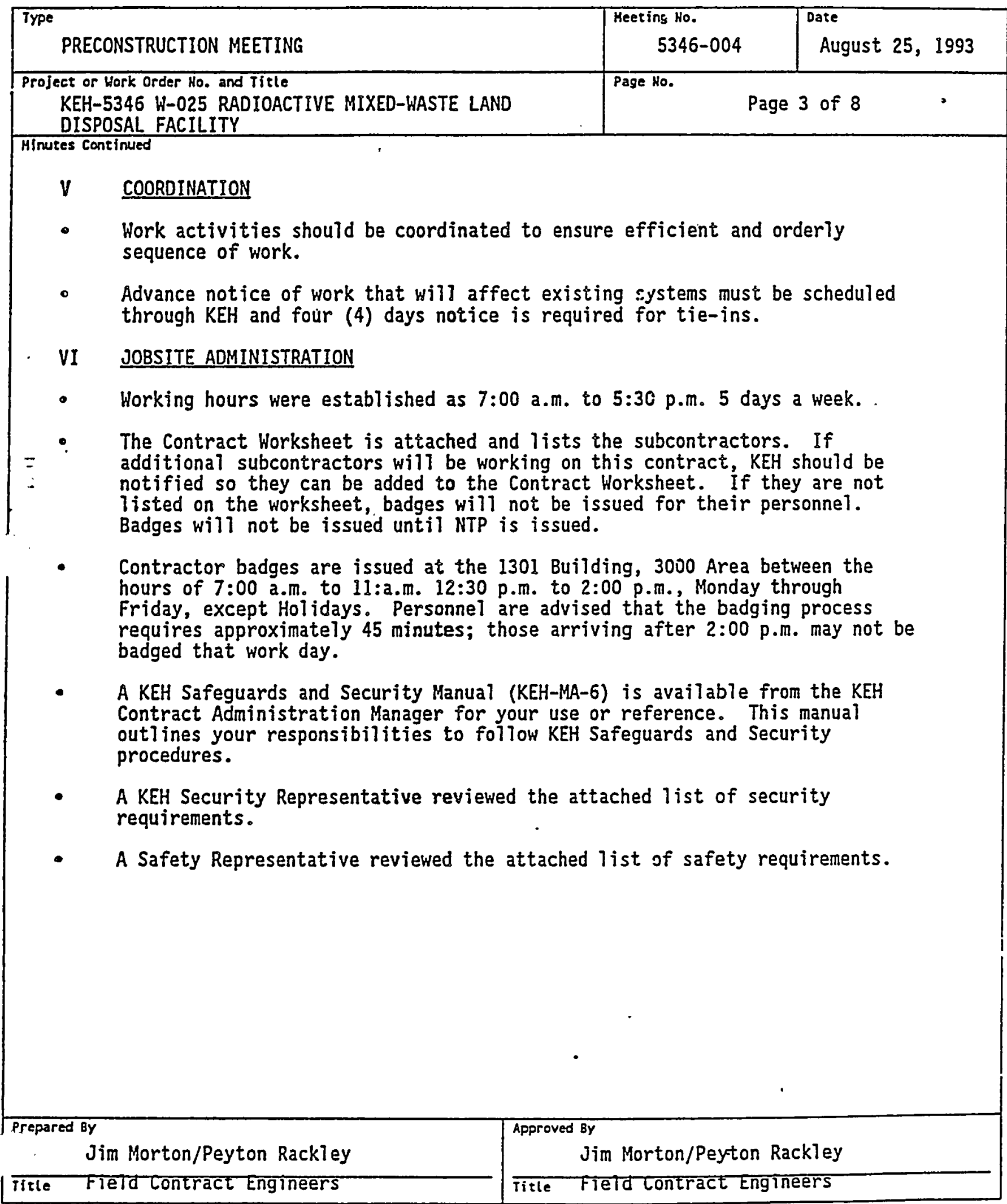

HPH_614.KEH 9/1/90

933-1354

C-1-3 
WHC-SD-W025-RPT-001, Rev. 0

KAISER ENEINEERS

HANFORD

MINUTES OF MEETING - CONTINUED

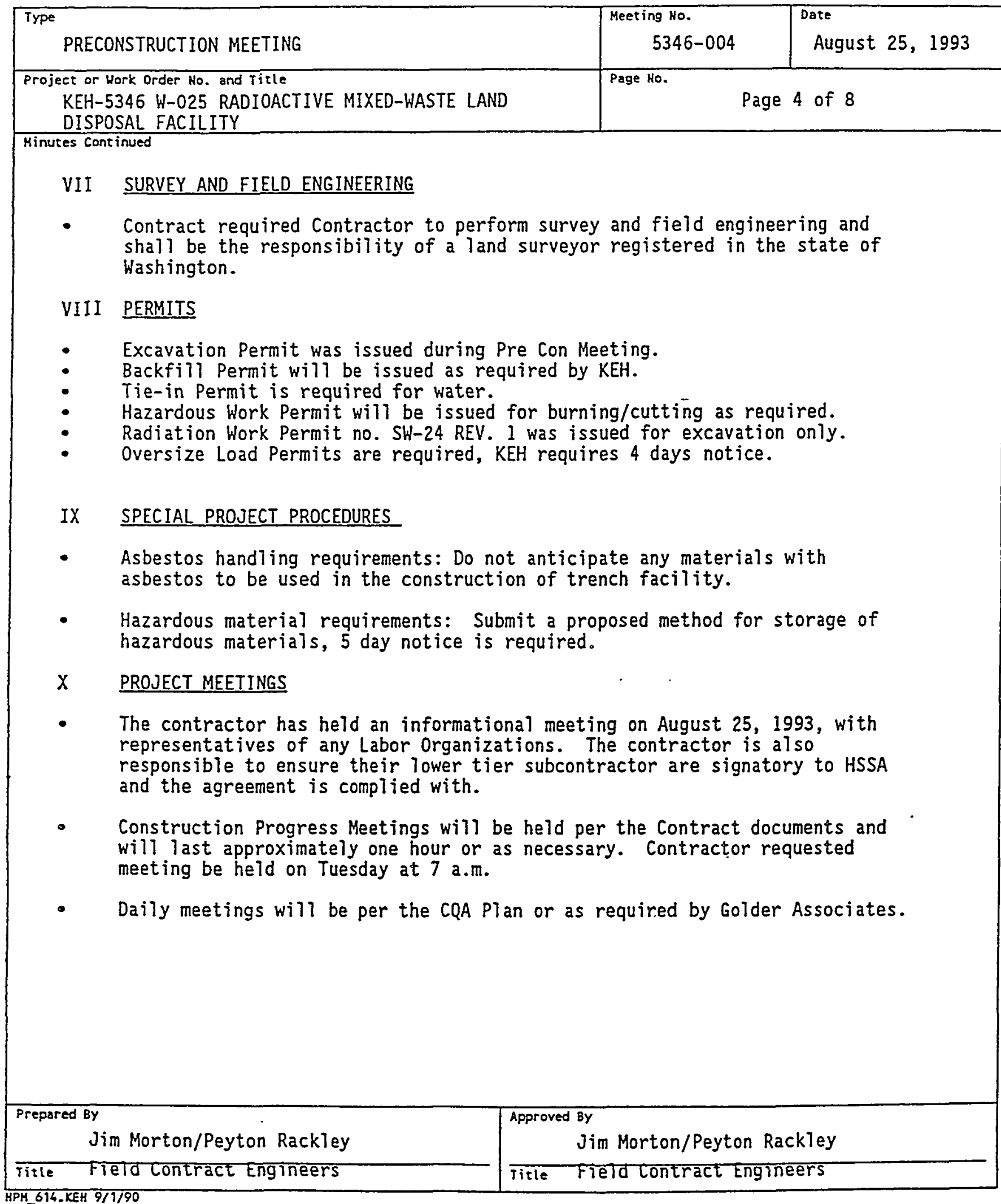

933-1354

C-1-4 
WHC-SD-W025-RPT-001, Rev. 0

KAISER ENEINEERS

HANFORD

MINUTES OF MEETING - CONTINUED

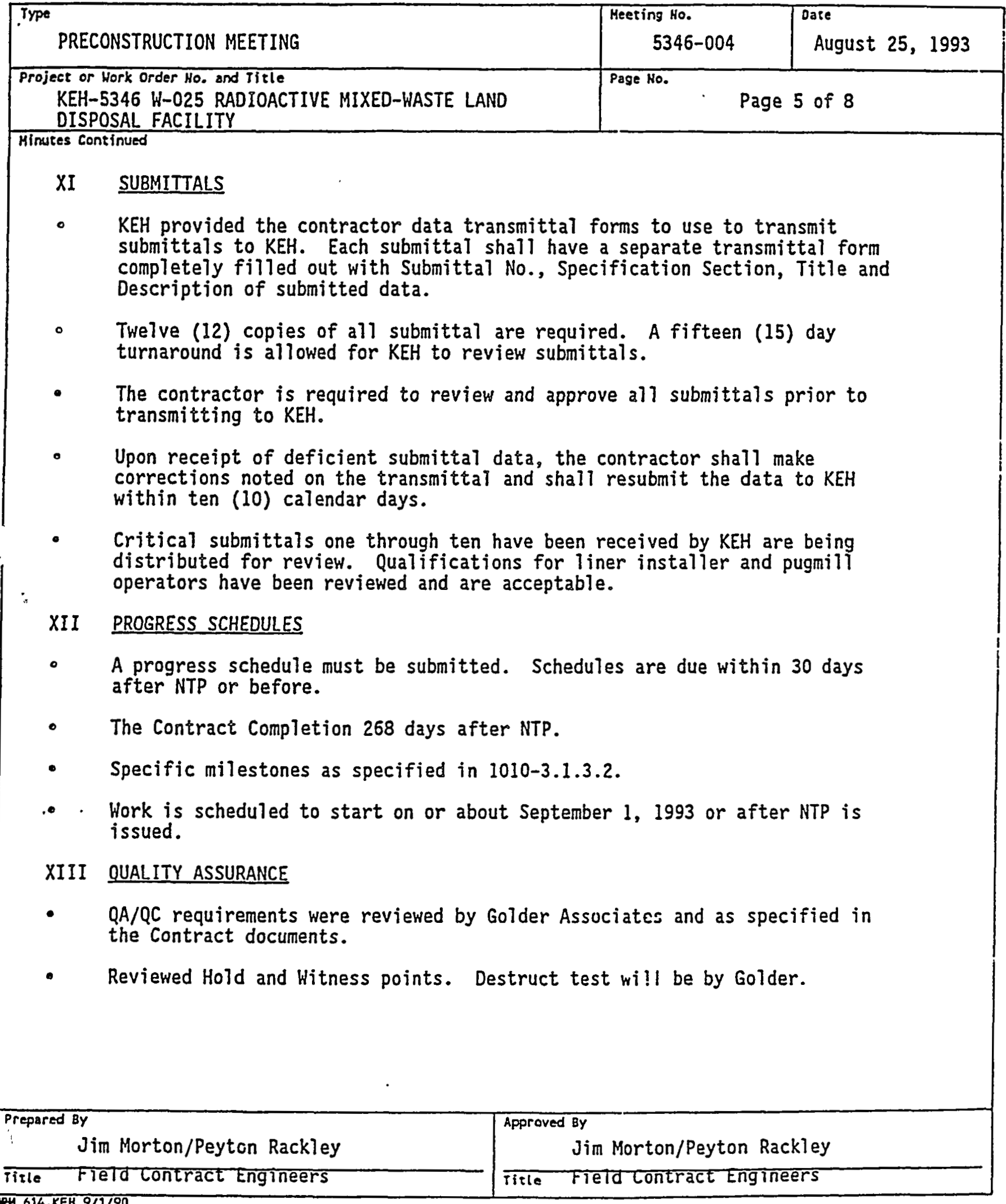

933-1354

C-1-5 
WHC-SD-W025-RPT-001, Rev. 0

KAISER ENGINEERS

HANFORD

MTNUTES OF MEETING - CONTINUED

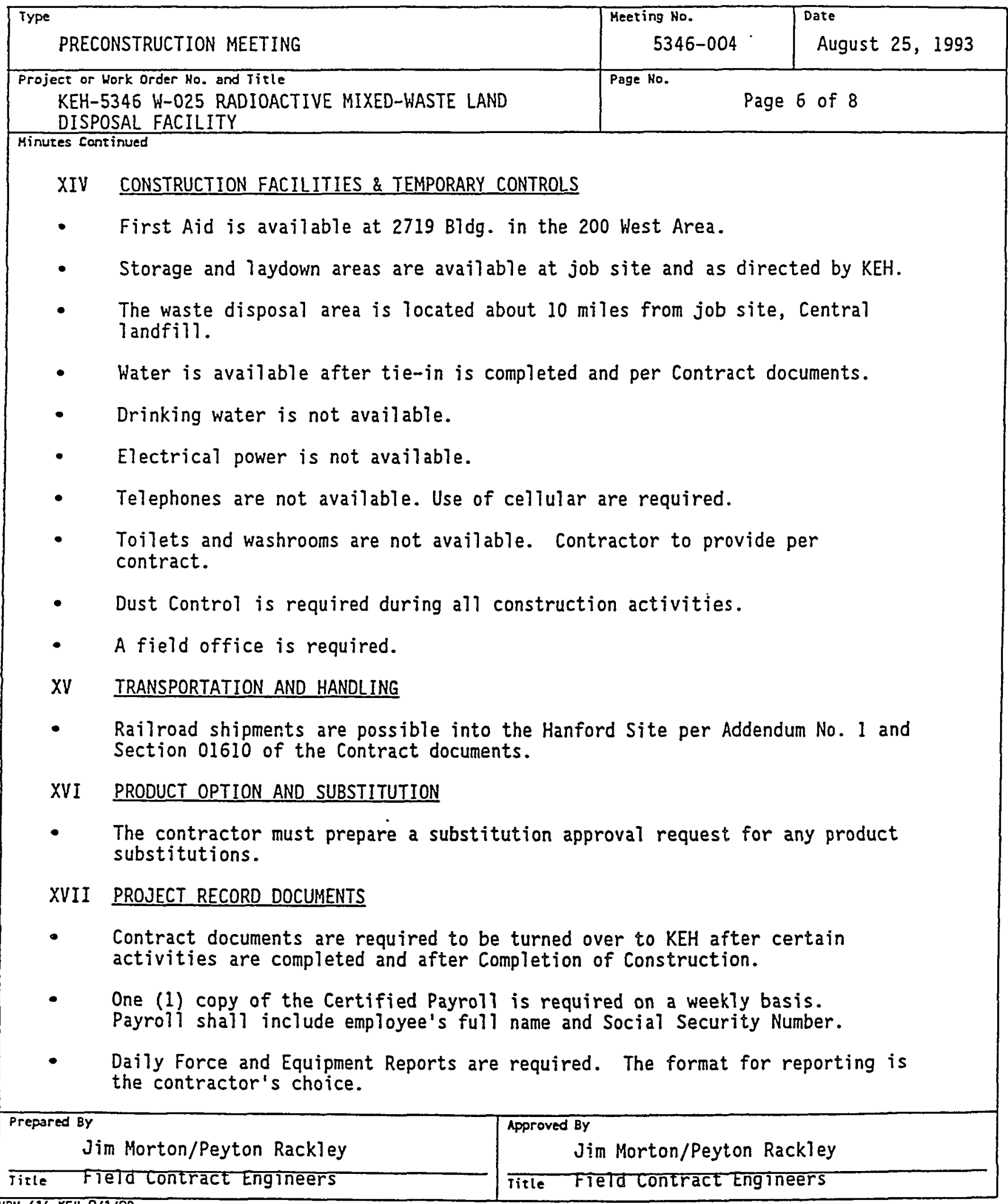

HPM_614-XEH 9/1/90

933-1354

C-1-6 
WHC-SD-W025-RPT-001, Rev. 0

KAISER ENGINEERS

HANFORD

MINUTES OF NIEETING - CONTINUED

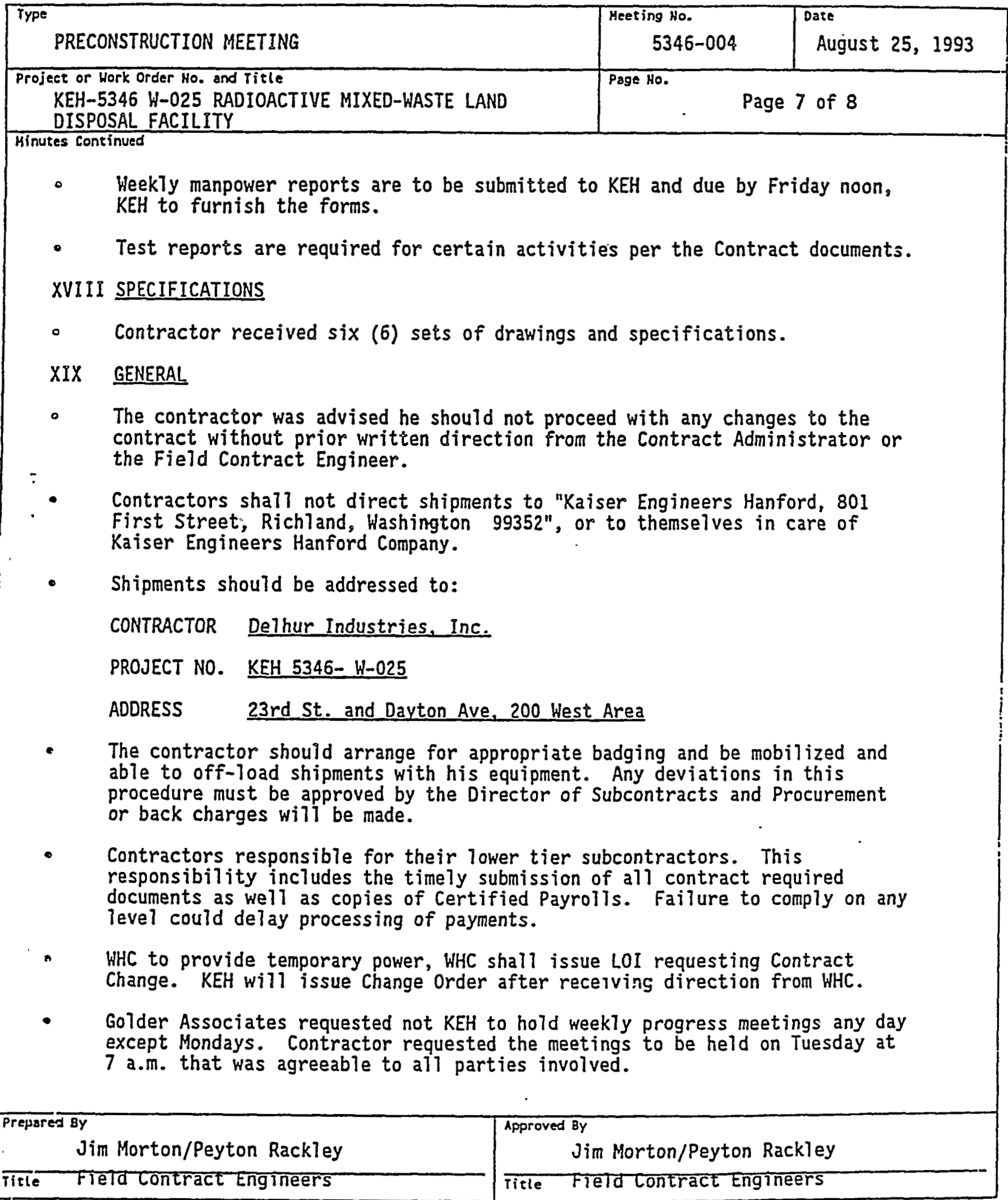


WHC-SD-W025-RPT-001, Rev. 0

KAISER ENGINEERS

HANFORD

MNUTES OF MEETNGG - CONTINUED

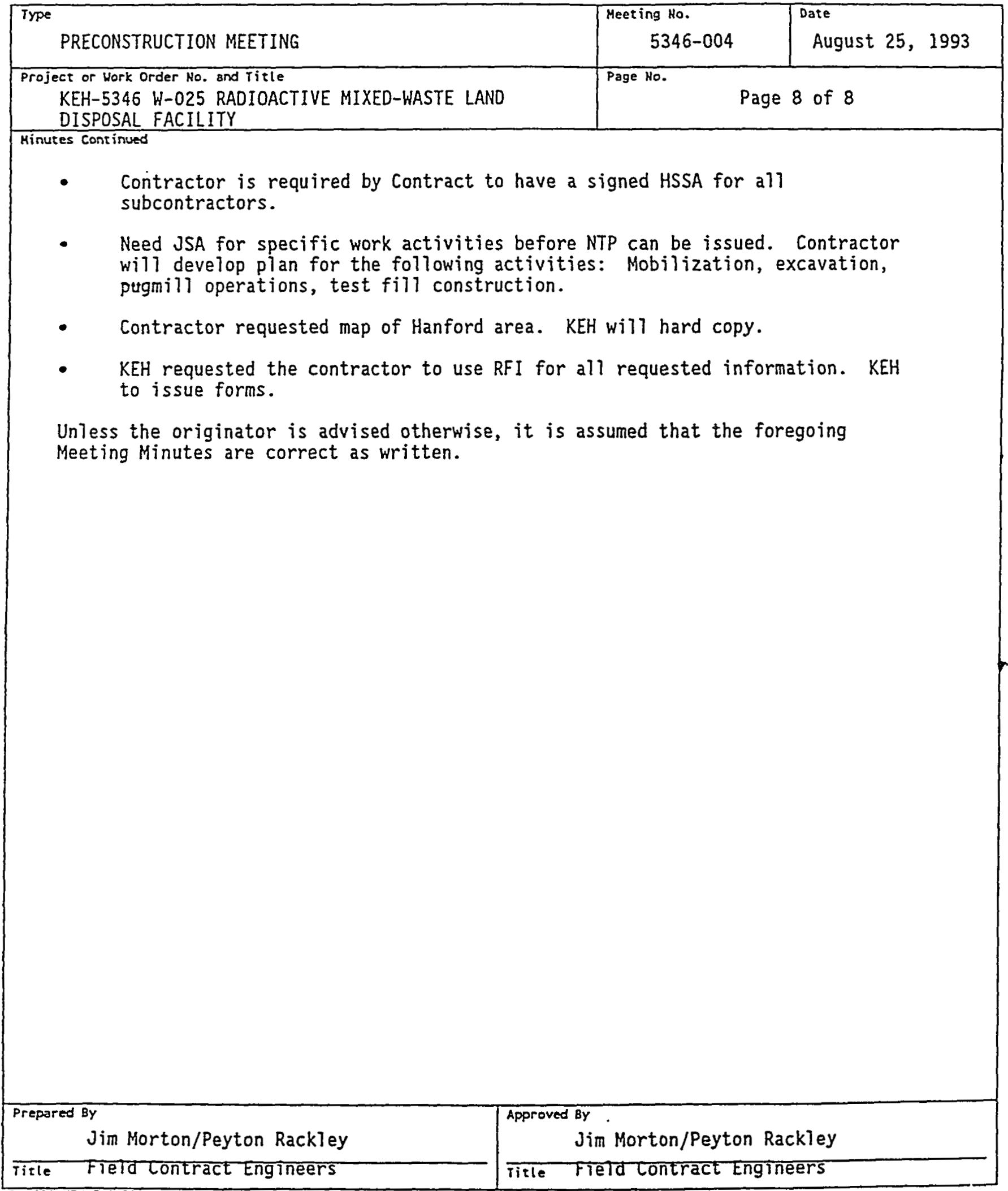


WHC-SD-W025-RPT-001，Rev。 0

APPENDIX C-2

GEOSYNTHETICS PRECONSTRUCTION MEETING MINUTES

$c-2$ 
WHC-SD-W025-RPT-001, Rev. 0

\author{
RMW LAND DISPOSAL FACILITY, NON DRAG-OFF \\ PRECONSTRUCTION MEETING WITH GEOMEMBRANE INSTALLER \\ NOVEMBER 17, 1993 \\ REF. NO. 933-1354
}

\title{
ATTENDANCE
}

NAME

DENNIS MARTIN

DAVE MATHIS

STEVE MILKA

SCOTT MATTHEES
REPRESENTING

GEOPACIFIC

GCS .

GCS

GCS

\section{GENERAL DISCUSSION}

Subgrade acceptance certificates will be required prior to deployment.

GeoPacific will install geomembrane on the slopes, then the floor, then in the sump.

Trial seams will be required every four hours, generally at the start of the day and after lunch. If a breakdown of a welding apparatus occurs, a new trial seam will be required after that piece of equipment is repaired (before being allowed to resume welding). During each seaming period, each technician who will be welding will be required to perform a trial seam with each welding apparatus he will be using.

Overlap for extrusion welds shall be at least 3 inches, and overlap for fusion welds shall be at least 5 inches.

Destructive test samples will be obtained at a minimum frequency of every 500 feet of welded seam. If a destructive test fails, bracketing destructive tests will be required at least ten feet on either side of the failing test. At a minimum, capped seams longer than 150 feet in length will destructively tested.

For welding below 40 degrees F., GCS will require additional trial seams, and will increase the frequency of destructive tests. GeoPacific should certify that their cold weather seamis will be equivalent in quality to seams produced at temperatures between 40 and 104 degrees $F$., and that such welding will not cause physical or chemical modifications such that the short-term or longterm performance of the liner will be adversely affected.

Resumes haive been received for the crew proposed for this project. If the crew which actually works on this project is different from the proposed crew, resumes of the new people will be required.

Generators must be fueled outside of the liner area, unless GeoPacific can construct suitable catch basins to catch spilled fuel and prevent spillage of the fuel on liner, even during windy conditions. 
WHC-SD-W025-RPT-001, Rev. 0

November 17, 1993

2

$933-1354$

Calibration for GeoPacific's field tensiometer must be supplied to GCS.

Calibration for the vacuum gauges to be used in vacuum testing extrusion welds must be supplied to GCS.

Calibration for the pressure gauges to be used in air pressure testing the fusion seams must be supplied to GCS. 
WHC-SD-W025-RPT-001, Rev。 0

APPENDIX C-3

ENGINEERING CHANGE NOTICES

$c-3$ 


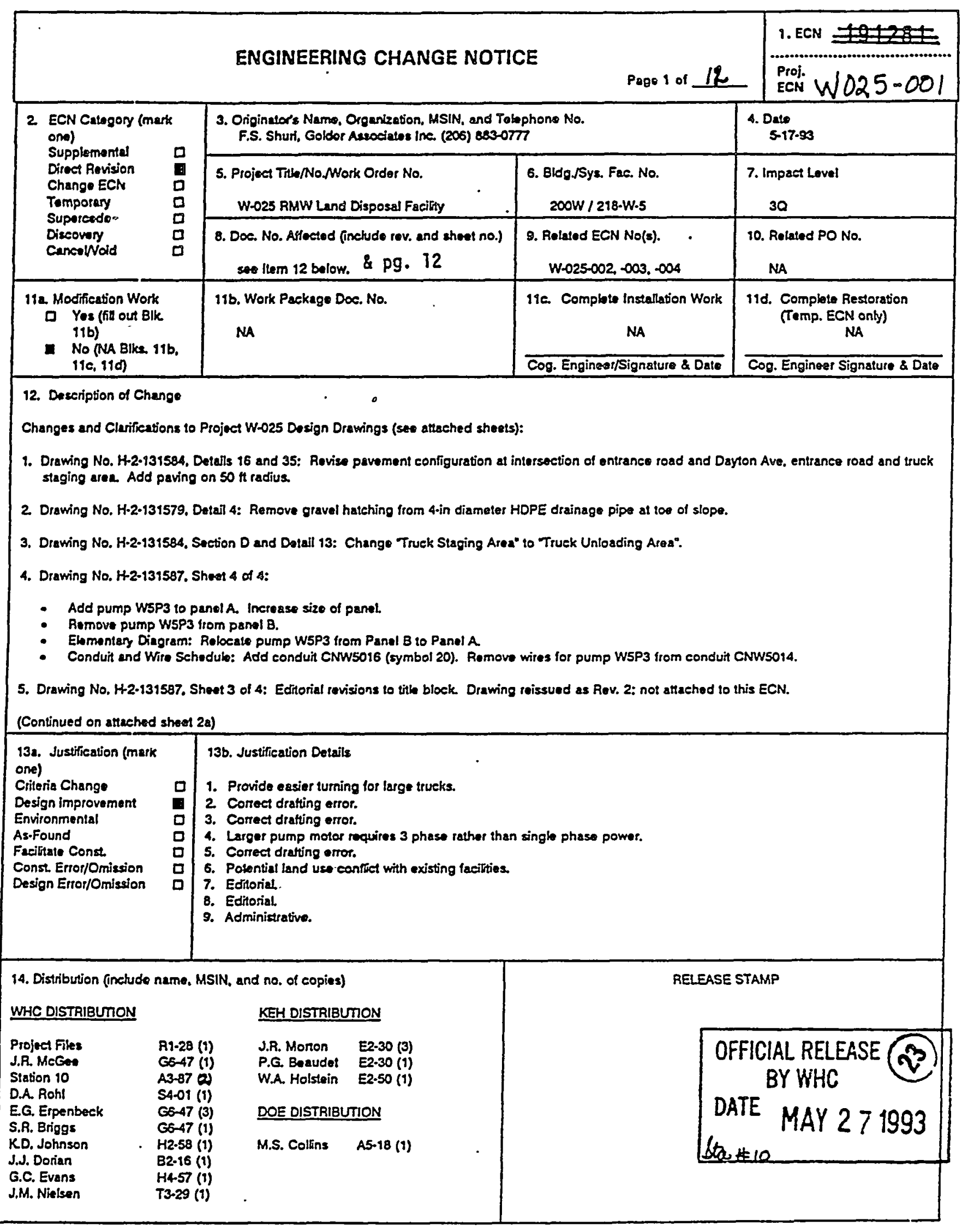




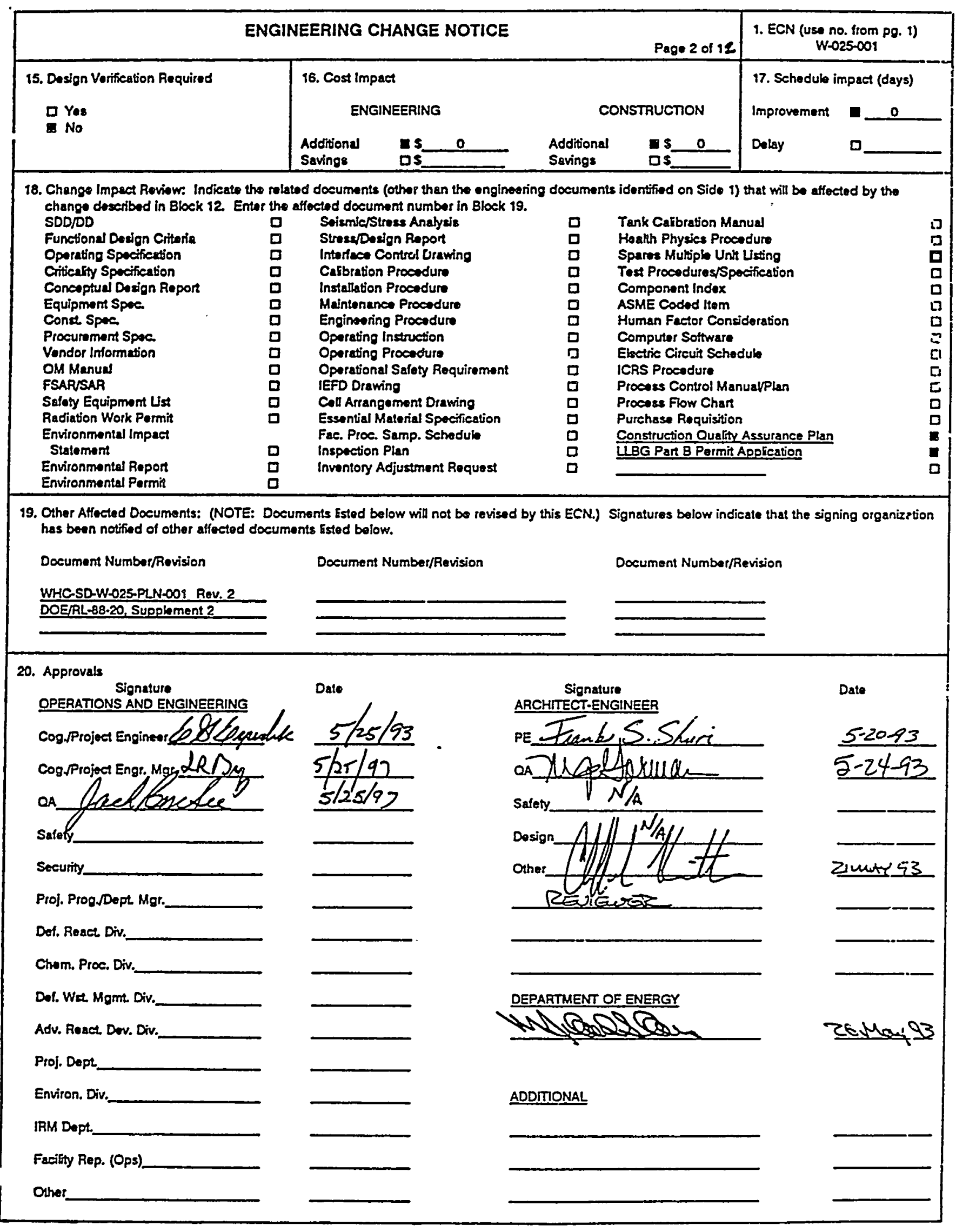


WHC-SD-W025-RPT-001, Rev. 0

ENGINEERING CHANGE NOTICE CONTINUATION SHEET

Comtinued from Section 12, page 1:

6. Drawing Nox. H-2-131575, H-2-131576, and H-2-131577: Remove "Exeavation Stockpilo Atos" and "Admix Prepatation Area" locations. Add noto that excavation clockpile ind admix preparation locations shall be approved by the Construction Managur.

7. Drawing No. H2.131587. Sheot 4 of 4. Elomentary Diagram: Change calbut for wathour meter to rofor to detail number 33.

8. Drawing Nor. H-2-131575, H-2-131576, and H-2-131577: Change CP4 Northing to 44121.29 .

9. Alt Place Golder Associates CAD fila number in appropriate bocation in tith block. 
ENGINEERING CHANGE NOTICE Modifies Dwg. No. H-2-131584. ECN No. $W-025-001$ Date 10-26-92 By FSS Ckd GM

Description of Change: Revise povement configuration at intersection of entrance road and Dayton Ave, entrance road and truck staging orea.

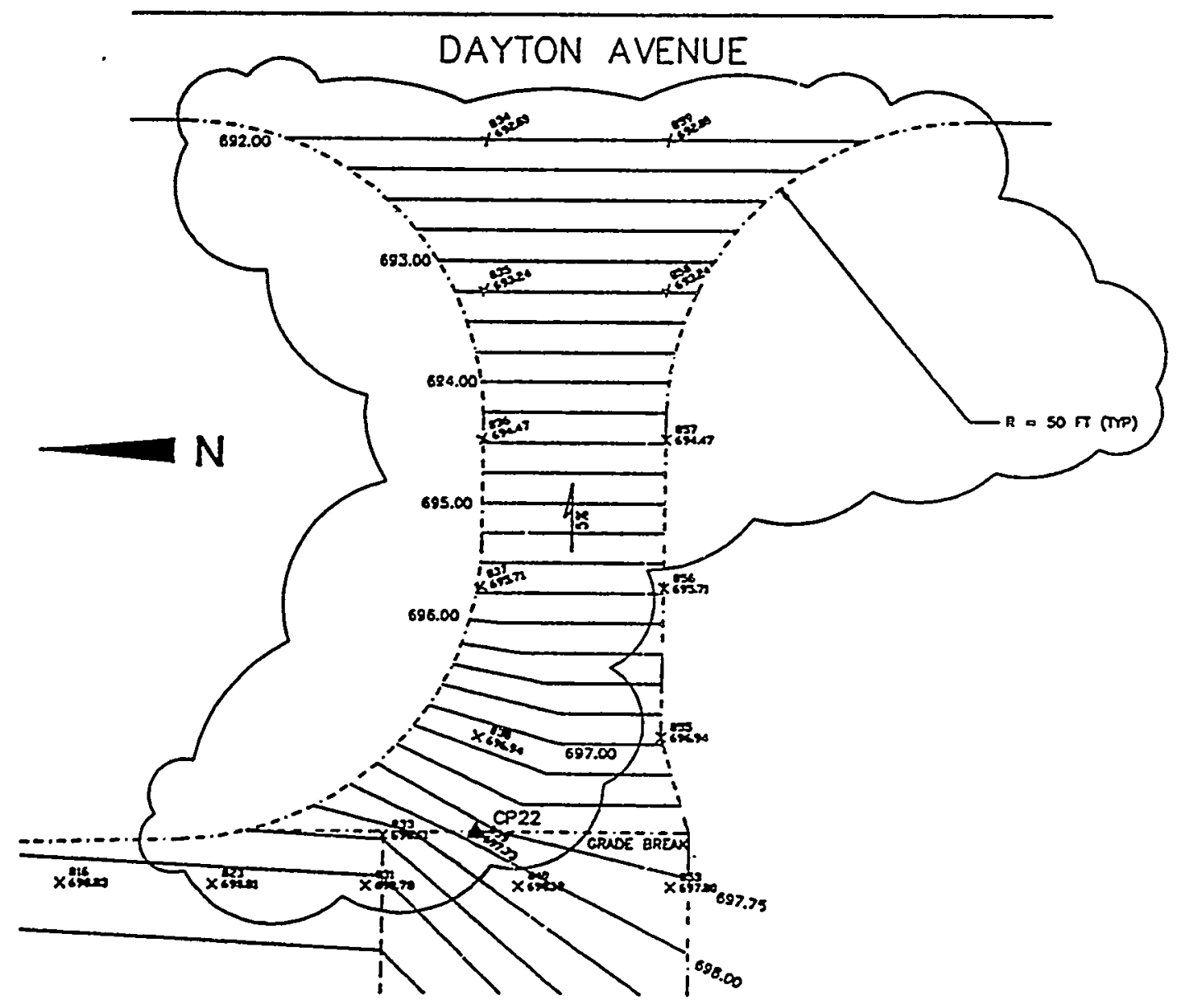

GRADING PLAN OF TRUCK STAGING AND UNLOADING AREAS $\frac{16}{\mathrm{H}-2-13158 \mathrm{~s}}$

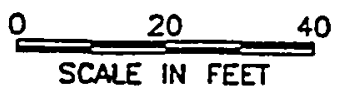

c ecn00101.dwg 
ENGINEERING CHANGE NOTICE Modifies Dwg. No. $\mathrm{H}-2-131584$ Dote 10-26-92 By FSS Ckd GM
ECN No. $W-025-001$

Poge 4 of 126846

Description of Change: Revise povement configuration ot intersection of entrance road and Doyton Ave, entrance road and truck staging oreo.

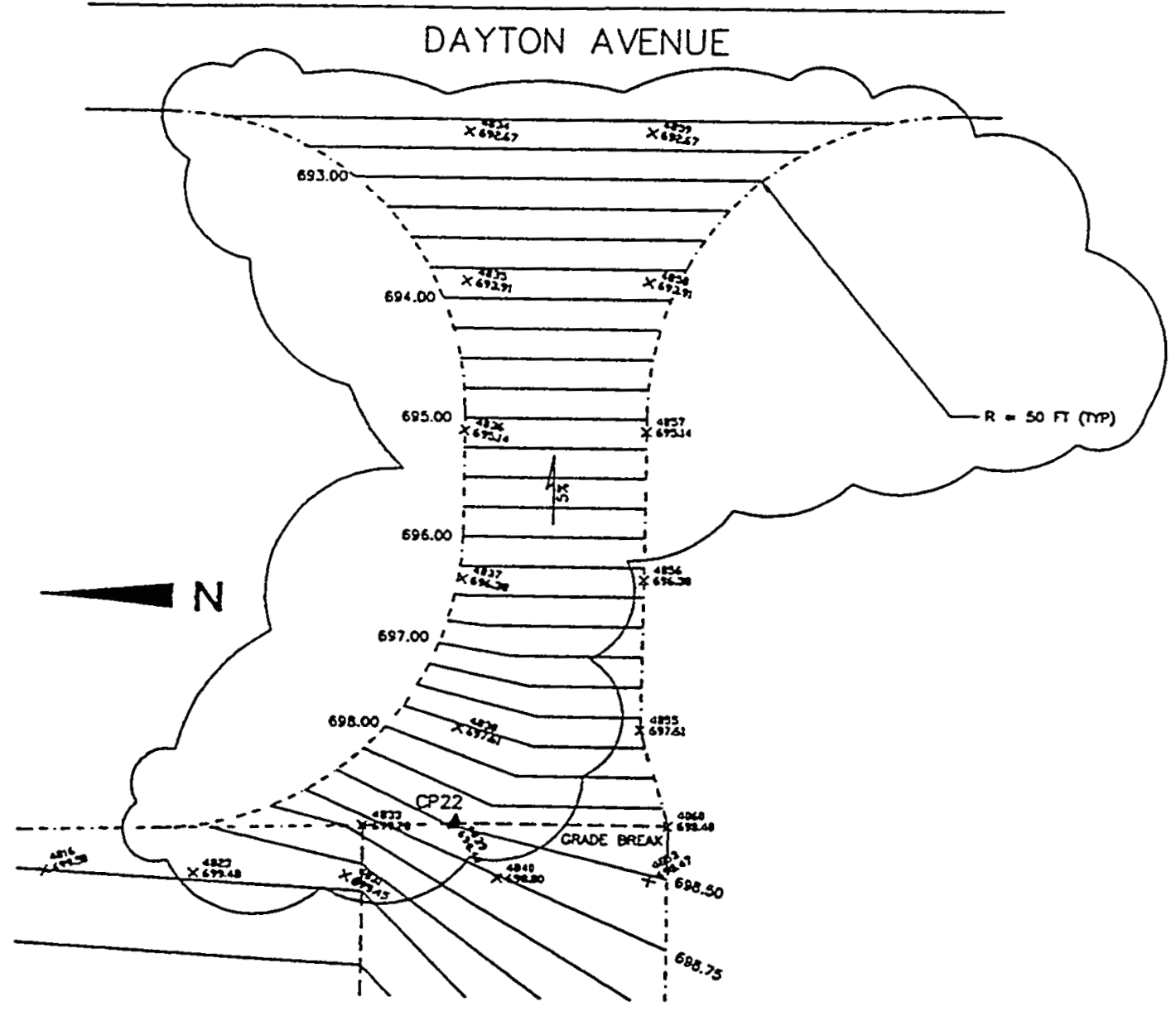

\section{PAVED CONTOURS OF TRUCK STAGING AND UNLOADING AREAS 35}

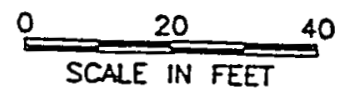




\section{ENGINEERING CHANGE NOTICE}

Modifies Dwg. No. H-2-131579

Date 10-27-92 By FSS Ckd GM

Description of Change: Remove grovel hatching from 4-in dia. HDPE drainage pipe. No other changes.

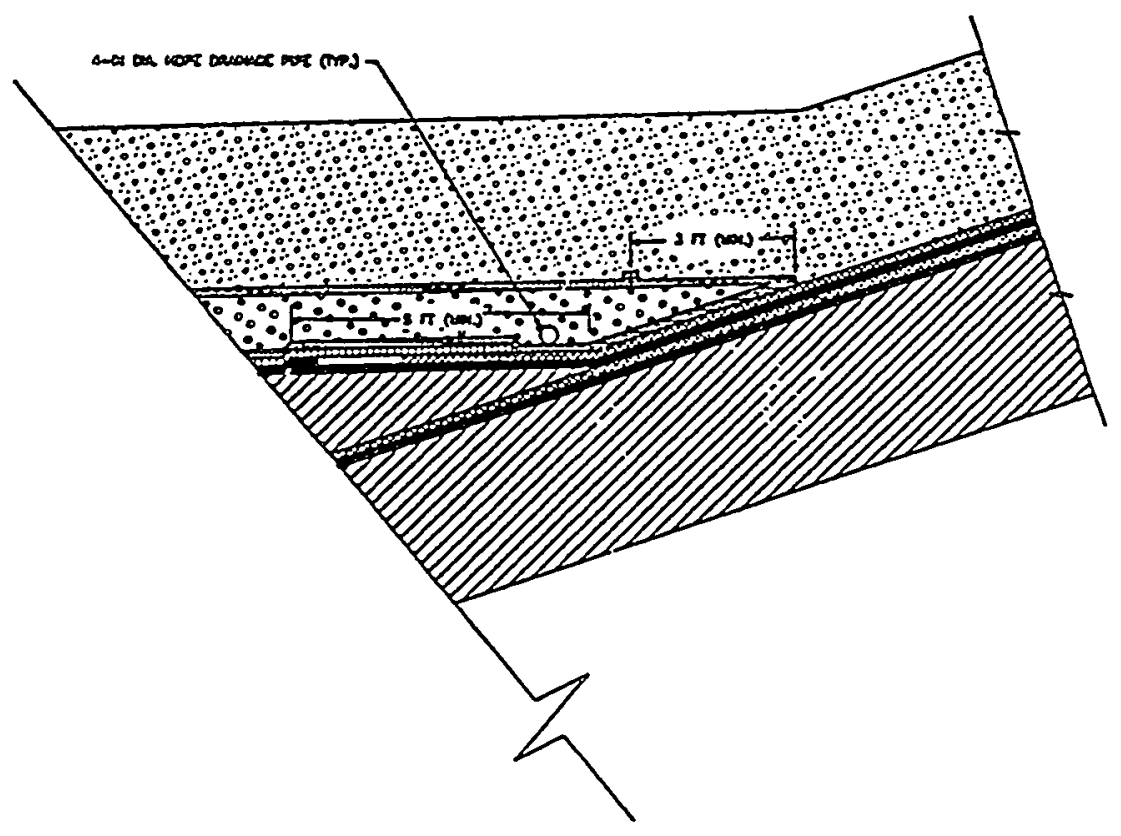

TYPICAL TOE OF SLOPE DETAIL $\frac{4}{4-2-1151592}$

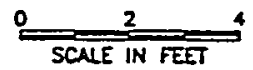

ecn00103.dwg 
ENGINEERING CHANGE NOTICE Modifies Dwg. No. H-2-131584

Dote 10-27-92

By FSS

Ckd GM
ECN No. W-025-001

Poge 6 of 126846

Description of Change: Change "Truck Staging Areo" to

"Truck Unloading Areo".

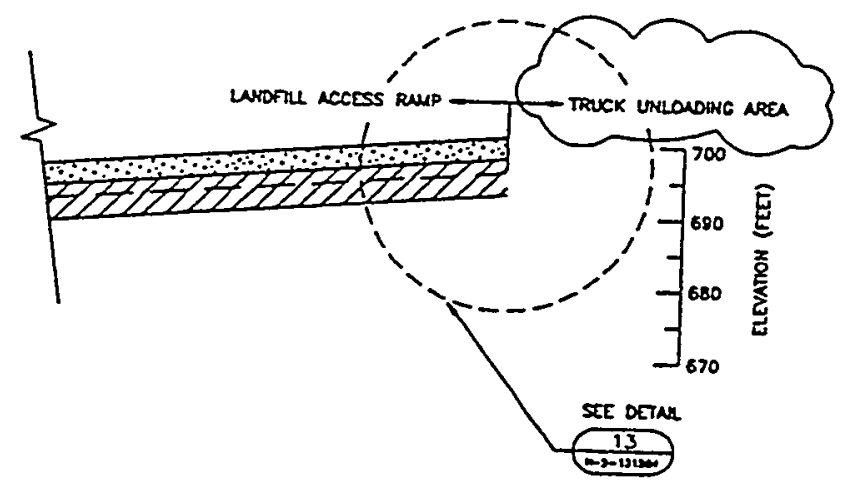

\section{ACCESS RAMP PROFILE}

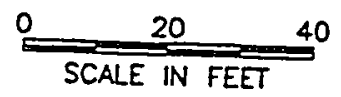


ENGINEERING CHANGE NOTICE Modifies Dwg. No. H-2-131584

Date 10-27-92 By FSS Ckd GM

Description of Change: Change "Truck Staging Area" to

"Truck Unloading Areo".

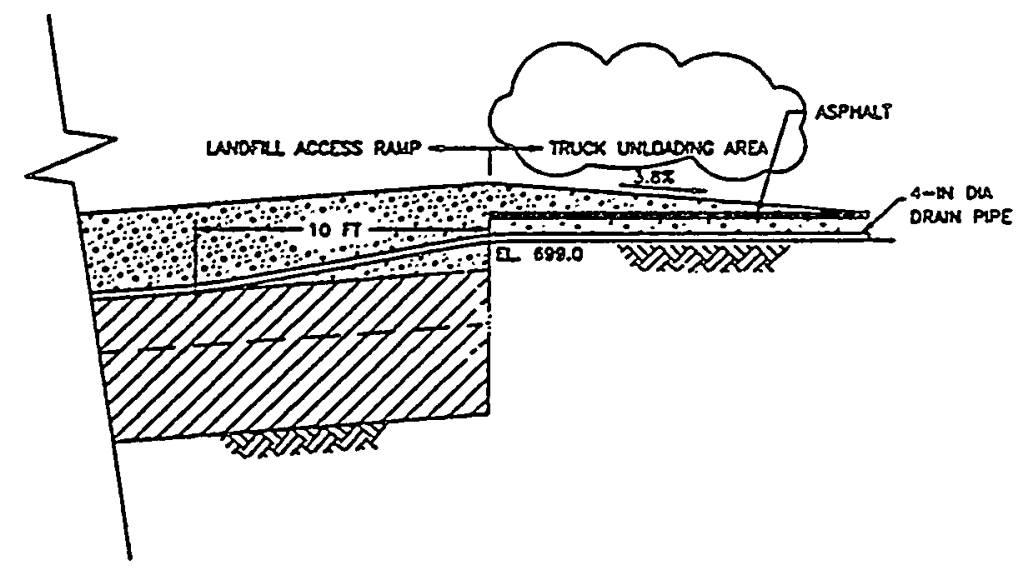

ACCESS RAMP/TRUCK

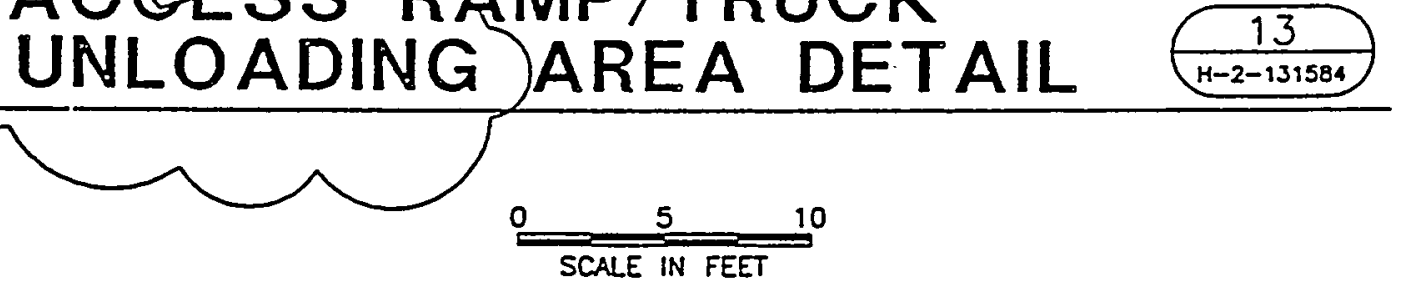

ecn00105.dwg

ECN No. W-025-001

Poge 7 of 12686 
ENGINEERING CHANGE NOTICE Modifies Dwg. No. H-2-131587

Date 10-27-92 By FSS Ckd GM

ECN No. $\quad W-025-001$

Page 8 of 12686

Description of Chonge: Add pump W5P3 to Ponel A. Increase size of panel.

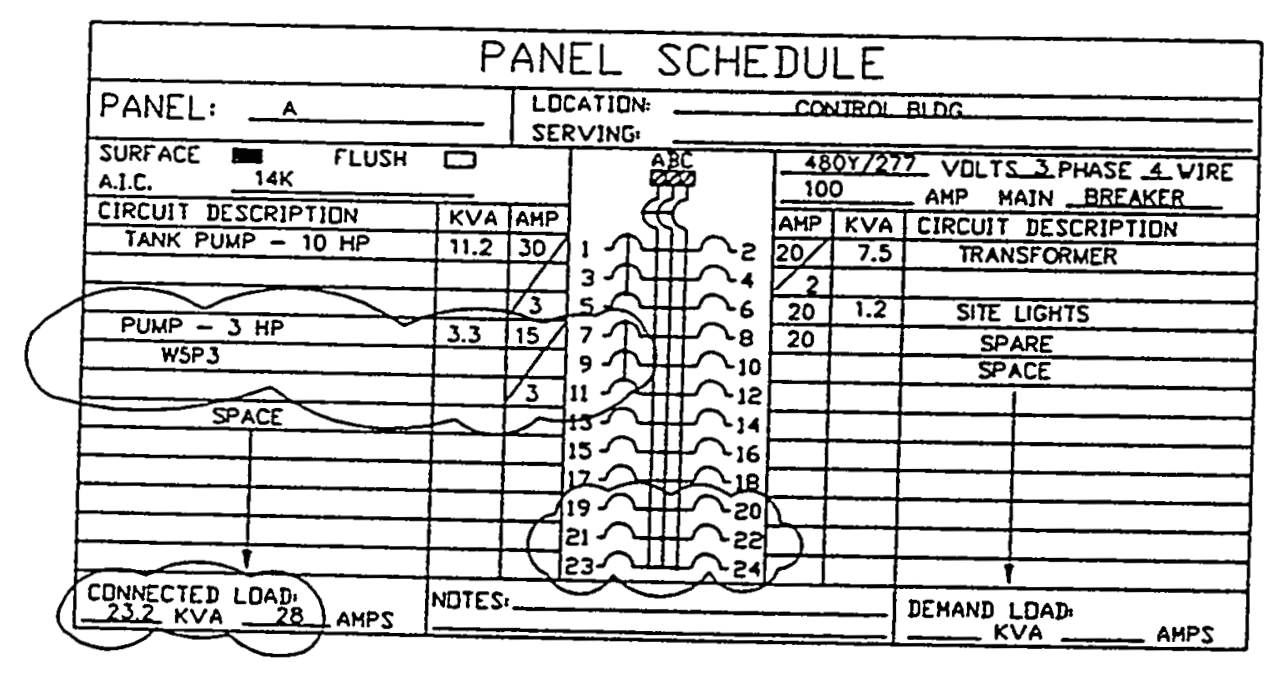

ecno0106.dwg 
ENGINEERING CHANGE NOTICE

Modifies Dwg. No. H-2-131587

Date 10-27-92 By FSS Ckd GM

Description of Change: Remove pump W5P3 from Ponel $B$.
ECN No. W-025-001

Page 9 of HTiz 6846.

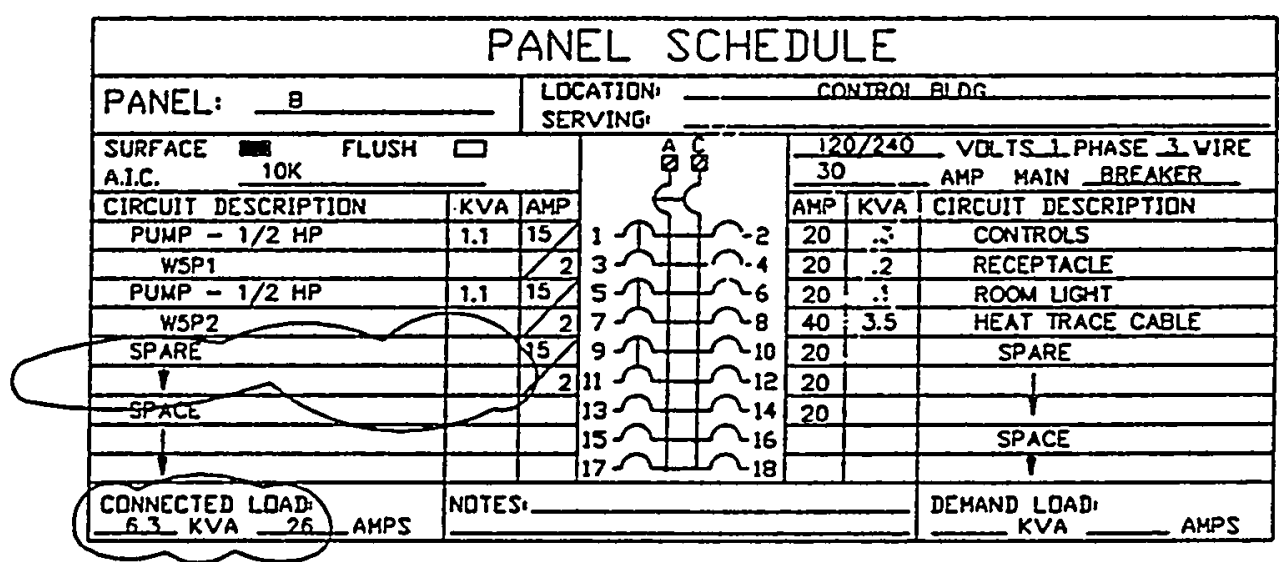

ecn00107.dwg 
ENGINEERING CHANGE NOTICE

Modifies Dwg. No. H-2-131587

Date 10-27-92 By FSS Ckd GM

Description of Change: Relocate pump W5P3 from Ponel B to Ponel A.

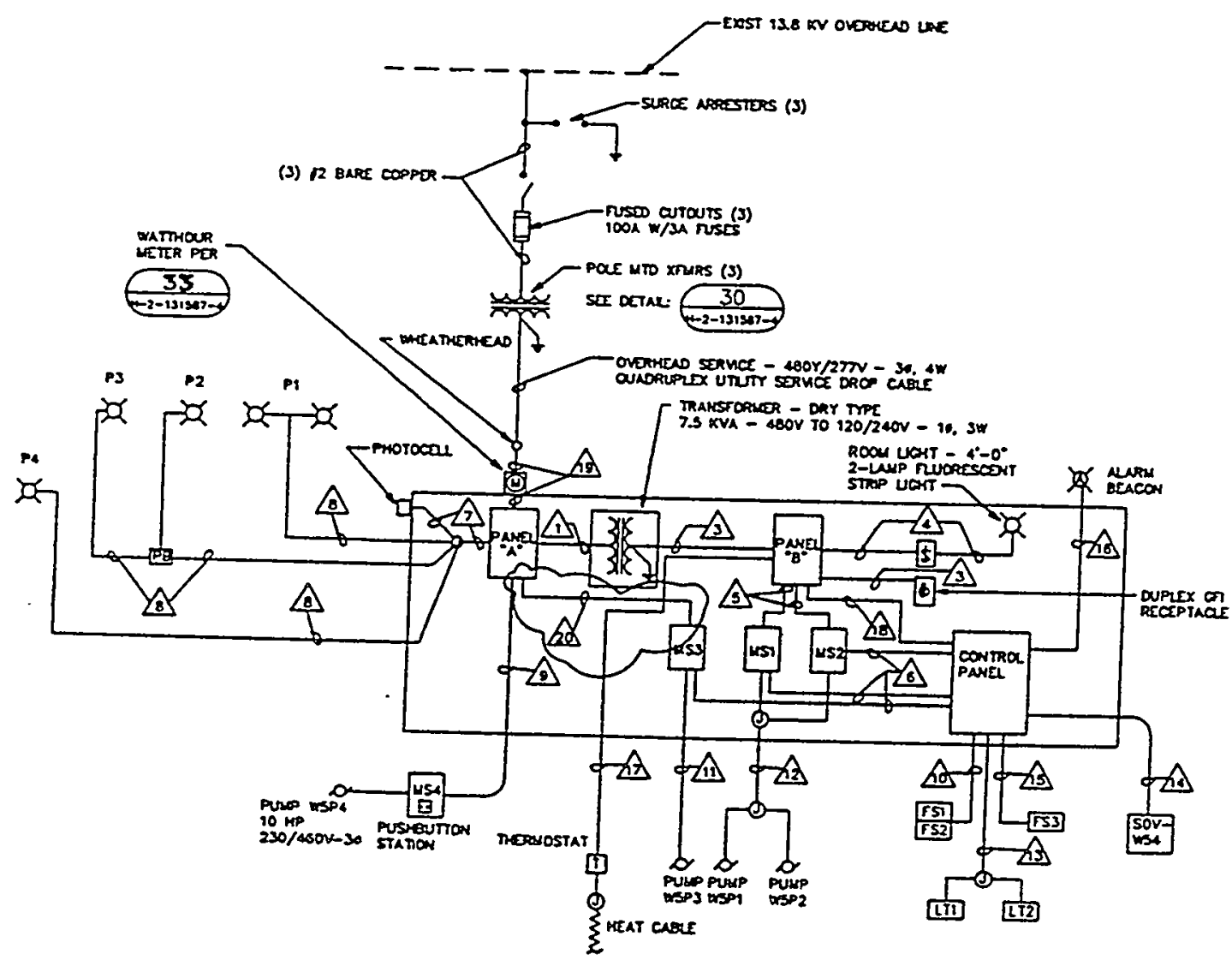

ELEMENTARY DIAGRAM
ECN No. $W-025-001$

Page 10 of $17 \cdot 12686$ 


\section{ENGINEERING CHANGE NOTICE}

Modifies Dwg. No. $\mathrm{H}-2-131587$

Date 10-27-92 By FSS Ckd GM

Description of Change: Add conduit CNW5016 (symbol 20).

Remove wires for pump W5P3 from conduit CNW5014.

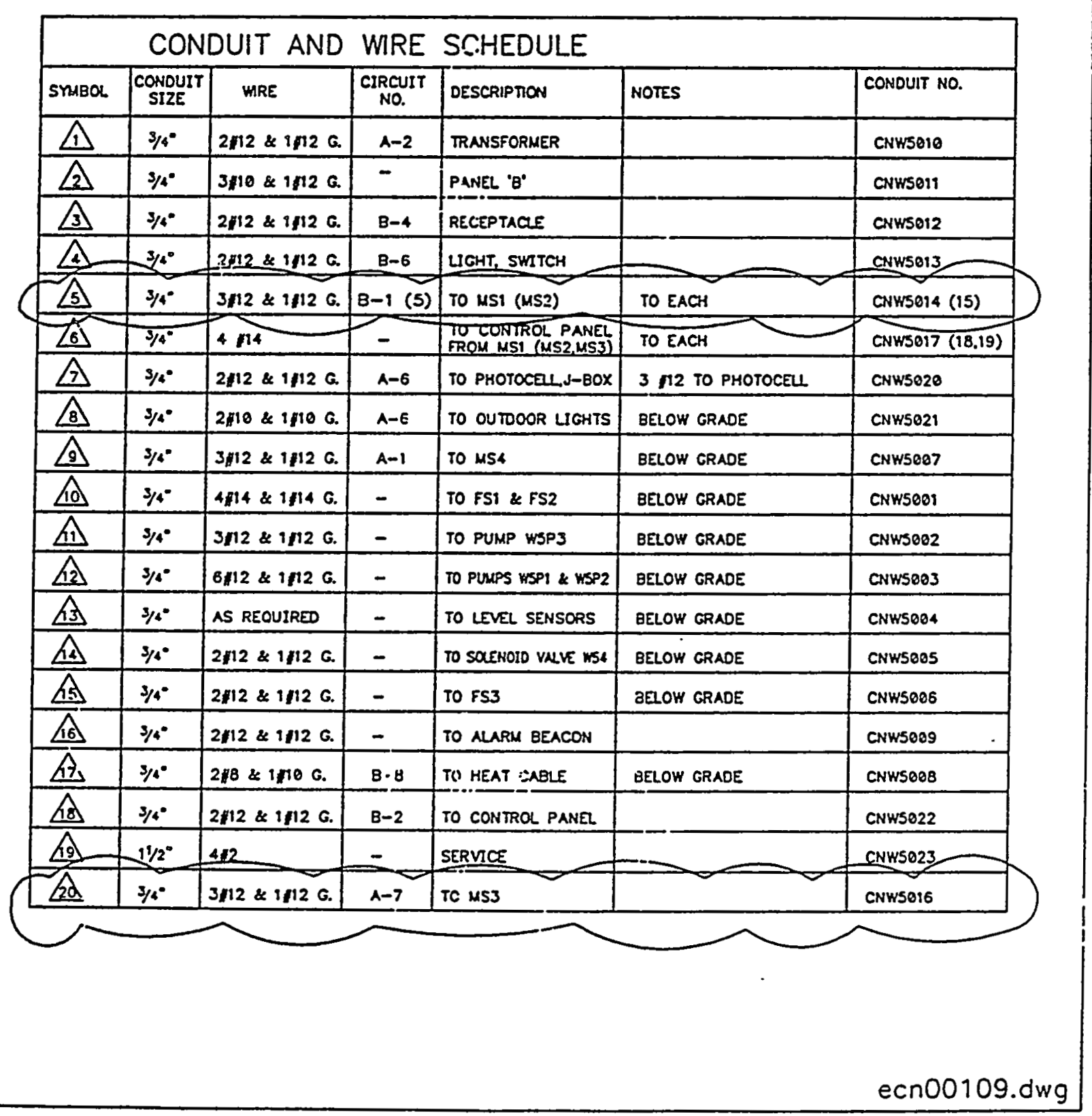




\begin{tabular}{l|l|l|l|l}
\hline ENGINEERING CHANGE NOTICE CONTINUATION SHEET & $\begin{array}{l}1 . \text { ECH } \\
\text { Poge } 12 \text { of } 12\end{array}$ & W-025-001 \\
\hline
\end{tabular}

GENERAL REVISION TO THE FOLLOWING DRAWINGS \& SPECIFICATIONS:

H-2-131573, "COVER SHEET," REV 1, SHEET I OF 1

H-2-131574, "SITE PLAN AND EXPLORATIONS," REY 1, SHEET I OF 1

$H-2-131575$, "GRADING PLAN," REV 1 , SHEET 1 OF 1

H-2-131576, "SECONDARY ADMIX CONTOURS," REV I, SHEET 1 OF 1

H-2-131577, "OPERATIONS LAYER CONTOURS," REV i, SHEET 1 OF 1

H-2-131578, "CROSS-SECTIONS," REV 1, SHEET 1 OF 1

H-2-131579, "LINER SYSTEM DETAILS," REV 1, SHEET 1 OF 1

H-2-131580, "SUMP LAYOUT," REV I, SHEET I OF I

H-2-131581, "SUMP CROSS-SECTIONS," REV 1, SHEET 1 OF 1

H-2-131582, "SUMP LEACHATE COLLECTION PIPES," REV 1 , SHEET 1 OF 1

H-2-131583, "SIDE SLOPE AND VERTICAL RISER PIPES," REV 1, SHEET 1 OF 1

H-2-131584, "TRUCK STAGING AREA AND ACCESS RAMP," REV 1, SHEET 1 OF 1

H-2-131585, "LEACHATE COLLECTION TANK AND PIPING, " REV 1 , SHEET 1 OF 1

H-2-131586, "SUMP PUMP DETAILS," REV 1, SHEET I OF 1

H-2-131587, "ELECTRICAL," REV I, SHEET' 1 OF 4

H-2-131588, "CONCEPTUAL FILL PLAN," REV 1, SHEET 1 OF 1

H-2-131589, "SUBGRADE GRID POINTS," REV 1, SHEET 1 OF 1

H-2-131590, "SECONDARY ADMIX GRID POINTS," REY 1, SHEET 1 OF 1

H-2-131591, "SECONDARY DRAINAGE GRAVEL GRID POINTS," REV 1 , SHEET 1 OF 1

H-2-131592, "PRIMARY DRAINAGE GRAVEL GRID POINTS," REV 1, SHEET 1 OF 1

H-2-131593, "OPERATIONS LAYER GRID POINTS," REV 1, SHEET 1 OF 1

H-2-131594, "PRIMARY ADMIX GRID POINTS," REV. 0, SHEET 1 OF 1 
$-\cdot$

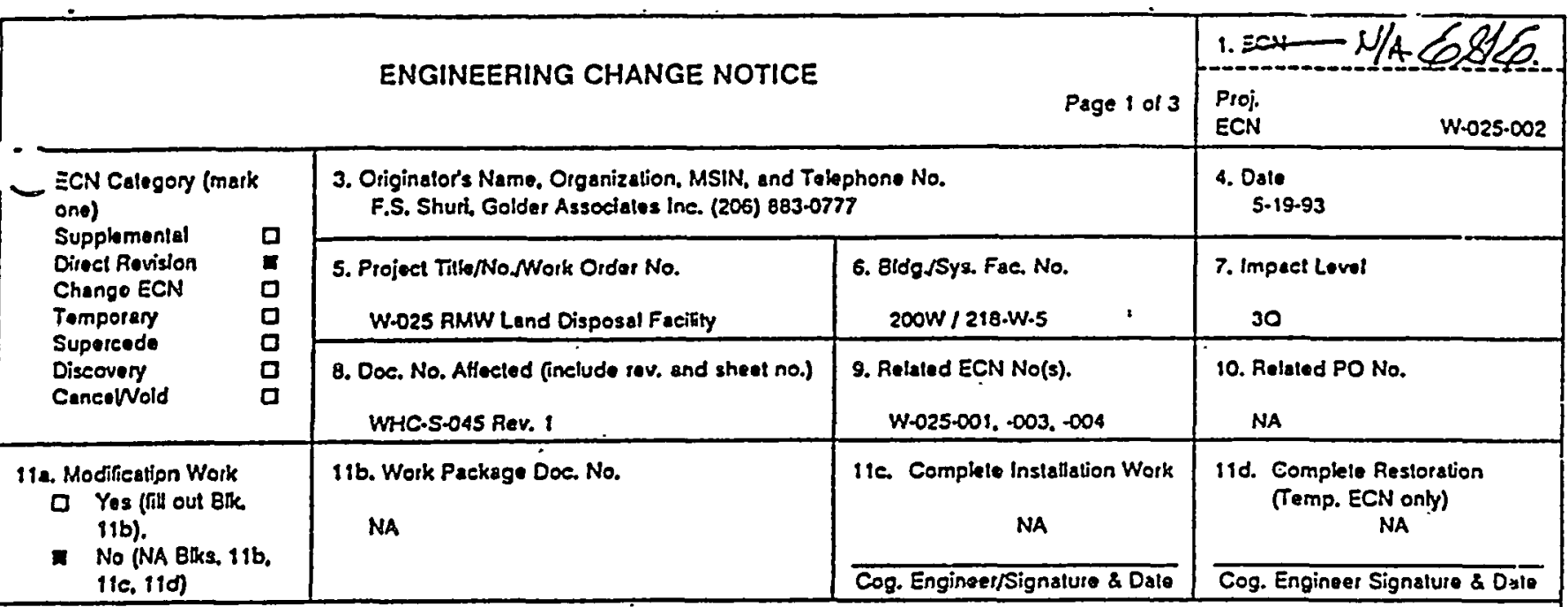

i2 Description ol Change

Cnenges and Clarifications to Project W-025 Speeifieations:

1. Section 02224, Pan 3.1 A, 1.: Delele "ASR 280E'. Delele "Production capabitty of 300 tons pet hour."

2. Section 02224. Part 3-1 A. 2.: Add -Ahernatively, belt seales on finished product bell only, and seale(s) for aceurately weighing soil and bentonile in small balches to the satis/action of the Construction Maneger and the COA Enginear prior to mixing."

3. Section 02224, Part 3-1 A.5.: Change to Vatiable speed hydrautic supply waler pumps capable of producing 300 tons per hour of sdmixture." *.

4. Section 02224. Part 3.1 F.: Add to end of paragraph The Contractor shatl provide means of testing for the percentage of bentonite by the melhylone blue inothod. A minimum of three tests shall be pertormed on each sample. At hast two test resulhs shall be within the specified imils of i bentonite content, and the thitd resull shall be within $0.5 \%$ of the specilied fmits."

(Liontinued on etteched sheet)

\begin{tabular}{l|l} 
13a. Justification (maik & 13b. Justifieation Details
\end{tabular}

one)

Crituris Change $\quad$ 1. Unnecessatily restrictive requitement.

Design improvement - $\quad \cdot \quad$ 2. Polentially lower cost option.

Environmental $\quad$ 3. Necessary requirement based on experience.

-As-Found - $\quad$ - 4. 4. Necessary requiremont based on exporience.

Faciflale Const. $\quad$ 5. Potentially not appleable and therelore misleading.

Const. Etror/Omission D 6. Clatilication.

Design Error/Omission $\square$ 7. Clarifiestion.

B. Additional requirement to tacifiate OA.

9. Additional requirements to improve material and work quality.

10. Additional requirement to improve work çuatity.

11. Clarification.

12. Additional requiuitements to improve maleria and work quatity.

13. Alresdy in design. therelore not required of Contractor.

14. Already in design. therefore not required ol Contractor.

15. Verify installation qualify.

16. Verify that material meets specifieations.

17. Verily material quafity.

18. More accuiate value.

19. Clarification.

20. Changes in industry spandards.
14. Distribution (include name. MSIN. and no. of copies)

\section{WHC DISTAIBUTION}

- Project Files

J.R. McGee

Station 10

D.A. Roh

E.G. Erpenbeck

S.R. Eriggs

K.D. Johnson

- Dorian

$\therefore$ Evans

J.M. Nielsen

\section{KEH DISTRIBUTION}

J.R. Morton E2.30 (3)

P.G. Beaudel E2-30 (1)

W.A. Holstein E2.50 (1)

A3. 87 (1)

S4-0t (1)

G6.47 (3)

G6.47 (1)

H2.58 (1)

82.16 (1)

H4.57 (1)

T3.29 (1)
DOE DISTRIBUTION

M.S. Colins A5.18 (1)
RELEASE STAMP

CFFICIAL RELEASE (20)
BY WHC
DATE HAY 271993
Sta. 10




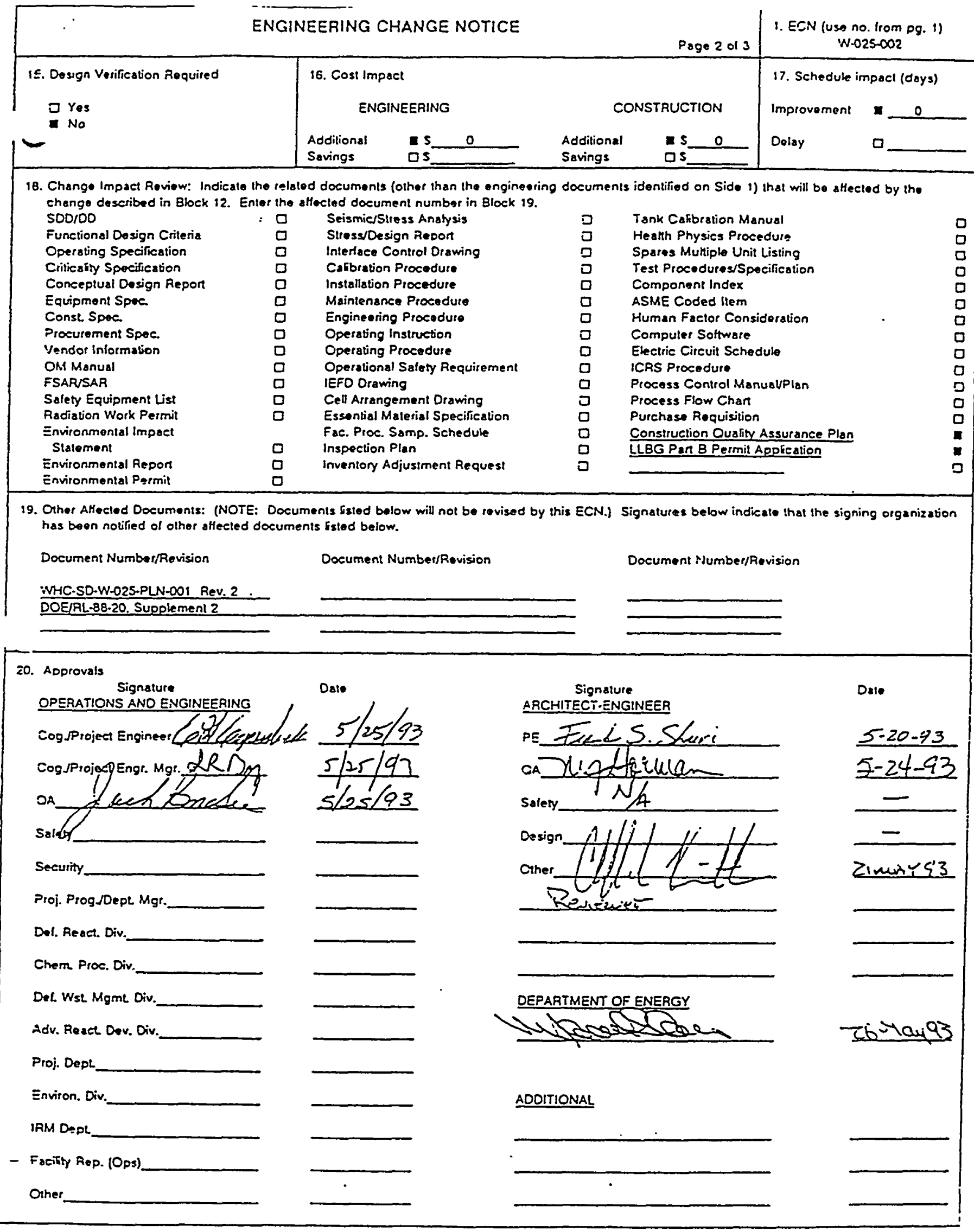




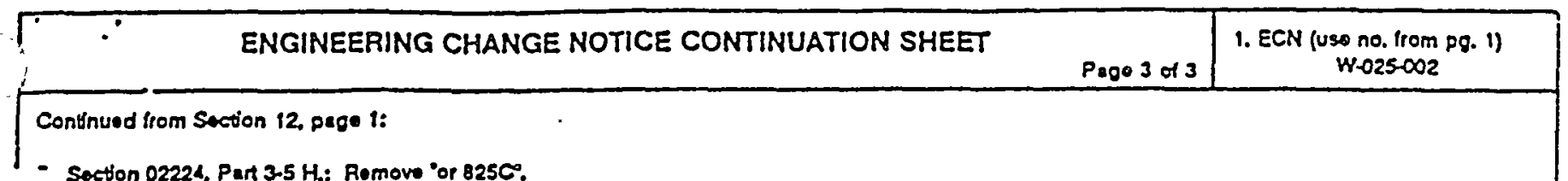

6. Secton 02220, Pen 3-1 F.: Revied lat sentence to reed "Greding equipment shall operate on no bas then 1 toot of dralnage layer matertai over any geosynthete tayer."

7. Section 02228, Part 3-1 A.3.: Revse last santence to read 'Grading equipment shall operate on a minimum of 2 foet of operations layer materiah'

8. Section 02275, Part 2-1. C.2.: Ruvise hirst buthot to read 'rol numbers and identification, resin lot and batch numbers; and".

D. Soction 02511: Add now part

Qg-5 SUBMTTALS

A. Submit esphalt concrate mix dedgn for approval prior to ordering materials.

B. Prior to construction, submit lor approval tho proposed methods lor plecing and corapacting top courso and asphak layers, and for applyng a!l primers and coatinge.

C. Submit in accordance with Soction 01300.

10. Section 02725, Part 1.3: Add new peragraph D. Submit quatifeatone of proposod loining personnelo

11. Section 02725, Part 3-4, C.: Add Fold lest pressure for 8 hour."

12. Section 03310, Part 1-2: Add new paregraphs

C. Submit concrete mix design for approval prior to ordering materials.

D. Submit manufactuters tochnical date on waterstope and proposed installeston mathods prior to ordering materiais.

E. Submit manufacturer's technical date on anchor bohs and proposed installation methods prior to ordoring materials."

jection 13340, Part 2-3: Debte "conforming to the requirements of Hanlord Site Standards".

14. Saction 16010, Part 1-4, B: Dolelo All appleablo Hanlord Plant Stendasde".

15. Section 02275, Part 3-1, E: Add new suberetion:

99. Containmant Basin Lnat Tasting

The ining of the contuinment basin under the bechate storage tank shall be lested to demonstrate that it is froe from baks. To perform the test. the besin shall be filind with chan water. The water bvol shall be measured to the nearest 0.01 inch over a minimum time of 48 hours. If the water bevel decreeses, the besin shall be drained and the bak repejred in eccordence with the procedures described in the previous section of these Specificatione. The lesting and repeir activites shall bo ropeeted until a pessing result is achieved. During the test meesures shel be iaken as necesery to provent precipitation from entering the basin. If the testing is portormed dutho hol weather, hinay be necessary is cover the water surfece to prevent eveporation.

16. Section 02226. Part 2.1: Add to end of first paragraph: The COA Engineer with perform a slake durabifty teat on the gravel samplos submitted by the Contractor. A lose in wolght of lass than $1 \%$ will be considered acceptablo."

17. Section 02224, Part 2-2: Add after first enntence: The bentonite shell have a troe swoll of $25 \mathrm{ml}$ per 2 gm or grester."

18. Secdon 02275. Part 2-1, B.2: Change-specifie gravity requirement from ${ }^{\circ}>0.94^{\circ}$ i0 $>0.935^{\circ}$.

19. section 02275, Part 2-1. B.5: Add Textured" bolore "geomembrane:

20. Section 02275, Part 2-1. tabie ol Required Geomembrane Properties:

- Change puncture resistance of Typo ll goomembrane to 80 lbs.

- Change puncture recistance of Type III goomembrane to $105 \mathrm{lb}$

- Change resistance to soil burial of Types II and III goomembrane to 9ox.

- Change emironmental stress arack of Typos II and III geomembrane to 2000 hrs.

$-$ 


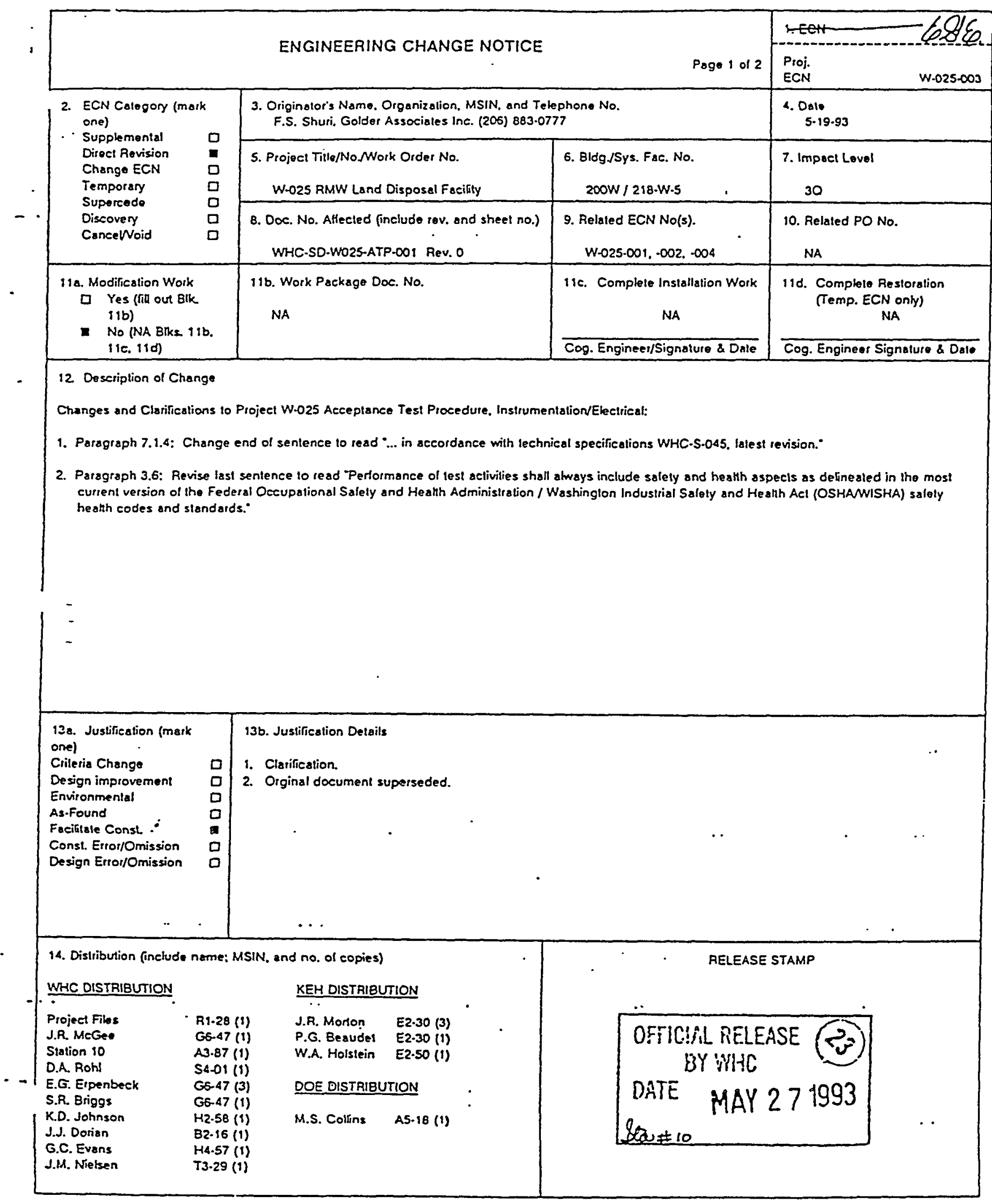


WHC-SD-W025-RPT-001, Rev. 0

\begin{tabular}{|c|c|c|c|c|c|c|c|c|c|}
\hline \multicolumn{7}{|c|}{ ENGINEERING CHANGE NOTICE } & \multicolumn{3}{|c|}{$\begin{array}{l}\text { 1. ECN (use no. from pg. 1) } \\
\text { W.025.003 }\end{array}$} \\
\hline \multirow[t]{2}{*}{$\begin{array}{l}\text { 15 Oasign "orification Required } \\
\text { • } \square \text { Yes } \\
\text { No No }\end{array}$} & & \multicolumn{3}{|c|}{$\begin{array}{l}\text { 16. Cost Impact } \\
\text { ENGINEERING }\end{array}$} & \multicolumn{2}{|c|}{ CONSTRUCTION } & \multicolumn{3}{|c|}{$\begin{array}{l}\text { 17. Schedule Impecl (days) } \\
\text { Improvement }\end{array}$} \\
\hline & & $\begin{array}{l}\text { Additional } \\
\text { Savings }\end{array}$ & as & 0 & $\begin{array}{l}\text { Additional } \\
\text { Savings }\end{array}$ & $\begin{array}{l}510 \\
05 \\
\end{array}$ & Delay & 口. & \\
\hline 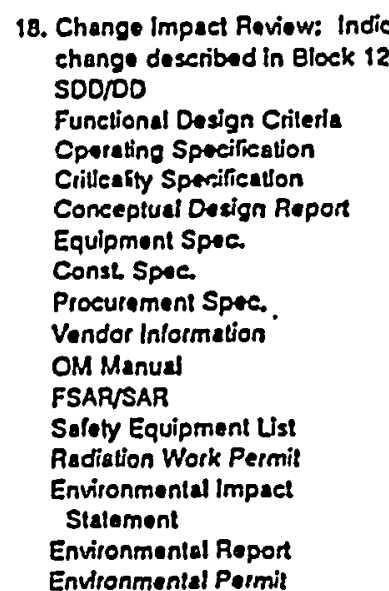 & 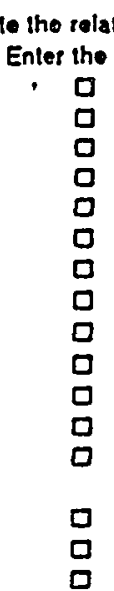 & 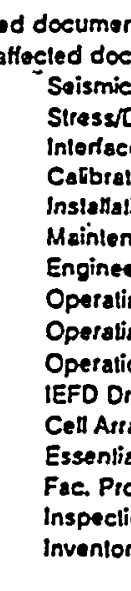 & $\begin{array}{l}\text { ss An } \\
\text { on Rep } \\
\text { intiol } \\
\text { Proced } \\
\text { Proend } \\
\text { Proe } \\
\text { Proce } \\
\text { istruct } \\
\text { roced } \\
\text { Salety } \\
\text { ig } \\
\text { ment } \\
\text { Jesial } \\
\text { amp. } \\
\text { Jan } \\
\text { justm }\end{array}$ & $\begin{array}{l}\text { the engine } \\
\text { in Block } \\
\text { is } \\
\text { sing } \\
\text { quirement } \\
\text { wing } \\
\text { cification } \\
\text { Redule } \\
\text { Request }\end{array}$ & $\begin{array}{l}a \\
0 \\
0 \\
a \\
0 \\
a \\
a \\
0 \\
0 \\
a \\
0 \\
0 \\
0 \\
0 \\
a \\
0\end{array}$ & $\begin{array}{l}\text { Tank Cafibration Ma } \\
\text { Heakh Physies Proe } \\
\text { Spares Muhiplo Unit } \\
\text { Tast Proceduros/Sp } \\
\text { Component index } \\
\text { ASME Coded liom } \\
\text { Human Faetor Cons } \\
\text { Compuler Soltware } \\
\text { Electric Circuil Sche } \\
\text { ICRS Procedure } \\
\text { Process Control Mar } \\
\text { Process Flow Chan } \\
\text { Purchase Requisitior } \\
\text { LLBG Part B Permit } \\
\text { Construction Ouality }\end{array}$ & 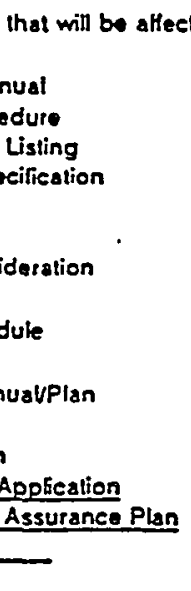 & dd by the & $\begin{array}{l}0 \\
0 \\
0 \\
0 \\
0 \\
0 \\
0 \\
0 \\
0 \\
0 \\
0 \\
0 \\
0 \\
0 \\
0 \\
0\end{array}$ \\
\hline
\end{tabular}

19. Othar AHected Documents: (NOTE: Documents ksted below will nol be tevised by this ECN.) Signalures below indicate that the signing organizalion has been notiried of other atfected documents fsted below.
Document Number/Rovision
Document Number/Fevision
Document Numbes/Revision

DOE/RL.88.20, Supplement 2 WHC.SD.WO25.DLN-DO1 ROV. 2

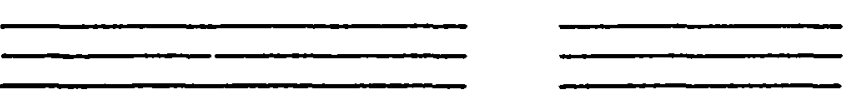

20. Approvals

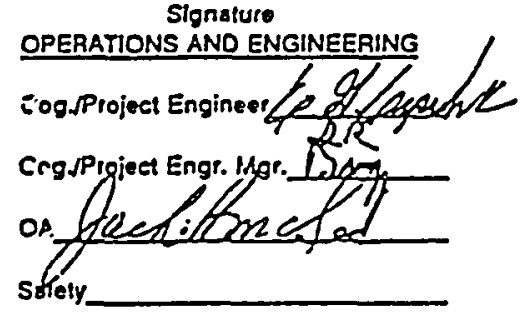

Dale

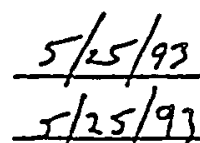

Se zurity.

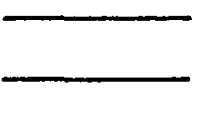

Proj. ProgfDept Mgr.

Signature
ARCHITECT.ENGINEER

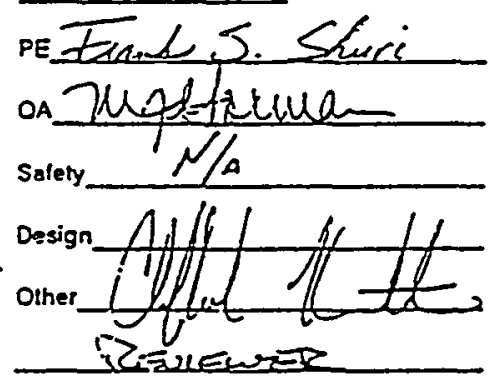

- Del. Resct. Div. . . . .

Chem, Proe. Dlv.

Del. Wst. Mgmt D.v:

Adv. Reset. Dev. Div.

Pioj. Depl.

Enviton. Div.

IRM Dept.

Facitty Rep. (Ops)

Othar.

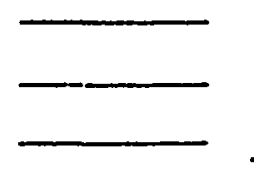

DEPARTMENT OF ENERGY

See T.TIE Page

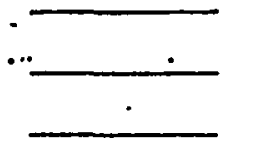

ADDITIONAL
Dits

$5-20: 93$

$5-24-93$

21 unte \&

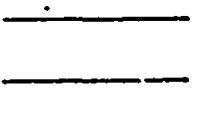

$5 / 25 / 93$ 


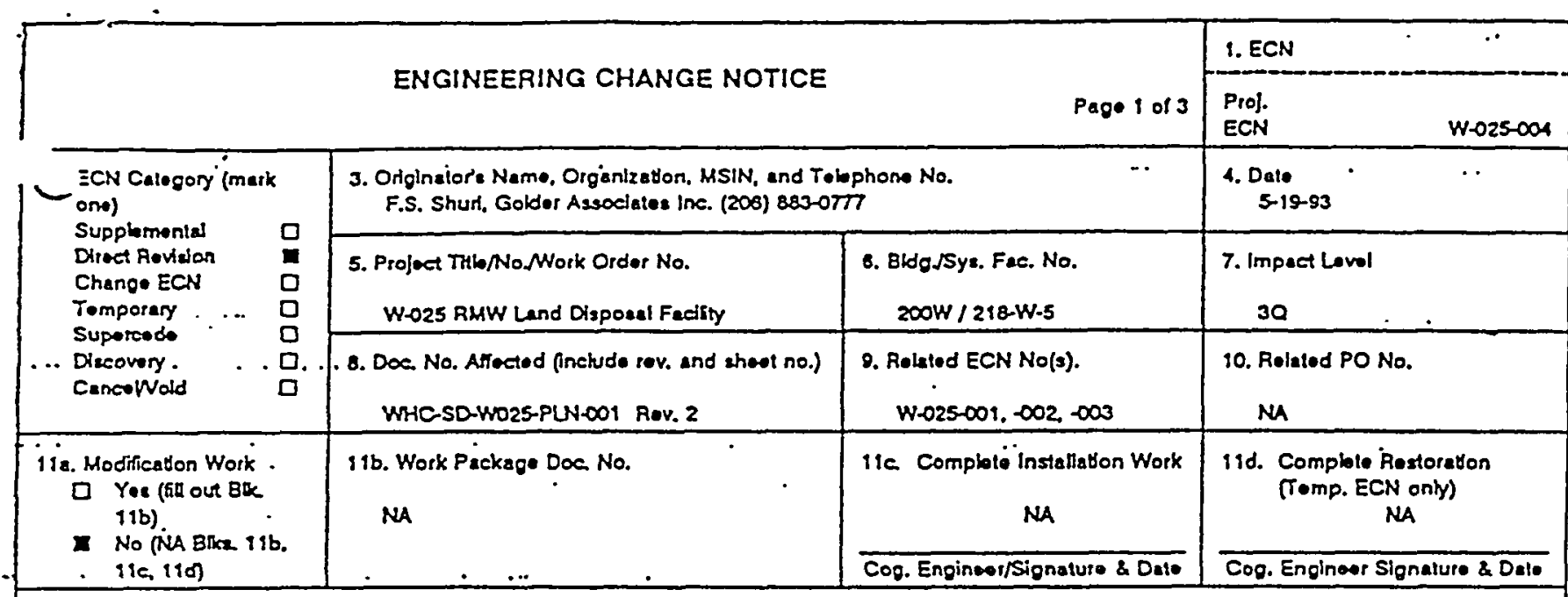

12. Deceription of Change

Chenges and Clatificabions to Project W-025 Construction Quefty Assurance Plan:

1. Appendix A, CQA Summary Requirements, Spocification Section 02224, page 1: Chenge acceptance criteria for Bentonite Conlent to teed "11\% to $14 \%$ dry wolght es determined by $\$ 200$ slowe:

2 Appendix A, caA Summary Requirements, Specification Section 02511, page 1: For first entry "Contractor submittal ... 2 ehange Specification Section a Paragraph 10 O2511 $1-5^{\circ}$.

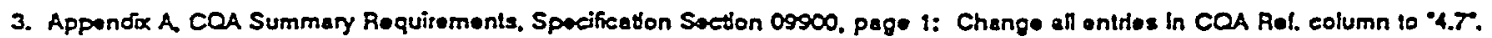

4. Section 4.423: Add new subsection:

Contelnment Basin Uner Testing

The ining of the containment baein under the bechate storage tenk shall be tested to demonstrate that it is free from loaks. To perform the test the besin shal be tillod with clean water. The water bovel shall be meesured to the neerest 0.01 inch over a minimum time of 48 hours. If the water bvel decreases, the besin shall be drelned and the bak repalred ln accordance with the proceduree doscribed in the Specifications. The lesting and repair

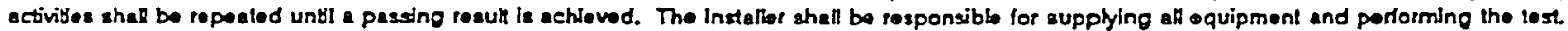
The result shall be submitted to the CQA Engineer for reviow prior to epproving the iner lnstallaton. During the tost, measures shall be laken as necessary to prevent precipitason from entering the besin. If the testing is performed during hot weather, it may be necessary to cover the water surfece to proveri eveporation. All such mesures shell be the instalbers responsibitity."

(Continuod on attached ahoel)

\begin{tabular}{l|l}
\hline i3e Justication (makk & 13b. Justification Doteils
\end{tabular}

one)

Crileria Change

Deslon improvemerd

Envionmental

As.Found

Facintate Const

Const Error/Omission

Dasion Errot/Oriksion

D 1. Ruvle to be consident with Specifications.

D. 2. Aeviee to be conuratent with Specificatione.

1. Editorial

0 4. Vority installation quatity.

z. S. Verify installation quaity.

6. Verify that material moets specifieations.

7. Verify that malorial moots spocifications.
14. Distribution (nclude name. MSIN, and no. of copies)

WHC DISTAIBUTION

Prolect Fios

J.R. McGoe

Slaton 10

D.A. Roh

SG. Erpenbeck

T. Brogs

. J. Johneon

J.J. Dorian

G.C. Evans

J.M. Niolson

\section{KEH OISTAIBUTION}

$R 1.28$ (1)

C5-47 (1)

A3.87 (1)

S4-0I (1)

G6-17 (3)

Co-47 (1)

H2.58 (1)

82-16 (1)

H4-57 (1)

T3-29 (i)

\section{J.R. Morton E2.30 (3) \\ P.G. Bosudil E2.30 (1) \\ W.A. Hoistoin E2.50 (1)}

DOE DISTRIBUTION

M.S. Coltins , A5-18 (1)
8. Verity matorial quaity.

9. Verify matorial qualty.

so. Verity assumed conditions.
RELEASE STAMP。

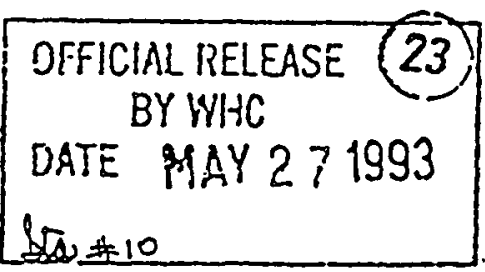




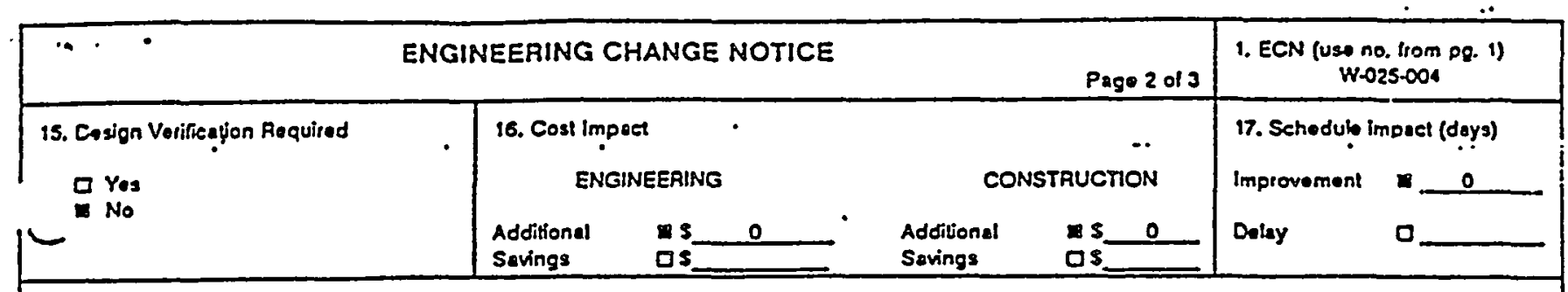

18. Chenge impact Roview: Indicate the relited documents (other then the engineering documents identified on Side 1) that will be alfectiod bij the chango describod in Block 12. Entes the affected document number in Block 19.

SODNO

Funetional Dasign Critorie

Opnrating Spocification

- Crilientity Spmeitication

Conceplual Design Poport

Equipment Spoc.

Const Spoc.

Vendor Inlormation . Operating Procidure

OM Manual

FSARSAR

Saloty Equipment Ust

Rediation Work Pormit

Environmental Impact

Statoment

Environmentel Roport

Environmental Permit

Inspeetion Plan
Tanik Caibration Menual

Heanh Physies Procedure

Sparos Muniplo Unil Listing

Test Procedures/Specification

Component Index

ASME Coded Item

Human Factor Considoration

Computer Soltwore

Electric Circuil Sehedule

ICRS Procedure

Process Control Manuaviplan

Process Flow Chart

Purcheso Requisition

LLBG Pant B Permil Appieation

Fac. Proc. Samp. Schedulo

Invantory Adjustment Request

19. Other Affected Documents: (NOTE: Documents fisted below will not be revised by this ECN.) Signatures below indieale that the signing organization hes been notified of other atfected documents Isted below.

- Document Number/Revision Document Number/Revision Document Number/Revision

\section{DOE/RL-88-20, SUpplemen: 2}

$\rightarrow$

20. Approval

20. Approvals signature

OPSRATIONS ANO ENGINEERING.

CogrProjoct Engr. Mgr. $Q R D$ is

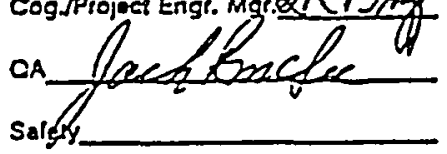

Security

Proj. Prog.Jept Mgr.

Del. React Div.

Chem. Proce Div.

Del. Wst Mgmt. Div.

Adv, Roset. Dov, Div.

Prof Dept

Environ. Div.

IRM DepL.

1

- Facitity Rep. (Ops)

Orher

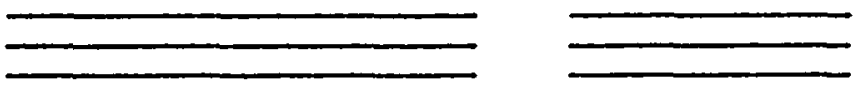


WHC-SD-W025-RPT-001, Rev. 0

\begin{tabular}{|c|c|c|c|}
\hline$\therefore$ & ENGINEERING CHANGE NOTICE CONTINUATION SHEET & P. & $\begin{array}{l}\text { 1. ECN (USA no. } 110 \mathrm{~m} \text { Do. I) } \\
\text { W.025-004 }\end{array}$ \\
\hline
\end{tabular}

Appendx A COA Summery Requirements, Specification Section 02275, paga A: Add requiremont lor conlainment besin Iner testing.

5. Section 4.3.3: Add requirement for proconstruction slake durabitity lesting.

7. Appenóx A COA Summery Requiremenis, Specification Section 02226. page 2: Add requirement for slake durability lesting.

8. Section 4.3.2: Add requirement lor free swiell testing.

9. Appendix A, COA Summary Requirements. Specification Soction 02224, page 1: Add requirement for free swrell testing.

10. Section 4.3.1: To socond bulhet add requirement for recording dele on geologie map duting construction, and nolitying COTA it unexpected or changed geobgle condilions äre encounitereö.

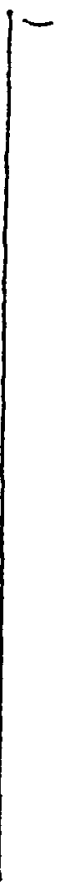

1<smiles>C1CCC1</smiles> 


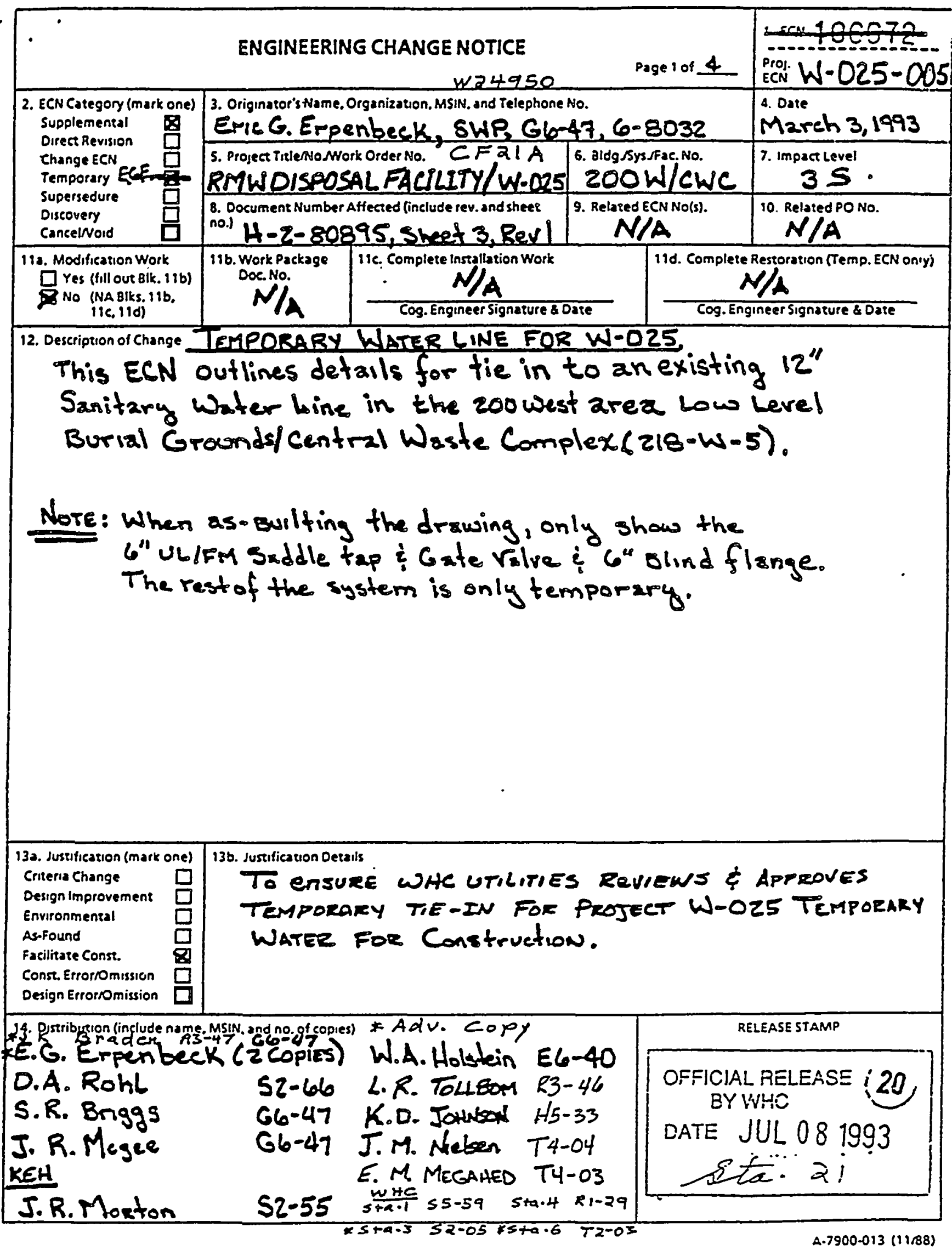




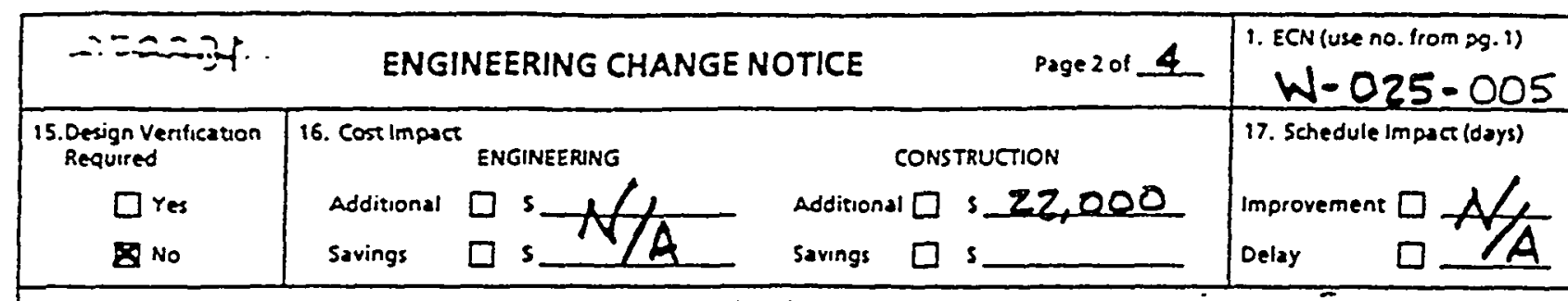

18. Change impact Review: Indicate the related documents (other than the engineenng documents identified on side i) that will be affecsed by the change described in Block 12. Entee the affected document number in Block 19. SDORO

Functional Design Criterio Operatung Specification Criticality Specification Conceptual Design Report Equipment Spec.

Const. Spec.

$\square$ Sersmiestress Analysis

rocurement Spec.

Vendor informazion OMManual

FSARSAR

Safety Equipment List Radiation Work Permit Environmenialimpact Statement Envionmental Report Environmental Permit

StresuDesign Report

Interface Control Drawing

Calibration Procedure

Installation Procedure

Maintenance Procedure

Engineernng Procedure

Operating Instruction.

Operating Procedure.

Operational Safety Requirement

- IEfo Drawing ‥

Cell Arrangement Drawing

Essentral Material 5 pecification

Fac. Proc. Samp. Schedule

Inspection Plan

Inventory Adjustment Request
Tank Calibration Manual

Health Physics Procedure

Spares Multiple Unit Listing

Test Procedures/Specificasion

Component index

ASME Coded item

Human Factor Consideration

- Computer Software

- Elecrnic Circuit Schedule

ICRS Procedure

- Pfocess Control Manualiplan

Process Flow Char.

Purchase Requistion.

19. Other Affected Documents: (NOTE: Documents lised below will not be revised by this ECN.) Signatures below indicate that the signing organization has been notified of other affected documents listed below. Document NumberRevisioner. . . . . .

ـ

20. Approvals

\section{Signature}

OPERATIONS AND ENGINEERING

Cog Projec: Engineer

Cogsprojec Engr Mgr

QA

sattit

Security

Proj.Prog Nepr. Mgr. .

Def.React Div.

Chem. Proc Div.

Def. Wr. Mgme. Div.

Adv. React. Dev. Div.

Proj. Dept

Environ. Div.

IRM Dept.

Faciliry Rep. (Ops)

Other
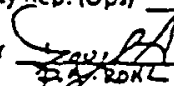

$\left(-\frac{1}{2}\right.$
Date

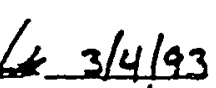

$3 / 16 / 90$
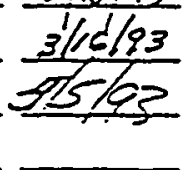

ARCTIYEC:.

PE

OA

Satery

Design

Orher

Documeni Number/Revision

\section{.}

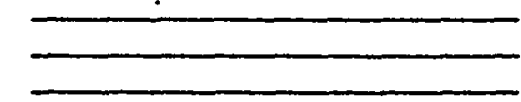

Date

$\square$
$\square$
$\square$
$\square$
$\square$
$\square$
$\square$
$\square$
$\square$
$\square$
$\square$
$\square$

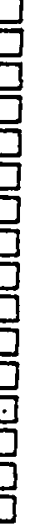




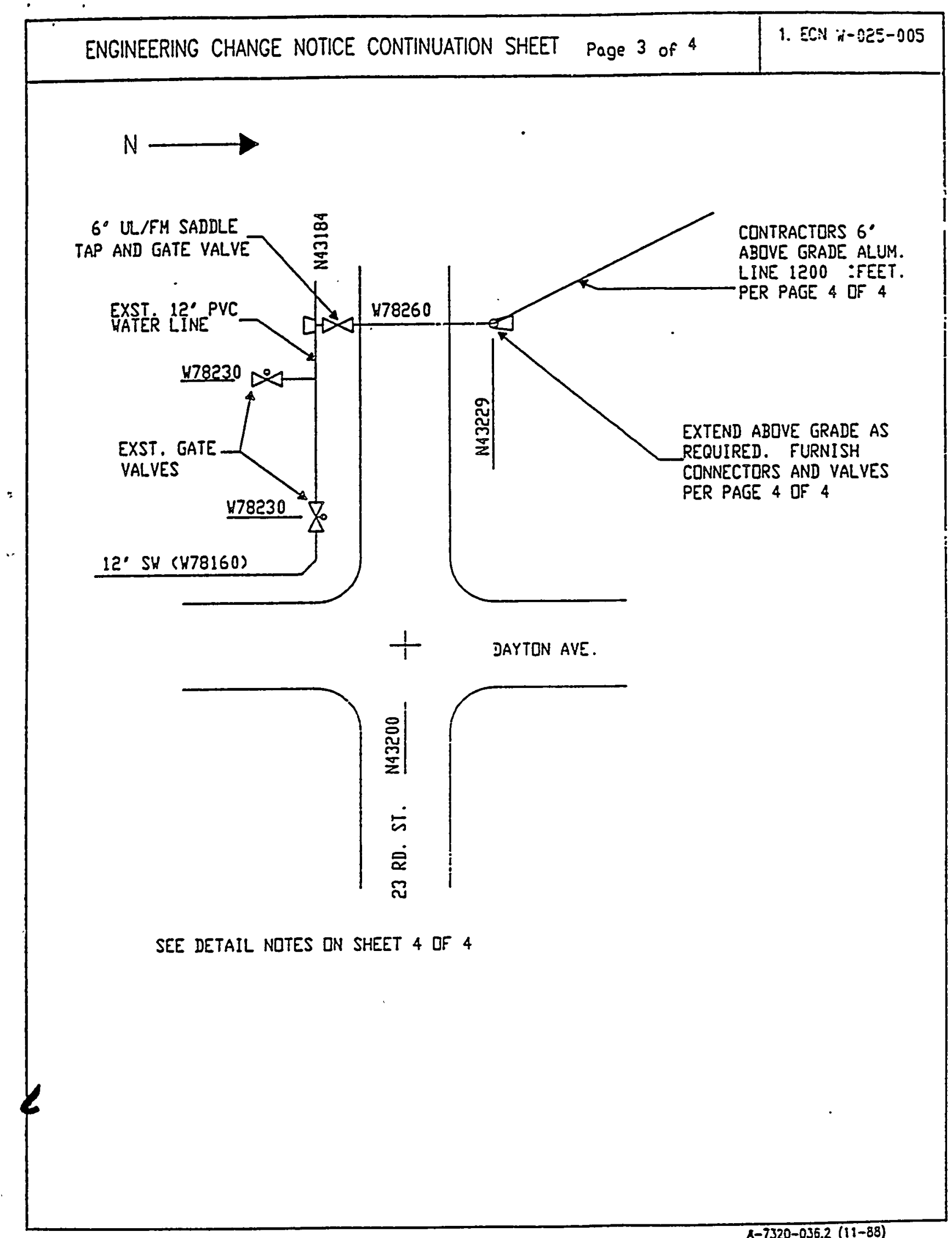




\section{ENGINEERING CHANGE NOTICE CONTINUATION SHEET Page 4 of $4 \quad \begin{gathered}1 . \text { ECN } \\ w-025-005\end{gathered}$}

\section{SPECIFICATIONS FOR TEMPORARY WATER LINE ON W-Q25}

\section{HO HORK TO COMMENCE PRIOR TO WHC RPPROVED TIE-IK PERMIT SEE SECTIOH O 1065}

1. Hend dig to locate'exieting 12 Inch Poly vimyl chlorlde (PVC) Iine. Wo digoing shall be inltitlated Without the excavation peralt. Permit to be provided by Vestinghowe Henford Compery (UHC).

2. Bury 6 Inch PVC line to weth existiog 12 Inch Sanitary vater line at te-in.

3. Instell all thrust blocks and restraints per Matlonol Fire Protection Ansoclatlon (MFPh) and Kalser Engineers hanford (KEH) upprovel.

6. The suddl tap and 6 inch gate valves shall be flange connection all other can be archanleal jolnt.

5. Provide and inctall valve box and cover at tie-ln. Kinture 40 inch goil cover.

6. After completion of construction remove all pipe and spourtenance to the acdl top and 6" Underurfters Laboratory Factory Nutual (UL/FM) gate valve. Install o 6 inch blind flange.

7. Sadtle tap and gate velve ihall be desloned for hot tap.

8. Inseall Per KFPA 26

9. Plpe shall conform to Amerlean Vater Vorks Association (Awsk) C900, 6 Inah.

10. Subilt manufucturers procedure for hot tap and instsllation, gutalt so bic purveyor and keK for approvel.

11. The following equipment shall be reaulied for the semporary construction water, above grede comections:

- 6 Inch slow opening gate valve, rising sten and 6 inch flow weter.

- Redued pressure beckflou preventer, siallar to Baeco-sirgap. KEHhHE approval.

- Lacking device on 6 inch gate valve to control Gallon Per Minute (opk) flow not to exceed 400 CPH.

12. Disinfection of componente ahell be in accordance with the Hanford Plant Standard, HPS-112-M, or AWWA C-650 and/or C-651. Hater purveyor will release water line for un aftor rocelipt of reaulta of bacteriological asalysis from kanford Envirormantal Health Foudation (HEHF).

13. The Reduced Pressure (RP) backflow prevention device shall be on the eurrent Vashinaton state Departinent of Health approved IIst. It shall be teated per UHC procedure S-CC-0046 or UHC water purveyor opproved equl. Systea ulll not be place into service prlor so whe muter purveyors reviet and acceptance of device test dite cheet. The Rp beckftow prevention device shell be akld or atand wonted, and axchored to prevent wovement. The device shall be manted horizontally on tremework suitable for upporsing the weight of the device and the shutoft valves (el and 2 ) supplied with the device ss realied by the Uashington state Departinent of Heal th.

16. Instaliation of the backflow prevention device shall be In occordance with the Atu, Paclfic Morthuest Seetion, Aecepted Procedure and Proctice In Cross-connetion Control Manul, latest adition.

15. Lhen onergizing the eross cantry line, she contruetor andfor emplorees sholl not open the 6 inch volve sore then four (6) pevolutions per winute on the valve-hand weel. IEK shall be notitled 6 hours in edvance of contrastor opening valve and wleneased by KEH to misure she above procedure is followed. Thls procedure aus be follaned for all operetions of the valve on and off.

16. All beckfllt ahall be coppeted to echleve a demalty of 955 of Modiffed Proctor Donsity (Astu 0.1557).

17. Contractor ahall anitize and flush (ollow 3 doys for testing) tonporary ueter apply line fron 12" tap to the bove ground comections.

18. Contractor shall provide, install and maintain intll completion of the project. 12002 (inear feet of 6 inch Aluminan line with alt new oostets and Jolnt clamps. Cross country line shall be leak free will in operation or repaired per UHC/KEH direselon. - 


\section{ENGINEERING CHANGE NOTICE}

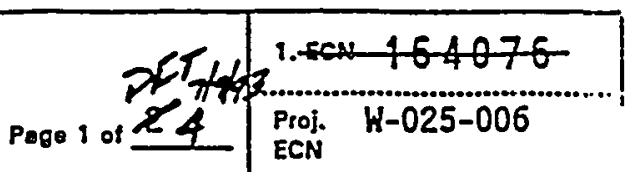

2. ECN Cangory (mark aro)
Supplomental
Diroct Rovidion
Change ECN
Tomporay
Stundby
Supornodur
CancoWold

3. Odglnatore Nam, Organization, MSIN, and Tobphon No

F.S. Shuri, Golder Associates Inc. (206) 883-0777

5. Project Tith/NosWork Order No.

W-025 RMW Land Disposal Facility

8. Document Numbers Changed by this ECN (includes sheet no. and $n v_{\text {.) }}$

Cancolvold

WHCS-045 Rov. 2

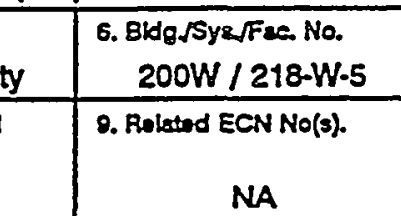

4. Dato

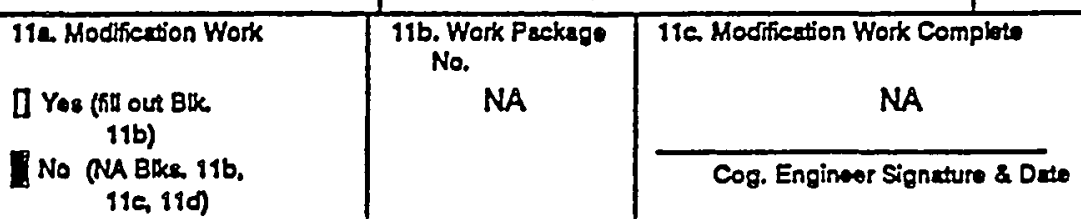

12. Daxiption of Change

Chengos to Project W-02S Specifications:

1. Section 02275-2. Part 1.7 'Submittals": Add now paragraph:

E Submil evidence of quatheations of organization that will zerve es Geosynthetes Installop. As a minimum, the Goosymtheties Inxtallor shall have succassfully complated at bust 10 projects consisting of installation of as bast 10,000,000 square feet (10ta) of HDPE iner. Projects shall include RCRA lendfilts and surfeco impoundmorts.

Submil quartications of koy personnel Including suportmensem, foreman, and had woldas. Kay parsonnol shall be full sime employeses of the Geosynthatics Indalles."

2. Section 16460 Dry Type Trandomers: Include enched Soction in the entirety (2 pages).

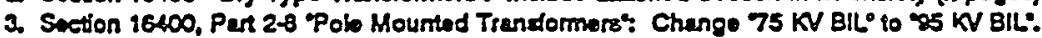

4. Section 16400. Purt 24 ${ }^{\circ}$ Open Fuse Cutouts - Pols Mounted": Add the folloming: Curtouts shall bo extra hesvy duty, 100 Amp continuous 5600 Amp Intormpting duty or botter. Cutouts shall be fused with 27 fuses."

5. Section 16450, Part 3-1 Grounding, Gentar: Add the following: Grounding shall be performed in accordance with the tequirements of the National Eloctrical Sufoty Code nules 95 and $97:$

13a Justification (mark Critoris Change [] Design Improvement [] Envionmental
on)

Asfound D Faciftate Const [] Const Error/Omission D Design Error/Omission

13b. Justifietion Dotails

1. Exelude unquafifed installation contractors

2 Conect omicsion.

3. Conform to current Hartord Sito etandards.

4. Clumitextion.

5. Corform to current Harford Site standards.

14. Ditribution (include name, MSIN, and no. of copies) WHC DISTRIBUTION KEH DISTRIBUTON

Projest Fils R1-2B (1) J.R.Morton E230 (3)

JRH HCGe G. G.47 (1) P.G. Boaudet E230 (1)

Stution 10 A3-87 (1) WA Holetein E2-50 (1)

DA Rohl S401 (1)

EG. Erponbeck G5-47 (3)

S.R. Brigge $\quad$ C6-47 (1)

KD. Johnion H2-58 (1)

DOE DISTRIBUTION

J. Dorian B2.96 (1)

M.S. Colins A5-18 (1)

G.C. Evans H4-57 (1)

J.M. Nolsen T3-29(1)

A.7900-013-2 (06/92) GEF095 
WHC-SD-W025-RPT-001, Rev. 0

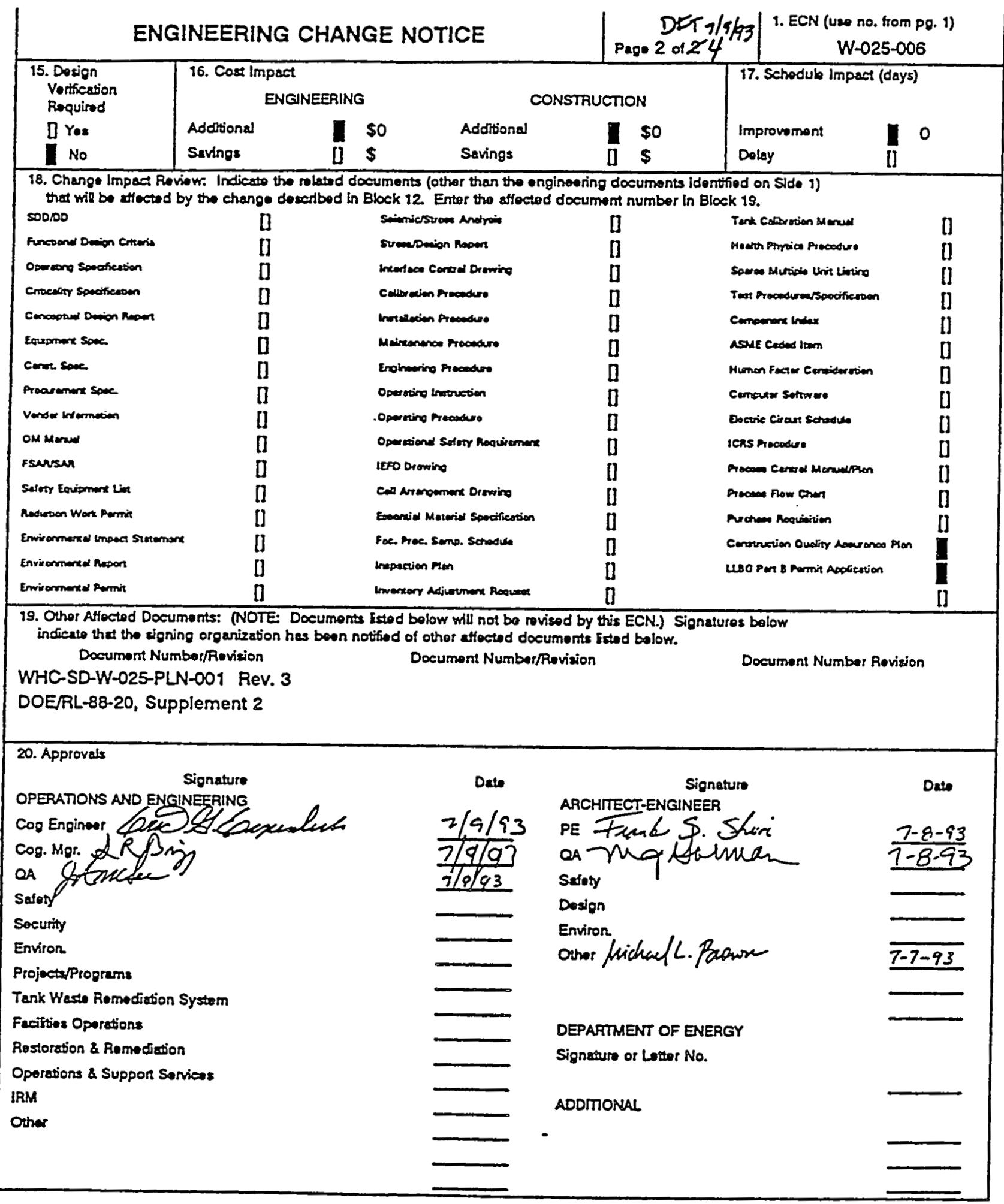

\section{A-7900-013-3 (05/92) GEFOS6}




\section{SECTION 16460 \\ DRY TYPE TRANSFORMERS}

\section{PART 1: GENERAL}

\section{1-1 DESCRIPTION}

Provide dry type transformers complete and fully operational. Transformers shall be U.I. listed and comply with NEMA Standard ST-20. Deliver transformers to site for testing 2 weeks prior to installation.

\section{1-2 SUBMTTALS}

A. Prepare and submit shop drawings for review prior to manufacture. Include dimensioned front plan and section views, wiring and connection diagrams, and bolting template.

B. Submit in accordance with Section 01300.

\section{PART 2: PRODUCTS}

\section{2-1 MANUFACTURER}

Tierney, Sorgel, Westinghouse, General Electric.

\section{2-2 ENCLOSURE}

A. Steel panel enclosure over core, coil, and terminal chamber with louvered openings for convection cooling.

B. Enclosure required for environment in which located.

\section{2-3 WINDINGS}

Separate primary and secondary. Windings shall have Class $\mathrm{H}$ insulation and shall be rated for continuous operation at rated KVA with temperature rise of not over 150 degrees $C$ above a 40 degree $C$ ambient, with a maximum hot spot temperature of 220 degrees $C$. Windings and core and coil assembly shall be treated and built to resist the effects of dirt and moisture.

\section{2-4 PRIMARY TAPS}

Minimum of two 2-1/2 percent below normal (rated) primary voltage.

\section{2-5 CAPACITY}

WHC NON-DRAG-OFF 
Continuous rating not less than size noted.

PART 3: INSTALLATION

\section{3-1 MOUNTING}

Mount on vertical mounting wall as noted or as required. Remove all shipping blocks prior to installation.

\section{3-2 CONNECTIONS}

A. 120/240 volt single phase secondary transformers shall be considered "grounded neutral separately derived systems" and neutral shall be grounded per code accordingly.

B. Transformer raceway connections shall be flexible metal conduit as specified in Section 16110.

C. Voltage Tap Connections: Connect all transformers at "normal" tap. After facility is completely energized, measure secondary voltages at all transformers and reconnect taps as subsequently directed.

\section{3-3 FIELD QUAITTY CONTROL}

Verify that transformer installation is in accordance with manufacturer's instructions and Drawings and Specifications. Verify correct wire identification and type of transformer. 
WHC-SD-W025-RPT-001, Rev. 0

.1 .5

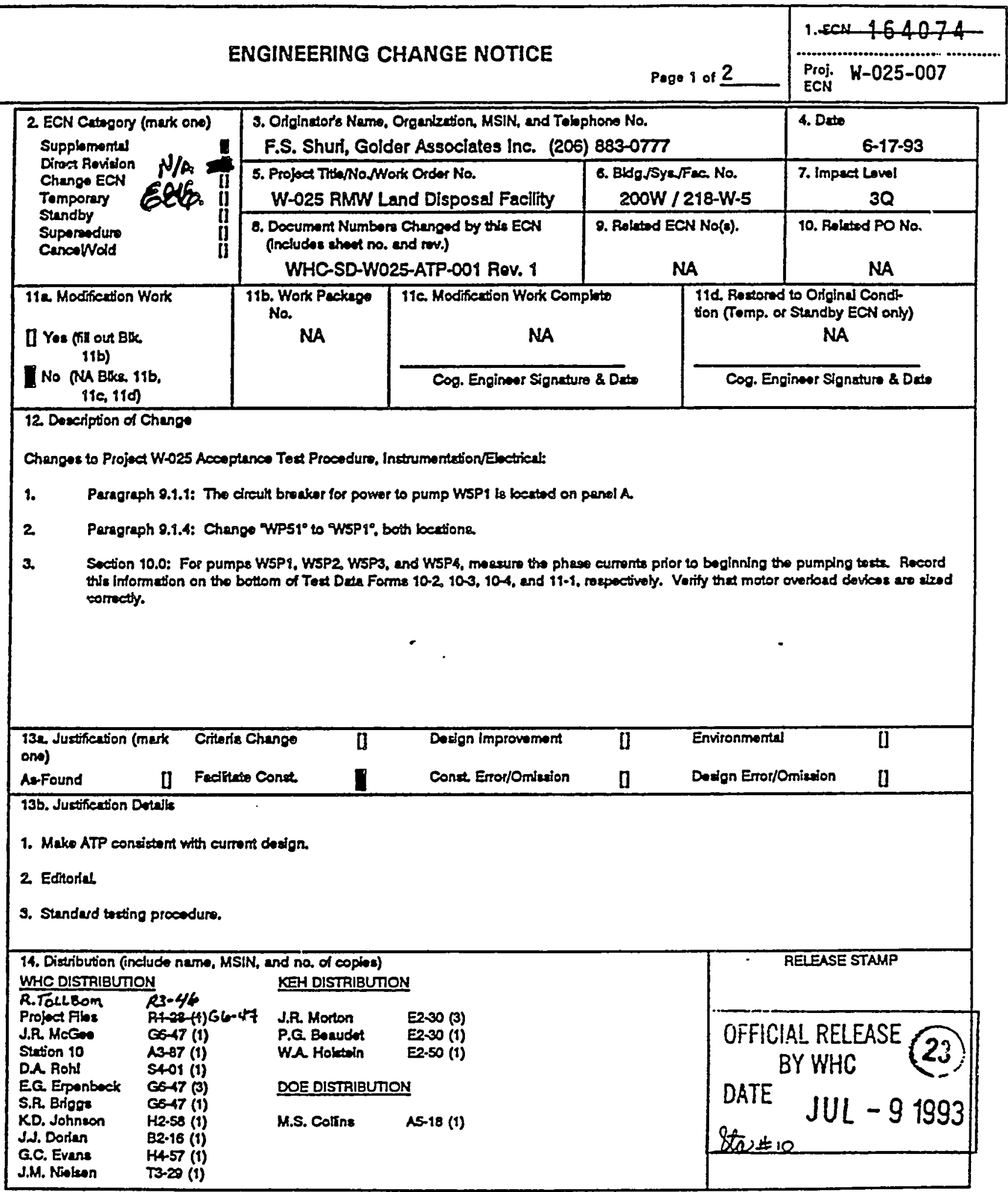

A-7000-013-2 (06/92) GEF095 
WHC-SD-W025-RPT-001, Rev. 0

\begin{tabular}{|c|c|c|}
\hline ENGINEERING CHANGE NOTICE & Pهge 2 of 2 & $\begin{array}{c}\text { 1. ECN (use no. from po. 1) } \\
\text { W-025-007 }\end{array}$ \\
\hline
\end{tabular}

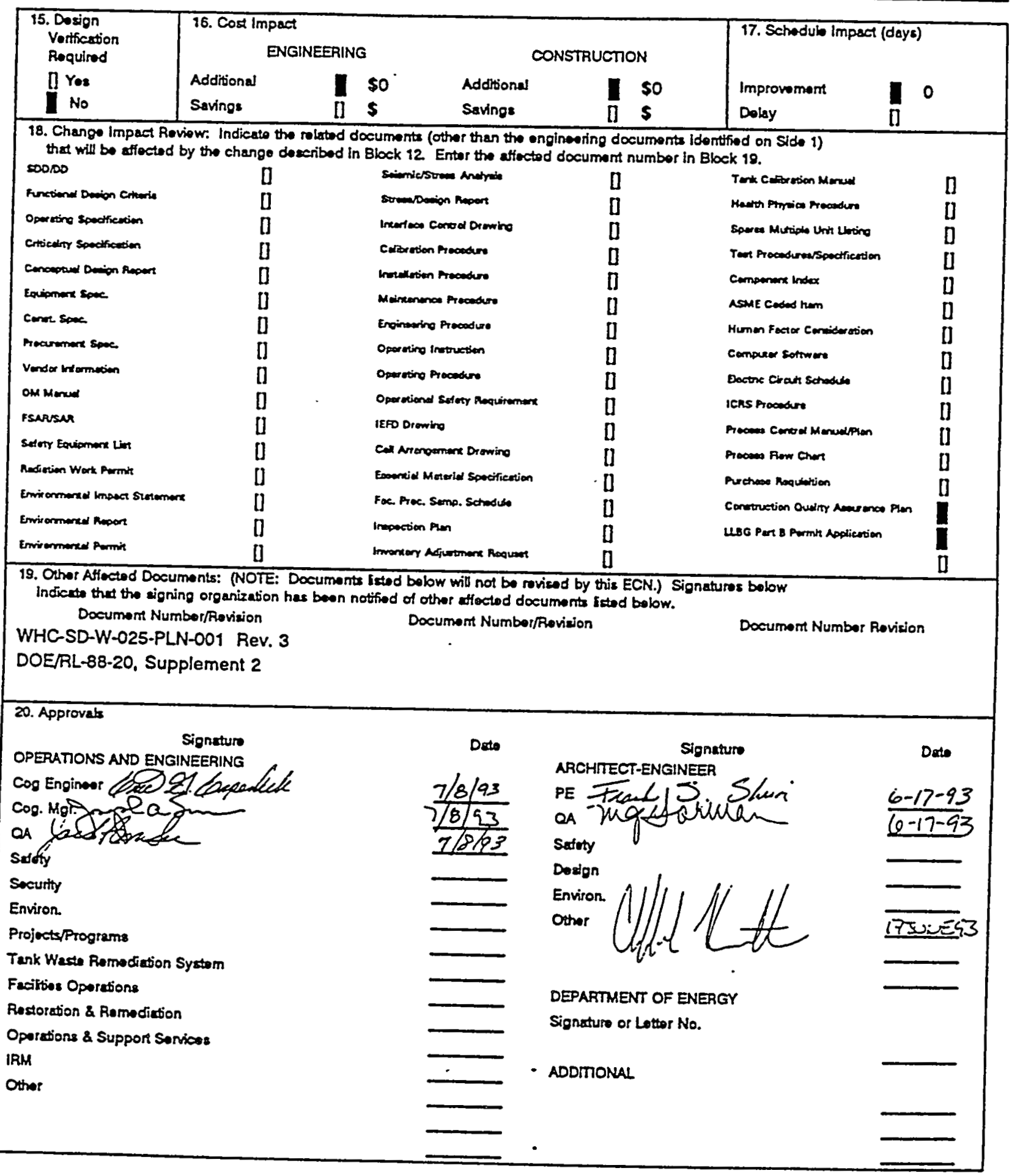


WHC-SD-W025-RPT-001, Rev. 0

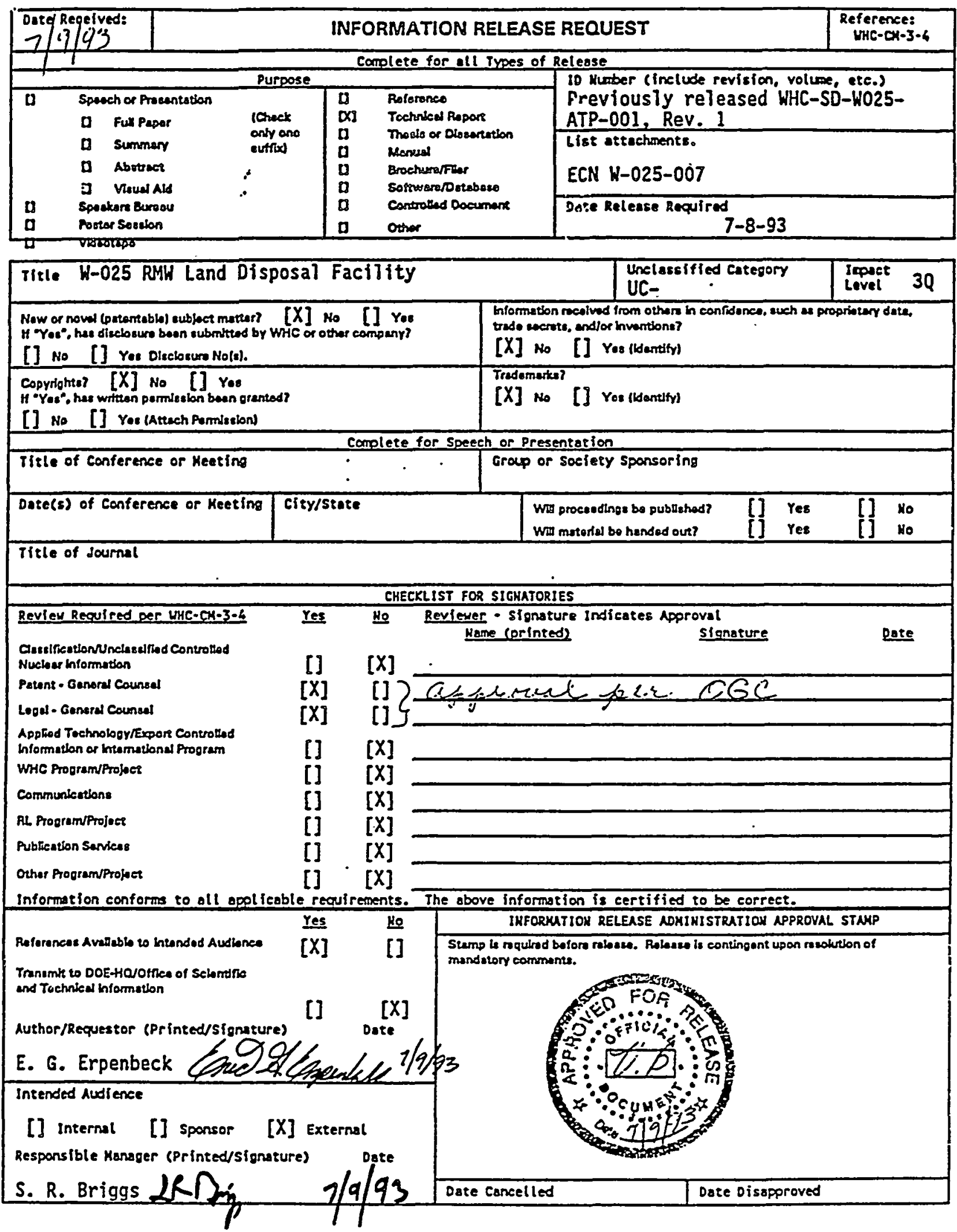




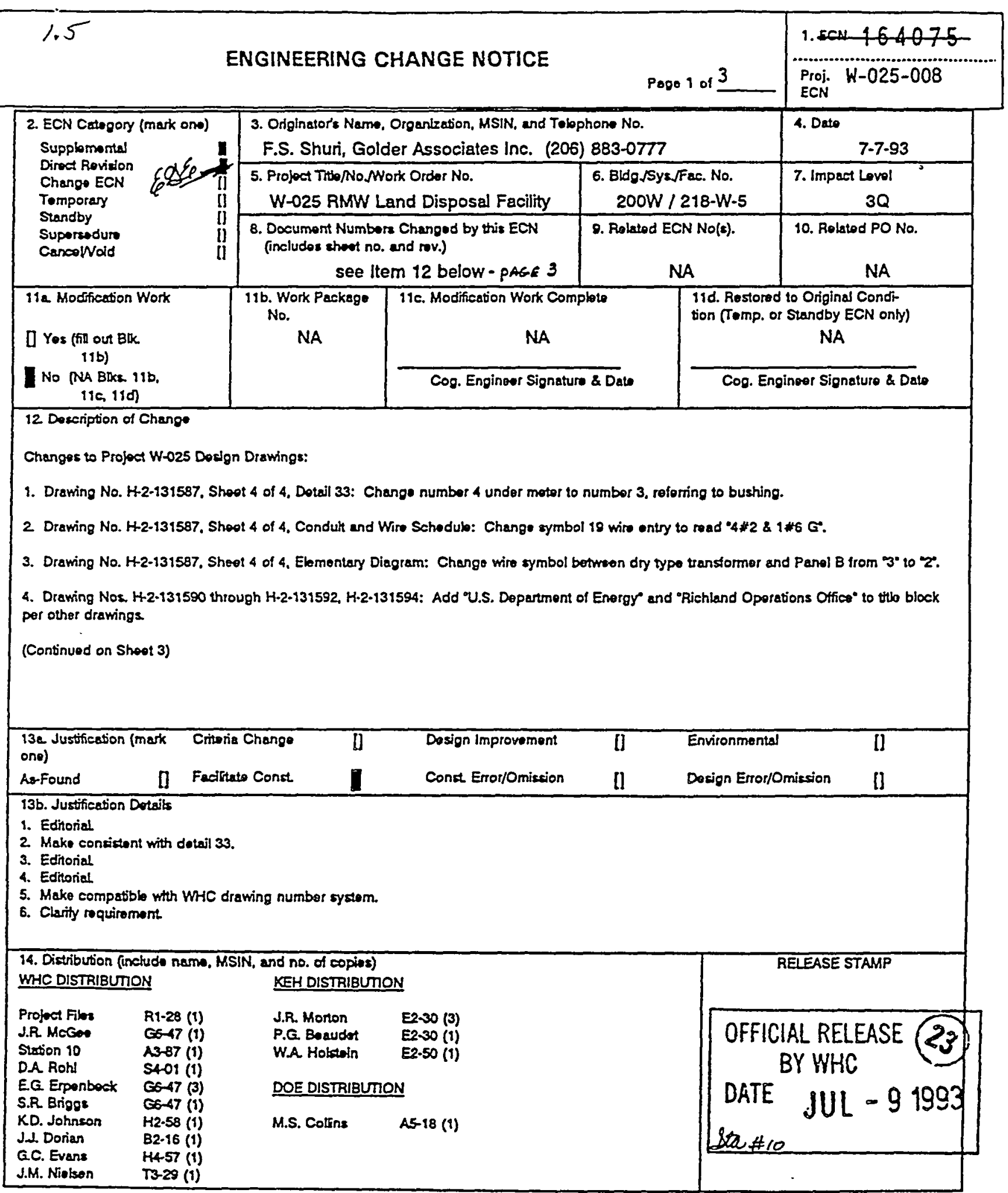

A-7900-013-2 (05/82) GEFO95 
WHC-SD-W025-RPT-001, Rev. 0

\begin{tabular}{|l|l|l|}
\hline ENGINEERING CHANGE NOTICE & Pago 2 of 3 & $\begin{array}{c}\text { 1. ECN (use no. from Pg. 1) } \\
\text { W-025-008 }\end{array}$ \\
\hline
\end{tabular}

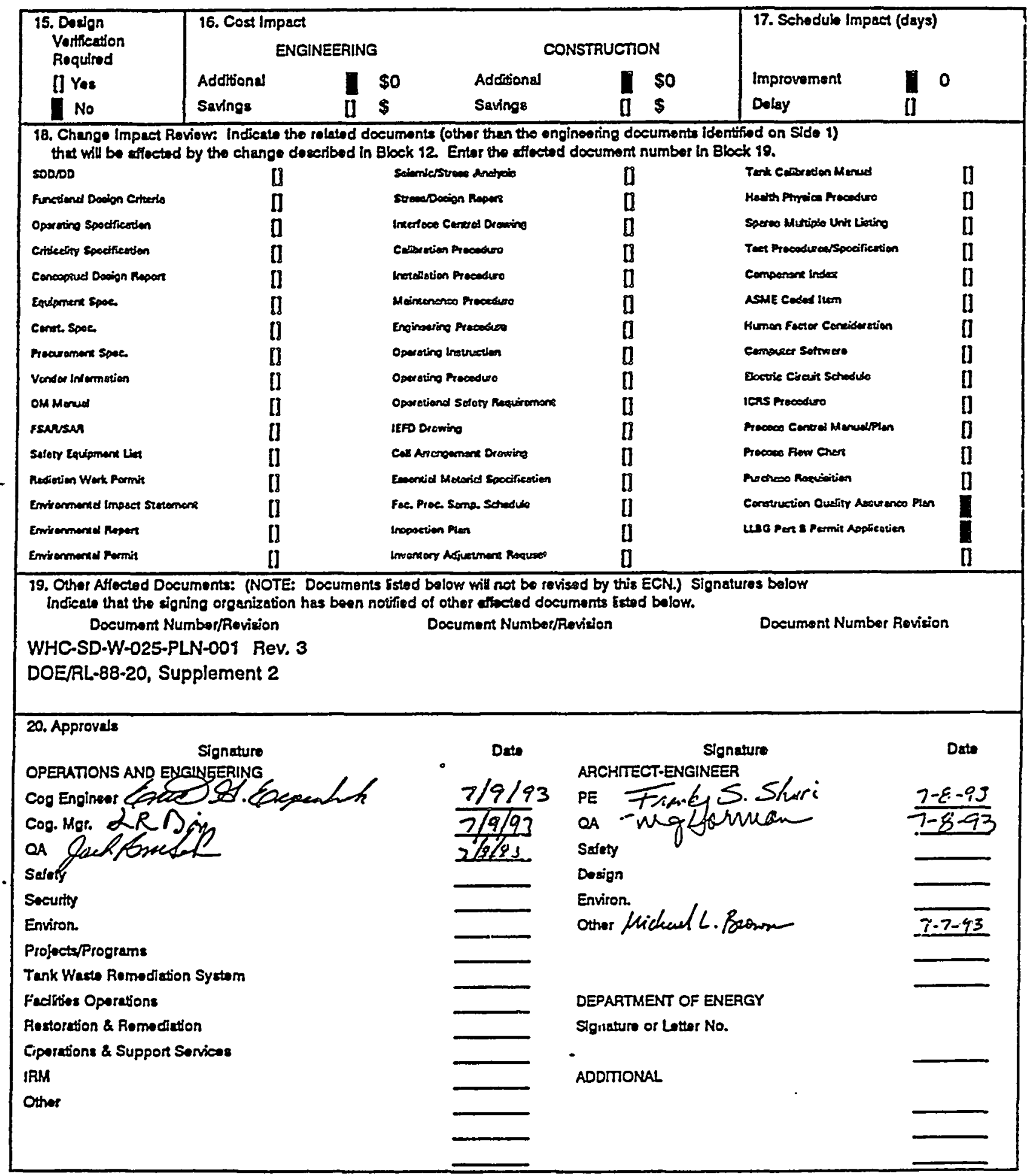

A-7900-013-2 (06/92) GEFOS6 


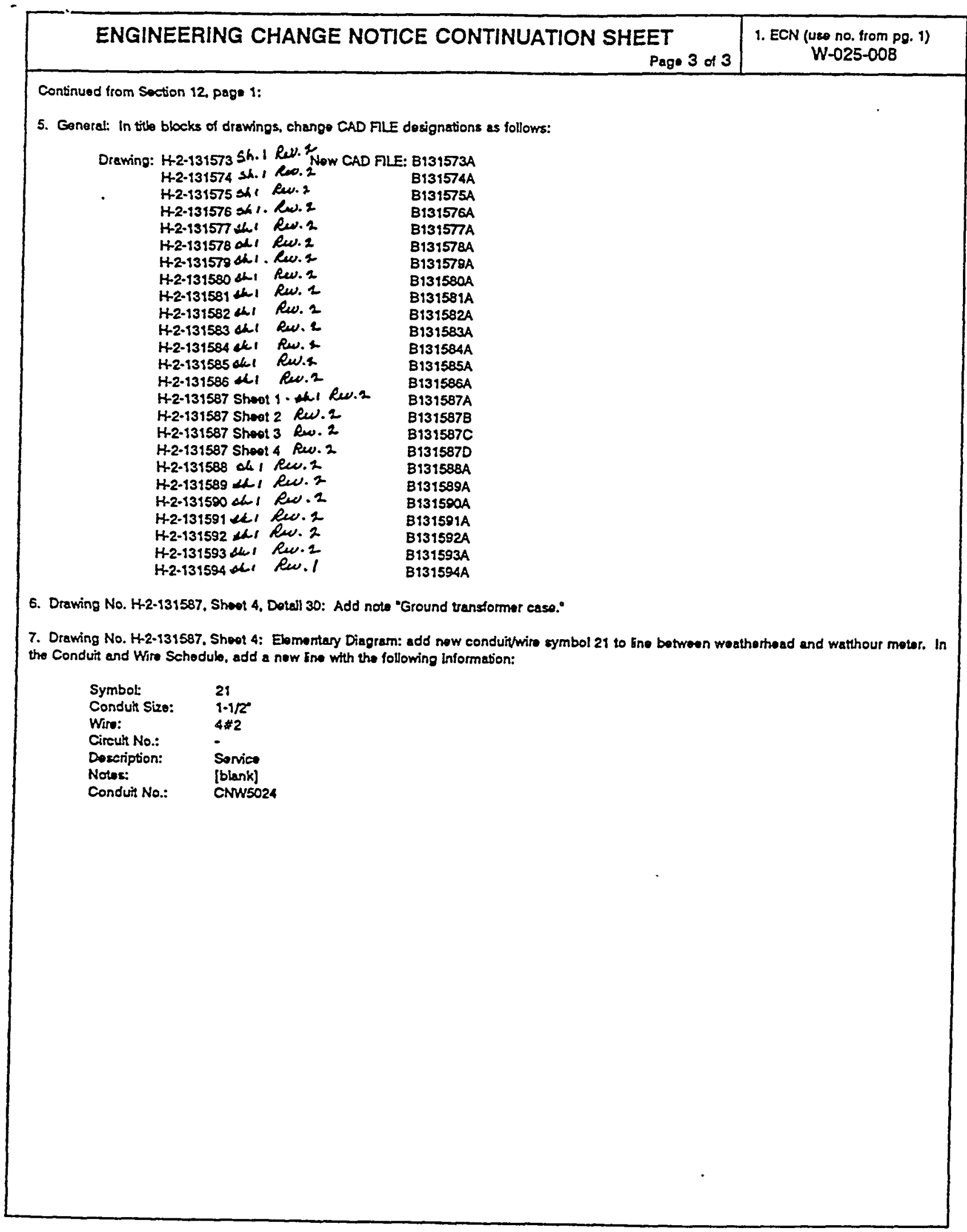


WHC-SD-W025-RPT-001, Rev. 0

ENGINEERING CHANGE NOTICE

Pags 1 of 3

$1.4-164077$

Proj. H-025-009 ECN

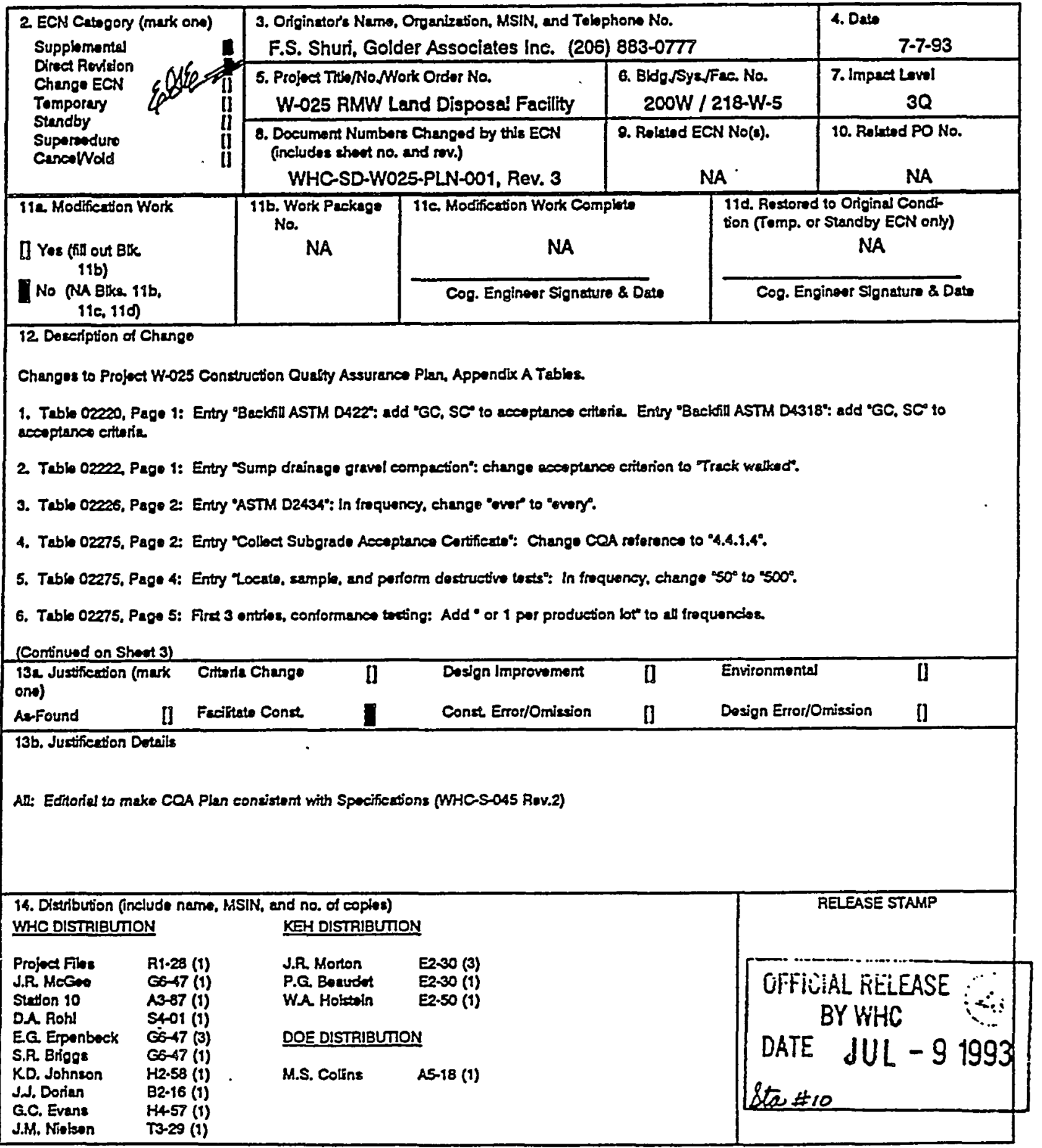

A.7900-013-2 (06/92) GEF095 


\begin{tabular}{|c|l|c|}
\hline ENGINEERING CHANGE NOTICE & 1. ECN (use no. from pg. 1) \\
W-025-009
\end{tabular}

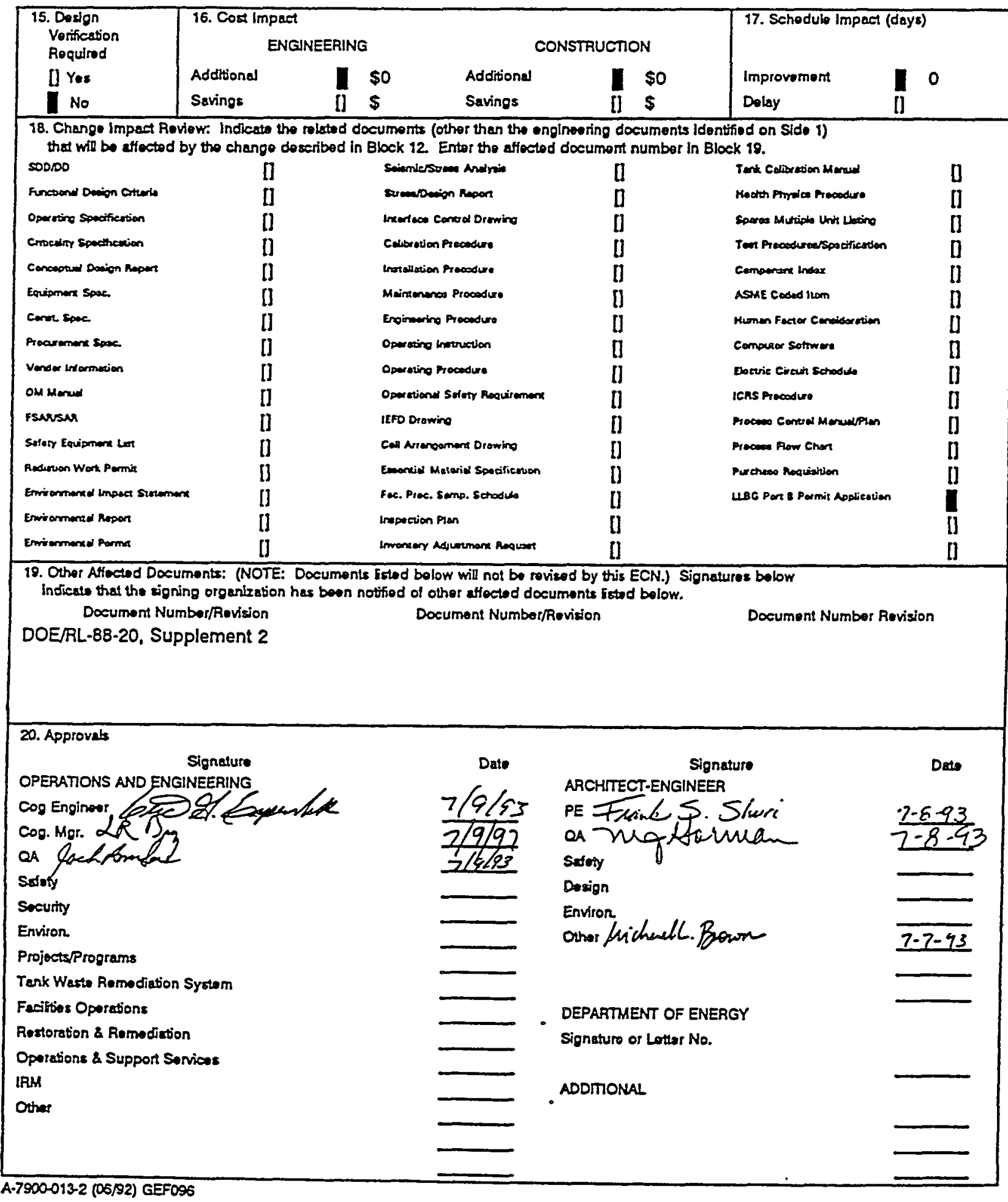




\section{ENGINEERING CHANGE NOTICE CONTINUATION SHEET}

Page 3 of 3
1. ECN (use no. from pg. 1) W-025-009

Continued from Section 12, page 1:

7. Table 02275, Page 5: Entry "Contirm that all geonot is covered": change COA roference to $4.5 .23^{\circ}$ and change specification saction and

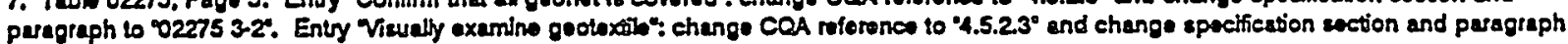
to $022753.2 \%$

8. Tablo 02511, Page 1: Entry "Contractot zubmittar: change trequancy to Pfior to ordoring (materiats) and prior to construetion (mathods)".

9. Tabb 02511, Page 1: Entry "Extraction/gradution": change specticestion saction and paragraph to "02511 2.1".

10. Tabl6 02511, Page 1: Entry Top coura ASTM D1557: change specification section and paragraph to D2511 3-1:.

11. Tabli 02511, Page 1: Entry Top coures ASTM D2922: change specifications section and paragraph to v2511 3-1".

12. Tablo 02511. Page 1: Entry "Asphak concrete construction tolatances": change spectication section and paragraph to "02511 3-5".

13. Tabio 02511. Page 1: Entry "Compaction of esphakr: change spoctication soction and paragraph to V2511 3-38".

14. Tablo 02720, Page 1: Entry "Ditch dimensions": change specticettion soction and paragraph to "02720 3".

15. Tablo 02727, Page 1: Entry "Fiser plpo tobrance": change epectication anction and paragraph to 02727-38:

16. Tablo 15050 to 15250, Page 1: Enty "Contretor submittar: In eppecitieation saction and paragraph, change "15100 1-20 10 "15100 1-3". Entry Recolving Inspection": in specticetion enction and paragraph, chenge" 15100 1-2" 10 "15100 i-3".

17. Tablo 16010 to 16859, Page 1: Entry "Contractor's es-buil drawings": In specifieation ection and paragraph, change "13340 1-58' to $13340166^{\circ}$. 
WHC-SD-W025-RPT-001, Rev. 0

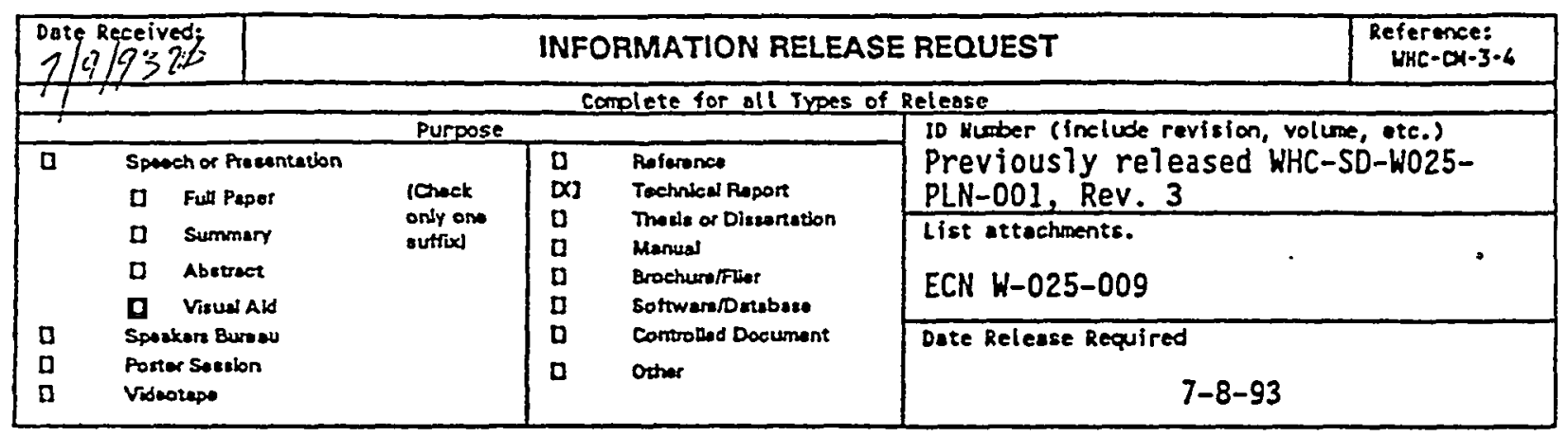

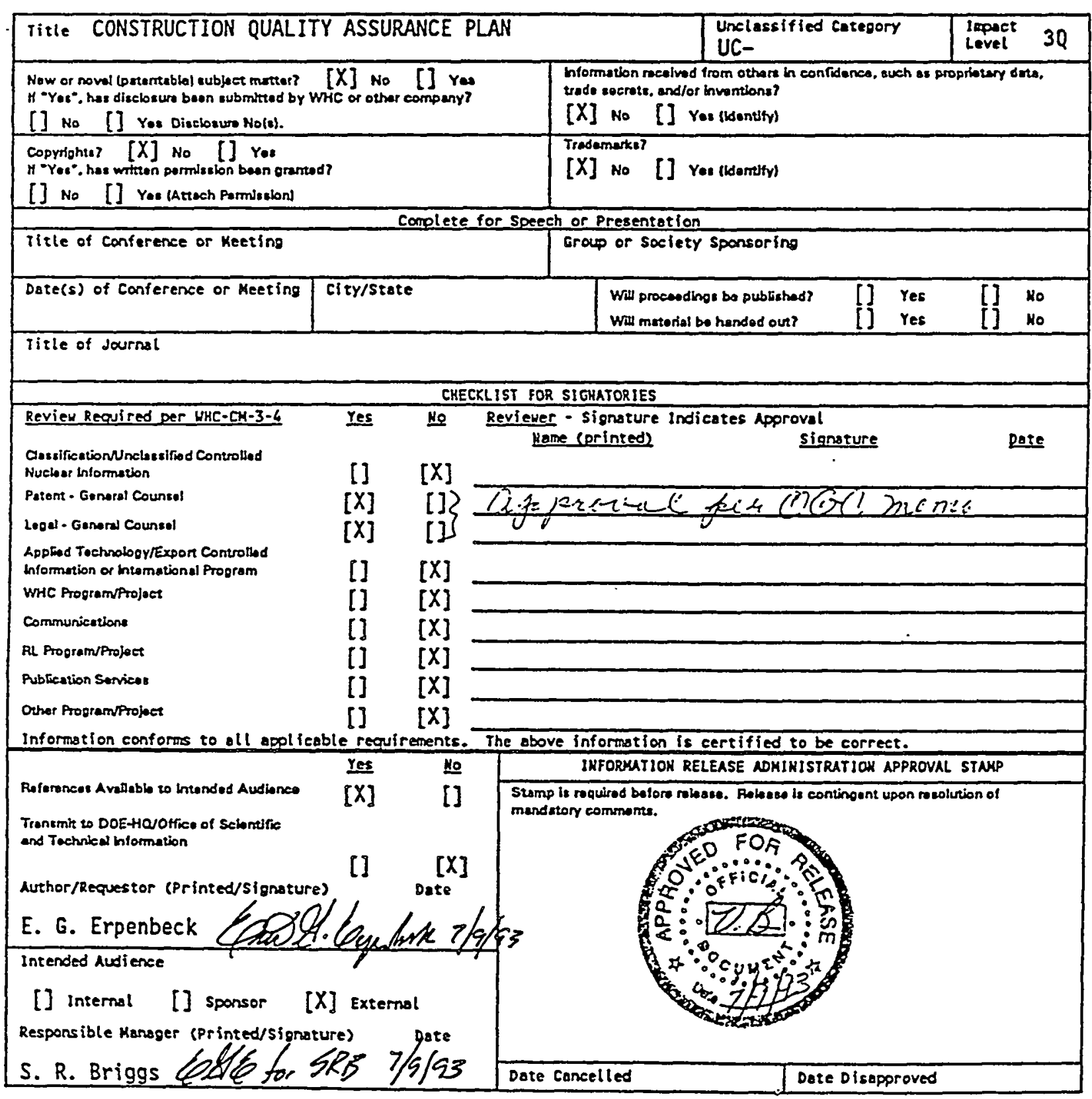


WHC-SD-W025-RPT-001, Rev. 0

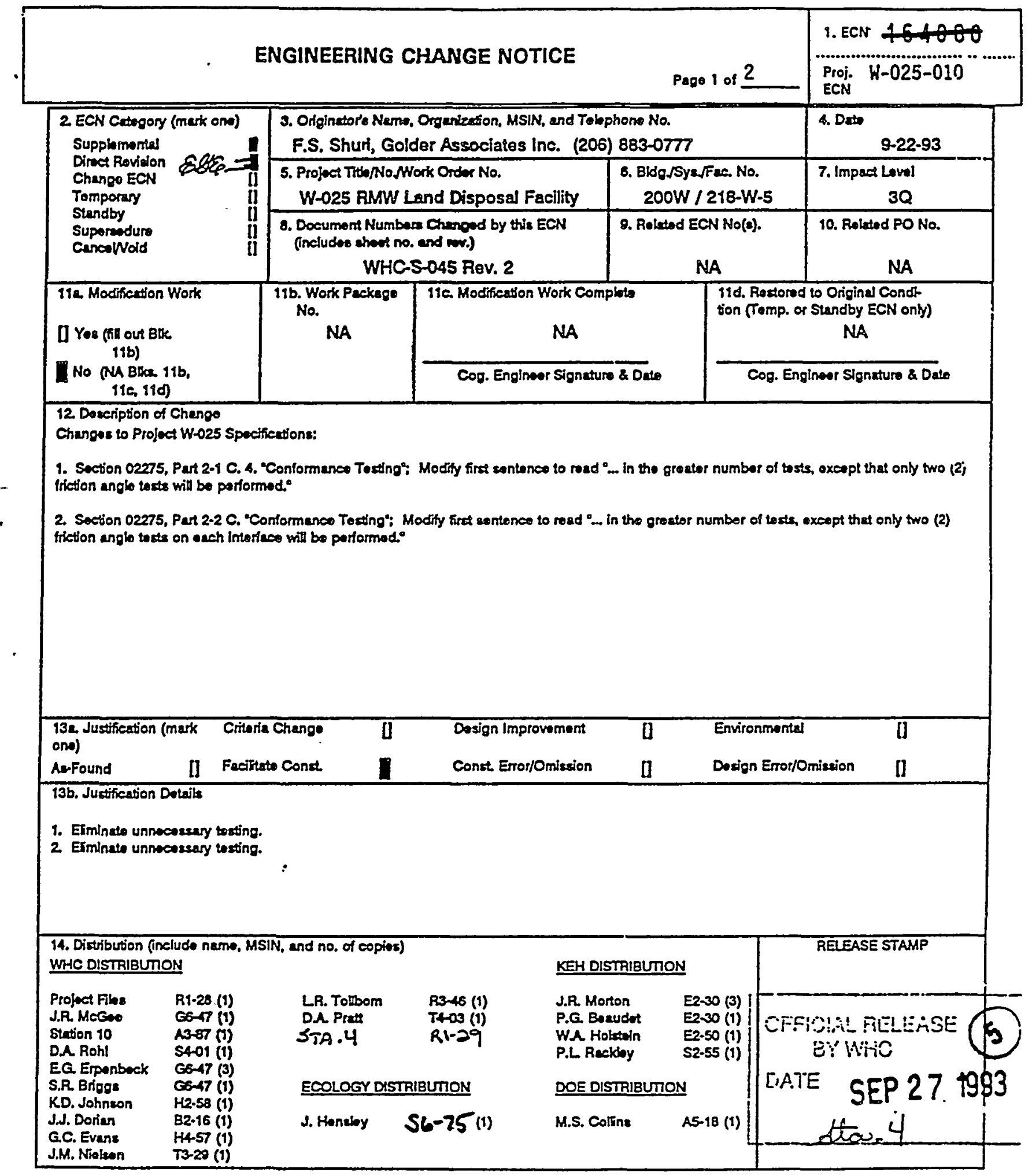

A-7900-013-2 (05/92) GEF095 
WHC-SD-W025-RPT-001, Rev. 0

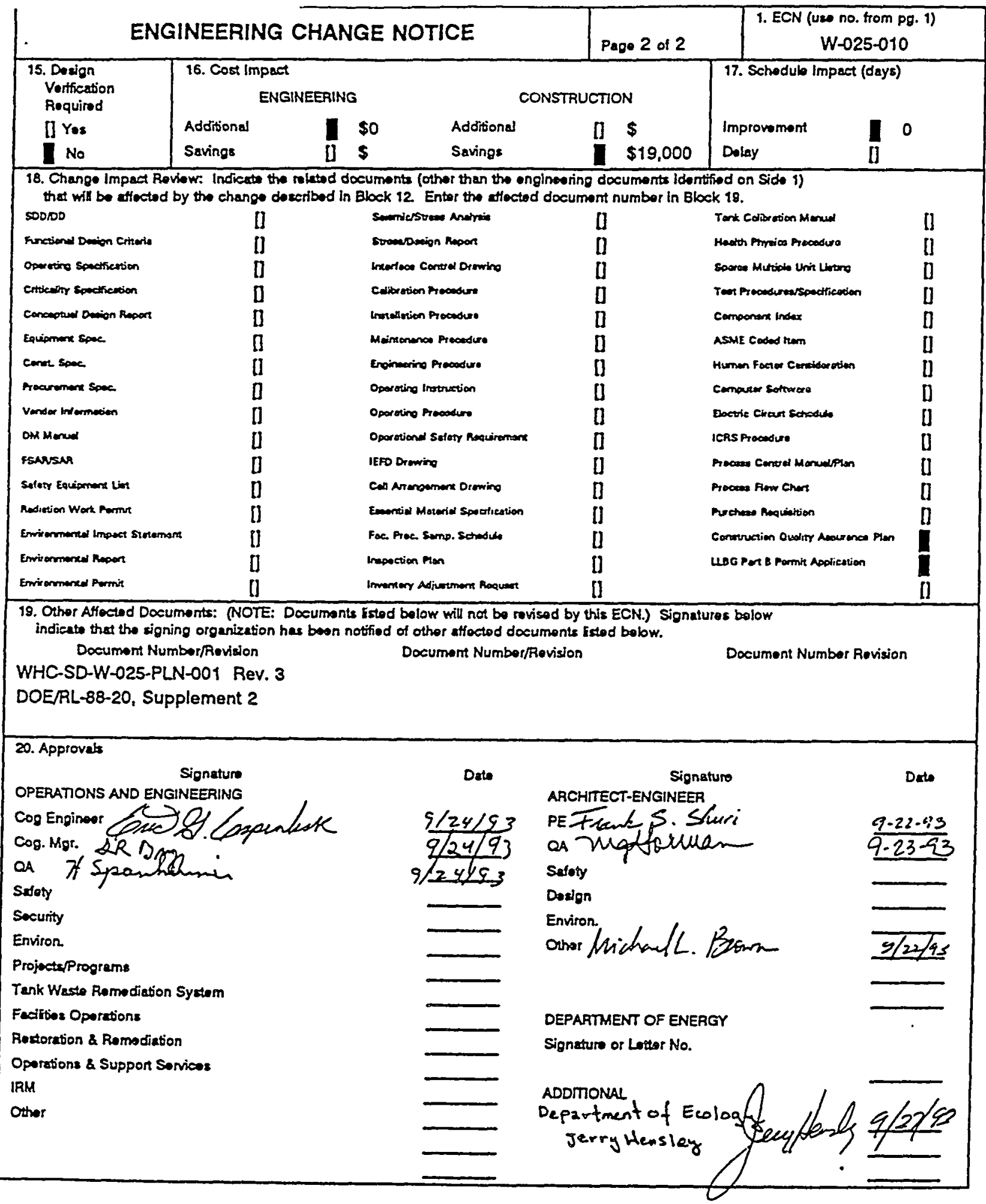




\begin{tabular}{|c|c|c|}
\hline & & 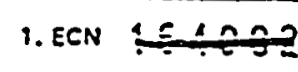 \\
\hline 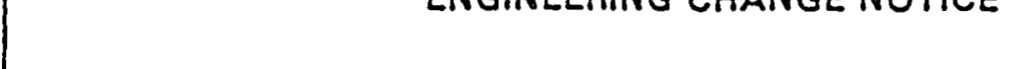 & Pogo 1 of _ & $\begin{array}{l}\text { Proj. } \quad K-025-011 \\
\text { ECN }\end{array}$ \\
\hline
\end{tabular}

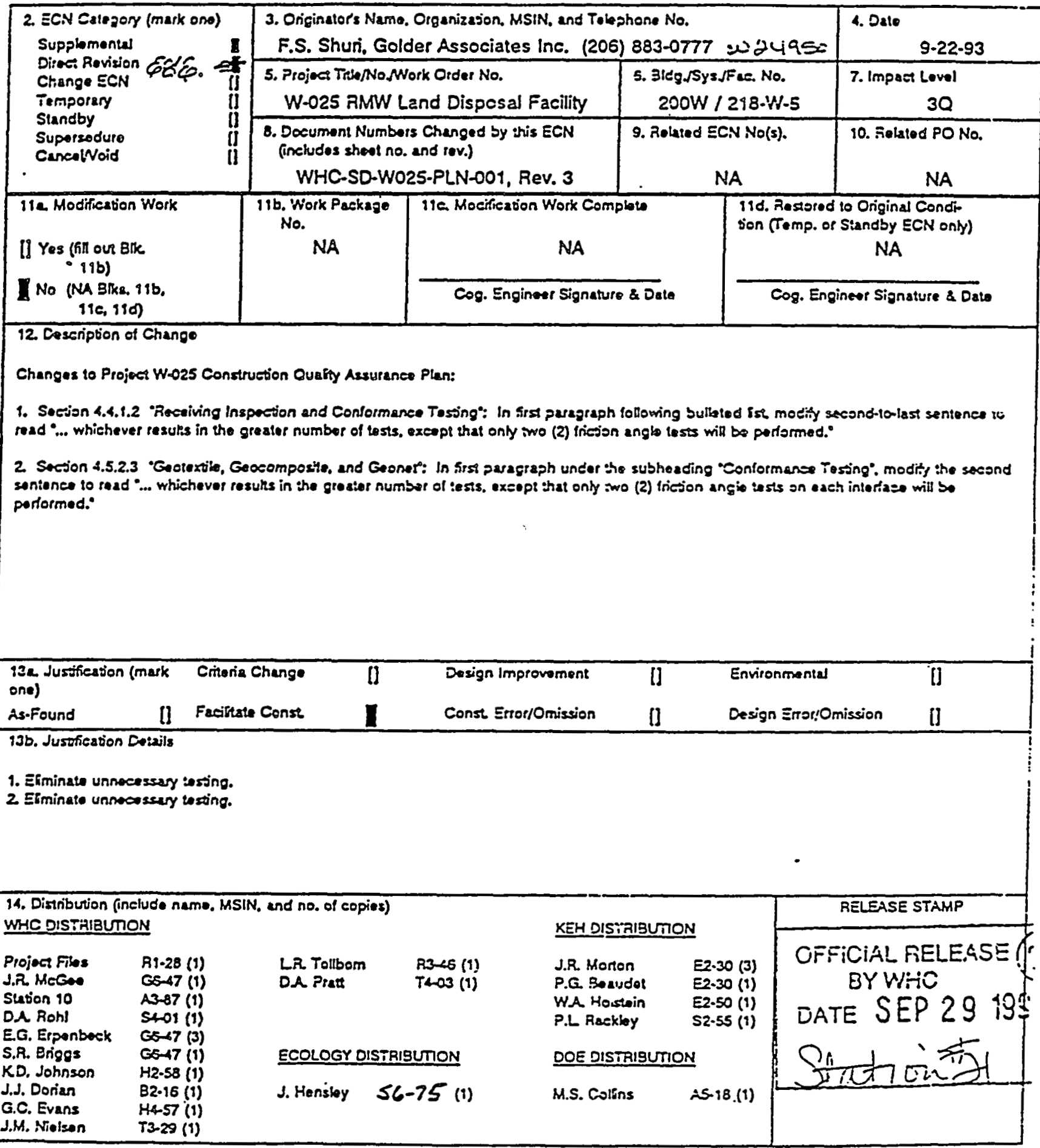

A.7900-013-2 (05;52) GEFOS5 
WHC-SD-W025-RPT-001, Rev. 0

\section{ENGINEERING CHANGE NOTICE}

Page 2 of 2

1. ECN (use no. trom po. i)

W-025-011

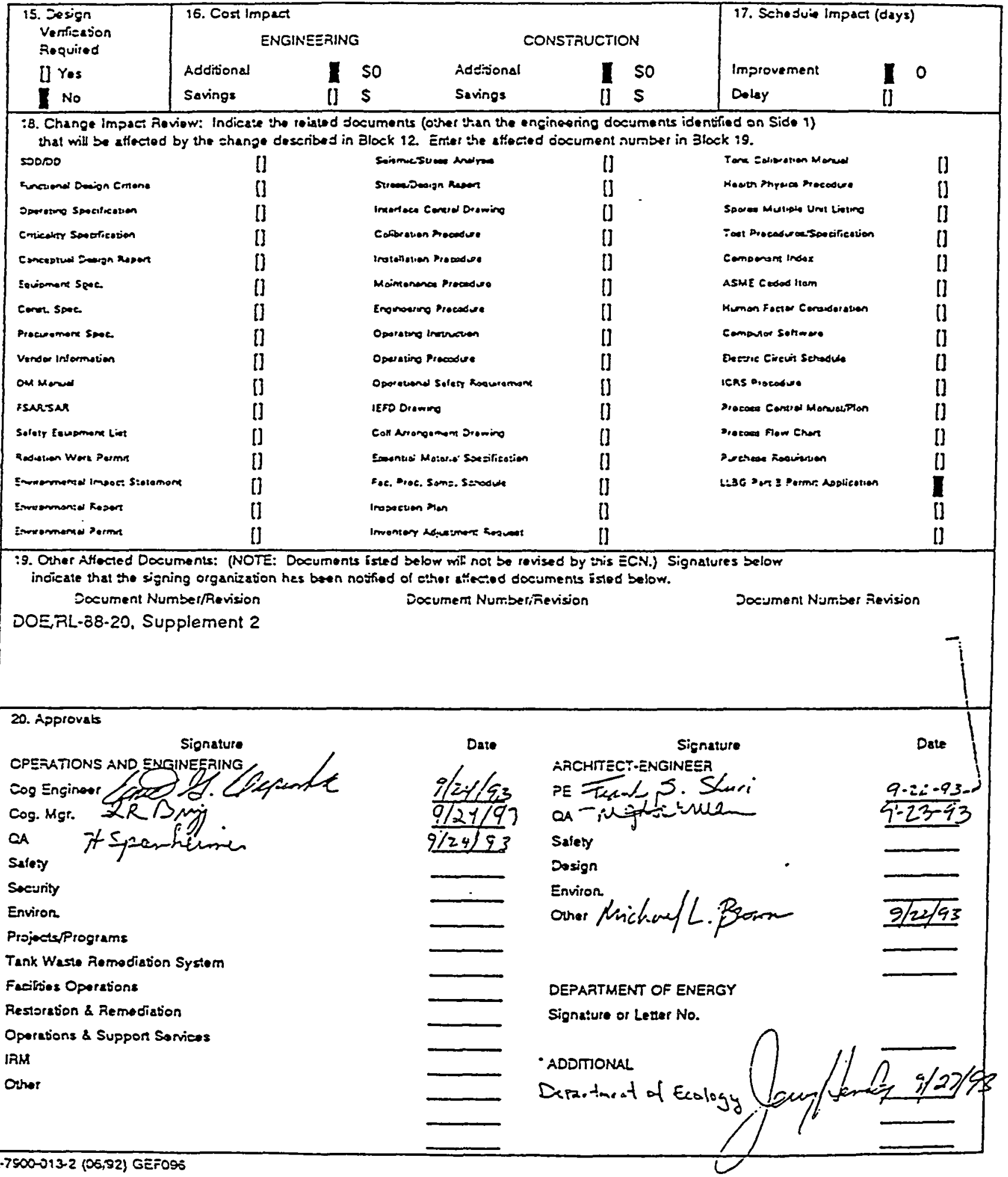


WHC-SD-W025-RPT-001, Rev. 0

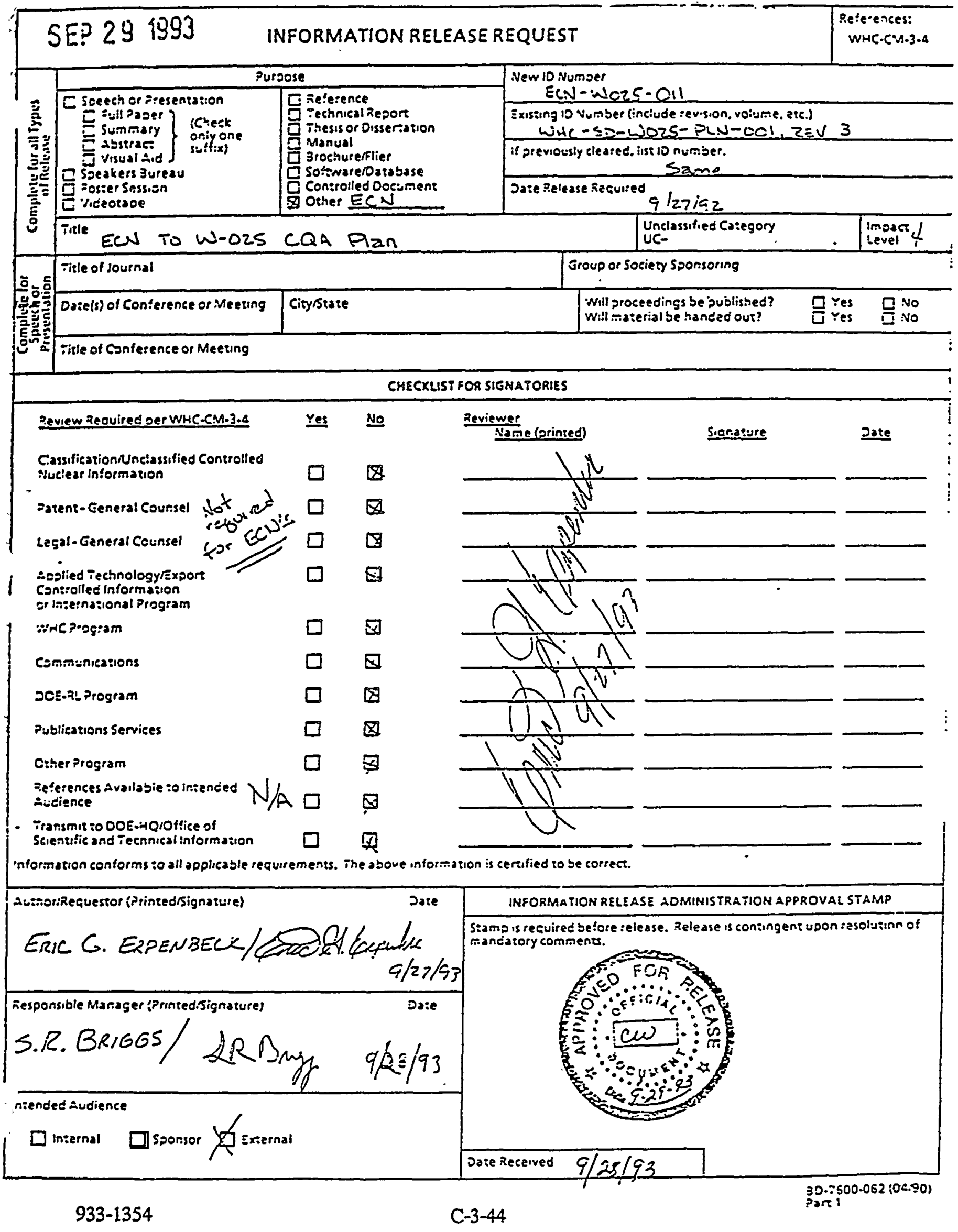




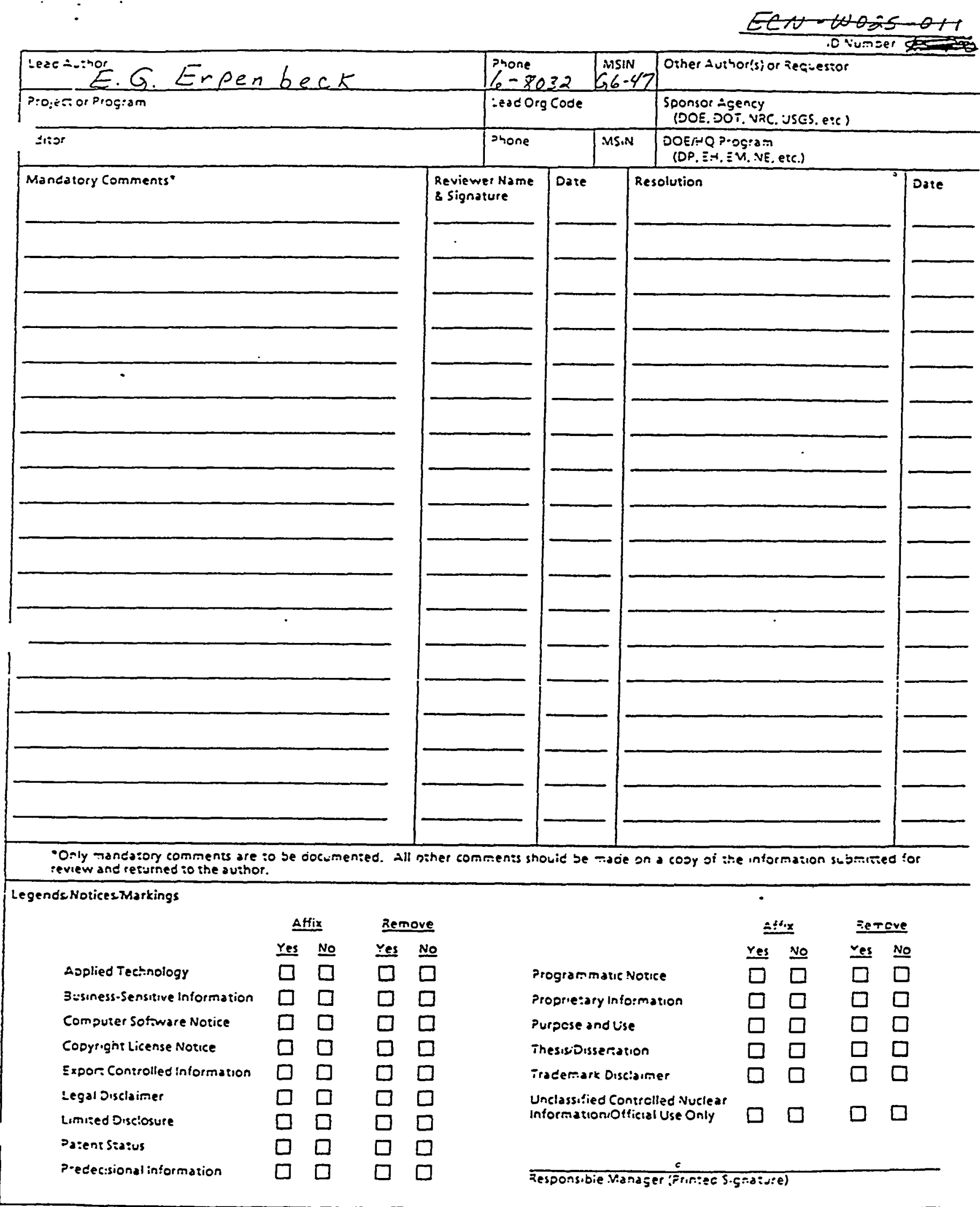


3. Origlnator's Name, Organlastion, MSIN, and Tolephone No.

4. Dato

F.S. Shuri, Golder Associates Ine. (206) 883-0777

9-22-93

\begin{tabular}{|c|}
\hline 112. Moorfication Work \\
\hline $\begin{array}{l}1 \text { Yos (fill out Blk } \\
\text { 11b) } \\
\text { No (NA Bllks. 11b, } \\
\text { 11c, 19d) }\end{array}$ \\
\hline
\end{tabular}

5. Project Tithonos Work Ordar No. W-025 RMW Land Disposal Facility

8. Documant Numbere Changed by this ECN

6. Bidg.Sya/fac. No. (includes shoot no. and rov.) see ltem 12 below

9. Rolated ECN No(s).

7. Impect Lovel

$3 Q$

see ltem 12 below

1b. Work Peckage

NA

No. NA

NA

11d. Rostored to Original Condf tion (Temp. or Standby ECN only)

NA

12. Doseription of Change

Changes to Project W-025 Dozign Drawings:

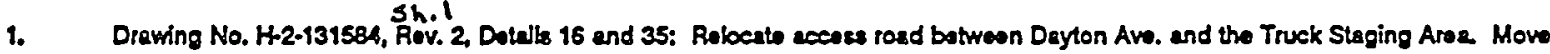

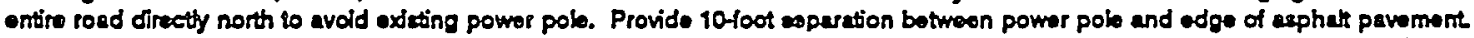

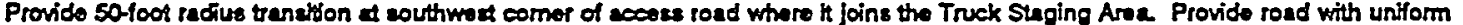
alopo betweon Truck Staging Aree and Dayton Avenue. Tranetton betwoen ecoass road and Truck Staging Aree ahall bo emooth and -ven. Do not pleco top couras until becation and prepared eubgrade have been approved by the Construction Manager. Rucord actual beation of road on arbull drawings.

Thls modificetion also applies to all other Project W-025 Design Drawings that show the eccess rosd.

\begin{tabular}{|c|c|c|c|c|c|}
\hline $\begin{array}{l}\text { 132. Justification (mark } \\
\text { one) }\end{array}$ & Critaria Change & Design improvement & [] & Environmental & [] \\
\hline ArFound II & Fecititete Conse & Const Error/Omiscion & [] & Dasign Errot/Omission & [] \\
\hline
\end{tabular}

1. Actual beation of powar pole interteres whth rosd. Location originally provided to Designar was not correct Moving road is antlcipated to be bes expantive and dituptive than relocating powror pole.

14. Distribution (include name, MSIN, and no. of copies) WHC DISTRIBUTION

Project Fibe

J.R. MCGe

Stition 10

DA RohI

EG. Erpenbeck

S.R. Brigge

KD. Johneon

J.J. Dorian

G.C. Evan:

J.M. Nisten
R1-24 (1)

Co-47 (1)

A3-87 (1)

5401 (1)

G6-47 (3)

G6-47 (1)

H2.58 (1)

B2-16 (1)

$H 457$ (1)

T3-20 (8)
Cog. Engines Signcture \& Date
Cog. Engineer Signature \& Dats

47900-013-2 (06/92) GEF095 
WHC-SD-W025-RPT-001, Rev. 0

\begin{tabular}{|c|c|c|}
\hline ENGINEERING CHANGE NOTICE & $P_{\text {age } 2 \text { of } 2}$ & $\begin{array}{c}\text { 1. ECN (urs no. trom pg. 1) } \\
\text { W-025-012 }\end{array}$ \\
\hline
\end{tabular}

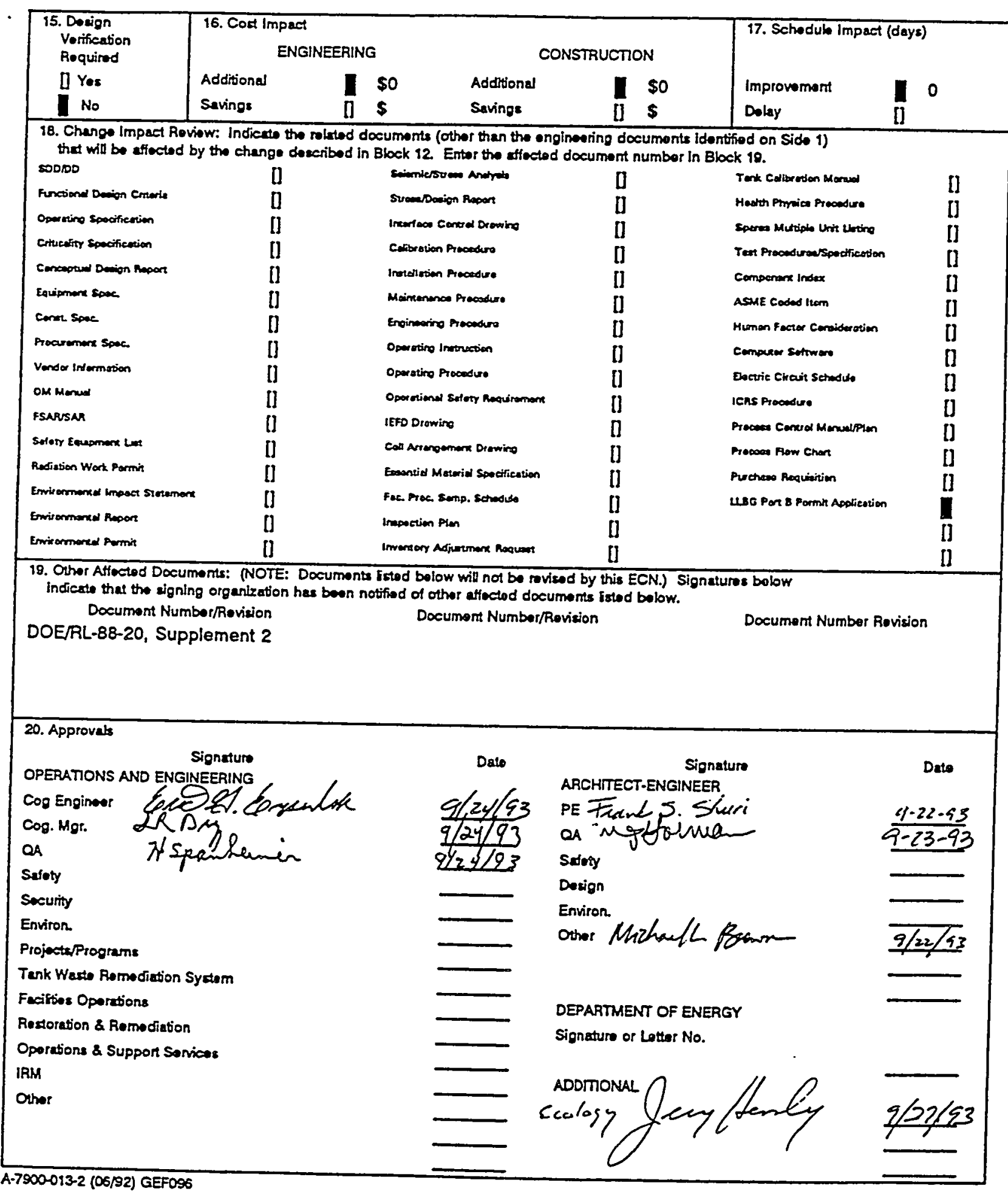




\section{ENGINEERING CHANGE NOTICE}

EG Erpenbes w24950 $66-47$ 376-8033

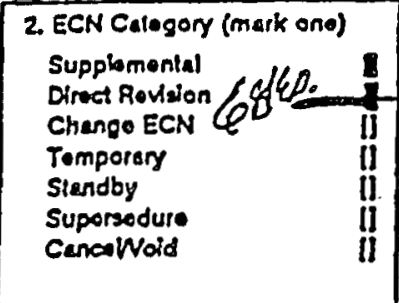

3. Originator's Namo, Organizaton, MSIN, and Tolephone No.

F.S. Shuri, Golder Assoclates inc. (206) $883-0777$

5. Project TrikNo Work Ordor No. GF $2 \overline{5}$

6. Bldg.Syeffac No.

Proj. W-025-013 ECN

4, Dale

, Dale

10-6.93

ECN

(includes sheot no. and rov.)

WHC-S-045 ReV. 2

9. Reluled ECN No(s).

7. Impact Level

avel

112. Modifieston Work

11b. Work Package 11c. Modificaton Work Complote

NA

10. Relaled PO No.

I] Yas (fil out Bile No.

$$
\text { 11b) }
$$

No NA BIKr, 116, 11c. 110 )

NA

Cog. Engineer Signature \& Dato i1d. Rostored to Orinal Condt Son (Tomp. or Slendby ECN only)

NA

\section{Doxcripton of Change}

Changes to Project W-025 Specifications:

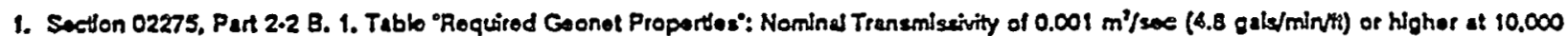

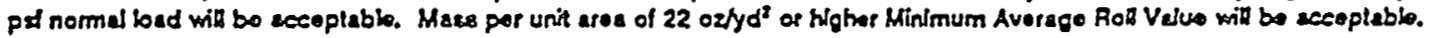

2. Secton 02275, Part 2.2 B. 2 Table "Required Geotextlo Propertes": Mesannit Aroe of 7.0 ozyd" or higher will be ecepleble lor Type A

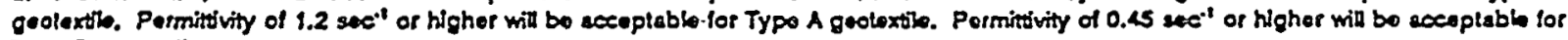
Type 3 geotextile.

3. Section 02725, Part 24 Sehedulo: For 1.S-inch-diameter submersible pump discharge piplng. SOR 11 wid also be aceeptable.

\begin{tabular}{|c|c|c|c|c|}
\hline $\begin{array}{l}\text { 132 Justification (mark } \\
\text { ono) }\end{array}$ & Criteria Change & Design Improvement & [] & Environmental \\
\hline As-Found II & Faciftato Const & Const Error/Omizsion & [] & Design Error/Onlsslon \\
\hline
\end{tabular}

136, Justification Deteils

Feduced cost, improved schedulo for delivery.

1. Exceeds required performance criterie. Ser attachod cakulation sheel

2. Exceeds required performence criteria Soe altached calculation sheot

3. Sfightly thicker wall does nol adversoly affect portormance.

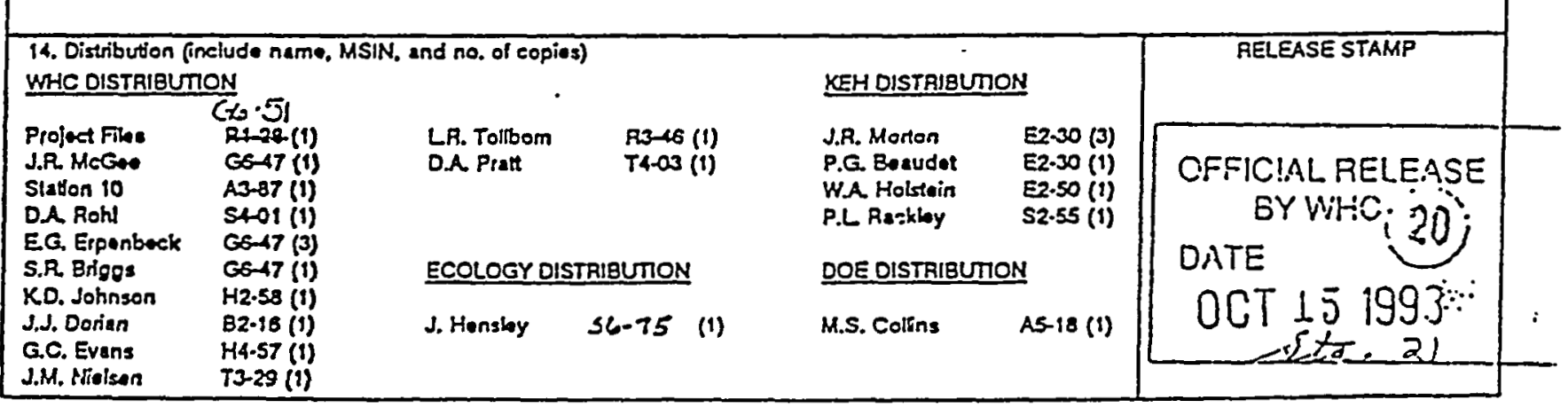

A.7900-013-2 (CS,92) GEF095 
WHC-SD-W025-RPT-001, Rev. 0

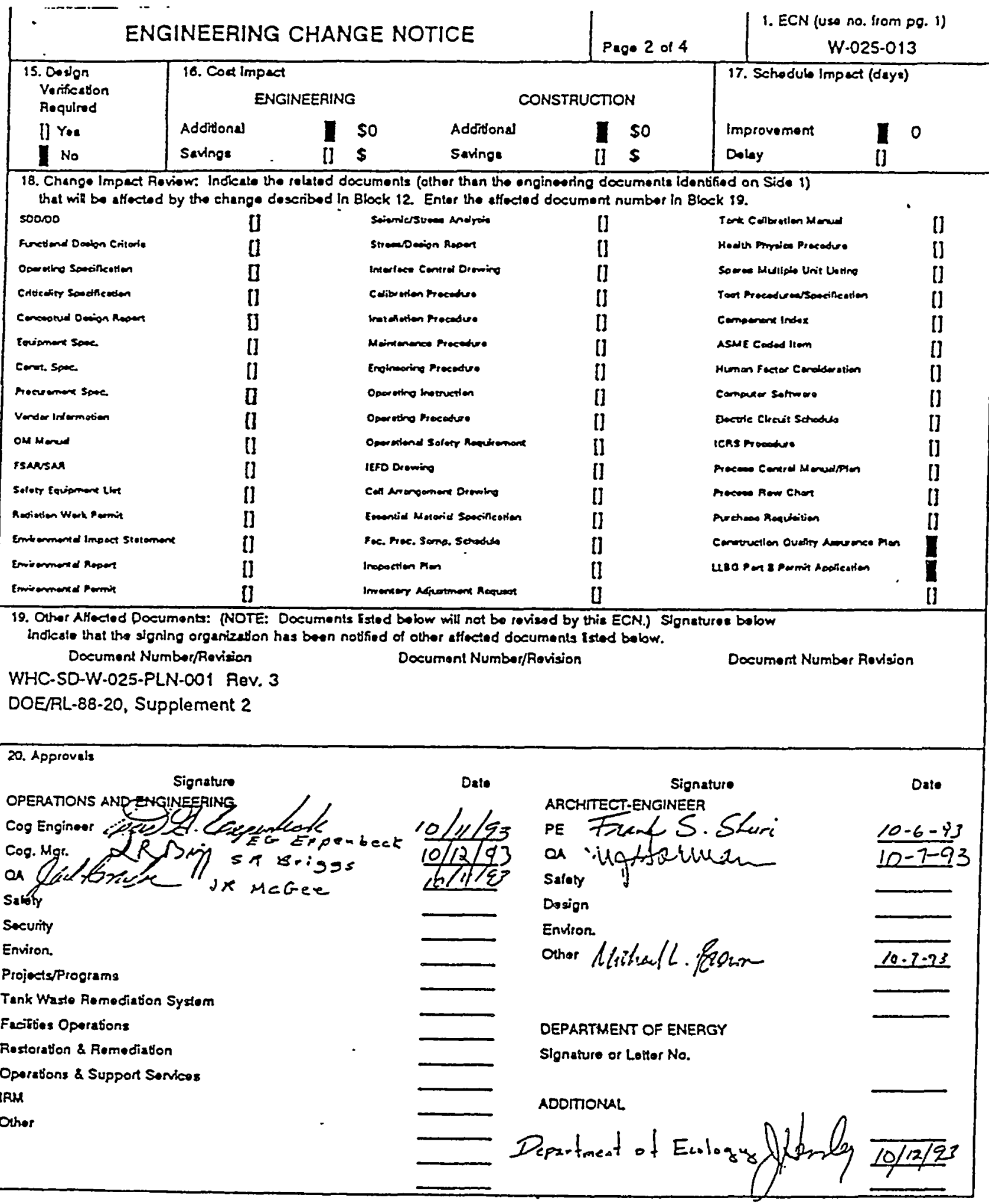


WHC-SD-W025-RPT-001, Rev. 0

wo25013 Sheed 3 of 4

\section{Golder}

Associales

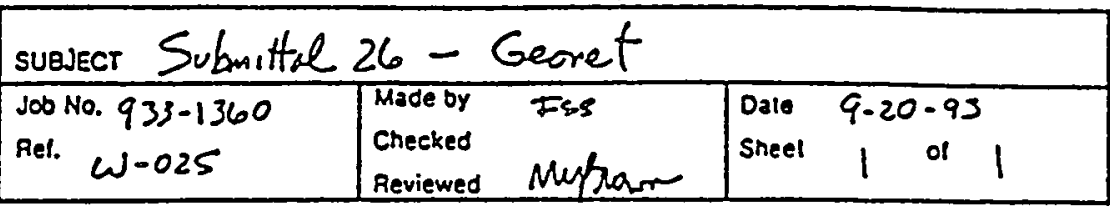

(1) Tranisinissivity

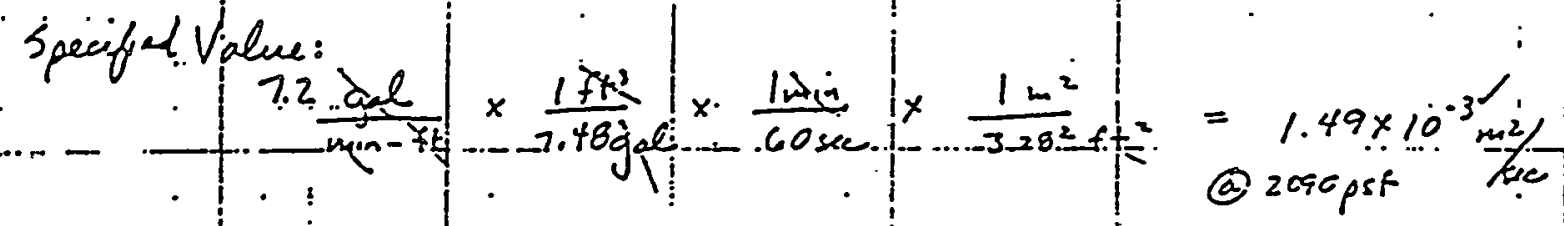
Proposed Valice: $1.0 \times 10^{-3} @ 10,000$ st:

Reguired Value: See Sectin 3, Page 7 of Vol II fo desig Repot. $Q_{\text {max }}=$ Flow at toe of slope $=0.62$ gpaifft:

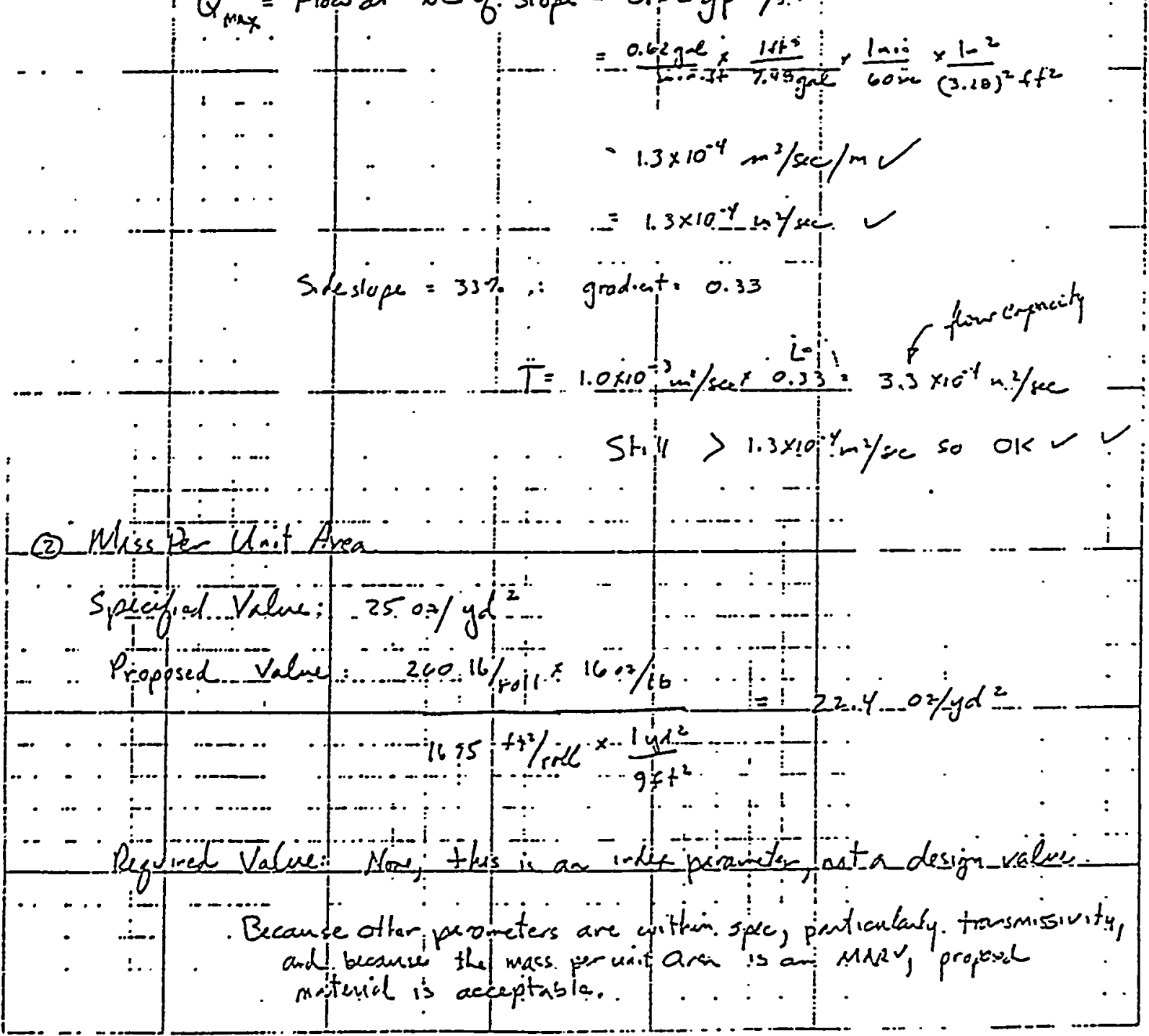


WHC-SD-W025-RPT-001, Rev. 0

wo $025+13$ that $40+4$

\section{Golder}

Associates

\begin{tabular}{|c|c|c|}
\hline subuect Sub. & \& 24 - Cretert & \\
\hline $\begin{array}{l}\text { Job No. } 933-1360 \\
\text { Rel. w.025 }\end{array}$ & $\begin{array}{l}\text { Made by } \text { FSS } \\
\text { Checked } \\
\text { Reviewed LWulgegun }\end{array}$ & \begin{tabular}{|cc|c|} 
Dale & $9-20-93$ \\
Sheol & 1 of
\end{tabular} \\
\hline
\end{tabular}

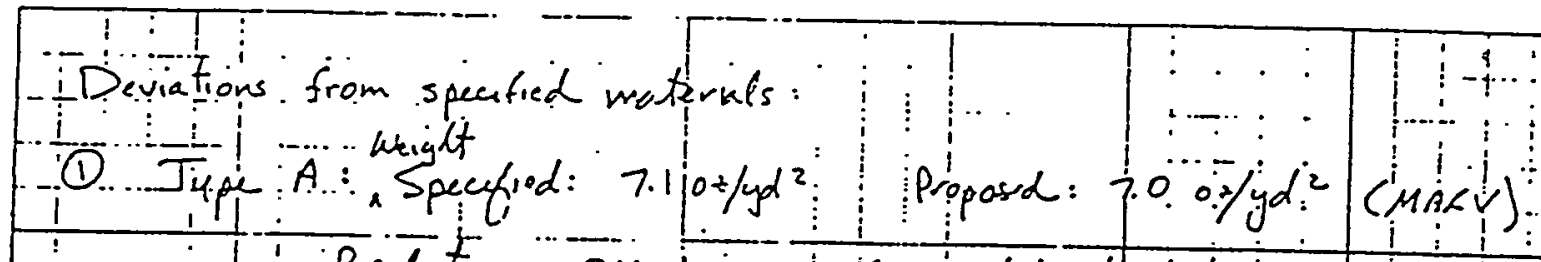

- Resolution: OK becanse Specs did ket state aveng on

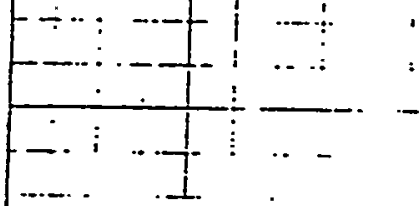

and.

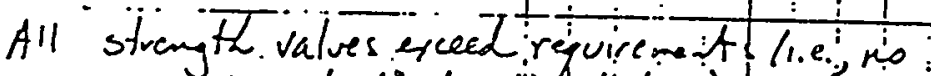
impact of ireduced oftickress)

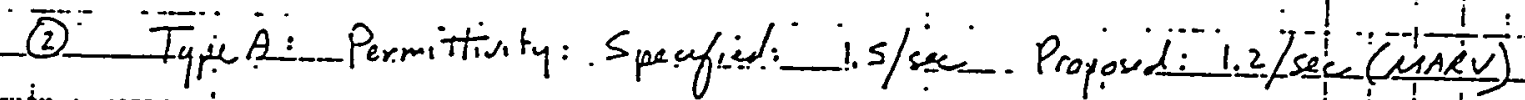

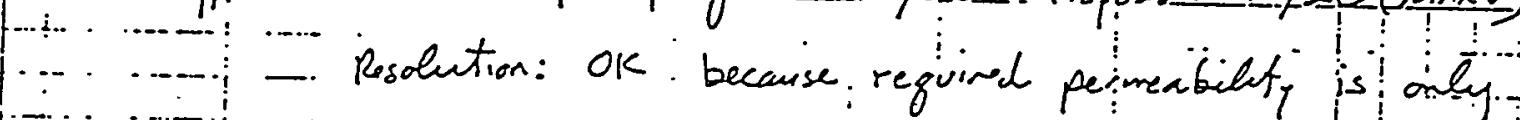

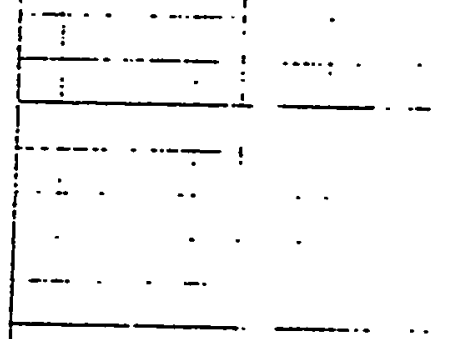

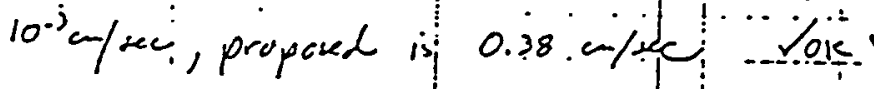

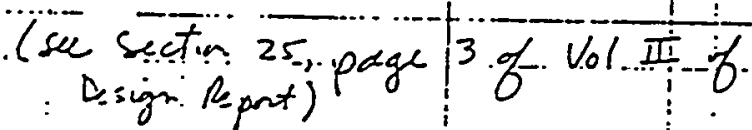
Note that $: P=\frac{k}{t}$ where $P=$ perifunty. $x=$-perimeliet

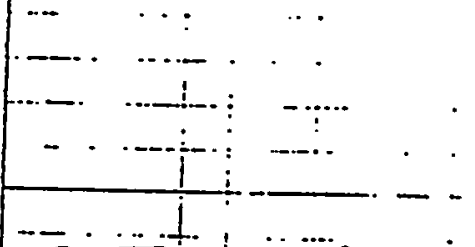
$\therefore$ in comparibly thich geotextiles, if

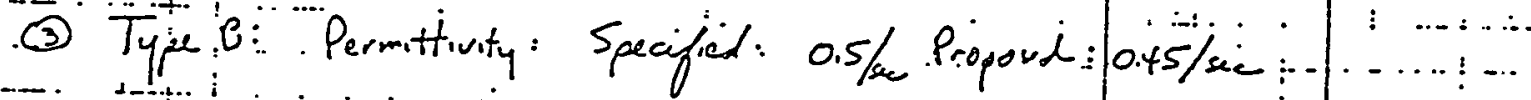

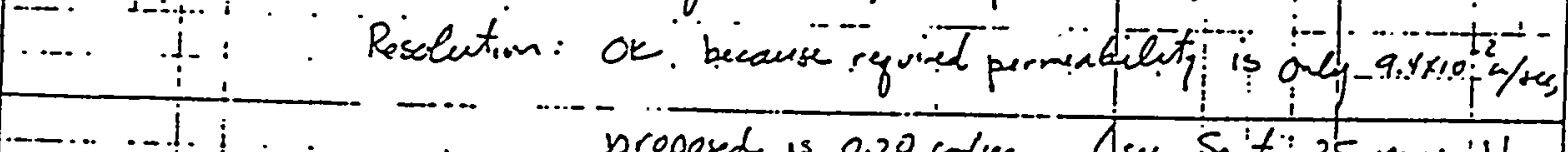

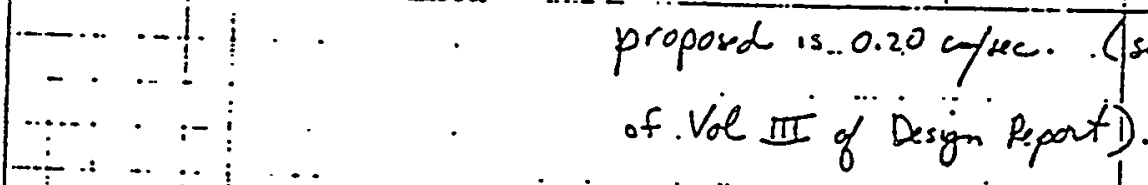

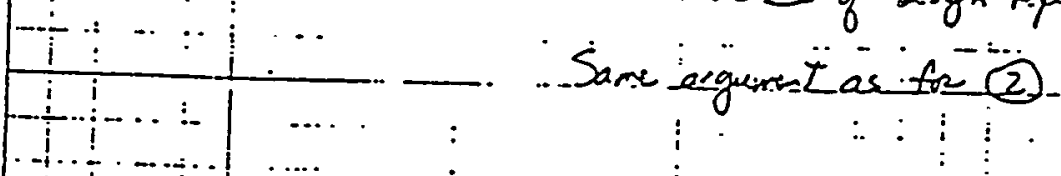




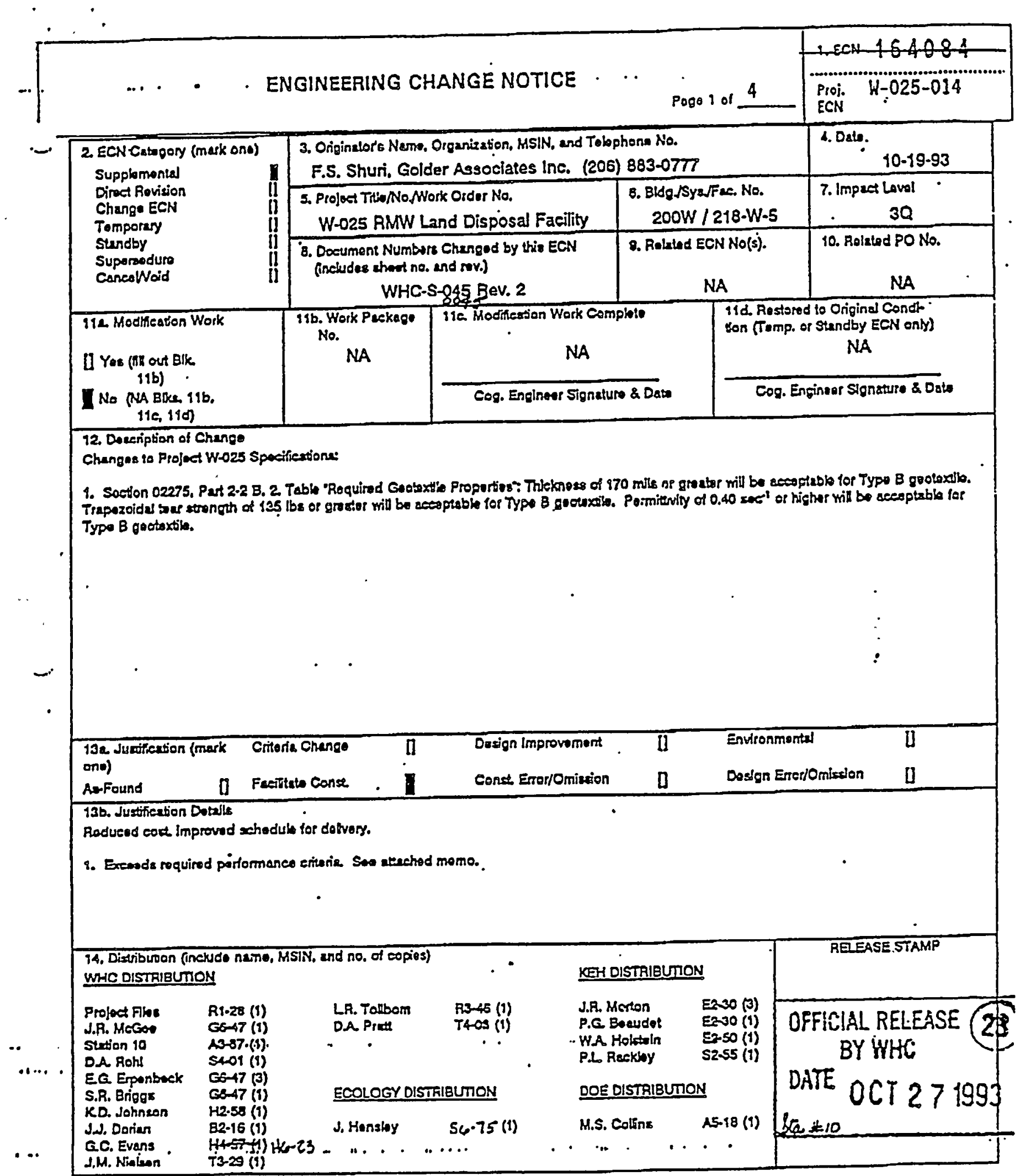

$\rightarrow$ A.7500013-2 (06/92) GEFOOS 
WHC-SD-W025-RPT-001, Rev. 0

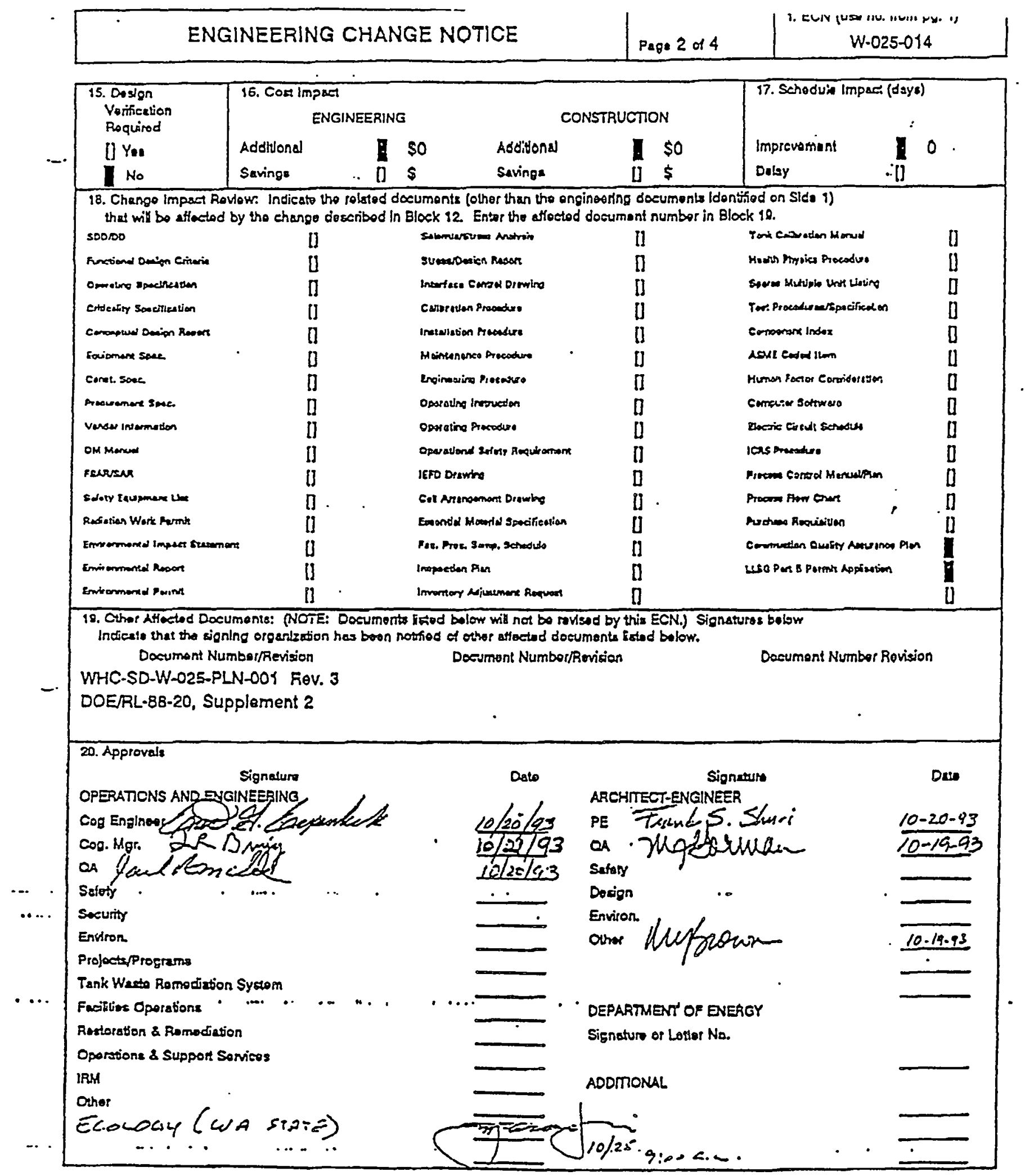


TO: Project File

October 19, 1993

ER: Frank Shuri, GAI Redmond T3S

RE: W-025 GEOTEXIILE CONFORMANCE ?ESIING IRESULTS, Job No. 933-1360

Conformance test results on several geotextile samples indicated property values that are slightly lower than the project specifications for the material. Specifically,

- Geotextile Type B: specified thickness: 185 mils, measured thickness: 175 and 176 mils

- Geotextile Type B: specified trapezoidal tear strength: $150 \mathrm{lb}$, measured trapezoidal tear strength: 146 and $147 \mathrm{lb}$

- Geotextile Type B: specified permittivity: $0.45 \sec ^{-3}$, measured penrittivity: 0.44 sect $^{-1}$

The impact of slightly low property values on geotextile performance was evaluated. With respect to Type B geotextile, thickness is not a primary consideration if the other material properties are within specification. Some compression of the geotextile may occur as it is wrapped into a soll. Thickness may affect the ability of a geotextile to provide cushioning, but for the W-025 Landfill, this is no longer a significant design concern. Originally, one of the purposes of the Type B geotextile was to cushion the primary geomembrane from the underlying secondary drainage gravel. However, the liner system has been redesignied so that a primary admix layer is now present between the geomembrane and the gravel. Therefore, cushioning the geomernbrane is no longer required.

With respect to slightly low trapezoidal tear strength, the W-025 Design Report (WHC-SD-W025FDR-D01), Calculation C.25 "Geotextile Selection" was reviewed to evaluate required design strengths. The required strength parameters (including.factors of safety) and those measured on the Type B geotextile are as follows:

\begin{tabular}{|c|c|c|c|}
\hline - & Burst Strength: & $\begin{array}{l}\text { Required: } \\
\text { Available: }\end{array}$ & $\begin{array}{l}51 \mathrm{psi} \\
850 \text { to } 918 \mathrm{psi}\end{array}$ \\
\hline$\therefore$ & $\begin{array}{l}\text { Grab Strength: } \\
. . . .\end{array}$ & $\begin{array}{l}\text { Required: } \\
\text { Available: -.. }\end{array}$ & $\begin{array}{l}216 \mathrm{lb} \\
507 \text { to } 745 \mathrm{lbs}\end{array}$ \\
\hline - & Puncture Strength: & $\begin{array}{l}\text { Required: } \\
\text { Available: }\end{array}$ & $\begin{array}{l}2 \mathrm{lb} \\
288 \text { to } 312 \mathrm{lb}\end{array}$ \\
\hline
\end{tabular}

Trapezoidal tear strength is not a specifically required parameter that models a physically realistic failure mode, hence there is no required design value. The above confomance test results indicate that the available strength of the Type B geotextile far exceeds the design requirements. Therefore, the slightly low trapezoidal tear strength is not a concern. 
WHC-SD-W025-RPT-001, Rev. 0

With respect to the slightly low permittivity value for the Type B geotextile, the measured value is still more than twice the value required for design purposes. The supporting calculations are presented in ECN W-025-13.

In summary, the conformance test results discussed above probably reflect normal variability in geotextile materials and will not adversely affect the performance of these materials as part of the $W-025$ liner system. This conclusion applies only to the test data evaluated in this memo and should not be applied to other conformance test results.

geotextw5I 


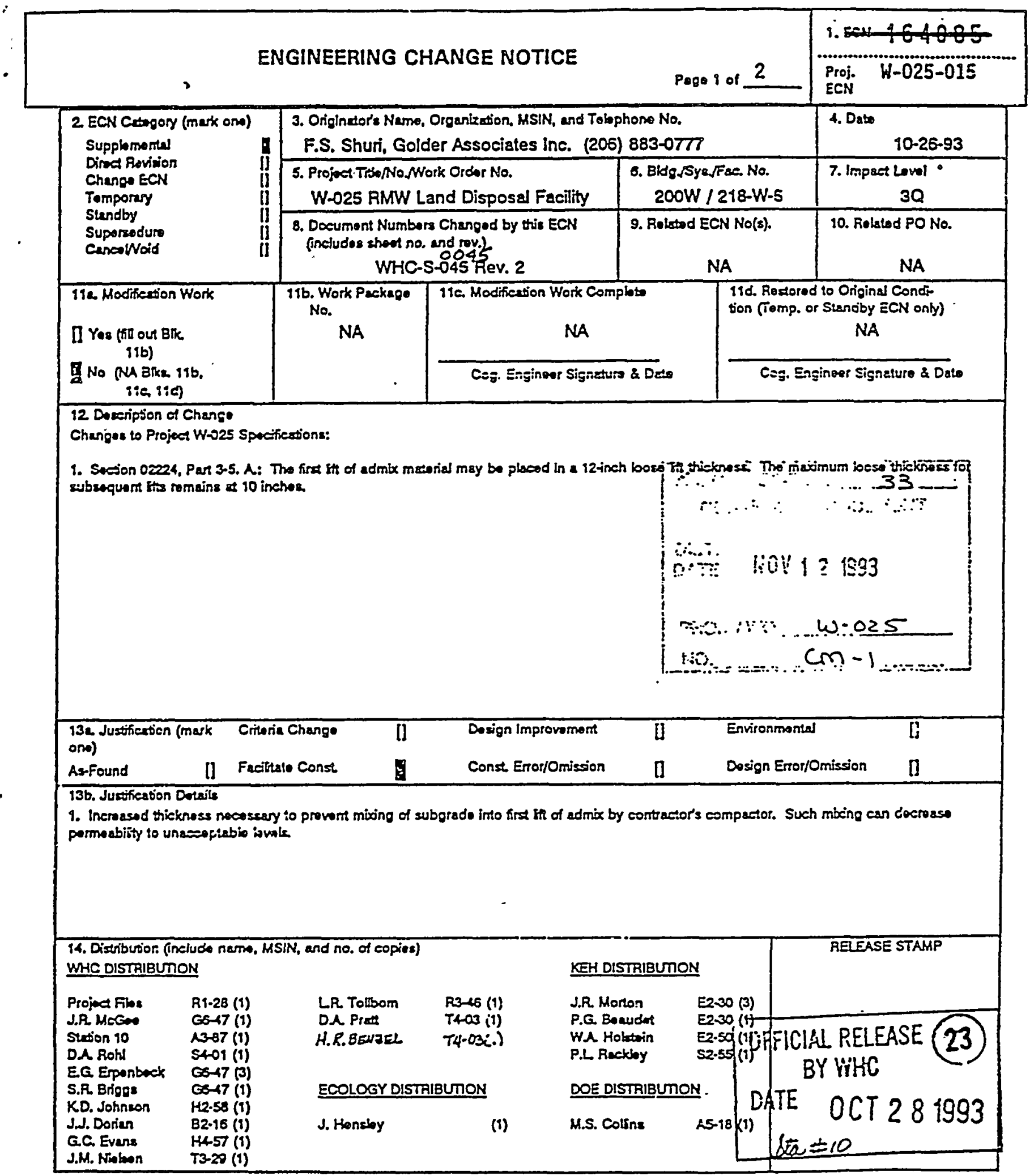

A.7000-013-2 (06/82) GEFOS5 
WHC-SD-W025-RPT-001, Rev. 0

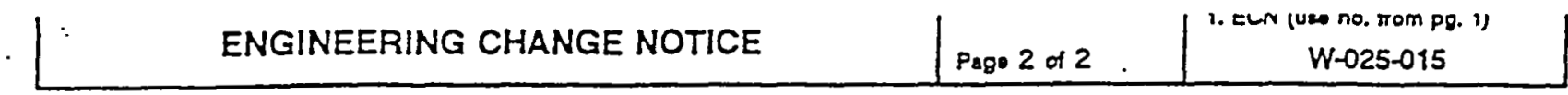

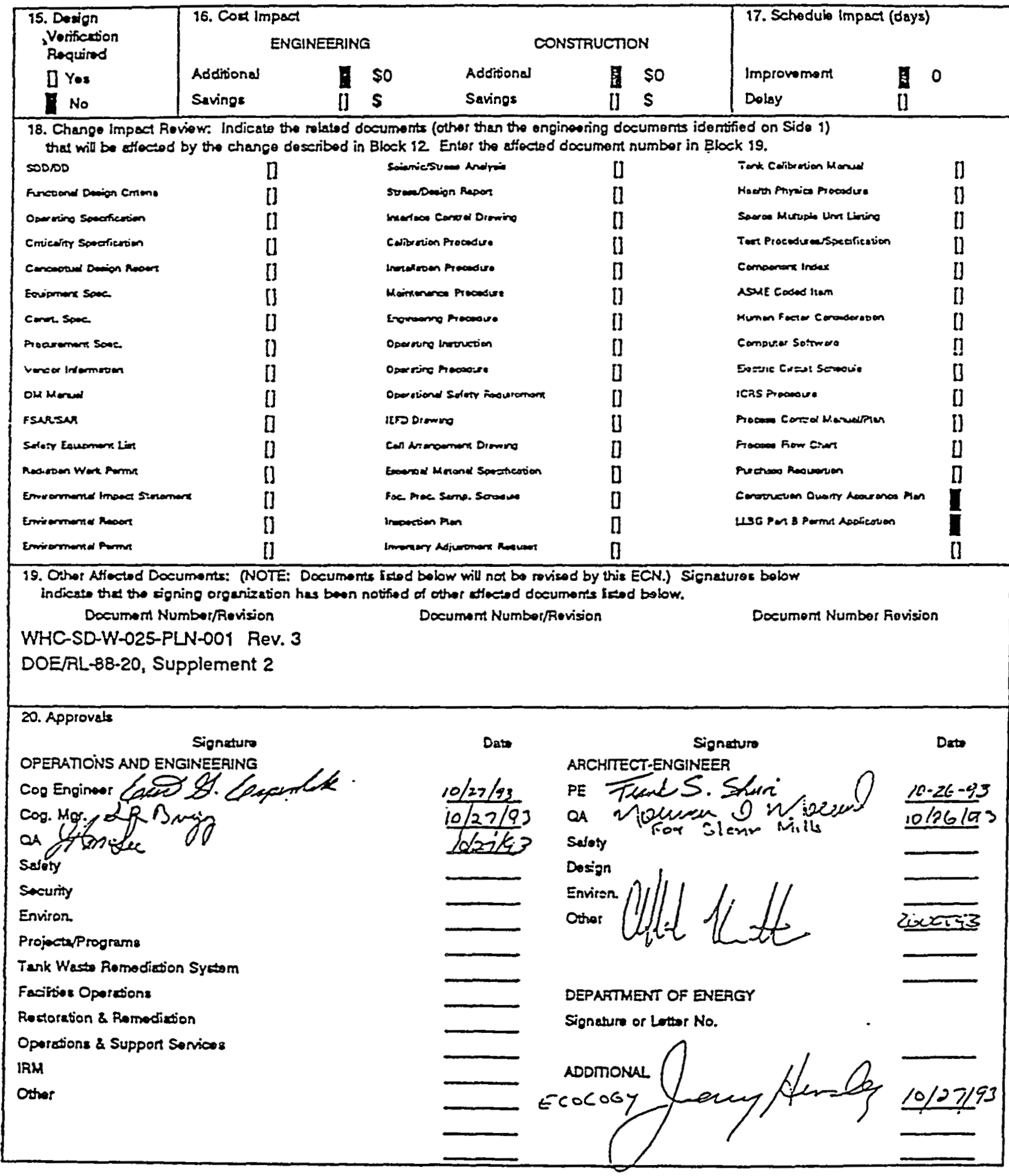




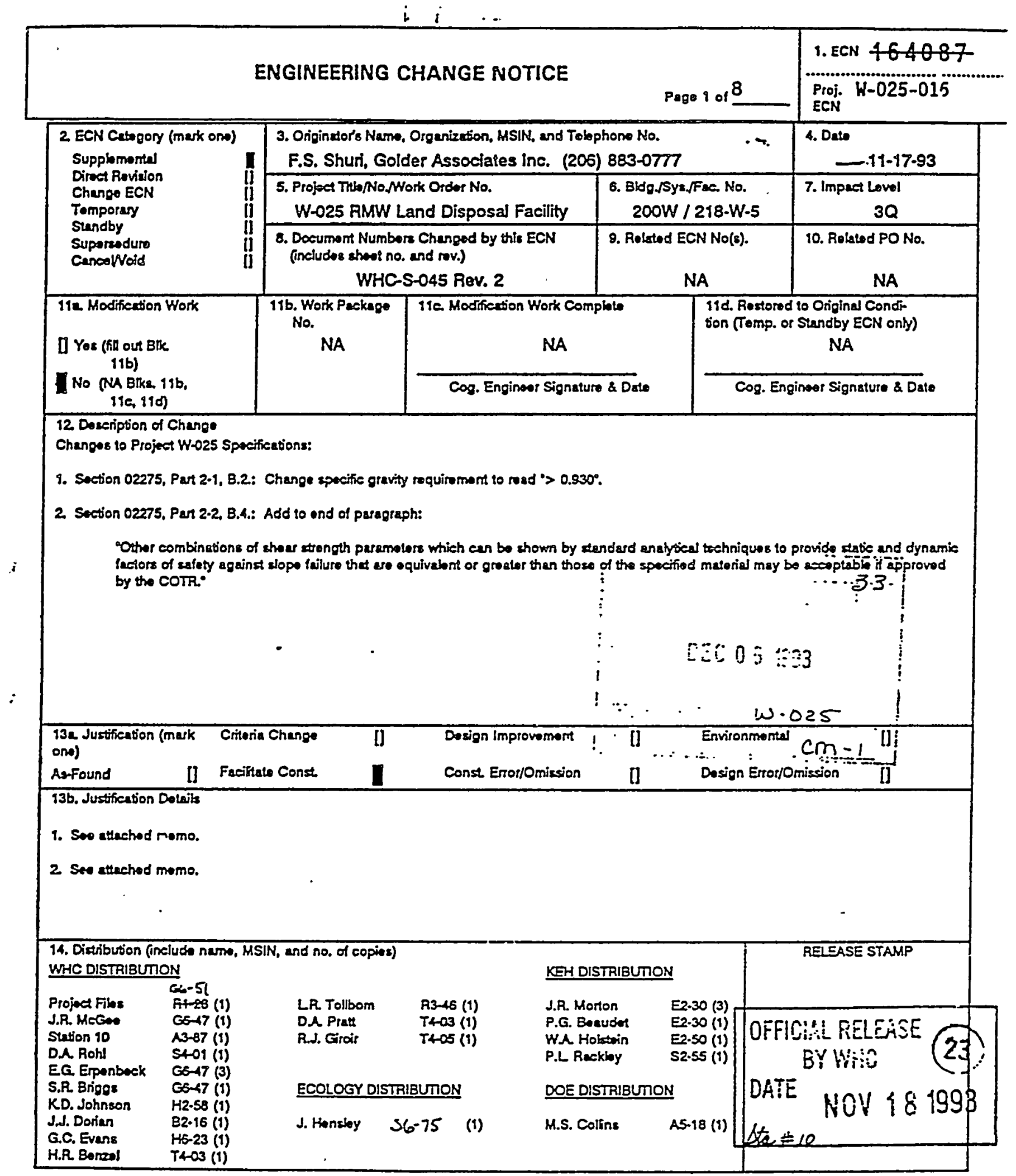

A-7900-013-2 (05/92) GEFO95 
WHC-SD-W025-RPT-001, Rev. 0

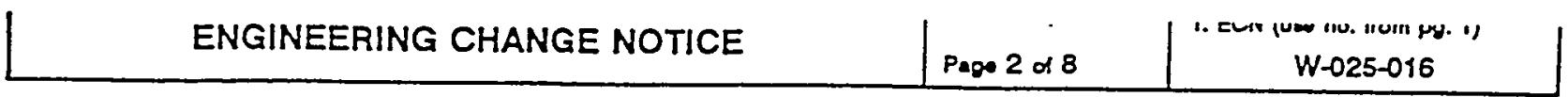

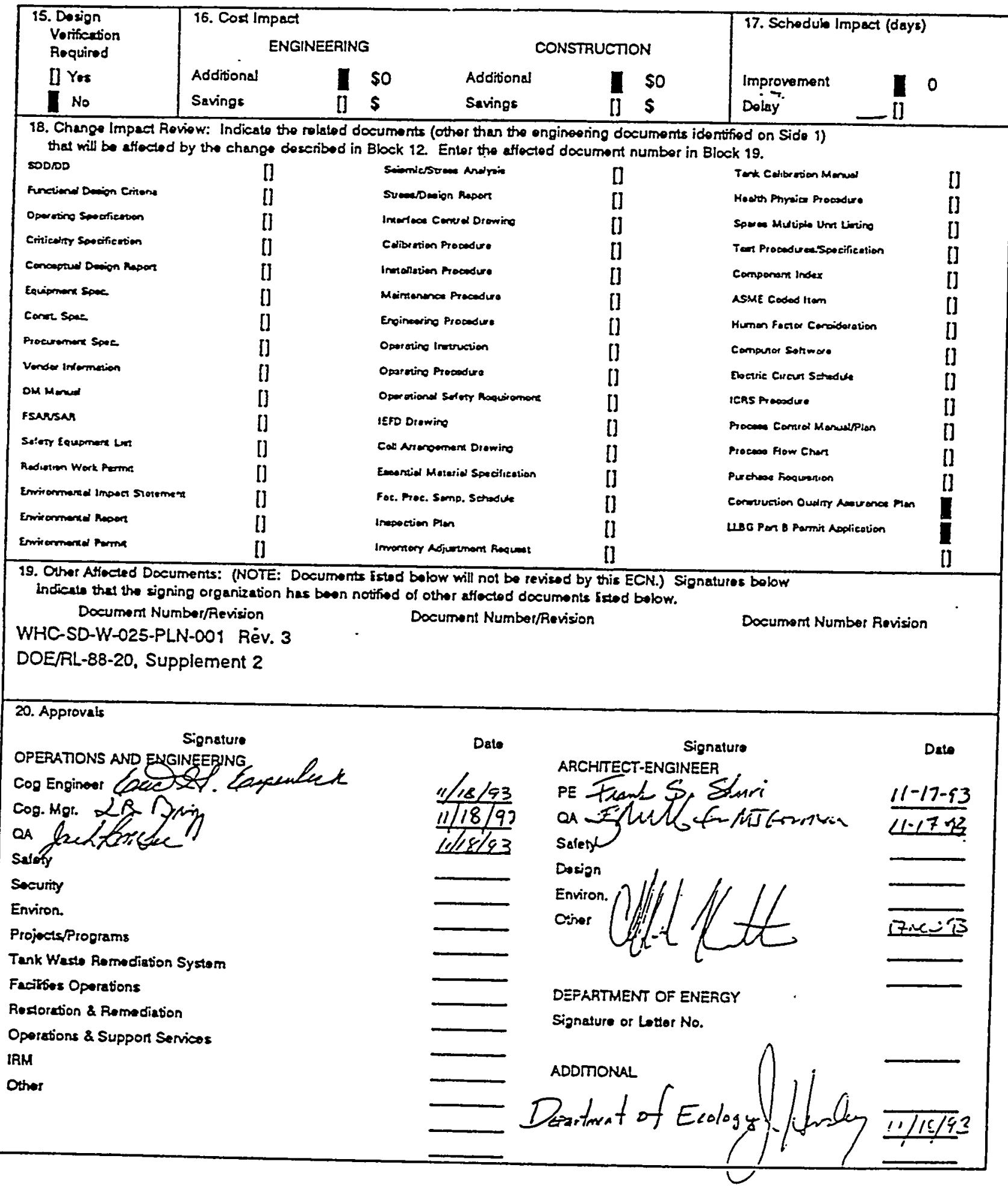


WHC-SD-W025-RPT-001, Rev. 0

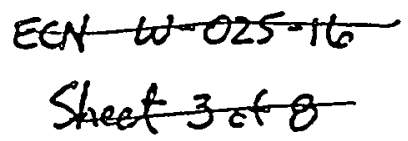

MEMORANDUM

TO: W-025 Landfill Project File, 933-1360

November 17, 1993

FR: FrankS. Shuri Fos

RE: CHANGES TO GEOMEMBRANE SPECIFICATIONS

1. Crystallinity. Specification Section 02275 , Part 2-1, B.4. requires a crystallinity of 50 to 60 percent, or as approved by the COTR. Crystallinity of the geomembrane proposed by the Contractor appears to be in the range of 40 to 50 percent. It is recommended that the COTR approve this material with respect to crystallinity for the following reasons:

(a) Per the attached letter from SLT North America, Inc., lower crystallinity provides an increase in elongation, resistance to stress cracking, and chemical resistance. Such changes will enhance the long-term performance of the liner and are therefore desirable.

(b) Because there are only minimal tensile loads on the liner, the slight decrease in tensile strength noted in the SLT letter is not a concern for this project.

(c) Measurement of crystallinity appears to be a relatively inexact process.

(d) Crystallinity is not a primary design parameter; it is included for "fingerprinting" purposes. As long as other physical properties conform to specifications, geomembrane ferformance is expected to be satisfactory.

2. Interface Shear Strength. Specification Section 02275, Part 2-2, B.4. requires a friction angle between the geocomposite and textured geomembrane of at least $27.5^{\circ}$ under a normal load of $400 \mathrm{psf}$ and a displacement of at least 2 inches. Test results indicate a friction angle of $18^{\circ}$ with an apparent cohesion of $0.6 \mathrm{psi}(86 \mathrm{psf})$. Slope stability analyses using the specified strength parameters (friction angle $=27.5^{\circ}$, cohesion $=0$ ) show a static factor of safety of 1.6 and a dynamic factor of safety of 1.1 (see attached sheet). The same stability analysis using the actual strength parameters (friction angle $=18^{\circ}$, cohesion $=86 \mathrm{psf}$ ) shows a static factor of safety of 1.8 and a dynamic íactor of safety of 1.3. For design purposes, factors of safety of 1.5 and 1.1 have been considered acceptable for static and dynamic loading conditions, respectively.

The factors of safety exhibited by the actual liner materials are well above both the minimum acceptable values and the values provided by a material with the specified strength parameters. Therefore, the Specifications should be expanded to allow any combination of parameters which can be shown by standard analysis to provide adequate factors of safety under expected loading conditions.

3. Resin Density. Specification Section 02275, Part 2-1, B.2. requires a resin specific gravity greater than 0.935 . Actual measurements range from 0.932 to 0.934 . However, the finished geomembrane density ranges from 0.942 to 0.945 , which is greater than the minimum required value of 0.94 . Because the finished geomembrane density is greater 
than required and because other physical properties satisfy the specification requirements, the slightly low resin density is not considered significant with respect to geomembrane performance. The Specifications should be revised to allow lower values for resin density.

ecn16mmo.w51 
INFINITE SLOPE STABILITY

Date: $\quad$ 10-Mar-92
Made by: FSS

Checked by: $R Q$. .'

Reviewed by:

Reference: Stability Analysis of Earth Slopes, Huang, Y.H., 1983

Infinite slope equation: $F S=\left((c / G d) \sec a+\left((1-r u) \cos a-C s^{*} \sin a\right) \tan P\right) /\left(\sin a+C s^{*} \cos a\right)$

Where:

$$
\begin{aligned}
& C=\text { effective cohesion } \\
& G=\text { unit weight of sliding mass } \\
& d=\text { vertical thickness of sliding mass } \\
& a=\text { slope angle } \\
& C s=\text { horizontal acceleration } \\
& P=\text { effective friction angle } \\
& r u=\text { pore pressure factor }
\end{aligned}
$$

$\begin{array}{lrl}G= & 120 \text { pct } \\ d= & 3 \text { ft } \\ a= & 18.43 \text { degrees } \\ C s= & 0.12 \mathrm{~g} \\ c= & 0 \text { psf } \\ P= & 27.5 \text { degrees } \\ r u= & 0\end{array}$

$\begin{array}{lll}\text { Static Analysis: } & \text { FS }= & 1.56 \\ \text { Dynamic Analysis: } & \text { FS }= & 1.10\end{array}$


INFINITE SLOPE STABILITY

Date: 17-Nov-93
Made by: FSS Checked by: Reviewed by:

Reference: Stability Analysis of Earth Slopes, Huang, Y.H., 1983

Infinite slope equation: $F S=\left((C / G O) \sec a+\left((1-r u) \cos a-C s^{*} \sin a\right) \tan P\right) /\left(\sin a+C s^{*} \cos a\right)$

Where: $\quad c=$ effective cohesion

$G=$ unit weight of sliding mass

$d=$ vertical thickness of sliding mass

$a=$ slope angle

Cs $=$ horizontal acceleration

$P=$ effective friction angle

$r u=$ pore pressure factor

$=$ (Volume of mass submerged $\cdot 62.4 \mathrm{pci}) /$

(Total volume of mass "unit weight of soil in pct)

$\begin{array}{lrl}G= & 120 \mathrm{pef} \\ d= & 3 \mathrm{ft} \\ \mathrm{a}= & 18.43 \text { degrees } \\ \mathrm{Cs}= & 0.12 \mathrm{~g} \\ \mathrm{C}= & 86 \mathrm{psf} \\ \mathrm{P}= & 18 \text { degrees } \\ \mathrm{ru}= & 0\end{array}$

Static Analysis: $\quad$ FS =

$1.77 \%$

Dynamic Analysis: $\quad F S=$

$1.27 \mathrm{~V}$ 
Page 1 of 9 +5en $+5+938$ Proj: $W-025-017$

\begin{tabular}{|c|c|c|c|c|c|}
\hline $\begin{array}{l}\text { 2. ECN Cangory (mark ono) } \\
\text { Supplomentul }\end{array}$ & \multicolumn{4}{|c|}{$\begin{array}{l}\text { 3. Originctors Nemo, Organization, MSIN, and Toiophone No. } \\
\text { F.S. Shuri, Golder Associates Inc. (206) 883-0777 }\end{array}$} & $1-20-94$ \\
\hline $\begin{array}{l}\text { Dreci Riewien } \\
\text { Chenge ECA } \\
\text { Temporay }\end{array}$ & \multicolumn{2}{|c|}{$\begin{array}{l}\text { 5. Project TiterNo, Work Order Na. } \\
\text { W-025 RMW Land Disposal Facility }\end{array}$} & \multicolumn{2}{|c|}{$\begin{array}{l}\text { 6. Budg-Sye-fFe No. } \\
\text { 200W / 218-W-5 }\end{array}$} & $\begin{array}{r}\text { 7. Impectend } \\
30\end{array}$ \\
\hline $\begin{array}{l}\text { Superdey } \\
\text { Suncurvoid }\end{array}$ & \multicolumn{2}{|c|}{$\begin{array}{l}\text { 8. Document Numbers Changed by this ECN } \\
\text { (ncudes ahe no and (nW.) } \\
\text { WHC-S-045 Rev. } 2\end{array}$} & \multicolumn{2}{|c|}{$\begin{array}{l}\text { 9. Rolad ECN No(s). } \\
\text { W-025-18 }\end{array}$} & $\begin{array}{l}\text { 10. Roluted PO Na. } \\
\text { NA }\end{array}$ \\
\hline $\begin{array}{l}\text { Q Yos (1II out Bue } \\
\text { 11b) }\end{array}$ & $\begin{array}{l}\text { 116. Work Packeq. } \\
\text { No. } \\
\text { NA }\end{array}$ & \multicolumn{2}{|c|}{ I1C Modifedton Work Compiots } & \multicolumn{2}{|c|}{ NA } \\
\hline 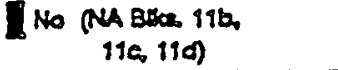 & & \multicolumn{2}{|c|}{ Cog Enpineer Signaure a Das } & \multicolumn{2}{|c|}{ Cog. Engines Signature \& Deto } \\
\hline
\end{tabular}

12. Ducipion of Change

Chenges to Projer Hozs Specifendon:

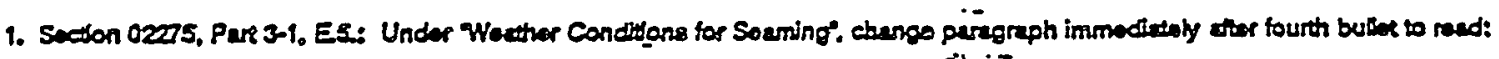
$\therefore \cdot-$.

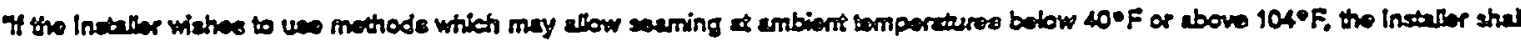

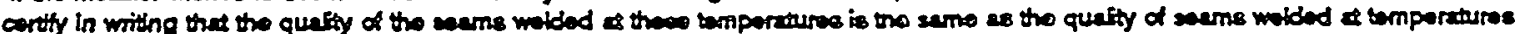

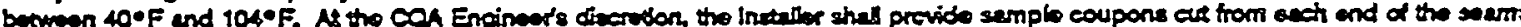

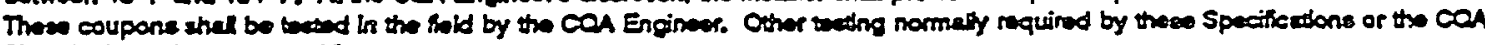
Plen shall alo be performed.

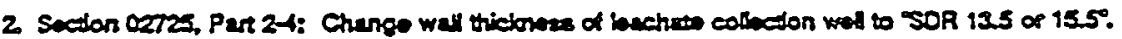

(Continind on Shent 3)

\begin{tabular}{|c|c|c|c|}
\hline $\begin{array}{l}\text { 13e Jutiticeston (matk } \\
\text { one) }\end{array}$ & Criteria Crenge & Dexign Improvement & Environmental \\
\hline Arfound $\quad$ I] & Faciltat Cont & Conte EratOminion & Dadign Entor/Omistion \\
\hline
\end{tabular}

13b. Juatidetion Oerrile

1. Etubliaher verification the

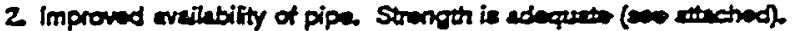

(Conthued on Shent 3)

14. Dietribution (include nume, MSin, and na of copies) WHC DISTRIBUTION

\begin{tabular}{|c|c|}
\hline 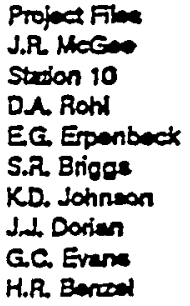 & 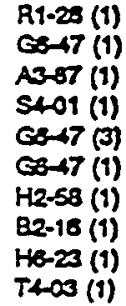 \\
\hline
\end{tabular}

A7900-013-2 (overa) Geross
DA Prat

Ru. Erair

R3218 (1)

Thas (1)

$\operatorname{Th} \alpha \cos (1)$

ECOLOGY DISTRIBUTION

J. Hencly

$56-15$

\section{KEH DISTRIBUTION}

J.R. Horton

P.G. Boutude

WA. Holvin

P.L Rectiry

DOE DISTAIBUTION

M.S. Collns
RELEASE STAMP

E2-30 (3)

2230 (1)

E2-50 (1)

$\$ 2-55$ (1)

N

A5-18 (1)
:

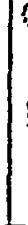

$\sin$
분

J 
WHC-SD-W025-RPT-001, Rev. 0

\section{ENGINEERING CHANGE NOTICE \\ Page 2 \& 9 \\ 1. ECN (use no. from po. 1) \\ W-025-017}

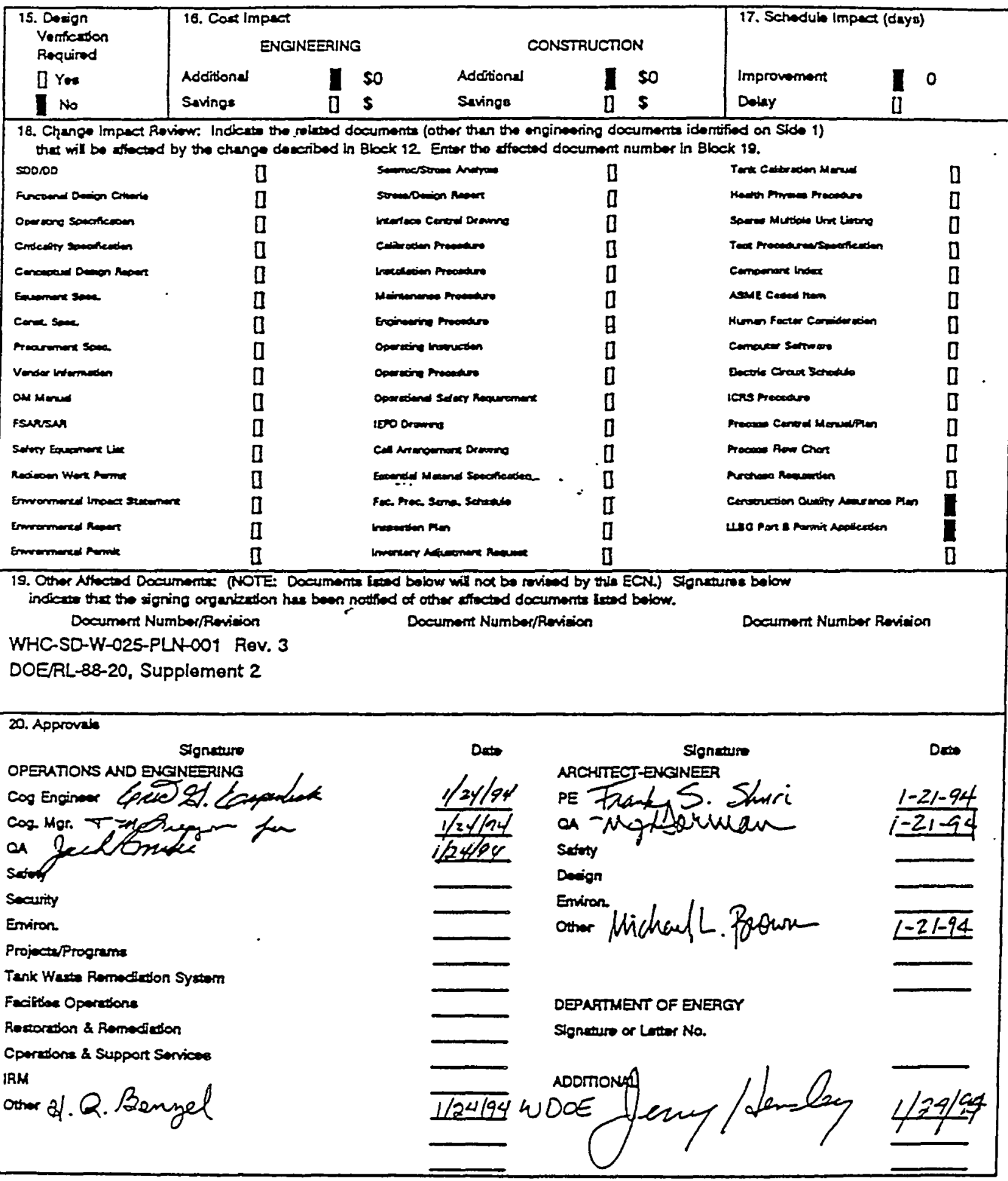


Continuad from Section 12, page 1:

3. Section 02725. Part 2-5: Chenge tite of Parr 2.5 to Coupings and End Caps:. Add paragraph:

c. Provide molded polyetrylane and cape for ends of all clanout ecoes pipes Cups shall provent entry of sail or debria into the chanou pipe and shall be nemoveble. Compoeiton of polyethylene shall be compatible with chanous pipes per

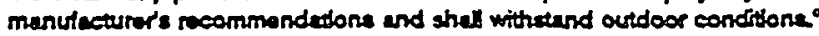

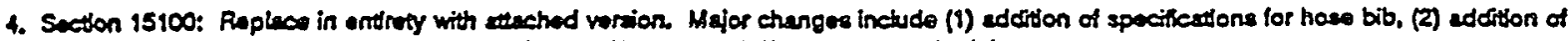

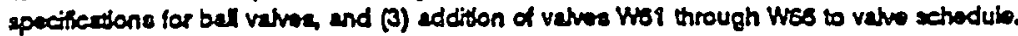

5. Secton 15104, Part 1-1: Roviso paragraph to raed

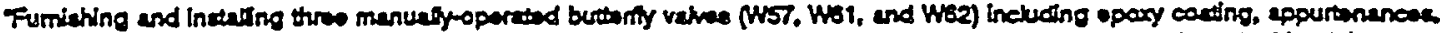

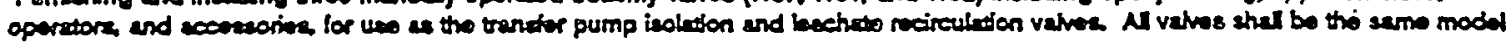
from the seme menufecturer."

8. Secton 15150, Pan 1-1: Revieo paragraph to read

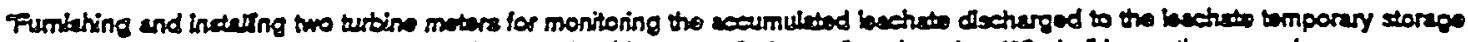

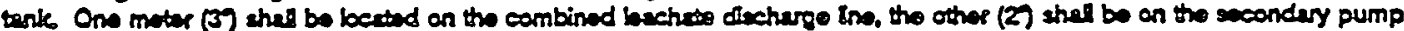

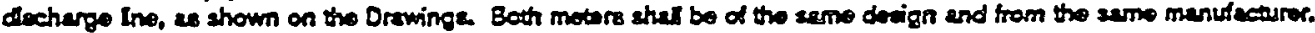

7. Suction 13340, Pat Z Add Pat 2-11:

2-19 LMTT SWTTCA

$$
\text { ... - - - . - }
$$

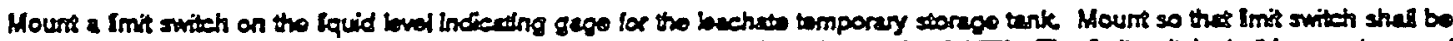

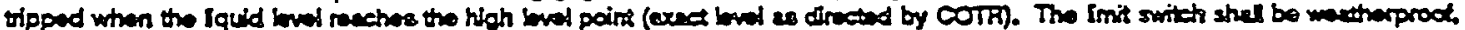

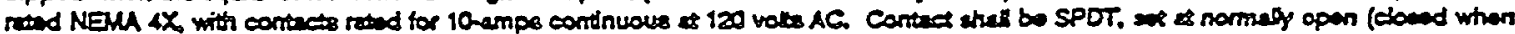

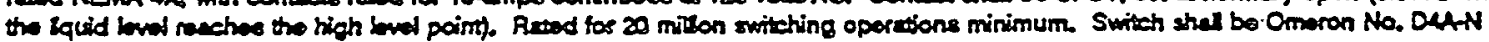
(with side rotary plenger of similes devicos) of approved equel.

Continued from Sedon 136, peos !:

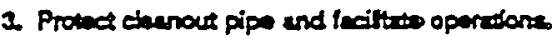

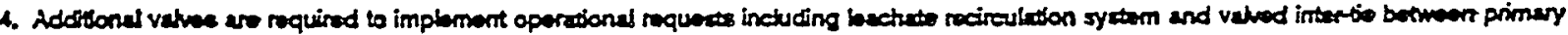
and excondary rumpe

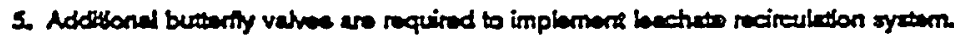

a. Alowe diectherge from anch eump to bo moveured indopendenty.

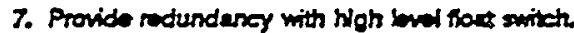


WHC-SD-W025-RPT-001, Rev. 0

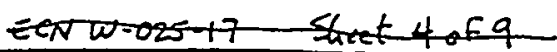

Golder Associates subuect Leachate Collection well Earthloadin

\begin{tabular}{|c|c|c|}
\hline $\begin{array}{l}\text { Joo No. } 933-1360 \\
\text { Ret. } \omega-025\end{array}$ & $\begin{array}{l}\text { Made by Fss } \\
\text { Checked } \\
\text { Reviewed Huffrown }\end{array}$ & $\begin{array}{lc}\text { Oate } & 10-13^{1} \cdot 93 \\
\text { Sheet } 1 & 1\end{array}$ \\
\hline
\end{tabular}

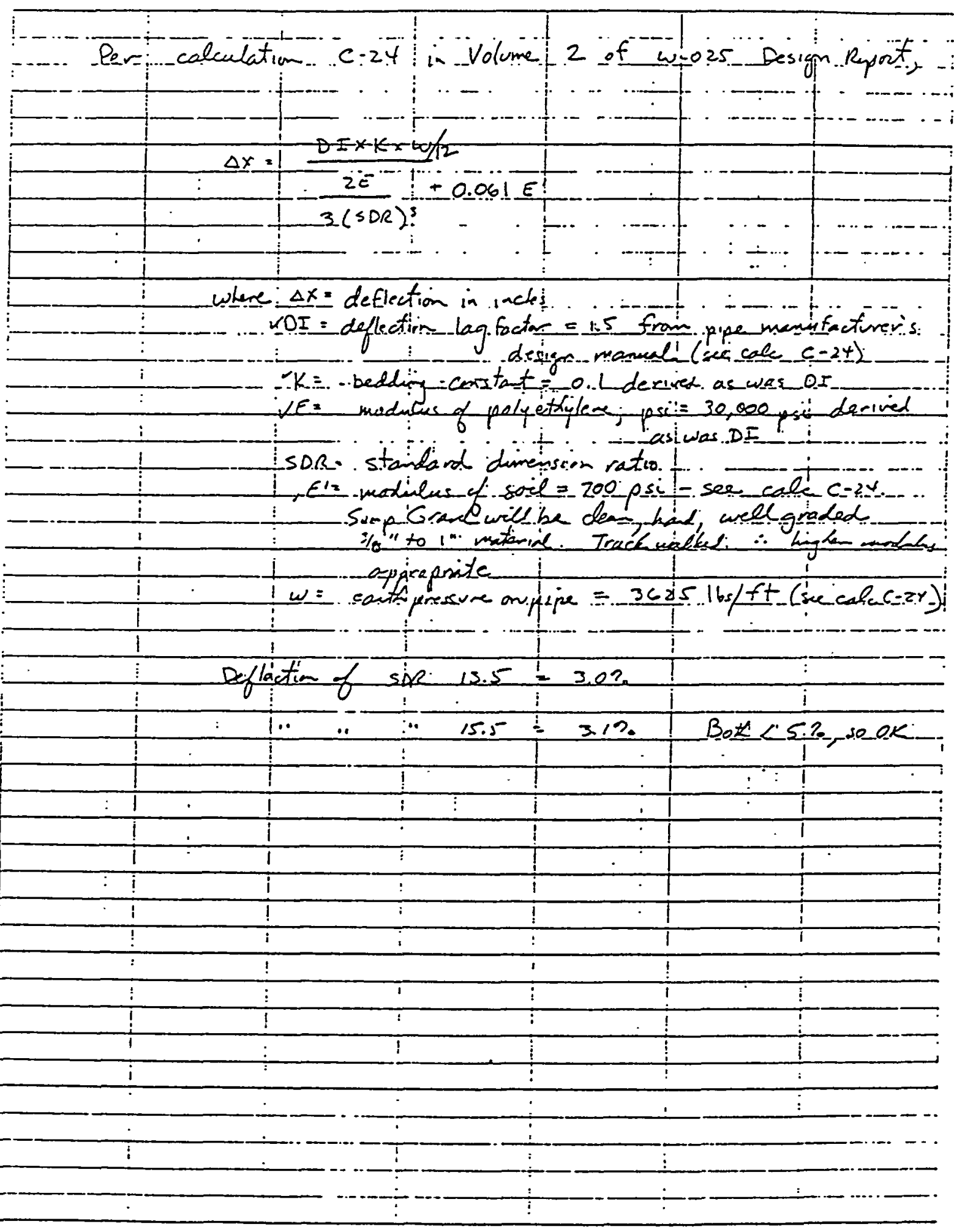


WHC-SD-W025-RPT-001, Rev. $0^{\circ}$ ECH to-035-17 Shest 5af9

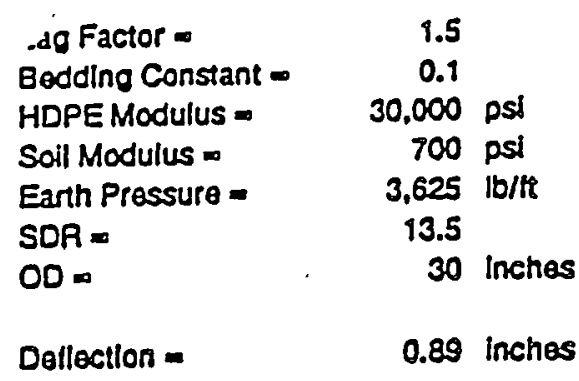

$3.0 \%$ 


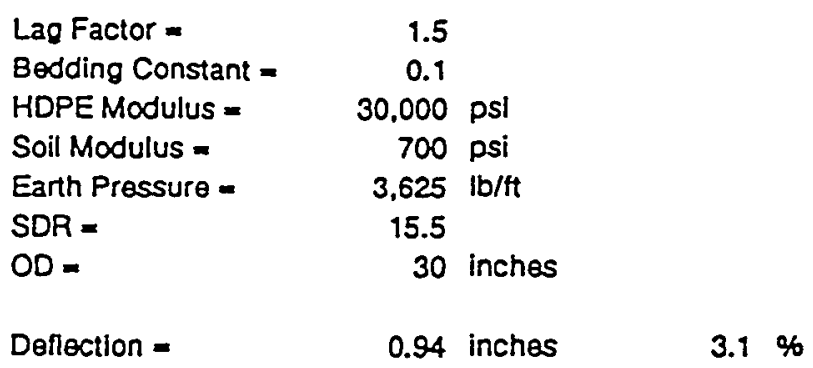


WHC-SD-W025-RPT-001, Rev. 0 Ein woo25-17 Sheet 7of9-

WHC=S-045 Rev.2

SECTION 15100

VALVES

\section{PART 1: GENERAL}

\section{1-1 SECTION INCLUDES}

All valves used for control of water and air, manual, electric, hydraulic, and pneumatic operators; epoxy coating; control units; and appurtenant work. Butterfly valves are covered separately under Section 15104.

\section{1-2 RELATED SECTIONS}

A. Section 1510 - Butterfly Valves

\section{1-3 SUBMITIALS}

A. Submit detailed manufacturer's information on all valves proposed for use.

B. Submit in accordance with Section 01300 .

\section{PART 2: PRODUCTS}

\section{2-1 VACUUM RELEASE VALVES}

Provide vacuum release valves on discharge line of submersible pumps and the transfer pump discharge line at locations shown on the Drawings. Connect to HDPE piping using service saddles. Connect to stainless steel pipe by direct tapping or other method approved by the COTR. Valves shall be constructed of corrosion resistant wetted parts.

\section{2-2 SOLENOID DRAIN VALVE AND PIPING}

Fumish and install an electric solenoid-operated valve for back-draining of leachate to prevent freezing. Enclosure shall be NEMA-4. Wetted parts shall be stainless or thermoplastic Orifice size $3 / 8^{n}$ minimum, $115 \mathrm{VAC}$ coil; use ASCO solenoid valve or approved equal. Tap HDPE pipe using type 101S saddle, available from Industrial Plastics, Inc, Washougal, WA or approved equal. All connecting piping shall be HDPE or type 316 stainless steel; all fittings shall be type 316 stainless steel. Drain piping shall be insulated but not heat traced. 
WHC-SD-W025-RPT-001, Rev. 0 ECN ivi=025-17 Steet 8 of 9

WATC-S=045 ReV.2

\section{2-3 SAMPLE COCKS}

Fumish and install 1/4" NPT sample cocks, fabricated of type 316 stainless steel, on tank discharge piping at the location(s) shown on the Drawings. Sample cocks shall be heat traced.

\section{2-4 HOSE BIB}

Furnish and install hose bib and $0.5^{\mathrm{m}}$ hose connection, fabricated of type 316 stainless steel, on tank discharge piping at the location shown on the drawings. Hose bib shall be heat traced.

\section{2-5 CHECK VALVES}

Furnish and install five check valves, one on each of the leachate sump pump discharge pipes and two on the leachate tank recirculation system, as shown on the Drawings. Wetted parts shall be type 316 stainless steel, rubber, thermoplastic, or other approved corrosion-resistant material.

\section{2-6 DRAIN DOWN VALVES AND PIPING}

Provide stainless steel or thermoplastic spring-actuated valve which will close against pump pressure, and open against gravity pressure allowing the piping to drain back into the sump. Connecting pipe and fittings shall be stainless steel Valve pipe size shall be $1 / 4^{\prime \prime}$ minimum.

\subsection{BALL VALVES}

Furnish and install two ball valves, one $1.5^{n}$ on the primary side slope discharge line (valve W66) and one $3^{\prime \prime}$ on the leachate line to the temporary storage tank (valve W65), as shown on the Drawings. Valves shall be manually operated. Wetted parts shall be type 316 stainless steel or other approved corrosion resistant material. Vaives shall be similar in quality to Watts Regulator Industrial Products Division series S-8XXX, Velan EE-1000, or approved equal.

\section{2-8 VALVE SCHEDULE}

Valve No.

W51

W52

W53

W54

W55

\section{Description}

$11 / 2$ " primary check valve $11 / 2^{\prime \prime}$ secondary check valve $3^{\prime \prime}$ primary check valve solenoid drain down value spring release, drain down valve, $1 /{ }^{\prime \prime}$ min. 
W56

W57

W58

W59

W60

W61

W62

W63

W64

W65

W66 spring release, drain down valve, $1 /{ }^{\prime \prime}$ min.

Transfer pump isolation valve, $4^{\text {" }}$ manually operated BF valve, lever actuator (see Section 15104)

Vacuum release valve, primary leachate discharge line

Vacuum release valve, secondary leachate discharge line

Vacuum release valve, transfer pump discharge line Leachate tank recirculation system, $4^{\prime \prime}$ manually operated BF valve, lever actuator (see section 15104)

Leachate tank recirculation system, $4^{\prime \prime}$ manually operated BF valve, lever actuator (see section 15104)

$4^{\text {n }}$ check valve for leachate tank recirculation system

$4^{n}$ check valve for leachate tank recirculation system

Leachate piping valved-intertie, $3^{n}$ manually operated ball valve

Leachate piping valved-intertie, $1.5^{n}$ manually operated ball valve

\section{2-9 VALVE MARKING}

Valves shall have stainless steel (SS) indented marker plates marked with the valve number, Panduit Corporation, Catalog No. MMP350-C or approved equal, held in place with SS ties, Panduit Corporation, Catalog No. MLT4S-CP or approved equal. Characters shall be $3 / 16$ " in height minimum, indented with indenter marker press, Panduit Corporation, Catalog No. IMP187, or approved equal.

\section{PART 3: EXECUTION}

\section{3-1 GENERAL}

All valves shall be installed in accordance with AWWA Standards and manufacturer's recommendations.

\section{3-2 SOLENOID DRAIN VALVE AND PIPING}

Install solenoid drain valve and piping around $3^{n}$ diameter check valve such that low spots are minimized. Locate solenoid and piping taps at an elevation about $1 / 2^{\prime \prime}$ above invert of $3^{n}$ piping.

$$
\text { - END. }
$$




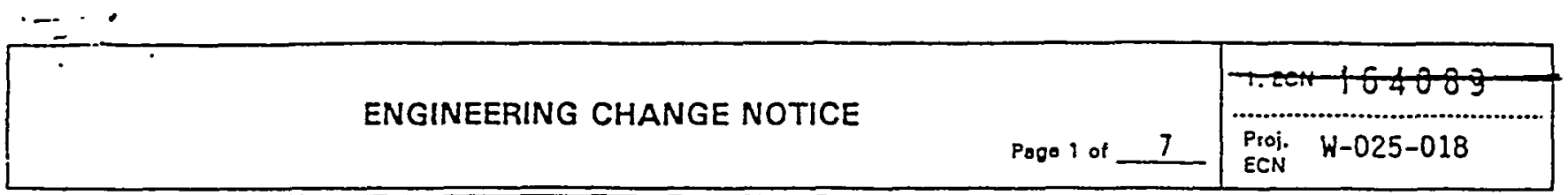

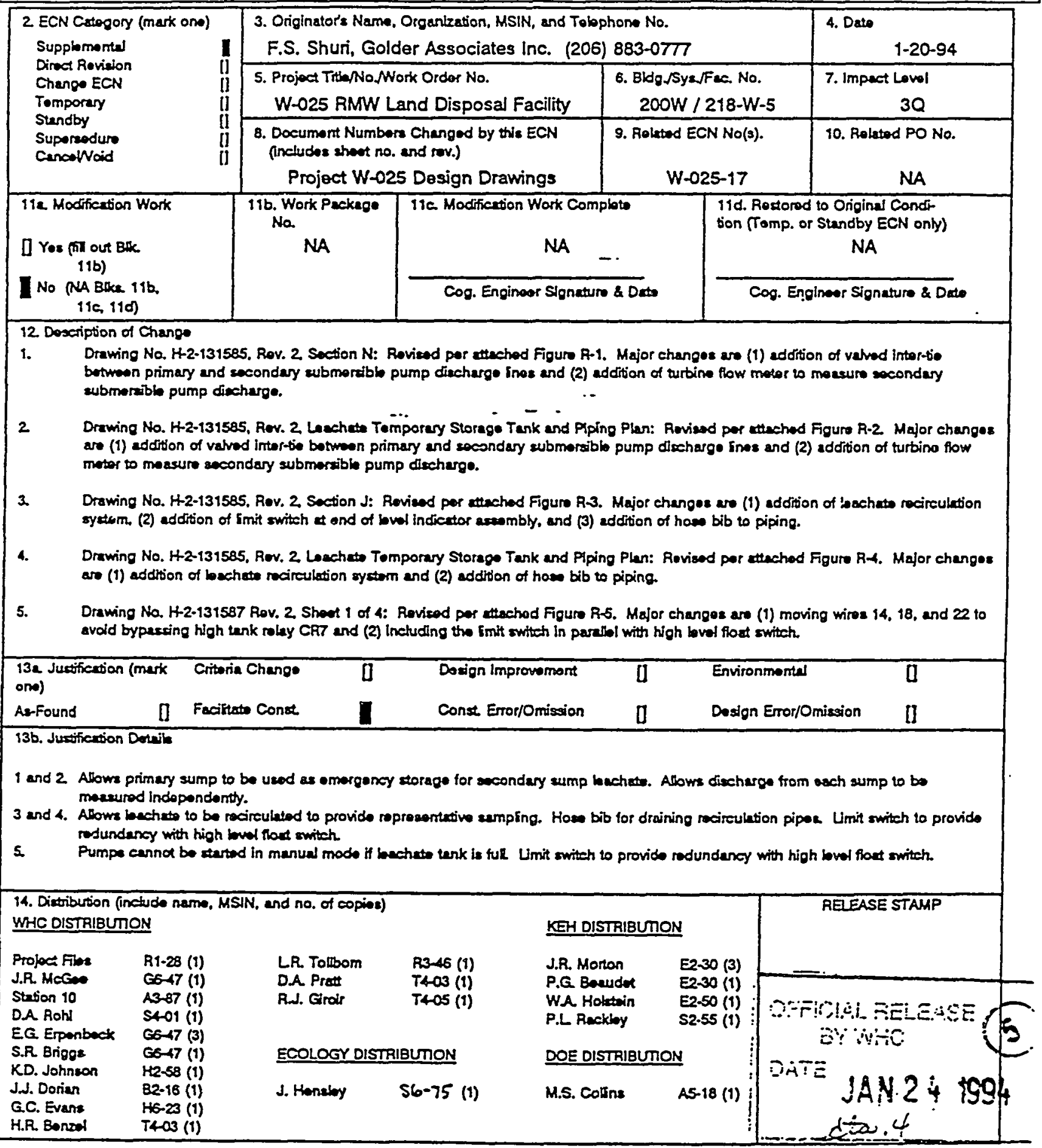

A-7900-013-2 (06/92) GEFO95 
WHC-SD-W025-RPT-001, Rev. 0

\begin{tabular}{|l|l|l|}
\hline$\cdot \cdot$ ENGINEERING CHANGE NOTICE & Pago 2 of 7 & $\begin{array}{c}\text { 1. ECN (uso no. trom pg. 1) } \\
\text { W-025-018 }\end{array}$ \\
\hline
\end{tabular}

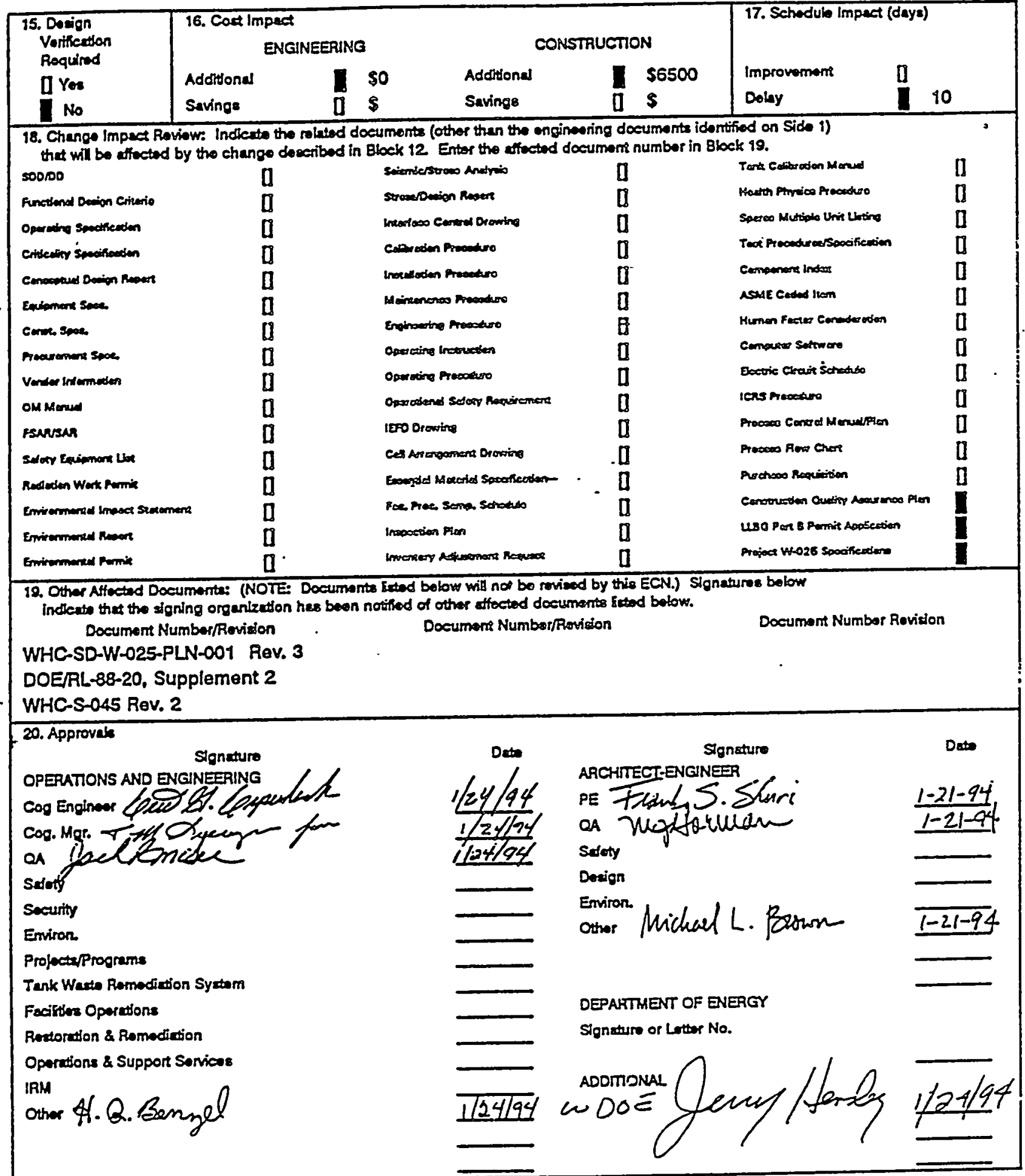


WHC-SD-W025-RPT-001, Rev. 0

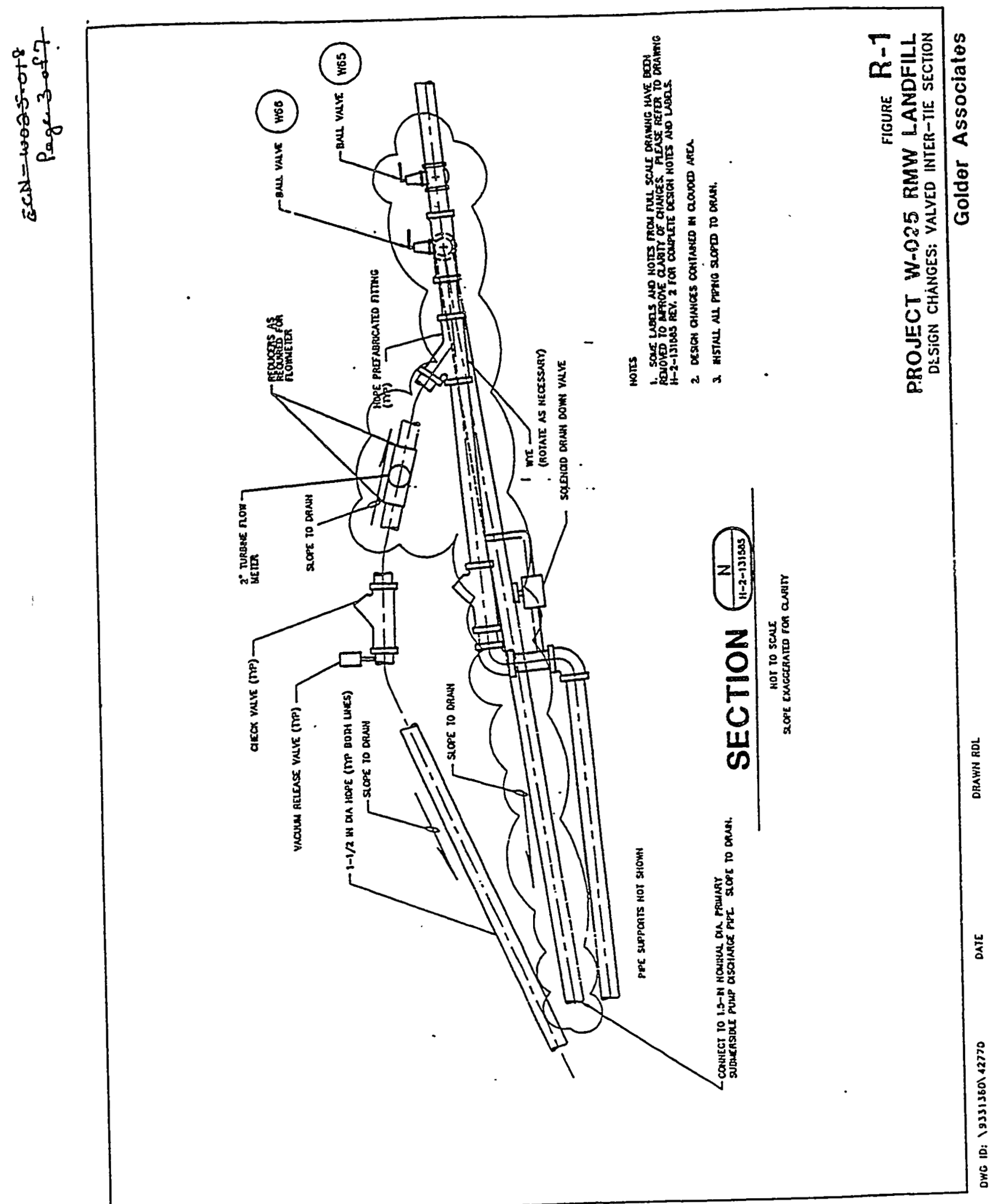


WHC-SD-W025-RPT-001, Rev. 0
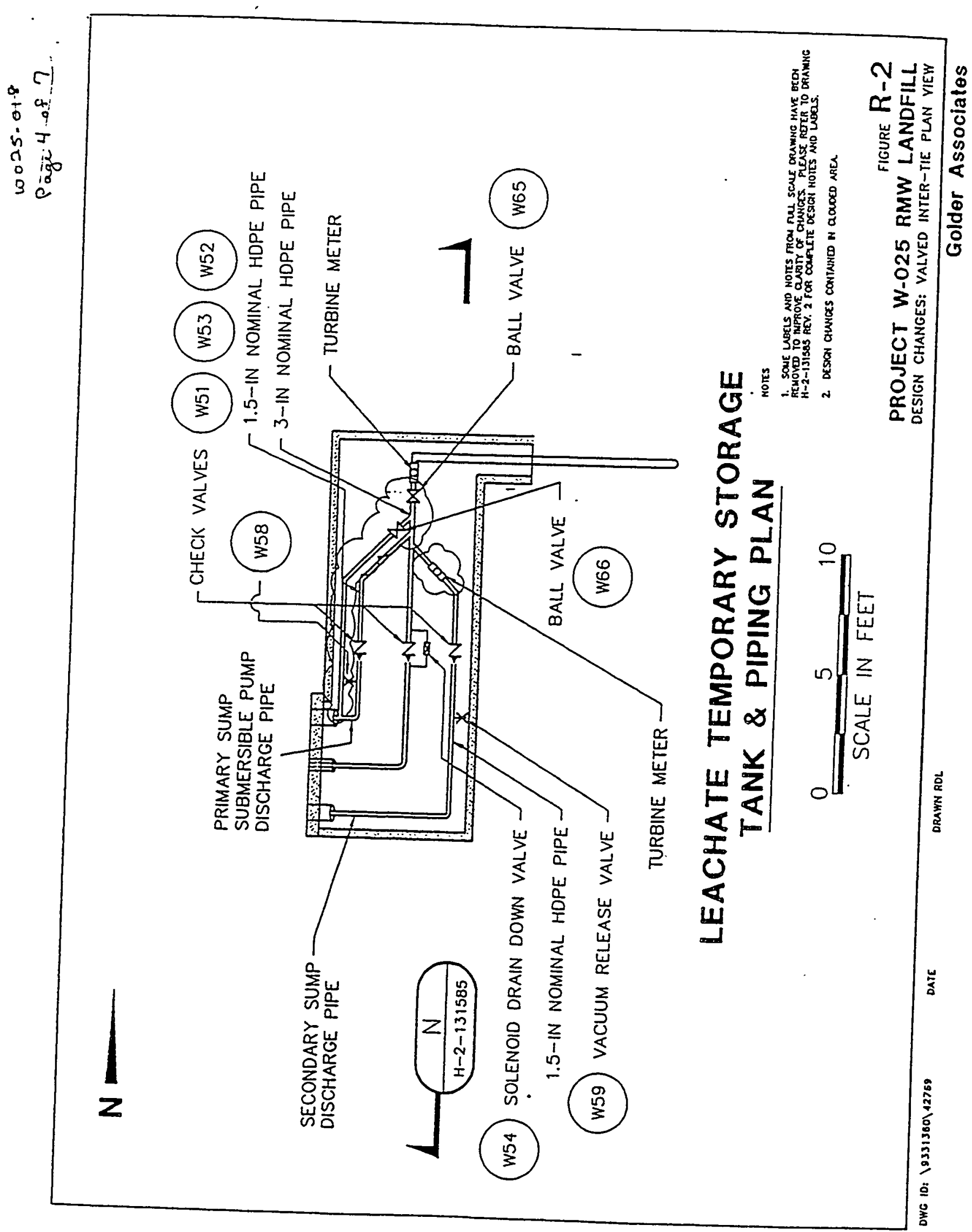
WHC-SD-W025-RPT-001, Rev. 0

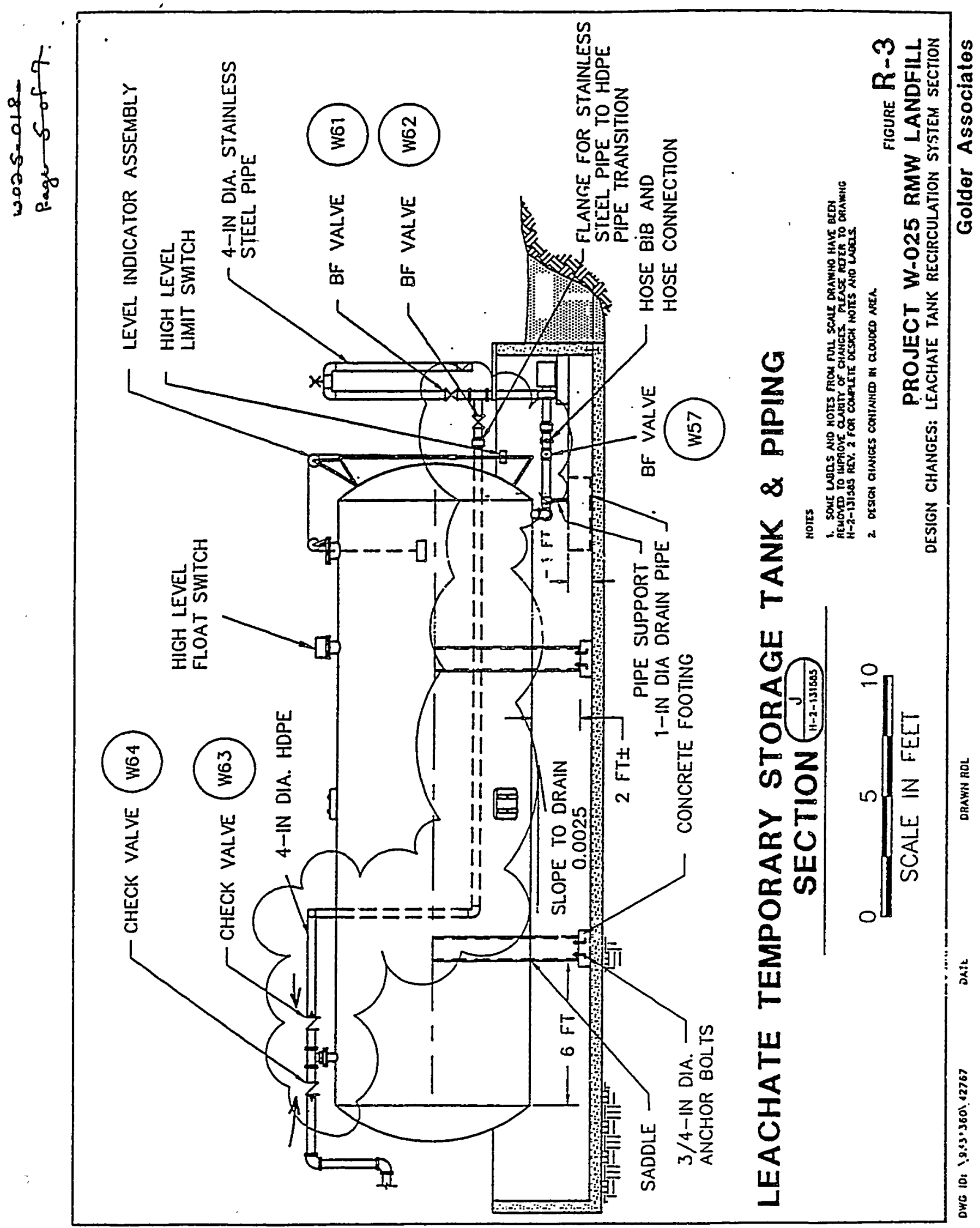


WHC-SD-W025-RPT-001, Rev. 0

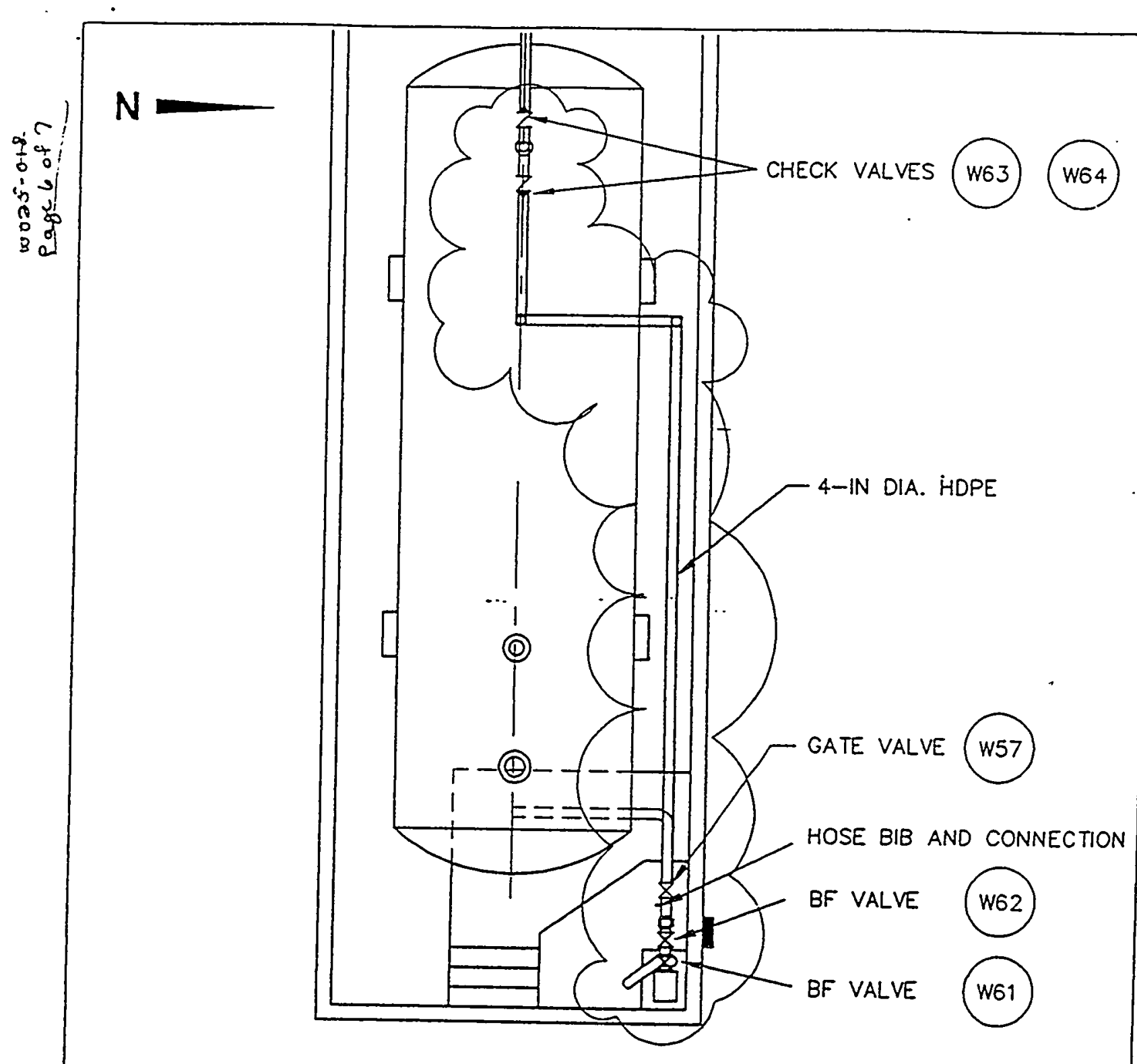

\section{LEACHATE TEMPORARY STORAGE TANK \& PIPING PLAN}
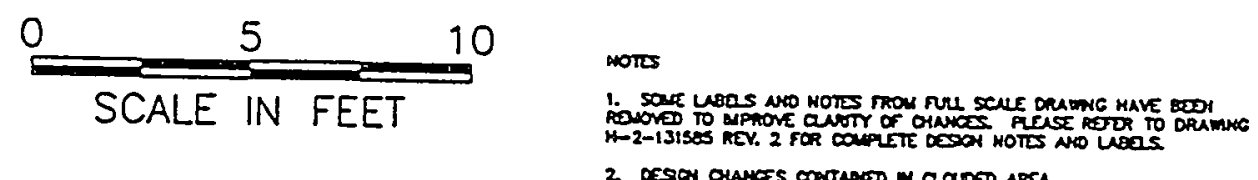

FIGURE $R-4$

PROJECT W-025 RMW LANDFILL DESIGN CHANGES: LEACHATE TANK RECIRCULATION SYSTEM PLAN VIEW 
WHC-SD-W025-RPT-001, Rev. 0

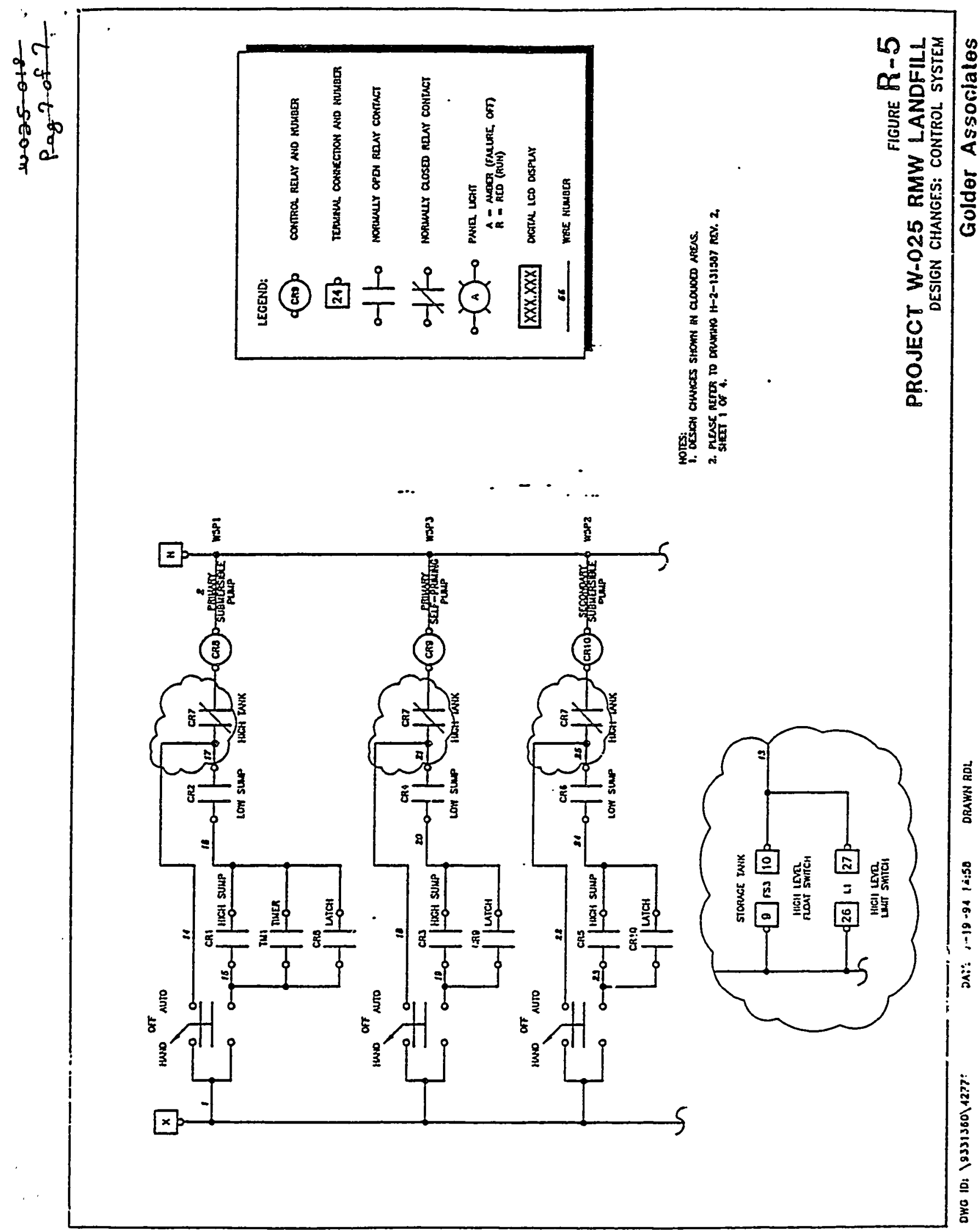


WHC-SD-W025-RPT-001, Rev. 0

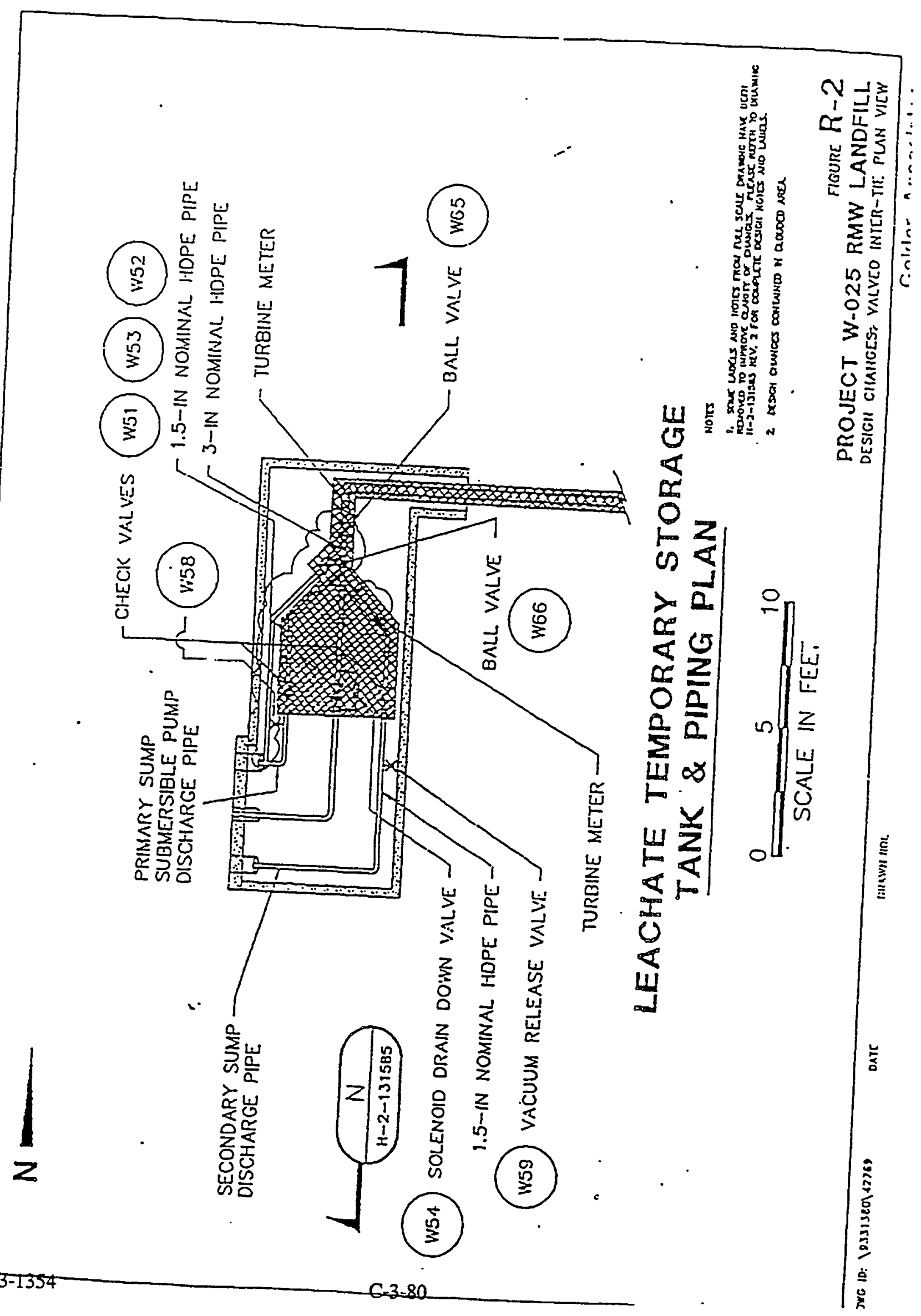


WHC-SD-W025-RPT-001, Rev่. 0

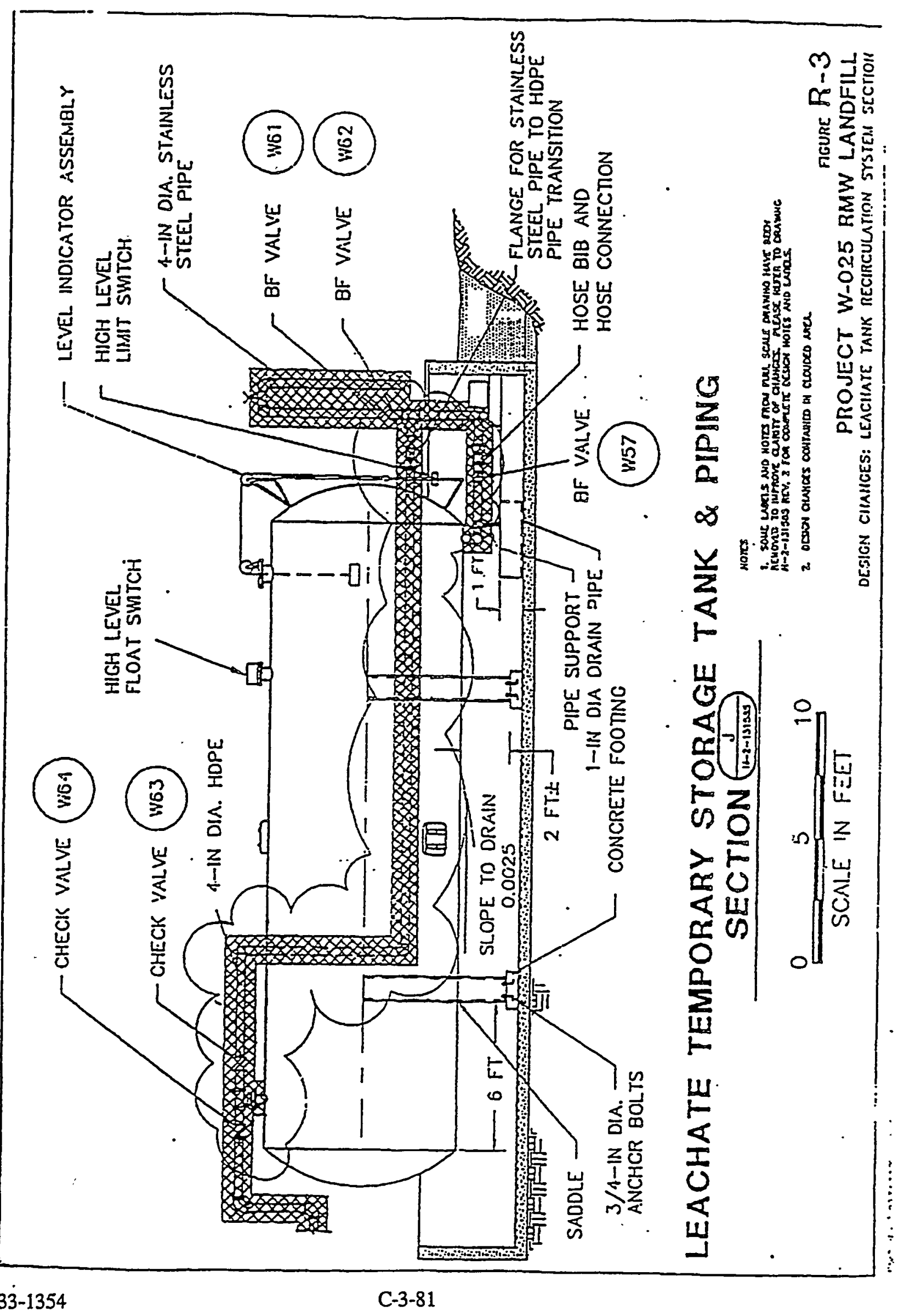




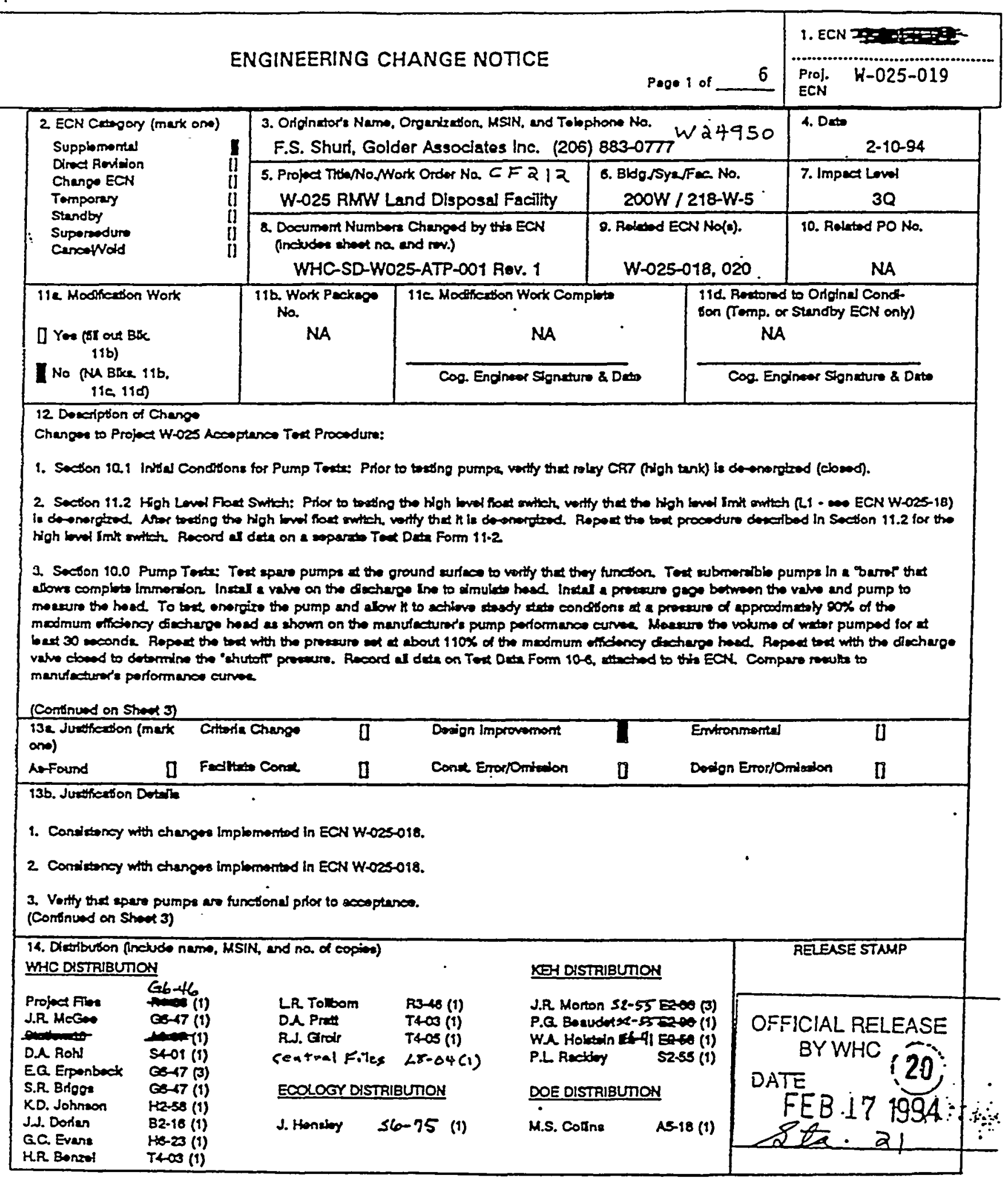

A-7900013-2 (108/82) GEFOS 


\begin{tabular}{|c|c|c|}
\hline ENGINEERING CHANGE NOTICE & 1. ECN (uno no. from po. 1) \\
W-025-019
\end{tabular}

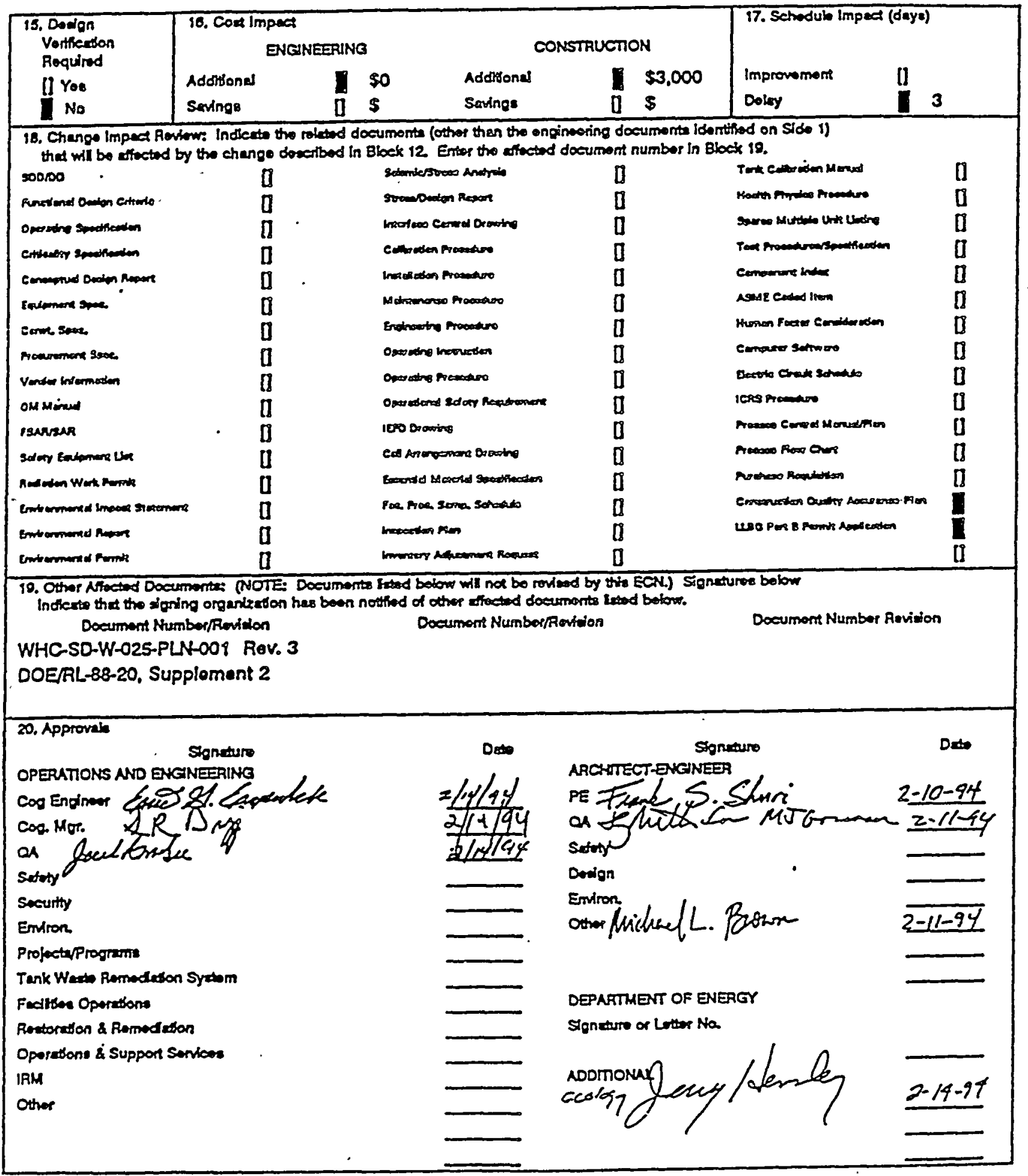


WHC-SD-W025-RPT-001, Rev. 0

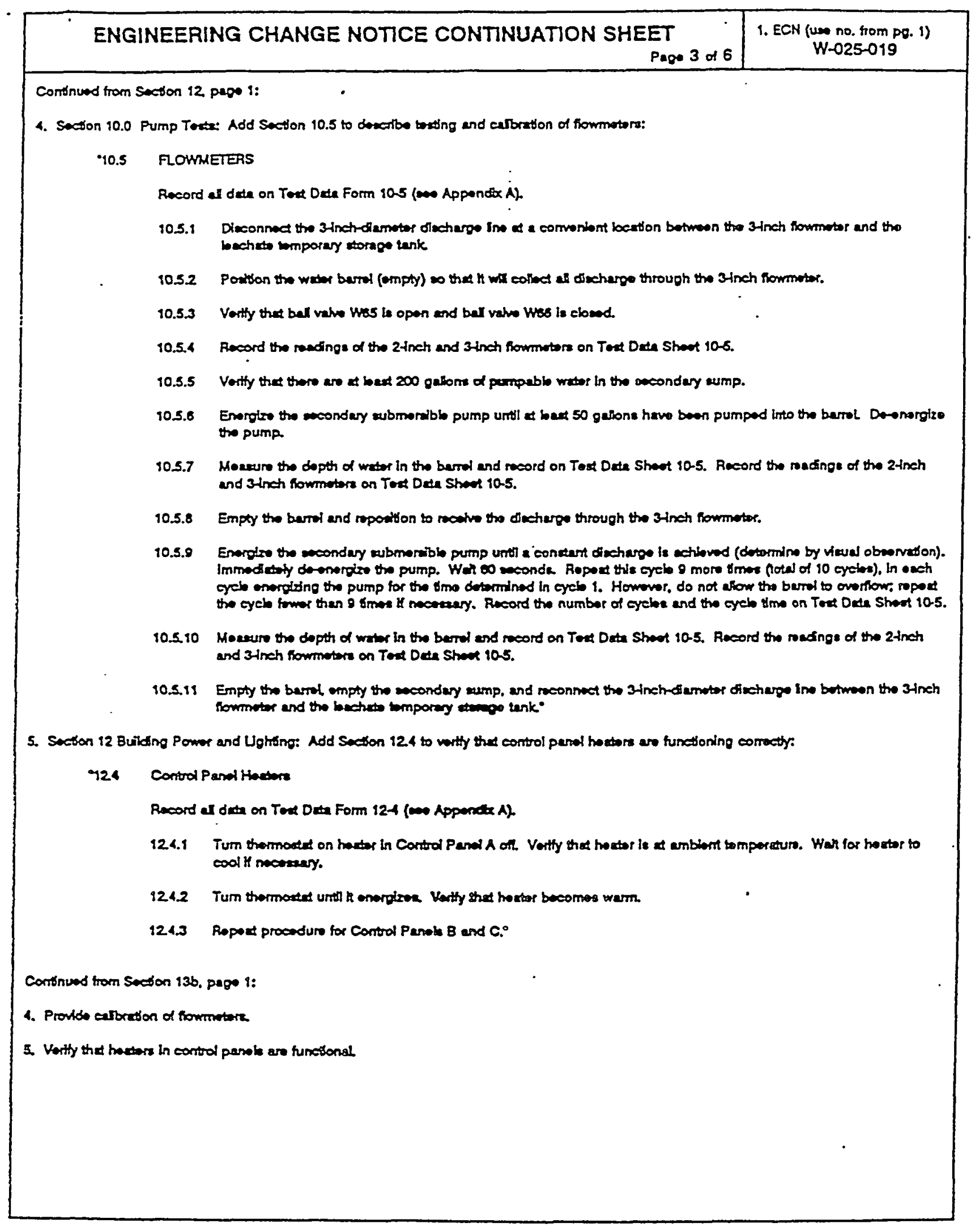




\section{IEST DATA FORM 10-5 \\ $P_{3}$. yto \\ PUMP TESTS - FLOWMETER CALIBRATION}

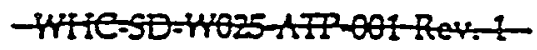

b-025-0T9

Barrel Diameter (units)

\begin{tabular}{|c|c|c|c|c|c|}
\hline Step No. & Activity & Verify & Reading & Date & By \\
\hline 10.5 .1 & Disconnect discharge line & & NA & & \\
\hline 10.5.2. & Position water barrel & & NA & & \\
\hline \multirow[t]{2}{*}{10.53} & Ball valve W65 open & & NA & & \\
\hline & Ball valve W66 closed & & NA & & \\
\hline \multirow[t]{2}{*}{10.5 .4} & 2-inch flowmeter reading & NA & & & \\
\hline & 3-inch flowmeter reading & NA & & & \\
\hline 10.55 & 200 gallons in secondary sump & & NA & & \\
\hline 10.5 .6 & Pump 50 gallons into barrel & & NA & & \\
\hline \multirow[t]{3}{*}{10.5 .7} & Depth of water in barrel & NA & & & \\
\hline & 2-inch flowmeter reading & NA & & & \\
\hline & 3-inch flowmeter reading & NA & & & \\
\hline 10.5 .8 & Empty barrel and reposition & & NA & & \\
\hline \multirow[t]{2}{*}{10.5 .9} & First cycle time & NA & & & \\
\hline & Total number of cycles (including ist) & NA & & & \\
\hline \multirow[t]{3}{*}{10.5 .10} & Depth of water in barrel & NA & & & \\
\hline & 2-irch flowmeter reading & NA & & & \\
\hline & 3-inch flowmeter reading & NA & & & \\
\hline \multirow[t]{3}{*}{10.5 .11} & Empty barrel & & NA & & \\
\hline & Empty secondary sump & & NA & & \\
\hline & Reconnect discharge line & & NA & & \\
\hline
\end{tabular}


WHC-SD-W025-RPT-001, Rev. 0

IEST DATA FORM $10-6$

PUMP TESTS - SPARE PUMPS

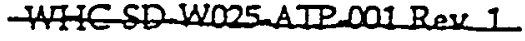
$-25=019$

$\mapsto, 5 / 6$

Pressure Gage Model and SN

\begin{tabular}{|c|c|c|c|c|c|}
\hline Pump Type and Serial No. & $\begin{array}{l}\text { Volume Pumped } \\
\text { (units) }\end{array}$ & Time & $\begin{array}{l}\text { Pressure Head } \\
\text { (units) }\end{array}$ & Date & By \\
\hline & & & & & \\
\hline & & & & & \\
\hline & & & & & \\
\hline & & & & & \\
\hline & & & & & \\
\hline & & & & & \\
\hline & & & & & \\
\hline & & & & & \\
\hline & & & & & \\
\hline & & & & & \\
\hline . & & & & & \\
\hline
\end{tabular}


WHC-SD-W025-RPT-001, Rev. 0

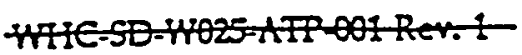

TEST DATA FORM $12-4$

$$
\begin{array}{r}
\text { Noz5-019 } \\
f_{5}-616
\end{array}
$$

BUILDING POWER AND LIGHIING - CONIROL PANEL HEATERS

\begin{tabular}{|l|l|c|c|c|c|}
\hline Step No. & \multicolumn{1}{|c|}{ Activity } & Verify & Reading & Date & By \\
\hline 12.4 .1 & Heater at ambient - Control Panel A & & NA & & \\
\hline 124.2 & Heater becomes warm - Control Panel A & & NA & & \\
\hline 124.3 & Heater at ambient - Control Panel B & & NA & & \\
\cline { 2 - 6 } & Heater becomes warm - Control Panel B & & NA & & \\
\cline { 2 - 6 } & Heater at ambient - Control Panel C & & NA & & \\
\cline { 2 - 6 } & Heater becomes wam - Control Panel C & & NA & & \\
\hline
\end{tabular}




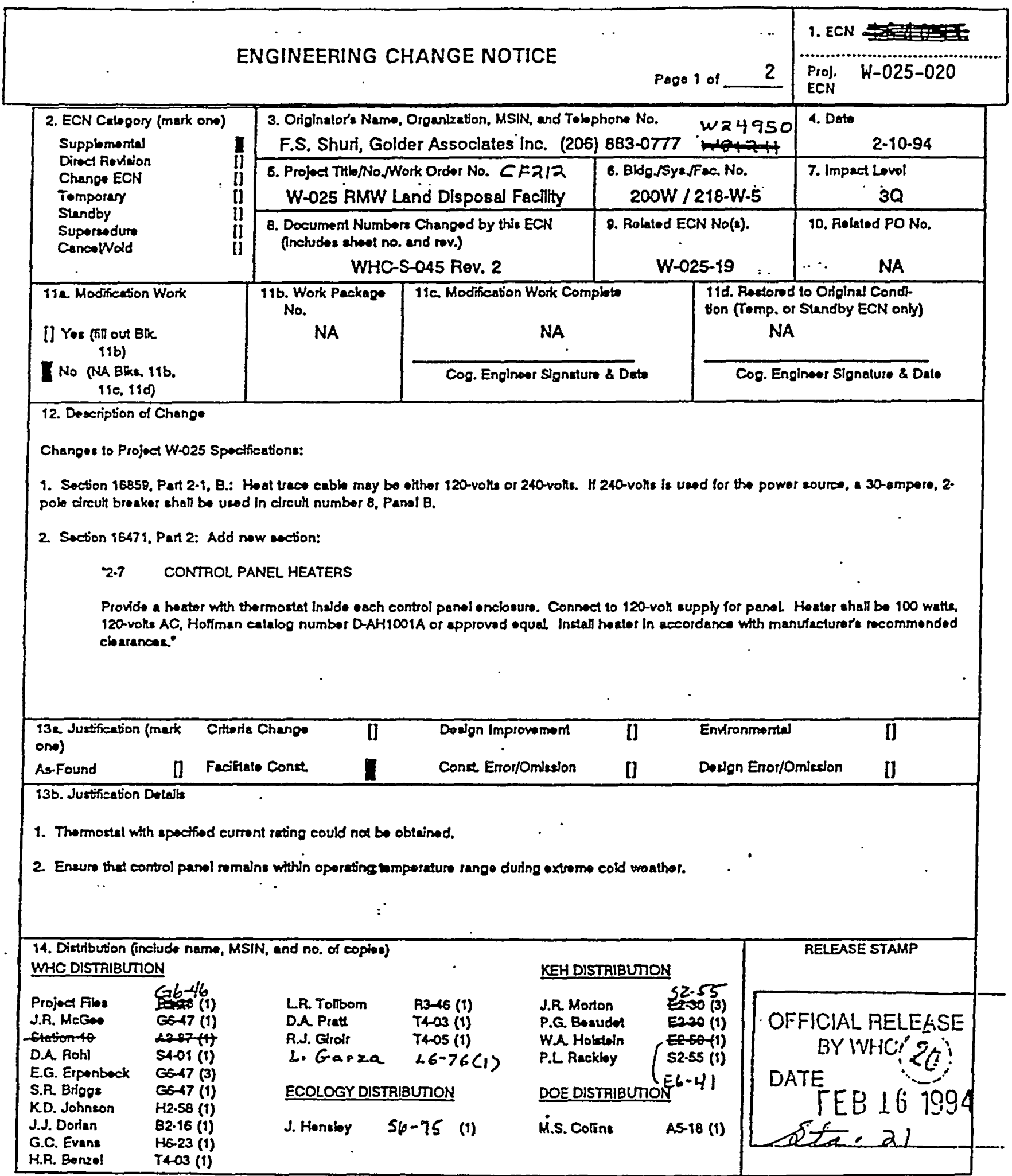

A-7000-013-2 (06/82) GEF085 
WHC-SD-W025-RPT-001,Rev。 0

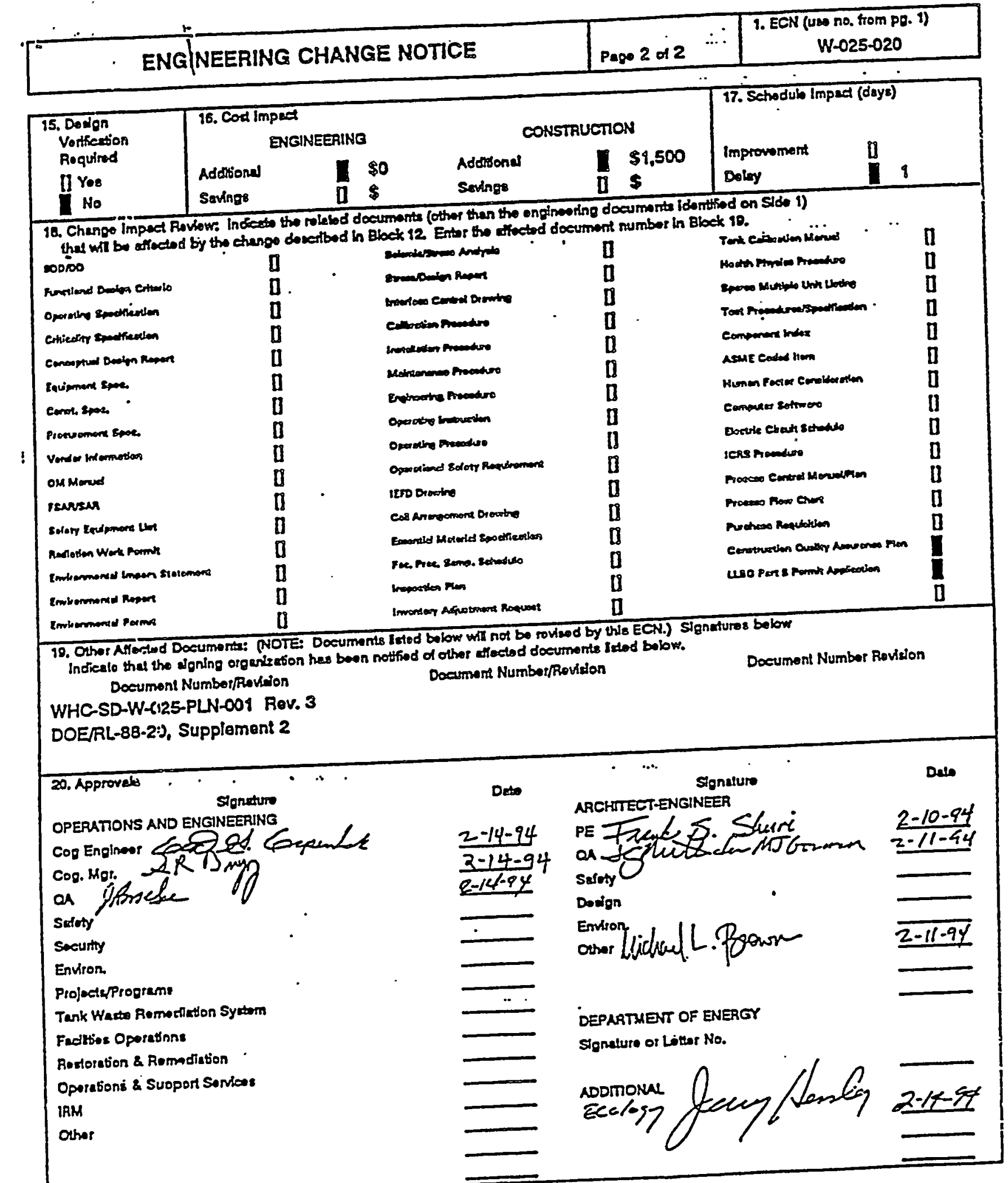


WHC-SD-W025-RPT-001, Rev. 0

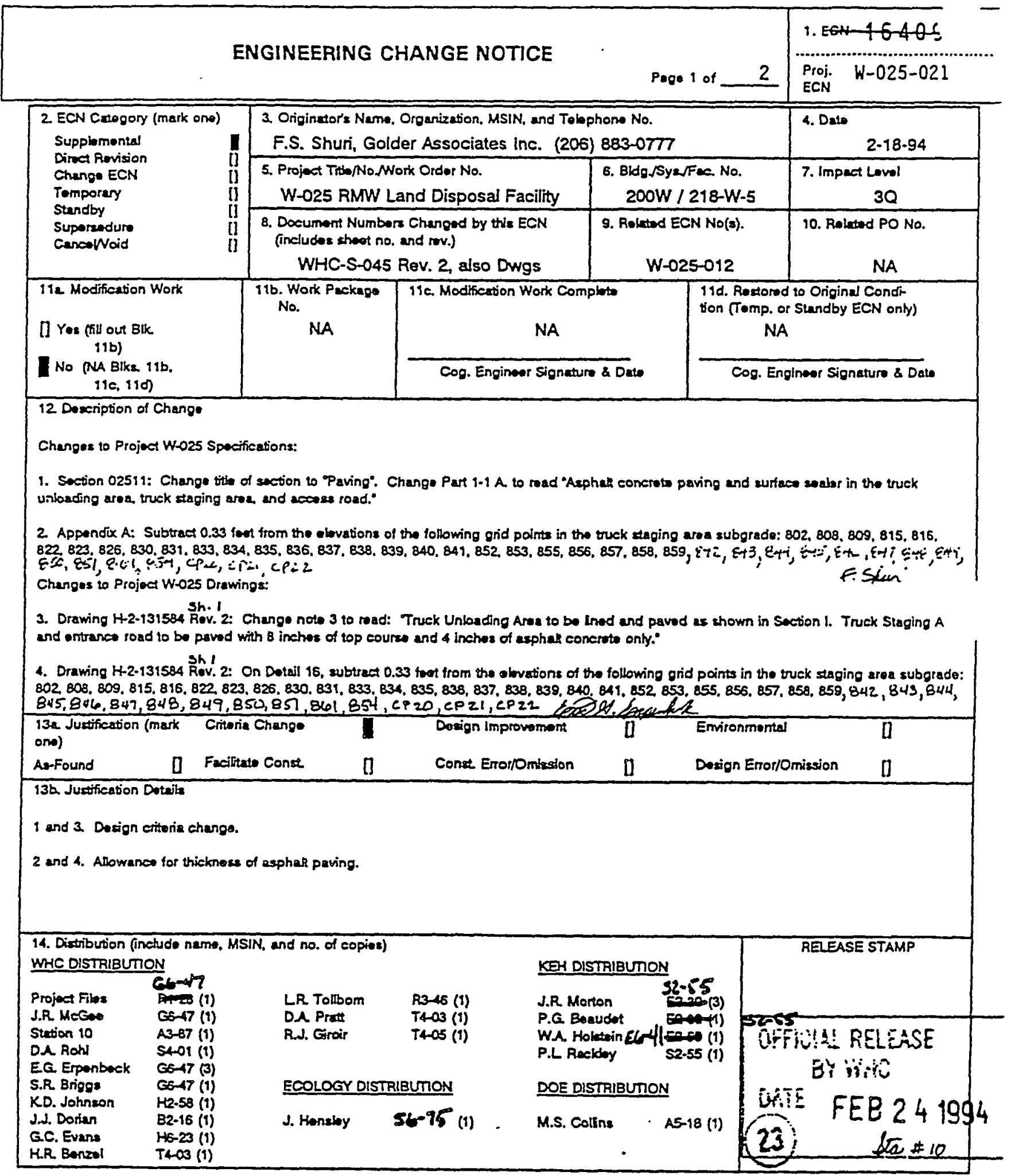

A-7900013-2 (06/82) GEF095 
WHC-SD-W025-RPT-001, Rev. 0

\begin{tabular}{|l|l|l|}
\hline ENGINEERING CHANGE NOTICE & $\begin{array}{c}\text { 1. ECN (uso no. from po. 1) } \\
\text { W-025-021 }\end{array}$ \\
\hline
\end{tabular}

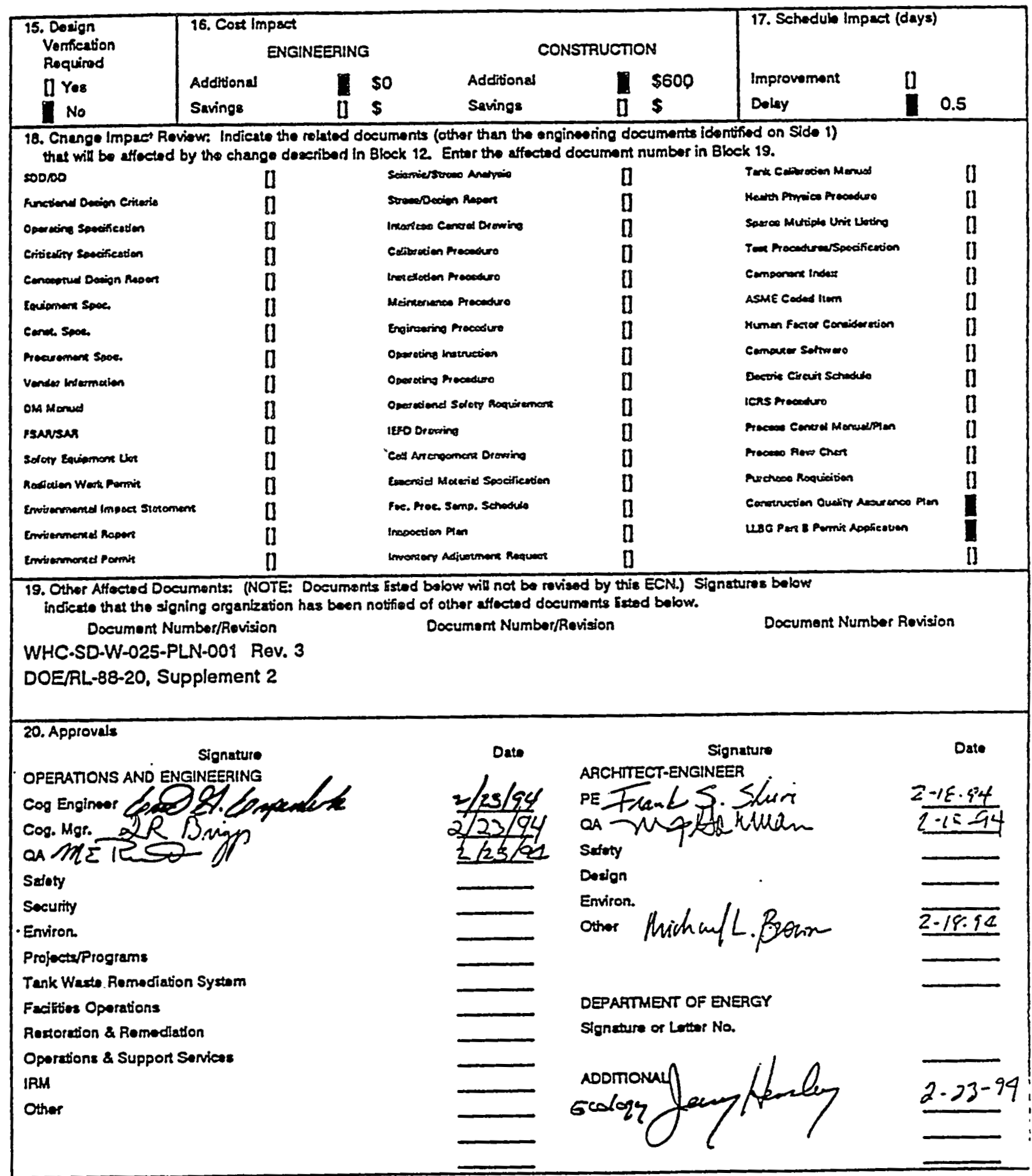


WHC-SD-W025-RPT-001, Rev. 0

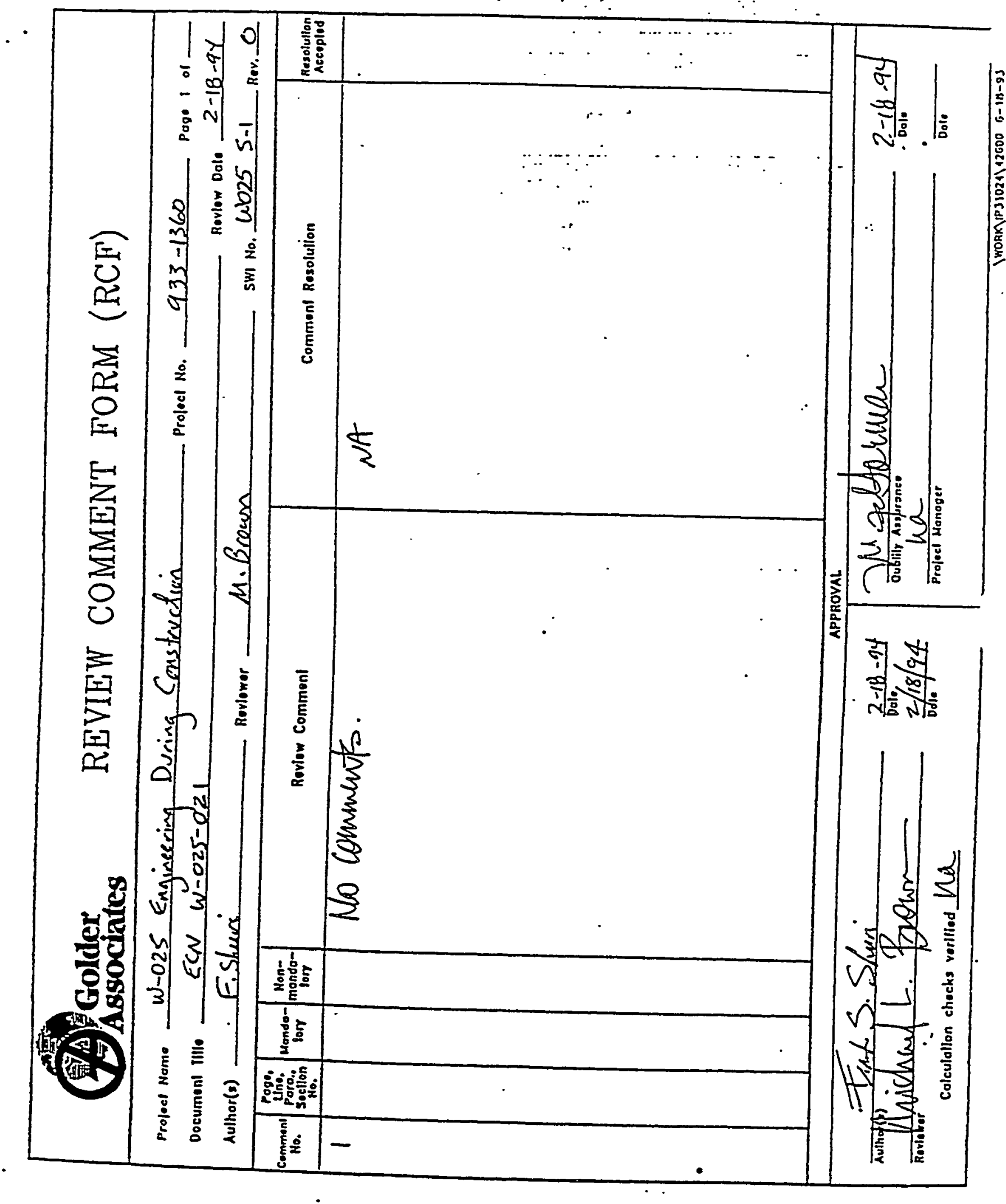


WHC-SD-W025-RPT-001, Rev. 0

\begin{tabular}{|c|c|c|c|c|c|}
\hline \multicolumn{5}{|c|}{ ENGINEERING CHANGE NOTICE } & $\begin{array}{l}\text { Prop. } \quad H-025-022 \\
\text { ECN }\end{array}$ \\
\hline 2. ECN Calogory (mark one) & \multicolumn{4}{|c|}{ 3. Odginatora Name, Organizention, MSIN, and Tolinghomo No. } & 4. Daie \\
\hline \multirow{3}{*}{$\begin{array}{l}\text { Supplomortal } \\
\text { Dioce Rovition } \\
\text { Chenge ECN } \\
\text { Tomporary } \\
\text { Stendby } \\
\text { Supureodure. } \\
\text { CencolWold }\end{array}$} & \multicolumn{4}{|c|}{ F.S. Shuri, Golder Assoclates Inc. (206) 883-0777 } & $4-20-94$ \\
\hline & \multicolumn{2}{|c|}{$\begin{array}{l}\text { 5. Project TibaNo.Work Ordor No. CF } 2 B 4 \\
\text { W-025 RMW Land Disposal Facility }\end{array}$} & \multicolumn{2}{|c|}{$\begin{array}{l}\text { 6. Bldo Syw Fac Na. } \\
200 W / 218-W-5\end{array}$} & 7. Impeat Lovel \\
\hline & \multicolumn{2}{|c|}{$\begin{array}{l}\text { 8. Documert Numbers Changed by the ECN } \\
\text { (includes them no. and rov.) } \\
\text { WHC-S-045 Rev. 2, Dosign Drawings }\end{array}$} & \multicolumn{2}{|c|}{ 9. Roleted ECN No(s). } & $\begin{array}{l}\text { 10. Ralated PO No. } \\
\text { NA }\end{array}$ \\
\hline $\begin{array}{l}\text { Ife Modificution Woik } \\
\text { II Yos (at out Ble } \\
\text { 11b) } \\
\text { No Bu Bke, isb, } \\
\text { 11c, 11d) }\end{array}$ & $\begin{array}{l}\text { 116. Work Peckege } \\
\text { No. } \\
\text { NA }\end{array}$ & \multicolumn{2}{|c|}{$\begin{array}{l}\text { I1a. Modricution Work Complate } \\
\text { NA } \\
\text { Cog. Englineor SIgneture \& Date }\end{array}$} & \multicolumn{2}{|c|}{$\begin{array}{l}\text { 11d. Rectored to Original Condt } \\
\text { Son (Tomp. or Standby ECN onlyl } \\
\text { NA }\end{array}$} \\
\hline
\end{tabular}

12. Desectpton of Change

Changes to Project W-ozs Spacitications:

१. Section 11210, Perr 1-1: Add now pasegraph:

-E The colk-priming conttlugal pump and waydy vecuum uead to romovo lquld from the encondery contalnment basin undor the bectiete tomporary toregs lenk.

2. Section 11210, Part 2: Add now Part:

26

UQUID REMOVAL PUMPS

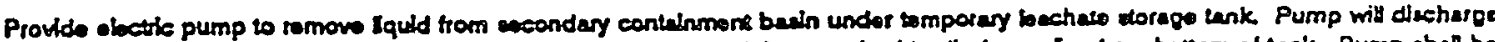

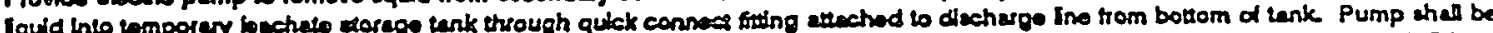

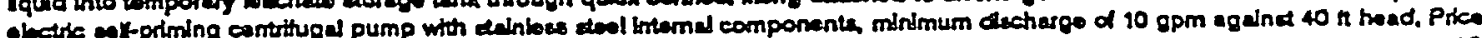

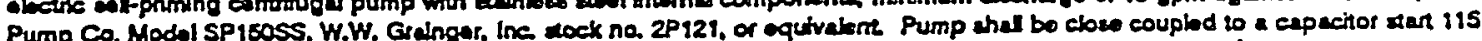

Vox eloctric motor:

(Continued on Shoet 3 )

\begin{tabular}{|c|c|c|c|c|c|c|}
\hline $\begin{array}{l}\text { 13e, Justifection (merk } \\
\text { (n)) }\end{array}$ & Criteria Chenges & T & Deslan Improwemert & D & Emitonmental & 【l \\
\hline AnFound & Fuditute Const & n & Const Error/Omialon & ! & Dedign Errot/Omiselon & !] \\
\hline
\end{tabular}

13b. Juatification Dolelis

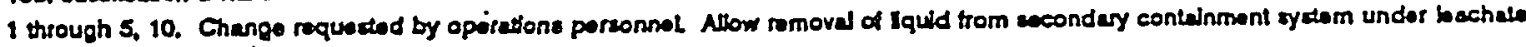
tomporary atogo tank

(Continued on Showt 3)

14. Didributon (inctude name, MSIN, and no. of copies) WHC DISTRIBUTION

\begin{tabular}{|c|c|}
\hline 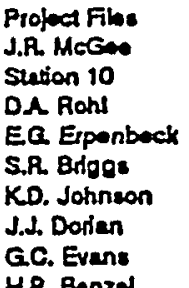 & 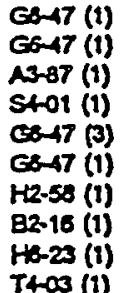 \\
\hline
\end{tabular}

HR. Banzed THOS (i)

$\begin{array}{ll}\text { LR. Tomom } & \text { R3-46 (1) } \\ \text { DA. Pret } & \text { T403 (1) } \\ \text { PuJ. Gidel } & \text { T40S (1) }\end{array}$

ECOLOGY DISTAIBUTON

J. Henciby

S\&75 (1)

\section{KEH DISTRIBUTON}

j.R. Morton $\$ 2.55$ (3)

P.G. Bacudat $\$ 2.55(1)$

WA Holdaln ES-11 (1)

P.L Anelyby 52.55 (1)

DOE OISTRIBUTION

M.S. Calns

15-10 (1)
RELEASE STAMP

OFFICIAI_RELEASE

BYWHIC:20)

DATE

-

APR 27.1994

A.7500013-2 (0692) GEF095 
WHC-SD-W025-RPT-001, Rev. 0

\begin{tabular}{|c|c|c|}
\hline ENGINEERING CHANGE NOTICE & Page 2 a 5 & $\begin{array}{c}\text { 1. ECN (uso no. fom pg. 1) } \\
\text { W-025-022 }\end{array}$ \\
\hline
\end{tabular}

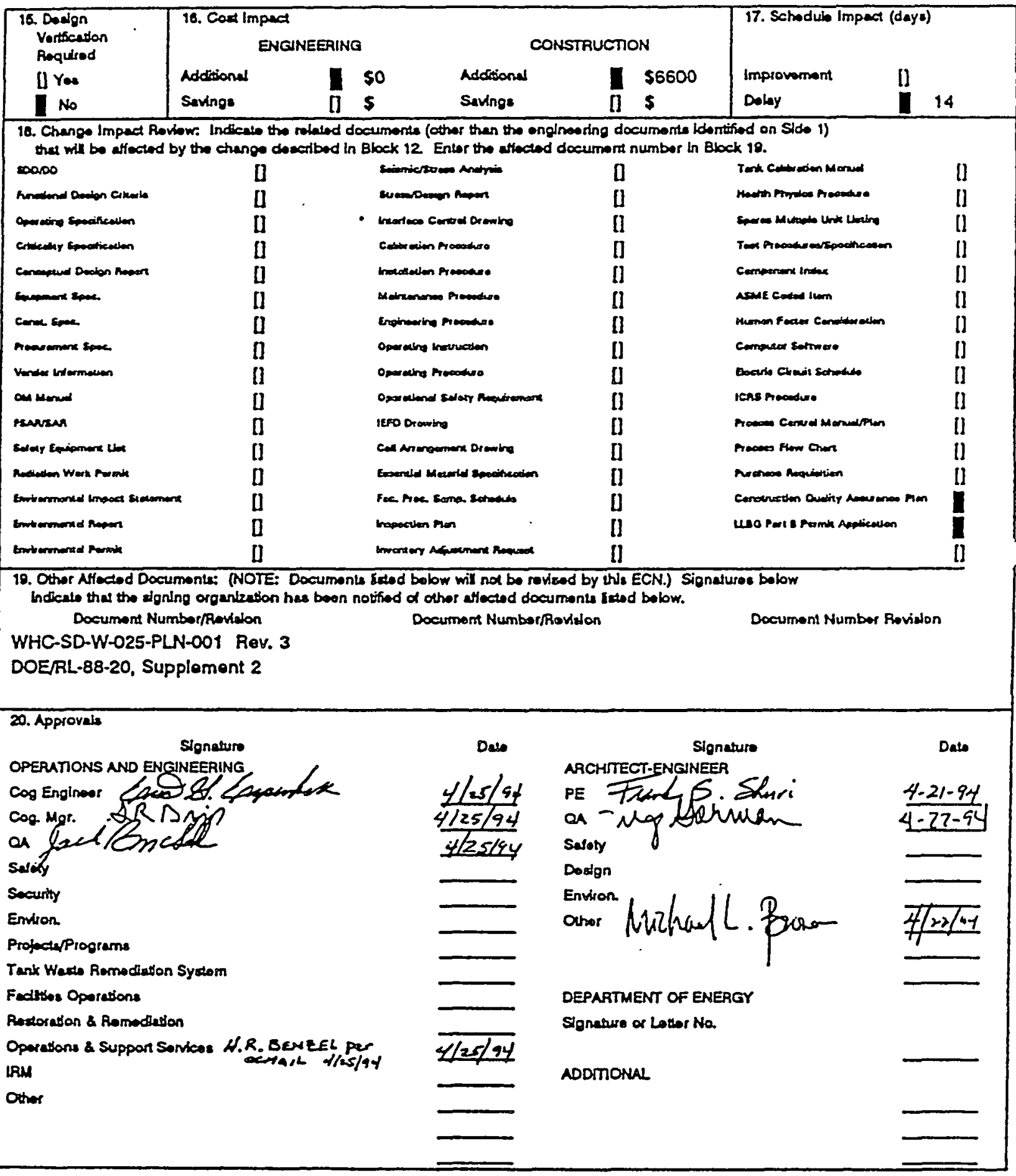


ENGINEERING CHANGE NOTICE CONTINUATION SHEET Pege 3 \& 5

Continued trom Section 12 page 1:

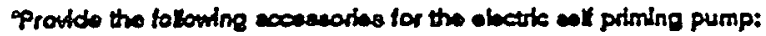

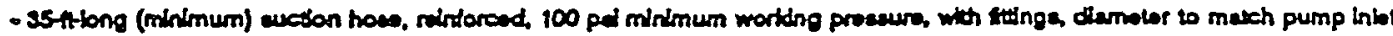
port heavy duty, nubber or equivelant

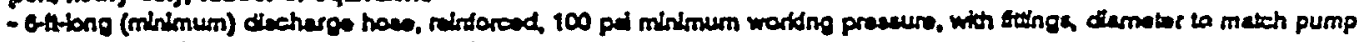
decherce port heavy dury, nubber or equiviant

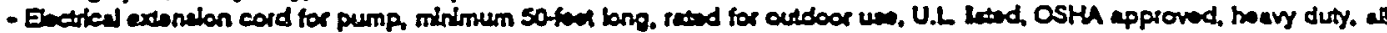

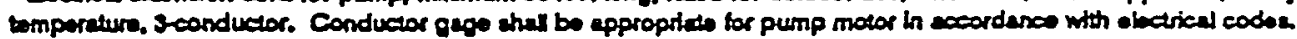

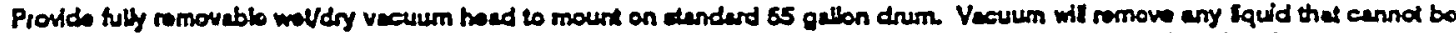

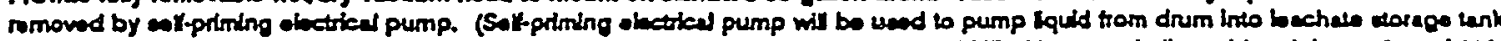

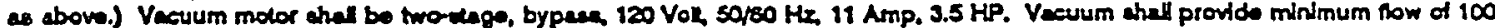

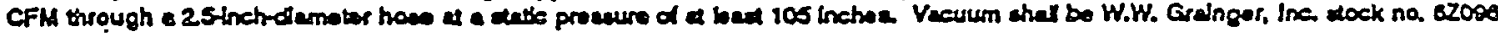
or equiviters

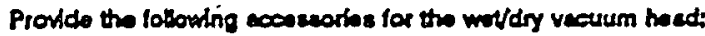

- 55 gellon drum cocted on lneldo to resitt orgente lquide.

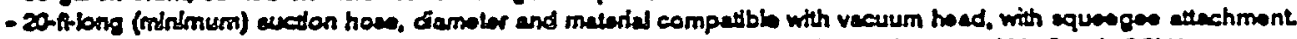

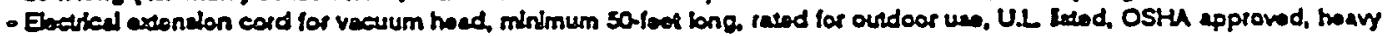

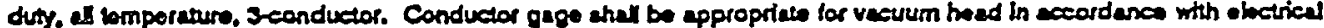
codes:

3. Secton 15100: Add now paregraph:

\section{2-10 QUROK CONNECTS}

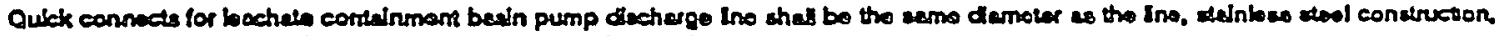

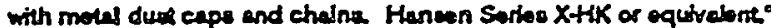

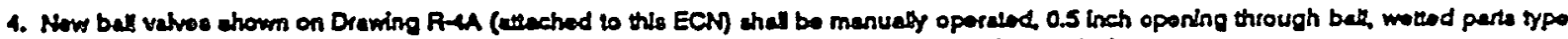

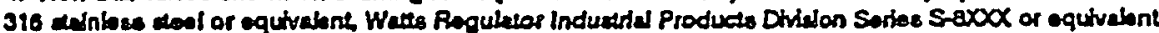

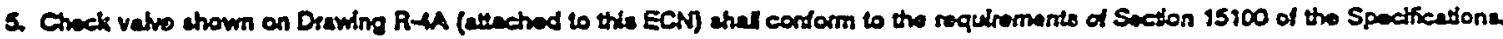

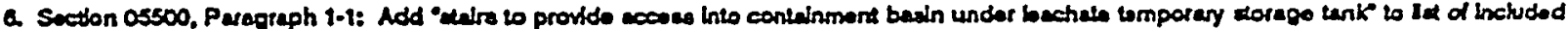
theme

7. Section 05500, Pan 2: Add now paragraph:

$$
\begin{aligned}
& \text { 2.5 STEE PIPE } \\
& \text { Sted plpo thal corform to ASTM AS3." }
\end{aligned}
$$

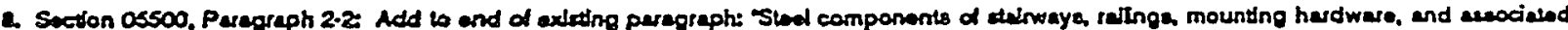
conctures ahill be gaverized in ecordanco wh ASTM A123."

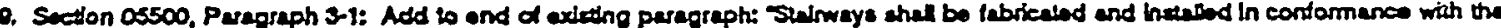

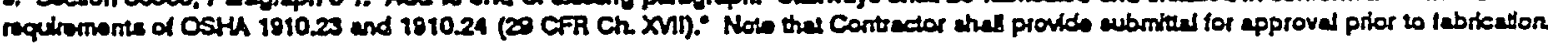

Cranges to Project Woes Doralgn Drendinga:

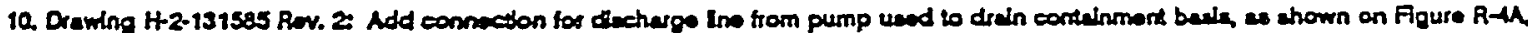
anched to this ECN.

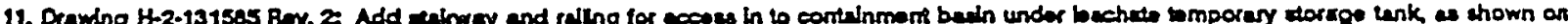

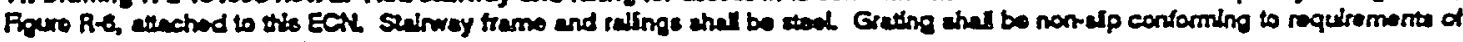
Spectications and this ECNe

Continued from Suction 13b, pape 1:

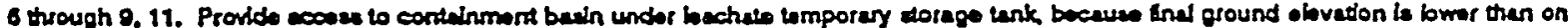
ortinal topography unod for dodgn. 


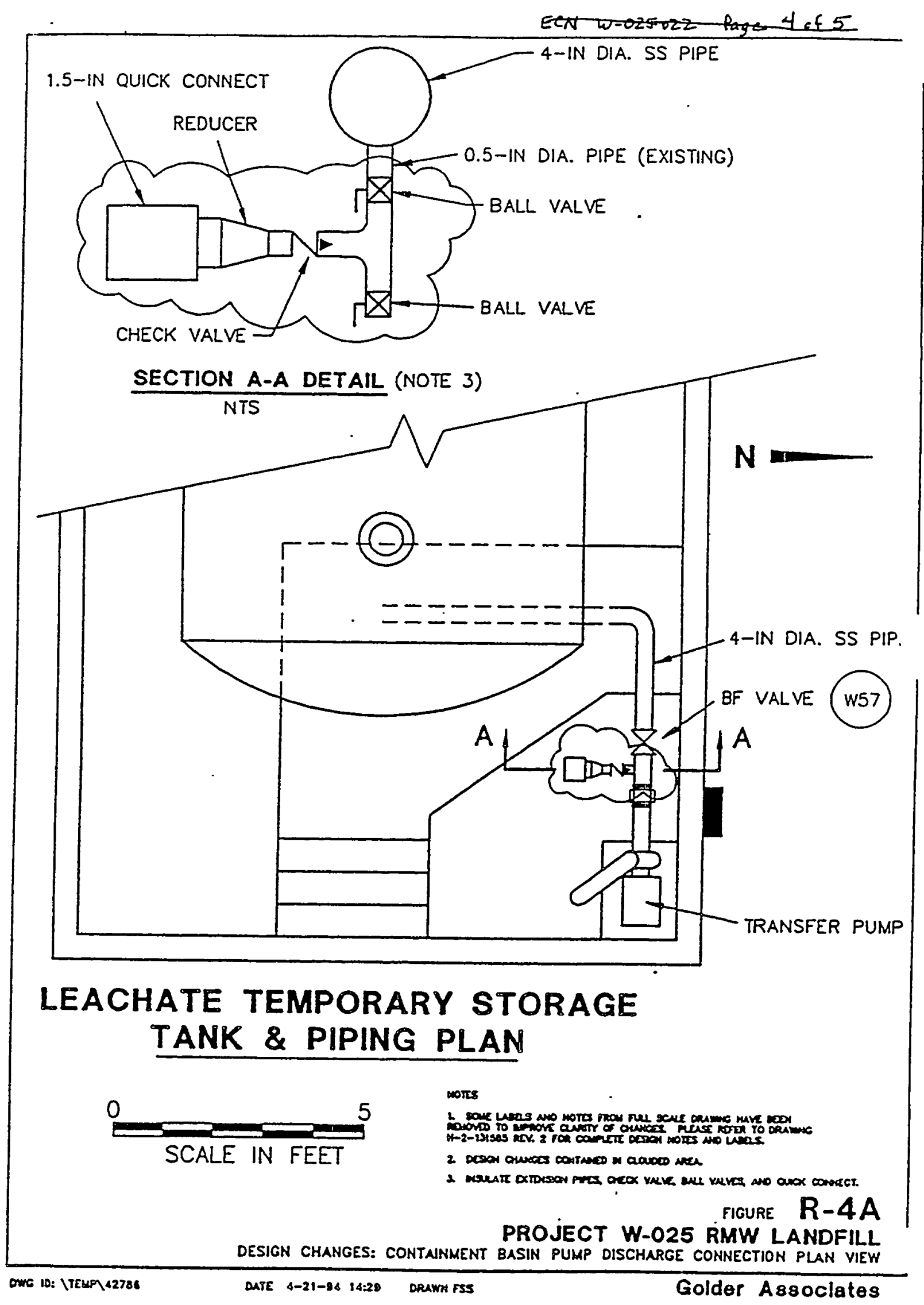


WHC-SD-W025-RPT-001, Rev. 0

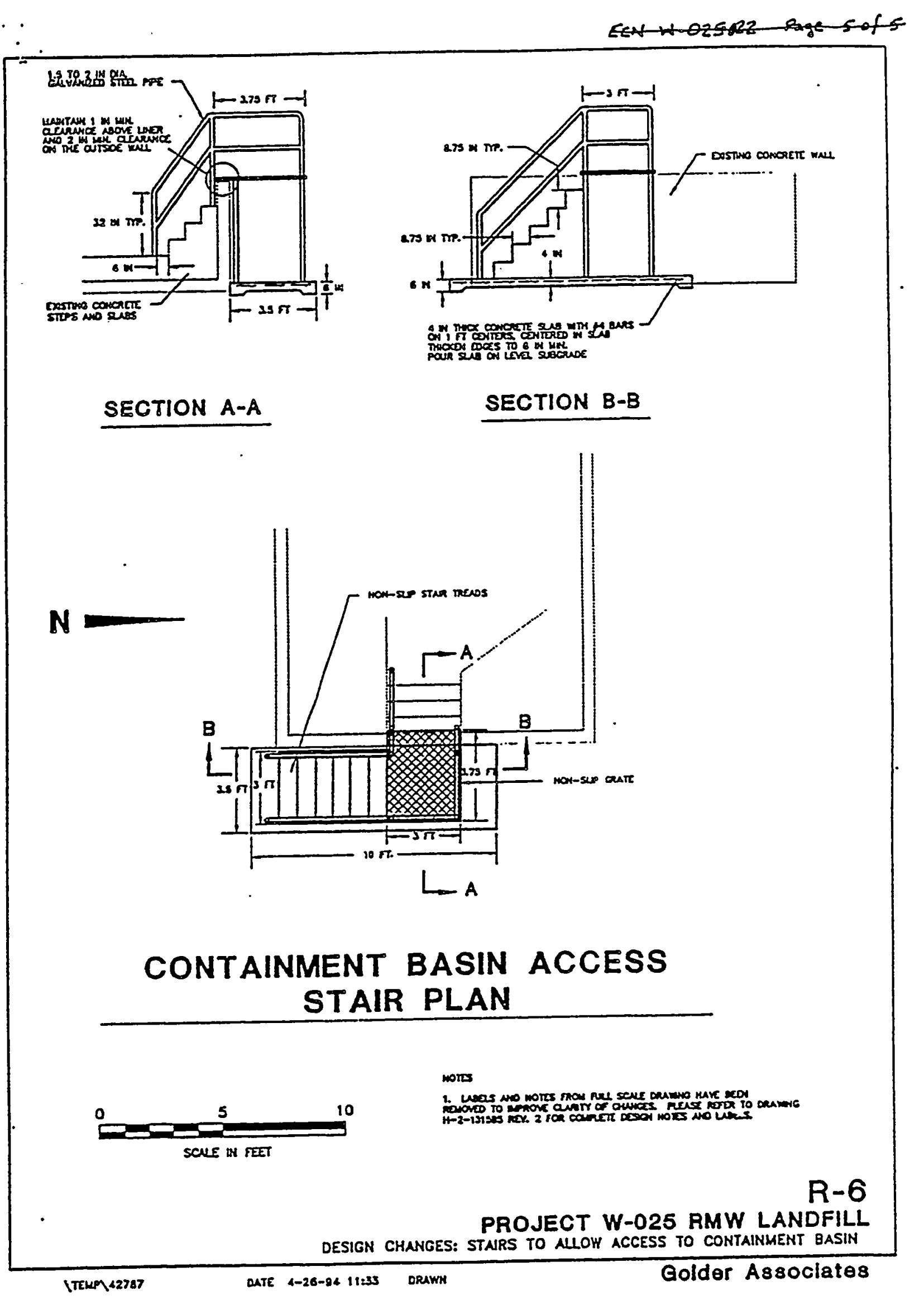


WHC-SD-W025-RPT-001, Rev. 0

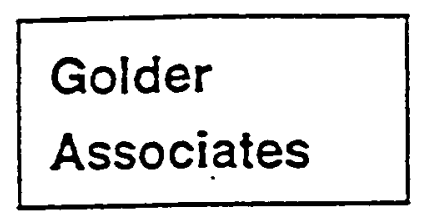

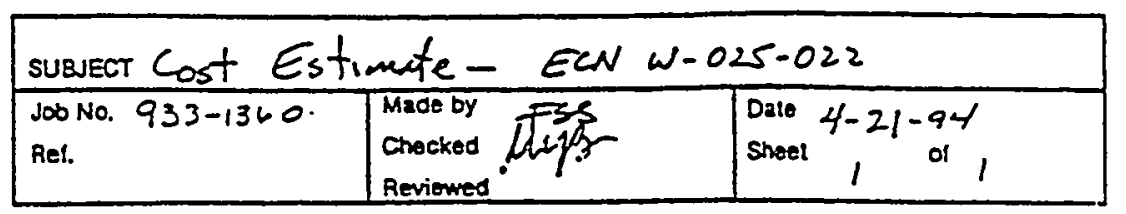

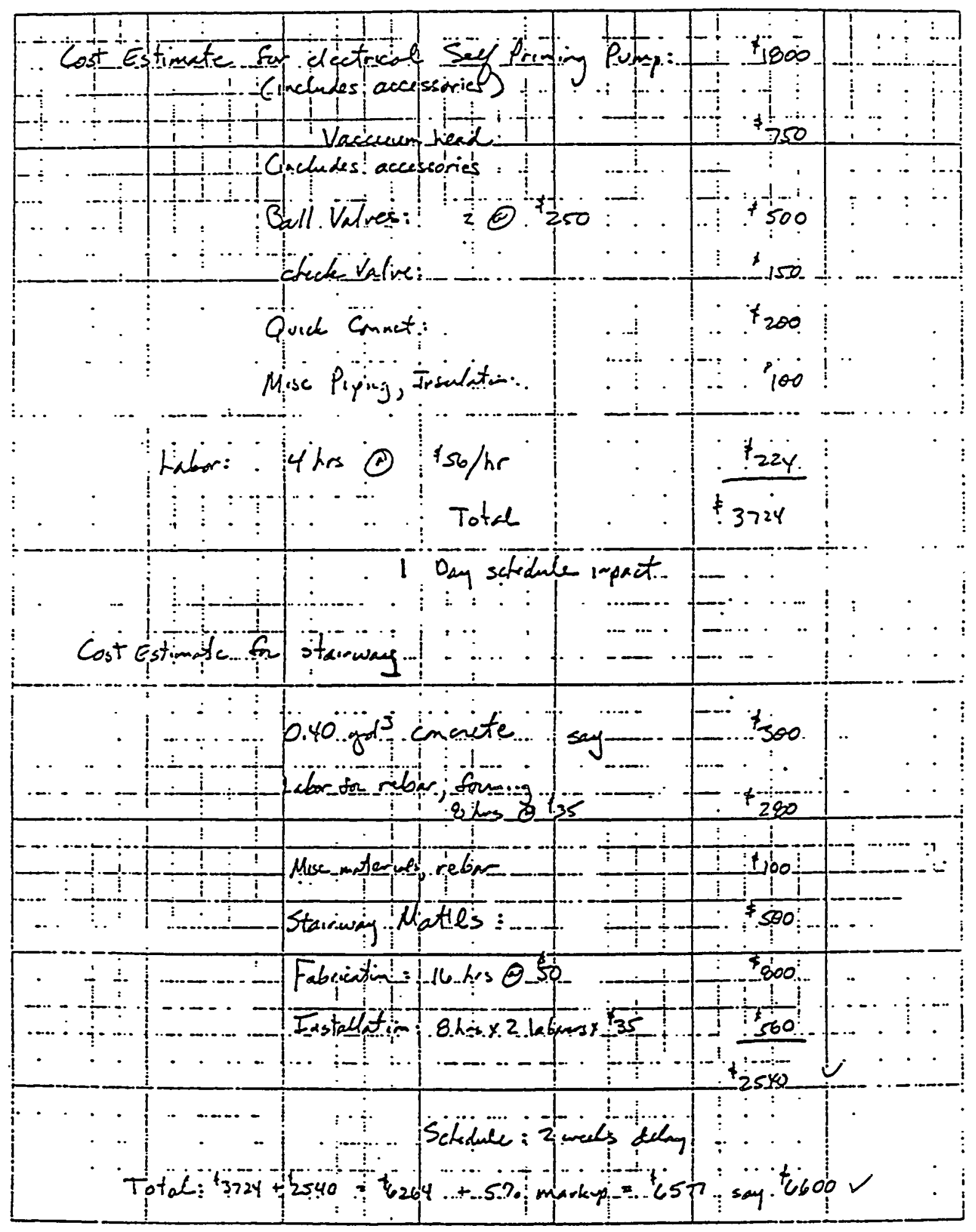

933-1354

C-3-98 
WHC-SD-W025-RPT-001, Rev. 0

$\because \because \because$

ENGINEERING CHANGE NOTICE

\begin{tabular}{|c|c|}
\hline & 1. ECN $+5=34$ \\
\hline ge 1 of 6 & $\begin{array}{l}\text { Proj. } \quad W-225-023 \\
\text { ECN }\end{array}$ \\
\hline
\end{tabular}

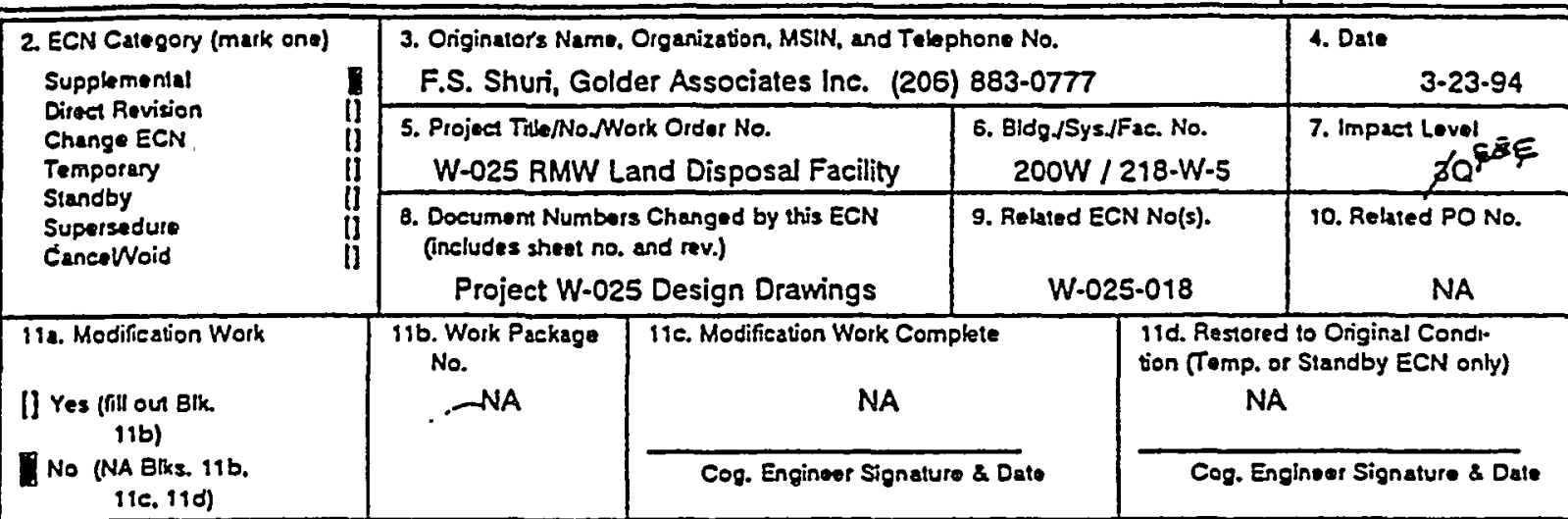

12. Description of Change

Changes to Projoct W-025 Design Drawings:

1. Draving No. H-2-131585. Rev. 2. Lewthato Tomporary Storage Tank \& Piping Plan: Add hoal trace and insulation to all piping. vahves llowmerers. and other piping system components bonween kachate temporary storage tank and check valves nearest t:rest wall, as designated by hatched area on Figure R.2A. atteched to this ECN. Heat trace and insulation shall conform to existing requirements in Spreifieations WHC.S-045 Rev. 2

2. Drawing No. H-2-131585. Rev. 2. Leachate Tomporay Storago Tank \& Fiping Soction J: Add heal trace and insulation to all piping. valvas. and other piping systom compononts associaled with loachate tomporary storage tank, designated by hatehed area on Figure R3A. anached to this ECN. Heat trace and insulation shall conform to axisting requirements in Specifications WHC-S-045 Rev. 2.

3. Drawing No. H.2-131585, Rev. 2. Leachate Tomporary Storage Tank \& Piping Section J: Add vacuum robease valve W67 12 inches upatream from chock valve at discharge pipe entrance to leachate lemporary sorage fank, as shown on Figure R.3B. attached to this ECN. Vacuum release valve shall eontorm to existing requirements in Specifications WHC.S-045 Rev. 2.

\begin{tabular}{|c|c|c|c|c|c|c|c|}
\hline $\begin{array}{l}\text { 132 Justifi } \\
\text { ono) }\end{array}$ & & Criteria Change & [] & Design Improvement & $\pi$ & Environmental & [] \\
\hline As-Found & [] & Facilitate Const. & [] & Const Enor/Omission & [] & Design Error/Omissien & 【] \\
\hline
\end{tabular}

13b. Justification Details

1 and 2. Prevent freezing of water in piping system.

3. Allow piping system to drain so that setr.priming pump will start as intended.

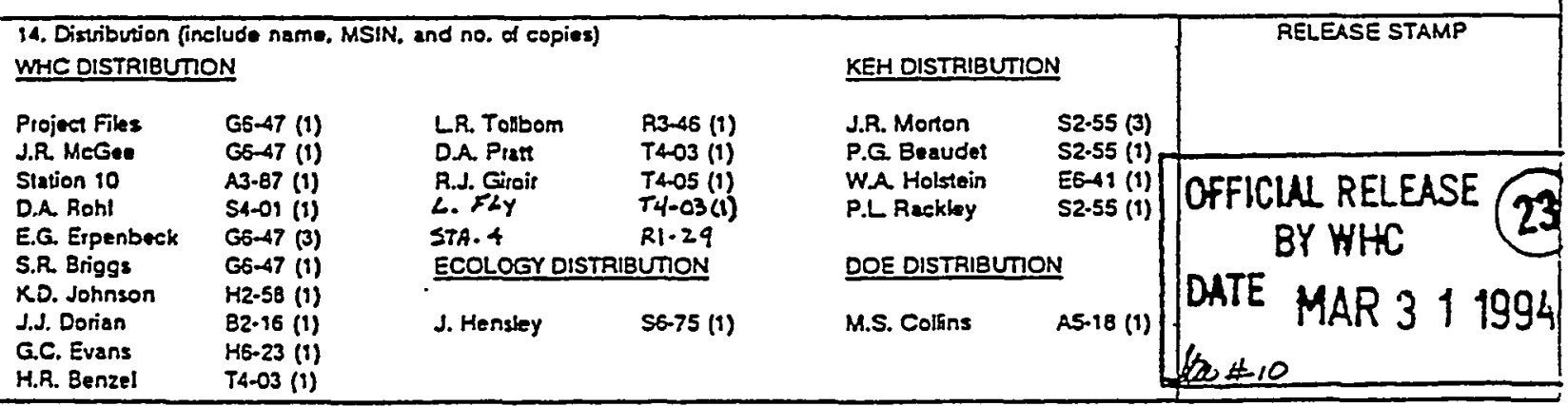

A.7900-013.2 (06/92) GEFO95 


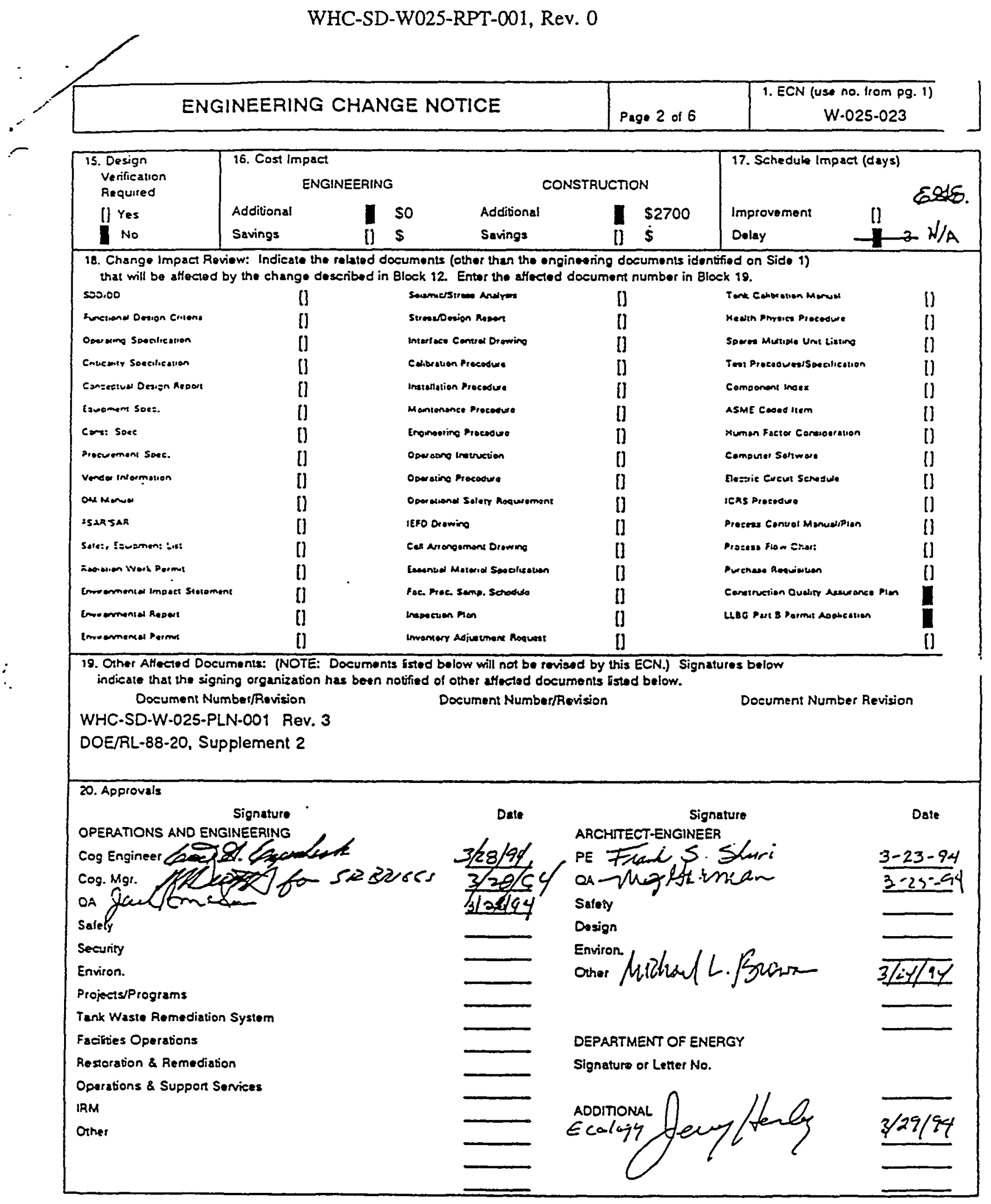


Continued trom Soction 12, page 1:

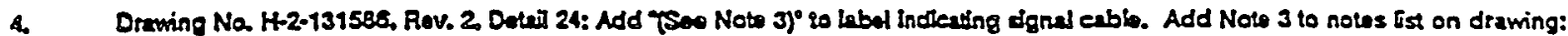

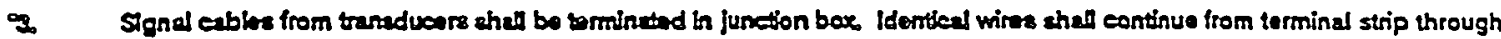
conduat to contral building:

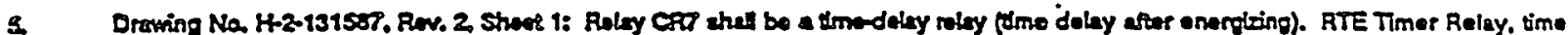

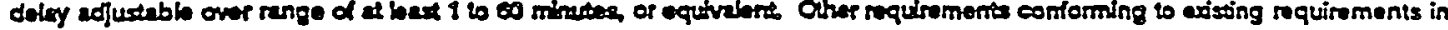

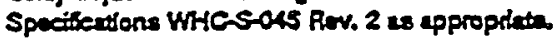

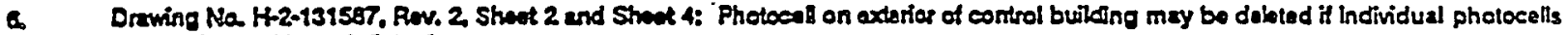
ise provided with eech figt foctur.

Corrinued fram Section 13b, page is

4. NHow transducars to be romoved for cafbratton op repleoment without removing signal eable from conduit and control panst.

5. Prwant pumps from cyaing id Equid invel in bechate tank sloshos

5. Equivalont altomativo to facitiato constrution.

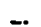

$-\cdot \cdot$

$-\cdot-\cdot$

-...


WHC-SD-W025-RPT-001, Rev. 0

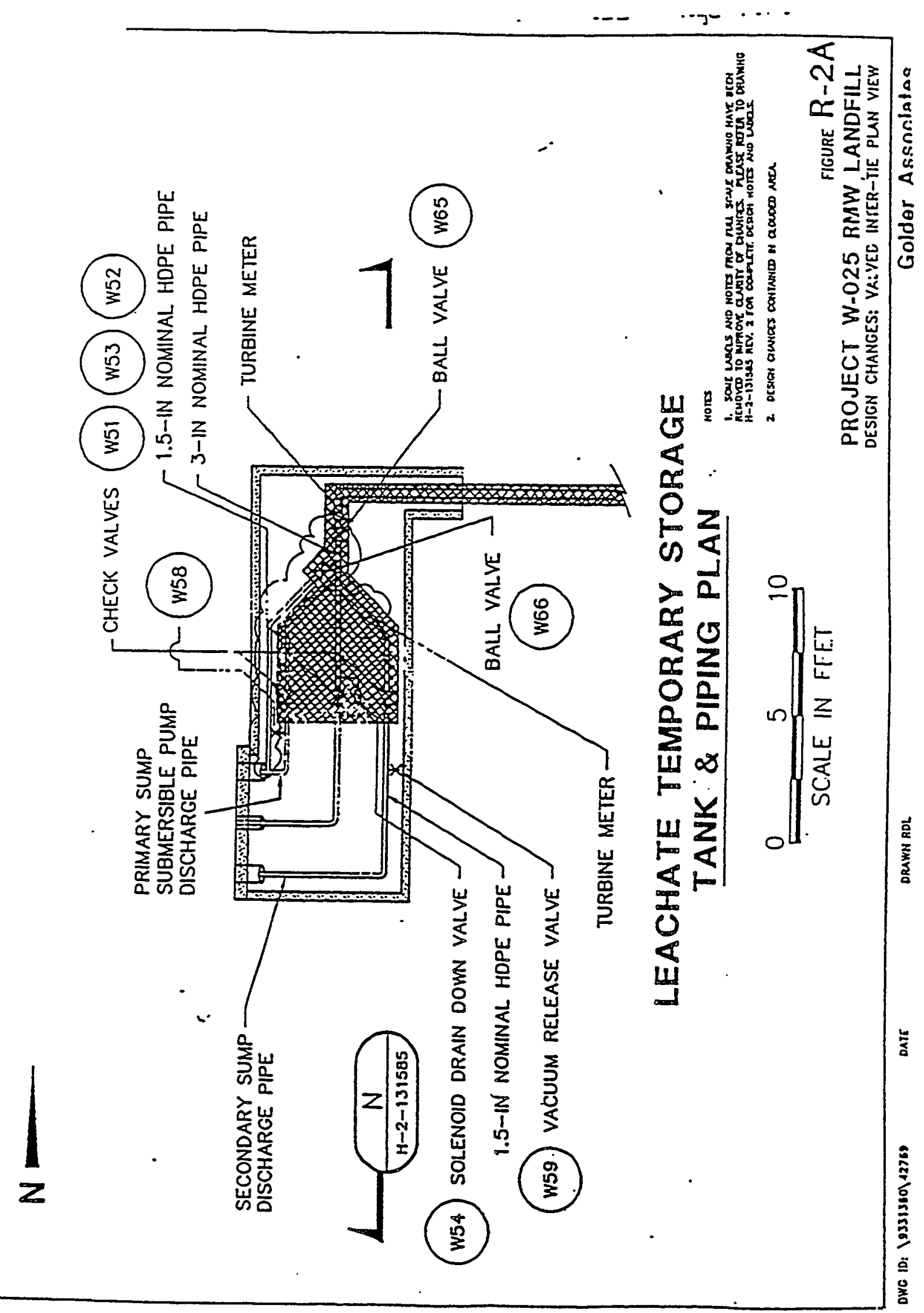


WHC-SD-W025-RPT-001, Rev. 0

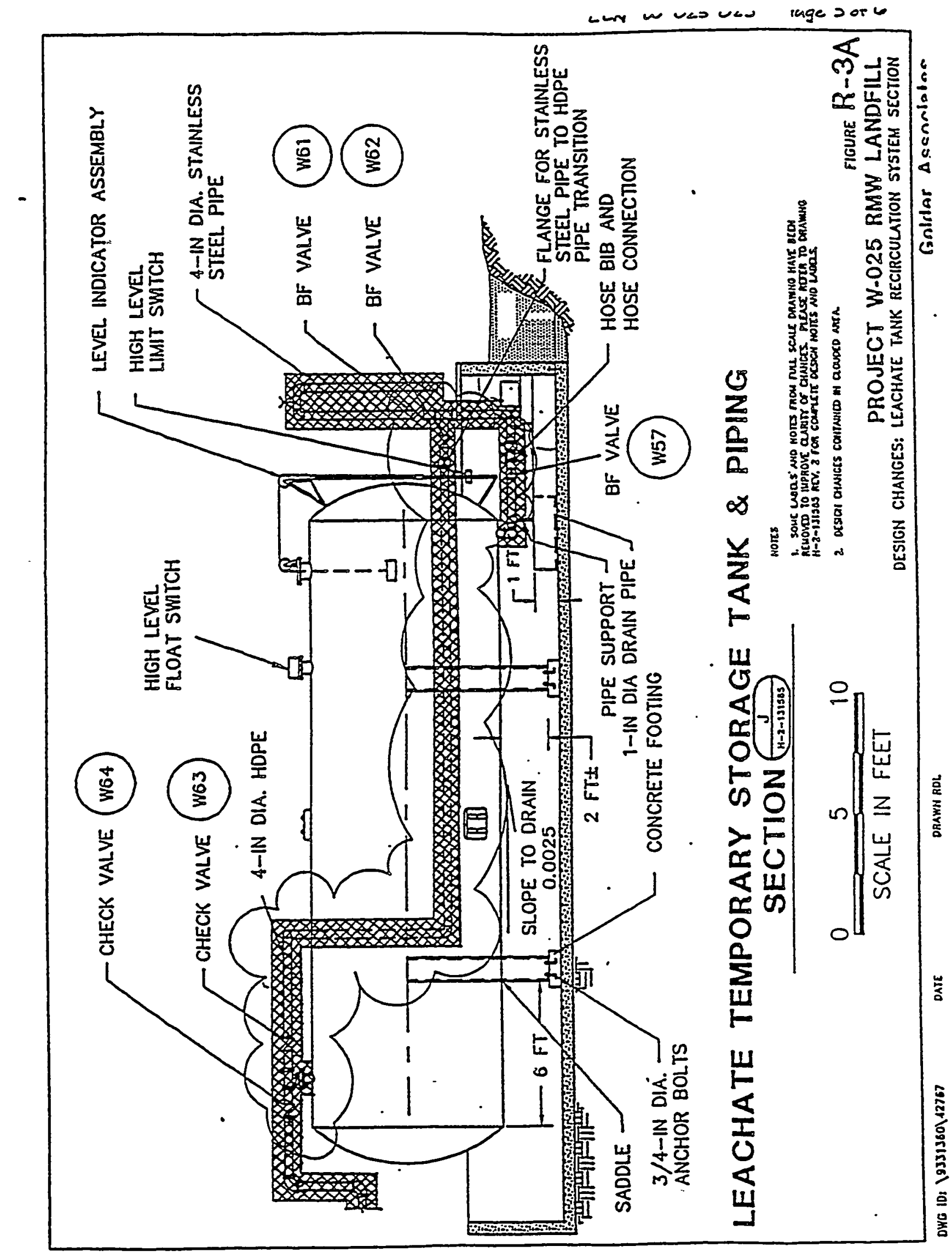


WHC-SD-W025-RPT-001, Rev. 0

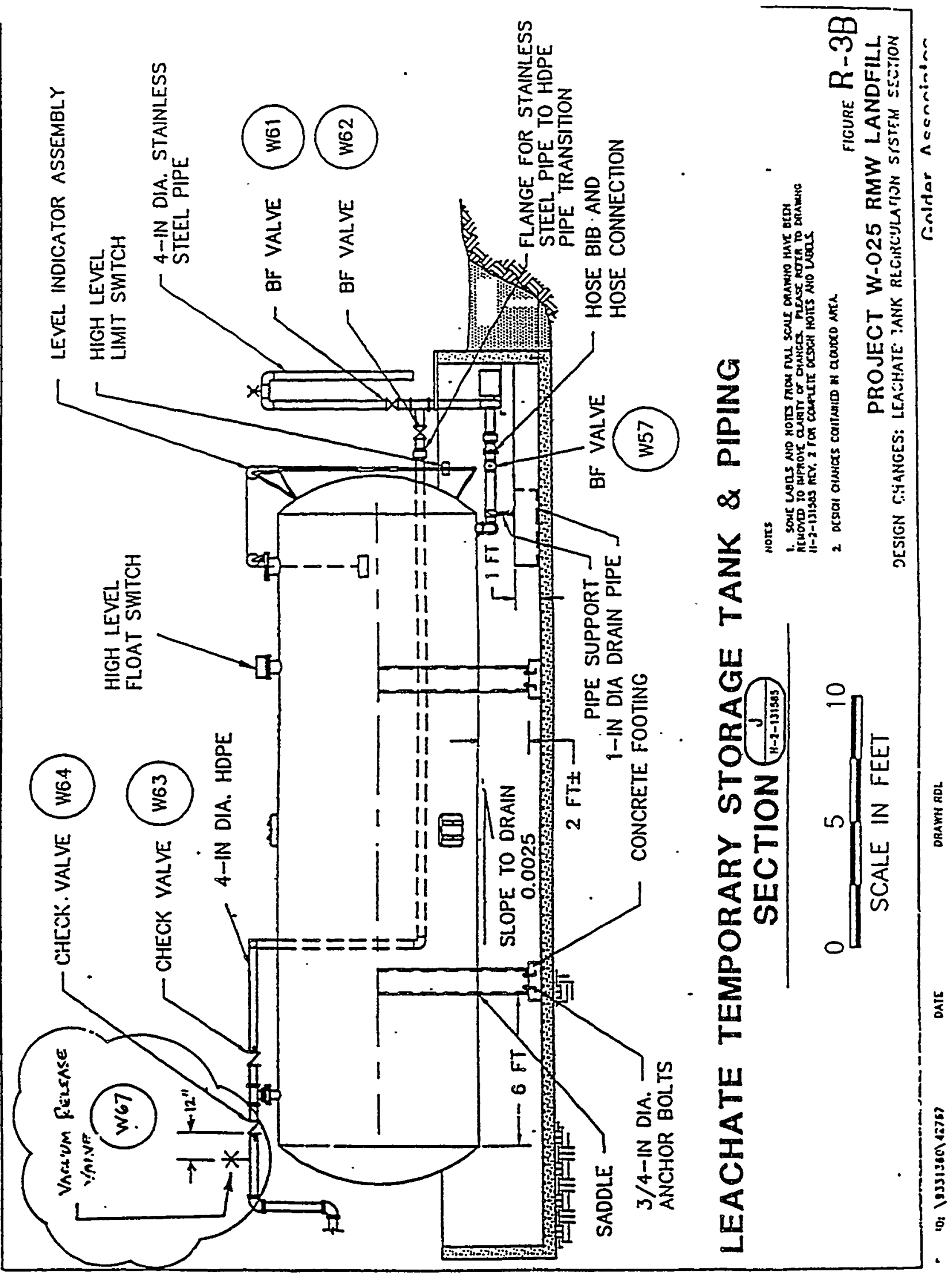


WHC-SḌ-W025-RPT-001, Rev. 0

\begin{tabular}{|c|c|c|c|}
\hline & suenect Cost Est & $A_{e}-E C N$ & $5-023$ \\
\hline Associates & $\begin{array}{l}100 \mathrm{Na}, 933-1360 \\
\text { Rel. }\end{array}$ & $\begin{array}{l}\text { Made oy FSS } \\
\text { Onucked Fol } \\
\text { Revienod }\end{array}$ & $\begin{array}{l}\text { Dale } 3-23-94 \\
\text { sheet } 1 \text { of } 1 \\
\end{array}$ \\
\hline
\end{tabular}

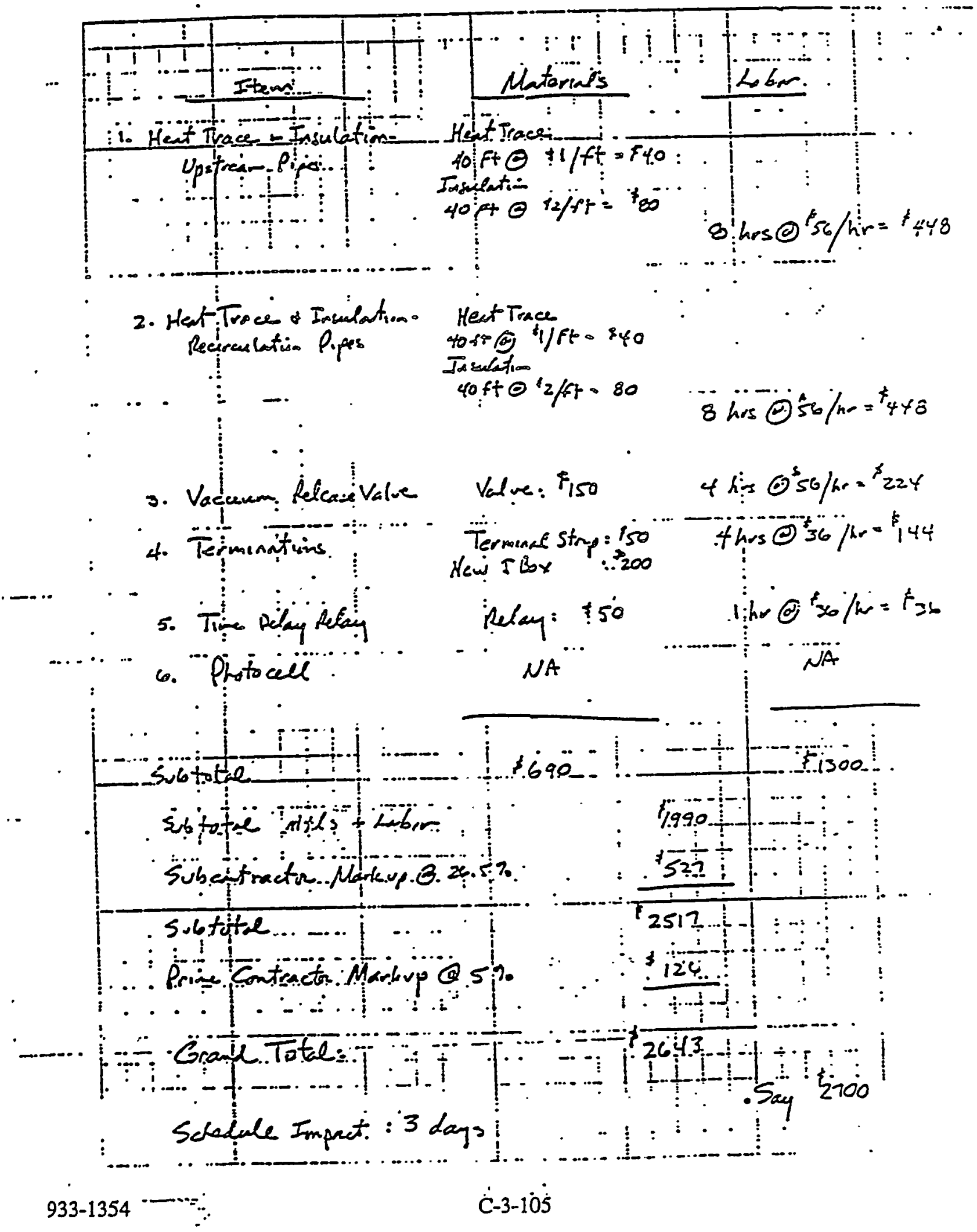


WHC-SD-W025-RPT-001, Rev. 0

$-\cdots \cdot$

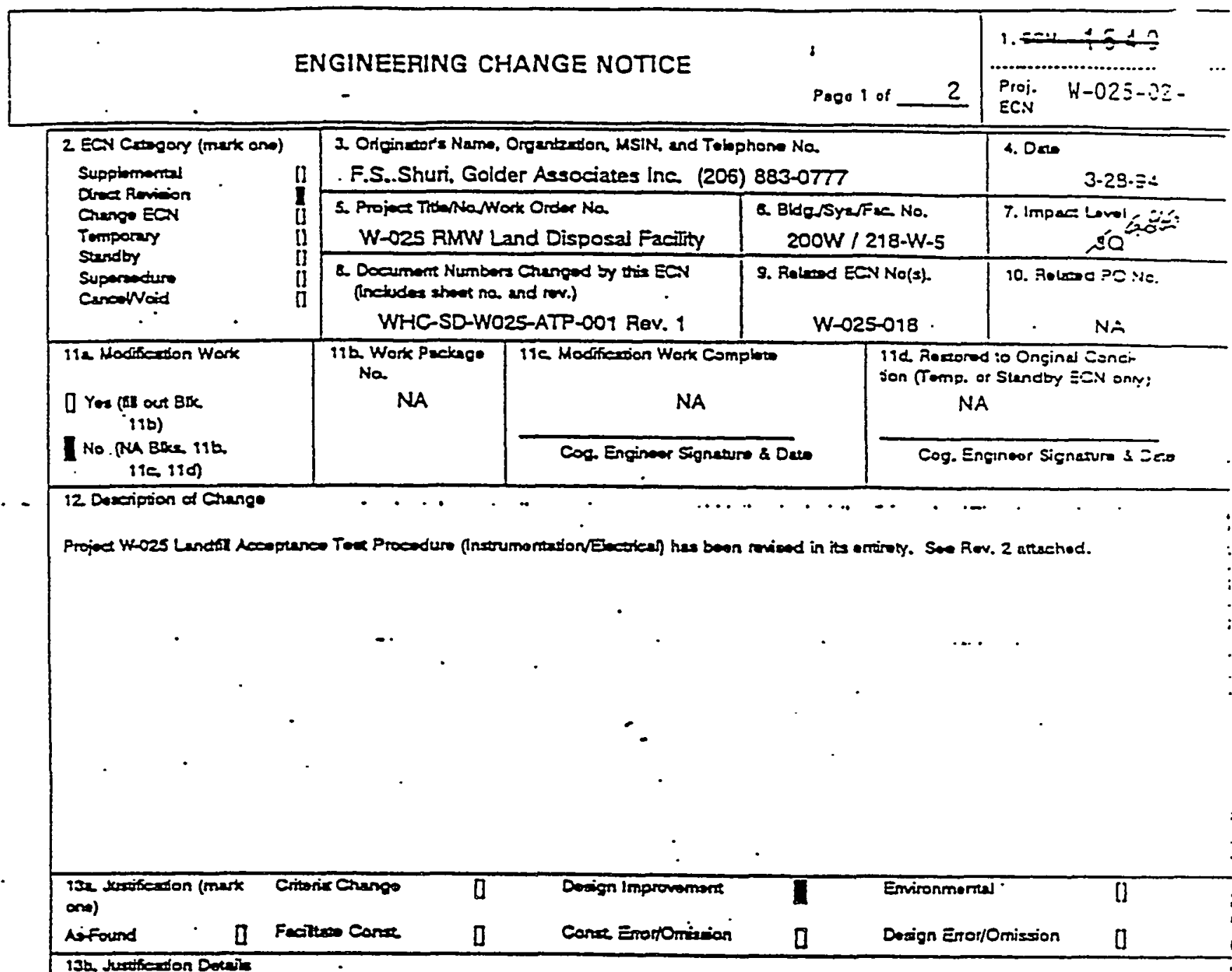

$\because \because \because \because \because$

13t.

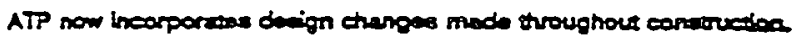

14. Qubibuton (nxcludo nama, HSIN, and na of copion) MAC DSTRIBUTION

\begin{tabular}{|c|c|}
\hline 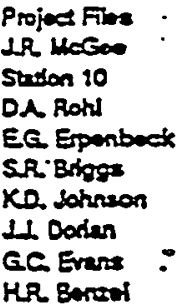 & 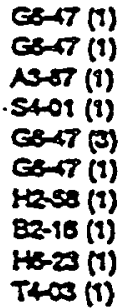 \\
\hline
\end{tabular}

A-7900-013-2:(06/92) GEF025

$$
4 \cos 20
$$

\section{KEH DISTRIBUTON}

LRTOHban R3-46 (9)

OA Prat TL-O3 (1)

RLGroir TSOS (1)

ECOROGY OISTRIBUTONON

1 Henciby S6-75 (I)
LR Morton 52.55 (3)

P.G. Bocudot $\$ 2.55$ (1)

WA Holntin

PL Ruckby

DOE DISTRABUTON

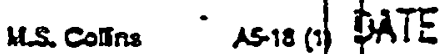

52.55 (1) OFFICIAL RELEASE 23 BY WHC

APR $0 \div 1994$

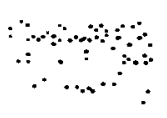


WHC-SD-W025-RPT-001, Rev. 0

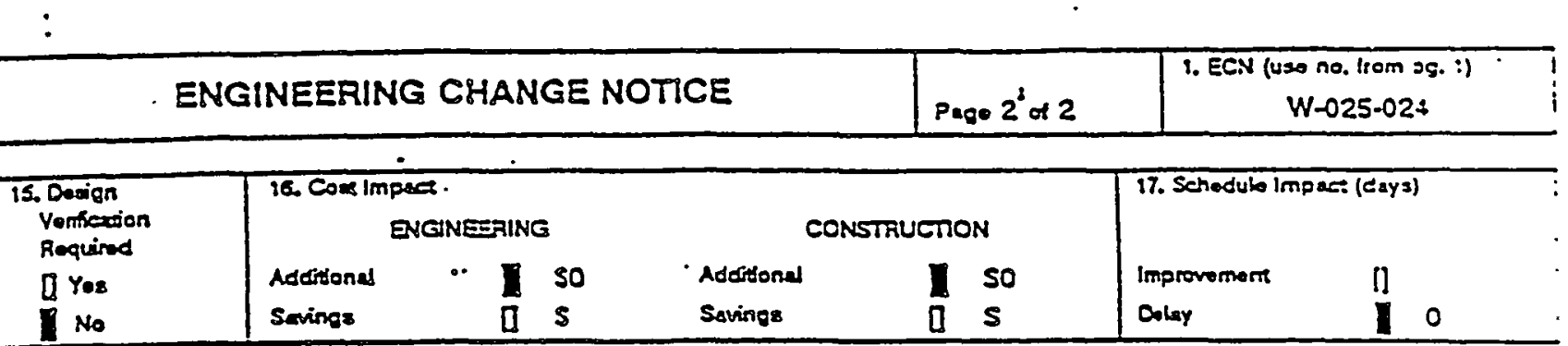

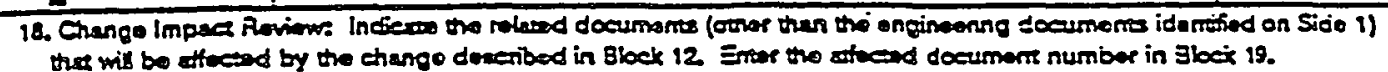

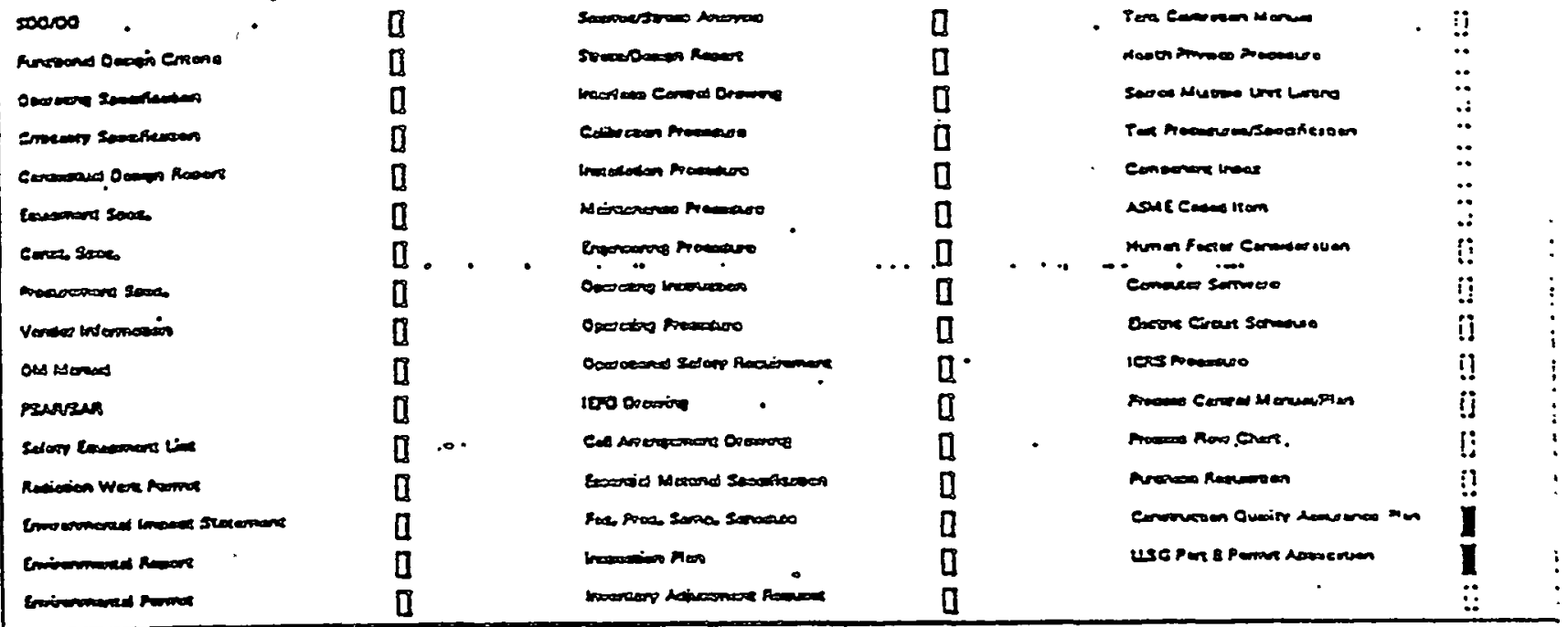

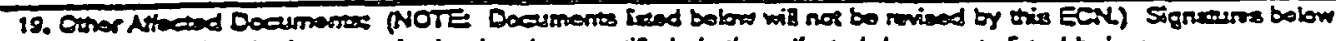

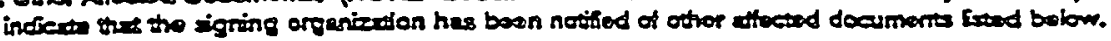

Document Number/Revieion Document NumborRovision

Dosumort Numbor Revsion

WHCSD-W-025-PLN-0OY Rev. 3

DOERRL-88-20, Supplement 2

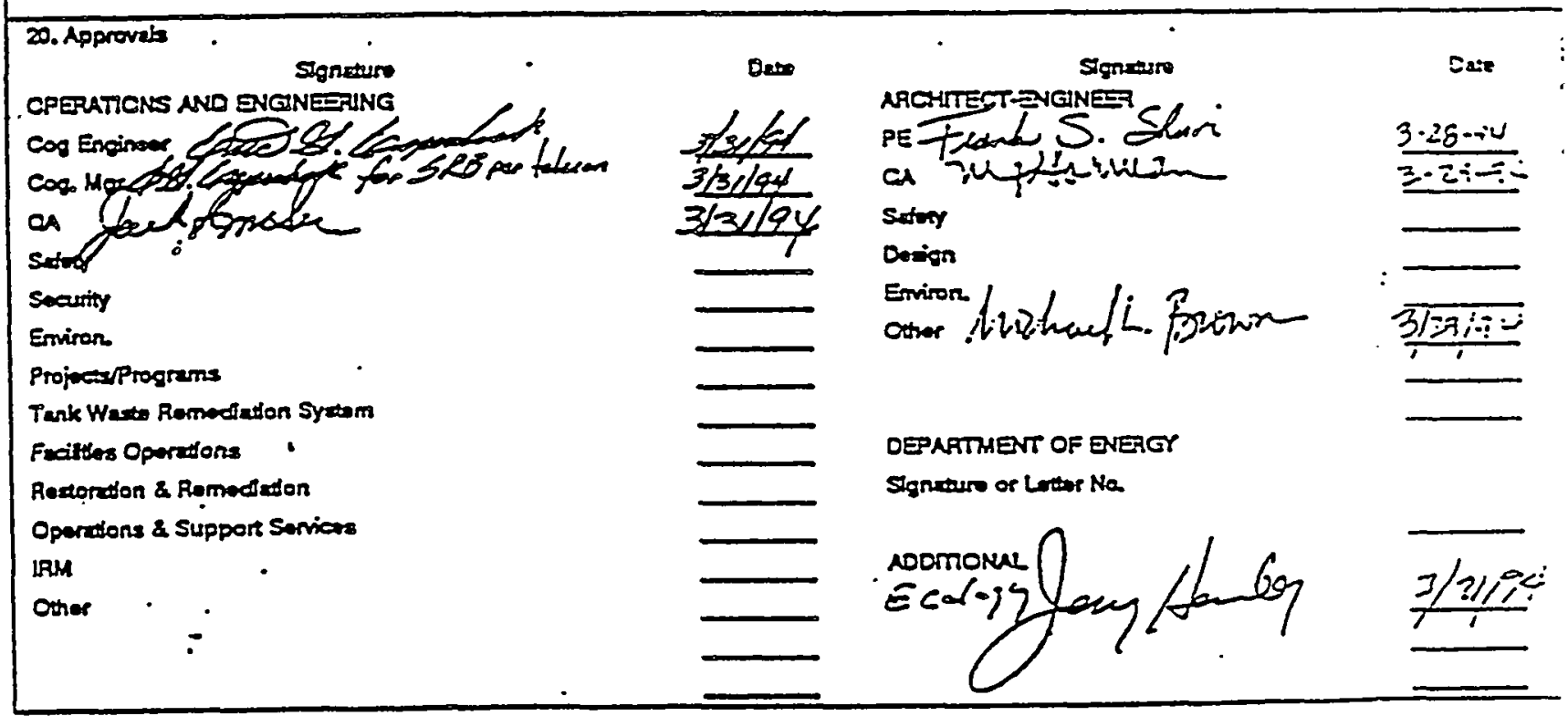


WHC-SD-W025-RPT-001, Rev. 0

UKIGINAL

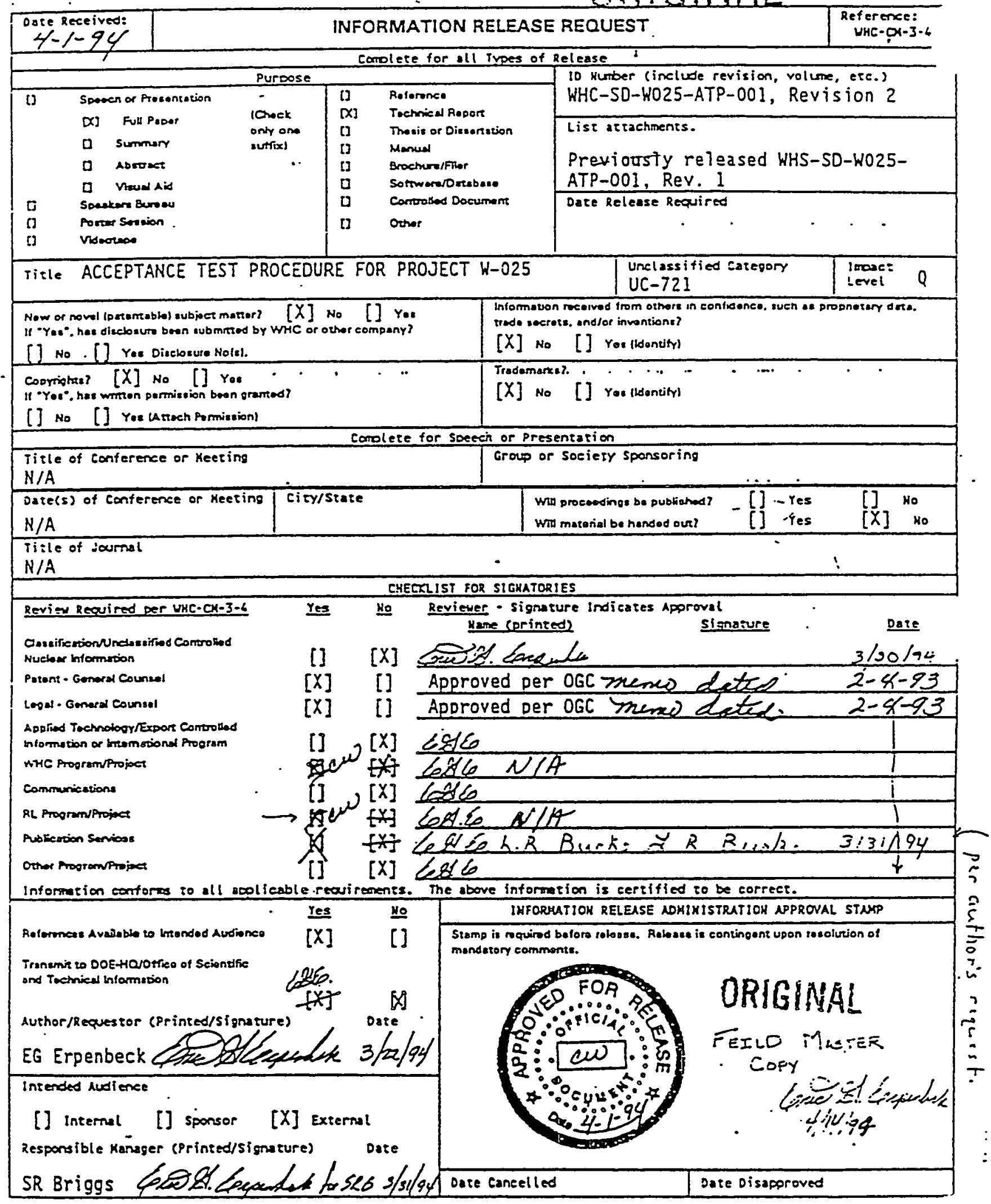

80-7600-062 (C8/91) UEF074 


\section{SUPPORTING DOCUMENT} 1

1. rotal pages if 4 ?

\begin{tabular}{|l|l|l|}
\hline $\begin{array}{l}\text { 2. Title } \\
\text { W-025. ACCEPTANCE TEST PROCEDURE }\end{array}$ & $\begin{array}{l}\text { 3. Number } \\
\text { WHC-SD-WO25-ATP-001 }\end{array}$ \\
\hline $\begin{array}{l}\text { 5. Key Words } \\
\text { ATP }\end{array}$ & $\begin{array}{l}\text { 6. Nuthor } \\
\text { Wame: EG ERPENBECK }\end{array}$ \\
\hline
\end{tabular}

\section{Abstract}

THIS ATP HAS BEEN PREPARED TO ESTABLISH FIELD TESTING PROCEDURES TO DEMONSTRATE THAT THE ELECTRICAL/INSTRUMENTATION SYSTEMS FUNCTION AS INTENDED BY DESIGN

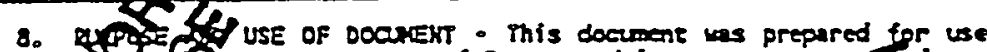
W I COS U.S. Deparzene of Esergy and its concractors its is to 2 - oly to perform direses or integrase tort under

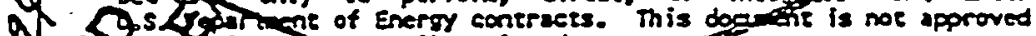

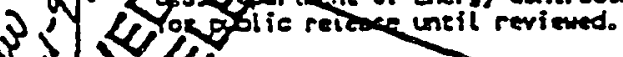

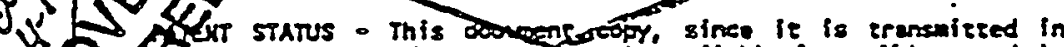
(D) Arance of patene elearance onsade avoileble in confidence solely Qf Cfor use in perforgunes of wont undep contrects with the

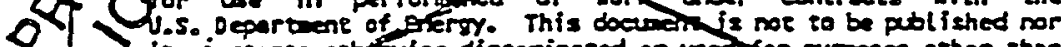

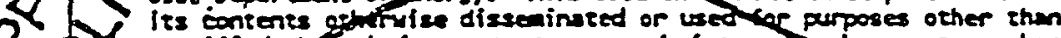

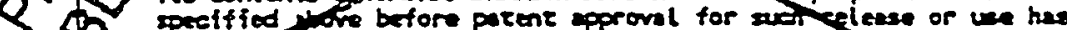

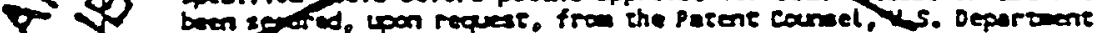

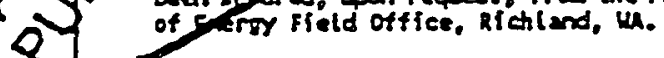

OIsculker - This report was prepared of en secount of work sponsored by an sgency of the Uni ted states Goverment. Kef ther the thited states Goverment nor amy agency thereof, nor any of their whloys nor amy of their contractors, subconeraetors or their aployes, nor any of their contreters, suoconeractors or ineir legal liability or responsibility for the accuracy, eorplezeness, or amy third perty's use or the results of such lase of any information, apara would not intrime privately aned rights. Reference herein to umy speciffic compertial product, process, or service by trade name. tredenark, naxufacturer, or otherwise, does not necessarity consisf tute or imply its exdorsenent, recocmendation, or favoring by the United states Goverment or any agency thereof or its conernctorz or sibecontraetors. The vieus and opinions of authors expressed herein do not secessaflly state or reflect those of the United states Goverment or ary agency thereof.

10. RELEXSE STAKP

OFFICLAL REIEASE BY IVHC DATE APR $0 \uparrow 1994$ $x=10$ 
WHC-SD-W025-RPT-001, Rev. 0

ACCETTANCE TEST PROCEDURE NO. WFC-SD-WO25-ATP-D01, REV. 2 DATE 3/28/94

SLITEC! NSTRUMENTATON/ELECIRICAL

LOCATION: 300 WEST

PROJECI :VLPBER W-O2S WORK ORDER TDD

PROJECT IIILE RMW LAVD DISPOSAI FACILTV

Prepared By

GOLDER ASSOCIATES INC.

Redmond, Washington

\section{ORIGINAL}

For the LiS Department of Energy

Contract DE-ACOS-89RL11615

Rev 2

\section{IEST PROCEDURE APPROVED}

GOLDER ASSOCIATES INC
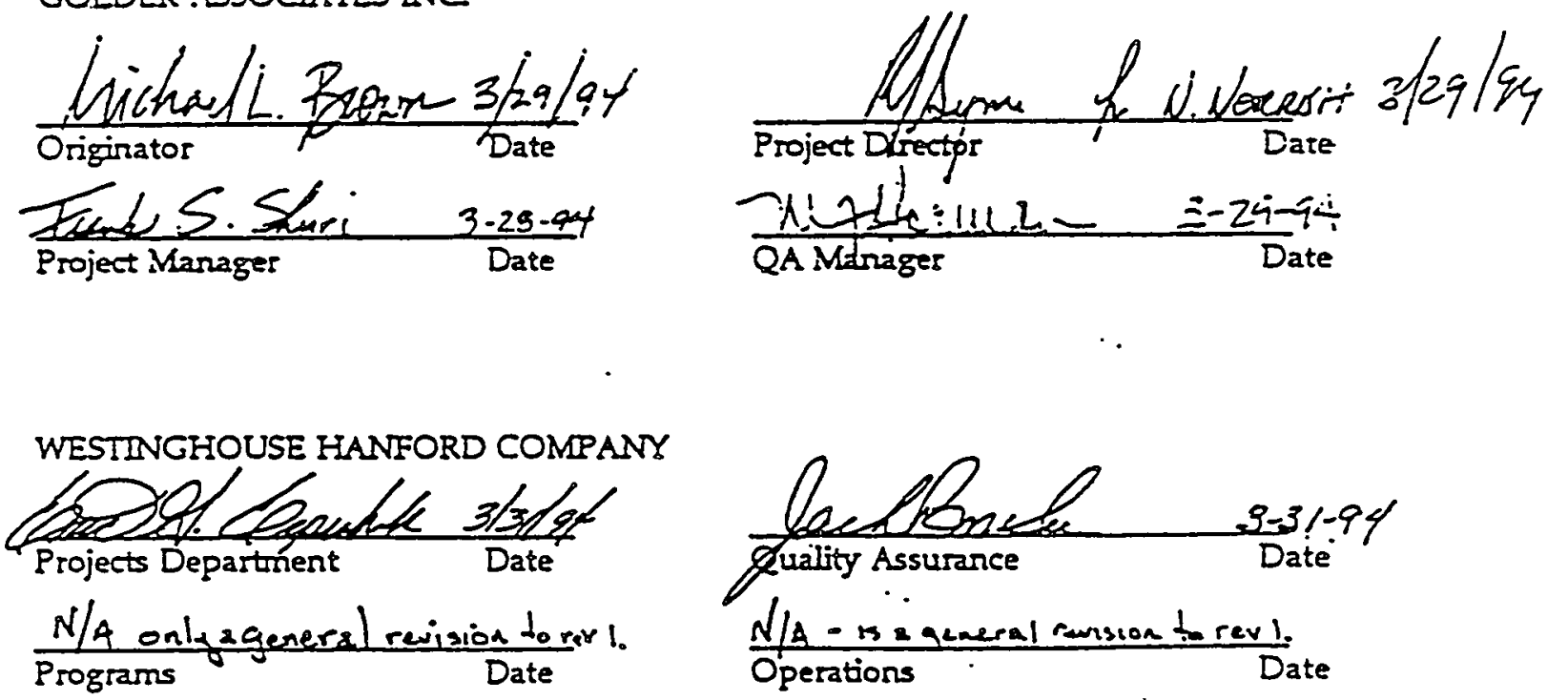
WHC-SD-W025-RPंT-001, Rev. 0

WHE-SD-WO25-ATP-001 Rev.2

CONTENTS

1.0 PURPOSE OF THE ACCEPTANCE TEST $\quad \cdots \quad-\cdots$

20 REFERENCE DRAWINGS AND SPECIFICATIONS 1

21 DRAWINGS 1

22. SPECIFICATIONS : 1

3.0 RESPONSIBILTIIES 1

3.1 WHC PROJECT ENGINEER

3.2 TEST DIRECTOR

3.3 WITNESSES 3

3.4 RECORDER

3.5 CONSTRUCTION CONTRACTOR 4

3.6 OCCUPATIONAL SAFETY AND HEALTH

4.0 ACCEPTANCE TEST PROCEDURE CHANGE CONTROL 4

5.0 IEST EXECUTION 5

5.1 WITHOUT EXCEPTION

5.2 WITH EXCEPTION/RESOLVED

5.3 WITH EXCEPTIONOUTSTANDING 5

6.0 RECORDING AND RESOLVING EXCEPTIONS 5

6.1 GENERAL

6.2 RECORDING 5

63 RETEST/RESOLUTION 6

6.4 APPROVAL AND ACCEPTANCE

6.5 DISTRIBUTION

7.0 TEST CONDITIONS AND EQUIPMENT REQUIRED 6

7.1 GENERAL 6

7.2 EQUIPMENT REQUIRED

8.0 ELESIRICAL HEAT TRACE SYSTEM

8.1 IEMPERATURE SENSOR

8.2 HEAT TRACE CABLE 9

9.0 TRANSDUCERS AND METER/CONTROLLERS 9

9.I PRIMARY SUMP 9

92 SECONDARY SUMP 
CONTENTS (cont)

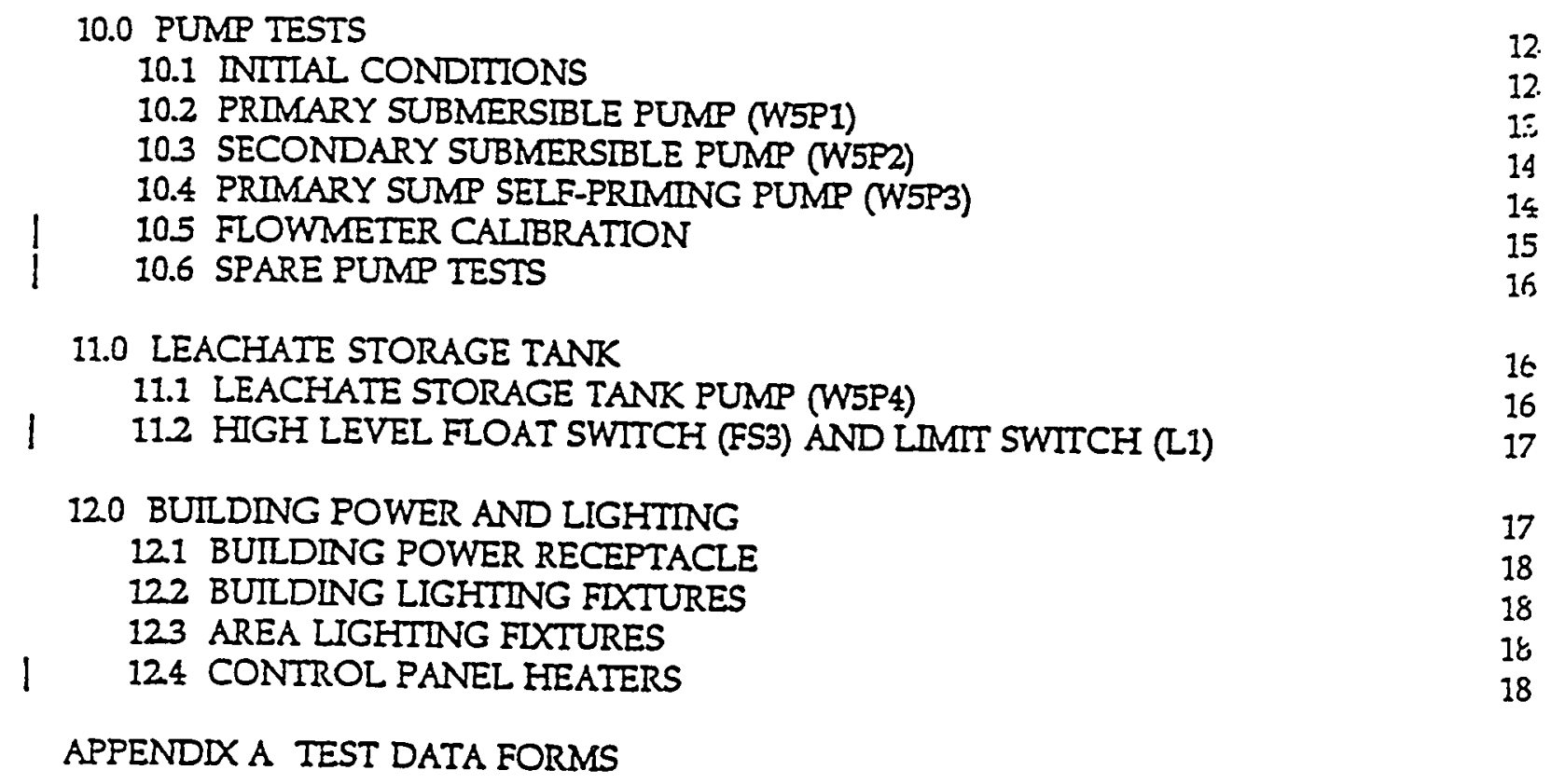

12.

12.

$1:$

14 14

APPENDIX A TEST DATA FORMS 
WḦC-SD-W025-RPT-001, Rev. 0

WIICSDWO25-ATP=001 ReV. 2

ACCEPTANCE IEST PROCEDURE

RMW LAND DISPOSAL FACILITY

PROIECT W-025

1.0 PURPOSE OF THE ACCEPTANCE TEST

This acceptance test procedure (ATP) has been prepared to establish field testing procedures to demonstrate that the Electrical/nstrumentation systems function as intended by the design.

20 REFERENCE DRAWINGS AND SPECIFICATIONS

21 DRAWINGS

H-2-131585 1 OF 1 Leachate Collection Tank \& Piping

H-2-131586 IOF 1 Sump Pump Details

H-2-131587 1 OF 4 Electrical - Control

H-2-131587 2 OF 4 Electrical - Tank \& Control Bldg.

H-2-131587 3 OF 4 Electrical Site Plan

H-2-131587 \& OF 4 Electrical Details

\section{SPECIFICATIONS}

WHC-S-045 Radioactive Mixed Waste Land Disposal Facility, Non-Drag-Off, Technical Specifications.

\subsection{RESPONSIBILITIES}

Each company or organization participating in the conduct of this ATP will designate personnel to assume the responsibilities and duties as defined herein for their respective roles. The names of these designees shall be provided to the Recorder for listing on the Recorder's copy of the Test Execution Sheet prior to the performance of any part of this ATP.

3.1 WHC PROJECT ENGINEER

\subsubsection{Designate a Test Director.}

3.12 Act as liaison between the participants in acceptance testing.

3.1.3 Distribute the approved testing schedule as soon as approval has been obtained (one or more weeks prior to testing).

\section{$x$}


3.1.4 Schedule and conduct a pre-ATP kickoff meeting with test participants ona or more weeks prior to start of testing.

3.1.5 Notify the persons performing and witnessing the test two days frior to the start of testing.

3.1.6 Notify all concerned parties when a change is made in the testing schedule.

3.1.7 Sign Test Execution Sheet when ATP is approved and accepted.

3.1.8 Take necessary action to clear exceptions to the ATP.

3.1.9 Sign Exception Sheet when exception has been resolved.

3.1.10 Provide a distribution list for the approved and accepted ATP.

\subsection{TEST DIRECTOR}

32. Coordinate all acceptance testing.

3.22 Distribute all documents including completed AIP, exceptions, resolutions and aprovisuls.

$3: 13$ Confirm that field testing and inspection of the system or portion of the system to be tested has been completed.

32.4 Stop any test which, in the judgement of the Director, may cause damage to the system until the test procedure has been revised.

325 Obtain revisions to the ATP, as recessary, to comply with authorized field changes or to accommodare existing field conditions.

32.6 Evaluate recorded data, discrepancies, and exceptions.

3.7.7 Obtain from Golder Associates Ine (GAi) any information related to this ATP or changes necessayy to clear or resolve objections.

328 'Sign Test Execution Sheet when ATP has been performed.

329 Sign Exception Sheet when retest has been executed and accepted.

3210 Obtain required signatures on the ATP Master prior to reproduction and distribution. 
WHC-SD-W025-RPT-001, Rev. 0

WIC-SD-W025-AIP-DOT ReV. 2

\subsection{WITNESSES}

Witnesses shall be provided by participating organizations.

3.3.1 Witness the tests.

3.3.2 Evaluate results of testing.

33.3 Assist the Test Director when requested.

3.3.4 Sign Test Execution Sheet as a Witness.

3.3.5 Sign Exception Sheet as a Witness when retest has been executed and accepted.

3.3.6 An Acceptance Inspector will witness the test for the Design Engineer.

\subsection{RECORDER}

The Recorder will be provided by Golder Construction Services.

3.4.1 Record names of all designated personnel on Recorder's copy of ATP prior to start of testing.

3.4.2 Observe tests and record test data.

3.4.3 Sign the Test Execution Sheet as the Recorder.

3.4.4 Iniaial and date every test step on the Recorder's copy as it is completed, next to the step number or on a table, when provided. On tables where there is not room for both the initial and date, date may be entered in space provided at bottom of colum

3.4.5 On the Exception Sheet, record objections or exceptions and test steps which are not performed. Have the information transferred in ink or typed to the Master of the Exception Sheet(s). Additional Exception Sheets are to be added as needed.

3.4.6 Orally notify the Test Director at time the objection is made.

3.4.7 Assign page numbers to Data Sheets and Exception Sheets, after AIP is complete. Record page numbers for these items and make corrections, as necessary, to page numbers shown for these pages in the index.

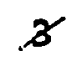


WHC-SD-W025-RPT-001, Rev. 0

WIICSDWW025.AIP.001 ReV.Z

3.4.8 Transfer changes and the final test results with Recorder's signature and dates for each step to the Master in ink or type. Submit the completed Master to Construction Document Control for approval signature routing. Also submit the Recorder's copy for record retention.

\subsection{CONSTRUCTION CONTRACTOR}

3.5.1 Organize and perform this acceptance test under coordination of the Tr:st Director.

3.5.2 Confirm that all equipment required for performing this test will be available at the start sf testing.

3.5.3 Provide equipment required for performing this acceptance test, which has not been designated as being provided by others.

3.5.4 Request in writing from the Project Engineer those services, materials, or equipment that have iuen designated as being supplied by the DOE or others.

\subsection{Or:UPATIONAL SAFETY \$ND HEALTH}

Individuals shall carry out their assigned work in a safe manner to protect themselves and others from undue hazards and to prevent damage to property and environment. Facility line managers shall assure the safety of all activities within their areas to prevent injury, property damage, or interruption of operation. Performance of test activities shall always include safety and hiealth aspects as delineated in the most current version of the Federal Occupational Safety and Health AdministrationWashington Industrial Safety and Health Act (OSHAWWISHA) safety health codes and standards.

\subsection{ACCEPTANCE TEST PROCEDURE CHANGE CONIROL}

A.:-eptance testing shall be conducted in accordance with the steps and requirements sfereified in this procedure. Any required changes must be authorized in accordance with approved change control procedures for this project and promptly accomplished. $P_{i}=c e d u r e$ changes during testing must be approved by the WHC project engineer, WI3C quality assurance, and the GAI inspectur (via initials). The GCS recorder shall note these changes as exceptions (see Sectior. 52), provided that these changes do not afert safety and health. The changes shall be incorporated in the final acceptunce test refort 
WHC-SD-W025-RPT-001, Rev. 0

WIE-SD-WO25-ATP-001 ReV.2

\subsection{TEST EXECUTION}

\subsection{WTIHOUT EXCEPTION}

5.1.1 Check applicable space on Test Execution Sheet to show that the AIP has been performed and no exceptions have been recorded.

5.12 Sign and date Test Execution Sheet in the spaces provided.

5.13 Distribute requisite copies and send master of ATP to the WHC Project Engineer.

\subsection{WITH EXCEPTION/RESOLVED}

5.2.1 Check applicable space on Test Execution Sheet to show that the AIP has been performed with exceptions recorded and resolved.

522 Sign and date Test Execution Sheet in the spaces provided.

5.2.3 Distribute requisite copies and send master of AIP to the WHC Project Engineex

\subsection{WITH EXCEPTIONVOUTSTANDING}

5.3.1 Check applicable space on Test Execution Sheet to show that the AIP has been performed with exceptions recorded, in part or all of which are presently outstanding and unresolved.

532 Sign and date Test Execution Sheet in the spaces provided.

5.3.3 Distribute requisite copies and send master of ATP to the WHC Project Engineer.

\subsection{RECORDING AND RESOLVING EXCEPTIONS}

\section{I GENERAL}

Exceptions to the AIP are sequentially numbered and recorded on individual Exception Sheets. This enables case-by-case resolution, recording, approval, and distribution of each exception.

\subsection{RECORDNG}

6.21 Number each exception sequentially as it occurs and record it on an Exception Sheet 
WHC-SD-W025-RPT-001, Rev. 0

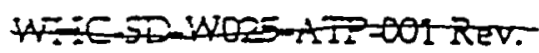

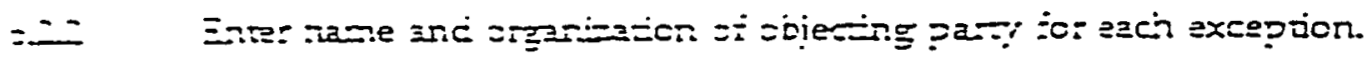

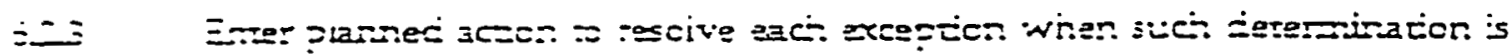
race.

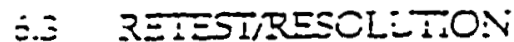

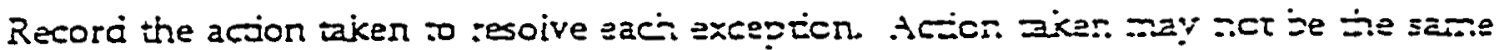
as planned action.

ó.1 When accion taken results in an acreptable retest, sign and cate Retest Execution and Aeseptance secsion of the Exception Sheet

53: When acion tiken coes not invoive an acseprable :etest, scike out the Retest Execution and Acceptance section of the Exception Sieet Resolve exception as shown uncer j... jelow.

\section{I.APROVALAWD ACCETAVE}

The WFC Project Engineer provides inai approval anc acseptance si exceptions jy checking one of the following on Exceprion Sineer

j.:. Retest Approved anc Acseprec: Appilabie winen Retest Execusion aric Acseparice seccion is sompietes

j.: $\quad$ Exception Acsepteci-as-is: Tequires serilec expiariacion.

j.j3 Other: Recuires cieriled explaration.

The Zroject Engineer signs and jares Sie Excepcion Sineer ard sobirs ther intermal approvais, if required.

$\therefore$ DISIRIBLTION

Distribute requisite copies of completed Exception Sheets to WHC personnel and deparments as directed by the WHC Project Engineer.

\subsection{IEST CONDIIONS AND EOUIPMENT REOUTRED}

\subsection{GENERAI}

The following conditions shall exist at the start of the acceptance testing for that portion of the system being tested. 
WHC-SD-W025-RPT-001, Rev. 0

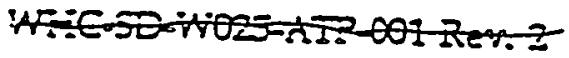

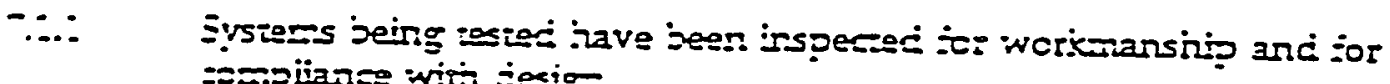 :=s:piance witi jexior-

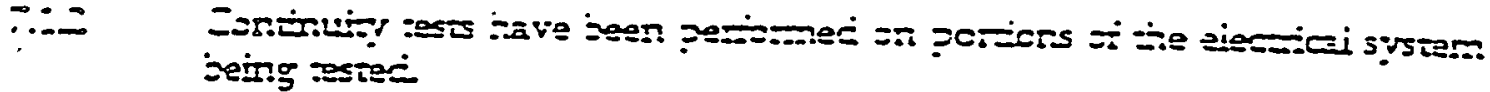

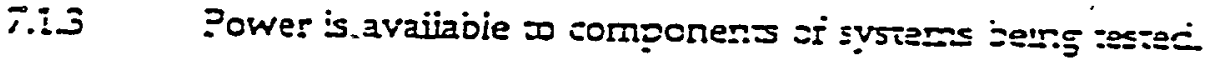
7.1.: Coninuity tests of instrumentation wiring iave been periormed in

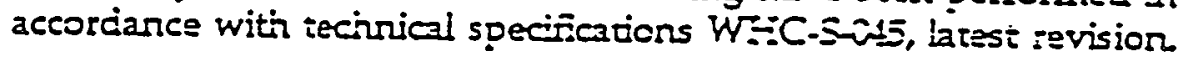
7.i. All test instruments have a vaiid calibraion stamp attacied that incicates a caibracion traceabie to the :Vational Institute or Standarcs and Iechnology.
i.1.j Mechods or water disposal usec in this AF have been approved by the izciities Management
… Personnei responsible ior direcing withessing anc periorning the tess

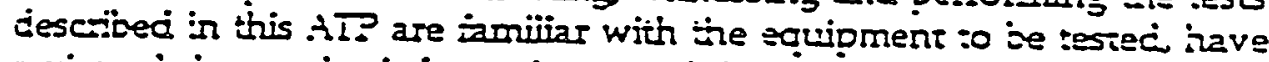 reviewed the vencior iniormation pertaining to the operation of tie equipment, and ase iamiiliar witi the requiremens of this acceptance test procedure

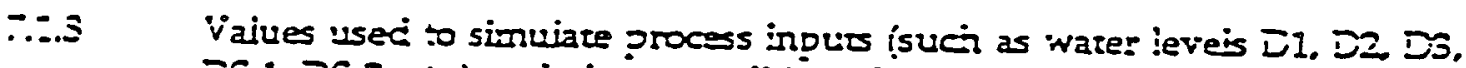

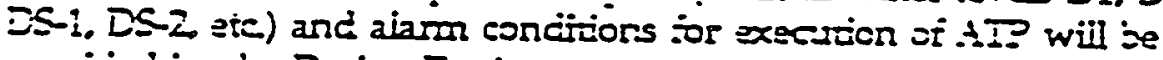 proviced jy the Design Engineer.

$\therefore \quad$ EQLTPMEVT REQLIRED

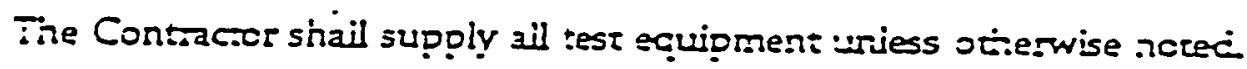

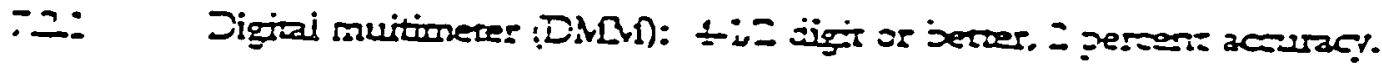

in Seven shoring junpers with ON-OFF switch, 14-0age wire, 12 inch approximate length

7.23 Clamp-on Ammeter (COA): Scale 0-6 amp.

724 One highway water tanker, 5,000 gallon capacity, with discharge capability, with comparable KAMVAIOK fitting 1673-A, 3 inches diameter.

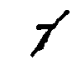


7.2.5 Water level transducer (WLI): $0-10$ psi, precalibrated with sufficient cable to reach from primary sump to control building (approximately 200 feet). Altematively, a water level indicator or other measuring device as approved by the Project Engineer may be used, provided that real-time communications with personnel in the control room are established.

-26 Measuring tape, measuring rod, folding scale, or equivalent: $6 \mathrm{ft}$ (r.Lin.), +10.5 in.

7.27 Dry Ice (summer only).

7.2.8 Hairdryer or equivalent forced air heater (winter).

729 Water Container (barrel), 60-inches deep minimum.

7.2 10 Receptacle Tester: Haind-held device which verifies correct connections of receptacie and simulates ground-fault condition. Ideal \#61-051 or equal.

7211 Valve for pump discharge line.

7212 Pressure gages for fump discharge line, 0 to 50 psi range (valved) and 0 to 200 psi range.

8.0 ELECTRICAL HEA IIIACE SYSTEM

Inis procedure will demonstrute the correct functioning of electrical heat trace system and the temperature sensing (thermostat) devices. Verify and record the folloving:

\subsection{TEMPERATURE SENSOR}

Record all data on Test Data Form \&-1 (see Appendix A).

8.1.1 Energize heat trace circuit (close breaker B8). Disconnect the power conductors to the thermostat.

8.1.8 Visually inspect the sensor and contacts for damage.

8.1.3 Using digital multimeter set to measure resistance (ohms), place connections on opposite sides of thermostat contacts.

8.14 Cool or heat (depending upon exterior temperature at time of testing) thermostat sensor to simulate outside temperature changes. 
WHC-SD-W025-RPT-001, Rev. 0.

WAIESDWO2S-ATP=001 ReV.2

8.1.5 Observe multimeter output during testing. When simulated temperature of themostat is above $40^{\circ} \mathrm{F}$ (approximately), the resistance should be high (above 500 ohms), indicating that the contacts are open. When the simulated temperature is below $40^{\circ} \mathrm{F}$ (approximately), the resistance should be low (below 2 ohms), indicating that the contacts are closed. Verify both conditions.

8.1.6 Remove meter connections and reconnect power conductors.

\subsection{HEAT TRACE CABLE}

Record all data on Test Data Form 8-2 (see Appendix A).

8.2 .1 Energize heat trace circuit (close breaker B8).

8.22 Manually force the contacts on the thermostat closed. Measure the current in the conductors to the heat trace cable using COA. Current should be 5 amps minimum (note: current is dependent upon temperature of heat trace cable. The current will increase as the temperature of the cable decreases).

8.23 Manually force the contants on the thermostat open. Measure the current in the conductors to the heat trace cable using COA. The current should be less than 0.1 amps.

\subsection{TRANSDUCERS AND METER/CONTROLLERS}

- This procedure will demonstrate the correct functioning of the level transducers and the associated meter/controllers and relays. See Figure 1 for definitions of water levels and associated pumps and relays. Verify and record the following.

\subsection{PRIMARY SUMP}

Record all data on Test Data Form 9-1 (see Appendix A).

9.1.1 Remove power to pumps W5P1 and W5P3 by opening circuit breakers B1 and A7.

9.1.2 Through the vertical riser pipe, install the water level transducer (WLT) in the bottom of the primary sump leachate collection well with remote readout in the control building. Verify that the reading is equal to the water level in the sump (if any), as measured with a tape measure, measuring rod, or similar device. Alternatively, measure the water level in the sump with the water level indicator and calculate the depth of water. 
WHC-SD-W025-RPT-001, Rev. 0

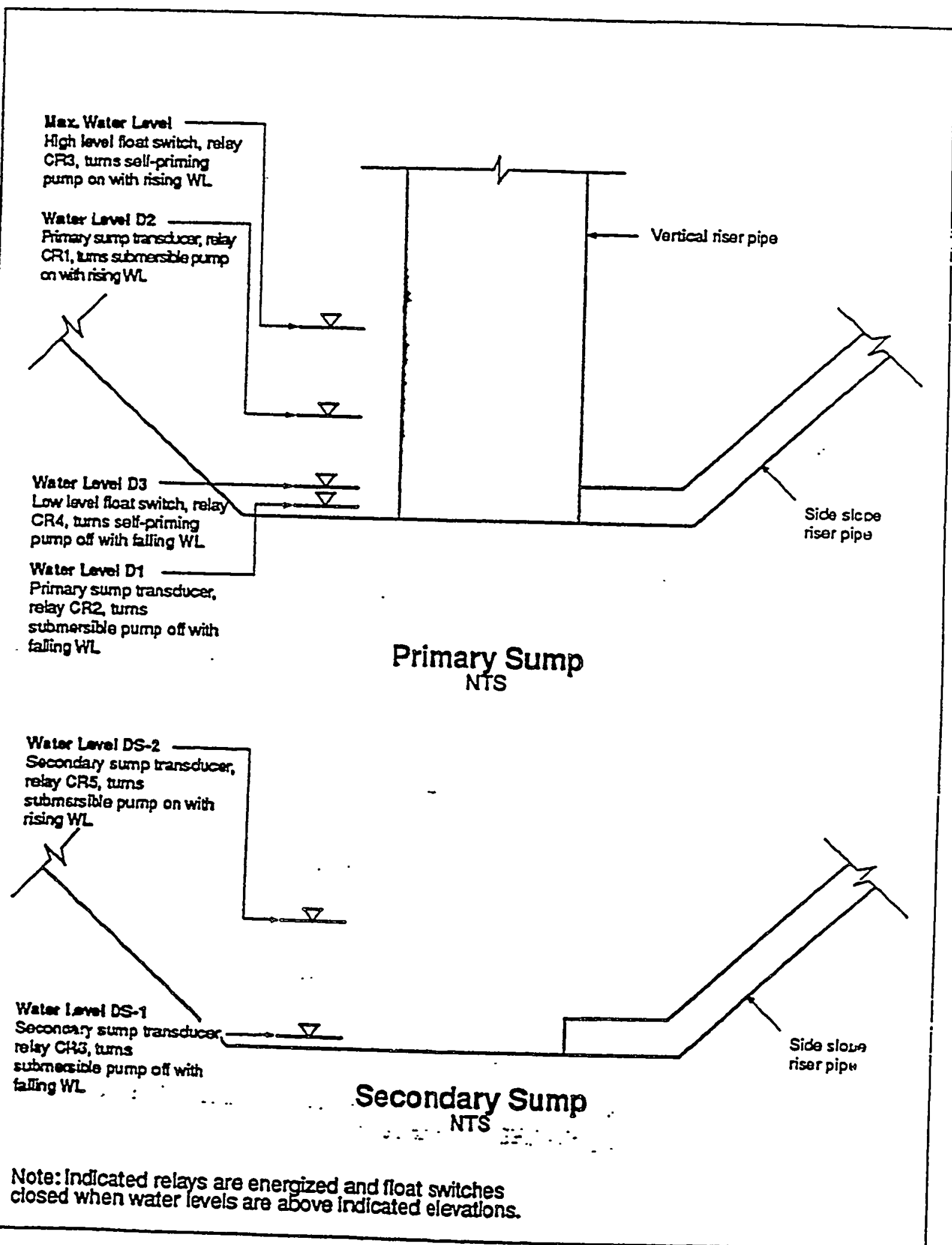

933 1354/49332/3-28-94

Figure I Water Level Definitions. 
WHC-SD-W025-RPT-001, Rev. 0

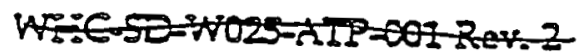

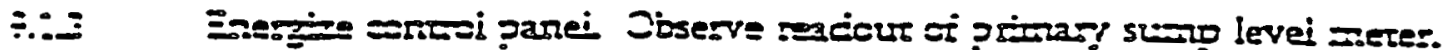

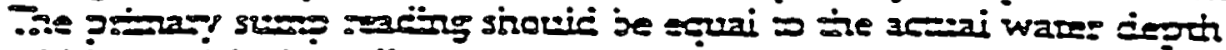

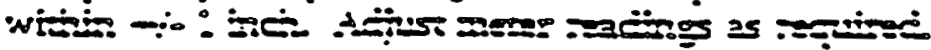

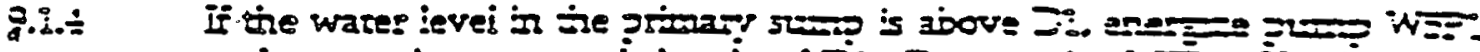

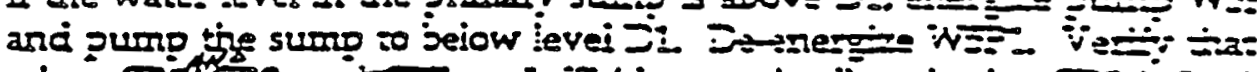

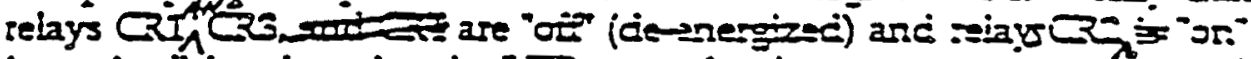
(energized) by oiserving the $T=D$ on eacin reiay.

9.1.5 Begin adding potable water to the primary sump througi the versical sise: pipe Add water at a wrirom rate so that the sise of the water level in the leaeizate collection weil is no nore war 2 ineines/ninute. Take care to avoic exessive nusuienes during illing. if necssary, siog alling at 2-irch intervis and allow the wateg level to scabiline Recorc the actual ise in the surno level and on the Dirnary Sump Levei Meter at $I$ inci intervis. Both reacings shouid be within $\div$ - I inci. Conminue througiout alling.

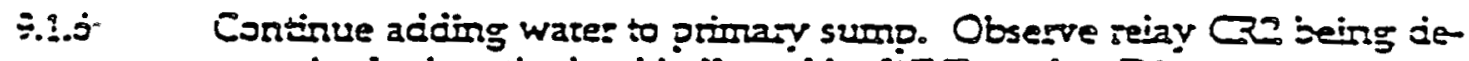
energized when the level inciared jy WT $_{\bar{i}}$ reacizes $\overline{\mathrm{D}}$

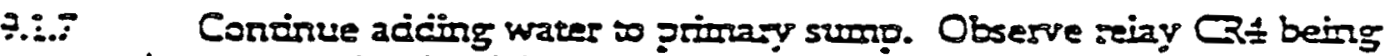
forl if de-energized at level Ds and jelay Cíl being energized ar leved DI Stop illing surno.

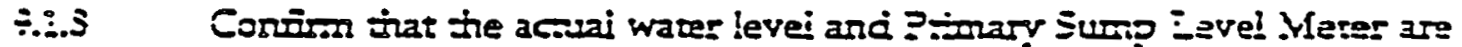
withir $\rightarrow-1$ incis

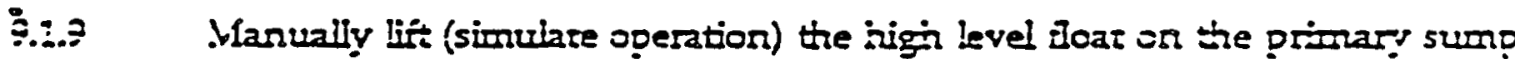

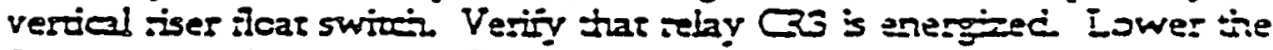

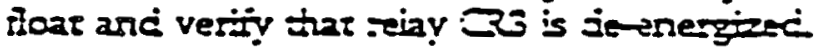

\section{TECONDARY STMP}

Record all data on Iest Data Form 22 (see Appendix A).

921 Pump the secondary sump to below level DS-1. Remove power to punp WSP2 by opening circuit breaker $B 5$.

922 Verify that relay CRS is "off" (de-nergized) and CRS is "on" (energized) by observing the LED on each relay. 
WHC-SD-W025-RPT-001, Rev. 0

WFE-SO-NOESATP-DI REV ?

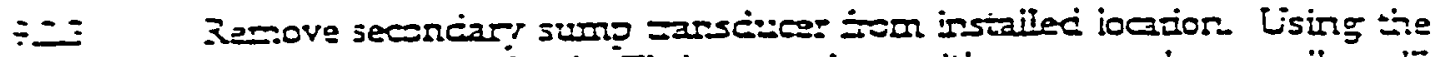

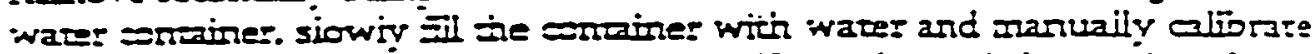

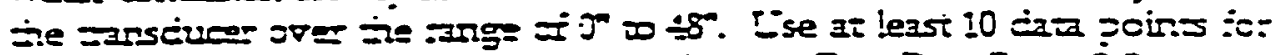

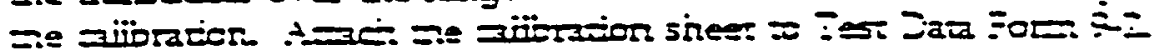

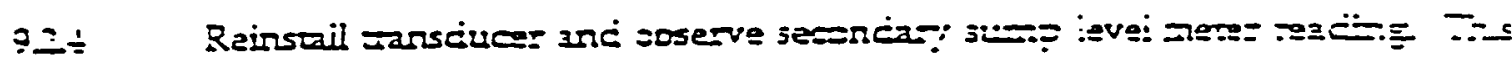
sirould be near DS-i.

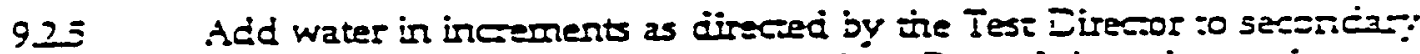
sump via the secondary slope siser pipe. Recorc ine voiume or wate: added to the sumo. Wait 2 sinutes arter each increment of water, and record the secondary surnp level meter reading Verify that the seconciar! sump level meter reading inceases as water is acded.

$9=5 \quad$ Continue adding wares in incements and observe that relay $C$ 36 is deenergized when the !evel is $\overline{D S}$ 1, and that $Q R$ is enezgized wisen the !eva! is DSE Stop alling sump.

\section{$: 0.0$}

PUNF TESIS

This procedure will demonstrate the conect iuncioning oi the punps and asssciatec

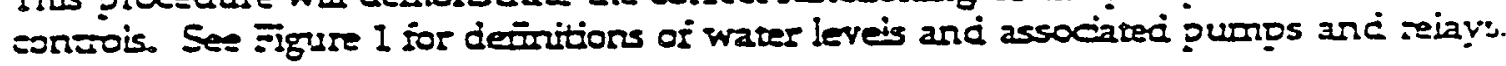

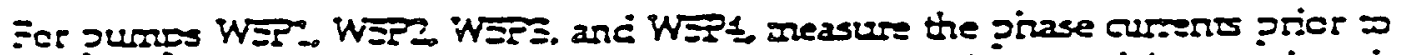

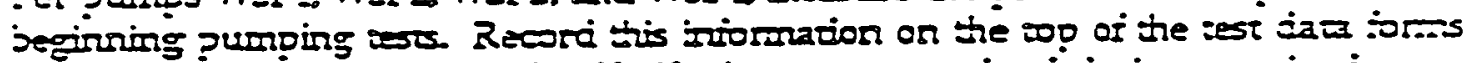

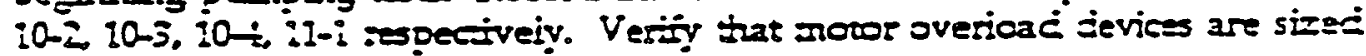
sorsecty.

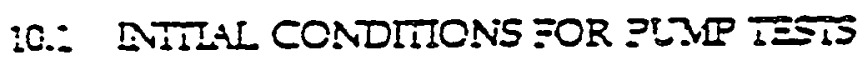

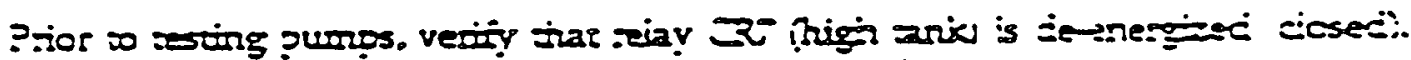

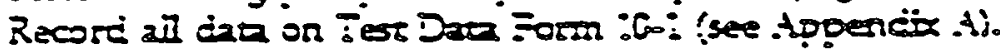

10.1.1 Rimary Sumeng

This testing should begin with water in the prinary sump above level D2 Verify the following concitions on the control panel:

2. CRI is energizer.

b. $\mathrm{QR2}$ is de-energized.

c $C^{3}$ is de-energized.

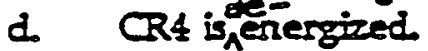

e. Pump filure light for the primary submessible punp is on

E. The roof alarm light is on is

g. The ganel lights associated with relays $C R I_{\lambda}$ 
WHC-SD-W025-RPT-001, Rev. 0

WICSE-WO25-AIT-001 keV.?

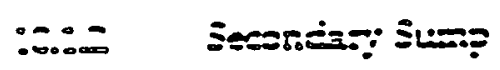

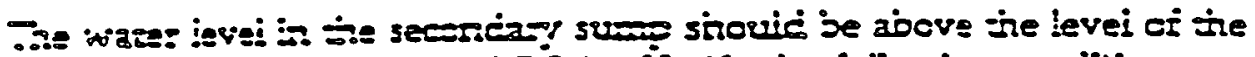

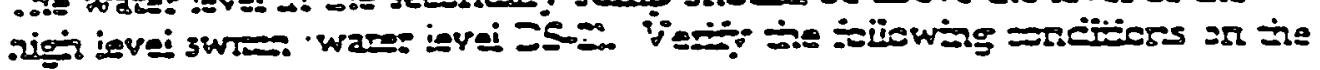
sorscoi pareis
2. CRS is energizec.
b. $R^{6}$ is de-energized
c Pump jillure ligint for the seconcary submessibie purno is $=\Omega$
d. The roor alaran ligint is on.
e. The panel light associated with relay $\mathrm{C} 5$ is on

\section{PRMARY STBWERSTBLE PUMP (WSPT)}

Record all dacs on Test Dat Form 10-2 (see Appendix s).

:0.: Tre switci on the concol panel for the punp should ise in the "vi" posicion. Close circii jreake: ji.

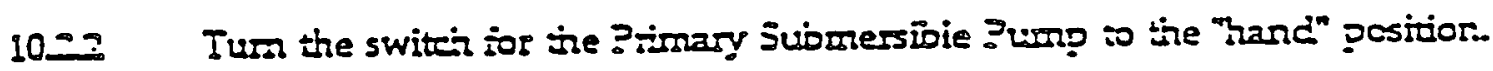

1093 Verify by airect visual ocservation that wares is being gumped jom the sump into the storge tank

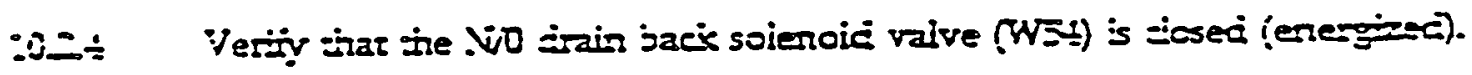

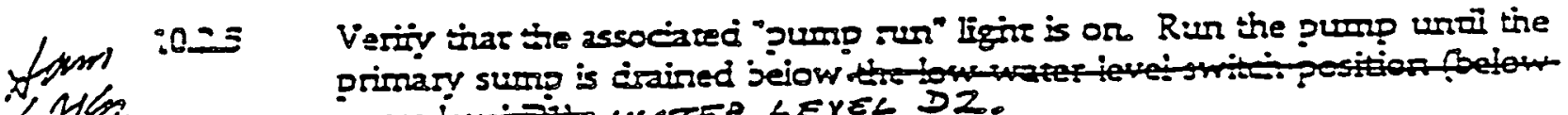

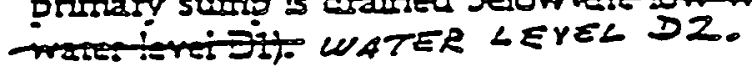

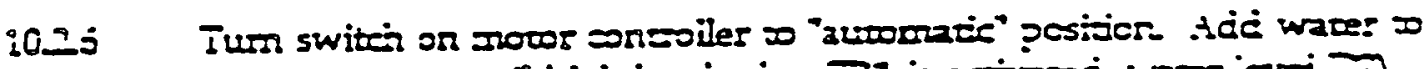
?.

10:- Vexily that the purn sars automatially and that the pump ren ligit is on

1028 Verify by direct visual observation that water is being pumped from the sump into the storage tank

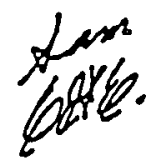

1029

Verify that when the water level in the sump reaches low level (water level D1), relay $\mathcal{R}_{2}$ is energized, and the pump is automationly shut off

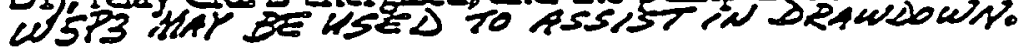

10210

Add water to the primary sump to above.level D1. Manually initiate operation of Timer TIMI and verify that the purnp is automationly started. 


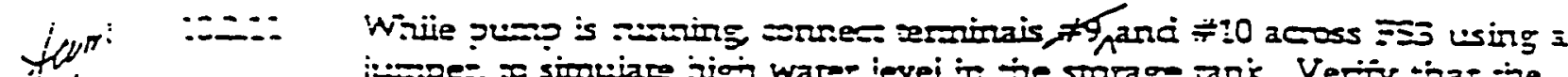

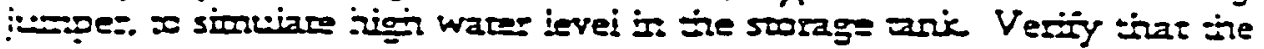

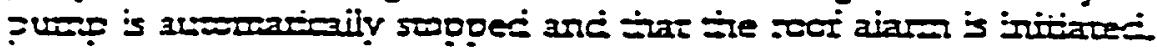

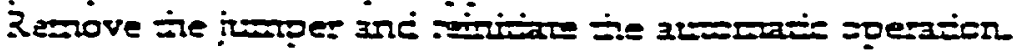

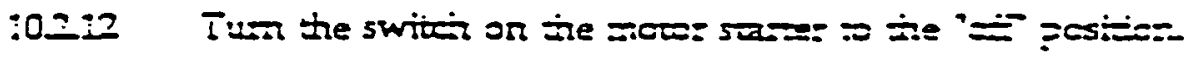

103 SECONDARY SUBMERSIBL ELAL (WEF)

Record all data on Test Data Form i0-3 (see Appendix $t$ ).

$$
10.2 \cdot 11
$$

Repeat steps 1021 througi 1023 and 10220 except for the seconcary suburasibie pump, accing water to the secondary sump through the secondary siser pis e as required

I0.: PRLARY SURP SEL =TRRING PT:RP (NSF3)

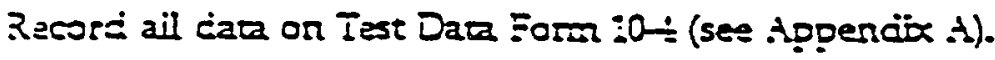

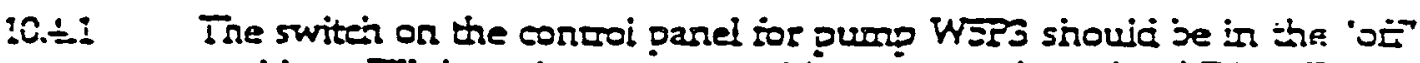
position Fill the prinagy sump witi water to above level I2 C.cse cireuit breaker

$: 0 .:$ Tun the switic on the contel pand to the hanc" position

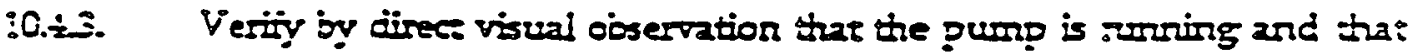

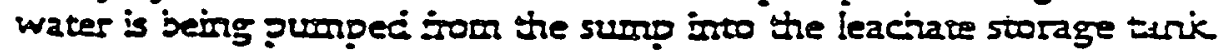

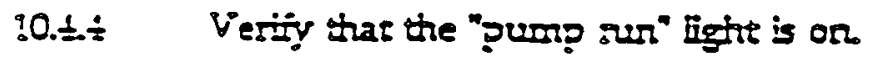

:0.15 Verijy operacion sor sne ạinure.

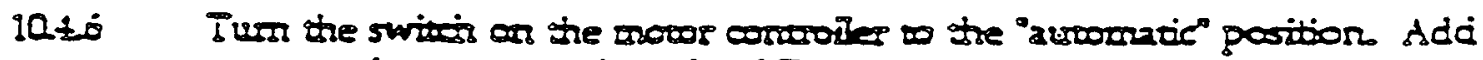
water so the semps to above leved D2.

ICII Manually raise the high level vertial niser float switeh to simulate high water condition.

10.4 .8 Verify that the punp is automationlly started.

10.4.9 Verify that the punp continues ronsing until the water level reaches the low level verticil niser Hoat switeh (water level D3), when the punp is automatiolly shut off.

$$
10.2 .11
$$

10.110 Add water to the primary sump and perform step 1029 lor this punp. 
WHC-SD-W025-RPT-001, Rev. 0

WHE-SD-W025-ATP-001 Rev.-2

\subsection{FLOWMETER CALIBRATION}

Record all data on Test Data Form 10-5 (see Appendix A).

10.5.1 Disconnect the 3-inch-diameter discharge line at a convenient location between the 3-inch flowmeter and the leachate temporary storage tank

10.5.2 Position the water barrel (empty) so that it will collect all discharge through . the 3-inch flowmeter.

10.5.3 Verify that ball valve $W 65$ is open and ball valve $W 66$ is closed.

10.5.4 Record the readings of the 2-inch and 3-inch flowmeters on Test Data Sheet 10-5.

10.5.5 Verify that there are at least 200 gallons of pumpable water in the secondary sump.

10.5.6 Energize the secondary submersible pump until at least 50 gallons have been pumped into the barrel. De-energize the pump.

10.5.7 Measure the depth of water in the barrel and record on Test Data Sheet 105. Record the readings of the 2-inch and 3-inch flowmeters on Test Data Sheet 10-5.

105.8 Empty the barrel and reposition to receive the discharge through the 3-inch flowmeter.

10.5.9 Energize the secondary submersible pump until a constant discharge is achieved (determine by visual observation). Immediately de-energize the pump. Wait 60 seconds. Repeat this cycle 9 more times (total of 10 cycles), in each cycle energizing the pump for the time determined in cycle 1 . However, do not allow the barrel to overflow; repeat the cycle fewer than 9 times if necessary. Record the number of cycles and the cycle time on the Test Data Sheet 10-5.

1C 5.10 Measure the depth of water in the barrel and record on Test Data Sheet 105. Record the readings of the 2-inch and 3-inch flowmeters on Test Data Sheet 10-5.

10.5.11 Empty the barrel, empty the secondary sump, and reconnect the 3-inchdiameter discharge line between the 3-inch flowmeter and the leachate temporary storage tank 
WHC-SD-W025-RPT-001, Rev. 0

WHCSD-WO2SAIR-DOI REV 2

\section{10.6 SPARE PUMP IESTS}

Test spare pumps at the ground surface to verify that they function. Test submersible pumps in a "barrel" that allows complete immersion. Record all data on Test Data Form 10-6.

12.6.1 Install the valve on the pump discharge line to simulate head.

1ii.6.2 Install the pressure gage between the valve and pump to measure the head.

2ij.5.3 Energize the pump and allow it to achieve steady state conditions at a pressure of approximately $90 \%$ of the maximum efficiency discharge head as shown on the manufacturer's pump pefformance curves. Measure the volume of water pumped for at least 30 seconds.

10.6.4 Repeat the test with the pressure set at about 110\% of the maximum efficiency discharge head.

13.65 Repeat the test with the discharge valve closed to determine the "shutoff" pressure.

10.6.6 Repeat steps 10.6.1 through 10.6.5 for all spare pumps.

\subsection{LEACHATE STORAGE TANK}

This procedure will demonstrate correst funcoioning of the transfer pump and controls associated with the leachate storage tarik. Yerifj and record the following.

\subsection{LEACHATE STORAGE TANK PUMP (W5F4)}

Record all data on Test Data Form I1-I (see Appendix A).

13.1.I Verify that the storage tank is at least 25 percent full of water. Iurn on circuit breaker AI for the pump.

11 1\% Using the DMM, measure the voltage at the motor terminals (I1, T2. T3). The voltage should read 0 between tarminals II \& $I 2, I 2 \& T 3$, and between $I I$ and the metal frame.

11.3.3 Push the "start" pushbutton on the motor starter. Verify that the rartor is operating correctiy with proper rotation. Verify by direct visual observation that water is being pumped from the tank. 
WHC-SD-W025-RPT-001, Rev. 0

WIE-SD-WO25-ATP=001 Rev.2

11.1.4 Using the DMM, measure the voltage at the motor terminals. The voltage should read between 460 and 500 volts between terminals $I I$ \& TZ and between terminals $T 2 \&$ T3.

11.1.5 Continue pumping for 2 minutes minimum. Push the "stop ${ }^{n}$ pushbutton and verify that the pump stops running.

\section{11.2. HIGH LEVEL FLOAT SWITCH (FS3) AND ITMIT SWITCH (LI)}

Record all data on Test Data Forms 11-2A and 11-2B (see Appendix A).

11.21 Observe that the red "high tank level" light on the control panel is off and that relay $C R T$ is de-energized. Disconnect limit switch $L 1$.

1122 Fill the storage tank with water to above the high level. Verify that FS3 operates at the selected "high water" level

11.2.3 Observe that relay $C R 7$ is energized and time delay is functional.

11.24 Observe that the red "high tank level" light on the control panel is on.

11.2.5 Observe that the roof alarm light is on and time delay is functional.

11.2.6 Verify that the submersible pumps and self primary pumps will not operate (3 pumps total) by temporarily tuming the appropriate control switches on the control panel to "hand" position and observing that the associated "pump run" light does not come on.

11.27 Lower the water level in the tank and observe that FS3 returns to normal condition (repeat step 1121).

11.2.8 Reconnect limit switch LI and disconnect the high level float switch FS3.

11.29 Repeat steps 11.21 through 11.2.7 to test L1. Record all data on Test Data Form 11-2B (see Appendix A).

11.210 Reconnect high level float switch FS3.

\subsection{BUILDING POWER AND LIGHTING}

This procedure will demonstrate the correct functioning of control building power and lighting, and area lighting. Verify and record, the following: 
WHC-SD-W025-RPT-001, Rev. 0

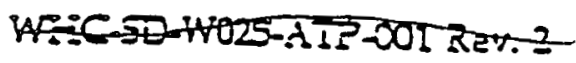

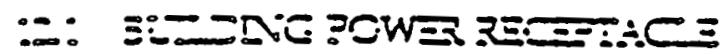

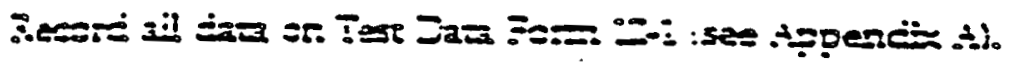

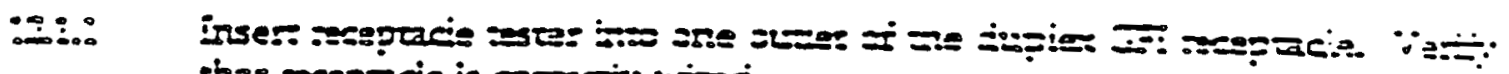
that seceptreis is sorstriy virs:

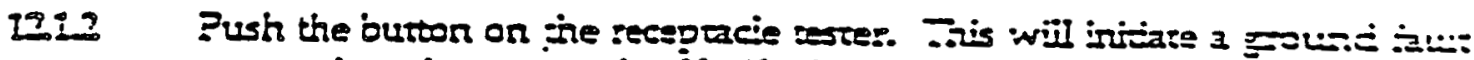

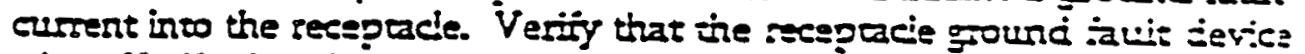
trips. Verify that the outler is now denergized (observe that ail ine ligins on the tester are now oin).

Igis Repeat Step IZII jor the ouher outlet

\section{9- BLILDING LIGESTIU FDTLRES}

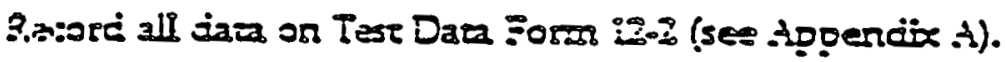

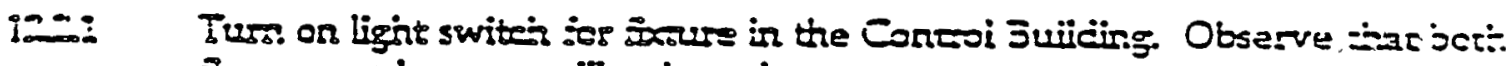
Ifuornsient lamps are illuminated

\section{:O" AREALIGITIVS FLIURES}

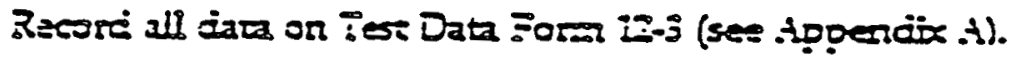

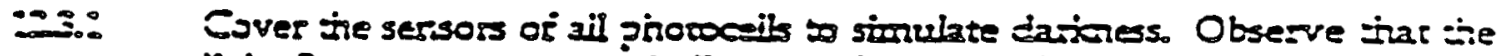

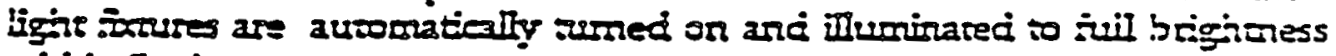
witrin 5 mirures.

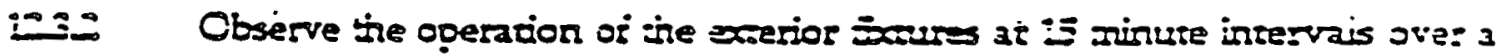

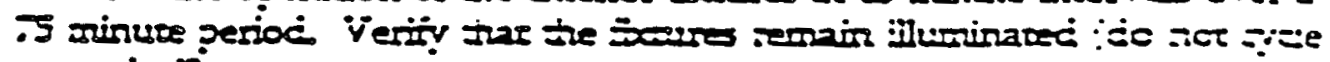
on anc बiने

Renove coves irous photocil sersors. Vexify that all fitures turs uff.

\section{I2: CONIROL PANEL HEATERS}

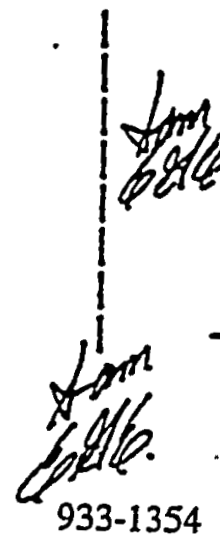

Record all data on Test Data Form 12-4 (see Appendix A).

1241 Turn thermostat on heater in Control Panel $X$ off Verify that heater is at ambient temperature. Wait for heater to coll if necessary.

1242 Turn themostat until it energizes. Verify that heater becomes warm.

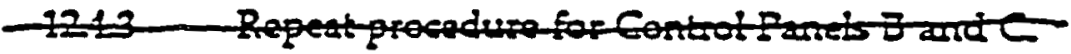

$$
18
$$




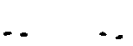

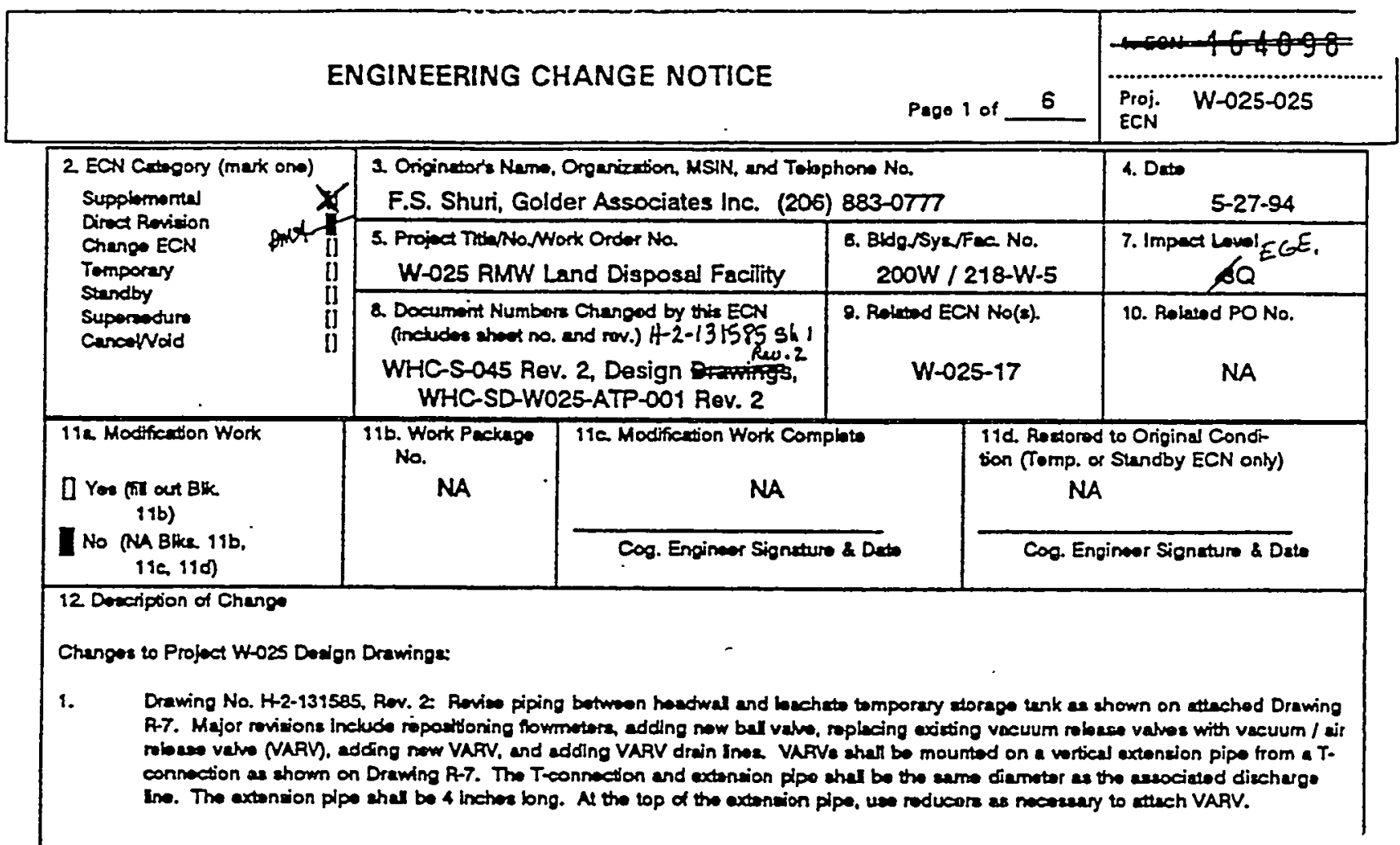

(Continued on Sheot 3)

\begin{tabular}{|c|c|c|c|c|c|c|}
\hline $\begin{array}{l}\text { 13e tustifi } \\
\text { ono) }\end{array}$ & & Criteria Change & & Design Improvement & D & Environmental \\
\hline Asfound & 0 & Fecifted Conse & Д & Cont Eror/Omiation & D & Dorign Emor/Omiecion \\
\hline
\end{tabular}

13b. Kufifertion Doteile

Improve occuracy of flowmetars and prowont ary bakago of bechate.

14. Divtribution (include name, MSIN, and no. of copios) WHC DISTRIBUTION

\begin{tabular}{|c|c|}
\hline 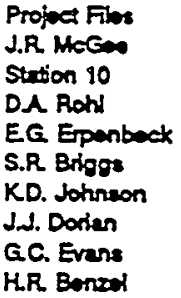 & $\begin{array}{l}G 6-47(1) \\
66-47(1) \\
A 3-67(1) 2 \\
5401(1) \\
G 5-47(3) \\
C 5-47(1) \\
H 2-58(1) \\
B 2-16(1) \\
46-23(1) \\
T 4-03(1)\end{array}$ \\
\hline
\end{tabular}

ATP00-013-2 (08/92) CEFOOS 


\section{ENGINEERING CHANGE NOTICE}

Page 2 of 6

1. ECN (use no, from pg. 1)

W-C25-025

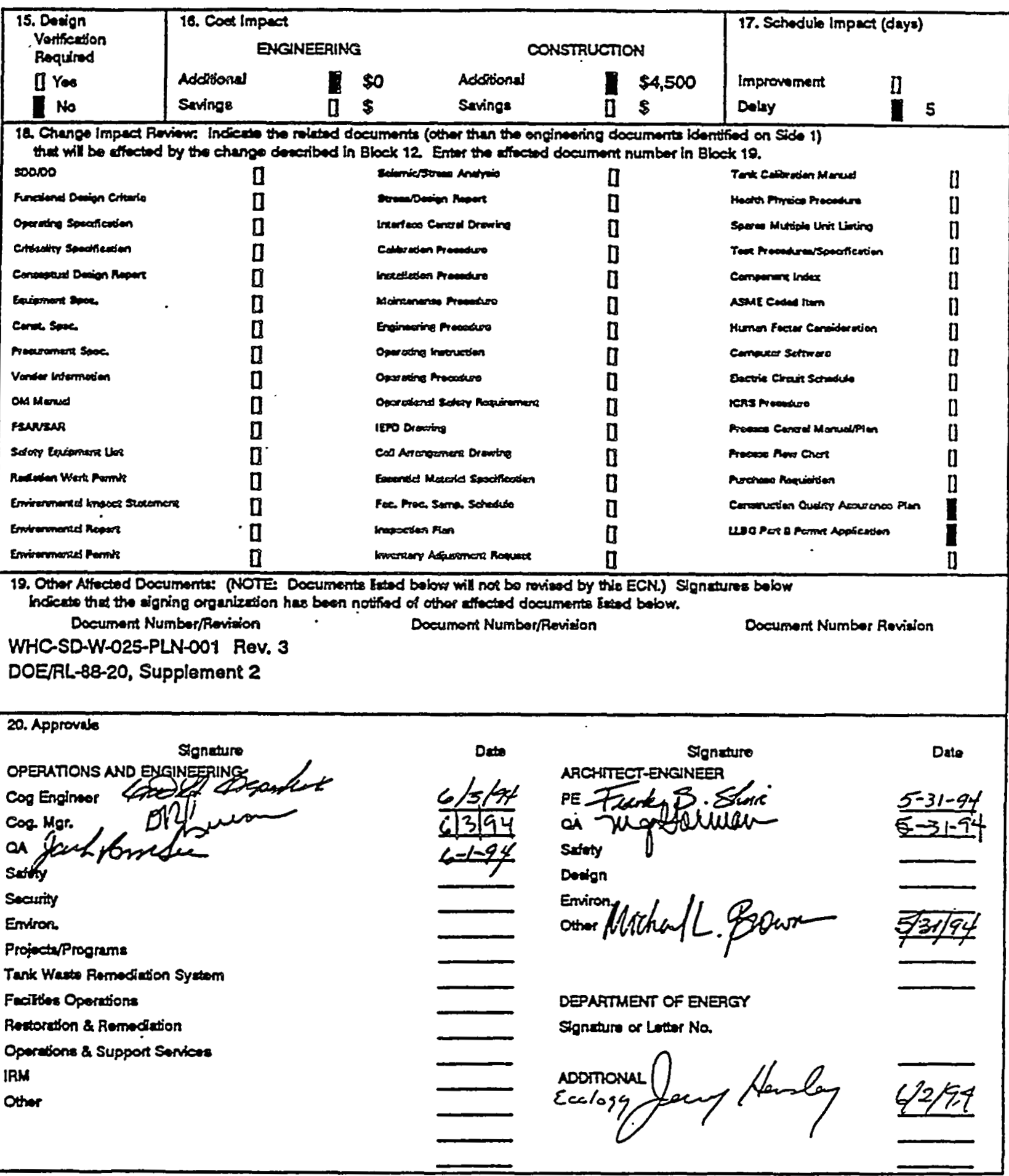


WHC-SD-W025-RPT-001, Rev. 0

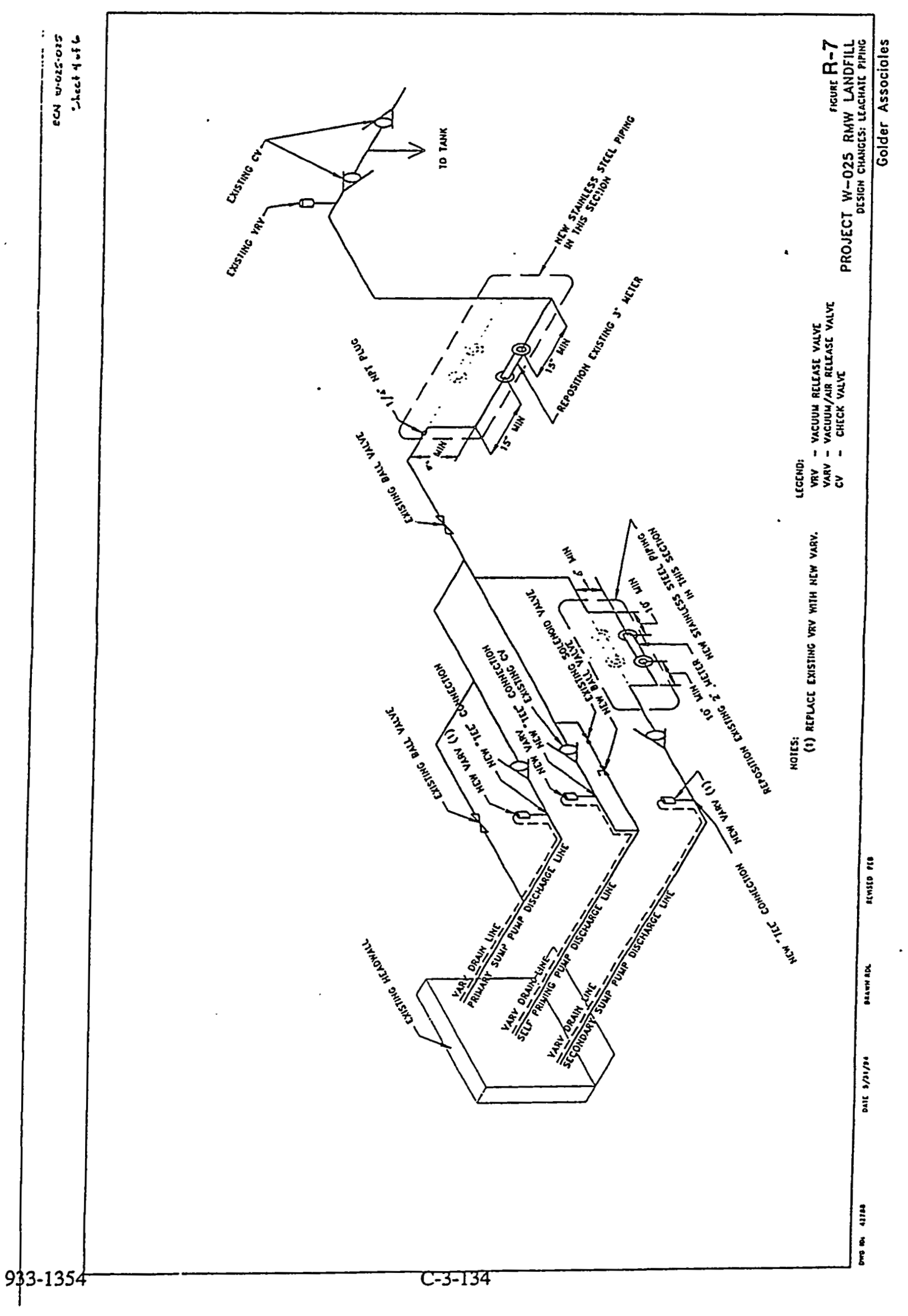


WHC-SD-W025-RPT-001, Rev. 0

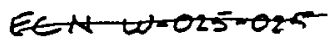

Sheets- 5 .

\subsection{FLOWMETER CALIBRATION}

Record all data on Test Data Form 10-5 (see Appendix A).

10.5.1 Verify that there is sufficient water in the secondary sump to pump 500 gallons and still maintain a final water level of at least 8 inches.

10.5.2 Verify that the water in the sump is clean and will not produce foam when pumped. Flush sump if necessary until clean, non-foaming water is obtained.

10.5.3 Verify that ball valve $W 65$ is open and ball valve $W 66$ is closed. Verify that the ball valve in series with the solenoid draindown valve is closed.

10.5.4 Measure the water level in the leachate temporary storage tank to the nearest 0.01 foot with a rigid rod. Measure through port in top of tank If necessary, add water until depth in tank is at least 0.3 feet.

10.5.5 Remove 1/4inch plug immediately upstream of 3-inch flowmeter. Energize secondary submersible pump. Pump until air no longer escapes through $1 / 4$ inch hole. Replace plug. De-energize pump.

10.5.6 Measure the water level in the leachate temporary storage tank as described in step 10.5.4. Record on Test Data Sheet 10-5.

10.5.7 Record the readings of the 2-inch and 3-inch flowmeters on Test Data Sheet 10-5.

10.5.8 Energize the secondary submersible pump and pump at least 500 gallons into the leachate temporary storage tank

10.5.9 Measure the water level in the leachate temporary storage tank as described in step 10.5.4. Record on Test Data Sheet 10-5.

10.5.10 Record the readings of the 2-inch and 3-inch flowmeters on Test Data Sheet 10-5. If the meter readings differ from the calculated volume (based on measurements of water depth in the tank) by more than 5 percent, repeat step 10.5 .5 using the self-priming pump (primary sump). Ensure that there is sufficient water in the primary sump prior to purging the discharge pipes. Continue the AIP from step 10.5.6 using the secondary submersible pump.

10.5.11 Repeat steps 10.5.8 through 10.5.10 twice, for a total pumped volume of about 1500 gallons. 
WHC-SD-W025-RPT-001, Rev. 0

Eentw-025-025

Shect 6 of

IEST DATA FORM 10-5

PUMP TESTS - FLOWMETER CALIBRATION

\begin{tabular}{|c|c|c|c|c|c|}
\hline Step No. & Activity & Verify & Reading & Date & By \\
\hline 10.5 .1 & Verify water volume & & NA & & \\
\hline 10.52 & Verify water clean & & NA & & \\
\hline \multirow[t]{3}{*}{10.5 .3} & Ball valve $W 65$ open & & NA & & \\
\hline & Ball valve W66 closed & & NA & & \\
\hline & Solenoid ball valve closed & & NA & & \\
\hline 10.5 .4 & Water level in tank & NA & & & \\
\hline 10.5 .5 & Purge air & & NA & & \\
\hline 10.5 .6 & Water level in tank & $\mathrm{NA}$ & & & \\
\hline \multirow[t]{2}{*}{10.5 .7} & 2-inch flowmeter reading & NA & & & \\
\hline & 3-inch flowmeter reading & NA & & & \\
\hline 10.5 .8 & Pump 500 gallons - 1st cycle & & NA & & \\
\hline 10.5 .9 & Water level in tank - Ist cycle & NA & & & \\
\hline \multirow[t]{3}{*}{10.5 .10} & $\begin{array}{l}\text { 2-inch flowmeter reading - 1st } \\
\text { Eycle }\end{array}$ & NA & & & \\
\hline & $\begin{array}{l}\text { B-inch flowmeter reading - Ist } \\
\text { Eycle }\end{array}$ & $N A$ & & & \\
\hline & Purge air & & NA & & \\
\hline \multirow[t]{8}{*}{10.5 .11} & Pump 500 gallons - 2nd cycle & & NA & & \\
\hline & Water level in tank - 2nd cycle & NA & & & \\
\hline & $\begin{array}{l}\text { Einch flowmeter reading - 2nd } \\
\text { Eycle }\end{array}$ & NA & & & \\
\hline & $\begin{array}{l}\text { 3-inch flowmeter reading - 2nd } \\
\text { Eycle }\end{array}$ & NA & & & \\
\hline & Pump 500 gallons - 3rd cycle & & NA & & \\
\hline & Water level in tank - 3rd cycle & NA & & & \\
\hline & $\begin{array}{l}\text { 2-inch flowmeter reading - 3rd } \\
\text { Eycle }\end{array}$ & NA & & & \\
\hline & $\begin{array}{l}\text { B-inch flowmeter reading - 3rd } \\
\text { kycle }\end{array}$ & NA & & & \\
\hline
\end{tabular}


WHC-SD-W025-RPT-001, Rey. 0

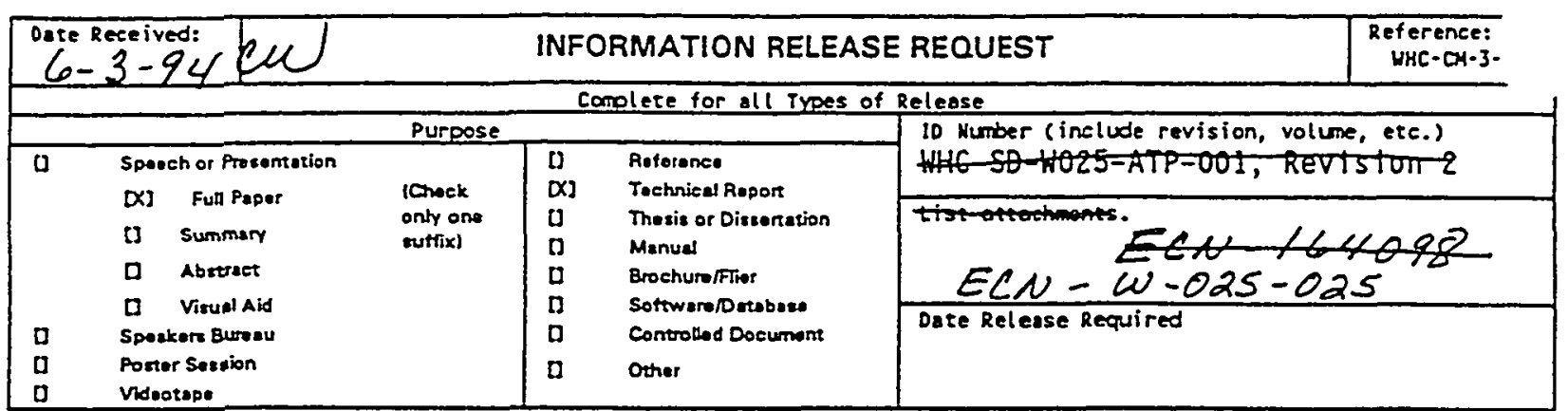

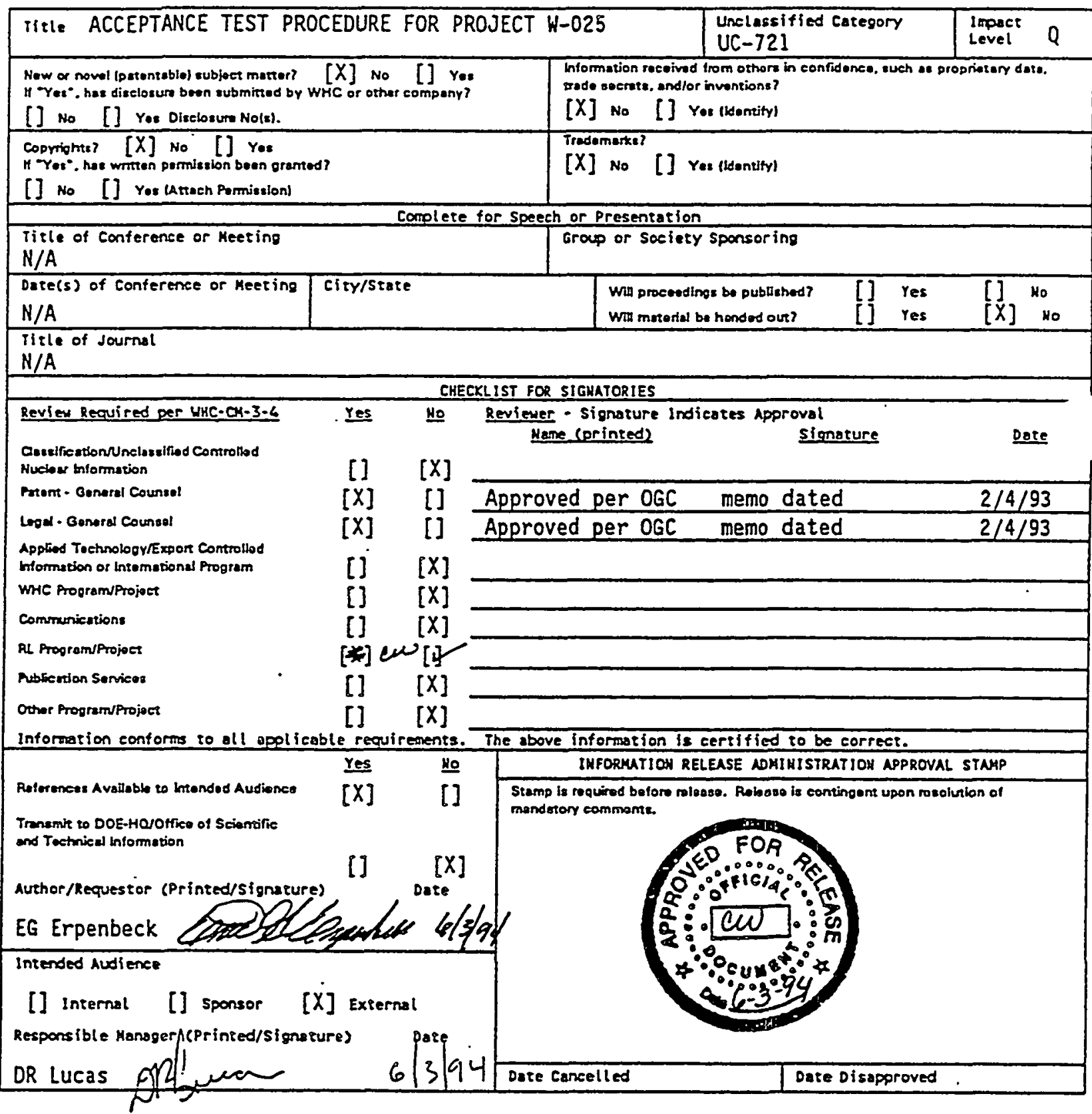


WHC-SD-W025-RPT-001, Rev. 0

\section{Golder}

Associates

surnect Cost Estimate- EN w-025.025 Job No. 923 -PO26

Rer.

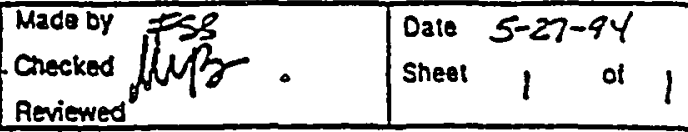

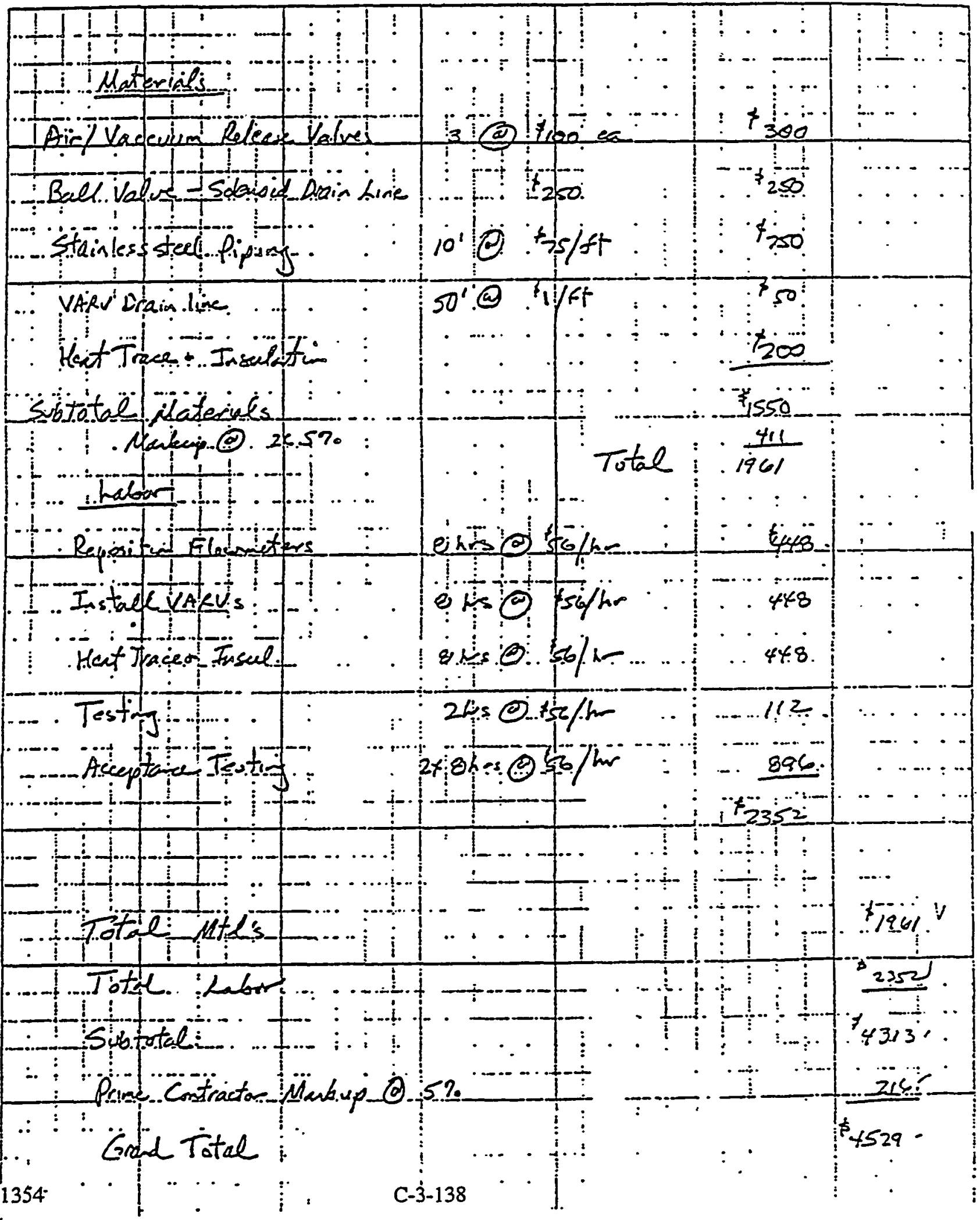


WTFDSOKF $2 B 5$

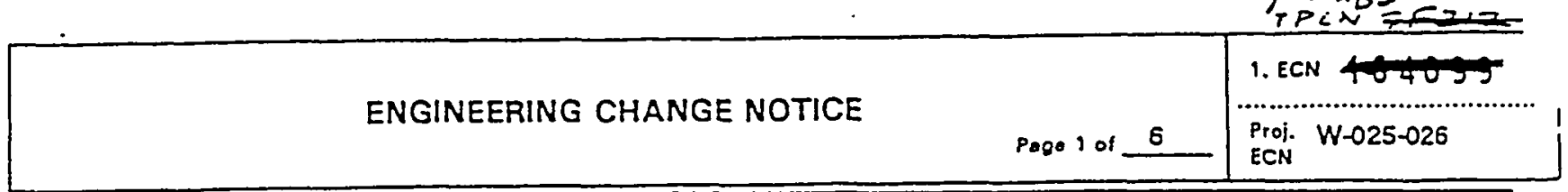

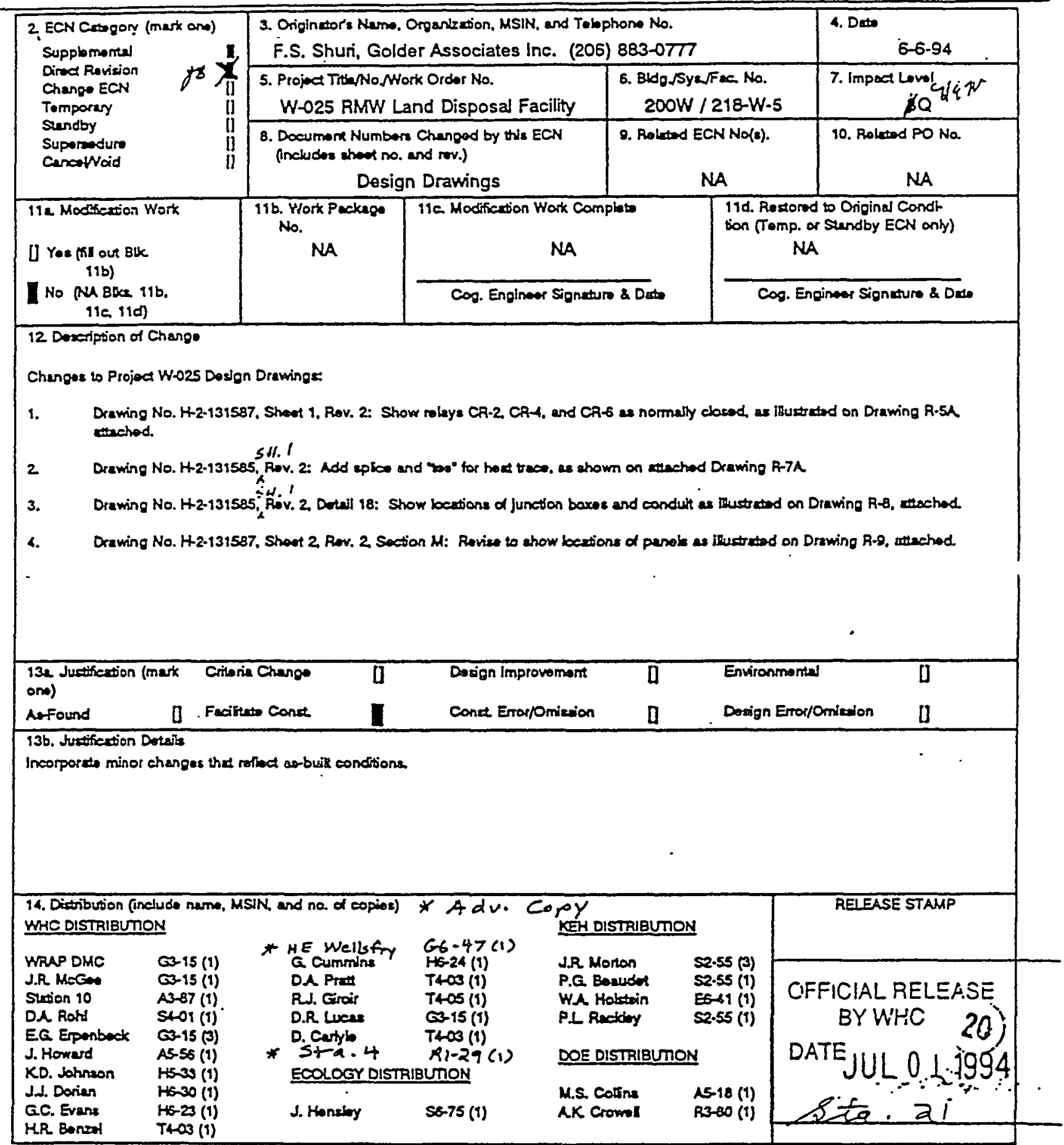

A-7500-013-2 (06/92) GEF095 


\begin{tabular}{c|c} 
Page $2 \propto 6$ & $\begin{array}{c}\text { 1. ECN (use no. tom PQ. 1) } \\
\text { W-025-026 }\end{array}$ \\
\hline
\end{tabular}

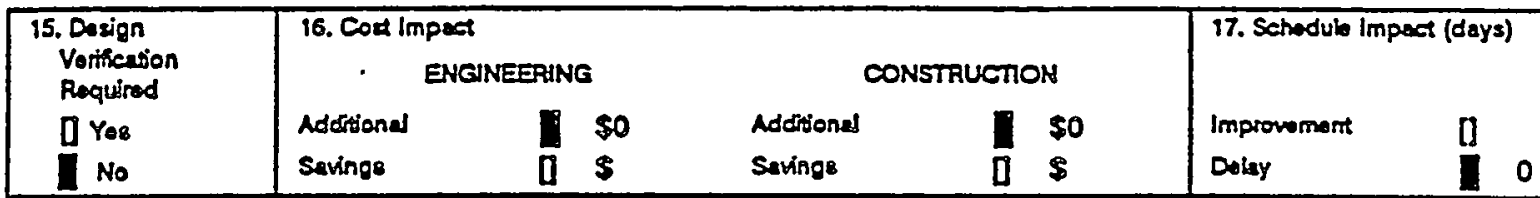

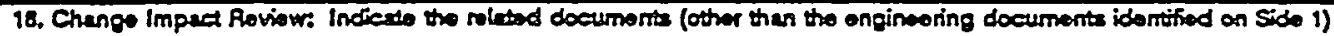
thet wil bo afiectad by the chango doscribed in Bbock 12 Enter the effected documert number in Block 19.

\begin{tabular}{|c|c|}
\hline $500 / 00$ & Satemiessom Anmina \\
\hline Probiond D-ion Cherle & somomion Raver \\
\hline Oporting sanifiention & 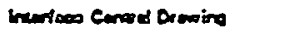 \\
\hline Criticeling samificotion & 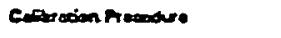 \\
\hline 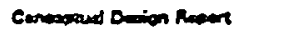 & 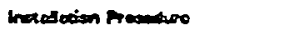 \\
\hline 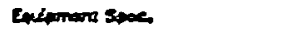 & 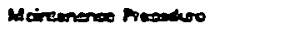 \\
\hline 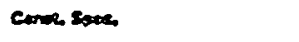 & 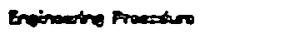 \\
\hline Provermans sooe. & overeting bounden \\
\hline Vonser intomotien & 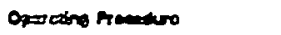 \\
\hline On Minus & 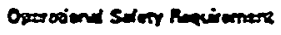 \\
\hline Feneser & Itro browins \\
\hline Suror Exipneri & 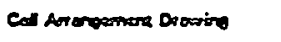 \\
\hline Rodiotien Wort romk & 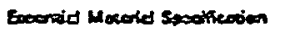 \\
\hline 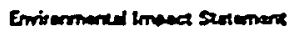 & 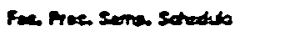 \\
\hline Enviormanted Rapart & meposion Mlos \\
\hline 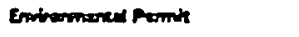 & 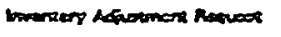 \\
\hline
\end{tabular}

Tent Curborion Hored

Hodth Mricies Preocturo

sooros Mutioso Unit linting

Teor frocoduressocoticuion

comeners budere

NSAE Cod ham

Menon fouer Cercidertion

comerer sernous

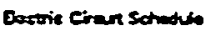

ICRS PTocoseso

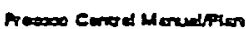

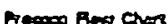

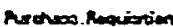

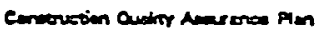

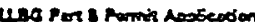

19, Othe Afrected Documente: (NOTE: Documents fed bolow will not bo revized by this ECN.) Signatures below

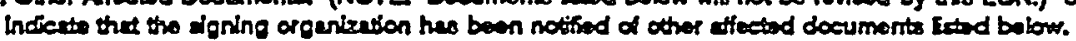
Doeumort NumbertRovizion
Documant NumbetRonision

Document Number Rovition

WHCSD-W-025-PLN-001 ReV. 3

DOE/RL-88-20, Supplement 2

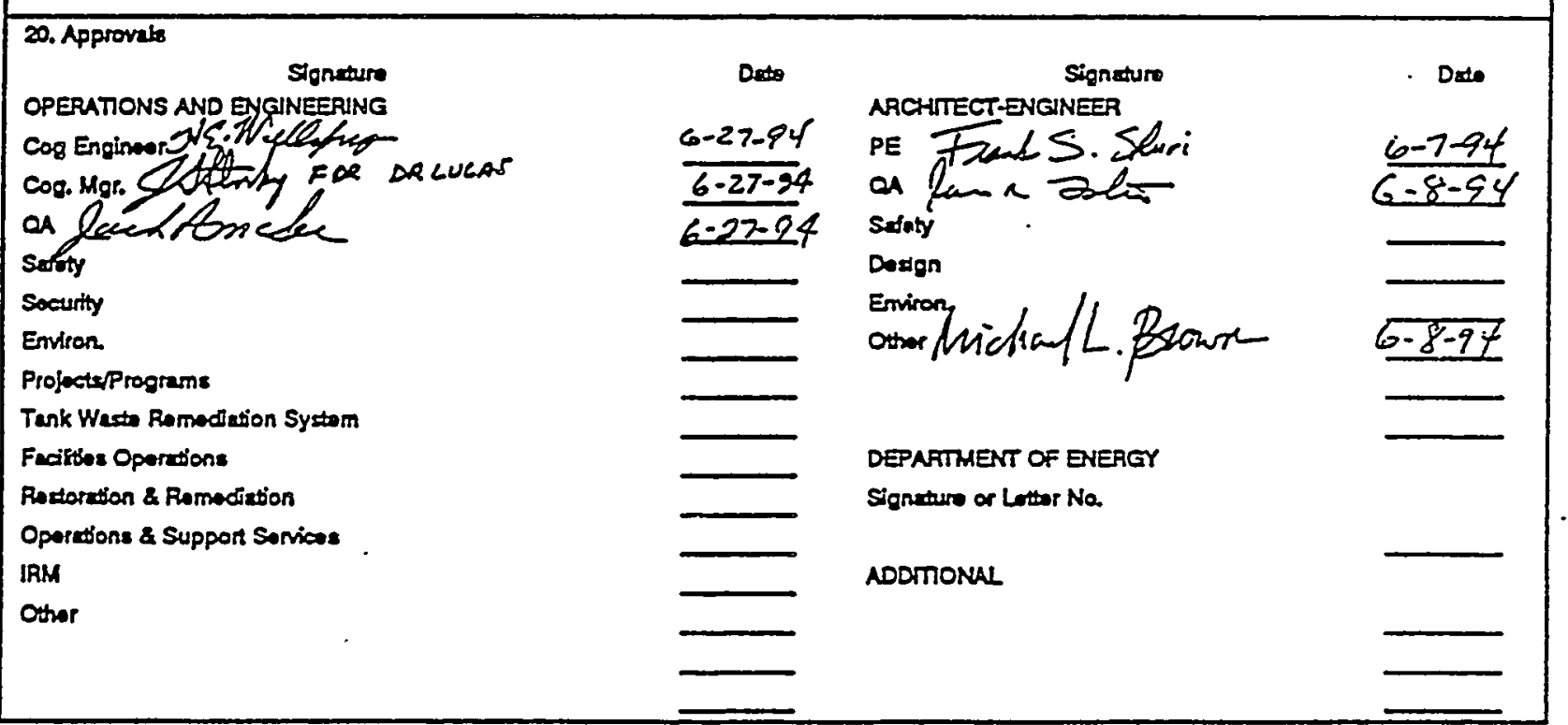


WHC-SD-W025-RPT-001, Rev. 0

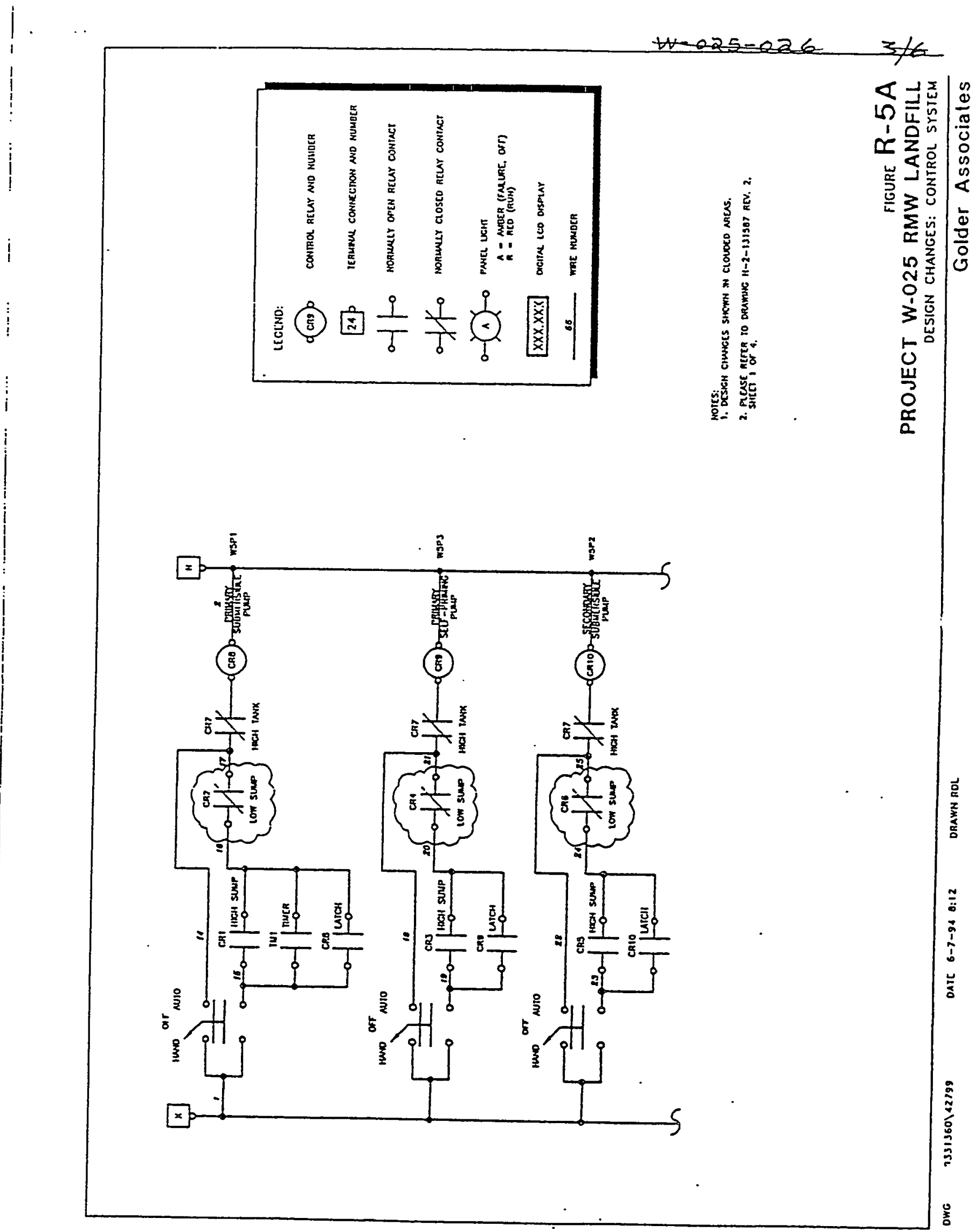


WHC-SD-W025-RPT-001, Rev. 0

$3-25-025 \quad 4 / 6$
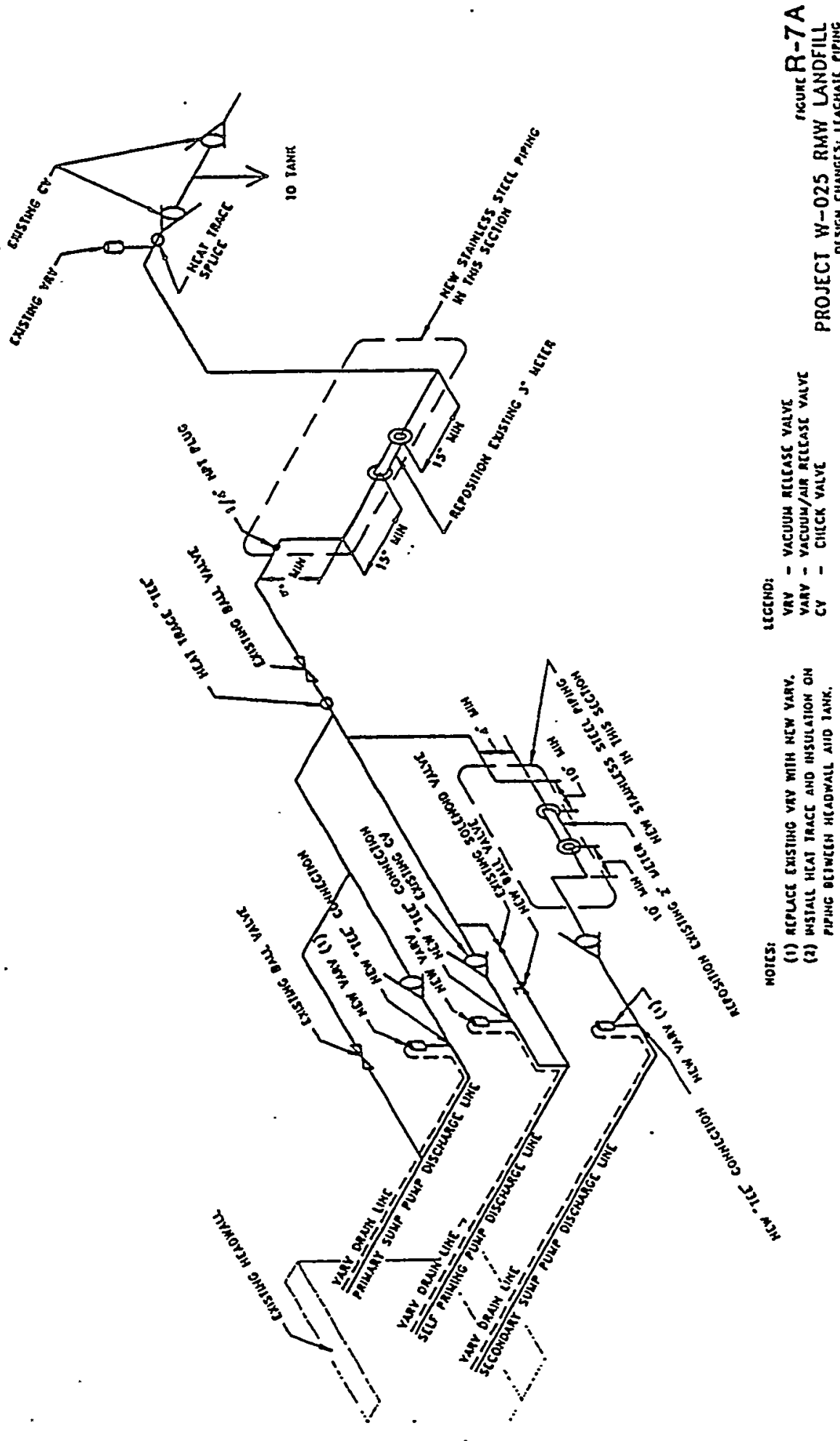

\% 
WHC-SD-W025-RPT-001, Rev. 0

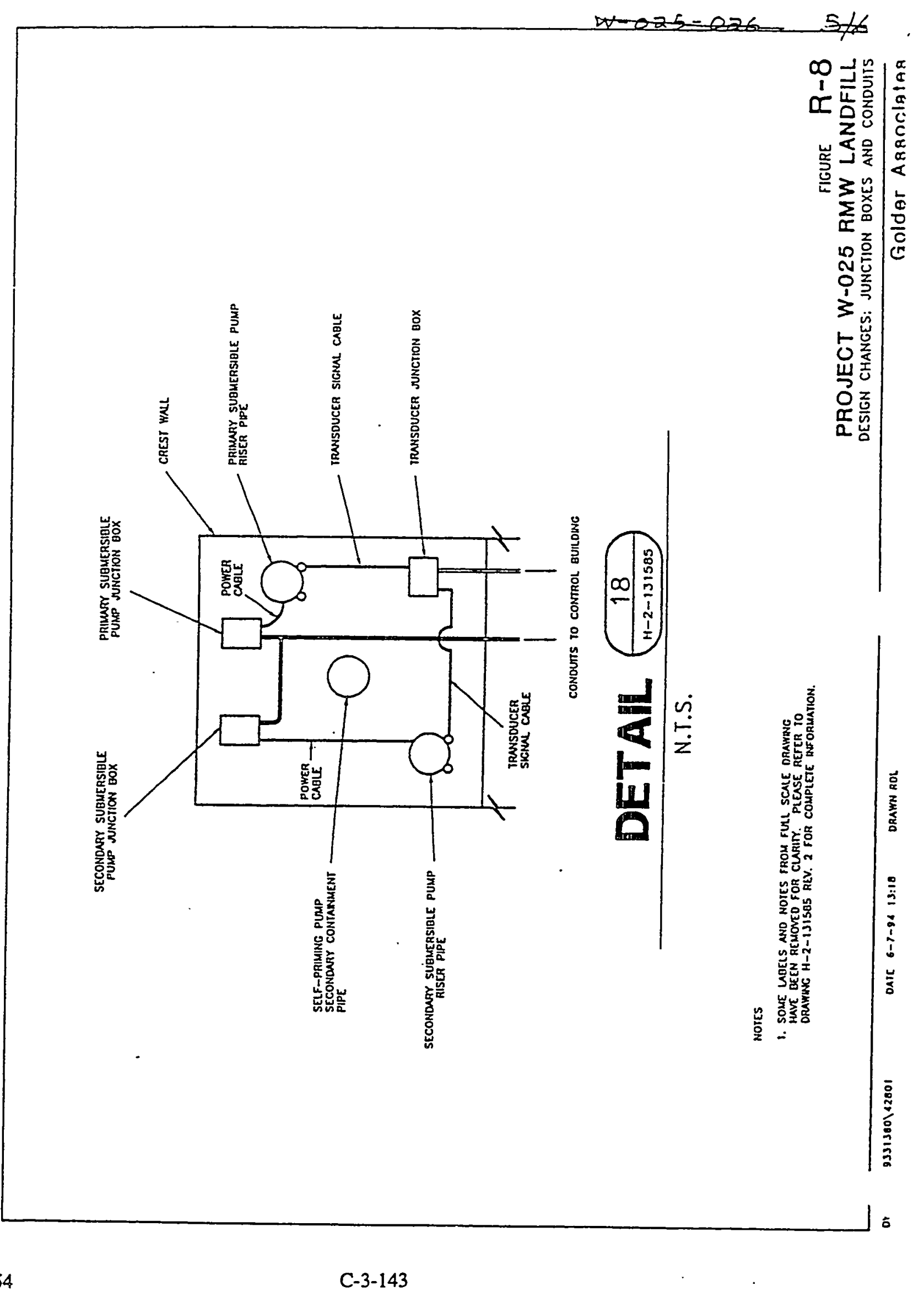


WHC-SD-W025-RPT-001, Rev. 0

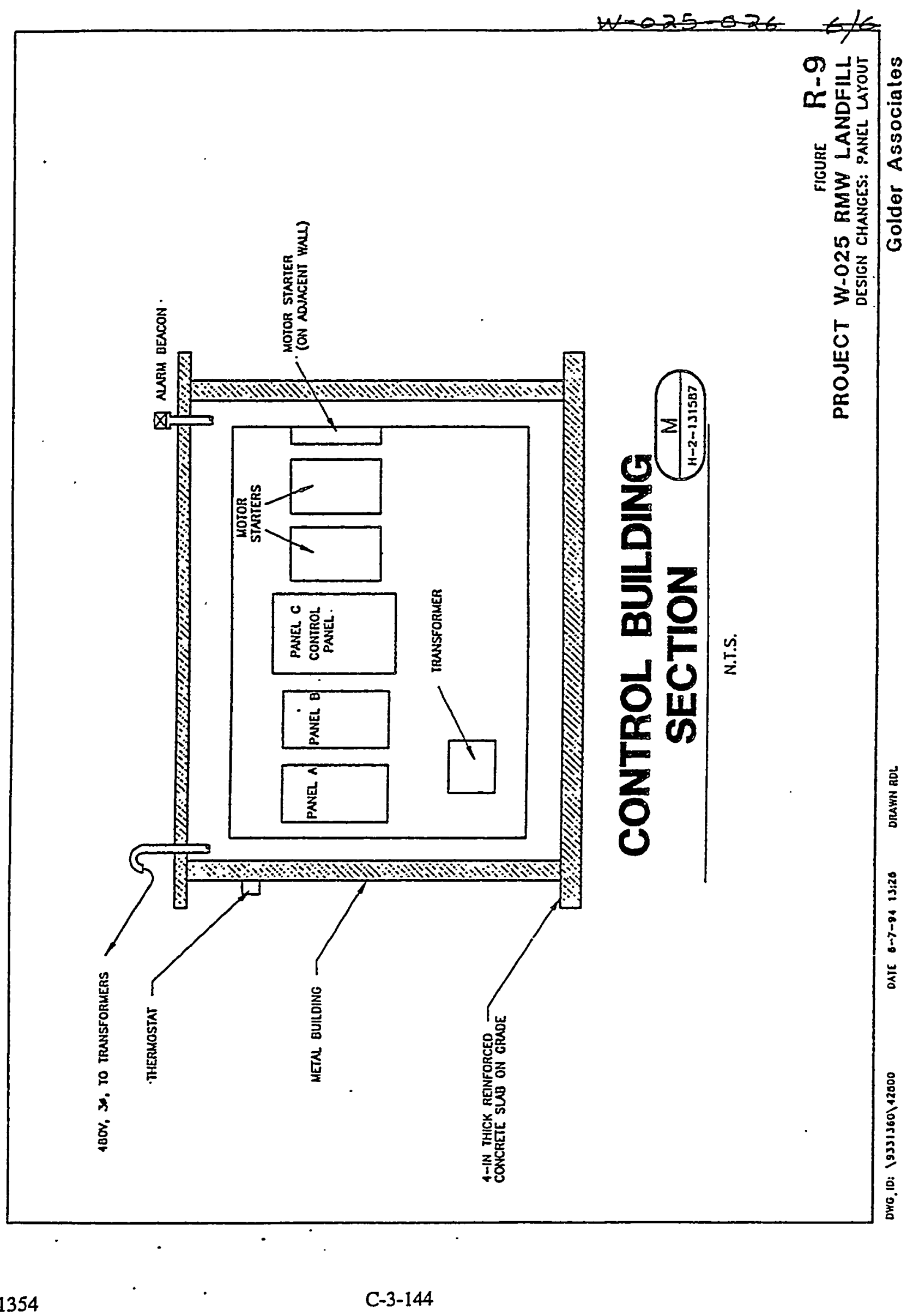




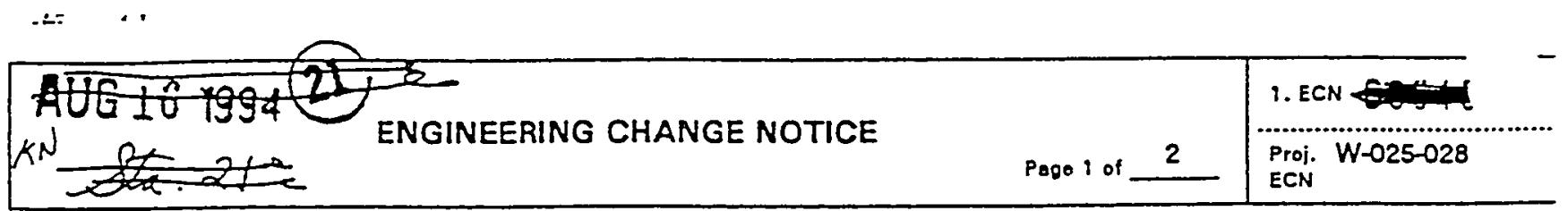

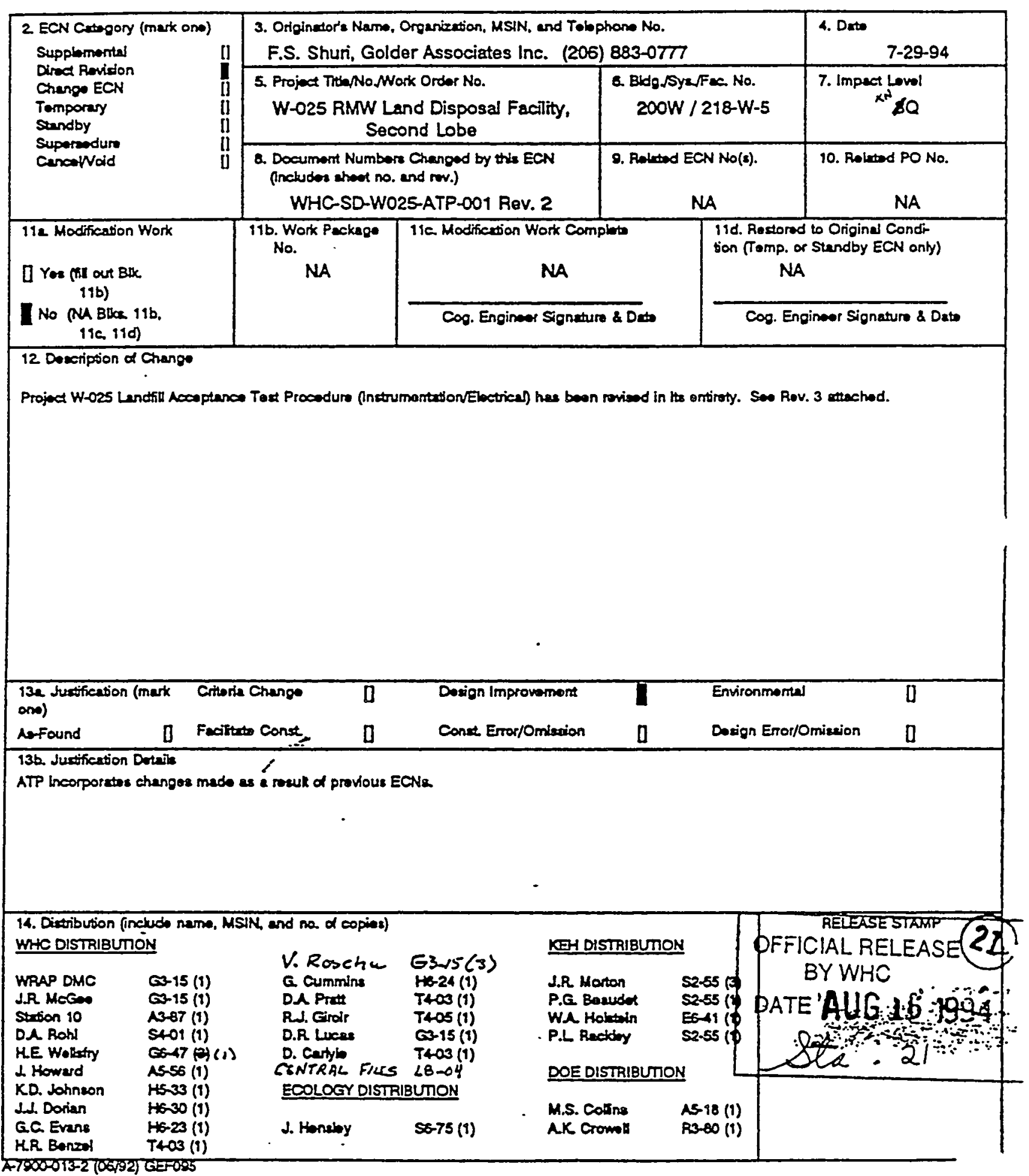




\begin{tabular}{|c|l|l|}
\hline ENGINEERING CHANGE NOTICE & $\begin{array}{c}\text { 1. ECN (uro no. from pg. 1) } \\
\text { W-025-028 }\end{array}$ \\
\hline
\end{tabular}

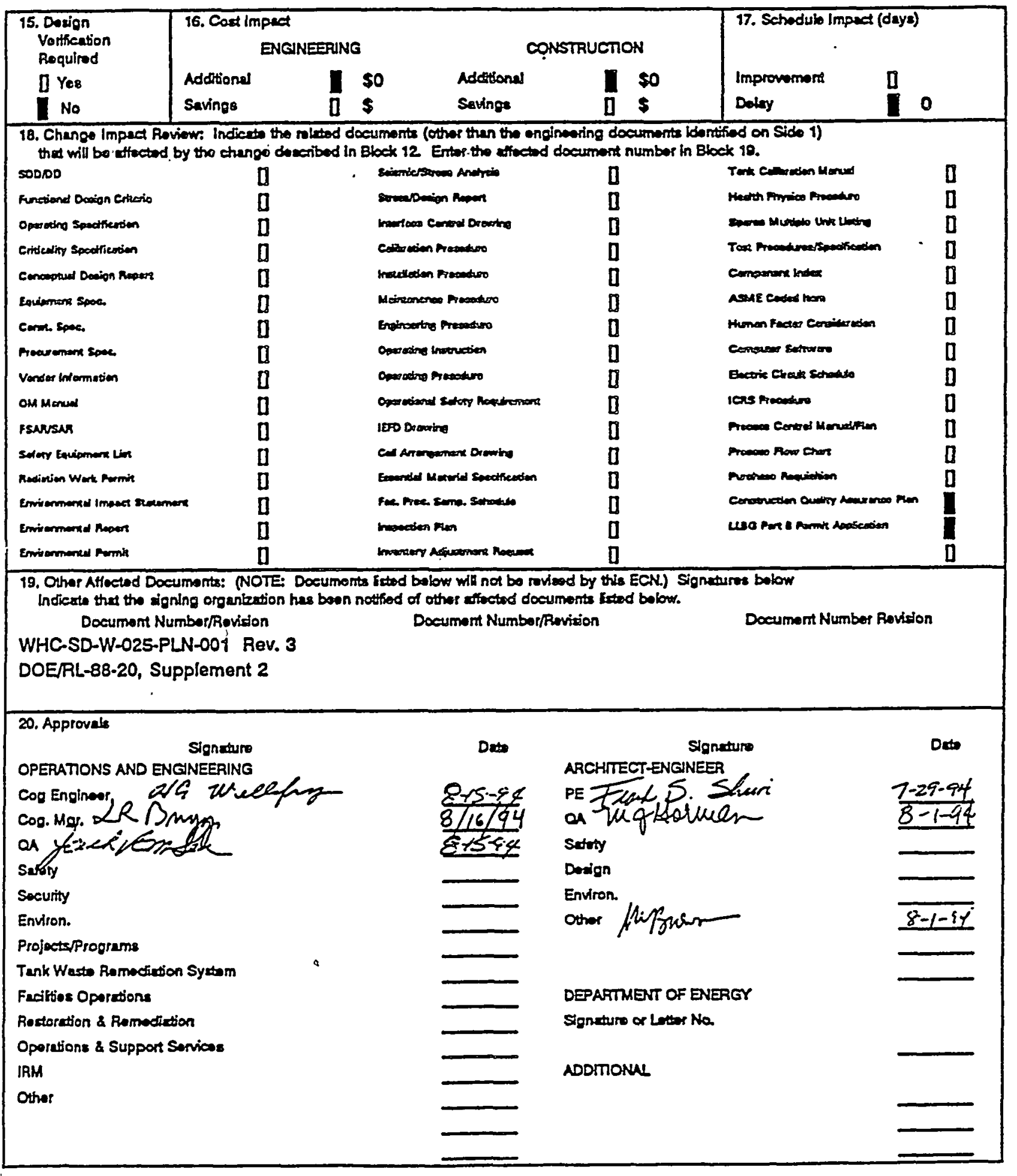


WHC-SD-W025-RPT-001, Rev. 0

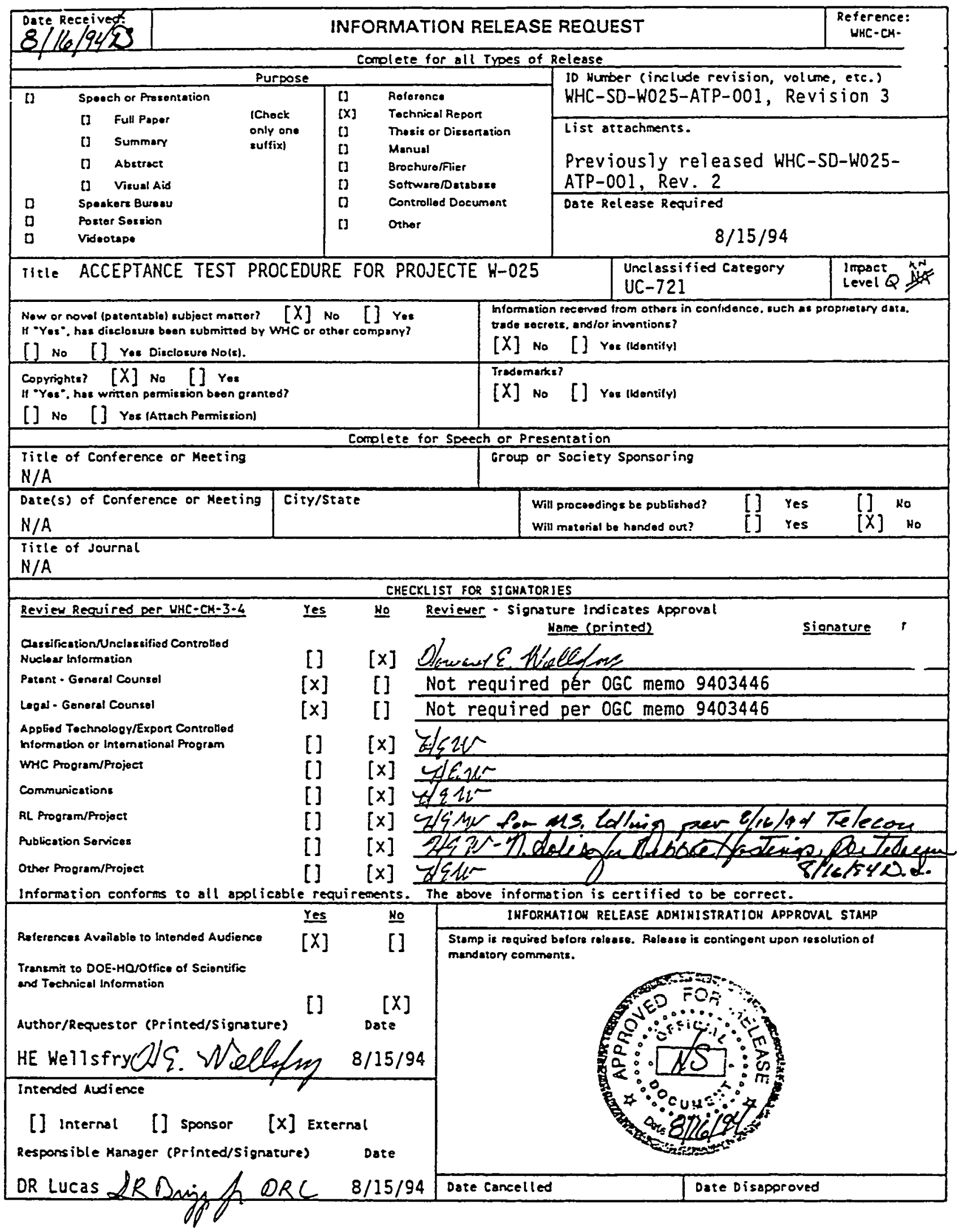




\section{SUPPORTING DOCUMENT}

1. Toral Pages 49

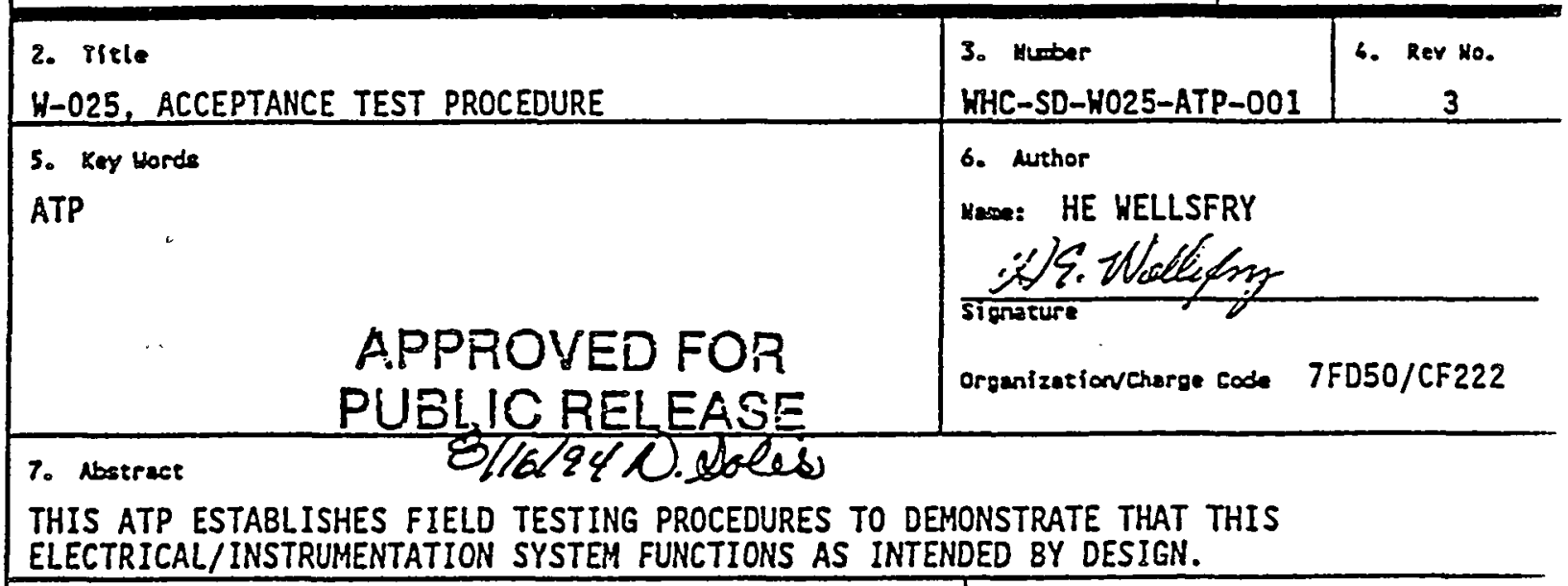

$\because \therefore \because$ $\because \cdots, \because, \cdots$

$\because \cdots, \cdots$ $\because \because \cdots$ $\because \because \because \because$
8. PURPOS AHD USE OF DOCAMENT - Thls documenty was prepered por use Hithin the U.S. Department of Enteray ind its dptractors. (t) is to be used only to perforn, direct, or integrate sork under U.S. Department of Energy cont sets. This docemey is hot approved - for pablic rokese unitil rey rued.

patent status - This ceument copy, since it is troseiteted in advance of patent $c$ - rance, is made avaitabl in confidoce solely for use in peporamee of work unde contrects ith the U.S. Department It Energy This document not to be publishod nop Its coneents hhervise dibeminated or yed for purposes otheh than specified gove before patenx approval for such release or use has been secured, upon request, from the gitent Counsel, U.S. Departeent of Eng.y Field Office, Richland, th.

DISCLUIMER - This report was prepared as an account of work sponsored by an agensy of the United States Government. Wel thep the United States Covernment nor any agency thereof, nor any of thele enployess, nor any of their contractors, subcontractors or their coployees, makes any karranty, express or implled, or assumes any legal llability or respansibility for the aecuracy, coupleteness, of any third party's use or the results of such use of any informition. apoaratus, product, or process diselosed, or represents that its use would not infringe privately aned rights. Reference harein to any specifie commereiul product, process, or service by trads nume. trademark, manufacturer, or otherwise, does not necessarily const fute or itsply its endorsecont, recormendation, or favoring by the United states Government or any ageney tharesf or its contractors or subcontratetors. The vieus and opinions of authors expressed herein do not necessarily state or roflect those of the United States Goverment or any saency thereof

9. Impaci Level Q
10.

RELEASE STMMP

CFF!:UR! TIELEASSE 5) vivilc

BAT: AUG 161994

Sta. 21 
WHC-SD-W025-RPT-001, Rev. 0

\begin{tabular}{|c|c|c|}
\hline RECORD OF REVISION & $\begin{array}{l}\text { (1) Documene Mutoer } \\
\text { WHC-SO-WO25-ATP-DOI }\end{array}$ & $: \quad \cdot$ \\
\hline $\begin{array}{l}\text { (2) rizle } \\
\text { ACCEPTANCE. TEST PROCEDURE }\end{array}$ & & \\
\hline
\end{tabular}

\section{EXANGE COATROL RECORO}

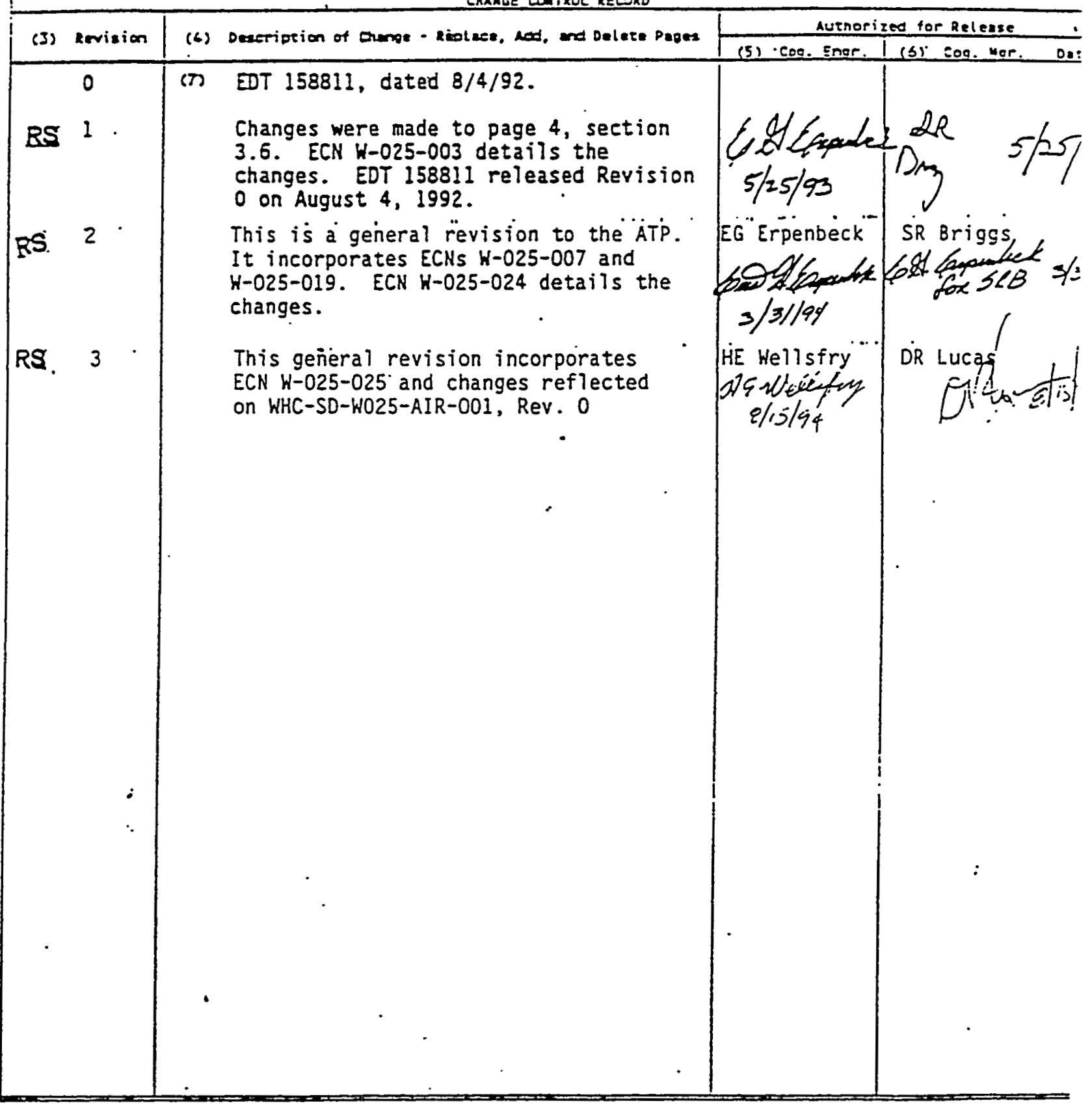

4-7320-005 (08/99) CEF) VEFI68 
WHC-SD-W025-RPT-001, Rev. 0

$\triangle C C E P T A N C E$ TEST PROCEDURE NO. WHC-SD-W025-ATP-001, REV. 3 DATE: 729/94

SUBJECT INSTRUMENTATTON/ELECTRICAL

LOCATION 200 WEST

PROJECT NUMBER

W-025

WORK ORDER NA

PROJECT TITLE RMW LAND DISPOSAL FACTLITY

\author{
Prepared By \\ GOLDER ASSOCIATES INC. \\ Redmond, Washington
}

For Westinghouse Hanford Company

Order MRY-SVV-301407

$\operatorname{Rev} 3$

IEST PROCEDURE APPROVED

GOLDER ASSOCIATES INC.
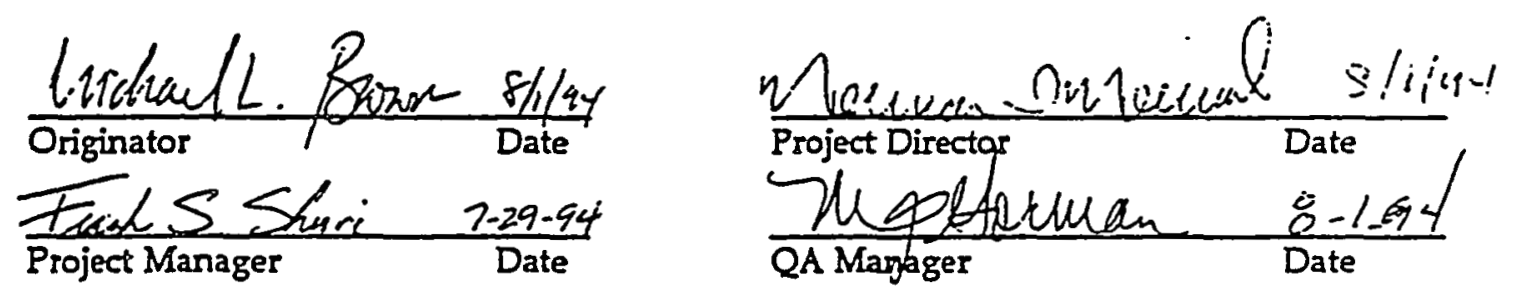

WESTINGHOUSE HANFORD COMPANY
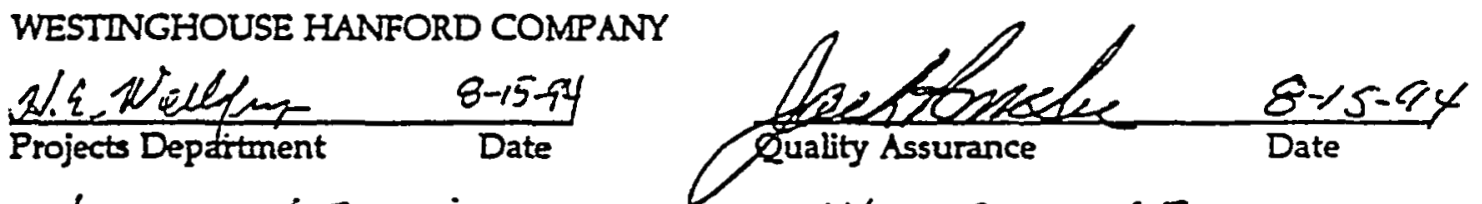

$\frac{\text { N/A General Revigion }}{\text { Programs }}$

$\frac{N / A \text { Geweret Eris-con }}{\text { Operations }}$ 


\section{CONIENTS}

1.0 PURPOSE OF THE ACCEPTANCE TEST

20 REFERENCE DRAWINGS AND SPECIFICATIONS

21 DRAWINGS

22 SPECIFICATIONS

3.0 RESPONSIBILITIES

3.1 WHC PROJECT ENGINEER

3.2 TEST DIRECTOR

3.3 WITNESSES

3.4 RECORDER

3.5 CONSTRUCTION CONTRACTOR

3.6 OCCUPATIONAL SAFETY AND HEALTH

4.0 ACCEPTANCE IEST PROCEDURE CHANGE CONTROL

5.0 TEST EXECUTION

5.1 WITHOUT EXCEPTION

5.2 WITH EXCEPMON/RESOLVED

5.3 WITH EXCEPTIONOUTSTANDING

6.0 RECORDING AND RESOLVING EXCEPTIONS

6.1 GENERAL

6.2 RECORDING

6.3 RETEST/RESOLUTION

6.4 APPROVAL AND ACCEPTANCE

6.5 DISTRIBUTION

7.0 TEST CONDITIONS AND EQUIPMENT REQUIRED

7.1 GENERAL

72 EQUIPMENT REQUIRED

8.0 ELECTRICAL HEAT TRACE SYSTEM

8.1 TEMPERATURE SENSOR

8.2 HEAT TRACE CABLE

9.0 TRANSDUCERS AND METER/CONTROLLERS

9.1 PRIMARY SUMP

9.2 SECONDARY SUMP 
WHC-SD-W025-RPT-001, Rev. 0

WHC-SD-W025-ATP-001 Rev. 3 -

$\therefore \quad:$

CONTENTS (cont)

10.0 PUMP TESTS $\quad 12$

10.1 INITIAL CONDITIONS 12

10.2 PRIMARY SUBMERSIBLE PUMP (W5P1) 13

10.3 SECONDARY SUBMERSIBLE PUMP (W5P2) 14

10.4 PRIMARY SUMP SELF-PRIMING PUMP (W5P3)

10.5 FLOWMETER CALIBRATION

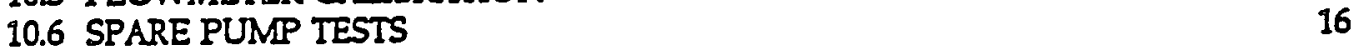

11.0 LEACHATE STORAGE TANK 16

11.1 LEACHATE STORAGE TANK PUMP (W5P4) 16

11.2 HIGH LEVEL FLOAT SWITCH (FS3) AND LMIT SWITCH (L1) 17

12.0 BUILDING POWER AND LIGHTING 18

12.1 BUILDING POWER RECEPTACLE

12.2 BUILDING LIGHTING FIXIURES

12.3. AREA.LIGHTING FIXTURES 18

$12 \&$ CONTROL PANEL HEATERS 18

APPENDIX A TEST DATA FORMS 
WHC-SD-W025-RPT-001, Rev. 0

WHCSDW025-ATP=-001-Rē. 3

\section{ACCEPTANCE TEST PROCEDURE}

\section{RMW LAND DISPOSAL FACILITY}

\section{PROIECT W-025}

\subsection{PURPOSE OF THE ACCEPTANCE TEST}

This acceptance test procedure (ATP) has been prepared to establish field testing procedures to demonstrate that the Electrical/nstrumentation systems function as intended by the design.

\section{REFERENCE DRAWINGS AND SPECIFICATIONS}

\section{DRAWINGS}

$\begin{array}{lll}\text { H-2-131585 } & \text { 1 OF 1 } & \text { Leachate Collection Tank \& Piping } \\ \text { H-2-131586 } & \text { 1 OF 1 } & \text { Sump Pump Details } \\ \text { H-2-131587 } & \text { 1 OF } 4 & \text { Electrical - Control } \\ \text { H-2-131587 } & \text { 2 OF } 4 & \text { Electrical - Tank \& Control Bldg. } \\ \text { H-2-131587 } & \text { 3 OF } 4 & \text { Electrical Site Plan } \\ \text { H-2-131587 } & \text { 4 OF } 4 & \text { Electrical Details }\end{array}$

\section{SPECIFICATIONS}

WHC-S-045 Radioactive Mixed Waste Land Disposal Facility, Non-Drag-Off, Technical Specifications.

\subsection{RESPONSIBILITIES}

Each company or organization participating in the conduct of this ATP will designate personnel to assume the responsibilities and duties as defined herein for their respective roles. The names of these designees shall be provided to the Recorder for listing on the Recorder's copy of the Test Execution Sheet prior to the performance of any part of this ATP.

\subsection{WHC PROJECT ENGINEER}

\subsubsection{Designate a Test Director.}

3.1. Act as liaison between the participants in acceptance testing.

3.1.3 Distribute the approved testing schedule as soon as approval has been obtained (one or more weeks prior to testing).

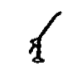


3.1.4 Schedule and conduct a pre-AIP kickoff meeting with test participants one or more weeks prior to start of testing.

3.1.5 Notify the persons performing and witnessing the test two days prior to the start of testing.

3.1.6 Notify all concerned parties when a change is made in the testing schedule.

3.1.7 Sign Test Execution Sheet when ATP is approved and accepted.

3.1.8 Take necessary action to clear exceptions to the AIP.

3.1.9 Sign Exception Sheet when exception has been resolved.

3.1.10 Provide a distribution list for the approved and accepted ATP.

\subsection{TEST DIRECTOR}

3.2.1 Coordinate all acceptance testing.

3.2. Distribute all documents including completed ATP, exceptions, resolutions and approvals.

3.23 Confirm that field testing and inspection of the system or portion of the system to be tested has been completed.

3.24 Stop any test which, in the judgement of the Director, may cause damage to the system until the test procedure has been revised.

3.2.5 Obtain revisions to the ATP, as necessary, to comply with authorized field changes or to accommodate existing field conditions.

3.26 Evaluate recorded data, discrepancies, and exceptions.

3.2.7 Obtain from Golder Associates Ine (GAD) any information related to this ATP or changes necessary to clear or resolve objections.

3.28 Sign Test Execution Sheet when ATP has been performed.

3.29 Sign Exception Sheet when retest has been executed and accepted.

3.2.10 Obtain required signatures on the ATP Master prior to reproduction and distribution. 
WHC-SD-W025-RPT-001, Rev. 0

WHCSEWO25-ATHET R

\section{WITNESSES}

Witnesses shall be provided by participating organizations.

3.3.1 Witness the tests.

3.3.2 Evaluate results of testing.

3.3.3 Assist the Test Director when requested.

3.3.4 Sign Test Execution Sheet as a Witness.

3.3.5 Sign Exception Sheet as a Witness when retest has been executed and accepted.

3.3.6 An Acceptance Inspector will witness the test for the Design Engineer.

\subsection{RECORDER}

The Recorder will be provided by Golder Construction Services.

3.4.1 Record names of all designated personnel on Recorder's copy of ATP prior to start of testing.

3.4.2 Observe tests and record test data.

3.4.3 Sign the Test Execution Sheet as the Recorder.

3.4.4 Initial and date every test step on the Recorder's copy as it is completed, next to the step number or on a table, when provided. On tables where there is not room for both the initial and date, date may be entered in space provided at bottom of column.

3.4.5 On the Exception Sheet, record objections or exceptions and test steps which are not performed. Have the information transferred in ink or typed to the Master of the Exception Sheet(s). Additional Exception Sheets are to be added as needed.

3.4.6 Orally notify the Test Director at time the objection is made.

3.4.7 Assign page numbers to Data Sheets and Exception Sheets, after ATP is complete. Record page numbers for these items and make corrections, as necessary, to page numbers shown for these pages in the index.

\section{.8}


WHC-SD-W025-RPT-00I, Rev. 0.

WIE-SD-WO25-ATP-DOT ReV. 3

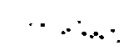

3.4.8 Transfer changes and the final test results with Recorder's signature and dates for each step to the Master in ink or type. Submit the completed Master to Construction Document Control for approval signature routing. Also submit the Recorder's copy for record retention.

\subsection{CONSTRUCTION CONIRACTOR}

3.5.1 Organize and perform this acceptance test under coordination of the Test Director.

3.5.2 Confirm that all equipment required for performing this test will be available at the start of testing.

3.5.3 Provide equipment required for performing this acceptance test, which has not been designated as being provided by others.

3.5.4 Request in writing from the Project Engineer those services, materials, or equipment that have been designated as being supplied by the DOE or others.

OCCUPATIONAL SAFETY AND HEALTH

Individuals shall carry out their assigned work in a safe manner to protect themselves and others from undue hazards and to prevent damage to property and environment. Facility line managers shall assure the safety of all activities within their areas to prevent injury, property damage, or intemuption of operation. Performance of test activities shall always include safety and health aspects as delineated in the most current version of the Federal Occupational Safety and Health Administration/Washington Industrial Safety and Health Act (OSHA/WISHA) safety health codes and standards.

Acceptance testing shall be conducted in accordance with the steps and requirements specified in this procedure. Any required changes must be authorized in accordance with approved change control procedures for this project and promptly accomplished. Procedure changes during testing must be approved by the WHC project engineer, WHC quality assurance, and the GAI inspector (via initials). The GCS recorder shall note these changes as exceptions (see Section 5.2), provided that these changes do not affect safety and health. The changes shall be incorporated in the final acceptance test report. 


\subsection{TEST EXECUTION}

\subsection{WITHOUT EXCEPTION}

5.1.1 Check applicable space on Test Execution Sheet to show that the ATP has been performed and no exceptions have been recorded.

5.1.2 Sign and date Test Execution Sheet in the spaces provided.

5.1.3 Distribute requisite copies and send master of ATP to the WHC Project Engineer.

5.2 WITH EXCEPTIONRESOLVED

5.21 Check applicable space on Test Execution Sheet to show that the ATP has been performed with exceptions recorded and resolved.

5.22 Sign and date Test Execution Sheet in the spaces provided.

5.23 Distribute requisite copies and send master of ATP to the WHC Project Engineer

\subsection{WTTH EXCEPTIONOUTSTANDING}

5.3.1 Check applicable space on Test Execution Sheet to show that the ATP has been performed with exceptions recorded, in part or all of which are presently outstanding and unresolved.

5.3.2 Sign and date Test Execution Sheet in the spaces provided.

5.3. Distribute requisite copies and send master of AIP to the WHC Project Engineer.

\subsection{RECORDING AND RESOLVING EXCEPTIONS}

\subsection{GENERAL}

Exceptions to the ATP are sequentially numbered and recorded on individual Exception Sheets. This enables case-by-case resolution, recording, approval, and distribution of each exception.

\subsection{RECORDING}

6.21 Number each exception sequentially as it occurs and record it on an Exception Sheet. 
WHC-SD-W025-RPT-001, Rev. 0

WTHE-SP-W025-ATP-001-Rev 3

622 Enter name and organization of objecting party for each exception.

6.23 Enter planned action to resolve each exception when such determination is made.

\subsection{RETEST/RESOLUTION}

Record the action taken to resolve each exception. Action taken may not be the same as planned action.

6.3.1 When action taken results in an acceptable retest, sign and date Retest Execution and Acceptance section of the Exception Sheet.

6.3.2 When action taken does not involve an acceptable retest, strike out the Retest Execution and Acceptance section of the Exception Sheet Resolve exception as shown under 6.4, below.

\subsection{APPROVAL AND ACCEPTANCE}

The WHC Project Engineer provides final approval and acceptance of exceptions by checking one of the following on Exception Sheet:

6.4.I Retest Approved and Accepted: Applicable when Retest Execution and Acceptance section is completed.

6.4.2 Exception Accepted-as-is: Requires detailed explanation.

6.4.3 Other: Requires detailed explanation.

The Project Engineer signs and dates the Exception Sheet and obtains other internal approvals, if required.

6.5 DISTRIBUTION

Distribute requisite copies of completed Exception Sheets to WHC personnel and departments as directed by the WHC Project Engineer.

\subsection{TEST CONDITIONS AND EQUIPMENT REQUIRED}

\subsection{GENERAL}

The following conditions shall exist at the start of the acceptance testing for that portion of the system being tested.

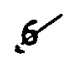


WHC-SD-W025-RPT-001, Rev. 0

WHE-SDWWO25ATPO01-ReV.S

7.1.1 Systems being tested have been inspected for workmanship and for compliance with design.

7.12 Continuity tests have been performed on portions of the electrical system being tested.

7.1.3 Power is available to components of systems being tested.

7.1.4 Continuity tests of instrumentation wiring have been performed in accordance with technical specifications WHC-S-045, latest revision.

7.1.5 All test instruments have a valid calibration stamp attached that indicates a calibration traceable to the National Institute of Standards and Technology.

7.1.6 Methods of water disposal used in this ATP have been approved by the Facilities Management.

7.1.7 Personnel responsible for directing, witnessing and performing the tests described in this ATP are familiar with the equipment to be tested, have reviewed the vendor information pertaining to the operation of the equipment, and are familiar with the requirements of this acceptance test procedure.

7.1.8 Values used to simulate process inputs (such as water levels D1, D2, D3, DS-1, DS-2, etc.) and alarm conditions for execution of ATP will be provided by the Design Engineer.

\subsection{EQUIPMENT REQUIRED}

The Contractor shall supply all test equipment unless otherwise noted.

7.21 Digital multimeter (DMM): $41 / 2$ digit or better, 2 percent accuracy.

7.22 Seven shorting jumpers with ON-OFF switch, 14-gage wire, 12 inch approximate length.

7.23 Clamp-on Ammeter (COA): Scale 0.6 amp.

724 One highway water tanker, 5,000 gallon capacity, with discharge capability, with comparable KAMVALOK fitting 1673-A, 3 inches diameter. 
725 Water level transducer (WLT): 0-10 psi, precalibrated with sufficient cable to seach from primary sump to control building (approximately 200 feet). Niternatively, a water level indicator or other measuring device as approved by the Project Engineer may be used, provided that real-time communications with personnel in the control room are established.

7.26 Measuring tape, measuring rod, folding scale, or equivalent: $6 \mathrm{ft}(\mathrm{min}$.$) ,$ $+1-0.5 \mathrm{in}$.

7.2.7 Dry Ice (summer only).

7.28 Hairdryer or equivalent forced air heater (winter).

729 Water Container (barre), 60-inches deep minimum.

7.2.10 Receptacle Tester: Hand-held device which verifies correct connections of receptacle and simulates ground-fault condition. Ideal \#61-05I or equal.

72.11 Valve for pump discharge line.

7.2.12 Pressure gages for pump discharge line, 0 to 50 psi range (valved) and 0 to 200 psi range.

\subsection{ELECIRICAL HEAT TRACE SYSTEM}

This procedure will demonstrate the correct functioning of electrical heat trace system and the temperature sensing (thermostat) devices. Verify and record the following:

\subsection{TEMPERATURE SENSOR}

Record all data on Test Data Form 8-1 (see Appendix A).

8.1.1 Energize heat trace circuit (close breaker B8). Disconnect the power conductors to the thermostat

8.1.2 Visually inspect the sensor and contacts for damage.

8.1.3 Using digital multimeter set to measure resistance (ohms), place connections on opposite sides of thermostat contacts.

8.1.4 Cool or heat (depending upon exterior temperature at time of testing) thermostat sensor to simulate outside temperature changes. 
WHC-SD-W025-RPT-001, Rev. 0

WIE-SD-WOZS-ATPDIRE.3

8.1.5 Observe multimeter output during testing. When simulated temperature of thermostat is above $40^{\circ} \mathrm{F}$ (approximately), the resistance should be high (above $500 \mathrm{ohms}$ ), indicating that the contacts are open. When the simulated temperature is below $40^{\circ} \mathrm{F}$ (approximately), the resistance should be low (below 2 ohms), indicating that the contacts are closed. Verify both conditions.

8.1.6 Remove meter connections and reconnect power conductors.

\subsection{HEAT TRACE CABLE}

Record all data on Test Data Form 8-2 (see Appendix A).

8.21 Energize heat trace circuit (close breaker B8).

8.22 Manually force the contacts on the thermostat closed. Measure the current in the conductors to the heat trace cable using COA. Current should be 5 amps minimum (note: current is dependent upon temperature of heat trace cable. The current will increase as the temperature of the cable decreases).

8.2.3 Manually force the contacts on the thermostat open. Measure the current in the conductors to the heat trace cable using COA. The current should be less than 0.1 amps.

\subsection{IRANSDUCERS AND METER/CONTROLLERS}

This procedure will demonstrate the correct functioning of the level transducers and the associated meter/controllers and relays. See Figure 1 for definitions of water levels and associated pumps and relays. Verify and record the following:

\subsection{PRIMARY SUMP}

Record all data on Test Data Form 9-1 (see Appendix A).

9.1.1 Remove power to pumps W5P1 and W5P3 by opening circuit breakers BI and $\mathrm{AT}$.

9.1.2 Through the vertical riser pipe, install the water level transducer (WLT) in the bottom of the primary sump leachate collection well with remote readout in the control building. Verify that the reading is equal to the water level in the sump (if any), as measured with a tape measure, measuring rod, or similar device. Altematively, measure the water level in the sump with the water level indicator and calculate the depth of water. 
WHC-SD-W025-RPT-001, Rev. 0

WHL-SOHO25-ATP-OOY,REV.3

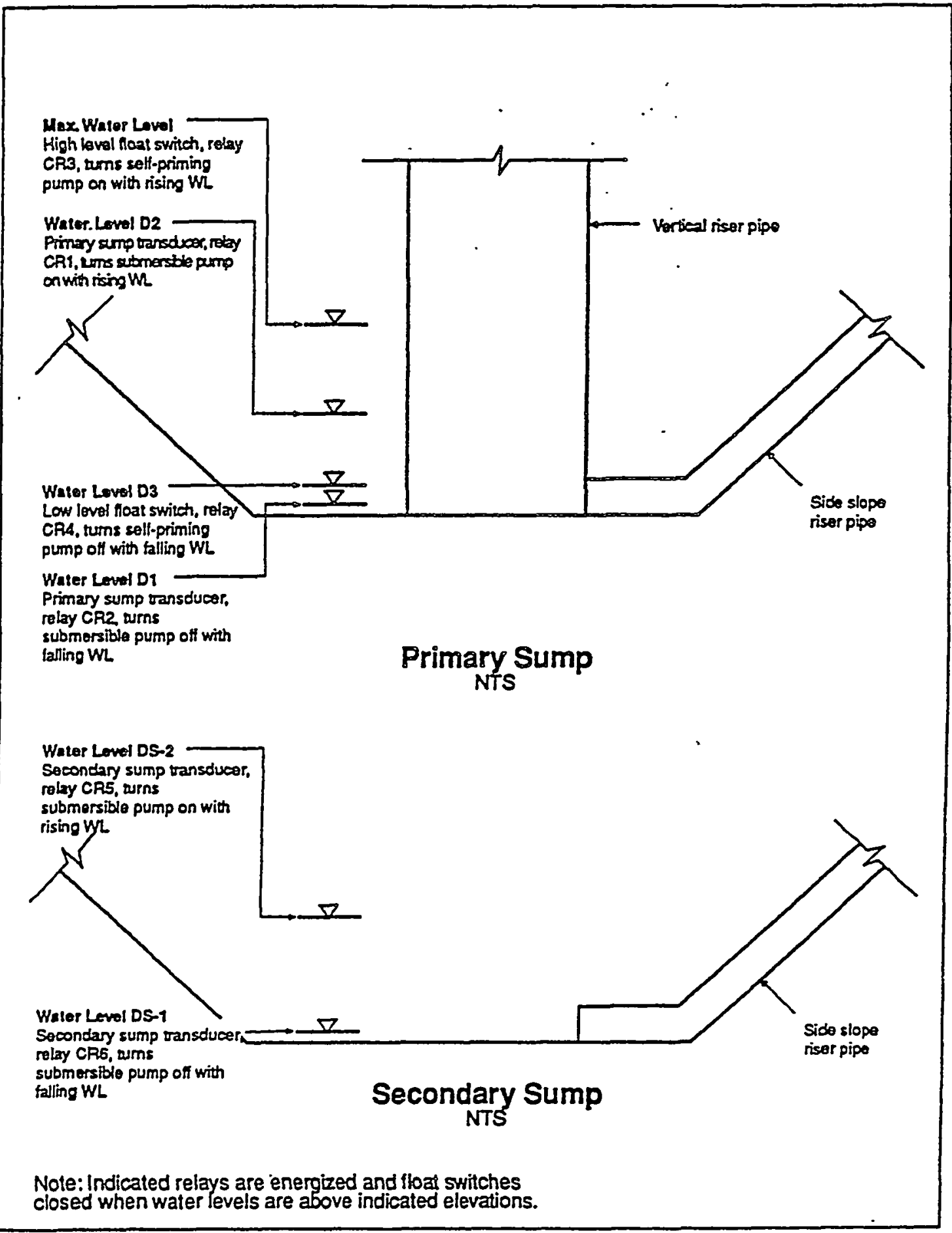

$2431576 / 5325017.29-94$

Figure I. Water Level Definitions. 
9.13 Energize control panel. Observe readout of primary sump level meter. The primary sump reading should be equal to the actual water depth within $+/-1$ inch. Adjust meter readings as required.

9.1.4 If the water level in the primary sump is above D1, energize pump W5P1 and pump the sump to below level D1. De-energize W5P1. Verify that relays CRI and CR3 are "off" (de-energized) and relays CR2 and CR4 are "on" (energized) by observing the LED on each relay.

9.1.5 Begin adding potable water to the primary sump through the vertical riser pipe. Add water at a uniform rate so that the rise of the water level in the leachate collection well is no more than 2 inches/minute. Take care to avoid excessive turbulence during filling. If necessary, stop filling at 2 inch intervals and allow the water level to stabilize. Record the actual rise in the sump level and on the Primary Sump Level Meter at 2 inch intervals. Both readings should be within $+/ .1$ inch. Continue throughout filling.

9.1.6 Continue adding water to primary sump. Observe relay $C R 2$ being deenergized when the level indicated by WLT reaches DI.

9.1.7 Continue adding water to primary sump. Observe relay CR4 being deenergized at level D3 and relay CR1 being energized at level D2. Stop filling sump.

9.1.8 Confirm that the actual water level and Primary Sump Level Meter are within $+/-1$ inch.

9.1.9 Manually lift (simulate operation) the high level float on the primary sump vertical riser float switch. Verify that relay $C R 3$ is energized. Lower the float and verify that relay $C R 3$ is de-energized.

\subsection{SECONDARY SUMP}

Record all data on Test Data Form 9-2 (see Appendix A).

921 Pump the secondary sump to below level DS-1. Remove power to pump W5P2 by opening circuit breaker B5.

922 Verify that relay CR5 is "off" (de-energized) and CR6 is "on" (energized) by observing the LED on each relay. 
9.23 Remove secondary sump transducer from installed location. Using the water container, slowly fill the container with water and manually calibrate the transducer over the range of $0^{n}$ to $48^{n}$. Use at least 10 data points for the calibration. Attach the calibration sheet to Test Data Form 9-2.

9.24 Reinstall transducer and observe secondary sump level meter reading. This should be near DS-1.

92.5 Add water in increments as directeci by the Test Director to secondary sump via the secondary siope riser pipe. Record the volume of water added to the sump. Wait 2 minutes after each increment of water, and record the secondary sump level meter reading. Verify that the secondary sump level meter reading increases as water is added.

9.26 Continue adding water in increments and observe that relay CR6 is deenergized when the level is DS-1, and that CR5 is energized when the level is DS-2. Stop filling sump.

\subsection{PUMP TESTS}

This procedure will demonstrate the correct functioning of the pumps and associated controls. See Figure 1 for definitions of water levels and associated pumps and relays.

For pumps W5P1, W5P2, W5P3, and W5P4, measure the phase currents prior to beginning pumping tests. Record this information on the top of the test data forms $10-2,10-3,10-4,11-1$ respectively. Verify that motor overload devices are sized correctly.

\subsection{INIILAL CONDITIONS FOR PUMP TESTS}

Prior to testing pumps, verify that relay CR7 (high tank) is de-energized (closed). Record all data on Test Data Form 10-1 (see Appendix A).

10.1.1 Primary Sump

This testing should begin with water in the primary sump above level D2 Verify the following conditions on the control panel:

a. CR1 is energized.

b. $\quad C R 2$ is de-energized.

c. CR3 is de-energized.

d. CR4 is de-energized.

e. Pump failure light for the primary submersible pump is on.

$$
12
$$


WHC-SD-W025-RPT-001, Rev. 0

WHC-SD-W025-ATP-DO1 Rev. 3

f. The roof alarm light is on.

g. The panel light associated with relay $C R 1$ is on and the lights associates with relays $C R 2, C R 3$, and $C R 4$ are off.

10.1.2 Secondary Sump

The water level in the secondary sump should be above the level of the high level switch (water level DS-2). Verify the following conditions on the control panel:

a. $\quad$ CR5 is energized.

b. CR6 is de-energized.

c. Pump failure light for the secondary submersible pump is on.

d. The roof alarm light is on.

e. The panel light associated with relay $\mathrm{CR} 5$ is on.

\subsection{PRIMARY SUBMERSIBLE PUMP (W5P1)}

Record all data on Test Data Form 10-2 (see Appendix A).

10.21 The switch on the control panel for the pump should be in the "off" position. Close circuit breaker BI.

10.22 Tum the switch for the Primary Submersible Pump to the "hand" position.

10.23 Verify by direct visual observation that water is being pumped from the sump into the storage tank.

10.24 Verify that the No drain back solenoid valve (W54) is closed (energized).

10.2.5 Verify that the associated "pump run" light is on. Run the pump until the primary sump is drained below the high water level (D2).

10.26 Tum switch on motor controller to "automatic" position. Add water to primary sump until high level relay CR1 is activated (water level D2).

10.27 Verify that the pump start automatically and that the pump run light is on.

10.28 Verify by direct visual observation that water is being pumped from the sump into the storage tank

10.29 Verify that when the water level in the sump reaches low level (water level D1), relay CR2 is energized, and the pump is automatically shut off. W5P3 may be used to assist in primary sump drawdown. 
10.210 Add water to the primary sump to above level D1. Manually initiate operation of Timer TMI and verify that the pump is automatically started.

10.211 While pump is running, connect terminals H2F and \#10 across FS3 using a jumper, to simulate high water level in the storage tank. Verify that the pump is automatically stopped and that the roof alarm is initiated. Remove the jumper and re-initiate the automatic operation.

10.212 Tum the switch on the motor starter to the "off" position.

10.3 SECONDARY SUBMERSIBLE PUMP (W5P2)

Record all data on Test Data Form 10-3 (see Appendix A).

Repeat steps 10.21 through 10.28 and 10.211 except for the secondary submersible pump, adding water to the secondary sump through the secondary riser pipe as required.

\subsection{PRIMARY SUMP SELF-PRDMING PUMP (W5P3)}

Record all data on Test Data Form 104 (see Appendix A).

10.4.1 The switch on the control panel for pump W5P3 should be in the "off" position. Fill the primary sump with water to above level D2. Close circuit breaker A7.

10.4.2 Turn the switch on the control panel to the "hand" position.

10.4.3. Verify by direct visual observation that the pump is running and that water is being pumped from the sump into the leachate storage tank

10.4.4 Verify that the "pump run" light is on.

10.4.5 Verify operation for one minute.

10.4.6 Tum the switch on the motor controller to the "automatic" position. Add water to the sump to above level D2

10.4.7 Manually raise the high level vertical riser float switch to simulate high water condition.

10.4.8 Verify that the pump is automatically started. 
10.4.9 Verify that the pump continues running until the water level reaches the low level vertical riser float switch (water level D3), when the pump is. automatically shut off.

10.4.10 Add water to the primary sump and perform step 10.211 for this pump.

\subsection{FLOWMETER CALIBRATION}

Record all data on Test Data Form 10-5 (see Appendix A).

10.5.1 Verify that there is sufficient water in the secondary sump to pump 500 gallons and still maintain a final water level of at least 8 inches.

10.5.2 Verify that the water in the sump is clean and will not produce foam when pumped. Flush sump if necessary until clean, non-foaming water is obtained.

10.5.3 Verify that ball valve $W 65$ is open and ball valve $W 66$ is closed. Verify that the ball valve in series with the solenoid draindown valve is closed.

10.5.4 Measure the water level in the leachate temporary storage tank to the nearest 0.01 foot with a rigid rod. Measure through port in top of tank. If necessary, add water until depth in tank is at least 0.3 feet.

10.5.5 Remove 1/4-inch plug immediately upstream of 3-inch flowmeter. Energize secondary submersible pump. Pump until air no longer escapes through $1 / 4$-inch hole. Replace plug. De-energize pump.

10.5.6 Measure the water level in the leachate temporary storage tank as described in step 10.5.4. Record on Test Data Sheet 10-5.

10.5.7 Record the readings of the 2-inch and 3-inch flowmeters on Test Data Sheet 10-5.

10.5.8 Energize the secondary submersible pump and pump at least 500 gallons into the leachate temporary storage tank.

10.5.9 Measure the water level in the leachate temporary storage tank as described in step 10.5.4. Record on Test Data Sheet 10-5.

10.5.10 Record the readings of the 2-inch and 3-inch flowmeters on Test Data Sheet 10-5. If the meter readings differ from the calculated volume (based on measurements of water depth in the tank) by more than 5 percent, repeat step 10.5 .5 using the self-priming pump (primary sump). Ensure that there is sufficient water in the primary sump prior to purging the 
discharge pipes. Continue the ATP from step 10.5 .6 using the secondary submersible pump.

10.5.11 Repeat steps 10.5.8 through 10.5 .10 twice, for a total pumped volume of about 1500 gallons.

\subsection{SPARE PUMP TESTS}

Test spare pumps at the ground surface to verify that they function. Test submersible pumps in a "barrel" that allows complete immersion. Record all data on Test Data Form $10-6$.

10.6.1 Install the valve on the pump discharge line to simulate head.

10.6.2 Install the pressure gage between the valve and pump to measure the head.

10.6.3 Energize the pump and allow it to achieve steady state conditions at a pressure of approximately $90 \%$ of the maximum efficiency discharge head as shown on the manufacturer's pump performance curves. Measure the volume of water pumped for at least 30 seconds.

10.6.4 Repeat the test with the pressure set at about $110 \%$ of the maximum efficiency discharge head.

10.6.5 Repeat the test with the discharge valve closed to determine the "shutoff" pressure.

10.6.6 Repeat steps 10.6.1 through 10.6 .5 for all spare pumps.

\subsection{LEACHATE STORAGE TANK}

This procedure will demonstrate correct functioning of the transfer pump and controls associated with the leachate storage tank Verify and record the following:

\subsection{LEACHATE STORAGE TANK PUMP (W5P4)}

Record all data on Test Data Form 11-1 (see Appendix A).

11.1.1 Verify that the storage tank is at least 25 percent full of water. Tum on circuit breaker $A 1$ for the pump.

11.1.2 Using the DMM, measure the voltage at the motor terminals $(T, T 2, T 3)$. The voltage should read 0 between terminals $T \& \& T 2, T 2 \& T$, and between $\mathrm{II}$ and the metal frame.

$$
16^{\circ}
$$


11.1. Push the "start" pushbutton on the motor starter. Verify that the motor is operating correctly with proper rotation. Verify by direct visual observation that water is being pumped from the tank

11.1.4 Using the DMM, measure the voltage at the motor terminals. The voltage should read between 460 and 500 volts between terminals $T 1 \& T 2$ and between terminals $\Gamma \mathbf{I} \& \mathbf{T}$.

11.1.5 Continue pumping for 2 minutes minimum. Push the "stop" pushbutton and verify that the pump stops running.

\subsection{HIGH LEVEL FLOAT SWITCH (FS3) AND LIMIT SWITCH (LL1)}

Record all data on Test Data Forms 11-2A and 11-2B (see Appendix A).

11.21 Observe that the red "high tank level" light on the control panel is off and that relay $C R 7$ is de-energized. Disconnect limit switch $L 1$.

11.2.2 Fill the storage tank with water to above the high level. Verify that FS3 operates at the selected "high water" level

11.2.3 Observe that relay $C R 7$ is energized and time delay is functional.

11.2.4 Observe that the red "high tank level" light on the control panel is on.

11.2.5 Observe that the roof alarm light is on and time delay is functional.

11.2.6 Verify that the submersible pumps and self primary pumps will not operate ( 3 pumps total) by temporarily tuming the appropriate control switches on the control panel to "hand" position and observing that the associated "pump run" light does not come on.

11.27 Lower the water level in the tank and observe that FS3 returns to nomal condition (repeat step 11.21).

11.28 Reconnect limit switch LI and disconnect the high level float switch FS3.

11.29 Repeat steps 11.21 through 11.27 to test L1. Record all data on Test Data Form 11-2B (see Appendix A).

11.210 Reconnect high level float switch FS3. 
WHC-SD-W025-RPT-001, Rev. 0

WHESDWW025ATP=01 ReV.3

\subsection{BUILDING POWER AND LIGHTING}

This procedure will demonstrate the correct functioning of control building power and lighting and area lighting. Verify and record the following:

\subsection{BUILDING POWER RECEPTACLE}

Record all data on Test Data Form 12-1 (see Appendix A).

121.1 Insert receptacle tester into one outlet of the duplex GFI receptacle. Verify that receptacle is correctly wired.

12.1. Push the button on the receptacle tester. This will initiate a ground fault current into the receptacle. Verify that the receptacle ground fault device trips. Verify that the outlet is now deenergized (observe that all the lights on the tester are now off).

121.3 Repeat Step 121.2 for the other outlet.

\subsection{BUILDING LIGHTING FDTURES}

Record all data on Test Data Form 12-2 (see Appendix A).

1221 Tum on light switch for fixture in the Control Building. Observe that both fluorescent lamps are illuminated.

\subsection{AREA LIGHTING FLXTURES}

Record all data on Test Data Form 12-3 (see Appendix $A$ ).

123.1 Cover the sensors of all photocells to simulate darkness. Observe that the light fixtures are automatically turned on and illuminated to full brightness within 5 minutes.

123.2 Observe the operation of the exterior fixtures at 15 minute intervals over a 75 minute period. Verify that the fixtures remain illuminated (do not cycle on and off).

123.3 Remove covers from photocell sensors. Verify that all fixtures turn off.

\subsection{CONTROL PANEL HEATERS}

Record all data on Test Data Form 12-4 (see Appendix A). 
WHC-SD-W025-RPT-001, Rev. 0

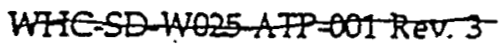

$1 \quad 124.1$ Turn thermostat on heater in Control Panel C off. Verify that heater is at ambient temperature. Wait for heater to cool if necessary.

124.2 Tum thermostat until it energizes. Verify that heater becomes warm.

124.3 Repeat procedure for Control Panels $\mathrm{A}$ and $\mathrm{B}$. 
WHC-SD-W025-RPT-001, Rev. 0

-WHC-SD-W025-ATP=001-Rev. 3

APPENDIX A

TEST DATA FORMS

R-T 
WHC-SD-W025-RPT-001, Rev. 0

WHC-SD-W025-ATP-001 Rev.3 -

\section{LIST OF FORMS}

TEST EXECUTION SHEET

EXCEPTION SHEET

TEST DATA FORM 8-1 HEAT TRACE SYSTEM - TEMPERATURE SENSOR TEST

TEST DATA FORM 8-2 HEAT TRACE SYSTEM - HEAT TRACE CABLE TEST

TEST DATA FORM 9-1 TRANSDUCERS AND METER/CONTROLLERS - PRIMARY SUMP

TEST DATA FORM 9-2 TRANSDUCERS AND METER/CONTROLLERS - SECONDARY SUMP

TEST DATA FORM 10-1 PUMP TESTS - INTIIAL CONDIIONS

TEST DATA FORM 10-2 PUMP IESTS - PRIMARY SUBMERSIBLE PUMP (W5P1)

TEST DATA FORM 10-3 PUMP TESTS - SECONDARY SUBMERSIBLE PUMP (W5P2)

TEST DATA FORM 10-4 PUMP TESTS - PRIMARY SUMP SELF-PRIMING PUMP (W5P3)

TEST DATA FORM 10-5 PUMP TESTS - FLOWMETER CALIBRATTON

TEST DATA FORM 10-6 PUMP TESTS - SPARE PUMPS

TEST DATA FORM 11-1 LEACHATE STORAGE TANK - TANK PUMP (W5P4)

TEST DATA FORM 11-2A LEACHATE STORAGE TANK - HIGH LEVEL FLOAT SWITCH (FS3)

TEST DATA FORM 11-2B LEACHATE STORAGE TANK - HIGH LEVEL LIMIT SWTTCH (L1)

TEST DATA FORM 12-1 BUILDING POWER AND LIGHTING - BUILDING POWER RECEPTACLE

TEST DATA FORM 12-2 BUILDING POWER AND LIGHIING - BUILDING LIGHTING FIXTURES

TEST DATA FORM 12-3 BUILDING POWER AND LIGHTING - AREA LIGHTING FLXTURES

TEST DATA FORM $12-4$ BUILDING POWER AND LIGHTING - CONTROL PANEL HEATERS 
WHC-SD-W025-RPT-001, Rev. 0

WHC-SD-W025-ATP-01 Rev.-3

TEST EXECUTION SHEET

TEST EXECUTION

ATP No.

Test No.

Installation Contractor Date

Recorder/Organization

Date

Test Director/Organization

Date

\section{TEST WITNESS}

\begin{tabular}{lll}
\hline Witness/Organization & Date & Witness/Organization
\end{tabular}

\begin{tabular}{llll}
\hline Witness/Organization & Date & Witness/Organization & Date
\end{tabular}

\section{IEST ACCEPTANCE}

Kaiser Engineers Hanford

$\begin{array}{ll}\begin{array}{l}\text { Without } \\ \text { Exception }\end{array} & \begin{array}{l}\text { With } \\ \text { Exception/Resolved }\end{array} \\ \text { Field Engineer } & \text { Date }\end{array}$

\begin{tabular}{llll}
\hline Design Engineer & Date & Project Engineer
\end{tabular}

\section{IEST APPROVAL AND ACCEPTANCE}

Westinghouse Hanford Company

$\begin{array}{lll}\text { Without } & \text { With } & \text { With } \\ \text { Exception } & \text { Exception/Resolved _ } & \text { Exception/Outstanding }\end{array}$

\begin{tabular}{llll}
\hline (Title or Department) & Date & (Title or Department) & Date \\
\hline (Title or Department) & Date & (Title or Department) & Date
\end{tabular}


WHC-SD-W025-RPT-001, Rev. 0

\section{EXCEPTION SHEET}

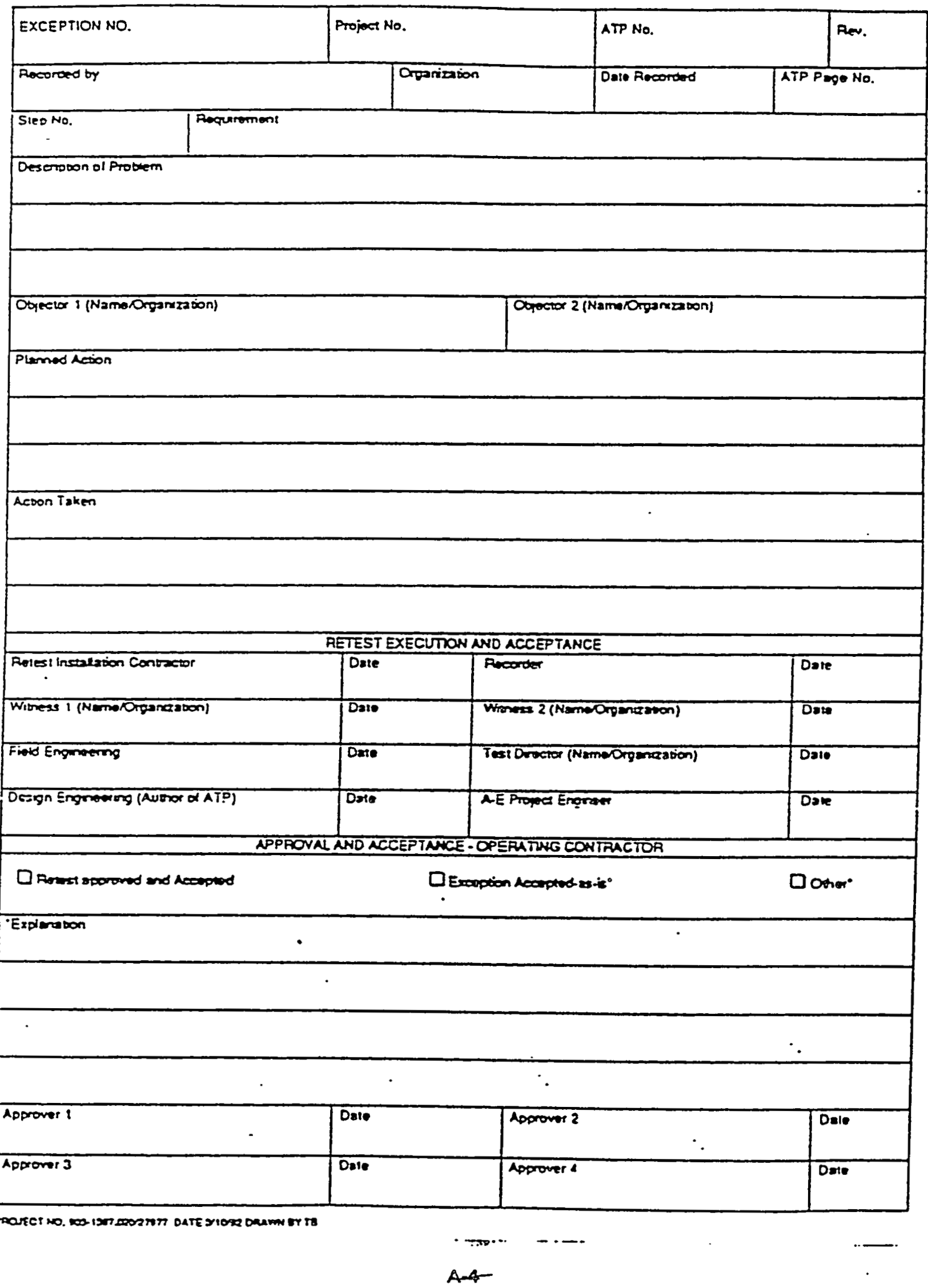


WHC-SD-W025-RPT-001, Rev. 0

WHE-SE-WO25-ATP=DI Rev. 3

IEST DATA FORM \&-1

HEAT TRACE SYSTEM - TEMPERATURE SENSOR TEST

Test Equipment: DMM Manufacturer and Model No.

Serial No.

Step No,
\begin{tabular}{|l|l|c|c|c|c||}
\hline \multicolumn{1}{|c|}{ Activity } & Verify & Reading & Date & By \\
\hline 8.1 .1 & Disconnect conductors & & NA & & \\
\hline 8.1 .2 & Visual inspection & & NA & & \\
\hline 8.1 .3 & Connect DMM & & NA & & \\
\hline 8.1 .4 & Cool or heat & & NA & & \\
\hline 8.1 .5 & Contacts open & NA & & & \\
\cline { 2 - 7 } & Contacts closed & NA & & & \\
\hline 8.1 .6 & Reconnect conductors & & NA & & \\
\hline
\end{tabular}


WHC-SD-W025-RPT-001, Rev. 0

WHCSDWO25-ATP-001-REV.3

\section{TEST DATA FORM \&-2}

HEAT TRACE SYSTEM - HEAT TRACE CABLE TEST

Test Equipment. COA Manufacturer and Model No. Serial No.

\begin{tabular}{|c|c|c|c|c|c|c|}
\hline Step No. & Activity & & Verify & Reading & Date & By \\
\hline 8.21 & Energize circuit & $\cdot \cdot$ & $\cdot$ & $\mathrm{NA}$ & & \\
\hline 8.22 & Contacts closed & & $\therefore \quad N A$ & & & \\
\hline 8.23 & Contacts open & & - - NA & - & & $\cdots$ \\
\hline
\end{tabular}

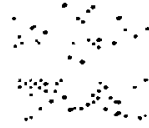

$\cdot \cdot$ 
WHC-SD-W025-RPT-001, Rev. 0

WIE-SD-WO2SATP-001 Rev.3

IEST DATA FORM 9.1

TRANSDUCERS AND METER/CONTROLLERS - PRIMARY SUMP

Test Equipment. WLI Manufacturer and Model No.

Serial No.

Water depths for pump controls: D1

D2

D3

\begin{tabular}{|c|c|c|c|c|c|}
\hline Step No. & Activity & Verify & Reading & Date & By \\
\hline 9.1.1 & Disconnect pump power & & NA & & \\
\hline \multirow[t]{3}{*}{9.12} & Install WLT & & NA & & \\
\hline & WLT reading & NA & & & \\
\hline & Sump water level & NA & & & \\
\hline \multirow[t]{2}{*}{9.1 .3} & Sump water level & NA & & & \\
\hline & Sump level meter reading & NA & & & \\
\hline 9.1 .4 & Verify relays are correct & & NA & & \\
\hline \multirow[t]{14}{*}{9.1 .5} & Water level \#1 & NA & & & \\
\hline & Sump level meter reading \# 1 & NA & & & \\
\hline & Water level \#2 & NA & & & \\
\hline & Sump level meter reading \#2 & NA & & & \\
\hline & Water level \#3 & NA & & & \\
\hline & Sump level meter reading \#3 & NA & & & \\
\hline & Water level \#4 & NA & & & \\
\hline & Sump level meter reading \#4 & NA & & & \\
\hline & Water level \#5 & NA & & & \\
\hline & Sump level meter reading \#5 & NA & & & \\
\hline & Water level \#6 & NA & & & \\
\hline & Sump level meter reading \#6 & NA & & & \\
\hline & Water level \#7 & NA & & & \\
\hline & Sump level meter reading \#7 & NA & & & \\
\hline
\end{tabular}

$x-7$ 
WHC-SD-W025-RPT-001, Rev. 0

WHESD W025-AIP-001-ReV.-3

IEST DATA FORM 9-1 (Cont)

\begin{tabular}{|c|c|c|c|c|c|}
\hline Step No. & Activity & Verify & Reading & Date & By \\
\hline & Water level \#8 & NA & & & \\
\hline & Sump level meter reading \#8 & NA & & & \\
\hline & Water level \#9 & NA & & & \\
\hline & Sump level meter reading \#9 & NA & & & \\
\hline & Water level \#10 & NA & & & \\
\hline & Sump level meter reading \#10 & NA & & & \\
\hline \multirow[t]{2}{*}{9.1 .6} & Verify CR2 de-energized & & NA & & \\
\hline & Water level & NA & & & \\
\hline \multirow[t]{4}{*}{9.1 .7} & Verify CR4 de-energized & & NA & & \\
\hline & Water level (CR4) & NA & & & \\
\hline & Venify CRI energized & & NA & & \\
\hline & Water level (CRI) & NA & & & \\
\hline \multirow[t]{2}{*}{9.1 .8} & Water level & NA & & & \\
\hline & Sump level meter reading & NA & & & \\
\hline \multirow[t]{2}{*}{8.1 .9} & Check high level float on & & NA & & \\
\hline & Check high level float off & & $\mathrm{NA}$ & & \\
\hline
\end{tabular}


IEST DATA FORM 9-2

TRANSDUCERS AND MEIER/CONTROLLERS - SECONDARY SUMP

Water depths for pump controls: DS-1

;

DS-2

\begin{tabular}{|c|c|c|c|c|c|}
\hline Step No. & Activity & Verify & Reading & Date & By \\
\hline 9.2 .1 & Disconnect pump power & & NA & & \\
\hline 9.22 & Verify relays are correct & & NA & & \\
\hline 9.23 & $\begin{array}{l}\text { Calibrate transducer } \\
\text { (attach calibration sheet) }\end{array}$ & & NA & & \\
\hline 9.2 .4 & Sump level meter reading & NA & & & \\
\hline \multirow[t]{20}{*}{9.25} & Water volume \#1 & NA & & & \\
\hline & Sump level meter reading \#1 & NA & & & \\
\hline & Water volume \#2 & NA & & & \\
\hline & Sump level meter reading \#2 & NA & & & \\
\hline & Water volume \#3 & NA & & & \\
\hline & Sump level meter reading \#3 & NA & & & \\
\hline & Water volume \#4 & NA & & & \\
\hline & Sump level meter reading \#4 & NA & & & \\
\hline & Water volume \#5 & NA & & & \\
\hline & Sump level meter reading \#5 & NA & & & \\
\hline & Water volume \#6 & NA & & & \\
\hline & Sump level meter reading \#6 & NA & & & \\
\hline & Water volume \#7 & NA & & & \\
\hline & Sump level meter reading \#7 & NA & & & \\
\hline & Water volume \#8 & NA & & & \\
\hline & Sump level meter reading \#8 & NA & & & \\
\hline & Water volume \#9 & NA & & & \\
\hline & Sump level meter reading \#9 & NA & & & \\
\hline & Water volume \#10 & NA & . & & $\cdot$ \\
\hline & Sump level meter reading \#10 & NA & & & \\
\hline
\end{tabular}


WHC-SD-W025-RPT-001, Rev. 0

WIE-SD-WO2S-ATP-DOTREV.3

IEST DATA FORM 9-2 (Cont)

\begin{tabular}{|l|l|c|c|c|c|}
\hline \hline Step No. & \multicolumn{1}{|c|}{ Activity } & Verify & Reading & Date & By \\
\hline 9.26 & Verify CR6 deenergized & $\cdot$ & NA & & \\
\hline & Sump level meter reading (CR6) & NA & & & \\
\hline & Verify CR5 energized & & NA & & \\
\hline & Sump level meter reading (CR5) & NA & & & \\
\hline
\end{tabular}

$A+0$ 
WHC-SD-W025-RPT-001, Rev. 0

WAIE-SDWOZAIP-DI REV.3

ATTACHMENT TO TEST DATA FORM 9-2

CALIBRATION SHEET - SECONDARY SUIMP TRANSDUCER

\begin{tabular}{|l|l|l|}
\hline Reading No. & Measured Water Depth & Transducer Reading \\
\hline & & \\
\hline & & \\
\hline & & \\
\hline & & \\
\hline & & \\
\hline & & \\
\hline & & \\
\hline & & \\
\hline & & \\
\hline & & \\
\hline & & \\
\hline & & \\
\hline & & \\
\hline
\end{tabular}

$\therefore \cdots \cdots \cdot \because$

$\therefore, \cdots, \because, \because, \cdots$

$\because \because \because{ }^{*} \because \cdots$

A- +4 
WHC-SD-W025-RPT-001, Rev. 0

WIE-SD-WO25-ATW1 RE.3

IEST DATA FORM 10-1

PUMP TESTS - INITIAL CONDITIONS

Water depths for pump controls: D1

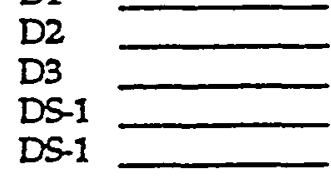

\begin{tabular}{|c|c|c|c|c|c|}
\hline Step No. & Activity & Verify & Reading & Date & By \\
\hline & Verify CR7 de-energized & & NA & & \\
\hline \multirow[t]{11}{*}{10.1 .1} & Primary sump water level & NA & & & \\
\hline & \multicolumn{5}{|l|}{ Verify on control panel: } \\
\hline & CRI energized & & NA & & \\
\hline & CR2 de-energized & & NA & & \\
\hline & CR3 de-energized & & NA & & \\
\hline & CR4 de-energized & & NA & & \\
\hline & Pump failure light on & & NA & & \\
\hline & Roof alarm light on & & NA & & \\
\hline & \multicolumn{5}{|l|}{ Relay indicator lights: } \\
\hline & CRI on & & NA & & \\
\hline & CR2, CR3, CR4 off & & NA & & \\
\hline \multirow[t]{8}{*}{10.1 .2} & Secondary sump water level & NA & & & \\
\hline & \multicolumn{5}{|l|}{ Verify on control panel: } \\
\hline & CR5 energized & & NA & & \\
\hline & CR6 de-energized & & NA & & \\
\hline & Pump failure light on & & NA & & \\
\hline & Roof alarm light on & & NA & & \\
\hline & \multicolumn{5}{|l|}{ Relay indicator lights on: } \\
\hline & CR5 & & NA & & \\
\hline
\end{tabular}


WHC-SD-W025-RPT-001, Rev. 0

HAIG-SD-WO25-ATP=0O1 Rev. 3

IEST DATA FORM 10-2

PUMP TESTS - PRIMARY SUBMERSIBLE PUMP (WSP1)

\begin{tabular}{|c|c|c|c|c|c|}
\hline Step No. & Activity & Verify & Reading & Date & By \\
\hline \multirow[t]{3}{*}{10.0} & \multirow[t]{3}{*}{ Phase current } & NA & & & \\
\hline & & NA & & & \\
\hline & & NA & & & \\
\hline 10.0 & Motor overload size & & NA & & \\
\hline 10.21 & Energize pump & & NA & & \\
\hline 10.22 & Switch to "hand" & & NA & & \\
\hline 10.23 & Verify pumping & & NA & & \\
\hline 10.24 & Verify W54 energized & & NA & & \\
\hline \multirow[t]{2}{*}{10.25} & Verify "run" light on & & NA & & \\
\hline & Drain sump below high level & & NA & & \\
\hline \multirow[t]{2}{*}{10.26} & Activate CRI & & NA & & \\
\hline & Water level at start & NA & & & \\
\hline \multirow[t]{2}{*}{10.2 .7} & Verify auto start & & NA & & \\
\hline & Verify "run" light on & & NA & & \\
\hline 10.28 & Verify pumping & & NA & & \\
\hline \multirow[t]{2}{*}{10.29} & Verify pump shut off & & NA & & \\
\hline & Water level at shut off & NA & & & \\
\hline 10.210 & Verify auto start & & NA & & \\
\hline \multirow[t]{2}{*}{10.211} & Verify automatic stop & & NA & & \\
\hline & Verify roof alarm & & NA & & \\
\hline 10.212 & Switch motor starter to "off" & & NA & & \\
\hline
\end{tabular}


WHC-SD-W025-RPT-001, Rev. 0

WHE-SD-W025-ATP-001 Rev. 3

IEST DATA FORM 10-3

PUMP TESTS - SECONDARY SUBMERSIBLE PUMP (W5P2)

\begin{tabular}{|c|c|c|c|c|c|}
\hline Step No. & Activity & Verify & Reading & Date & By \\
\hline \multirow[t]{3}{*}{10.0} & \multirow[t]{3}{*}{ Phase current } & NA & & & \\
\hline & & NA & & & \\
\hline & & NA & & & \\
\hline 10.0 & Motor overioad size & & NA & & \\
\hline 10.3 .1 & Energize pump & & NA & & \\
\hline 10.3 .2 & Switch to "hand" & & NA & & \\
\hline 10.3 .3 & Verify pumping & & NA & & \\
\hline \multirow[t]{2}{*}{10.3 .4} & Verify "run" light on & & NA & & \\
\hline & Drain sump below low level & & NA & & \\
\hline \multirow[t]{2}{*}{10.3 .5} & Activate CR5 & & NA & & \\
\hline & Water level at start & NA & & & \\
\hline \multirow[t]{2}{*}{10.3 .6} & Verify auto start & & NA & & \\
\hline & Verify "run" light on & & NA & & \\
\hline 10.3 .7 & Verify pumping & & NA & & \\
\hline \multirow[t]{2}{*}{10.3 .8} & Verify pump shut off & & NA & & \\
\hline & Water level at shut off & NA & & & \\
\hline \multirow[t]{2}{*}{10.3 .10} & Verify automatic stop & & NA & & \\
\hline & Verify roof alarm & & NA & & \\
\hline
\end{tabular}


WHC-SD-W025-RPT-001, Rev. 0

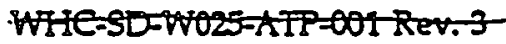

TEST DATA FORM 10-4

PUMP TESTS - PRIMARY SUMP SELF-PRIMING PUMP (W5P3)

\begin{tabular}{||l|l|l|l|l|l|}
\hline Step No. & \multicolumn{1}{|c|}{ Activity } & Verify & Reading & Date & By \\
\hline 10.0 & Phase current & NA & & & \\
\cline { 3 - 6 } & & NA & & & \\
\hline & & & & & \\
\hline 10.0 & Motor overload size & NA & & \\
\hline 10.4 .1 & Add water to sump & NA & & & \\
\cline { 2 - 6 } & Energize pump & & NA & & \\
\hline 10.4 .2 & Switch to "hand" & & NA & & \\
\hline 10.4 .3 & Verify pumping & & NA & & \\
\hline 10.4 .4 & Verify "run" light on & & NA & & \\
\hline 10.4 .5 & Verify pumping 1 minute & & NA & & \\
\hline 10.4 .6 & Switch to "auto" & & NA & & \\
\cline { 2 - 7 } & Add water to sump & NA & & & \\
\hline 10.4 .7 & Raise high level switch & & NA & & \\
\hline 10.4 .8 & Verify auto start & & NA & & \\
\hline 10.4 .9 & Verify pump shut off & & NA & & \\
\hline & Water level at shut off & NA & & & \\
\hline 10.4 .10 & Verify automatic stop & & NA & & \\
\hline & Verify roof alarm & NA & & \\
\hline & & & & \\
\hline
\end{tabular}


WHC-SD-W025-RPT-001, Rev. 0

WFC-SD=W025-ATP-O01Rer.3-

IEST DATA FORM 10-5

PUMP TESTS - FLOWMETER CALIBRATION

\begin{tabular}{|c|c|c|c|c|c|}
\hline Step No. & Activity & Verify & Reading & Date & By \\
\hline 10.5 .1 & Verify water volume & & NA & & \\
\hline 10.5 .2 & Verify water clean & & $N A$ & & \\
\hline \multirow[t]{3}{*}{10.5 .3} & Ball valve W65 open & & NA & & \\
\hline & Ball value W66 closed & & NA & & \\
\hline & Solenoid ball valve closed & & NA & & \\
\hline 10.5 .4 & Water level in tank & $\mathrm{NA}$ & & & \\
\hline 10.5 .5 & Purge air & & NA & & \\
\hline 10.5 .6 & Water level in tank & NA & & & \\
\hline \multirow[t]{2}{*}{10.5 .7} & 2-inch flowmeter reading & NA & & & \\
\hline & B-inch flowmeter reading & $\mathrm{NA}$ & & & \\
\hline 10.5 .8 & Pump 500 gallons - 1st cycle & & NA & & \\
\hline 10.5 .9 & Water level in tank - 1st cycle & NA & & & \\
\hline \multirow[t]{3}{*}{10.5 .10} & $\begin{array}{l}\text { 2-inch flowmeter reading - 1st } \\
\text { Eycle }\end{array}$ & NA & & & \\
\hline & $\begin{array}{l}\text { 3-inch flowmeter reading - 1st } \\
\text { Eycle }\end{array}$ & NA & & & \\
\hline & Purge air & & NA & & \\
\hline \multirow[t]{8}{*}{10.5 .11} & Pump 500 gallons - 2nd cycle & & NA & & \\
\hline & Water level in tank - 2nd cycle & $\mathrm{NA}$ & & & \\
\hline & $\begin{array}{l}\text { 2-inch flowmeter reading - } 2 \text { nd } \\
\text { Eycle }\end{array}$ & NA & & & \\
\hline & $\begin{array}{l}\text { 3.inch flowmeter reading - } 2 \text { nd } \\
\text { Eycle }\end{array}$ & NA & & & \\
\hline & Pump 500 gallons - 3rd cycle & & NA & & \\
\hline & Water level in tank - 3rd cycle & $N A$ & & & \\
\hline & $\begin{array}{l}\text { 2-inch flowmeter reading - } 3 \text { rd } \\
\text { Eycle }\end{array}$ & NA & & & \\
\hline & $\begin{array}{l}\text { 3-inch flowmeter reading - 3rd } \\
\text { Eycle }\end{array}$ & NA & & & \\
\hline
\end{tabular}

Anto 
WHC-SD-W025-RPT-001, Rev. 0

WHC-SD-W025-ATP-DO1-Rev. 3-

IEST DATA FORM $10-6$

PUMP IESTS - SPARE PUMPS

Pressure Gage Model and SN

\begin{tabular}{|l|l|l|l|l|l|}
\hline $\begin{array}{c}\text { Pump Type and } \\
\text { Serial No. }\end{array}$ & $\begin{array}{c}\text { Volume } \\
\text { Pumped } \\
\text { (units) }\end{array}$ & Time & $\begin{array}{c}\text { Pressure } \\
\text { Head } \\
\text { (units) }\end{array}$ & Date & By \\
\hline & & & & & \\
\hline & & & & & \\
\hline & & & & & \\
\hline & & & & & \\
\hline & & & & & \\
\hline & & & & & \\
\hline & & & & & \\
\hline & & & & & \\
\hline & & & & & \\
\hline & & & & & \\
\hline
\end{tabular}


WHC-SD-W025-RPT-001, Rev. 0

WHIESA-WO25-ATPOOI ReV.3

IEST DATA FORM 11-1

LEACHATE STORAGE TANK - TANK PUMP (W5P4)

DMM Manufacturer and Model No.

Serial No.

\begin{tabular}{|c|c|c|c|c|c|}
\hline Step No. & Activity & Verify & Reading & Date & By \\
\hline \multirow[t]{3}{*}{10.0} & \multirow[t]{3}{*}{ Phase current } & NA & & & \\
\hline & & NA & & & \\
\hline & & NA & & & \\
\hline 10.0 & Motor overload size & & NA & & \\
\hline 11.1 .1 & Check tank; activate breaker & & NA & & \\
\hline \multirow[t]{4}{*}{11.1 .2} & \multicolumn{5}{|l|}{ Voltage between terminals: } \\
\hline & $I 1$ and $T 2$ & NA & & & \\
\hline & $\mathrm{I} 2$ and $\mathrm{T} 3$ & NA & & & \\
\hline & 11 and metal frame & NA & & - & \\
\hline \multirow[t]{2}{*}{11.1 .3} & Verify motor rotation & & NA & & \\
\hline & Verify pumping & & NA & & \\
\hline \multirow[t]{3}{*}{ 11.1.4 } & \multicolumn{5}{|l|}{ Voltage between terminals: } \\
\hline & $\mathrm{N} 1$ and $\mathrm{T} 2$ & NA & & & \\
\hline & 12 and 13 & NA & & & \\
\hline 11.1 .5 & Verify pump shut off & & NA & & \\
\hline
\end{tabular}


WHC-SDD-W025-RPT-001, Rev. 0

WHE-SD-W025-ATP-001 ReV.3

IEST DATA FORM 11-2A

LEACHATE STORAGE TANK - HIGH LEVEL FLOAT SWTTCH (FS3)

Tank high water level

\begin{tabular}{|c|c|c|c|c|c|}
\hline Step No. & Activity & Verify & Reading & Date & By \\
\hline \multirow[t]{2}{*}{1121} & Verify "high tank level" light & & NA & & \\
\hline & Verify CR7 de-energized & & NA & & \\
\hline \multirow[t]{2}{*}{11.22} & Verify FS3 & & NA & & \\
\hline & Water level at FSS tum on & NA & & & \\
\hline 11.23 & $\begin{array}{l}\text { Verify CR7 energized and } \\
\text { record time delay }\end{array}$ & & & & \\
\hline 1124 & Verify "high tank level" light & & NA & & \\
\hline 11.25 & $\begin{array}{l}\text { Verify roof alarm and record } \\
\text { time delay }\end{array}$ & & & & \\
\hline \multirow[t]{4}{*}{11.26} & Verify pumps inoperable: & & & & \\
\hline & W5P1 & & NA & & \\
\hline & W5P2 & & NA & & \\
\hline & W5P3 & & NA & & \\
\hline \multirow[t]{3}{*}{11.27} & Verify "high tank level" light & & NA & & \\
\hline & Verify $C R 7$ de-energized & & NA & & \\
\hline & Water level at FS3 tum off & NA & & & \\
\hline
\end{tabular}


WHC-SD-W025-RPT-001, Rev. 0

WTE-SD-W25-ATP-DO1_Rev_3

IEST DATA FORM 11-2B

LEACHATE STORAGE TANK - HIGH LEVEL LIMTT SWITCH (Li)

Tank high water level

\begin{tabular}{|c|c|c|c|c|c|}
\hline Step No. & Activity & Verify & Reading & Date & By \\
\hline \multirow[t]{2}{*}{11.21} & Verify "high tank level" light & & NA & & \\
\hline & Verify CR7 de-nergized & & NA & & \\
\hline \multirow[t]{2}{*}{11.2 .2} & Verify LI & & NA & & \\
\hline & Water level at L1 turn on & NA & & & \\
\hline 11.23 & $\begin{array}{l}\text { Verify CR7 energized and } \\
\text { record time delay }\end{array}$ & & & & \\
\hline 11.24 & Verify "high tank level" light & & NA & & \\
\hline 11.2 .5 & $\begin{array}{l}\text { Verify roof alarm and record } \\
\text { time delay }\end{array}$ & & & & \\
\hline \multirow[t]{4}{*}{11.2 .6} & Verify pumps inoperable: & & & - & \\
\hline & W5P1 & & NA & & \\
\hline & W5P2 & & NA & & \\
\hline & W5P3 & & NA & & \\
\hline \multirow[t]{3}{*}{11.27} & Verify "high tank level" light & & NA & & \\
\hline & Verify CR7 de-energized & & NA & & \\
\hline & Water level at II turn off & NA & & & \\
\hline
\end{tabular}


WHC-SD-W025-RPT-001, Rev. 0

WHC.SDWW25-ATP=001ReV. 3

IEST DATA FORM 12-1

BUILDING POWER AND LIGHTING - BUILDING POWER RECEPTACLE

Test Equipment Receptacle Tester Manufacturer and Model No. Serial No.

\begin{tabular}{|l|l|c|c|c|c|}
\hline Step No. & \multicolumn{1}{|c|}{ Activity } & Verify & Reading & Date & By \\
\hline 12.1 .1 & Verify receptacle wiring & & NA & & \\
\hline 12.1 .2 & $\begin{array}{l}\text { Verify that GFI trips } \\
\text { (outlet 1) }\end{array}$ & & NA & & \\
\hline 12.1 .3 & $\begin{array}{l}\text { Verify that GFI trips } \\
\text { (outlet 2) }\end{array}$ & & NA & & \\
\hline
\end{tabular}


WHC-SD-W025-RPT-001, Rev. 0

WHE-SD-W025-ATPDO1_ReV 3

IEST DATA FORM $12-2$

BUILDING POWER AND LIGHTING - BUILD̈ING LIGHTING FXXTURES

\begin{tabular}{|l|c|c|c|c|c|}
\hline Step No & Activity & Verify & Reading & Date & By \\
\hline 1221 & Verify lights function & $\ddots$ & $\cdot$ NA & $\cdots$ & $\cdots \ldots$ \\
\hline
\end{tabular}

A-22 
WHC-SD-W025-RPT-001, Rev. 0

WHE-SD-WO25-ATTP-OOTRE. R

TEST DATA FORM 12-3

BUILDING POWER AND LIGHTING - AREA LIGHTING FIXTURES

\begin{tabular}{|c|c|c|c|c|c|}
\hline Step No. & Activity & Verify & Reading & Date & By \\
\hline 123.1 & \multicolumn{5}{|c|}{ Verify initial illumination: } \\
\hline & P1a & & NA & & \\
\hline & $\mathrm{P} 1 \mathrm{~b}$ & & $\mathrm{NA}$ & & \\
\hline & $\mathbf{P 2}$ & & $\mathrm{NA}$ & & \\
\hline & P3 & & $\mathrm{NA}$ & & \\
\hline & $\mathrm{P4}$ & & NA & & \\
\hline \multirow[t]{19}{*}{123.2} & \multicolumn{5}{|c|}{ Verify illumination over time: } \\
\hline & Time \#1 & NA & & & \\
\hline & P1a & & $\mathrm{NA}$ & & \\
\hline & P1b & & NA & & \\
\hline & $\mathrm{P2}$ & & NA & & \\
\hline & P3 & & $\mathrm{NA}$ & & \\
\hline & $\mathrm{P} 4$ & & $\mathrm{NA}$ & & \\
\hline & Time \#2 & NA & & & \\
\hline & P1a & & NA & & \\
\hline & P1b & & NA & & \\
\hline & $\mathrm{P2}$ & & NA & & \\
\hline & P3 & & $\mathrm{NA}$ & & \\
\hline & $\mathrm{P4}$ & & NA & & \\
\hline & Time \#3 & NA & & & \\
\hline & P1a & & NA & & \\
\hline & $\mathrm{P} 1 \mathrm{~b}$ & & $\mathrm{NA}$ & & \\
\hline & $\mathrm{P2}$ & & $\mathrm{NA}$ & & \\
\hline & P3 & & NA & & \\
\hline & $\mathrm{P4}$ & & $\mathrm{NA}$ & & \\
\hline
\end{tabular}


WHC-SD-W025-RPT-001, Rev. 0

WIIESD-W025-AIT-001-ReV. 3-

IEST DATA FORM $12-3$ (Cont)

\begin{tabular}{|c|c|c|c|c|c|}
\hline Step No. & Activity & Verify & Reading & Date & By \\
\hline & Time \#4 & $\mathrm{NA}$ & & & \\
\hline & Pla & & NA & & \\
\hline & P1b & & NA & & \\
\hline & P2 & & NA & & \\
\hline & P3 & & NA & & \\
\hline & P4 & & NA & & \\
\hline & Time \#5 & NA & & & \\
\hline & $\mathrm{Pla}$ & & NA & & \\
\hline & Pib & & $\mathrm{NA}$ & & \\
\hline & P2 & & NA & & \\
\hline & P3 & & NA & & \\
\hline & P4 & & NA & & \\
\hline 123.3 & Verify fixtures tum & & & & \\
\hline & Pia & & NA & & \\
\hline & P1b & & NA & & \\
\hline & $\mathrm{P} 2$ & & NA & & \\
\hline & P3 & & NA & & \\
\hline & P4 & & $\mathrm{NA}$ & & \\
\hline
\end{tabular}


WHC-SD-W025-RPT-D01, Rev. 0

-WIE-SE-W025-ATP-OOT ReY.3.

TEST DATA FORM 124

BUILDING POWER AND LIGHIING - CONTROL PANEL HEATERS

\begin{tabular}{|c|c|c|c|c|c|}
\hline Step No. & Activity & Verify & Reading & Date & By \\
\hline 124.1 & Heater at ambient - Control Panel C & & NA & & \\
\hline 124.2 & $\begin{array}{l}\text { Heater becomes warm - Control } \\
\text { Panel C }\end{array}$ & & NA & & \\
\hline \multirow[t]{4}{*}{124.3} & Heater at ambient - Control Panel A & & NA & & \\
\hline & $\begin{array}{l}\text { Heater becomes warm - Control } \\
\text { Panel A }\end{array}$ & & NA & & \\
\hline & Heater at ambient - Control Panel B & & NA & & \\
\hline & $\begin{array}{l}\text { Heater becomes warm - Control } \\
\text { Panel B }\end{array}$ & & NA & & \\
\hline
\end{tabular}


WHC-SD-W025-RPT-001, Rev. 0

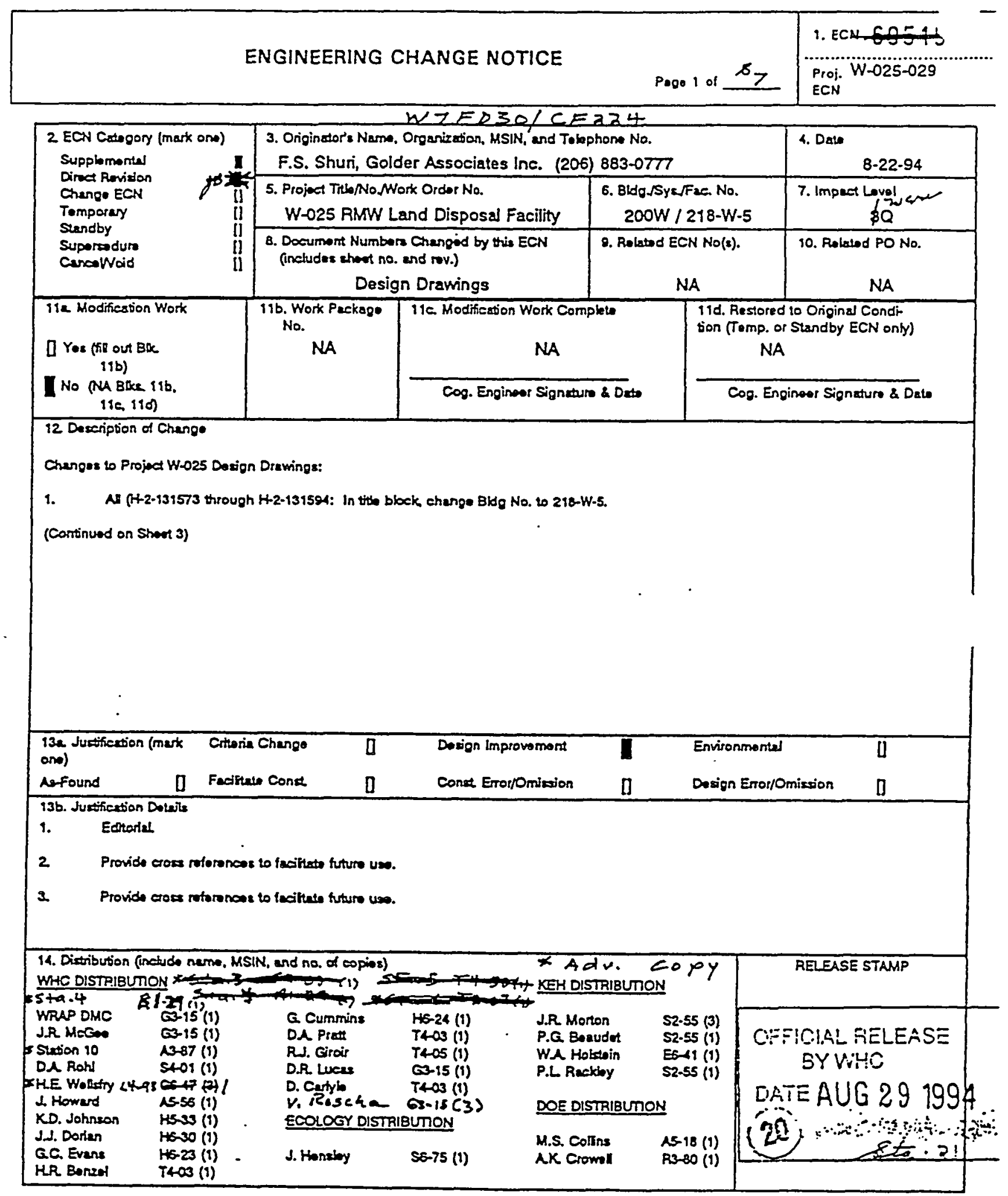

A-7000-013-2 (06/82) GEFOS5 


\section{ENGINEERING CHANGE NOTICE} Pago 2 \& 867

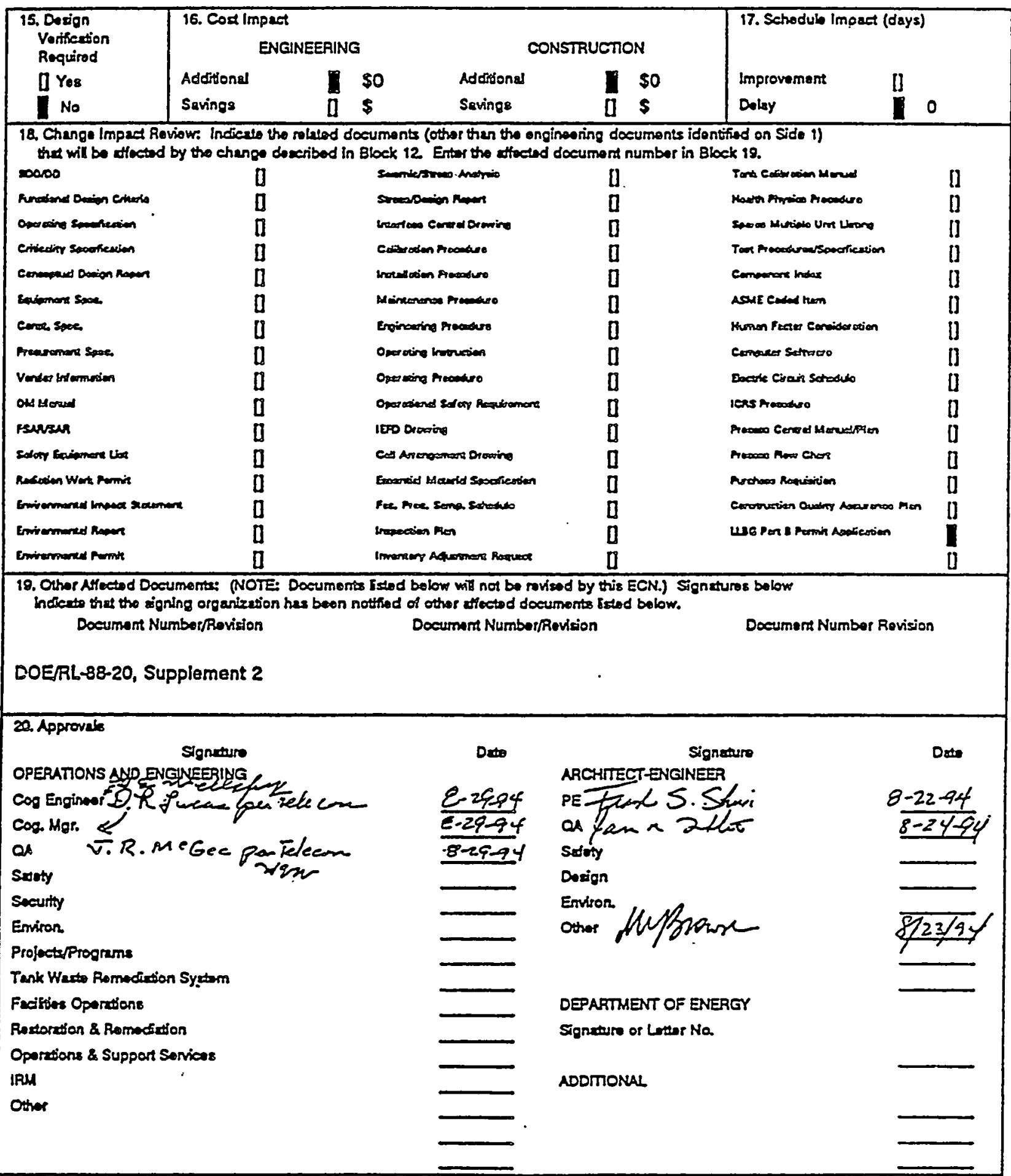


WHC-SD-W025-RPT-001, Rev. 0

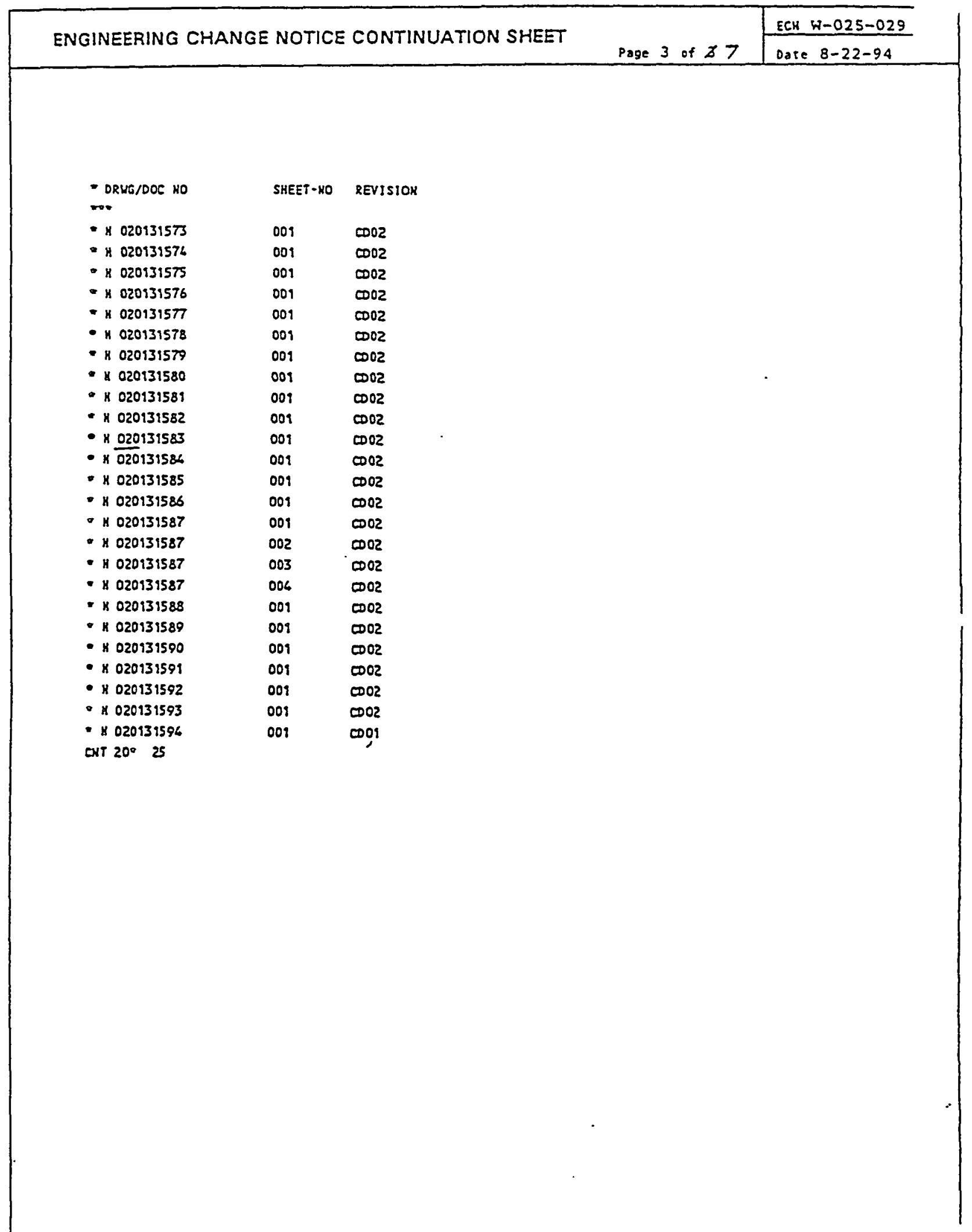

A-7900-013.6 (04/96) GEF096 


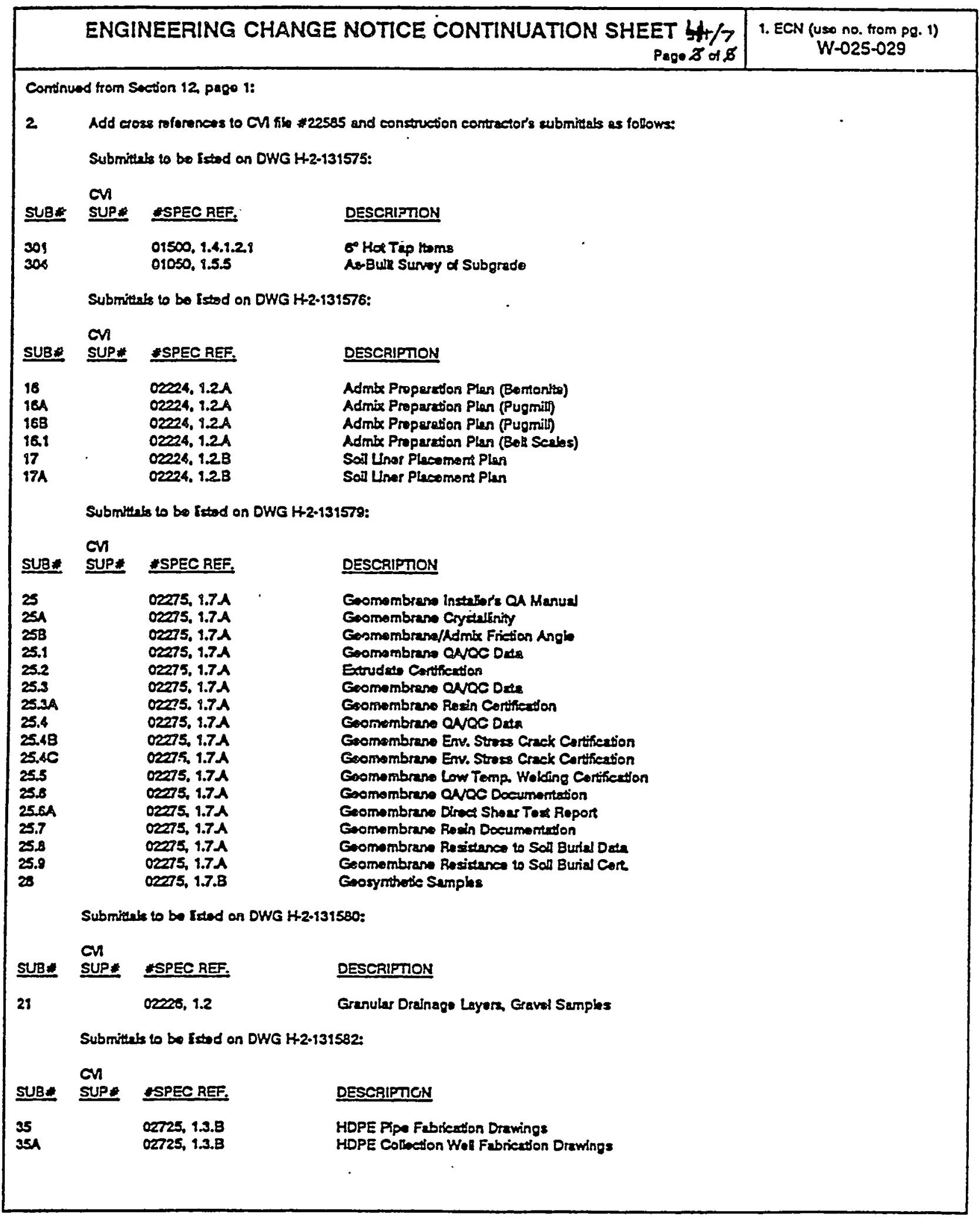




\begin{tabular}{|c|c|c|c|c|}
\hline & \multicolumn{3}{|c|}{ 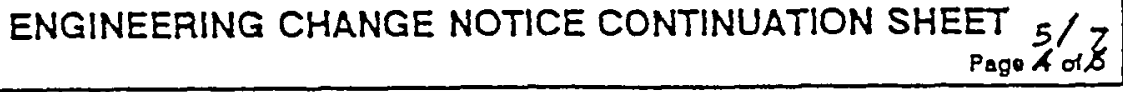 } & $\begin{array}{l}\text { 1. ECN (uso no. from po. 1) } \\
\text { W-025-029 }\end{array}$ \\
\hline \multicolumn{5}{|c|}{ Continued ham Soction 12 papo 3 : } \\
\hline \multicolumn{5}{|c|}{ Submitals to be tetod an DWG H-2-131583: } \\
\hline SUB: & $\begin{array}{l}\text { CV } \\
\text { SUP. }\end{array}$ & \#SPEC REF. & DESCRIPTION & \\
\hline 37 & & $02727,1.2 \mathrm{~A}$ & Vertical Piser Pipe & \\
\hline $37 A$ & & $02727,1.21$ & Procast Concruls Pipe & \\
\hline 38 & & $03210,1.2 \mathrm{~A}$ & Robas and Costing & \\
\hline 48 & & $09900,1.6 \wedge$.2 & Manhole Coating Manufacturer's Specifications & \\
\hline \multirow[t]{2}{*}{184} & & $08900,1.64 .2$ & Manhol Costing Manufacturers Specifications & \\
\hline & \multicolumn{4}{|c|}{ Submitals to be fatod on DWG H-2-131584: } \\
\hline SUB: & $\begin{array}{l}\text { CV } \\
\text { SUP: }\end{array}$ & \#SPEC REF. & DESCRIPTION & \\
\hline \multirow{3}{*}{$\begin{array}{l}31 \\
31.1\end{array}$} & & $02511,9.5$ & Asphat Mlx Design & \\
\hline & & 02511.1 .5 & Asphat Soal Coat & \\
\hline & \multicolumn{4}{|c|}{ Submittats to be fadod on DWG H-2-131585; } \\
\hline SUB: & $\begin{array}{l}\text { CV } \\
\text { SUP: }\end{array}$ & ESPEC REF. & DESCRIPTION & \\
\hline \$2 & & $03210,1.2 .3$ & Rober Shop Drawings & \\
\hline$\$ 0$ & & 03310.1 .21 & Ait $\rightarrow$ mitrining Admix Corticication & \\
\hline 41 & & 03310.1 .2 & Conerute Mla Dasign & \\
\hline$\$ 2$ & & $03310,1.2$ & Anehor Bolle & \\
\hline $\mathbf{s}$ & & 03310.12 & Watertops & \\
\hline 47 & & $00000,1.6 \Lambda 1$ & Pain Coating Syatome Lit & \\
\hline$\$ 8.1$ & & $08900,1.6 \Lambda 2$ & Tark Couting Menuiacturers Spocticutions & \\
\hline ss. & & $13205,13 \lambda$ & Laches Tank Fabriceston Drawings & \\
\hline s24 & & $13205,1.31$ & Lachut Tank Fabrication Drawings & \\
\hline $53 B$ & & 13205.1 .31 & Loachate Tank Liquid Loval Indiestor & \\
\hline 53.1 & & 13205.131 & Lachate Tank Fabricution Draminge - Vort & \\
\hline 53.2 & & 13205,131 & Lach to Tank Fabrication Drawings - Ebow & \\
\hline 53.3 & & 13205.1 .31 & Loachato Tank Fabrication Drawings & \\
\hline 53.4 & & 13205.1 .31 & Loachato Tank Fabrication Drawings - Lottes & \\
\hline 53.5 & & $13205,9.31$ & Lasch to Task Fubriextion Dwgk - ASME Cort & \\
\hline 53.8 & & $13205,1.31$ & Lonehato Task Fabricaton Drawings & \\
\hline 57 & & $15000,1.4 \hat{1}$ & Discharo Pipe - Shop Drawings & \\
\hline 58 & & $\$ 5000,9.4 .8$ & Laschate Tank Fabrication Drawings + Sholl & \\
\hline 59 & & 15050 , 1.4.C & Shinbes Stol Pipe Cortification & \\
\hline$\infty$ & & $15165,1.24$ & Hengora, Brackets Supports - Tochrical Dete & \\
\hline ฯ & & $15066,1.28$ & Pipo and Condut Support Sydom - Calculations & \\
\hline 62 & & $15068,1.2 \mathrm{C}$ & Pipo and Conduk Support Sytom - Drevings & \\
\hline$\infty$ & & 15100,131 & Vecuum Robess Valves & \\
\hline 64 & & 95900,921 & Solondd Drain Valve and Piping & \\
\hline Q.1 & & $15100,1.3 \AA$ & Solondid Drain Velve and Piping & \\
\hline os & & $15100,1.31$ & Sample Cocks & \\
\hline$\infty$ & & $95100,9.31$ & Chock Valves & \\
\hline 5 & & $15900,1.3 \hat{A}$ & Automatie Diain Valve - Menut. Information & \\
\hline 6 & & $15104,1.21$ & Buterity Valves & \\
\hline Eed & & 15104.121 & Butung Valves & \\
\hline$\infty$ & & $15150,1.2 A$ & Turbine Motor & \\
\hline & & $15150.1 .3 \AA$ & Turtion Moter - Installetion, Oparation, and Muintonenco Menual & \\
\hline 70 & & 15250. $1.3 \hat{A}$ & Leach to Tank and Pipe Insuletion - Menuf. Info. & \\
\hline 71 & & $15250,1.3 .8$ & Leachat Tank and Pipe Insulation - Cartifeation & \\
\hline
\end{tabular}




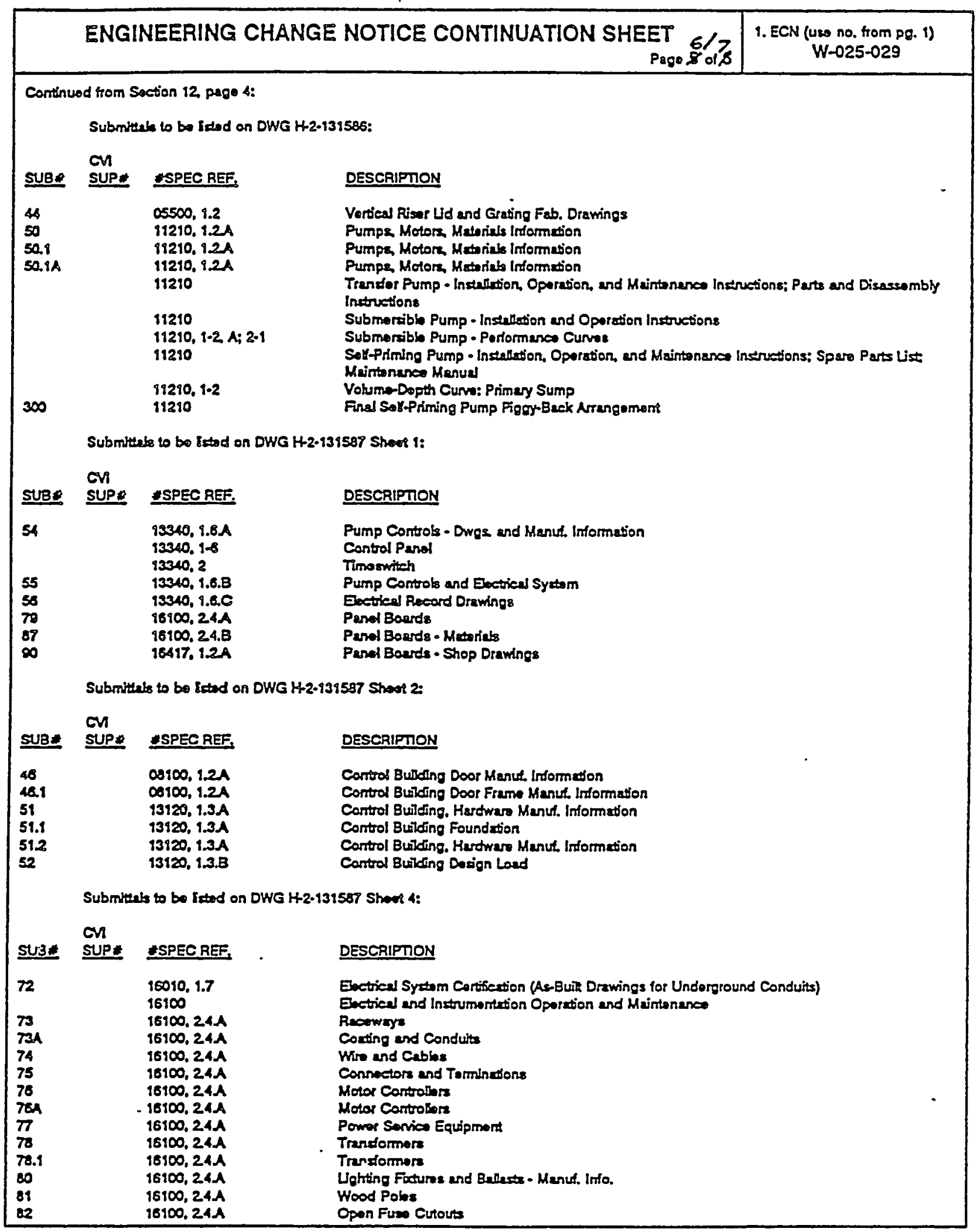


WHC-SD-W025-RPT-001, Rev. 0

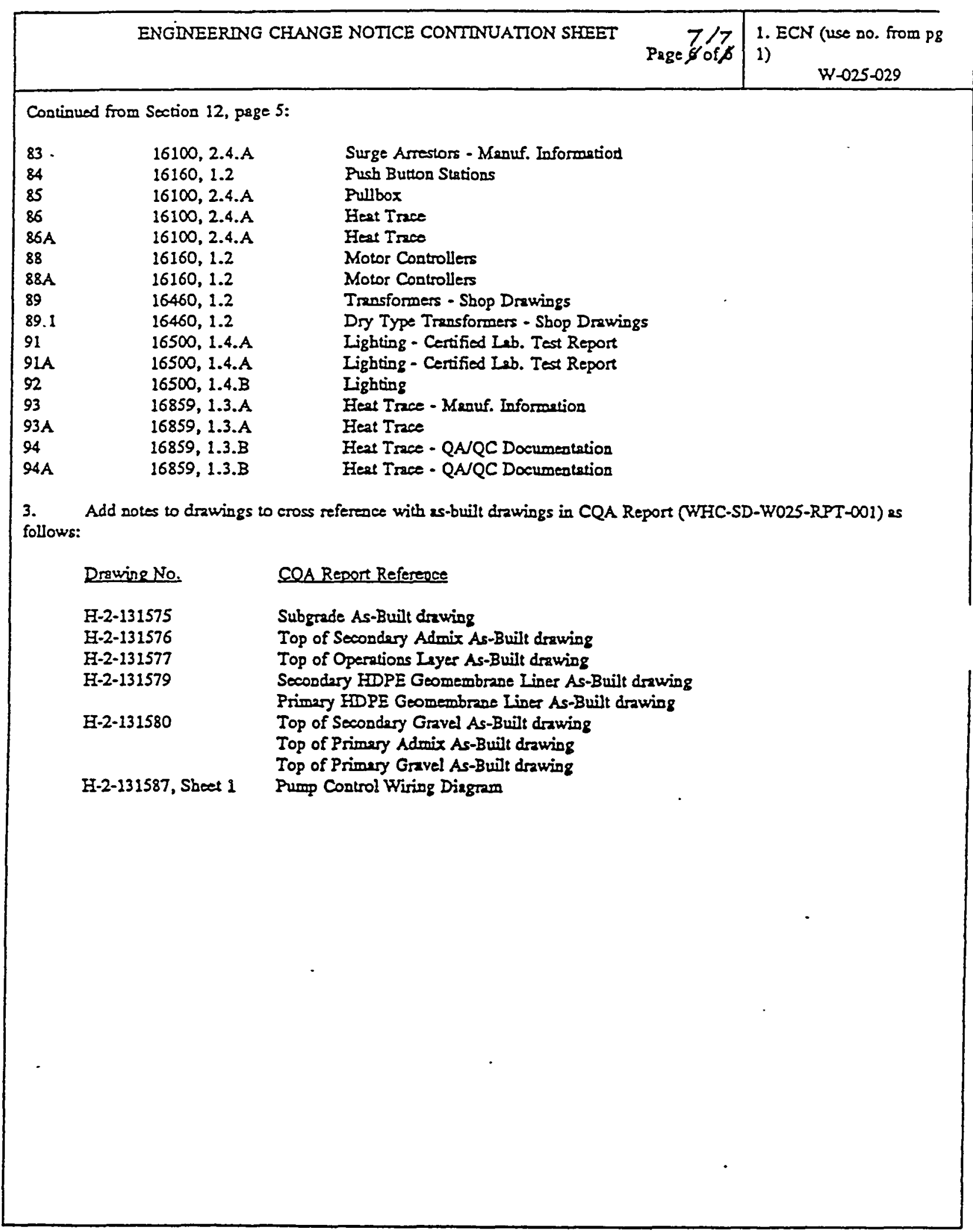


WHC-SD-W025-RPT-001, Rev. 0

\section{ENGINEERING CHANGE NOTICE}

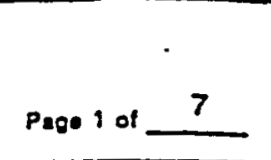

1. $\varepsilon C N=-\infty=-2=Z$

$\therefore$

Proj. W-025-031

ECN

WフะกZR

\begin{tabular}{|l|l}
\hline 2. ECN Category (mark ono) & 3. Origintors Namo, Organizaton, MSIN and Telophono No
\end{tabular}

Supplemental

Direat Rovision

Chengo ECN

Tomporary

Sindby

Suparsedure

cenconoid

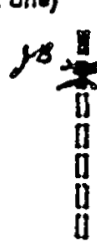

(206) 883-0777

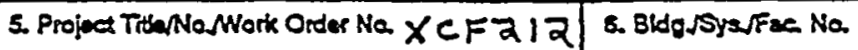
W-025 RMW Land Disposal Facility

6. Documon Numbers Changed by this ECN (nceludos theor na and rov.)

Design drawings. WHCS-045 Rev. 2

9. Roleted ECN No(s).

4. Date

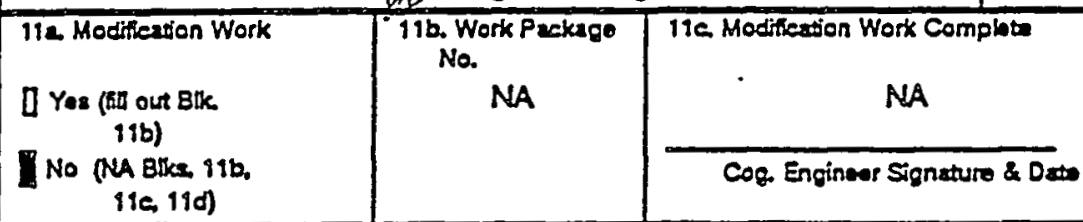

12. Dexcription of Change

Changes to Project W-025 Dasign Drawings:

1. Orawing No. 1-2-131585: sheet 1, Rev. 2 , tes

Louchato Tomparay Storago Tank and Plping Pian: Change TURBINE METER io FLOWMETER throughout

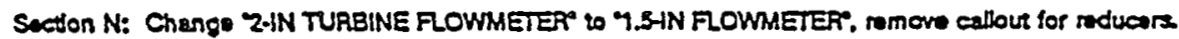

Sacton J: Chango TURBINE METER' wo "FLWMETER".

Dotal 38: Modfly per wehed Skatches 1 through 3.

Changes to Project W-025 Speciticutions:

2 Soetion 15150: Roplace in entirnty with etrached

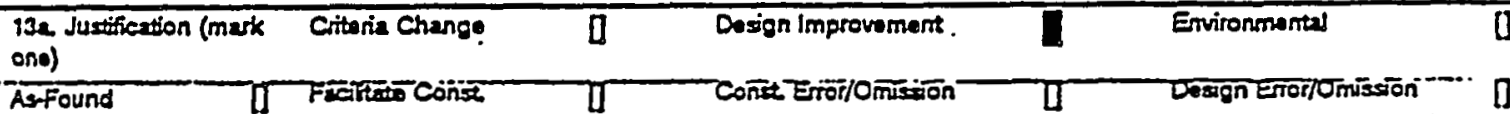

13b. Juxtificrion Detaits

1. Rofiect change in fowmeter typo and dimansions

2. incroas flowmetor acuracy.

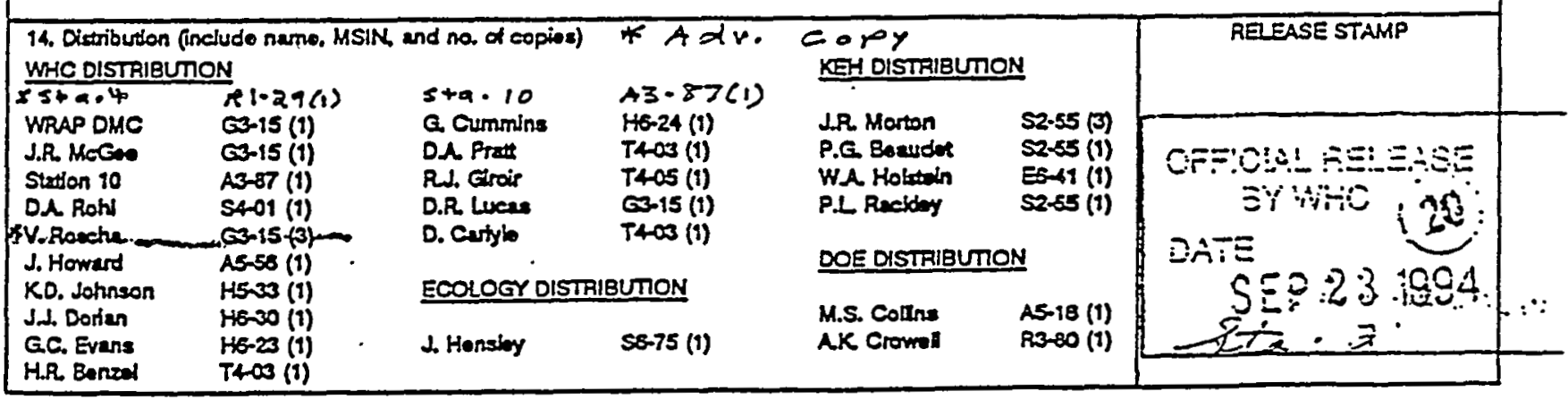

A.7900-013-2 (06/92) GEFO95 
WHC-SD-W025-RPT-001, Rev. 0

\begin{tabular}{|l|l|c|}
\hline ENGINEERING CHANGE NOTICE & Page 2 of 7 & $\begin{array}{c}\text { 1. ECN (uso no. trom pg. 1) } \\
\text { W-025-031 }\end{array}$ \\
\hline
\end{tabular}

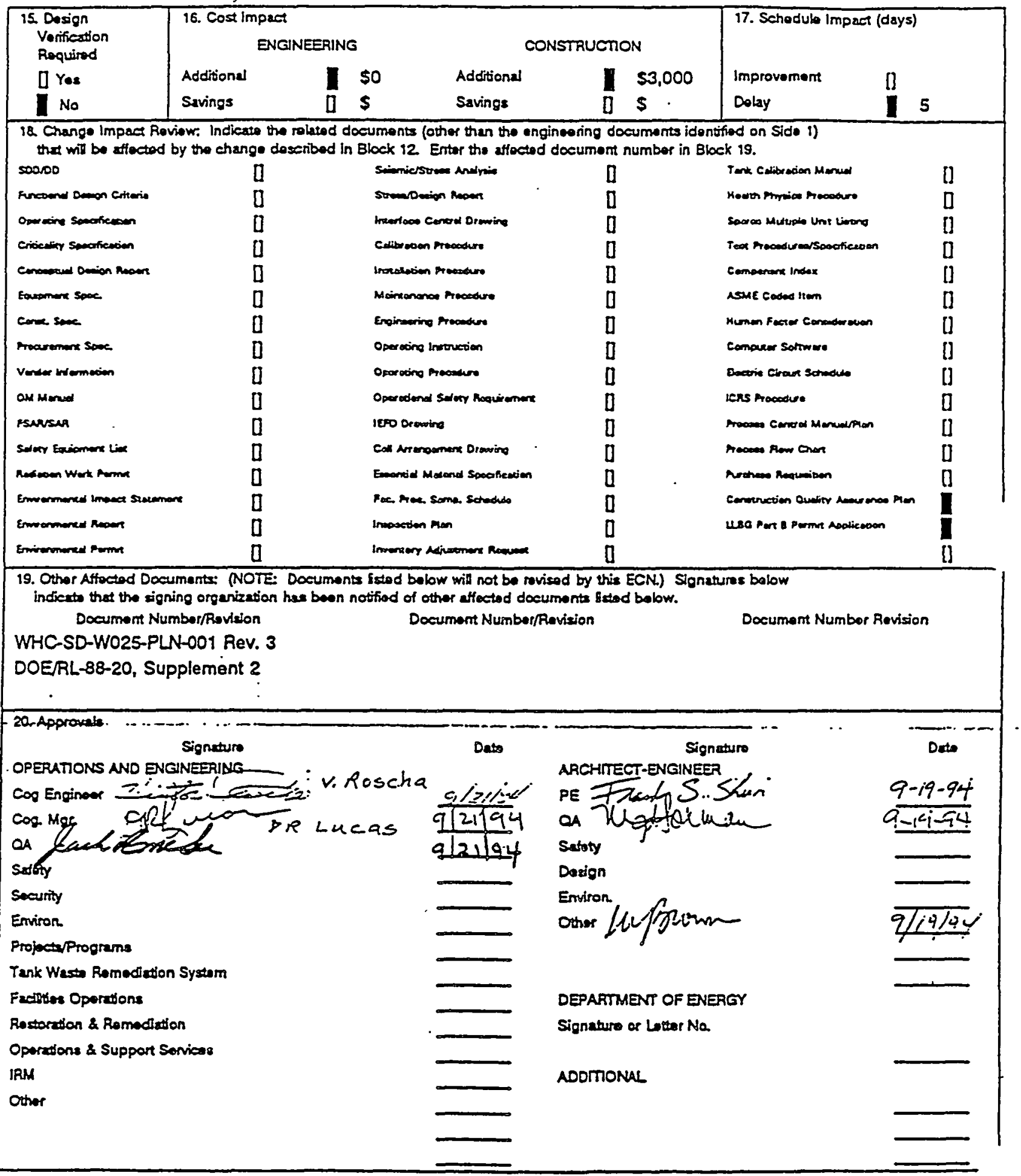


WHC-SD-W025-RPT-001, Rev. 0

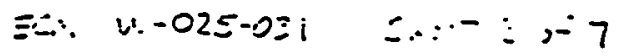

ià

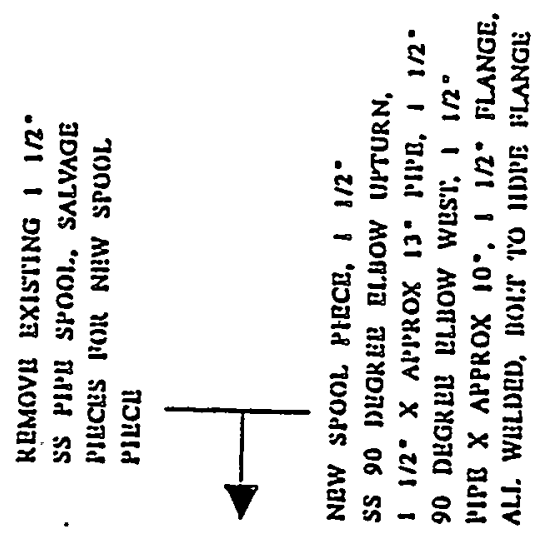

$\underset{I}{x}$

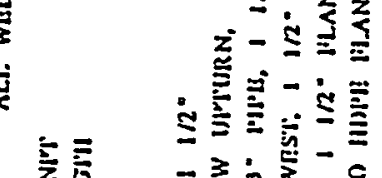

市焦

高

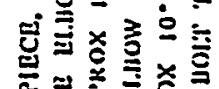

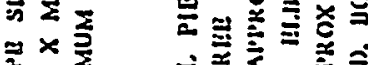

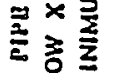

的高空

는

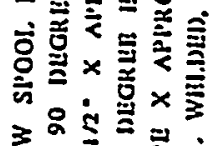

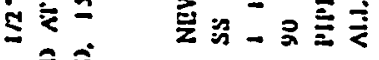

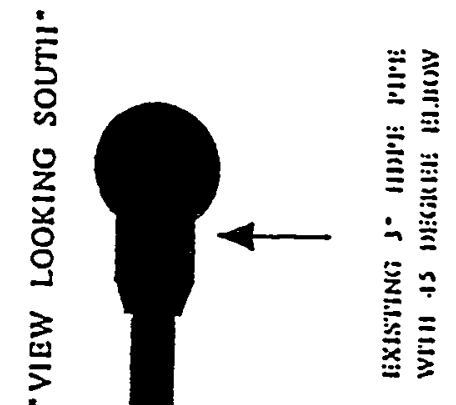

产言言

$\bar{m}$

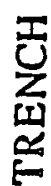

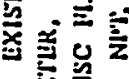

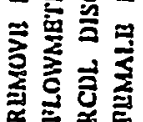
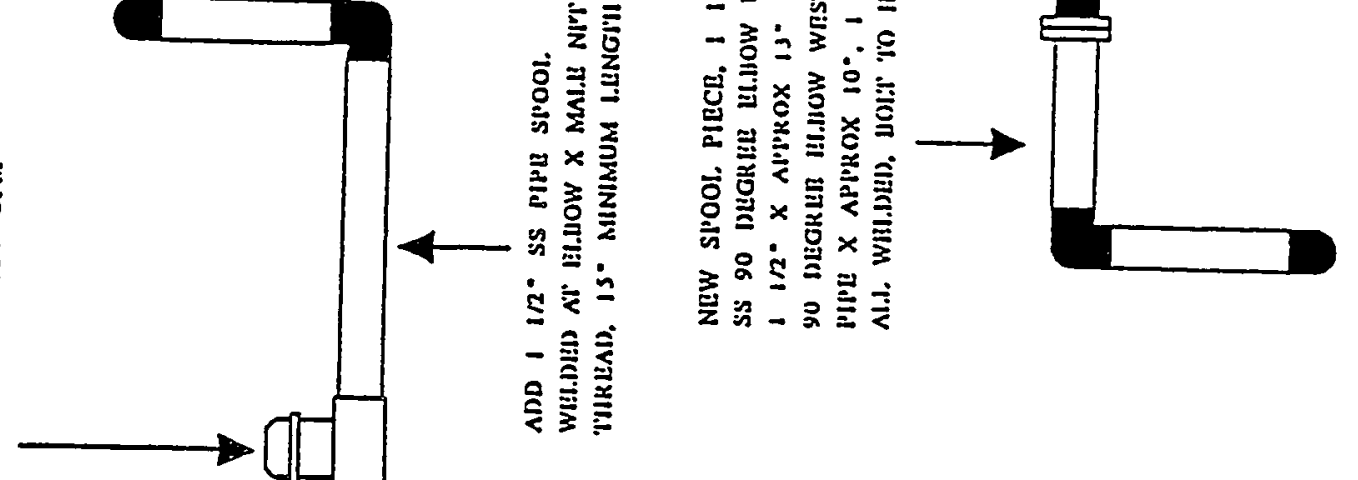

$\because \because \cdots$
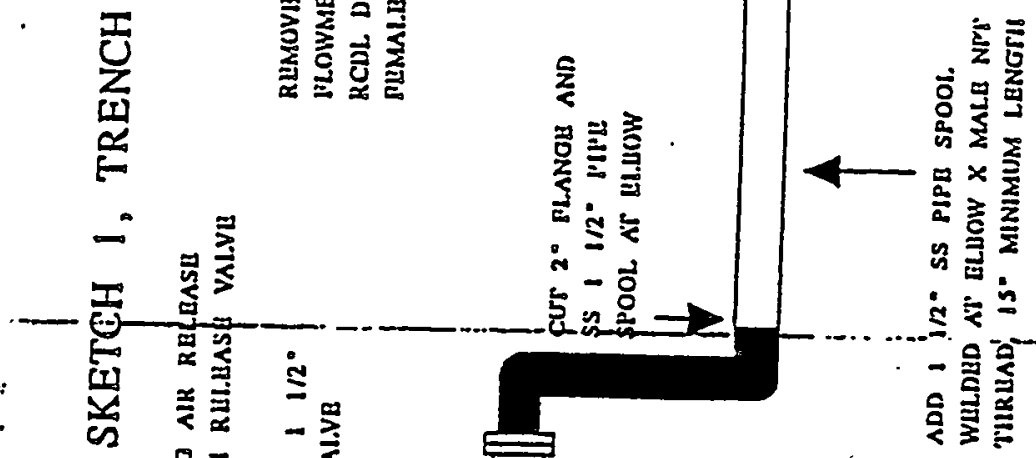

is

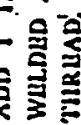

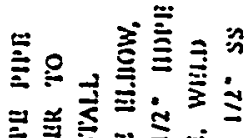

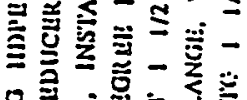

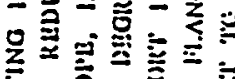

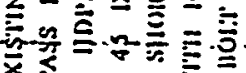

音录 -

总蓄

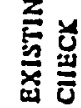

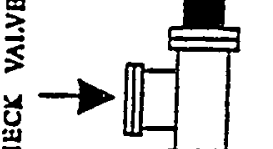

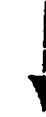

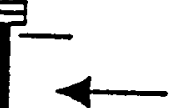

$\dot{\Sigma}$

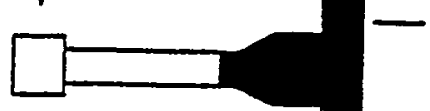

i

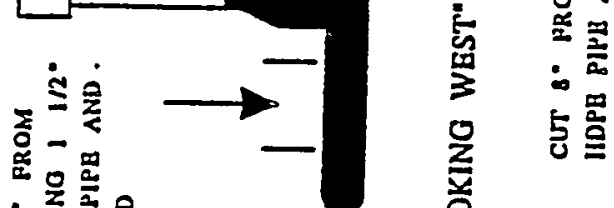

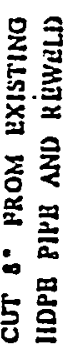

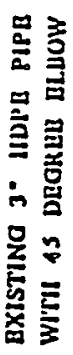

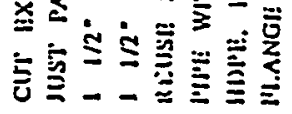

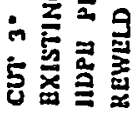

옹

㝕

亭 
WHC-SD-W025-RPT-001, Rev. 0

EC.1, N-0ZE:-

率

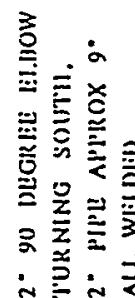

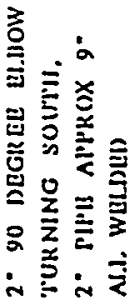
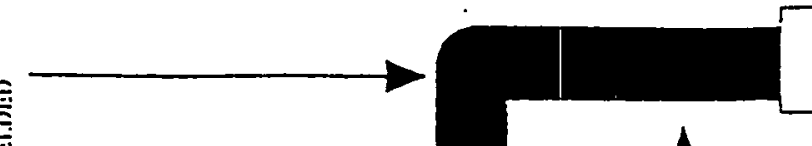

(O)

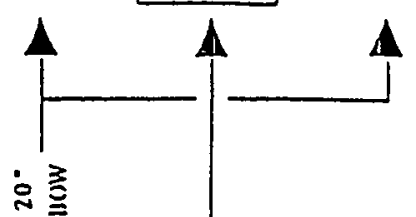

.
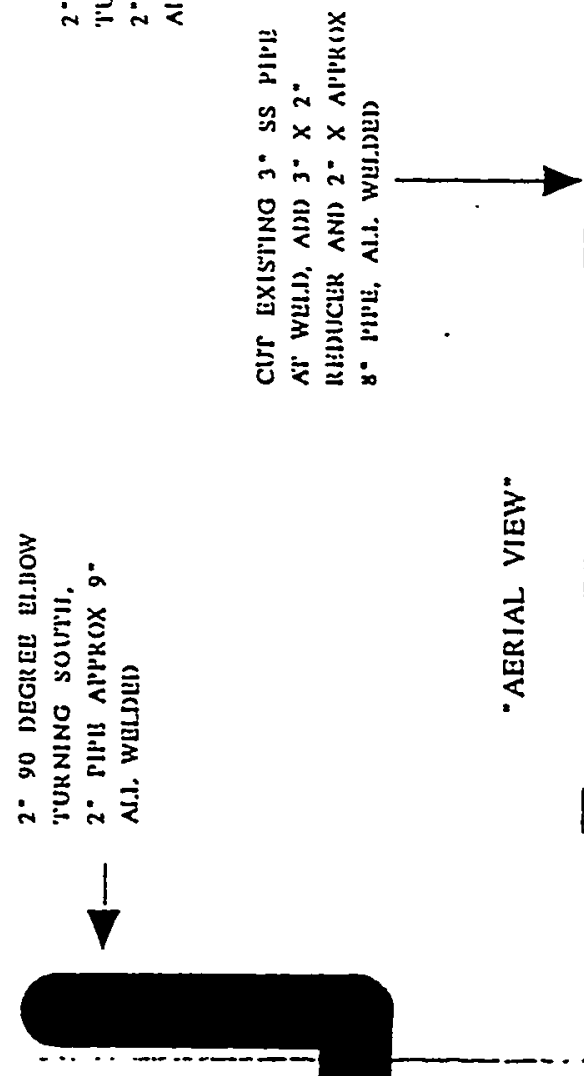

高

芦只”

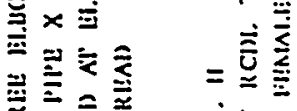

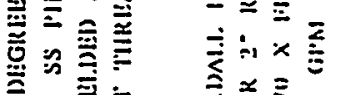

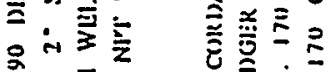

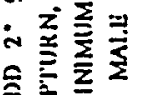

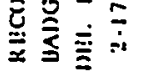

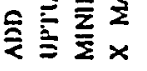

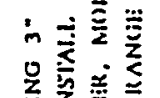

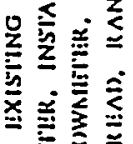

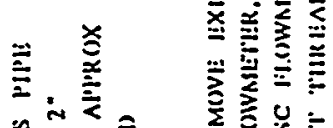
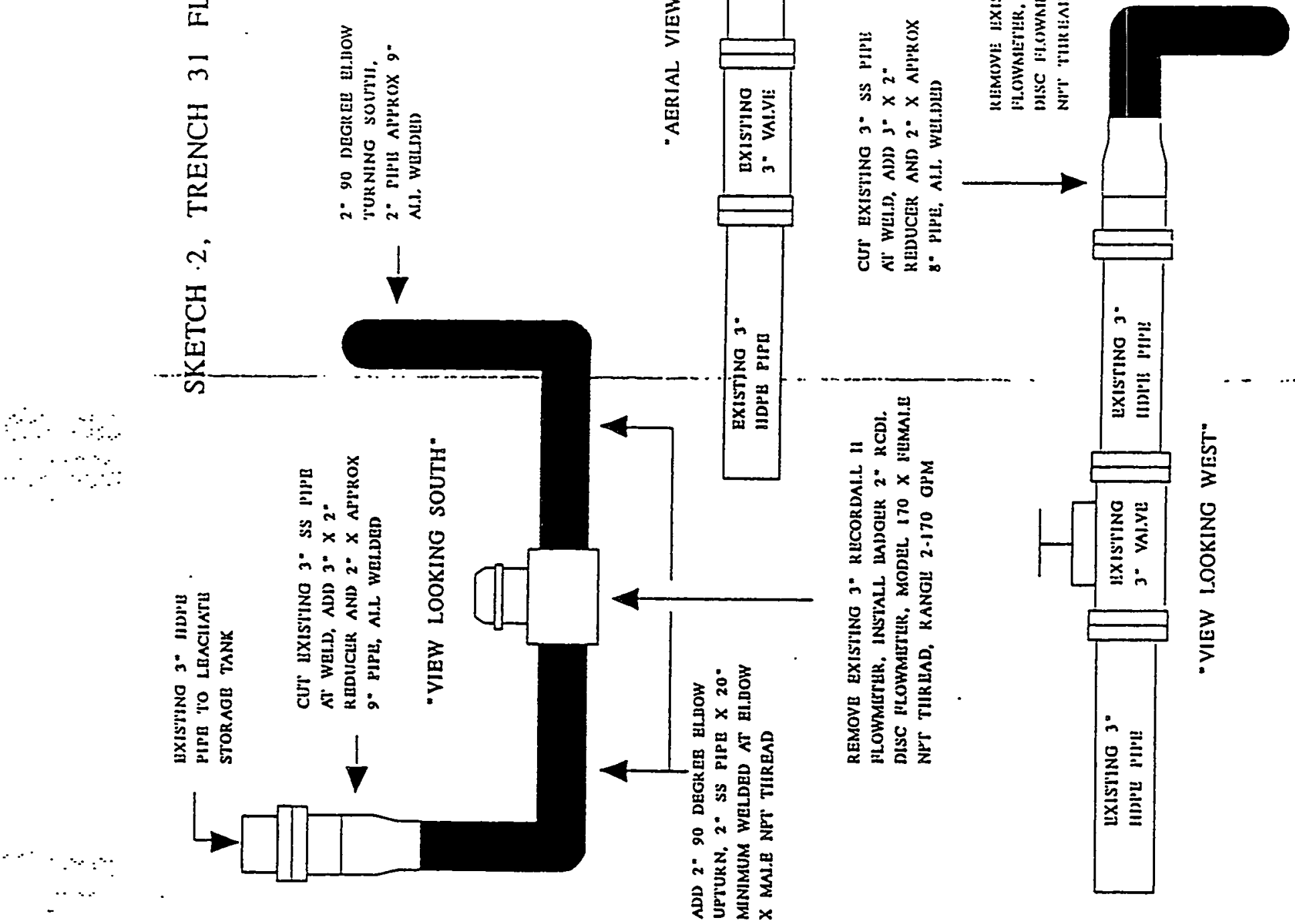

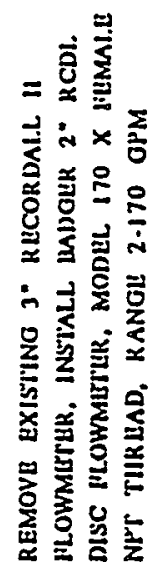

$\min \dot{x}$

呈食玄玄

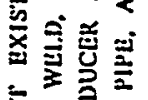

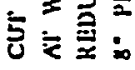

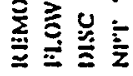

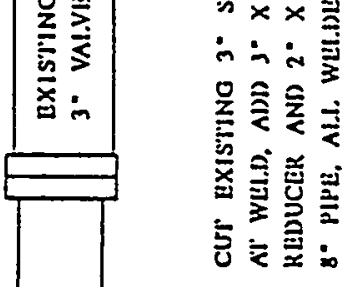

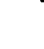


WHC-SD-W025-RPT-001, Rev. 0

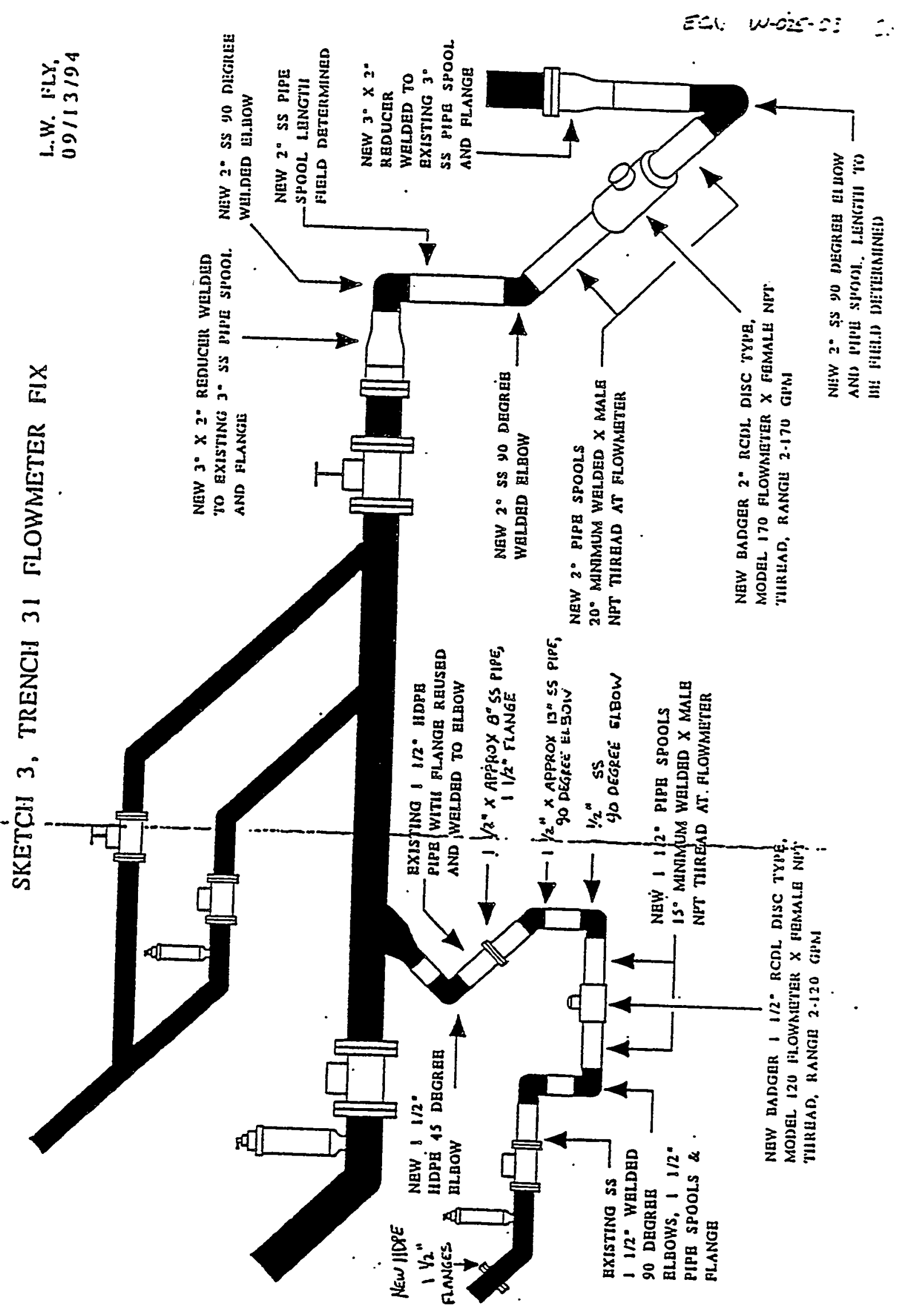


WHC-SD-W025-RPT-001, Rev. 0

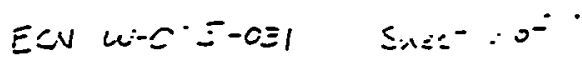

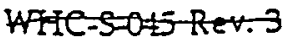

SECTION 15150

FLOWMETERS

\section{PART 1: GENERAL}

\section{I-1 SECIION INCLUDES}

Fumishing and installing two flowmeters for monitoring the accumulated leachate discharged to the leachate temporary storage tank One meter shall be located on the combined leachate discharge line, the other shall be on the secondary pump discharge line, as shown on the Drawings. Both meters shall be of the same design and from the same manufacturer.

\section{1-2 SUBMITTALS}

A Submit product information showing conformance with specifications contained herein.

B. Submit proposed procedure for calibrating flowmeters.

C. Submit calibration data and certification of calibration.

D. Submit in accordance with Section 01300 .

\section{PART 2: PRODUCTS}

\section{2-I PERFORMANCE}

The flowmeter shall be capable of measuring the accumulated flow through the meter with an accuracy of $\pm-1.5 \%$-or betterat-nominat-low-rates from 5 -gpm-to-160-gpm (combined discharge line) and $5 \mathrm{gpm}$ to $60 \mathrm{gpm}$ (secondary pump discharge line). A flow rate indicator is not required. Fiping upstream and downstream of flowmeters shall be straight and of same diameter as flowmeter for at least 10 diameters.

\section{2-2 MANUFACIURERS}

Hersey, Rockwell, Badger or approved equal

\section{PART 3: EXECUIION}

\section{3-I CALIBRAIION}

Provide certified calibration over full range of flow rates to demonstrate that flowmeter performance satisfies the requirements of these specifications. Actual calibration of each 
WHC-SD-W025=RPT-001, Rëv. 0

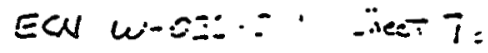

WHC-S-045Rev. 3

flowmeter will be required; manufacturer's generic performance data will not be acceptable.

Calibration procedure shall be approved by COTR grior to use.

3-2 INSTALIATION

Install flowmeters in accordance with manufacturer's recommendations.

-END . 
WHC-SD-W025-RPT-001, Rev. 0

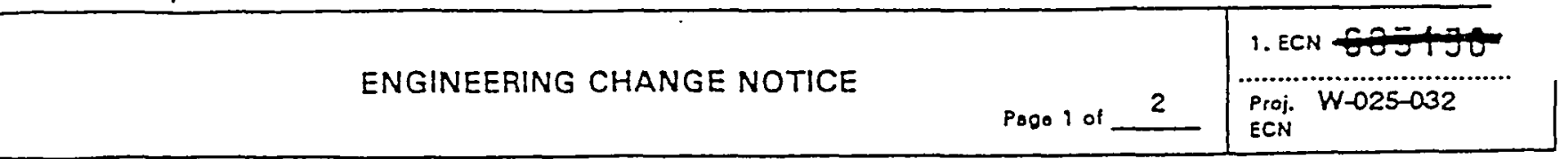

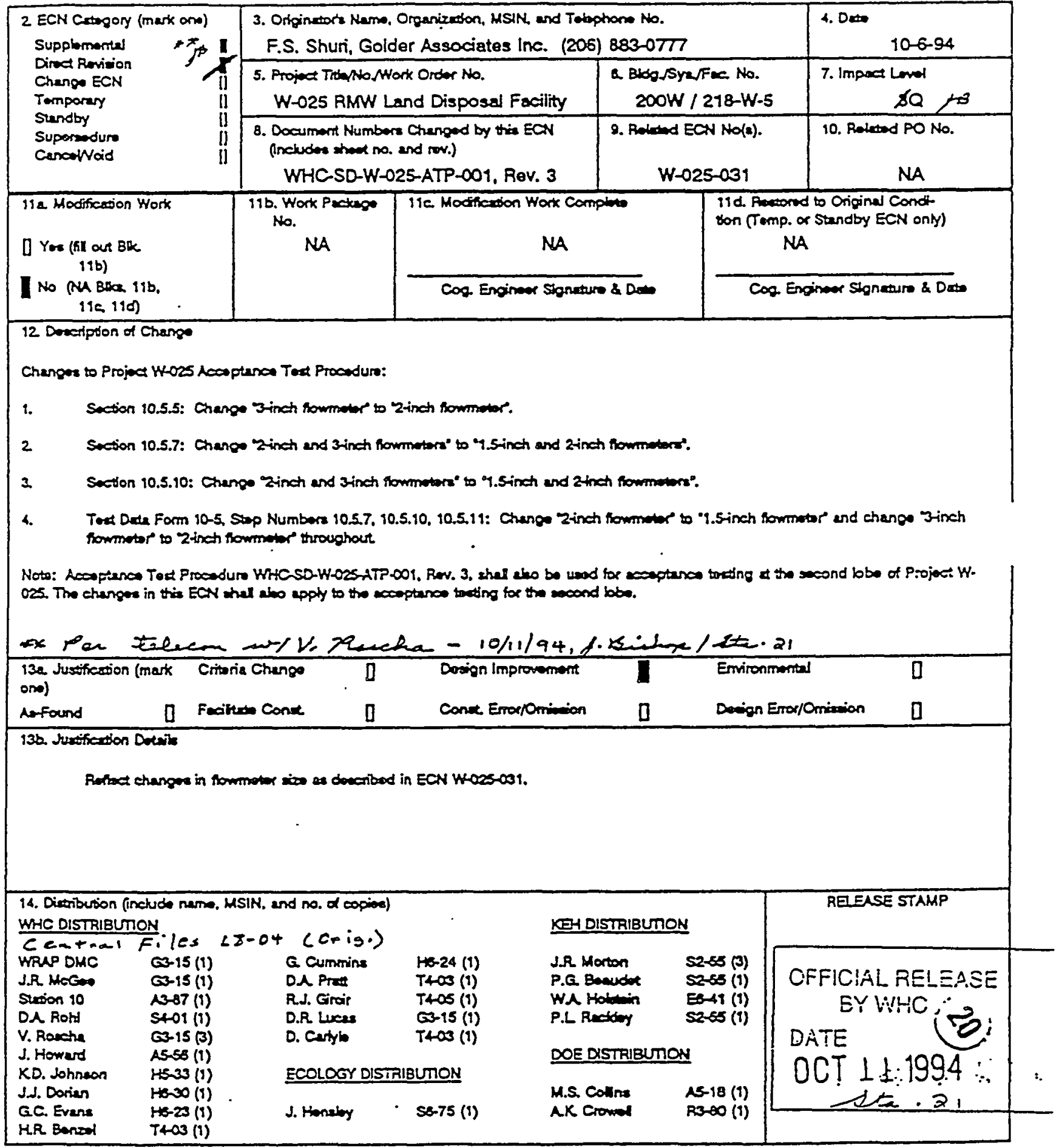

A-7900-013-2 (06/92) GEFDOS 


\begin{tabular}{|c|c|c|}
\hline ENGINEERING CHANGE NOTICE & Page 2 of 2 & $\begin{array}{c}\text { 1. ECN (uno no. from po. 1) } \\
\text { W-025-0.32 }\end{array}$ \\
\hline
\end{tabular}

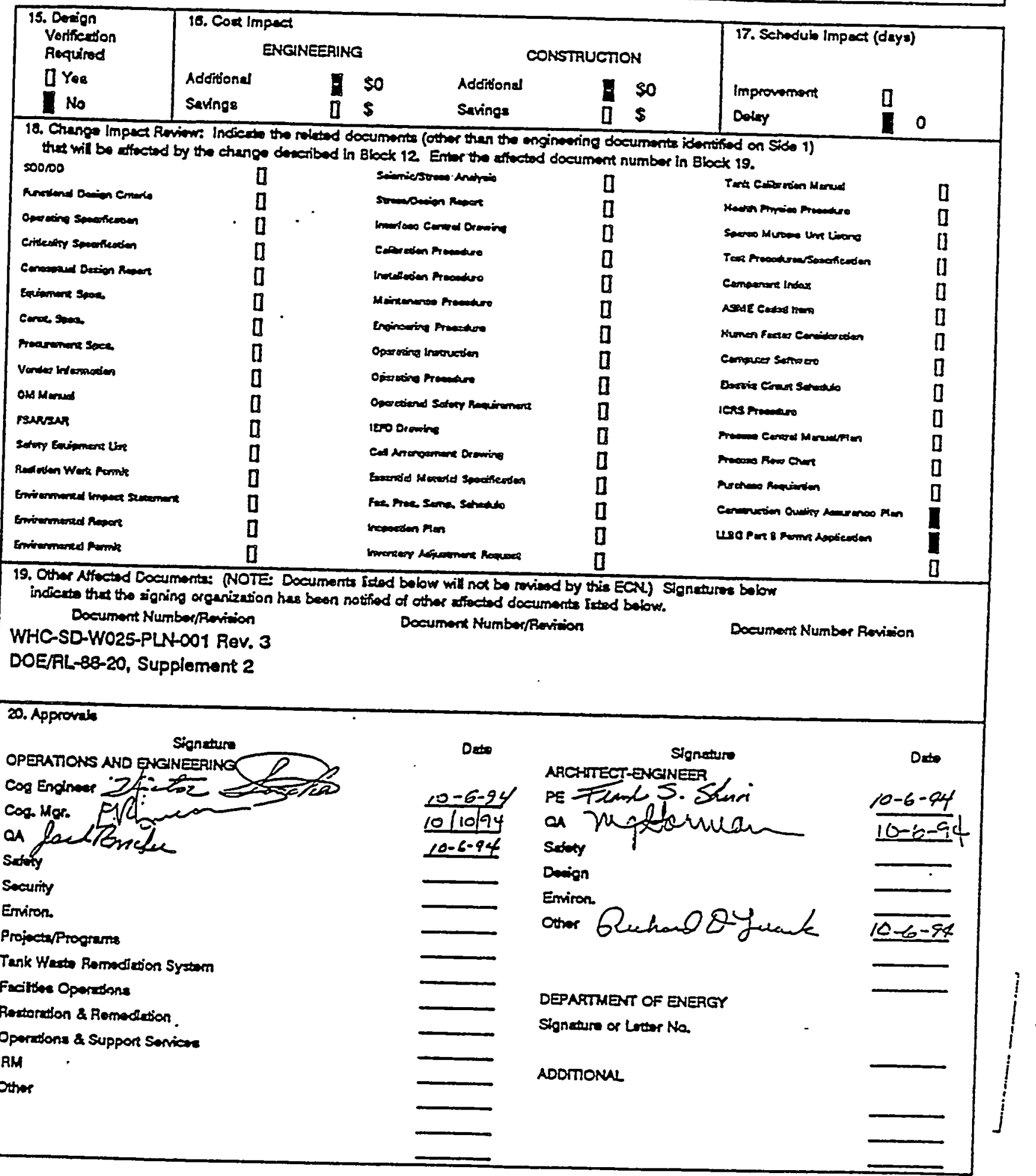


WHC-SD-W025-RPT-001, Rev. 0

\section{INFORMATION RELEASE REQUEST PUBLIC DOCUMENT CHANGE/REVISION \\ (Short Form No. 1)}

1. Base Document ID Number: ECN-W025-032 (SD-W025-ATP-001 REV 3)

2. Base Document Title:

Acceptance Test Procedure $\mathrm{H}-025$

\section{Change/Revision Number: 3}

4. Change/Revision Date: $\quad 08 / 16 / 94$

5. Unclassified Category: ${ }^{\bullet}$ UC - N/A

6. Budget \& Reporting Code:" B\&R - N/A

I verify this change/revision to the base document indicated above:

- Complies with the provisions of WHC-CM-3-4

- Contains no Limited-Use information

- Contains no classified or Applied Technology references

- Does not change the intent or meaning of the base document

- And, the base document itself is approved for public release.

7. Responsible Manager: DR Lucas

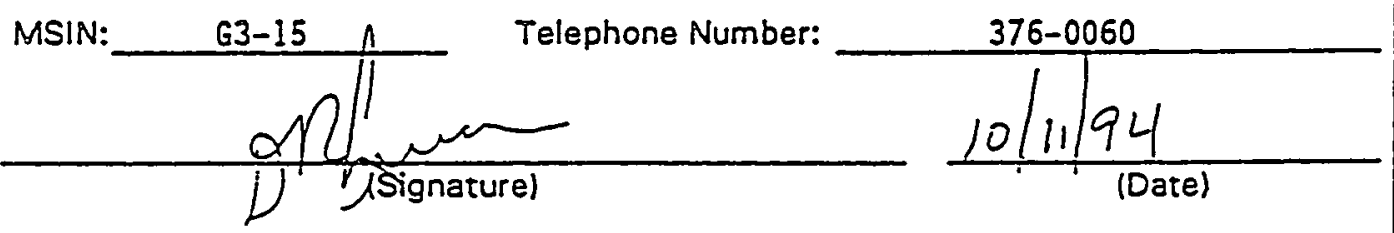

8. Information Release Administration Specialist: * .

"Required only for full revisions transmitted to OSTI.

- Required only for full revisions. 
WHC-SD-W025-RPT-001, Rev. 0

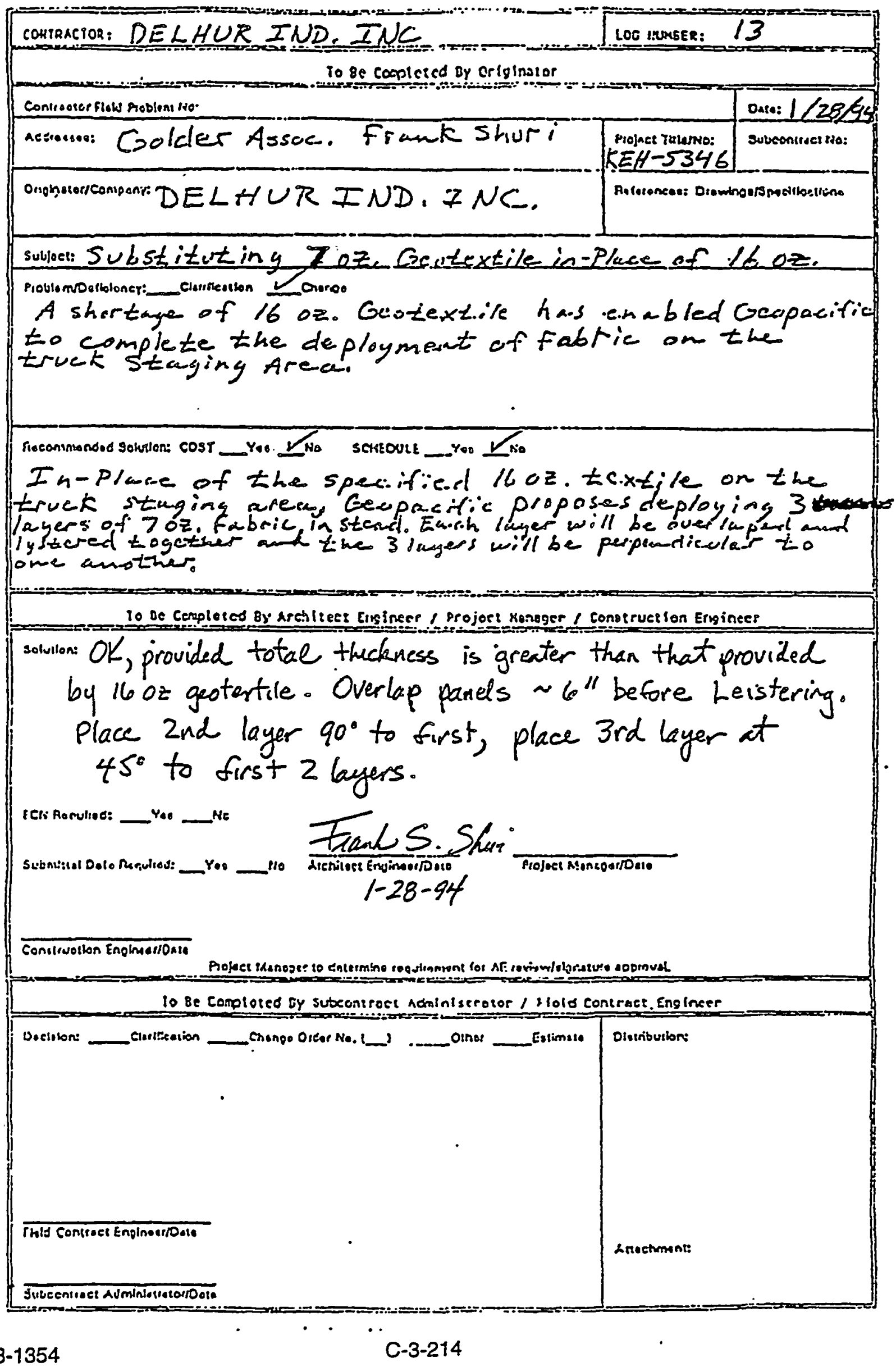


WHC-SD-W025-RPT-001, Rey. 0

APPENDIX D

SOIL MATERIALS LABORATORY TEST RESULTS 
WHC-SD-W025-RPT-001，Rev。0

APPENDIX D-1

EXCAVATION AND BACKFILL

$D-1$ 
WHC-SD-W025-RPT-001, Rev. 0

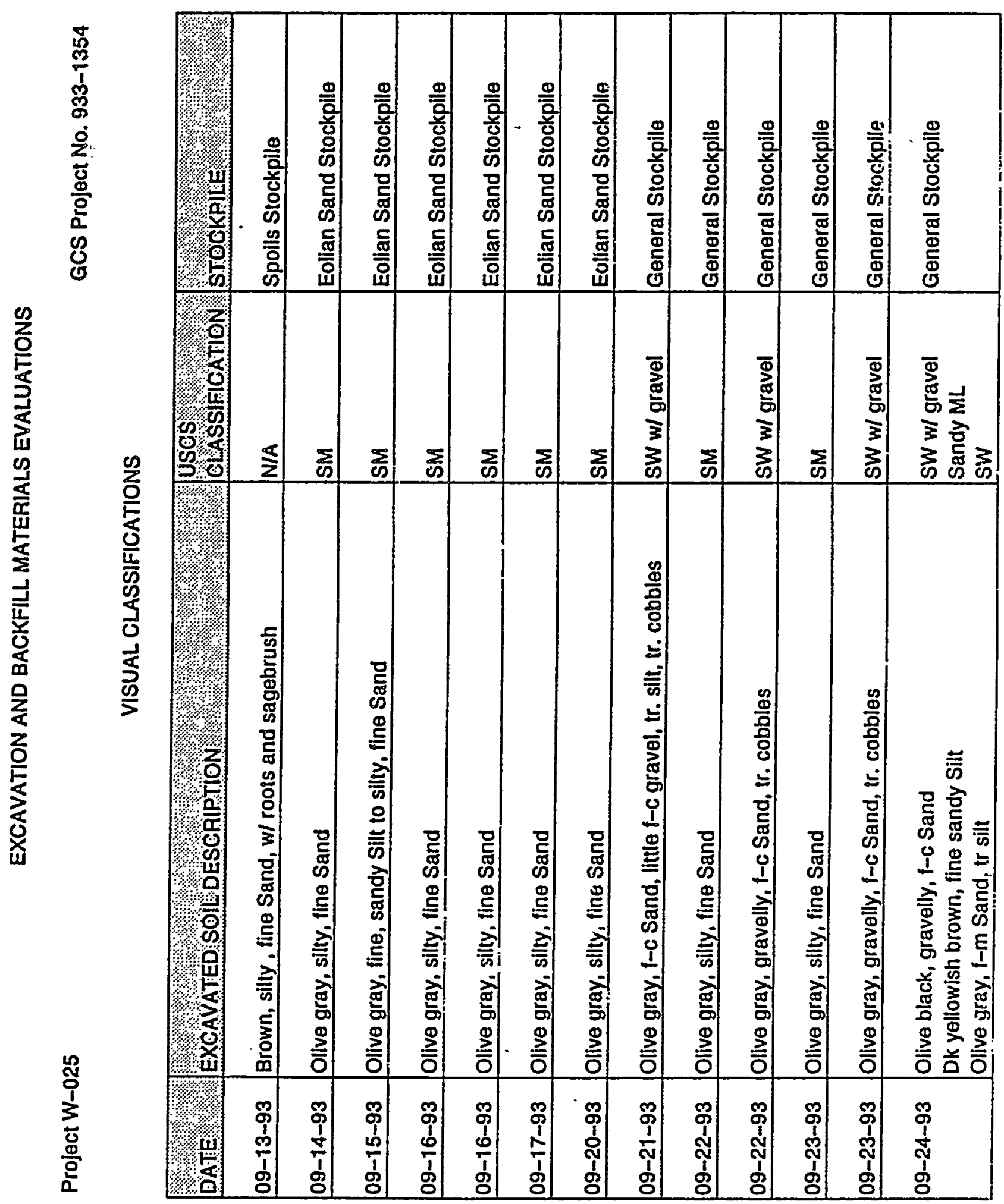


WHC-SD-W025-RPT-001, Rev. 0

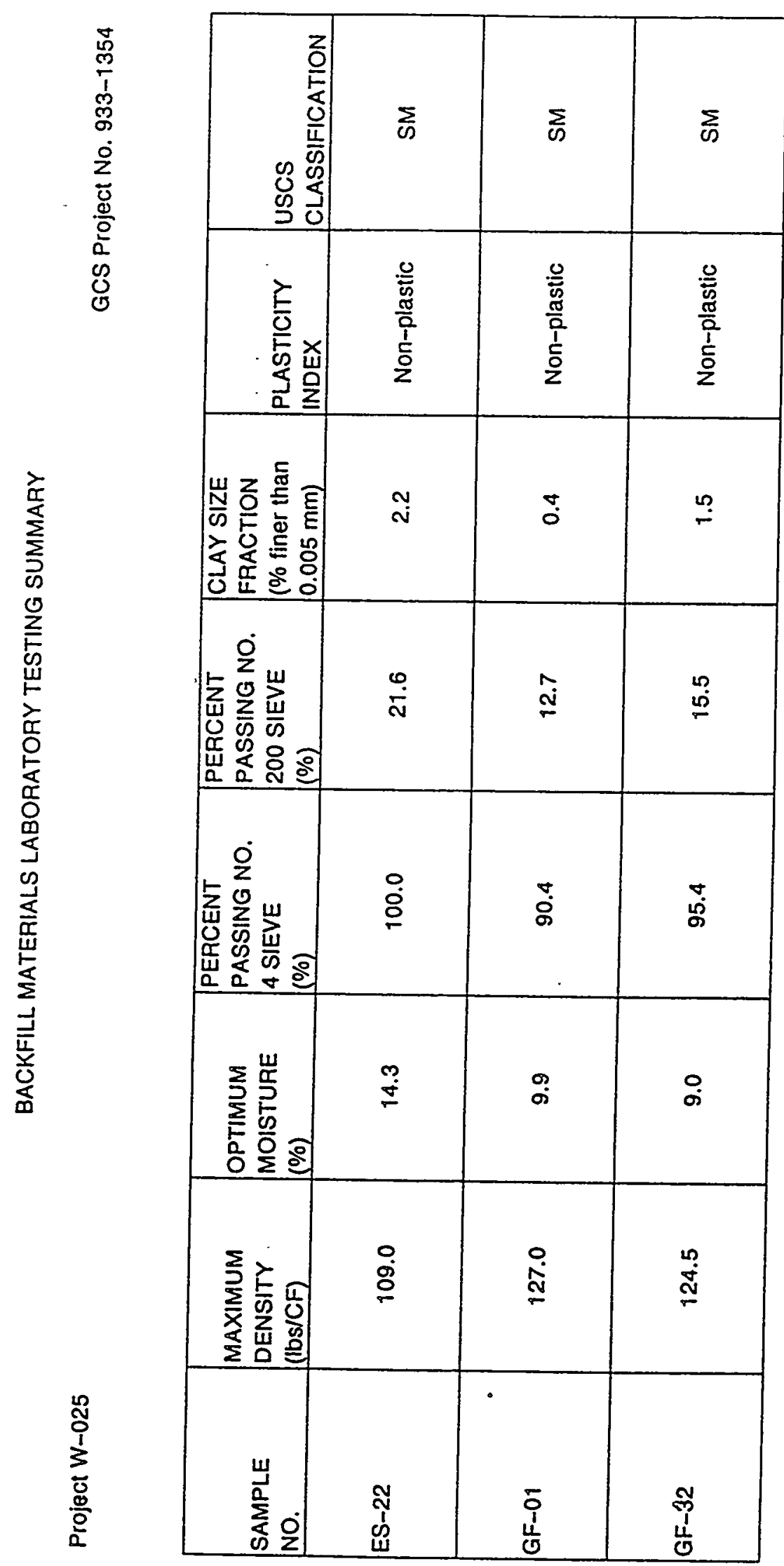

D-1-2 
WHC-SD-W025-RPT-001，Rey。0

APPENDIX D-2

EOLIAN SAND

D-2 
WHC-SD-W025-RPT-001, Rev. 0

Page-7

EOLIAN SAND

LABORATORY TESTING SUMMARY

PROJECT W-025

GCS PROJECT NO. 933-1354

\begin{tabular}{|c|c|c|c|}
\hline ४ै। SAMPLE $\%$ NO $\%$ \% & 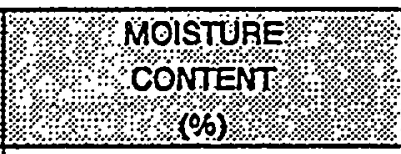 & 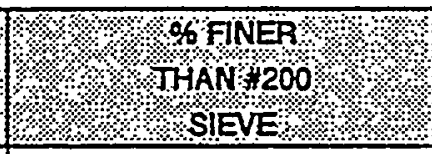 & $\begin{array}{l}1 \% 6 \mathrm{CLAY} \mathrm{SIZE} \\
1 \% \\
\text { FRACTION }\end{array}$ \\
\hline ES-01 & 19.3 & 22.1 & 3.2 \\
\hline ES-02 & 3.5 & 19.8 & 20 \\
\hline ES-03 & 5.9 & 17.2 & 1.7 \\
\hline ES-04 & 3.5 & 23.3 & 1.1 \\
\hline ES-05 & 6.3 & 25.2 & 2.1 \\
\hline ES-06 & 4.3 & 18.1 & 2.1 \\
\hline ES-07 & 3.6 & 28.3 & 1.9 \\
\hline ES-08 & 4.7 & 29.4 & 1.4 \\
\hline ES-09 & 4.9 & 19.1 & 1.9 \\
\hline ES-10 & 5.0 & 19.4 & 0.7 \\
\hline ES-11 & 3.6 & 21.8 & 1.7 \\
\hline ES-12 & 4.4 & 21.5 & 1.5 \\
\hline ES-13 & 4.8 & 19.0 & 1.1 \\
\hline ES-14 & 4.7 & 19.3 & 0.7 \\
\hline ES-15 & 4.3 & 17.4 & 0.6 \\
\hline ES-16 & 7.3 & 20.4 & 0 \\
\hline ES-17 & 3.6 & 21.3 & 1.1 \\
\hline ES-18 & 3.2 & 22.0 & 0.7 \\
\hline ES-19 & 4.1 & 20.7 & 0.2 \\
\hline ES-20 & 5.9 & 19.8 & 0 \\
\hline ES-21 & 4.0 & 20.4 & 0 \\
\hline ES-22 & 4.8 & 21.6 & 2.2 \\
\hline ES-23 & 4.9 & 21.9 & 2.8 \\
\hline ES-24 & 4.2 & 20.0 & 2.8 \\
\hline
\end{tabular}


WHC-SD-W025-RPT-001, Rev. 0

\section{EOLIAN SAND \\ LABORATORY TESTING SUMMARY}

PROJECT W-025

GCS PROJECT NO. 933-1354

\begin{tabular}{|c|c|c|c|}
\hline SNAPLA & $\begin{array}{c}\text { MOISTURE } \\
\text { CONTENT } \\
\text { (\%) } \\
1 \%\end{array}$ & 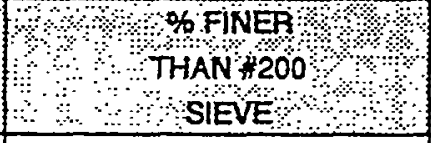 & $\begin{array}{c}\% \mathrm{CCAYSIZE} \\
\mathrm{FAACTON} \\
(005 \mathrm{~mm})\end{array}$ \\
\hline ES-25 & 4.9 & 19.3 & 2.5 \\
\hline ES-26 & 5.4 & 19.2 & 2.6 \\
\hline ES-27 & 6.5 & 20.1 & 2.8 \\
\hline ES-28 & 4.6 & 21.6 & 2.8 \\
\hline ES-29 & 4.5 & 19.5 & 3.1 \\
\hline ES-30 & 4.9 & 19.2 & 2.9 \\
\hline ES-31 & 4.8 & 20.1 & 2.6 \\
\hline ES-32 & 5.3 & 25.0 & 2.8 \\
\hline ES-33 & 4.2 & 19.6 & 2.7 \\
\hline ES-34 & 4.7 & 20.3 & 2.1 \\
\hline ES-35 & 4.4 & 20.5 & 1.7 \\
\hline ES-36A & N/A & 25.1 & 2.2 \\
\hline ES-36 & 4.4 & 18.5 & 2.2 \\
\hline ES-37 & 4.6 & 19.5 & 1.2 \\
\hline ES-38A & N/A & 24.6 & 0 \\
\hline ES-38 & 4.4 & 34.1 & 0 \\
\hline ES-39 & N/A & 19.0 & 2.4 \\
\hline ES-40 & 5.1 & 26.3 & 0 \\
\hline
\end{tabular}


WHC-SD-W025-RPT-001, Rev. $0^{\circ}$

APPENDIX D-3

BENTONITE 
APPENDIX D-3.1

CQA LABORATORY TEST RESULTS

D-3.1 
WHC-SD-W025-RPT-001, Rev. 0

BENTONITE PRECONSTRUCTION TEST SUMMARY

\begin{tabular}{|c|c|c|c|c|c|}
\hline 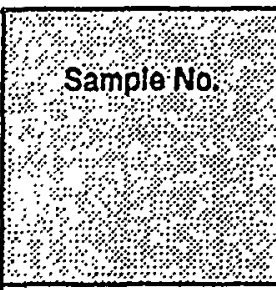 & 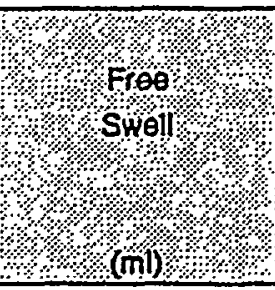 & 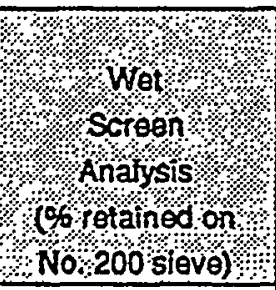 & 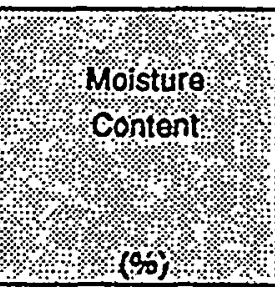 & 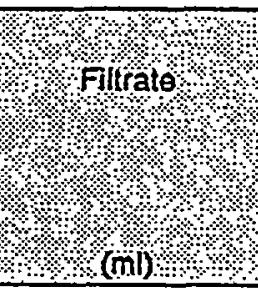 & 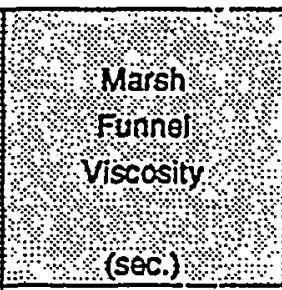 \\
\hline Specificalions & $25 \mathrm{ml}$ minimum & $4.0 \%$ maximum & $10.0 \%$ maximum & $15 \mathrm{ml}$ maximum & 36.5 sec. minimum \\
\hline$B-1$ & 25 & 2.8 & 7.9 & 15 & 69 \\
\hline $\mathrm{B}-2$ & 26 & 28 & 7.3 & 15 & 98 \\
\hline$B-3$ & 26 & 2.1 & $8 . \varepsilon$ & 83.5 & 165 \\
\hline$B-4$ & 27 & 2.8 & 6.0 & 15 & 208 \\
\hline B-5 & 26 & 1.9 & 7.5 & 15 & 91 \\
\hline$B-6$ & 25 & 1.2 & 7.6 & 15 & 80 \\
\hline$B-7$ & 26 & 1.4 & 7.2 & 13.5 & 84 \\
\hline$B-8$ & 26 & 1.4 & 6.6 & 13.6 & 83 \\
\hline B-9 & 26 & $2 . T$ & 7.1 & 15 & $\$ 15$ \\
\hline
\end{tabular}


WHC-SD-W025-RPT-001，Rev。0

APPENDIX D-3.2

VISCOSITY CORRELATION

$D-3.2$ 


\section{PACIFIC \\ PROCESS \\ \& LINER, INC.

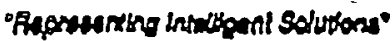

- FAX TRANSMITTAL.

Date:

$8 / 30 / 93$

Pages:

5 (Including Cover)

To:

Cliff Kriltep

Colder Assocletes

Fax Phone:

208-882-5488

From:

Jim Coskey

Sublect:

Coprelation-Vlsoosity/barral yleld/marsh iunnel

If there are any problems in transmlssion, plaase call 503.928 .4400 .

Clif,

There is a qualltative correlation between vlscosity, barrel yleld and the marsh funnel test. The barrel yield can be approximated via marah funnel testing to determine vlecosity of a given qurnitty bentonite in a glven volume water.

Using the attached graph and appropriate calculations the following approximation might be made:

A slury with 22.5 grams of bentonite/350 $c 0$ of weter and a viscosity of 15 or greater will have a barrel yleld of 91 or greater.

Explanation: a 15 centlpose slurry of $22.5 \mathrm{~g} / 350 \mathrm{Cc} \mathrm{H2O}$ equals 91 BBY. The minimum 15 centlpose vlscosity is determined by timing the required volume of slurry thru the Marsh funnel in approximately 36 to 37 seconds (per the attached graph). Keep in mind, thls is only an approximatlon end a viscometer is requlred to confirm these. measurements to assure soouracy. 
WHC-SD-W025-RPT-001, Rev. 0

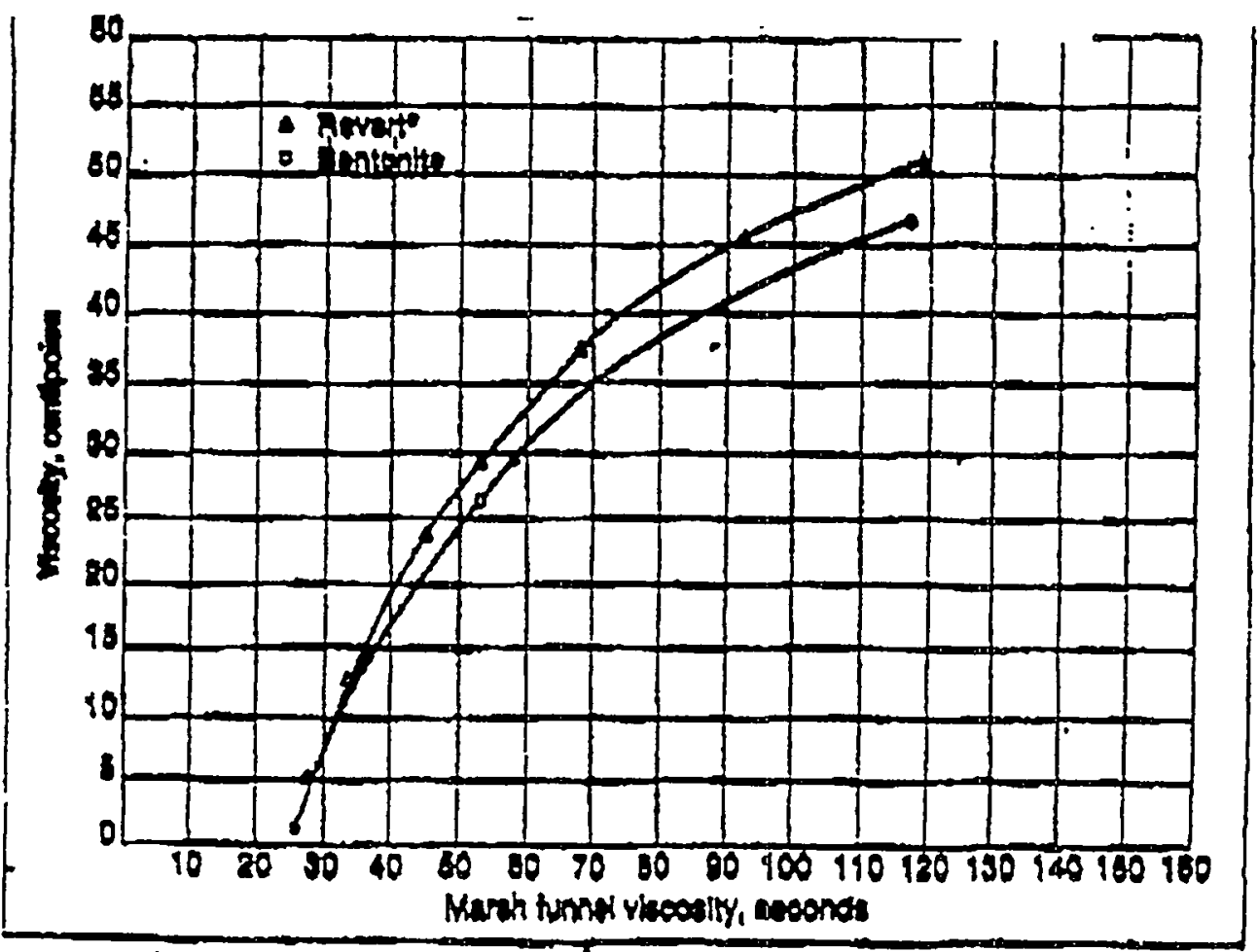

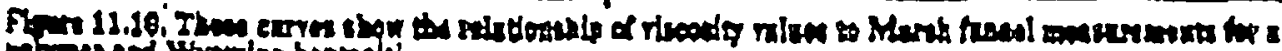

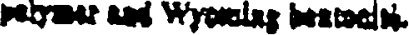


WHC-SD-W025-RPT-001, Rev. 0

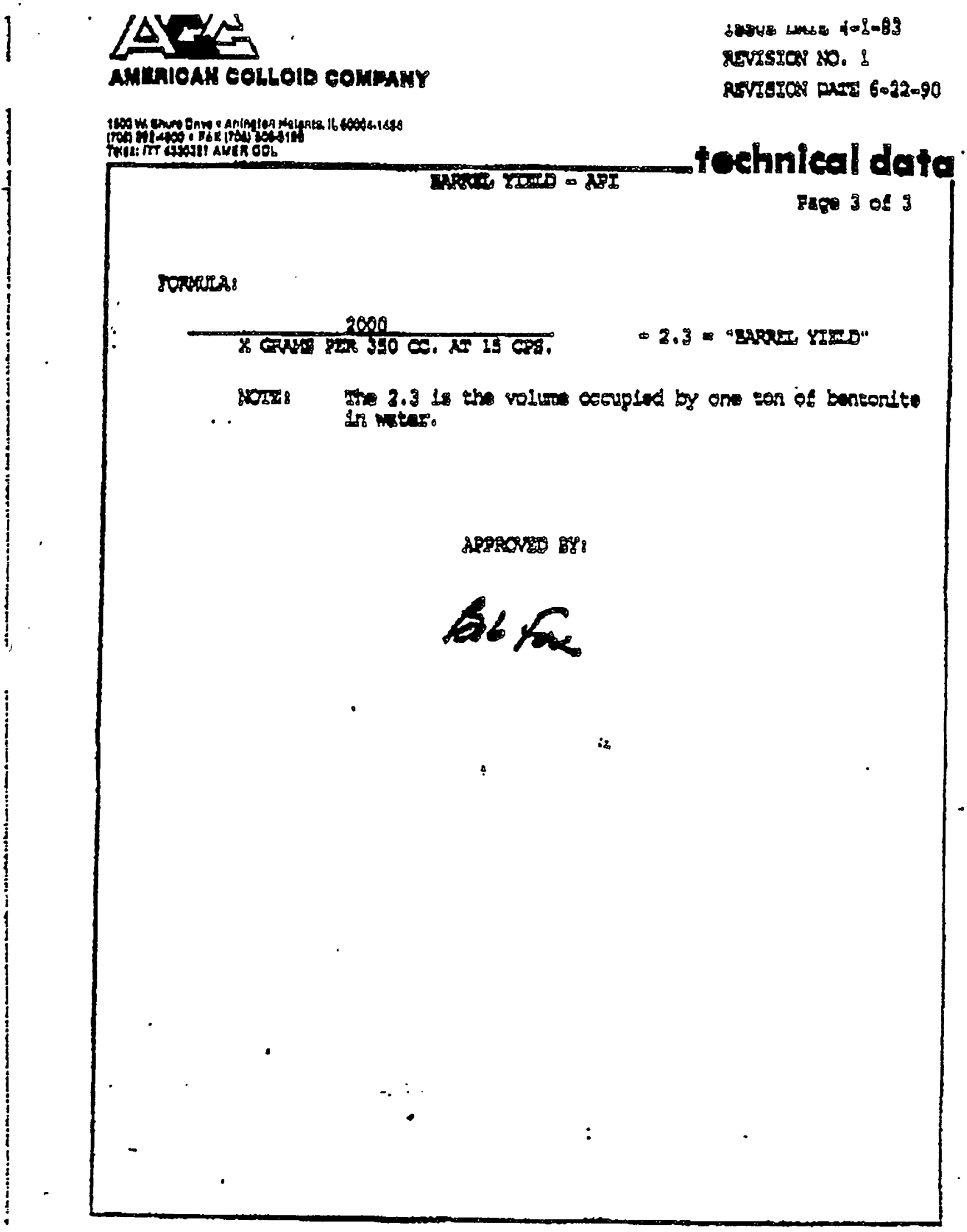

933-1354

D-3.2-3 
WHC-SD-W025-RPT-001, Rev. 0

IUTers and $4-1-83$

Rurdston No. I

REIISIOA OATE 6-22-90

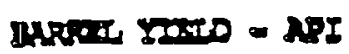

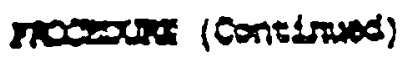

rape 2 of 3

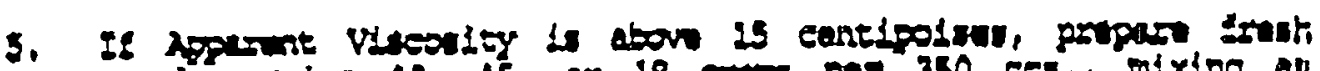

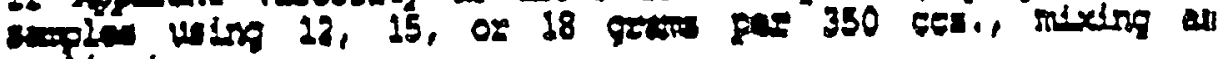
auslind,

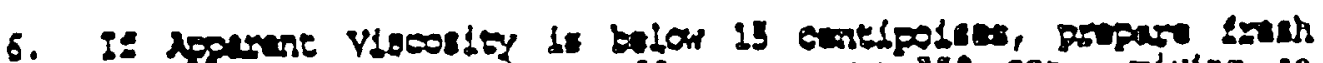

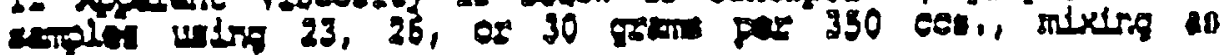
outelend.

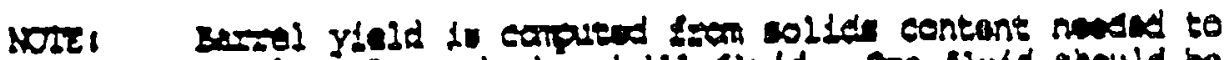

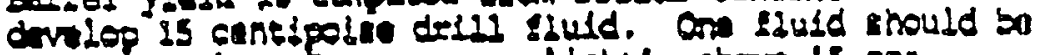

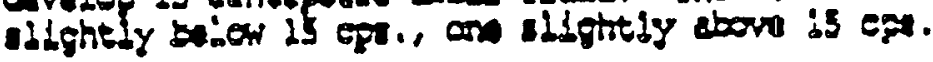

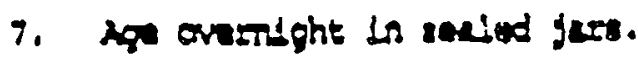

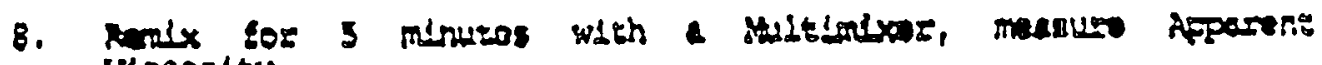
Vluesestby.

ararerrear

romostex

\section{5}

$20.1 \mathrm{R}$.

$11.23 \mathrm{G}$.

$13.5 \mathrm{Gr}$.

$13.0 \mathrm{E.}$

$21.3 \mathrm{ER}$.

18.0 G.

$20.0 \mathrm{cR}$.

$27.3 \mathrm{E}$.

$25.0 \mathrm{G}$.

30.0 a.

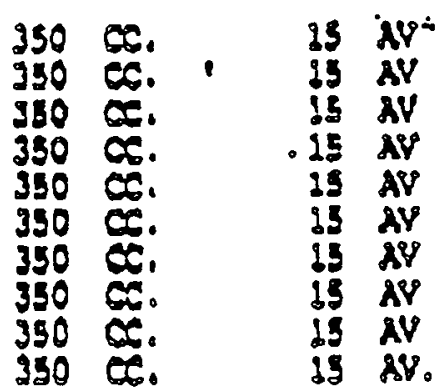

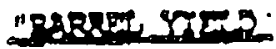

$200 \mathrm{Ber}$,

280 gal.

130 Bat.

236 照?

239 8E.

$113 \mathrm{BEZ}$.

103 REZ.

g1 $3 \mathrm{Bi}^{\circ}$

82 B표,

69 DRL.

AV $=600$ rpin dial reading +2 


\section{8rCoron 8 \\ VPSCOEITY AND GEL STRENGTH}

\section{Dusiptien}

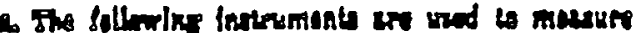

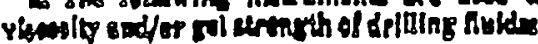

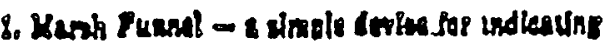

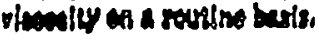

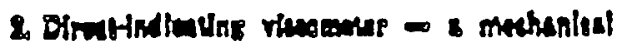

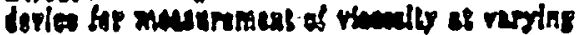
Herroung

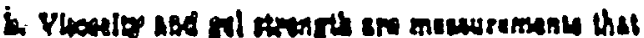

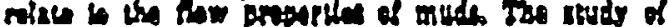

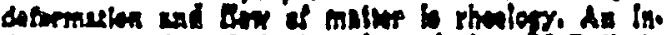

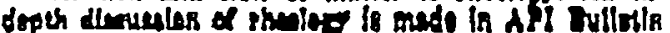

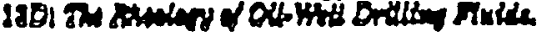

\section{MABUS FUANTL}

\section{Epripanat}

$$
\text { a. Minta Jonnd }
$$

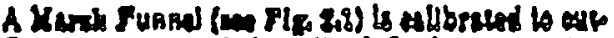

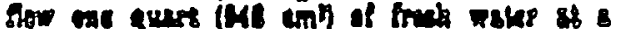

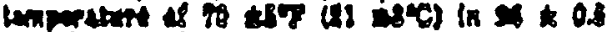

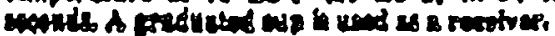

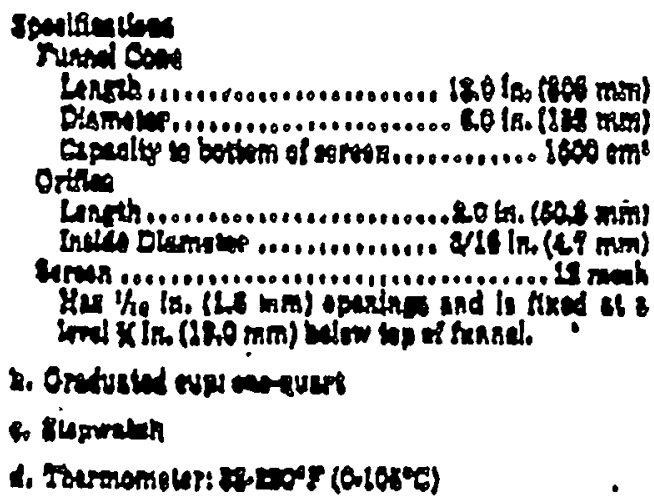

\section{Procdure}

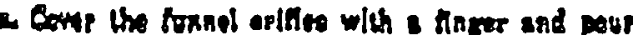

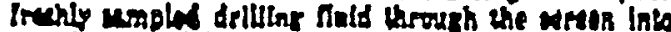

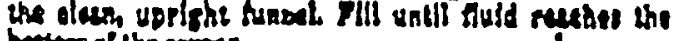
bertom al utu corwen.

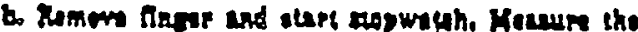

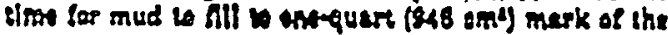
tuly.

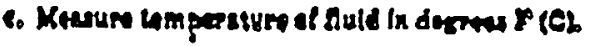

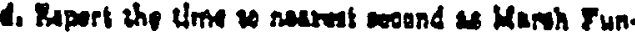

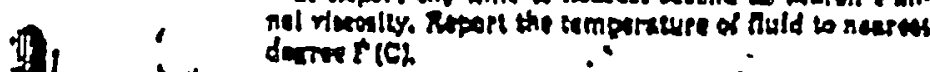

\section{DIRESTHDICATHE VIBCOMETES}

\subsection{Eutpment}

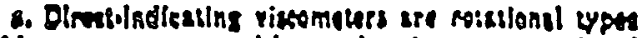
of Instruntrals powaped by on siretrle ropet op a hand

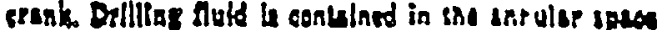

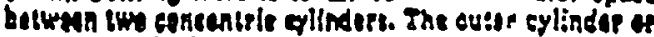

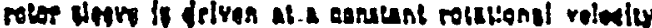

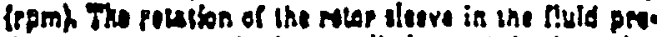

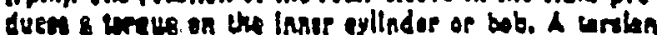

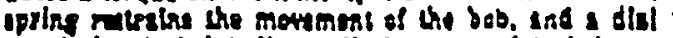

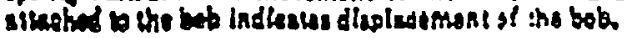

inateumant eanstuned hem beth cdivilot wo that oluth viluoly and ylald polke te obuind by using reeding

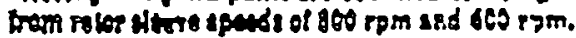

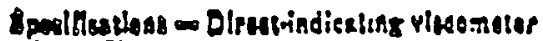
Feses Ilom

imide dientier. $1.49 \cdot 1.14 .13 \mathrm{~mm}$ Tuill hisk $\$ .183$ in. $187.00 \mathrm{man}$ enthed in

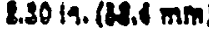
Two rowe of $\mathrm{h}$ ia. $(3.11 \mathrm{~mm})$ holes speced ito

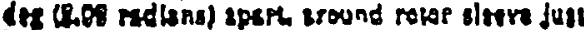
tolow entiod line

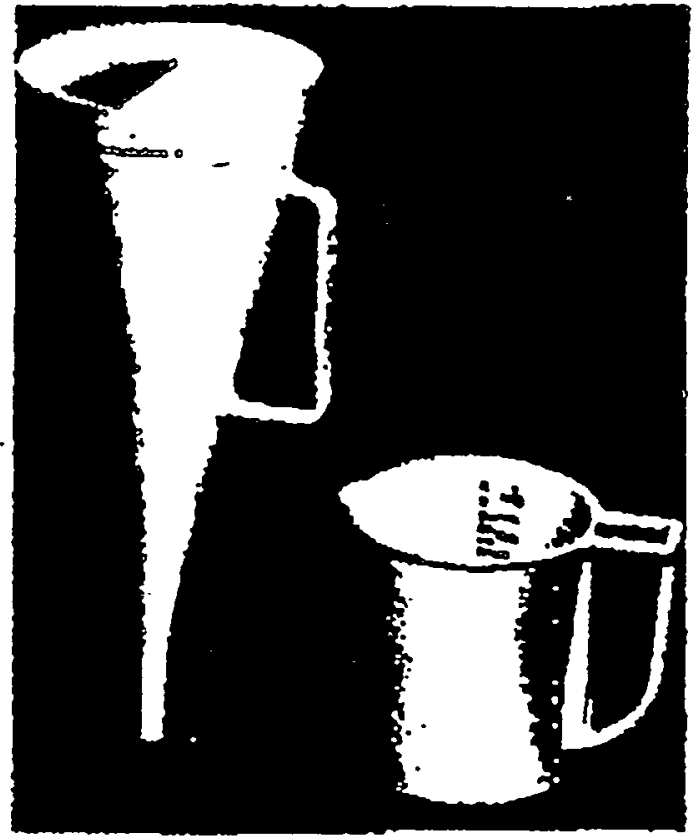

Fia.21

MAITK FUKNEL AND CLP 
WHC-SD-W025-RPT-001, Rev. 0

APPENDIX D-3.3

CERTIFICATES OF ANALYSIS

$D-3.3$ 
WHC-SD-W025-RPT-001, Rev. 0

BENTONITE RAILCAR INVENTORY

Project H-025

GCS Project No. 933-1354

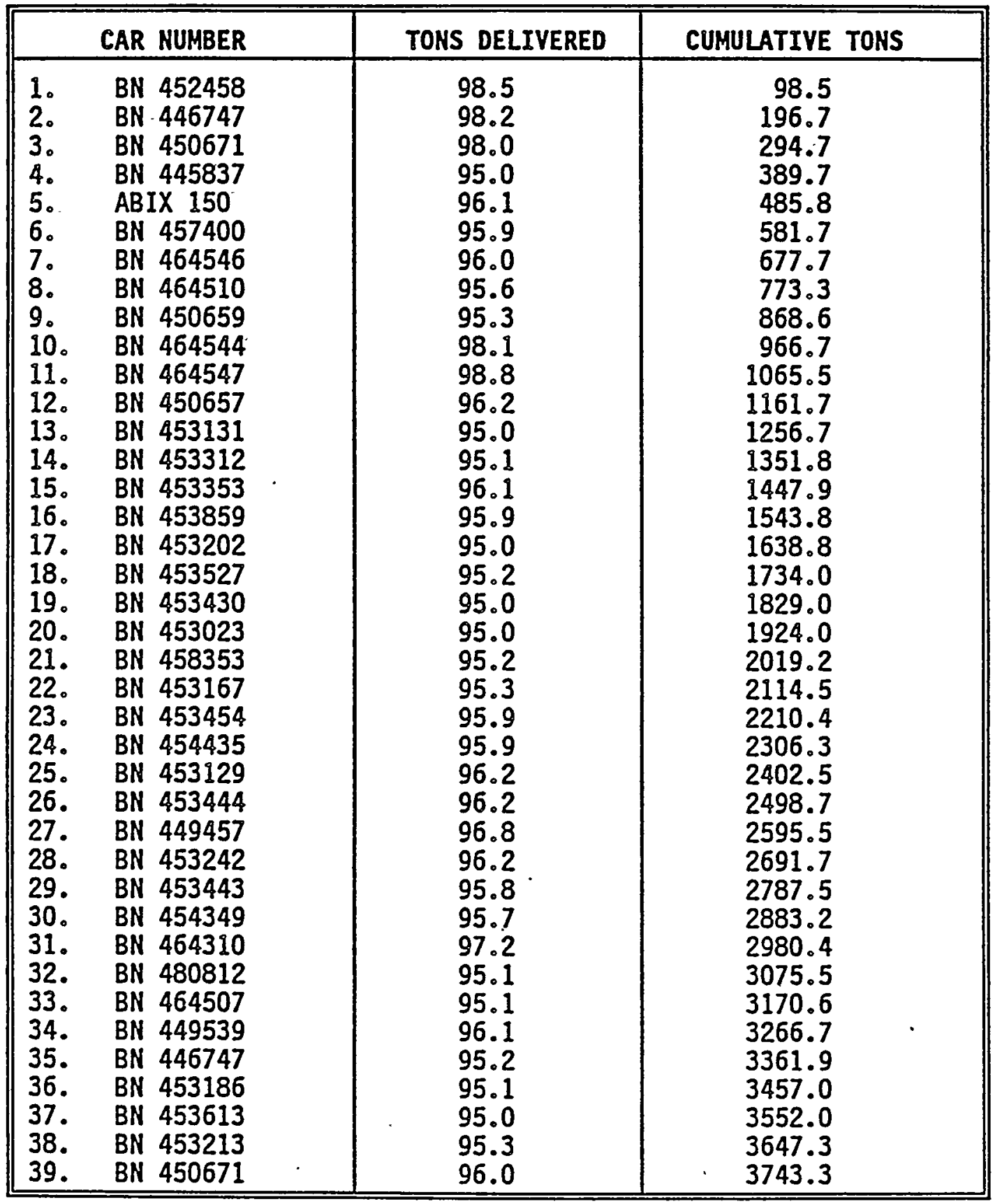




\section{CERTIFICATE OF ANALYSIS \\ AMERICAN COLLOID COMPANY 1500 W. SHURE DRIVE ARLINGTON HEIGHTS, ILLINOIS 60004}

TO:

$$
\frac{\text { Delhur Industries }}{\frac{\text { P.0.Bax } 1116}{\text { Part Angeles, WA. } 93620=0.201}}
$$$$
\text { DATE } 10-11-93
$$

ATTN: Rick Hevworth

A shipment of Cf5-200 left our Uptow plant in BN452458 on $10-11-93$ as requested on your order number

A sample from this shipment was tested, and gave the following results

\section{IEST}

Visc

PV/UPRatio

FL

Free swell

moiature

-200 mesh

BBL Yield
SPECIFICATION

$15.0 \mathrm{~min}$

3 to 1 max

$15.0 \max$

nons

$12.0 \% \cdot \max$

$70 / 200 \mathrm{~min}$. $90 \mathrm{~min}$
RESULTS

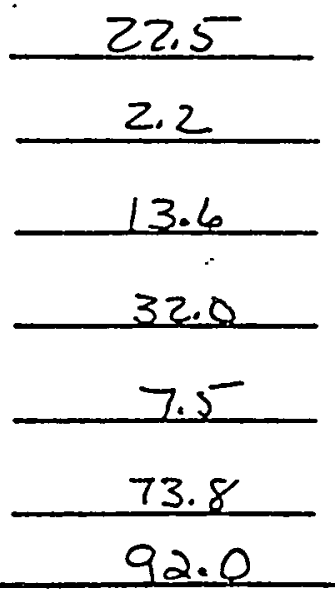

We hereby certify that the test results shown above represent this shipment. Tests were conducted using American Colloid Company and/or customer approved laboratory procedures.

TEST CONDUCTED BY: WG APPROVED BY: LeW. AMERICAN COLLOID COMPANY

In any correspondence regarding this shipment, please refer to our Order No. $0 / 9125$. 


\section{CERTIFICATE OF ANALYSIS \\ AMERICAN COLLOID COMPANY \\ ¿500 W. SHURE DRIVE \\ ARLINGTON HEIGHTS, ILLINOIS 60004}

TO: $\frac{\text { Delhur Industries }}{\frac{\text { P. B. Bax } 116}{\text { Part Angeles, Wa } 93620=0201}}$

DATE $10-13-93$

ATTN: Rick Hurworth

A shipment of Cf5-200 left our Uptor plant in BN4506.71 on $10-13-93$ as requested on your order number

A sample from this shipment was iested, and gave the following results

\begin{tabular}{|c|c|c|}
\hline IEST. & SPECIFICATION & RESULTS \\
\hline Vise & $15.0 \mathrm{~min}$ & $22: 5$ \\
\hline PV/UPRatio & 3 to 1 max & 2.1 \\
\hline EL & $15.0 \mathrm{max}$ & 13.4 \\
\hline Fire swell & none & 24.0 \\
\hline mointure & $12.0 \%$ max & 9.8 \\
\hline-200 mesh & $70 / 200 \mathrm{~min}$. & 70.6 \\
\hline$B B L$ Yield & $90 \mathrm{~min}$ & 92.0 \\
\hline
\end{tabular}

We hereby certify that the test results shown above represent this shipment. Tests were conducted using American Colloid Company and/or customer approved laboratory procedures.

TEST CONDUCTED BY: jee

APPROVED BY: $\frac{\mathcal{f} . \omega}{\text { AMERICAN COLLOID COMPANY }}$

In any correspondence regarding this shipment, please refer to our Order No. $01913 \mid$. 


\section{CERTIFICATE OF ANALYSIS}

A.MERICAN COLLOID COMPANY 1500 W. SHURE DRIVE ARLINGTON HEIGHTS, ILLINOIS 60004

TO: Delhur Industries

DATE $10-14-93$

P. 0 . Bax 1116
Port Angeles, WA. $93620=0201$

ATTN: Bick Hurworth

A shipment of C $f 5-200$ left our Uprov plant in BN457400 on $10-14-93$ as requested on your order number

A sample from this shipment was tested, and gave the following results

\section{IEST}

- Vise

PV/UPRatio

EL

Free swell

moisture

-200 mesh

BBL Yield
SPECIFICATION

$15.0 \mathrm{~min}$

3 to 1 max

$15.0 \max$

nons.

$12.0 \%$ max

$70 / 200 \mathrm{~min}$. $90 \mathrm{~min}$
BESULTS

\begin{tabular}{c}
$\frac{22.0}{2.4}$ \\
\hline$\frac{13.6}{28.0}$ \\
\hline 8.1 \\
\hline 73.2 \\
\hline 92.0
\end{tabular}

We hereby certify that the test results shown above represent this shipment. Tests were conducted using American Colloid Company and/or cusiomer approved laboratory procedures.

TEST CONDUCTED BY: Sm

APPROVED BY: LeO

AMERICAN COLLOID COMPANY

In any correspondence regarding this shipment, please refer to our Order No. 019134. 


\section{CERTIFICATE OF ANALYSIS \\ AMERICAN COLLOID COMPANY \\ 1500 W. SHURE DRIVE \\ ARLINGTON HEIGHTS, ILLINOIS 60004}

To: $\frac{\text { Delhur Industries }}{\text { P. B. Bax } 1116}$

DATE $10-15-93$

Part Angeles, WA. $93620=0201$ RECEIVED

OCT 281993

ATTN: Rick Huvworth

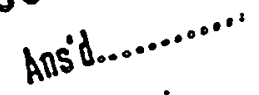

A shipment of

Cf $5-200$

left our Upton

plant in ABIX 150

on $10-15-93$ as requested on your order number

A sample from this shipment was tested, and gave the following results

\section{IEST.}

Visc

PV/UPRatio

FL

Free swell

moisture

-200 mesh

$B B L$ Yield
SPECIFICATION

$15.0 \mathrm{~min}$

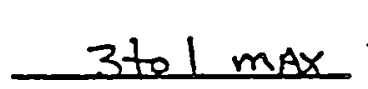

$15.0 \max$

nons

$12.0 \%$ max

$70 / 200 \mathrm{~min}$.

$90 \mathrm{~min}$
RESULTS

24.0

2.4

14.2

26.6

8.1

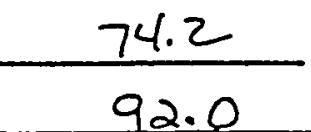

We hereby certify that the test results shown above represent this shipment. Tests were conducted using American Colloid Company and/or customer approved laboratory procedures.

TEST CONDUCTED BY: \& $M$

APPROVED BY: $\frac{\mathcal{L} W}{\text { AMERICAN COLLOID COMPANY }}$

In any correspondence regarding this shipment, please refer to our Order No. 019136. 


\section{CERTIFICATE OF ANALYSIS}

AMERICAN COLLOID COMPANY 1500 W. SHURE DRIVE

ARLINGTON HEIGHTS, ILLINOIS 60004

TO: Delhur Industries

DATE $10-16-93$

P. O. Bax 1116

Part Angeles, Wo. $93620=0201$

ATTN: Rick Hurwarth

A shipment of Cf5-200 left our Uptow plant in BN450659 on $10-16-93$ as requested on your order number

A sample from this shipment was tested, and gave the following results

IEST

Visc

PV/uPRatio

FL

Free swell

moisture

-200 Mesh

BBL Yield
SPECIFICATIQN

$15.0 \mathrm{~min}$

3 tol max.

$15.0 \max$

nons

$12.0 \%$ max

$70 / 200 \mathrm{~min}$.

$90 \mathrm{~min}$
RESULTS, 28.0

2.7

14.0

30,0

7.8

71.8

92.0

We hereby certify that the test results shown above represent this shipment. Tesis were conducted using American Colloid Company and/or customer approved laboratory procedures.

\section{TEST CONDUCTED BY: BE}

RECEIVED

OCT 251993

APPROVED BY:

Lev-

AMERICAN COLLOID COMPANY

Ansid.

In any correspondence regarding this shipment, please refer to our Order No. 019137. 


\section{CERTIFICATE OF ANALYSIS \\ AMERICAN COLLOID COMPANY \\ 1500 W. SHURE DRIVE \\ ARLINGTON HEIGHTS, ILLINOIS 60004}

TO: Delhur Industries

DATE $10-16-93$

P. O. Bax 1116

Port Angeles; Wh. $93620=0201$

ATTN: Rick Huvworth

A shipment of CfS-200 left our UptoN plant in BN 464544 on $10-16-93$ as requested on your order number

A sample from this shipment was tested, and gave the following results

\section{IEST}

Visc

PV/YPRatio

FL

Free swell

moisture

-200 mesh

$B B L$ Yield
SPECIFICATION

$\frac{15.0 \min }{3+1 \max }$

$15.0 \max$

none

$12.07 . \max$

$70 / 200 \mathrm{~min}$.

$90 \mathrm{~min}$
RESULTS

\begin{tabular}{c}
$\frac{31.0}{2.8}$ \\
\hline$\frac{14.0}{26.0}$ \\
\hline 7.6 \\
\hline 71.6 \\
\hline 92.0
\end{tabular}

We hereby certify that the test results shown above represent this shipment. Tests were conducted using American Colloid Company and/or customer approved laboratory procedures.

TEST CONDUCTED BY: IMC

APPROVED BY: $-\mathcal{L}$

AMERICAN COLLOID COMPANY

In any correspondence regarding this shipment, please refer to our Order No. 019140. 


\section{CERTIFICATE OF ANALYSIS}

AMERICAN COLLOID COMPANY

1500 W. SHURE DRIVE

ARLINGTON HEIGHTS, ILLINOIS 60004

TO:

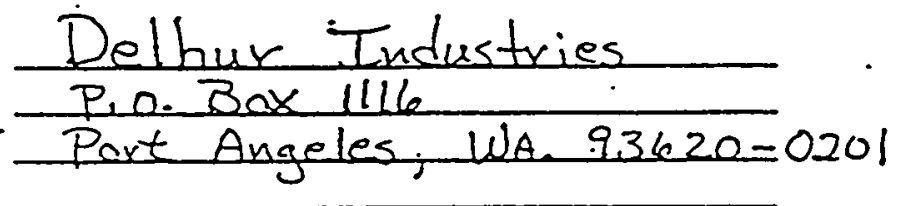

$$
\text { DATE } 15-16-93
$$

ATTN: Rick Herworth

A shipment of Cf5-200 left our Uprow plant in BN464547 on $10-16-93$ as requested on your order number

A sample from this shipment was tested, and gave the following results

\section{IEST}

- $V_{\text {isc }}$

PV/YP.Ratio

FL

Free swell

moisture

-200 mesh

BBL Yield
SPECIFICATION

$\frac{15.0 \text { min }}{3 \text { to } 1 \text { max. }}$

$15.0 \max$

nene

$12.0 \%$ max

$70 / 200 \mathrm{~min}$.

$90 \mathrm{~min}$

\section{RESULTS}

$\frac{\frac{31.5}{2.8}}{\frac{14.0}{26.0}}$

7.6

72.2

92.0

We hereby certify that the test results shown above represent this shipment. Tests were conducted using American Colloid Company and/or customer approved laboratory procedures.

TEST CONDUCTED BY: WOS

APPROVED BY: Ses-

AMERICAN COLLOID COMPANY

In any correspondence regarding this shipment, please refer to our Order No. $0 / 9133$. 


\section{CERTIFICATE OF ANALYSIS \\ AMERICAN COLLOID COMPANY 1500 W. SHURE DRIVE ARLINGTON HEIGHTS, ILLINOIS 60004}

TO:

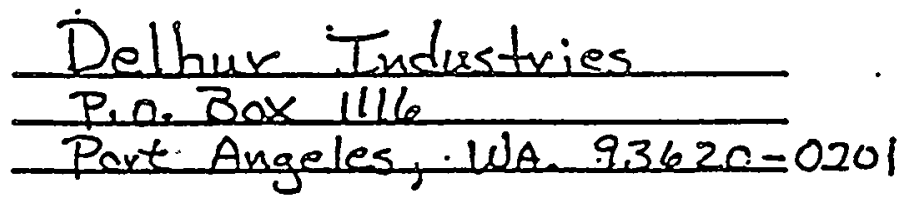

DATE $10-18-93$

ATTN: Rick Hevworth

A shipment of Cf5-200 left our Uprow plant in BN 464510 on $10-18-93$ as requested on your order number

- A sample from this shipment was tested, and gave the following results

IEST

Vise

PV/UP Ratio

FL

Eree swell

moisture

-200 mesh

BBL Yield
SPECIFICATION

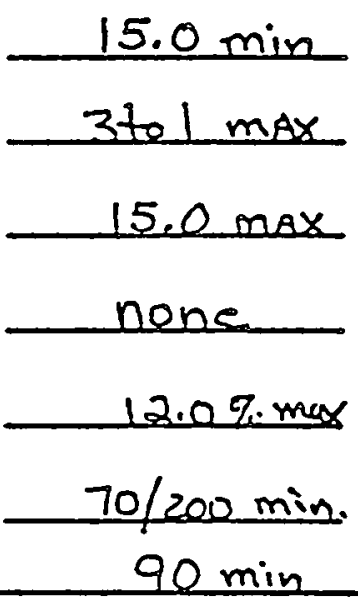

RESULTS

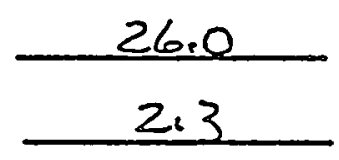

13,8

$24,0^{\circ}$

8.6

76.4

92.0

We hereby certify that the test results shown above represent this shipment. Tests were conducted using American Colloid Company and/or customer approved laboratory procedures.

TEST CONDUCTED BY: $L M$

APPROVED BY: \&u.AMERICAN COLLOID COMPANY

In any correspondence regarding this shipment, please refer to our Order No. 019138 . 
WHC-SD-W025-RPT-001, Rev. 0

\section{CERTIFICATE OF ANALYSIS \\ AMERICAN COLLOID COMPANY \\ 1500 W. SHURE DRIVE \\ ARLINGTON HEIGHTS, ILLINOIS 60004}

TO: Delhur Industries

DATE $10-18-93$

P. O. Bax 1116

Port Angeles, Wa. $93620=0201$

ATTN: Rick Herworth

A shipment of CfS-200 left our Uptow plant in BN464546 on $10-18-93$ as requested on your order number

A sample from this shipment was tested, and gave the following results

IEST

Visc

PV/UPRatio

FL

Free swell

moisture

-200 mesh

$B B L$ Yield
SPECIFICATION

$\frac{15.0 \text { min }}{3+01 \text { max }}$

15.0 max

none

$12.0 \%$ max

$70 / 200 \mathrm{~min}$.

$90 \mathrm{~min}$
RESULTS

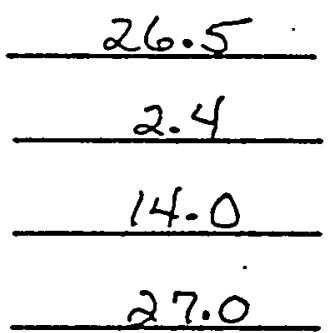

7.8

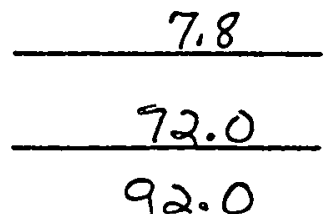

We hereby certify that the test results shown above represent this shipment. Tests were conducted using American Colloid Company and/or customer approved laboratory procedures.

$$
\begin{aligned}
& \text { TEST CONDUCTED BY: LM } \\
& \text { APPROVED BY: } \\
& \text { Lw. } \\
& \text { AMERICAN COLLOID COMPANYY }
\end{aligned}
$$

In any correspondence regarding this shipment, please refer to our Order No. 19139. 
RECEIVED

NOV 011993

Ans. 0

\section{CERTIFICATE OF ANALYSIS \\ AMERICAN COLLOID COMPANY 1500 W. SHURE DRIVE \\ ARLINGTON HEIGHTS, ILLINOIS 60004}

TO: Delhur Industries

DATE $10-19-93$ P.O. Bax 1116

Port Angeles, Wa. $93620=0201$

ATTN: Rick Huvworth

A shipment of CfS-200 left our Uptos plant in BN450657 on $10-19-93$ as requested on your order number

A sample from this shipment was tested, and gave the following results

IEST

Visc

PV/YPRatio

FL

Eree swel!

moisture

-200 mesh

$B B L$ Yield
SPECIFICATION

$15.0 \mathrm{~min}$

3tol max

15.0 max

none

$12.0 \%$ max

$70 / 200 \mathrm{~min}$.

$90 \mathrm{~min}$

RESULTS

30.0

3.0

14.0

28.0

8.1

71.2

92.0

$\dot{W}$ hereby certify that the test results shown above represent this shipment. Tests were conducted using American Colloid Company and/or customer approved laboratory procedures.

TEST CONDUCTED BY: Im

APPROVED BY: Lew.

AMERICAN COLLOID COMPANY

In any correspondence regarding this shipment, please refer to our Order No. $019 \sqrt{4} \mathrm{~L}$. 


\section{CERTIFICATE OF ANALYSIS \\ AMERICAN COLLOID COMPANY 1500 W. SHURE DRIVE ARLINGTON HEIGHTS, ILLINOIS 60004}

TO: Delhur Industries

DATE $10-20-93$

$$
\text { P. O. Bax } 1116
$$

Port Angeles, WA. $9.3620-0201$ RECEIVER

ATTN: Rick Huvworth

A shipment of C $15-200$ lett our UproN plant in BN 45313] on $10-20-93$ as requested on your order number

A sample from this shipment was tested, and gave the following results

\section{IEST}

Visc

PV/YPRatio

FL

Free swell

moisture

-200 mesh

BBL Yield
SPECIFICATION

$15.0 \mathrm{~min}$

3 to 1 max

$15.0 \max$

none

$12.0 \% \max$

$70 / 200 \mathrm{~min}$.

$90 \mathrm{~min}$
RESULTS

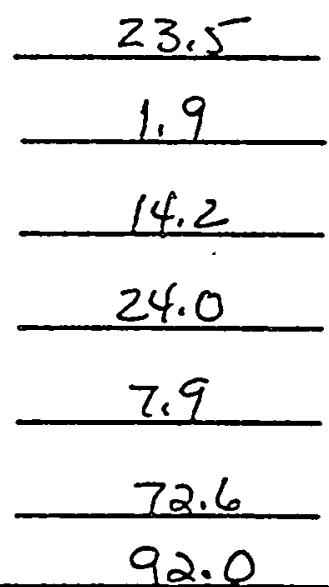

We hereby certify that the test results shown above represent this shipment. Tests were conducted using American Colloid Company and/or customer approved laboratory procedures.

TEST CONDUCTED BY: LM

APPROVED BY:

AMERICAN COLLOID COMPANY

In any correspondence regarding this shipment, please refer to our Order No. 019142 


\section{CERTIFICATE OF ANALYSIS \\ AMERICAN COLLOID COMPANY 1500 W. SHURE DRIVE ARLINGTON HEIGHTS, ILLINOIS 60004}

TO: Delhur Industries

DATE M.PB.:3

P. D. Bax 1116
Part Angeles; Wa .93620-0201

ATTN: Bick Hurworth

A shipment of C $15-200$ left our Upten plent in BN\&530.23 on $10-16-93$ as requested on your order number

A sample from this shipment was tesied, and gave the following results

TEST

Visc

PV/UPRatio

FL

Free swell

mointere

-200 mesh

BBL Uield
SPECIFICATION

$15.0 \mathrm{~min}$

3 te 1 max

$15.0 \max$

none

$12.0 \%$ max

$70 / 200 \mathrm{~min}$.

$90 \mathrm{~min}$
RESULTS

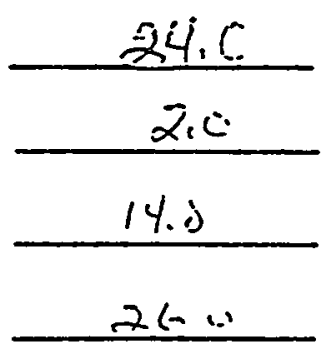

$\% .3 \%$

72.

92.0

We hereby certify that the test results shown above represent this shipment. Tests were conducted using American Colloid Company and/or customer approved laboratory procedures.

TEST CONDUCTED BY: $i\left(i_{2}\right.$

APPROVED BY:

AMERICAN COLLOID COMPANY

In any correspondence regarding this shipment, please refer to our Order No.

1 i"ilici. 


\section{CERTIFICATE OF ANALYSIS \\ AMERICAN COLLOID COMPANY 1500 W. SHURE DRIVE ARLINGTON I:EIGHTS, ILLINOIS 60004}

TO: Delhur Indistries

DATE $10 \cdot 26.93$

$$
\frac{\text { P. . Box 1116 }}{\text { Pout Angeles; Wla 93620=0201 }}
$$

ATTN: Rick Hurworth

A shipment of C $15-200$ left our Upton plant in BN45316? on $\frac{10-26-93}{}$ as requested on your order number

A sample from this shipment was tested, and gave the following results

\section{IEST}

Visc

PV/UPRatio

FL

Free swell

moisture

-200 mesh

BBL Yield
SPECIFICATION

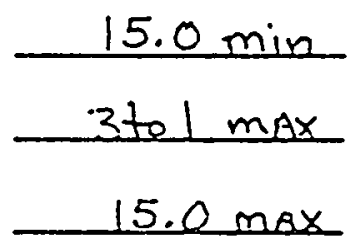

none

$12.09 \cdot \max$

$70 / 200 \mathrm{~min}$. $90 \mathrm{~min}$
RESULTS

$\frac{30.5}{2.7}$

13.2

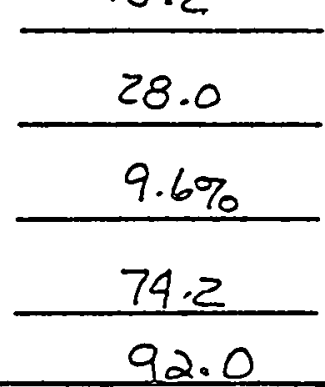

We hereby certify that the test results shown above represent this shipment. Tests were conducted using American Collcid Company and/or customer. approved laboratory procedures.

TEST CONDUCTED BY: $\omega .6$.

APPROVED BY:

AMERICAN COLLOID COMPANY

In any correspondence regarding this shipment, please refer to our Order No. 019153. 


\section{CERTIFICATE OF ANALYSIS \\ AMERICAN COLLOID COMPANY 1500 W. SHURE DRIVE ARLINGTON HEIGHTS, ILLINOIS 60004}

TO: $\frac{\text { Delhur Industries }}{\frac{\text { P. D. Bax } 1116}{\text { Port Angeles; Wa } .93620=0201}}$

DATE 10.26 .93

ATTN: Rick Herworth

A shipment of CfS-200 left our Upton plant in 12,N 453202 on $10-26$ as recsested on your order number

A sample from this shipment was tested, and gave the following results

IEST

Visc

PV/UPRatio

FL

Eree swell

moiature

-200 Mesh

$B B L$ Yield
SPECIFICATION

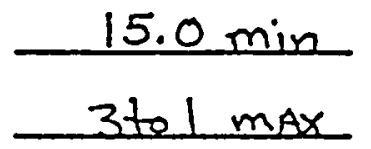

$15.0 \max$

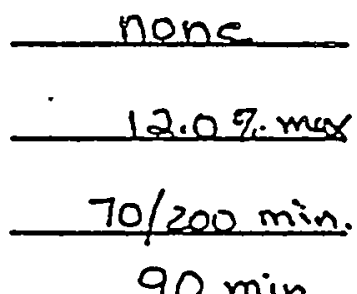

RESULTS.

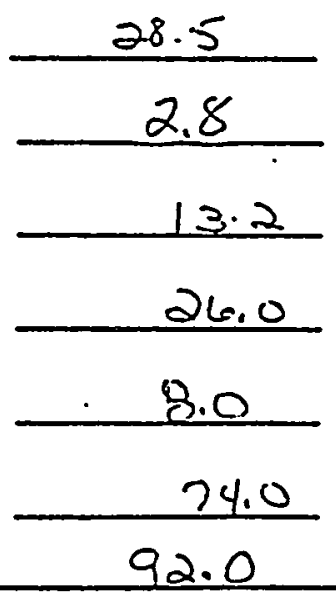

We hereby cerify that the test results shown above represent this shipment. Tests were conducted using American Colloid Company and/or customer approved laboratory procedures.

TEST CONDUCTED BY:

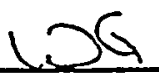

- APPROVED BंY: $\mathcal{L W}$ AMERICAN COLLOID COMPANY

In any correspondence regarding this shipment, please refer to our Order No. 19149 


\section{CERTIFICATE OF ANALYSIS \\ AMERICAN COLLOID COMPANY 1500 W. SHURE DRIVE \\ ARLINGTON HEIGHTS, ILLINOIS 60004}

TO: Delhur Industries

DATE $i_{i}-26-93$

$$
\begin{aligned}
& \text { P.0. Bax 1116 } \\
& \text { Part Angeles, Wa } 93620=0201
\end{aligned}
$$

ATTN: Rick Hevworth

A shipment of C $75-200$ left cur Uprow plant in BN 4533i2 on $10-26-93$ as requested on your order number

A sample from this shipment was tested, and gave the following results

\section{TEST}

Visc

PV/UPRatio

EL

Free swiel!

moisture

-200 mesh

BBL Yield
SPECIFICATION

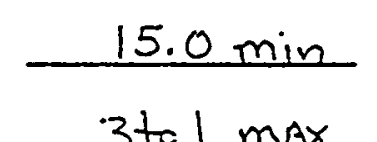

3 to 1 max

$15.0 \max$

none

12.07 . max

$70 / 200 \mathrm{~min}$.

$90 \mathrm{~min}$
RESULTS

$\frac{28.0}{2.3}$

24.0

8.1

71.1

92.0

We hereby certify that the test results shown above represent this shipment. Tesis were conducted using American Colloid Company and/or customer approved laboratory procedures.

$$
\begin{aligned}
& \text { TEST CONDUCTED BY: LUG } \\
& \text { APPROVED BY: } \frac{\text { LUi. }}{\text { AMERICAN COLLOID COMPANY }}
\end{aligned}
$$

In any correspondence regarding this shipment, please refer to cur Order No. 019144 


\section{CERTIFICATE OF ANALYSIS \\ AMERICAN COLLOID COMPANY 1500 W. SHURE DRIVE \\ ARLINGTON HEIGHTS, ILLINOIS 60004}

TO: Delhur Industries

DATE $10 \cdot 2 i 1 \cdot ; 3$ P.n. Bax lille

Part Angeles, WA $9.362 c=0201$

ATTN: Rick Huvworth

A shipment of CA $5-200$ left our Upton plant in BN1453.3.53 on $10 \cdots 2 b_{0}$ as requested on your order number

A sample from this shipment was tested, and gave the following results

IEST

Yisc

PV/UPRatio

FL

Free swell

moisture

-200 mesh

$B B L$ Yield
SPECIFICATION

$\frac{\frac{15.0 \mathrm{~min}}{3+1 \mathrm{max}}}{15.0 \mathrm{max}}$

nons.

$12.0 \% \cdot \operatorname{mex}$

$70 / 200 \mathrm{~min}$.

$90 \mathrm{~min}$
RESULTS

$\frac{.30 .5}{2.60}$

$\therefore$ is

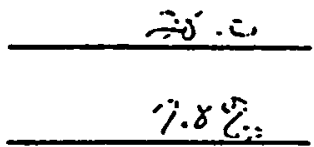

$1.2 \%$

92.0

We hereby certify that the test results shown above represent this shipment. Tests were conducted using American Colloid Company and/or customer approved laboratory procedures.

TEST CONDUCTSD BY:

(is

APPROVED BY: LU.

AMERICAN COLLOID COMPANY

In any correspondence regarding this shipment, please refer to our Order No.

19,44 


\section{CERTIFICATE OF ANALYSIS \\ AMERICAN COLLOID COMPANY 1500 W. SHURE DRIVE ARLINGTON HEIGHTS, ILLINOIS 60004}

TO: Delhur Industries

DATE $10-26.97=$ P. O. Bax 1116

Port Angeles; WA. $9.3620=0.201$

ATTN: Bicks Hevworth

A shipment of C $15-200$ left our Upron plant in 1344534.30 on. 10.26 as requested on your order number

A sample from this shipment was tested, and gave the following results

\section{TEST}

- $y_{i s c}$

PV/UPRatio

FL

Free swell

moisture

-200 mesh

BBL Yield
SPECIFICATION

$15.0 \mathrm{~min}$

3 to 1 max

15.0 max

none

$12.0 \% \max$

$70 / 200 \mathrm{~min}$.

$90 \mathrm{~min}$
RESULTS

29.0

2.1

13.4

24.6

$7,9 \%$

7.3. 6

92.0

We hereby certify that the test results shown above represent this shipment. Tests were conducted using American Colloid Company and/or customer approved laboratory procedures.

TEST CONDUCTED BY: lox APPROVED BY: Lew. AMERICAN COLLOID COMPANY

In any correspondence regarding this shipment, please refer to our Order No. 191,51 . 


\section{CERTIFICATE OF ANALYSIS \\ AMERICAN COLLOID COMPANY 1500 W. SHURE DRIVE ARLINGTON HEIGHTS, ILLINOIS 60004}

TO: Delhur Industries

DATE $10 \cdot 36-93$ P. O. Bex 1116

Part Angeles; Wa. $.93620=0201$

ATTN: Rick Herworth

A shipment of Cf5-200 left our Upton plant in BN 453454 on $10-26-93$ as requested on your order number

- A sample from this shipment was tested, and gave the following results

IEST

Vise

PV/YPRatio

FL

Free swell

moisture

-200 Mesh

$B B L$ Yield
SPECIFICATION

$\frac{15.0 \mathrm{~min}}{3 \text { to } 1 \text { max }}$

15.0 max

none

$12.0 \%$ max

$70 / 200 \mathrm{~min}$.

$90 \mathrm{~min}$
RESULTS

29.0

.2 .5

$13 \cdot 2$

28.0

$\frac{8.1}{73.6}$

We hereby certify that the test results shown above represent this shipment. Tests were conducted using American Colloid Company and/or customer approved laboratory procedures.

TEST CONDUCTED BY: S.M,

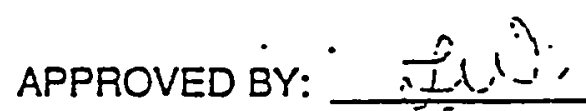

AMERICAN COLLOID COMPANY

In any correspondence regarding this shipment, please refer to our Order No. 019151 . 


\section{CERTIFICATE OF ANALYSIS \\ AMERICAN COLLOID COMPANY 1500 W. SHURE DRIVE ARLINGTON HEIGHTS, ILLINOIS 60004}

To: Delhur Industries

DATE $10.21 .-43$ P. 0. Bax 1116

Port Angeles; Wla $9.3620=0201$

ATTN: Rick Hevworth

A shipment of Cf $5-200$ left our plant in $3 / 1453527$ on $10 \cdot 26$ as requested on your order number

A sample from this shipment was tested, and gave the following results

\section{IEST}

Visc

PV/uPbatio

FL

Free swell

moisture

-200 mesh

BBL Yield
SPECIFICATION

$\frac{15.0 \min }{3 \text { to } 1 \text { max }}$

$15.0 \max$

none

$12.0 \% \max$

$70 / 200 \mathrm{~min}$.

$90 \mathrm{~min}$
RESULTS

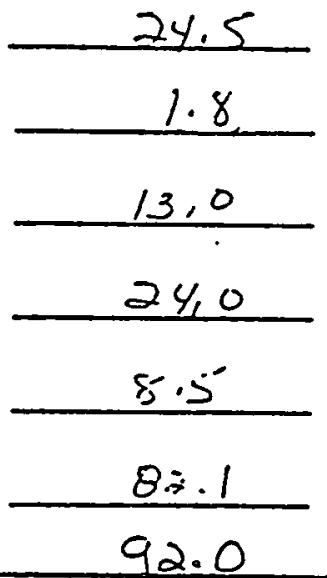

We hereby certify that the test results shown above represent this shipment. Tests were conducted using American Colloid Company and/or customer approved laboratory procedures.

TEST CONDUCTED BY:

L.S

APPROVED BY: L LW.

AMERICAN COLLOID COMPANY

In any correspondence regarding this shipment, please refer yo our Order No. 19150. 


\section{CERTIFICATE OF ANALYSIS \\ AMERICAN COLLOID COMPANY \\ 1500 W. SHURE DRIVE \\ ARLINGTON HEIGHTS, ILLINOIS 60004}

TO: Delhur Industries P.n. Bax 11116

Part Angeles; WA $9.3620=0201$

ATTN: Rick Hurworth

A shipment of Cf $5-200$ left our Upron DATE $1 C, 20 \%$ on $10 \cdot 76$ as requested on your order number

A sample from this shipment was tested, and gave the following results

TEST

Visc

PV/uPRatio

FL

Free swell

mainture

-200 mesh

BBL vield
SPECIFICATION

$15.0 \mathrm{~min}$

3 to 1 max

15.0 max

none

$12.0 \%$ max

$70 / 200 \mathrm{~min}$.

$90 \mathrm{~min}$

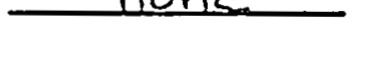
plant in 18, 1 45:3.59

We hereby certify that the test results shown above represent this shipment. Tests were conducted using American Colloid Company and/or customer approved laboratory procedures.

TEST CONDUCTED BY: RESULTS

74.5

MiY\%.

APPROVED BY:

AMERICAN COLLOID COMPANY

In any correspondence regarding this shipment, please refer to our Order No.

19148 
WHC-SD-W025-RPT-001, Rev. 0

\section{CERTIFICATE OF ANALYSIS \\ AMERICAN COLLOID COMPANY 1500 W. SHURE DRIVE ARLINGTON HEIGHTS, ILLINOIS 60004}

TO: Delhur Industries

DATE $10-36-93$ P.O. Bax 1116

Part Angeles; WA $.93620-0201$ M.2Y U 81993

An'

ATTN: Rick Huvwerth

A shipment of Cf $15-200$ left our Uprow plant in $\beta_{M} 454435$

on $10-36-93$ as requested on your crder number

A sample from this shipment was tested, and gave the following results

\section{IEST}

Vise

PV/UPRatio

FL

Free swell

moisture

-200 mesh

BBL Yield
SPECIFICATION

$15.0 \mathrm{~min}$

3 to 1 max

$15.0 \max$

none

$12.0 \%$ max

$70 / 200 \mathrm{~min}$.

$90 \mathrm{~min}$
RESULTS

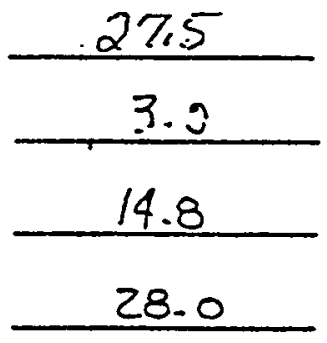

8.7

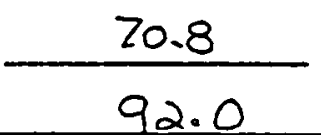

We hereby certify that the test results shown above represent this shipment. Tests were conducted using American Colloid Company and/or customer approved laboratory procedures.

TEST CONDUCTED BY: L.M.

APPROVED BY:

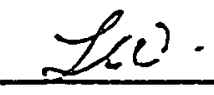

AMERICAN COLLOID COMPANY.

In any correspondence regarding this shipment, please refer to our Order No. 019270. 


\section{CERTIFICATE OF ANALYSIS \\ AMERICAN COLLOID COMPANY 1500 W. SHURE DRIVE ARLINGTON HEIGHTS, ILLINOIS 60004}

TO: Delhur Tndistries

DATE $10 \div 1 \ldots, \ldots$

P. O. Bax 1116

Part Angeles; Wa. 9.3620-0201

ATTN: Rick Huvworth

A shipment of Cf5-200 left our Upton plant in Bus458353 on $10-i b$ as requested on your order number

A sample from this shipment was tested, and gave the following results

IEST

SPECIFICATION

RESULTS

Visc

$15.0 \mathrm{~min}$

PV/UPRatio

3 te 1 max

$15.0 \max$

none

Free swell

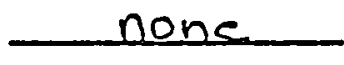

moisture

$12.0 \%$ max

-200 Mesh

BBL Yield

$70 / 200 \mathrm{~min}$.

$90 \mathrm{~min}$

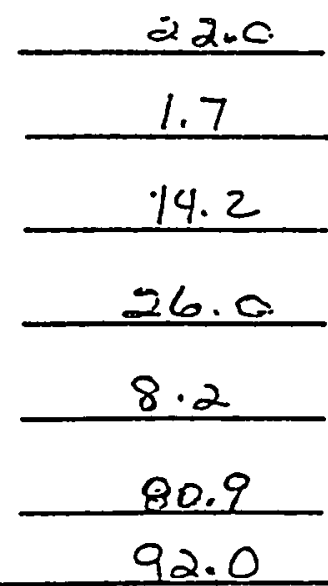

We hereby certify that the test results shown above represent this shipment. Tests were conducted using American Colloid Company and/or customer approved laboratory procedures.

TEST CONDUCTED BY: $\omega^{\prime} G$

APPROVED BY: Lw.

AMERICAN COLLOID COMPANY

In any correspondence regarding this shipment, please refer to our Order No. $14 ; 1455$. 


\section{CERTIFICATE OF ANALYSIS \\ AMERICAN COLLOID COMPANY 1500 W. SHURE DRIVE ARLINGTON HEIGHTS, ILLINOIS 60004}

TO: Delhur Industries

DATE $11-i-93$

$$
\begin{aligned}
& \text { P. } 0 . \text { Bax } 1116 \\
& \text { Port Angeles; Wha } 9362 \theta=0201
\end{aligned}
$$

ATTN: Rick Hevworth

A shipment of ( $15-200$ left our Upton plent in BN 480812 on $11-1-93$ as requested on your order number

A sample from this shipment was tested, and gave the following results

\section{TEST}

Visc

PV/YPRatio

FL

Free swell

moisture

-200 mesh

BBL Yield

\section{SPECIFICATION}

$15.0 \mathrm{~min}$

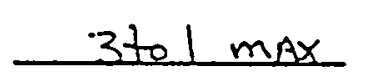

$15.0 \max$

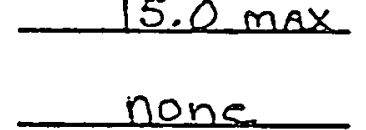

$12.0 \%$ max

$70 / 200 \mathrm{~min}$.

$90 \mathrm{~min}$
RESULTS

30.0

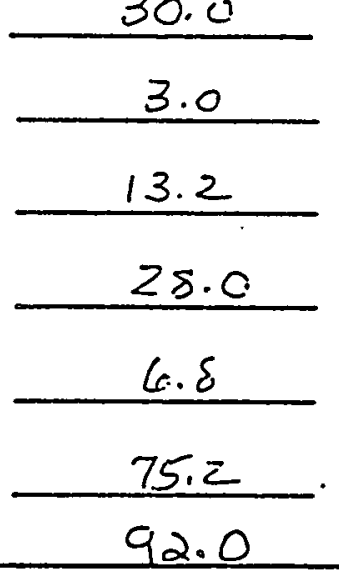

We hereby certify that the test results shown above represent this shipment. Tests were conducted using American Colloid Company and/or customer approved laboratory procedures.

TEST CONDUCTED BY: Lile

APPROVED BY: 。 Jeer.

AMERICAN COLLOID COMPANY

In any correspondence regarding this shipment, please refer to our Order No. 019163 . 
WHC-SD-W025-RPT-001, Rev. 0

\section{CERTIFICATE OF ANALYSIS \\ AMERICAN COLLOID COMPANY \\ 1500 W. SHURE DRIVE \\ ARLINGTON HEIGHTS, ILLINOIS 60004}

TO: Delhur Industries

DATE ir:Sill: :

$$
\frac{\text { P. O. Box } 1116}{\text { Pout Angeles, Wa. } 93620=0201}
$$

ATTN: Rick Huvwarth

A shipment of C $75-200$ left our Upton plant in BN453/29 on leislas as requested on your order number

A sample from this shipment was iesied, and gave the following results

IEST

Yisc

PV/UPRatio

FL

Free swell

moisture.

-200 Mesh

BBL vield
SPECIFICATION

$\frac{15.0 \min }{3+1 \max }$

$15.0 \max$

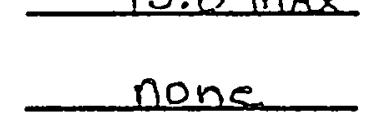

$12.07 . \max$

$70 / 200 \mathrm{~min}$. 96 min
RESULTS

$\frac{24.0}{2.4} \frac{14.0}{28.0}$

250.0

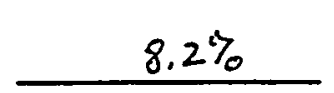

$72.5 / 200$

92.0

We hereby certify that the test results shown above represent this shipment. Tests were conducted using American Colloid Company and/or customer approved laboratory procedures.

TEST CONDUCTED BY: $\bar{H}$

APPROVED BY: fer.

AMERICAN COLLOID COMPANY

In any correspondence regarding this shipment, please refer to our Order No. 
WHC-SD-W025-RPT-001, Rev. 0

\section{CERTIFICATE OF ANALYSIS \\ AMERICAN COLLOID COMPANY \\ 1500 W. SHURE.DRIVE \\ ARLINGTON HEIGHTS, ILLINOIS 60004}

TO: Delhur Tridustries

DATE

$$
\frac{\text { P.n. Box 1116 }}{\text { Port A:igeles; Wla. } 93620-0201}
$$

ATTN: Fick Herworth

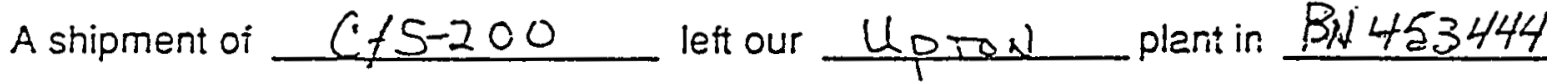
on as requested on your order number

A sample from this shipment was tested, and gave the following results

IEST

Visc

PV/uPRatio

FL

Free surell

mointure

-200 mesh

BBL Yield
SPECIFICATION

$15.0 \mathrm{~min}$

3 to 1 max

15.0 max

none

$12.09 . \operatorname{mex}$

$70 / 200 \mathrm{~min}$.

$90 \mathrm{~min}$
RESULTS

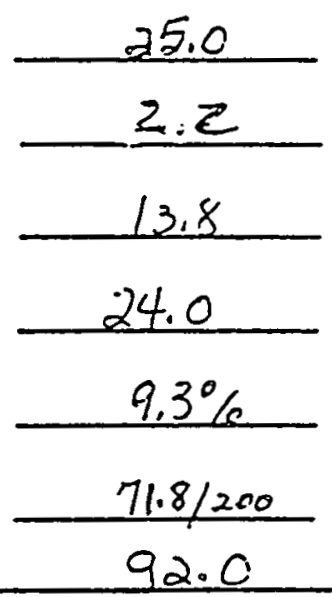

We hereby certify thai the test results shown above represent this shipment. Tests were conducted using American Colloid Company and/or customer approved laboratory procedures.

$$
\begin{aligned}
& \text { TEST CONDUCTED BY: } \\
& \text { APPROVED BYY: } \\
& \text { AMERICAN COLLOID COMPANY }
\end{aligned}
$$

In any correspondence regarding this shipment, please refer to our Order No. 


\section{CERTIFICATE OF ANALYSIS \\ AMERICAN COLLOID COMPANY \\ 1500 W. SHURE DRIVE \\ ARLINGTON HEIGHTS, ILLINOIS 60004}

TO:

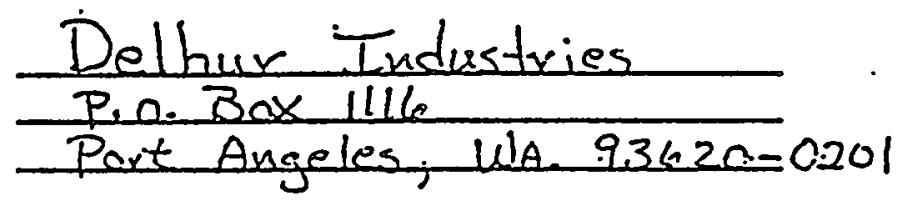

DATE $11-1-9.3$

ATTN: Rick Hevworth

A shipment of C $75-200$ left our Uprod plant in BN 464310 on $11-1-93$ as requested on your order number

A sample from this shipment was iested, and gave the following results

IEST

Visc

PV/YPRatio

FL

Free swell

moisture

-200 mesh

BBL Yield
SPECIFICATION

$15.0 \mathrm{~min}$

3 to 1 max

$15.0 \max$

nons.

$12.0 \%$ max

$70 / 200 \mathrm{~min}$. $90 \mathrm{~min}$
RESULTS

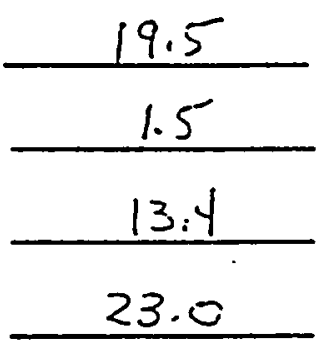

7.0

81.4

92.0

We hereby certify that the test results shown above represent this shipment. Tests were conducted using American Colloid Company and/or customer approved laboratory procedures.

TEST CONDUCTED BY: LiC

APPROVED BY: $\frac{\text { JeD }-}{\text { AMERICAN COLLOID COMPANY }}$

In any correspondence regarding this shipment, please refer to our Order No. $019 / 62$. 


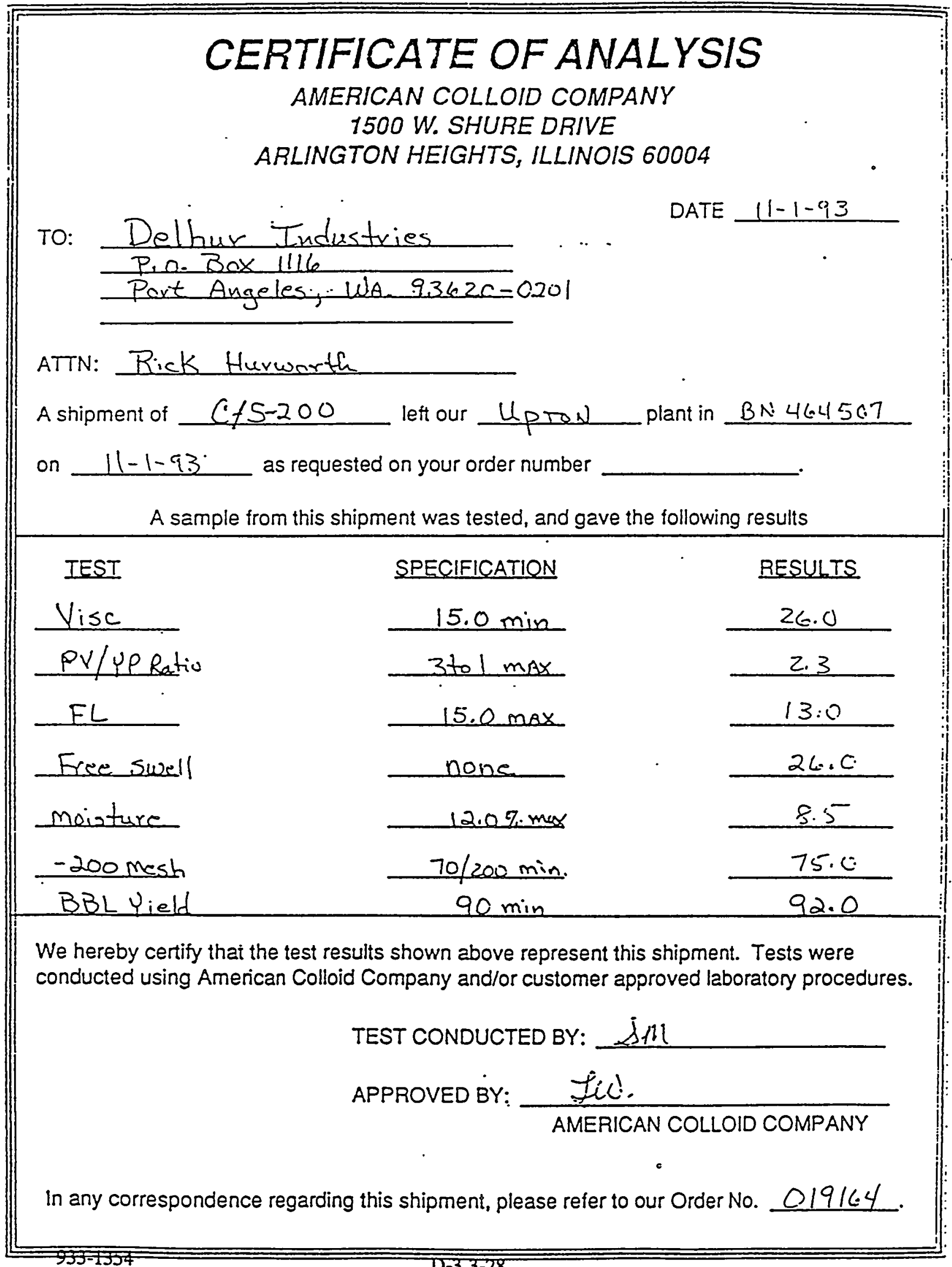




\section{CERTIFICATE OF ANALYSIS \\ AMERICAN COLLOID COMPANY 1500 W. SHURE DRIVE ARLINGTON HEIGHTS, ILLINOIS 60004}

TO: Delhur Industries

DATE MPI:AT:

$$
\text { P.n. Bax lllle }
$$

ATTN: Bick Hevworth

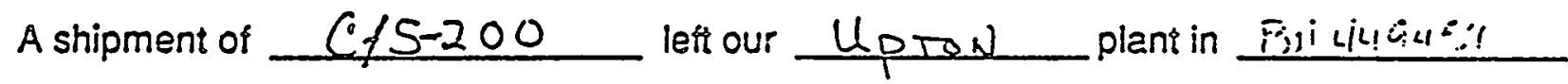
on 10 iaj $1 k_{i}:$ as requested on your order number

A sample from this shipment was iested, and gave the followirg results

\section{TEST}

Vise

PV/UPRatio

FL

Free swell

misinture

-200 mesh

BBL Yield
SPECIFICATION

$15.0 \mathrm{~min}$

3 to 1 max

$15.0 \max$

none

$13.0 \%$ max

$70 / 200 \mathrm{~min}$.

$90 \mathrm{~min}$
RESULTS

28.0

2.7

13.4

24.0

$8.3 \%$

$75.8 / 200$

92.0

We hereby certify that the test results shown above represent this shipment. Tests were conducted using American Colloid Company and/or customer approved laboratory procedures.

$$
\begin{aligned}
& \text { TESTT CONDUCTED BY: } \frac{\text { ) }}{\text { ! }} \\
& \text { APPROVED BY: } \mathcal{f}(l) \text {. } \\
& \text { AMERICAN COLLOID COMPANY }
\end{aligned}
$$

In any correspondence regarding this shipment, please refer to our Order No. 


\section{CERTIFICATE OF ANALYSIS \\ AMERICAN COLLOID COMPANY \\ 1500 W. SHURE DRIVE \\ ARLINGTON HEIGHTS, ILLINOIS 60004}

TO: Delhur Industries

DATE $11-2 \cdot 93$

$$
\frac{\text { P. n. Bax llle }}{\text { Part Angeles, Wa } .93620=0201}
$$

1:ECEIVED

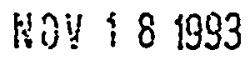

ATTN: Rick Hurworth

Ans'd.

A shipment of CfS-200 lett our Uprow plant in BN2/53/86 on $11-2-i 3$ as requested on your order number

A sample from this shipment was tested, and gave the following results

IEST

Visc

PV/UPRatio

FL

Eree swell

moisture

-200 Mesh

BBL Yield
SPECIFICATION

$\frac{\frac{15.0 \mathrm{~min}}{3 \text { to } 1 \text { max }}}{15.0 \mathrm{max}}$

nons

$12.0 \%$ max

$70 / 200 \mathrm{~min}$.

$90 \mathrm{~min}$
RESULTS

27.0

\begin{tabular}{c}
27.0 \\
\hline 2.5 \\
\hline 14.0 \\
\hline 28.0 \\
\hline 7.1 \\
\hline 72.4 \\
\hline 92.0
\end{tabular}

We hereby certify that the test results shown above represent this shipment. Tests were conducted using American Colloid Company and/or customer approved laboratory procedures.

TEST CONDUCTED BY: \& B

APPROVED BY: . Les.

AMERICAN COLLOID COMPANY

In any correspondence regarding this shipment, please refer to our Order No. 019160. 


\section{CERTIFICATE OF ANALYSIS \\ AMERICAN COLLOID COMPANY \\ 1500 W. SHURE DRIVE \\ ARLINGTON HEIGHTS, ILLINOIS 60004}

TO: Delihur Industries

DATE 144193 P. O. Box IIlle

Part Angeles, Wh. $93620=0201$

ATTN: Rick Huvworth

A shipment of C C $5-200$ left our Uptow plant in BN 453213 on $11 / 4 / 43$ as requested on your order number

A sample from this shipment was tested, and gave the following results

IEST

Visc

PV/up latio

FL

Free swell

moisture

-200 mesh

$\mathrm{BBL}$ vield
SPECIFICATION

$15.0 \mathrm{~min}$

3 to 1 max

$15.0 \max$

none

$12.0 \%$ mex

$70 / 200 \mathrm{~min}$. $90 \mathrm{~min}$
BESULTS
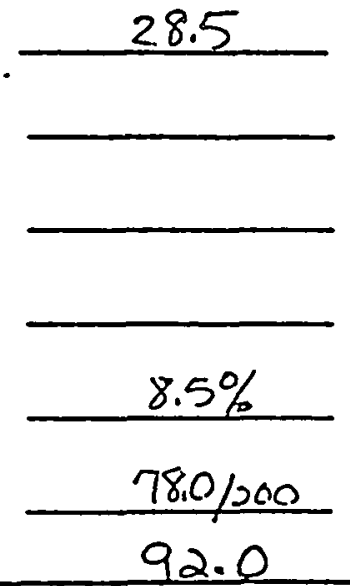

We hereby certify that the test results shown above represent this shipment. Tests were conducted using American Colloid Company and/or customer approved laboratory procedures.

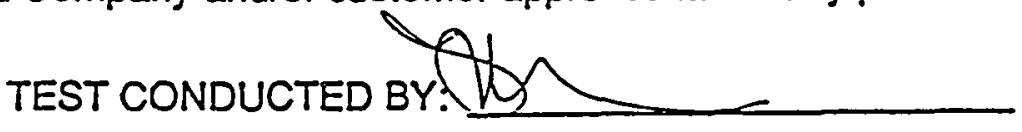
APPROVED BY: -

AMERICAN COLLOID COMPANY

In any correspondence regarding this shipment, please refer to our Order No. 81916 ?. 
WHC-SD-W025-RPT-001, Rev. 0

\section{CERTIFICATE OF ANALYSIS \\ AMERICAN COLLOID COMPANY 1500 W. SHURE DRIVE ARLINGTON HEIGHTS, ILLINOIS 60004}

TO: Delhur Industrie,

DATE $11-2.93$ P.n. Bax 1116

Part Angeles; Wa $93620=0201$ B. B.

Hoy $\mathrm{i} 81993$

ATTN: Rick Huvworth 4nsid........... A shipment of CfS-200 left our UptoN plant in BN446747 on $11-2-93$ as requested on your order number

A sample from this shipment was tested, and gave the following results

TEST

Visc

PV/UPRatio

EL

Free suxil

mointure

-200 mesh

BBL Yield
SPECIFICATION

$15.0 \mathrm{~min}$

3 to 1 max

$15.0 \max$

none

$12.0 \%$ max

$70 / 200 \mathrm{~min}$.

$90 \mathrm{~min}$
RESULTS

27.0

2.5

13.6

28.0

7.8

81.4

92.0

We hereby certity that the test results shown above represent this shipment. Tests were conducted using American Colloid Company and/or customer approved laboratory procedures.

TEST CONDUCTED BY: Z Lu

APPROVED BY: Lev.

AMERICAN COLLOID COMPANY

In any correspondence regarding this shipment, please refer to our Order No. $01916 /$. 
WHC-SD-W025-RPT-001, Rev。 0

\section{APPENDIX D-4}

ADMIX/SOIL LINER

$D-4$ 
WHC-SD-W025-RPT-001, Rev. 0

ADMIXPRECONSTRUCTON TESTING SUMMARY

Project W-025

GCS Project No. 933-1354

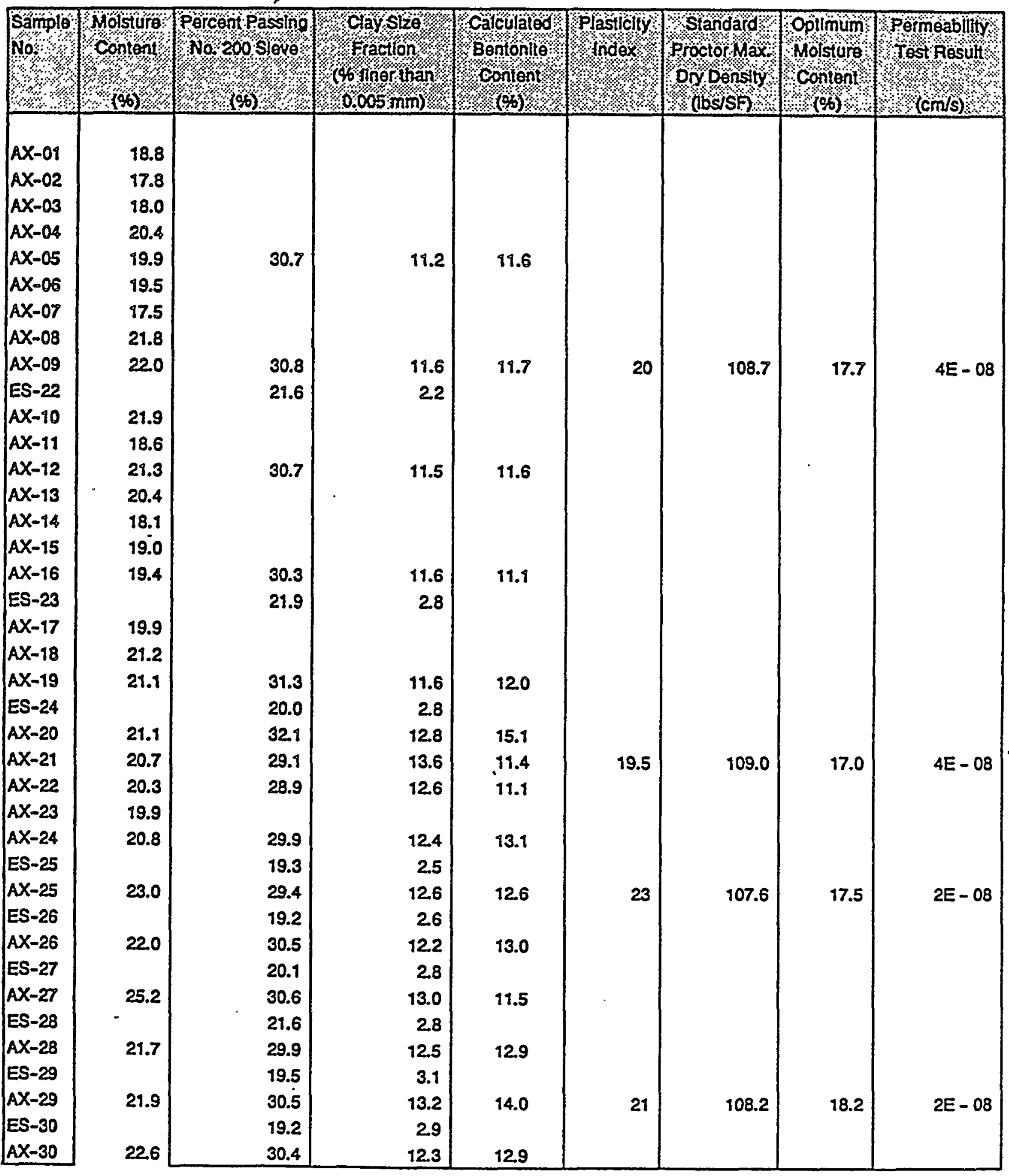


WHC-SD-W025-RPT-001, Rev. 0

ADMIX PRECONSTRUCTION TESTING SUMMARY

\begin{tabular}{|c|c|c|c|c|c|c|c|c|}
\hline Sample & $\begin{array}{l}\text { Moisture } \\
\text { Content: } \\
+ \\
(\%, 6)\end{array}$ & $\begin{array}{l}\text { Percent Passing } \\
\text { No. } 200 \text { Sleve } \\
3 \\
(\%)\end{array}$ & $\begin{array}{c}\text { ctay Slies } \\
\text { Fraction } \\
(\% \text { finer than } \\
0.005 \mathrm{~mm})\end{array}$ & $\begin{array}{l}\text { Calculatod } \\
\text { Bentonite } \\
\text { content } \\
\text { (\%) }\end{array}$ & $\begin{array}{l}\text { Plasticity } \\
\text { frofex } \\
+4\end{array}$ & $\begin{array}{l}\text { Standard } \\
\text { Proctor Max } \\
\text { ory Density } \\
\text { obs/SF) }\end{array}$ & $\begin{array}{l}\text { Oplimum } \\
\text { Motsture } \\
\text { Conterits } \\
(\%) \%\end{array}$ & $\begin{array}{l}\text { Permeability } \\
\text { Test Rosult } \\
\% \text { (cm/s) }\end{array}$ \\
\hline$E S-31$ & & 20.1 & 2.6 & & & & & \\
\hline$A X-31$ & 21.6 & 33.9 & 12.9 & 11.9 & & & & \\
\hline ES-32 & & 25.0 & 2.8 & & & & & \\
\hline$A X-32$ & 22.3 & 30.5 & 12.0 & 13.6 & & & & \\
\hline ES-33 & & 19.6 & 2.7 & & & & & \\
\hline$A X-33$ & 20.7 & 29.7 & 12.4 & 11.8 & & & & \\
\hline ES-34 & & 20.3 & 2.1 & & & & & \\
\hline$A X-34$ & 21.1 & 29.3 & 11.0 & 11.1 & & & & \\
\hline ES-35 & & 20.5 & 1.7 & & & & & \\
\hline$A X-35$ & 19.3 & 35.5 & 12.1 & 13.9 & 33 & 109.0 & 18.0 & $2 E-O B$ \\
\hline ES-36A & & 25.1 & 2.2 & & & & & \\
\hline ES-36 & & 18.5 & 2.2 & & & & & \\
\hline$A X-36$ & 22.6 & 30.8 & 12.2 & 14.0 & & & & \\
\hline ES-37 & & 19.5 & 1.2 & & & & & \\
\hline$A X-37$ & 22 & 34.5 & 11.7 & 13.1 & 23 & 109.0 & 18.0 & $2 E-0 B$ \\
\hline ES-38A & & 24.6 & 0 & & & & & \\
\hline ES-38 & & 34.9 & 0.0 & & & & & \\
\hline$A X-38$ & 21.1 & 28.6 & 11.4 & 11.9 & & & & \\
\hline ES-39 & & 19.0 & 2.4 & & & & & \\
\hline$A X-39$ & 21.6 & 34.6 & 9.2 & 11.3 & & & & \\
\hline$E S-40$ & & 26.3 & 0.0 & & & & & \\
\hline
\end{tabular}

Notes:

A Sample No. with a prefix of AX denotes an admix sample obtained during the production of the admix. Permeability tests performed on the AX samples were laboratory recompacted permeability tests.

A Sample No. with a prefix of ES indicates an eollan sand sample obtained just prior to mixing with bentonite.

A Sample No. with a suffix of $A$ indicates a retest. 
WHC-SD-W025-RPT-001, Rev, 0

Pago:

ADMIX PRECONSTRUCTION TESTING SUMMARY

Project W-025

GCS Project No. 933-1354

EXAMPLE CALCULATION OF BENTONITE CONTENT:

PROBLEM - Determine the percentages of the sand and bentonite components of the admixture based on dry welghts of the materials.

KNOWN - $\quad 30.5 \%$ of the admix (A) passes the \#200 sleve.

$20.2 \%$ of the sand (S) passes the \#200 sieve.

ASSUME - $\quad 100.0 \%$ of the bentonite (B) passes the $\# 200$ sieve.

Based on the \%'s passing the \#200 sieve for each of the components (A, B, \& S), the \%'s of sand and bentonite comprising the admix can be determined as follows:

(B $\times \%$ passing $\# 200)+(S \times \%$ passing $\# 200)=(A \times \%$ passing $\# 200)$

$(B \times 100.0)+(S \times 20.2)=(A \times 30.5)$ or $100.0 B+20.2 S=30.5 A$

$A=100 \%$ of the admix; and bentonite $(B) \rightarrow$ sand $(S)=100 \%$ of the admix, so $B+S=100 ;$ and $S=100-B$

Therefore: $100.0 B+[20.2 \times(100-B)]=100 \times 30.5$

$100.0 B+2020-20.2 B=3050$

Solving the equation for $B$, the bentonite content, $B=12.91$.

The bentonite comprises $12.91 \%$ of the admix. 
WHC-SD-W025-RPT-001, Rev. 0

SOIL LINER LABORATORY TESTING SUMMARY

Project W-025

GCS Project No. 933 1354

\begin{tabular}{|c|c|c|c|c|}
\hline Sample & Porcent Passing & 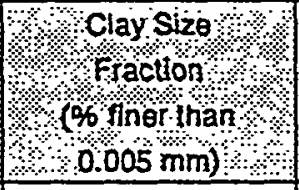 & \% & $\begin{array}{l}\text { Permeabllty } \\
\text { Test hosull } \\
\text { (cm/s) } \\
\text { (c) }\end{array}$ \\
\hline$S L-03$ & 32.3 & 12.6 & $N / A$ & N/A \\
\hline$S L-12$ & 30.9 & 11.5 & 25 & $1 E-08$ \\
\hline SL-55 & 32.5 & 13.1 & 22 & $5 E-08$ \\
\hline$S L-86$ & 31.3 & 11.5 & 18 & $1 E-06$ \\
\hline$S L-86 A$ & 33.8 & 11.3 & 24 & $1 E-08$ \\
\hline SL-129 & 31.2 & 11.9 & 21 & $3 E-08$ \\
\hline$S L-136$ & 33.6 & 12.5 & 32 & $3 E-08$ \\
\hline$S L-153$ & 33.6 & 11.6 & 23 & $2 E-08$ \\
\hline$S L-154$ & 32.3 & 11.0 & 25 & $8 E-09$ \\
\hline$S L-160$ & 34.5 & 11.3 & 29 & $3 E-08$ \\
\hline$S L-161$ & 33.5 & 10.4 & 27 & $3 E-08$ \\
\hline$S L-162$ & 30.8 & 10.9 & 28 & $5 E-09$ \\
\hline$P L-15$ & $N / A$ & N/A & $N / A$ & $1 E-08$ \\
\hline
\end{tabular}

Notes:

A Sample No. with a prefix of SL indicates a sample from the compacted secondary soil liner.

Permeability tests were performed on relatively undisturbed Shelby tube samples of this material.

A Sample No. with a prefix of PL indicates a sample from the compacted primary soil liner.

Permeabllity test was performed on a relatively undisturbed Shelby tube sample of this material.

A Sample No. with a suffix of $A$ indicates a retest. 
WHC-SD-W025-RPT-001, Rev。 0

APPENDIX D-5

GRANULAR DRAINAGE MATERIALS

D-5 
WHC-SD-W025-RPT-001, Rev. 0

경

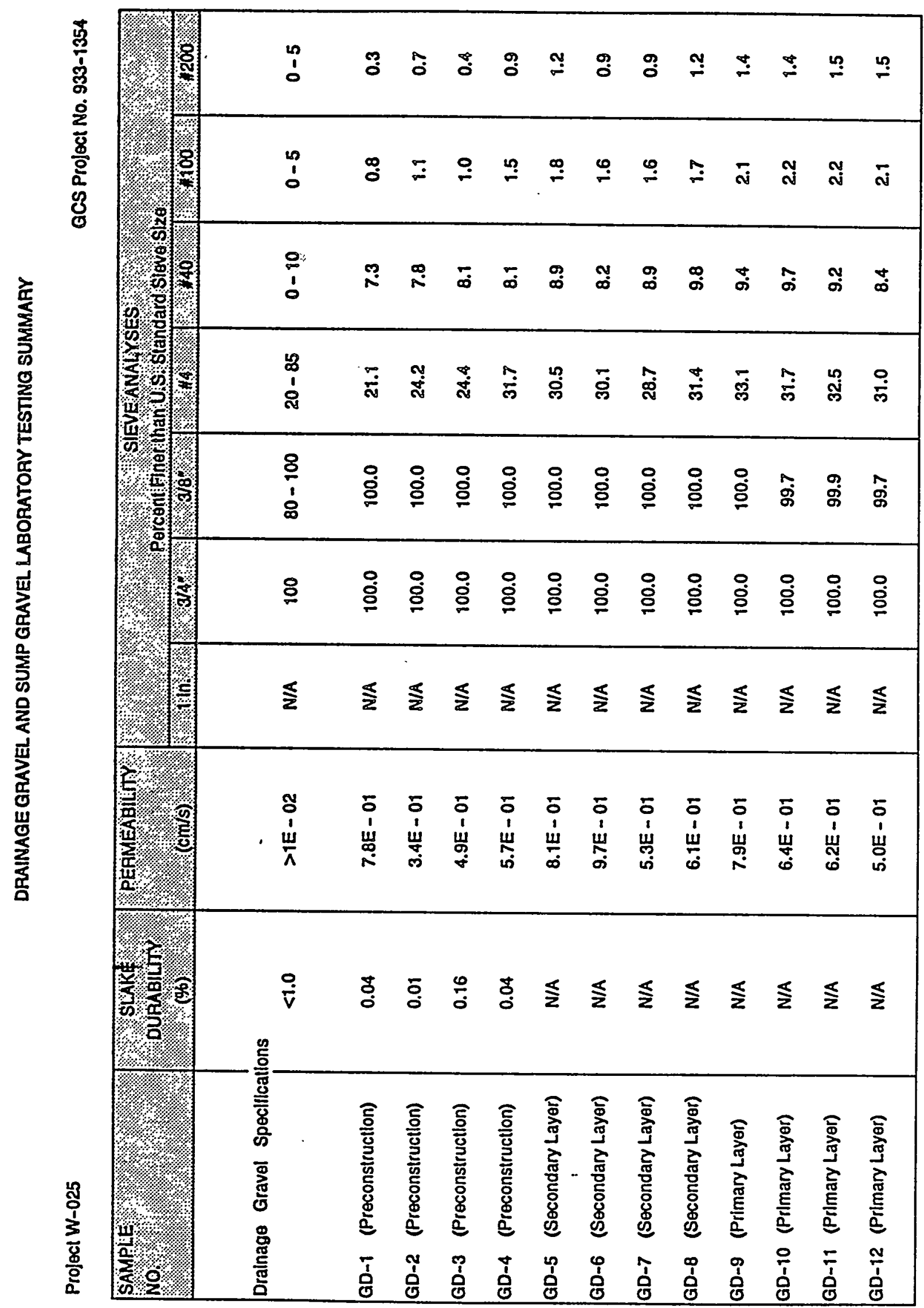


WHC-SD-W025-RPT-001, Rev. 0

$\sim$
$\stackrel{8}{\square}$
0
0

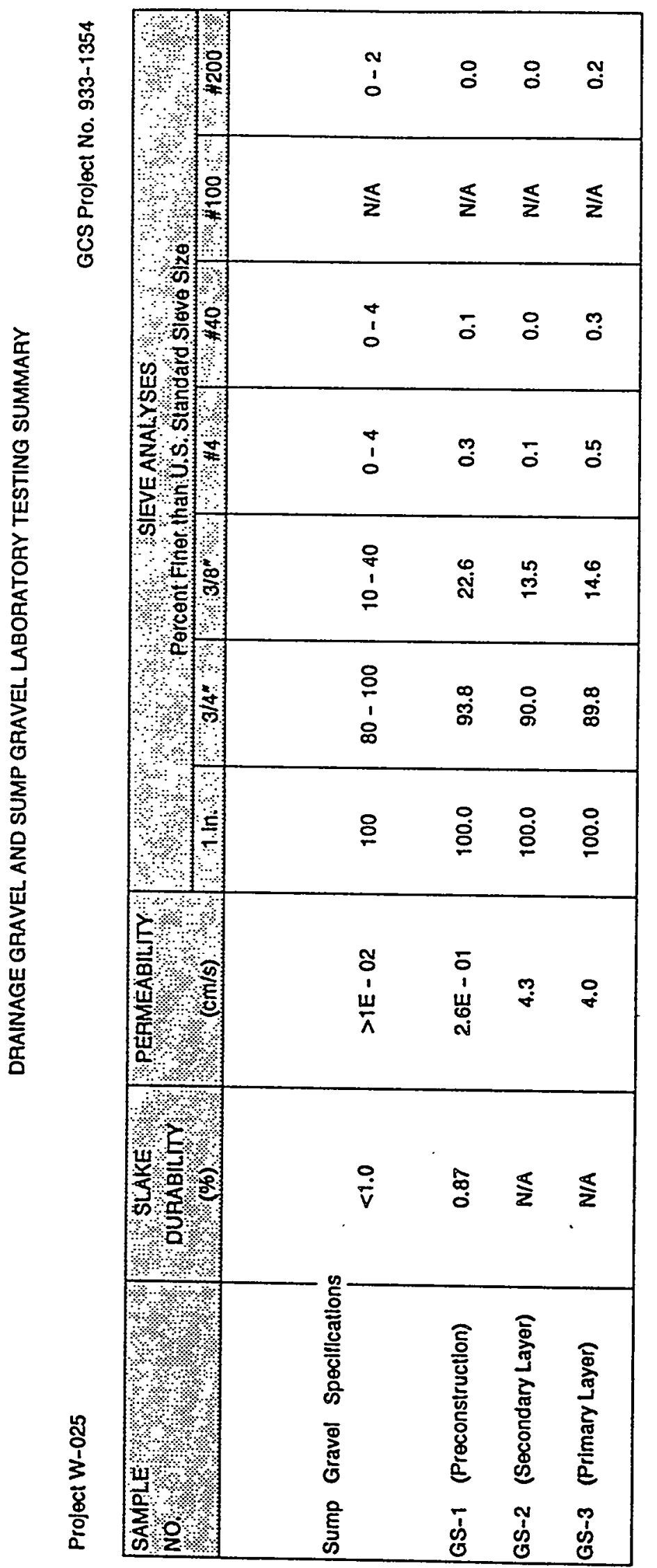

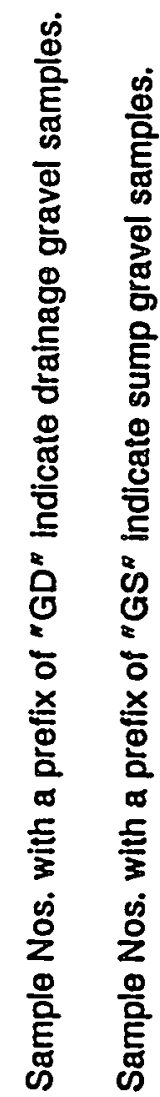


WHC-SD-W025-RPT-001, Rev。0

APPENDIX D-6

TOP COURSE

$D-6$ 
WHC-SD-W025-RPT-001, Rev. 0

\begin{tabular}{|c|c|c|c|c|c|c|c|}
\hline \multirow{7}{*}{ 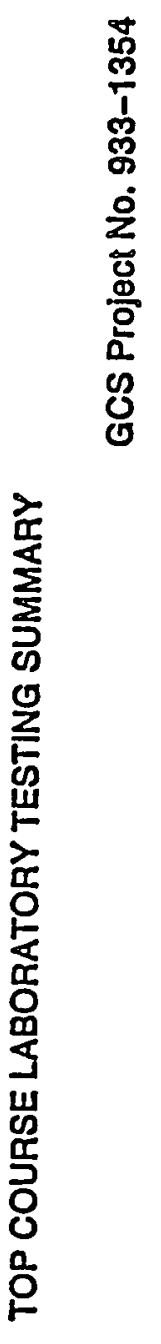 } & 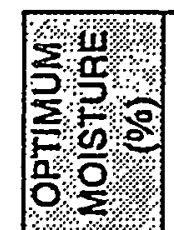 & 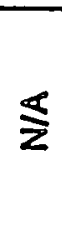 & $\bar{\infty}$ & $\frac{\varsigma}{z}$ & $\hat{\mathbf{z}}$ & $\underset{\infty}{\infty}$ & $\bar{\infty}^{-}$ \\
\hline & 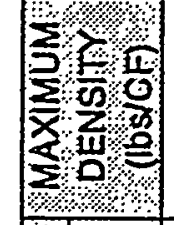 & $\frac{\widehat{s}}{\mathrm{z}}$ & 占 & $\S$ & $\S$ & 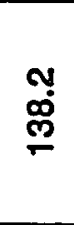 & $\stackrel{\infty}{\circ}$ \\
\hline & 的 & o & $\overbrace{i \infty}^{\infty}$ & $\stackrel{\leftrightarrow}{*}$ & $\underset{\sigma 0}{*}$ & 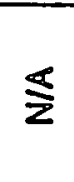 & $\frac{\mathbf{s}}{\mathbf{z}}$ \\
\hline & $\mid \begin{array}{ll}\mid \begin{array}{l}5 \\
\frac{1}{2}\end{array} \\
\frac{1}{2}\end{array}$ & \begin{tabular}{l}
\multirow{+}{*}{} \\
1 \\
$\infty$
\end{tabular} & ઼ָ & 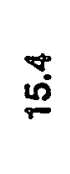 & $\stackrel{\circ}{\circ}$ & $\frac{\varsigma}{z}$ & 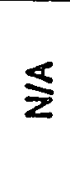 \\
\hline & 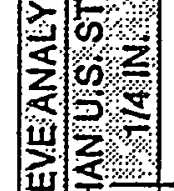 & $\begin{array}{l}2 \\
1 \\
1 \\
20\end{array}$ & $\begin{array}{l}0 \\
\stackrel{0}{\circ} \\
\stackrel{\leftrightarrow}{\circ}\end{array}$ & ஜூ & 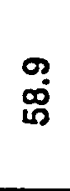 & 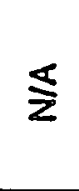 & 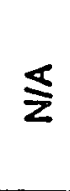 \\
\hline & का & 오 & 용 & : & 용 & 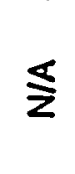 & $\widehat{s}$ \\
\hline & 네 & ঃ & 용 & 용 & ஜ̊ & $\lesssim$ & 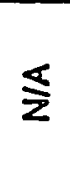 \\
\hline \multirow{2}{*}{ 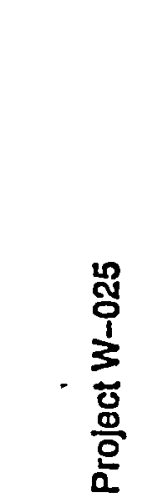 } & 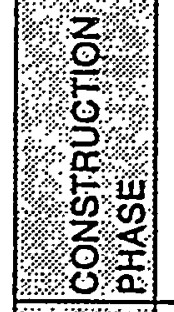 & 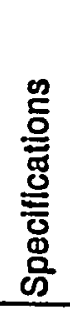 & 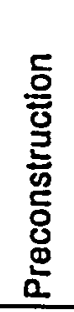 & 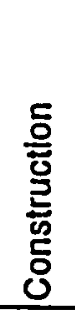 & 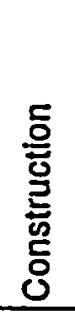 & 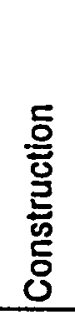 & 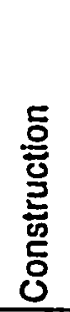 \\
\hline & (4) & & $\begin{array}{l}\overline{1} \\
0 \\
0\end{array}$ & $\begin{array}{l}\text { o } \\
\vdots \\
\vdots\end{array}$ & $\begin{array}{l}0 \\
1 \\
0 \\
1\end{array}$ & \begin{tabular}{l}
\multirow{D}{1}{} \\
0 \\
1
\end{tabular} & $\begin{array}{l}00 \\
1 \\
0\end{array}$ \\
\hline
\end{tabular}


WHC-SD-W025-RPT-001, Rev。 0

APPENDLX D-7

OPERATIONS LAYER

D-7 
WHC-SD-W025-RPT-001, Rev. 0

Page-t

\section{OPERATIONS LAYER \\ PRECONSTRUCTION LABORATORY TESTING SUMMARY}

Project W-025

GCS Project No. 933-1354

\begin{tabular}{|c|c|c|c|c|c|}
\hline अ SAMPLE & $\begin{array}{l}\text { MOISTURE } \\
\text { CONTENT } \\
\%(\%)\end{array}$ & $\begin{array}{l}\text { OOFINER } \\
\% \\
\% \text { THAN" } \\
\text { SIEVE }\end{array}$ & $\begin{array}{l}\text { OOFFINER } \\
\text { THAN:\$200 } \\
\text { FIEVE }\end{array}$ & $\begin{array}{l}\% \text { CEAY SIZE } \\
\% \text { FRACTION } \\
\%(005 \mathrm{~mm})\end{array}$ & $\begin{array}{l}\text { औै } \\
\text { CLASSIFICATION } \\
\text { CLSS }\end{array}$ \\
\hline ES-01 & 11.3 & 100.0 & 22.1 & 3.2 & $S M$ \\
\hline ES-02 & 3.5 & 100.0 & 19.8 & 2.0 & $S M$ \\
\hline ES-03 & 5.9 & 100.0 & 17.2 & 1.7 & $S M$ \\
\hline ES-04 & 3.5 & 100.0 & 23.3 & 1.1 & $S M$ \\
\hline ES-05 & 6.3 & 100.0 & 25.2 . & 2.1 & $S M$ \\
\hline ES-06 & 4.3 & 100.0 & 18.1 & 21 & $S M$ \\
\hline ES-07 & 3.6 & 100.0 & 28.3 & 1.1 & $S M$ \\
\hline ES-08 & 4.7 & 100.0 & 21.4 & 1.4 & $S M$ \\
\hline ES-09 & 4.9 & 100.0 & 19.1 & 1.9 & $S M$ \\
\hline ES-10 & 5.0 & 100.0 & 19.4 & 0.7 & $S M$ \\
\hline ES-11 & 3.6 & 100.0 & 21.8 & 1.7 & $S M$ \\
\hline$E S-12$ & 4.4 & 100.0 & 21.5 & 1.5 & SM \\
\hline ES-13 & 4.8 & 100.0 & 19.0 & 1.1 & SM \\
\hline ES-14 & 4.7 & 100.0 & 19.3 & 0.7 & $S M$ \\
\hline ES-15 & 4.3 & 100.0 & 17.4 & 0.6 & $S M$ \\
\hline ES-16 & 7.3 & $100.0^{\circ}$ & 20.4 & 0 & $S M$ \\
\hline ES-17 & 3.6 & 100.0 & 21.3 & 1.1 & $S M$ \\
\hline ES-18 & 3.2 & 100.0 & 22.0 & 0.7 & $S M$ \\
\hline ES-19 & 4.1 & 100.0 & 20.7 & 0.2 & $S M$ \\
\hline ES-20 & 5.9 & 100.0 & 19.8 & 0 & $S M$ \\
\hline ES-21 & 4.0 & 100.0 & 20.4 & 0 & $S M$ \\
\hline GF-01 & 2.4 & 90.4 & 12.7 & 0.4 & $S M$ \\
\hline GF-32 & 6.6 & 95.4 & 15.5 & 1.5 & $S M$ \\
\hline
\end{tabular}


WHC-SD-W025-RPT-001, Rev. 0

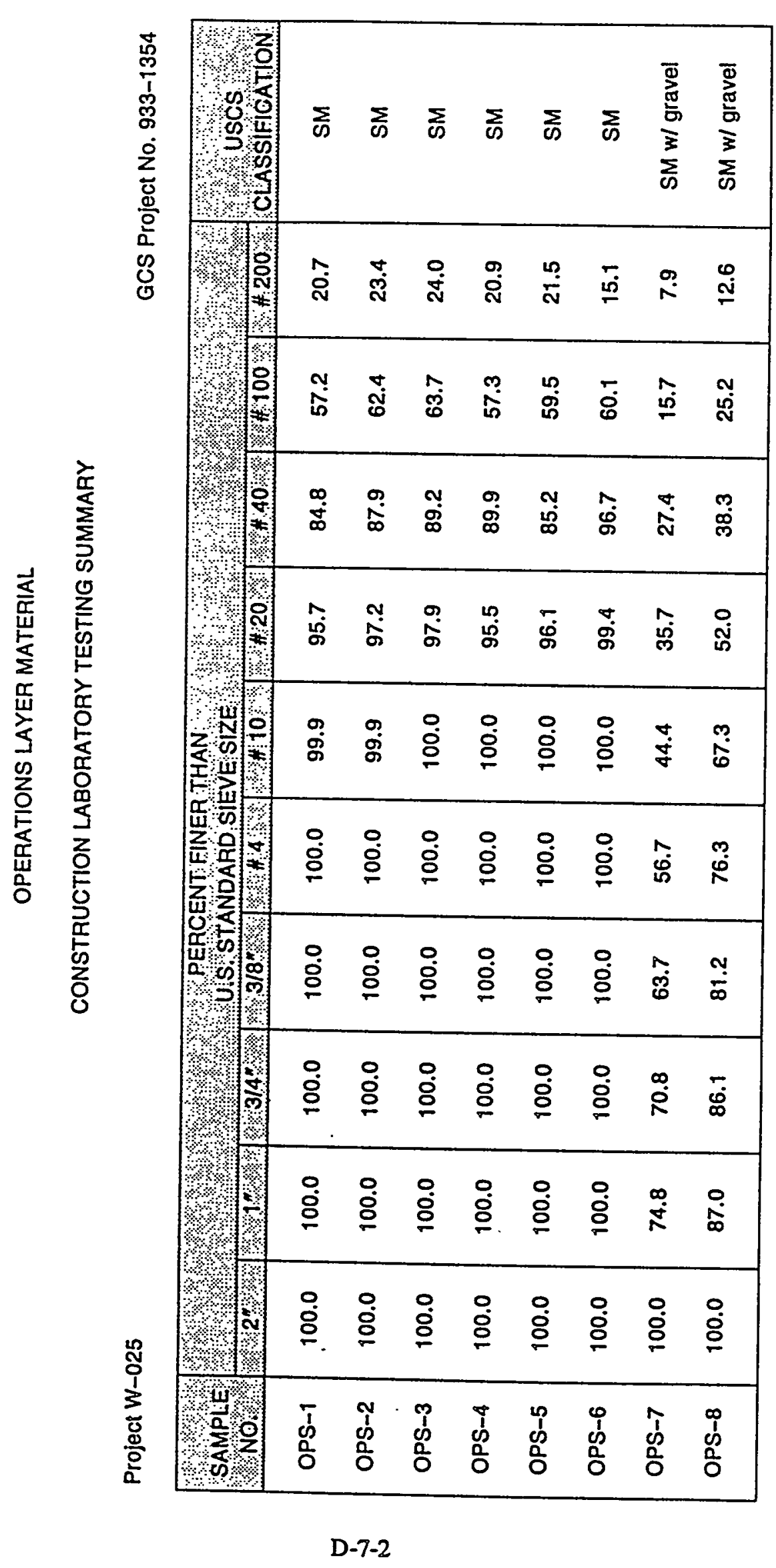


WHC-SD-H025-RPT-001。Rev。0

APPENDIX E

GEOMEMBRANE CONFORMANCE TESTS 
WHC-SD-W025-RPT-001, Rev. 0

APPENDIX E-1

SURVELLANCE INSPECTION REPORTS

$E-1$ 
Inspected Activity or Test: OBSERVE MANUFACTURING DF HDPE GEONIEMBRANE AFT SLTAMERICA (HOUSTON)

Job Number: $933-1354$

Personnel Contacted: BOB SCHULER (LEAD NLAN), RICARDO IBABRA (SELOND) \& NATHAN IVY (LAB TECH.)

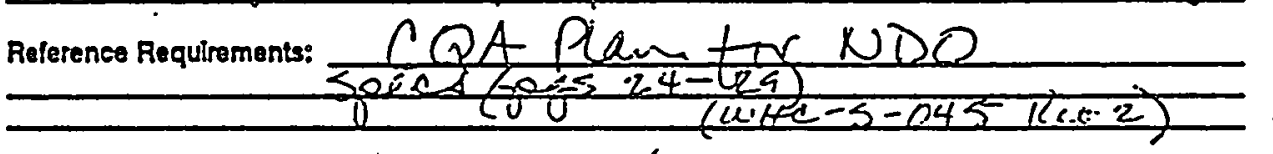
Insp. Date: Qc+.16,1993start Time: 2:40 pm End Time: 6:25 pr Equipment Conditioncelibration status: Fewler 5 A 175 Qutside Mlicvometer - Cal,bretion due mireh, 94

Observation and Comments (Use Extra Shoets as Required):

\begin{tabular}{l}
\hline \\
\hline \\
\hline NCIR Refarenco (f any): \\
NIONE
\end{tabular}

Action Items Requining Correction (Uso Extra Sheots as Required):

Nore

\begin{tabular}{l}
\hline Inspector: Durwood H. Gregory \\
Closed 8y QA Manager: Date: $\frac{0 c t .18,1993}{10-21-93}$ \\
Action ltem Cosure Data: \\
Comments: \\
\hline
\end{tabular}


DOE/NDO CQA/WA

IN-PI_ANT CQA REPORT

Durwood H. Gregory of Golder Associates arrived on site at the SLT, America, Inc. plant (Hardy Rd.) at 1445 hrs. on Saturday, Oct. 16, 1993 and met with Bob Schuler (lead man) and Ricardo Ibarra (second) at $2: 48 \mathrm{pm}$. The scope of work was to observe manufacture of $60 \mathrm{mil}$ HDPE geomembrane for project (DOE/NDO $\mathrm{CQA} / \mathrm{WA})$, Project \# 933-1354 at the Hanford Nuclear Reservation in Eastern Washington, SIT customer \# 8491 for Hanford/ Geopacific (contractor). At the time of arrival, SLT was just completing roll \#AC-7679 for another project, and was projecting to start roll \#AC-7680 at 3:00 for this project. Mr. Gregory walked to lab at 2:55 pm, met with Nathan Ivy, (laboratory technician), and discussed project, (only 3 personnel in-plant at this time). At $3: 00$, the end cut of roll -7679 and start of -7680 was observed.

A slight ribbing finish was observed on the bottom side of geomembrane, a micrometer reading at this area (approximately 3 it wide at approximately the middle of roll (width)), even at the "valley" still read a $64 \mathrm{mil}$ reading. Lead man (Bob) had already observed said incongruity, and adjusted the heat to rectify the situation.

Micrometer readings were taken along trail edge of roll -7679 . The low of $62.5 \mathrm{mil}$, and the average was $63 \mathrm{mil}$ thickness.

At 3:12, Mr. Gregory walked around "machine", observed process, machinery and hopper, and noticed regrind through observation window and around hopper distributor. He brought this to the attention of the lead-man, who explained that this regrind was from edging they trim off each side ( 1.25 in. wide) which was sent through a regrinder and, then sent in measured portions back into the hopper. It appeared that the regrind constituted no more than $1 \%$ of raw resin, but no documentation was available. At $3: 20$, the ribbing was observed to have increased in width, (not intensity). The lead-man told of turning down the temperature.

Mr. Gregory observed the cut of roll -7680 , at 3:35 and start of roll-7681; The trailing edge of roll -7680 was measured using a micrometer to be $62.5 \mathrm{mil} \mathrm{min}$. and $63.5 \mathrm{mil}$ avg. The roll length was 480 ft and the sheet width was $23.5 \mathrm{ft}$ wide.

The remainder of the manufacturing process for roll -7681 was observed and the ribbing had completely disappeared by end of 7681 , $(4: 10)$. At $4: 15$, coupons and dog-bones were observed cut from the sample from roll \#AC-7681. Testing procedures and results observed were: impact, tensile's, tear resistance, puncture and dimensional stability.

page 2 of 3 
At the control panel, the computer readings were observed:

time: Avg. Max. Min. Std. Dev. Stas
$4: 33$
63.59
65.92
61.26
.53
24
$4: 50$
63.83
65.32
61.52
.55
5
(Scans = computer "micrometer" scanning across sheet.

Mr. Gregory walked around "machine.", observed process, machinery and hopper, observe cut. of AC-7682 at $4: 47$ and start of -7683 . To $l a b$ at 5:00, take micrometer readings, on trail -7682, low 62.5, average $63.5 \mathrm{mil}$ thick, prepare paper work, observe resting.

Mr. Gregory returned to the manufacturing line at $5: 15$, observe cut of AC-7683 at 5:23. The trailing edge of roll -7683 was measured to be $63.5 \mathrm{~min}$. and high of 64 , with an average reading of $64 \mathrm{mil}$ thick.

At control panel, readings:

time:

Avg. Max. Min. Std. Dev. Sas

$\begin{array}{llllll}5: 25 & 63.98 & 65.22 & 62.20 & .45 & 4 \\ 5: 47 & 63.61 & 65.22 & 61.41 & .51 & 30 \\ 5: 52 & 63.59 & 65.22 & 61.41 & .50 & 36\end{array}$

The trailing edge cut was observed at 5:57 (1757 hrs) of roll \#AC-7684 (the fifth (5th) and final roll of this project today). Mr. Gregory followed sample from roll \# AC-7684 to the lab at 6:03, micrometer readings showed a low of $63 \mathrm{mil}$, a high of 64 mil, with an average of $63.5 \mathrm{mil}$ thick. Observed partial testing of this roll.

Mr. Gregory received the raw resin letter certificate. The RolI Test Data Reports (certifications) will be available Monday (Oct. 18), along with a list of previously accepted rolls. *

Off-site at $6: 25 \mathrm{pm}$ to office.

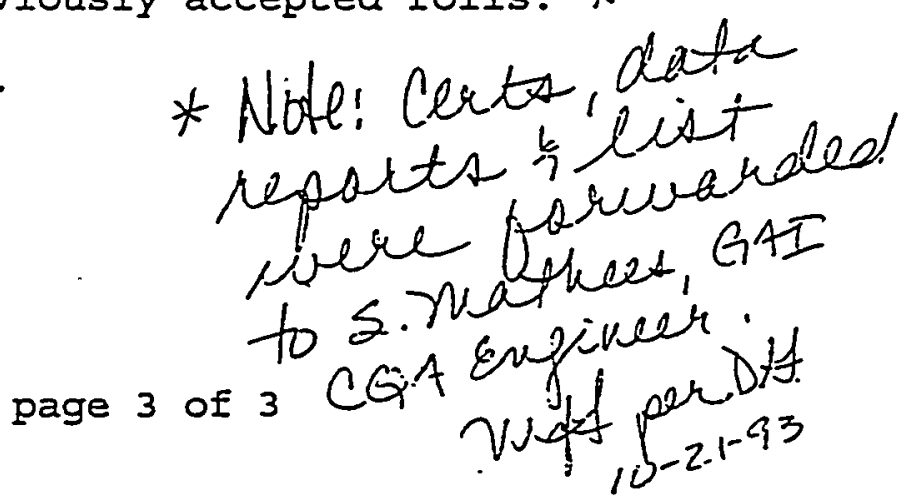




\section{SURVEILLANCE INSPECTION REPORT}

Page 1 of 3

No.: 057

Inspected Activity or Test: OBSERVE MAMUPACTURING OF

HDPE GEONEMBRANE HT SLT, AMETCIEA (HOLSTON)

Job Number:

933.1354

Personnel Contacted: Havold Hendersun (Plant monager)

RAY Johnson (LEAD), Jeff Debrows ki (second) \& Mélody ADaws

Reference Requirements: $\frac{\text { CQA Plans for NDQ }}{1 / \text { specipiestina }(2024-29)}$

(LAB inaNAGER) (Wtes-04 Rar2)

Insp. Date: 0ct, 19,1993 start Time: 10:35 am End Time: 2:50 pu

Equipment Condition/Calibration Status: Fowler 5 A175 Qutside Microweter - Calibeation due marchig.

Observation and Comments (Use Extra Sheets as Required):

SEE SHEES 2 हो 3 of 3

NCIR Reference (if any):

None

Action Items Requiring Correction (Use Extra Sheets as. Required): SEE REPORT L follow, Pages $2 \xi 3$ of 3 , tegording Fecyeled Polymat.

inspector: Durwood H. Gregory

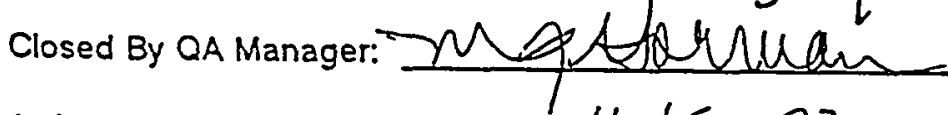

Date: Oct. 19,1993

Action llem Closure Date:

$11-15-93$

Comments: Copr os letter dated Oetrber 22,1993

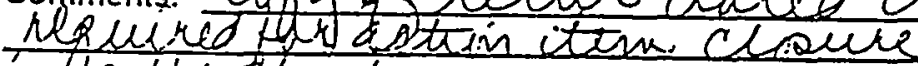

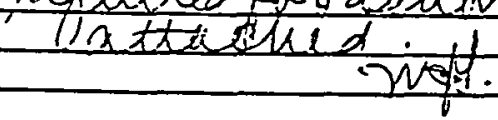

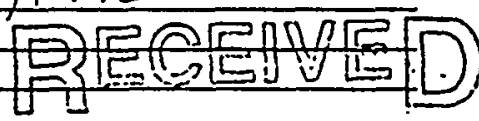

OCT 201993 
DOE/NDO CQA/WA

IN-PLANT CQA REPORT

Durwood H. Gregory of Golder Associates arrived on site at the SLT, America, Inc. plant (Hardy Rd.) at 1035 hrs. on Tuesday, Oct. 19, 1993 and met with Harold Henderson (plant manager), Ray Johnson (lead), and Jeff Dobrowski (second) at 2:48 pm. The scope of work was to observe manufacture of $80 \mathrm{mil}$ HDPE geomembrane for project (DOE/NDO CQA/WA), Project \# 933-1354 at the Hanford Nuclear Reservation in Eastern Washington, SLT customer \# 8491 for Hanford/ Geopacific (contractor). At the time of arrival, SLT was just completing roll \# AC-7788 for another project, and was projecting to start roll \# AC-7789 shortly for this project. Mr. Gregory walked to lab at 10:40 pm, met with Melody Adams, (laboratory manager), and discussed scope. At 10:55, the end cut of roll -7788 and start of -7789 was observed.

Micrometer readings were taken along trail edge of roll -7788 . The low of $82 \mathrm{mil}$, and the average was $82.5 \mathrm{mil}$ thickness.

At 11:02, Mr. Gregory walked around "machine", observed process, machinery and hopper, and noticed an exorbitant amount of regrind being dispensed into the hopper distributor. He observed dispensing for four (4) hopper loads, and determined this to be above the "Iess than $2 \%$ " as stated in the specifications. He brought this to the attention of the plant manager, asking $M r$. Henderson how much regrind was being added, stating the possible conflict with the specifications. Mr. Henderson said they can shut the regrind off, however, this would take two (2) full rolls to clean out, and would re-start for this project in an hour or so. Mr. Henderson also stated that the edging referenced in Surveillance Inspection Report (SIR) No. 056 constituted only 1\% of the volume.

Offsite at $11: 20 \mathrm{pm}$, to go to the office to call Mr. Scott Matthees (Golder - Project CQA Engineer and Receiving Inspector), and Ms. Joey Gorman (Golder - EA Vianajer fust $10-21.93$

Durwood H. Gregory of Golder Associates arrived on site at the SLT, America, Inc. plant (Hardy Rd.) at 1315 hrs. on Tuesday, Oct. 19, 1993 and met with Ray Johnson (lead).

Mr. Johnson informed Mr. Gregory that SLT was assigning this roll (AC-7793) to this project along with the next roll that Mr. Gregory would observe. Mr. Gregory observed the trail cutting of roll -7793, at $1: 46$ and start of roll -7794; The trailing edge of roll -7793 was measured using a micrometer to be $82 \mathrm{mil}$ min. and $83 \mathrm{mil}$ avg. The roll. length was $360 \mathrm{ft}$ and the sheet width was 23.5 ft wide.

$$
\text { page } 2 \text { - } 3
$$


Mr. Gregory spoke with Mr. Henderson regarding documentation assuring the previous rolls (60mil) accepted for this project do meet the specifications, specifically the fifth bullet on page 26, of WHC-SD-W025-PLN-001, Rev. 3, dated May 21, 1993, stating recycled polymer not exceed $2 \%$ by weight. Mr. Henderson retrieved the "daily production report" (SIT) and explained to Mr. Gregory that the reports for those two days showed zero recycled polymer (regrind) was used. Mr. Henderson also stated that Mr. Bill walling (Director of Engineering Services) will certify/ensure in a letter this fact. Mr. Gregory should be able to receive this letter on Wednesday, Oct. 20, 1993.

At the control panel, the computer readings were observed:

time: Avg. Max. Min. Std. Dev. Sts

$\begin{array}{llllll}1355 & 80.85 & 82.71 & 79.30 & .50 & 15 \\ 1409 & 80.82 & 82.71 & 79.24 & .44 & 32 \\ 1415 & 80.77 & 82.71 & 77.63 & .49 & 39\end{array}$

(Scans = computer "micrometer" scanning across sheet .

The remainder of the manufacturing process for roll -7794 was observed and was completed without action items requiring correction. At 2:21, roll AC-7794 was sampled for SLT laboratory testing and quality assurance. Mr. Gregory tracked this sample to the laboratory and observed the following tests: impact, tensile's, and tear resistance.

Mr. Gregory received the Roll Test Data Report forms from SLT, including the raw resin letter certificate. $*$

Off-site at 2:50 pm to office.

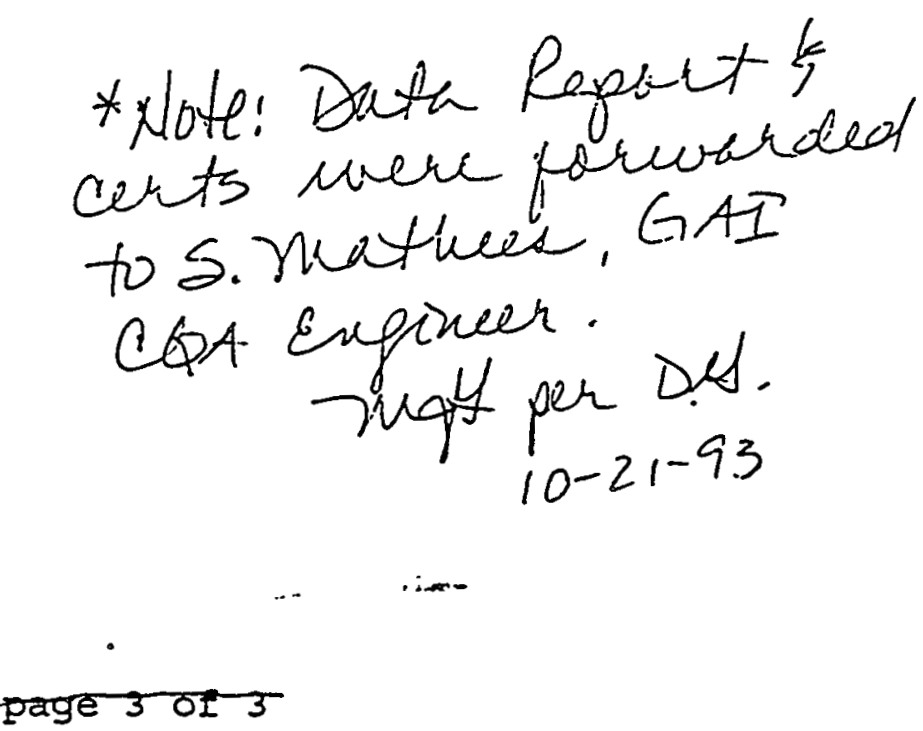


WHC-SD-W025-RPT-001, Rev. 0

L2].

NORTH AMERICA, INC.

A serocest oc

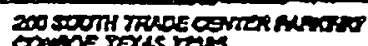

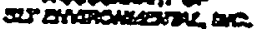

connos pous rias TE: res

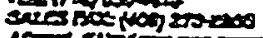

actoser. 22, 1993

Geopacific Iiaing Irea

1300 Dexter ATE. N. Snive 250

Sectile, Fashington 98109

Atres

E. Hahosere

BE:

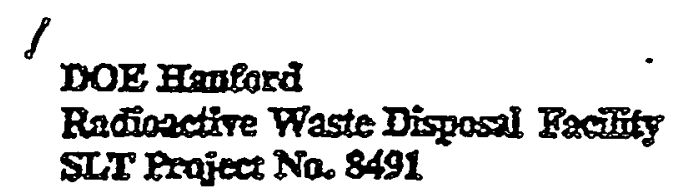

\section{Certifiction}

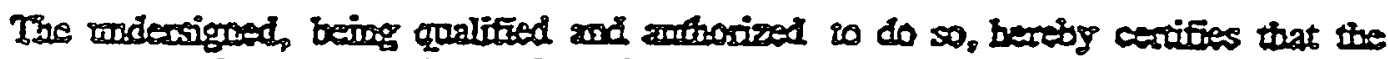

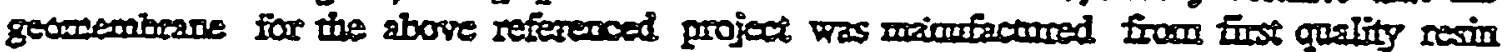
which contains no reclsined polymer and les thas $2 \%$ of recycled trim.

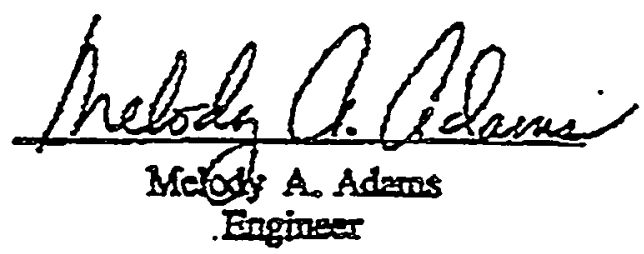

MAN/m

For emironmentel lening solutions... the wordd comes to SUT." 
Page 1 of 9

No.: 058

Inspected Activity or Test: Obsevve Texturing or "Friction Fleveing" of HDPE Geomembrane at the SLT Amence Plaut (convoe, To)

Job Number: Q $33-1354$

Personnel Contacted: Don ( lewellyn (mGre of manyf), Steve Westerman (morn. Crew Leader), And Chuch Bervossks (Atter Csewlodor) Reference Requirements: CQH Plon fom NDO Aftzched Specifications (6poses) (Sheets 4-9.f9) Insp. Date: $0 \mathrm{ct} .22,93$ start Time: $1345 \mathrm{hrs}$ End Time: $1740 \mathrm{hvs}$ Equipment Condition/Calibration Status: None

Observation and Comments (Use Extra Sheets as Required): See sheets $2 \xi 309$

NCIR Reference (if any): Nowe

Action Items Requiring Correction (Use Extra Sheets as Required):

Nowe

Inspector: Durwad dl Gregory Closed By QA Manager: Mgefoluha. Date: Oct.25,1993 Action Item Closure Date: ne

Comments: 
WHC-SD-W025-RPT-001, Rev. 0 ,

DOE/NDO CQA/WA

IN-PLANT CQA REPORT SIR NO. 058

Durwood H. Gregory of Golder Associates arrived on site at the Conroe (North) plant of SLT, America, Inc. at 1330 hrs. on Friday, Oct. 22, 1993 and met with Dan Llewellyn (manager of manufacturing), and Steve Westerman (Crew Leader) at 1:45 pm. The scope of work was to observe the texturing or "Friction Flex"ing of $60+$ mil HDPE geomembrane previously manufactured at the south plant (Hardy Rd.). This texturing of geomembrane is for project (DOE/NDO CQA/WA), Project \# 933-1354 at the Hanford Nuclear Reservation in Eastern Washington, SLT customer \# 8491 for Hanford/ Geopacific (contractor). At the time of arrival, SIT was spraying roll \# AC-6910, which is to be the first roll for this project.

At 1:55, Mr. Gregory began observing the machinery and process, to be described below.

The rolls AC-6904 through AC-6917, were shipped to the Conroe plant from the Hardy plant to be "Flexed", as per FAX from Melody Adams (Lab Manager) on October, 18, 1993. Mr. Gregory was informed that; when produced, a roll of geomembrane that is to be Flexed, is run twice as long and assigned two roll numbers. This is to make the Flexing process run more expediently by cutting the "changing of rolls" time in half. Mr. Gregory observed the following rolis in the yard... AC-6904/6905, 6906/6907, $6908 / 6909,6912 / 6913$, and 6916/6917. One roll was mislabeled, and found to be AC-6914/6915.

Based on a telephone conversation with Claudia Blackman, (production), Mr. Gregory was informed that there will be twentynine (29) rolls Friction-Flexed and three (3) rolls of smooth $(60+\mathrm{mil})$. It was determined that all rolls were not on site at the time.

The process for Friction Flexing consists of un-rolling the premanufactured double length rolls of geomembrane through a set of rollers and between two spraying devices, assigned to spraying HDPE specs or Flex onto the geomembrane. The two sets of sprayers (one on each side of geomembräne) are affixed to carriages set on tracks that are trolled back and forth spraying the Flex, as the geomembrane is pulled through.

Shift" change occurred at 2:30 pm, bringing on Chuck Bernoski as Crew Leader. 
WHC-SD-W025-RPT-001, Rev. 0

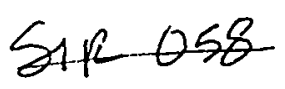

The "Flex"ing is a slow process, taking 2.7 ft per minute, with rolis running 475 ft. Iong, taking approximately 3 hours per roll.

At $3: 20 \mathrm{pm}$, Mr. Gregory was informed of a clogged nozzle; SLT would have to shut down and cool the line to clean or blow-out the nozzles, then heat back up. This procedure should take approximately $I$ hour and is a standard problem that typically happens with very high humidity. At 4:35 pm the process was back on line. Mr. Gregory observed the area where "spitting" or "splattering" occurred, and could not discern any significant difference.

Mr. Gregory left production line at 5:30, and was away from plant at $5: 40 \mathrm{pm}$. 
WHC-SD-W025-RPT-001, Rev. 0

\section{SPECS}

Sar o58 4069

WHG.SOA5 Rev. 2

\section{I6 GEOCOMPOSITE}

Geocomposite consisis of geonet with a layer of geotextile themally bonded to each side. Gecomposite is used on the side slopes of the landfill above the primary and sceondary geomembrane.

\subsection{SUBMIITALS}

So Manufactusers of the various geosynthetic materinks shill subrrit all required . quality assurance and quality control documentation as described in the following sections and the CQA Plan.

B. The Contractor shall submit samples of all proposed geosynthetie materials for "fingerprinting" analyses and conformance testing to defermine acceptability. Sampling shall be preformed in aceordance with the following sections of this specification and the CQA Plan Conformance testing will be performed by the CQA Engineer. Acceptability shall be determined by the COTR. Unacceptable materials shall be temoved and disposed of at Contractor? expense.

C. The Geosynthetios Installer shall submit a quality assurance program for installation of geosynthetic materials as well as a list of proposed geomembrane seaming personnel and their expertence recordi.

D. All submittals shall corform to the requirements of Section 01300

PART 2: PRODUCTS

\section{GEOMEMBRANE LINER}

\section{A. General}

Three types of geomembrane are shown on the Drawings: TYPE I geomembrane will be used for both primary and secondary FML on the landifll side slopes and access ramp. TYPE II geomembrane will be used for both primary and secondary FML on the base of the landfill. TYPE III geomembrene will be uned to line the truck usloading ares at the entrance to the landill access ramp and to line the containment basin under the leachate storage tark. The three TYPES are defined ax follows:

TYPE I - 60 mil thickness, textured (roughened) both sides

TYPE II. - 60 mil thickness, smooth both sides

TYPE III - $\quad 80 \mathrm{mil}$ thickness, smooth both sides 


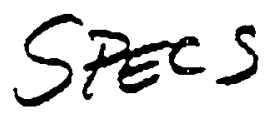

WHC.SOL5 Rev. 2

\begin{abstract}
All geomembrane, regardless of type, shull consist of unreinforced high density polyethylene (IDOPE) sheet.

The Geomembrare Manufacturer stall be a commercial entity nomally engaged in manufacture of HDPE geomembrans for landfill appieations. Examples of potentid munufacturess indude SLT Environmental Inc, Hourton, Texs; Gundle Lining Systems Inc, Houston, Texas; and Poly-Flex, Inc, Grand Prairie, Texas. Use of these examples is not intended to restrict potential munufacturers.
\end{abstract}

\title{
B. Specific FMI Properties
}

1. The raw material shall be new polyethylene resin cortaining no more than $2 \%$ clean recycled polymer by weight.

2 The resin shall meet the following specifiations:

- Specific Gravity (ASTM DT92 Method A or ASTM D1505): >0.935

- Melt Index (ASTM DI238 Condition 199216): $0.1-1.18 / 10 \mathrm{~min}$

3. Physical, mechenical, and environmental properties shall meet or exceed the valuet specified in the table contained in this Part of the Specifications.

4. Geomembrane shall have a cryseallinity of 50 to 60 percers, or as approved by the COTR, as determined by Differential Sanning Calorimetry (DSC).

5. The residual friction angle between the textured geomembrane and the soilbentonite admix used in the landfill lines (see Section 0222A) shall have a minimum value of $27.5^{\circ}$ under a normal losd of 400 psf \pm 50 psf and a displacement of at leasit 2 inches. Friction angle shall be detemined by direct shear testing.

C. Manufacturing Quality Control

1. Geomembrane Manufacturing

Quality control testing shall be carried out by the Manufacturer to demionstrate that the product meets the specifications listed in Part 2-1.B. Additional testing may be arried ourt for purposes of determining conformance by the CQA Engineer. If the result of the Manuficturer's and the CQA Engineer's terting differ significanty, the testing shall be repented by the CQA Engineer, and the Manufacturer shall be sllowed to monitor this terting. The results of this latter series of test will prevall, provided that the applicable test methods have been followed. 


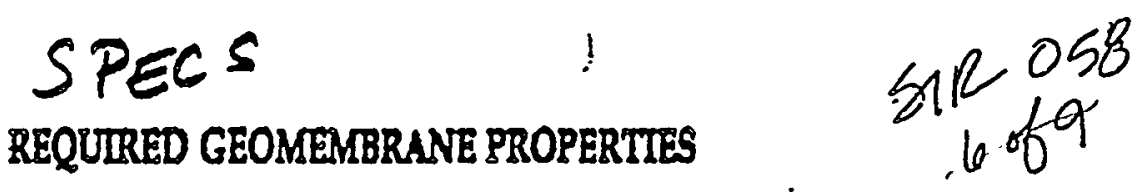

Physical Properties

Thickness

Thickness

Specific Gravity

Melt Index

Mechanical Properties

Tensile Properties

(each direction)

range $\quad 8 / 10 \mathrm{~min} \quad 0.1-1.1 \quad 0.1-1.1 \quad 0.1-1.1$

1. Force per unit width at yield

2 Tensile strength minimum Bain (forse per unit width at break)

3. Elongation at yield

4. Elongation at minimum क break

Tear Resistance minimum $\quad$ b

Puncture Resistance minimum it

minimum $\%$

$\%$

$\mathbb{b}$

Ib

Environmental Properties

Low Temperature maximum

$\mathbf{F}$

$\%$.

Carbon Black Content range

Carbon Black

NA

Dispersion

Dimensional Stability - max change $\%$

(each direction)

Resistance to Soil $\%$ strength

Burial

Environmental Stress minimum Crack hrs
$\%$

\section{$\%$}

$\begin{array}{ll}60 & 60 \\ 54 & 5 t \\ 0.54 & 0.54\end{array}$

0.1-1.1 $\mathbf{8 0}: \cdot$.
$\mathbf{2 n}$.

$0.1-1.1$ ed

.

\section{TYPE I TYPE II TYPE II}

\section{TEsT}




\section{SPECS}

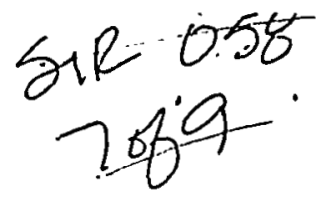

WHC.SOLS ReV. 2

Prior to the delivery of any geomembrane material, the Manufacturer shall provide the COIR and the CQA Engineer with the following information:

- the origin (Resin Supplier's name, resin production plant), identifieation (brand name, number) and production date of the resin;

- a list of quantities and descriptions of materials other than the base polymer which comprise the geomembrane;

- a copy of the quality control certificates issued by the Rerin Supplier,

- reports on the tests conducted by the Marufactures and/or the CQA Engineer to confirm that the quality of the resin used to manufacture the geomembrane satisfies these Specifieations [these tests shall include specific grevity (ASTMS DT92 Method $A$ or A5TM D1505) and melt index (ASTM D1238 Condition 190216)l:

- a statement that no recycled polymer is added to the resin or that recycled polymer is clean and does not exceed $2 \%$ by weight

- a properties sheet including at a minimum, all specined properties on the table in Section 2-1.B, messured using test methods indicated in the specifications, or equivalent:

- report on the tests, induding sampling procedures, conducted by the Manufacturer and/or the CQA Engineer to confirm that the geomembrane meets the project specifications;

- a certifieation that property values given in the properties sheet are guaranteed by the Geomembrare Menufacturer.

The CQA Engineer will review these doruments and report any discrepancies with the above requirements to the COTR. The CQA Engineer will confirm that:

- the property values certified by the Geomembrane Manufacturer meet all of the specifientions; and

- the measurements of properties by the Geomembrane Manufacturer are property documented and that the test methods used are acceptable 
In addition, the CQA Engineer may, at the request of the COIR, visit the manufacturing plant, preferably during the production of the particular geomembrane for this project, in order to evaluate the Manuractures's quality control procedures.

\section{Rolls}

Pror to shipment the Geomembrene Minnufactures shall provide the COIR ard the CQA Engineer with a quality control certificate for each roll of geomembrene provided. The quality control certificate shall be signed by a gesponsible party employed by the Geomembrane Manufactures, such as the production manager. The quality control certificate shall include:

- roll numbers and identification, resin lot and batch numbers; and

- samping procedures and sesuits of quality control testr. As a mirionum, results shall be given for thickness, tensile strength, and tear pesistance, evaluated in aceordance with the methods indieated in these Specifications or equivalent methods approved by the Design Engineer. Tests shail be conducted on each production lot of geomembrane or every 50,000 squsare feet, whichever results in the greater number of test.

The CQA Engineer will:

- confirm that the quality control certifieates have been provided at the specified frequency for all rolls, and that exch certifieste identifies the rolls reinted to it, and

- review the quality control certificates and confirm that the certified roll properties meet the specifications.

\section{Manufacturing Plant Visit}

The Manufacturer shall allow the COTR or his designated representative to visit the manufacturing plant, if the COIR so chooses. If possible, the visit shall be prior to or during the manufacturing of the geomembrane solls for the specific project The COTR or his desigrated representative shall review the manufacturing process, quality control, baboratory facilibes, and testing procedures. 


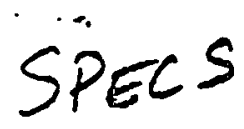<smiles>CC(C)(C)CC(CC1CC1)(C(=O)NC(C)(C)C)C1CC1</smiles>

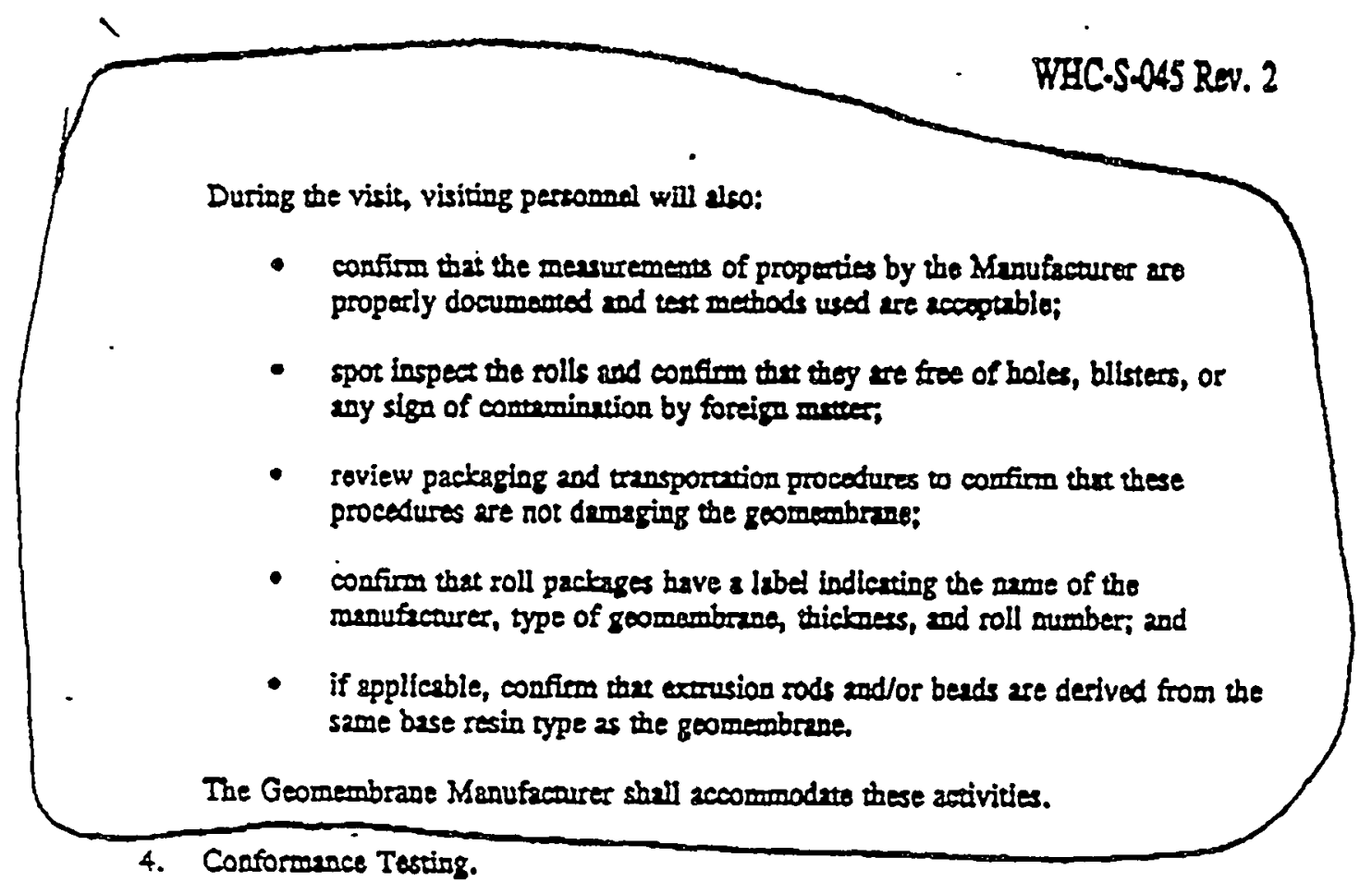

2. Tests

Upon dellvery of the rolls of geomembizne, the CQA Engines will obtrin samples at a frequency of one per production lot or one per 30,000 squere fees

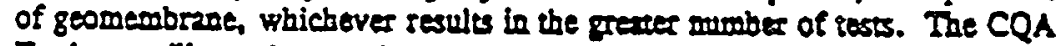
Engineer will test the samples to determine conformance 20 both the design specifications and the list of gureread properdes.

As a minimun, lests to determine the following chaneteristics will be perforsed on geomerioranes:

- specific gravity (ASTM D792 Method A or ASTM D1505):

- carbon blect conrear and virud inspection to evaluato earbos black disperrion (ASTM D1603);

- inlckeess (ASTM D1593);

- tensile charactaristics (yield strength, elongation af yield, break strength, elongation at breat, ASTM D638); and 


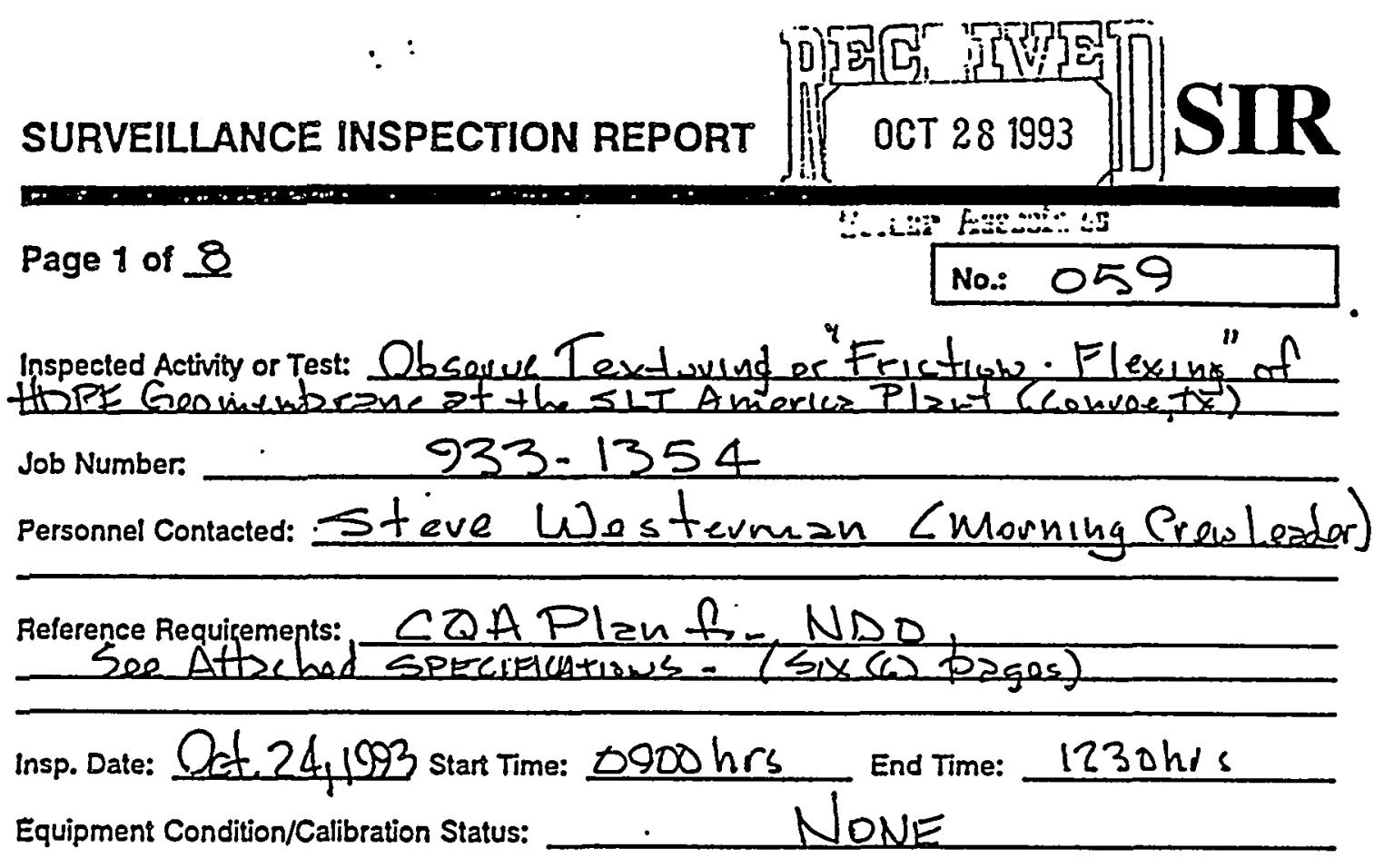

Equipment Condition/Calibration Status:

$\cdot$

Observation and Comments (Use Extra Sheets as Required): SEE Shoets 2 ddh of 8

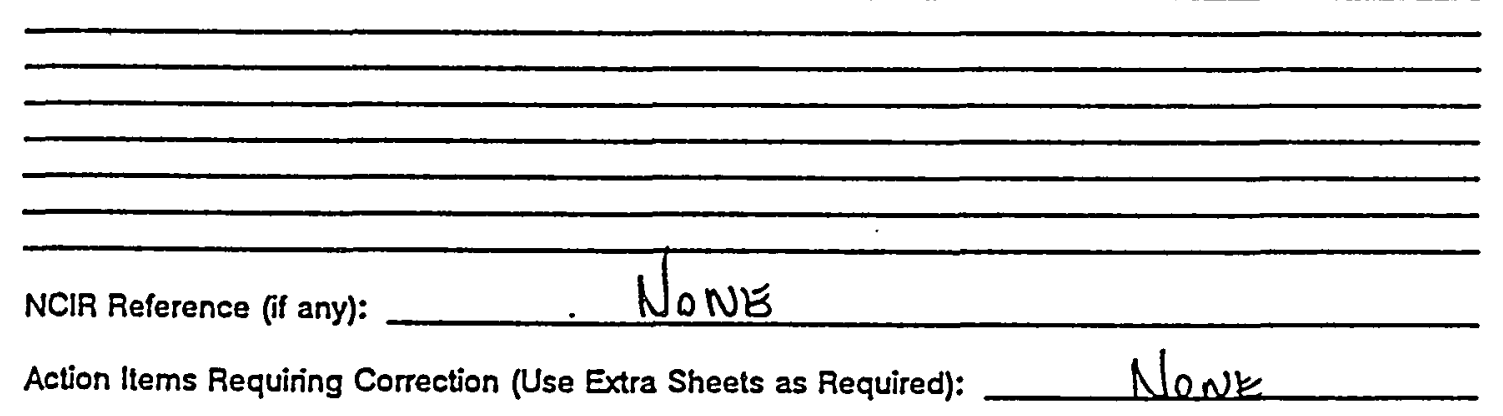

Action ltems Requining Correction (Use Extra Sheets as Required):

inspector. Durwood H. Gregory

Closed By QA Manager: Dhe hequar Date: Oct. 25, 1993

Action Item Closure Date:

Date: $11-5.93$

Comments:

GOLDER ASSOCIATES INC. 
WHC-SD-W025-RPT-001, Rev. 0

DOE/NDO CQA/WA

IN-PLANT CQA REPORT SIR NO. 059

Durwood H. Gregory of Golder Associates arrived on site at the Conroe (North) plant of SLT, America, Inc. at 0900 hrs. on Sunday, Oct. 24, 1993 and met with Steve Westerman (Crew Leader). The scope of work was to observe the texturing or "Friction Flex"ing of 60+ mil HDPE geomembrane previously manufactured at the South plant (Hardy). This texturing of geomembrane is for project (DOE/NDO CQA/WA), Project \# 933-1354 at the Hanford Nuclear Reservation in Eastern Washington, SLT customer \# 8491 for Hanford/ Geopacific (contractor). At the time of arrival, SLT was spraying rolI \# AC-6912, which is to be the fifteenth roll for this project.

After reviewing the two previous days production report, $\mathrm{Mr}$. Gregory began observing the machinery and process. Roll \# 6912 was in the process of being Flexed.

The following rolls had been Friction Flexed over Friday, Saturday and up to now... AC-6910\&6911, AC-6914\&6915, 6927 \& 6928, AC-6916\&6917, AC-6929\&6930, AC-6931\&6932, and $6898 \&$ 6899 .

At 11:14am, Mr. Gregory observed the trail cutting of roll \# AC6912 and the lead of roll \#AC-6913.

Mr. Gregory observed the manufacturing process for the next 45 minutes; at $1200 \mathrm{hrs}, \mathrm{Mr}$. Gregory was informed of a clogged nozzle. SLT would have to shut down and cool the line to clean or blow-out the nozzles, then heat back up. This procedure should take approximately $I$ hour and is a standard problem that typically happens in rainy weather.

Mr. Gregory left production line at $12: 20 \mathrm{pm}$ and was away from plant at 1230 hrs.

page- 2 of 8 
WHC-SD-W025-RPT-001, Rev. 0
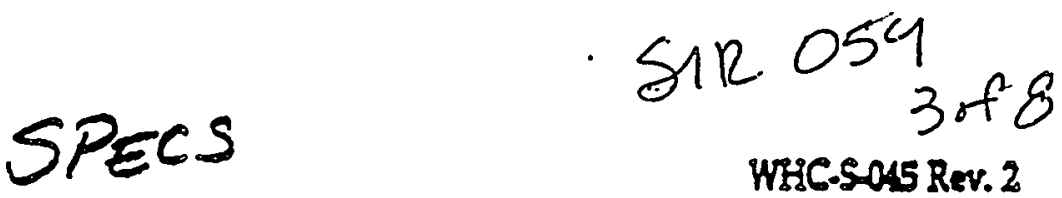

\section{if GEOCOMPOSIIE}

Geocomposite consists of geonet with a layer of geotextile thermally bonded to each side. Goocomponite is und on the side slopes of the hundfill above the primsary and secondary geamembrane.

\section{I.7 SUBMIITALS}

S. Manufacturess of the various geosynthebic matrinbs shall submit all raquired . quality asurance and quality contral documentation as deseribed is the following rections and the CQA Flan

B. The Contractor shall submit samples of all proposed geosynthetic materials for "fingerprinting" analyses and confomance testing to detemine acceptability. Sempling shall be preformed in acrerdane with the following section of this specifiation and the CQA Plan Conformance tenting will be pessormed by the CQA Engineer. Acceptability shall be determined by the COTR. Unaceeptable materials shall be removed and disposed of at Contractor's expense.

C. The Ceosynthetios Installer shull submit a quality assurance progran for installation of geosynthetic materivis as well as a list of proposed geomernbrane seaming personnel and their expertence recordh.

D. All submittals shall corform to the requirements of Sertion 01300

\section{PARI 2: PRODUCTS}

\section{I GEOMEMBRANE LINER}

\section{A Generi}

Three typer of geomembrane are shown an the Drawiagr: TYPE ! gernembrane will be used for both primary and secondary FML on the landen side slopes and ramp. TYPE II geomembrane will be uned for both pristary and secondary FML on the base of the lendfill. TYPE II geomembrene will be weed to line the truck unioading ares at the entrance to the landfill acess ramp and to line the containnent basin under the leactute storage tank The thres TMPES are defined al folbown:

TYPE I - 60 mil thickness, textured (roughened) both sides

TYPE II . 60 mil thickness, smooth both side

TYPE III - 80 mil thickness, smooth both sides

WHC NON-DRAGOFF 

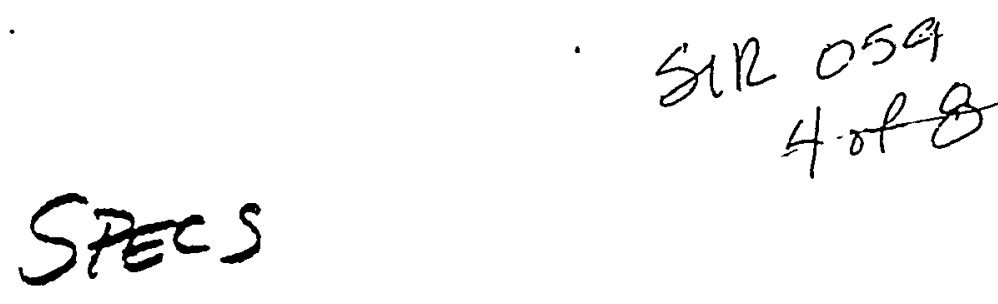

WHC.SOLS Rev. 2

All geomembrane, regardless of type, stull consist of unreinforeed high density polyettrylene (HDPE) sheet.

The Ceomembrane Manufacturer shall be a commercial entity narmally angaged in manufactare of HDPE geamembrase for landfill applications. Exampies of potential menufacturess indude SIT Environmental Ine, Hourtan, Texs; Gundle Lining System Inc, Houstor, Texus; and Poly-Flex, Ine, Grand Prairie, Teons. Use of these eamples is not intended to rexriet potential manufacturess.

\section{B. Specific FML Properties}

1. The raw materal shall be new polyethylene resin containting no more than $2 \%$ dean recyeled polymer by weight.

2 The resin shall meet the following specibertions:

- Specific Gravity (ASTM DTS2 Method A or NTTM DI505): >0.935

- Melt Index (ASTM DI238 Condition 190216): 0.1 - I.I $870 \mathrm{~min}$

3. Phyrical, mechnical and environmental properties shall meet or exceed the value specified in the table contained in this Part of the Specifientions.

4. Ceomembrane shall have a crysallinity of 50 to 60 percent, or as approved by the COIR, as detersained by Differemint Sasaning Caloximetry (DSO).

3. The residual friction angle between the textured geomenbrane and the soilbentonite adrix used in the landfin liner (see Section 02224) shall have a minimum value of $275^{\circ}$ under a normal load of $400 \mathrm{psf}=50$ psf and a disphoernert of at leart 2 inches. Frition angle strall be deterswined by direct shear toting

C Musufacturing Qunlity Contral

1. Geomembrane Manufacturing

Quality control terting shall be arried out by the Manufacturer to

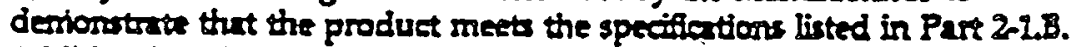
sdditonal besting may be arried out for purposes of determining corformenen by the CQA Engineer. If the result of the Menuficturet's and the CQA Enginer's testing differ significantly, the terting shull be repented by the CQA Engineer, and the Manuficturer shall be allowed to monitor this testing. The results of thin latter series of terts will prevall provided that the appllable test methods have been followed. 
WHC-SD-W025-RPT-001, Rev. 0

$$
\begin{aligned}
& \text { SPEC S : } \\
& \text { BEQUTRED GEOMEMBRANE PROPERTIES }
\end{aligned}
$$

\begin{tabular}{|c|c|c|c|c|c|}
\hline $\begin{array}{l}\text { Thiderness } \\
\text { Thickernas } \\
\text { Specifle Gravity }\end{array}$ & $\begin{array}{l}\text { miniroum avg } \\
\text { minimtum } \\
\text { mirimem }\end{array}$ & $\begin{array}{l}\text { mils } \\
\text { mils } \\
\text { NAt }\end{array}$ & $\begin{array}{l}60 \\
54 \\
\text { Qs }\end{array}$ & $\begin{array}{l}60 \\
58 \\
0.94\end{array}$ & $\begin{array}{l}\text { og } \\
72 \\
\text { ast }\end{array}$ \\
\hline
\end{tabular}

Physieal Propertion

Melt index

$$
\text { range } 810 \mathrm{mir}
$$

Q.8.1.8

$0.1-1.2$

Q.2-1.20

Mechanical Peperties

Tensile Properties

(exch direction)

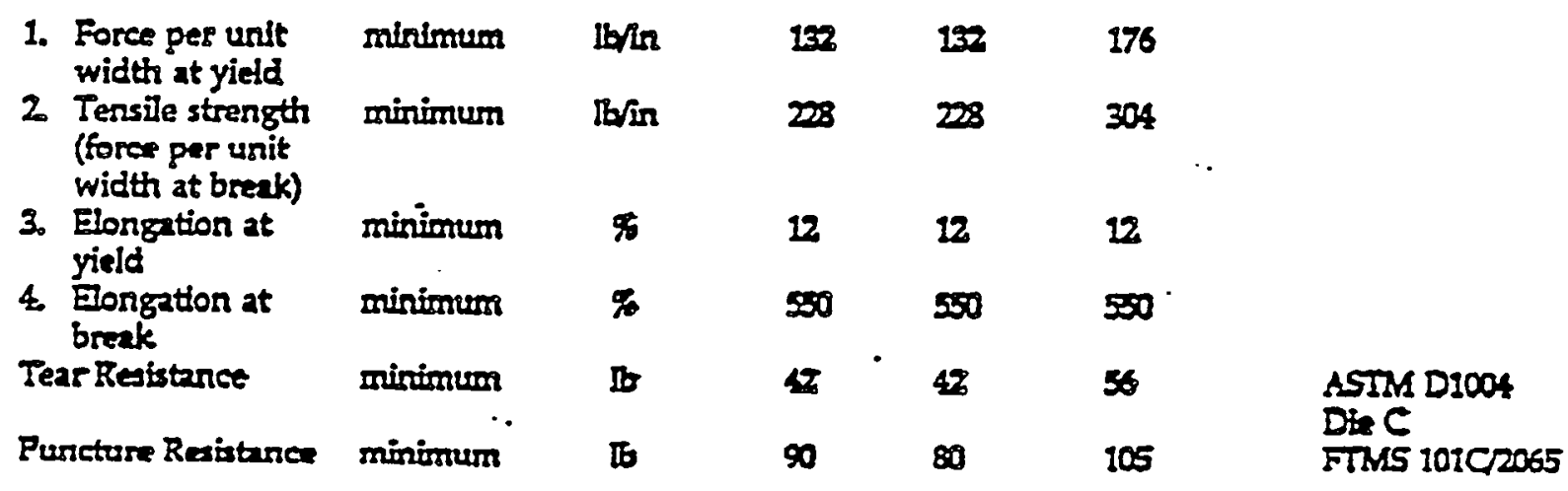

Envirenmental Properties

Low Temperature moximum $F$

Carbon Black Content nange

Cutorn Blind

Dispersion

NA

Dimensional Stability max diange $\%$

(ench direction)

Resistence to Soil $\%$ strength retained

Burial

5

AI/A

$.75 \quad-75$

$-73$

ASTM D746

Procodure $B$

$23 \quad 2.3 \quad 25$

AVAZ AYAZ AYAZ

ASTM DTERS

ASTM DEN15

$\pm 1 \quad \pm 1 \quad \pm 1$

ASTM DIOSP

9590

90

15TM D3083 (ar modithed in NSF Appendix

\section{Environmental Stress minimum ho Crack}

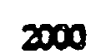

2000
A) ATTM DIGS3 (a) modifind in NSF Appendlx $A$ Condition $C$ at 1000 
Prot to the delivery of any geomembrane material, the Menufacturer shall provide the COTR and the CQA Engineer with the following information:

- the origin (Resin Suppliar's name, reain production plant), identification (brand rume, number) and production date of the resirit

- I list of quantitien and descriptions of materials other than the buse polymer which cormprise the geomernbrane;

- copy of the quality control certifiates isued by the Rexin Suppller,

- reports on the tests conducted by the Manufacturet and/or the CQA Engineer to corfirm that the quality of the resin uned to manufactare the geomembrane satisfies these Specifications [there torts shall indude specific gravity (ASTM DTO2 Method A or ASTM D15COS) and melt index (ASTM DI238 Condition 190216)]:

- a statement that no recycled polymer is added to the resin or that recyded polymer is dean and does not exced $2 \%$ by weight,

- 2 properties sheet including at a minimuon all specified properties on the table in Section 2-1.B, measured using test methods indicated in the specifieations, or equivalent:

- reports on the terts, induding sampling procedures, onducted by the Manufactures and/or the CQA Engineer to confirm that the geomembrane meets the project specifications;

- crtifieation that property values given in the properties sheet are gmantred by the Ceomembrane Manufacturer.

The Cen Engineer will review these documents and report any discrepancies with the above requirements to the COTR. The CQA Engineer will confirm that:

- the property values certified by the Geomembrane Manusterser mont all of the specifientions; and

- the measurements of properties by the Ceomeribrant Manuficturer are property documented and that the test methods used are aprable 


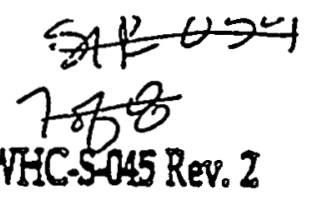

In addition, the CQA Engineep may, at the requext of the COTR, visit the manufacturing plant, preferably during the production of the particular geomembrane for this project, in order to evaluate the Manufacturer's quality control procedures.

\section{Rolb}

Pror to shipment the Ceomembrene Manufacturer shall provide the COTR and the CQA Engirieer with a quility control certifente for each roll of geamembrene provided. The quelify control certifica stuall be signed by a responsible purty enployed by the Geornembrane Manufactures, such as the production marager. The quality control cestifen shall include:

- roll numbers and identifiation, resir lot and batch numbers; and

- sxumpling procedure und results of quality control tests is a minionum, results shall be given for thickress, tensile strength, and tear resistonce, evaluated in acoordance with the methods indiented in these Specifications or eguivalent methods approved by the-Design Engineer. Tests shall be conducted on exch production lot of geomembrane or every 50,000 square foct, whichever results in the grenter sumber of serts.

The CQA Engineer will:

- confirm that the quality control certiontes have been provided at the specilied frequency for all rolls, and that ench cortiface identifies the rolle related to it and

- seview the quality control certifiates and confirm that the certified soll properties areet the specifications.

3. Manufuctiring Plant Visit

The Manufieturer shall allow the COTR or his desigrated ropresntative to viait the munuficturing plant if the COTR so chooses. If posisible, the visit shall be prior to or during the menufacturing of the geomearbrane solls for the specifie projext. The COTR or hin designaterd representative shall reviow the manufucturing process, quality control, laboratery facilities, and testing grocentures. 
WHC-SD-W025-RPT-001, Rev. 0
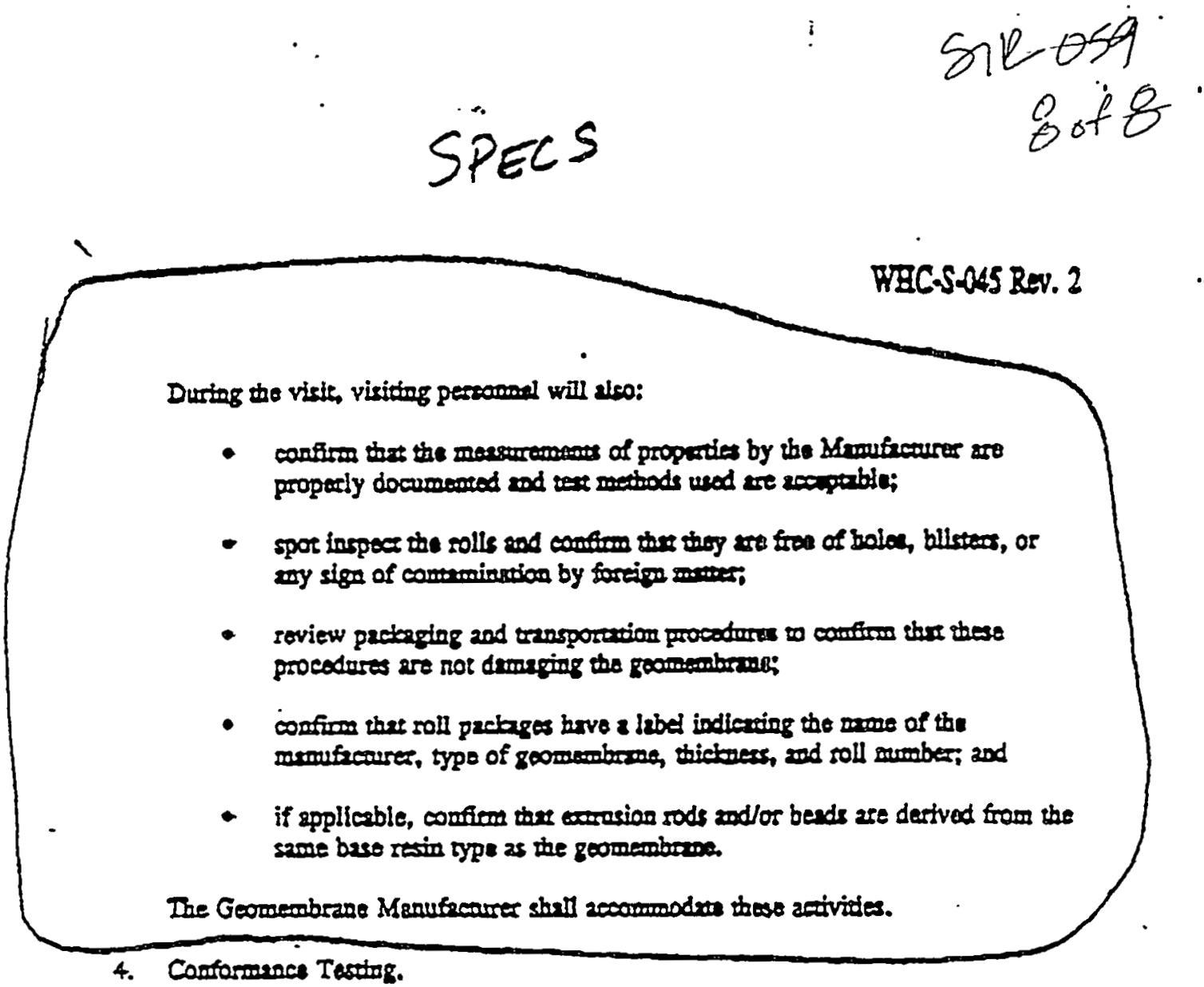

$2 T$

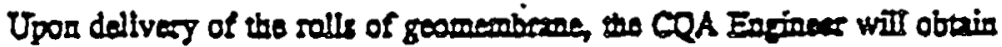

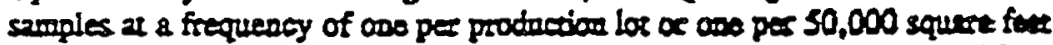

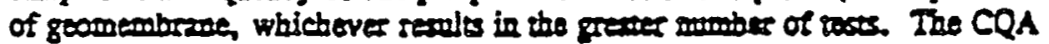

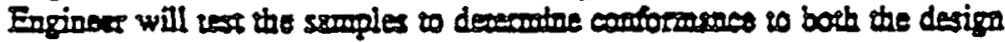

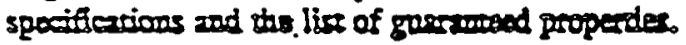

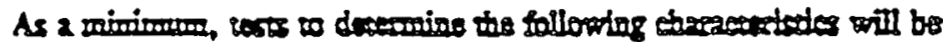
As on geoment:

- specifle gravity (ASTM DT92 Method A or ASTM DIS0S):

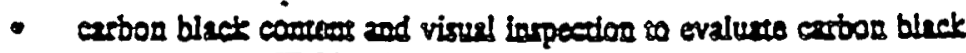
disperion (ASTM D1603);

- thicher (ASTM DI593):

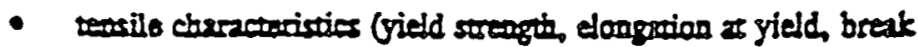
strength, elorgarion at breats ASTS6 D638); and 
HHC-SD-W025-RPT-001, Rev. 0

APPENDIX E-2

CQA CONFORMANCE TESTS 
WHC-SD-W025-RPT-001, Rev. 0

GOLDER CONSTRUCTION SERVYCES, INC.

933-1354

GEOMEMBRANE INVENTORY CONTROL RECORD

$05 / 90 / 94$

DEPARTHENT OF ENERGY

Page: 1

NON-DRAG OFF LANDFILL FACILITY

HANDFORD NUCLEAR RESERVATION

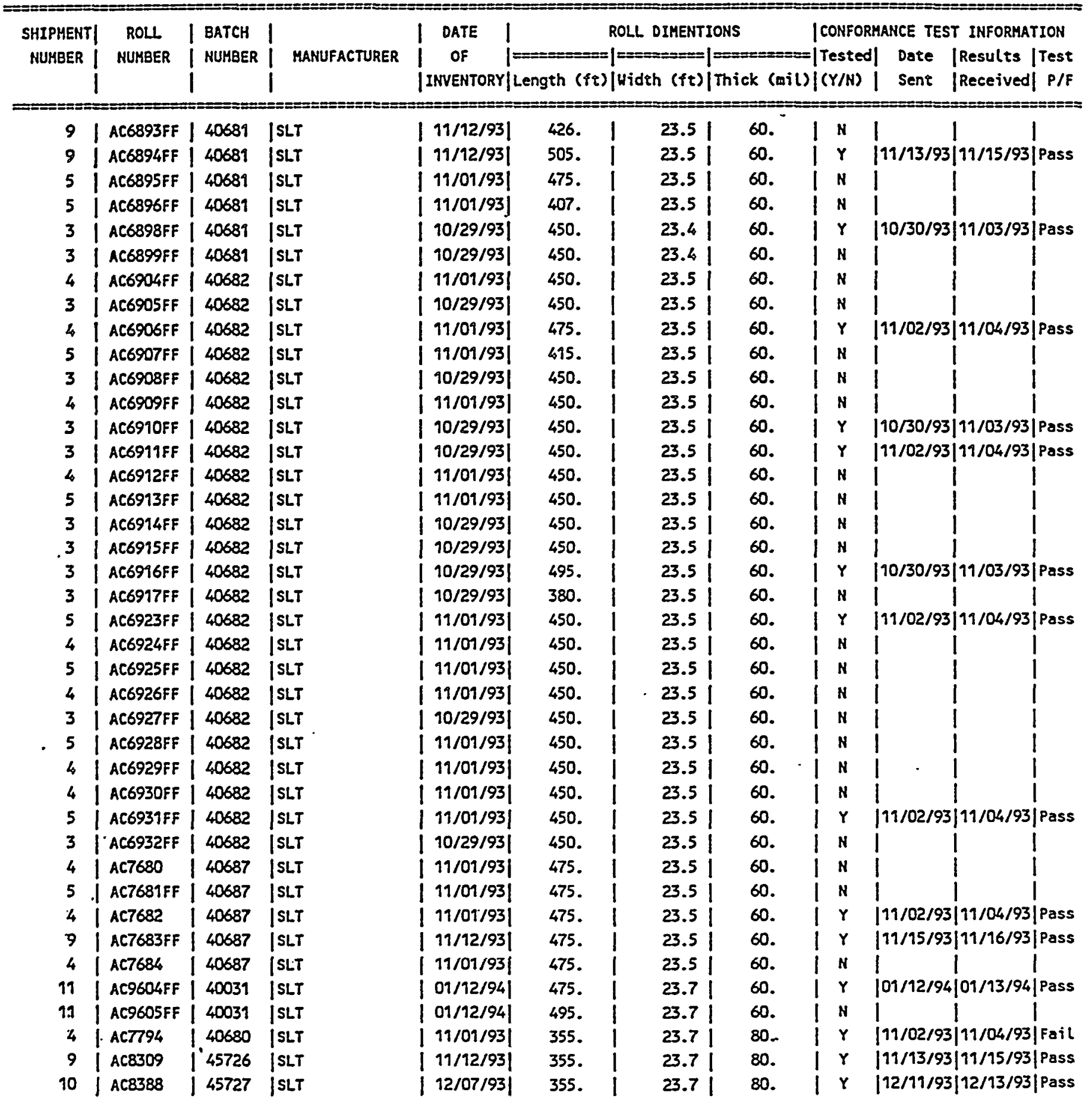

Square Feet Delivered $=420685$. 
WHC-SD-W025-RPT-001, Rev. 0

NOVEMBER 1993

\begin{tabular}{|c|}
\hline $\begin{array}{c}\text { SUMMARY OF TEXTURED GEOMEMBRANE } \\
\text { CONFORMANCE TEST RESULTS }\end{array}$ \\
DEPARTMENT OF ENERGY \\
NON DRAG-OFF CQA \\
WASHINGTON
\end{tabular}

833-1354

ROLL

DESIGNATION AC-6898 AC-6910 AC-6916 AC-6808 AC-6911 AC-6923 AC-6931 AC-6894 AC-7683 AC-9604

\begin{tabular}{|c|c|c|c|c|c|c|c|c|c|c|}
\hline $\begin{array}{l}\text { THICKNESS } \\
\text { (mils) } \\
\text { ASTM D } 1593\end{array}$ & 67.6 & 67.2 & 67.7 & 66.8 & 67.5 & 67.8 & 67.0 & 67.5 & 68.4 & 68.3 \\
\hline $\begin{array}{l}\text { DENSITY } \\
(g / c c) \\
\text { ASTM D } 1505\end{array}$ & 0.843 & 0.844 & 0.943 & 0.844 & 0.943 & 0.943 & 0.843 & 0.942 & 0.844 & 0.845 \\
\hline STRENGTH AT & & & & & & & & & & \\
\hline YIELD (ppi) & 156.8 & 159.7 & 157.6 & 156.7 & 155.8 & 154.8 & 154.4 & 156.6 & 161.4 & 168.8 \\
\hline $\begin{array}{l}\text { MDTD (1) } \\
\text { ASTM D } 638\end{array}$ & 153.5 & 160.5 & 152.7 & 157.6 & 155.2 & 156.0 & 155.8 & 154.4 & 161.6 & 166.3 \\
\hline STRENGTH AT & & & & & & & & & & \\
\hline BREAK (ppi) & 338.2 & 326.8 & 321.8 & 333.5 & 348.1 & 320.6 & 341.5 & 315.9 & 318.6 & 319.2 \\
\hline $\begin{array}{l}\text { MDTTD (1) } \\
\text { ASTM D } 638\end{array}$ & 327.0 & 307.0 & 332.7 & 328.4 & 338.7 & 318.3 & 318.4 & 320.4 & 308.8 & 304.5 \\
\hline ELONGATION & & & & & & & & & & \\
\hline AT YIELD (\%) & 22.1 & 23.7 & 22.5 & 25.5 & 24.2 & 22.9 & 22.8 & 25.5 & 22.1 & 24.2 \\
\hline $\begin{array}{l}\text { MD/TD (1) } \\
\text { ASTM D } 638\end{array}$ & 23.1 & 23.9 & 21.2 & 24.7 & 24.1 & 23.1 & 23.4 & 24.8 & 21.1 & 25.3 \\
\hline ELONGATION & & & & & & & & & & \\
\hline AT BREAK (\%6) & 838 & 774 & 808 & 792 & 830 & 822 & 836 & 770 & 788 & 810 \\
\hline $\begin{array}{l}\text { MDTTD (1) } \\
\text { ASTM D } 638\end{array}$ & 816 & 760 & 822 & 832 & 798 & 838 & 862 & 798 & 788 & 780 \\
\hline $\begin{array}{l}\text { MELT INDEX } \\
\text { (g/10min) } \\
\text { ASTM D } 1238\end{array}$ & 0.55 & - & 0.58 & - & - & - & - & - & - & - \\
\hline CARBON BLACK & & & & & & & & & & \\
\hline $\begin{array}{l}\text { CONTENT (\%) } \\
\text { ASTM D } 1603 .\end{array}$ & 2.80 & 2.86 & 2.80 & 2.81 & 2.84 & 2.84 & 2.82 & 2.74 & 2.85 & 2.76 \\
\hline CARBON BLACK & & & & & & & & & & \\
\hline $\begin{array}{l}\text { DISPERSION(2) } \\
\text { ASTM D } 3015\end{array}$ & A1 & A1 & A1 & A1 & A1 & A1 & A1 & A1 & A1 & A1 \\
\hline $\begin{array}{c}\text { CRYSTALLINITY } \\
\text { (\$6) } \\
\text { ASTM D } 3417\end{array}$ & 44 & - & 44 & - & - & - & - & - & - & - \\
\hline
\end{tabular}

(1) MDrTD corresponds to Machine Direction / Transverse Direction.

(2) Carbon black dispersion was tested according to ASTM D3015 and reported according to the ASTM D2663 dispersion classification chart. 
WHC-SD-W025-RPT-001, Rev. 0

NOVEMBER 1993

\begin{tabular}{|c|c|}
\hline$\cdots, \quad$ SUMMARY OF SMOOTH GEOMEMBRANE: $\ldots$ \\
CONFORMANCE TEST RESULTS \\
DEPARTMENT OF ENERGY \\
NON DRAG-OFF COA \\
WASHINGTON
\end{tabular}

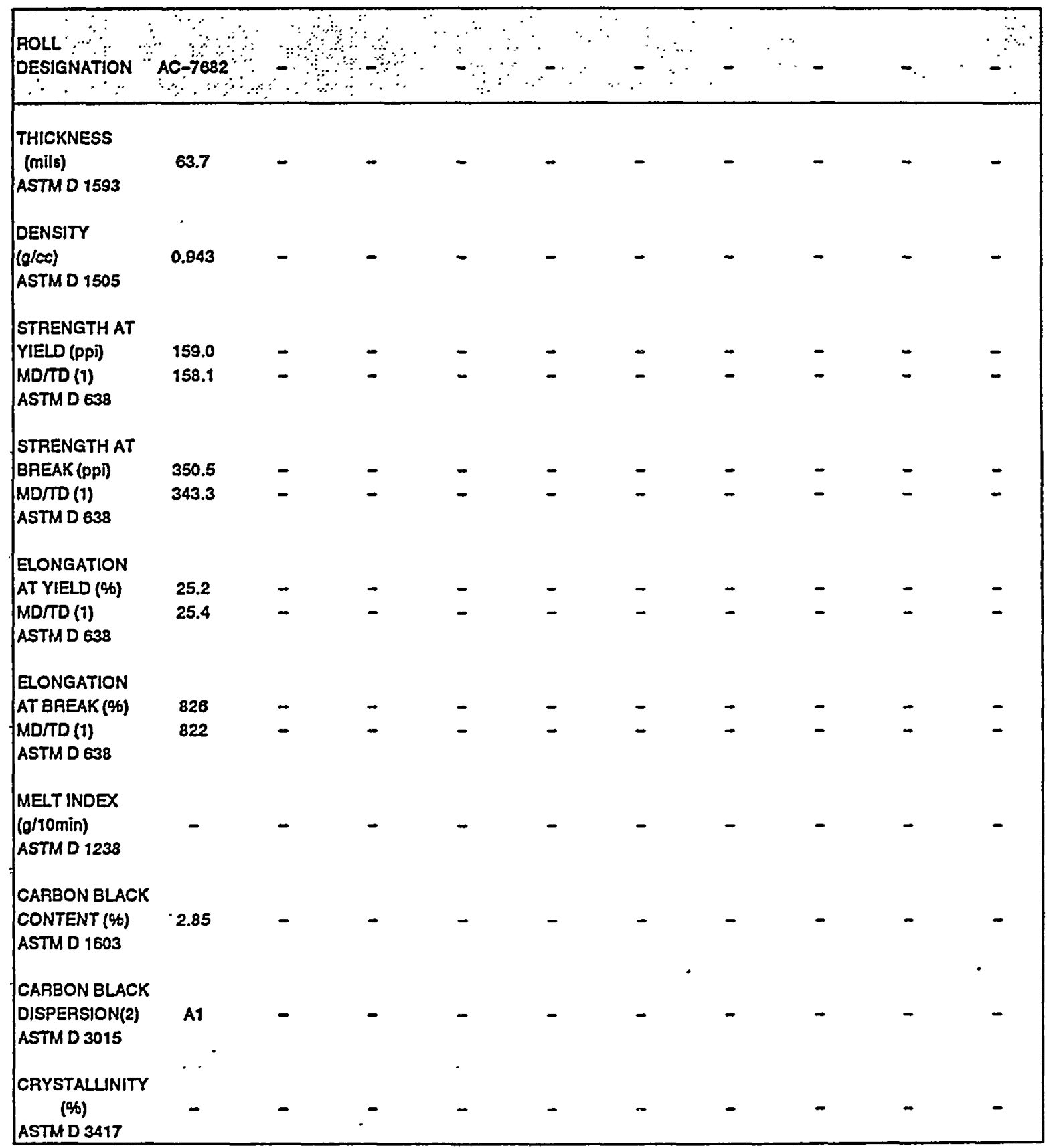

(1) MD/TD corresponds to Machine Direction / Transwerse Direction.

(2) Carbon black dispersion was tested according to ASTM D3015 and reported according to the ASTM D2663 dispersion classification chart. 
WHC-SD-W025-RPT-001, Rev. 0

NOVEMBER 1993

\begin{tabular}{|c|}
\hline $\begin{array}{c}\text { SUMMARY OF SMOOTH GEOMEMBRANE } \\
\text { CONFORMANCE TEST RESULTS }\end{array}$ \\
DEPARTMENT OF ENERGY \\
NON DRAG-OFF CQA \\
WASHINGTON
\end{tabular}

\begin{tabular}{|c|c|c|c|c|c|c|c|c|c|c|}
\hline $\begin{array}{l}\text { ROLL } \\
\text { DESIGNATION }\end{array}$ & AC- 7794 & $A C-8309$ & $A C-8388$ & - & - & - & - & - & - & - \\
\hline $\begin{array}{l}\text { THICKNESS } \\
\text { (mils) } \\
\text { ASTM D } 1593\end{array}$ & 81.8 & 81.1 & 81.0 & - & - & - & - & - & - & - \\
\hline $\begin{array}{l}\text { DENSITY } \\
\text { (g/cc) } \\
\text { ASTM D } 1505\end{array}$ & 0.938 & 0.940 & 0.942 & - & - & - & - & - & - & - \\
\hline STRENGTH AT & & & & & & & & & & \\
\hline YIELD (ppi) & 184.9 & 181.5 & 200.4 & - & - & - & - & - & - & - \\
\hline $\begin{array}{l}\text { MD/TD (1) } \\
\text { ASTM D } 638\end{array}$ & 183.6 & 186.9 & 195.8 & - & - & - & - & - & - & - \\
\hline STRENGTH AT & & & & & & & & & & \\
\hline BREAK (ppi) & 382.0 & 411.0 & 437.7 & - & - & - & - & - & - & - \\
\hline $\begin{array}{l}\text { MD/TD (1) } \\
\text { ASTM D } 638\end{array}$ & 414.9 & 441.9 & 410.8 & - & - & - & - & - & - & - \\
\hline ELONGATION & & & & & & & & & & \\
\hline AT YIELD (\%) & 22.9 & 23.2 & 27.2 & - & - & - & - & - & - & - \\
\hline $\begin{array}{l}\text { MDTTD (1) } \\
\text { ASTM D } 638\end{array}$ & 23.1 & 22.6 & 28.0 & - & - & - & - & - & - & - \\
\hline ELONGATION & & & & & & & & & & \\
\hline AT BREAK (\$) & 874 & 852 & 854 & - & - & - & - & - & - & - \\
\hline $\begin{array}{l}\text { MD/TD (1) } \\
\text { ASTM D } 638\end{array}$ & 870 & 888 & 850 & - & - & - & - & - & - & - \\
\hline $\begin{array}{l}\text { MELT INDEX } \\
\text { (g/10min) } \\
\text { ASTM D } 1238\end{array}$ & - & - & - & - & - & - & - & - & - & - \\
\hline CARBON BLACK & & 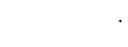 & & & & & & & & \\
\hline $\begin{array}{l}\text { CONTENT (\$6) } \\
\text { ASTM D } 1603\end{array}$ & 1.74 & 2.21 & 2.54 & - & - & - & - & - & - & - \\
\hline CARBON BLACK & & & & & & & & & & \\
\hline $\begin{array}{l}\text { DISPERSION(2) } \\
\text { ASTM D } 3015\end{array}$ & A1 & A1 & A1 & - & - & - & - & - & - & - \\
\hline $\begin{array}{l}\text { CRYSTALLINITY } \\
\text { (\%) } \\
\text { ASTM D } 3417\end{array}$ & - & - & - & - & - & - & - & - & - & - \\
\hline
\end{tabular}

(1) MDTD corresponds to Machine Direction / Transwerse Direction.

(2) Carbon black dispersion was tested according to ASTM 03015 and reported according to the ASTM D2663 dispersion classification chart. 
WHC-SD-W025-RPT-001, Rev. 0

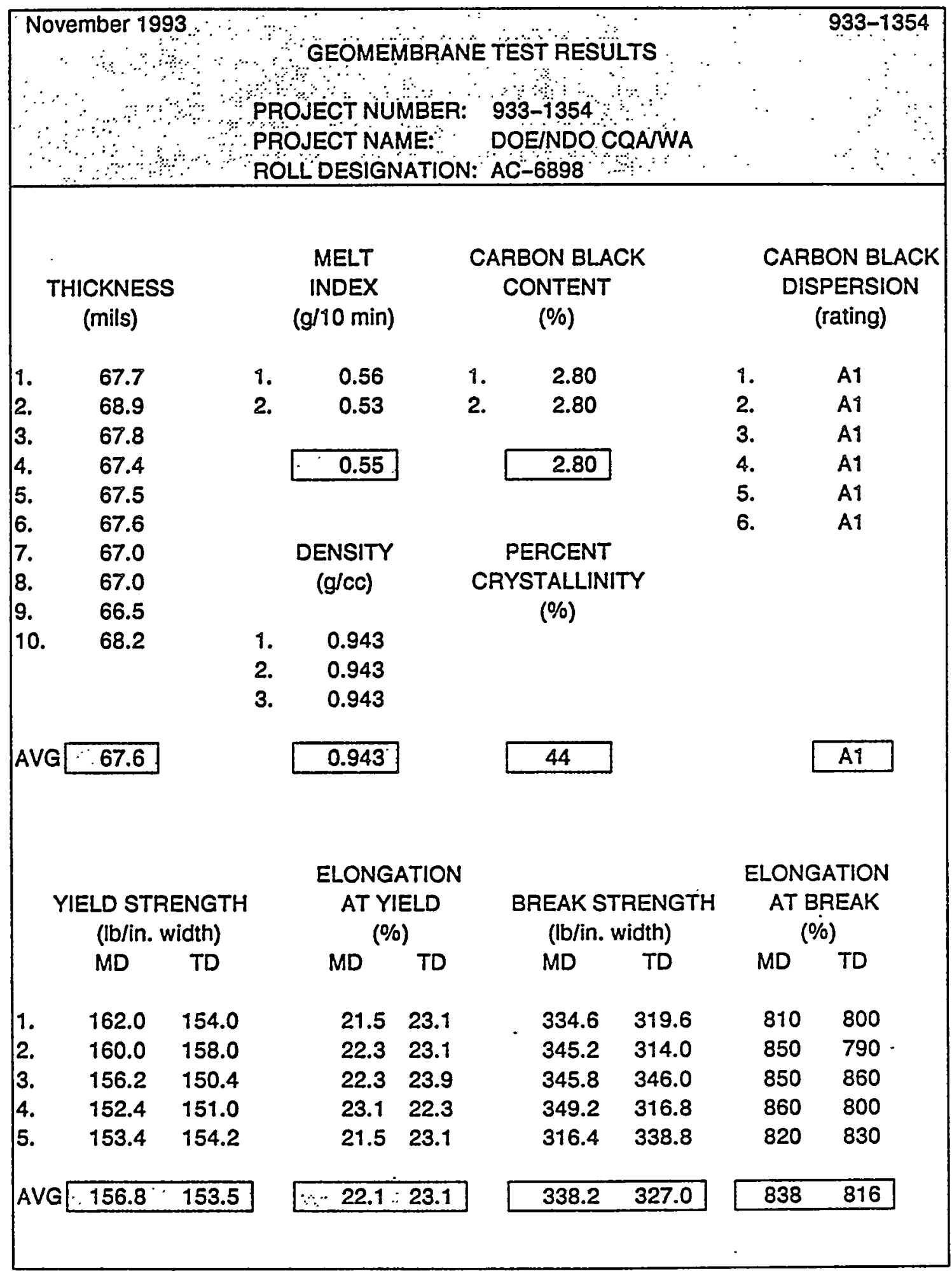


WHC-SD-W025-RPT-001, Rev. 0

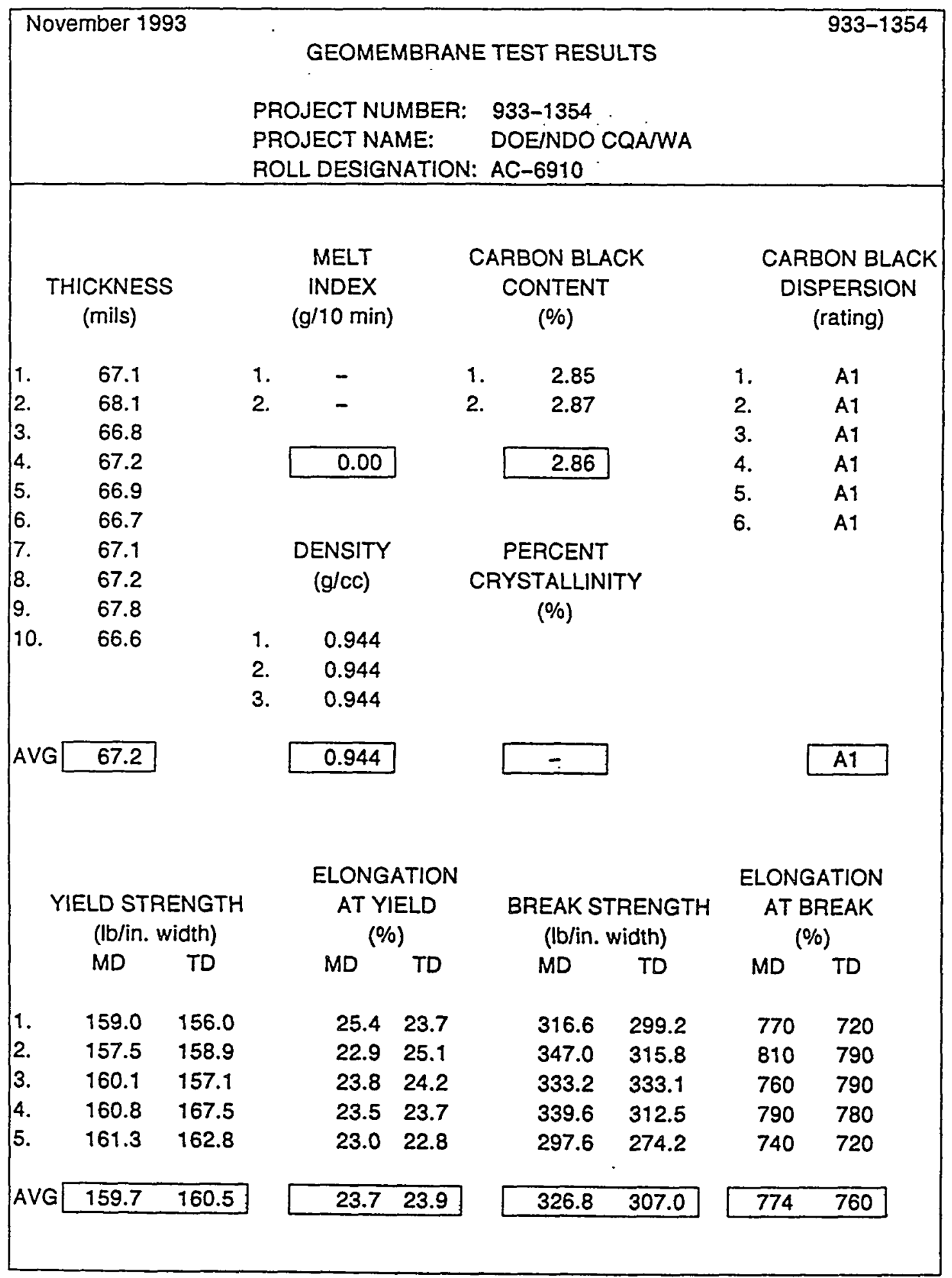




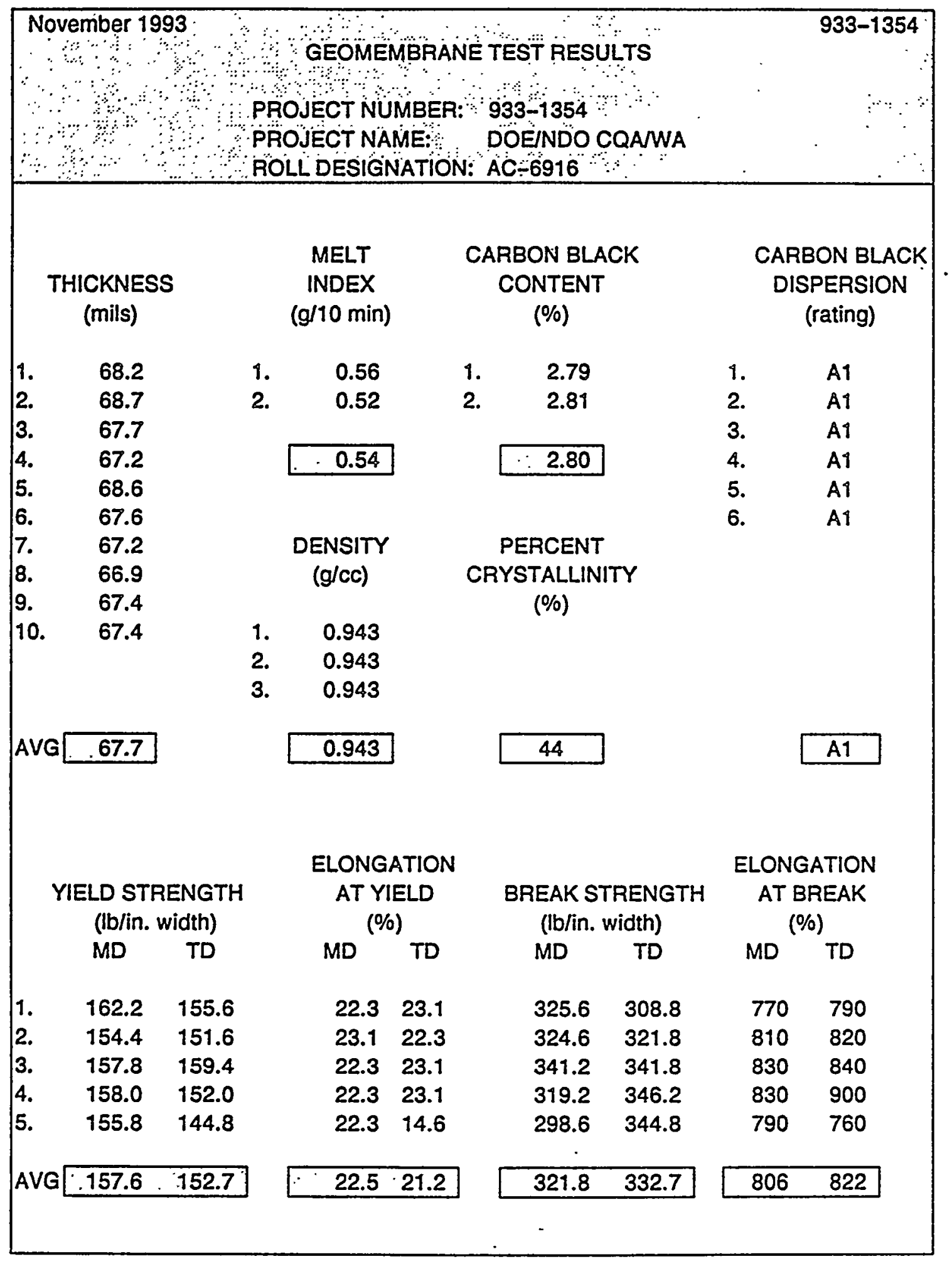


WHC-SD-W025-RPT-001, Rev. 0

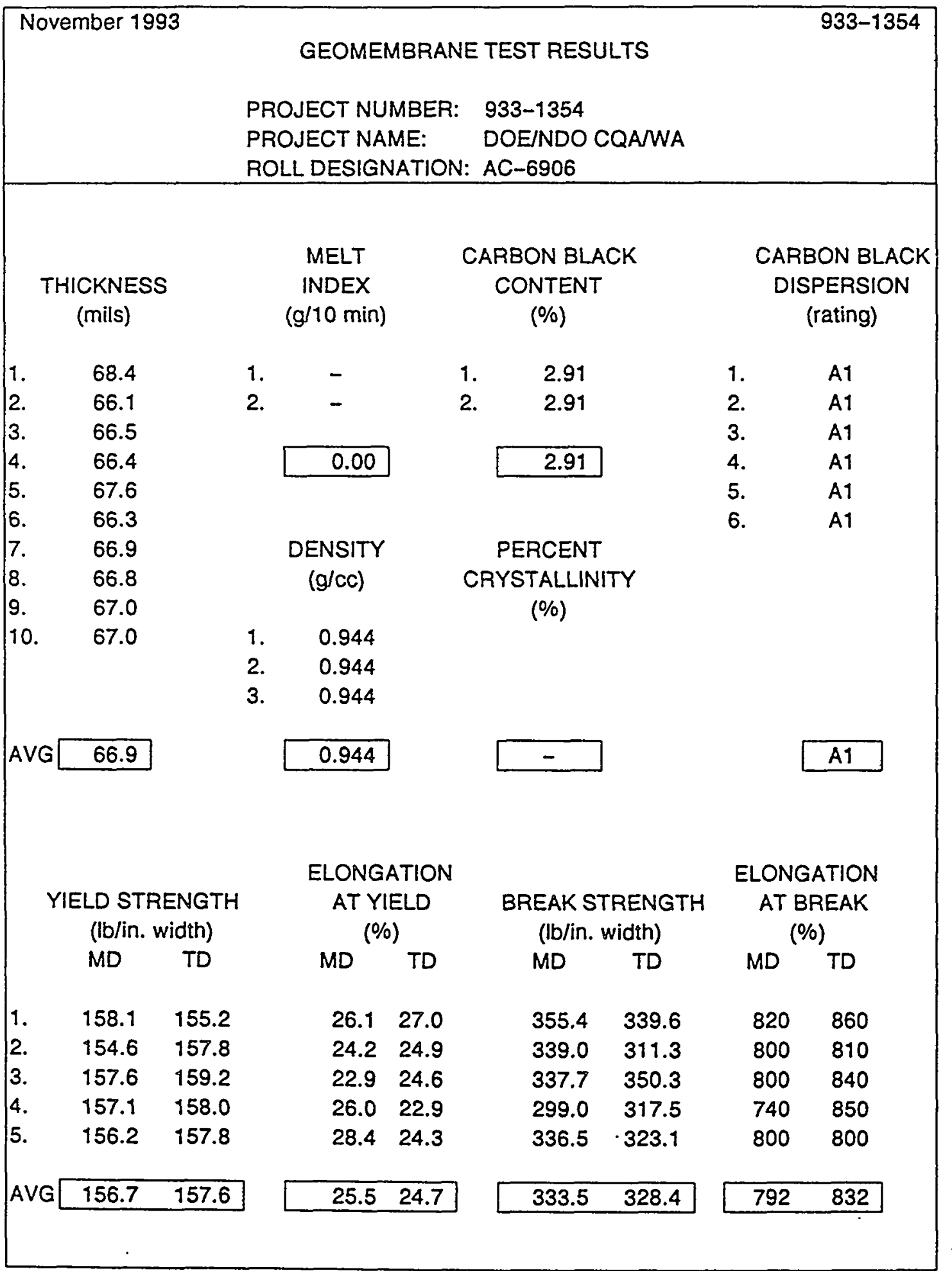




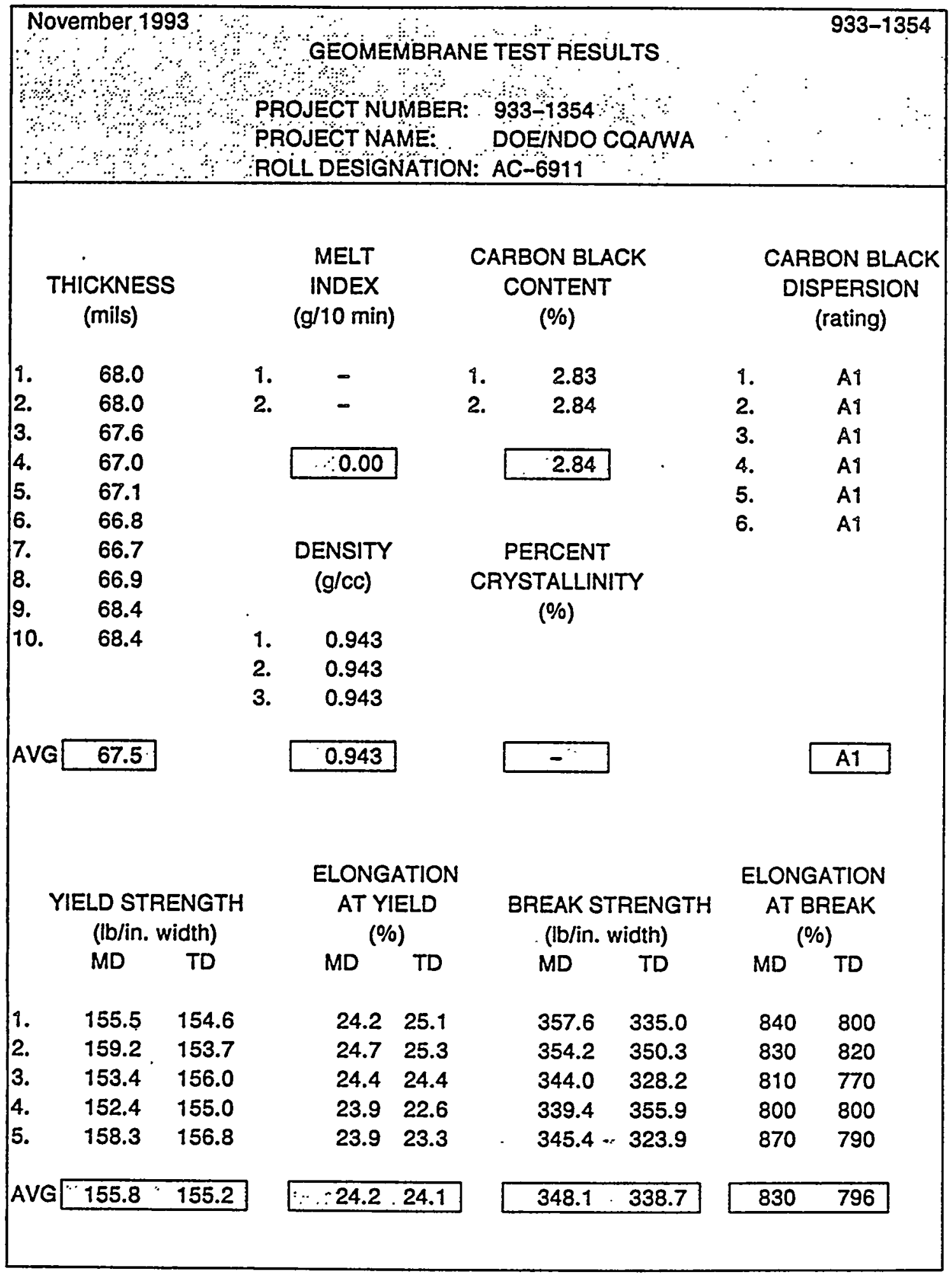


WHC-SD-W025-RPT-001, Rev. 0

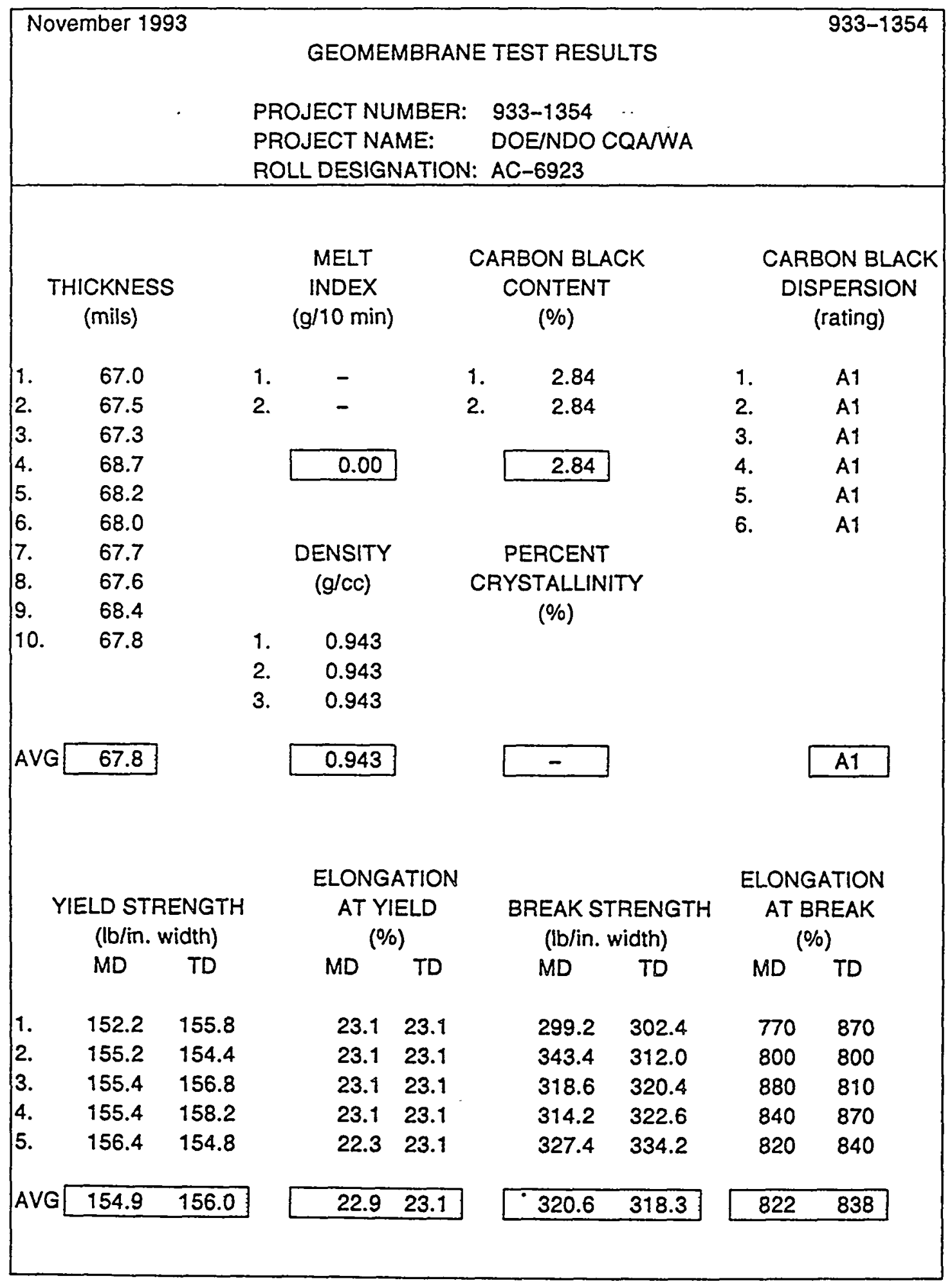




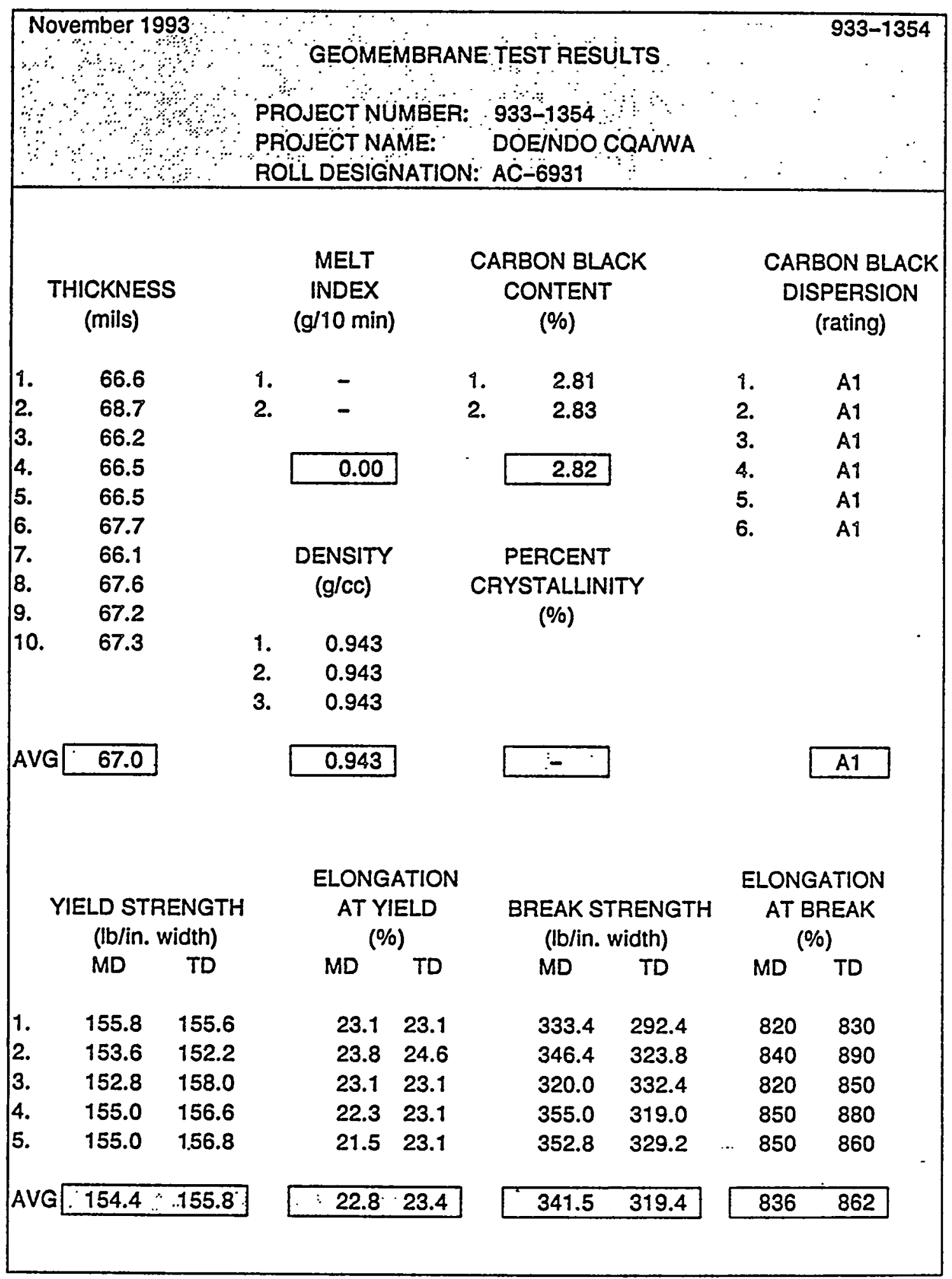


WHC-SD-W025-RPT-001, Rev. 0

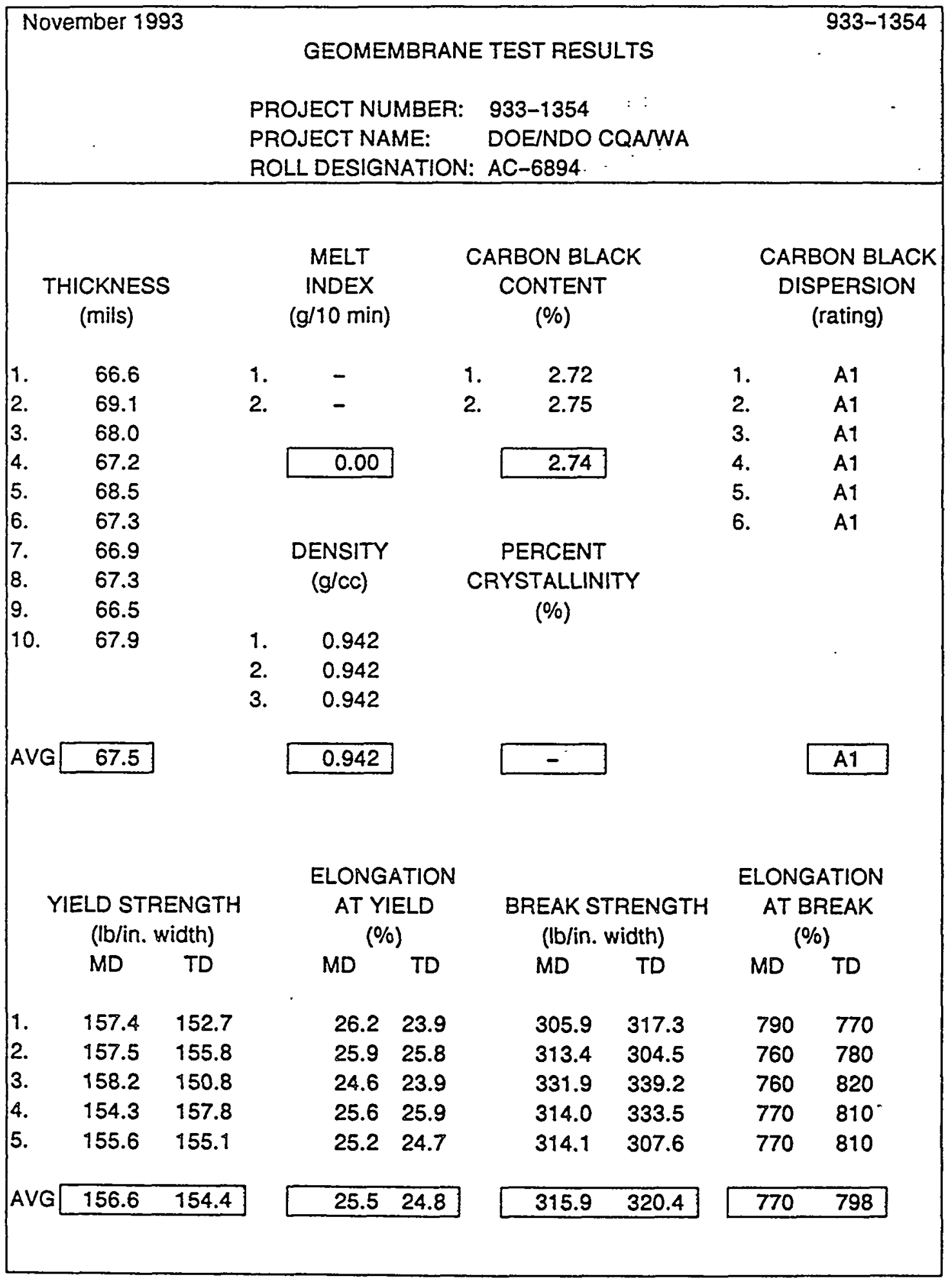


WHC-SD-W025-RPT-001, Rev. 0

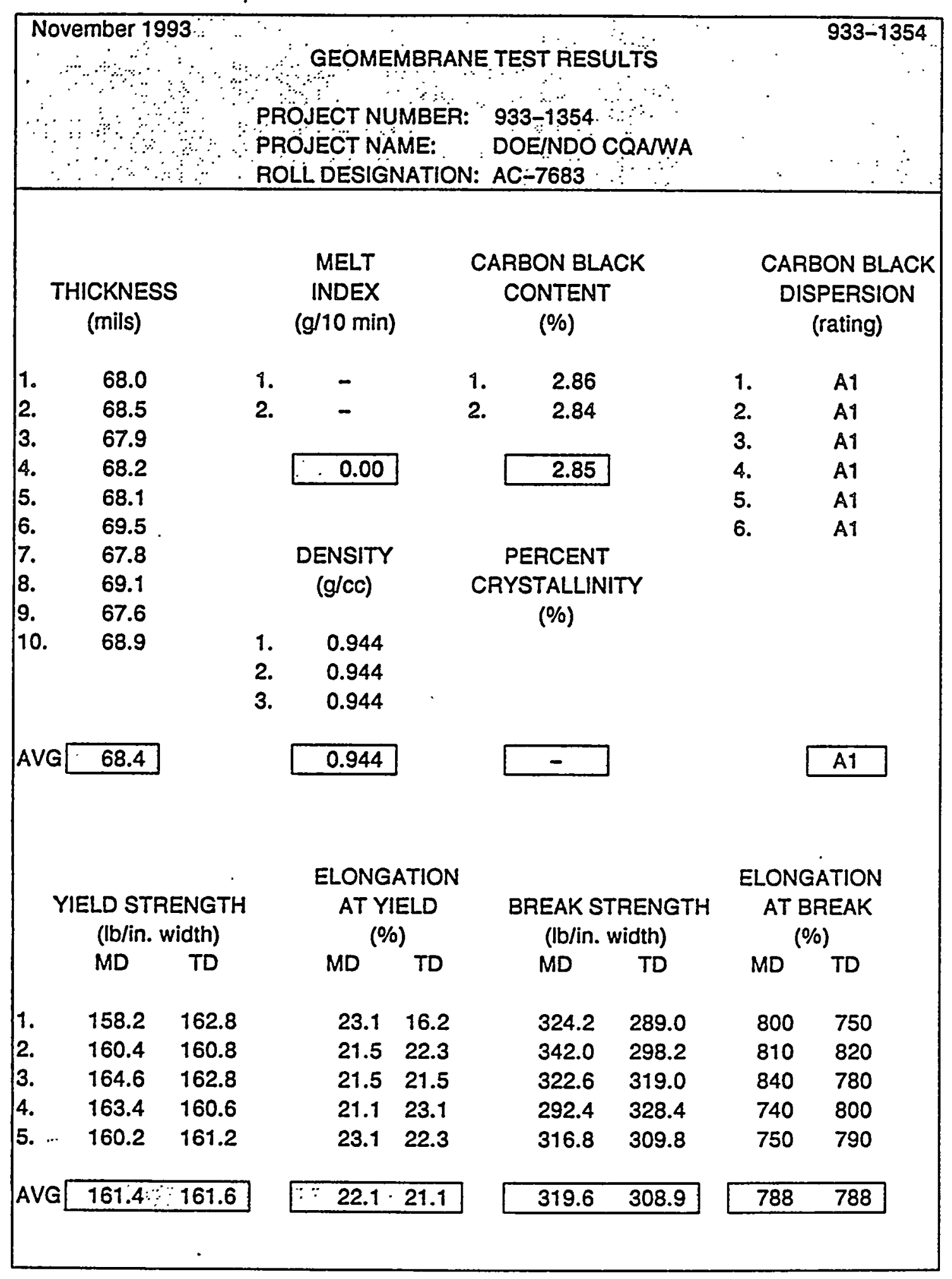


WHC-SD-W025-RPT-001, Rev. 0

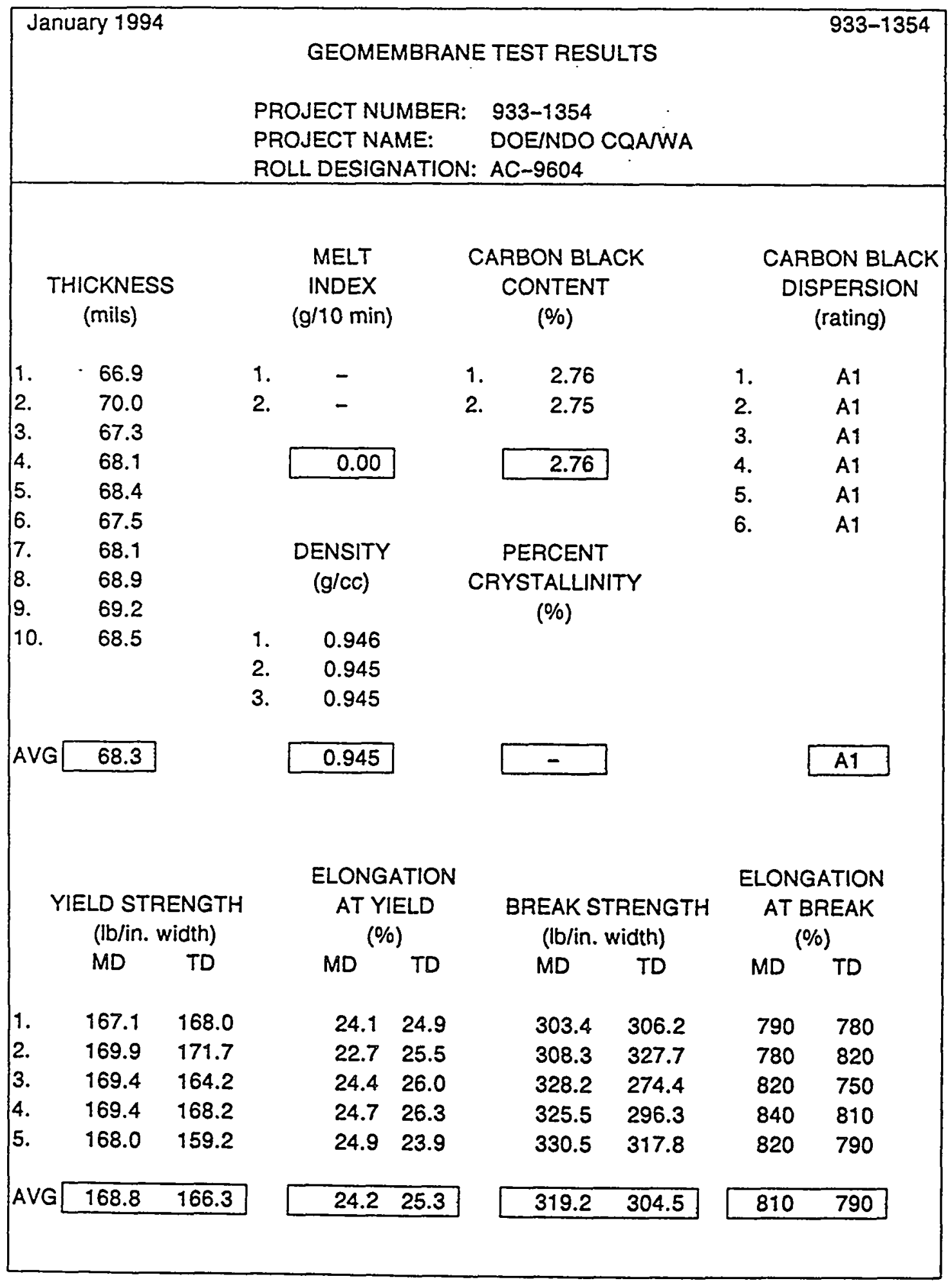


WHC-SD-W025-RPT-001, Rev. 0

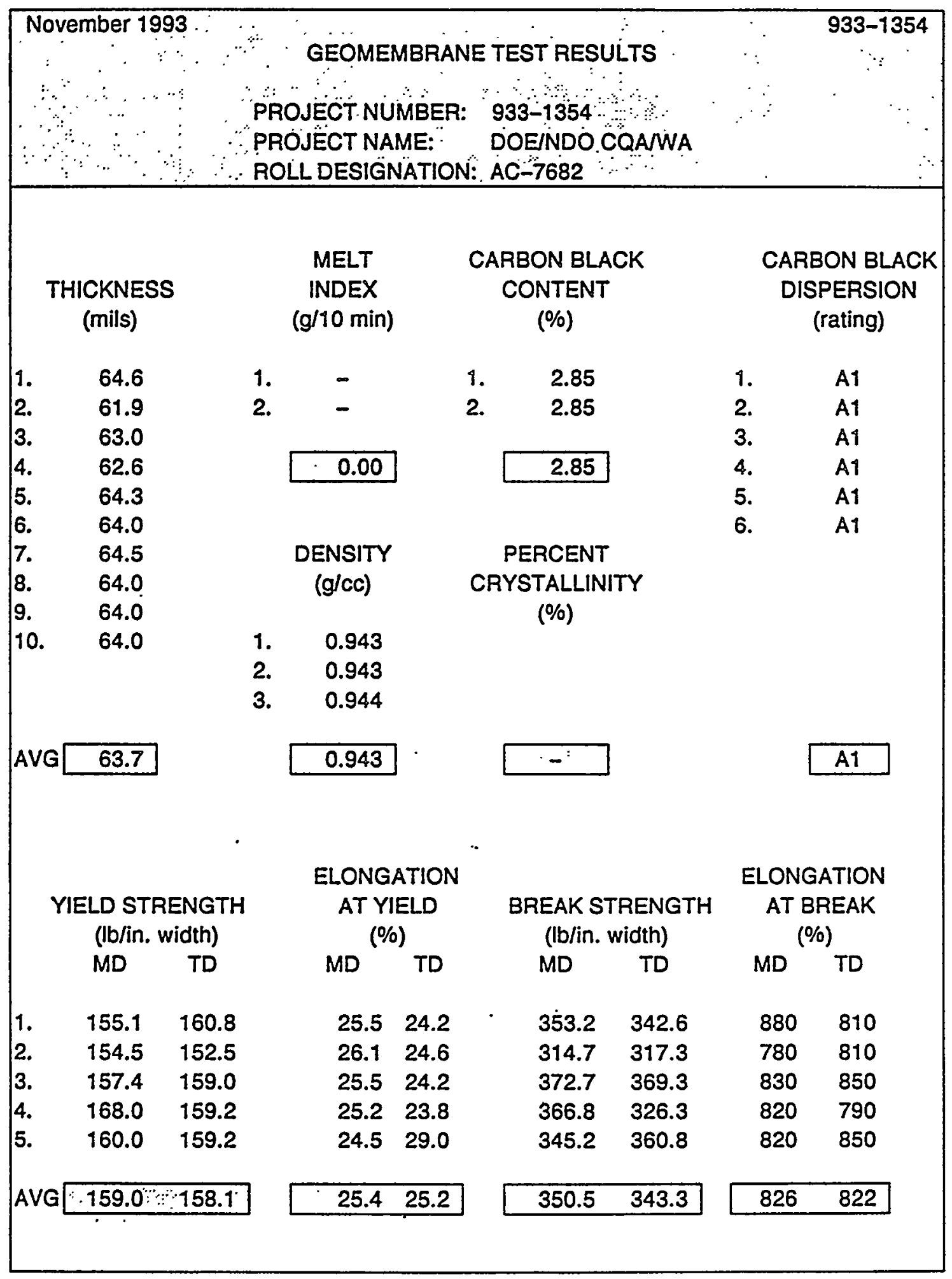


WHC-SD-W025-RPT-001, Rev. 0

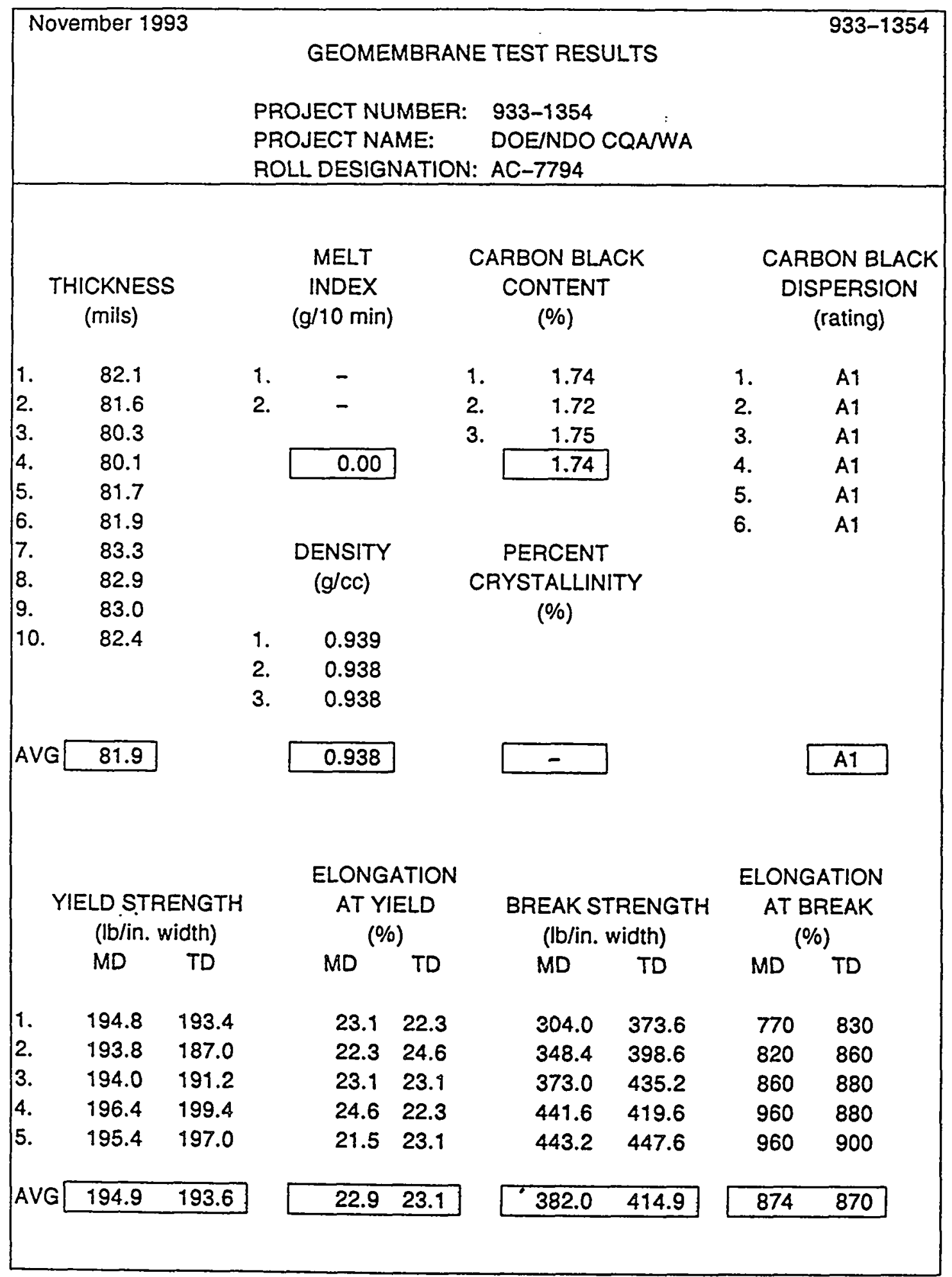




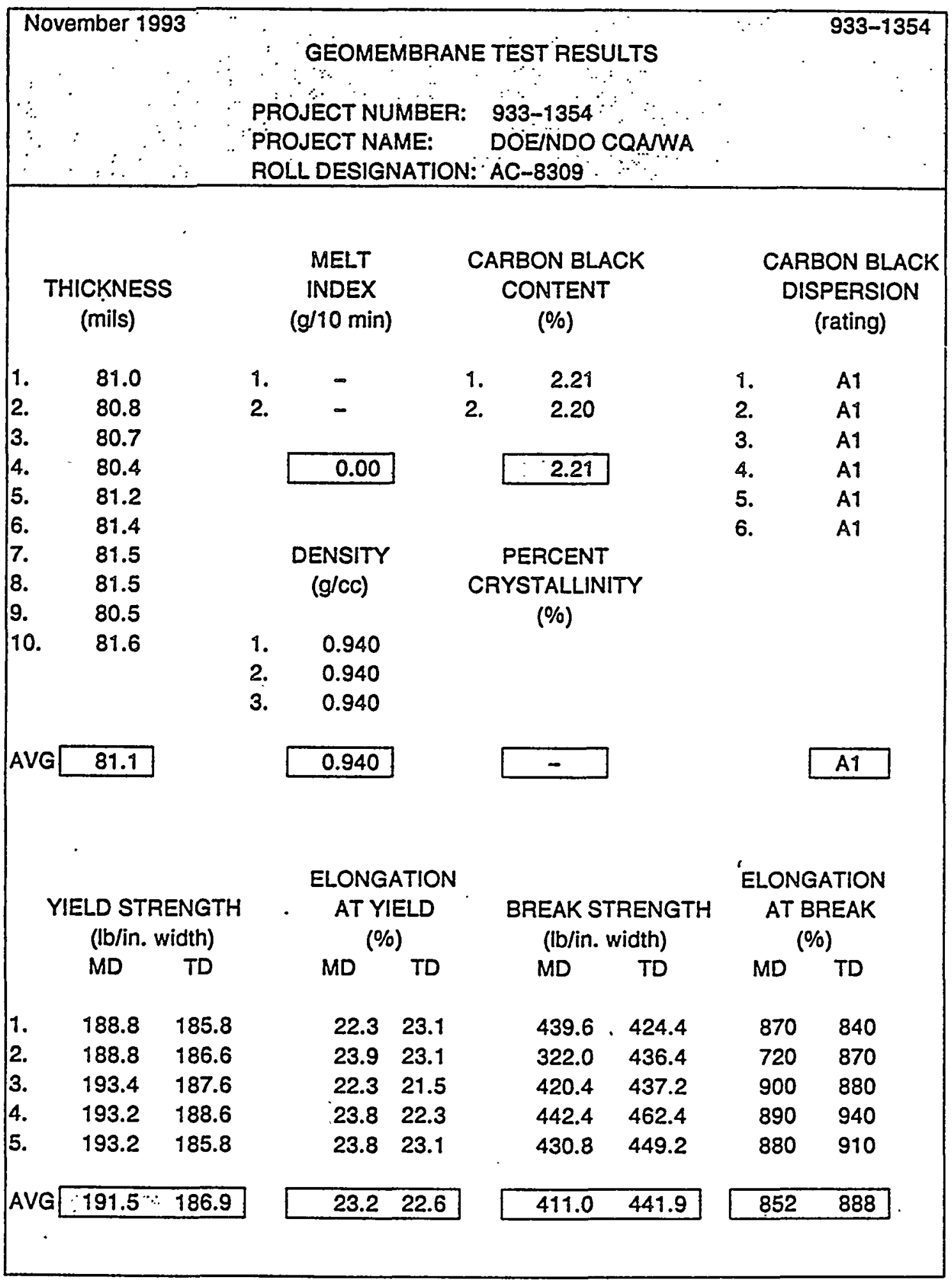


WHC-SD-W025-RPT-001, Rev. 0

\begin{tabular}{|c|c|c|c|c|c|c|c|c|}
\hline \multirow{2}{*}{\multicolumn{3}{|c|}{ December 1993}} & \multicolumn{4}{|c|}{ GEOMEMBRANE TEST RESULTS } & \multicolumn{2}{|r|}{$933-1354$} \\
\hline & & & \multicolumn{2}{|c|}{$\begin{array}{l}\text { PROJECT NUMBER: } \\
\text { PROJECT NAME: } \\
\text { ROLLDESIGNATION: }\end{array}$} & $\begin{array}{r}933-1354 \\
\text { DOEINDO } \\
\text { I: AC-8388 }\end{array}$ & QAWA & & \\
\hline \multicolumn{3}{|c|}{$\begin{array}{l}\text { THICKNESS } \\
\text { (mils) }\end{array}$} & \multicolumn{2}{|l|}{$\begin{array}{c}\text { MELT } \\
\text { INDEX } \\
(g / 10 \mathrm{~min})\end{array}$} & \multicolumn{2}{|c|}{$\begin{array}{c}\text { CARBON BLACK } \\
\text { CONTENT } \\
(\%)\end{array}$} & \multicolumn{2}{|c|}{$\begin{array}{c}\text { CARBON BLACK } \\
\text { DISPERSION } \\
\text { (rating) }\end{array}$} \\
\hline 1. & 81.6 & & 1. & & 2.53 & & 1. & A1 \\
\hline 2. & 81.6 & & 2. & & 2.55 & & 2. & A1 \\
\hline 3. & 80.8 & & & & & & 3. & A1 \\
\hline 4. & 80.3 & & 0.00 & & 2.54 & & 4. & A1 \\
\hline 5. & 81.3 & & & & & & 5. & A1 \\
\hline 6. & 81.1 & & & & & & 6. & A1 \\
\hline 7. & 80.6 & & DENSITY & & PERCENT & & & \\
\hline 8. & 80.6 & & $(g / c c)$ & & CRYSTALLIN & & & \\
\hline 9. & 81.1 & & & & $(\%)$ & & & \\
\hline \multirow[t]{3}{*}{10.} & 81.4 & & 0.942 & & & & & \\
\hline & & & 0.942 & & & & & \\
\hline & & & 0.942 & & & & & \\
\hline \multirow[t]{4}{*}{ AVG [} & 81.0 & & 0.942 & & - & & & A1 \\
\hline & \multirow{2}{*}{\multicolumn{2}{|c|}{$\begin{array}{l}\text { IELD STRENGTH } \\
\text { (lb/in. width) }\end{array}$}} & \multirow{2}{*}{\multicolumn{2}{|c|}{$\begin{array}{l}\text { ELONGATION } \\
\text { AT YIELD } \\
(\%)\end{array}$}} & \multirow{2}{*}{\multicolumn{2}{|c|}{$\begin{array}{l}\text { BREAK STRENGTH } \\
\text { (lb/in. width) }\end{array}$}} & \multirow{2}{*}{\multicolumn{2}{|c|}{$\begin{array}{l}\text { ELONGATION } \\
\text { AT BREAK } \\
(\%)\end{array}$}} \\
\hline & & & & & & & & \\
\hline & $M D$ & TD & $M D$ & TD & MD & TD & $M D$ & TD \\
\hline 1. & 200.0 & 195.6 & 26.6 & 27.2 & 402.4 & 387.9 & 810 & 800 \\
\hline 2. & 198.3 & 197.4 & 25.8 & 28.2 & 444.4 & 448.8 & 880 & 880 \\
\hline 3. & 205.1 & 198.4 & 27.2 & 29.0 & 426.0 & 447.2 & 840 & 870 \\
\hline 4. & 199.8 & 194.8 & 28.1 & 28.4 & 444.4 & 323.0 & 860 & 780 \\
\hline 5. & 198.6 & 193.0 & 28.2 & 27.3 & 479.2 & 447.2 & 880 & 920 \\
\hline AVG & 200.4 & 195.8 & 27.2 & 28.0 & 437.7 & 410.8 & 854 & 850 \\
\hline
\end{tabular}




\section{\begin{tabular}{|l|l|l|}
\hline SOIL ADMIX/TEXTURED HDPE ROLL 6898 & Figuro
\end{tabular}}

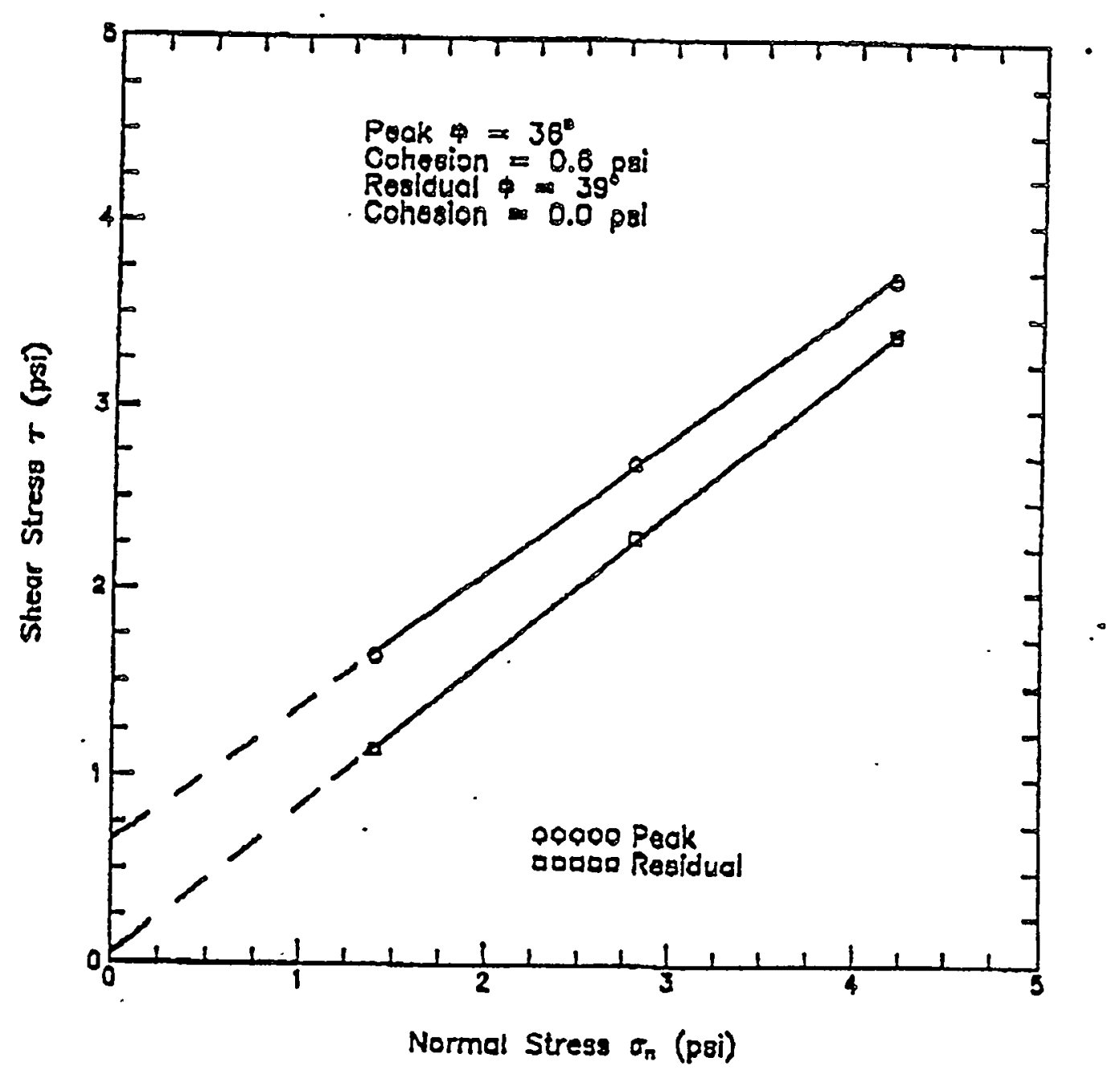


WHC-SD-W025-RPT-001, Rev. 0

Figuro
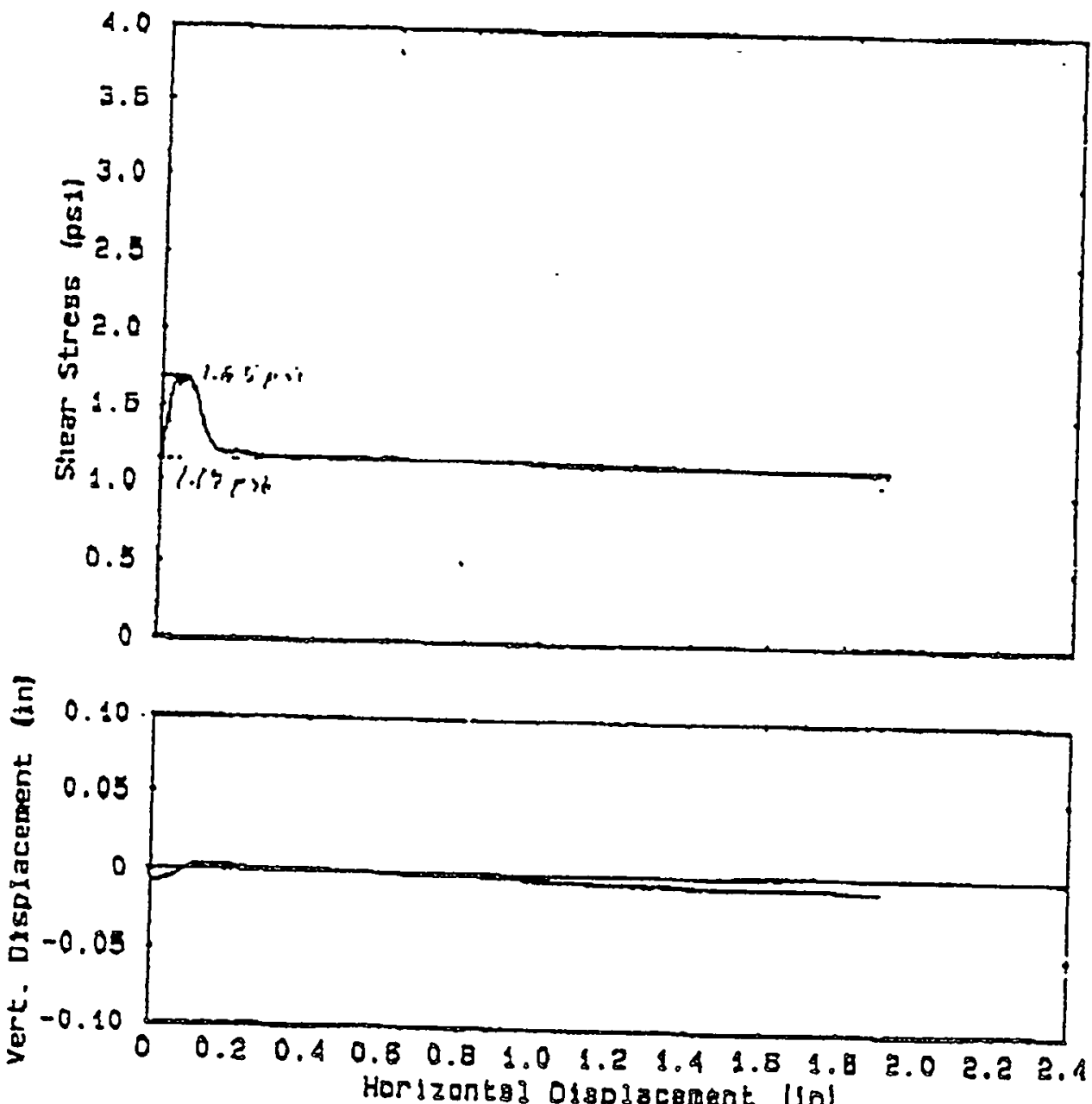

Direct Shear Test

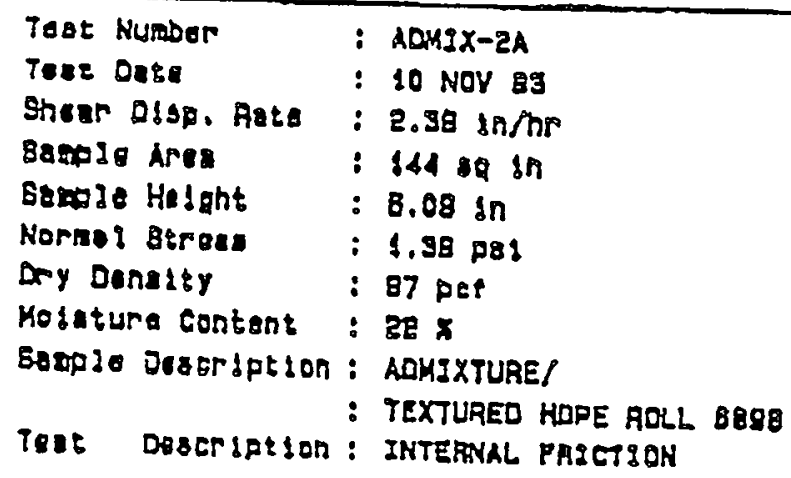


WHC-SD-W025-RPT-001, Rev。 0

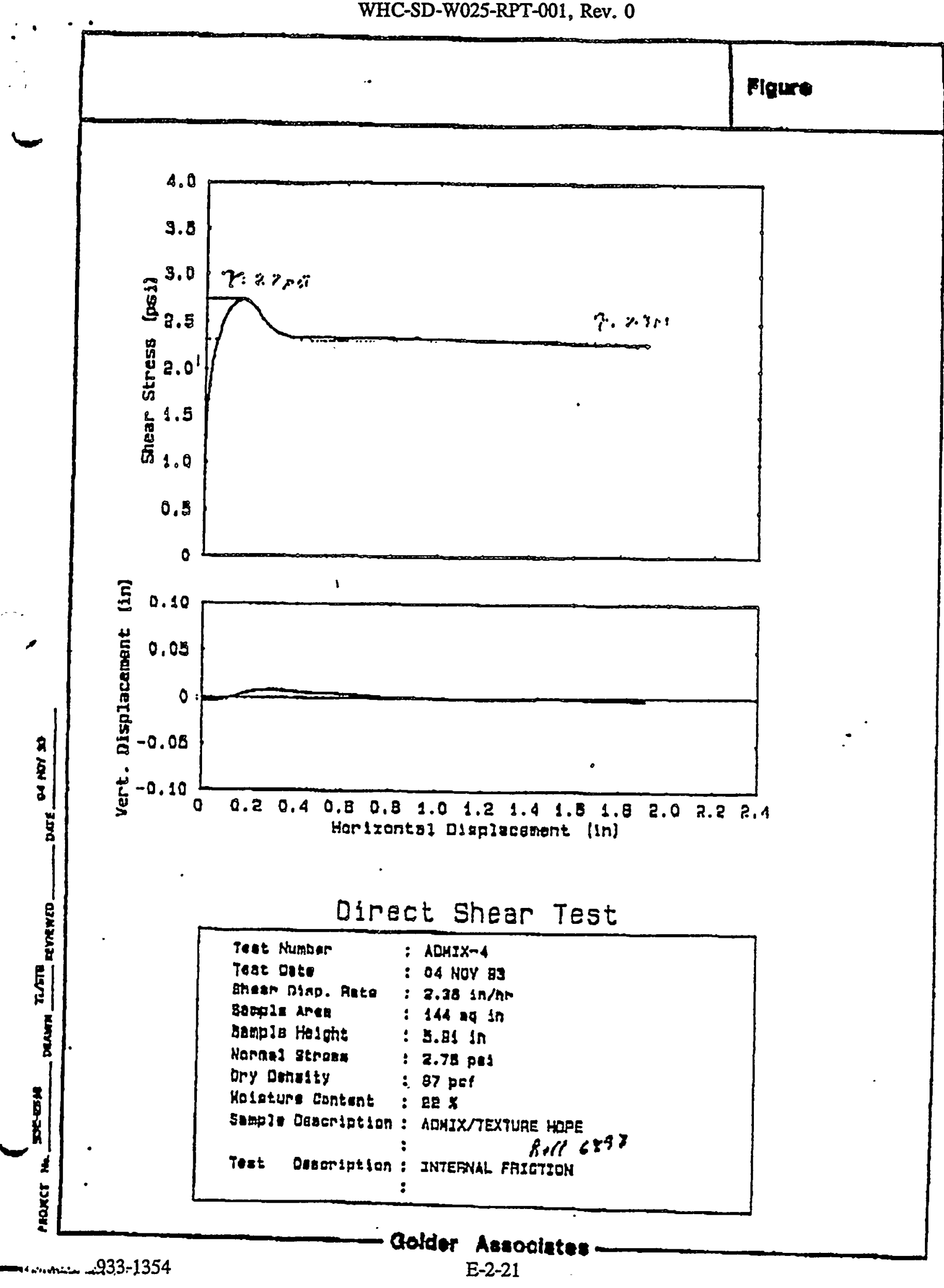




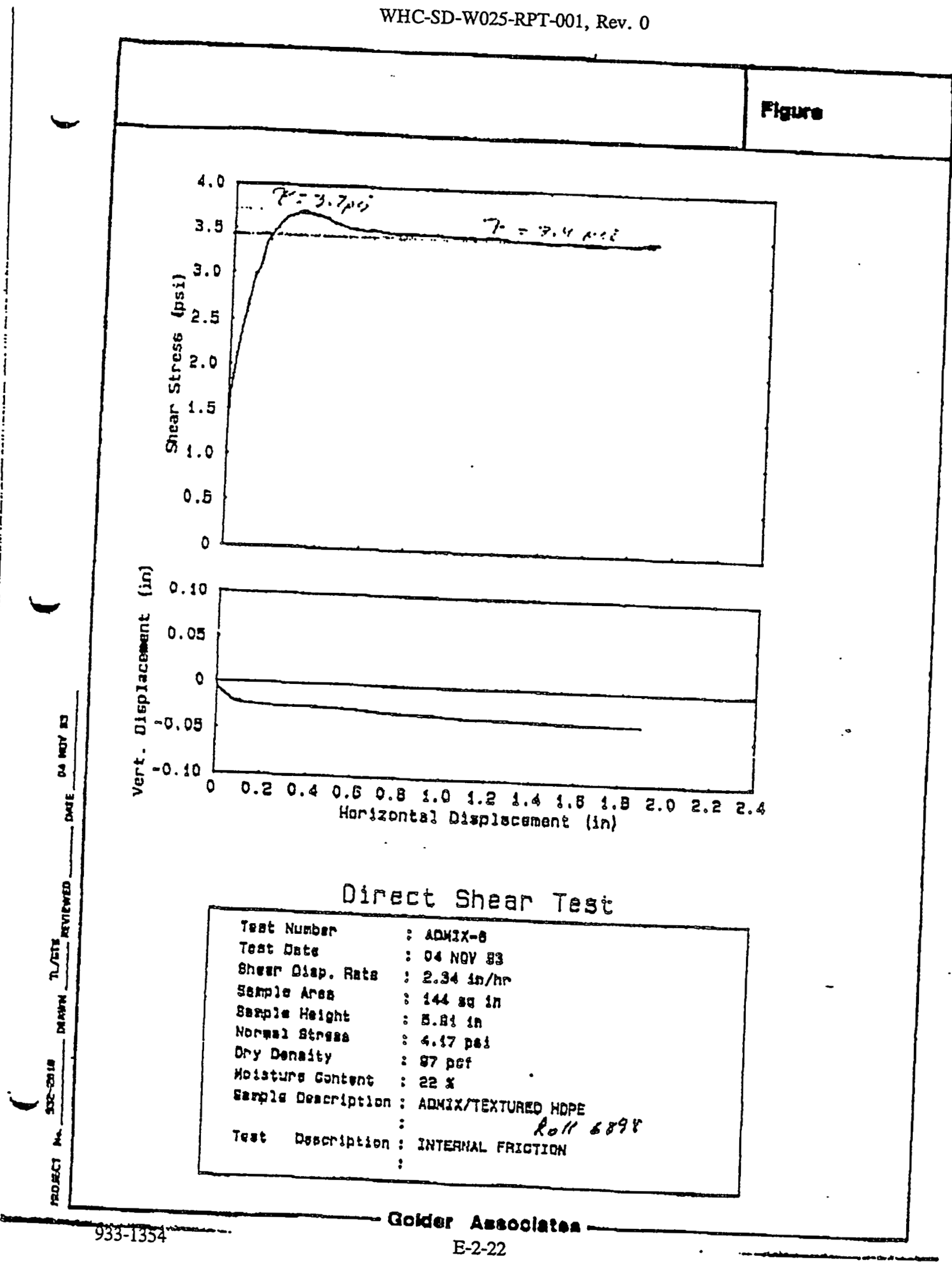




\section{SOIL ADMIX/TEXTURED HDPE ROLL 6916}

\section{Flopro}

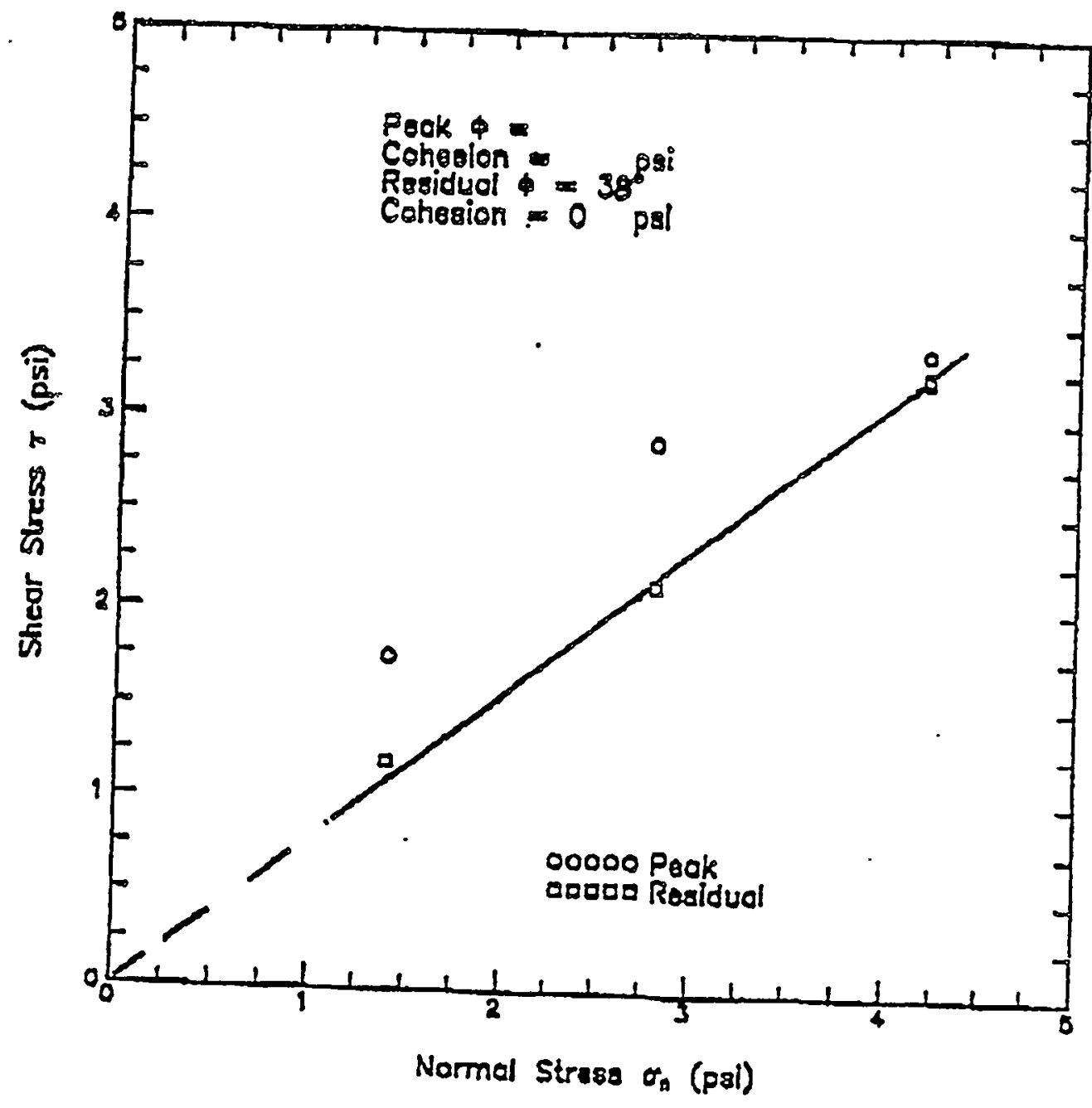


WHC-SD-W025-RPT-001, Rev. 0

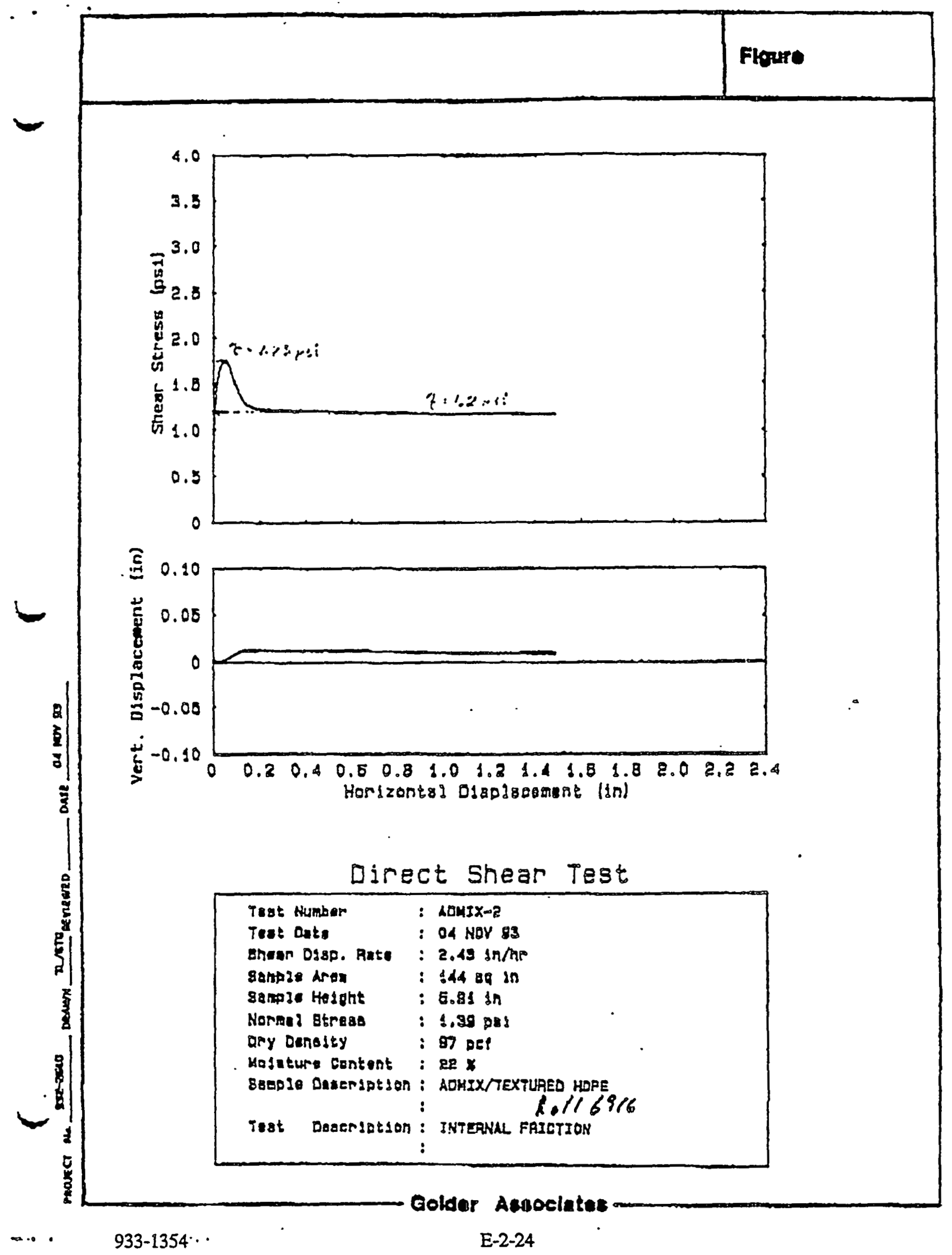




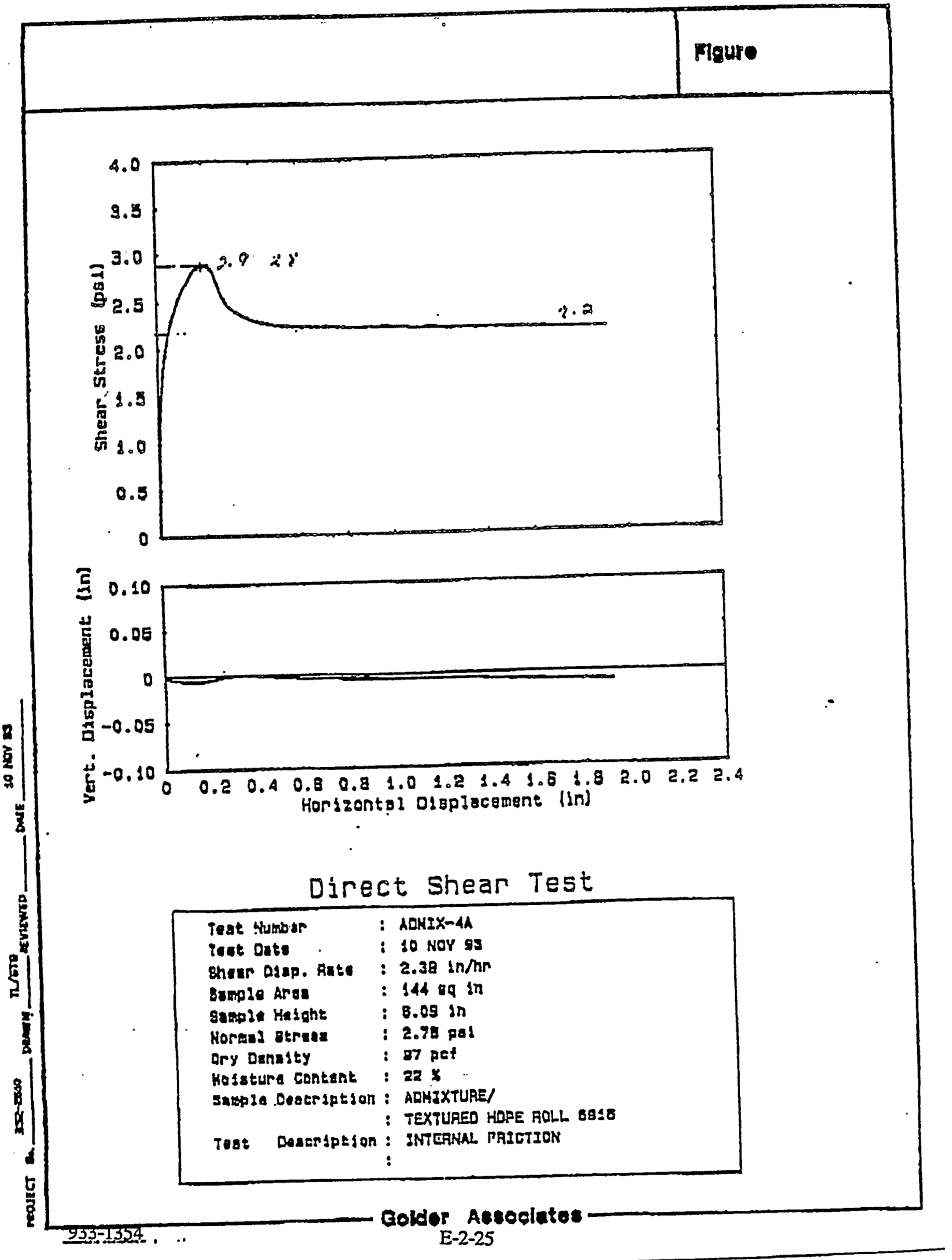




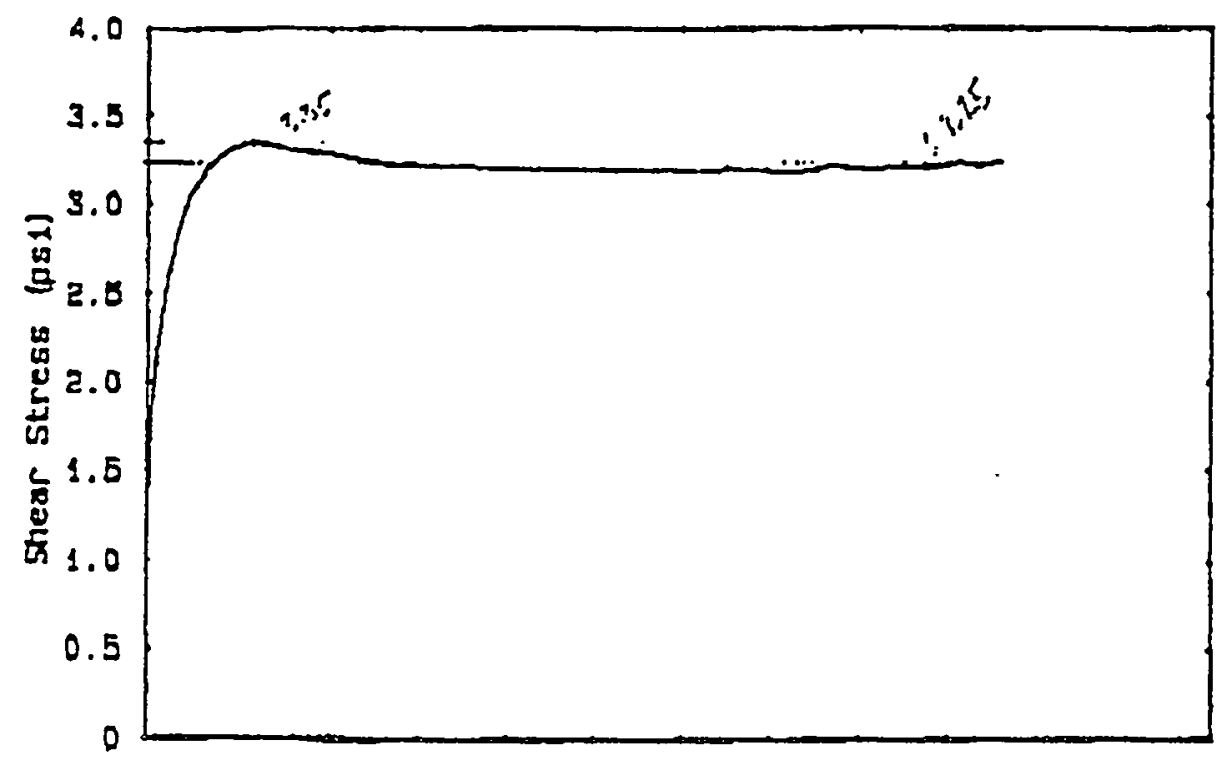

E

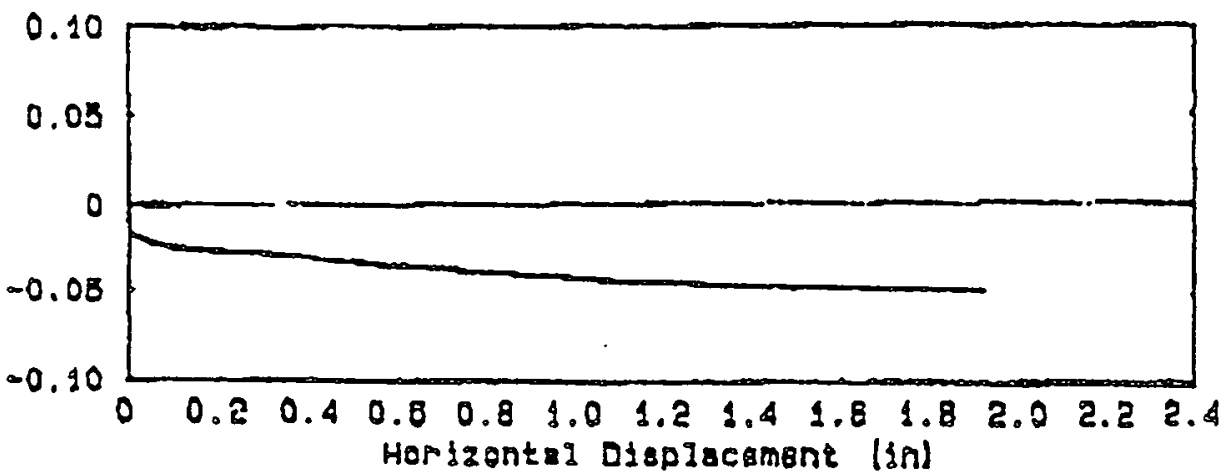

\section{Direct Shear Test}

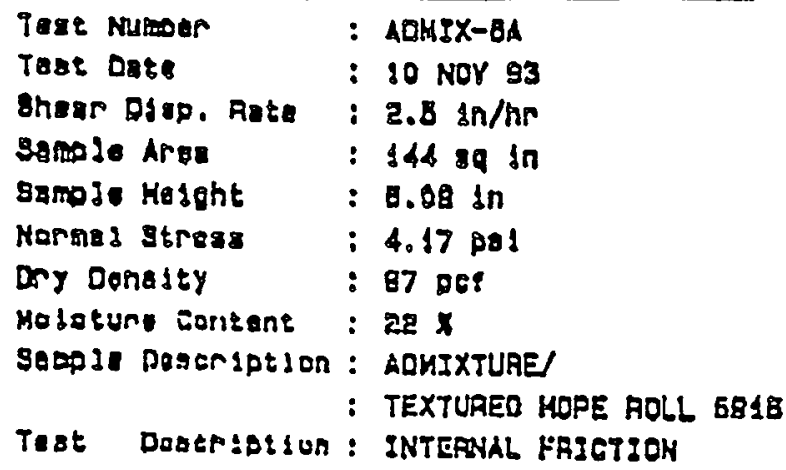


WHC-SD-H025-RPT-001, Rev。 0

\begin{abstract}
APPENDIX E-3
MANUFACTURER'S AND INSTALLER'S QC DOCUMENTATION
\end{abstract}




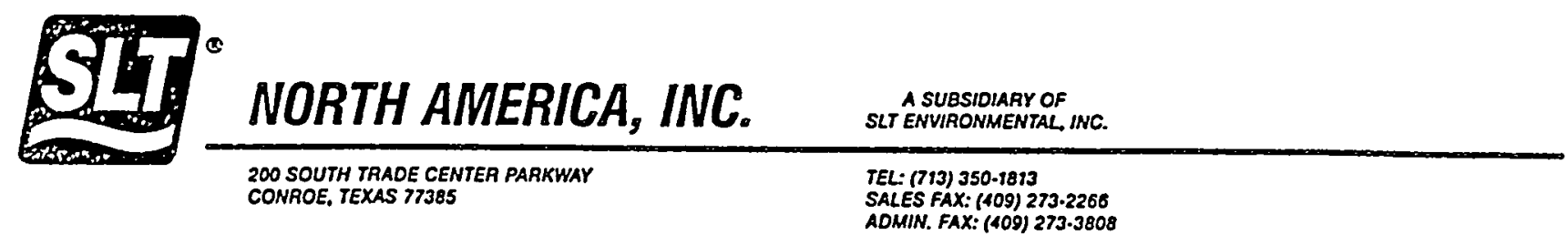

September 24, 1993

GeoPacific Lining Inc.

1300 Dexter Ave. North, Suite 250

Seattle, Washington 98109

ATTN: El Haluschak

RE: $\quad$ D.O.E Hanford

Radioactive Waste Disposal Facility

SLT Project No. 93-8491

Regarding comment 1 of the specification review by Golder Associates, Inc. for submittal 25 dated September 21,1993, a value of $45-50 \%$ for the crystallinity of the HDPE resin rather than $50-60 \%$ will not effect the mechanical properties, chemical resistance or the life expectancy of the material.

\section{Crystallinity}

In order to see a significant effect on material properties the difference in crystallinity must be greater than $15 \%$. As crystallinity decreases elongation, chemical resistance and environmental stress crack resistance increases; in turn, the tensile strength decreases slightly. Once again, the difference in crystallinity must be greater than $15 \%$ for any significant effect to be of concern.

The measurement of crystallinity can be determined in basically three different manners: (a) DSC - Differential Scanning Calorimetry, (b) Calculation based on density or (c) X-ray diffraction. The values provided by SLT North America, Inc. were determined using the DSC method which cannot measure the complete content of crystallites in the material.

This method consists of heating the sample over a temperature range of $50^{\circ} \mathrm{C}$ to $200^{\circ} \mathrm{C}$ the crystallinity is determined by integrating the peak area of the DSC curve

For environmental lining solutions...the world comes to SLT. ${ }^{\circ}$ 
GeoPacific Lining Inc.

9/24/93

Page 2

generated. Polyethylene has some small crystals which melt at room temperature $\left(22.7^{\circ} \mathrm{C}\right)$ and therefore, are not included in the value derived from this method. The following is the equation used:

$$
\mathrm{K}=\left(\Delta \mathrm{H} / \Delta \mathrm{H}_{\mathrm{K}}\right) * 100 \quad[\mathrm{~J} / \mathrm{g}] /[\mathrm{J} / \mathrm{g}]
$$

Where $\Delta \mathrm{H}_{\mathrm{K}}$ is the theoretical enthalpy of fusion for $100 \%$ crystalline polyethylene. In addition to small crystals not being measured, the theoretical value for enthalpy of fusion (heat of fusion) can be chosen from values ranging from 55 to $70 \mathrm{cal} / \mathrm{g}$ leading to varied results which are all considered legitimate.

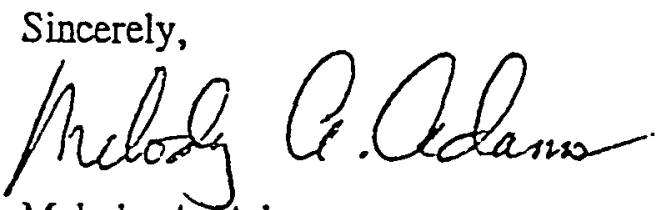

Melody A. Adams

Engineer

maa 


\begin{tabular}{|c|c|c|c|c|}
\hline $\begin{array}{l}\text { SLT } \\
\text { Hanfo } \\
\text { SL }\end{array}$ & $\begin{array}{l}\text { orth America, } \\
\text { d Radioactive Was } \\
\text { Project No. } 8491 \\
\text { loil No. AC6898 } \\
\text { - Test Method }\end{array}$ & Value & Units & 04-Nov-93 \\
\hline $\begin{array}{l}\text { Density } \\
\text { Crystallinity } \\
\text { Low Load Meit Flow (D) } \\
\text { High Load Melt Flow (IS) } \\
\text { Melt Flow Ratio }\end{array}$ & $\begin{array}{l}\text { ASTM D 792 } \\
\text { ASTM D } 1238 \mathrm{E} \\
\text { ASTM D } 1238 \mathrm{E} \\
\text { ASTM D } 1238 \mathrm{E}\end{array}$ & $\begin{array}{c}0.945 \\
35.96 \\
0.499 \\
1.494 \\
3.0\end{array}$ & $\begin{array}{c}{[g / c \infty]} \\
{[g]} \\
{[g / 10 \mathrm{~min}]} \\
{[g / 10 \mathrm{~min}]}\end{array}$ & \\
\hline
\end{tabular}

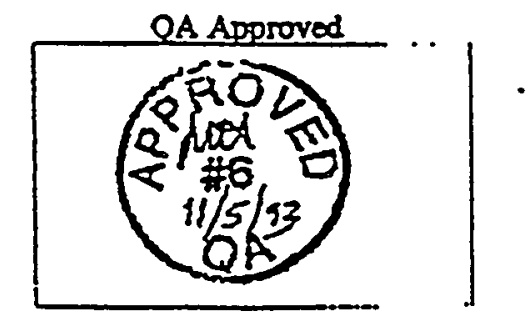

Acess 2183 
WHC-SD-W025-RPT-001, Rev. 0

进

NORTH AMERICA, INC.

A SUBSIDIAAY OF SLTENVIRONMENTAL INC.

200 SOUTH TRADE CENTER PARKWAY

TEL: (713) 350-1813

CONROE, TEXAS 77305

TEL: (713) 350.1813
SALES FAX: (409) 273.2268 ADWIN. FAX. (109) 273.3808

November 19, 1993

TO: El Haluschak

GeoPacific Lining, Inc.

FROM: Melody Adams $\mu$ it

SLT North America, Inc.

RE: Crystallinity Test Results - Hanford

Recently we supplied test results for crystallinity of our HDPE liner specifically roll number AC6898. The result was $35.96 \%$ which did not meet the required $45-50 \%$ as accepted by GeoPacific. If you would reference the letter I sent dated September 24 of this year which discussed crystallinity and how it is determined. I had noted that the theoretical $100 \%$ crystalline value used in calculating crystallinity was in fact a range, 55 to $70 \mathrm{cal} / \mathrm{g}$ (230 to $293 \mathrm{~J} / \mathrm{g}$ ). When the test was performed here in the lab, the technician who made the calculation was new and was not aware of the range of constants that are used. Therefore, we ran two more tests and came up with a range of acceptable values:

$$
\begin{array}{r}
K=36.2 \text { to } 45.7 \% \\
35.2 \text { to } 44.8 \%
\end{array}
$$

The constant used to calculate the original value of $35.96 \%$ was $290 \mathrm{~J} / \mathrm{g}$ so the other end of the range will be $45.34 \%$ based on $230 \mathrm{~J} / \mathrm{g}$. Therefore, depending on what constant is used for the heat of fusion for $100 \%$ crystalline material you will obtain a range of values that are all acceptable.

If you have any questions concerning this explanation please feel free to call me at (713)847-9188. I apologize for the delay in this response. 


\section{SL} NORTH AMERICA, INC.

A SUBSIOIARY OF SLT ENMRONMENTAL, IHC.

200 SOUTH TRADE CENTER PARKWAY

TEL: (7I3) J50.1813
SALES FAX: $(409) 273.2260$

CONAOE, TEXAS 77385

AOUIN. FAX: (409) 273.380 .

November 19, 1993

GeoPacific Lining, Inc.

1300 Dexter Ave. North, Siute 250

Seattle, Washington 98109

Attn: $\quad$ El Haluschak

RE: Crystallinity Test Results- Hanford

Recently we supplied test results for crystallinity of our HDPE liner specifically roll number AC6898. The result submitted was calculated using the incorrect constant for heat of fusion of a $100 \%$ crystallinity material. The constant to be used is $230 \mathrm{~J} / \mathrm{g}$ to result in a crystallinity of $45.3 \%$. We also performed two more tests on the sample roll using the same constant as stated above to get the following:

\section{$45.7 \%$ and $44.8 \%$}

If you have any questions concerning this explanation please feel free to call me at (713)$847-9188$.

Sincerely,

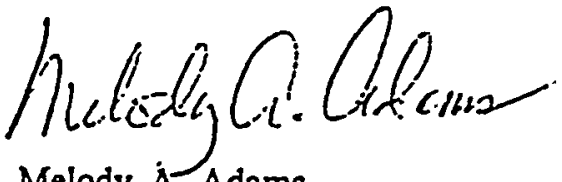

Melody A. Adams

Project Engineer/Laboratory Supervisor

For environmental lining solutions...the world comes to SLT. ${ }^{\circ}$ 
Mr. Tony Samples

DELHUR Industries, Inc.

P.0. Box 1116

Port Angeles, Washington 98362

RECEIVED

Subject: Final Report JAN 191994

Interface Direct Shear Testing

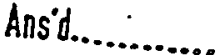

W-025 Landfill - Hanford Site

Eastern Washington

Dear Mr. Samples:

GeoSyntec Consuitants (GeoSyntec) is pleased to present the results of the interface direct shear testing program recently performed for DELHUR Industries, Inc. (DELHUR). The interface direct shear testing was conducted at GeoSyntec's Geomechanics and Environmental Laboratory in Atlanta, Georgia. This letter report was prepared by Mr. Robert H. Swan, $\mathrm{Jr}$. and $\mathrm{Dr}$. Zehong Yuan, both of GeoSyntec. The repo: \pm was reviewed by Dr. Gary R. Schmertmann, P.E. (Georgia) also of GeoSyntec, in accordance with the internal peer review policy of the firm.

The testing program was conducted in accordance with the test procedures defined in DELHUR Purchase Order No. 25818DHI, issued to GeoSyntec on 22 September 1993. GeoSyntec understands that the purpose of the testing program was to evaluate the interface shearing resistance between: (i) a soil-bentonite admix soil and 60-mil (1.5-mm) thick SLT North America, Inc. (SLT) textured high density polyethylene (HDPE) geomembrane; (ii) a SLT double-sided geocomposite and 60-mil (1.5-mm) thick SLT textured HDPE geomembrane; and (iii) an operations layer material and SLT double-sided geocomposite, using the direct shear method. The SLT double-sided geocomposite consisted of a nonwoven geotextile heat-bonded to each side of a geonet component. GeoSyntec also understands that the sample preparation procedures and testing conditions used in the testing program were selected by DELHUR based on the project specifications. The interface direct shear tests were conducted under two types of moisture conditions: (i) "as-placed" where the geomembrane was tested dry and the soil was tested under as-placed moisture conditions; and (ii) "saturated" where the entire interface was thoroughly moistened with tap water under fully submerged conditions.

\section{GL3479/GEL93300}

Corporrate Olice:

621 N.W. 53rd Street - Suire 650

Boca Raton, Florida 33487 . USA

TCl. (407) $995-0900 *$ Fax (407) 995-0925
Regional Ortices: Allanta, GA - Boca Raton, FL Columbia, MD $\bullet$ Huntington Beach, CA Walnut Creck, $\mathrm{CA} \bullet$ Brussels, Belgium

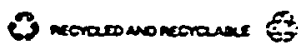
E-3-6 
Mr. Tony Samples

14 January 1994

Page 2

The remaining sections of this letter report present: (i) a description of the configuration of the test specimens used in the interface direct shear tests; (ii) the testing procedures used in the interface direct shear tests; and (iii) the test results.

\section{CONFIGURATION OF THE TEST SPECIMEN}

Three interface direct shear test series were conducted to evaluate the shearing resistance of the selected soil-geomembrane, geocompositegeomembrane, and soil-geocomposite interfaces under as-placed or saturated moisture conditions. Each test series consisted of three interface direct shear tests with each test conducted at a different level of normal stress ranging from 200 to 600 psf (10 to $29 \mathrm{kPa}$ ) using a freshly prepared test specimen. Table l summarizes the general testing conditions that were used for the interface direct shear tests. The configurations of the test specimens in the three test series were as follows:

- Test Series Number 1: interface between soil-bentonite admix soil and 60-mil (1.5-mm) thick SLT textured HDPE geomembrane. From top to bottom, each test specimen consisted of:

- soil-bentonite admix soil;

- 60-mit (1.5-mm) thick SLT textured HDPE geomembrane; and

- concrete sand.

- Test Series Number 2: interface between SLT double-sided geocomposite and 60-mi $(1.5-\mathrm{mm})$ thick SLT textured HDPE geomembrane (under saturated conditions). From top to bottom, each test specimen consisted of:

- porous rigid substrate;

- SLT double-sided geocomposite;

- 60-mit (1.5-mm) thick SLT textured HDPE geomembrane; and

- soil-bentonite admix soil. 
WHC-SD-W025-RPT-001, Rev. 0

GeoSyntec Consultants

Mr. Tony Samples

14 January 1994

Page 3

- Test Series Number 3: interface between operations layer material and SLT double-sided geocomposite (under saturated conditions). From top to bottom, each test specimen consisted of:

- operations layer material;

- SLT double-sided geocomposite; and

- soil-bentonite admix soil.

Bulk samples of the geomembrane, geocomposite, soil-bentonite admix soil, and operations layer material were provided to GeoSyntec by DELHUR. The concrete sand was provided by GeoSyntec to fill the lower shear box and serve as a bedding layer below the test interface in Test Series 1.

\section{TESTING PROCEDURES}

The interface direct shear tests were performed in general accordance with the American Society for Testing and Materials (ASTM) Standard Test Method D 5321, "Determining the Coefficient of Soil and Geosynthetic or Geosynthetic and Geosynthetic Friction by the Direct Shear Method." The tests were conducted in a large direct shear device containing an upper and lower shear box. The upper shear box measures 12 in. by $12 \mathrm{in.}(300$ mm by $300 \mathrm{~mm}$ ) in plan and $3 \mathrm{in.}(75 \mathrm{~mm})$ in depth. The lower shear box measures $12 \mathrm{in}$. by $14 \mathrm{in.}(300 \mathrm{~mm}$ by $350 \mathrm{~mm}$ ) in plan and $3 \mathrm{in} .(75 \mathrm{~mm}$ ) in depth.

A summary of the test equipment and conditions used to conduct the interface direct shear tests is presented in Table 2. This table indicates the size of the shear box, the initial compaction condition for the soil components (i.e., dry unit weight and moisture content), the normal stress applied during consolidation, time for consolidation, the moisture content of the soil at the completion of testing, the normal stress applied to the soil or rigid substrate during shearing, and the horizontal displacement rate for each test.

GL3479/GEL93300 
Mr. Tony Samples

14 January 1994

Page 4

For each test series, fresh geosynthetic specimens were used for each normal stress condition. For each test, the test specimens were set up and tested as described below to achieve the desired moisture conditions and to cause shearing to occur at the desired interface.

- Test Series Number 1: a fresh geomembrane specimen was cut from the bulk sample of geomembrane provided by DELHUR and attached to the lower shear box with mechanical compression clamps. Fresh specimens of the soil-bentonite admix soil were moistureconditioned and compacted away from the geomembrane specimen by hand tamping to the reported dry unit weight for each normal stress condition, as specified by DELHUR. The compacted admix soil specimen was then placed on the geomembrane for testing. This set-up caused shearing to occur at the interface between soi?-bentonite admix soil and geomembrane. Each test specimenwas tested under as-placed moisture conditions.

- Test Series Number 2: fresh specimens of the soil-bentonite admix soil were moisture-conditioned and compacted into the lower shear box by hand tamping to the reported dry unit weight for each normal stress condition, as specified by DELHUR. Fresh geocomposite. and geomembrane specimens were cut from the bulk samples of geosynthetic materials provided by DELHUR and attached to the upper and lower shear box, respectively, with mechanical compression clamps. This set-up caused shearing to occur at the geocomposite-geomembrane interface. For each test, the test specimen was saturated by pouring tap water into the upper shear box. The tap water flowed through the porous rigid substrate and geocomposite specimen and drained at the geocomposite-geomembrane interface. Pouring was continued until the geocomposite and geomembrane specimens were completely submerged in the tap water. The normal stress used for shearing was applied to the test specimen prior to submerging the test specimen in the tap water. Each test specimen was then immediately sheared after it was completely submerged in the tap water.

GL3479/GEL93300 
Mr. Tony Samples

14 January 1994

Page 5 .

- Test Series Number 3: fresh specimens of the soil-bentonite admix soil were moisture -conditioned and compacted into the lower shear box by hand tamping to the reported dry unit weight for each normal stress condition, as specified by DELHUR. A fresh geocomposite specimen was cut from the bulk sample of geocomposite provided by DELHUR and attached to the lower shear box with mechanical compression clamps. Fresh specimens of the operations layer material at its as-received moisture content were compacted directly on top of the geocomposite specimen by light hand tamping to the reported dry unit weight for each normal stress condition. This set-up caused shearing to occur at the interface between operations layer material and geocomposite. For each test, the test specimen was saturated by pouring :ap water on the top surface of the compacted operations layer material. The tap water flowed through the specimen and drained at the test interface. Pouring was continued until the test specimen was completely submerged in the tap water. The normal stress used for shearing was applied to the test specimen prior to submerging the test specimen in the tap water. Each test specimen was then immediately sheared after it was completely submerged in the tap water.

For each test series, the target dry unit weight and moisture content for the soil-bentonite admix soil were specified by DELHUR based on the project specifications. The target dry unit weight and moisture content for the admix soil were $97 \mathrm{lb} / \mathrm{ft}^{3}\left(15.3 \mathrm{kN} / \mathrm{m}^{3}\right)$ and 21 to 22 percent. DELHUR did not specify any target dry unit weight and moisture content conditions for the operations layer material which was used in Test Series 2 and 3. The reported values of dry unit weight for each soil specimen were determined by measuring the as-placed volume of the soil and dividing this volume into the calculated total dry weight of the moist soil specimen. 
Mr. Tony Samples

14 January 1994

Page 6

Other features of the testing procedure included the following:

- a freshly remolded 3-in. (75-mm) thick layer of concrete sand was used as a bedding layer below the test interface in Test Series 1 ; the concrete sand was compacted by hand tamping to a relatively dense state under dry conditions;

- each test specimen was sheared at a constant displacement rate immediately after application of the normal stress used for shearing;

- the direction of shear for each test was in the direction of manufacture (machine direction) of the geosynthetic samples;

- each test was performed using a constant effective sample area (i.e., the plan area of the geosynthetic specimens were larger than that of the upper shear box), therefore, no area correction was required when computing normal and shear stresses; and

- each test was sheared until a constant, residual shear load was recorded.

\section{TEST RESULTS}

The total-stress interface shearing resistance was evaluated for each applied normal stress. The test data were plotted on a graph of shear force versus horizontal displacement. The resulting plots are presented in Attachment 1 to this report. The peak value of shear force was used to calculate the peak shear strength. For this report, the residual shear strength was assumed to be equal to the stabilized, post-peak shear force measured at the end of each test.

The total-stress peak and residual shear strengths derived from the plotted test results are summarized in Table 3 . These strengths were plotted on a graph of shear stress versus normal stress and the results

\section{GL3479/GEL93300}


Mr. Tony Samples

14 January 1994

Page 7

were used to evaluate total-stress peak and residual strength envelopes. A best-fit straight 7 ine was drawn through the three data points from each test series to obtain total-stress peak and residual friction angles and adhesions. The coefficient of correlation $\left(R^{2}\right)$, a standard statistical indicator of how well the best-fit line matches the test data, was obtained for each best-fit line. The plot of the shear stress versus normal stress for each test series is also presented in Attachment 1. The interface friction angles, adhesions, and $R^{2}$ values derived from the plotted test results are summarized in Table 4.

For each test series, it is noted that the reported adhesion is the shear stress axis intercept of the best-fit straight line drawn through the test data on the plot of shear stress versus normal stress. This value may not be the "true adhesion" of the interface and caution should be exercised in using this adhesion value for applications involving normal stresses outside the range of stresses covered by the test series.

The reported results apply only to the materials and test conditions used during the laboratory program. The results do not necessarily apply to other materiais or test conditions. The test resuits should not be used in engineering analyses unless the test conditions model the anticipated field conditions. The testing was performed in accordance with general engineering testing standards and requirements. This testing report is submitted for the exclusive use of DELHUR. 
WHC-SD-W025-RPT-001, Rev. 0

\section{GeoSyntec Consultants}

Mr. Tony Samples

14 January 1994

Page 8

CLOSURE

GeoSyntec appreciates the opportunity to conduct interface direct shear testing for DELHUR. If you have any questions about this report, or if you require additional information, please do not hesitate to contact any of the undersigned.

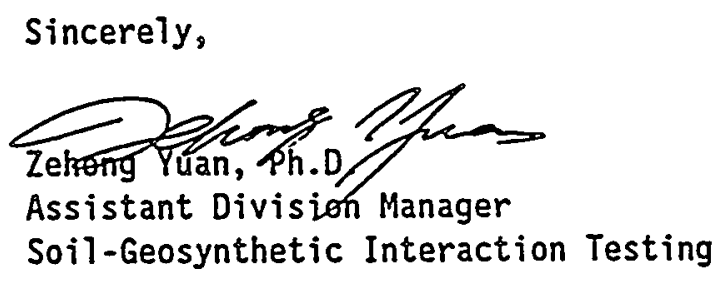

Attachments
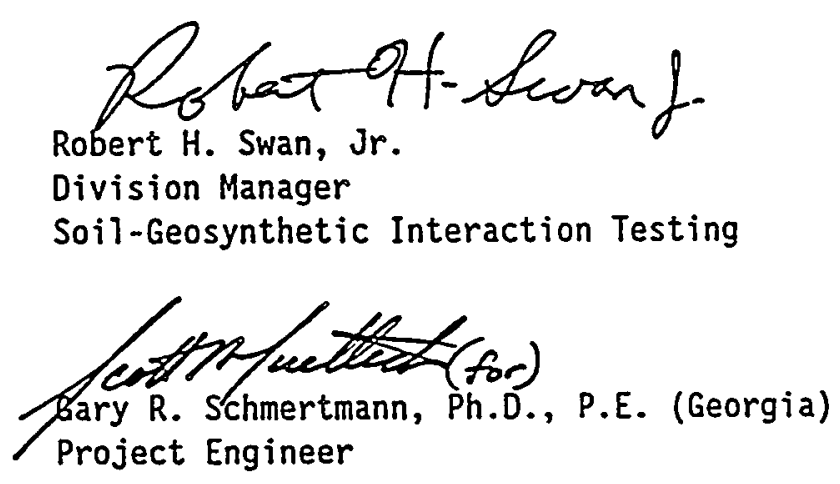

GL3479/GEL93300 
WHC-SD-W025-RPT-001, Rev. 0

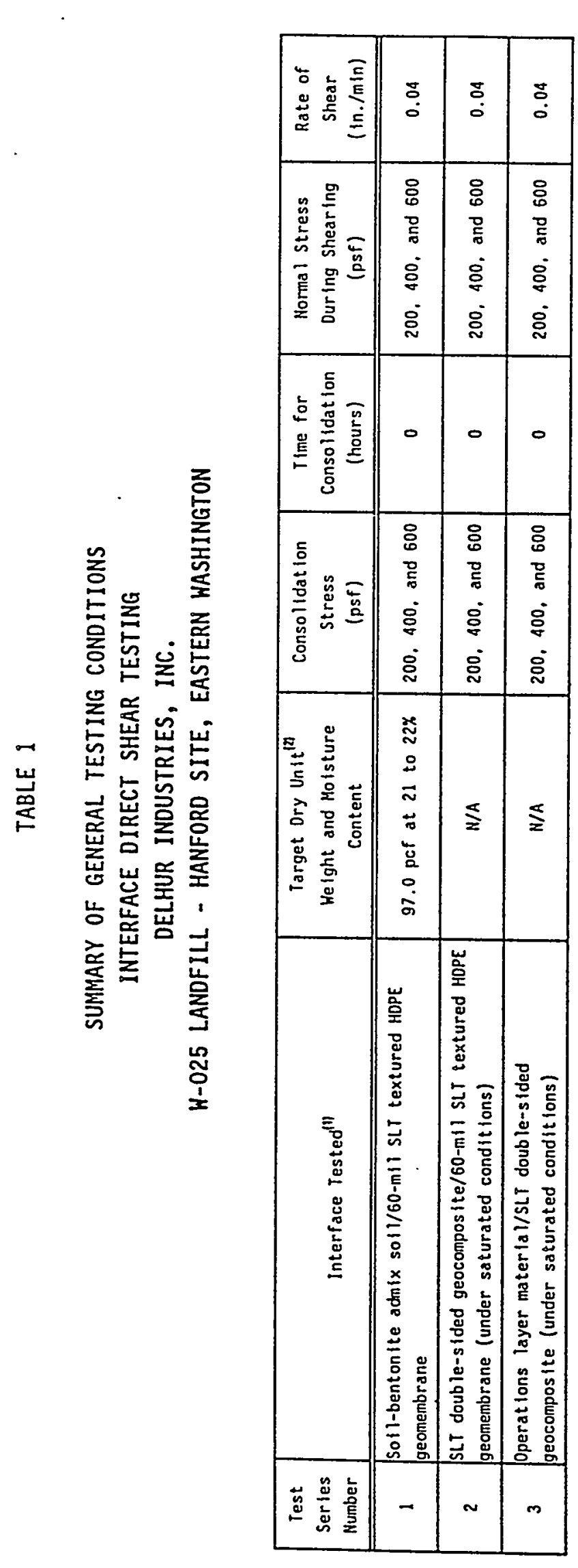

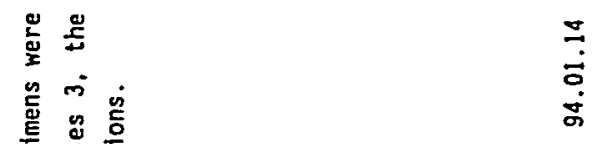

总吉泀

要要

兽京莺

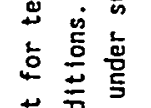

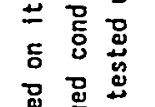

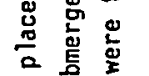

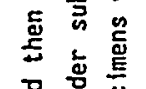

몽 홀

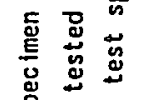

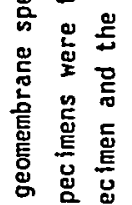

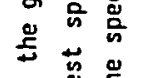

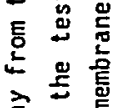

产 橧

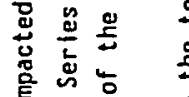

尊

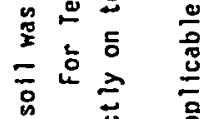

药

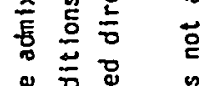

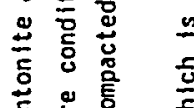

至造言

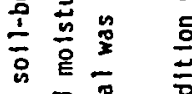

突

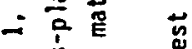

造施离

这喜要

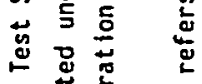

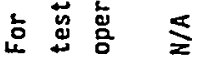

$\equiv \bar{\Xi}$

蒙

옹 
WHC-SD-W025-RPT-001, Rev. 0

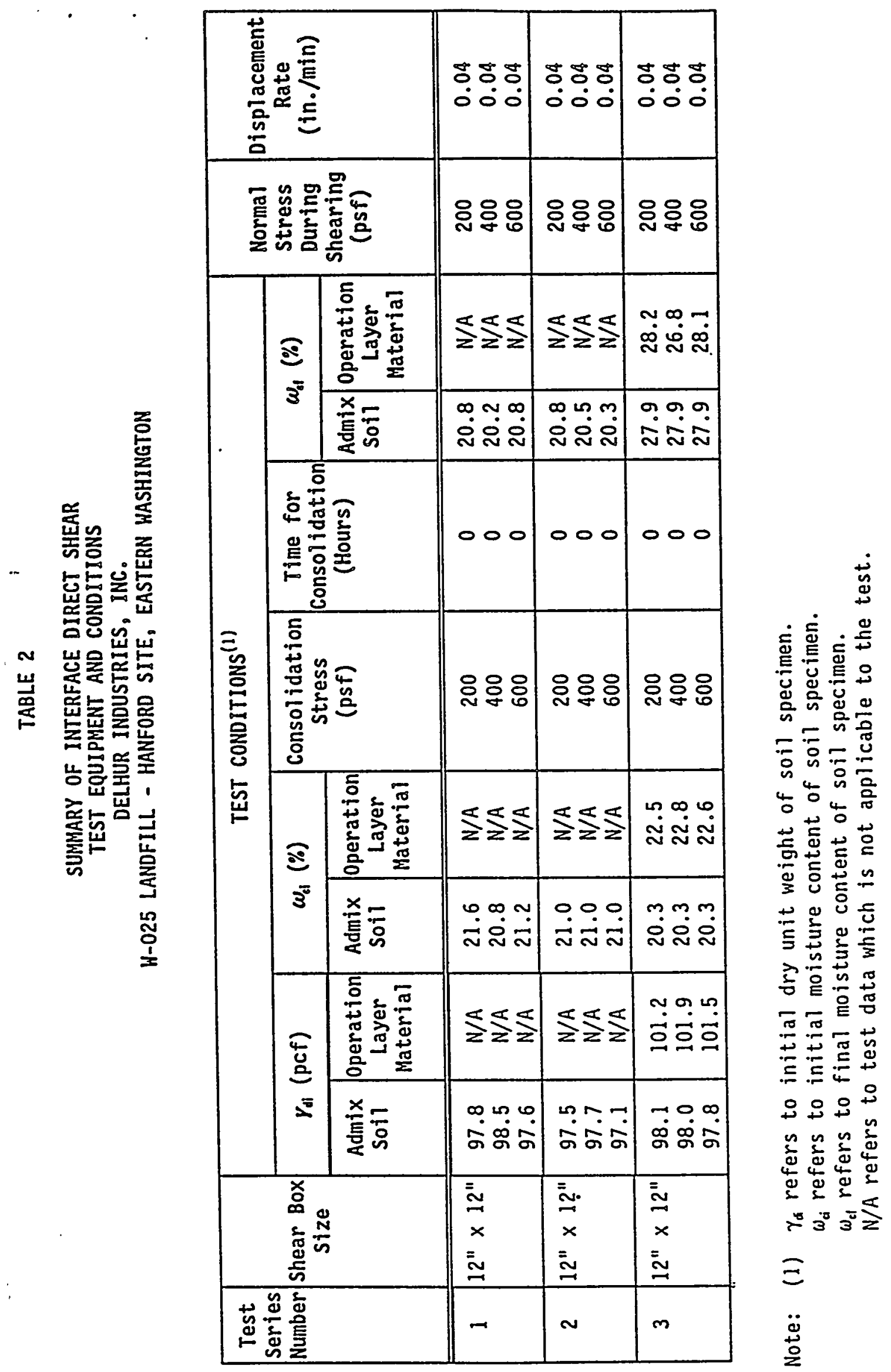


TABLE 3

INTERFACE DIRECT SHEAR TEST RESULTS

MEASURED PEAK AND RESIDUAL TOTAL SHEAR STRENGTHS

DELHUR INDUSTRIES, INC.

W-025 LANDFILL-HANFORD SITE, EASTERN WASHINGTON

\begin{tabular}{|c|c|c|c|c|}
\hline $\begin{array}{l}\text { Test } \\
\text { Series } \\
\text { Number }\end{array}$ & $\begin{array}{c}\text { Normal }^{(1)} \\
\text { Stress } \\
\text { (psf) }\end{array}$ & $\begin{array}{c}\text { Measured Peak } \\
\text { Shear Strength } \\
\text { (psf) }\end{array}$ & $\begin{array}{c}\text { Heasured Residual } \\
\text { Shear Strength } \\
\text { (psf) }\end{array}$ & $\begin{array}{c}\text { Reference } \\
\text { Appendix } \\
\text { Figure Number }\end{array}$ \\
\hline 1 & $\begin{array}{l}200 \\
400 \\
600 \\
\end{array}$ & $\begin{array}{l}225 \\
381 \\
527\end{array}$ & $\begin{array}{l}205 \\
371 \\
507\end{array}$ & $1-1$ and $1-2$ \\
\hline 2 & $\begin{array}{l}200 \\
400 \\
600\end{array}$ & $\begin{array}{l}297 \\
518 \\
684\end{array}$ & $\begin{array}{l}168 \\
255 \\
343\end{array}$ & $1-3$ and $1-4$ \\
\hline 3 & $\begin{array}{l}200 \\
400 \\
600\end{array}$ & $\begin{array}{l}172 \\
302 \\
472\end{array}$ & $\begin{array}{l}142 \\
251 \\
396\end{array}$ & $1-5$ and $1-6$ \\
\hline
\end{tabular}

Note: (1) Test specimens were sheared immediately after application of normal stress used for shearing. 
WHC-SD-W025-RPT-001, Rev. 0

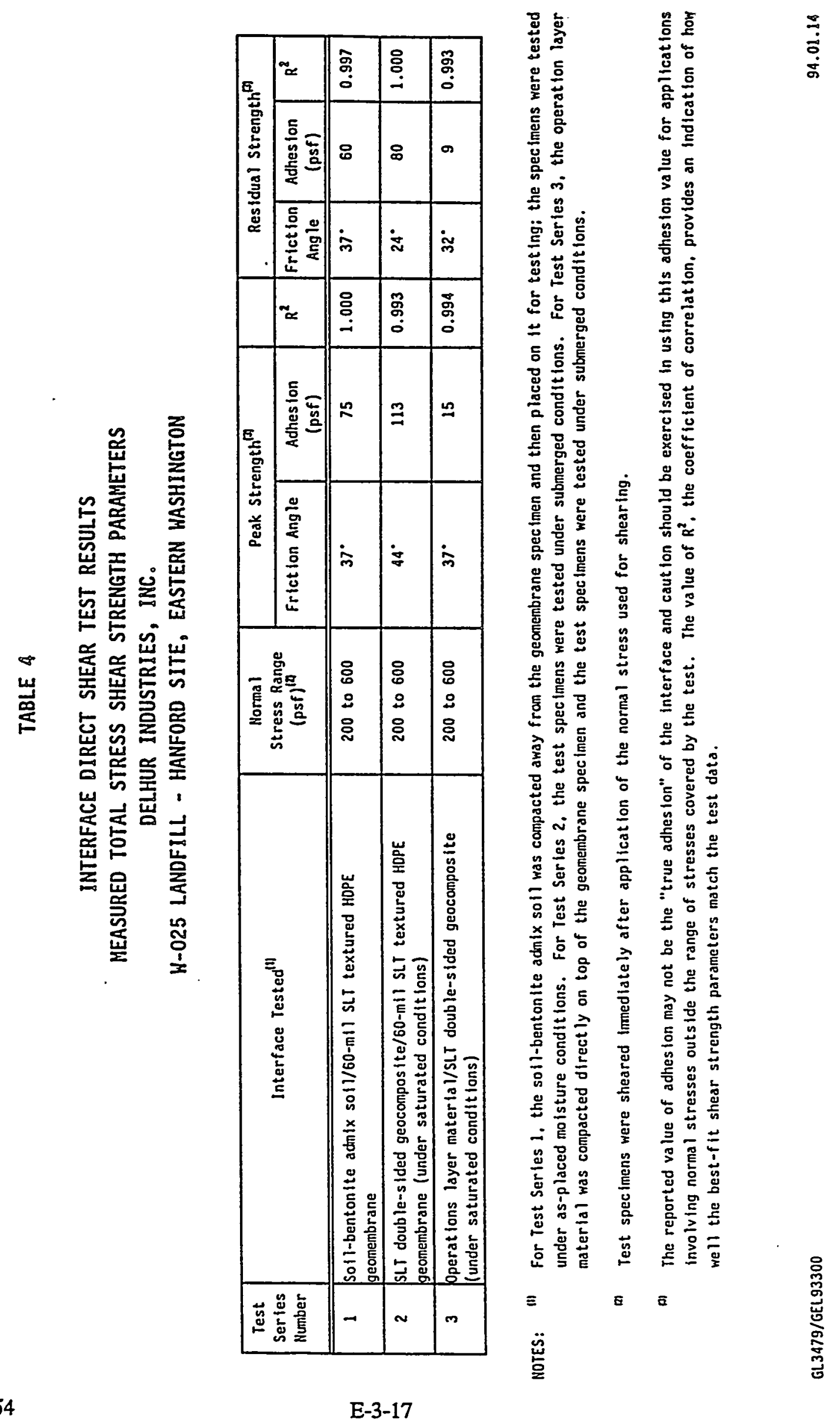


WHC-SD-W025-RPT-001, Rev. 0

\section{ATTACHMENT 1 \\ INTERFACE DIRECT SHEAR TEST RESULTS}




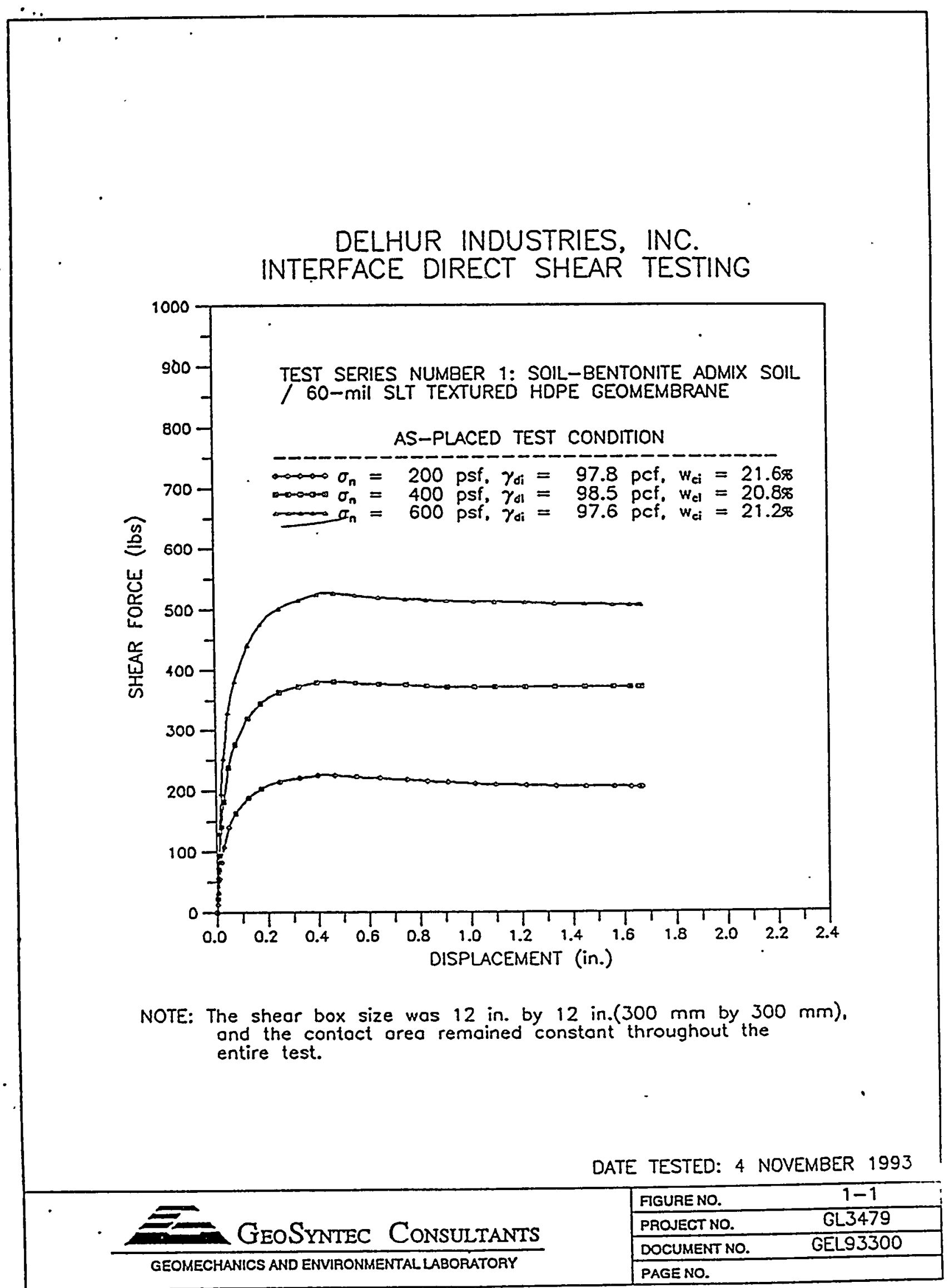




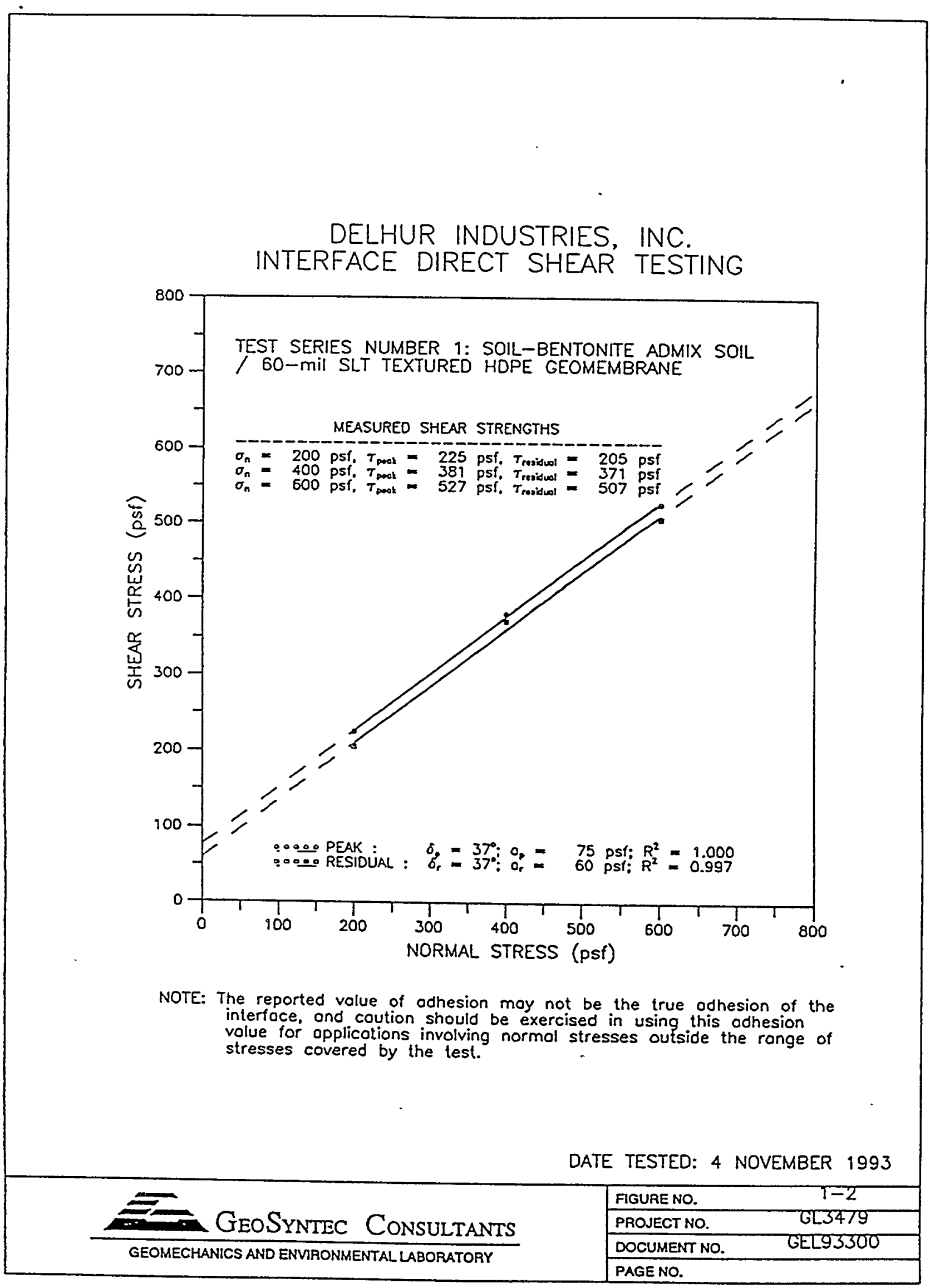




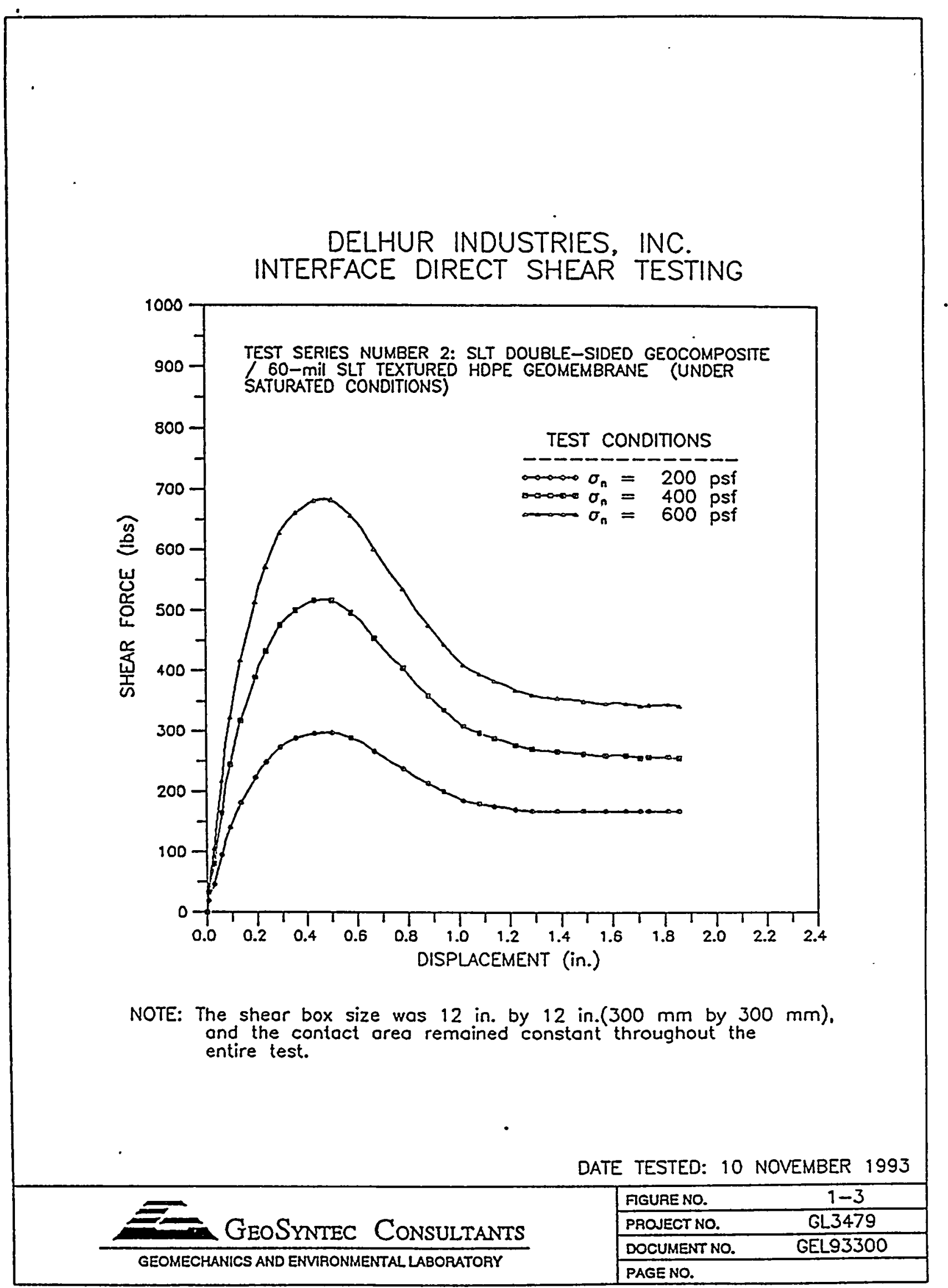




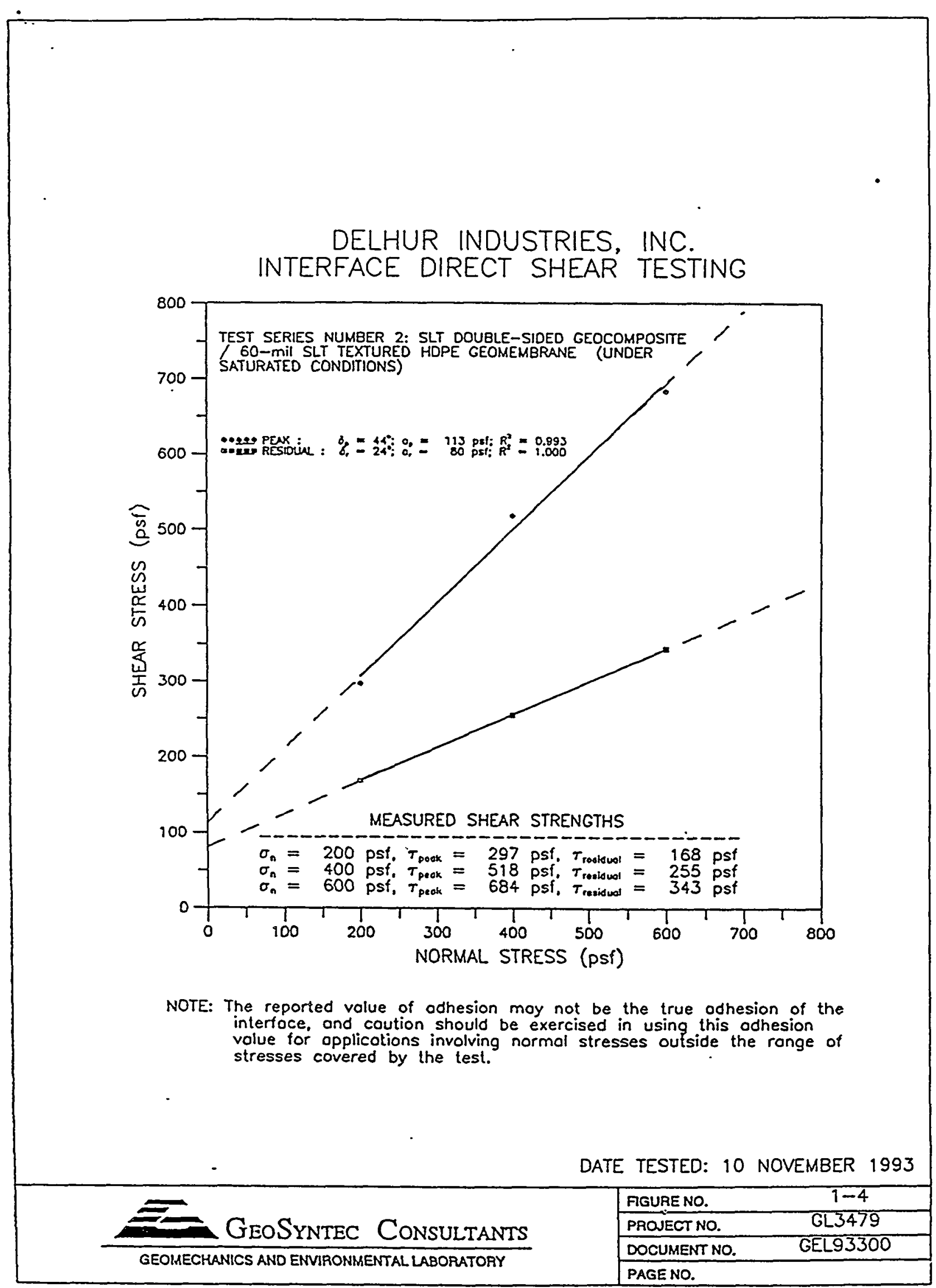




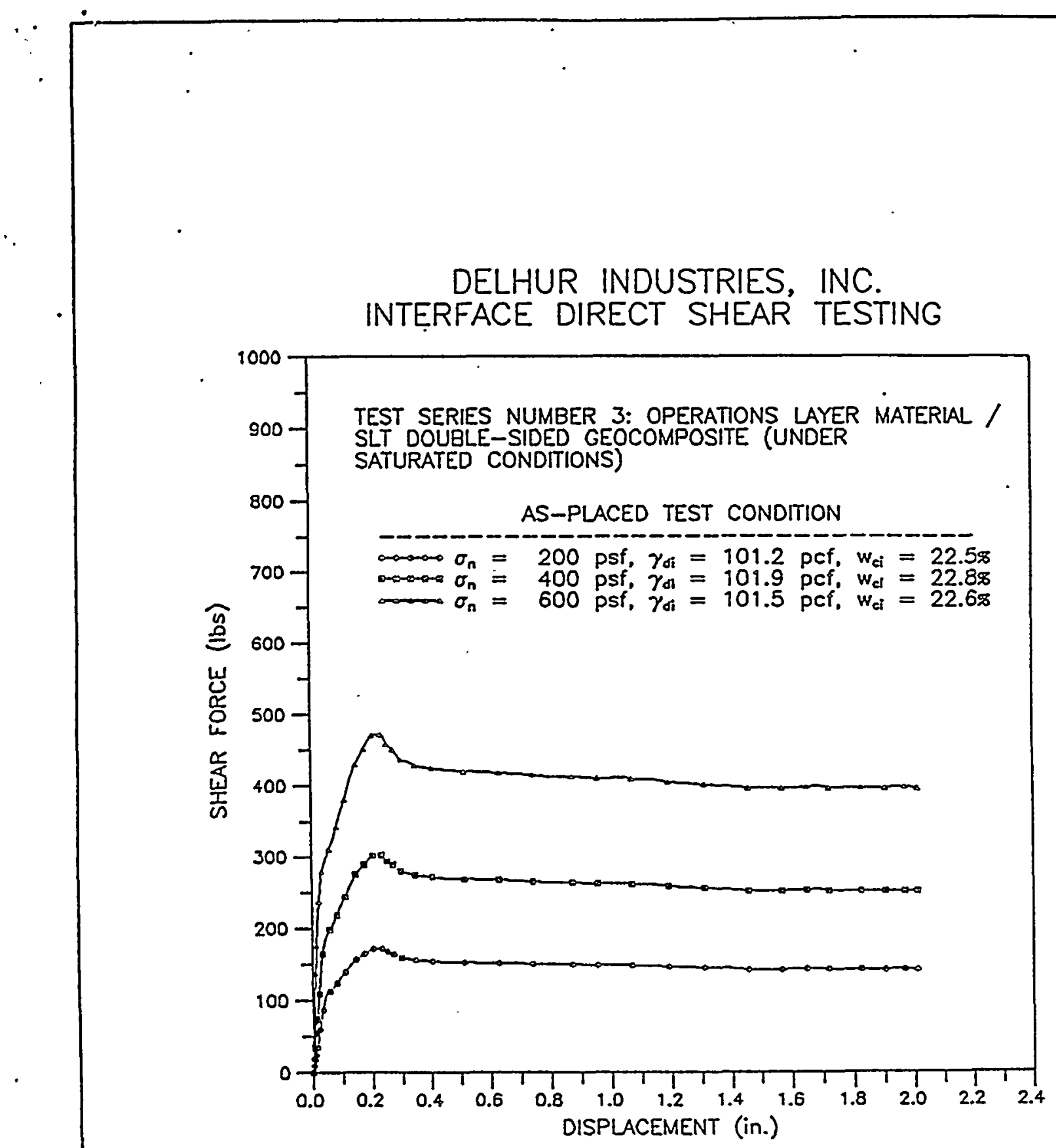

NOTE: The shear box size was 12 in. by 12 in. $(300 \mathrm{~mm}$ by $300 \mathrm{~mm}$ ), and the contact areo remoined constant throughout the entire test.

DATE TESTED: 9 NOVEMBER 1993

\begin{tabular}{|c|c|c|}
\hline \multirow{3}{*}{$=$ GeoStrntec Consultants } & FGUURE NO. & $1-5$ \\
\hline & PROJECTNOO. & GL3479 \\
\hline & DOCUMENT NO. & GEL93300 \\
\hline
\end{tabular}




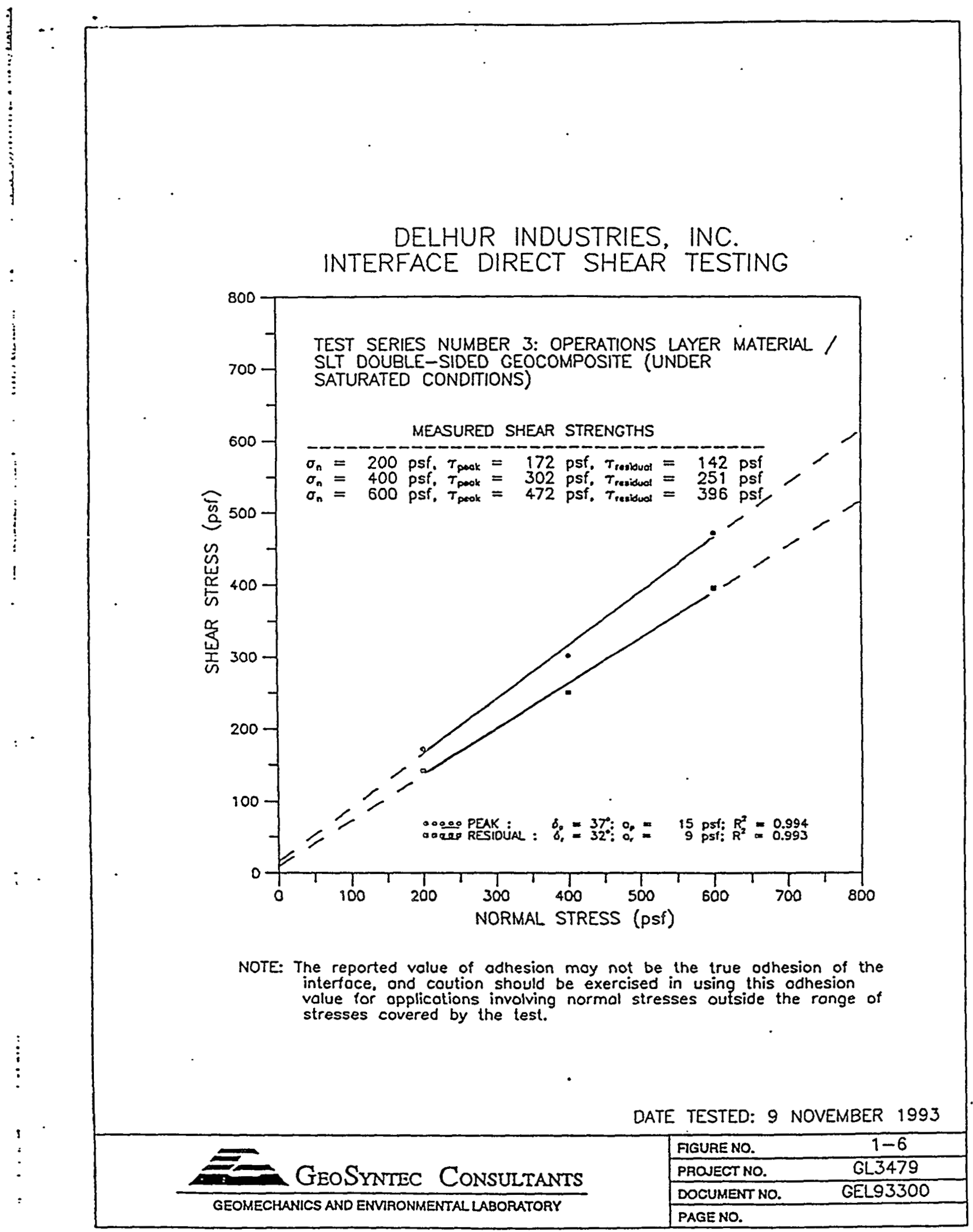


A stastonati of

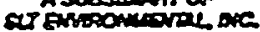

Noveonber 4. 1993

GeoPacific Lining, Ine.

1300 Dew Ave. N. 250

Seattle, Wash. 98109

Attr: El Haluschak

RE: $\quad$ D.O.E Eanford Radiozctive Waste

SLT Project No. 8491

\section{Certification}

The undersigned, being qualified and authorized to do so, hereby certifies that the SLT EyperFlex mamufactured for the above referenced project will meet or exceed the minimim specifications as listed on page $02275-4$ of the customer specifications.

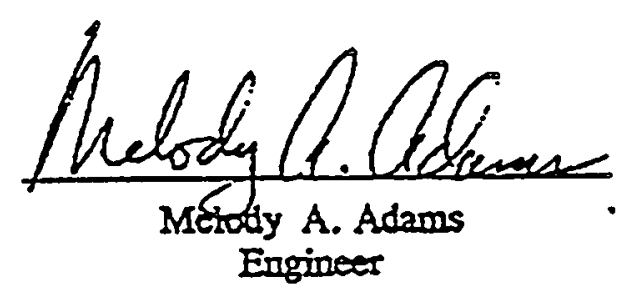




\section{$\dot{S} L \bar{T}$}

ROLL NO. AC-6893FF

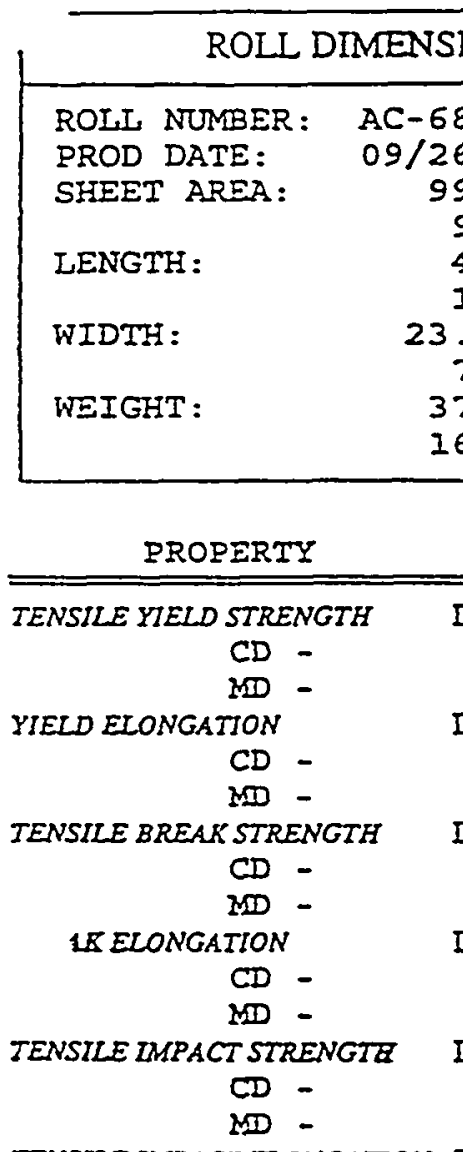

TENSILE IMPACT ELONGATION D1822 CD -

INITIAL TEARING STRENGTH CD ND -

PLNCTURE STRENGTH SHRINKAGE

$$
\text { CD - }
$$

CARBON BLACK DISPERSION

CARBON BLACK CONTENT GEOMEMBRANE DENSITY

ESCR

THICKNESS

NOMINAL

MNIMUM

AVG. THICKNESS
Roll Test Data Report

\section{Roll Test Data Report}

\section{$\%$}

WHC-SD-W025-RPT-O01, Rev. 0 
$\mathrm{LT}$

Roll Test Data Report

ROLL NO. AC-6894FF

\begin{tabular}{|l|l|}
\hline \multicolumn{3}{|c|}{ ROLL DIMENSIONS $\approx \pm 1 \%$} \\
\hline ROLL NUMBER: & AC $-6894 \mathrm{FF}$ \\
PROD DATE: & $09 / 26 / 93$ \\
SHEET AREA : & 11847 sq.ft. \\
& 1101 sq. meters \\
LENGTH: & 505 feet \\
& 154 meters \\
WIDTH: & 23.46 feet \\
WEIGHT: & 7.1 meters \\
& 3860 pounds \\
& 1751 kilograms
\end{tabular}

\begin{tabular}{|c|c|c|c|}
\hline \multicolumn{4}{|c|}{ RESIN LOT INFORMAIION } \\
\hline $\begin{array}{l}\text { LOT NUMBER } \\
\text { RESIN TYPE }\end{array}$ & $\begin{array}{l}S-40681 \\
600 \text { SERI }\end{array}$ & ES & \\
\hline PROPERTY & UNITS & METHOD & RESULT \\
\hline $\begin{array}{l}\text { DENSITY } \\
\text { MOISTURE } \\
\text { BRITTLENESS } \\
\text { MELT INDEX }\end{array}$ & $\begin{array}{l}g / c c \\
\circ \\
\circ \mathrm{C} \\
g / 10 \mathrm{~min} .\end{array}$ & $\begin{array}{l}D 792 \\
D 570 \\
D 746 \\
D 1238\end{array}$ & $\begin{array}{l}0.932 \\
<0.09 \\
<-70 \\
0.551\end{array}$ \\
\hline
\end{tabular}

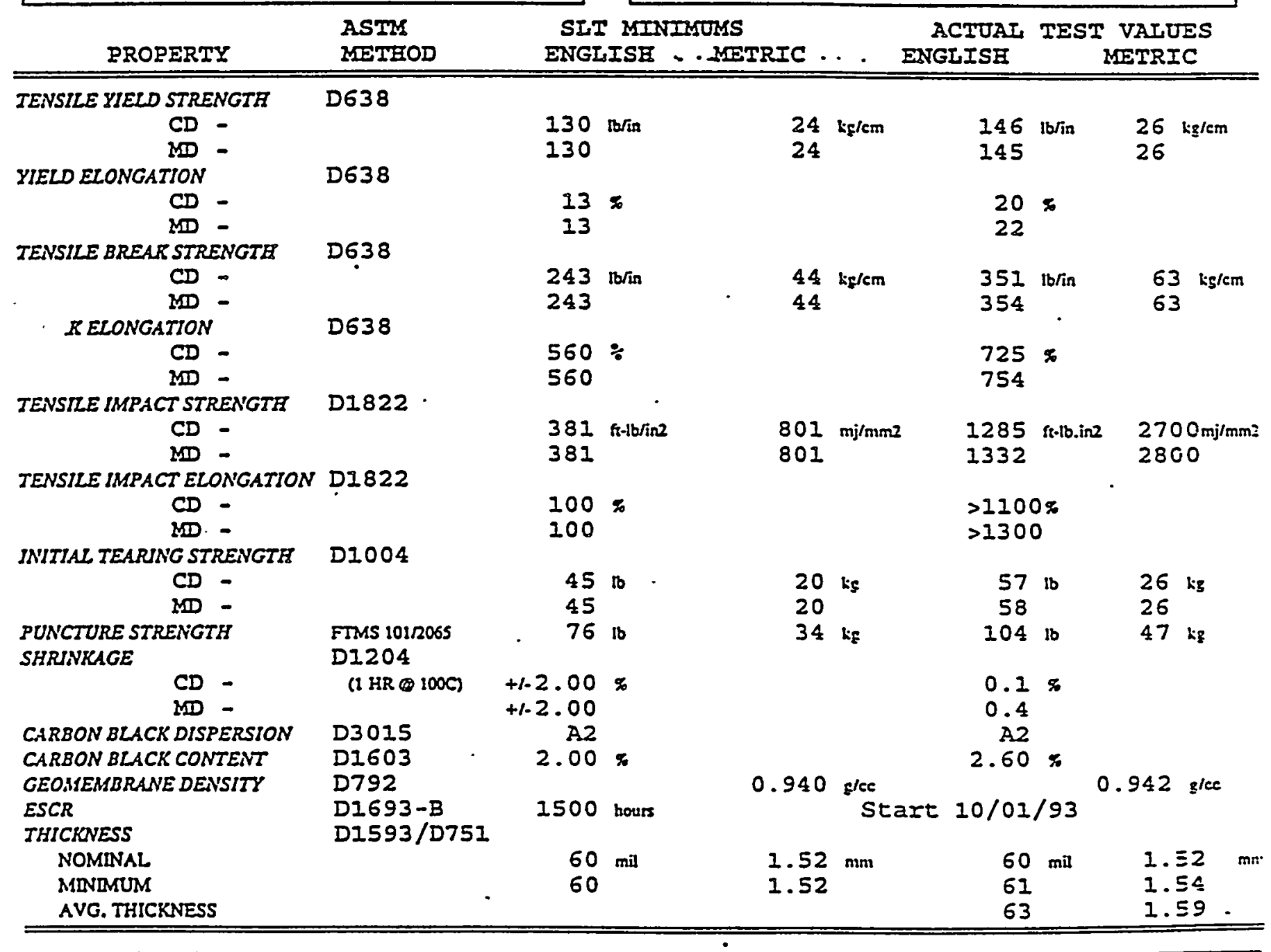

TEST NOTES: MD=MACHINE DIRECTION CD=CROSS DIRECTION ESCR START DATE IS GIVEN -FOR TENSILE ELONGATION TEST GAUGE LENGTH $=1.3$ ON YIELD. 2.5 ON BREAK ALL STRENGTH VALUES BASED ON NOMINAL THICXNESS

USTOMER:

JOB NO.

DATE SHIPPED

COMMENTS 


\begin{tabular}{|c|c|c|}
\hline \multicolumn{3}{|c|}{ ROLI DIMENSIONS $\approx \pm 1 \%$} \\
\hline ROLI NUMBER: & $A C-6895 F F$ & \\
\hline PROD DATE: & $09 / 26 / 93$ & \\
\hline SHEET AREA: & $\begin{array}{r}11144 \\
1036\end{array}$ & $\begin{array}{l}\text { sq. ft. } \\
\text { sq. meters }\end{array}$ \\
\hline IEENGTH: & $\begin{array}{l}475 \\
145\end{array}$ & $\begin{array}{l}\text { feet } \\
\text { meters }\end{array}$ \\
\hline WIDTH: & $\begin{array}{r}23.46 \\
7.1\end{array}$ & $\begin{array}{l}\text { feet } \\
\text { meters }\end{array}$ \\
\hline WEIGHT: & $\begin{array}{l}3880 \\
1760\end{array}$ & $\begin{array}{l}\text { pounds } \\
\text { kilograms }\end{array}$ \\
\hline
\end{tabular}

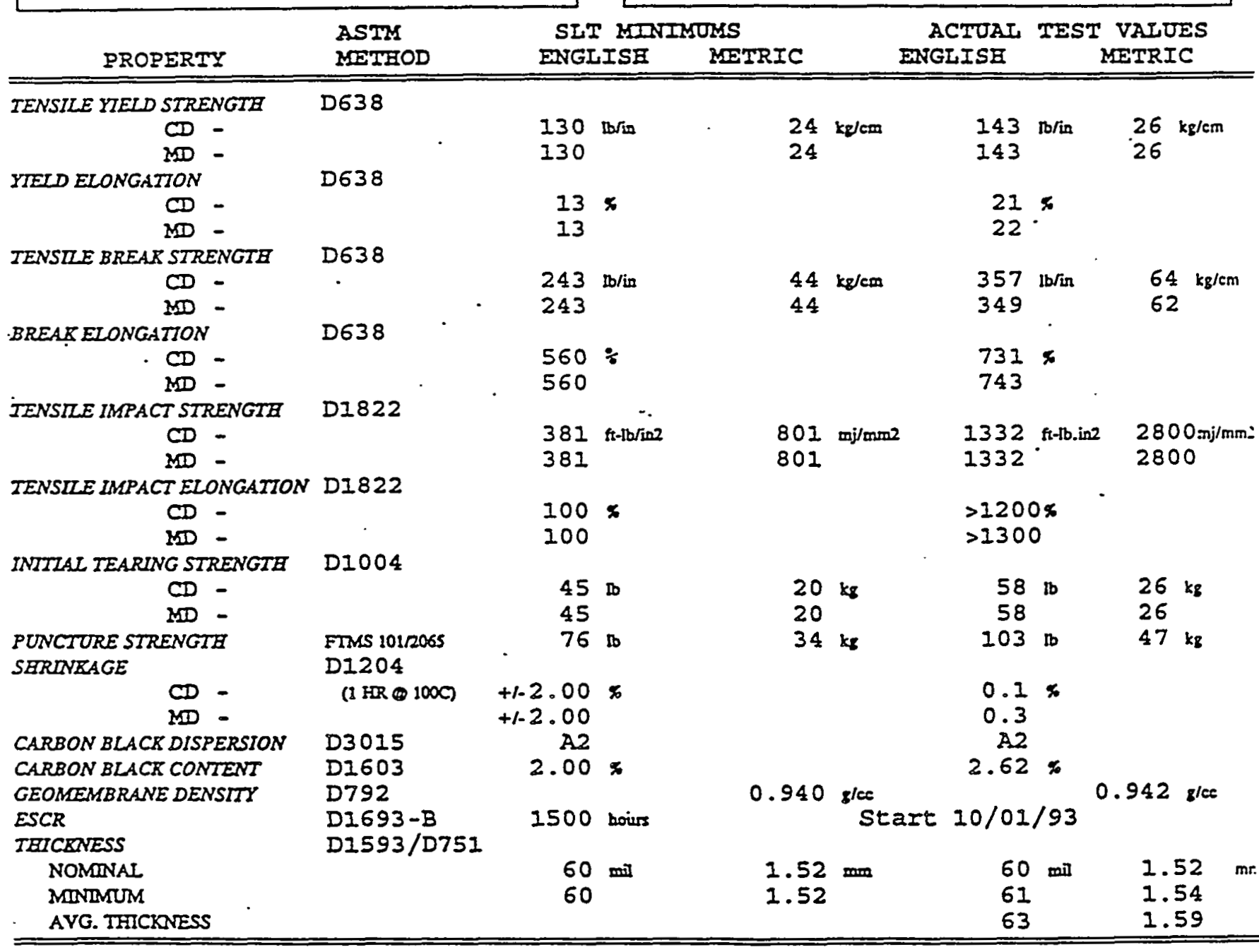

TEST NOTES: MD=MACFINE DIRECTION CD=CROSS DIRECTION ESCR START DATE IS GIVEN -FOR TENSIIE ELONGATION TEST GAUGE LENGTH $=1.3$ ON YDED. 2.5 ON BREAK ALL STRENGTH VALUES BASED ON NOMINAL THLCRNESS.

CUSTOMER : JOB NO. COMMENTS
DATE SHIPPED

\section{RESIN LOT INFORMATION}

LOT NUMBER S-4068I

RESIN TYPE . 600. SERIES

PROPERTY UNITS METHOD RESULT

$\begin{array}{llll}\text { DENSITY } & \text { G/CC } & D 792 & 0.932\end{array}$

MOISTURE $\%$ D570 $<0.09$

BRITTLENESS ${ }^{\circ} \mathrm{C} \quad$ D746 $<-70$

MELT INDEX $g / 10 \mathrm{~min}$. D1238 0.551 
SLT

$\because$ Roll Test Data Report

-

ROLI NO. AC-6896FF

\begin{tabular}{|lc|}
\hline \multicolumn{3}{|c|}{ ROIL DIMENSIONS $\approx \pm 1 \%$} \\
\hline ROIT NUMBER: & AC-6896FF \\
PROD DATE: & $09 / 26 / 93$ \\
SHEET AREA : & 9548 sq.ft. \\
& 887 sq. meters \\
IEENGTH: & 407 feet \\
& 124 meters \\
WIDTH: & 23.46 feet \\
& 7.1 meters \\
WEIGHT: & 3160 pounds \\
& 1433 kilograms
\end{tabular}

$\because$

AS
PROPERTY MIE

ASIM SIT MINIMUMS

RESIN LOT INFORMATION

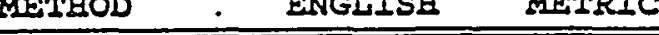

ACTUAY TEST VALUES

IENSILE IMPACT STRENGTH D1822

$$
\text { CD - }
$$

$\mathrm{MD}$ -

TENSILE IMPACT ELONGATION D1822

CD -

$\mathrm{MD}$ -

INITZAL TEARING STRENGTE D1004

$\mathrm{CD}$ -

ND -

PUNCTURE STRENGTE

SHRINRAGE

$$
\mathrm{CD} \text { - }
$$

CARBON BLACK DISPERSION

CARBON BLACK CONTENT

GEOMEMBRANE DENSITY

ESCR

THICENESS

NOMMNAL

MANIMUM

AVG. THICXNESS

$\begin{array}{lll} & 45 \mathrm{~b} & 20 \mathrm{~kg} \\ & 45 & 20 \\ \text { FTMS 1012065 } & 75 \mathrm{bb} & 34 \mathrm{~kg}\end{array}$

$130 \mathrm{bo} / \mathrm{in}$

130

$13 \%$

13

243 th/in

243

$560 \%$

560

$381 \mathrm{ftabinz}$.

381

$100 \%$

100

DI204

(I HR ब 1000) $+1-2.00 \%$

D3015

D1603

D792

D1693-B

DI593/D75I

A2

$2.00 \%$

1500 hours
$60 \mathrm{mil}$

60

\section{S-40681}

600 SERIES

ONTTS - METHOD RESULT

D792 0.932

$D 570<0.09$

D746 <-70

. D1238 0.551

IEST NOTES: MD=MACFINE DIRECTION CD=CROSS DRRECTON ESCR START DATE IS GIVEN -FOR TENSILE ELONGATION TEST GAUGE LENGTH=1.3 ON YIELD. 2.5 ON BREAR ALI STRENGTH VALUES BASED ON NOMENAL THICKONESS

CUSTOMER:

DATE SHIPPED

JOB NO.

COMMENTS

$24 \mathrm{~kg} / \mathrm{em}$

24

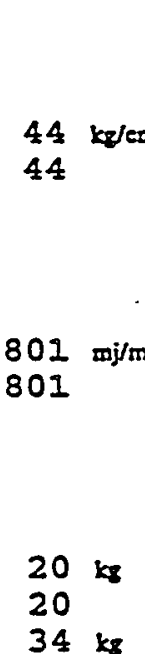

$44 \mathrm{~kg} / \mathrm{em}$

(34) $\begin{array}{ll}140 \mathrm{rb} / \mathrm{in} & 25 \mathrm{~kg} / \mathrm{cm} \\ 141 & 25\end{array}$

$22 \%$

22

$351 \mathrm{tb} / \mathrm{in}$

341

$63 \mathrm{~kg} / \mathrm{cm}$

61

$730 \%$

722

$332 \mathrm{ftb} . \mathrm{in2} 2800 \mathrm{mj} / \mathrm{mr}$

$1332 \quad 2800$

$>1200 \%$

$>1300$

$\begin{array}{rrr}58 \mathrm{lb} . & 26 \mathrm{~kg} \\ 57 & 26 & \\ 104 \mathrm{lb} & 47 \mathrm{~kg}\end{array}$

$0.1 \%$

0.2

A2

$2.65 \%$
$0.940 \mathrm{~g} / \mathrm{ee}$
Start $10 / 01 / 93$
$0.942 \mathrm{~g} / \mathrm{ec}$

1.52 m

60 min

61

63

1.52

1.54

1.59 


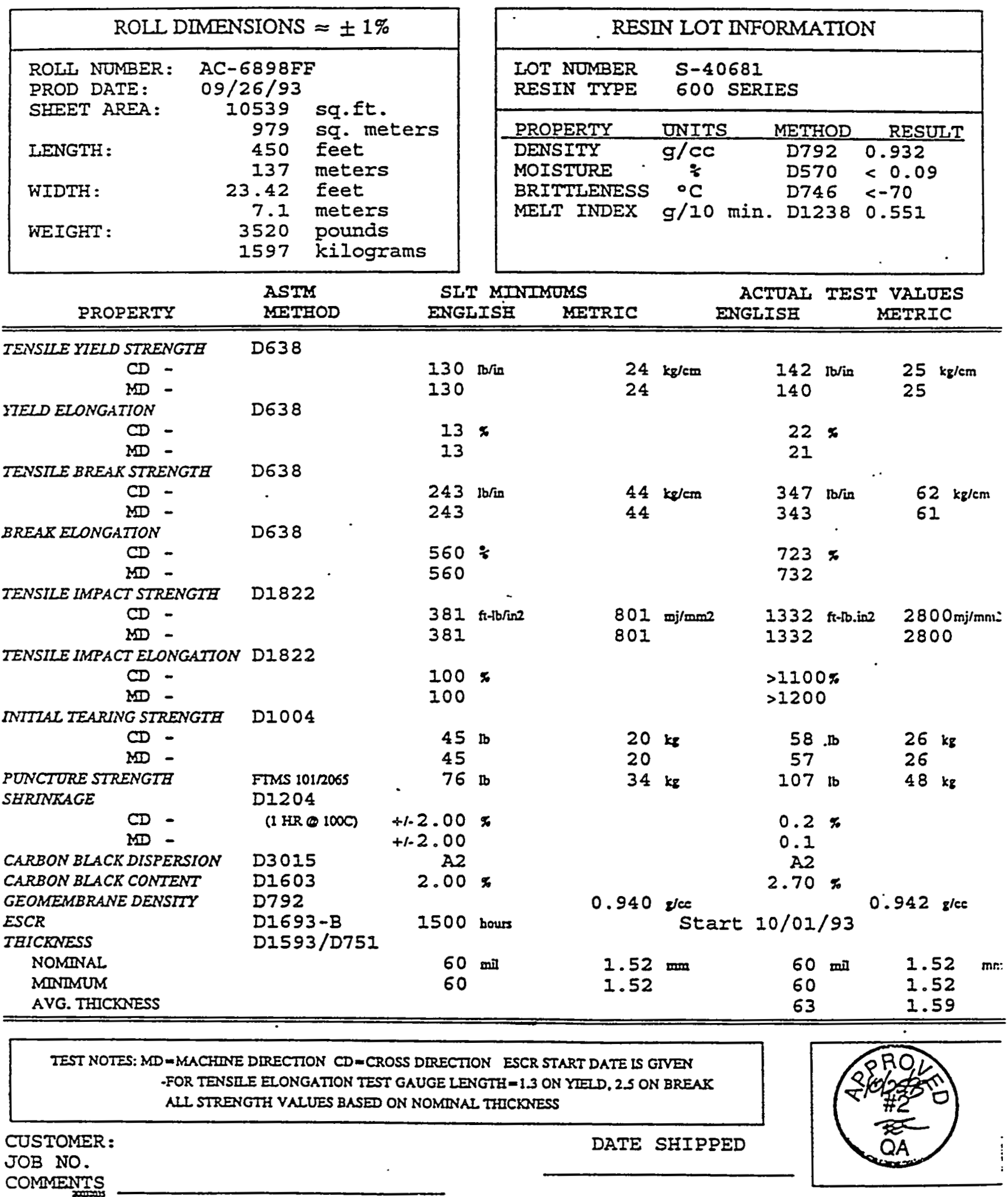




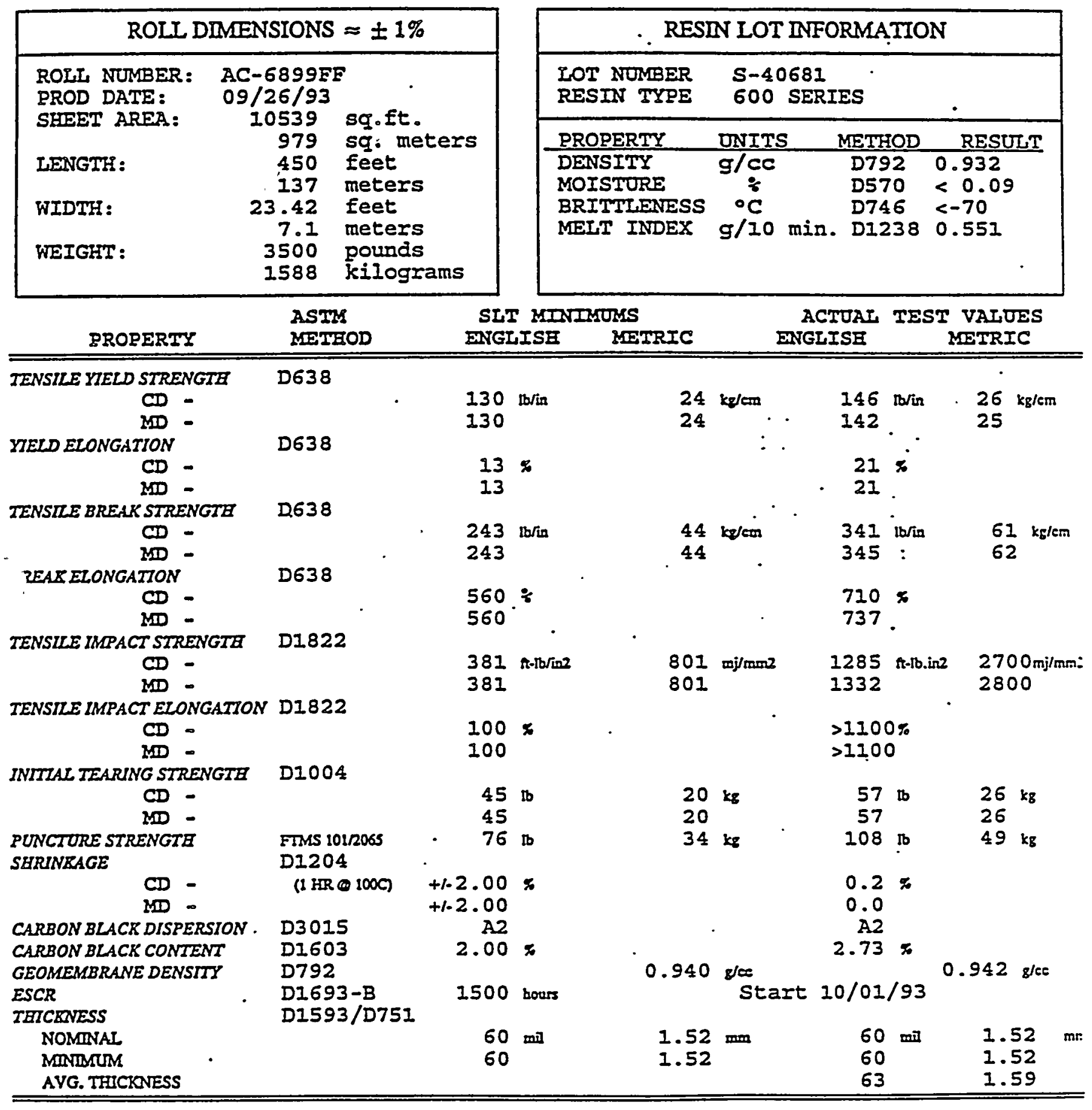

TEST NOTES: MO = MACHINE DIRECTION CD =CROSS DIRECTION ESCR START DATE IS GIVEN -FOR TENSILE EIONGATION TEST GAUGE LENGTH=1.3 ON YIEID. 2.5 ON BREAR ALI STRENGTH VALUES BASED ON NOMRNAI TFICXNESS

USTOMER:

DATE SHIPPED

JOB NO.

COMMENTS 
ENSIONS $\approx \pm 1 \%$

C-6904FF

/26/93

10557

sq. meters

450 feet

137 meters

23.46 feet

7.1 meters

3720 pounds

1687 kilograms

\begin{tabular}{|c|c|c|c|}
\hline \multicolumn{4}{|c|}{ RESIN LOT INFORMATION } \\
\hline $\begin{array}{l}\text { LOT NUMBER } \\
\text { RESIN TYPE }\end{array}$ & $\begin{array}{l}S-40 \\
600\end{array}$ & $\begin{array}{l}682 \\
\text { SERIES }\end{array}$ & $\cdot$ \\
\hline PROPERTY & UNITS & METHOD & RESULT \\
\hline $\begin{array}{l}\text { DENSITY } \\
\text { MOISTURE } \\
\text { BRITILENESS } \\
\text { MELT. INDEX }\end{array}$ & $\begin{array}{c}g / c c \\
\div \\
\circ \mathrm{C} \\
g / 10\end{array}$ & $\begin{array}{c}D 792 \\
D 570 \\
D 746 \\
\text { min. DI238 }\end{array}$ & $\begin{array}{l}0.933 \\
<0.09 \\
<-70 \\
0.555\end{array}$ \\
\hline
\end{tabular}

ASTM

MEIRIC

ENGLISE

MATRIC

D638

$D 638$

$130 \mathrm{tb} / \mathrm{in}$

130

$. .24 \mathrm{~kg} / \mathrm{cm}$
24

$150 . \mathrm{db} / \mathrm{in} \cdot-27 . \mathrm{kg} / \mathrm{cm}$
150

$13 \%$

13

$21 \%$

D638

D638

$243 \mathrm{Ib} / \mathrm{in}$.

243

21

$560 \%$

560

D1822

\section{$381 \mathrm{ft}+\mathrm{b} / \mathrm{inz}$}

381

$100 \%$

100

D1004

FTMS 101/2065 D1204

- (1 HR 100C)

D3015

D1603

D792

D1693-B

D1593/D751

$45 \mathrm{bb}$
45
$76 \mathrm{bb}$
$+1.2 .00 \%$
+1.2 .00
2.00
2.00
1500 bours
$60 \mathrm{mil}$
60

$60 \mathrm{mil}$
60
$44 \mathrm{~kg} / \mathrm{crs}$

44

$344 \mathrm{lb} / \mathrm{in}$

. 374

$7.13 \%$

774

$801 \mathrm{mj} / \mathrm{mm}^{2}$ 801 1332 ft-lb.in2
1285

$2800 \mathrm{mj} / \mathrm{mm}^{2}$ 2700

$>1000 \%$

$>1000$

\section{AVG. IHICKNESS}

TEST NOTES: MD = MACHIVE DIRECTION CD=CROSS DIRECTION ESCR START DATE IS GIVEN -FOR TENSILE ELONGATION TEST GAUGE LENGTH $=1.3$ ON YIEID. 2.5 ON BREAK AII STRENGTH VALUES BASED ON NOMDNAL THCCKNESS

$\begin{array}{rlr}58 \mathrm{~kb} & 26 \mathrm{~kg} \\ 59 & 27 \\ 111 \mathrm{~kb} & 51 \mathrm{ks}\end{array}$

$0.0 \%$

0.2

A.2

$2.83 \%$

$0.940 \mathrm{~g} / \mathrm{ce} \quad 0.944 \mathrm{~g} / \mathrm{cc}$ Start $10 / 01 / 93$

$1.52 \mathrm{~mm}$

1.52

$60 \mathrm{mil}$

61

1.60 
WHC-SD-W025-RPT-001, Rev. 0

SLT:

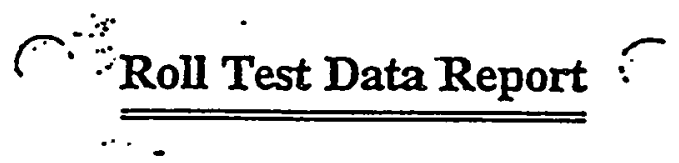

ROLI NO. AC-690SFF

\begin{tabular}{|lc|}
\hline \multicolumn{3}{|c|}{ ROLI DIMENSIONS $\approx \pm 1 \%$} \\
\hline ROLI NUMBER: & AC- $-6905 F F$ \\
PROD DATE: & $09 / 26 / 93$ \\
SHEET AREA : & 10557 sq.Et. \\
& 981 sq.- meters \\
LENGTH: & 450 feet \\
& WI37 meters \\
WIDTH : & 23.46 feet \\
& 7.1 meters \\
WEIGHT: & 3280 pounds \\
& 1488 kilograms \\
\hline
\end{tabular}

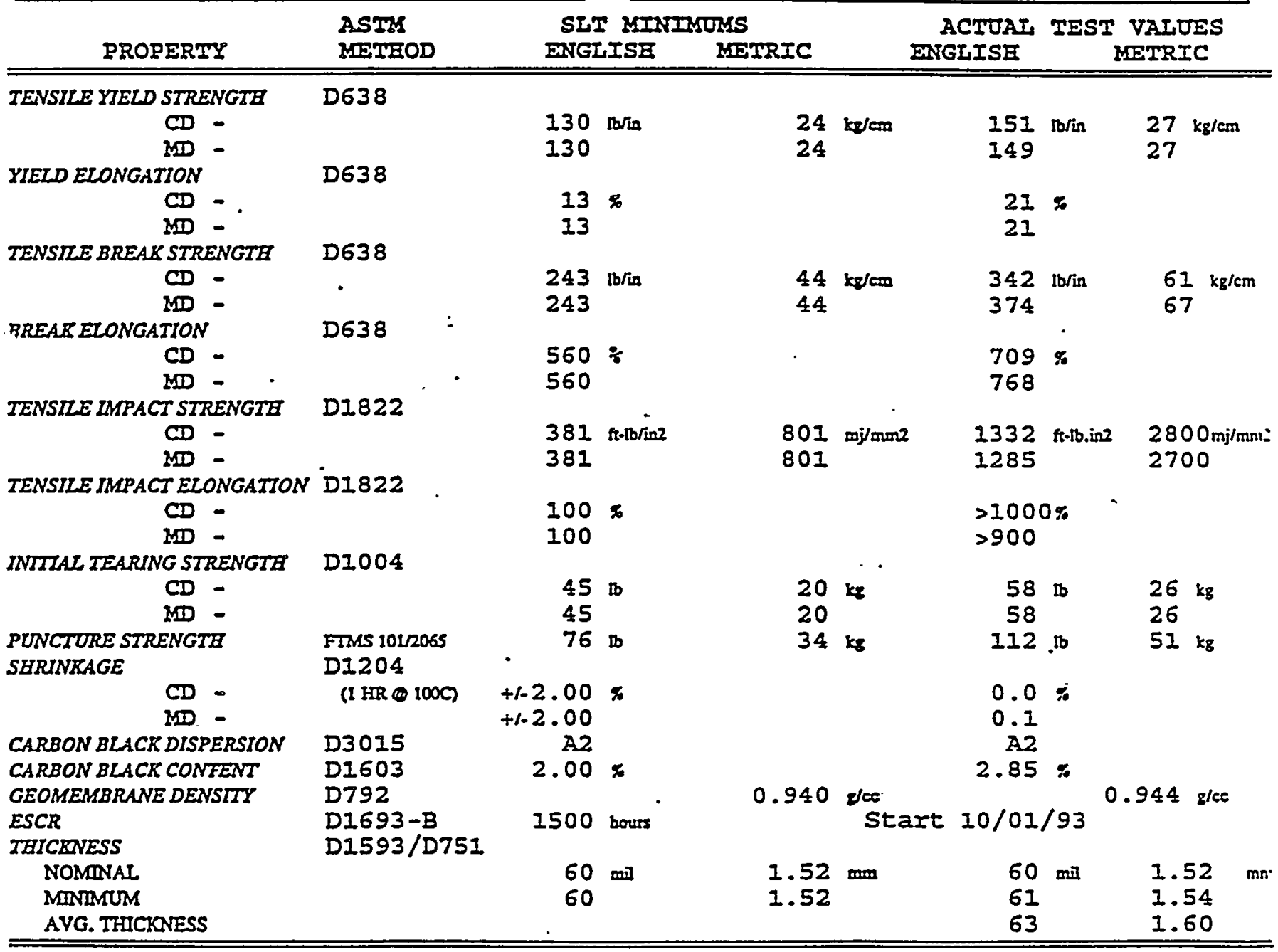

TEST NOTES: MD=MACHINE DIRECTION CD=CROSS DRRECTION ESCR START DATE IS GIVEN -FOR TENSLLE ELONGATION TEST GAUGE LENGTH =1.3 ON YIELD. 2.5 ON BREAR ALI STRENGTH VALUES BASED ON NOMINAL THCXNESS

USTOMER:

DATE SHIPPED

OB NO.

COMMENTS 
SLì

Roll Test Data Report

ROLI NO. AC-6906FF

ROLL DIME
ROII NUMBER: AC
PROD DATE: 09
IEET AREA:
WIDTH:
WEIGLT:
PETH:
PROPERTY
IENSILE YIELD STRENGTH
CD -
MD -
CD -
MD -
CIELD ELONGATION -
MD -
CD -
MD -

TENSILE IMPACT STRENGTH CD $M D$ -

TENSILE LMPACT ELONGATION D1822 $C D$ MD -

INITIAL TEARING STRENGTH $\mathrm{CD}$ MD -

PUNCTURE STRENGTH SERINRAGE

$$
\text { CD - }
$$

CARBON BLACK DISPERSION

CARBON BLACX CONTENT

GEOMEMBRANE DENSITY

ESCR

THICKNESS

- NOMINAL

MONIMUM

AVG. THICENESS

Asm sq. ft.

sq. meters

feet

145 meters
3.46 feet

7.1 meters

3540 pounds

\begin{tabular}{|c|c|c|c|}
\hline \multicolumn{4}{|c|}{ RESIN LOT INFORMATION . } \\
\hline $\begin{array}{l}\text { IOT NOMBER } \\
\text { RESIN TYPE }\end{array}$ & $\begin{array}{l}S-40682 \\
600 \quad \text { SERI }\end{array}$ & ES & \\
\hline PROPERTY & UNITS & METHOD & RESULT \\
\hline $\begin{array}{l}\text { DENSITY } \\
\text { MOISTURE } \\
\text { BRITTLENESS } \\
\text { MELT INDEX }\end{array}$ & $\begin{array}{l}g / \mathrm{cc} \\
\circ \div \\
\circ \mathrm{C} \\
g / 10 \mathrm{~min} .\end{array}$ & $\begin{array}{l}\text { D792 } \\
\text { D570 } \\
D 746 \\
D 1238\end{array}$ & $\begin{array}{l}0.933 \\
<0.09 \\
<-70 \\
0.556\end{array}$ \\
\hline
\end{tabular}

1606 kilograms MINIMUMS METRIC ENGLISE METHOD D638

D638

$130 \mathrm{tb} / 10$

130

$13 \%$

13

D 638

D638

DI822

\begin{tabular}{|c|c|c|}
\hline & 45 & Ib \\
\hline $\begin{array}{l}\text { FTMS 101/2065 } \\
\text { D1204 }\end{array}$ & ${ }^{76}$ & to \\
\hline$(1 \mathrm{HR} \oplus 1000)$ & $\begin{array}{l}+1.2 .00 \\
+1.2 .00\end{array}$ & 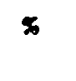 \\
\hline $\begin{array}{l}D 3015 \\
D 1603 \\
D 792\end{array}$ & $\begin{array}{r}A 2 \\
2.00\end{array}$ & 5 \\
\hline $\begin{array}{l}\mathrm{D} 1693-\mathrm{B} \\
\mathrm{D} 1593 / \mathrm{D} 751\end{array}$ & 1500 & bours \\
\hline & $\begin{array}{l}60 \\
60\end{array}$ & mil \\
\hline
\end{tabular}

D1004

$243 \mathrm{lb} / \mathrm{in}$

243

$560 \%$

560

$381 \mathrm{ft}-1 \mathrm{~b} / \mathrm{in} 2$

381

$100 \%$

100

SE

(2)

AVG. THICANESS

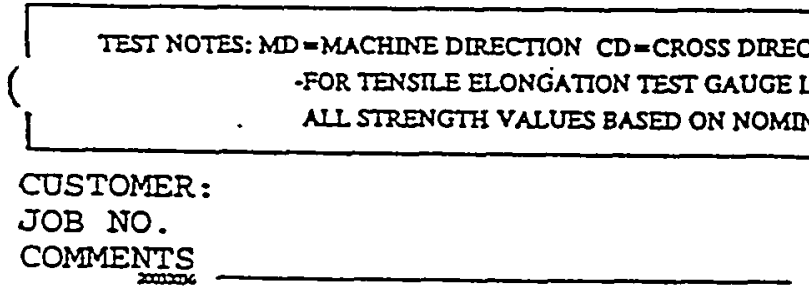

$24 \mathrm{kglm}$

24 ...

$151 \mathrm{~b} / \mathrm{in}$. 150

$27 \mathrm{~kg} / \mathrm{cm}$ 27

$\begin{array}{llll} & 20 \% & & \\ & 21 & & \\ 44 \mathrm{~kg} / \mathrm{cm} & 341 \mathrm{~b} / \mathrm{in} & 61 \mathrm{~kg} / \mathrm{cm} \\ 44 & 371 & 66\end{array}$

$708 \%$
762

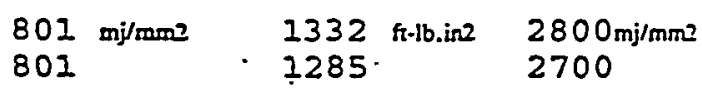

$>1000 \%$ $>900$

$\begin{array}{ll}20 & \mathrm{~kg} \\ 20 & \\ 34 & \mathrm{~kg}\end{array}$

$58 \mathrm{lb}$
57
$113 \mathrm{lb}$

$27 \mathrm{~kg}$ 26 $51 \mathrm{~kg}$

$0.0 \%$

0.1

A2

$2.87 . \%$

0.940 vec Start $10 / 01 / 93$

$0.944 \mathrm{~g} / \mathrm{cc}$
$60 \mathrm{mil}$

61

63
1.52

I. 54

1.59 $\mathrm{mm}$

1. 52

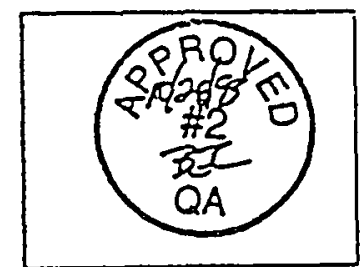




\section{ROLL DIMENSIONS $\approx \pm 1 \%$}

ROLL NUMBER: AC-6907FE :

PROD DATE: 09/26/93

SEIEET AREA:

LENGTH :

WIDTH:

WEIGHT :
9736

415 feet

126 meters

23.46 feet

7.1 meters

3280 pounds

1488 kilograms

\begin{tabular}{|c|c|c|c|}
\hline \multicolumn{4}{|c|}{ RESIN LOT INFORMATION } \\
\hline $\begin{array}{l}\text { IOT NOMBER } \\
\text { RESIN TYPE }\end{array}$ & $\begin{array}{l}S-40682 \\
.600 \mathrm{SERI}\end{array}$ & CES & - \\
\hline PROPERTY & UNITS & METHOD & RESULT \\
\hline $\begin{array}{l}\text { DENSITY } \\
\text { MOISTURE } \\
\text { BRITILENESS } \\
\text { MELT INDEX }\end{array}$ & $\begin{array}{l}\text { g/cc } \\
\frac{\mathrm{c}}{5} \\
{ }^{\circ} \mathrm{C} \\
\mathrm{g} / 10 \mathrm{~min} .\end{array}$ & $\begin{array}{l}D 792 \\
D 570 \\
D 746 \\
D 1238\end{array}$ & $\begin{array}{l}0.933 \\
<0.09 \\
<-70 \\
0.556\end{array}$ \\
\hline
\end{tabular}

ASTM SLT MININOMS

ACTUAT TEST VALUES METEOD

$D 638$

TENSILE YTELD STRENGTE $\mathrm{CD}$ -

YIELD ELONGATION

$\mathrm{CD}$ -

TENSILE BREAR STRENGTH $\mathrm{CD}$ -

REARELONGATION

CD -

SID -

TENSILE IMPACT STRENGTE D1822 CD -

TENSTE IMPACT ELONGATION DI822 CD -

INITLAL TEARING STRENGTA CD ND -

PUNCTURE STRENGTE SHRINRAGE

D638

$130 \mathrm{rb} / \mathrm{in}$

130

\section{$13 \%$}

13

D638

D638

D638

D1004

FTMS 1012065

D1204

$$
\text { CD - }
$$

CARBON BLACK DISPERSTON

CARBON BLACK CONTENT

GEOMEMBRANE DENSTTY

ESCR

TAICRNESS

NOMNAL

MINIMOM

AVG. THICKNESS
(I FR 1000) $+1.2 .00 \%$

$+1.2 .00$

D3015

$D I 603$

D792

D1693-B

D1593/D751

$2.00 \times$
$60 \mathrm{mil}$

60
ENGLISE METRIC

\section{$243 \mathrm{rb} / \mathrm{in}$}

243

$560 \div$

560

381

$100 \%$

100
381 th-1b/inz

$24 \mathrm{~kg} / \mathrm{cm}$

24 ENGIISE

encis

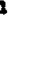

$151 \mathrm{~b} / \mathrm{in}$
150

METRIC

IEIRIC

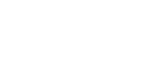

$44 \mathrm{~kg} / \mathrm{cm}$ 44

\section{5}

21

$356 \mathrm{db} / \mathrm{ia}$

372

$64 \mathrm{~kg} / \mathrm{cm}$

67

742

764

$801 \mathrm{mj} / \mathrm{mm} 2$ 801

1332 ft-tb.ing

1285

$2800 \mathrm{mj} / \mathrm{mm}:$ 2700

$27 \mathrm{~kg} / \mathrm{cm}$ 27

$>1000 \%$

$>900$

$\begin{array}{lrl}20 \mathrm{~kg} & 58 \mathrm{~kb} & 26 \mathrm{~kg} \\ 20 \mathrm{~kg} & 57 & 26 \\ 34 \mathrm{~kg} & 113 \mathrm{~kb} & 51 \mathrm{~kg}\end{array}$

$0.0 \%$

0.2

A2

$2.89 \%$

$0.940 \mathrm{z} / \mathrm{ce}$

1500 bours

Start 10/01/93

$0.944^{g / c e}$
$1.52 \mathrm{~mm}$

1.52
60 mil

61

63 \begin{tabular}{l}
1.52 \\
1.54 \\
1.59 \\
\hline
\end{tabular}

TEST NOTES: $M D=$ = MACHRNE DIRECTION CD=CROSS DIRECTION ESCR START DATE IS GIVEN .FOR TENSILE EIONGATION TEST GAUGE LENGTR $=1.3$ ON YIED. 2.5 ON BREAK ALL STRENGTH VALUES BASED ON NOMINAL THHCKNESS

USTOMER :

DATE SHIPRED

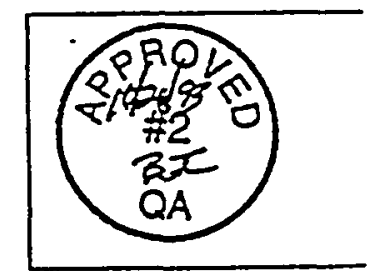

SOB NO.

COMMENTS 


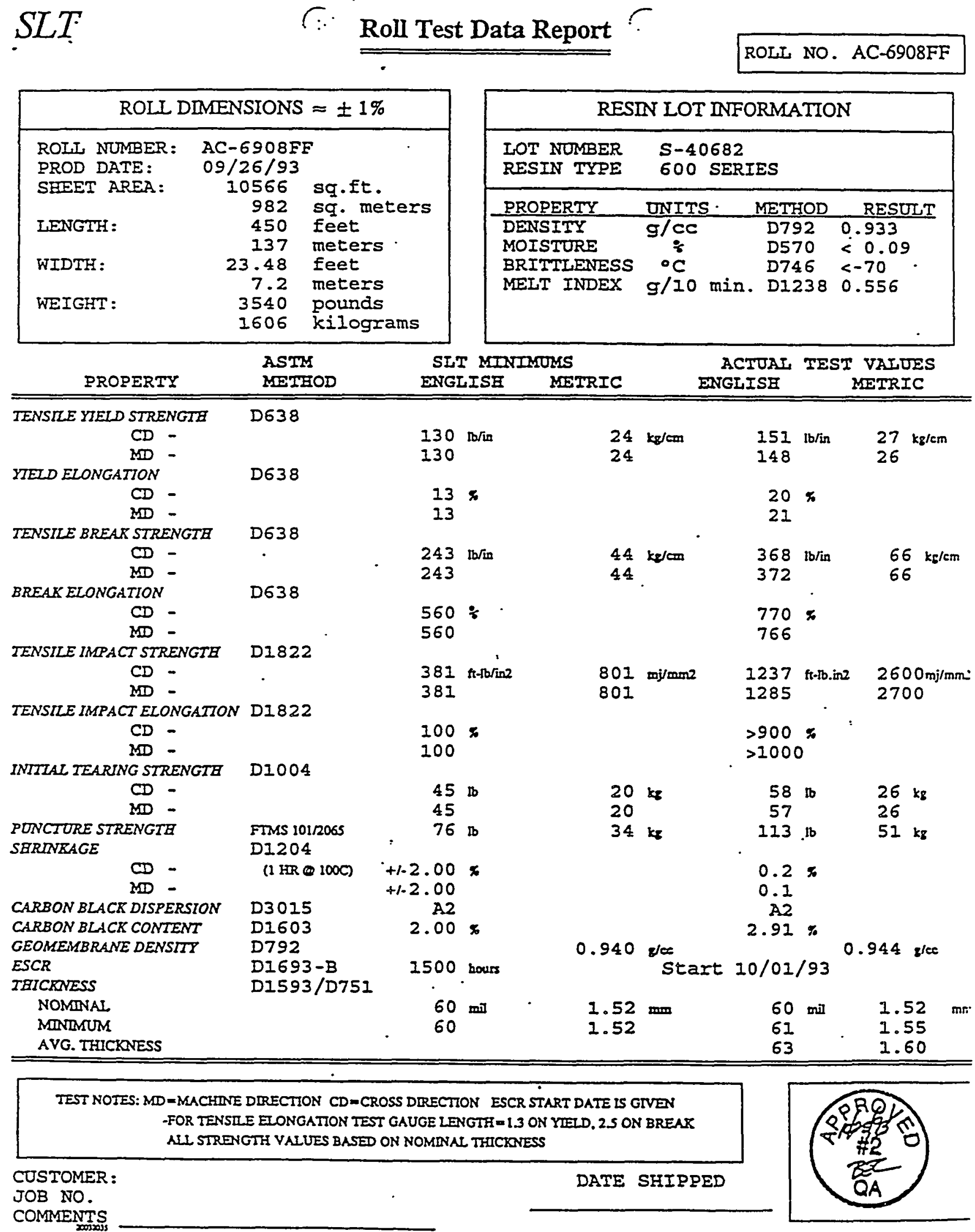


WHC-SD-W025-RPT-001, Rev. 0

LT.

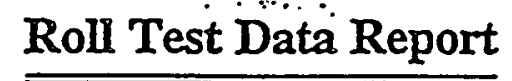

ROLI NO. AC-6909FF

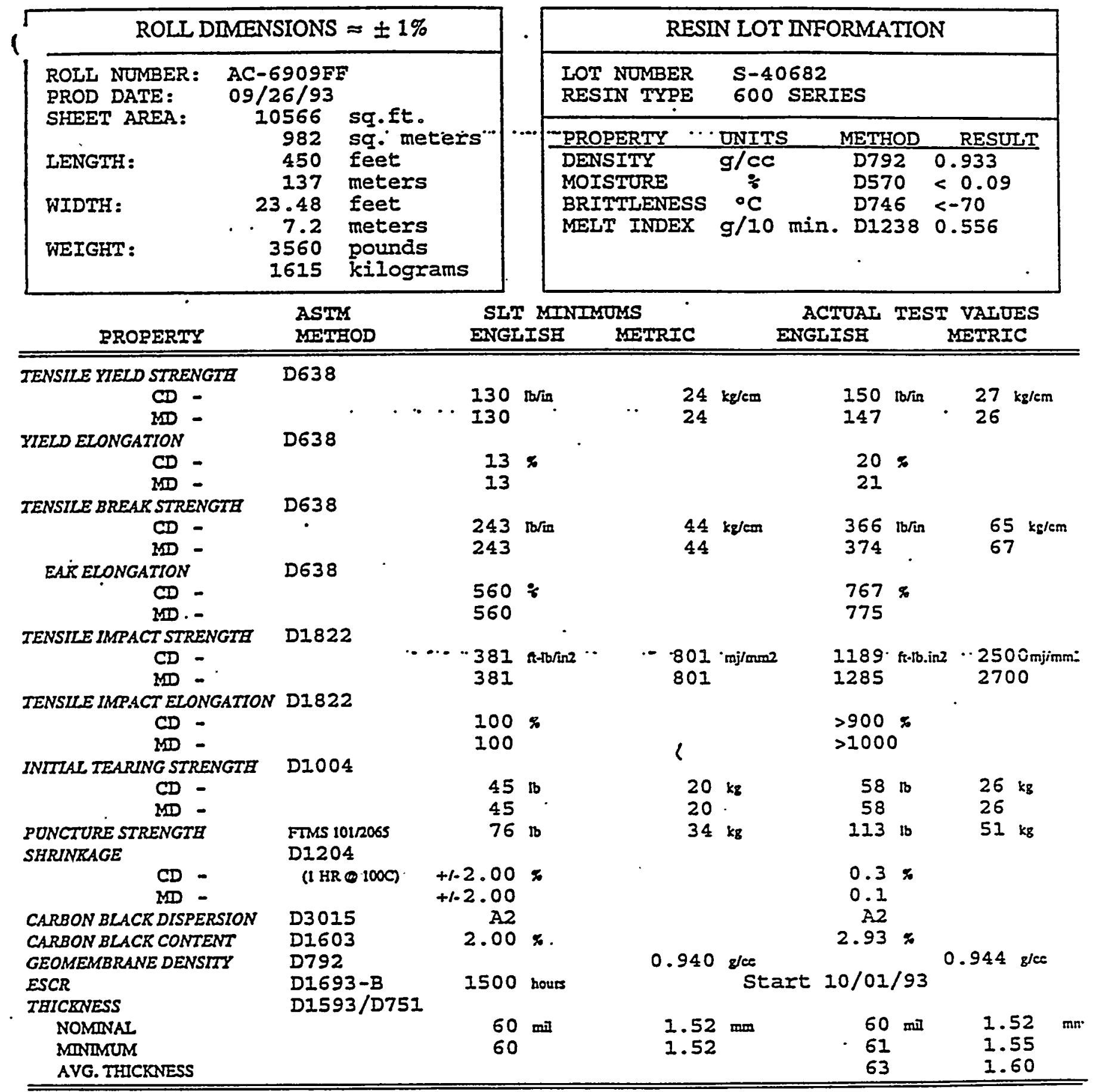

TEST NOTES: MD = MACHDNE DRECTION CD=CROSS DRECTION ESCR START DATE IS GIVEN .FOR TENSILE ELONGATION TEST GAUGE LENGTH=1.3 ON YIEID, 2.5 ON BREAK ALL STRENGTH VALUES BASED ON NOMRNAL THICKNESS

JSTOMER:

. $\quad \cdots \cdot \cdot \cdots-D A I E \cdot S F I P P E D$

JOB NO.

COMMENTS

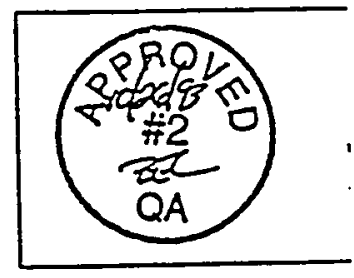




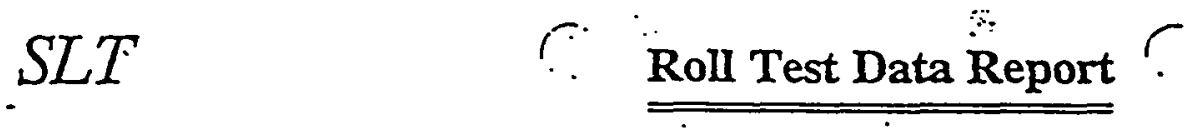

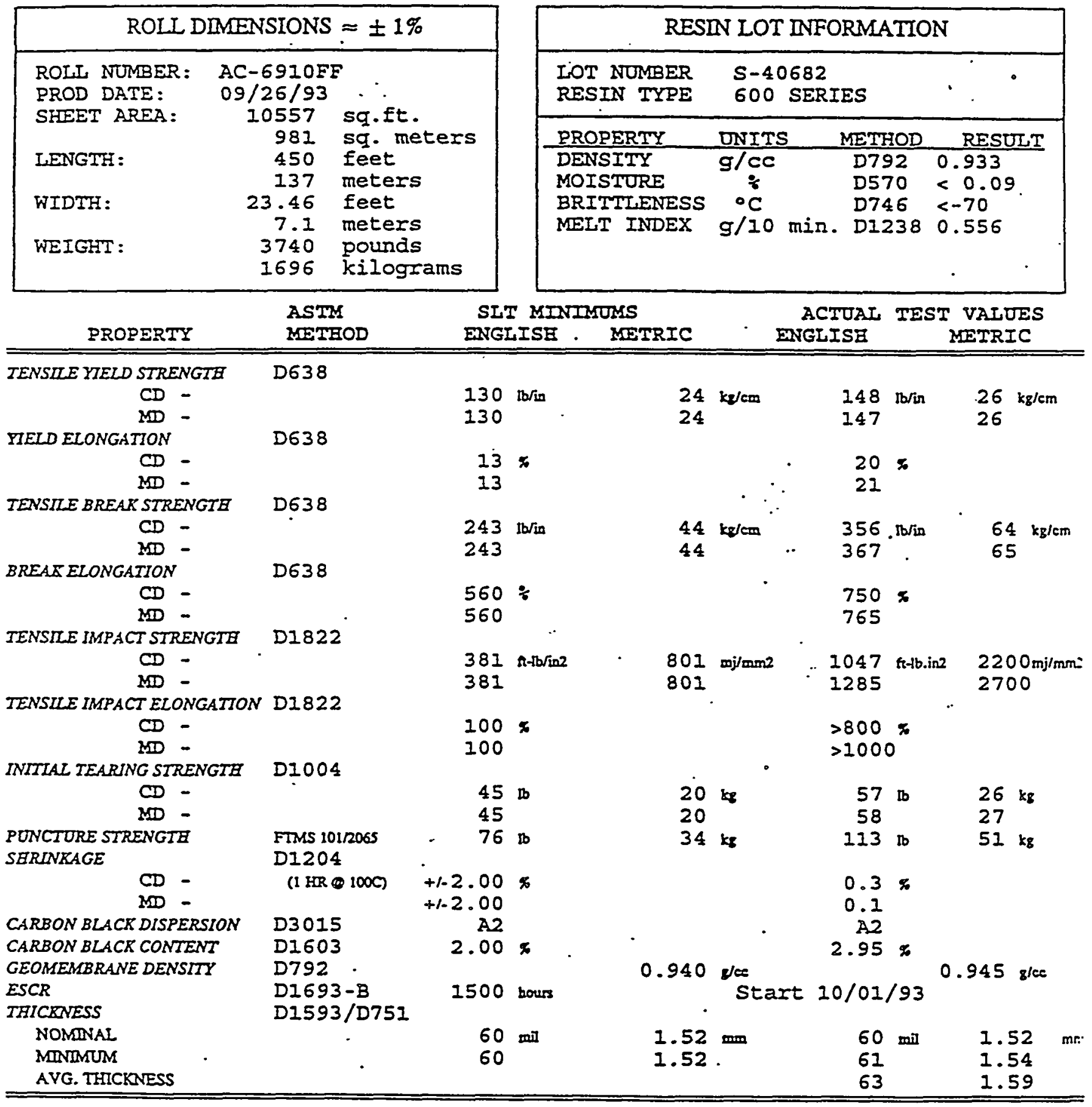

TEST NOTES: $M D=M A C F I N E$ DIRECTION CD=CROSS DIRECTION ESCR START DATE IS GIVEN -FOR TENSIIE ELONGATION TEST GAUGE LENGTH $=1.3$ ON YIELD. 2.5 ON BREAK ALI STRENGTH VALUES BASED ON NOMINAI THICKONESS

CUSTOMER :

JOB NO.

COMMENTS
DATE SHIPQED

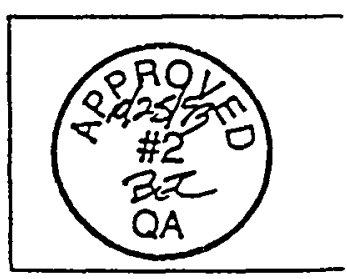




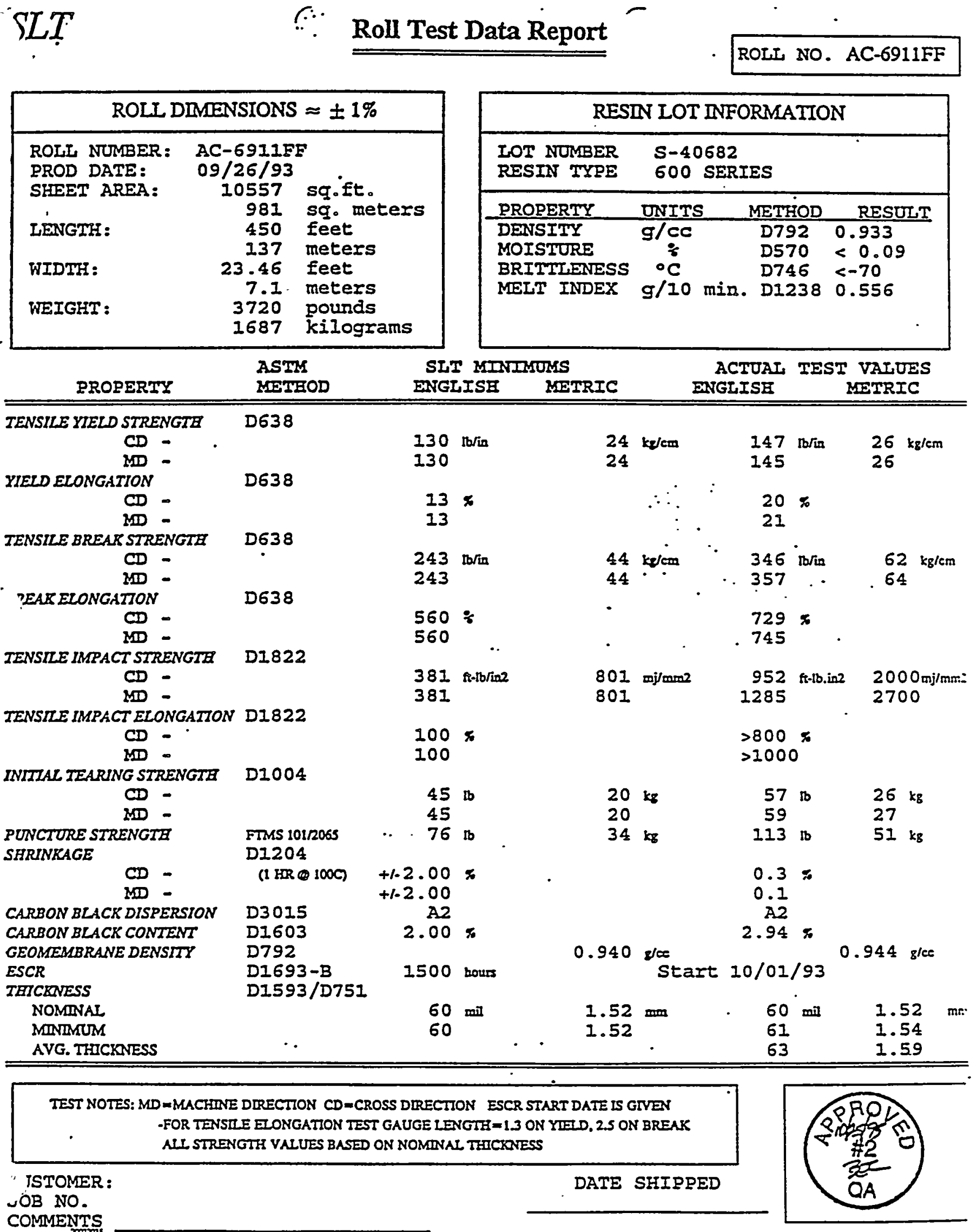


WHC-SD-W025-RPT-001, Rev. 0

'SLT

\section{Roll Test Data Report}

ROLI NO. AC-6912FF

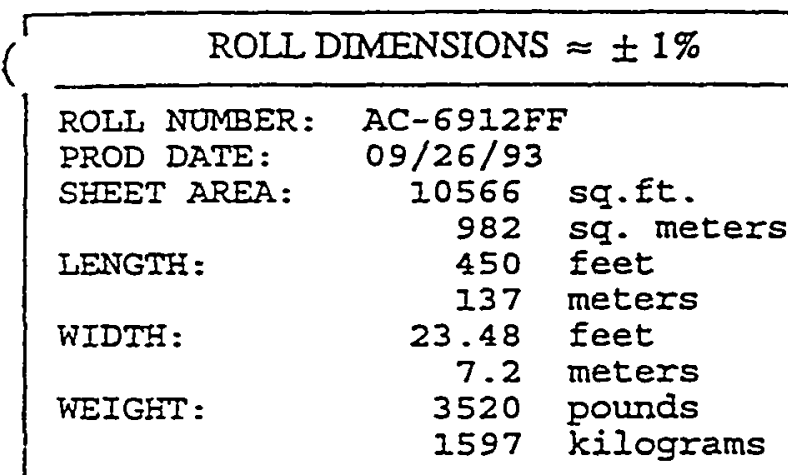

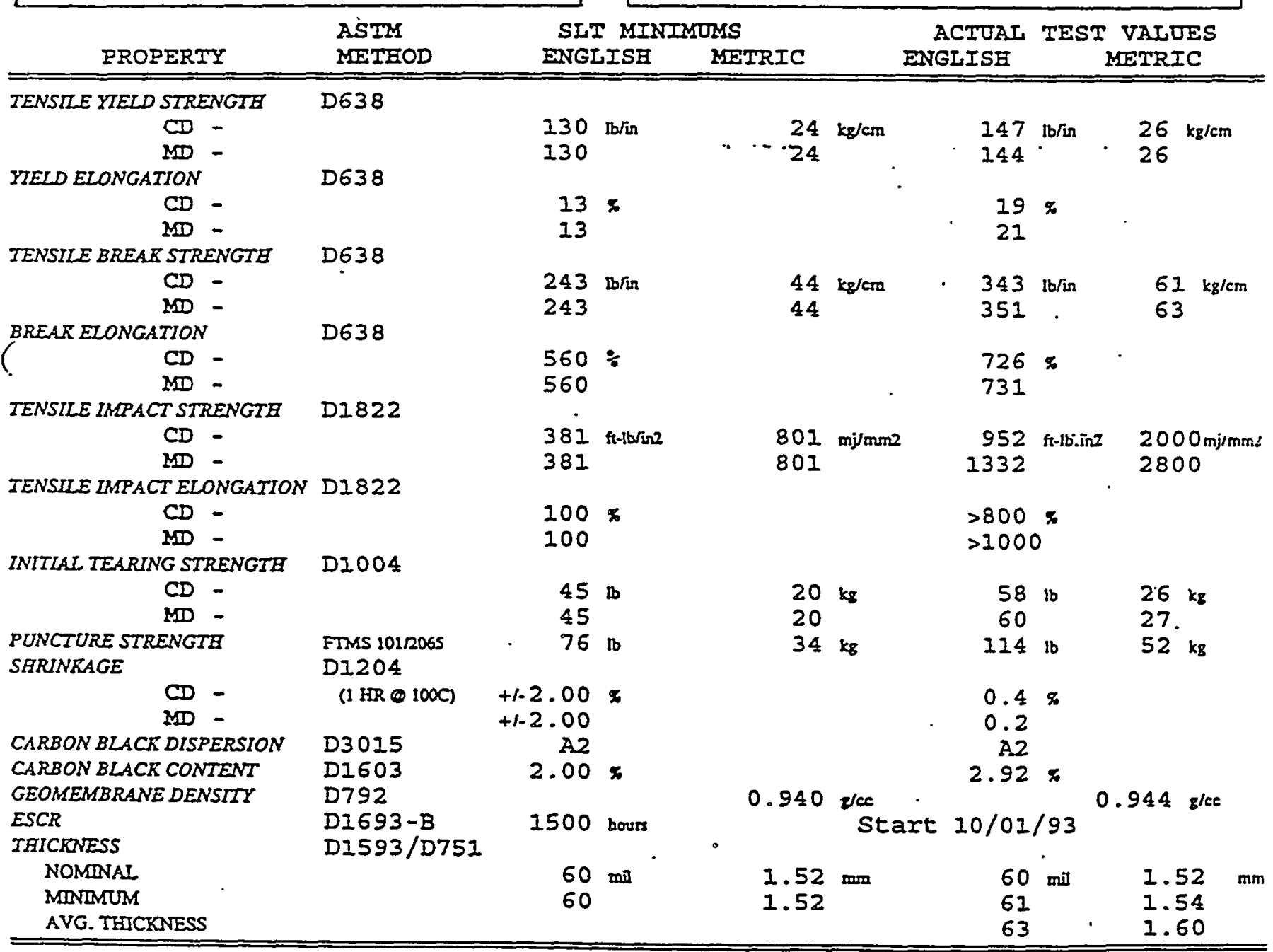

\footnotetext{
TEST NOTES: MD=MACHINE DIRECTION CD=CROSS DLRECTI
-FOR TENSTIE ELONGATION TEST GAUGE
ALI STRENGTH VALUES BASED ON NOMD
} 
WHC-SD-W025-RPT-001, Rev. 0

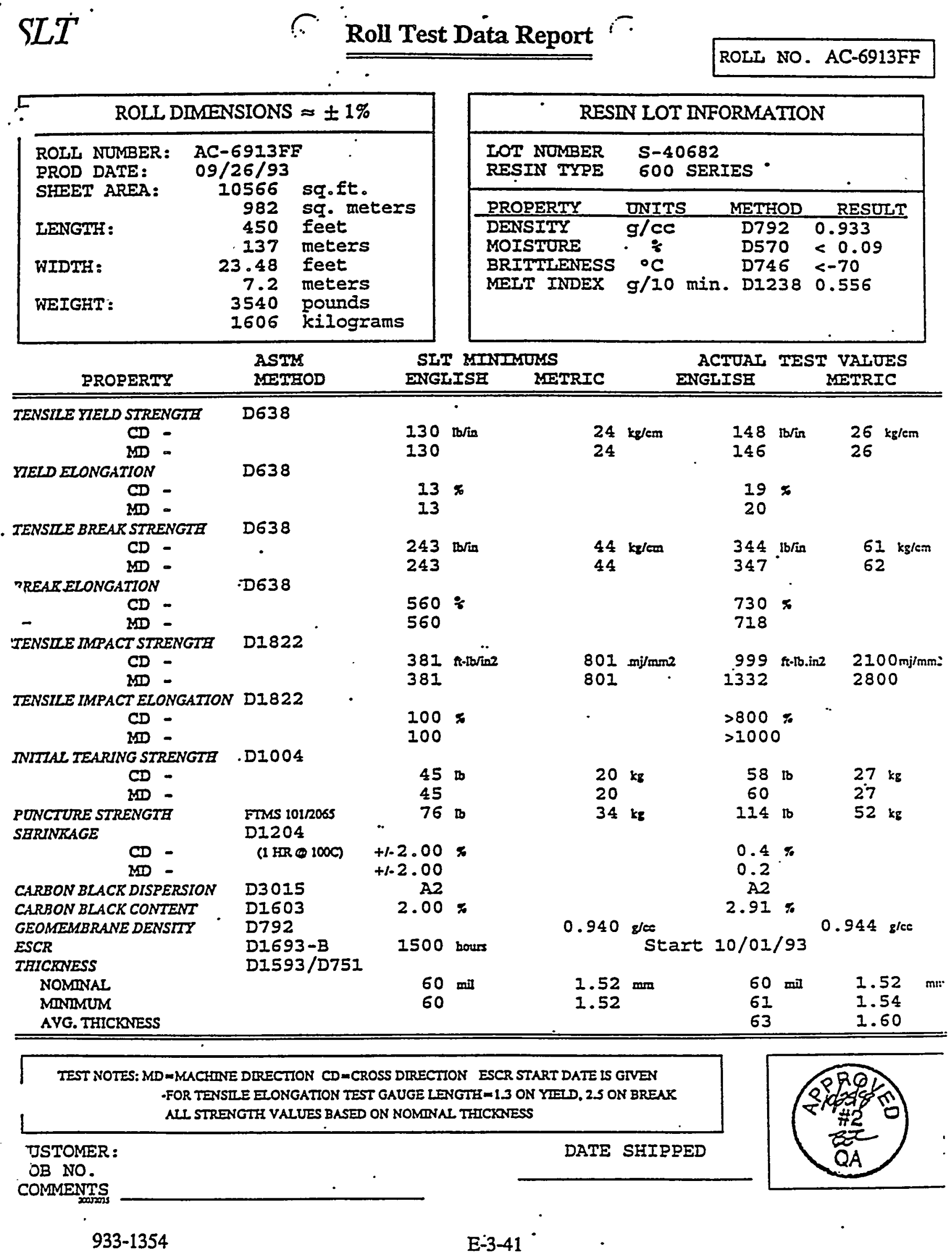




\begin{tabular}{|l|l|}
\hline \multicolumn{3}{|c|}{ ROLI DIMENSIONS $\approx \pm 1 \%$} \\
\hline ROLI NUMBER: & AC-6914FF \\
PROD DATE: & $09 / 26 / 93$ \\
SHEET AREA: & 10566 sq.ft. \\
& 982 sq. meters \\
LENGTH: & 450 feet \\
& 137 meters \\
WIDTH: & 23.48 feet \\
& 7.2 meters \\
WEIGHT: & 3740 pounds \\
& 2696 kilograms \\
\hline
\end{tabular}

\begin{tabular}{|c|c|c|c|}
\hline \multicolumn{4}{|c|}{ RESIN LOT INFORMATION } \\
\hline $\begin{array}{l}\text { LOT NOMBER } \\
\text { RESIN TYPE }\end{array}$ & $\begin{array}{l}\text { S-40682 } \\
600 \text { SERI }\end{array}$ & IES & \\
\hline PROPERTY & UNITS & METHOD & RESULT \\
\hline $\begin{array}{l}\text { DENSITY } \\
\text { MOISTURE } \\
\text { BRITTLENESS } \\
\text { MELT INDEX }\end{array}$ & $\begin{array}{l}\text { g/cc } \\
\vdots \\
\circ \mathrm{C} \\
\mathrm{g} / \mathrm{I} 0 \mathrm{~min} .\end{array}$ & $\begin{array}{l}D 792 \\
D 570 \\
D 746 \\
D 1238\end{array}$ & $\begin{array}{l}0.933 \\
<0.09 \\
<-70 \\
0.556\end{array}$ \\
\hline & & & \\
\hline
\end{tabular}

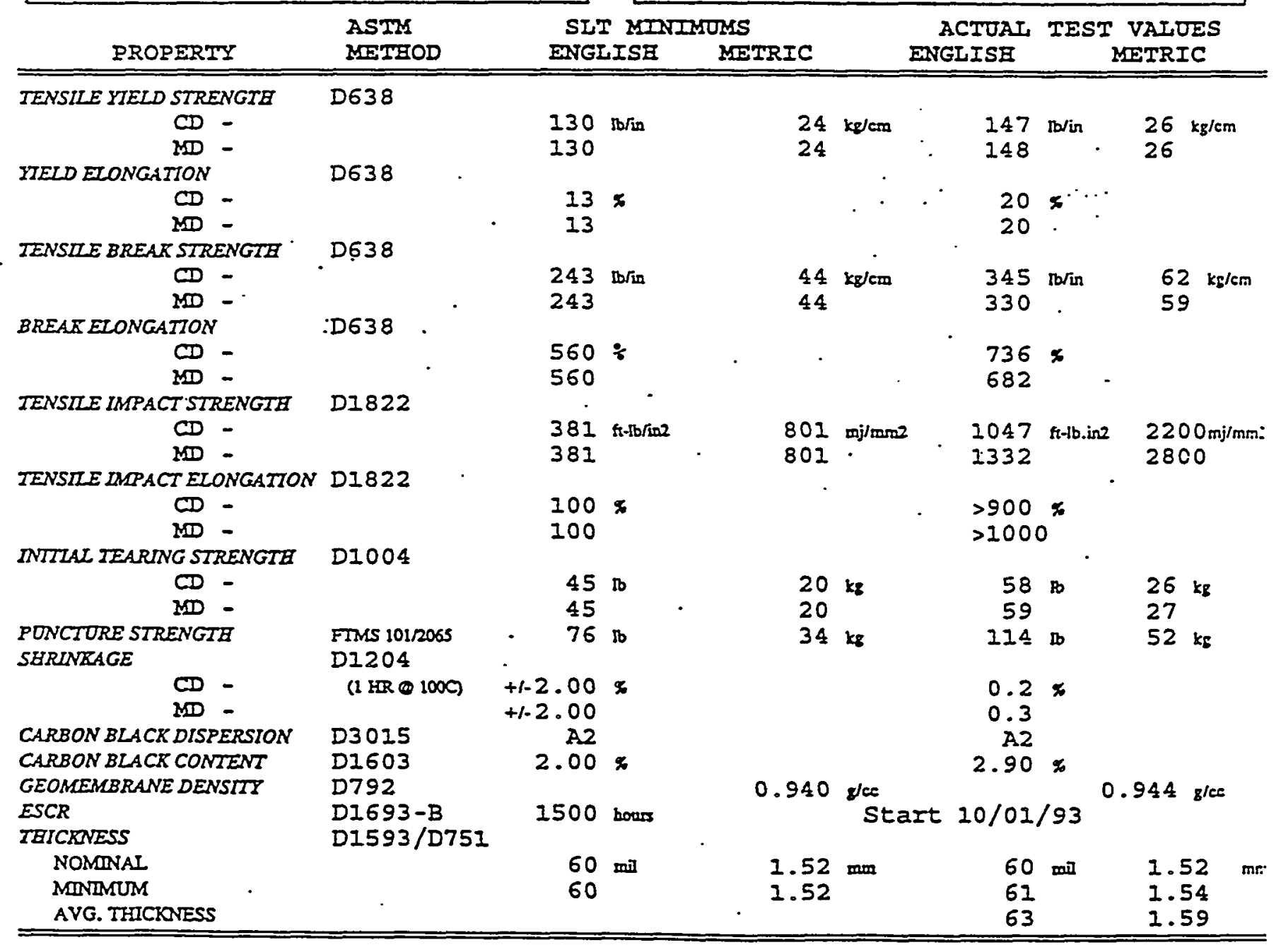

IEST NOTES: SDD -MUCFINE DIRECTION CD=CROSS DIRECTION ESCR START DATE IS GTVEN -FOR TENSILE ELONGATION TEST GAUGE LENGTH=1.3 ON YIELD, 2.5 ON BREAK ALL STRENGTH VALUES BASED ON NOMINAL THICKNESS 


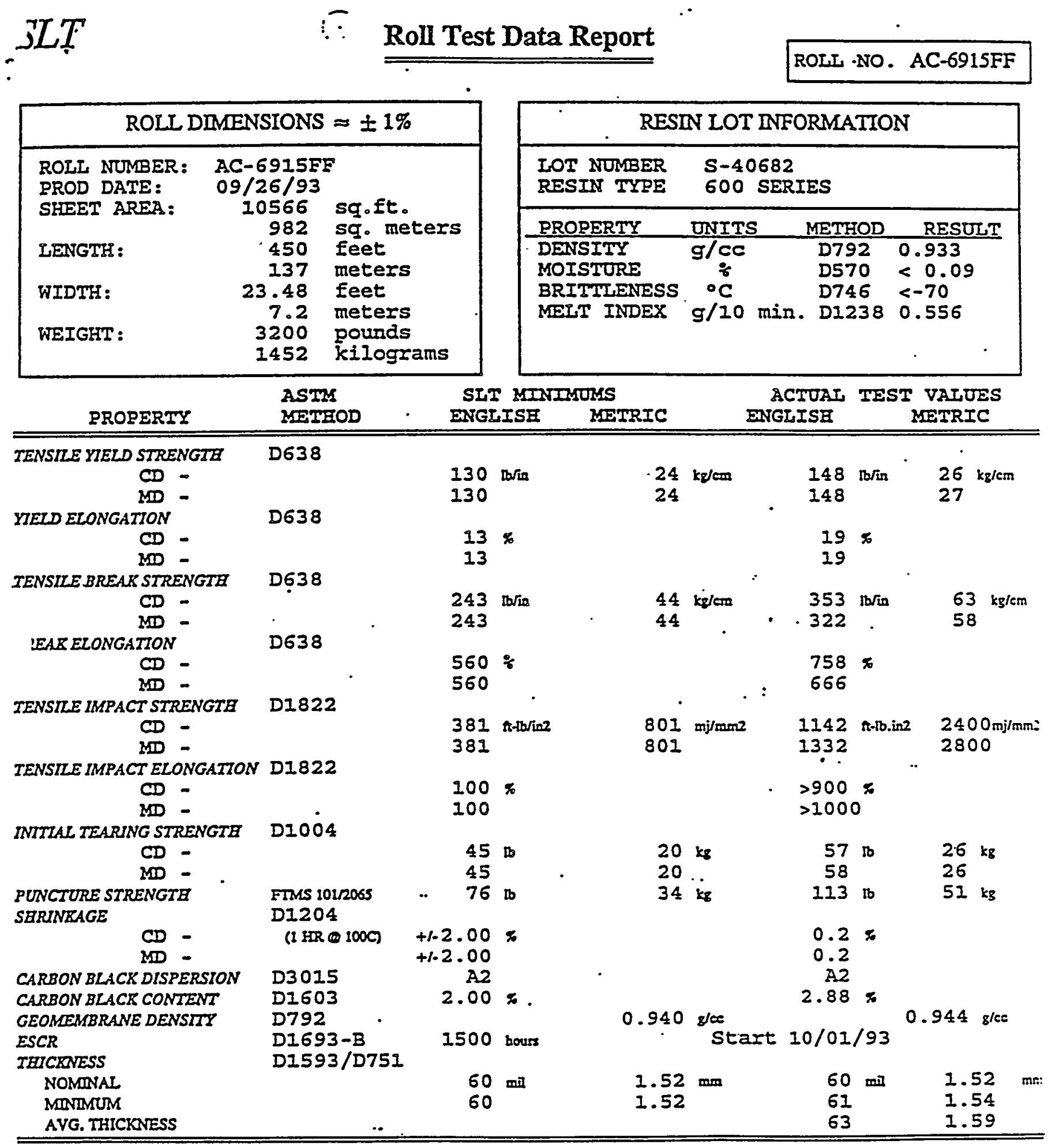

TEST NOTES: MD = MACHINE DIRECTION CD=CROSS DIRECTION ESCR START DATE IS GIVEN -FOR TENSILE ELONGATION TEST GAUGE LENGTH $=1.3$ ON YIETD. 2.5 ON BREAK ALI STRENGTH VALUES BASED ON NOMENAL THICKNESS

JSTOMER :

DATE SHIPPED

JOB NO.

COMMENTS 


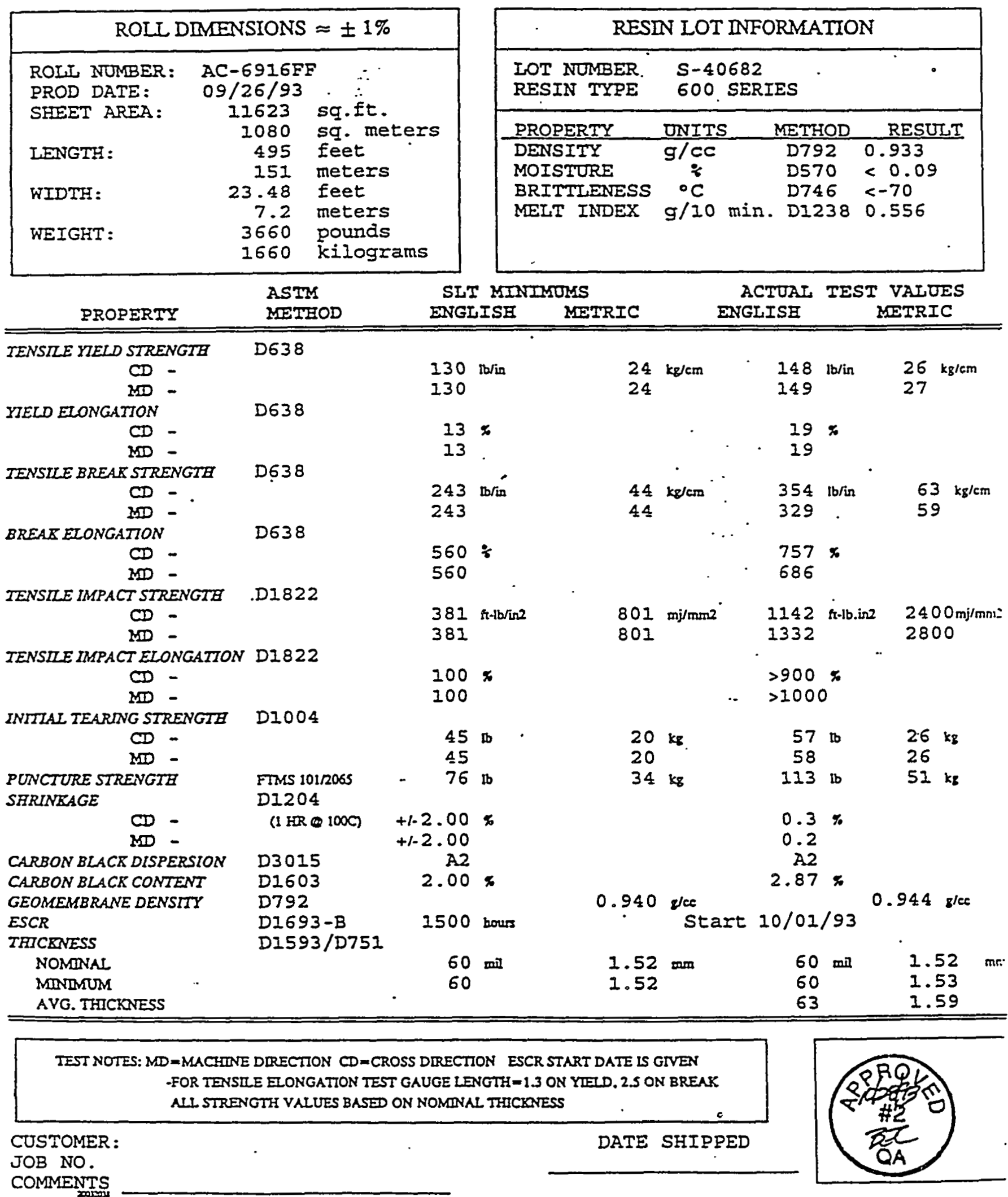




\begin{tabular}{|c|c|c|}
\hline \multicolumn{3}{|c|}{ ROLL DIMENSIONS $\approx \pm 1 \%$} \\
\hline $\begin{array}{l}\text { ROLL NUMBER: } \\
\text { PROD DATE: } \\
\text { SEEET AREA: } \\
\text { LENGTH: } \\
\text { WIDTHE: } \\
\text { WEIGHT: }\end{array}$ & $\begin{array}{c}A C-6917 F F \\
09 / 26 / 93 \\
8922 \\
829 \\
380 \\
116 \\
23.48 \\
7.2 \\
2840 \\
1288\end{array}$ & $\begin{array}{l}\quad \\
\text { sq.ft. } \\
\text { sq. meters } \\
\text { feet } \\
\text { meters } \\
\text { feet } \\
\text { meters } \\
\text { pounds } \\
\text { kilograms }\end{array}$ \\
\hline
\end{tabular}

\begin{tabular}{|c|c|c|c|}
\hline \multicolumn{4}{|c|}{ RESIN LOT INFORMATION } \\
\hline $\begin{array}{l}\text { LOT NOMBER } \\
\text { RESIN TYPE }\end{array}$ & $\begin{array}{l}S-40682 \\
600 \text { SERI }\end{array}$ & IES & \\
\hline PROPERTY & ONITS & METHOD & RESULT \\
\hline $\begin{array}{l}\text { DENSITY } \\
\text { MOISTURE } \\
\text { BRITTLENESS } \\
\text { MEIT INDEX }\end{array}$ & 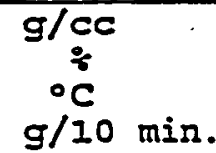 & $\begin{array}{l}D 792 \\
D 570 \\
D 746 \\
D 1238\end{array}$ & $\begin{array}{l}0.933 \\
<0.09 \\
<-70 \\
0.556\end{array}$ \\
\hline
\end{tabular}

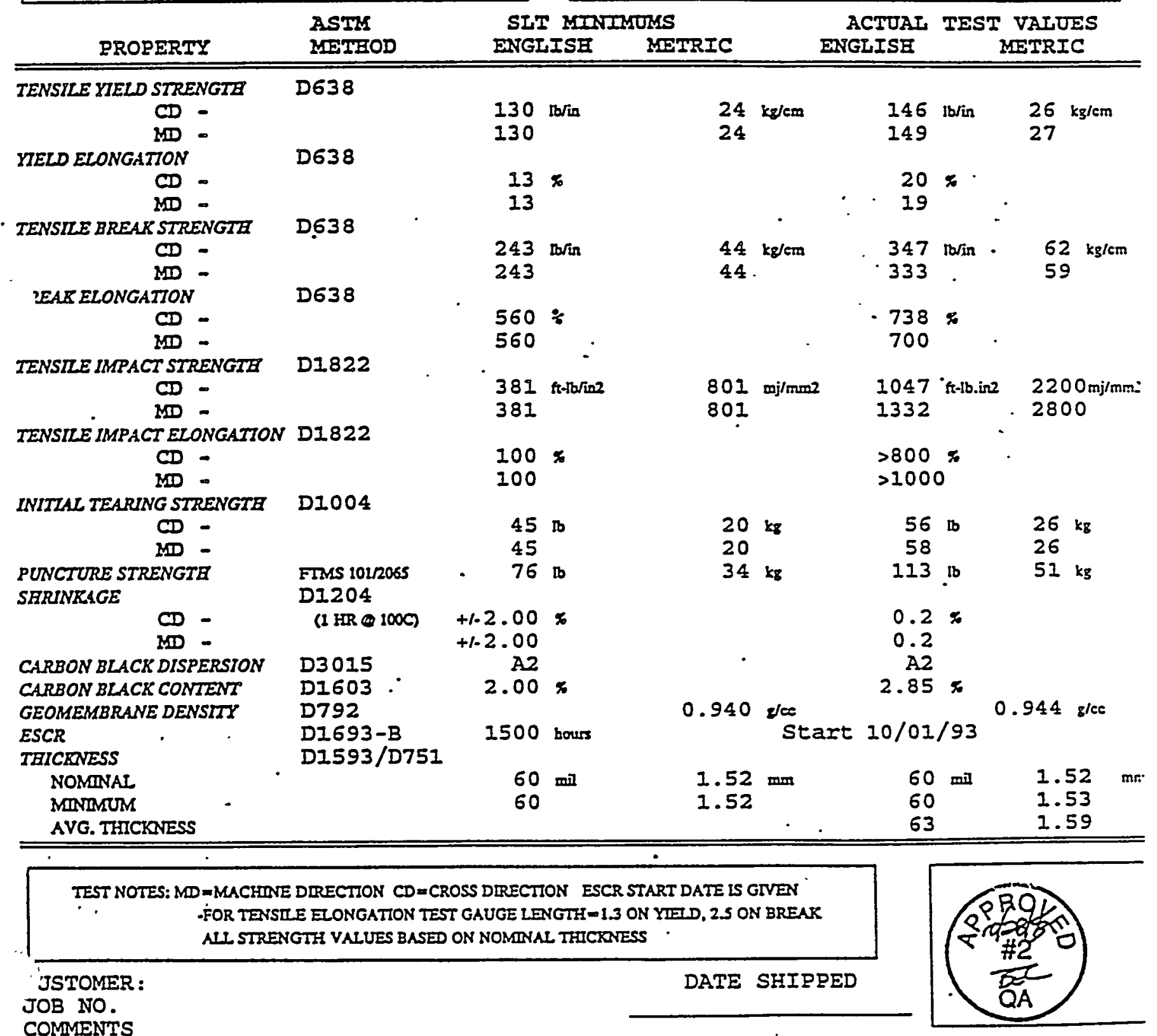




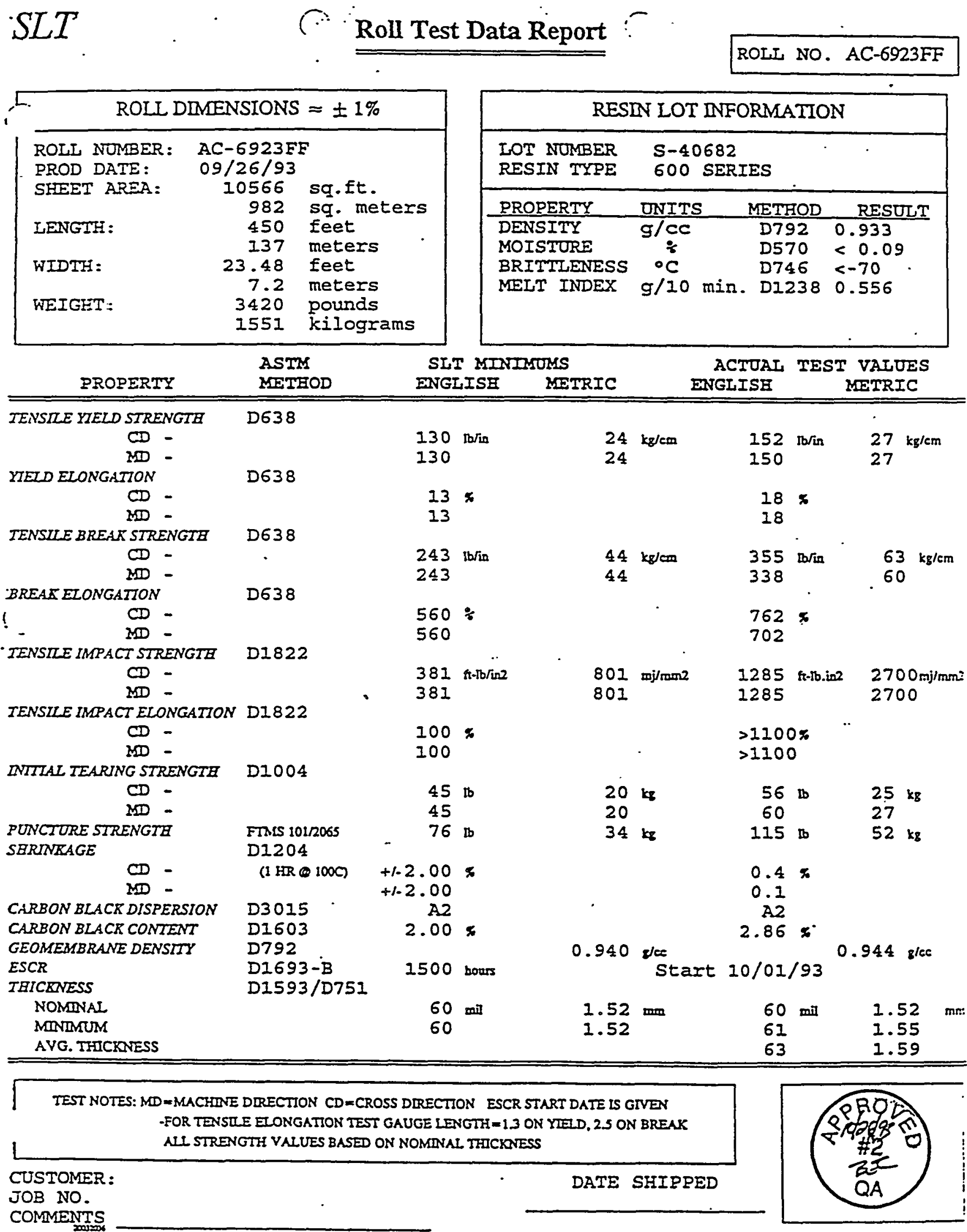




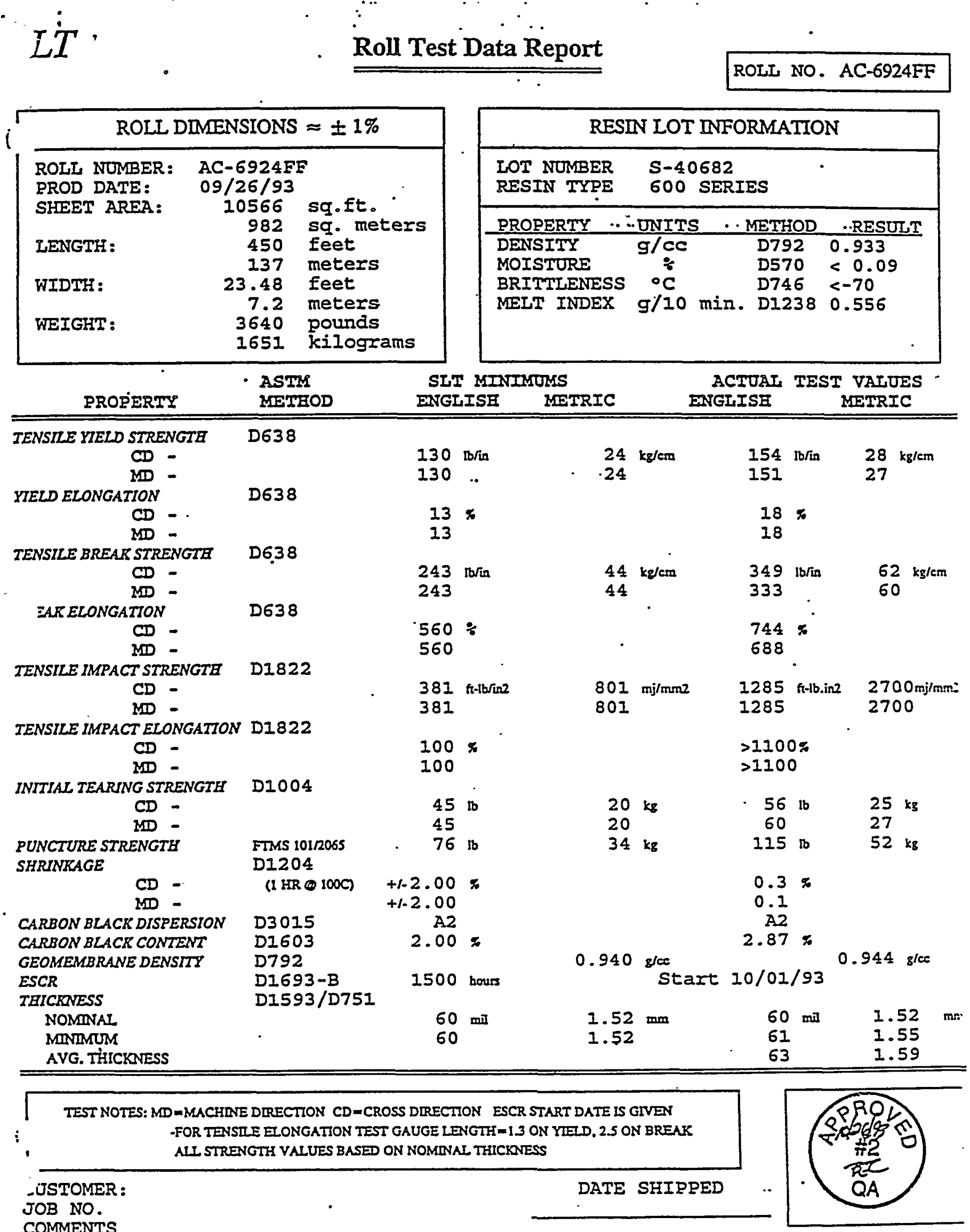




\begin{tabular}{|c|c|c|}
\hline \multicolumn{3}{|c|}{ ROLL DIMENSIONS $\approx \pm 1 \%$} \\
\hline ROLI NUMBER: & $\begin{array}{l}A C-6925 F E \\
09 / 26 / 93\end{array}$ & \\
\hline SHEET AREA: & $\begin{array}{r}10566 \\
982\end{array}$ & $\begin{array}{l}\text { sq.ft. } \\
\text { sq. mete }\end{array}$ \\
\hline LENGTH: & $\begin{array}{l}450 \\
137\end{array}$ & $\begin{array}{l}\text { feet } \\
\text { meters }\end{array}$ \\
\hline WIDTH: & $\begin{array}{r}23.48 \\
7.2\end{array}$ & $\begin{array}{l}\text { feet } \\
\text { meters }\end{array}$ \\
\hline WEIGHT: & $\begin{array}{l}3780 \\
1725\end{array}$ & $\begin{array}{l}\text { pounds } \\
\text { kilograms }\end{array}$ \\
\hline
\end{tabular}

\begin{tabular}{|c|c|c|c|c|c|c|c|c|}
\hline \multirow[b]{2}{*}{ PROPERTY } & \multirow{2}{*}{$\begin{array}{l}\text { ASTM } \\
\text { METEOD }\end{array}$} & \multicolumn{3}{|c|}{ SIT MINIMOMS. } & \multirow{2}{*}{\multicolumn{2}{|c|}{$\begin{array}{l}\text { ACTUAL } \\
\text { ENGLISE }\end{array}$}} & \multirow{2}{*}{\multicolumn{2}{|c|}{$\begin{array}{l}\text { TEST VALUES } \\
\text { METRIC }\end{array}$}} \\
\hline & & ENGL & UISE & METRIC & & & & \\
\hline IENSILE YIELD STRENGTH & $D 638$ & & & & & & & \\
\hline $\begin{array}{l}C D- \\
M D\end{array}$ & & 130 & torin & 24 & $\mathrm{~kg} / \mathrm{cm}$ & 153 & $\mathrm{Da} / \mathrm{in}$ & $27 \mathrm{~kg} / \mathrm{cm}$ \\
\hline YTELD ELONGATION - & D638 & 130 & & 24 & & 151 & & 27 \\
\hline CD & & 13 & $x$ & & & 18 & $x$ & \\
\hline LD - & & 13 & & & & 18 & & \\
\hline TENSILE BREAK STRENGTH & $D 638$ & & & & & & & \\
\hline $\begin{array}{l}C D- \\
M D\end{array}$ & $\cdot$ & $\begin{array}{l}243 \\
243\end{array}$ & Ib/in & $\begin{array}{l}44 \\
44\end{array}$ & $\mathrm{~kg} / \mathrm{cms}$ & $\begin{array}{l}346 \\
335\end{array}$ & $\mathrm{Ib} / \mathrm{in}$ & $\begin{array}{l}62 \mathrm{~kg} / \mathrm{cm} \\
60\end{array}$ \\
\hline BREAI ELONGATION & $D 638$ & & & & & & & \\
\hline . $\quad C D=$ & & $\begin{array}{l}560 \\
560\end{array}$ & $\%$ & - & & $\begin{array}{l}729 \\
691\end{array}$ & $x$ & \\
\hline TENSILE IMPACT STRENGTH & D1822 & & & & & & & \\
\hline $\begin{array}{l}C D- \\
M D\end{array}$ & & $\begin{array}{l}381 \\
381\end{array}$ & $\{t-1 b / i m 2$ & $\begin{array}{l}801 \\
801\end{array}$ & $\operatorname{mj} / \mathrm{mma} 2$ & $\begin{array}{l}1285 \\
1332\end{array}$ & thalb.ing & $\begin{array}{l}2700 \mathrm{mj} / \mathrm{mm} \\
2800\end{array}$ \\
\hline TENSILE IMPACT ELONGATTOI & $D 2822$ & & & & & & & \\
\hline $\begin{array}{l}C D- \\
\mathbb{N D}-\end{array}$ & & $\begin{array}{l}100 \\
100\end{array}$ & $\%$ & & & $\begin{array}{l}>1100 \\
>1100\end{array}$ & $0 \%$ & \\
\hline INITLAL TEARING STRENGTH & D1004 & & & & & & & \\
\hline $\begin{array}{l}\mathrm{CD}- \\
\mathrm{MD}-\end{array}$ & & $\begin{array}{l}45 \\
45\end{array}$ & b & $\begin{array}{l}20 \\
20\end{array}$ & $\mathrm{~kg}$ & $\begin{array}{l}56 \\
60\end{array}$ & tb & $\begin{array}{l}25 \mathrm{~kg} \\
27\end{array}$ \\
\hline $\begin{array}{l}\text { PUNCTURE STRENGTH } \\
\text { SERINRAGE }\end{array}$ & $\begin{array}{l}\text { FTMS 101/2065 } \\
\text { D1204 }\end{array}$ & 76 & $\mathbb{b}$ & 34 & kg & 115 & $\mathfrak{b}$ & $52 \mathrm{~kg}$ \\
\hline CD - & (1 HR $100 \mathrm{C})$ & $\begin{array}{l}+1.2 .00 \\
+1.2 .00\end{array}$ & $\%$ & & & $\begin{array}{l}0.3 \\
0.1\end{array}$ & \% & \\
\hline CARBON BLACR DISPERSION & $D 3015$ & A2 & & & & A2 & & $\cdot$ \\
\hline CARBON BLACK CONTENT & D1603 & 2.00 & x & & & 2.89 & $\%$ & \\
\hline $\begin{array}{l}\text { GEOMEMBRANE DENSITY } \\
\text { ESCR }\end{array}$ & D792 & & & 0.940 & E/ee & & & 0.944 v/ce \\
\hline $\begin{array}{l}\text { ESCR } \\
\text { THICENESS }\end{array}$ & $\begin{array}{l}D 1693-B \\
D 1593 / D 751\end{array}$ & 1500 & bours & & star & It $10 / 01 /$ & 193 & \\
\hline $\begin{array}{l}\text { NOMUNAI } \\
\text { MINMMUM } \\
\text { AYG. THICKNESS }\end{array}$ & & $\begin{array}{l}60 \\
60\end{array}$ & $\min$ & $\begin{array}{l}1.52 \\
1.52\end{array}$ & $m$ & $\begin{array}{l}60 \\
61 \\
63\end{array}$ & mil & $\begin{array}{l}1.52 \\
1.55 \\
1.59\end{array}$ \\
\hline
\end{tabular}

TEST NOTES: MD=MACFINE DIRECTION CD=CROSS DIRECTION ESCR START DATE IS GIVEN -FOR TENSLE ELONGATION TEST GAUGE LENGTH $=1.3$ ON YIEDD. 2.5 ON BREAK ALI STRENGTH VALUES BASED ON NOMINAL TFICKONESS

CUSTOMER:

JOB NO.

COMMENTS

\begin{tabular}{|llll|}
\hline \multicolumn{4}{|c|}{ RESIN LOT INFORMATION } \\
\hline IOT NOMBER & S-40682 & $\cdot$ \\
RESIN TTYE & 600 & SERIES \\
\hline PROPERTY & UNITS & METHOD & RESULT \\
\hline DENSITY & g/CC & D792 & 0.933 \\
MOISTURE & $\%$ & D570 & $<0.09$ \\
BRITTLENESS & $\circ \mathrm{C}$ & D746 & $<-70$ \\
MEIT INDEX & g/I0 min. & DI238 & 0.556 \\
& & & \\
& & & \\
\hline
\end{tabular}

TEST VALTES

15310

$27 \mathrm{~kg} / \mathrm{cm}$

$18 \%$

$346 \mathrm{rb} / \mathrm{in} \quad 62 \mathrm{~kg} / \mathrm{cm}$ 335 
$" s \grave{T}$.

Roll Test Data Report

ROLI NO. AC-6926FF

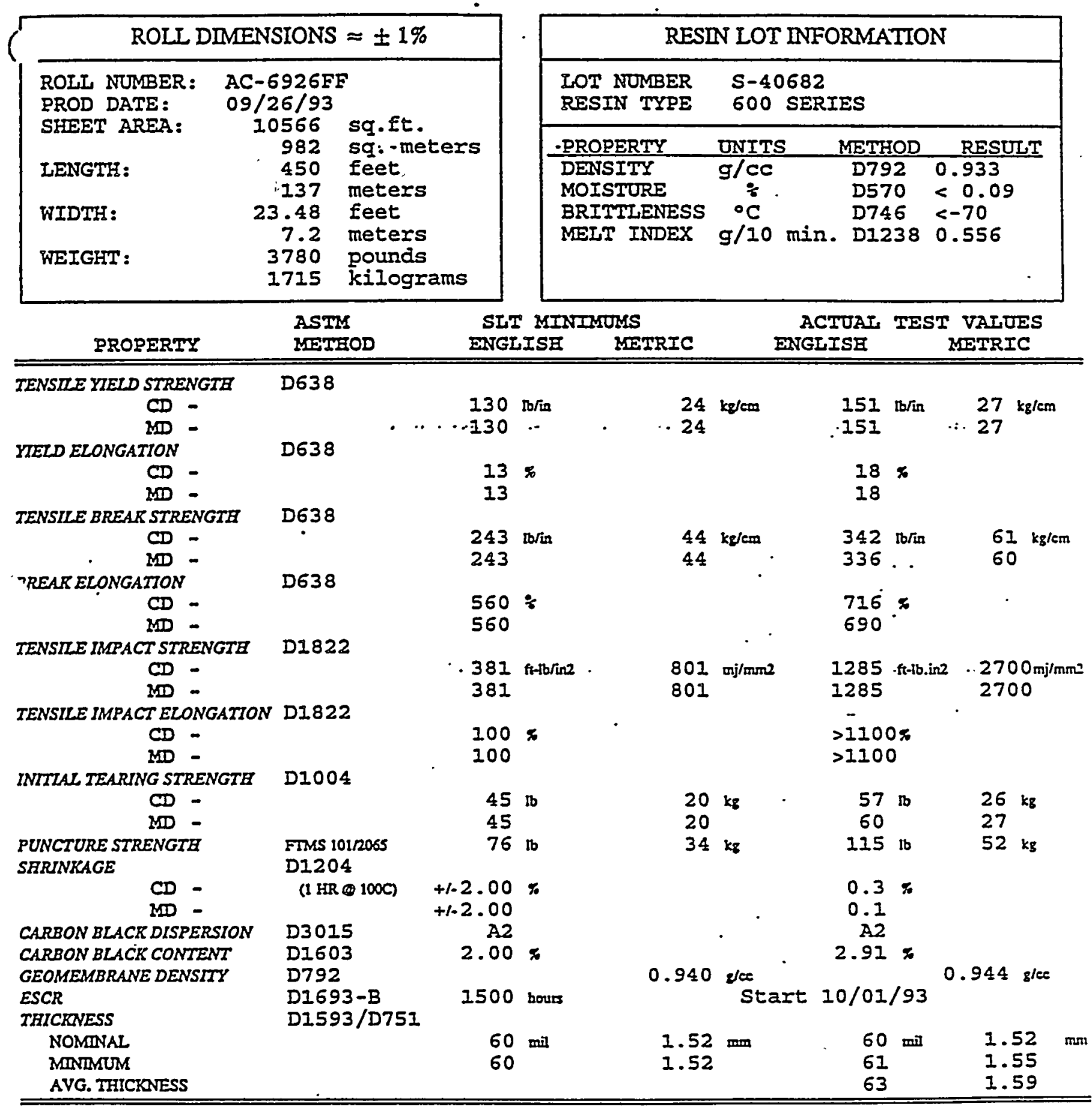

TEST NOTES: MD - MUCHINE DIRECTION CD-CROSS DIRECTION ESCR STTART DATE IS GIVEN .FOR TENSLLE ELONGATION TEST GAUGE LENGTH $=1.3$ ON YIELD. 2.5 ON BREAK ALL STRENGTH VALUES BASED ON NOMINAL THLCKNESS

TUSTOMER : ...DATE SHIPPED JOB NO. COMMENTS

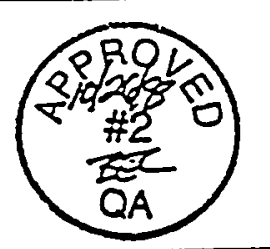


SLT

ก Roll Test Data Report

ROLI, NO. AC-6927FF

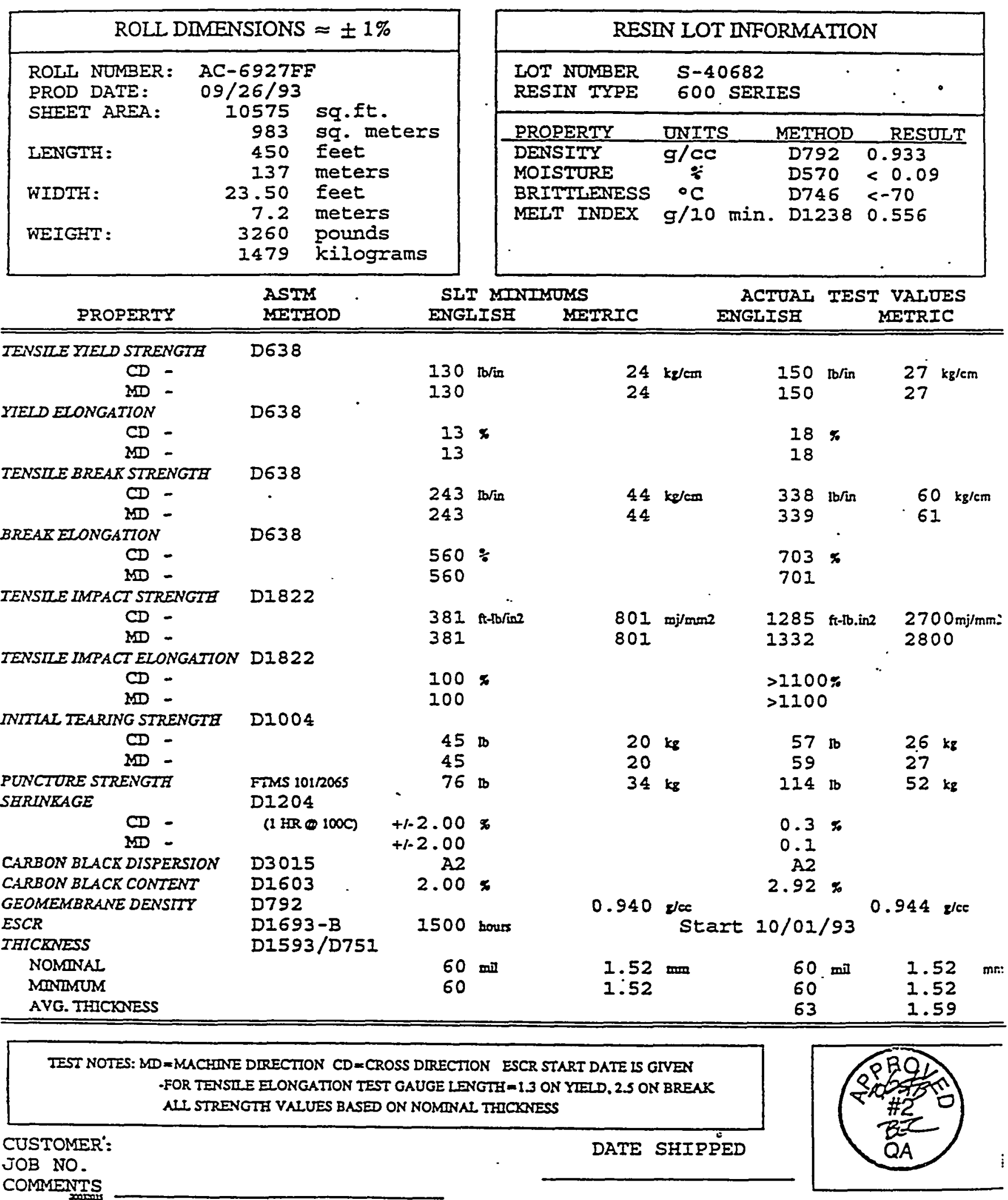


WHC-SD-W025-RPT-001, Rev. 0

SLT

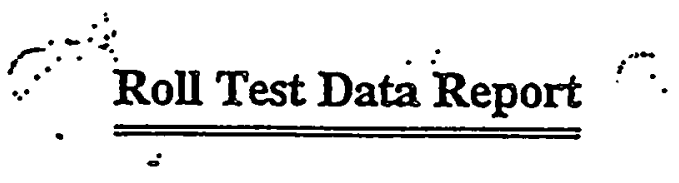

ROLI NO. AC-6928FF

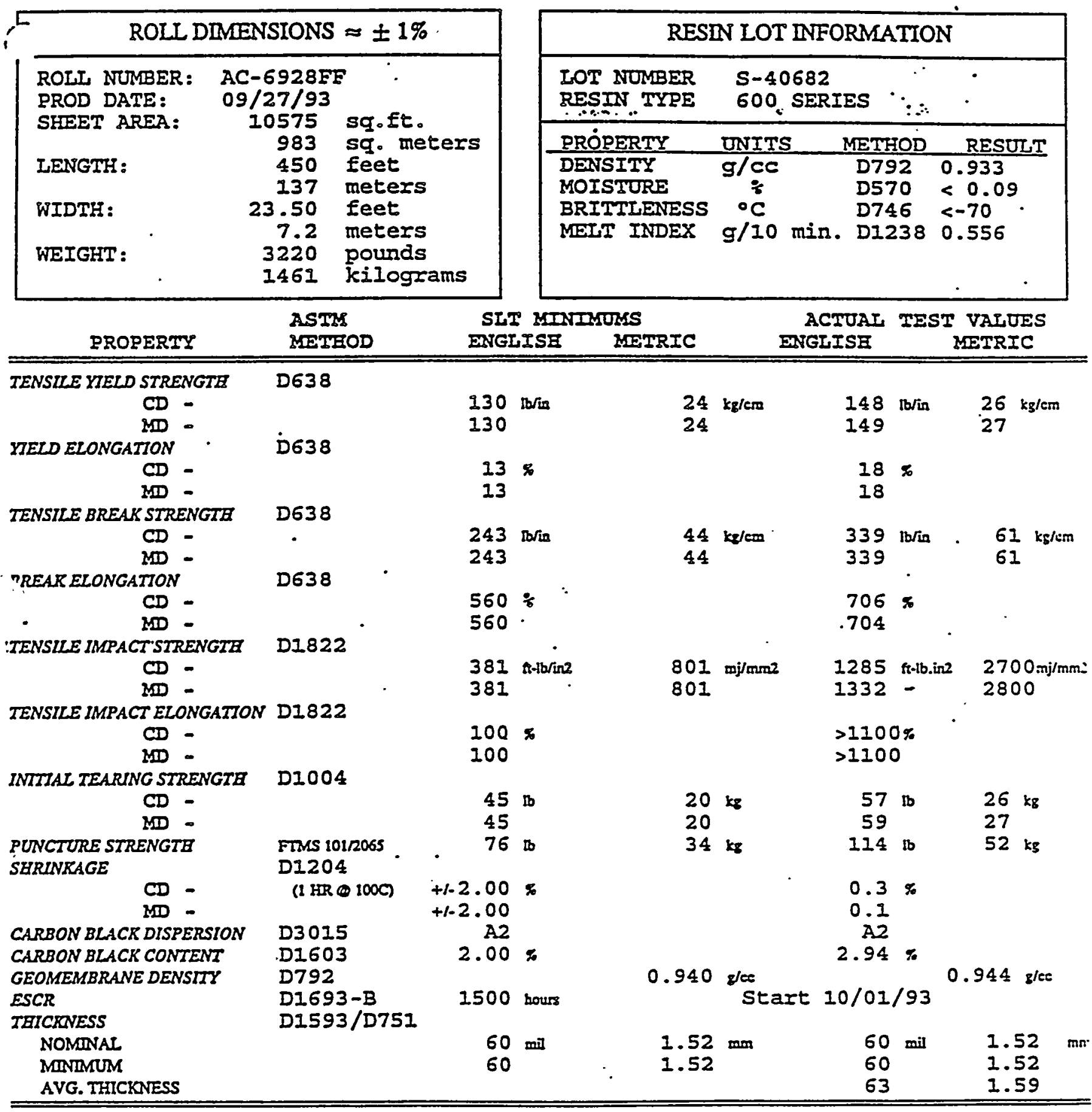

TEST NOTES: MD =MACHINE DIRECTION CD=CROSS DRECTION ESCR START DATE IS GIVEN -FOR TENSILE EIONGATION TEST GAUGE LENGTH-1.3 ON YIEID. 2.5 ON BREAK ALL STRENGTH YALUES BASED ON NOMENAL THICKNESS

USTOMER :

DATE SHIPPED

.OB NO.

COMMENTS

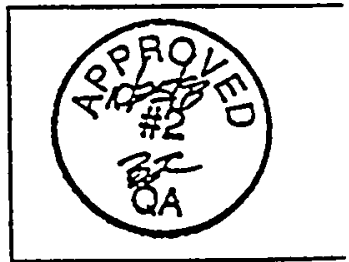




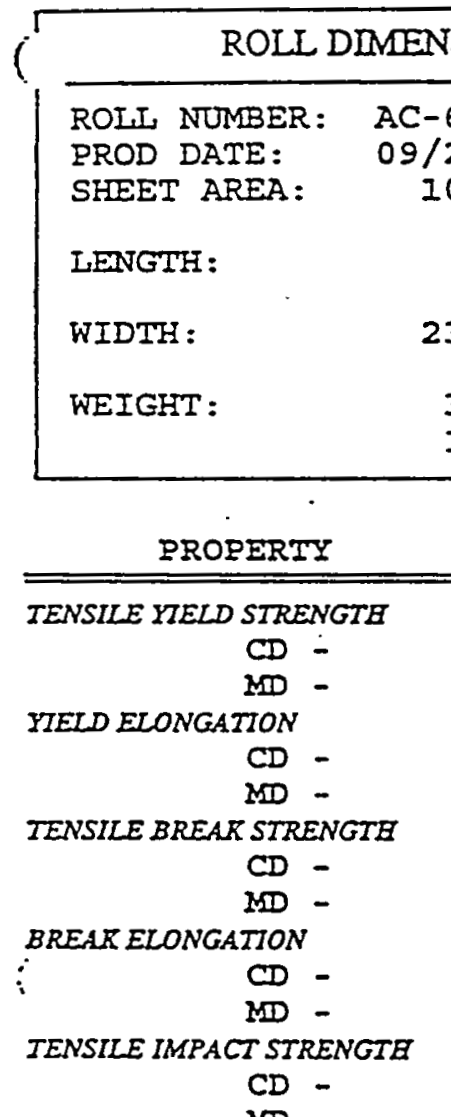

$\mathrm{ND}$ -

TENSILE IMPACT ELONGATION DI822

$\mathrm{CD}$ -

INITIAL TEARING STRENGTE $\mathrm{CD}$ $\mathrm{MD}$ -

PUNCTURE STRENGTH SHRINKAGE

$$
\text { CD - }
$$

CARBON BLACK DISPERSION CARBON BLACK CONTENT GEOMEMBRANE DENSTTY ESCR TEICKNESS NOMINAI MINIMUM AVG. THICKNESS

D638

D638

D638

D638

$D 1822$

D1004

DI204

D3015

\begin{tabular}{|c|c|c|c|}
\hline \multicolumn{4}{|c|}{ RESIN LOT INFORMATION } \\
\hline $\begin{array}{l}\text { LOT NUMBER } \\
\text { RESIN TYPE }\end{array}$ & $\begin{array}{l}S-40682 \\
600 \text { SERI }\end{array}$ & IES & \\
\hline PROPERTY & DNITS & METHOD & RESULT \\
\hline $\begin{array}{l}\text { DENSITY } \\
\text { MOISTURE } \\
\text { BRITTLENESS } \\
\text { MELT INDEX }\end{array}$ & $\begin{array}{l}g / c c \\
\frac{0}{*} \\
\circ \mathrm{C} \\
g / 10 \mathrm{~min} .\end{array}$ & $\begin{array}{l}\text { D792 } \\
D 570 \\
D 746 \\
D 1238\end{array}$ & $\begin{array}{l}0.933 \\
<0.09 \\
<-70 \\
0.556\end{array}$ \\
\hline
\end{tabular}

D1603

D792

$$
1696 \text { kilograms }
$$

137 meters

23.50 feet

7.2 meters

3740 pounds

ASTM SIT MININUMS

METHOD
ACTUAL TEST VATUES ENGIISE METRIC

ENGLISE METRIC

$24 \mathrm{~kg} / \mathrm{cm}$

$\begin{array}{lr}147 \mathrm{rb} / \mathrm{in} & 26 \mathrm{~kg} / \mathrm{cm} \\ 149 . . & .27\end{array}$

130

\section{$13 \%$}

13

$243 \mathrm{rb} / \mathrm{ill}$

243

$560 \div$

560

$\begin{array}{ll}381 & \mathrm{ft} \\ 38 \mathrm{~b} / \mathrm{in} 2\end{array}$
801

$100 \%$

100 .

$\begin{array}{ll} & 45 \mathrm{bb} \\ & 45 \\ \text { FTMS 101/2065 } & 76 \mathrm{bb}\end{array}$

(I $\operatorname{LR} \odot 1000)$

$+1.2 .00 \%$

$+1.2 .00$

D1693-B

D1593/D751

$$
2.00 \%
$$

1500 bours

$\begin{array}{ll}60 \mathrm{~min} \cdot & 1.52 \mathrm{~mm} \\ 60 & 1.52\end{array}$

24

$44 \mathrm{~kg} / \mathrm{cm}$

44

$801 \mathrm{mi} / \mathrm{mm} 2$

$$
\begin{aligned}
& 19 \% \\
& 19
\end{aligned}
$$

$346 \mathrm{lb} / \mathrm{in}$

344 .

$62 \mathrm{~kg} / \mathrm{cm}$ 62

$725 \%$

722

1285 fr-lb.in 2 1285

$2700 \mathrm{mj} / \mathrm{mm} 2$ 2700

$>1100 \%$ $>2200$

$$
20
$$

$34 \mathrm{~kg}$

$\begin{array}{rlr}57 \mathrm{~kb} & 26 \mathrm{~kg} \\ 59 & 27 \\ 115 \mathrm{~kb} & 52 \mathrm{~kg}\end{array}$

$0.3 \%$

0.1

A2

$2.95 \%$

Start $10 / 01 / 93$

\subsection{5 gice}$$
1.52
$$

\footnotetext{
TEST NOTES: MD=MACHINE DIRECTION CD=CROSS DIRECII
-FOR TENSLIE ELONGATION TEST GAUGE
ALL STRENGTH VALUES BASED ON NOMINA
CUSTOMER:
JOB NO.
COMMENTS
}

\begin{tabular}{l}
$60 \mathrm{mil}$ \\
$61.52 \mathrm{~mm}$ \\
$63 \quad \cdot 1.55$ \\
\hline
\end{tabular}

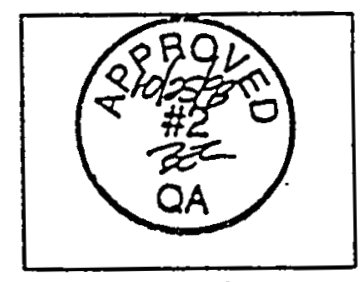


SLìi

Roll Test Data Report

ROLL NO. AC-6930FF

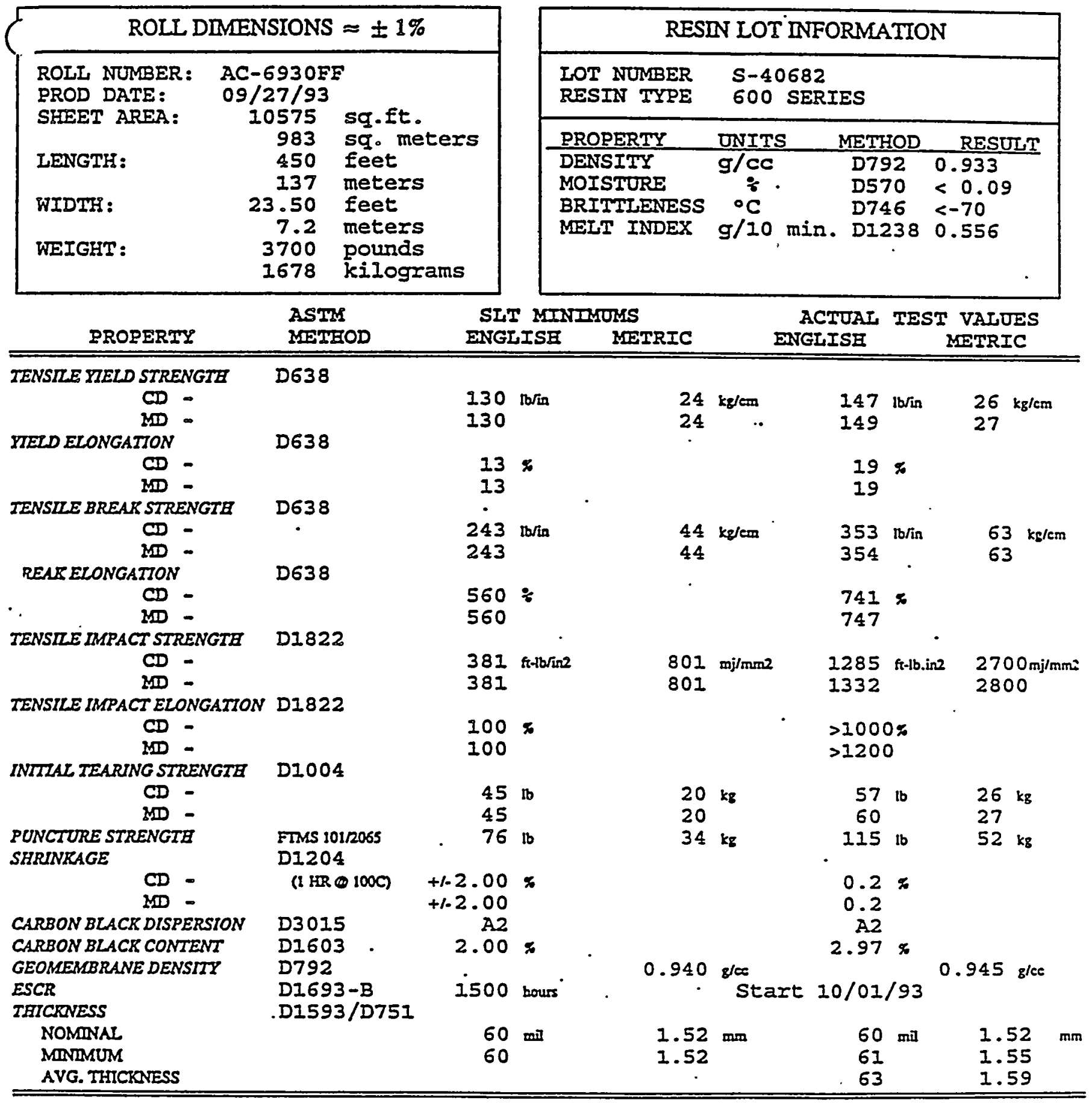

TEST NOTES: MD=MACFINE DIRECTION CD=CROSS DIRECTION ESCR START DATE IS GIVEN -FOR TENSILE ELONGATION TEST GAUGE LENGTH = 1.3 ON YIEDD, 2.5 ON BREAK ALL STRENGTF YALUES BASED ON NOMENAL TEUCKNESS

OSTOMER :

JOB NO.

DATE SHIPPED

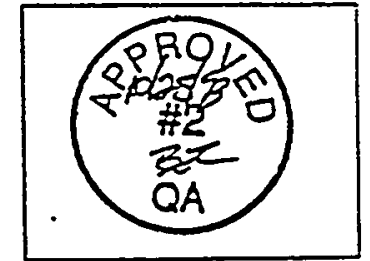

COMMENTS 


\begin{tabular}{|l|l|}
\hline \multicolumn{3}{|c|}{ ROLI DIMENSIONS $\approx \pm 1 \%$} \\
\hline ROII NUMBER: & AC-6931FF \\
PROD DATE: & $0.9 / 27 / 93$ \\
SHEET AREA : & 10575 sq.ft. \\
& 983 sq. meters \\
IENGTH: & 450 feet \\
& 137 meters \\
WIDTH : & 23.50 feet \\
& 7.2 meters \\
WEIGHT: & 3740 pounds \\
& 1696 kilograms
\end{tabular}

\begin{tabular}{|c|c|c|c|}
\hline \multicolumn{4}{|c|}{ RESIN LOT INFORMATION } \\
\hline $\begin{array}{l}\text { LOT NUMBER } \\
\text { RESIN TYPE }\end{array}$ & $\begin{array}{l}S-40682 \\
600 \text { SERI }\end{array}$ & & \\
\hline $\begin{array}{l}\text { PROPERTY } \\
\text { DENSITY } \\
\text { MOISTURE } \\
\text { BRITTLENESS } \\
\text { MEIT INDEX }\end{array}$ & $\begin{array}{l}\text { UNITS } \\
\text { g/CC } \\
\vdots \\
\stackrel{\circ}{C} \\
\text { g/I0 min. }\end{array}$ & $\begin{array}{l}\text { METHOD } \\
\text { D792 } \\
\text { D570 } \\
\text { D746 } \\
\text { D1238 }\end{array}$ & $\begin{array}{l}\quad \text { RESULT } \\
0.933 \\
<0.09 \\
<-70 \\
0.556\end{array}$ \\
\hline
\end{tabular}

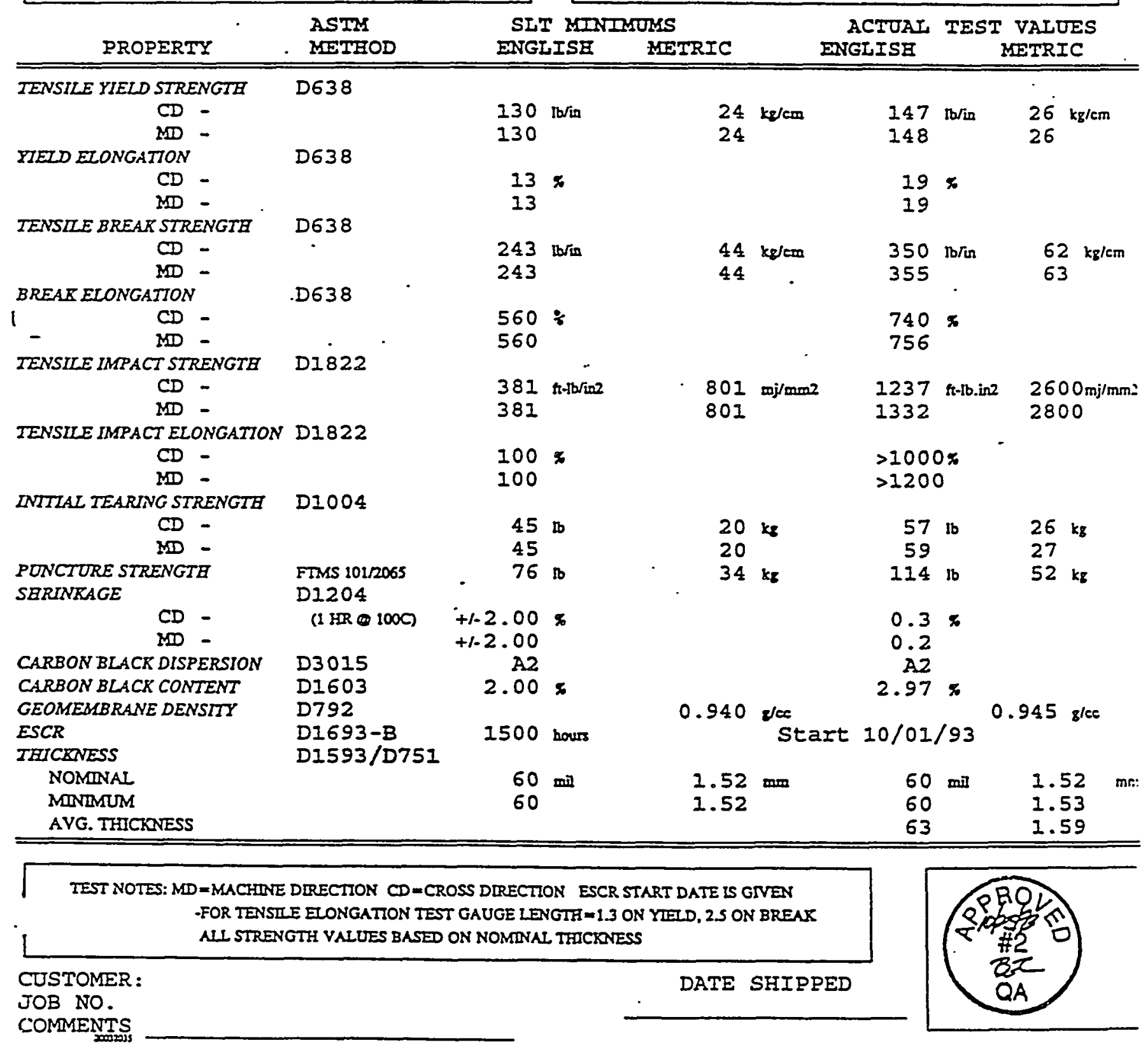




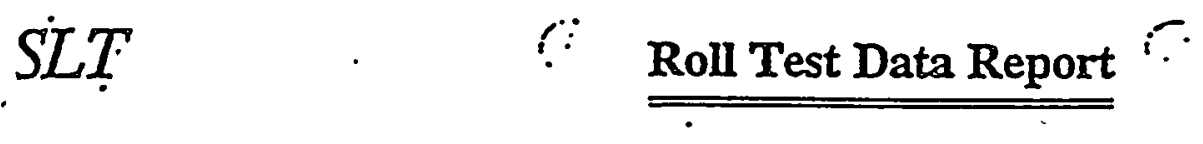

ROLI NO. AC-6932FF

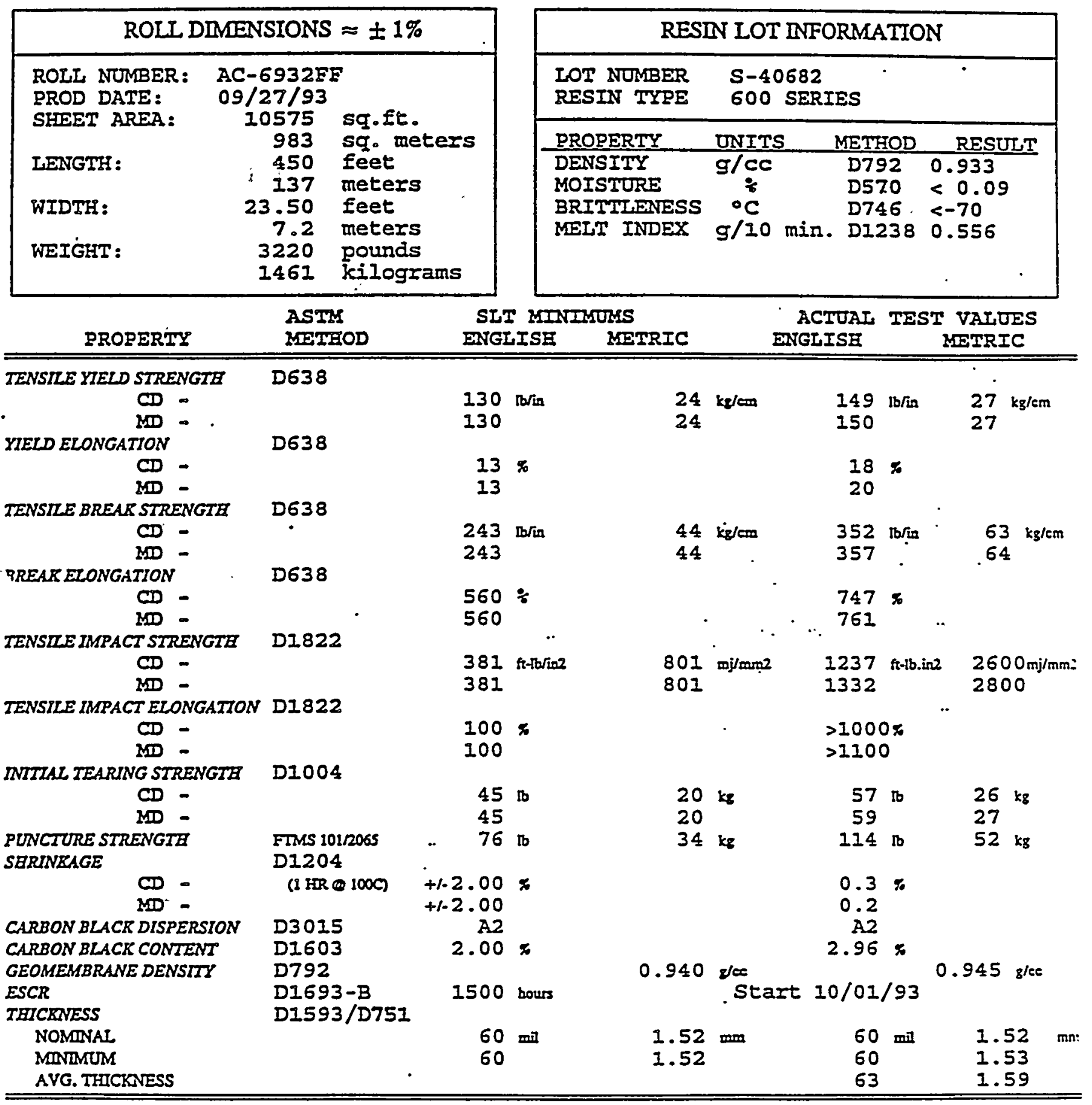

TEST NOTES: MD=MACFINE DIRECTION CD=CROSS DRRECTION ESCR START DATE IS GIVEN -FOR TENSLE ELONGATION TEST GAUGE LENGTH=1.3 ON YIED. 2.5 ON BREAK ALI STRENGTH VALUES BASED ON NOMINAL THICXNESS

USTOMER:

DATE SHIPPED

SOB NO.

COMMENTS

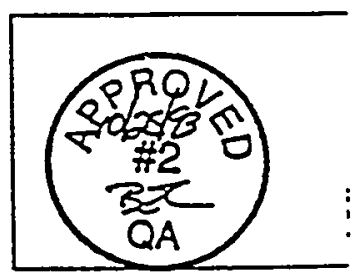


SLí

Roll Test Data Report

ROLI NO. AC-7680

\begin{tabular}{|c|c|c|}
\hline \multicolumn{3}{|c|}{ ROLI DIMENSIONS $\approx \pm 1 \%$} \\
\hline ROII NUMBER: & $A C-7680$ & \\
\hline PROD DATE: & $10 / 16 / 93$ & \\
\hline SHEET AREA: & $\begin{array}{r}-11163 \\
1037\end{array}$ & $\begin{array}{l}\text { sq. ft. } \\
\text { sq. meters }\end{array}$ \\
\hline IENGTH: & $\begin{array}{l}475 \\
145\end{array}$ & $\begin{array}{l}\text { feet } \\
\text { meters }\end{array}$ \\
\hline WIDTH: & $\begin{array}{r}23.50 \\
7.2\end{array}$ & $\begin{array}{l}\text { feet } \\
\text { meters }\end{array}$ \\
\hline WEIGHT: & $\begin{array}{l}3548 \\
1609\end{array}$ & $\begin{array}{l}\text { pounds } \\
\text { kilograms }\end{array}$ \\
\hline
\end{tabular}

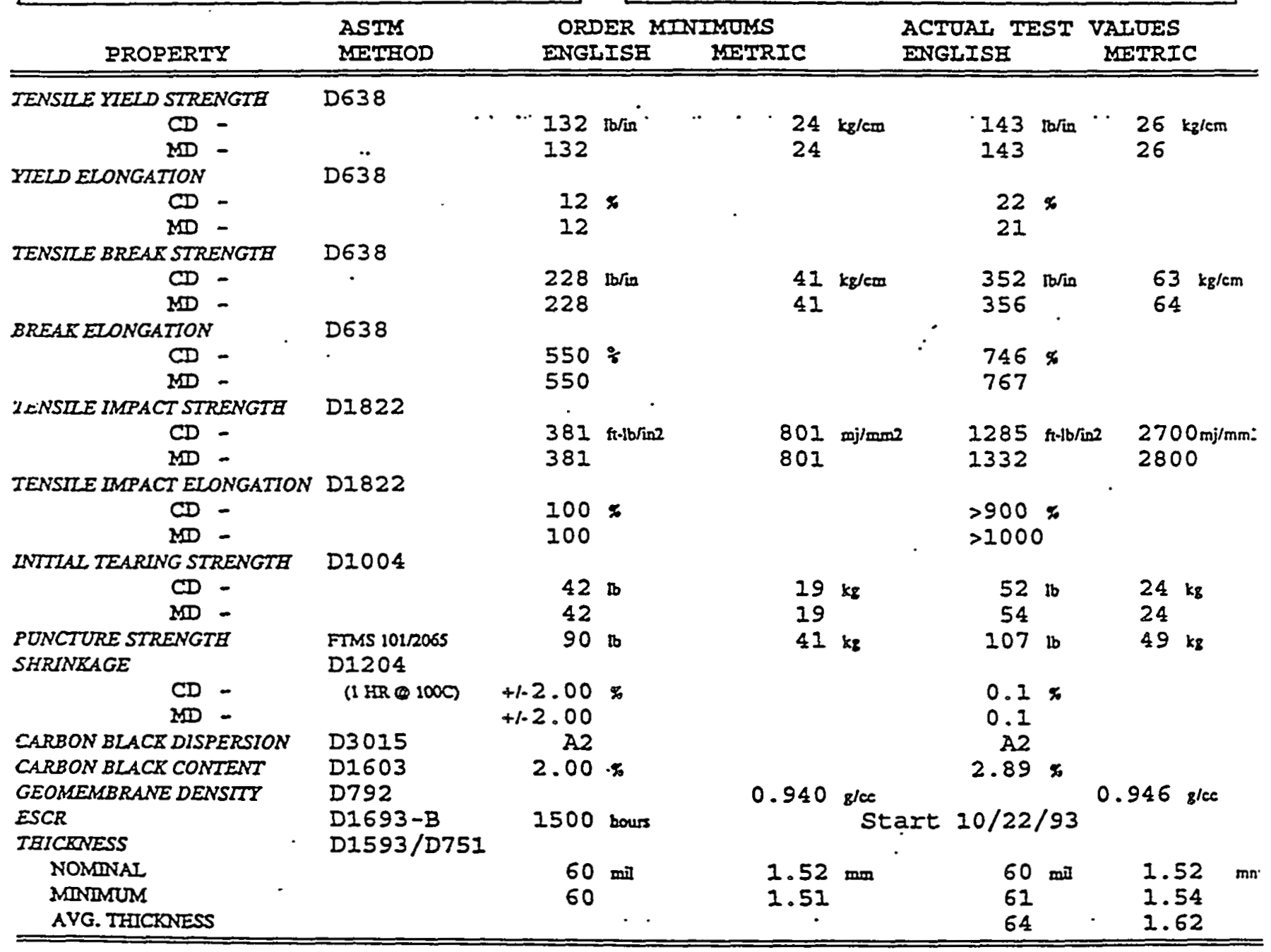

TEST NOTES: MD =MACHIN DIRECTION CD=CROSS DIRECTION ESCR START DATE IS GIVEN -FOR TENSILE ELONGATION TEST GAUGE LENGTH - 1.3 ON YIELD. 2.5 ON BREAK ; ALI STRENGTH VALUES BASED ON NOMINAL THICRNESS

CUSTOMER : HANFORD/GEOPACIFIC JOB NO. 8491

DATE SHIPPED

\begin{tabular}{lccll}
$\begin{array}{l}\text { IOT NUMBER } \\
\text { RESIN TYPE }\end{array}$ & \multicolumn{2}{l}{$\begin{array}{l}\text { S-40687 } \\
005\end{array}$ SERIES } \\
\hline PROPERTY & UNITS & METHOD & RESULT \\
\hline DENSITY & $9 / C C$ & D792 & 0.934 \\
MOISTURE & $\%$ & D570 & $<0.09$ \\
BRITTLENESS & $\circ \mathrm{C}$ & D746 & $<-70$ \\
MELT INDEX & $\mathrm{g} / 20 \mathrm{~min}$ & D1238 & 0.550
\end{tabular}


:SLì

Roll Test Data Report

ROLL NO. AC-7682

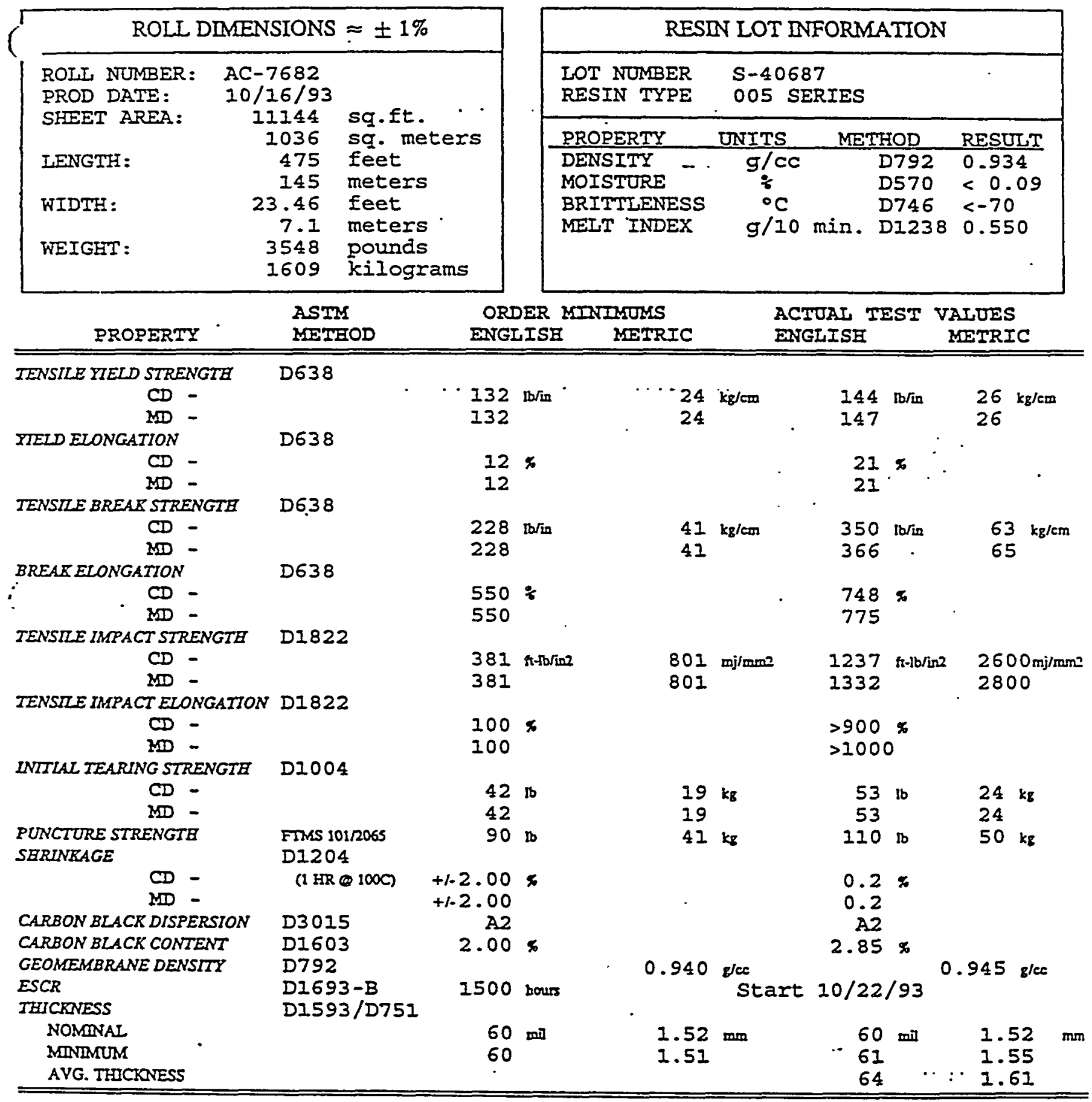

TEST NOTES: ND=KACHDVE DIRECTION CD=CROSS DIRECTION ESCR START DATE IS GIVEN -FOR TENSILE ELONGATION IEST GAUGE LENGTH = 1.3 ON YIEID. 2.5 ON BREAR AII STRENGTH VALUES BASED ON NOMDAI THHCENESS

CUSTOMER : HANEORD/GEOPACIFIC

JOB NO. 8491

DATE SHIPPED

COMMENTS

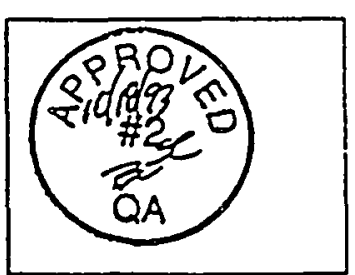


$\dot{S} L \ddot{T}$

Roll Test Data Report

-

ROLL NO. AC-7683FF

\begin{tabular}{|l|l|}
\hline \multicolumn{3}{|c|}{ ROLL DIMENSIONS $= \pm 1 \%$} \\
\hline ROLI NUMBER: & AC-7683FF \\
PROD DATE: & $10 / 16 / 93$ \\
SHEET AREA : & 1153 sq. ft. \\
& 1037 sq. meters \\
LENGTH: & 475 feet \\
WIDTH: & 145 meters \\
& 23.48 feet \\
WEIGHT: & 7.2 meters \\
& 3780 pounds \\
& 1715 kilograms \\
\hline
\end{tabular}

\begin{tabular}{|c|c|c|c|}
\hline \multicolumn{4}{|c|}{ RESIN LOT INFORMATION } \\
\hline $\begin{array}{l}\text { LOT NUMBER } \\
\text { RESIN TYPE }\end{array}$ & $\begin{array}{l}S-40687 \\
005 \text { SERI }\end{array}$ & & \\
\hline PROPERTY & UNITS & METHOD & RESULT \\
\hline $\begin{array}{l}\text { DENSITY } \\
\text { MOISTURE } \\
\text { BRITTLENESS } \\
\text { MEIT INDEX }\end{array}$ & $\begin{array}{c}\text { g/cc } \\
: \\
: C \\
g / 10 \mathrm{~min} .\end{array}$ & $\begin{array}{l}\text { D792 } \\
\text { D570 } \\
\text { D746 } \\
\text { D1238 }\end{array}$ & $\begin{array}{l}0.934 \\
<0.09 \\
<-70 \\
0.550\end{array}$ \\
\hline
\end{tabular}

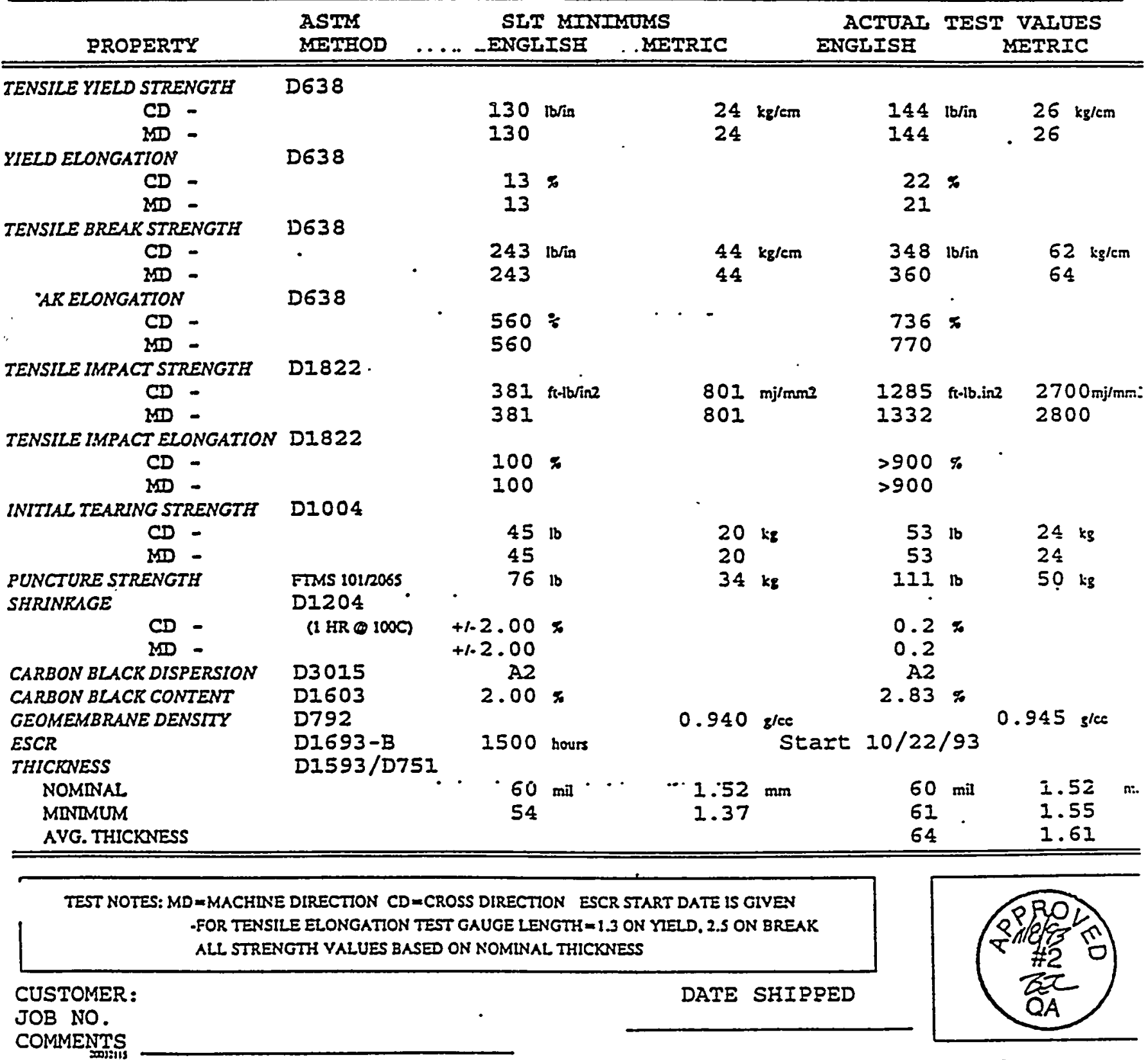


¿SLi :"

\section{Roll Test Data Report}

ROLI NO. AC-7684

\begin{tabular}{|c|c|c|}
\hline \multicolumn{3}{|c|}{ ROLI DIMENSIONS $\approx \pm 1 \%$} \\
\hline ROLI NUMBER: & $A C-7684$ & \\
\hline PROD DATE: & $10 / 16 / 93$ & \\
\hline SHEET AREA: & $\begin{array}{r}11163 \\
1037\end{array}$ & $\begin{array}{l}\text { sq.ft. } \\
\text { sq. meters }\end{array}$ \\
\hline IENGTH: & $\begin{array}{l}475 \\
145\end{array}$ & $\begin{array}{l}\text { feet } \\
\text { meters }\end{array}$ \\
\hline WIDTE: & $\begin{array}{r}23.50 \\
7.2\end{array}$ & $\begin{array}{l}\text { feet } \\
\text { meters }\end{array}$ \\
\hline WEICHT : & $\begin{array}{l}3552 \\
1611\end{array}$ & $\begin{array}{l}\text { pounds } \\
\text { kilograms }\end{array}$ \\
\hline
\end{tabular}

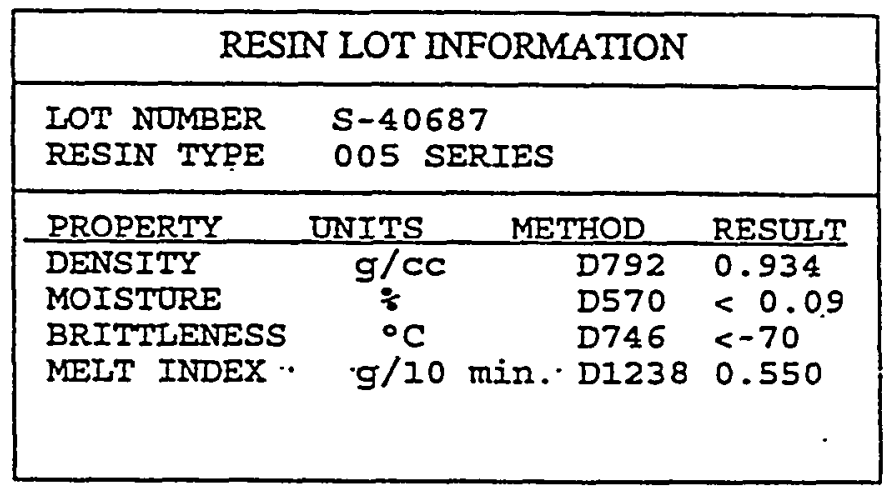

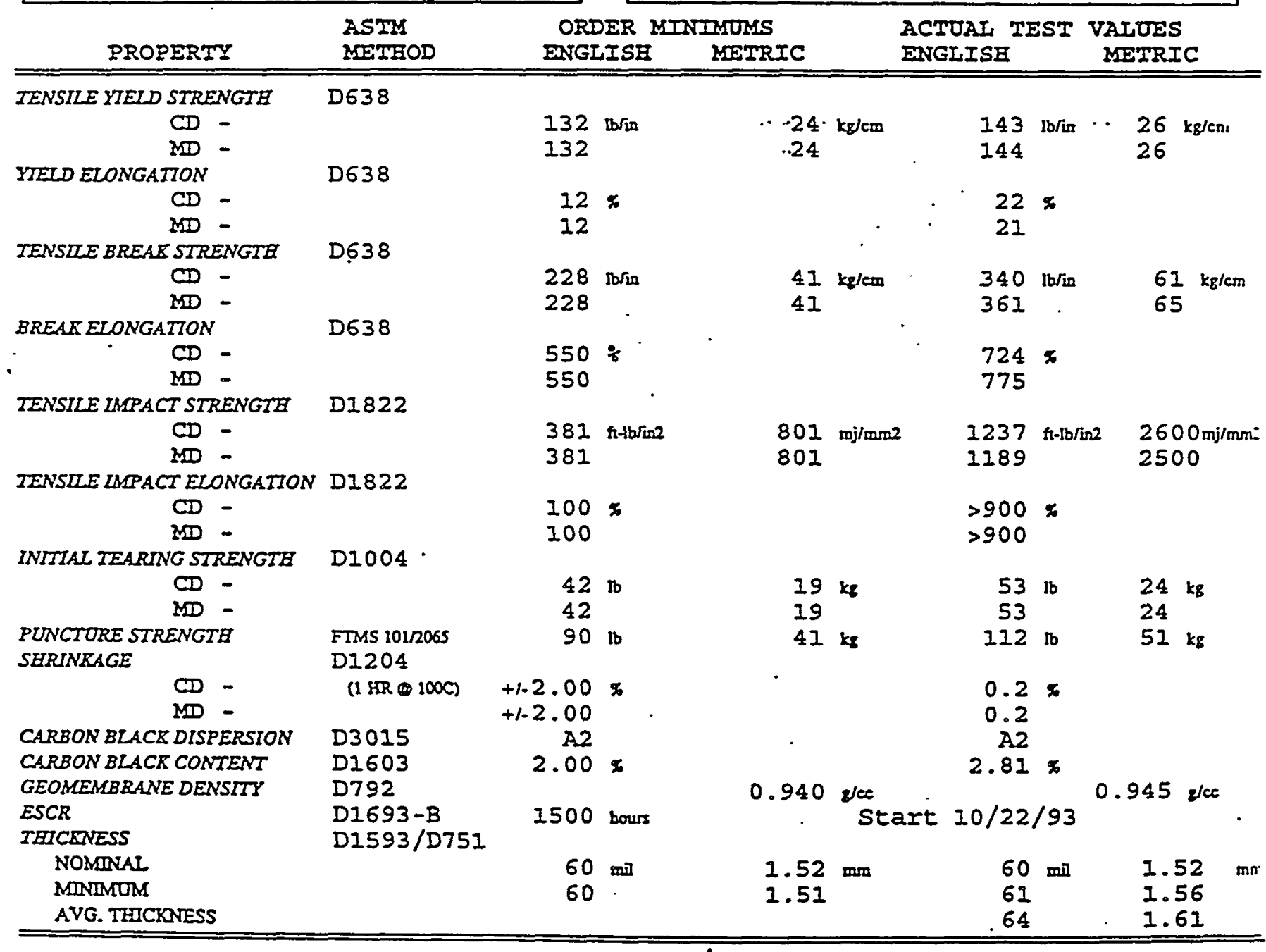

TEST NOTES: MD=MACHINE DIRECTION CD=CROSS DIRECTION ESCR START DATE IS GTVEN -FOR TENSILE ELONGATION TEST GAUGE LENGTH = 1.3 ON YTELD. 2 S ON BREAK AII STRENGTH VALUES BASED ON NOMONAL TFICKONESS

L

CUSTOMER : HANFORD/GEOPACIFIC JOB NO. 8491

COMMENTS
DATE SHIPPED

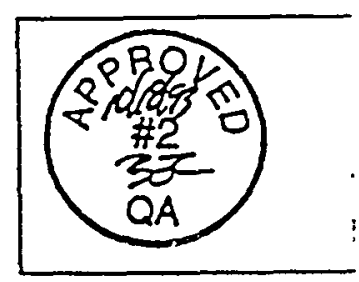


$\operatorname{SLT} \cdot$

Roll Test Data Report

ROLL NO. AC-7794

\begin{tabular}{|l|l|}
\hline \multicolumn{3}{c|}{ ROLI DIMENSIONS $\approx \pm 1 \%$} \\
\hline ROLI NOMBER: & AC-7794 \\
PROD DATE: & $10 / 19 / 93$ \\
SHEET AREA: & $8410 \mathrm{sq.ft.}$ \\
& 782 sq. meters \\
IENGTH: & 355 feet \\
& 108 meters \\
WIDTH: & 23.69 feet \\
WEIGHT: & 7.2 meters \\
& 3424 pounds \\
& 1553 kilograms
\end{tabular}

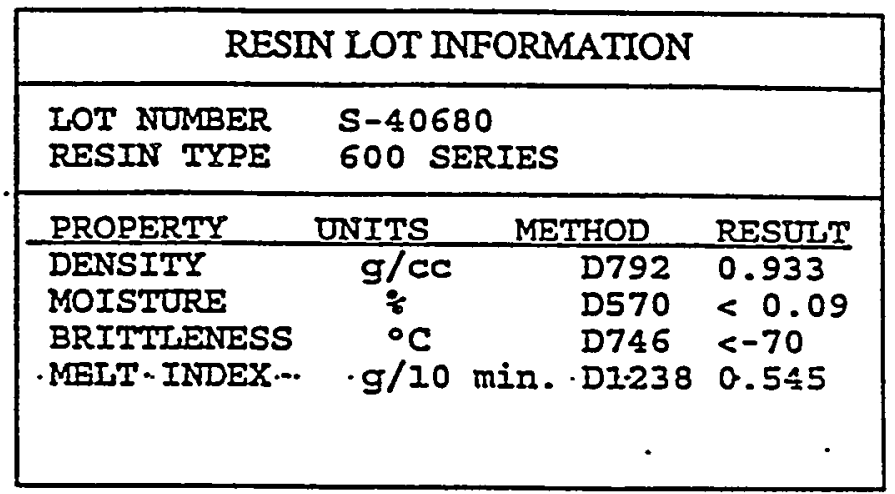

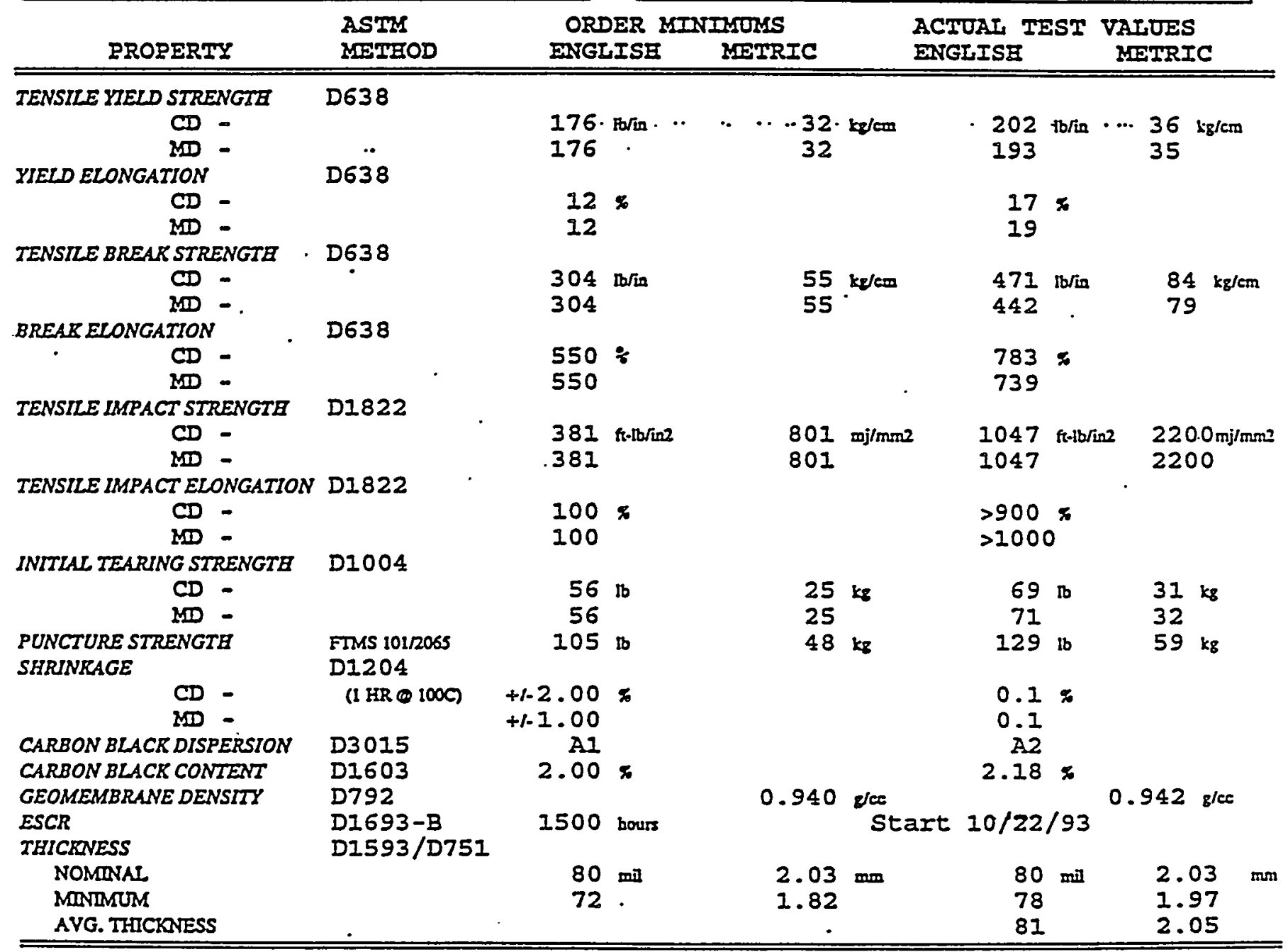

TEST NOTES: MD=MACHINE DRECTION CD=CROSS DIRECTION ESCR START DATE IS GVEN

-FOR TENSLE ELONGATION TEST GAUGE LENGTH 1.3 ON YIELD, 2.5 ON BREAK
ALI STRENGTH VALUES BASED ON NOMINAL THICKNESS

-FOR TENSLE ELONGATION TEST GAUGE LENGTH 1.3 ON YIELD, 2.5 ON BREAK
ALI STRENGTH VALUES BASED ON NOMINAL THICKNESS

"TJSTOMER : HANEORD/GEORACIFIC

JB NO. 8491

DATE SHIPPED

COMMENTS

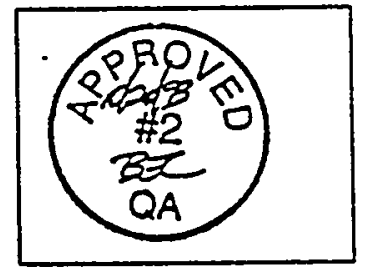


WHC-SD-W025-RPT-001, Rev. 0

$S L T$

Roll Test Data Report

ROLI NO. AC-8309

ROLL DIMENSIONS $\approx \pm 1 \%$

\begin{tabular}{lcl}
\hline ROLI NOMBER: & AC -8309 & \\
PROD DATE: & $10 / 31 / 93$ & \\
SHEET AREA : & 8396 & sq. \\
& 780 & sq. meters \\
LENGTH: & 355 feet \\
& 108 meters \\
WIDTH : & 23.65 feet \\
& 7.2 meters \\
WEIGHT: & 3376 pounds \\
& 1531 kilograms
\end{tabular}

\begin{tabular}{|c|c|c|c|}
\hline \multicolumn{4}{|c|}{ RESIN LOT INFORMATION } \\
\hline $\begin{array}{l}\text { LOT NUMBER } \\
\text { RESIN TYPE }\end{array}$ & $\begin{array}{c}\cdots-S-45726 \\
600 \text { SERIES }\end{array}$ & & - \\
\hline PROPERTY & UNITS & IETHOD & RESULT \\
\hline $\begin{array}{l}\text { DENSITY } \\
\text { MOISTURE } \\
\text { BRITILENESS } \\
\text { MELT INDEX }\end{array}$ & $\begin{array}{l}g / \mathrm{cc} \\
\% \\
\circ \mathrm{C} \\
g / 10 \mathrm{~min} .\end{array}$ & $\begin{array}{l}D 792 \\
D 570 \\
D 746 \\
D 1238\end{array}$ & $\begin{array}{l}0.932 \\
<.0 .09 \\
<-70 \\
0.551\end{array}$ \\
\hline
\end{tabular}

SLT MINTMOMS ENGIISE MEIRIC

ACTUAT TEST VAIUES ENGLISH METRIC

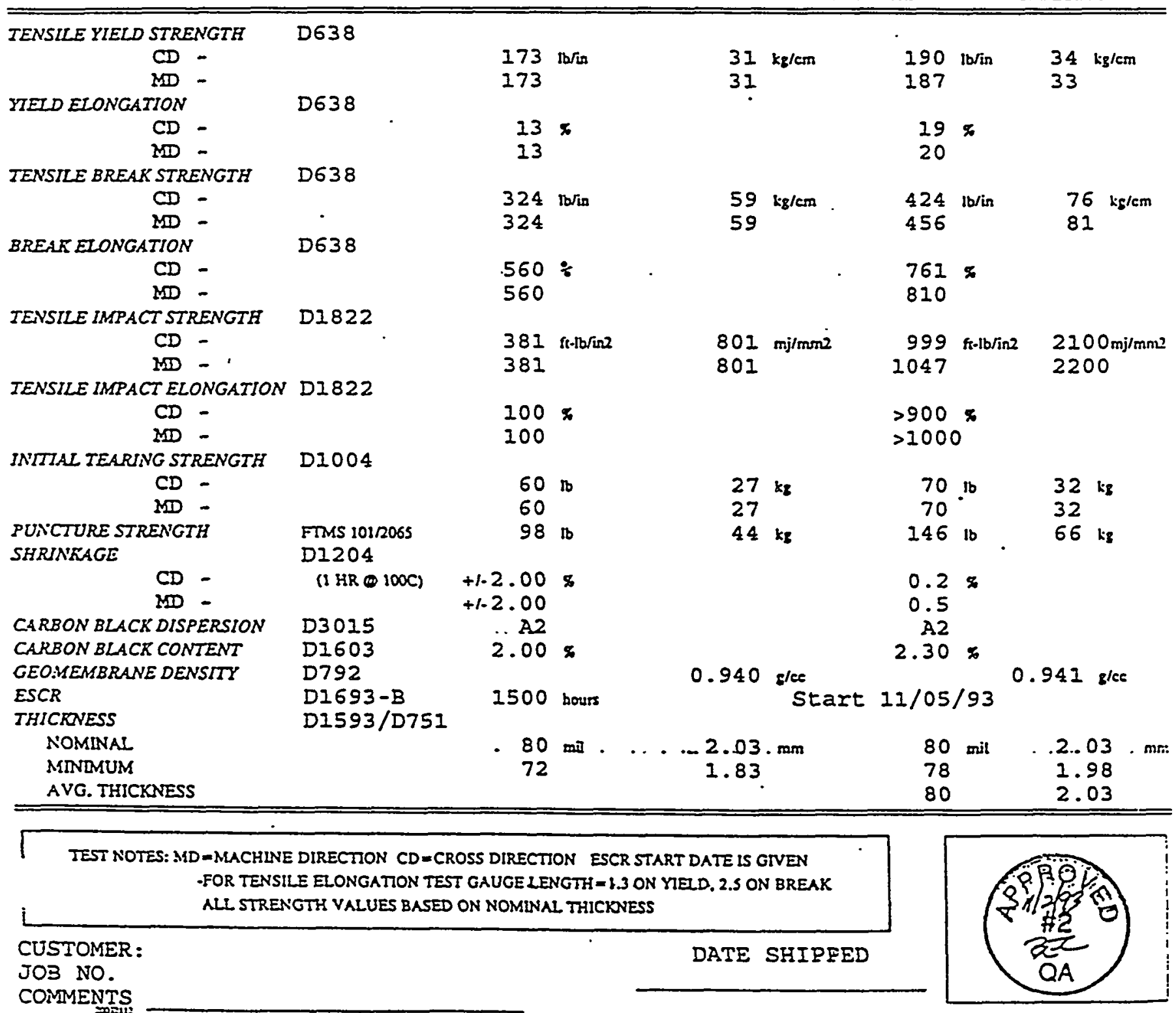




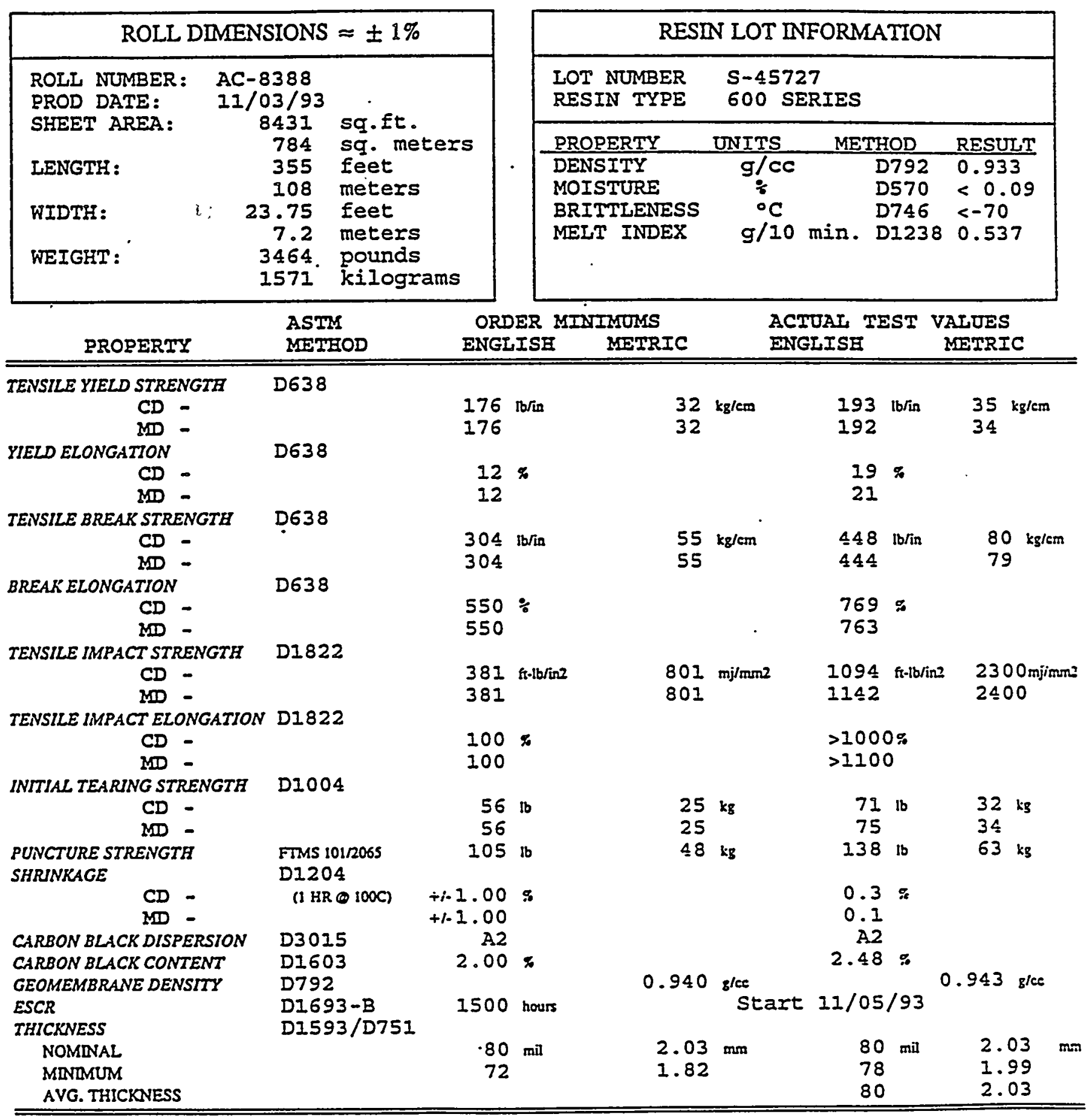

TEST NOTES: MD = MACHINE DIRECTION CD =CROSS DIRECTION ESCR START DATE IS GIVEN .FOR TENSTLE ELONGATION TEST GAUGE LENGTH $=1.3$ ON YTELD. 2.5 ON BREAK ALI STRENGTH VALUES BASED ON NOMINAL THICKNESS 
ROLI DIMENSTONS $\approx \pm 1 . \%$

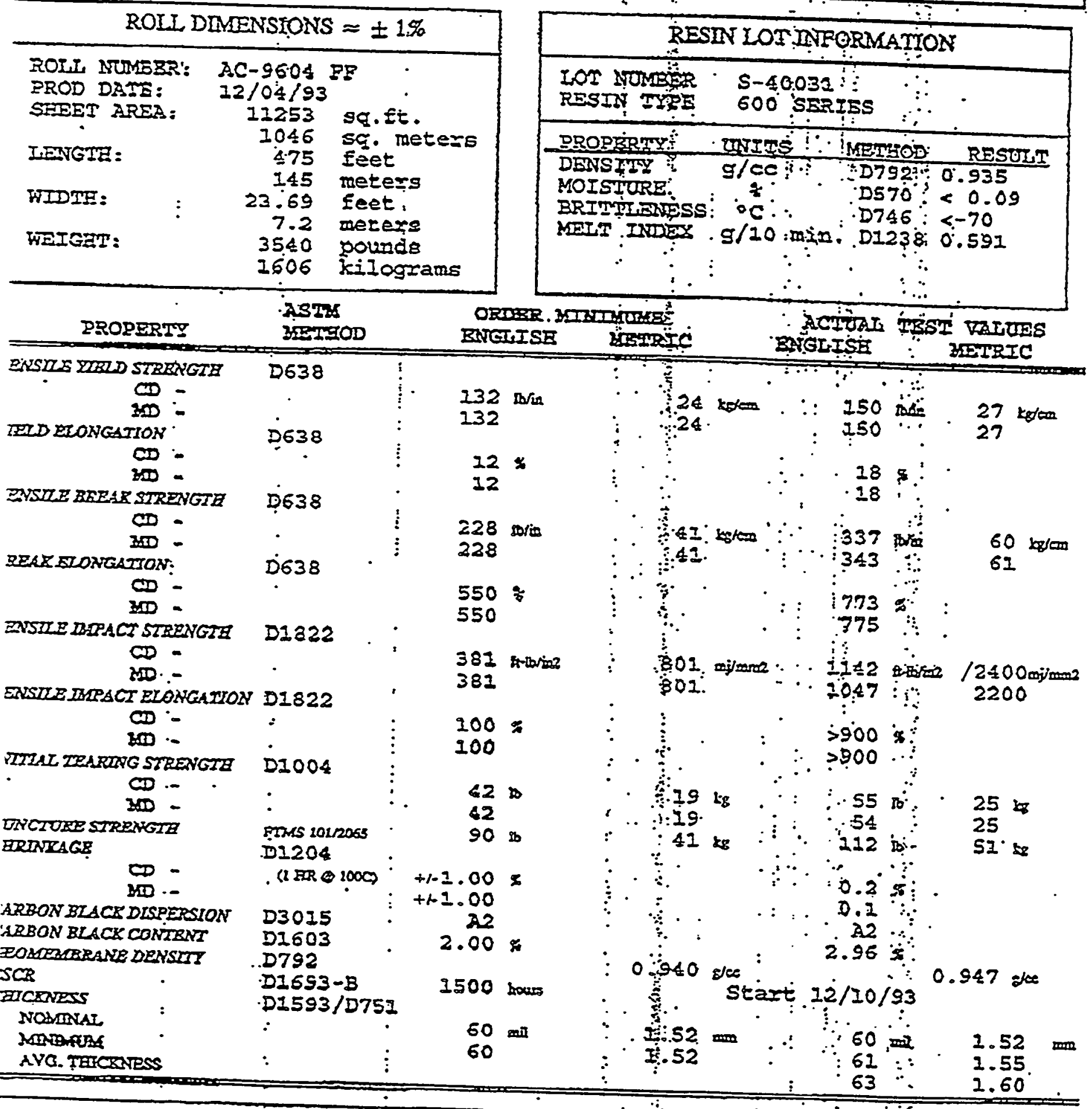

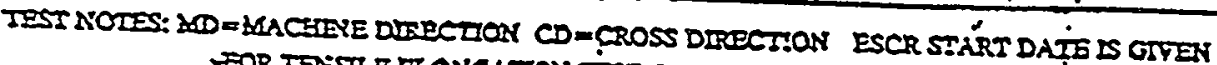

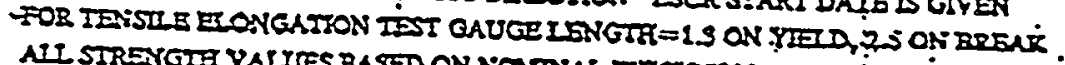

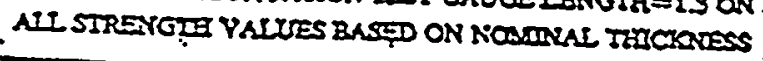

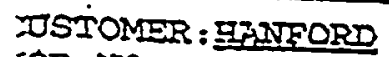

rOB NO. 8491

2CHISINTS
DX广TE SIIPEDD

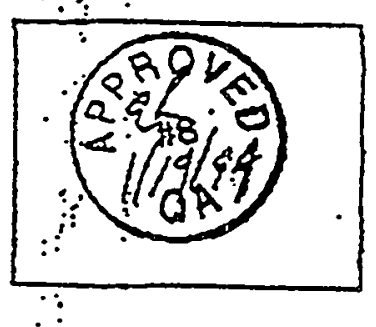




\begin{tabular}{|c|c|c|}
\hline \multicolumn{3}{|c|}{ ROIL DILENSIONS $= \pm 1 \%$} \\
\hline 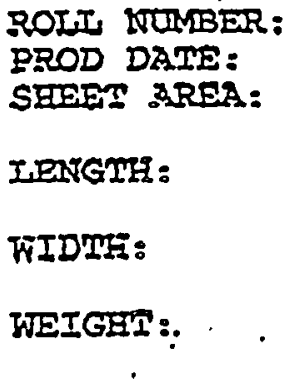 & $\begin{array}{c}\text { AC- }-9605 \\
12 / 04 / 93 \\
11727 \\
1090 \\
495 \\
151 \\
23.69 \\
7.2 \\
3540 \\
1606\end{array}$ & $\begin{array}{l}\text { sq. } \\
\text { sqti. } \\
\text { sq. meters } \\
\text { feot: } \\
\text { meteris } \\
\text { feet: } \\
\text { meters } \\
\text { pounds } \\
\text { jilograms }\end{array}$ \\
\hline
\end{tabular}

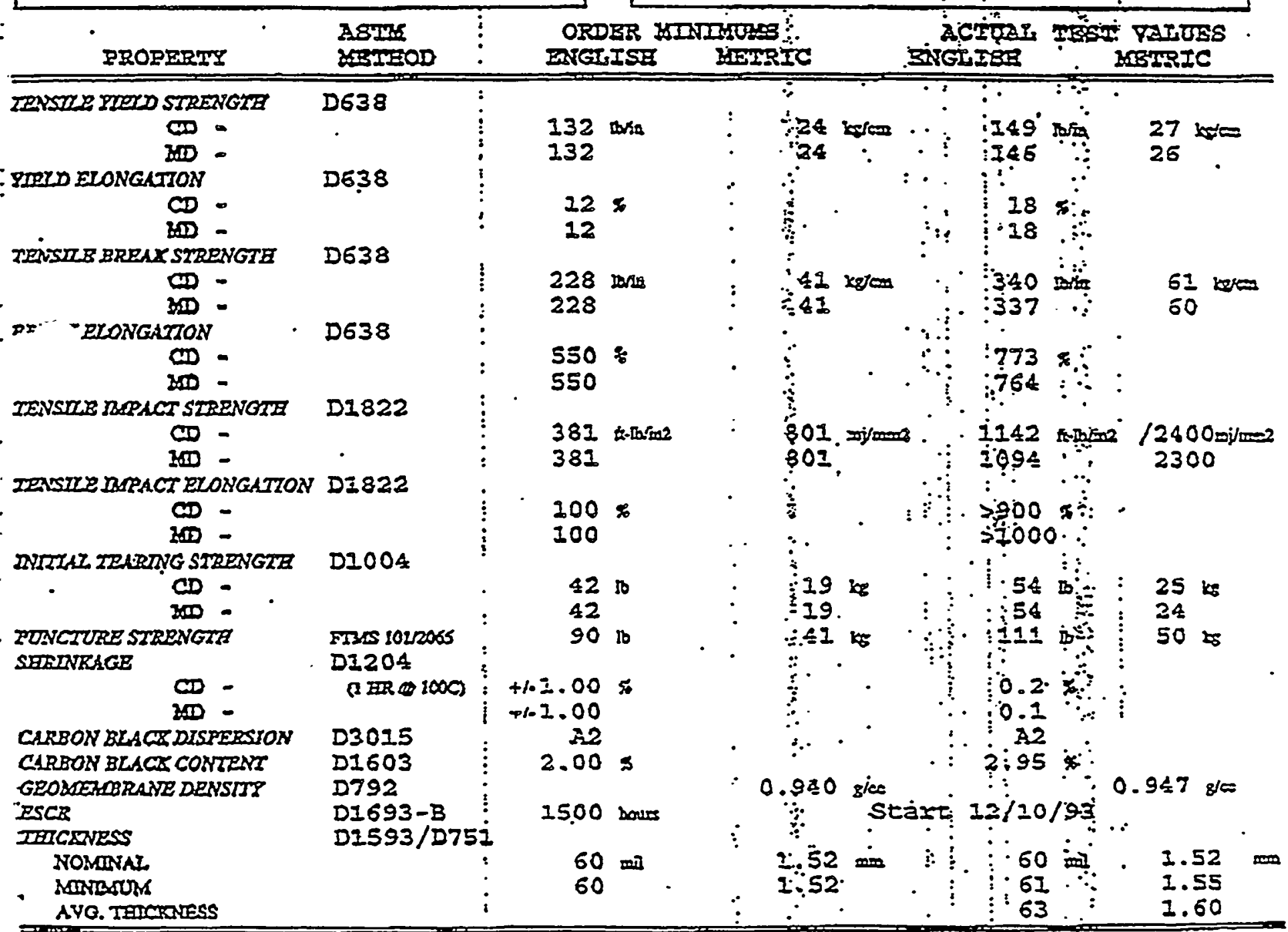

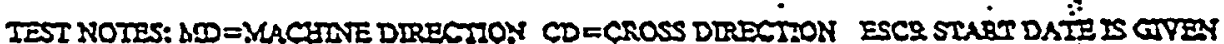
-FOR TEYSOEE ELONOATTON TEST OAUGE IENGJHE=1.3 ON YDED. 2.5 ON BREANK

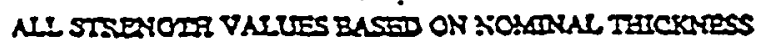

ULOFOMER : FINANORD

JOB NO. 8491

COMIBNISS
RESTIY LOT:MTPQRMATIOTN

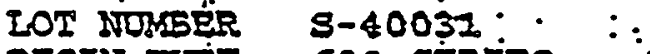

RESIN TYPE 600 BERTiSS

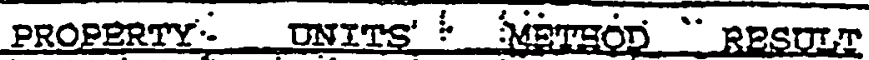

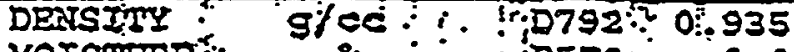

MOISTURE: $\quad: \div: 2570 \%<0.09$

BRTTLLENBSS ${ }^{\circ} \mathrm{C} \quad .^{\circ} \cdot \mathrm{D} 746 \div<-70$

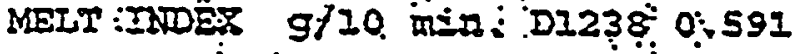

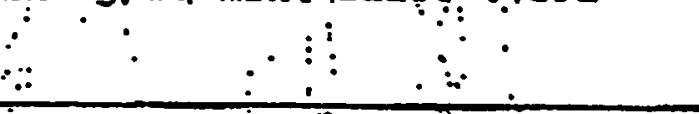

acter ICBTHIC 


\section{WHC-SD-W025-RPT-001, Rev. 0 \\ STAR... Safety Through Accountability and Recognition}

\section{Mobil Polyethylene \\ Certificate of Analysis \\ "QUALITY PEOPLE MAKING QUALITY PRODUCTS"}

Marc Vanover

SLT North America Inc.

August 17, 1993

200 S. Trade Center Pkwy

Conroe, TX 77385

Mobil Chemical, a major supplier of quality polyethylene, has shipped a lot of Material to your facility. The lot identification and analysis are listed below. This data has been supplied by our Quality Control Laboratory located here in Beaumont. If you should need further information or another copy of this report please call your sales office in Irving, TX 214-580-1040.

\begin{tabular}{|c|c|c|c|}
\hline Resin Ident & & Resin Analysis & Lot Data \\
\hline $\begin{array}{l}\text { Product Type } \\
\text { Lot Number } \\
\text { Hopper Car Number } \\
\text { Quantity Shipped } \\
\text { Date of Manufacture } \\
\text { P.O. Number } \\
\qquad \\
\text { Location St } \\
\text { Aldine, TX }\end{array}$ & $\begin{array}{l}\text { HDA-601 } \\
\text { S-40680 } \\
\text { RUSX-8040 } \\
165,350 \\
17-\text { Aug-93 }\end{array}$ & $\begin{array}{l}\text { Melt Index (gms/10 min) I } \\
\text { Density }(g m s / c c)\end{array}$ & $\begin{array}{l}0.56 \\
0.934\end{array}$ \\
\hline
\end{tabular}

Thank you for your business. If you need additional assistance, please do not hesitate to contact our Sales Representative in your area.

Sincerely,

WAS Sintit

W. H. Pintsch

Quality Assurance Coordinator

- mproveras

FAX 10 713en7-205s AWire. TX 


\section{Mobil Polyethylene Certificate of Analysis "QUALITY PEOPLE MAKING QUALITY PRODUCTS"}

Bill Walling

SLT North America Inc.

September 22, 1993

200 S. Trade Center Pkwy

Conroe, TX 77385

Mobil Chemical, a major süplier-of-quality polyethylene, has shipped a lot of Material to your facility. The lot identficiculom and angidysis are:listed below. This data has been

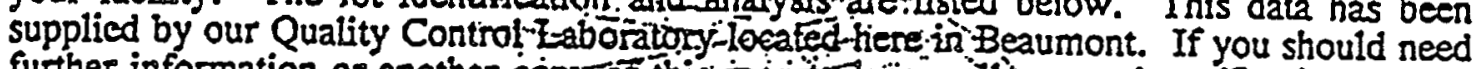
further information or another copy of this repoit please ceify your sales office in Irving, IX 214-580-1040,

\begin{tabular}{|c|c|c|}
\hline Resin Identification & unt & Lot Data \\
\hline 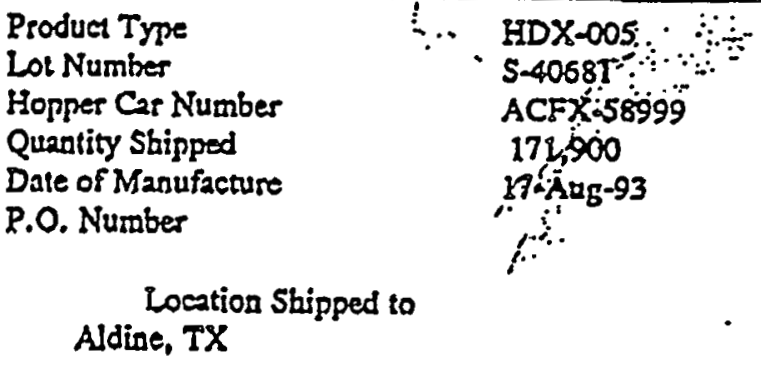 & $\begin{array}{l}\text { Melt Index (gms/10 min) } \mathrm{I}_{2} \\
\text { Density (gms/cc) }\end{array}$ & $\begin{array}{l}0.56 \\
0.934\end{array}$ \\
\hline
\end{tabular}

Thank you for your business. If you need additional assistance, please do not hesitate to contact our Sales Representative in your area.

Sincerely,

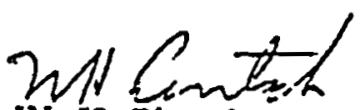

W. H. Pintsch

Quality Assurance Coordinator 


\section{STAR... TE}

\section{Mobil Polyethylene Certificate of Analysis \\ "QUALITY PEOPLE MAKING QUALITY PRODUCTS"}

Bill Walling

SLT North America Inc.

September 22, 1993

200 S. Trade Center Pkwy

Conroe, TX 77385

Mobil Chemical, a major supplier of quality polyethylene, has shipped a lot of Material to your facility. The lot identification and analysis are listed below. This data has been supplied by our Quality Control Laboratory located here in Beaumont. If you should need further information or another copy of this report please call your sales office in Irving,

TX 214-580-1040.

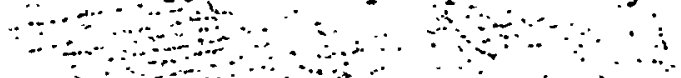

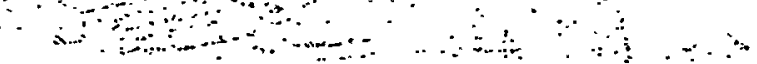

\begin{tabular}{|c|c|c|c|}
\hline Resin Identification & $\begin{array}{cc}\cdots & \cdots \\
\cdots & \ddots\end{array}$ & Test $: \because{ }^{\text {Resin Analysis }}$ & Lot Data \\
\hline $\begin{array}{l}\text { Product Type } \\
\text { Lot Number } \\
\text { Hopper Car Number } \\
\text { Quantity Shipped } \\
\text { Date of Manufacture } \\
\text { P.O. Number } \\
\qquad \\
\text { Location Shipped to } \\
\text { Aldine, TX }\end{array}$ & $\begin{array}{l}\text { HDX-005 } \\
\text { S-40682 } \\
\text { MOBX-52368 } \\
171,750 \\
17-\text { Aug-93 }\end{array}$ & $\begin{array}{l}\text { Melt Index (gms/10 } \mathrm{min} \text { ) Iz } \\
\text { Density (gms/cc) }\end{array}$ & $\begin{array}{l}0.56 \\
0.934\end{array}$ \\
\hline
\end{tabular}

Thank you for your business. If you need additional assistance, please do not hesitate to contact our Sales Representative in your area.

Sincerely,

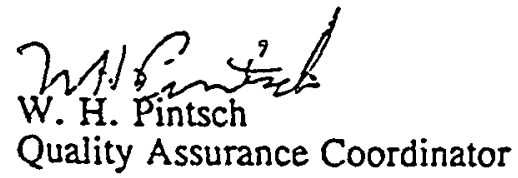




\section{STAR.. Lafety Through Accountability and Recognition}

\section{Mobil Polyethylene \\ Certificate of Analysis \\ "QUALITY PEOPLE MAKING QUALITY PRODUCTS"}

Bill Walling

SLT North America Inc.

September 22, 1993

200 S. Trade Center Pkwy

Conroe, TX 77385

Mobil Chemical, a major supplier of quality polyethylene, has shipped a lot of Material to your facility. The lot identification and analysis are listed below. This data has been supplied by our Quality Control Laboratory located here in Beaumont. If you should need further information or another copy of this report please call your sales office in Irving, TX 214-580-1040.

\begin{tabular}{|c|c|c|c|}
\hline Resin Identification & & Resin Analysis & \\
\hline $\begin{array}{l}\text { Product Type } \\
\text { Lot Number } \\
\text { Hopper Car Number } \\
\text { Quantity Shipped } \\
\text { Date of Manufacture } \\
\text { P.O. Number }\end{array}$ & $\begin{array}{l}\text { HDX-005 } \\
\text { S-40687 } \\
\text { ACFX-52401 } \\
175,550 \\
18-\text { Aug } 93\end{array}$ & $\begin{array}{l}\text { Melt Index (gms/10 } \mathrm{min}) \mathrm{I}_{2} \\
\text { Density (gms/cc) }\end{array}$ & $\begin{array}{l}0.55 \\
0.934\end{array}$ \\
\hline $\begin{array}{l}\text { Location Shipped to } \\
\text { Aldine, TX }\end{array}$ & & & \\
\hline
\end{tabular}

Thank you for your business. If you need additional assistance, please do not hesitate to contact our Sales Representative in your area.

Sincerely,

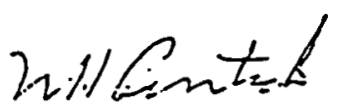

W. H. Pintsch

Quality Assurance Coordinator 


\section{WHC-SD-W025-RPT-001, Rev. 0 \\ STAR... Safety Through Accountability and Recognition}

\section{Mobil Polyethylene \\ Certificate of Analysis \\ "QUALITY PEOPLE MAKING QUALITY PRODUCTS"}

Bill Walling

SLT North America Inc.

October 20, 1993

200 S. Trade Center Pkwy

Conroe, TX 77385

Mobil Chemical, a major supplier of quality polyethylene, has shipped a lot of Material to your facility. The lot identification and analysis are listed below. This data has been supplied by our Quality Control Laboratory located here in Beaumont. If you should need further information or another copy of this report please call your sales office in Irving, TX 214-580-1040.

\begin{tabular}{|c|c|c|c|}
\hline Resin Identification & & Test $\quad$ Resin Analysis & Lot Data \\
\hline $\begin{array}{l}\text { Product Type } \\
\text { Lot Number } \\
\text { Hopper Car Number } \\
\text { Quantity Shipped } \\
\text { Date of Manufacture } \\
\text { P.O. Number } \\
\qquad \begin{array}{l}\text { Location Shipped to } \\
\text { Aldine, TX }\end{array}\end{array}$ & $\begin{array}{l}\text { HDA-601 } \\
\text { S-45726 } \\
\text { MOBX-52233 } \\
174,350 \\
13-A \text { Aug }-93\end{array}$ & $\begin{array}{l}\text { Melt Index (gms/10 } \mathrm{min}) \mathrm{I}_{2} \\
\text { Density (gms/cc) }\end{array}$ & $\begin{array}{l}0.54 \\
0.934\end{array}$ \\
\hline
\end{tabular}

Thank you for your business. If you need additional assistance, please do not hesitate to contact our Sales Representative in your area.

Sincerely,

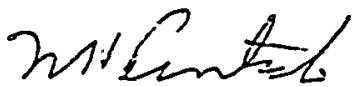

W. H. Pintsch

Quality Assurance Coordinator 


\section{WHC-SD-W025-RPT-001, Rev. 0 \\ STAR... Safety Through Accountability and Recognition}

\section{Mobil Polyethylene Certificate of Analysis "QUALITY PEOPLE MAKING QUALITY PRODUCTS"}

Bill Walling

SLT North America Inc.

October 20, 1993

200 S. Trade Center Pkwy

Conroe, TX 77385

Mobil Chemical, a major supplier of quality polyethylene, has shipped a lot of Material to your facility. The lot identification and analysis are listed below. This data has been supplied by our Quality Control Laboratory located here in Beaumont. If you should need further information or another copy of this report please call your sales office in Irving, TX 214-580-1040.

\begin{tabular}{|c|c|c|c|}
\hline Resin Identification & & Resin Analysis & Lot Data \\
\hline $\begin{array}{l}\text { Product Type } \\
\text { Lot Number } \\
\text { Hopper Car Number } \\
\text { Quantity Shipped } \\
\text { Date of Manufacture } \\
\text { P.O. Number } \\
\text { Location Shipped to } \\
\text { Aldine, TX }\end{array}$ & $\begin{array}{l}\text { HDA-601 } \\
\text { S-45727 } \\
\text { ACFX-52300 } \\
173,550 \\
13-\text { Auy }-93\end{array}$ & $\begin{array}{l}\text { Melt Index (gms/10 min) Iz } \\
\text { Density (gms/cc) }\end{array}$ & $\begin{array}{l}0.54 \\
0.934\end{array}$ \\
\hline
\end{tabular}

Thank you for your business. If you need additional assistance, please do not hesitate to contact our Sales Representative in your area.

Sincerely,

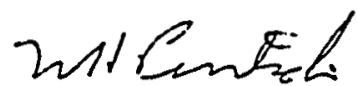

W. H. Pintsch

Quality Assurance Coordinator 


\section{Mobil Polyethylene Certificate of Analysis "QUALITY PEOPLE MAKING QUALITY PRODUCTS"}

Marc Vanover

SLT North America Inc.

200 S. Trade Center Pkwy

Conroe, TX 77385

Mobil Chemical, a major supplier of quality polyethylene, has shipped a lot of Material to your facility. The lot identification and analysis are listed below. This data has been supplied by our Quality Control Laboratory located here in Beaumont. If you should need further information or another copy of this report please call 'your sales office in Irving, TX 214-580-1040.
August 31, 1993

\begin{tabular}{|c|c|c|c|}
\hline Resin Identification & $\quad \therefore$ & $\therefore$ Test $\quad$ Resin Analysis & Lot Data \\
\hline $\begin{array}{l}\text { Product Type } \\
\text { Lot Number } \\
\text { Hopper Car Number } \\
\text { Quantity Shipped } \\
\text { Date of Manufacture } \\
\text { P.O. Number } \\
\text { Location Shipped to } \\
\text { Aldine, TX }\end{array}$ & $\begin{array}{l}\text { HDG-600 } \\
\text { S-40031 } \\
\text { TLCX-38077 } \\
181,450 \\
25-\operatorname{Jan}-93 \\
\therefore\end{array}$ & $\begin{array}{l}\text { Melt Index }(\mathrm{gms} / 10 \mathrm{~min}) \text { I2 } \\
\text { Density (gms/cc) }\end{array}$ & $\begin{array}{l}0.59 \\
0.935\end{array}$ \\
\hline
\end{tabular}

Thank you for your business. If you need additional assistance, please do not hesitate to contact our Sales Representative in your area.

Sincerely,

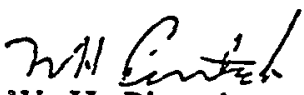

W. H. Pinisch

- Quality Assurance Coordinator 


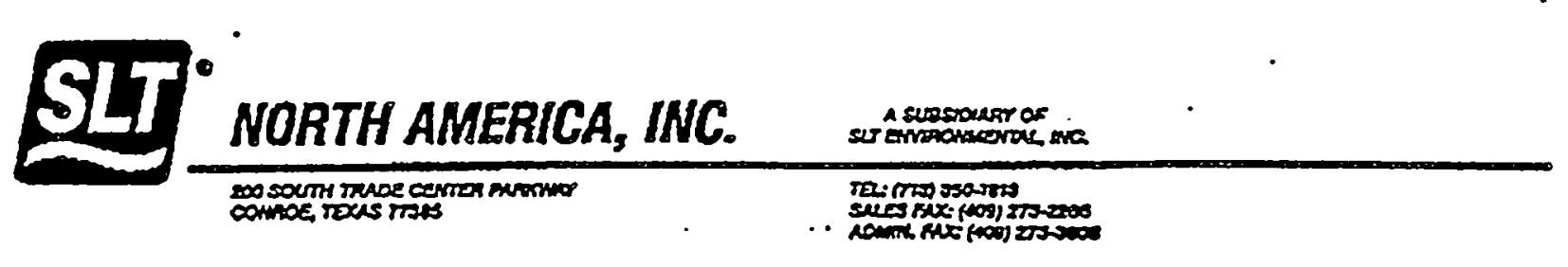

Occober 22, 1993

GeoPacific Ilining Inc. 1300 Dexter Ave. N., Suite 250

Seattle, Washington 98109

Attr: El Earuschak

RE: $\quad /$ DOE Hanford Radioactive Waste Disposal Facility SLT Project No. 8491

\section{Certification}

The undersigned, being qualified and anthorized to do so, bercby certifies that the geomenone for the above referenced project was mamractured from finst quality resin which contains no reclaimed polymer and less than $2 \%$ of recyeled trim.

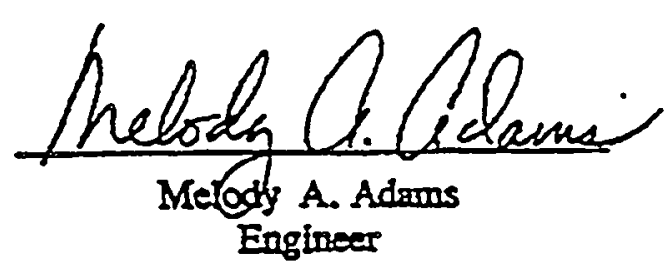

MAN/ma

For environmental lining solutions...the world comes to SLT. 
WHC-SD-W025-RPT-001, Rev. 0

近

NORTH AMERICA, INC.

A SUBSIDIARY OF SLT ENVIRONMENTAL, INC

200 SOUTH TRADE CENTER PARKWAY

TEL: (713) 350.5813

SALES FAX: (409) $273-2266$

ADMIN. FAX: (400) 273.3808

January 31,1994

GeoPacific Lining, Inc.

1300 Dexter Avenue \#205

Seattle, WA 98109

Attn: $\quad$ El Haluschak

RE: $\quad$ D.O.E Hanford Radioactive Waste

SLT Project No. US/93-8491

\section{Certification}

The undersigned, being qualified and authorized to do so, hereby certifies that the SLT HyperFlex ${ }^{\otimes}$ liner manufactured and supplied to the above referenced project has passed the required 2000 hours for ASTM D 1693 Condition B, Environmental Stress Crack Resistance:
AC6895
AC 6905
AC6910
AC6915
AC6925
AC6930
AC7682
AC6896
AC6906
AC6911
AC6916
AC6926
AC6931
AC7684
AC6899
AC6907
AC6917
AC6927
AC6932
AC6913
AC6923
AC6928
AC7680
AC6904
AC6909
AC6924
AC6929
AC7681

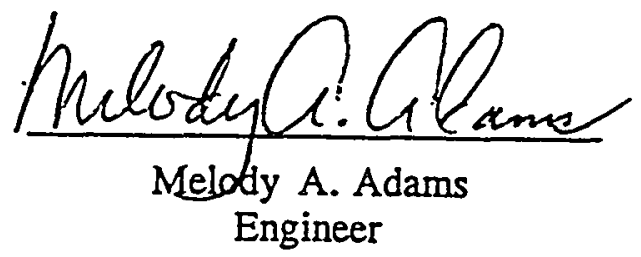


WHC-SD-W025-RPT-001, Rev. 0

近

NORTH AMERICA, INC. . Asussounroe

200 SOUTH TRUDE CENTER PARKXAY TEL: (TTJ) 250.1ETS

CONROE, TDAS T7SES

SALES PAX: (400) 27.2286

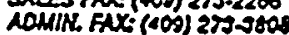

March 14, 1994

GeoPacific Lining, Inc.

1300 Dexter Ave. N, Suite 250

Seattle; WA 98109

Atta: El Kraluscbak

RE: $\quad$ D.O.EHanford Radioactive Waste

SLT Project No。 US/93-8491

\section{Certification}

The undersigned, being qualified and authorized to do so, hereby certifies that the SLT HyperFlex liner manufactured and supplied to the above referenced project has passed the required 2000 hours for ASTM D 1693 Condition B, Environmental Stress Crack Resistance: .

AC6893

AC6894
AC7683

AC8309
AC8388

AC9604

AC9605

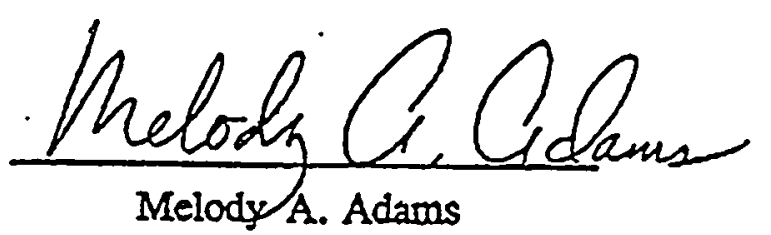

Engineer 
March 14, 1994

GeoPacific Iining Inc.

1300 Dexter Ave. $N$, Suite 250

Seattle, WA 98109

Attn: El Haluschak

RE: $\quad$ D.O.E.Hanford Radioactive Waste SLT Project No. 8491

\section{Certification \\ (Revised)}

The undersigned, being qualified and authorized to do so, hereby certifies that the Hyperflex liner for the above referenced project will meet the requirement of $90 \%$ strength retained for Resistance to Soil Burial, ASTM 3083.

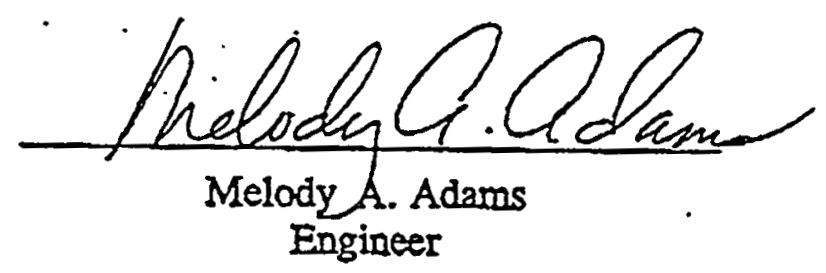


November 3, 1993

GeoPacific Lining, Inc.

1300 Dexter Ave. N. \# 250

Seattle, Wash. 98109

Attn: El Haluschak

RE: $\quad$ D.O.E Hanford Radioactive Waste

SLT Project No. 8491

\section{Certification}

The undersigned, being qualified and authorized to do so, hereby certifies that the welding extrudate (rod and resin) manufactured by Blackwell Plastics for use by SLT and its customers, is made from the same base resin type and will exhibit similar physical characteristics as the membrane.

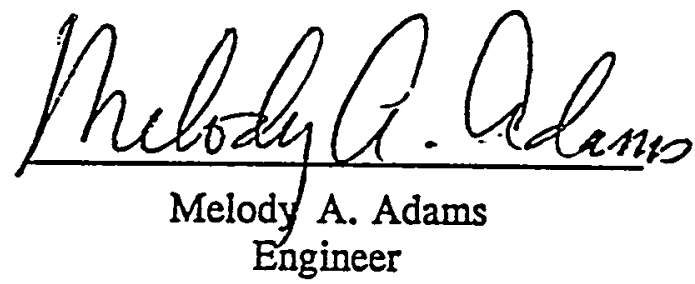




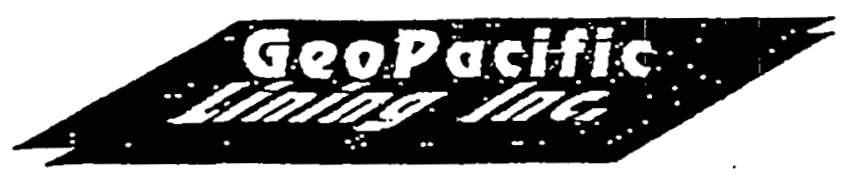

Dec. $2 / 93$
INST ALLATION CONTRACTOR FOR YOUR CEOSNTHEIIC NEEDS

File no.: $354 / 93$

Delhur Industries, Inc.

Port Angeles, WA

RE: Cold weather seaming between 30 and 40 degrees. Fianford Site - KEF-5346 (W-025)

Attention: Tony

In reply to the GCS's field data form dated: 11-20-93

- Accept this letter as certification to the statements:

$n$ the quality of the seams welded at temperatures below 40 degrees $F$. and above 30 degrees $F$. are of the same quality of seam as those welded at temperatures between 40 degrees $F$. and 104 degrees F.."

$\pi$ For seams weIded below 40 degrees $F .$, a sample coupon shall be provided from each end of the seam. AII other testing required by the specifications wiIl be performed."

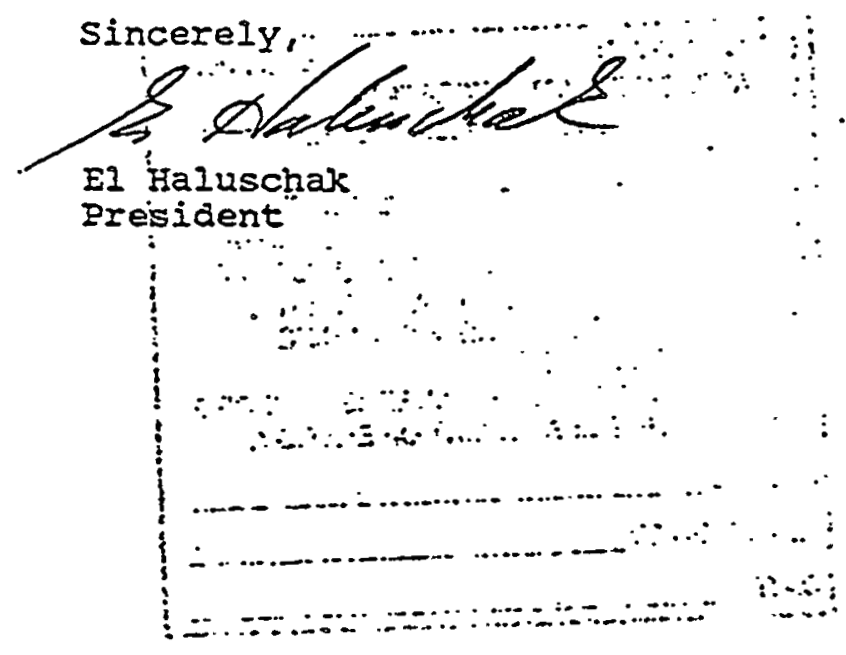

Ste. 205 - 1300 Dexter Avenue North, Seattle, WA 98109 • Phone: (206) 284-2378 - Fax: (206) 283706S 
$\therefore$

16 August 1994

Scott Matthews

Golder and Associaies

Re: SLT Job $\$ 94-9538$

Fax: $509 / 373-9484$

\section{Certifieation}

The undersigned, being qualified and authorized to do so hereby certifies that SIT Hyperflex ${ }^{\oplus}$ is made of high quality, virgis polyethylene resir. The finished product is composed of $97-98 \%$ polyethylene and $2-3 \%$ carbon black for uv stability. SLT has adhered to this formulation for all material carrying the HyperFlex trade name.

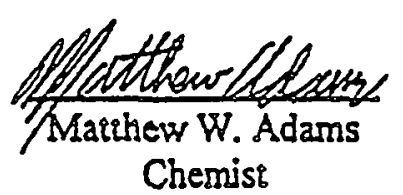

Chemist 
WHC-SD-W025-RPT-001, Rev. 0

S4] NORTH AMERICA, INC.

A SUBSIDIARY OF SLT ENVAONLENTAL ING.

200 SOUTH TRIOE CENTER PAAKKAY

16 August 1994

Scott Matthews

Golder and Associates

Re: SLT Job \# 94-9538

Fax: 509/373-9484

\section{Certification}

The undersigned, being qualified and authorized to do so hereby certifies that SLI HyperFlex ${ }^{\infty}$ is made of high quality, virgin polyethylene resin. The resin used for manufacturing all Hyperflex geomembranes was developed for the express purpose of geomembrane production and is supplied by a single reisn manufacturer to one, consistent specification All material refered to as SI I HyperFlex has been made with this resin.

Further, due to the consistency of raw materials used in the production of HyperFlex, all HyperFlex material exhibits similar physical properties and chemical resistance.

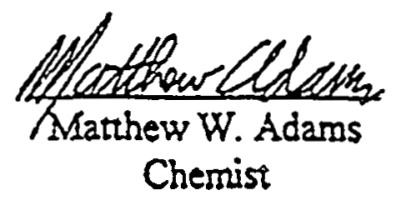


WHC-SD-W025-RPT-001，Rev。 0

APPENDIX F

GEONET CONFORMANCE TESTS

$F-i$ 
WHC-SD-W025-RFT-001, Rev. 0

APPENDIX F-1

CQA CONFORMANCE TESTS

$F-1$ 
WHC-SD-W025-RPT-001, Rev. 0

GOLDER CONSTRUCTION SERVICES, INC.

933-1354

GEONET INVENTORY CONTROL RECORD

05/10/94

DEPARTHENT OF ENERGY

Page: 1

NON-DRAG OFF LANDFILL FACILITY

HANDFORD MUCLEAR RESERYATION

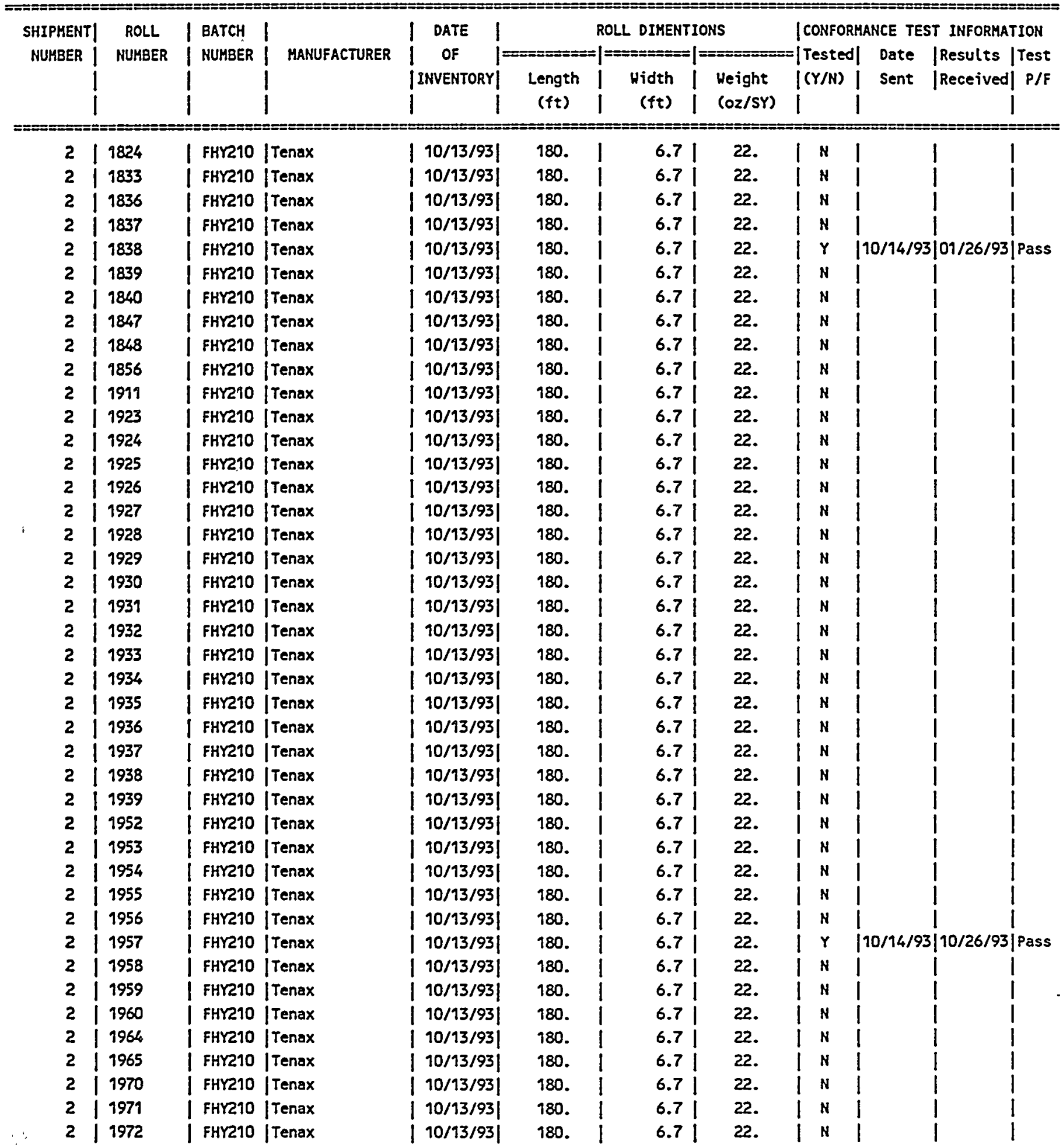


WHC-SD-W025-RPT-001, Rev. 0

Square Feet Delivered $=50652$. 
WHC-SD-W025-RPT-001, Rev. 0

OCTOBER 1993

SUMMARY OF GEONET CONFORMANCE TEST RESULTS

DEPARTMENT OF ENERGY

NON DRAG-OFF CQA

WASHINGTON

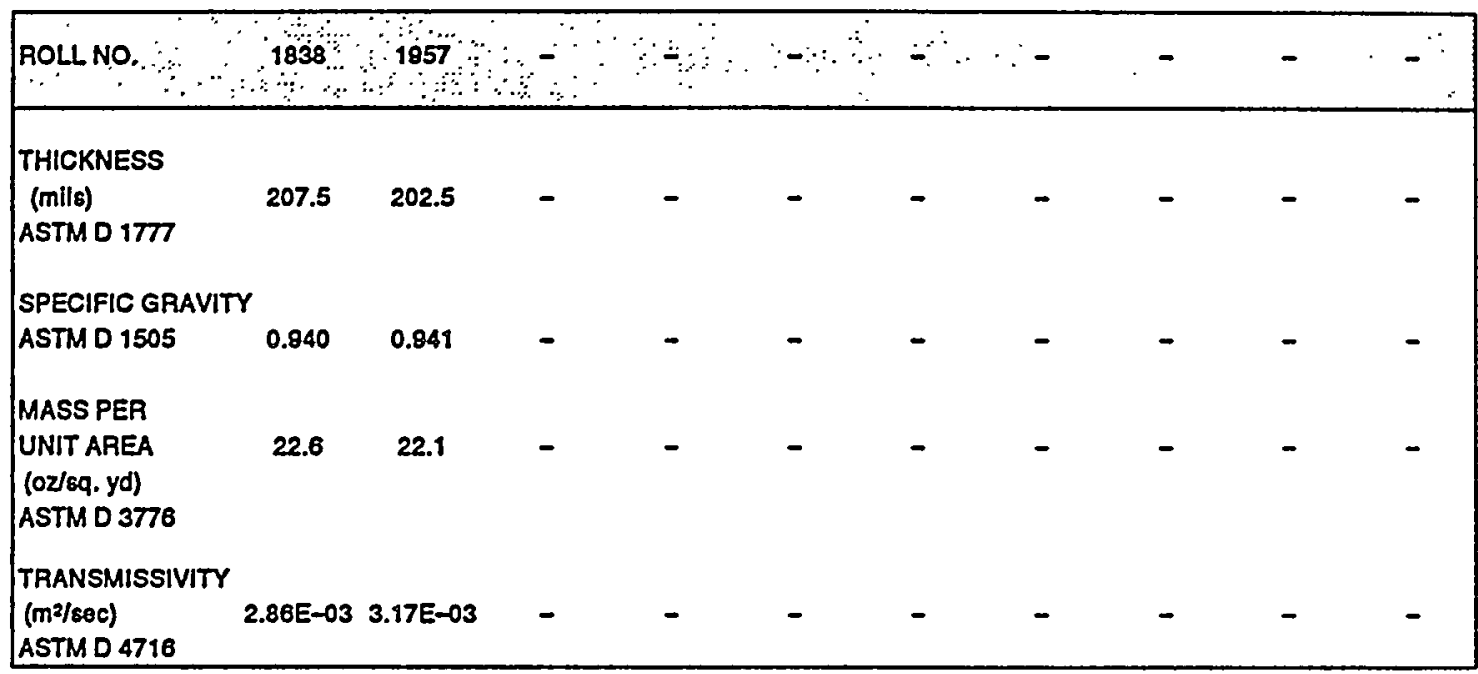


WHC-SD-W025-RPT-001, Rev. 0

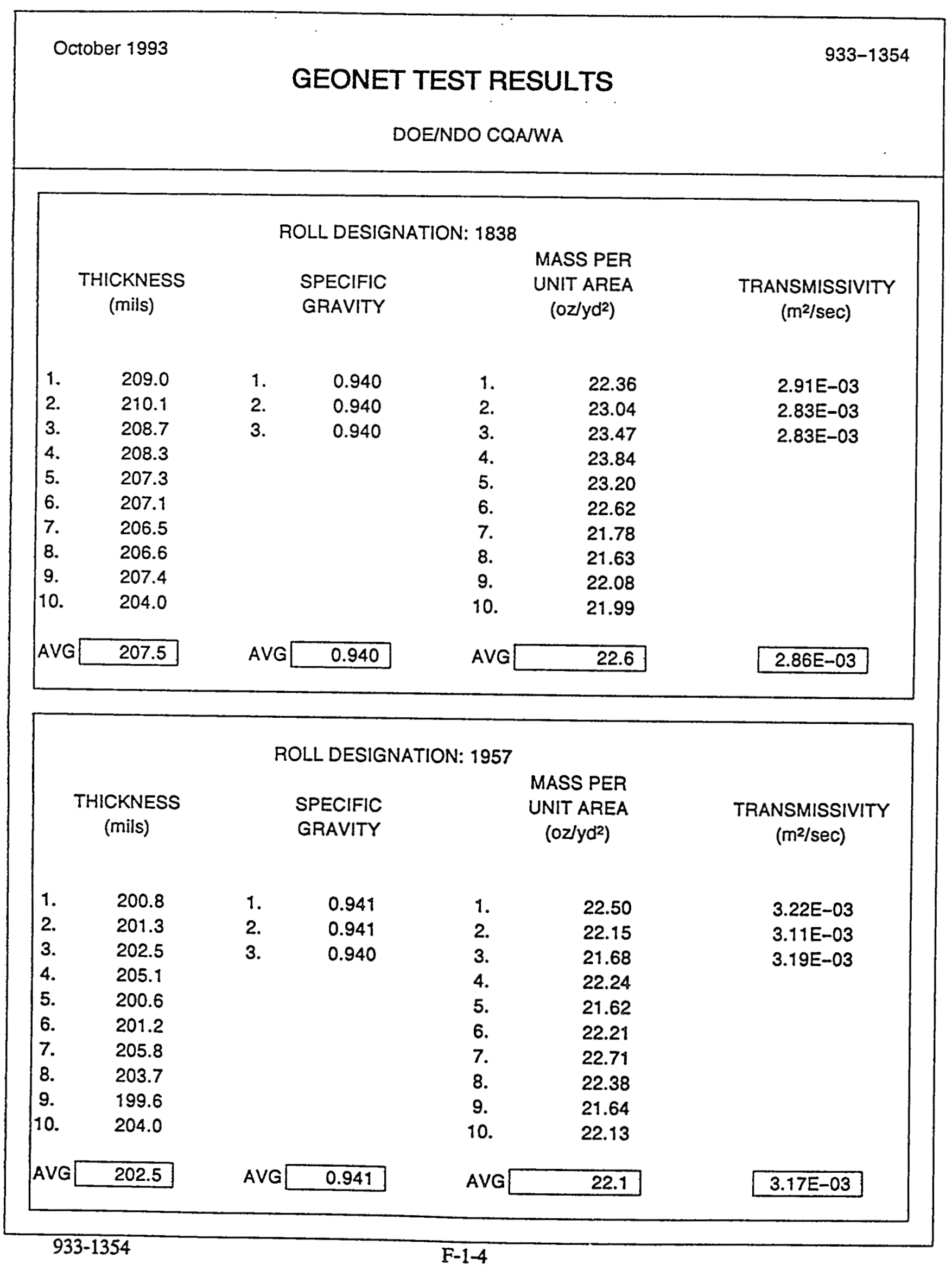


WHC-SD-H025-RPT-001, Rev. 0

APPENDIX F-2

MANUFACTURER'S QC DOCUMENTATION

$F-2$ 
September 3, 1993

MI. El Haluschak

Geo Pacific Iining, Inc.

106-1687 West Broadway

Vancouver, B.C. V6J $1 \times 2$

RE: Hanford Landfill Project

Dear MI. Haluschak;

TEYAX Corporation certifies that all the rolls of CI-2 Geonet and TNT-206064 Geocomposite to be delivered to the above reierenced project will, be in accordance with the attached specifications aated $9 / 3 / 93$ and $7 / 20 / 93$.

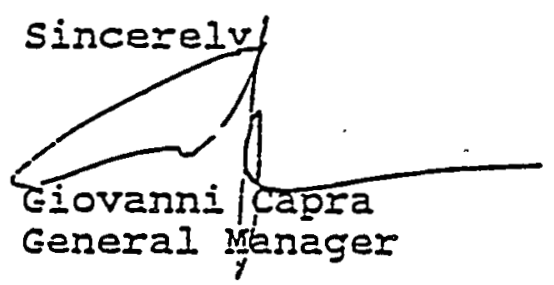

Enclosures:

TENAX CE-2 Series Geonet specifications dated $9 / 3 / 93$ TENAX TNT-206064 Geocomposite specification daied $7 / 20 / 93$ 


\begin{tabular}{|c|c|c|c|}
\hline \multirow{2}{*}{090393} & \multicolumn{3}{|c|}{ 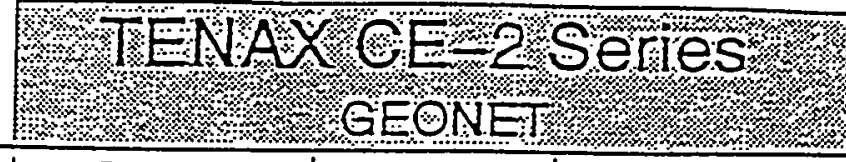 } \\
\hline & TEST & UNIT & VALUES \\
\hline \multicolumn{4}{|l|}{ PERFORMANCE } \\
\hline TENSILE STRENGTH & ASTM D4595 & $\mathrm{lb} / \mathrm{ft}$ & 450 \\
\hline $\begin{array}{l}\text { HYDRAULIC TRANSMISSIVITY } \\
@ \mathrm{i}=1 \text { and vertical sUress }\end{array}$ & ASTM D47:6 & $\begin{array}{c}\mathrm{m}^{2} / \mathrm{sec} \\
\text { psi }\end{array}$ & $\begin{array}{c}0.001 \\
@ 10.000\end{array}$ \\
\hline \multicolumn{4}{|l|}{ COMPOSITION } \\
\hline POLYETHYLENE CONTENT & ASTM Di248 & $\%$ & 98 \\
\hline DENSITY & ASTM D1505 & g/cm3 & .940 \\
\hline MEIT FLOW INDEX & ASTM D1238 & $\mathrm{g} / 10 \mathrm{~min}$ & 0.3 \\
\hline FOAMING AGENT & - & - & No \\
\hline CARBON BLACK CONTENT & ASTM D1603 & $\%$ & 2 \\
\hline \multicolumn{4}{|l|}{ DIMENSIONS } \\
\hline GEONET THICKNESS & ASTM DITT & in & .20 \\
\hline ROLL WIDTT: & - & it & 0.7 \\
\hline ROLL LENGTH & - & ti & 250 \\
\hline ROLL AREA & - & $t^{2}$ & 1675 \\
\hline ROLL WEIGHTT & - & lb & 260 \\
\hline
\end{tabular}

Geonet iensiite sirength and transmissivity are reported as represantative typical (average) roll values.

All cther reporied properies are minimum average roll values in $\equiv$ ech principal cirection. 
WHC-SD-W025-RPT-001, Rev. 0

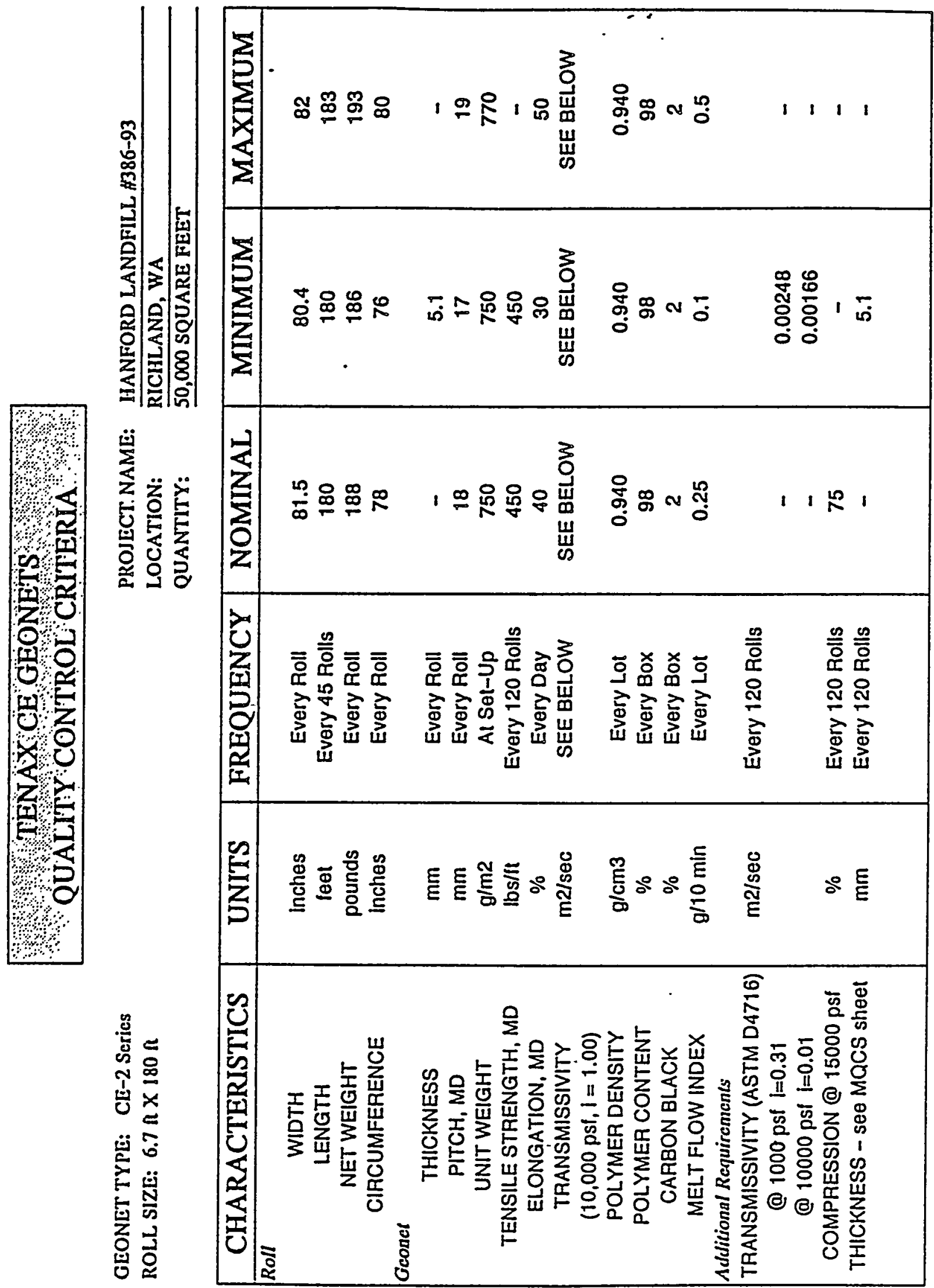




\section{MASS POLYMERS CORPORATION}

198 WEST HIEH STRET

June 3, 1993

Mr Mario Tonani

Tenax Corporation

B291 Patuxent Range Road

Jessup, MD 20794

Dear Mario,

As per your P.O. 2454, the specifications for Novacor FHY-210-A Lot 7-1-15217-2 32 are as follows:

$\begin{array}{ll}\text { Helt Index } & 0.23 \mathrm{~g} / 10 \mathrm{~min} \\ \text { Density } & 0.935 \mathrm{~g} / \mathrm{cc} \\ \text { Ship Date } & -1 / 6 / 93\end{array}$

Test Method ASTM D1238

Test Method ASTM D792

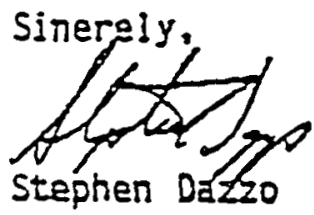


WHC-SD-W025-RPT-001, Rev. 0

HANQC.XLS

\begin{tabular}{|c|c|c|c|c|c|c|c|c|}
\hline & PROJECT & JAME: & IHANFORD & & & & & \\
\hline & PROJECT & & $386-93$ & & & & & \\
\hline & & & & & & & & \\
\hline & & & . & & & & & \\
\hline & & TEN & NAX QUALI & Y CONTR & ROLPROGR & & & \\
\hline & & ERTIFIC & ATION OF & IINIMUM F & PROPERTY & VALUES & & \\
\hline & & & & & & & & \\
\hline & & $\mathrm{CE}$ & GEONET & RODUCTI & IONDATAB & ISE & & \\
\hline & & & & & & & & \\
\hline & EXTRUDE & & (4) & & & & & \\
\hline & PRODUCT & & CE 2 & & CARBON E & LACK: & & $\%$ \\
\hline & POLYMER & TYPE: & IMDPE & & ROLLLEN & TH: & 180 & \\
\hline & FOAMING & GENT: & IN/A & & ROLL WEI & HT: & 188 & Ibs \\
\hline & THICKNES & & 5.2 & $\mathrm{~nm}$ & ROLL WID & & 80.4 & \\
\hline & & & & & & & & $=$ \\
\hline LINE & DATE OF & ROLL\# & THICK. & WIDTH & WEIGHT & CIRC. & PITCH & \\
\hline & PROD. & & {$[\mathrm{mm}]$} & [in] & [lbs] & [in] & {$[\mathrm{mm}]$} & \\
\hline 1 & $9 / 19 / 93$ & 1824 & 5.2 & 81.5 & 194.31 & 75 & 16 & \\
\hline 2 & $9 / 19 / 93$ & 1833 & 5.3 & 80.5 & $193.2 !$ & 76.5 & 161 & \\
\hline 3 & 9/19/93 & 1836 & 5.28 & 80.5 & 192.1 & 76 & 16 & \\
\hline 4 & $9 / 19 / 93$ & 1837 & 5.26 & 80.5 & 192.1 & 76 & 161 & \\
\hline 5 & $9 / 19 / 93 \mid$ & 1838 & 5.23 & 81 & 193.41 & 75 & 16 & \\
\hline 6 & $9 / 49 / 931$ & 1839 & 5.25 & 81 & 1931 & 74.75 & 16 & \\
\hline 71 & $9 / 19 / 931$ & 18401 & 5.3 & 80.75 & 192.9 & 74.75 & 16 & \\
\hline 8 & 9/19/93 & 1847 & 5.3 & 80.625 & 194.11 & 74.75 & 16 & \\
\hline 9 & $9 / 19 / 93$ & 1848 & 5.28 & 80.75 & 192.61 & 74.75 & 16 & \\
\hline 101 & $9 / 20 / 93$ & 1856 & 5.2 & 81.33 & 193.61 & 76.25 & 16 & \\
\hline 11 & $9 / 21 / 93$ & 1911 & 5.321 & 81.25 & 189.71 & 74.5 & 171 & \\
\hline 12 & $9 / 22 / 93$ & 1923 & 5.2 & 81 & 189.61 & 74.75 & 16 & \\
\hline 13 & $9 / 22 / 931$ & 1924 & 5.1 & 81 & 188.71 & 75 & 16 & \\
\hline 14 & $9 / 22 / 93$ & 1925 & 5.1 & 81 & 189.7 & 74.5 & 161 & \\
\hline 15 & $9 / 22 / 93 \mid$ & 1926 & 5.1 & 81 & 189 & 75 & 161 & \\
\hline 16 & $9 / 22 / 93 \mid$ & 1927 & 5.3 & 81 & 189.3 & 75.5 & 16 & \\
\hline 17) & $9 / 22 / 93$ & 1928 & 5.1 & 80.5 & 188.5 & 74 & 161 & \\
\hline 18 & $9 / 22 / 93$ & 19291 & 5.2 & 80.75 & 188.9 & 74.5 & 171 & \\
\hline 19 & $9 / 22 / 93$ & $\overline{19301}$ & 5.21 & 80.75 & 190.6 & 74.75 & 17 & \\
\hline 20 & $9 / 22 / 93$ & 1931 & 5.24 & 80.75 & 189.6 & 74.5 & 17 & \\
\hline 21 & $9 / 22 / 93$ & 1932 & 5.21 & 80.75 & 189.8 & 74.5 & 17 & \\
\hline 22 & $9 / 22 / 93$ & 1933 & 5.17 & 80.75 & 190.4 & 74.5 & 16 & \\
\hline 23 & $9 / 22 / 93$ & 1934 & 5.18 & 80.75 & 1901 & 74.625 & 16 & \\
\hline 24 & 9/22/93 & 1935 & 5.24 & 80.75 & 189.9 & 74.5 & 16 & \\
\hline 25 & $9 / 22 / 93$ & 1936 & 5.22 & 80.875 & 192.11 & 74.75 & 16 & \\
\hline 26 & $9 / 22 / 93$ & 1937 & 5.27 & 81 & 202.11 & 75.5 & 161 & \\
\hline 27 & $9 / 22 / 93 \mid$ & 1938 & 5.3 & 81.125 & 202.2 & 75.75 & 16 & \\
\hline 28 & 9/22/93 & 1939 & 5.25 & 81 & 200.31 & 75.5 & 16 & \\
\hline 29 & $9 / 23 / 93 \mid$ & $1952 !$ & 5 & 80.5 & 189.61 & 74.5 & 171 & \\
\hline 301 & $9 / 23 / 93 \mid$ & $1953 \mid$ & 5.2 & 80.5 & 189.81 & 75 & 17 & \\
\hline 311 & $9 / 23 / 93 \mid$ & 19541 & 5.3 & 80.5 & 1901 & 75 & 171 & \\
\hline 32 & $9 / 23 / 93$ & 19551 & 5.2 & 80.5 & 1911 & 75.5 & 171 & \\
\hline 33 & $9 / 23 / 93 \mid$ & $1956 !$ & 5 & 80.75 & 189 & 75 & 171 & \\
\hline 34 & $9 / 23 / 93 \mid$ & $1957 !$ & 5.21 & 80.5 & 188.9 & 75 & 161 & \\
\hline
\end{tabular}


WHC-SD-W025-RPT-001, Rev. 0

HANQC.XLS

\begin{tabular}{|r|r|r|r|r|r|r|r|r|}
\hline 35 & $9 / 23 / 93$ & 1958 & 5.1 & 80.5 & 187.9 & 75 & 16 & \\
\hline 36 & $9 / 23 / 93$ & 1959 & 5.1 & 80.75 & 187.6 & 74.5 & 16 & \\
\hline 37 & $9 / 23 / 93$ & 1960 & 5.2 & 80.75 & 188.8 & 74.75 & 16 & \\
\hline 38 & $9 / 23 / 93$ & 1964 & 5.17 & 81 & 190.2 & 75.25 & 17 & \\
\hline 39 & $9 / 23 / 93$ & 1965 & 5.2 & 81 & 191.3 & 75 & 16 & \\
\hline 40 & $9 / 23 / 93$ & 1970 & 5.2 & 81 & 187.3 & 75 & 17 & \\
\hline 41 & $9 / 23 / 93$ & 1971 & 5.2 & 81 & 188.7 & 75 & 16 & \\
\hline 42 & $9 / 23 / 93$ & 1972 & 5.25 & 81 & 183.3 & 74.5 & 16 & \\
\hline & & & & & & & & \\
\hline & & & & & & & & \\
\hline
\end{tabular}


WHC-SD-W025-RPT-001, Rev. 0

\section{MANUFACTURING QUALITY CONTROL RESULTS for the HANFORD LANDFILL}

WIDE WIDTH TENSILE ASTM D4595

Submittal Date: 10/26/93

Customer: GeoPacific Lining, Iric.

PRODUCT ROLLNO. PROPERTY TESTMETHOD TESTRESULTS SPECS UNITS

TER CE-2

\begin{tabular}{|c|}
\hline $\begin{array}{c}\text { Tenax CE-2 } \\
\text { Geonet }\end{array}$ \\
\hline
\end{tabular}

1931 Wide Width Tensile ASTM D4595 $519 \quad 450 \quad$ Ib/ft

2051 Wide Width Tensile $\quad$ ASTM D4595 $\quad 536 \quad 450 \quad$ lb/ft

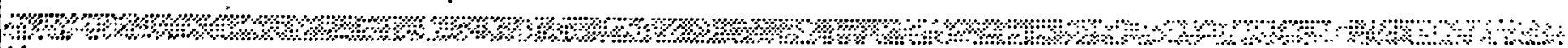
Notes: 


\section{MANUFACTURING QUALITY CONTROL RESULTS for the HANFORD LANDFILL}

CARBON BLACK

ASTM D4218

\begin{tabular}{|c|c|c|c|c|c|c|}
\hline \multicolumn{7}{|c|}{$\begin{array}{l}\text { Submittal Date: } 10 / 26 / 93 \\
\text { Customer: GeoPacific Lining, Inc. }\end{array}$} \\
\hline PRODUCT & ROLL NO. & PROPERTY & TEST METHOD & TEST RESULTS & SPECS & UNITS \\
\hline \multirow{3}{*}{$\begin{array}{c}\text { Tenax CE-2 } \\
\text { Geonet }\end{array}$} & 1811 & Carbon Black & ASTM D4218 & 2.14 & 2.0 & $\%$ \\
\hline & 1931 & Carbon Black & ASTM D4218 & 2.15 & 2.0 & $\%$ \\
\hline & 2051 & Carbon Black & ASTM D4218 & 2.27 & 2.0 & $\%$ \\
\hline
\end{tabular}
Notes: 


\section{MANUFACTURING QUALITY CONTROL RESULTS for the HANFORD LANDFILL \\ COMPRESSION BEHAVIOR \\ ASTM D1621}

Submittal Date: 10/26/93

Customer: Geopacific Lining, Inc.

\begin{tabular}{|ccccccc} 
PRODUCT & ROLLNO. & PROPERTY & TEST METHOD & TEST RESULTS & SPECS & UNITS \\
$\begin{array}{c}\text { Tenax CE-2 } \\
\text { Geonet }\end{array}$ & 1811 & Compression & ASTM D1621 & 77 & 75 & $\%$ \\
& 1931 & Compression & ASTM D1621 & 75 & 75 & $\%$ \\
& 2051 & Compression & ASTM D1621 & 76 & 75 & $\%$
\end{tabular}


MANUFACTURING QUALITY CONTROL RESULTS for the

HANFORD LANDFILL

MASS PER UNIT AREA

ASTM D3T76

Submittal Date: 10/26/93
Customer: GeoPacific Lining, Inc.

$$
\text { mist }
$$

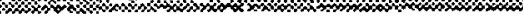

PRODUCT ROLL NO. PROPERTY

\begin{tabular}{|ccccccc}
$\begin{array}{c}\text { Tenax CE-2 } \\
\text { Geonet }\end{array}$ & 1811 & Mass/Unit Area & ASTM D3776 & 24.4 & N/A & oz/sy \\
& 1931 & Mass/Unit Area & ASTM D3776 & 24 & N/A & oz/sy \\
& 2051 & Mass/Unit Area & ASTM D3776 & 24.1 & N/A & oz/sy \\
& & & & & &
\end{tabular}

$\because$ Pि Notes: 
WHC-SD-W025-RPT-OC1, Rev. 0

\section{MANUFACTURING QUALITY CONTROL RESULTS for the \\ HANFORD LANDFILL}

SPECIFIC GRAVITY

ASTM D1505

.

Submittal Date: 10/26/93

Customer: GeoPacific Lining, inc.

1.

PRODUCT ROLLNO. PROPERTY TEST METHOD TEST RESULTS SPECS UNITS

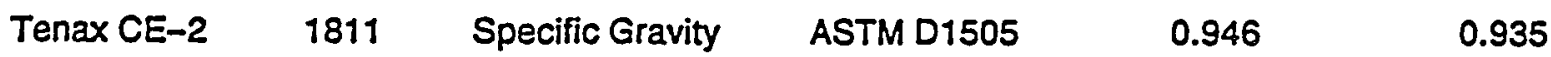

Geonet

$\begin{array}{lllll}1931 & \text { Specific Gravity } & \text { ASTM D1505 } & 0.946 & 0.935 \\ 2051 & \text { Specific Gravity } & \text { ASTM D1505 } & 0.946 & 0.935\end{array}$

\% Notes: 
WHC-SD-W025-RPT-001, Rev. 0

MANUFACTURING QUALITY CONTROL RESULTS for the

HANFORD LANDFILL

MINIMUM THICKNESS

ASTM D751

Submittal Date: 10/26/93

Customer: GeoPacific Lining, Inc.

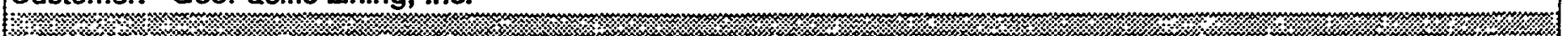

PRODUCT ROLLNO. PROPERTY TESTMETHOD TESTRESULTS SPECS UNITS

13 .

$\begin{array}{lllllll}\text { Tenax CE-2 } & 1811 & \text { Thickness } & \text { ASTM D751 } & 0.210 & 0.200 & \text { in }\end{array}$

Geonet

1931 Thickness ASTM D751 $0.211 \quad 0.200 \quad$ in

2051 Thickness ASTM D751 $0.211 \quad 0.200 \quad$ in

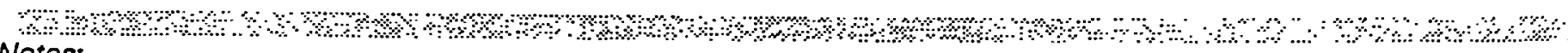
Notes: 
WHC-SD-W025-RPT-001, Rev. 0

\title{
MANUFACTURING QUALITY CONTROL RESULTS for the HANFORD LANDFILL
}

\author{
TRANSMISSIVITY TEST RESULTS \\ ASTM D4716
}

NORMAL LOAD: 1,000 psf

HYDRAULIC GRADIENT: 0.31

momomom

Submittal Date: 10/26/93

Customer: GeoPacific Lining, Inc.

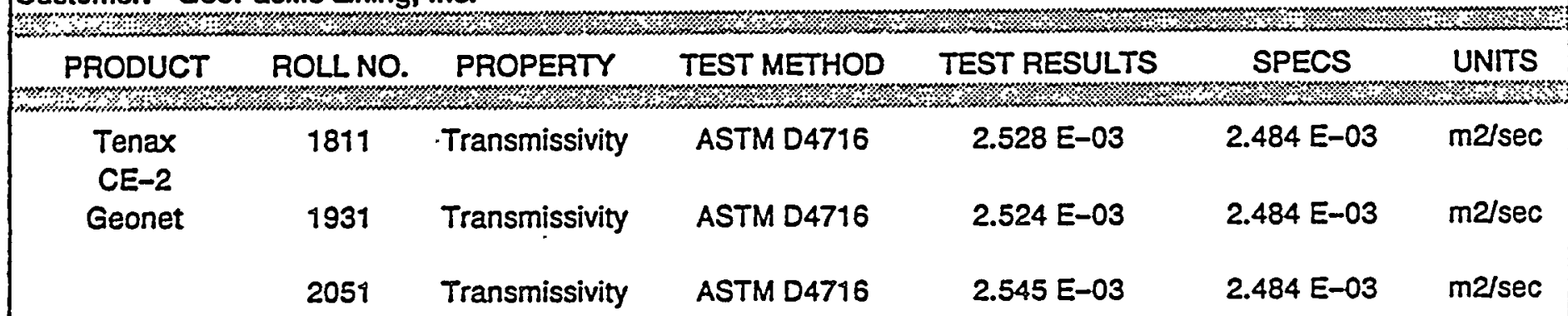

Notes: 
WHC-SD-W025-RPT-001, Rev. 0

\section{MANUFACTURING QUALITY CONTROL RESULTS for the HANFORD LANDFILL}

TRANSMISSIVITY TEST RESULTS

ASTM D4716

NORMAL LOAD: 10,000 psf

HYDRAULIC GRADIENT: 0.01

\begin{tabular}{|c|c|c|c|c|c|c|}
\hline \multicolumn{7}{|c|}{$\begin{array}{l}\text { Submittal Date: 10/26/93 } \\
\text { Customer: GeoPacific Lining, Inc. }\end{array}$} \\
\hline PRODUCT & ROLL NO. & PROPERTY & TEST METHOD & TEST RESULTS & SPECS & UNITS \\
\hline $\begin{array}{l}\text { Tenax } \\
\text { CE-2 }\end{array}$ & 1811 & Transmissivity & ASTM D4716 & 7.224 E-03 & $1.656 E-03$ & $\mathrm{~m} 2 / \mathrm{sec}$ \\
\hline Geonet & 1931 & Transmissivity & ASTM D4716 & 7.188 E-03 & $1.656 E-03$ & $\mathrm{~m} 2 / \mathrm{sec}$ \\
\hline & 2051 & Transmissivity & ASTM D4716 & $7.248 E-03$ & $1.656 E-03$ & $\mathrm{~m} 2 / \mathrm{sec}$ \\
\hline
\end{tabular}

Notes: 
Noventber 22, 1993

Mr. Tony Sample

Delluur Industrics, Ine.

4333 Tum Access Rd.

Port Angeles, WA 98362

\section{RE: Hanfurd/TENAX CE-2 Gconel}

Dear Mr. Sample:

This leller is to address the discrepancies between the results of the confornunce lests performed by the quulity assurance consulunt for the above referenced projeet and product nod the results provided from ous interaal quality control program.

It seems that the major concem is that the test resuits from our internal $Q C$ program indieate a specific gravily of 0.946 while the QA consultant's results indicate a specific gravity of 0.940 and 0.941 . Since there are many possible causes for variations in a property like specific gravity, it is impossible to deternine exactly what caused the diserepancies seen bere. However, the most probable causes are interlaboratory precision and the aceuracy of the standard test method. Cunsidering that there are many possible enuses of variation, it is not unusual to see the small variation seen here $(0.006$ and 0.005$)$ between two independent laboratories.

Additionally, there seems to be some concern about the CE-2; geonet's specific gravity selative to the seomenibrane's specific gravity. Since two different testing laboratorics performed the seusitive specific gravily tests on the CE-2 geonet, it would be most appropriate to consider only the test results produced by one of the laboratories when a comparison of the specific gravity of the geonet to the specifie gravity of the geonembrane is necessnry. Considering that inter-laboratory precision is a problem with a test like specific gravity, comparing laboratory "A's" geonet specific gravity with laboratory "B's" geomenubrane specific gravity would not be the most logical approach. The QA consultant's tests confirm that the geonel's specific gravity $(0.940$ and 0.941$)$ is less that the geomembrane's specific gravity $(0.942$ to 0.944$)$. Therefore, these should te no cause for concern about the compatibility of the geonet and the geomenibrane.

Mr. Sample, if you have any questions at all. please feel free to contact me. I will be glad to assist you in any way possible.

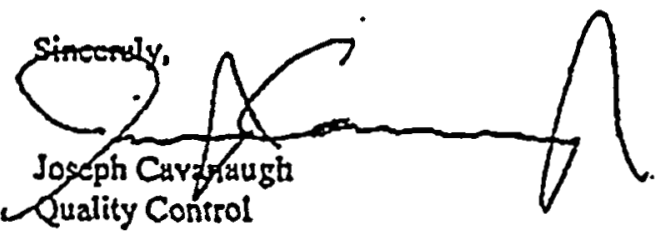


WHC-SD-W025-RPT-001, Rev. 0

APPENDIX G

GEOTEXTILE CONFORMANCE TESTS 
WHC-SD-H025-RPT-001, Rev. 0

APPENDIX G-1

CQA CONFORMANCE TESTS 
WHC-SD-W025-RPT-001, Rev. 0

GOLDER CONSTRUCTION SERVICES, INC.

933-1354

GEOTEXTILE INVENTORY CONTROL RECORD

05/10/94

DEPARTMENT OF ENERGY

Page: 1

NON-DRAG OFF LANDFILL FACILITY

HANDFORD NUCLEAR RESERVATION

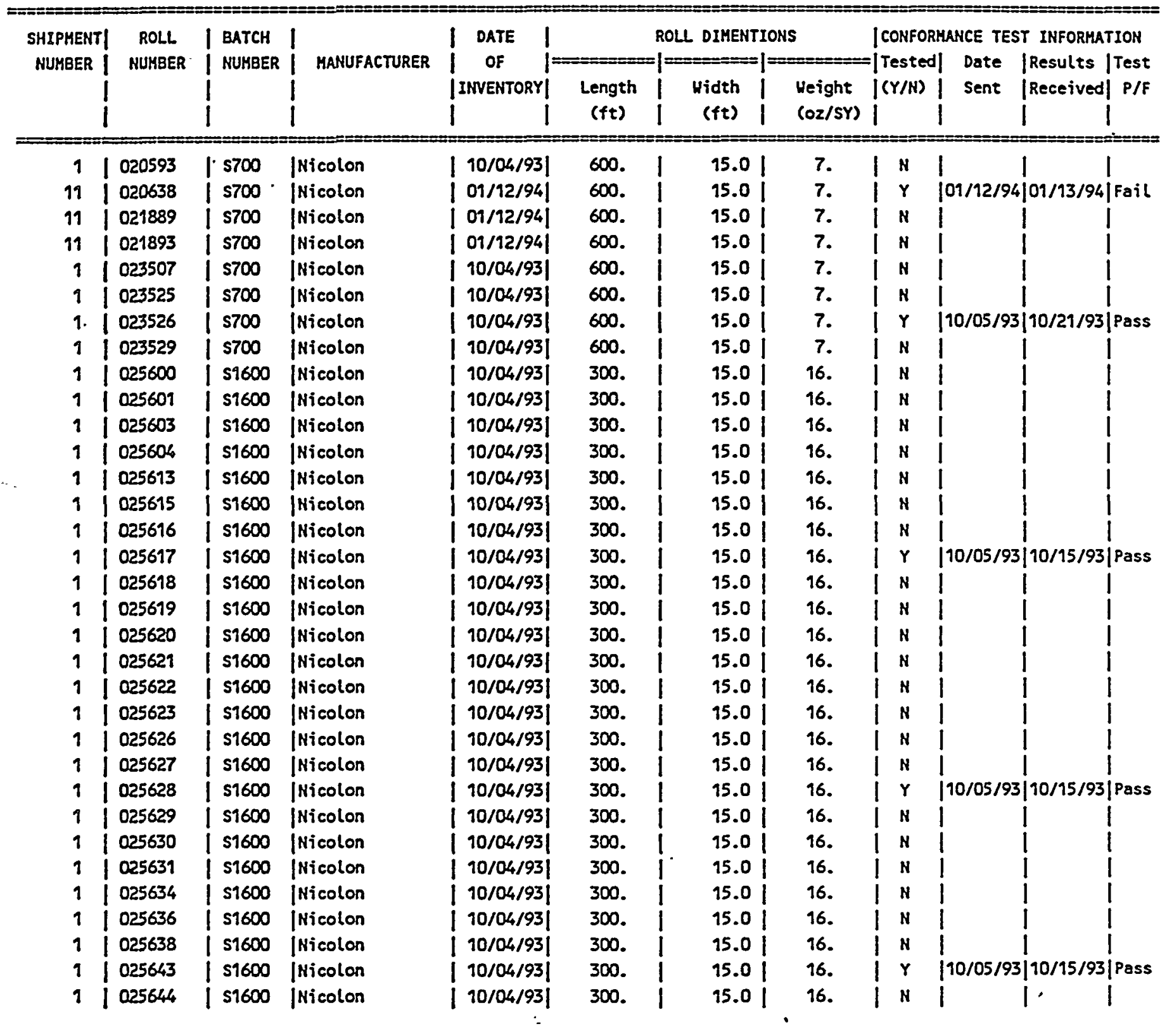

Square Feet Delivered $=184500$. 
WHC-SD-W025-RPT-001, Rev. 0

OCTOBER 1993

\begin{tabular}{|c|}
\hline SUMMARY OF GEOTEXTILE \\
CONFORMANCE TEST RESULTS \\
\hline DEPARTMENT OF ENERGY \\
NON DRAG-OFF COA \\
WASHINGTON \\
\hline
\end{tabular}

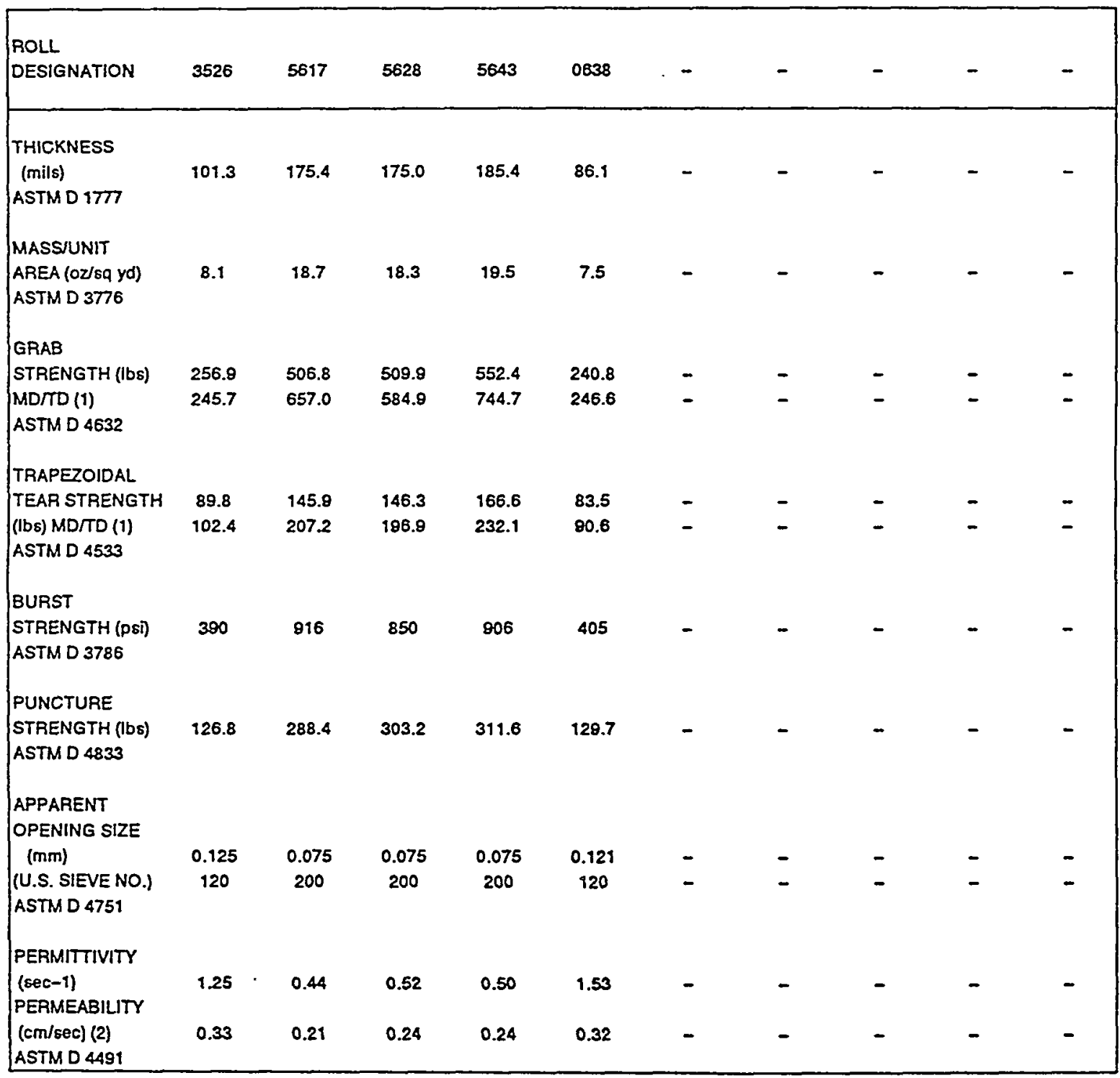

(1) MD/TD corresponds to Machine Direction / Transverse Direction.

(2) Permeability calculated by multiplying measured thickness by permittivity. 


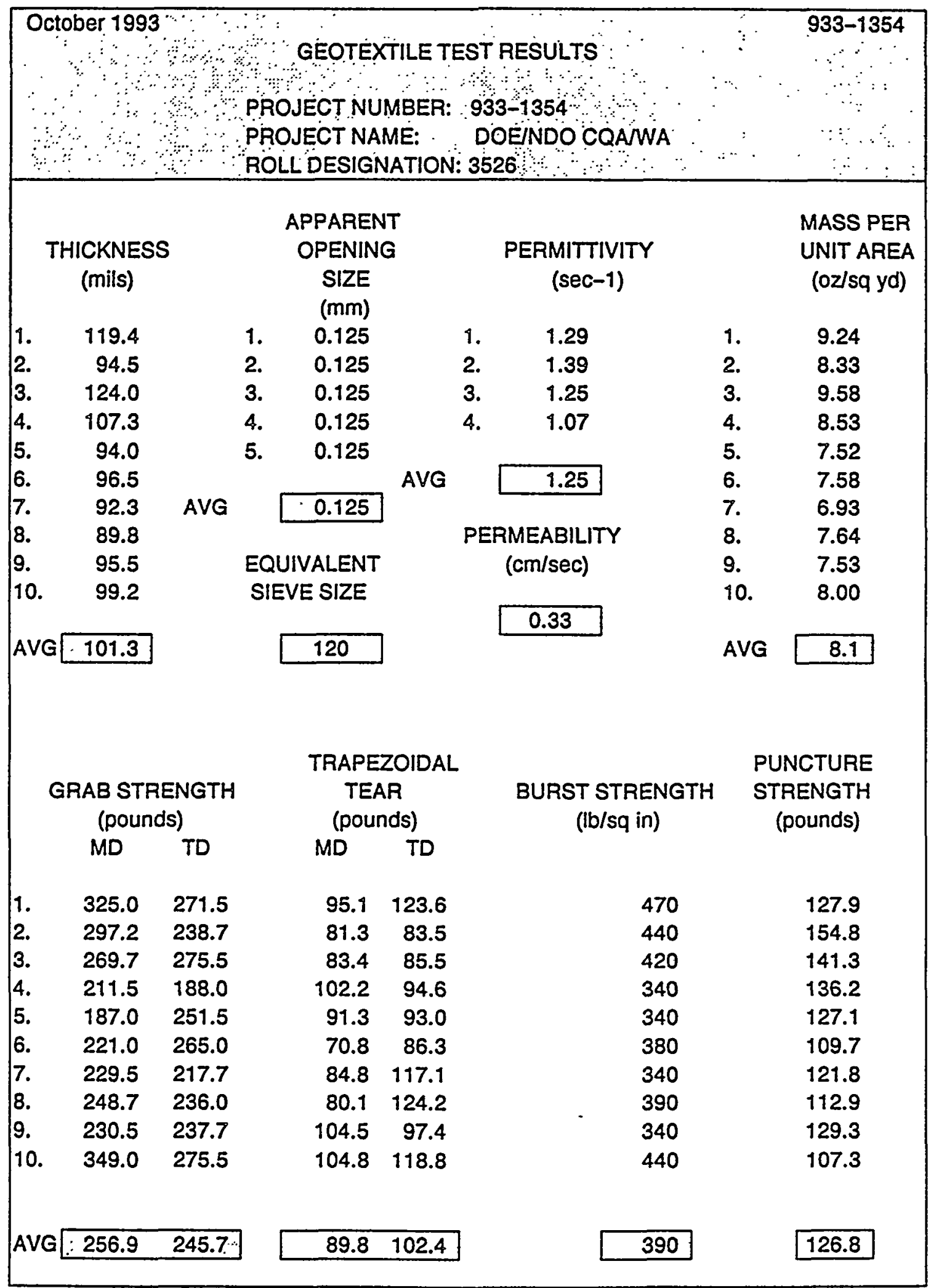


WHC-SD-W025-RPT-001, Rev. 0

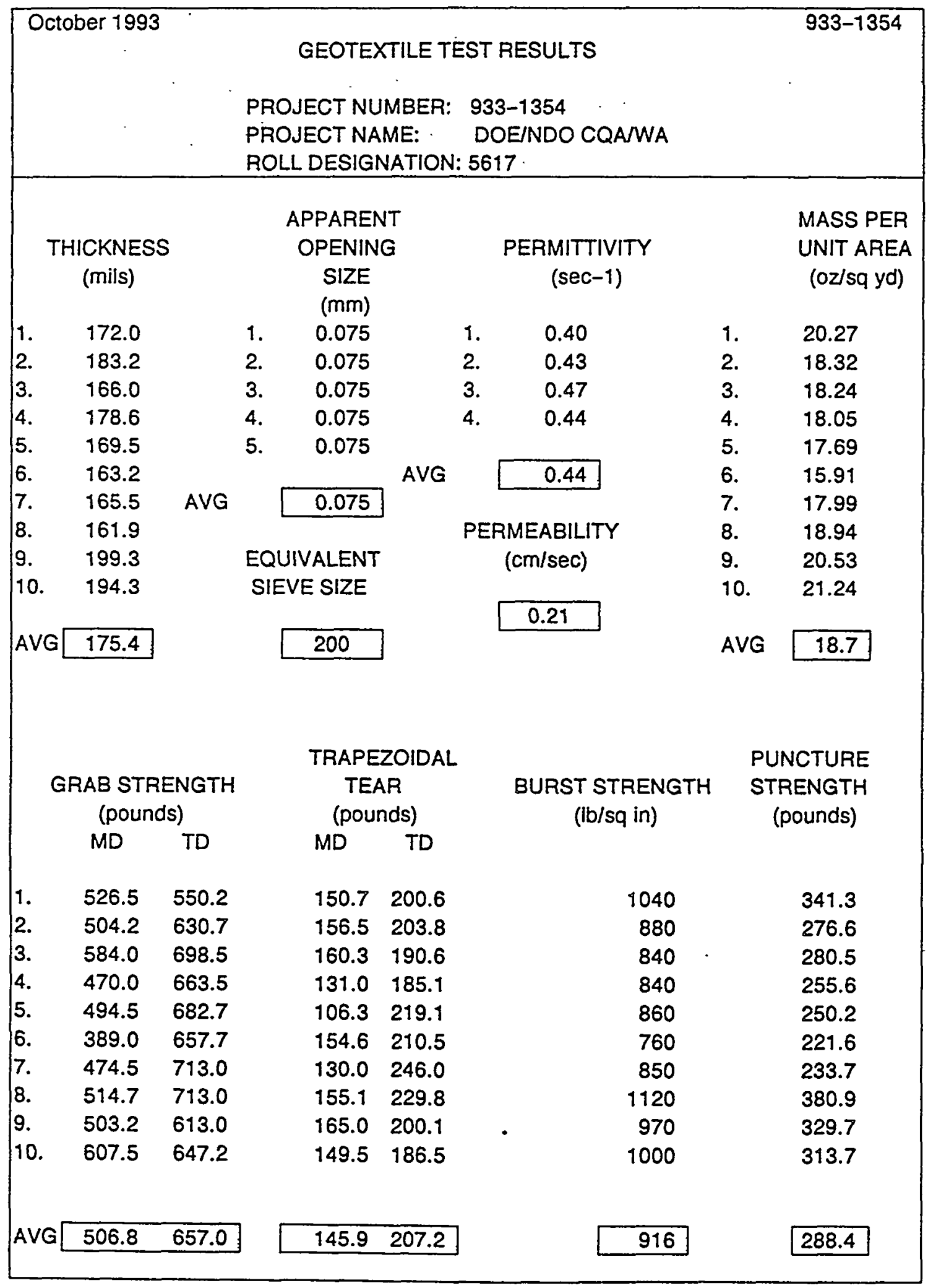


WHC-SD-W025-RPT-001, Rev. 0

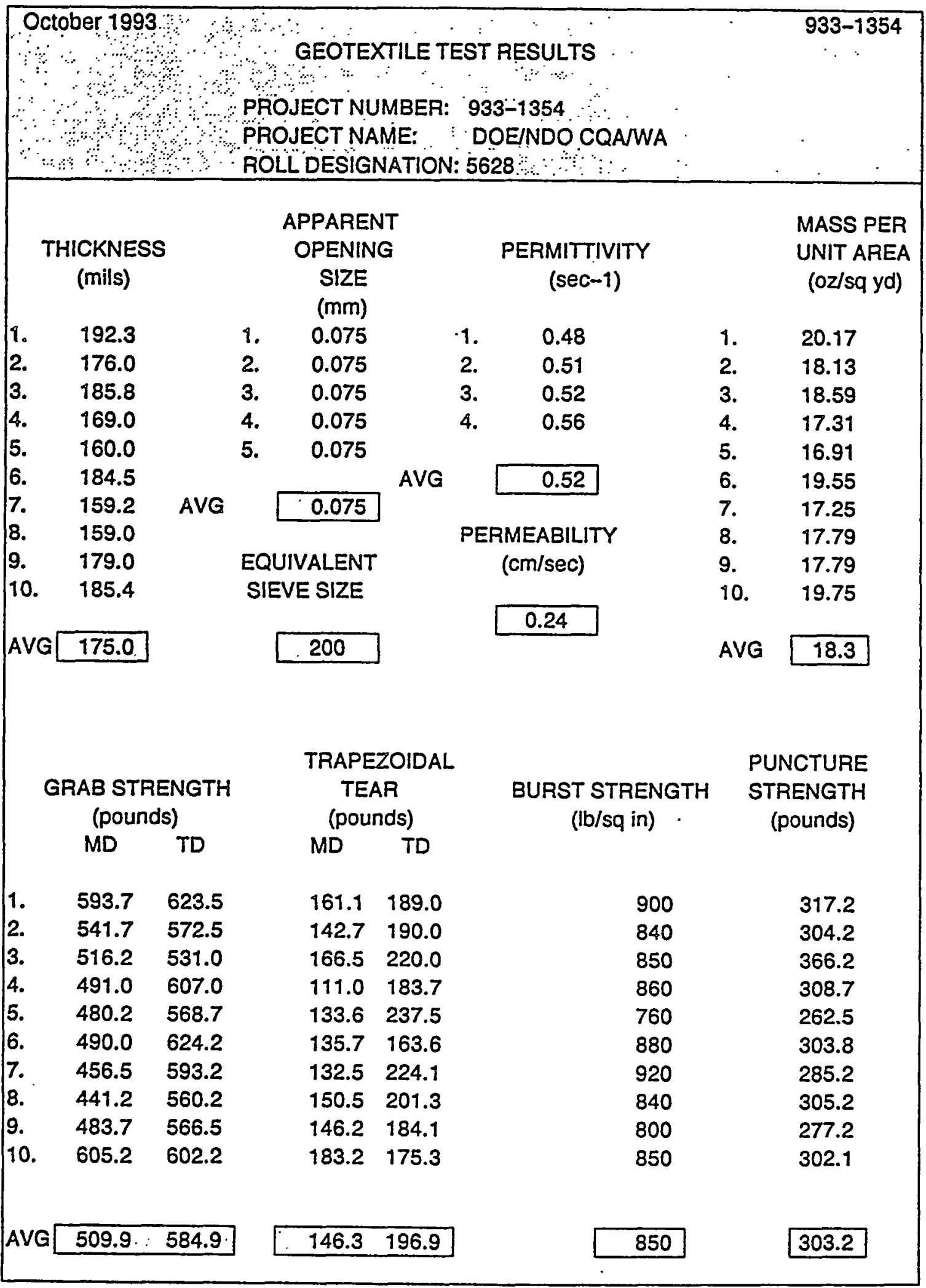


WHC-SD-W025-RPT-001, Rev. 0

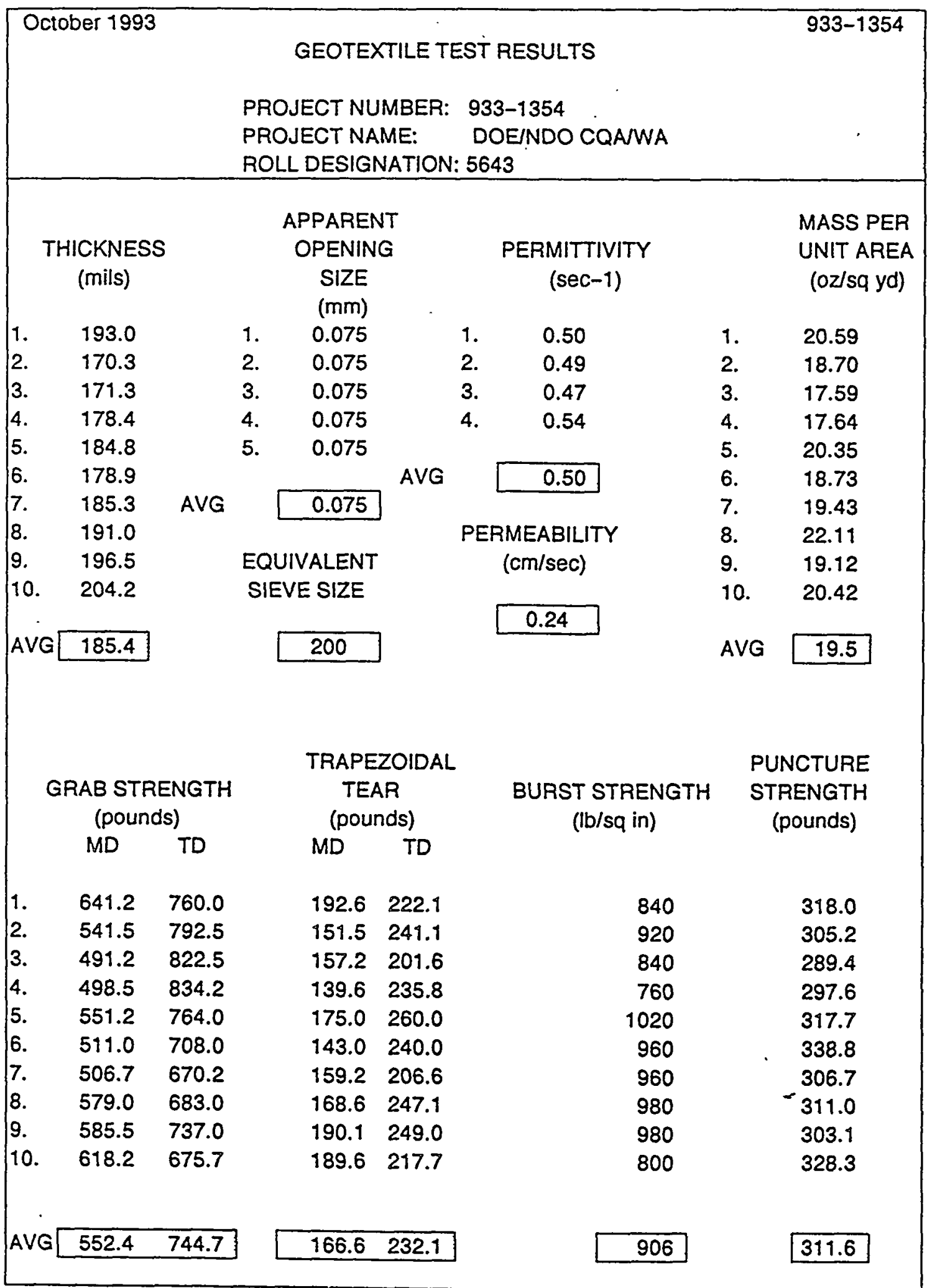




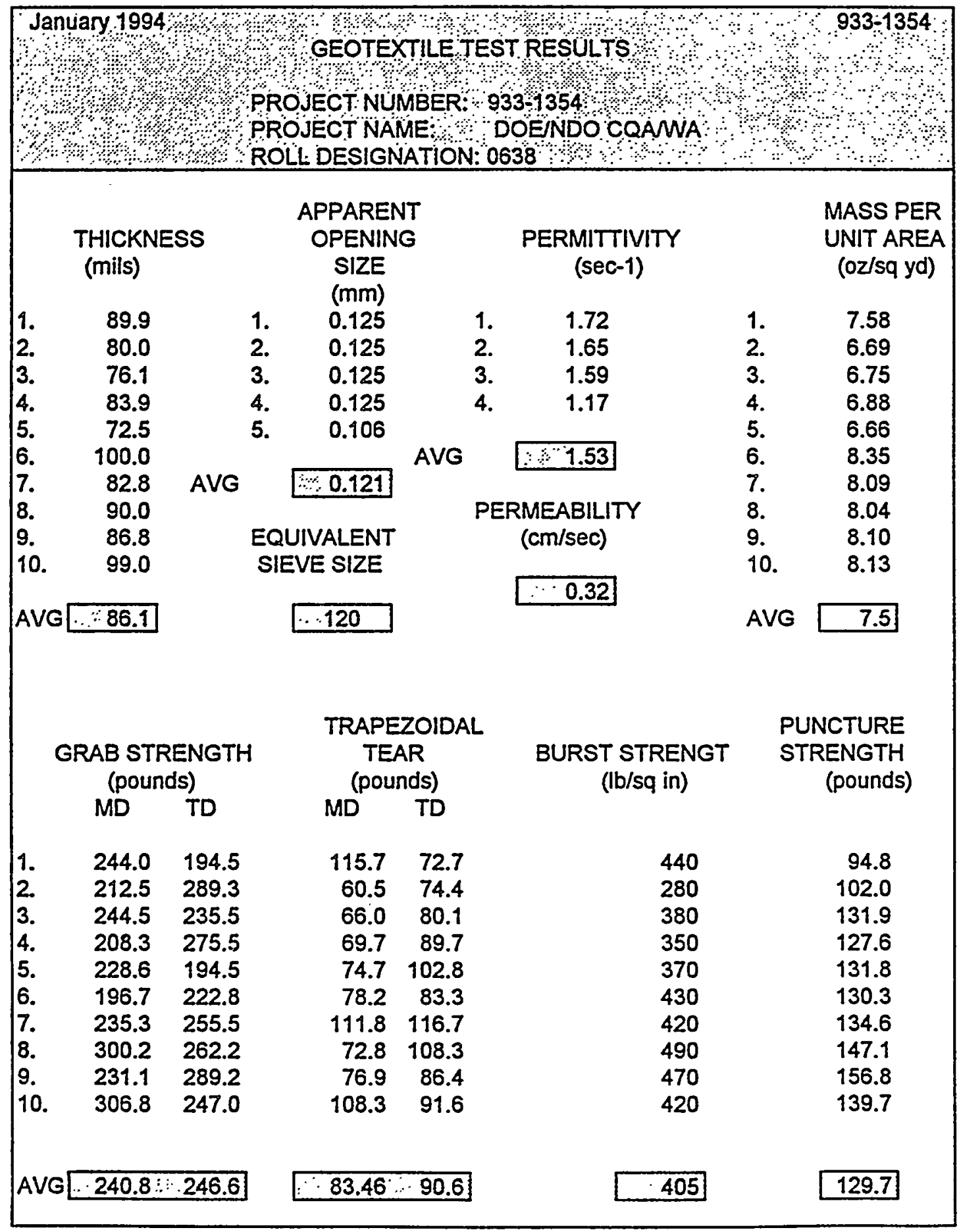


WHC-SD-W025-RPT-001, Rev。0

\section{APPENDIX G-2}

MANUFACTURER'S QC DOCUMENTATION 
Seprember 14, 1993

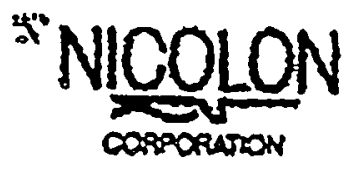

MS5. Don Hilderband

SLT Nonth America

200 S. Trade Ctr. Pky

Conroe, $T X$

RE: $\quad \mathbf{S 7 0 0}$

Project Name : North zanover D.O.T.

Deat Dan:

This is to certify thad Hicolon 5700 is a $100 \%$ polypropylens nonwoven needlepunched fabric. Nicolon 5700 is resistant to degradation due to ultraviolet exposure and resists commonly encounteted soil chemicais, insects, mildow and is nxan-biodegradshla. Polypropylene is stablo within a ph range of 2 to 13. Nicolon $\$ 700$ conforms to the physical properties listed in the following lebie

Eabric Prosty

Weight

Thickness

Grab Tensile

Grab Elangation

Trapezoid Toat

Punceure

Mullea Burst

AOS

Parmilltivily

Waler Flow Rate

Permeability

U.Y. Resistanco
Tes Metrod

ASTM D3776

ASTM DITT

ASTM D 6632

ASTM D6632

ASTMA D4533

ASTM D4833

ASTMS D3786

ASTM D4751

ASTM DA491

ASTM D4491

ASTM D4491

ASTM D4355
Inits

orisg.yd.

rils

bs.

$\%$

lbs.

$16 s$.

pos

US Stove\{nm)

$1 / \sec$

gpro/sg.n.

cmisec

$\%$
SA.R.Y.

7.0

110.

200 .

so.

80

115

400

70

1.2

95

.38

70

Notc: MARV - Minimum Average Roll Values. These are defined as toro (2) standard ceviations below the mean and represent a 95\% confidence kevel.

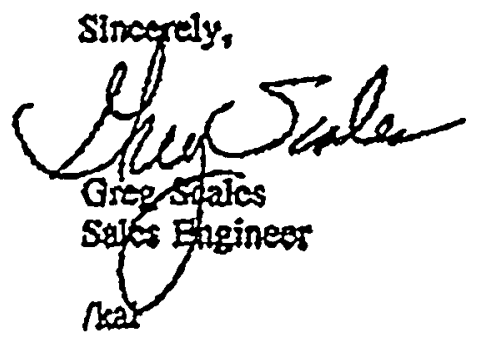

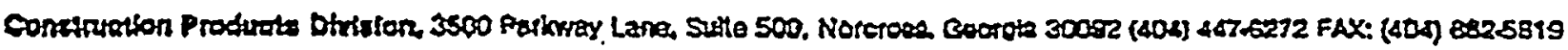


September 14, 1993

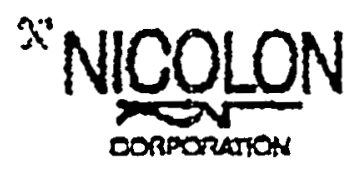

Mr. Don Hilderbrand

RE; $\quad 5700$

SLT North America

200 S. Trade Or. Pky

Contoe, TX

Project Name : North Hanover D.O.T.

Deas Don:

This is to certify that Nicolon $\$ 1600$ is a $1005 \%$ polypropylene nonwoven acediepunebed fabric. Nicaton SIEOO is resistant to degradation due to viluaviolet exposure and resists commonly encormered soll chemicals, insecis, mildew and is ron-blodegradable. Polypropylene is stable within a ph range of 2 to 13 . Nicolon $\$ 1600$ conforms to the physical properties listed in the following tabie.

\begin{tabular}{|c|c|c|c|c|}
\hline Eabric Propty & Test Mathad & Inits & MAS & V \\
\hline Weight & ASTMA D3T76 & oz/sq.yd. & 16.0 & \\
\hline Thickness & ASTM DIT77 & mits & 183 & \\
\hline Grab Tensile & ASTH D4632 & Itos. & 500. & \\
\hline Grab Elongation & ASTM D4632 & $\%$ & 60 & \\
\hline Trap Tear Streagth & ASTAK D4533 & los. & 160 & \\
\hline Puncture & ASTM D4833 & ths. & 245 & \\
\hline Mullen Burat & ASTM D3786 & $\mathrm{pri}$ & 780 & \\
\hline Waler Flow Rato & ASTM D449I & gal/nialat $t^{2}$ & 35 & \\
\hline Permeability & ASTM D4491 & $\mathrm{cm} / \mathrm{sec}$ & .20 & \\
\hline Permiluivily & ASTM D4491 & $\sec ^{\cdot 1}$ & .45 & • \\
\hline U.V. Resistance & $\begin{array}{l}\text { ASTM DA355 } \\
(\otimes 150 \text { hrs) }\end{array}$ & $\%$ & 70 & \\
\hline A.O.S. & ASTM DA75! & US Siove & 100 & \\
\hline
\end{tabular}

Note: MARV: Minimum Average Roll Valucs. These are defined as two (2) slandard devialions below the nuean and repreberl a $95 \%$ confidence level.

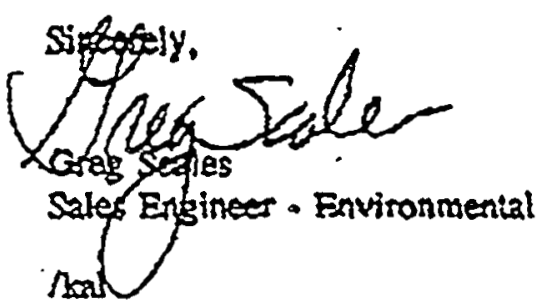

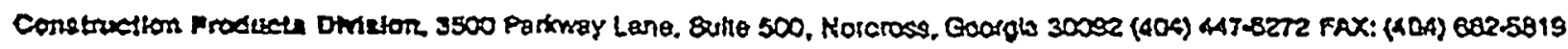




\section{ATLANTIC THREAD AND SUPPLY CO., INC. \\ P.0. $80 \times 87395$ - BALTHORE. MATMLND $21239^{\circ}$ \\ PHONE NO: $410-488-4040$ \\ FAXNO: 410-483-4008}

Ocrober 20, 1993

35. AI Haluchek

GHO PACIPIC LIRIRG, IKC.

suste 250

1300 pexter hyenue, North

seatcle. HA 98109

\section{CERTIFICATIOX}

Dear MF. Zalucbek,

ITEK: POLYPRO, RQLYZRORYLENE SEKIXG THREAD

SIZE TES 210

QUARTITY. 14 LBS.

Ho hereby cextify this thread is made of 1008 polypropylene siber and demonstrates the bame chemlcal resistance as che polypropyleme fabrte being used for this geocextile project.

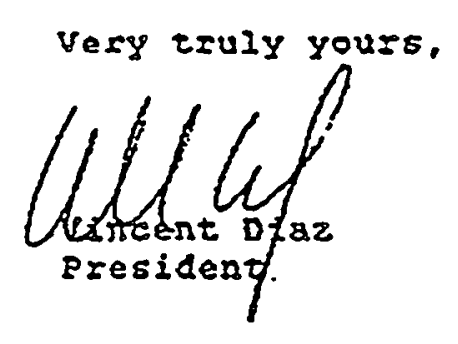

VD\&pab 
WHC-SD-W025-RPT-001, Rev. 0

September 30,1993

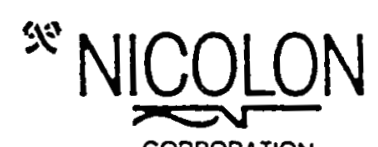

RE: NICOLON Style 5700

ORDER \# 3005

QUANTITY: $5000 \mathrm{SG.YA.}$

SHIP DATE: September 30, 1993.

PROJECT NAME : Handford waste Disposal

CUSTOMER ORDER \#: HT-31938

JOB : 8491

Ms. Colleen Murphy

SIT North America

200 s. Trade ctr. Pkwy

Conroe, TX 77385

Dear Ms. Murphy,

This is to certify that NICOLON 5700 is a $100 \%$ polypropylene nonwoven needle-punched fabric. NICOLON 5700 is resistant to degradation due to ultraviolet exposure and resists commoniy encountered soil chemicals, insects, mildew and is non-biodegradable. Polypropylene is stable within a pH range of 2 to 13. NICOLON style s700 conforms to the physical properties listed in the following table:

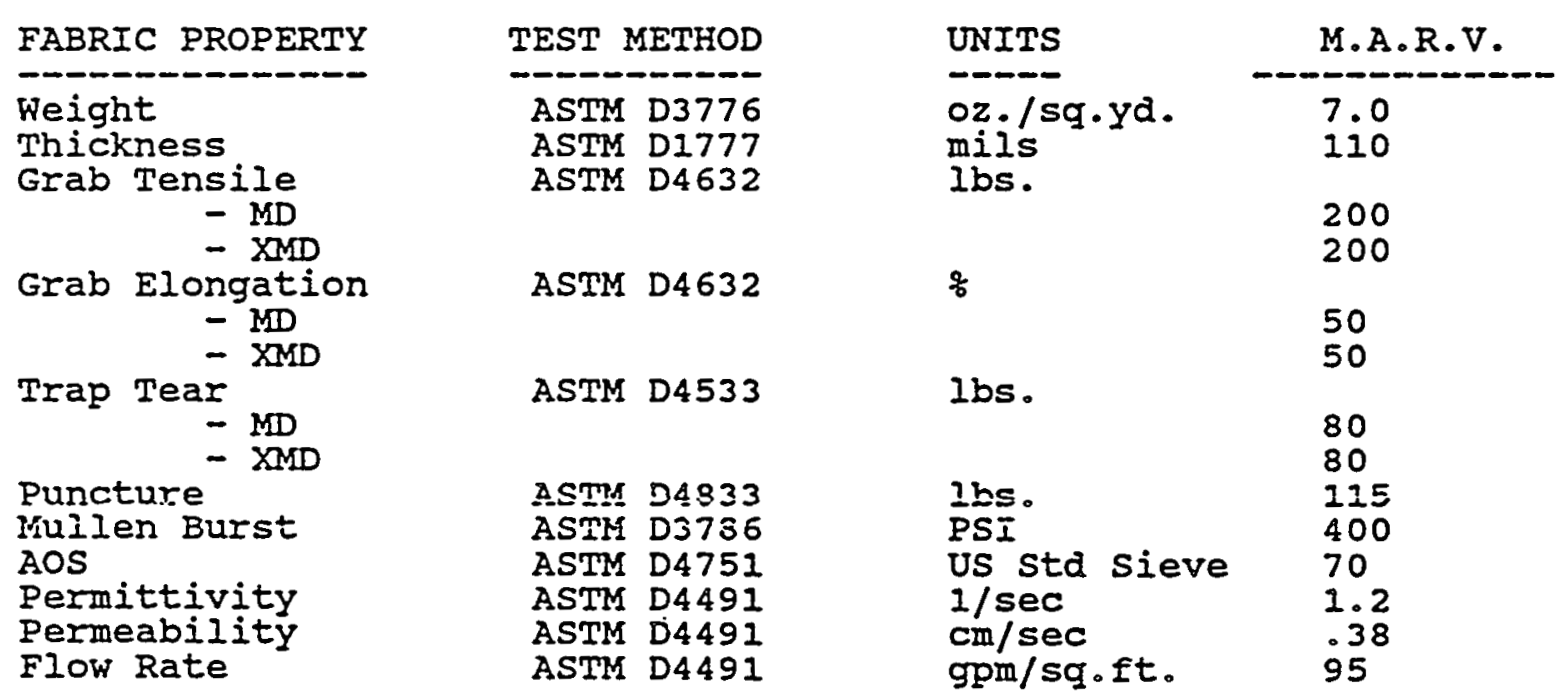

Attached are actual test values of rolls in shipment.

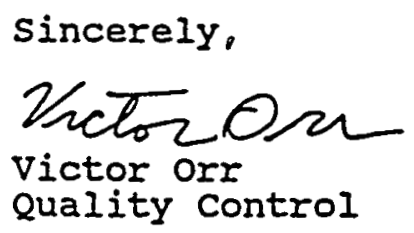

Sworn and subscribed to before me this 30 th day of September, 1993. Vicki Bentrele

Notary Public Signature

Nicolon Corporation, P.O. Box 1979 . Cleveland Road, Cornelia, Georgia 30531 (706) 778-9794 FAX. (706) 778-2043 
WHC-SD-W025-RPT-001, Rev. 0

PATE 9

10:28:06 30 SEP 9993

ROLL.. STYLE. ACTUAL THICK GRAB... GRAB... GRAB. GRAB. TRAP TRAP. PUMC BURST AOS PERHIT PERMEA FLON. MEIGHT HESS TEHSILE TEHSILE ELONG ELOHG TEAR TEAR TURE TIVITY BILITY RATE

$\begin{array}{lllll}\text { MHCH } & \text { XUACH } & \text { MUCH } & \text { XUCH MACH XHACH } \\ \text { DIR } & \text { DIR } & \text { DIR DIR DIR DIR }\end{array}$

$\begin{array}{llllllllllllllll}020593 & 5700 & 7.8 & 131 & 225 & 245 & 61 & 69 & 94 & 192 & 944 & 481 & 120 & 9.648 & .568 & 128 \\ 023507 & \mathbf{5 7 0 0} & 9.1 & 146 & 252 & 264 & 89 & 99 & 907 & 962 & 132 & 430 & 100 & 9.730 & .633 & 136 \\ 023525 & 5700 & 8.2 & 136 & 250 & 235 & 77 & 66 & 145 & 145 & 135 & 451 & 100 & 9.768 & .651 & 137 \\ 023526 & 5700 & 8.1 & 136 & 250 & 235 & 77 & 64 & 145 & 145 & 135 & 451 & 100 & 9.768 & .651 & 137 \\ 023529 & 5700 & 7.8 & 136 & 250 & 235 & 77 & 64 & 145 & 145 & 135 & 451 & 100 & 9.768 & .651 & 137\end{array}$

5 Rous Processed 
WHC-SD-W025-RPT-001, Rev. 0

September 30,1993

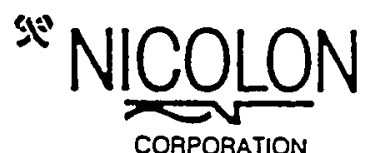

RE: NICOLON Style $\$ 1600$

ORDER \#3005

QUANTITY: $12500 \mathrm{Sq} . \mathrm{Yd}$.

SHIP DATE: September 30, 1993

PROJECT NAME : Handford Waste Disposal

CUSTOMER ORDER : HT-31938

JOB : 8491

Ms. Colleen Murphy

SLT North America

200 S. Trade Ctr. Pkwy.

Conroe, TX 77385

Dear Ms. Murphy,

This is to certify that NICOLON 51600 is a $100 \%$ polypropylene nonwoven needle-punched fabric. NICOLON S1600 is resistant to degradation due to ultraviolet exposure and resists commonly encountered soil chemicals, insects, mildew and is non-biodegradable. Polypropylene is stable within a pH range of 2 to 13. NICOLON style $\$ 1600$ conforms to the physical properties listed in the following table:

FABRIC PROPERTY

Weight

Thickness

Grab Tensile

- MD

- XMD

Grab Elongation

- MD

- XMD

Trap Tear

- MD

- XMD

Puncture

Mulien Burst

AOS

Permittivity

Permeability

Flow Rate

\begin{tabular}{ll} 
TEST METHOD \\
\hline ASTM D 3776 \\
ASTM D 1777 \\
ASTM & D4632 \\
& \\
ASTM & $D 4632$ \\
& \\
ASTM & $D 4533$ \\
& \\
ASTM & $D 4833$ \\
ASTM & $D 3786$ \\
ASTM & $D 4751$ \\
ASTM & $D 4491$ \\
ASTM & $D 4491$ \\
ASTM & $D 4491$
\end{tabular}

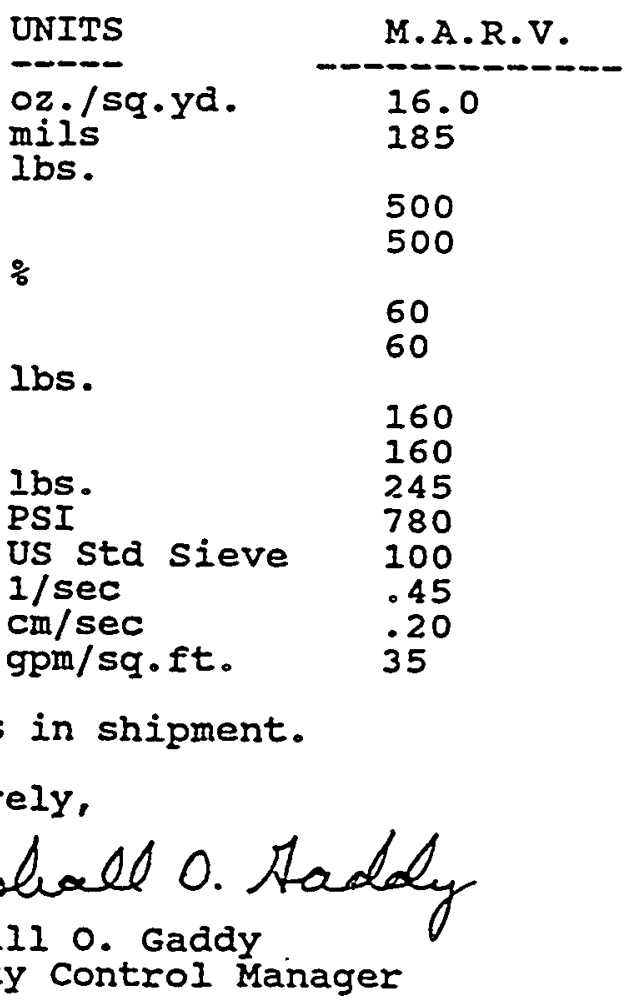

Sworn and subscriped to before me this 30th day of september, 1993. Notary public signature

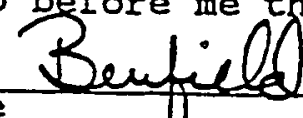
My Commission Expires Aul.

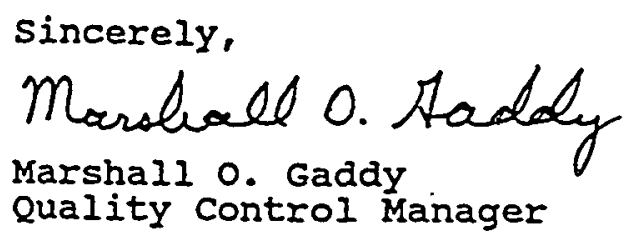

Nicolon Corporation, P.O. Box 1979 - Cleveland Road, Cornelia, Georgia 30531 (706) 778-9794 FAX. (706) 778-2048 


\section{WHC-SD-W025-RPT-001, Rev. 0}

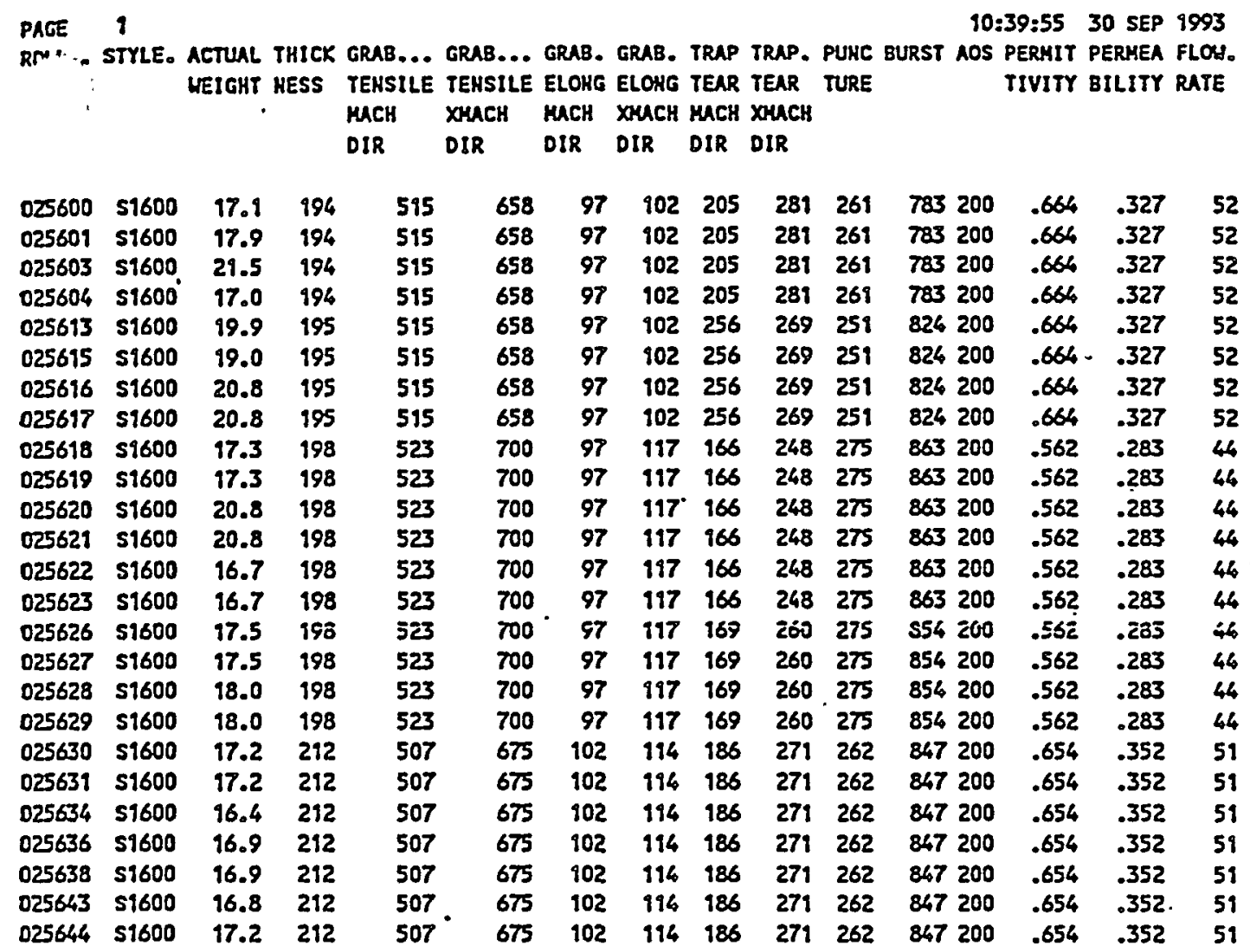

... ' is Processed 
February 10, 1994

RE: NICOLON Style 5700

QUANTITY: $3,000 \mathrm{sq}$. Vas.

Ms. Erin Huitt

SIT North America

200 S. Trade ctr. Pkwy.

Conroe, TX 77385

Dear Ms. Huitt,

This is to certify that Nicolon 5700 is a $100 \%$ polypropylene nonwoven needle-punched fabric. Nicolon 5700 is resistant to degradation due to ultraviolet exposure and resists commonly encountered soil chemicals, insects, milde' and is non-biodegradable. Polypropylene is stable within a pH range of 2 to 13 . Nicolon style s700 conforms to the physical proparties isted in the following table:

\section{FABRIC PROPERTY \\ Weight \\ Thickness \\ Grab Tensile \\ - MD \\ Grab Elongation \\ - $X D$ \\ Trap Tear \\ - MDD
- XIDD \\ Puncture \\ Burst \\ A.OS \\ Permittivity \\ Permeability \\ Flow Rate}

\begin{tabular}{|c|c|}
\hline $\begin{array}{l}\text { ASTM } \\
\text { ASTM } \\
\text { ASTM }\end{array}$ & $\begin{array}{l}\text { D } 377 \\
D 177 \\
D 463\end{array}$ \\
\hline STM & $D 463$ \\
\hline ASTM & D453 \\
\hline $\begin{array}{l}\text { ASTM } \\
\text { ASTM } \\
\text { ASTH } \\
\text { ASTM } \\
\text { ASTM } \\
\text { ASTH }\end{array}$ & $\begin{array}{l}\text { D48 } \\
D 37 \\
D 47 \\
D 44 \\
D 44 \\
D 44\end{array}$ \\
\hline
\end{tabular}

\begin{tabular}{ll} 
UNITS & M.A.R.V. \\
\hdashline oz./sg.yd. & 7.0 \\
mils & 110 \\
Ibs. & 200 \\
& 200 \\
& 50 \\
& 50
\end{tabular}

Ibs.

$\begin{array}{ll} & 80 \\ \text { Ibs。 } & 80 \\ \text { PSI } & 115 \\ \text { US std sieve } & 400 \\ \text { I/sec } & 70 \\ \mathrm{~cm} / \mathrm{sec} & 1.2 \\ \mathrm{gpm} / \mathrm{sq} . \mathrm{ft} . & 938\end{array}$

Attached are actual test value:; of rolls in shipments.

sincerely,

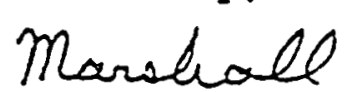

Marshall Gaddy Quality Control Manager

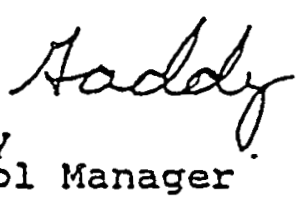
- Thorn and subscribed to before me this loth day of February, 1994.

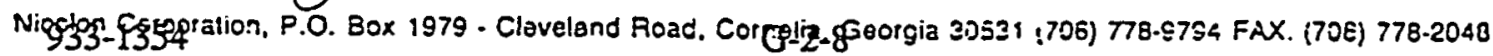


WHC-SD-W025-RPT-001, Rev. 0

PACE 1 14:26:05 10 FEB 1996 ROLL.HO STYLE. ACTURL.. THICK GRAB... GRAB... GRAB. GRAB. TRAP TRAP. PUNC BURST AOS PERHIT PERMEA FLON. WEIGHP HESS TEHSILE TENSILE ELOHG ELOHG TEAR TEAR TURE TIVITY BILITY RATE MACH XНАCH МАCH ХНАCH НАCH ХНАCH OIR OIR DIR OIR OIR DIR

$\begin{array}{llllllllllllllll}020638 & 5700 & 7.7 & 937 & 245 & 276 & 69 & 76 & 905 & 96 & 149 & 453 & 920 & 1.747 & .489 & 136 \\ 021889 & 5700 & 9.1 & 149 & 274 & 346 & 72 & 73 & 99 & 99 & 148 & 424 & 100 & 1.592 & .570 & 122 \\ 021893 & 5700 & 8.4 & 136 & 237 & 289 & 76 & 76 & 99 & 99 & 132 & 426 & 100 & 1.726 & .596 & 133\end{array}$

3 Row: Processed 
WHC-SD-H025-RPT-00\%, Rev. 0

APPENDIX H

GEOCOMPOSITE CONFORMANCE TESTS

$H-i$ 
WHC-SD-W025-RPT-001, Rev. 0

\section{APPENDIX H-1}

\section{CQA CONFORMANCE TESTS}

$H-1$ 
WHC-SD-W025-RPT-001, Rev. 0

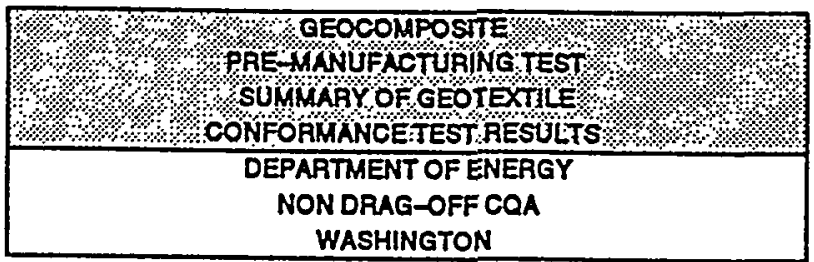

$933-1354$

ROL $1 \%$

DEGIGNATION

APPARENT

OPENING SIZE

(mm)

(U.S. SIEVENO.)

ASTM D 4751

$\begin{array}{cccc}0.140 & - & - & - \\ 100 & - & -\end{array}$

(1) MD/TD corresponde to Machine Direction / Traneveree Direction.

(2) Permeablity calculated by multiplying measured thicknose by pormittivity. 
WHC-SD-W025-RPT-001, Rev. 0

GOLDER CONSTRUCTION SERVICES, INC.

933-1354

GEOCOMPOSITE INVENTORY CONTROL RECORD

$05 / 10 / 94$

DEPARTHENT OF ENERGY

Page: 1

NON-DRAG OFF LANDFILL FACILITY

HANDFORD NUCLEAR RESERVATION

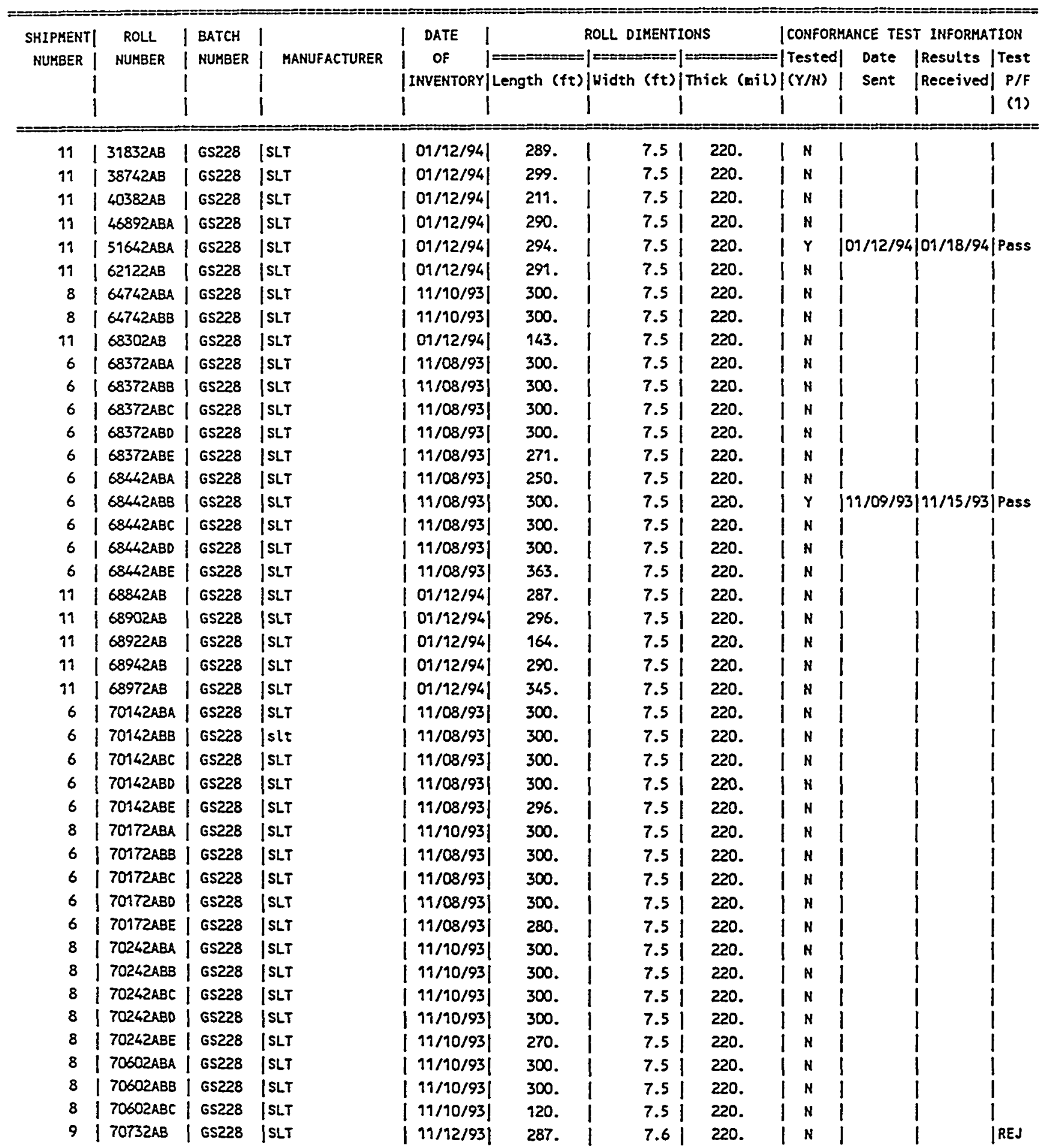


WHC-SD-W025-RPT-001, Rev. 0

GOLDER CONSTRUCTION SERVICES, INC.

933-1354

GEOCOKPOSITE INVENTORY CONTROL RECORD

05/10/94

DEPARTHENT OF ENERGY

Page: 2

NON-DRAG OFF LANDFILL FACILITY

HANDFORO NUCLEAR RESERVATYON

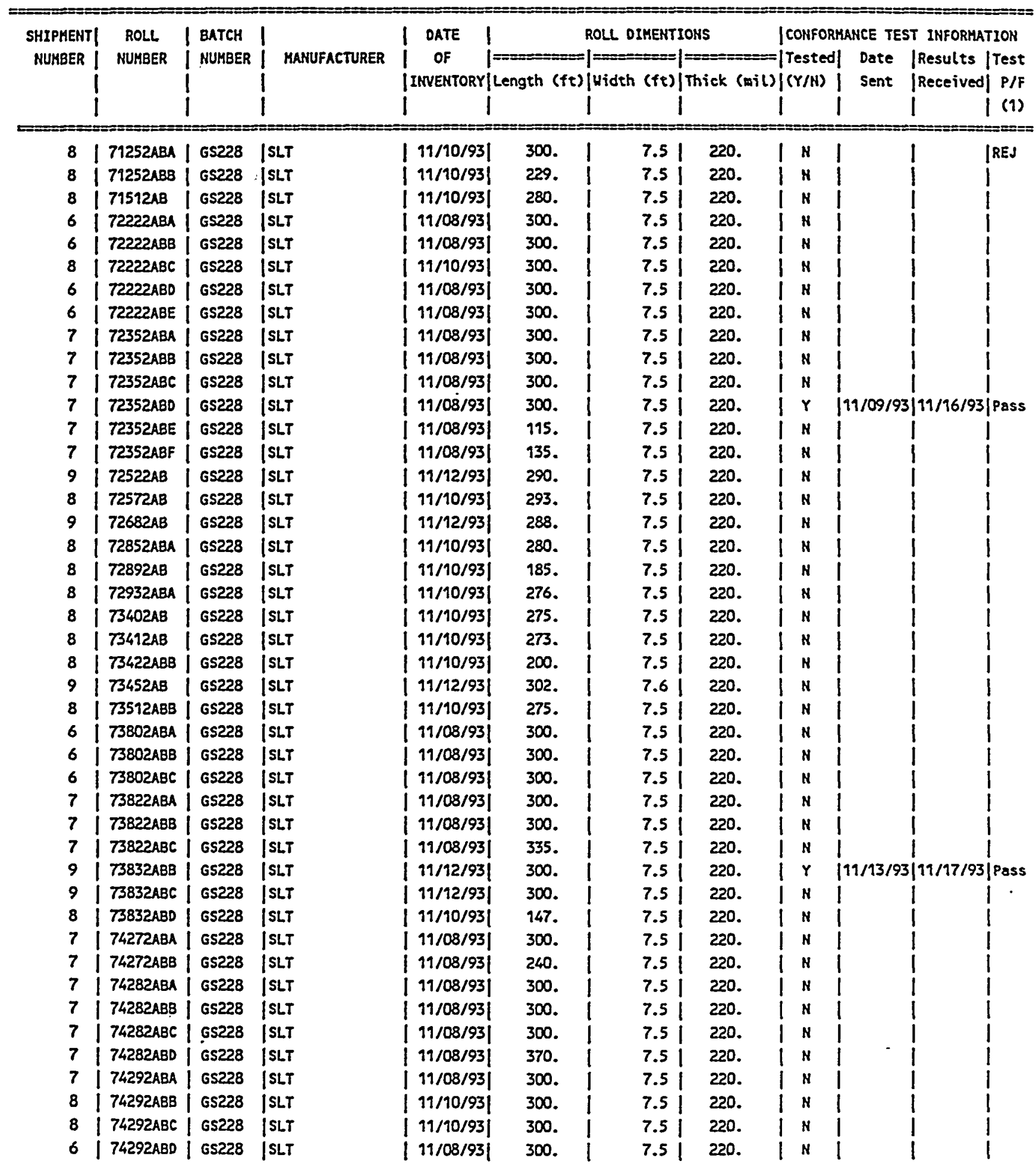


WHC-SD-W025-RPT-001, Rev. 0

GOLDER CONSTRUCTION SERVICES, INC.

933-1354

GEOCOMPOSITE INVENTORY CONTROL RECORD

05/10/94

DEPARTMENT OF ENERGY

Page: 3

NON-DRAG OFF LANDFILL FACILITY

HANDFORD NUCLEAR RESERVATION

\begin{tabular}{|c|c|c|c|c|c|c|c|c|c|c|c|}
\hline \multirow{4}{*}{$\begin{array}{r}\text { SHIPMENT } \\
\text { NUMBER } \\
\mid\end{array}$} & \multirow{4}{*}{$\begin{array}{l}\text { ROLL } \\
\text { NUMBER }\end{array}$} & \multirow{4}{*}{$\begin{array}{l}\text { BATCH. } \\
\text { NUMBER } \\
\mid\end{array}$} & \multirow{4}{*}{$\begin{array}{c}\text { MANUFACTURER } \\
\cdot\end{array}$} & \multirow{3}{*}{$\mid \begin{array}{l}\mid \text { DATE } \\
\mid \text { OF } \\
\mid \text { INVENTORY } \mid\end{array}$} & \multirow{3}{*}{$\begin{array}{l}\mid=========1 \\
\text { Length }(f t)\end{array}$} & \multicolumn{2}{|c|}{ ROLL DIMENTIONS } & \multicolumn{4}{|c|}{ | CONFORMANCE TEST INFORMATION } \\
\hline & & & & & & $\mid=x====x=1$ & $=== \pm=-==-1$ & |Tested| & Date & |Results | & | Test \\
\hline & & & & & & $\mid$ Width $(t t) \mid$ & hick (mil) & $|(Y / N)|$ & Sent & |Received| & $P / F$ \\
\hline & & & & 11 & 11 & $1 \quad 1$ & & 1 & & & | (1) \\
\hline 7 & 74292ABE & GS228 & |SLT & $|11 / 08 / 93|$ & 300. & 7.51 & 220. & N & & | & | \\
\hline 7 & $74302 A B A$ & G\$228 & ISLT & $|11 / 08 / 93|$ & 250. & 7.51 & 220. & N & & i & \\
\hline 7 & $74302 A B B$ & GS228 & |SLT & | 11/08/93| & 300. & $7.5 i$ & 220. & N & & i & \\
\hline 7 & $74302 A B C$ & GS228 & jSLT & | $11 / 08 / 93 \mid$ & 300. & 7.51 & 220. & $F$ & $11 / 09 / 93$ & $|11 / 16 / 93|$ & jPass \\
\hline 7 & $74302 A B D$ & GS228 & ¡SLT & | 11/08/93| & 300. & 7.51 & 220. & N & & 1 & \\
\hline 7 & 74302ABE & GS228 & |SLT & | 11/08/93| & 294. & 7.51 & 220. & N & & I & | \\
\hline 6 & $74312 \mathrm{ABA}$ & G\$228 & |SLT & | 11/08/93| & 300. & 7.51 & 220. & N & & l & \\
\hline 7 & 74312ABB & GS228 & ISLT & | 11/08/93| & 300. & 7.51 & 220. & N & & i & i \\
\hline 7 & 74312ABC & GS228 & |SLT & | 11/08/93| & 300. & 7.51 & 220. & $N$ & & $1 \quad 1$ & \\
\hline 6 & $74312 A B D$ & GS228 & ISLT & | 11/08/93| & 300. & $7.5 i$ & 220. & $\mathbf{F}$ & $11 / 09 / 93$ & $|11 / 16 / 93|$ & jPass \\
\hline 6 & 74312ABE & | GS228 & jSLT & | 11/08/93| & 287. & $7.5 i$ & 220. & $N$ & & $i \quad i$ & i \\
\hline 7 & $74322 A B A$ & G5228 & iSLT & | 11/08/93| & 300. & $7.5 i$ & 220. & N & & i & \\
\hline 7 & 74322ABB & GS228 & ISLT & | 11/08/93| & 300. & 7.51 & 220. & $\mathbf{Y}$ & $11 / 09 / 93$ & $|11 / 16 / 93|$ & |Pass \\
\hline 7 & $74322 \mathrm{ABC}$ & GS228 & |SLT & | 11/08/93 | & 300. & 7.51 & 220. & N & & & \\
\hline 7 & $74322 A B D$ & | Gs228 & |SLT & | 111/08/93| & 270. & $7.5 i$ & 220. & N & & i & i \\
\hline 7 & 74322ABE & | GS228 & ¡SLT & | 11/08/93| & 350. & 7.51 & 220. & M & & i & i \\
\hline 7 & $74332 \mathrm{ABA}$ & GS228 & |SLT & | 11/08/93 & 300. & 7.51 & 220. & N & & & \\
\hline 7 & 74332ABB & | GS228 & |SLT & | 11/08/93| & 300. & 7.51 & 220. & $N$ & & I & i \\
\hline 7 & 74332ABC & | GS228 & ISLT & | 111/08/93| & 300 & 7.51 & 220. & N & & i & \\
\hline 7 & I 74332ABD & GS228 & |SLT & | 11/08/93| & 300. & 7.51 & 220. & N & & I & \\
\hline 7 & I 74332ABE & GS228 & |SLT & | 19/08/93| & 324. & $7.5 i$ & 220. & N & & i & i \\
\hline 9 & I 74342ABA & GS228 & |SLT & $|11 / 12 / 93|$ & 300. & 7.51 & 220. & N & & I & \\
\hline 6 & $74342 \mathrm{ABB}$ & GS228 & |SLT & | 11/08/93| & 300. & 7.51 & 220. & H & & i & i \\
\hline 7 & 74342ABC & GS228 & |SLT & | 11/08/93 & 300. & 7.51 & 220. & N & & i & i \\
\hline 7 & $74342 A B D$ & GS228 & |SLT & | 111/08/93 & 300. & $7.5 i$ & 220. & N & & & \\
\hline 6 & I 74352ABA & GS228 & |SLT & | 11/08/93| & 300. & 7.51 & 220. & $Y$ & $11 / 09 / 93$ & $|11 / 16 / 93|$ & |Pass \\
\hline 6 & 1 74352ABB & GS228 & ISLT & | 11/08/93| & 300. & 7.51 & 220. & N & & $1 \quad 1$ & 1 \\
\hline 7 & 74352ABC & GS228 & |SLT & | 19/08/93 & 300. & $7.5 i$ & 220. & N & & i & i \\
\hline 6 & 74352ABD & GS228 & |SLT & | 91/08/93| & 300. & 7.51 & 220. & N & & 1 & i \\
\hline 9 & I 74352ABE & GS228 & |SLT & $|11 / 12 / 93|$ & 335. & 7.51 & 220. & N & & 1 & i \\
\hline 9 & 74362ABA & GS228 & |SLT & | 11/12/93| & 300. & 7.51 & 220. & N & & i & \\
\hline 9 & 74362ABB & GS228 & |SLT & | 11/12/93| & 300. & 7.51 & 220. & N & & & I \\
\hline 9 & I 74362ABC & GS228 & |SLT & | 19/12/93| & 300. & $7.5 i$ & 220. & H & & i & i \\
\hline 6 & 74362ABD & 65228 & $\mid S L T$ & | 11/08/93| & 300. & 7.51 & 220. & $N$ & & I & i \\
\hline 6 & I 74362ABE & GS228 & |SLT & | $11 / 08 / 93$ | & 334. & 7.51 & 220. & N & & i & i \\
\hline 8 & I $74832 \mathrm{ABA}$ & 65228 & |SLT & i 11/10/93| & 115. & 7.51 & 220. & N & & i & i \\
\hline 9 & I 74832ABB & 65228 & ISLT & $|11 / 12 / 93|$ & 300. & $7.5 i$ & 220. & $N$ & & I & \\
\hline 6 & I 74832ABC & GS228 & |SLT & | 11/08/93| & 300. & 7.51 & 220. & H & & 1 & \\
\hline 9 & I 74832ABD & GS228 & |SLT & | 11/12/93| & 300. & 7.51 & 220. & N & & 1 & \\
\hline 8 & I 74832ABE & 65228 & |SLT & [19/10/93| & 300. & 7.51 & 220. & H & & I & \\
\hline 7 & I 74832ABF & Gs228 & |SLT & | 11/08/93| & 220. & $7.5 i$ & 220. & N & & 1 & 1 \\
\hline 7 & I 74852ABA & 65228 & $\mid S L T$ & | 19/08/93| & 300. & 7.51 & 220. & N & & 1 & \\
\hline 7 & I 74852ABB & 65228 & ISLT & | 11/08/93| & 235. & $7.5 i$ & 220. & N & & I & \\
\hline 81 & | 74862ABA & 65228 & |SLT & | 11/190/93| & 300. & $7.5 i$ & 220. & $\mathbf{N}$ & & i & \\
\hline
\end{tabular}


WHC-SD-W025-RPT-001, Rev. 0

GOLOER CONSTRUCTION SERVICES, INC.

933-9356

GEOCOMPOSITE INVENTORY CONTROL RECORD

05/10/96

DEPARTHENT OF ENERGY

Page: 4

NON-DRAG OFF LANDFILL FACILITY

HANDFORD NUCLEAR RESERVATION

\begin{tabular}{|c|c|c|c|c|c|c|c|c|c|c|c|}
\hline \multirow{4}{*}{$\begin{array}{c}\text { SHIPMENT! } \\
\text { NUKBER }\end{array}$} & \multirow{4}{*}{$\begin{array}{l}\text { ROLL } \\
\text { NUMBER }\end{array}$} & \multirow{4}{*}{$\begin{array}{l}\text { BATCH } \\
\text { NUMBER }\end{array}$} & \multirow{4}{*}{ MANUFACTURER } & \multirow{4}{*}{\begin{tabular}{|}
$\left|\begin{array}{c}\text { DATE } \\
\text { OF } \\
\mid \text { INVENTORY }\end{array}\right|$ \\
$\mid$
\end{tabular}} & \multirow{2}{*}{$1=0===0==$} & \multicolumn{2}{|c|}{ ROLL DIMENTIONS } & \multicolumn{4}{|c|}{ |CONFORHANCE TEST INFORMATION } \\
\hline & & & & & & $=1========01$ & $========01$ & |Tested| & Date I & |Results | & Test \\
\hline & & & & & |Length $(f t)$ | & $\mid$ Hidth $(f t) \mid$ & Thick (mil) & $j(Y / N) \mid$ & Sent & |Received| & $P / F$ \\
\hline & & & & & & $i \quad i$ & $i$ & i & & & (1) \\
\hline & -1 & tent & & & I & & & & & & $=$ \\
\hline 8 & 74862ABB & GS228 & |SLT & | 11/10/93| & 352. & 7.5 & 220. & H & & 1 & REJ \\
\hline 8 & $74872 A B B$ & GS22B & ¡SLT & | 11/10/93| & 300. & 7.5 & 220. & H & & j & REJ \\
\hline 8 & 74872ABC & GS228 & ¡SLT & | $11 / 10 / 93$ | & 300. & 7.5 & 220. & $N$ & & i & 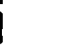 \\
\hline 8 & $74872 A B D$ & GS228 & ןSLT & | 11/10/93| & 215. & 7.5 & 220. & k & & i & 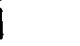 \\
\hline 9 & 74882ABA & G5228 & ¡SLT & | 11/12/93| & 300. & 7.5 & 220. & N & & i & 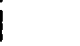 \\
\hline 8 & 74882AB8 & G5228 & |SLT & | 19/10/93| & 62. & 7.5 & 220. & $N$ & & i & 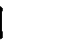 \\
\hline 8 & 74892ABA & GS228 & |SLT & | 11/10/93| & 300. & 7.5 & 220. & N & & i & i \\
\hline 8 & 74892ABB & GS228 & |SLT & | 11/10/93| & 300. & 7.5 & 220. & N & & i & $i$ \\
\hline 8 & 74892ABC & GS228 & |SLT & | 11/10/93| & 300. & 7.5 & 220. & N & & 1 & 1 \\
\hline 8 & 74892ABD & GS228 & ISLT & | 11/10/93| & 371. & 7.5 & 220. & N & & | & REJ \\
\hline 8 & $74902 A B C$ & GS228 & |SLT & | 11/10/93| & 280. & 7.5 & 220. & $N$ & & i & REJ \\
\hline 8 & 74902ABD & GS228 & |SLT & | 19/10/93| & 100. & 7.5 & 220. & $N$ & & i & REJ \\
\hline 8 & 74912ABA & GS228 & |SLT & | 11/10/93| & 300. & 7.5 & 220. & $N$ & & $1 \quad 1$ & REJ \\
\hline 8 & 74912ABB & GS228 & |SLT & | 11/10/93| & 140. & 7.5 & 220. & $\mathbf{Y}$ & $19 / 13 / 93$ & $|11 / 17 / 93|$ & Pass \\
\hline 8 & 74912ABC & GS228 & |SLT & | 11/10/93| & 50. & 7.5 & 220. & N & & i $\quad i$ & 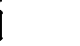 \\
\hline 8 & $74912 A 80$ & GS228 & ISLT & | 11/10/93| & 233. & 7.5 & 220. & $\mathbf{N}$ & & i & 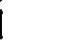 \\
\hline 9 & 74922АBA & G5228 & |SLT & | 11/12/93| & 300. & 7.5 & 220. & N & & l & 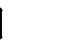 \\
\hline 9 & $74922 A B B$ & 65228 & ISLT & | 19/12/93| & 304. & 7.5 & 220. & N & & ] & 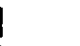 \\
\hline 11 & T778ZABA & GS228 & |SLT & | 01/12/94| & 300 & 7.5 & 220. & $N$ & & 1 & 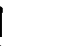 \\
\hline 11 & T7782ABB & GS228 & |SLT & | 01/12/94| & 210. & 7.5 & 220. & N & & i & 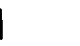 \\
\hline 11 & $77782 A B C$ & GS228 & |SLT & | 01/12/94| & 93. & 7.5 & 220. & $\mathbf{N}$ & & i & $i$ \\
\hline 11 & 80082ABA & 65228 & |SLT & | 01/12/94| & 300. & 7.5 & 220. & $N$ & & 1 & 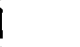 \\
\hline 11 & $80082 A B B$ & GS228 & |SLT & | 01/12/94| & 197. & 7.5 & 220. & $N$ & & i & i \\
\hline 11 & $80082 A B C$ & GS228 & |SLT & | 01/12/94| & 300. & 7.5 & 220. & $N$ & & i & 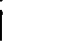 \\
\hline 11 & $80082 A B D$ & GS228 & |SLT & | 01/12/94| & 300. & 7.5 & 220. & $Y$ & $01 / 12 / 94 \mid$ & $|01 / 18 / 94|$ & Pass \\
\hline 11 & BOOBZABE & GS228 & |SLT & | 01/12/94| & 300. & 7.5 & 220. & N & & i $\quad i$ & 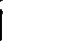 \\
\hline 11 & 80082ABF & GS228 & |SLT & | 01/12/94| & 127. & 7.5 & 220. & $N$ & & i & 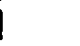 \\
\hline 19 & 80132ABA & GS228 & |SLT & | 01/12/94| & 285. & 7.5 & 220. & N & & i & 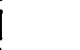 \\
\hline 11 & 80132ABB & GS228 & |SLT & | 01/12/94| & 379. & 7.5 & 220. & $N$ & & 1 & 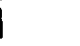 \\
\hline 11 & $82572 A B A$ & GS228 & |SLT & | 01/12/94| & 289. & 7.5 & 220. & $n$ & & I & 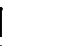 \\
\hline 11 & $82822 A B$ & GS228 & |SLT & $|01 / 12 / 94|$ & 300. & 7.5 & 220. & N & & i & 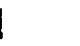 \\
\hline 19 & 83532AB & GS228 & isLT & | 01/12/94| & 192. & 7.5 & 220. & $N$ & & i & 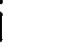 \\
\hline 11 & $83632 A B$ & GS228 & |SLT & | 01/12/94| & 283. & 7.5 & 220. & N & & 1 & I \\
\hline 11 & $83652 A B$ & GS228 & |SLT & | 01/12/94| & 290. & 7.5 & 220. & $\mathbf{N}$ & & I & 1 \\
\hline 11 & 83972AB & GS228 & |SLT & | 01/12/94| & 287. & $7.5 i$ & 220. & N & & I & 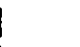 \\
\hline 11 & $83982 A B$ & GS228 & |SLT & | 01/12/94| & 344. & 7.51 & 220. & H & & 1 & 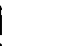 \\
\hline 97 & 83982ABB & GS228 & |SLT & | 01/12/94| & 300. & 7.51 & 220. & N & & 1 & I \\
\hline 11 & 83982ABD & GS228 & jSLT & | 01/12/94| & 400 & $7.5 i$ & 220. & $N$ & & 1 & \\
\hline
\end{tabular}


WHC-SD-W025-RPT-001, Rev. 0

Square Feet Delivered $=355686.9$

NOTES

$=$

(1) TEST P/F note of "REJ" indicates a roll of material which was rejected and removed from the site because the Manufacturer's QC test data indicated that the material did not meet the manufacturer's specifications. 
WHC-SD-W025-RPT-001, Rev. 0

NOVEMBER 1003

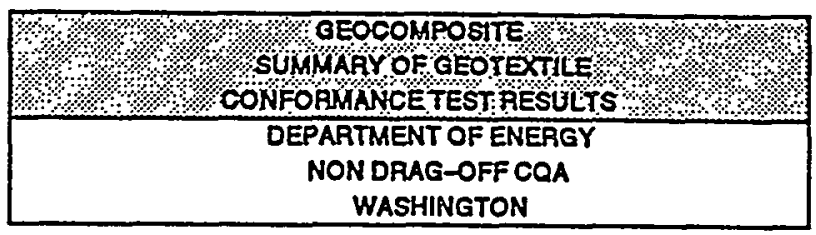

033-1354 CONFORMANCETESTRESUTS
OEPARTMENT OF ENERGY
NON DAAG-OFF CQA WASHINGTON

\begin{tabular}{|c|c|c|c|c|c|c|c|c|c|c|c|c|}
\hline $\begin{array}{l}\text { HOLI } \\
\text { DESIGNATION }\end{array}$ & \%8448 & Bosto & $\begin{array}{l}72 \% \text {. } \\
70 \% \\
\text { Top }\end{array}$ & 80, & $\begin{array}{l}74328 \\
\text { Top }\end{array}$ & $\begin{array}{l}74326 \\
\text { Botiom }\end{array}$ & 8435 & $\begin{array}{l}\%, 7435 \% \\
\text { Botroms }\end{array}$ & $\begin{array}{l}73838 \\
\text { Top }\end{array}$ & $\begin{array}{l}73838 \\
\text { Battom }\end{array}$ & $\begin{array}{r}74018 \\
\text { Top }\end{array}$ & $\begin{array}{l}\text { } 7491 \mathrm{a} \\
\text { Bottom }\end{array}$ \\
\hline
\end{tabular}


WHC-SD-W025-RPT-001, Rev. 0

JANUARY 1094

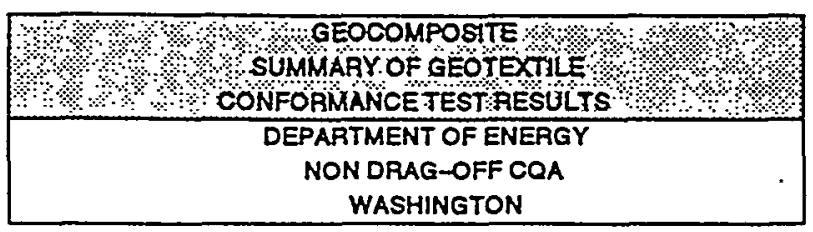

933-1354

ON DRAG-OFF COA

WASHINGTON

\begin{tabular}{|c|c|c|c|c|c|c|c|c|c|c|c|c|}
\hline $\begin{array}{l}\text { ROLI } \\
\text { DESIGNATION }\end{array}$ & Top: & $\begin{array}{l}\text { } 5164 \\
\text { Botion }\end{array}$ & $\begin{array}{l}80 \\
6008 \\
\text { Top }\end{array}$ & $\begin{array}{l}8 \% \\
800 \text { : } \\
\text { Bottom }\end{array}$ & & 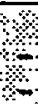 & & 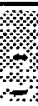 & 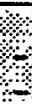 & & $\%$ & at: \\
\hline \multicolumn{13}{|l|}{ APPARENT } \\
\hline $\begin{array}{l}\text { OPENING SIZE } \\
(\mathrm{mm})\end{array}$ & 0.108 & 0.117 & 0.117 & 0.108 & - & - & - & - & - & - & - & - \\
\hline (U.S. SIEVE NO.) & 140 & 120 & 120 & 140 & - & - & - & - & - & - & - & - \\
\hline ASTM D 4751 & & & & & & & & & & & & \\
\hline
\end{tabular}




\section{GEOTEXTILE TEST RESULTS}

PROJECT NAME: DOEINDO CQAWA:

PROJECT NUMBER: 933-1354.

ROLL DESIGNATION: S-700

APPARENT

OPENING

SIZE

(mm)

1. 0.150

2. 0.150

3. 0.125

4. 0.150

5. 0.125

AVG 0.140

EQUIVALENT

SIEVE SIZE

100

ROLL DESIGNATION:

APPARENT

OPENING

SIZE

$(\mathrm{mm})$

1. -

2. -

$3 . \quad-$

$4 . \quad-$

5. -

AVG $\quad 0.000$

EQUIVALENT

SIEVE SIZE

$-$ 
WHC-SD-W025-RPT-001, Rev. 0

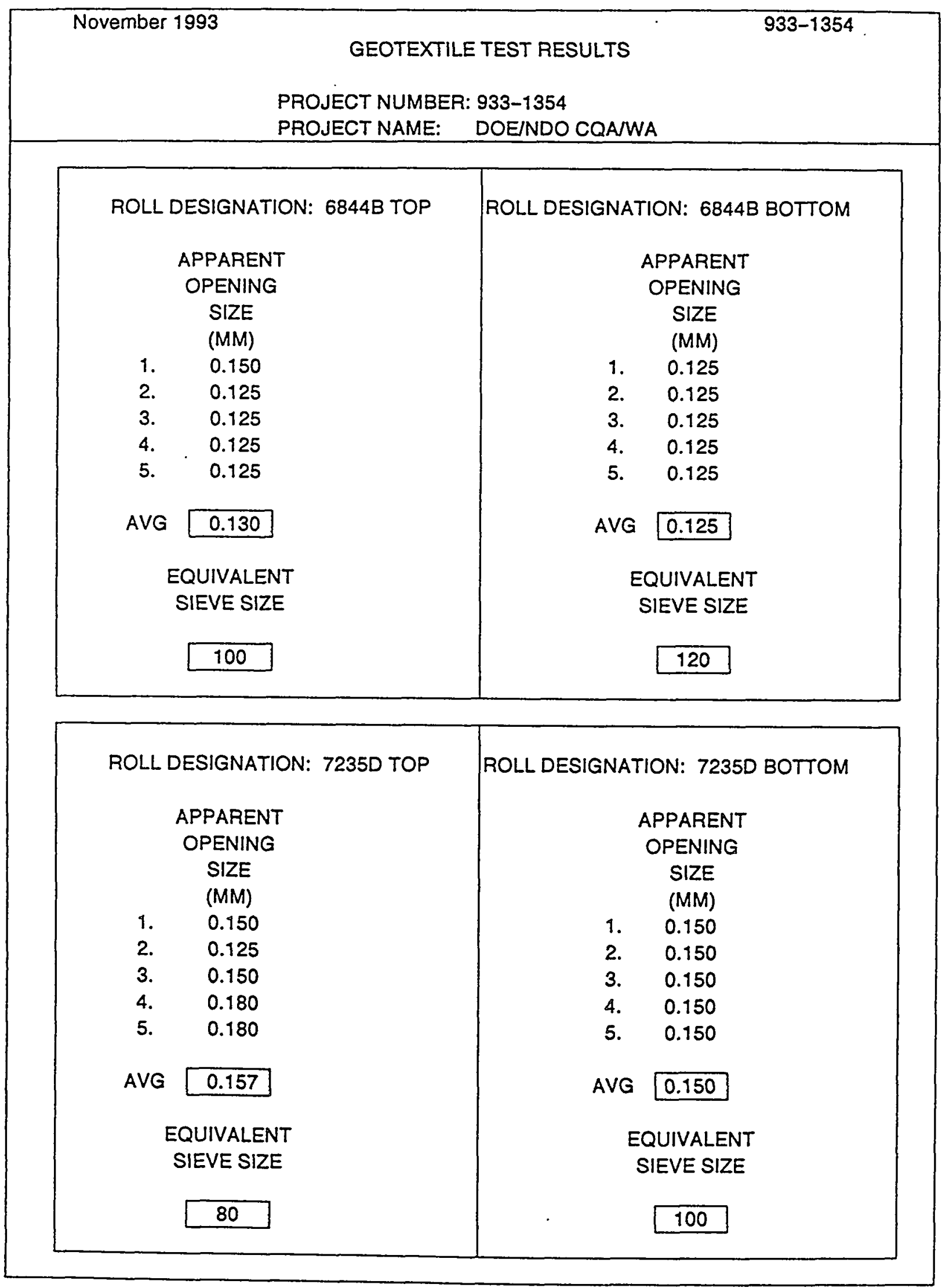


WHC-SD-W025-RPT-001, Rev. 0

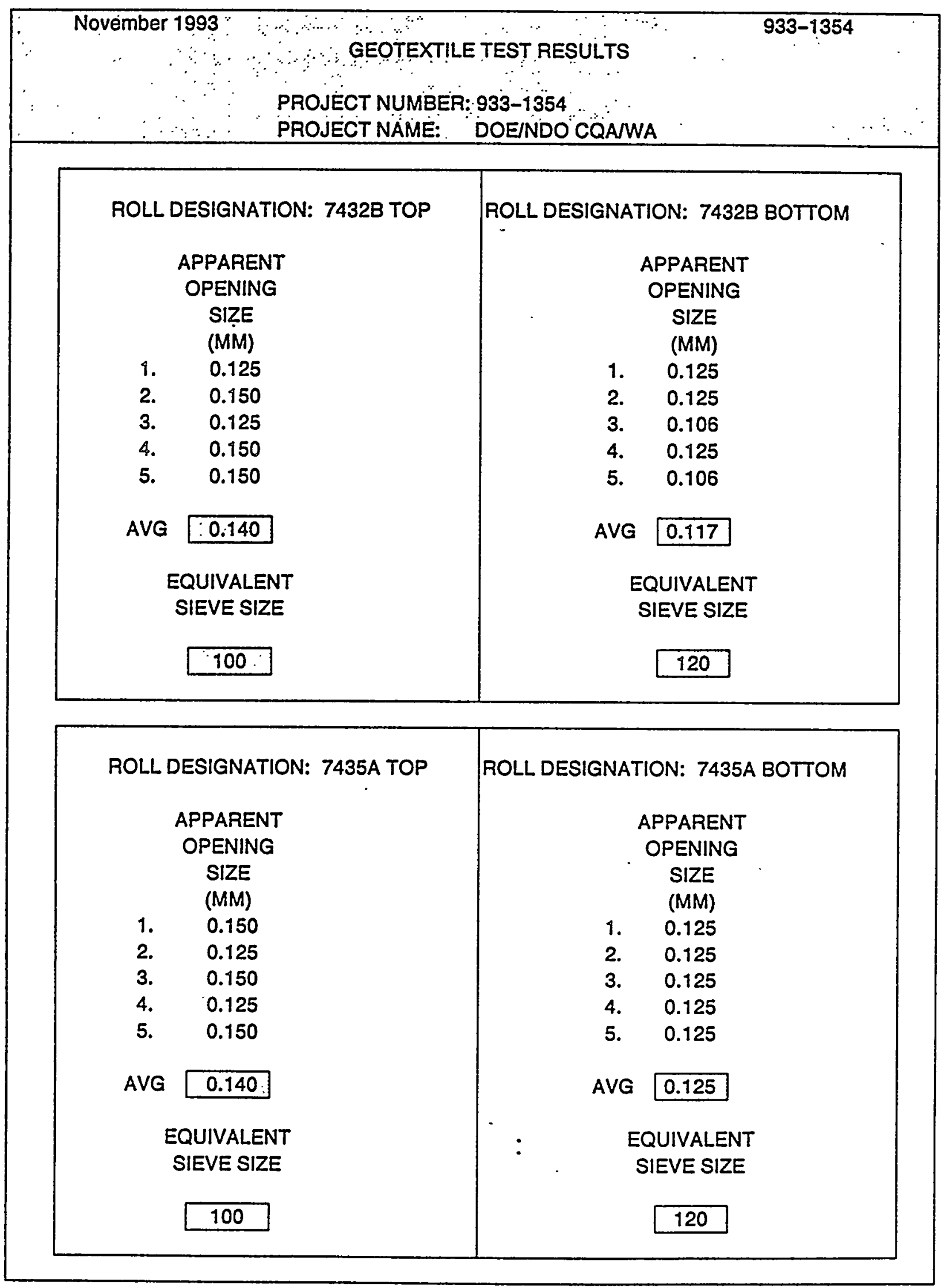


WHC-SD-W025-RPT-001, Rev. 0

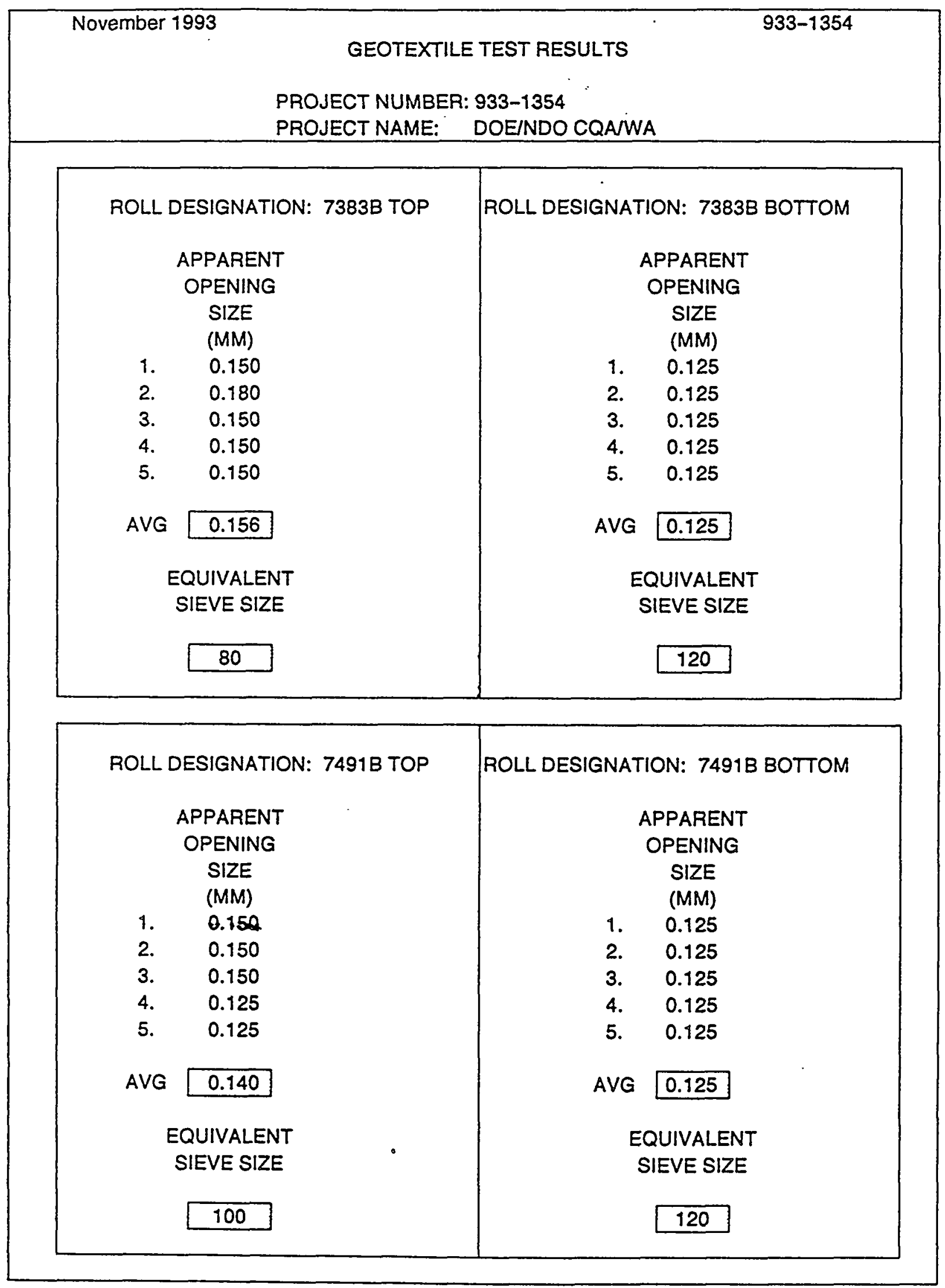


WHC-SD-W025-RPT-001, Rev. 0

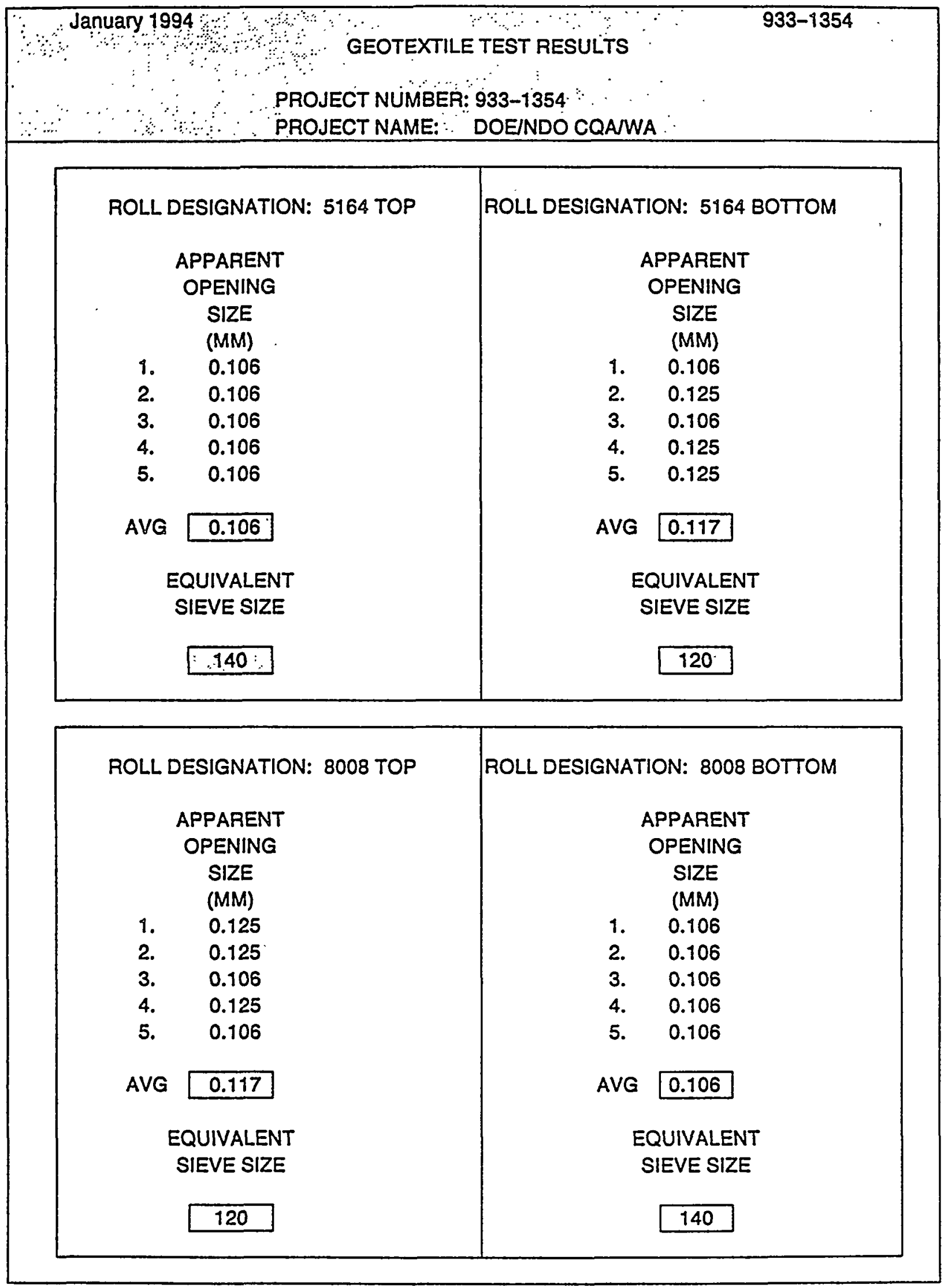


WHC-SD-W025-RPT-001, Rev. 0

7017 Farrell Road S.E.

Calgaxy, Alberta, T2H-0T3

Telephone: $1-(403)-259-4280$

Pacsimile: $1-(403)-253-1715$

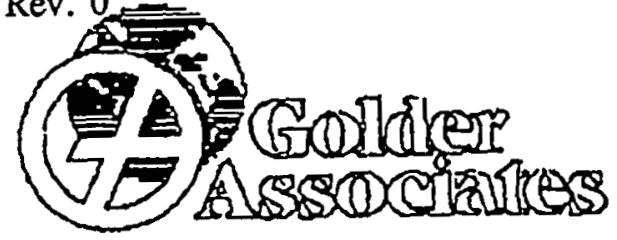

RAX TRANSHITIRE

November 16, 1993Ref. Ho. 932-2610

TorCliff Knitter fax no. I (206) 882-5498

Fr:Gord Guest / Deve Horsfleld / Thanh Iam

RE: TEST RESULTS TO DATE

\section{MESSAGE :}

Atteched please Eisd the results of the geocomposite roll 74310 textured HDPE roll 6916 and geccomposite roll $7430 \mathrm{C}$ - textured HDPE ro11 6898 interface friction tests completed to date. Detalled stress - deformation plots for each of the groups of 3 tests is provided along with peak and residual shear strength envelope Bummery.

In each of the serles the test results are in good agreement with one-another. All tests showed an initial paak in the shear stresB, occurring at within 0.4 inches of displacement. post peak softening was repid with all three results in each series tending toward a stable large strain (or regidual) shear strength beyond horizontal displacemento of 1.5 inches. The paek and resldual shoar strength envelopes are defined as follows!

\section{geocomposite $7430 \mathrm{C} / \mathrm{HDPE} 6898$}

- peak friction angle $40^{\circ}$, cohesion $0.6 \mathrm{psi}$

- residual friction angle $18^{\circ}$, cohesion $0.6 \mathrm{psi}$

geocomposite 74310 / HDPB 6916

- peak Eriction angle $34^{\circ}$, cohesion 1.2 psi

- residual friction angle $18^{\circ}$, cohesion $0.7 \mathrm{psi}$

We have completed both of the soil - geocomposites seriss of tests and expect to have the reaults to you this afternoon.

If you have any questions or further requirements please give us a call.

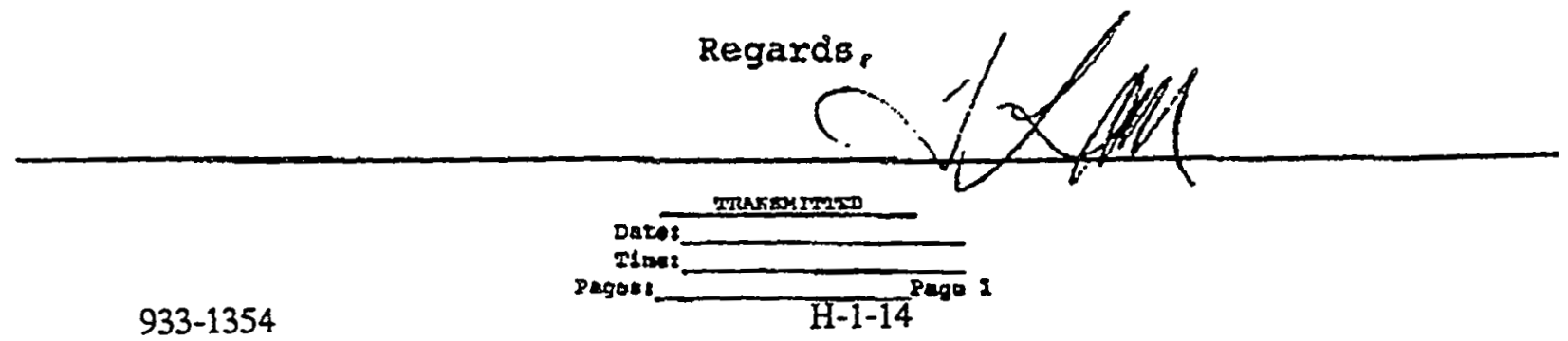


WHC-SD-W025-RPT-001, Rev: 0

GEOCOMPOSITE ROLL 7431D/

TEXTURED HDPE ROLL 6916

Figure

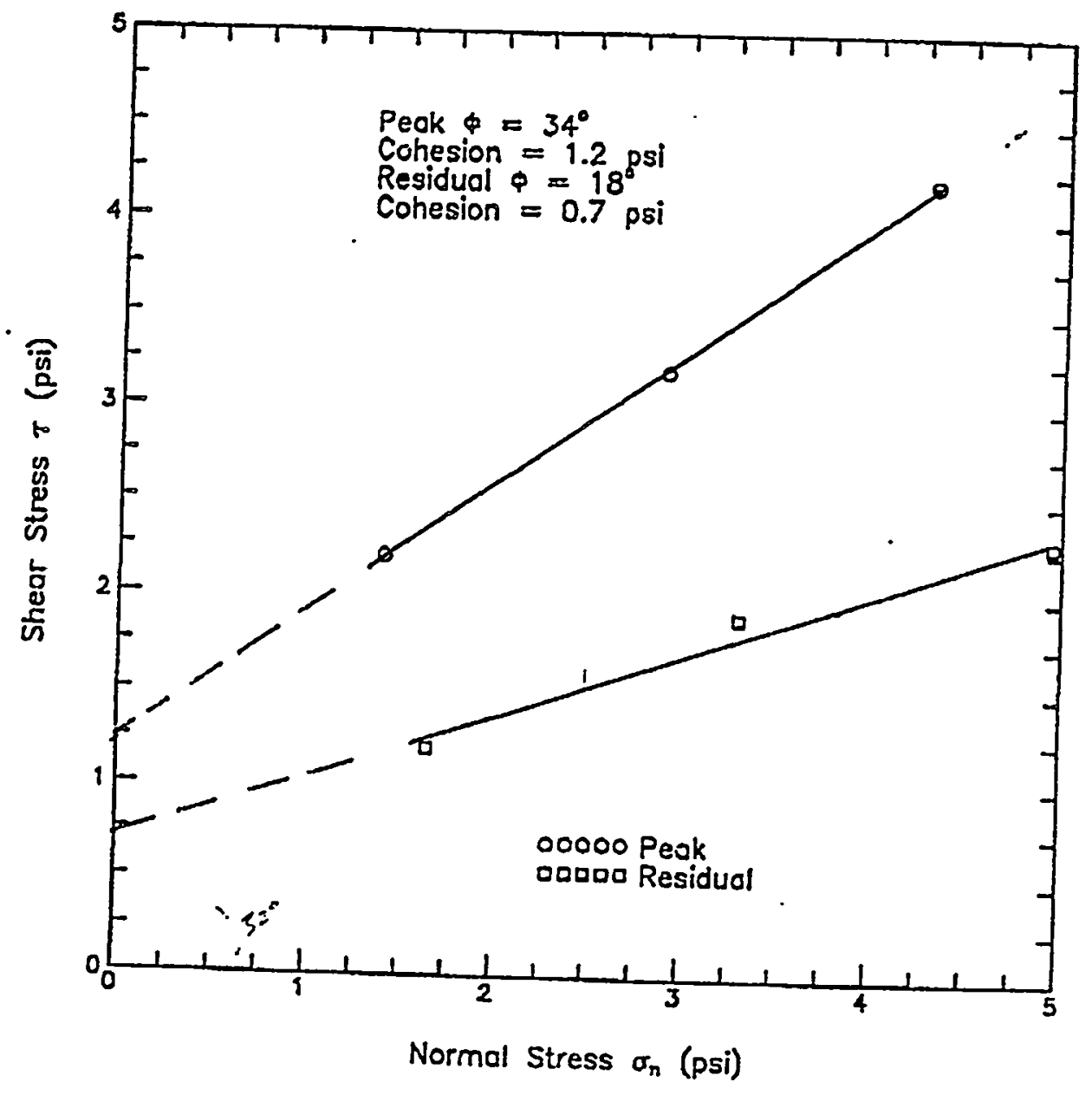

Golder Assoclates 
WHC-SD-W025-RPT-001, Rev. 0

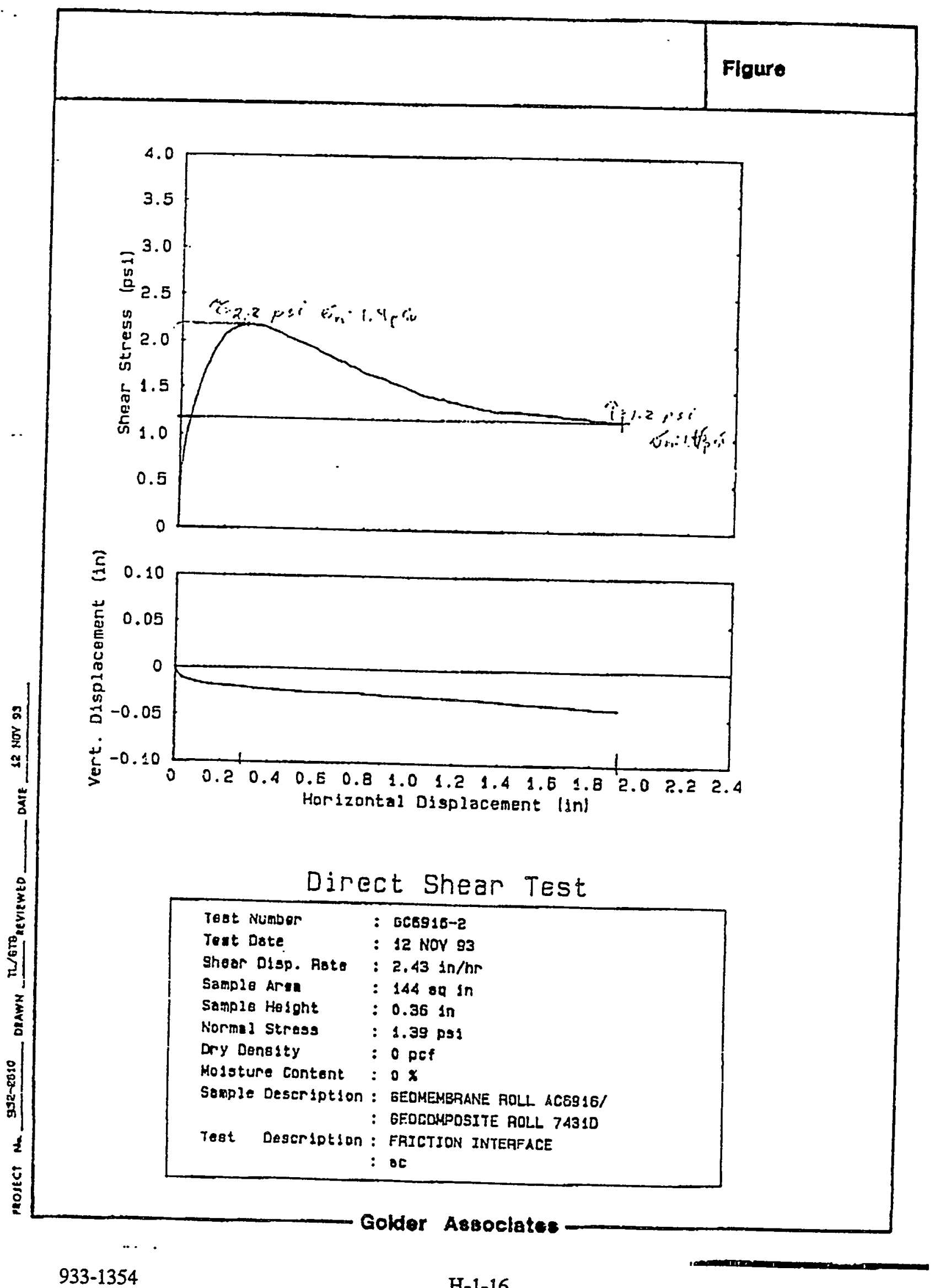




\section{Figure}
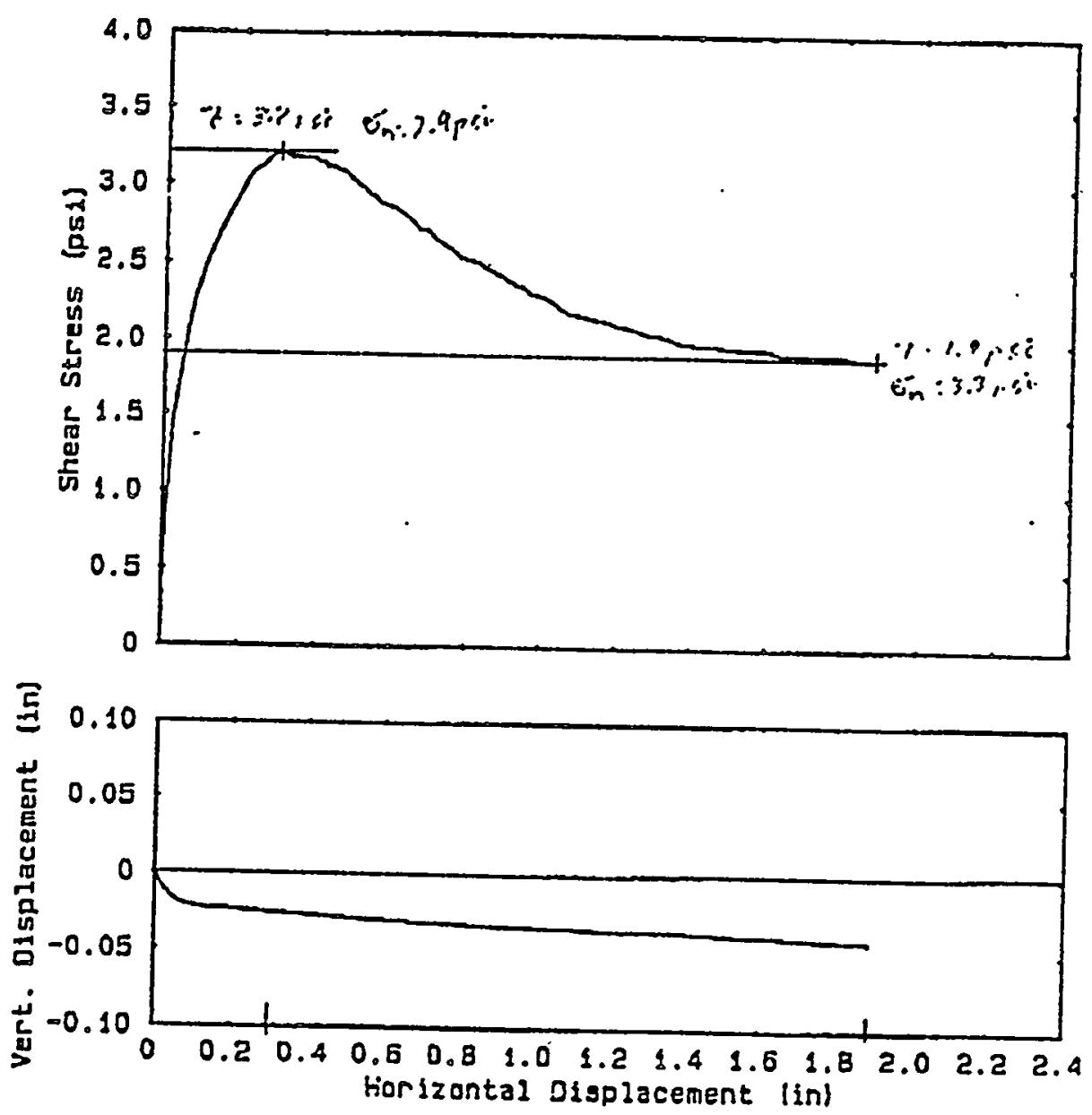

\section{Direct Shear Test}

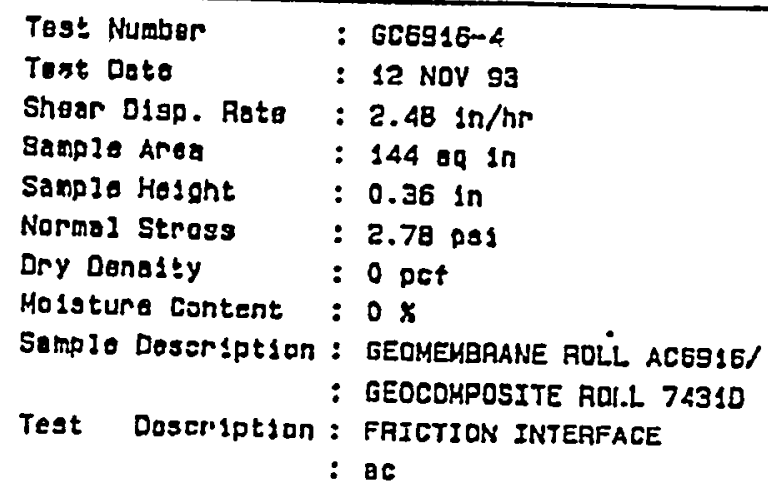

\section{Golder Assoclates}


WHC-SD-W025-RPT-001, Rev. 0

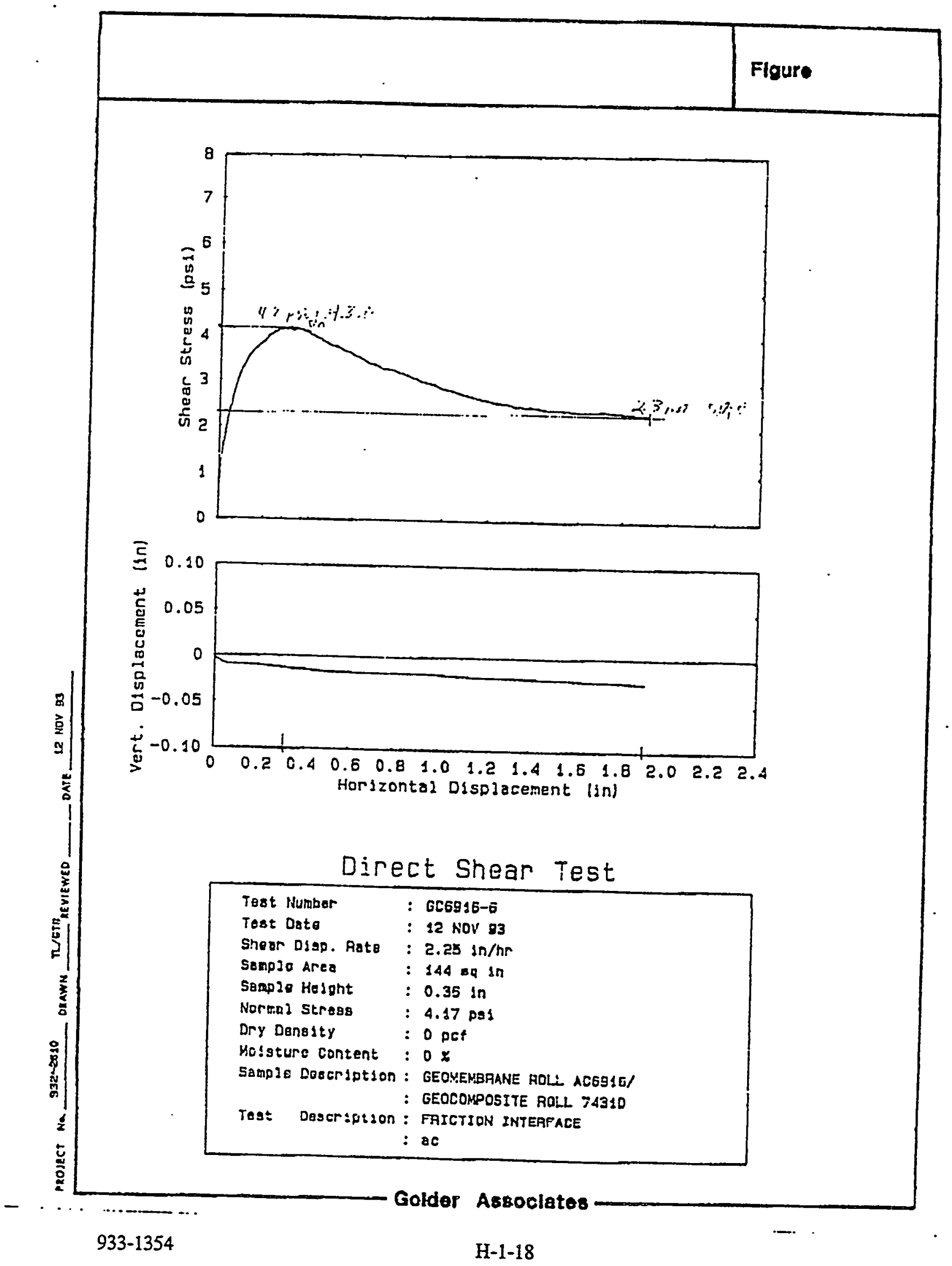


WHC-SD-W025-RPT-001, Rev. 0

\section{GEOCOMPOSITE ROLL 7430C/}

TEXTURED HDPE ROLL 6898

Figure

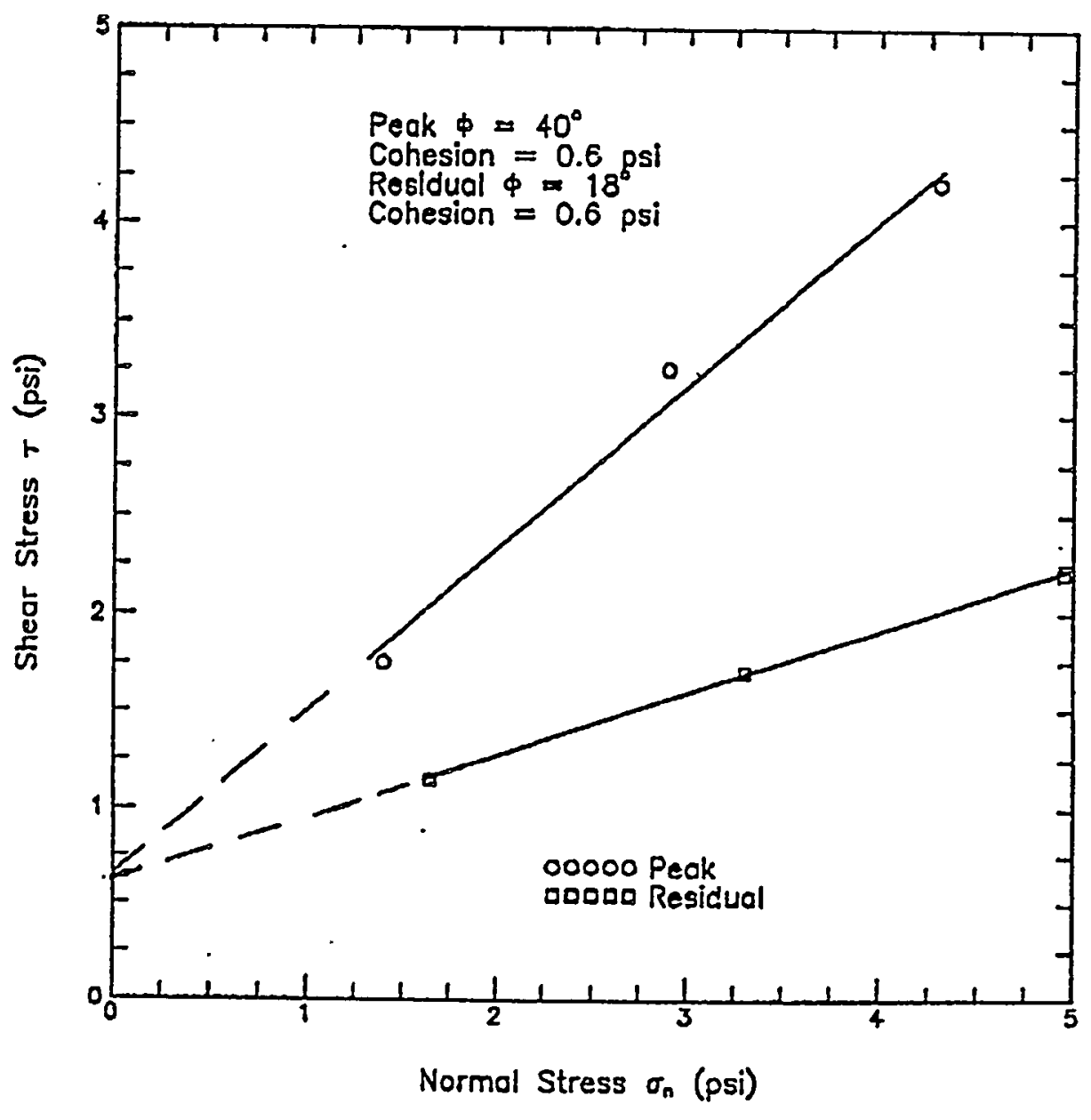

Golder Assoclates 
WHC-SD-W025-RPT-001, Rev. 0

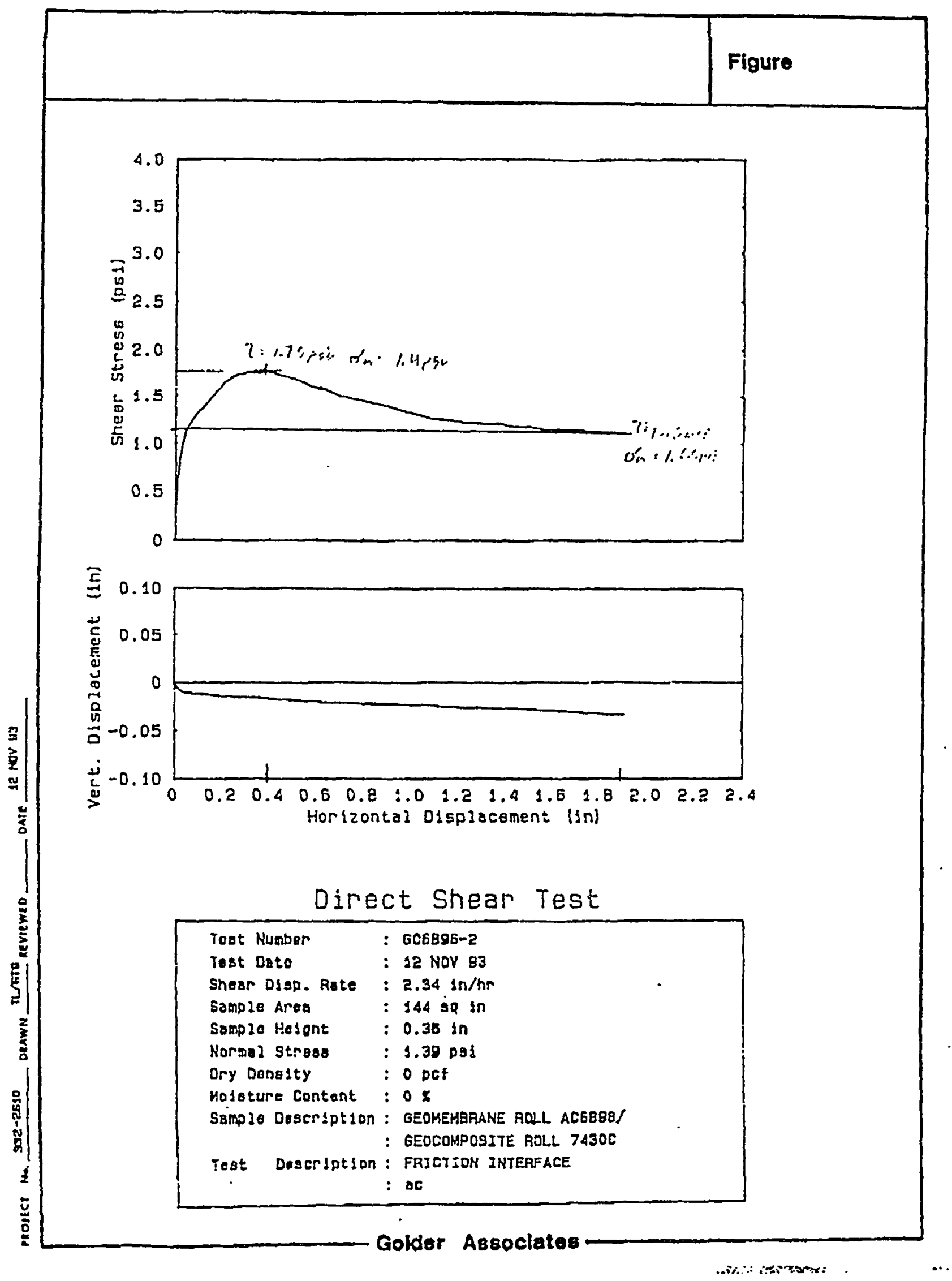


WHC-SD-W025-RPT-001, Rev. 0

FIgure

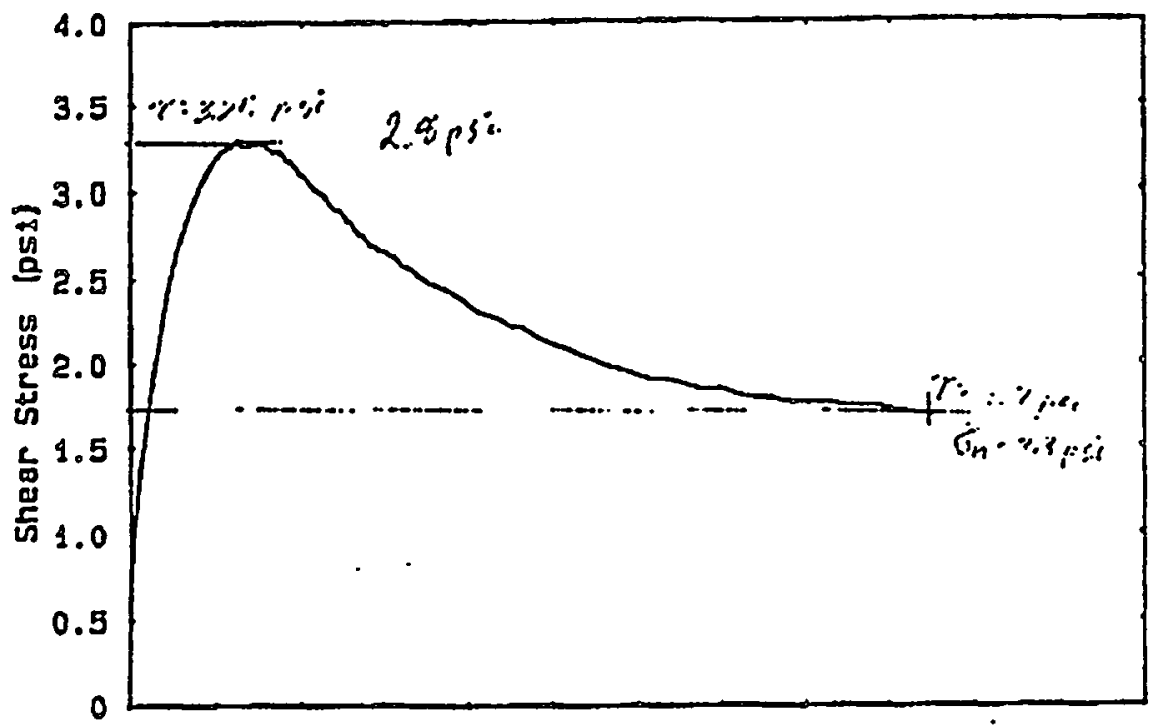

$\bar{E}$

0.10

荡 0.05

Direct Shear Test

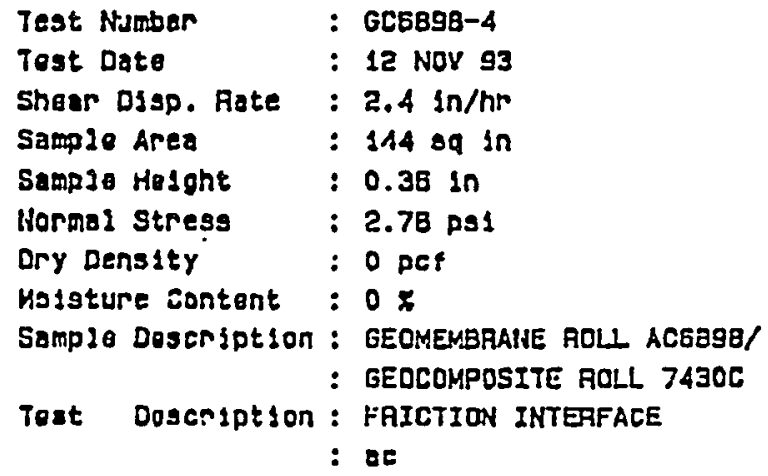

\section{Golder Assoclates}


WHC-SD-W025-RPT-001, Rev. 0

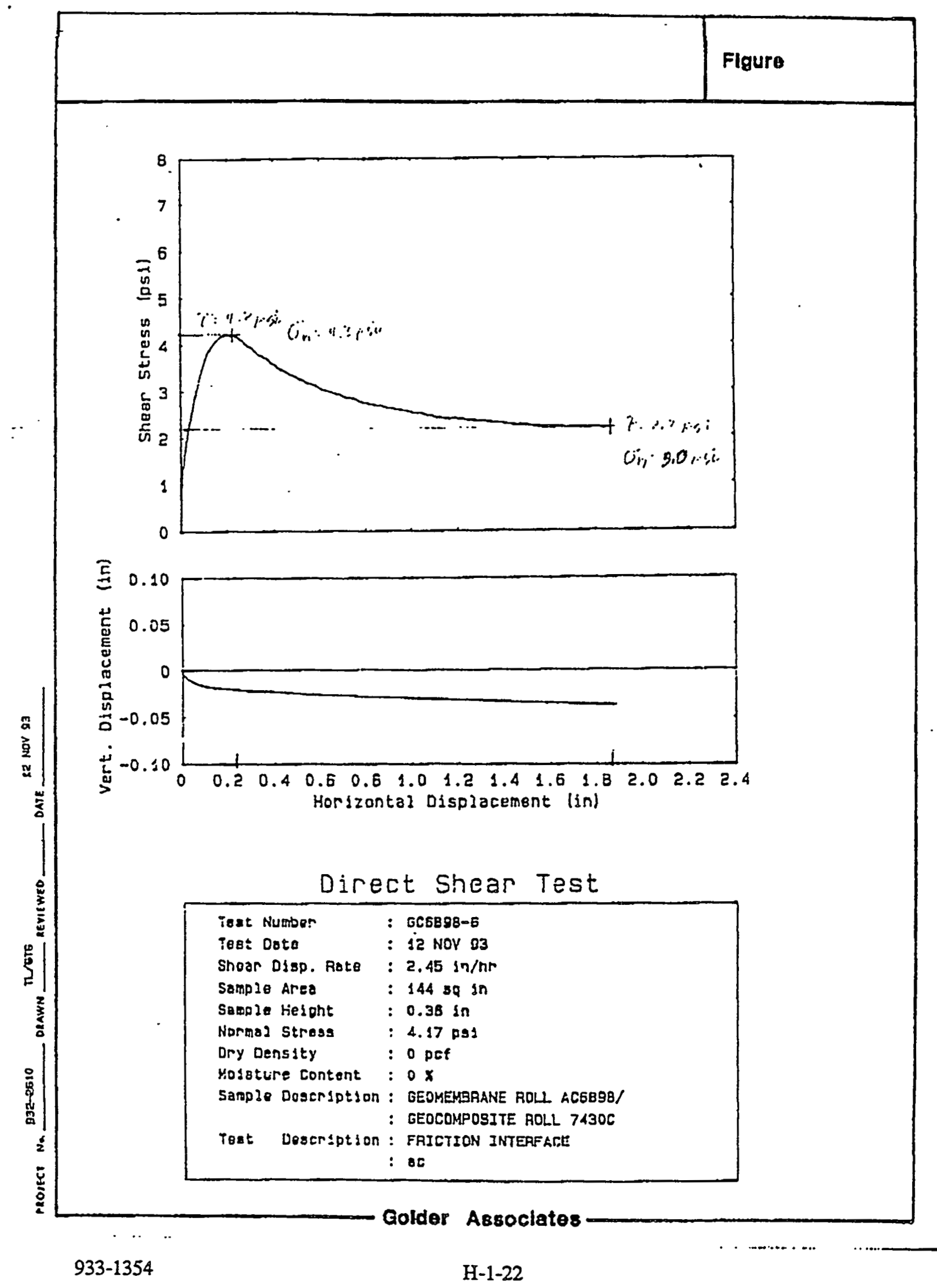


WHC-SD-W025-RPT-001, Rev. 0

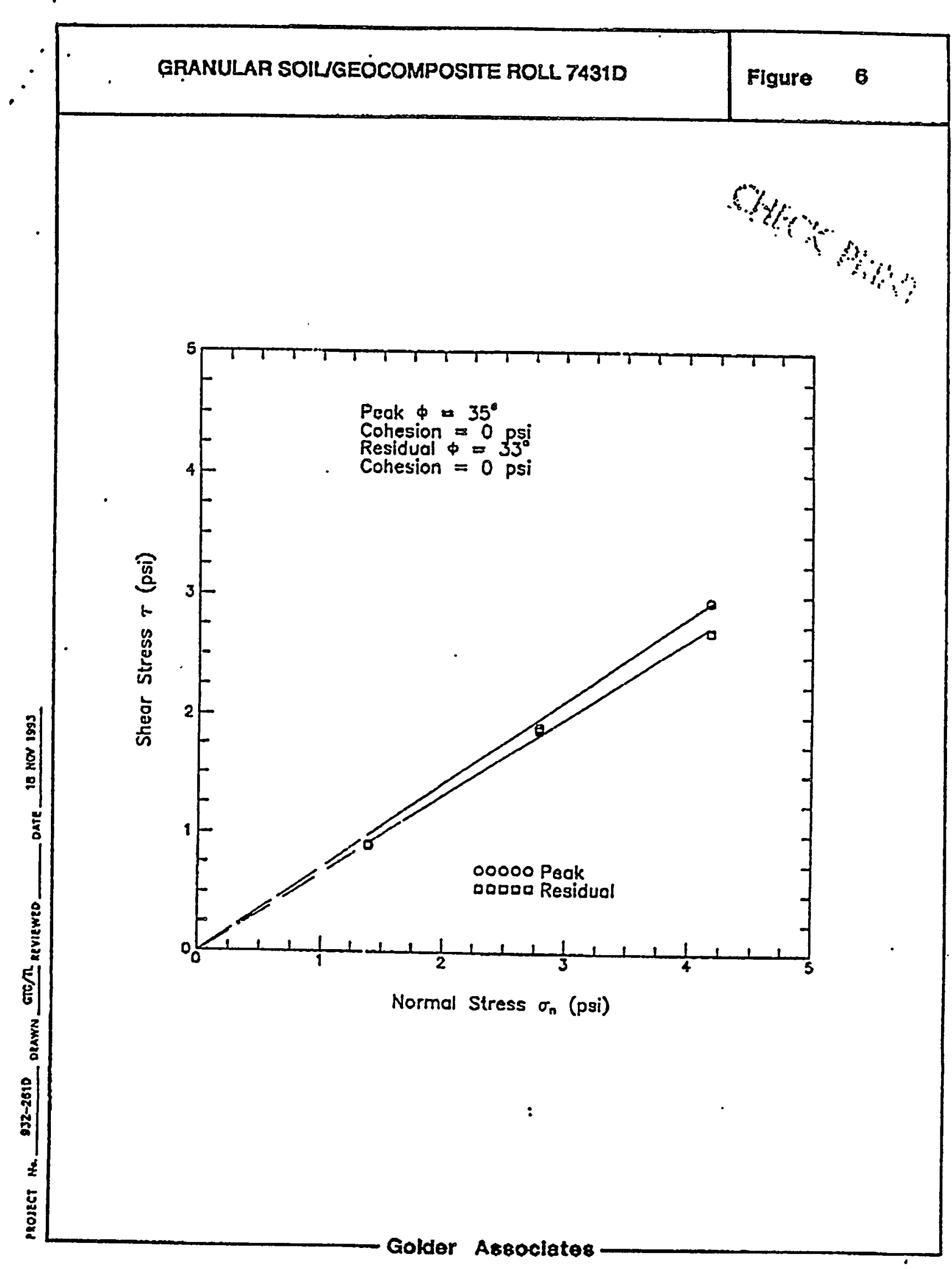

H-1-23 
WHC-SD-W025-RPT-001, Rev. 0

\section{Plouro}

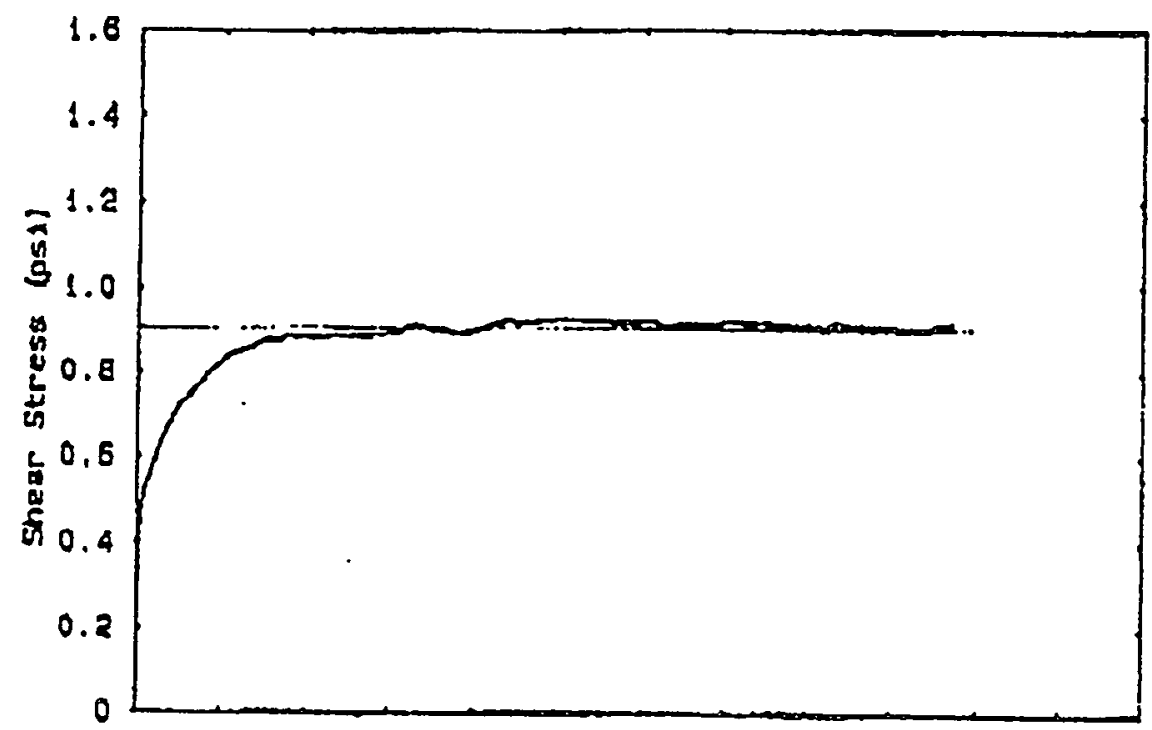

ఏ

0.10

范 0.05

造 0

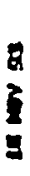

0

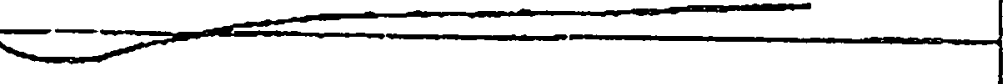

$-0.05$

$\dot{y}_{2}^{\prime}-0.10$

$\begin{array}{llllllllllllllll}0 & 0.2 & 0.4 & 0.0 & 0.8 & 1.0 & 1.2 & 1.4 & 1.6 & 1.8 & 2.0 & 2.2 & 2.4\end{array}$ Horlzonta) D!splacement (In)

Direct Shear. Test

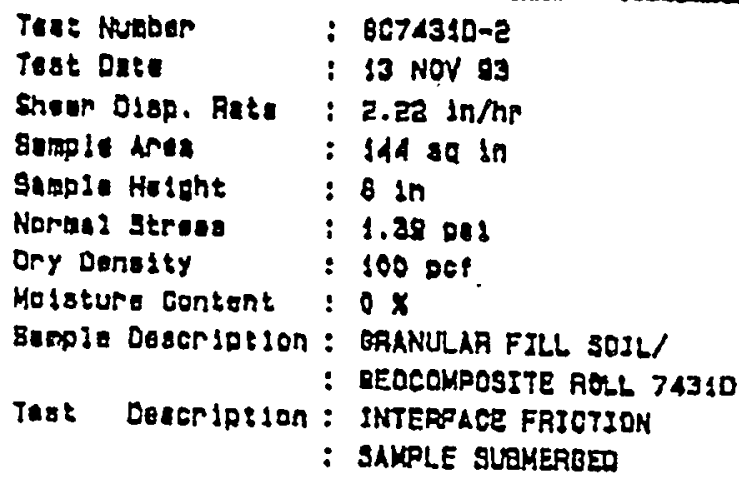


WHC-SD-W025-RPT-001, Rev. 0
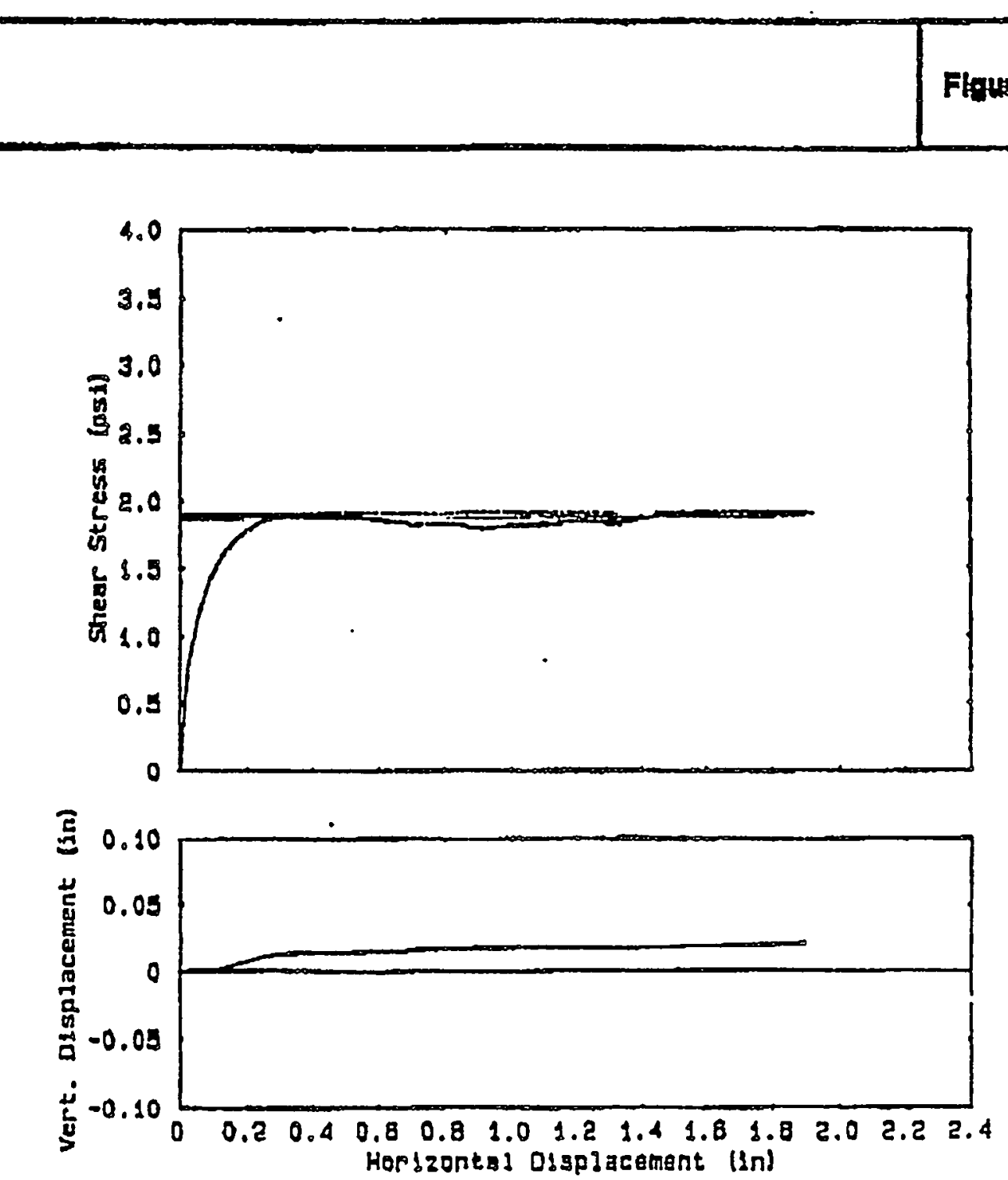

Direct Shear Test

\begin{tabular}{|c|c|c|c|}
\hline 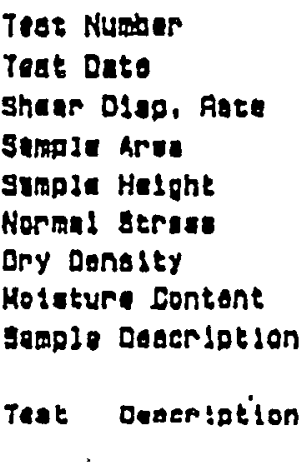 & $\begin{array}{l}\text { : } \\
: \\
: \\
: \\
: \\
: \\
: \\
: \\
:\end{array}$ & 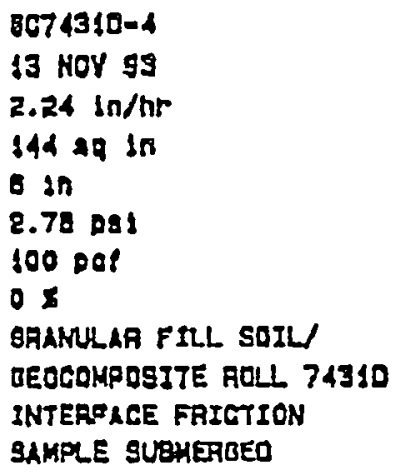 & c \\
\hline
\end{tabular}

\section{Golder Assoclates}


WHC-SD-W025-RPT-001, Rev. 0

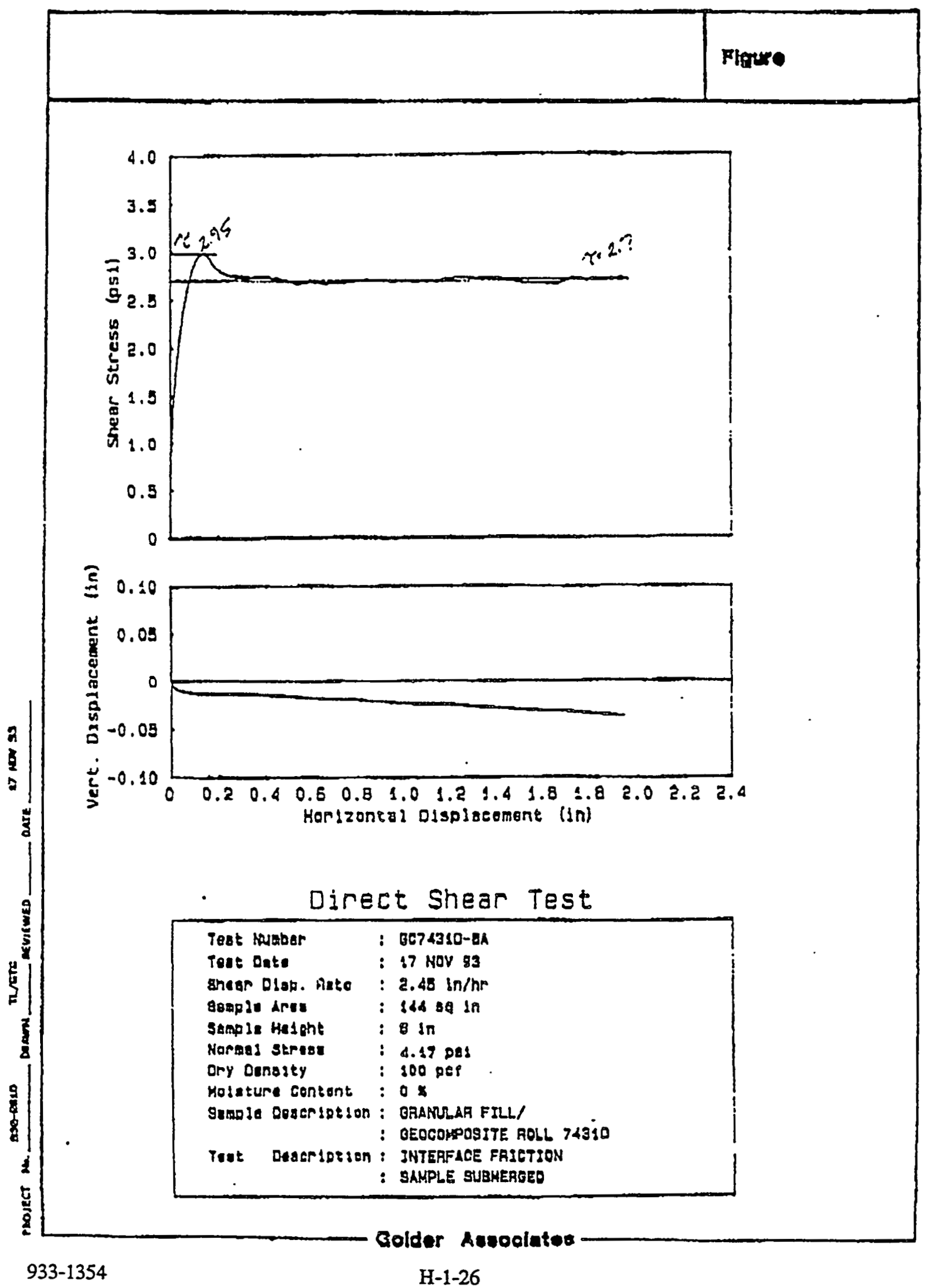


WHC-SD-W025-RPT-001, Rev. 0

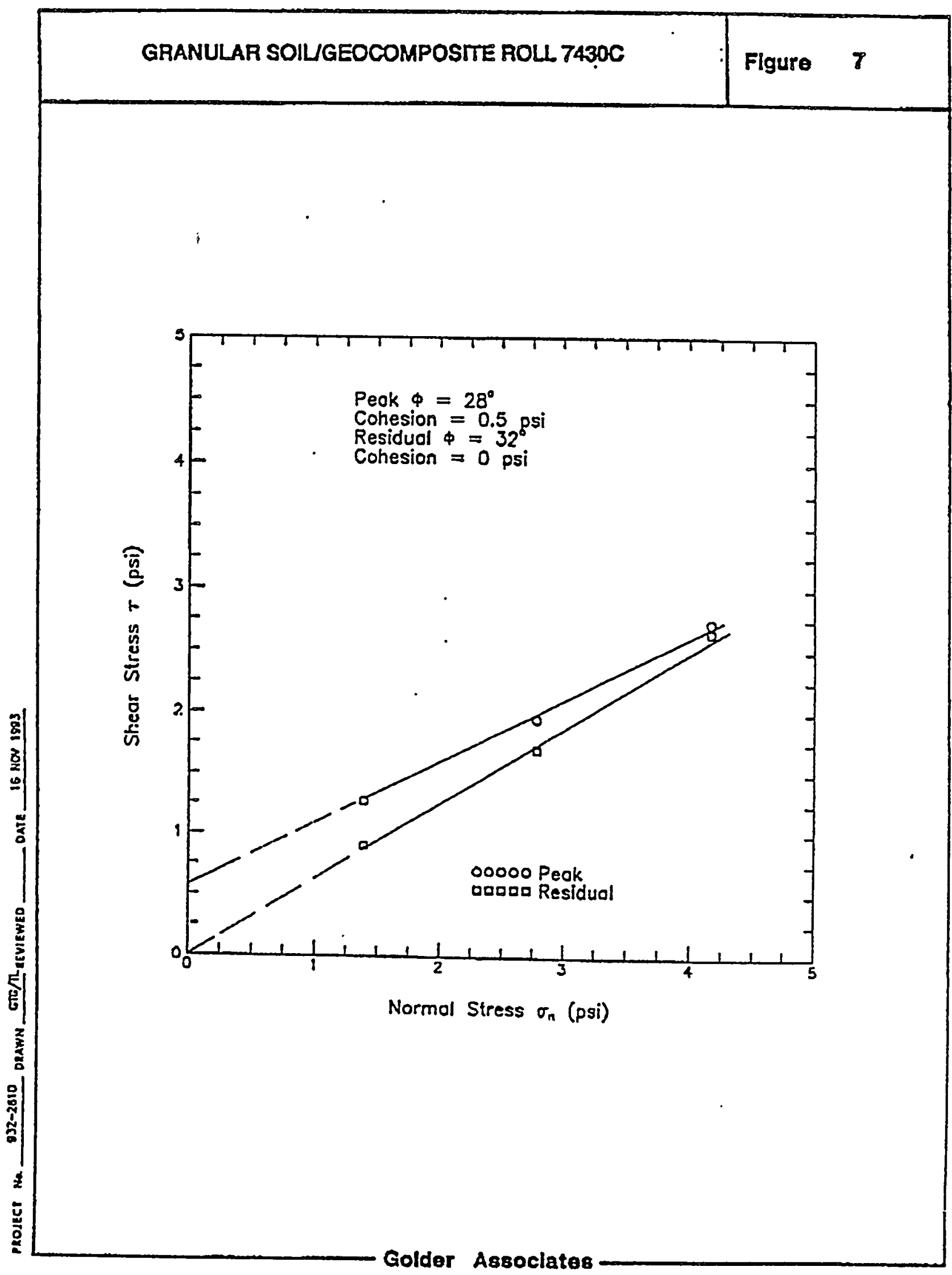


WHC-SD-H025-RPT-001, Rev. 0

\section{APPENDIX H-2 \\ MANUFACTURER'S QC DOCUMENTATION}

$h-2$ 


\title{
.
}

\author{
Doe Franford \\ Radioactive Waste WHCS-045 Rer 2 \\ Specification Review \\ Section 02275 \\ Geosynthetic Transrnission Media
}

B. Specific Geosynthetic Transmission Media Properties

1. SITS GS-228 will meet or exceed the propertics shown on pp. 33.

2. Type A Fabric, Nicolon S.700 has been approved.

3. The individual product properties which make up the gecompositc will meet the values specitied or previoushy approped as separate products.

4. SLT will provided material for direct shear testing. However, a frictional value of $275^{\circ}$ cannot be certified without the tests being performed.

I\% \& 6. SIT will provide test results and or certification to the specified or previousily approved materials.

7. Yes.

All other items, as spelled out in this section, as related to the geonet, geotextle and geocomposite are acceptable.

The properties of the net as a single product and the properties of the fabric as a single profuct make up the geccomposite. The only additional property used for geacomposite performance is Ply Adhesion. The SLT 7 oz composite will exhibit a Fly Adhesion in accordance with ASTM F904, 2" $\times 8^{n}$ @ 2 ipm of 1.0 poundsinci average.

For environmental lining solutions...the world comes to SLT." 

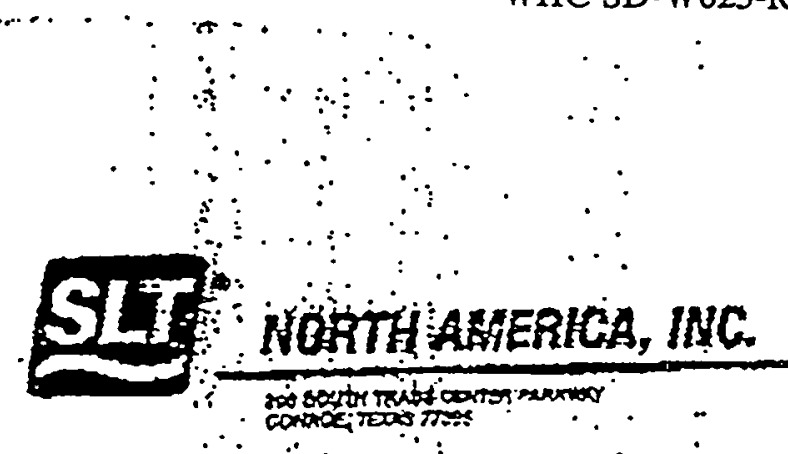

Decout $6,1993: \therefore$

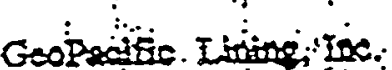

1300 Dejer Ave. It: 250 .

Serthi सiosi 98160

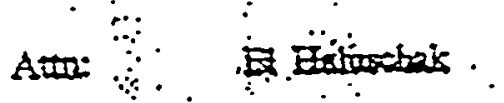

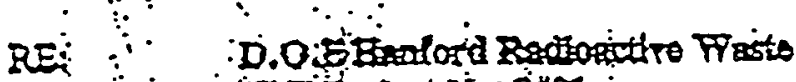

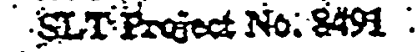

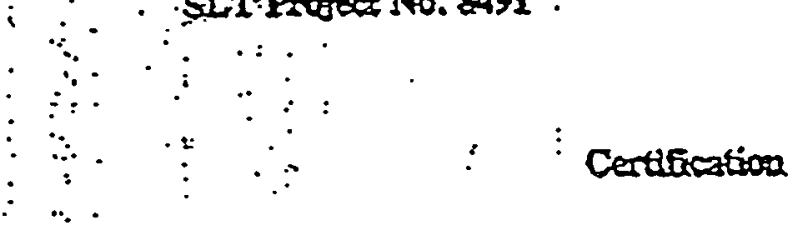

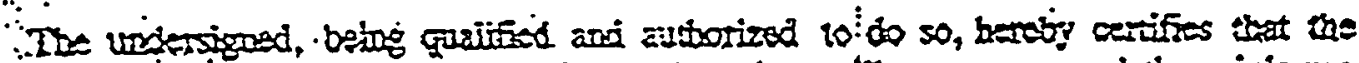

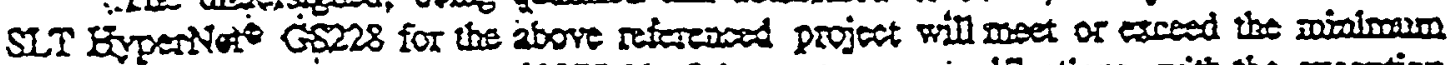

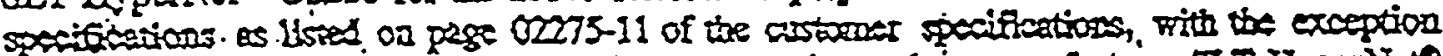

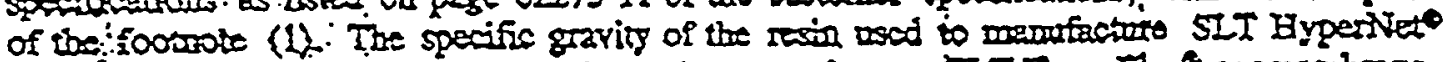

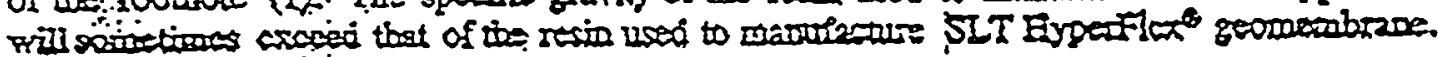

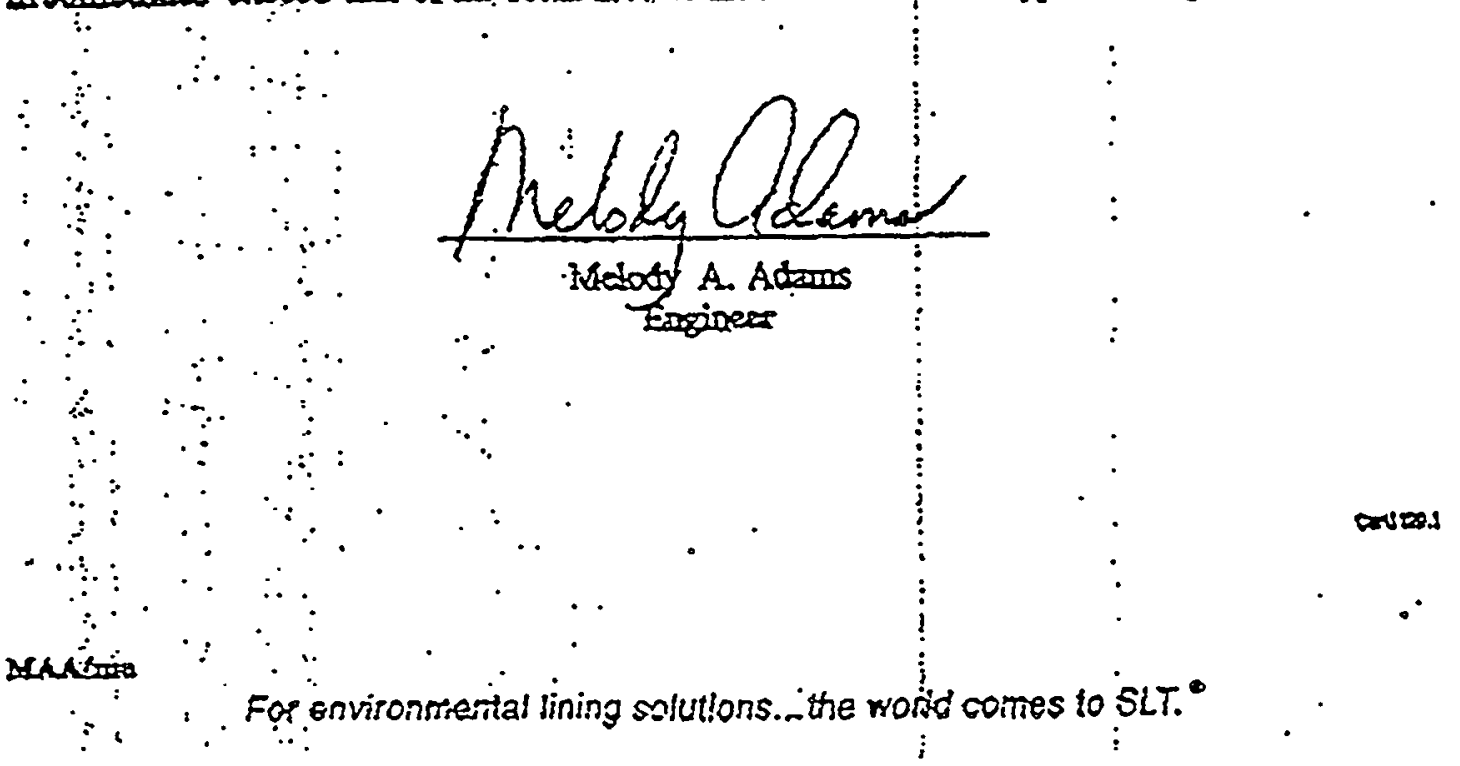




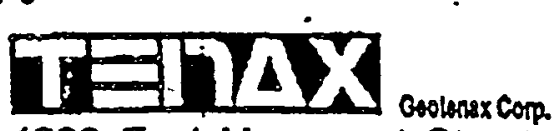

4800 East Monument Streot Baltimore, Maryland 21205

Ofiro: (110) 522.7000 order line: (800) 3558495

Far: (110) 522.7015 Wasta Hot (200) US-GRDOS

Noventher 22, 1993

Mc. Tony Sample

Delliur Industrics, Inc.

4333 Tum Access Rd.

Port Angeles, WA 98362

RE: Hanfurd / TENAX CE.2 Gconet

Dear Mr. Samplc:

This letter is to address the discrepancies between the results of the confornunce tests performed by the quulity assurance consultant for the above referenced project and product and the results provided from our internal quality control program.

It seems that the major concem is that the test results from our internal $Q C$ program indicate a specific gravity of 0.946 while the $Q A$ consultant's results indicate a specific gravity of 0.940 and 0.941 . Since there are many possible causcs for variations in a propenty like specific gravity, it is impossible to determine exactly what caused the discrepancies seen bere. However, the most probable causes are interlaboratory precision and the accuracy of the strudned test method. Considering that there are many possible cruses of variation, it is not unusual to see the small variation seen here $(0.006$ and 0.005$)$ between swo independeal laboralorics.

Additionally, there seems to be some concem abrout the CE-2 geonet's specific gravity relative to the geonrenabrane's specific gravity. Since two different testing laboratorics performed the seusitive specinic gravity tests on the CE-2 geonet, it would be most approprinte to consider only the test results produced by one of the laboratories when a comparison of the specific gravity of the geonet to the specinie gravity of the geonrembrane is necessary. Cunsidering that inter-laboratory precision is a problem with a test like specific gravily, comparing laboratory " $A$ 's" gconet specific gravity uith laborntory "B's" gcomenibrane specific gravity would not be the most Jugical approach. The $Q A$ consultani's lests confirm that the geoncl's specific gravity $(0.940$ and 0.941$)$ is less than'the geomembrane's specific gravity (0.942 to 0.944). Therefore, these should be no cause for concem about the compatibility of the geonet and the geomenbrane.

Mr. Sample, if you have any questions at all. please feel free to contuct me. I will be glad to assist you in any way possible.

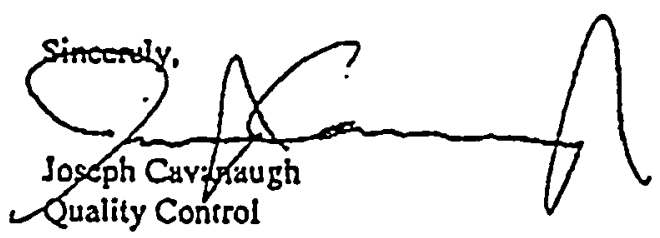


E1 fialuschak

Geopacific Iining Inc.

1300 Dexter Averre 205

seattle, Washingtar 98109

RE: Test Results and Certificatlon

IoI Geocomposite ano Hypernet

D.0.z. Bandford Radicactive Waste

SLT Project 8491

DeaI HI. Ealuschak:

As requested to lollow is the above referenced information outlined in our response to section 02275, Geosynthetic Transmissioni Media, item 5 and 6 . You will notice the geocomposite information is the actal test results based an data for every other roll. The eypernet reflects a letter of certification.

Please call me should you have any questions or need edditional informetion..

Best Regards,

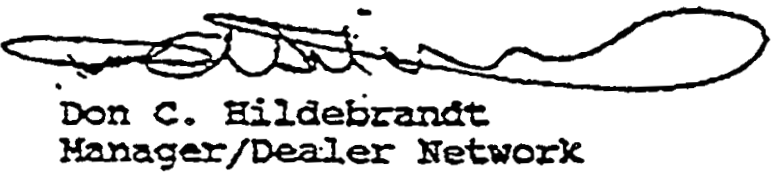




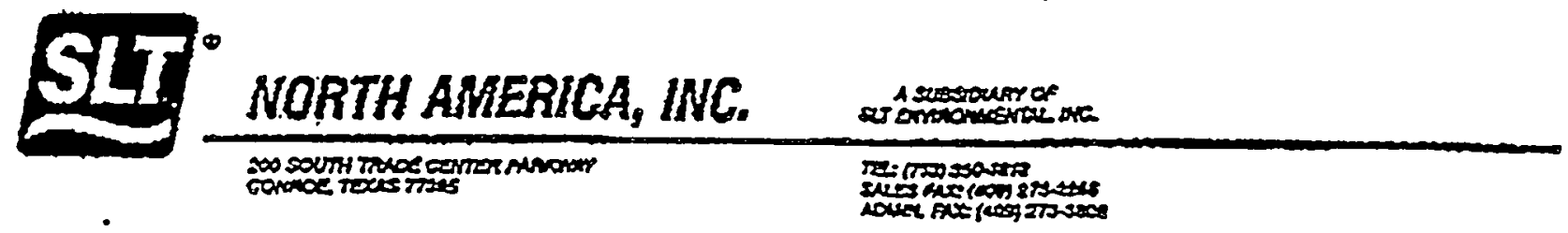

Hanford Composite Test Results

01-Nov-93

Peel - 2"X0" Sampie ASTMK D413/F904

\begin{tabular}{|c|c|c|c|c|c|c|c|c|}
\hline Roll No. & & 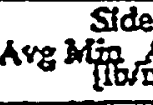 & $\sin$ & Avg Mide & $M$ & $\begin{array}{c}\text { Side } 1 \\
\text { Avepel } \\
\text { Dofint }\end{array}$ & $\begin{array}{l}\text { Side } 2 \\
\text { Aygrefel } \\
\text { [Ifin] }\end{array}$ & STATUS \\
\hline $\begin{array}{l}7235-2 A B-A \\
7235-2 A B-C \\
7235-2 A B-E\end{array}$ & : & $\begin{array}{l}5.4 \\
4.0 \\
52\end{array}$ & $\begin{array}{l}37.7 \\
19.3 \\
14.3\end{array}$ & $\begin{array}{l}5.3 \\
5.3 \\
5.3\end{array}$ & $\begin{array}{l}28.3 \\
27.4 \\
23.2\end{array}$ & $\begin{array}{r}21.6 \\
11.6 \\
9.7\end{array}$ & $\begin{array}{l}16.8 \\
16.3 \\
14.3\end{array}$ & $\begin{array}{l}\text { Pass } \\
\text { Pass } \\
\text { Pass }\end{array}$ \\
\hline $7383-2 A B B-C$ & & 32 & 5.1 & 15 & 3.7 & 42 & 2.6 & Pass \\
\hline $\begin{array}{l}7428-2 \mathrm{AB}-\mathrm{A} \\
7428-2 \mathrm{AB}-\mathrm{C}\end{array}$ & & $\begin{array}{l}5.6 \\
5.3\end{array}$ & $\begin{array}{l}49.0 \\
27.3\end{array}$ & $\begin{array}{l}5.3 \\
5.3\end{array}$ & $\begin{array}{l}40.9 \\
49.9\end{array}$ & $\begin{array}{l}27.3 \\
16.3\end{array}$ & 23.1 & $\begin{array}{l}\text { Pass } \\
\text { Pass }\end{array}$ \\
\hline $\begin{array}{l}7429-2 A B-A \\
7429-2 A B-C \\
7429-2 A B-E\end{array}$ & & $\begin{array}{l}52 \\
53 \\
52\end{array}$ & $\begin{array}{r}5.2 \\
29.4 \\
26.4\end{array}$ & $\begin{array}{l}5.4 \\
5.2 \\
5.4\end{array}$ & $\begin{array}{l}42.3 \\
33.2 \\
21.7\end{array}$ & $\begin{array}{r}52 \\
17.3 \\
15.8\end{array}$ & $\begin{array}{l}23.8 \\
19 \frac{2}{5} \\
135\end{array}$ & $\begin{array}{l}\text { Pass } \\
\text { Pass } \\
\text { Pass }\end{array}$ \\
\hline $\begin{array}{l}7430-2 A B-A \\
7430-2 A B-C \\
7430-2 A B-E\end{array}$ & : & $\begin{array}{l}5.4 \\
5.3 \\
5.3\end{array}$ & $\begin{array}{l}25.8 \\
24.9 \\
24.5\end{array}$ & $\begin{array}{l}5.2 \\
5.1 \\
5.2\end{array}$ & $\begin{array}{l}38.7 \\
37.0 \\
39.5\end{array}$ & $\begin{array}{l}15.6 \\
15.8 \\
14.9\end{array}$ & $\begin{array}{l}22.0 \\
21.1 \\
22.3\end{array}$ & $\begin{array}{l}\text { Pass } \\
\text { Pass } \\
\text { Pass }\end{array}$ \\
\hline $\begin{array}{l}7431-24 B-A \\
7431-2 A B-C \\
7431-2 A B-E\end{array}$ & : & $\begin{array}{l}5.4 \\
5.4 \\
5.2\end{array}$ & $\begin{array}{l}29.5 \\
31.6 \\
26.6\end{array}$ & $\begin{array}{l}5.3 \\
5.3 \\
.5 .1\end{array}$ & $\begin{array}{l}46.9 \\
16.9 \\
13.2\end{array}$ & $\begin{array}{l}17.5 \\
18.5 \\
15.9\end{array}$ & $\begin{array}{r}26.1 \\
11.1 \\
9.1\end{array}$ & $\begin{array}{l}\text { Pass } \\
\text { Pass } \\
\text { Pass }\end{array}$ \\
\hline $\begin{array}{l}7432-2 A B-A \\
7432-2 A B-C \\
7432-2 A B-E\end{array}$ & . & $\begin{array}{l}5.4 \\
5.2 \\
5.3\end{array}$ & $\begin{array}{l}36.8 \\
24.8 \\
50.2\end{array}$ & $\begin{array}{l}5.3 \\
5.3 \\
5.4\end{array}$ & $\begin{array}{l}34.4 \\
25.5 \\
26.8\end{array}$ & $\begin{array}{l}21.1 \\
15.0 \\
27.7\end{array}$ & $\begin{array}{l}19.9 \\
15.4 \\
16.1\end{array}$ & $\begin{array}{l}\text { Pass } \\
\text { Pass } \\
\text { Pass }\end{array}$ \\
\hline $\begin{array}{l}7433-2 A B-A \\
7433-2 A B-C \\
7433-2 A B-E\end{array}$ & & $\begin{array}{l}5.5 \\
5.2 \\
5.6\end{array}$ & $\begin{array}{l}41.3 \\
22.6 \\
21.5\end{array}$ & $\begin{array}{l}5.3 \\
5.1 \\
5.2\end{array}$ & $\begin{array}{l}30.6 \\
27.7 \\
34.2\end{array}$ & $\begin{array}{l}23.3 \\
13.9 \\
13.5\end{array}$ & $\begin{array}{l}17.9 \\
16.4 \\
19.7\end{array}$ & $\begin{array}{l}\text { Pass } \\
\text { Piss } \\
\text { Pass }\end{array}$ \\
\hline $\begin{array}{l}7434-2 A B-A \\
7434-2 A B-C\end{array}$ & & $\begin{array}{l}5 . \hat{3} \\
5.1\end{array}$ & $\begin{array}{l}30.5 \\
31.0\end{array}$ & $\begin{array}{l}5.4 \\
5.4\end{array}$ & $\begin{array}{l}35.4 \\
36.7\end{array}$ & $\begin{array}{l}17.9 \\
18.0\end{array}$ & $\begin{array}{l}20.4 \\
21.1\end{array}$ & Far \\
\hline $\begin{array}{l}743 S-2 A B-A \\
74=5-j \mathrm{i} B-C \\
7435-2 A B-E\end{array}$ & - & $\begin{array}{l}1.3 \\
1.0 \\
5.2\end{array}$ & $\begin{array}{r}4.5 \\
4.4 \\
19.4\end{array}$ & $\begin{array}{l}2.7 \\
1.5 \\
5.2\end{array}$ & $\begin{array}{r}5.8 \\
4.4 \\
24.0\end{array}$ & $\begin{array}{r}2.9 \\
2.7 \\
12.1\end{array}$ & $\begin{array}{r}4.2 \\
2.9 \\
14.6\end{array}$ & $\begin{array}{l}\text { Pass } \\
\text { Pass } \\
\text { Pass }\end{array}$ \\
\hline $\begin{array}{l}7436-2 A B-A \\
7436-2 A B-C \\
7436-2 A B-E\end{array}$ & & $\begin{array}{l}2.2 \\
1.9 \\
1.6\end{array}$ & $\begin{array}{l}3.5 \\
4.2 \\
3.7\end{array}$ & $\begin{array}{l}2.7 \\
2.5 \\
2.0\end{array}$ & $\begin{array}{l}6.2 \\
6.3 \\
4.5\end{array}$ & $\begin{array}{l}2.8 \\
3.1 \\
2.6\end{array}$ & $\begin{array}{l}4.5 \\
4.4 \\
3.2\end{array}$ & $\begin{array}{l}\text { Pass } \\
\text { Pass } \\
\text { Poss }\end{array}$ \\
\hline $\begin{array}{l}7483-2 A B-A \\
7 \angle 83-2 A B-C \\
7 \angle 83-2 A B-E\end{array}$ & & $\begin{array}{l}5.2 \\
5.2 \\
5.4 \\
\end{array}$ & $\begin{array}{l}35.7 \\
32.4 \\
2 . .1 \\
\end{array}$ & $\begin{array}{l}5.2 \\
5.3 \\
5.2 \\
\end{array}$ & $\begin{array}{r}17.5 \\
352 \\
30.0 \\
\end{array}$ & $\begin{array}{l}20.6 \\
18.8 \\
14.8 \\
\end{array}$ & $\begin{array}{l}11.3 \\
20.8 \\
17.6 \\
\end{array}$ & $\begin{array}{l}\text { Pass } \\
\text { Pass } \\
\text { Pass }\end{array}$ \\
\hline
\end{tabular}

NOTE: The following solls have PASSED the required minimum averoge of $1.0 \mathrm{lb}$ in ped velue:

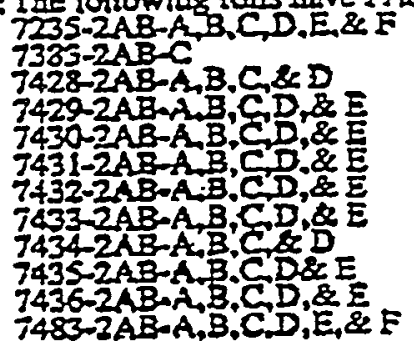

(Only resived a sorapte for 7383-2AB-C) 
Hanford Composite Test Results

02-Nov-93

Peel - 2"X9" Sample ASTM D413/5904

\begin{tabular}{|c|c|c|c|c|c|c|c|c|}
\hline Roll No. & $\begin{array}{l}\text { Side } \\
\text { Avg Minf } \\
\text { Ifori }\end{array}$ & $\operatorname{sax}$ & $\begin{array}{l}\text { Sid } \\
\text { Avg Mind }\end{array}$ & $\max$ & & 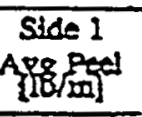 & 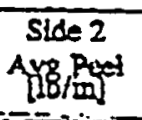 & STATUS \\
\hline $\begin{array}{l}6837-2 A B-A \\
6837-2 A B-C \\
6837-2 A B-E\end{array}$ & $\begin{array}{l}1.7 \\
1.7 \\
0.8\end{array}$ & $\begin{array}{l}4.5 \\
3.6 \\
2.3\end{array}$ & $\begin{array}{l}2.5 \\
2.6 \\
1.4\end{array}$ & $\begin{array}{l}5.9 \\
4.9 \\
4.2\end{array}$ & i & $\begin{array}{l}3.1 \\
2.6 \\
1.5\end{array}$ & $\begin{array}{l}4.2 \\
3.7 \\
2.7\end{array}$ & $\begin{array}{l}\text { Pass } \\
\text { pass } \\
\text { Pass }\end{array}$ \\
\hline $\begin{array}{l}7017-2 A B-A \\
7017-2 A B-C\end{array}$ & $\begin{array}{l}1.2 \\
0.8\end{array}$ & $\begin{array}{l}4.1 \\
2.7\end{array}$ & $\begin{array}{l}2.1 \\
2.0\end{array}$ & $\begin{array}{l}5.6 \\
5.0\end{array}$ & : & $\begin{array}{l}2.7 \\
1.8\end{array}$ & $\begin{array}{l}3.1 \\
3.5 .\end{array}$ & $\begin{array}{l}\text { Perss } \\
\text { Pars }\end{array}$ \\
\hline $\begin{array}{l}7380-2 A B-A \\
7380-2 A B-C\end{array}$ & $\begin{array}{l}0.9 \\
1.0\end{array}$ & $\begin{array}{l}3.4 \\
3.5\end{array}$ & $\begin{array}{l}2.3 \\
1.2\end{array}$ & $\begin{array}{l}6.1 \\
4.2\end{array}$ & : & $\begin{array}{l}2.2 \\
1.8\end{array}$ & $\begin{array}{l}4.2 \\
2.7\end{array}$ & $\begin{array}{l}\text { Pass } \\
\text { Pass }\end{array}$ \\
\hline $\begin{array}{l}7382-2 A B \\
7382-2 A B\end{array}-A$ & 1.3 & $\begin{array}{l}4.2 \\
4.4\end{array}$ & $\begin{array}{l}2.8 \\
2.6\end{array}$ & $\begin{array}{l}6.8 \\
5.1\end{array}$ & & $\begin{array}{l}2.8 \\
3.0\end{array}$ & $\begin{array}{l}4.8 \\
3.8\end{array}$ & $\begin{array}{l}\text { Pass } \\
\text { Pass }\end{array}$ \\
\hline $7427-2 A B-A$ & 1.0 & 2.5 & 2.8 & 6.2 & & 1.7 & 4.5 & Pass \\
\hline $7485-2 A B-A$ & 18. & 3.3 & 2.7 & 5.7 & & 2.5. & .4 .2 & Boss \\
\hline
\end{tabular}
NOTE:The following rolds have PASSED the required average of 1.0 lt/in peel value: 7017-2AB-A.B.AC $7380-2 \mathrm{AB}-\mathrm{A}, \mathrm{B}, \&$ $7382-2 A B-A, B, \&$ $7485-2 A B-A \& B$

There are 18 good rolls in this group that can be sblpped. 
A suasoun of

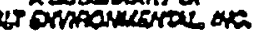

Hanford Compostte Test Results

03-Nov-93

Peel - 2".9" Sample ASTM D413/F904

\begin{tabular}{|c|c|c|c|c|c|c|c|}
\hline \multirow{2}{*}{$\frac{\text { Roll No. }}{6844-2 A B-A}$} & \multicolumn{2}{|c|}{ 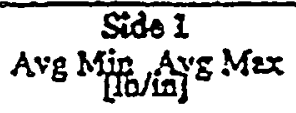 } & \multicolumn{2}{|c|}{$\begin{array}{c}\text { Side } 2 \\
\text { Avg Miq Avg MEx }\end{array}$} & \multirow{2}{*}{ 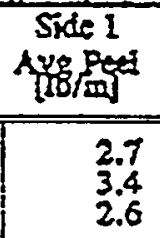 } & \multirow{2}{*}{ 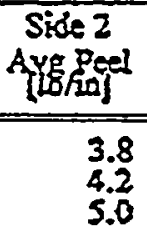 } & \multirow{2}{*}{$\begin{array}{c}\text { STATUS } \\
\text { Pess } \\
\text { Pass } \\
\text { Pass }\end{array}$} \\
\hline & $\begin{array}{l}1.3 \\
2.4 \\
3.4\end{array}$ & $\begin{array}{l}4.1 \\
4.5 \\
3.7\end{array}$ & $\begin{array}{l}2.1 \\
2.7 \\
2.5\end{array}$ & $\begin{array}{l}5.5 \\
5.7 \\
7.4\end{array}$ & & & \\
\hline $\begin{array}{l}7014-2 A B-A \\
7014-2 A B-C \\
7014-2 A B-E\end{array}$ & $\begin{array}{l}1.3 \\
2.1 \\
1.2\end{array}$ & $\begin{array}{l}3.8 \\
4.3 \\
2.8\end{array}$ & $\begin{array}{l}2.3 \\
1.7 \\
2.4\end{array}$ & $\begin{array}{l}5.8 \\
3.7 \\
5.3\end{array}$ & $\begin{array}{l}2.6 \\
32 \\
2.0\end{array}$ & $\begin{array}{l}3.1 \\
2.7 \\
3.8\end{array}$ & $\begin{array}{l}\text { Pass } \\
\text { pass } \\
\text { Pass }\end{array}$ \\
\hline $7017-2 A B-E$ & 1.2 & 2.8 & 2.4 & 5.3 & 2.0 & 3.8 & Pass \\
\hline $\begin{array}{l}702 C-2 A B-A \\
7024-2 A B-C \\
7024-2 A B-E\end{array}$ & $\begin{array}{l}1.9 \\
1.6 \\
1.4\end{array}$ & $\begin{array}{l}3.2 \\
4.4 \\
2.8\end{array}$ & $\begin{array}{l}3.5 \\
2.6 \\
2.0\end{array}$ & $\begin{array}{l}6.7 \\
3.1 \\
4.2\end{array}$ & $\begin{array}{l}2.6 \\
3.0 \\
2.1\end{array}$ & $\begin{array}{l}5.1 \\
3.8 \\
3.2\end{array}$ & $\begin{array}{l}\text { Pass } \\
\text { Pass } \\
\text { Dass }\end{array}$ \\
\hline $\begin{array}{l}7222-2 A B-A \\
7222-2 A B-C \\
7222-2 A B-E\end{array}$ & $\begin{array}{l}1.5 \\
1.7 \\
2.3\end{array}$ & $\begin{array}{l}3.3 \\
3.3 \\
3.3\end{array}$ & $\begin{array}{l}2.5 \\
2.9 \\
2.9\end{array}$ & $\begin{array}{l}6.3 \\
4.9 \\
5.8\end{array}$ & $\begin{array}{l}2.4 \\
2.5 \\
2.7\end{array}$ & $\begin{array}{l}4.4 \\
3.9 \\
6.3\end{array}$ & $\begin{array}{l}\text { Pass } \\
\text { Pass } \\
\text { Pass }\end{array}$ \\
\hline
\end{tabular}

NOTE: The following rolls have PASSED the required ayerage of 1.0 Ib/in peel valac:

6844-2AB-A.B.C.D.\& E

$7014-2 A B-B, B, D, \&$ E

7017-2AB-D,\& E

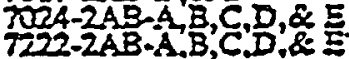

There ers 22 gcod rolls in this grols ihe can be shipped.

For environmental lining solutions...the world comes to SLT. 


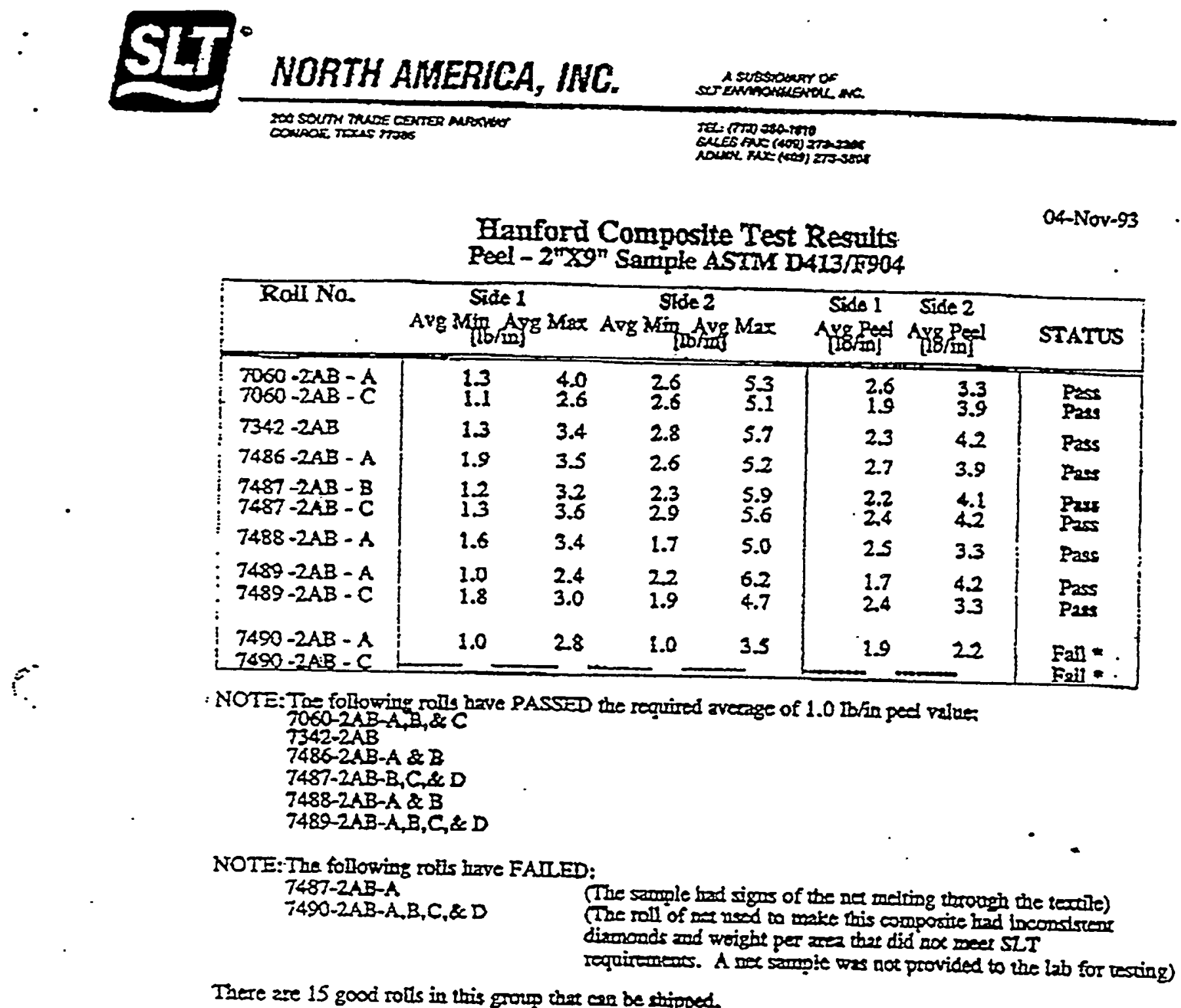

For environmentai lining solutions...the world comes to $S L T^{\infty}$ 


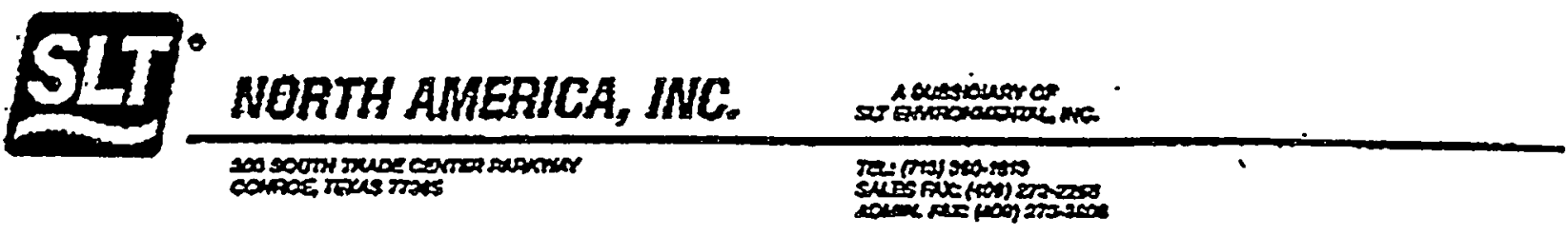

Hanford Composite Test Results

04-Nor-93 Peel - 2"X9" Sample ASTMI D413/F904

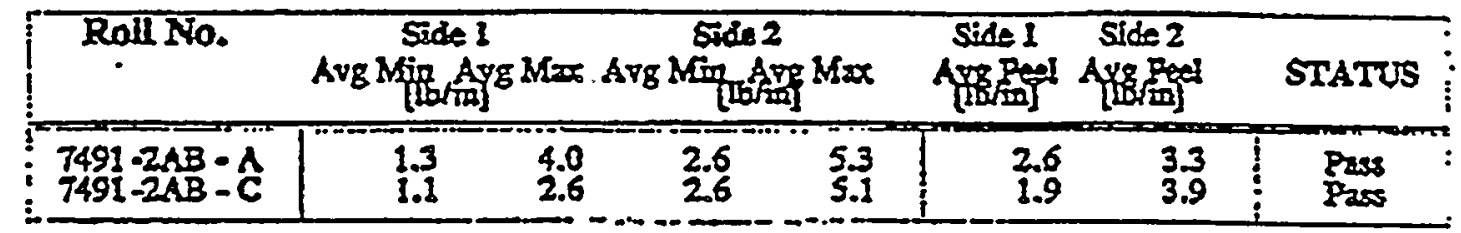

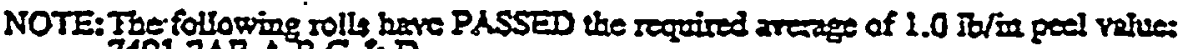
7491-2AB-A, B, C, \& D

There are 4 good rolls in this group thas can be shoped.

For environmental lining solutions...the world comes to SLT.9 
Peel - 2"XY" Sample ASTM D413/F904

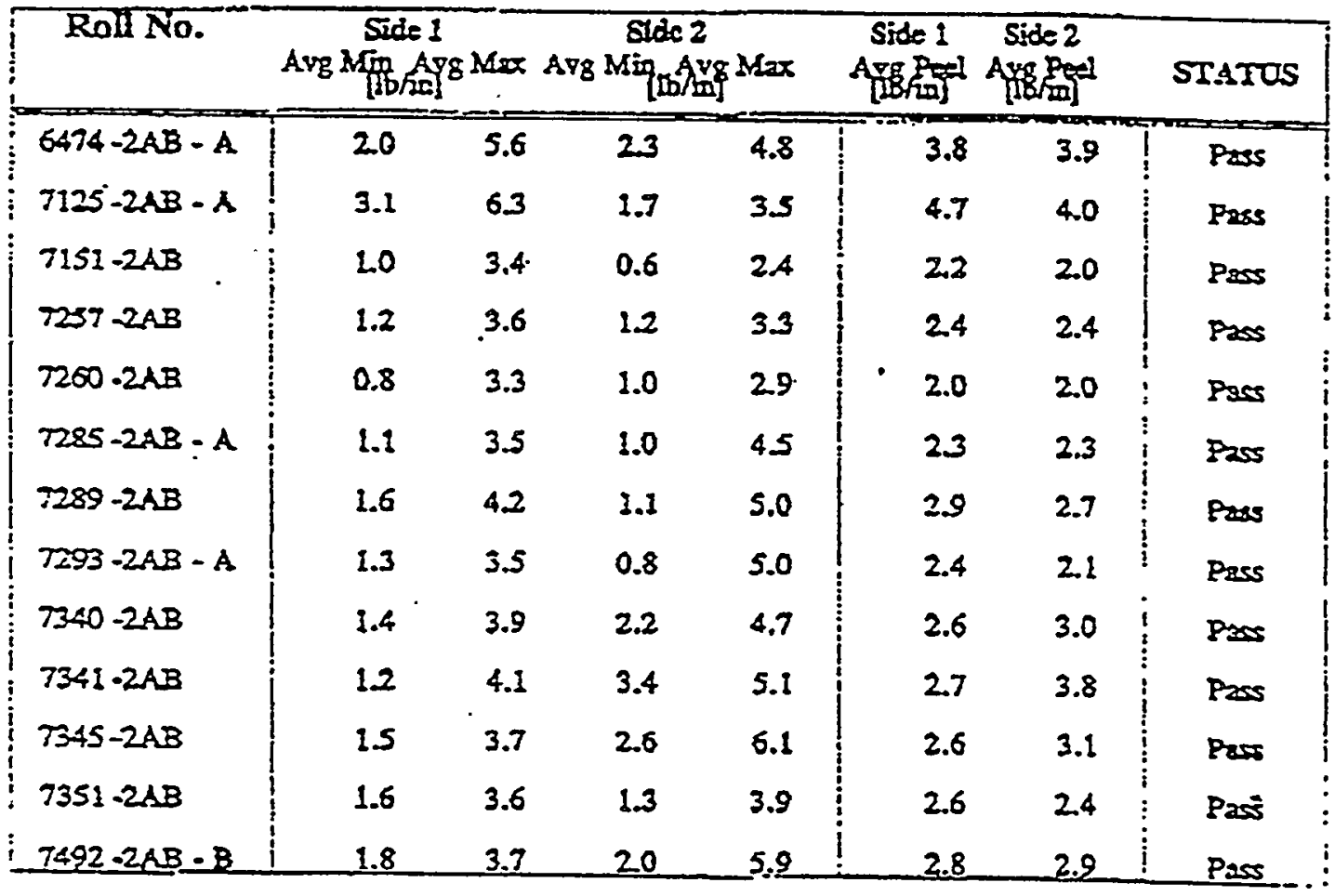

NOTE-The following rolls have $2 A S S E D$ the required average of 1.0 Jo/in peal value: 6474-2AB-A \& B

$7125-2 A B-A \& B$

7151-2AB

$7257-248$

T260-2AB

$7285-24 B-A \& B$

T2O-2AB

T293-2AB-A \& $B$

$7340-2 A B$

$7341-2 A B$

T345-2AB

T351-2AB

7492-2AB-A \& B

There aro 18 good rolls in ifts groug that can be shipped.

For anvironmental lining solutions...the world comes to SLT. 
Hanford Composite Test Results

08-Nov-93

Peel - 2"X9" Sample ASTM D413/F904

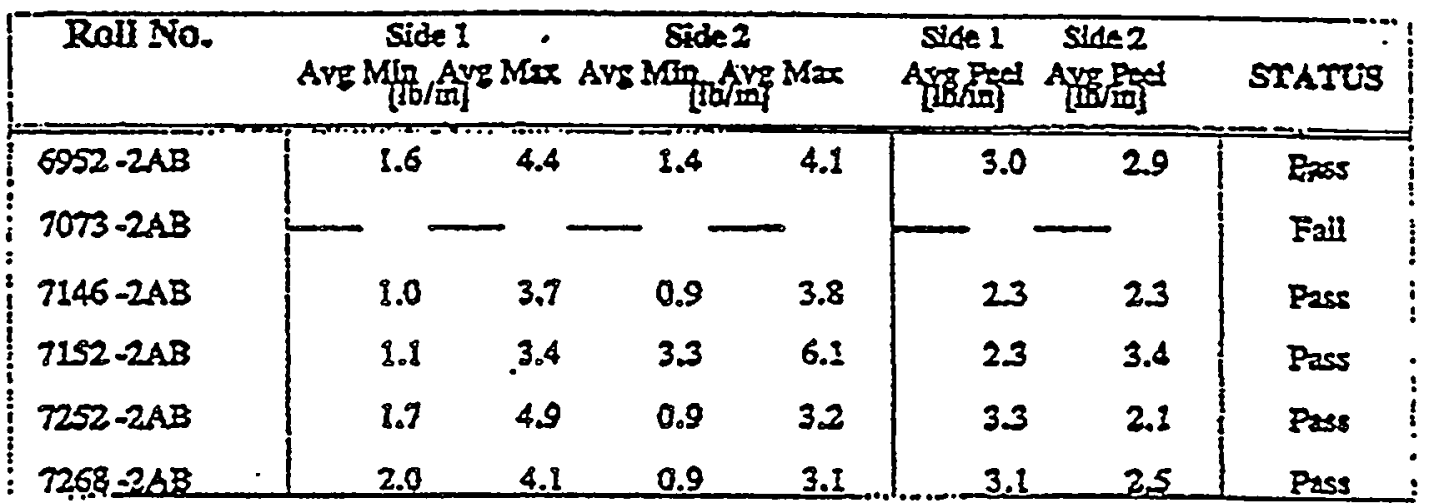

NOTE: The foltowing rolls bave \$ASSED the regaired aveage of 1.0 Iblan peet valice. 6952248

$7146-2 A B$

$7152.24 \mathrm{~B}$

T252-24B

$7268-2 A B$

There are 5 good rolls in tifis group that cen be shipped.

NOTE: The followiog rolls have FAll ED:

7073-2AB (Fallare due to rediting of texcilo). 
Hanford Composite Test Results

18-Jan-94

Peel - 2"X9" Sample ASTM D413/F904

\begin{tabular}{|c|c|c|c|c|c|c|c|}
\hline \multirow{2}{*}{$\begin{array}{r}\text { Roll No. } \\
3874-2 A B\end{array}$} & \multicolumn{2}{|c|}{$\begin{array}{c}\text { Side } 1 \\
\text { Avg Mij/Avg Max } \\
{[[\mathrm{b} / \mathrm{in}]}\end{array}$} & \multicolumn{2}{|c|}{$\begin{array}{c}\text { Side } 2 \\
\text { Avg Miq Avg Max } \\
{[\mathrm{lb} / \mathrm{in}\}}\end{array}$} & \multirow{2}{*}{$\begin{array}{c}\begin{array}{c}\text { Side 1 } \\
\text { Ayg Peel } \\
\text { [1B/in] }\end{array} \\
3.8\end{array}$} & \multirow{2}{*}{$\begin{array}{c}\begin{array}{c}\text { Side 2 } \\
\text { Ayg Peel } \\
\text { [18/in] }\end{array} \\
4.1\end{array}$} & \multirow{2}{*}{$=$} \\
\hline & 2.1 & 5.4 & 2.8 & 5.2 & & & \\
\hline $4038-2 A B$ & 2.4 & 5.4 & 2.3 & 4.2 & 3.9 & 3.8 & Pass \\
\hline $5177-2 A B$ & 1.2 & 2.5 & 2.5 & 5.2 & 1.8 & 2.5 & Fail \\
\hline $6884-2 \mathrm{AB}$ & 2.6 & 5.2 & 0.7 & 1.8 & 3.9 & 2.9 & Pass \\
\hline $6894-2 A B$ & 2.5 & 4.8 & 0.9 & 2.0 & 3.7 & 2.9 & Pass \\
\hline $8008-2 \mathrm{AB}-\mathrm{A}$ & 3.7 & 6.1 & 1.3 & 2.1 & 4.9 & 3.7 & Pass \\
\hline $8008-2 \mathrm{AB}-\mathrm{C}$ & 4.6 & 3.4 & 3.4 & 5.7 & 4.0 & 3.4 & Pass \\
\hline $8008-2 A B-E$ & 1.9 & 4.3 & 2.6 & 5.2 & 3.1 & 3.4 & Pass \\
\hline $8257-2 A B-A$ & 0.8 & 2.0 & 2.5 & 4.6 & 1.4 & 2.3 & Pass \\
\hline $8398-2 A B-A$ & 2.2 & 5.3 & 1.6 & 3.7 & 3.8 & 3.5 & Pass \\
\hline $8398-2 A B-C$ & 2.7 & 5.1 & 1.8 & 4.3 & 3.9 & 3.4 & Pass \\
\hline
\end{tabular}

NOTE: The following rolls have PASSED the required average of $1.0 \mathrm{lb} / \mathrm{in}$ peel value:

3183-2AB

3874-2AB

4038-2AB

$5164-2 A B$

6884-2AB

6890-2AB

$6892-2 A B$

$6894-2 \mathrm{AB}$

6897-2AB

$8008-2 A B-A, B, C, D, E \& F$

8257-2AB-A

8398-2AB-A,B,C \&D

There are 20 good rolls that can be shipped. 
Hanford Composite Test Results

18-Jan-94

Peel - 2"X9" Sample ASTM D413/F904

\begin{tabular}{|c|c|c|c|c|c|c|c|}
\hline Roll No. & $\begin{array}{c}\text { Sid } \\
\text { Avg Mip } \\
\text { (Ib) }\end{array}$ & & $\begin{array}{r}\text { Sid } \\
\text { Avg Min }\end{array}$ & $\operatorname{Max}$ & $\begin{array}{c}\text { Side } 1 \\
\text { Ayg Peel } \\
\text { [16/in] }\end{array}$ & $\begin{array}{c}\text { Side } 2 \\
\text { Ayg Peel } \\
\text { [16/in] }\end{array}$ & STATUS \\
\hline $7778-2 A B-A$ & 1.0 & 3.7 & 1.1 & 2.9 & 2.3 & 2.4 & Pass \\
\hline $7778-2 \mathrm{AB}-\mathrm{C}$ & 1.3 & 4.0 & 1.0 & 4.3 & 2.7 & 2.5 & Pass \\
\hline $8013-2 A B-A$ & 0.6 & 2.8 & 0.7 & 2.2 & 1.7 & 1.8 & Pass \\
\hline $8353-2 A B$ & 0.7 & 1.9 & 2.9 & 6.8 & 1.3 & 2.4 & Pass \\
\hline $8363-2 A B$ & 0.7 & 2.3 & 3.1 & 8.2 & 1.5 & 2.7 & Pass \\
\hline $8365-2 A B$ & 0.9 & 3.1 & 3.6 & 7.4 & 2.0 & 3.3 & Pass \\
\hline $8379-2 \mathrm{AB}$ & 0.6 & 2.4 & 3.5 & 6.8 & 1.5 & 2.9 & Pass \\
\hline
\end{tabular}

NOTE: The following rolls have PASSED the required average of $1.0 \mathrm{lb} / \mathrm{in}$ peel value based on above test results:

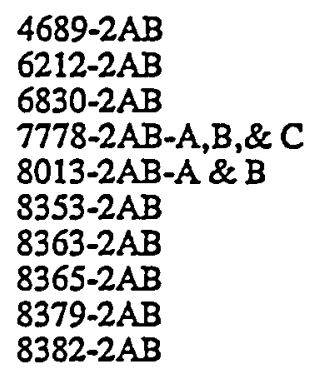

There are 13 good rolls that can be shipped. 
HHC-SD-W025-RPT-001, Rev. 0

\section{APPENDIX I}

PIPE MANUFACTURER'S QC DOCUMENTATION 
WHC-SD-W025-RPT-001, Rev. 0

\section{APPENDIX I-1}

\section{HDPE PIPE}

I-1 


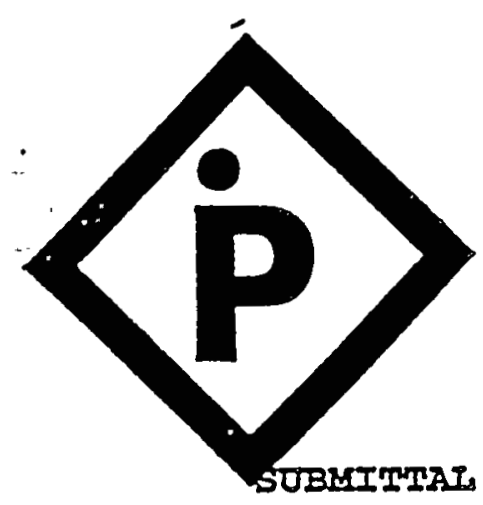

WHC-SD-W025-RPT-001, Rev. 0

\section{INDUSTRIAL PLASTICS, INC.}

740 S. 28th Street - Washougal, WA $98671-2597$

Phone (206) 835-2129 or (503) $227-5588$

1-800-634-5082 FAX (206) 835-3521

CTSTOMER NLAME DELFUR TNDUSIRIES

ORDER NUWBER PER QDOTATION DATED 7/23/93

PROJECT IDENTIFICATION FANEORD RMWD FACIIITY - 200 WEST AREA

PROJECT LOCATION RER 5346

FAANEORD, WASHINGTON

PIPE SIZES INCLUDING SDR OR WAII TEICSNESS:

SECTION 02725 FDPE PIPE \& FITIINGS

SOLID \& PERFORATED PER SPECIFICATIONS

4.5" OD X 3.68" ID X.409" WAIE, SDR 11, 160 PSI

8.625" OD $\times 7.05^{\prime \prime}$ ID X.784" WAII, SDR 11, 160 PSI

2.375" OD $X 1.93^{\prime \prime}$ ID $x .216^{\prime \prime}$ WAII, SDR 11,160 PSI

$3.500^{\prime \prime}$ OD $x 2.86^{\prime \prime}$ ID $x .318^{\prime \prime}$ WAII, SDR 11,160 PSI

$1.90^{\prime \prime}$ OD X1.55" ID $X .173^{\prime \prime}$ WAII, SDR 11, 160 RSI

FITTINGS TO BE OE LIRE METERIAI.

The above listed material for this project is certified to be manufactured from a PE 3408 Polyethylene compound as defined in ASTM D-1248 and is identified as:

TYPE

CAIEGORY
Crass

GRADE

$\frac{C}{P 34}$

This pipe compound has a cell classification according to ASTM D-3350 of $345434 \mathrm{C}$.

Special Iistings FULI PPI \& SPI MEMBERSEIP \& IISTING.

\begin{tabular}{ll}
\hline CERTIFICATION PREPARED BY \\
b:specs/I0
\end{tabular}

933-1354

I-1-1 


\section{P. Familian INDUSTRIAL PLASTICS, INC.}

740 S. 28th Street - Washougal, WA $98671-2597$

Phone (206) 835-2129 or (503) 227-5588

1-800-634-5082 FAX (206) 835-3521

SUBNITIAI AND CERTIFICATION OF MATERIAI SFECIFICATION

COSTOYER NAME DELHUR INDUSTRIES

ORDER NUDEER S.0.27307, P.0.25817

PROJECT IDENTIFICATION FANRORD RMWD FACILITY - 200 WEST AREA

PROJECT IOCATION

FANTFORD, WA

PIPE SIZES INCLUDING SDR OR WAII THICKNBSS:

IEACHATE COLIECTIOA WELI;

$30 "$ O.D. X 26.01" I.D. X 1.935" WALI SDR 15.5, 110 2SI

PER ATTACERD DRAWING

6.625"0.D. X 5.61"I.D. X. 491" KAII, SDR 13.5, 130 PSI

HDPE FLATSTOCK PER ATTACEED SPBC SHEET

The above 11sted material for this project is certified to be manufactured from a $\mathrm{FZ} 3408$ Polyethylene compound as defined in ASTM D-1248 and is identified ag:

TYPE III

CAIEGORI

5

Crass

GRADE

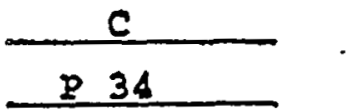

This pipe compound has a cell classificetion accordiag to ASTA $D-3350$ of $345434 C$.

Special Iistings SEE ATTACHED

$b=8 \operatorname{secs} / 20$

$933-1354$
CERTIFICATION RREPARED BY

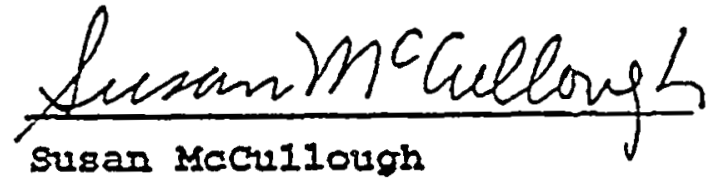

Saleg

Date: October 8, 1993 
October 12, 1893

FAMILIAN INDUSTRIAL PIJATICS

740 South 28 th street

Weshougal, HA 98671-2597

Re: Delhur Industries

Your P.O. \$4716

\begin{tabular}{rllll}
\hline $2800^{\prime}$ & $4^{\prime \prime}$ & $x$ & $40^{\prime}$ & SDRII \\
$160^{\prime}$ & $3^{\prime \prime}$ & $x$ & $40^{\prime}$ & SDRII \\
$400^{\prime}$ & $8^{\prime \prime}$ & $x$ & $40^{\prime}$ & SDRII \\
$800^{\prime}$ & $2^{\prime \prime}$ & $\times$ & $40^{\prime}$ & SDRII \\
$300^{\prime}$ & $1^{\prime \prime}$ & $\times$ & $300^{\prime}$ & SDRII \\
& & NSF COII
\end{tabular}

Datel Mat? $10883 \mathrm{NB}$ $91293 Y B$ $82793 Y B$ $61993 Y B$ 31093YB

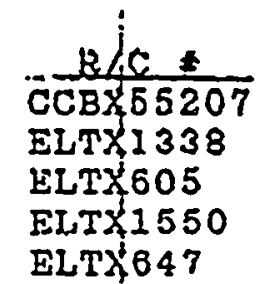

16360 Roscos Blyd, sulid $12 \%$

Vàn Nuys, Callornia 91406

(818) 893-1112

Wats Callomia Only (800) 252.7473

Facsimile (818) 892.0695

\section{Dear Sir:}

This letter is to certify that the DURATUFFei HDPE pipe produced by polarla pipe Co. for the above project: conforms to ASTM material apeciflcation D1248, Type III, Class C, Category 5; Grade P34. The pipo made from this material also conforms to cell classification PE345434C per ASTM specification D3350.

All resin/pellets were used from sources of HDPE material that are listed both with the National Sanjtation fopndation (NSF) and the Plastics Pipe Institute (IPI).
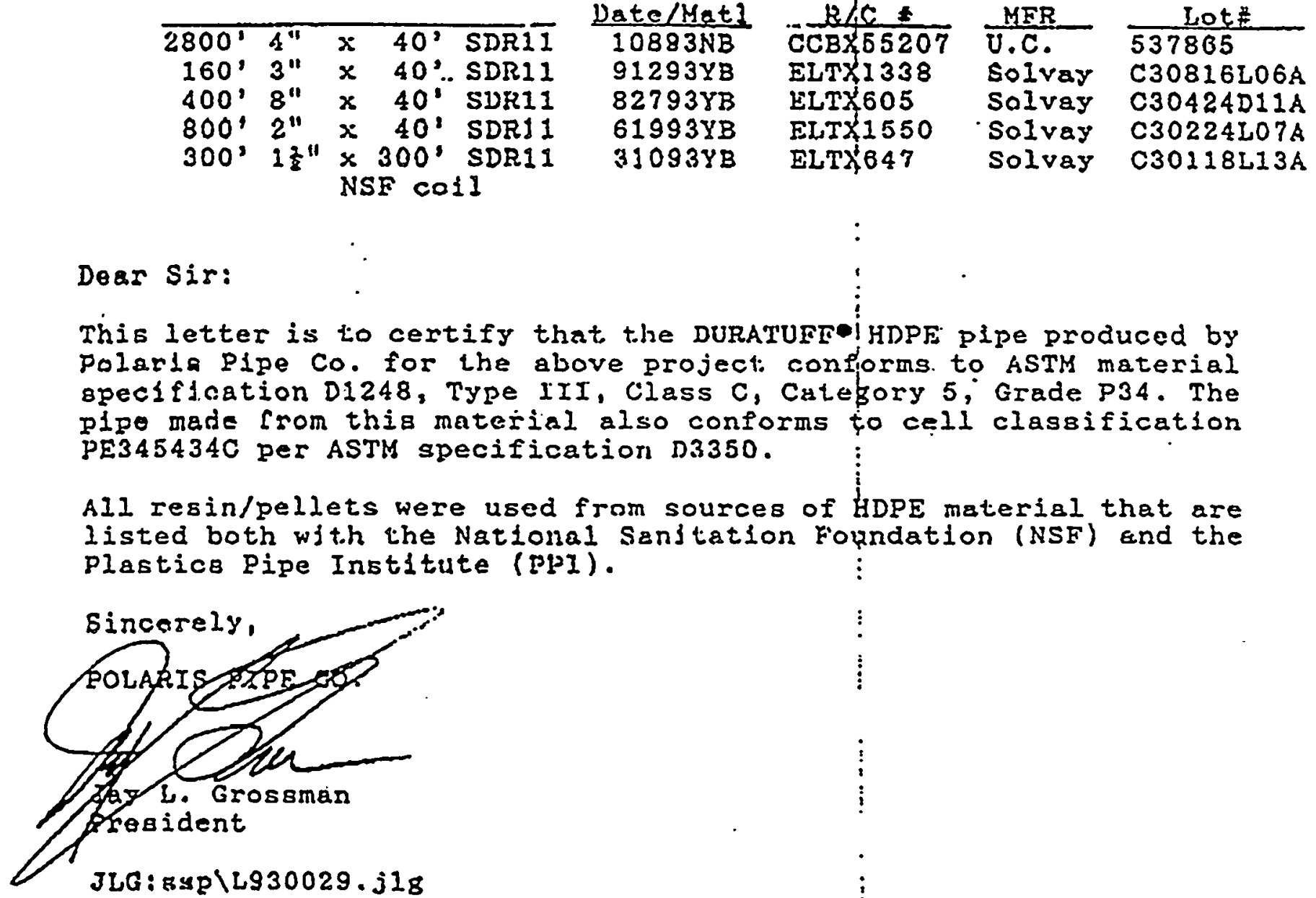

JLG: हsp $\backslash L 930029 \cdot 318$ 


\section{Polncis Pipe \\ Monufacturers of Plostic Pipe to.}

VIA FACSIMILE TO (206) 835=3521

November 30,1993

David Schlege 1

FAMILIAN INDUSTRIRL PLASTICS

740 south 28 th Street

Hashougal, WA 98671-2597

Re: Deinur Industriss

$$
\text { Your PO \$2121 06/02/92 }
$$$$
\text { SO \# } 13834
$$

$$
25^{\prime} 6^{\prime \prime} \times 25^{1} \overline{\text { SOR } 13.5} \text { HOPE PIDE }
$$

16380 Roscoo Blvd., Sulto 120

Van Nuys, California 91406

(818) 893 .

Wats Callfomia Only (800) 252 .

Facsimlis (B18) 892.0.

Dear Dave:

This letter is to certify that the bURATUFFo HDPE pipe oroduced by Polaris Pipe Co. for the above project conforms to ASTM material specification D1248, Type III, Clas's C, Catégory B, Grade P34. The pipe made from this material also conforms ito cell classification PE346434C per ASTM Epeciflcation D3350.

All resin/pellets were used from sources of iHDPE materlal that are listed both with the National Santtation Foundation (NSF) and the Plastics Pipe Institute (PPI).

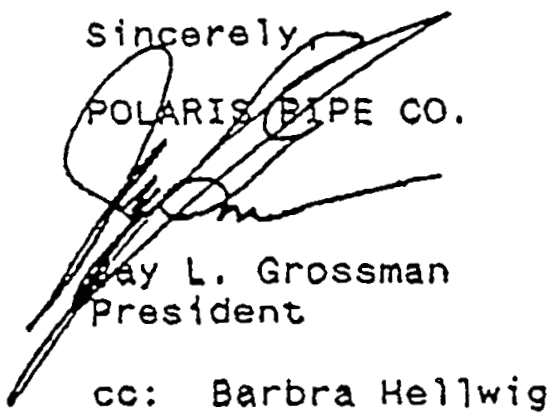

JLO:A201L830002.318 
WHC-SD-W025-RPT-001, Rev. 0

TYPICAL PHYSICAL PROPERTIES
TEST METHODS

PROCESSING
Preperty (Dolcmined on compression.

golded speciman)

Mechanieal Propertas.

Tensile Strength fortzk).

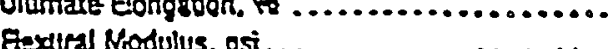

Tonsile Strength (vield). osi $\ldots \ldots \ldots \ldots \ldots$

Thermai ?ropertice -

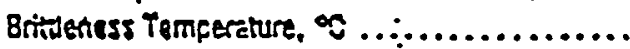

Malt index. $g / 10$ minutes. ...................

.

High Load Melt index, g/10 minutes

$4+\ldots \ldots \ldots$

ose induedon Temperature, $\alpha$

OSC induesion Tume, minutes

at 2:00 (10üs Oxygen)

- Meltirg Foint.

(n)

Crystifltagton Pçine. ocs

$\ldots \ldots \ldots$

c.

Vicat Softening Tamoerature, $\varnothing^{\circ}$

Miscallaneous Properties

Oensily. g/cms (oasa resin)

Density. olcm (compound)

Environmental Stress Crack Resistanct.

P. Hours

Thermally Aged Emironmental

Stress Crack Resistince." F F. Howis

Ourometer Hardness, Shors "

Lineas Mitmal Ccefficient of Expansion. insin $1^{\circ} \mathrm{C}\left(-30^{\circ} \mathrm{C}\right.$ w +30。 $\left.\mathrm{c}\right)$

bod Impact th-tos In netch at 234

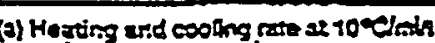

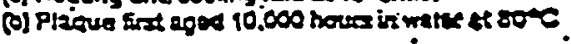

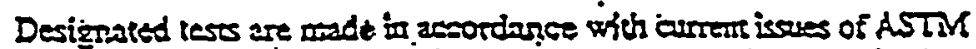

Standard Twing Mietiods. Cogies of these are avibble from tho Annerican Sociery for Testing and Malerisis, 1916 Race Stres, Pteiadulghis, PA 19103. on seques: Ite Union Caride ratiods are avaitble upon requesc.

LGDB-2480 blacis 4865 en be iendify prosessel by any of the current pipo extrusion methods in all pipe sies at rates considend corrocestilly sereptibla. Fowere, to artrure proper plastiesion end pertormance of the profuct wo rwommend tie following key poines be adbered to dosely. The

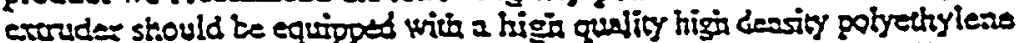
serew (prefersibly a barrite type to insure complete meiong), as well as a meit thermocouplo sid presere gauge for control.. 
WHC-SD-W025-RPT-001, Rev. 0

\section{Durauff.}

$\curvearrowleft \quad$ Polyethylene Pipe HDPE

PHYSICAL PROPERTIES DATA 989

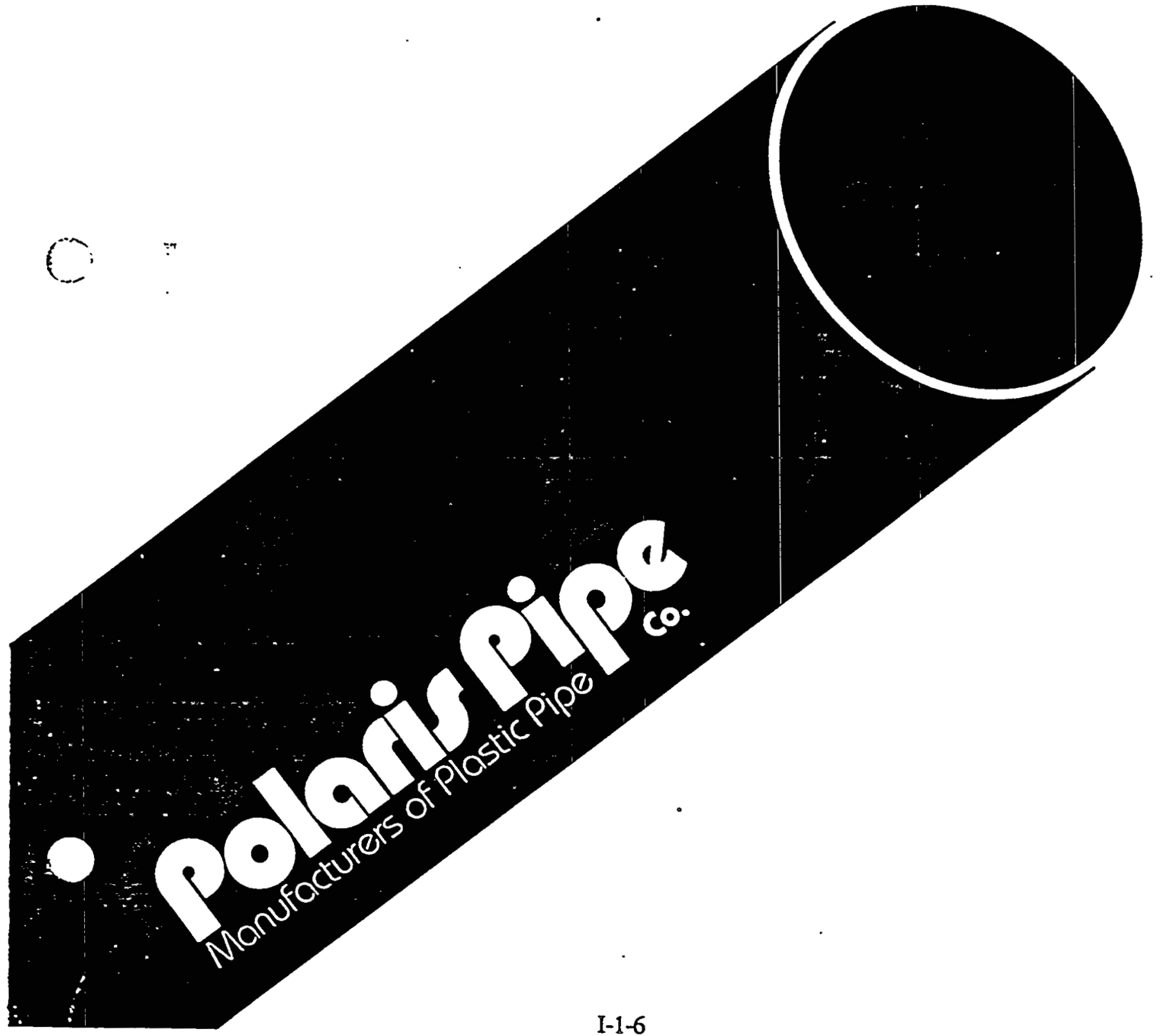




\section{DUraार्यf: polyethylene pipe}

STRAIGHT LENGTHS

Polaric Pipe

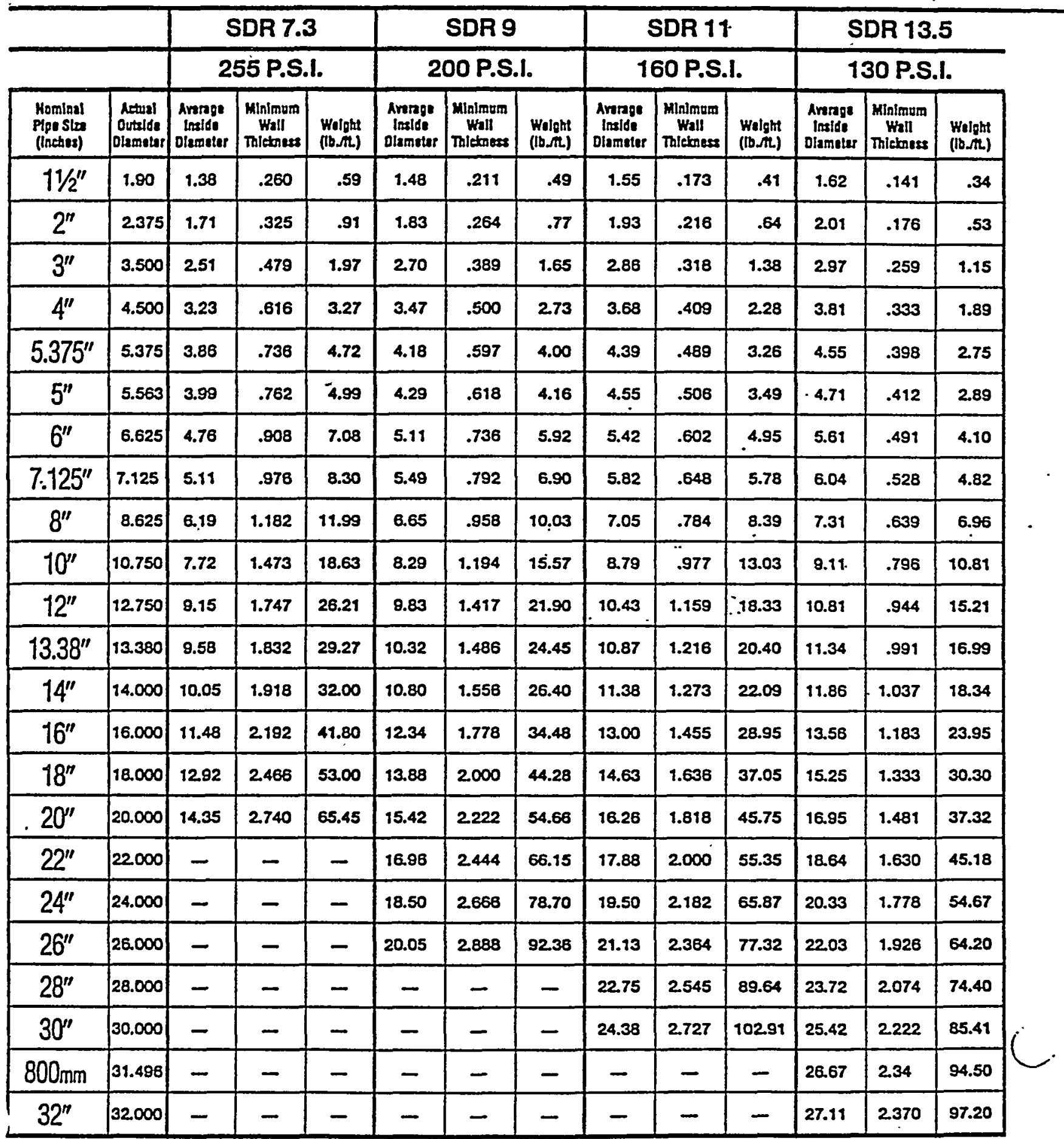

ALL WEIGHTS ARE CALCULATED IN ACCORDANCE WITH PPI-TR-7.

933-139ference ASTM Standard F714 / D3035 for-Pipe Specifications \& Dimensions. 


\section{Polncipipipe}

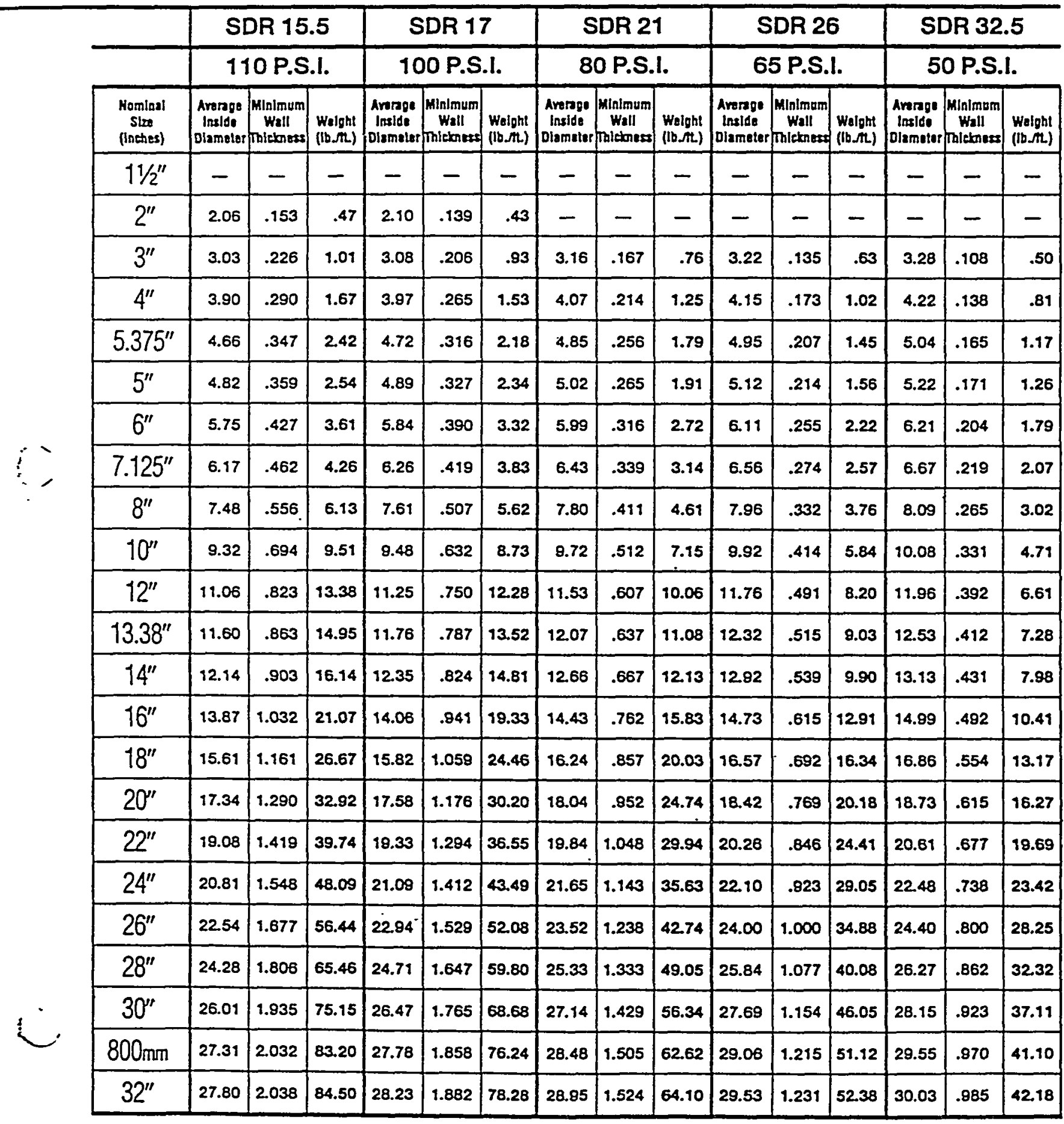

ALL WEIGHTS ARE CALCULATED IN ACCORDANCE WITH PPI-TR-7. 


\section{POLYETHYLENE FEATURES AND BENEFITS}

Polyethylene pipe is specifically designed to fulfill the needs of industry. The following are proven, cost effective applications for HDPE piping;

- Acid Lines

- Aoration

- Caustic Lines

- Chllled Water Lnes

- Cooling Water Lines

- Corrosive Wastes

- Dewatering Lines

- Drainage Lines

- Dredging Lines

- Electrical Conduit

- Fy Ash Lines

\author{
a Gas Lnes \\ - Irrigation Lnes \\ - Extractive Metallurgy \\ - Mud Lines \\ $\because$ Oil Patch \\ - Sewer Lines \\ - Sludge Lines \\ - Slurry Lines \\ - Tailing Lines \\ - Water Lines
}

Polaris polyethyene pipe is $10 \%$ to $30 \%$ lighter in weight than most other piping materials of the same size. The physical properties of Polaris polyethylene pipe make it a natural for systems in difficult terrain where conditions of harsh environment, caustic soil conditions and extremes of temperature make other piping materials impractical and at best short lived.

The flexibility of Polaris polyethylene pipe allows for installation without the use of many fittings and assembly can be done at a more suitable area for fusion. Pipe can then be pulled to installation site and dropped into trench or, if conditions are impossible for trenching, pipe can be installed above ground without fear of damage from ultraviolet attack, corrosion, rot or rust.

\section{PHYSICAL PROPERTIES DATA FOR POLARIS HIGH DENSITY, HIGH MOLECULAR WEIGHT PE 3408 POLYETHYLENE PRODUCTS.}

The material used by Polaris Pipe $C_{0}$. in the manufacturing of high density polyethylene pipe products conforms to ASTM D1248 is Type III Class C, Category 5. Grade P34. The following represents standard test values achieved at $73.4^{\circ} \mathrm{F}$ according to'standard industry practice.

\begin{tabular}{|c|c|c|}
\hline D.1505 & Density (Black Resin) & $0.955 \mathrm{~g} / \mathrm{cm}^{3}$ \\
\hline D.1505 & Density (natural resin) & $0.944 \mathrm{~g} / \mathrm{cm}^{3}$ \\
\hline D.1238 & Melt Index (Condition $\boxminus$ & $0.14 \mathrm{~g} / 10 \mathrm{~min}$ \\
\hline D.1238 & Melt Index (Condition F) & $11.0 \mathrm{~g} / 10 \mathrm{~min}$ \\
\hline D-1525. & Melting Point Cvicat softening point & $257^{\circ}$ Fahrenheit \\
\hline D.746 & Brittleness Temperature & $-180^{\circ}$ Fahrenheit \\
\hline D-696 & Thermal Expansion & $0.8 \times 10^{-4} \mathrm{in} / \mathrm{in} /{ }^{\circ} \mathrm{F}$ \\
\hline C-177' & Thermal Conductivity & $\left.3.5 \mathrm{Btu}-\mathrm{in} / \mathrm{ft}^{2}-\mathrm{hr}-{ }^{-} \mathrm{F}\right)$ \\
\hline D-638 & Tensile Strength, Yield (2 in/min) & $>3300$ psi \\
\hline D-638 & Eongation at Break & 800 percent \\
\hline D.638 & Modulus of Elasticity & 120,000 psi \\
\hline D-790 & Flexural Modulus & 140,000 psi \\
\hline D.3350 & Cell Classification & $345434 \mathrm{C}$ \\
\hline D-2837 & Hydrostatic Design Basis (HDB) $73^{\circ} / 100,000$ hrs & $1600 \mathrm{psi}$ \\
\hline D-2B37 & Hydrostatic Design Stress (HDS) $73 \% 100,000$ hrs & 800 psi \\
\hline D.2240 & Hardness-Shore D & $63-$ \\
\hline D-1693 & Environmental Stress Crack Resistance (ESCR). Condition C & $>5000$ Fzohrs \\
\hline D-1603 & Carbon Black & $2 \% \min$ \\
\hline
\end{tabular}

The information herein cortained is based upon data to be thoroughty refiable, but no quaranty or warranty with respect to acarrex, completeness, or product result is implied and no liability is assumed. Users should verthy suitabitity for their particular use or purpose of any information contained herein in view of the mamy uses of this material, and the ditferent equipment and tectniques used, we canct guarantee results in specific instances. No statement contained herein should be construed as a recommendation or ficarse to use our products in a mamer that would constitute thitiongement of amy patent. 


\section{INDUSTRIAL PLASTICS, INC.}

740 S. 28th Street - Washougal, WA $98671-2597$

Phone (206) 835-2129 or (503) 227-5588

1-800-634-5082 FAX (206) 835-3521

\section{HANFORD MIXED-WASTE DISPOSAL FACILITY 200- WEST AREA, HANFORD SITE, RICHLAND, WA}

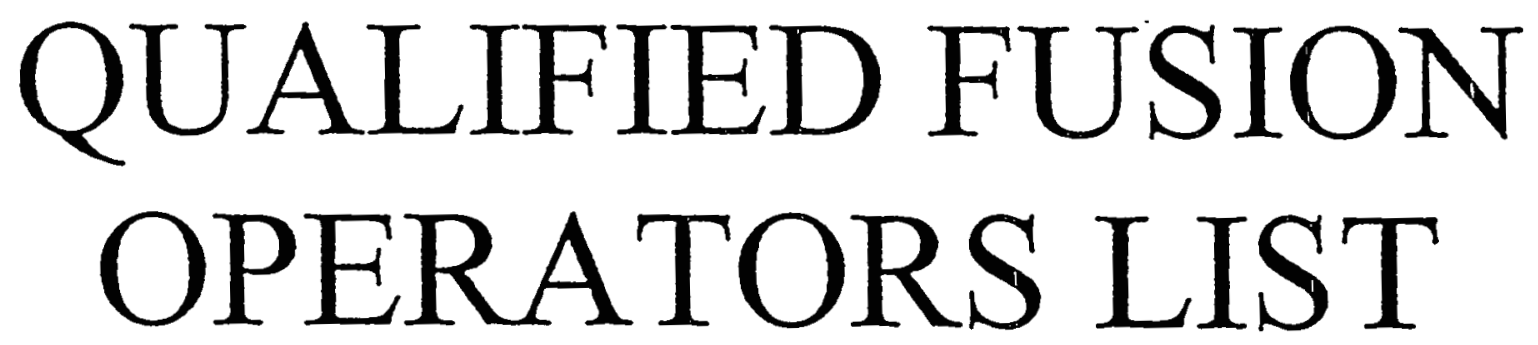




\section{WHC-SD-W025-RPT-001; Rev. 0}

TO: DOULE LRABTREE

FREIH: JIM-JALOBS

LISTEN BEOM ARE THE TEET RESULTS OK FUSIORS OH PIPE SAYPLLES

SUBKITIEI BY IKDUSTRIAL PLASTIES, IKE.

f. SDR 29 PEJ4OB BUTT FUSTOK SAMPLES

PIPE MARKIKE BURST TET GOLGGATIOH TET BET BACX IETT

OHE SPELIKEH THO SPECIYEHS - THREE SPELIMEXS

\begin{tabular}{|c|c|c|c|}
\hline STEYE HAYRICX & $\begin{array}{l}\text { PASSEI } \\
\text { PIPE FALLID }\end{array}$ & $\begin{array}{l}\text { PASSED } \\
\text { EOMGATIOH } 3100 \%\end{array}$ & PASSEI \\
\hline DAK HULAH & $\begin{array}{l}\text { PASSEI } \\
\text { PIPE FAILE }\end{array}$ & $\begin{array}{l}\text { PASSED } \\
\text { EOHEATIOK }>100 \%\end{array}$ & PASSED \\
\hline RANDY SALSBERRY & $\begin{array}{l}\text { PASSEI } \\
\text { PIPE FAILED - }\end{array}$ & $\begin{array}{l}\text { PASSE } \\
\text { EONGATIOK }>100 \%\end{array}$ & PASSEี \\
\hline ERIAK YUIRRAY & $\begin{array}{l}\text { PASSED } \\
\text { PIPE FAILED }\end{array}$ & $\begin{array}{l}\text { PASEE } \\
\text { EOKEATIOK }>100 Z\end{array}$ & PASSEI] \\
\hline RY BOYE & $\begin{array}{l}\text { PASEET } \\
\text { PIPE FAILED }\end{array}$ & $\begin{array}{l}\text { PASEבZ } \\
\text { EINGATIOH }>100 Z\end{array}$ & PASSEI \\
\hline Đ SALSBEERY & $\begin{array}{l}\text { PASSEI } \\
\text { PIPE FAILED }\end{array}$ & $\begin{array}{l}\text { PASSED } \\
\text { EDNGATIOK } 2100 \%\end{array}$ & PASSE \\
\hline RALPH FORD & $\begin{array}{l}\text { PASSED } \\
\text { PIPE FAILED }\end{array}$ & $\begin{array}{l}\text { PAS5T } \\
\text { EOHGATIOH }>100 \%\end{array}$ & PASSE] \\
\hline
\end{tabular}

LAB TIKE: 5 HRS

OATE TESTED: 20 DEE 90

TESTED BY: JIM JACUBS 


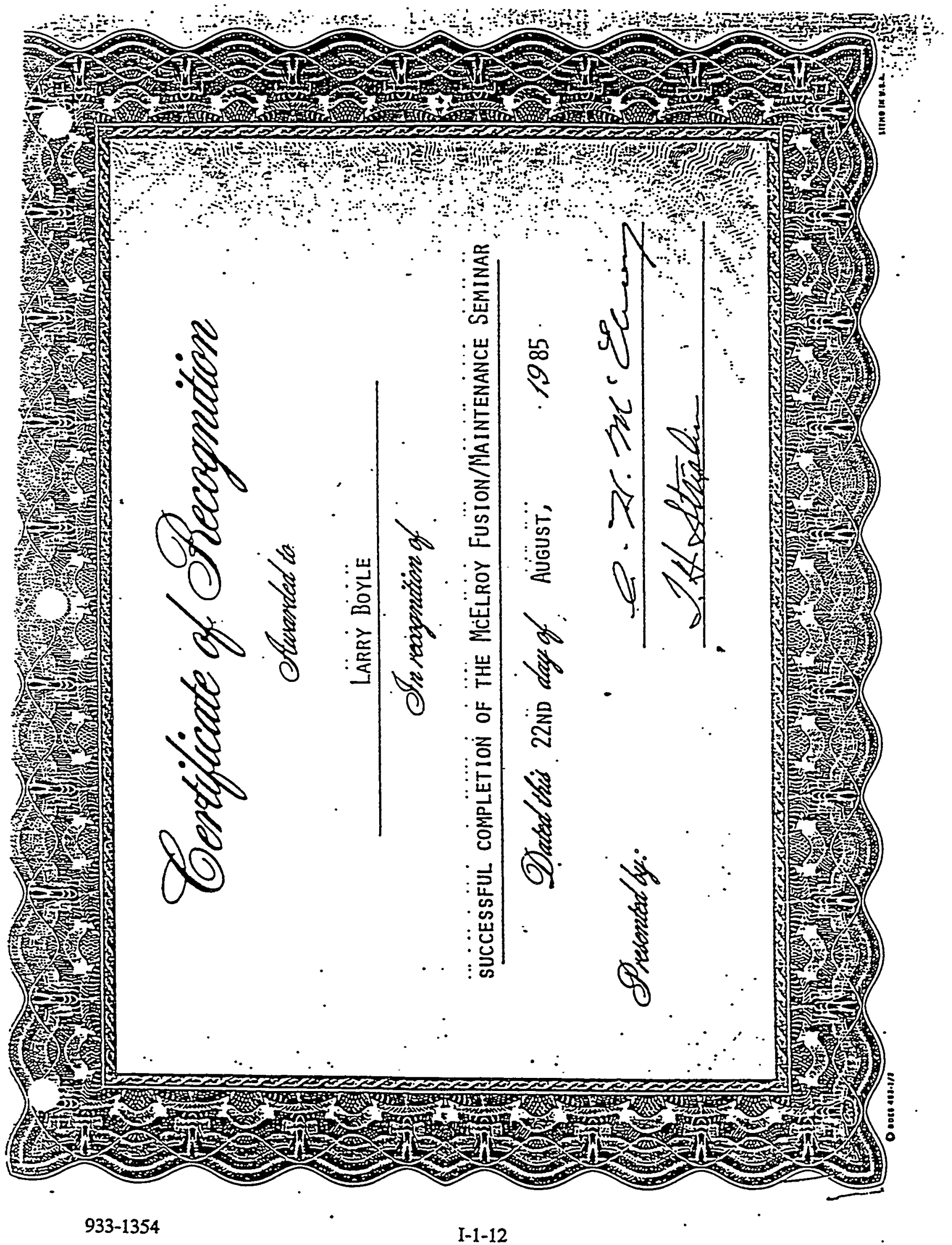




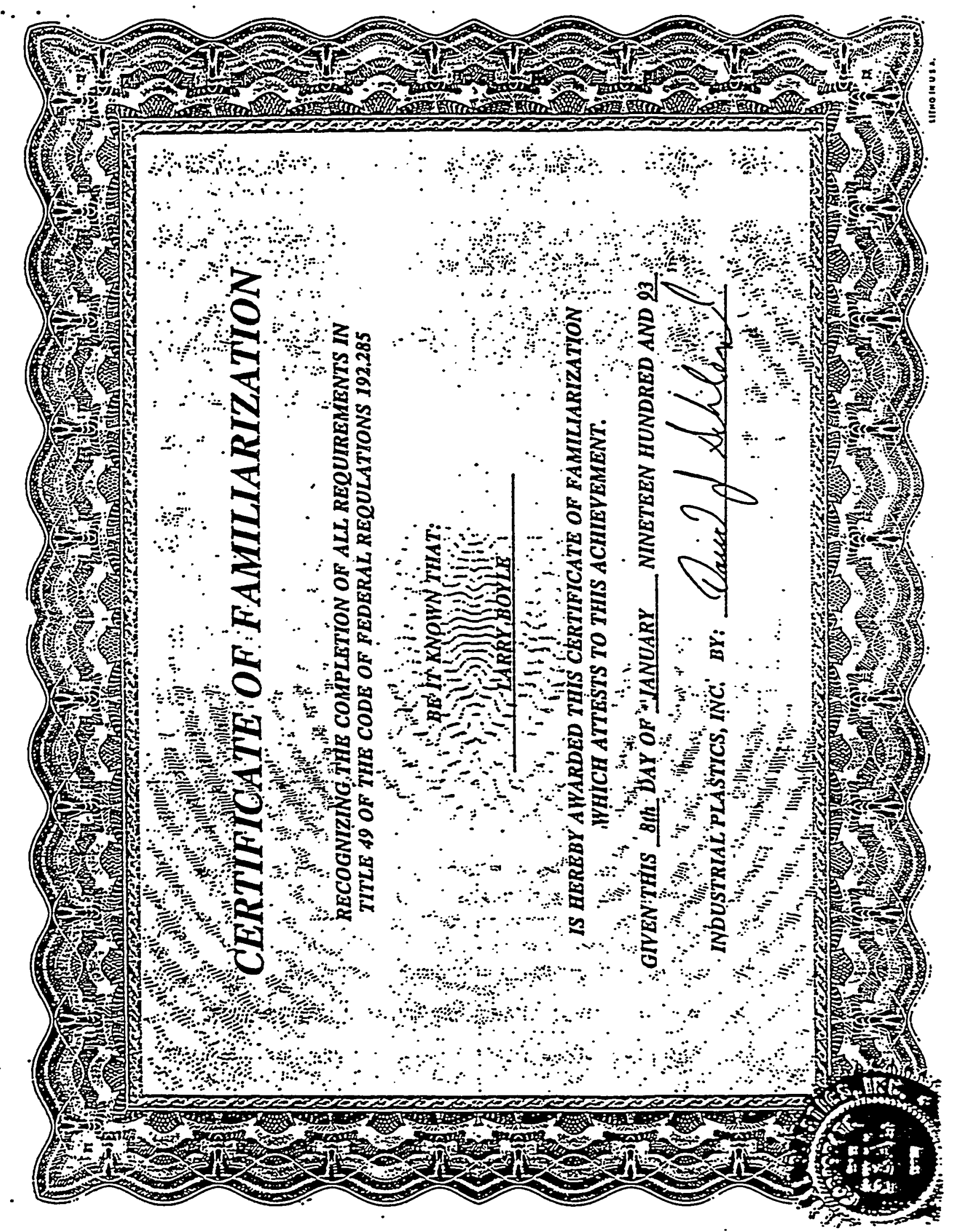




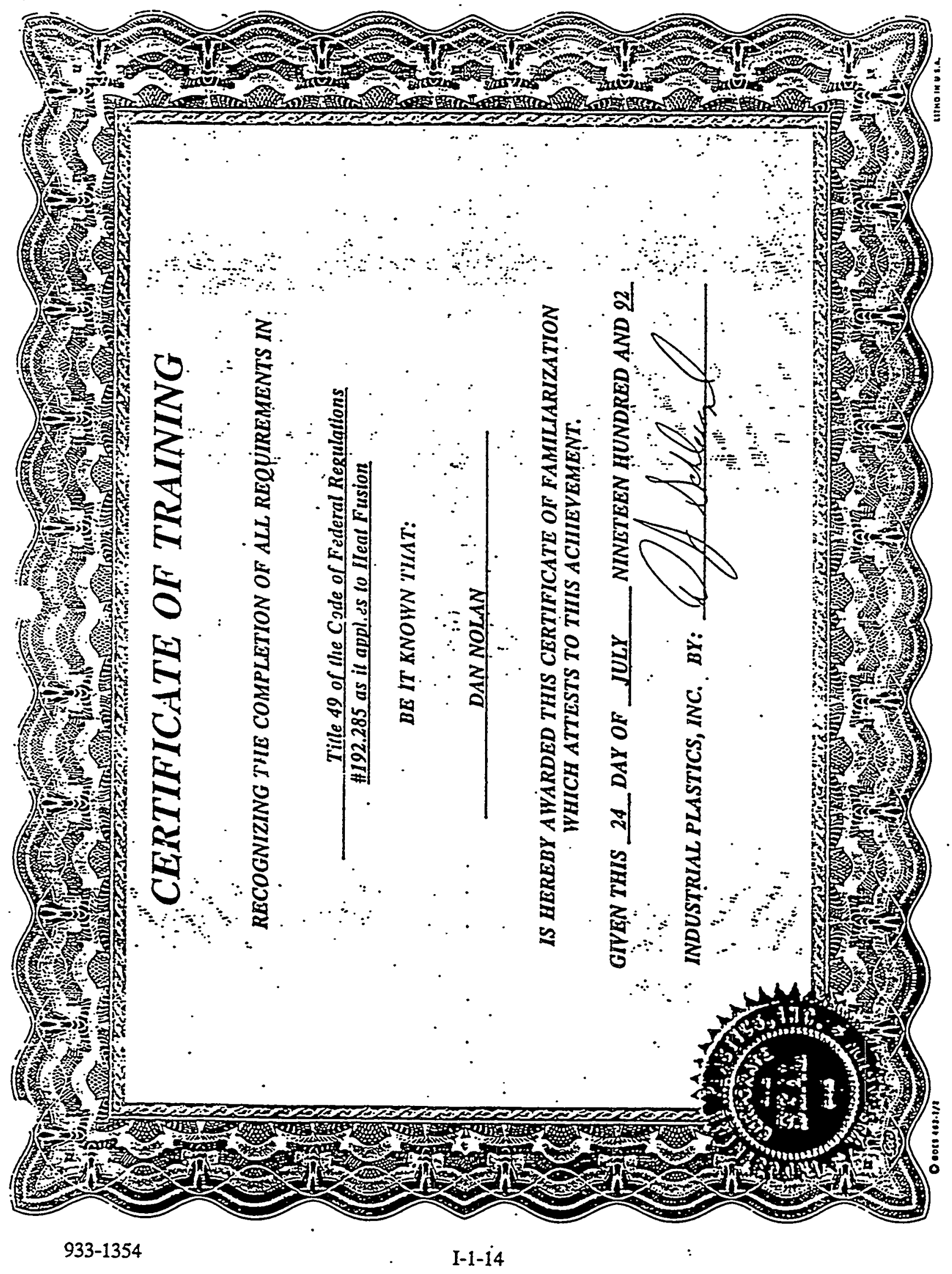




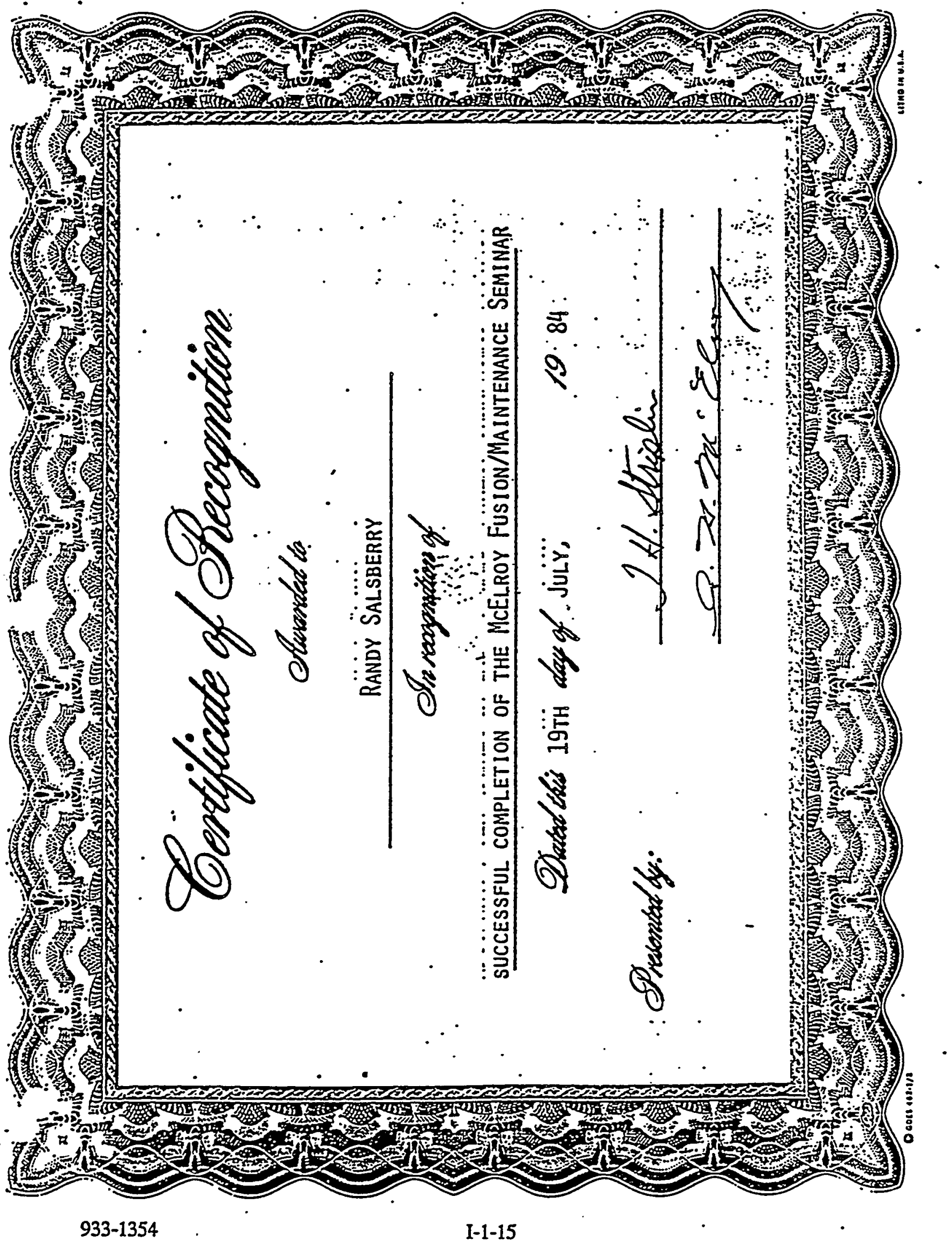




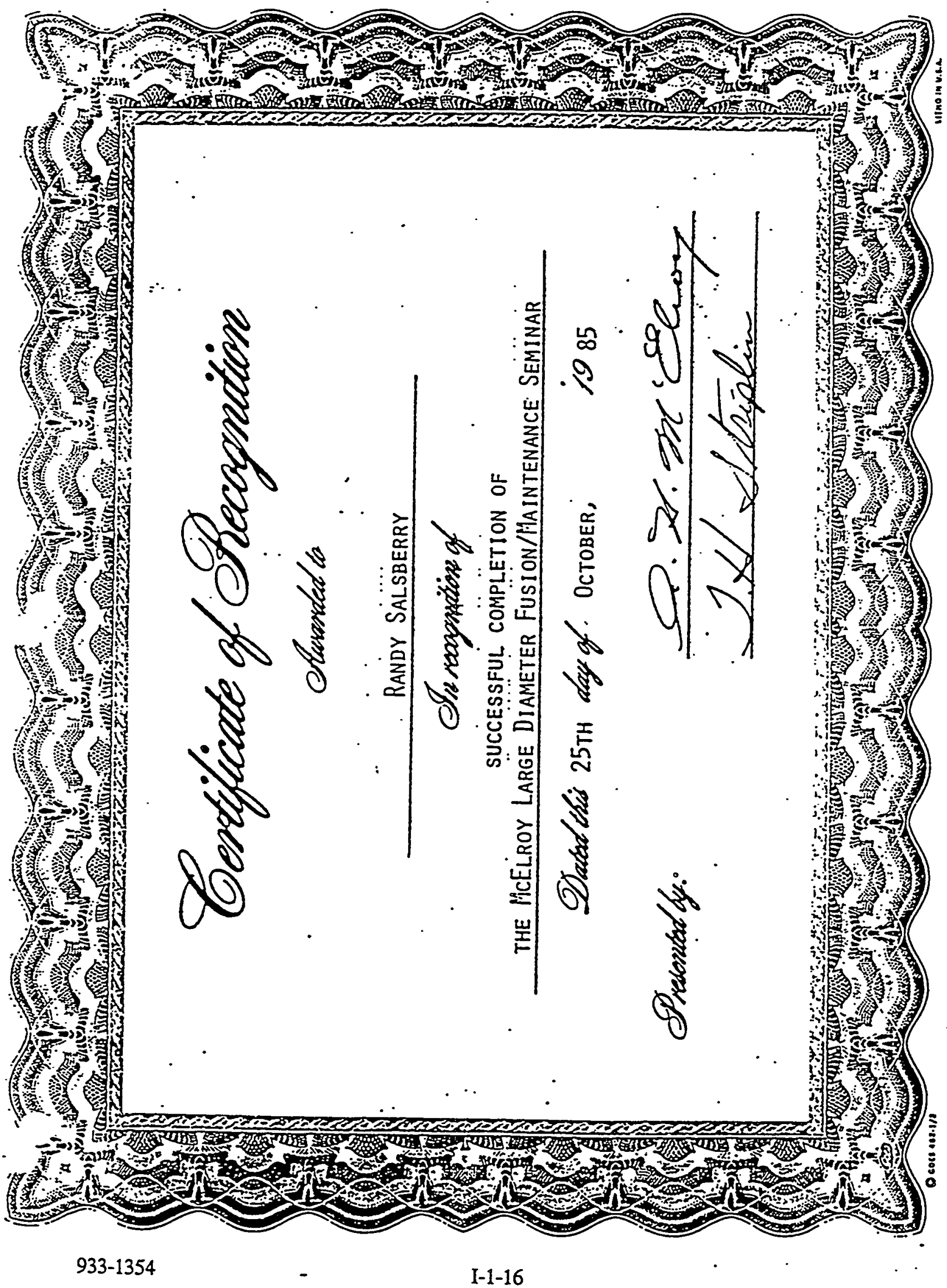




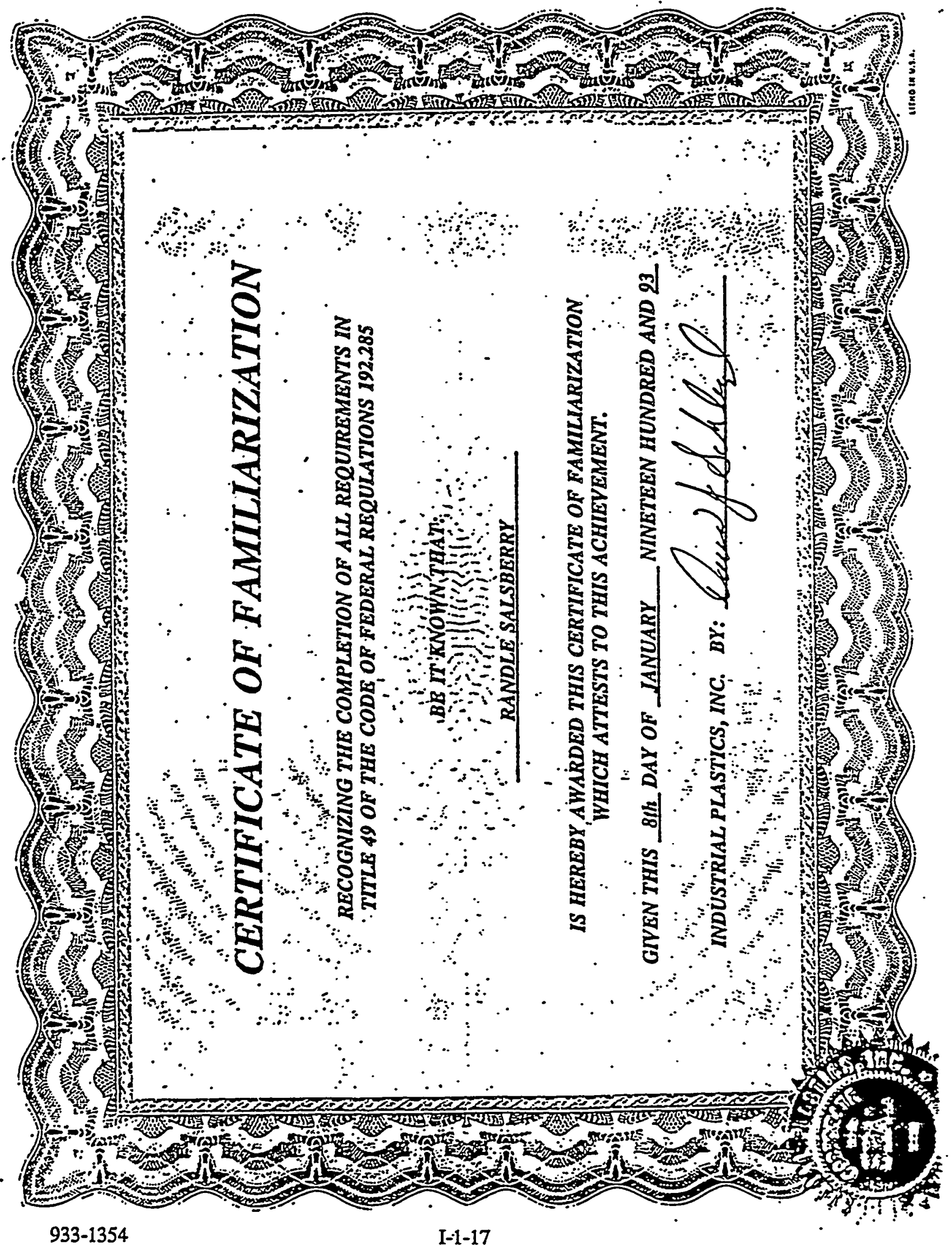


WHC-SD-W025-RPT-001, Rev. 0

\section{INDUSTRIAL PLASTICS, INC. \\ 740 S. 28th Street - Washougal, WA 98671-2597 \\ Phone (206) $835-2129$ or (503) 227.5588 \\ 1-800-634-5082 FAX (206) 835-3521}

\section{HANFORD MIXED-WASTE DISPOSAL FACILITY 200- WEST AREA, HANFORD SITE, RICHLAND, WA}

\section{QUALIFIED FUSION OPERATORS LIST}


WHC-SD-W025-RPT-001, Rev. 0

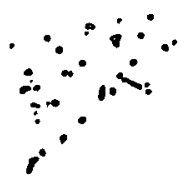

TD: DUS CRABTREE

FRIII: JIX-JALOBS

LISTEI BEOK ARE THE TEST RESLTS OK FUSIOHS DH PIPE SALPLES SUBMITIED BY IFOUSTRIAL PLASTICS, IKC.

4" SOR 21 PESHOB BITT FUSION SAYPLES

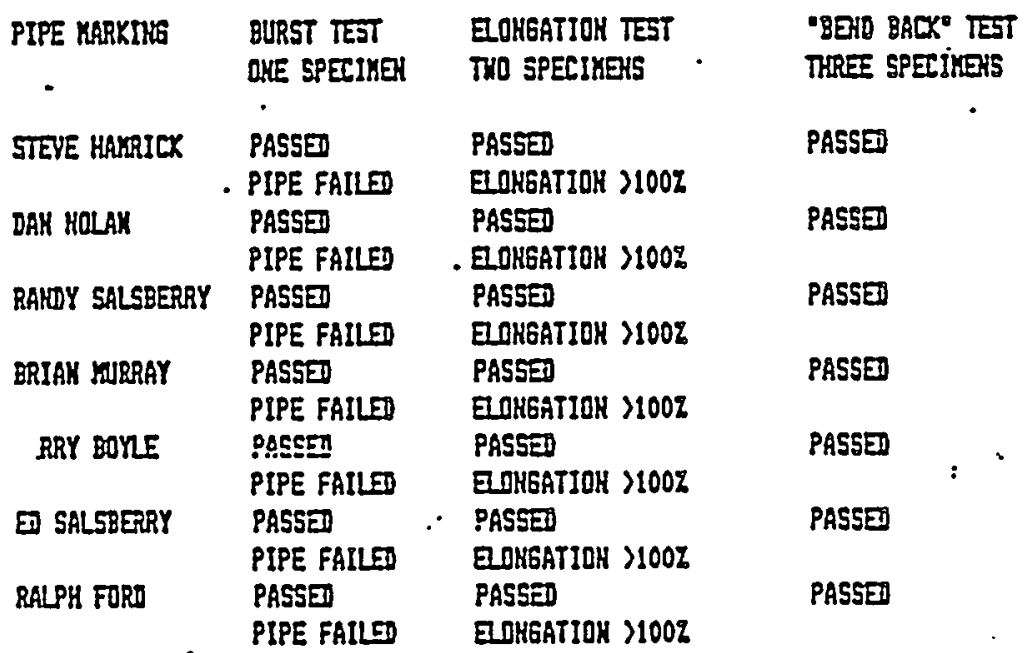

LAB TIIE: 5 YRS

DATE TETED: 20 DEC 90 TESTE BY: JIK JACORS 


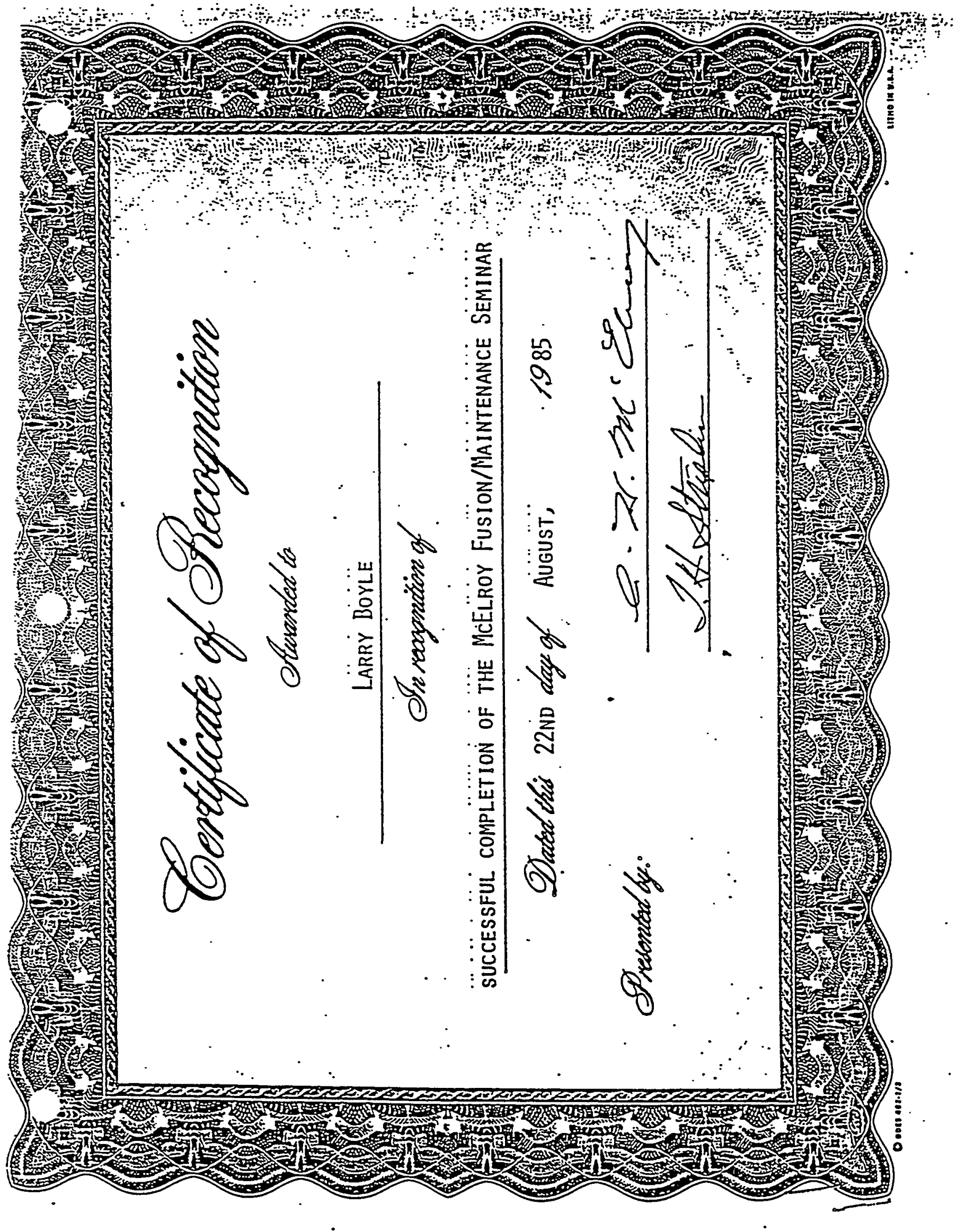

933-1354 


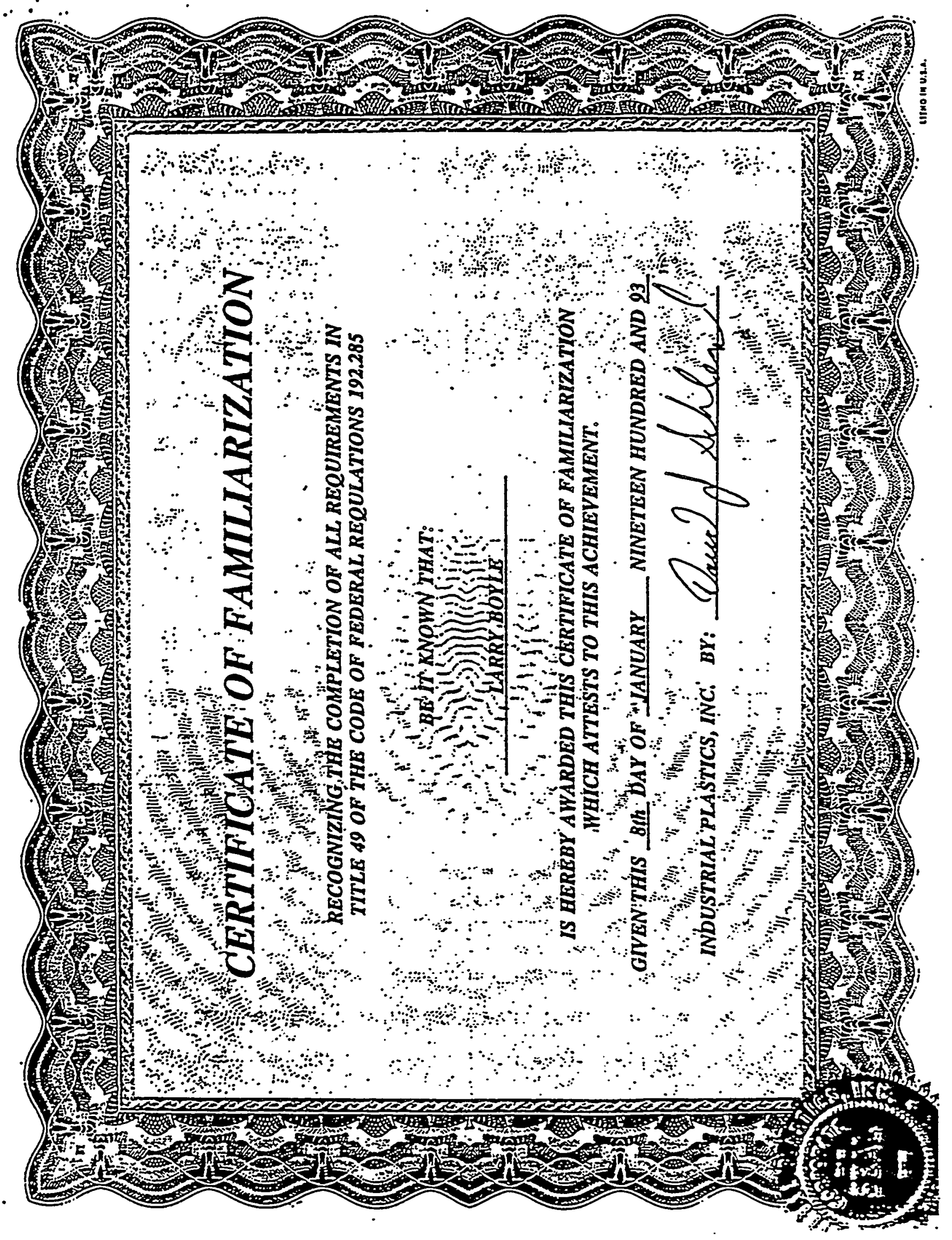




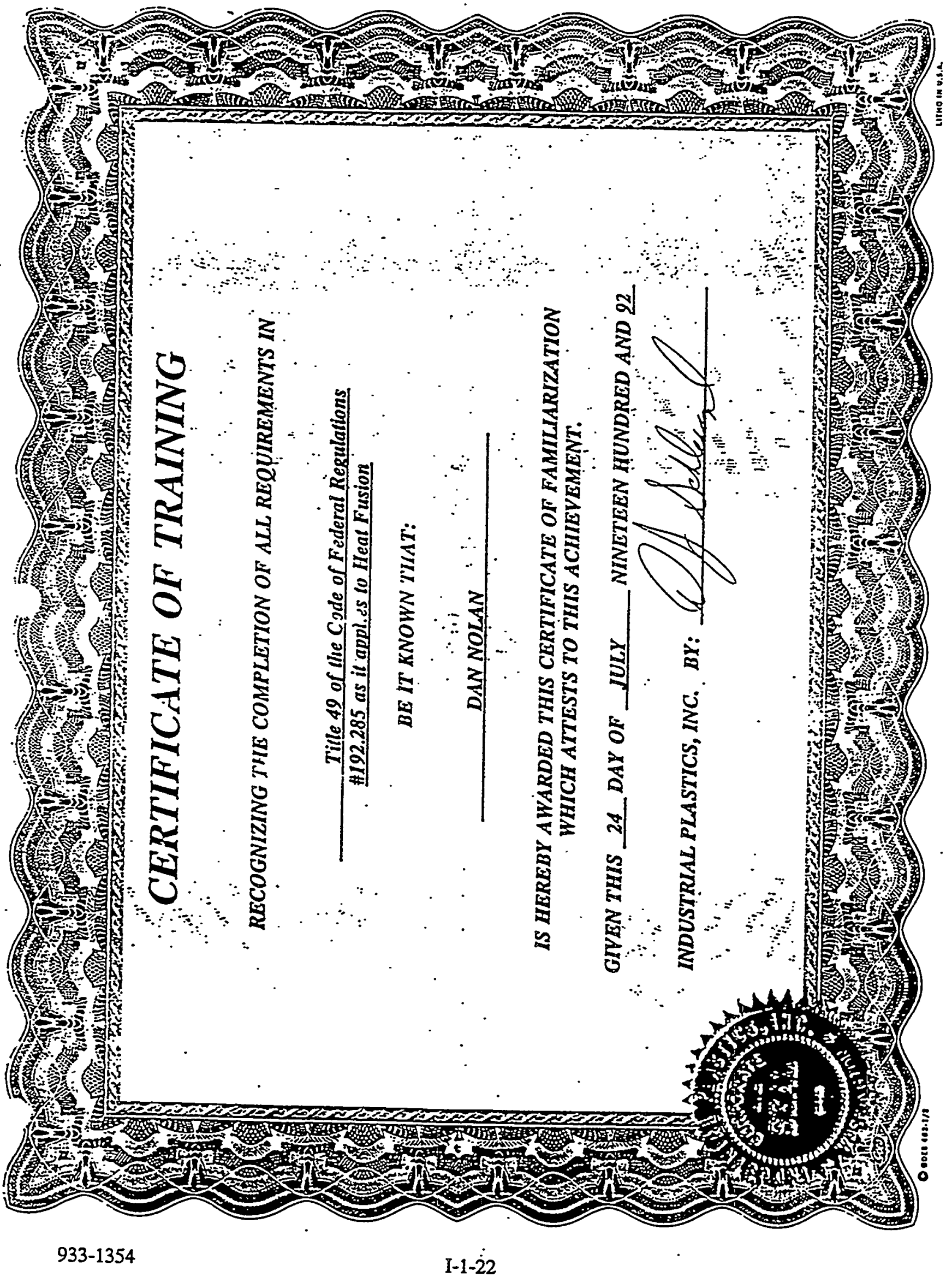




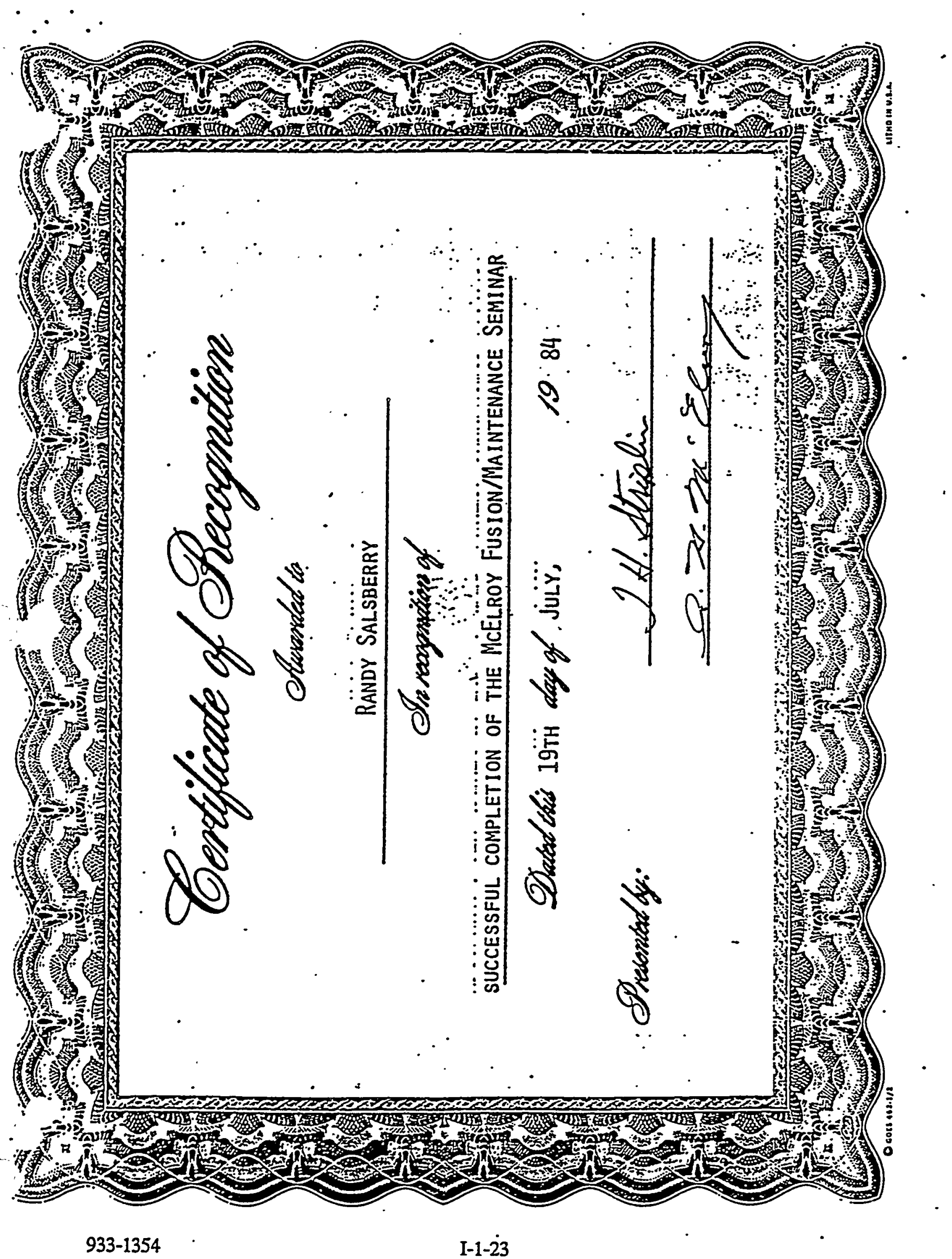




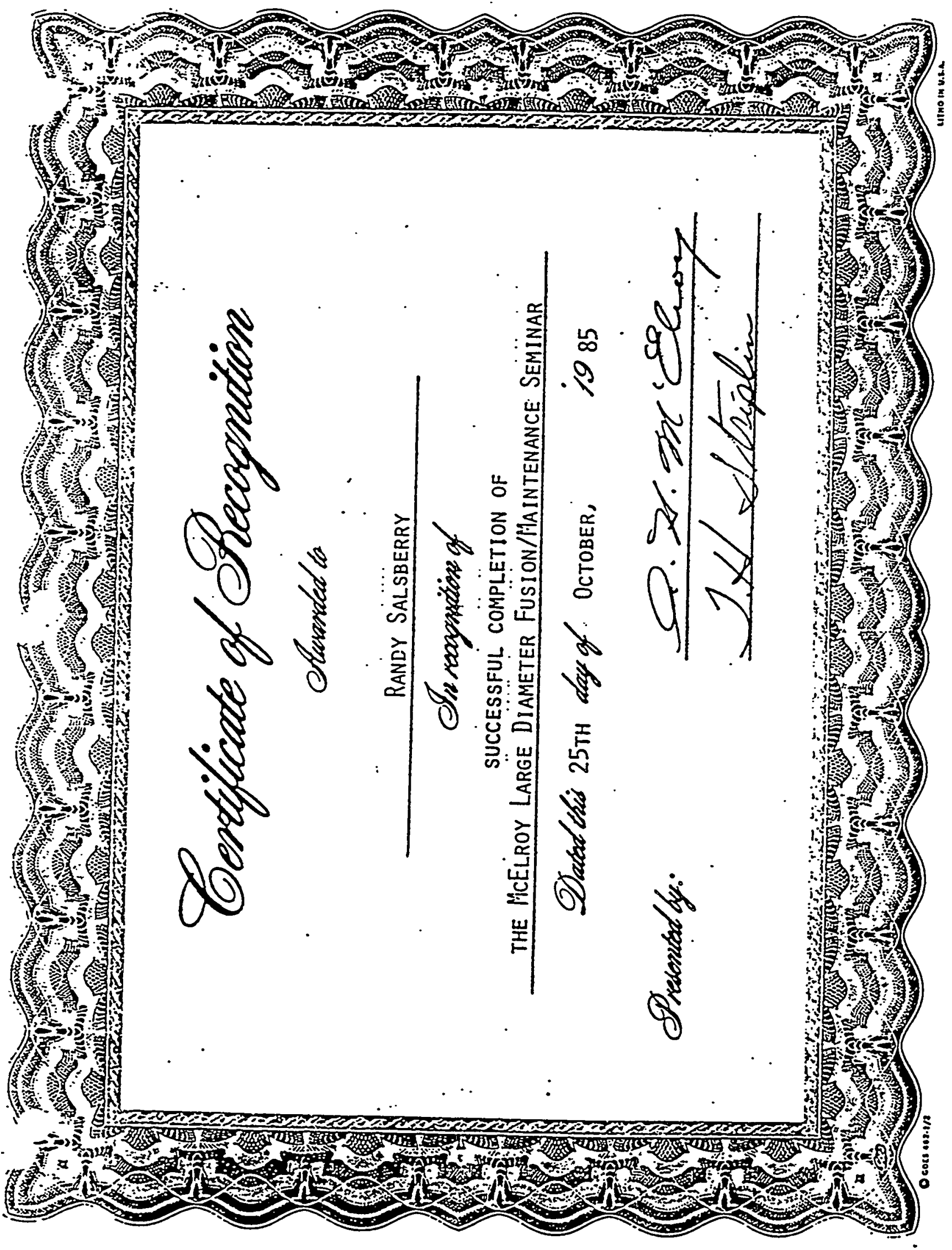




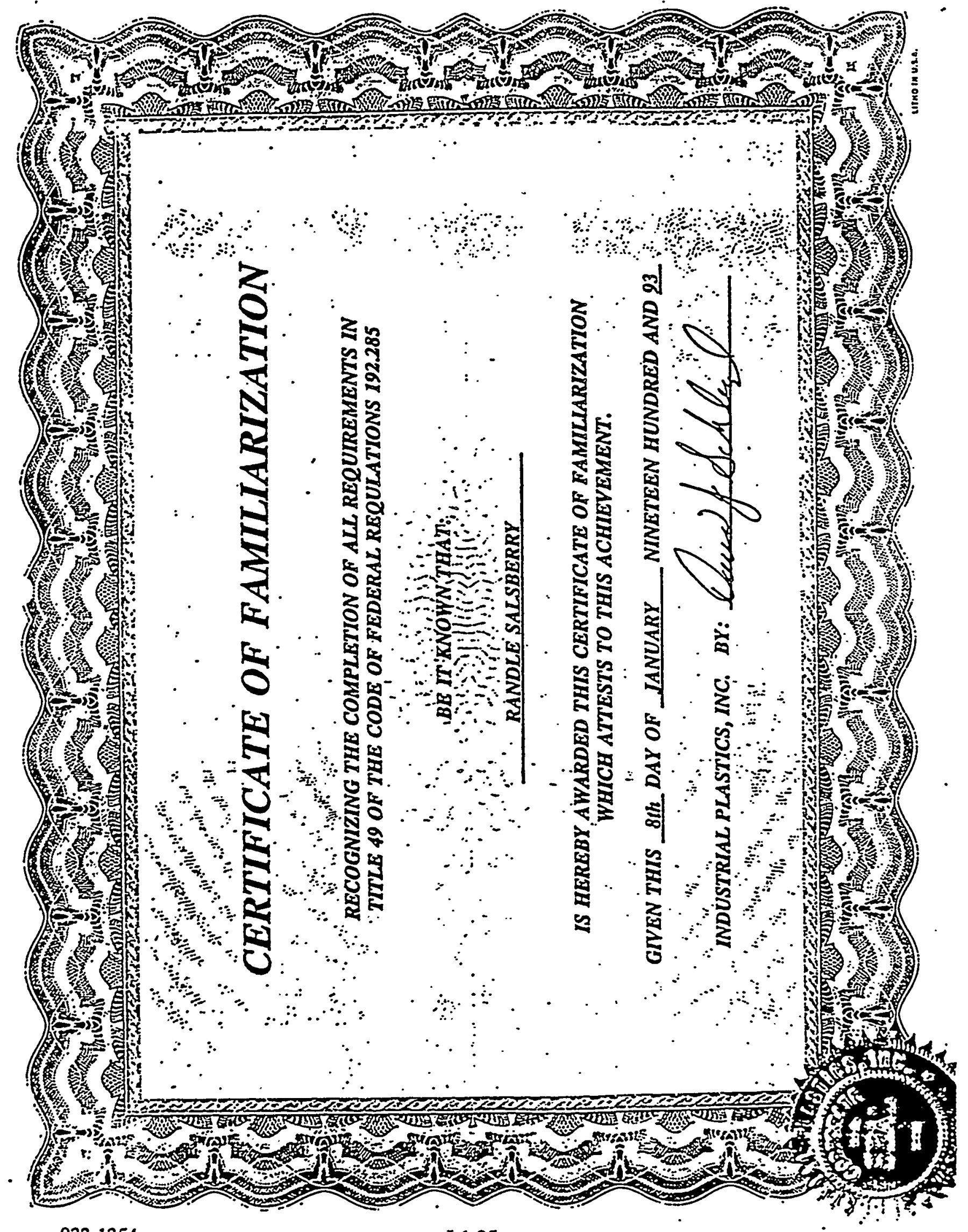


WHC-SD-W025-RPT-001, Rev. 0

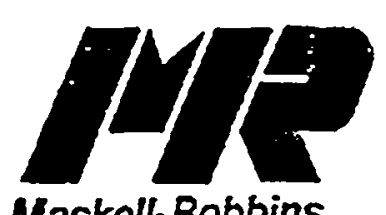

Maskel-Robbins

Date: AUGUST 1993

INCOAPOAATED

\section{MCELROY FUSION MACHINE TRAINING TO JOIN PHILLIPS DRISCOPIPE}

This Letter of Accreditation serves to attest that on the above date, Maskell-Robbins, Inc. performed complete hands-on McElroy fusion unit training for:

$\frac{\text { JOHN ELLINGWOOD }}{\text { Name }}$
$\frac{\text { DELHUR INDUSTRIES }}{\text { Company Name (if applicable) }}$

Training was given using a McEloy \# 1236 Unit on 20" SDR 21 Phillips Driscopipe 1000 (Pipe size/SDR)

Training was given using a MCEroy * Unit on Phillips Driscopipe (Pipe size/SDR)

Training was given using a McElroy \# Unit on Phillips Driscopipe

(Pipe size/SDR)

Training consisted of:

1. Procedures required to but fuse High Density Polyethylene (HDPE) pipe and fittings manufactured by Phallips Driscopipe only. No other brands of pipe were included.

2. Actual hands-on fusion by trainees unt it was deemed by the Maskell-Robbins technician that the trainee is proficient in performing proper butt fusion.

3. Fusing polyethylene pipe in accordance with Phillips Driscopipe recommendations and Title 49 of the Code of Federal Regulations 192.283, 192.285 and 192.287

4. Safety features were explained and emphasized.

This qualification will be in effect for a one year period from date of issue. At that time a refresher course will be required. This Letter of Accreditation is not a warrantee of the workmanship of the individual named above or any of the products worked upon by said individual.

Atrest:

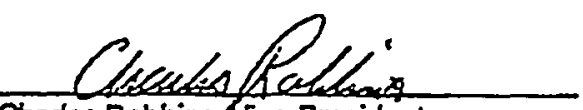

Charles Robbins, Vice-President

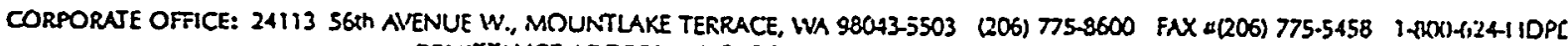
REAITYANCE ADDRESS: P.O. BOX 12590 , SEATILE, WA 98 III-4590

RECIONAL OFFICES 6 HIANGAR MAAY \$F, WAISONVILLE CA 95076 • 12450 GALVESTON ROAL, WEBSTER, TX 7598

9541 S. BACLEY PARK RD., SAIT LAKE CITY, LT 84088 • 524 W. INT'L AIRPORT RD. 4200 , ANCHORACE, AK 99518

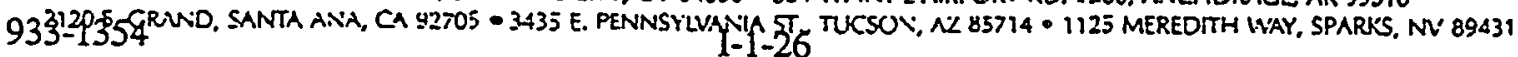


WHC-SD-W025-RPT-001, Rev. 0

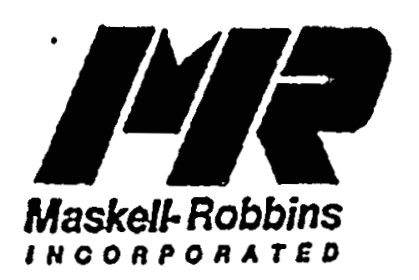

Date: Augusr 1993

\section{MCELROY FUSION MACHINE TRAINING TO JOIN PHILLLIPS DRISCOPIPE}

This Letter of Accreditation serves to attest that on the above date, Maskell-Robbins, Inc. performed complete hands-on McElroy fusion unit training for:

$\frac{\text { JOHN ELLINGWOOD }}{\text { Name }}$
$\frac{\text { DELHUR INDUSTRIES }}{\text { Company Name (fi applicable) }}$

Training was given using a McEroy * 1236 Unit on 20" SDR 2I Phillips Driscopipe 1000

- Training was given using a McElroy \# Unit on Phillips Driscopipe

(Pipe size/SDR)

Training was given using a McElroy \# Unit on Phillips Driscopipe

(Pipe size/SDR)

Training consisted of:

1. Procedures required to burt fuse High Density Polyethyiene (HDPE) pipe and fitings manufactured by Phillips Driscopipe only. No other brands of pipe were included.

2 Actual hands-on fusion by trainees until it was deemed by the Maskeli-fobbins technician that the trainee is proficient in performing proper butt fusion.

3. Fusing polyethylene pipe in accordance with Philips Driscopipe recommendations and Title 49 of the Code of Federal Regulations 192.283, 1922225 and 192.287

4. Safery features were explained and emphasized.

This qualification will be in effect for a one year period from date of issue. At that time a refresher course will be required. This Letter of Accreditation is not a warrantee of the workmanship of the - individual named above or any of the products worked upon by said individual.

Artest:

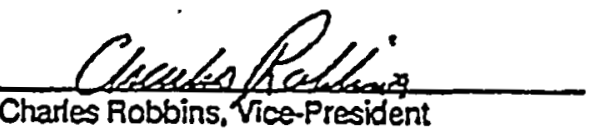

(1)

CORPORATE OFFICE: 24113 S6Th AVENUE W., MOUNTLAKE TERRACE, WA 98043-5503 (206) 775-8600 FAX 4006$) 775.5458$ 1-8011-624-110PE REMITTANCE ADDRESS: P.O. BOX 12590, SEATILL, WEA $98 \mathrm{i} 11$-4590 RECIONAL OFFCES: 6 HIANCAR WHY $2 F$, WATSONULLE CA 95076 - 1245O GALVESTON ROAL, WEBSTER, TX TI598 9541 5. BAGLEY PARK RO. S4IT LAKE CITY, LT 84098 - 524 W. INT'L AIRTORT RD. 4200 . ANCHORACE, AK 99518 933-135. S. GRND. SANTA ANA, CA 92705 - 3435 E. PENNSYLVANIA ST- TLKSON, AZ 83714 - 1125 MEREDTH IWAY, SPARKS, NV 89431 $[-1-27$ 
WHC-SD-W025-RPT-001, Rev. 0

APPENDIX I-2

STAINLESS STEEL PIPE 
4

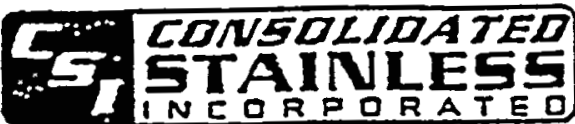

P.O. BOX 834 - DOVER, N.J. 07801

PHONE (201) 361-4014 - FAX (201) 361.3104

: CERTIFICATEOF

QUALITY CONTROL

SIZE: $2^{n}$ S.CH 40 ,

THFE: $396 / 3162$

HEAT $: 915333$

SFECIFICATION= ASTH A312-Z2A ASME SAక̈12-32A

CHEITICAL ANALYSIS $*$

\begin{tabular}{|cccccccc}
\hline$C$ & $M n$ & $F$ & $S$ & $S i$ & $N_{i}$ & $C_{r}$ & Mo \\
.018 & 1.75 & .028 & .017 & $\ddots .38$ & 11.22 & 16.36 & 2.05
\end{tabular}

- MECHanical FFOFERTIES

HARDDHESS, FIE YIELD STREHGTH,FSI ULTIMATE STRENGHT, FISI XELONG 84 48800

85500

TESTS

\begin{tabular}{|c|c|c|c|c|c|}
\hline $\begin{array}{c}\text { HYDFO, }: 5 I \\
1900\end{array}$ & $\begin{array}{l}\text { FI ATTEN } \\
\text {. OK }\end{array}$ & $\begin{array}{l}\text { FEFU. FLPTTEN } \\
\text { Ol: }\end{array}$ & $\begin{array}{l}\text { F:AFFE } \\
\text { OK }\end{array}$ & $\begin{array}{l}\text { FL finge } \\
\text { ox }\end{array}$ & $\begin{array}{l}\text { EDDY CURERENT } \\
\text {.ol }\end{array}$ \\
\hline
\end{tabular}

DATE

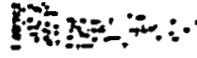




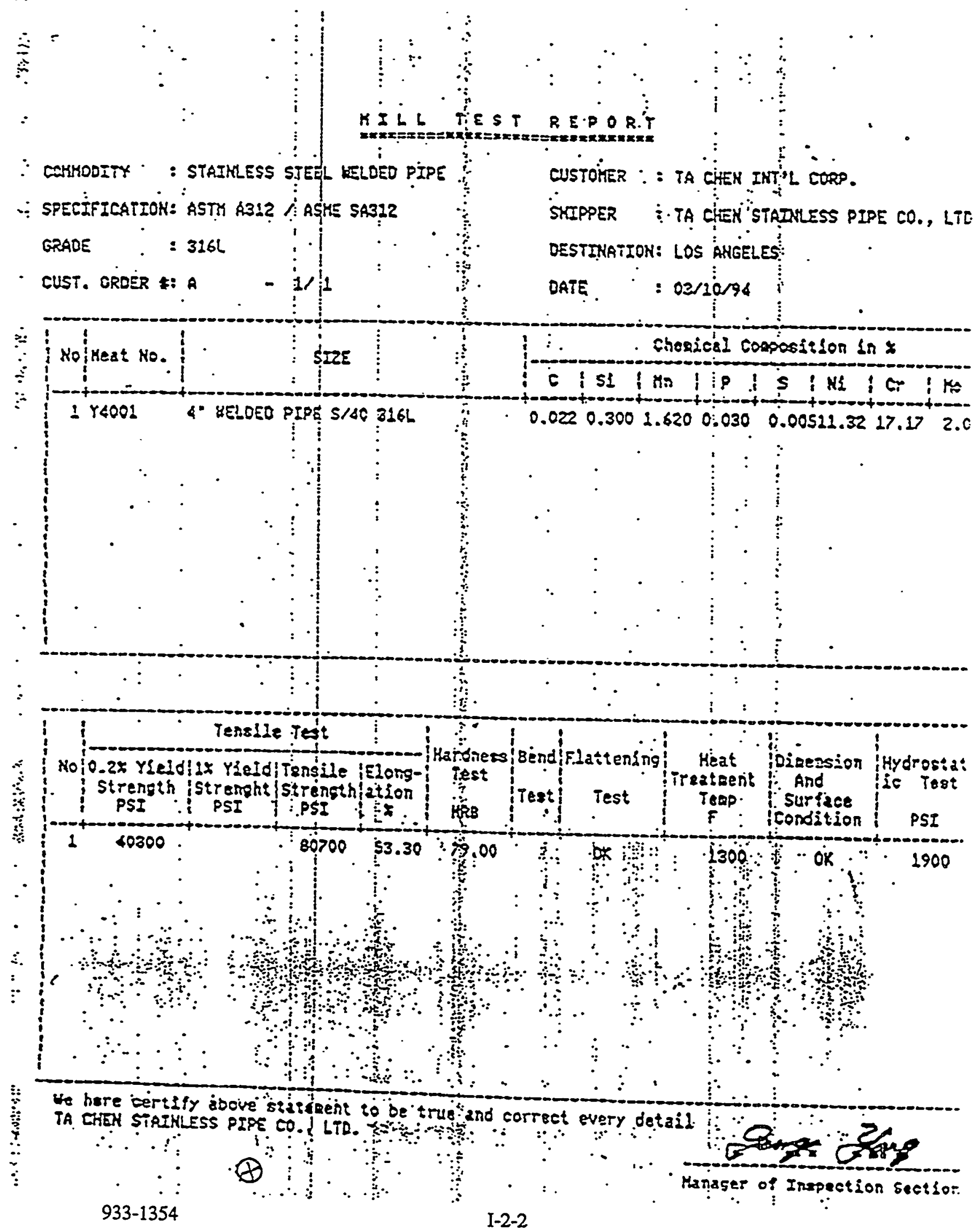


WHC-SD-H025-RPT-001, Rev。 0

APPENDIX $\mathbf{J}$

SUBGRADE AND GENERAL FILL MOISTURE-DENSITY TESTS

$j-i$ 
GOLDER CONSTRUCTION SERVICES INC.

SUMMARY OF SUBGRADE MOISTURE-DENSITY TESTS •

DEPARTHENT OF EMERGY 05/04/94

NON-DRAG OFF LANDFILL FACILITY

Page 1

HANFORD NUCLEAR RESERVATIOH

SUBGRADE

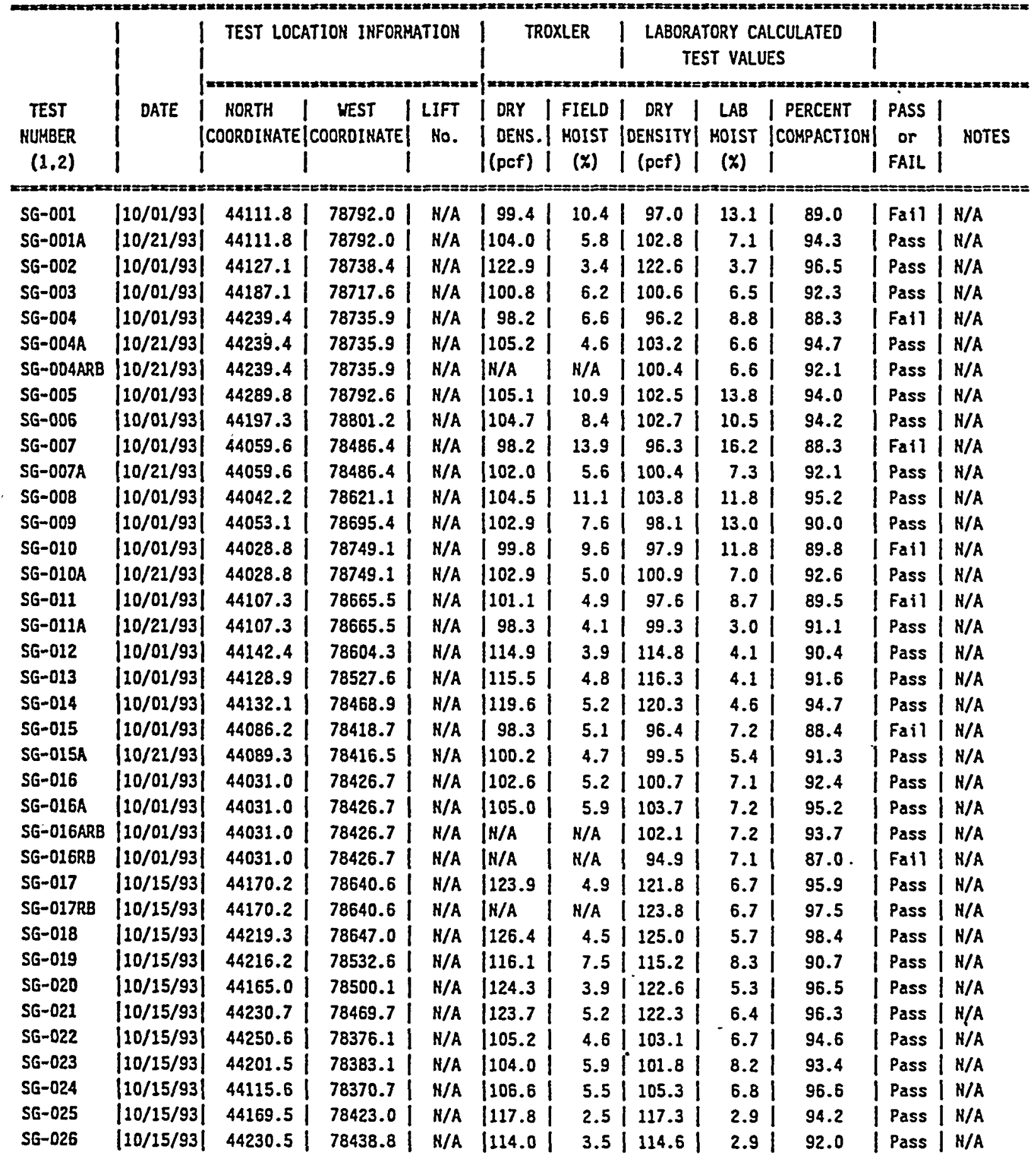


GOLDER CONSTRUCTIOH SERVICES INC. SUMMARY OF SUBGRADE MOISTURE-DENSITY TESTS DEPARTMENT OF EMERGY

NON-DRAG OFF LANDFILL FACILITY

Page 2

HAHFORD HUCLEAR RESERVATIOK

SUBGRADE

\begin{tabular}{|c|c|c|c|c|c|c|c|c|c|c|c|}
\hline & 1 & TEST LOCA & TION IKFORH & LATIOH & TRO & IXLER & $\begin{array}{r}\text { LABORA } \\
\text { TE }\end{array}$ & $\begin{array}{l}\text { ATORY CA } \\
\text { EST VALU }\end{array}$ & $\begin{array}{l}\text { ALCULATED } \\
\text { JES }\end{array}$ & 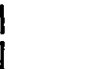 & \\
\hline & 1 & 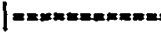 & tats & $x= \pm$ & $\mid=m w=$ & $\operatorname{mentan=0}$ & 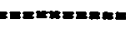 & cemmen=" & 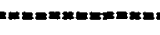 & $=m=0$ & 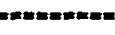 \\
\hline TEST & DATE & NORTH & WEST & LIFT & DRY & FIELD & DRY & LAB & PERCEKT & PASS & \\
\hline KUABER & 1 & |COORDINATE| & COORDIKATE & No. & DEHS. & HOIST & |DEMSITY| & MOIST & |COMPACTIOK & or & NOTES \\
\hline$(1.2)$ & 1 & 1 & & & $|(p c f)|$ & $(x)$ & $\mid$ (pcf) $\mid$ & | (x) & 1 & FAIL & \\
\hline & & & & & & & $=m=x=$ & & & & \\
\hline SG-027 & $|10 / 15 / 93|$ & 44263.6 & 78491.8 & H/A & |114.4 & 4.4 & 111.4 & 7.3 & 89.5 & Fall & H/A \\
\hline SG-027A & $|10 / 21 / 93|$ & 44263.6 & 78491.8 & H/A & 100.8 & 6.1 & 100.31 & 6.6 & 92.0 & Pass & N/A \\
\hline SG-028 & $|10 / 15 / 93|$ & 44270.4 & 78570.5 & $H / A$ & |115.5 & 2.8 & 115.3 & 3.0 & 92.6 & Pass & $H / A$ \\
\hline SG-029 & $|10 / 15 / 93|$ & 44274.9 & 78666.3 & K/A & |114.3 & 2.6 & 113.0 & 3.8 & 90.8 & Pass & H/A \\
\hline SG-030 & $|10 / 15 / 93|$ & 44309.6 & 78740.4 & $N / A$ & $|106.2|$ & 5.7 & 105.2 & 6.7 & 96.5 & Pass & H/A \\
\hline SG-031 & $|10 / 15 / 93|$ & $44314.4 \mid$ & 78623.4 & H/A & |107.9 & 5.3 & $105.9 \mid$ & 6.3 & 98.0 & Pass & $\mathrm{H} / \mathrm{A}$ \\
\hline $56-032$ & $|10 / 15 / 93|$ & 44322.4 & $78457.9 \mid$ & K/A & $|100.7|$ & 5.7 & $|100.0|$ & 6.4 & 91.7 & | Pass & K/A \\
\hline
\end{tabular}

(1) Test numbers with a suffix of " $A$ " indicate retests of falling moisture-density tests.

(2) Test numbers with a suffix of "RB" indicate rubber balloon tests. 
WHC-SD-W025-RPT-001, Rev. 0

GOLDER CONSTRUCTION SERVICES INC.

SUMHARY OF SUBGRADE MOISTURE-DEHSITY TESTS

$933-1354$

DEPARTHENT OF ENERGY

$05 / 01 / 94$

NON-DRAG OFF LANDFILL FACILITY

Page 1

HANFORD NUCLEAR RESERVATION

GEMERAL FILL SUBGRADE

\begin{tabular}{|c|c|c|c|c|c|c|c|c|c|c|c|}
\hline & 1 & \multicolumn{3}{|c|}{ TEST LOCATIOH IHFORHATIOH } & \multicolumn{2}{|c|}{ TROXLER } & \multicolumn{3}{|c|}{$\begin{array}{l}\text { LABORATORY CALCULATED } \\
\text { TEST VALUES }\end{array}$} & \multirow{2}{*}{\multicolumn{2}{|c|}{$\left.\right|_{1} ^{\mid}$}} \\
\hline & & \multirow{2}{*}{\multicolumn{9}{|c|}{ 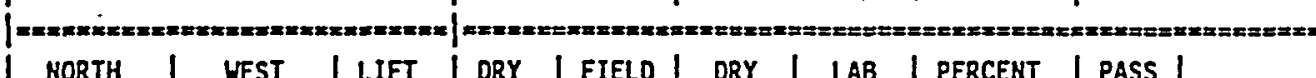 }} & \\
\hline TEST & DATE & | HORTH I & WEST $\quad$ I & LIFT & DRY & FIELD & | DRY | & LAB & | PERCENT | & | PASS & \\
\hline $\begin{array}{r}\text { NUMBER } \\
(1,2)\end{array}$ & i & |COOROINATE & COORDIMATE | & No. & $\begin{array}{l}\mid \text { DENS. } \mid \\
\mid \text { (pcf) } \mid\end{array}$ & $\begin{array}{c}\text { HOIST } \\
(x)\end{array}$ & $\begin{array}{l}\mid \text { DERSITY| } \\
\mid \text { (pcf) } \mid\end{array}$ & $\begin{array}{c}\text { MOIST } \\
(\bar{x})\end{array}$ & $\mid$ |COMPACTION| & or & $\begin{array}{l}\text { NOTES } \\
(3)\end{array}$ \\
\hline \multicolumn{12}{|c|}{ 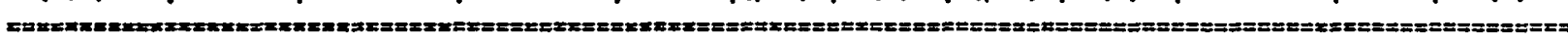 } \\
\hline GFSG-001 & $|10 / 08 / 93|$ & $|43929.4|$ & $78357.6 \mid$ & $N / A$ & $|112.8|$ & 4.0 & $|107.5|$ & 9.1 & $\mid$ & Pass | & $N / A$ \\
\hline GFSG-002 & $|10 / 08 / 93|$ & 43948.1 & 78220.1 & H/A & |108.8 & 4.1 & 106.0 & 6.9 & 97.2 & Pass & N/A \\
\hline GFSG-003 & $|10 / 08 / 93|$ & 44012.6 & 78308.2 & $H / A$ & 1104.2 & 5.7 & 102.1 & 7.8 & 93.7 & Pass & N/A \\
\hline GFSG-004 & $|10 / 08 / 93|$ & 44051.3 & 78314.1 & H/A & $\mid 110.4$ & 4.6 & 108.5 & 6.5 & 99.6 & Pass & N/A \\
\hline GFSG-005 & $|10 / 08 / 93|$ & 43984.6 & 78391.2 & N/A & |106.8 & 5.7 & 104.4 & 8.1 & 95.8 & Pass & $N / A$ \\
\hline GFSG-DOE & $|10 / 13 / 93|$ & 44112.1 & 78286.3 & H/A & 106.5 & 9.4 & 104.2 & 11.8 & 95.6 & Pass & N/A \\
\hline GFSG-007 & $|10 / 13 / 93|$ & 44178.0 & 78291.1 & H/A & |106.4 & 7.0 & 103.3 & 10.2 & 94.7 & Pass & N/A \\
\hline GFSG-008 & $|10 / 13 / 93|$ & 44253.2 & 78295.6 & H/A & ¡105.6 & 7.7 & 102.6 & 10.4 & 94.2 & Pass & $N / A$ \\
\hline GFSG-009 & $|10 / 13 / 93|$ & 44391.3 & 78733.8 & N/A & |103.4 & 11.3 & 101.1 & 13.9 & 92.7 & Pass & H/A \\
\hline GFSG-010 & $|10 / 13 / 93|$ & 44388.5 & 78632.2 & $H / A$ & 108.5 & 8.1 & 106.2 & 10.5 & 97.4 & Pass & N/A \\
\hline GFSG-011 & $|10 / 13 / 93|$ & 44382.6 & 78427.1 & H/A & |105.2 & 9.0 & 103.1 & 11.3 & 94.5 & Pass & $\mathrm{H} / \mathrm{A}$ \\
\hline GFSG-012 & $|10 / 13 / 93|$ & 44007.9 & 78467.2 & $M / A$ & ¡104.7 & 6.0 & 102.3 & 8.5 & 93.9 & Pass & N/A \\
\hline GFSG-012RB & $3|10 / 13 / 93|$ & 44007.9 & 78467.2 & H/A & IN/A & $N / A$ & 100.5 & 8.5 & 92.2 & Pass & H/A \\
\hline GFSG-013 & $|10 / 13 / 93|$ & 43982.3 & 78616.6 & $H / A$ & $|109.6|$ & 9.8 & 107.8 & 11.6 & 98.9 & Pass & $N / A$ \\
\hline GF5G-014 & $|10 / 13 / 93|$ & 44032.5 & 78798.1 & H/A & $|109.4|$ & 6.6 & 107.4 & 8.6 & 98.5 & Pass & N/A \\
\hline
\end{tabular}

(1) Test numbers with suffixes of RB indicate rubber balloon tests.

(2) See General Fill test no. GF-009RB for the rubber balloon test performed on 10/08/93. 
GOLDER CONSTRUCTION SERVICES INC.

SUMMARY OF GENERAL FILL MOISTURE-DENSITY TESTS • DEPARTMENT OF ENERGY 05/01/94

NON-DRAG OFF LANDFILL FACILITY $\quad$ Page 1

HANFORD NUCLEAR RESERVATION

GENERAL FILL

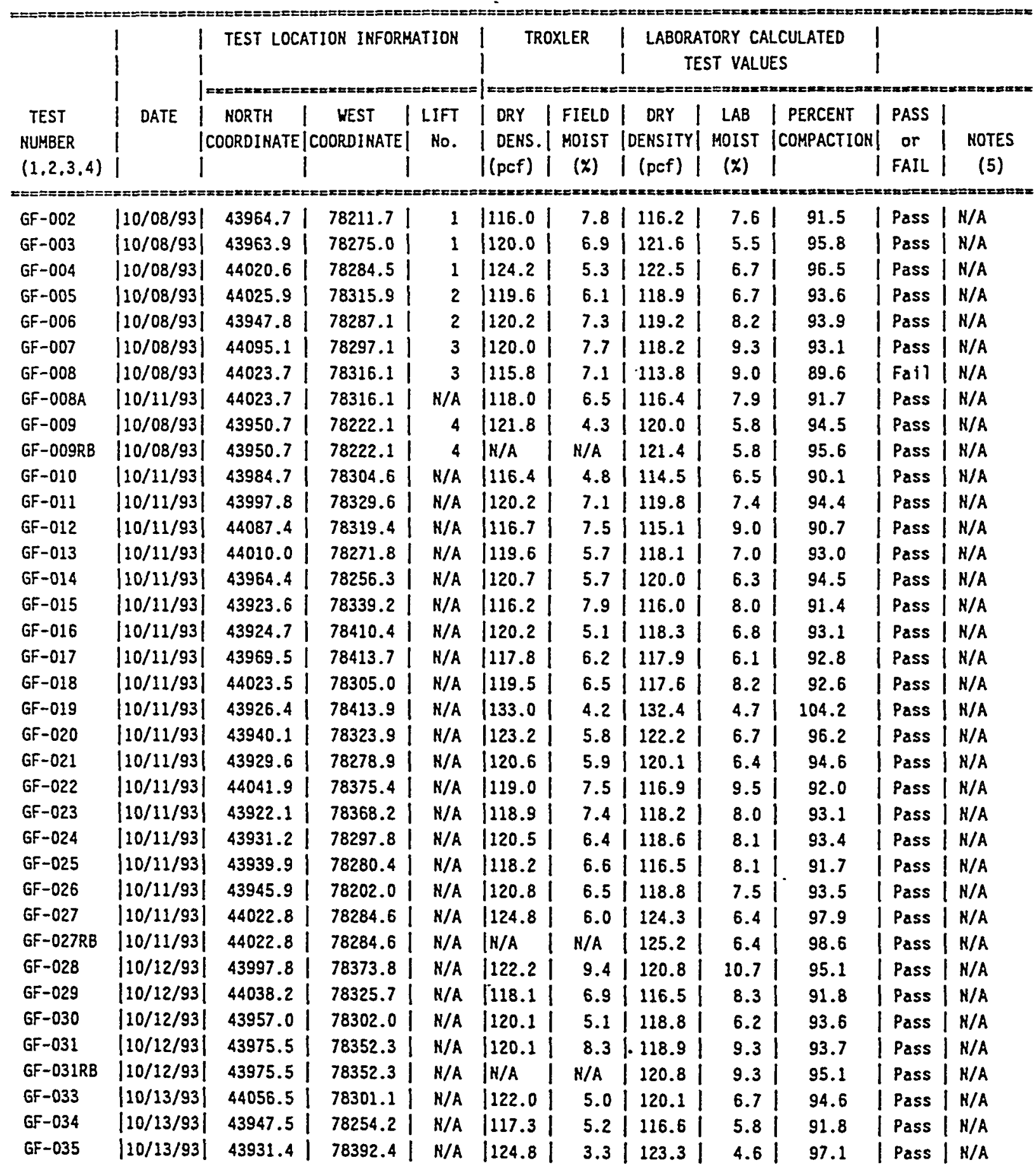


GOLOER COHSTRUCTIOH SERVICES IHC.

SURAARY OF GENERAL FILL HOISTURE-DEHSITY TESTS 933-1354 DEPARTHEMT OF EMERGY 05/01/94

MOH-DRAG OFF LAMDFILL FACILITY Page 2

HAHFORD HUCLEAR RESERVATIOH'

GEKERAL FILL

\begin{tabular}{|c|c|c|c|c|c|c|c|c|c|c|c|}
\hline \multirow[b]{2}{*}{$\begin{array}{l}\text { TEST } \\
\text { MURBER } \\
(1,2,3,4)\end{array}$} & \multirow[b]{2}{*}{ OATE } & \multicolumn{3}{|c|}{$\begin{array}{l}\text { TEST LOCATIOH IKFORHATIOK } \\
\text { | }\end{array}$} & \multicolumn{2}{|c|}{ TROXLER } & \multicolumn{3}{|c|}{$\begin{array}{l}\text { LABORATORY CALCULATED } \\
\text { TEST VALUES }\end{array}$} & \multicolumn{2}{|c|}{ | } \\
\hline & & KORTH $\mid$ & $\begin{array}{c}\text { WEST | } \\
\text { COORDIKATE }\end{array}$ & $\begin{array}{l}\text { LIFT } \\
\text { Ho. }\end{array}$ & $\left|\begin{array}{l}\text { ORY } \\
\mid \text { OEKS. } \\
\mid \text { (pcf) }\end{array}\right|$ & $\begin{array}{l}\text { FIELD } \\
\text { HOIST } \\
(x)\end{array}$ & $\begin{array}{l}\text { DRY } \mid \\
\mid \text { DENSITY } \mid \\
\mid \text { (pcf) } \mid\end{array}$ & $\begin{array}{l}\text { LAB } \\
\text { MOIST } \\
(x)\end{array}$ & $\begin{array}{l}\text { | PERCEHT } \\
\text { | СОМPACTIOK] }\end{array}$ & PASS & $\begin{array}{l}\text { HOTES } \\
\text { (5) }\end{array}$ \\
\hline$G F-036$ & $|10 / 13 / 93|$ & 44161.8 & 78276.0 & H/A & |123.1 | & 5.4 & 121.8 & 6.6 & 95.9 & Pass & M/A \\
\hline $6 F-037$ & $|10 / 13 / 93|$ & 44255.0 & 78272.5 & $H / A$ & |23.9 & 6.5 & 123.2 & 7.8 & 97.0 & Pass & K/A \\
\hline GF-038 & $|10 / 13 / 93|$ & 44377.9 & 78367.9 & $\mathrm{H} / \mathrm{A}$ & 122.4 & 6.1 & 119.9 & 8.3 & 94.4 & Pass & $H / A$ \\
\hline GF-039 & $|10 / 13 / 93|$ & 44393.3 & 78738.6 & $\mathrm{H} / \mathrm{A}$ & |122.6 & 3.1 & 122.1 & 3.4 & 96.2 & Pass & $\mathrm{H} / \mathrm{A}$ \\
\hline$G F-040$ & $|10 / 13 / 93|$ & 44388.6 & 78621.4 & $\mathrm{H} / \mathrm{A}$ & |121.8 & 4.0 & 120.9 & 4.8 & 95.2 & Pass & $B / A$ \\
\hline GF-041 & $|10 / 13 / 93|$ & 44222.2 & 78309.2 & $H / A$ & |125.1 & 4.4 & 124.5 & 4.9 & 98.0 & Pass & $\mathrm{M} / \mathrm{A}$ \\
\hline$G F-042$ & $|10 / 13 / 93|$ & 44377.8 & 78623.3 & $\mathrm{H} / \mathrm{A}$ & |107.5 & 4.0 & 104.9 & 6.7 & 96.2 & Pass & K/A \\
\hline$G F-043$ & $|10 / 13 / 93|$ & 44360.5 & $78446.2^{\circ}$ & $M / A$ & |107.8 & 7.6 & 106.4 & 9.0 & 97.6 & Pass & H/A \\
\hline $6 F-044$ & $|10 / 14 / 93|$ & 44071.6 & 78746.3 & H/A & |110.6 & 4.3 & 108.6 & 6.3 & 99.6 & Pass & $H / A$ \\
\hline$G F-045$ & $|10 / 14 / 93|$ & 43980.0 & 78597.5 & H/A & 104.3 & 3.6 & 102.5 & 5.5 & 94.0 & Pass & H/A \\
\hline GF-046 & $|10 / 14 / 93|$ & 43993.9 & 78584.5 & $\mathrm{H} / \mathrm{A}$ & 1116.0 & 3.3 & 114.5 & 4.7 & 90.2 & Pass & $\mathrm{H} / \mathrm{A}$ \\
\hline$G F-047$ & $|10 / 14 / 93|$ & 43973.3 & 78785.5 & $\mathrm{H} / \mathrm{A}$ & |106.5 & 3.5 & 104.9 & 5.1 & 96.3 & Pass & H/A \\
\hline GF- 048 & $|10 / 14 / 93|$ & 43996.7 & 78861.7 & $H / A$ & 105.2 & 4.1 & 103.9 & 5.4 & 95.3 & Pass & H/A \\
\hline GF-04BRB & $|10 / 14 / 93|$ & 43996.7 & 78861.7 & H/A & IN/A & $K / A$ & 101.0 & 5.4 & 92.7 & Pass & $H / A$ \\
\hline$G F-049$ & $|01 / 31 / 94|$ & 44044.9 & 78355.6 & 1 & |104.1 & 4.3 & 203.0 & 5.4 & 94.5 & Pass & H/A \\
\hline GF-050 & $|01 / 31 / 94|$ & 43984.7 & 78357.2 & 2 & |108.1 & 4.8 & 105.6 & 7.3 & 96.9 & Pass & H/A \\
\hline $6 F-051$ & $|01 / 31 / 94|$ & 44025.7 & 78405.9 & 3 & $\mid 105.4$ & 6.2 & 106.8 & 5.8 & 98.0 & Pass & $\mathrm{H} / \mathrm{A}$ \\
\hline GF-052 & $|01 / 31 / 94|$ & 44052.0 & 78390.5 & 4 & |104.3 & 4.2 & 102.8 & 5.7 & 94.3 & Pass & N/A \\
\hline GF-052RB & $|01 / 31 / 94|$ & 44052.0 & 78390.5 & 4 & IK/A & $N / A$ & 201.4 & 5.7 & 93.0 & Pass & $\mathrm{H} / \mathrm{A}$ \\
\hline$G F-053$ & $|02 / 09 / 94|$ & 44200.9 & 78354.1 & $H / A$ & 100.3 & 3.5 & 98.8 & 5.2 & 90.6 & Pass & $\mathrm{H} / \mathrm{A}$ \\
\hline GF-054 & $|02 / 09 / 94|$ & 44211.5 & 78348.5 & $\mathrm{H} / \mathrm{A}$ & |102.4 & 2.7 & 99.8 & 5.4 & 91.6 & Pass & $\mathrm{H} / \mathrm{A}$ \\
\hline GF-054RB & $|02 / 09 / 94|$ & 44211.5 & 78348.5 & H/A & $\begin{array}{l}98.3 \\
\end{array}$ & $H / A$ & 98.3 & 5.4 & 90.2 & Pass & H/A \\
\hline$G F-055$ & $|03 / 02 / 94|$ & 43982.2 & 78325.1 & $H / A$ & |114.5 & 5.0 & 112.7 & 6.7 & 90.5 & Pass & 5 \\
\hline GF-055RB & $|03 / 02 / 94|$ & 43982.2 & 78325.1 & $\mathrm{H} / \mathrm{A}$ & i112.0 & $M / A$ & 112.3 & 6.7 & 90.2 & Pass & 5 \\
\hline$G F-056$ & $|03 / 07 / 94|$ & 44060.1 & 78323.2 & 1 & |104.9 & 6.4 & 111.6 & $M / A$ & 102.4 & Pass & 5 \\
\hline 6F-056RB & $|03 / 07 / 94|$ & 44060.1 & 78323.2 & 1 & in/A & $H / A$ & 108.4 & H/A & 99.4 & Pass & 5 \\
\hline $6 F-057$ & $|03 / 11 / 94|$ & 44247.4 & 78355.0 & 1 & 100.7 & 1.5 & 99.3 & 2.9 & 91.1 & Pass & 5 \\
\hline GF-057RB & $|03 / 11 / 94|$ & 44247.4 & 78355.0 & 1 & 99.0 & H/A & 98.9 & 2.9 & 90.8 & Pass & 5 \\
\hline $\mathrm{GF}-05 \mathrm{~B}$ & $|03 / 23 / 94|$ & 44022.4 & 78434.3 & 1 & |106.2 & 3.3 & 105.6 & 3.8 & 96.9 & Pass & 5 \\
\hline GF-058RB & $|03 / 23 / 94|$ & 44022.4 & 78434.3 & 1 & IH/A & $N / A$ & $107.6 i$ & 3.8 & 98.7 & Pass & 5 \\
\hline
\end{tabular}


WHC-SD-W025-RPT-001, Rev. 0

NOTES

$== \pm=$

(1) Test numbers with suffix of " $A$ " indicate retests of failing moisture-density tests.

(2) Test numbers with suffixes of RB indicate rubber balloon tests.

(3) See General F1ll Subgrade test no. GFSG-012RB for rubber balloon test performed on 10/13/93.

(4) Test numbers GF-001 and GF-032 were assigned to laboratory tested samples of general fill materials.

(5) Rubber balloon verification tests were performed on general fill materials on days during which mo isture-density tests were performed on Top Course material. 
WHC-SD-W025-RPT-001, Rev。O

APPENDIX K

SOIL LINER MOISTURE-DENSITY TESTS

$k-i$ 
WHC-SD-W025-RPT-001, Rev. 0

\section{APPENDIX K-1}

SECONDARY SOIL LINER

$k-1$ 
GOLDER CONSTRUCTION SERVICES INC.

SUMMARY OF SOIL LIMER MOISTURE-DENSITY TESTS

933-1354

DEPARTHENT OF EMERGY

NON-DRAG OFF LANDFILL FACILITY

05/01/94

HAMFORD MUCLEAR RESERVATION

Page 1 SECONDARY SOIL LIMER

\begin{tabular}{|c|c|c|c|c|c|c|c|c|c|c|c|}
\hline \multirow[b]{2}{*}{$\begin{array}{l}\text { TEST } \\
\text { MUMBER } \\
(1,2,3)\end{array}$} & \multirow[b]{2}{*}{ DATE } & \multicolumn{3}{|c|}{ TEST LOCATIOK IMFORMATION } & \multicolumn{2}{|c|}{ TROXLER } & \multicolumn{3}{|c|}{$\begin{array}{l}\text { I LABORATORY CALCULATED } \\
\text { I TEST VALUES }\end{array}$} & \multicolumn{2}{|c|}{$!$} \\
\hline & & $\begin{array}{l}\text { HORTH } \\
\mid \text { COORDINATE }\end{array}$ & $\begin{array}{l}\text { WEST I } \\
\text { |COORDIHATE| }\end{array}$ & $\begin{array}{l}\text { LIFT } \\
\text { Ho. }\end{array}$ & $\begin{array}{l}\text { | ORY } \\
\text { | DENS. } \\
\text { I (pcf) }\end{array}$ & $\begin{array}{c}\text { FIELD } \\
\text { MOIST } \\
(x)\end{array}$ & $\begin{array}{l}\left|\begin{array}{l}\text { ORY } \\
\text { ODERSITY| } \\
\mid \text { (pcf) }\end{array}\right|\end{array}$ & $\begin{array}{c}\text { LAB } \\
\text { MOIST } \\
(x)\end{array}$ & $\begin{array}{l}\text { I PERCENT } \\
\text { | COMPACTION }\end{array}$ & $\left\{\begin{array}{l}\text { PASS } \\
\text { or } \\
\text { FAIL }\end{array}\right.$ & $\begin{array}{l}\text { NOTES } \\
\text { (4) }\end{array}$ \\
\hline SL-001 & $1 / 10 / 30 / 931$ & 843080 & $78739 ?$ & 1 & 097 & $30 ?$ & 002 & 101 & 018 & & No \\
\hline SL-002 & $|10 / 30 / 93|$ & 44262.3 & 78707.2 & 1 & 101.1 & 20.6 & 100.7 & 21.1 & 93.0 & Pass & N/A \\
\hline SL-003 & $|10 / 30 / 93|$ & 44280.1 & 78615.3 & 1 & | 99.8 & 22.0 & 100.7 & 21.0 & 93.0 & Pass & $\mathrm{H} / \mathrm{A}$ \\
\hline SL-004 & $|10 / 30 / 93|$ & 44241.5 & 78601.4 & 1 & | 105.7 & 18.9 & 103.9 & 21.0 & 96.0 & Pass & N/A \\
\hline SL-004RB & $|10 / 30 / 93|$ & 44241.5 & 78601.4 & 1 & $\mathrm{H} / \mathrm{A}$ & $\mathrm{N} / \mathrm{A}$ & 104.1 & 21.0 & 96.2 & Pass & $\mathrm{N} / \mathrm{A}$ \\
\hline SL-005 & $|10 / 31 / 93|$ & 44328.9 & 78640.5 & 1 & | 105.4 & 19.3 & 104.3 & 20.5 & 96.4 & Pass & $\mathrm{N} / \mathrm{A}$ \\
\hline SL-006 & $|10 / 31 / 93|$ & 44325.3 & 78705.7 & 1 & | 103.7 & 20.0 & 103.3 & 20.3 & 95.5 & Pass & N/A \\
\hline$S L-007$ & $|10 / 31 / 93|$ & 44326.7 & 78402.2 & 1 & $\begin{array}{l}99.8 \\
\end{array}$ & 21.4 & 101.8 & 19.0 & 94.1 & Pass & $\mathrm{H} / \mathrm{A}$ \\
\hline SL-DDB & $|10 / 31 / 93|$ & 44312.7 & 78462.3 & 1 & 102.4 & 21.2 & 104.0 & 19.2 & 96.1 & Pass & H/A \\
\hline SL-D0O & $|10 / 31 / 93|$ & 44291.5 & 78548.0 & 1 & 101.9 & 19.9 & 102.1 & 19.6 & 94.4 & Pass & $\mathrm{N} / \mathrm{A}$ \\
\hline SL-010 & $|10 / 31 / 93|$ & 44240.7 & 78448.0 & 1 & 105.7 & 19.5 & 105.9 & 19.3 & 97.8 & Pass & $N / A$ \\
\hline SL-011 & $|10 / 31 / 93|$ & 44222.1 & 78418.8 & 1 & 101.1 & 21.5 & 103.3 & 19.0 & 95.5 & Pass & $N / A$ \\
\hline SL-012 & $|10 / 31 / 93|$ & 44262.0 & 78555.9 & 1 & 102.9 & 21.0 & 103.8 & 19.9 & 95.9 & Pass & She lby \\
\hline SL-013 & $|10 / 31 / 93|$ & 44198.6 & 78415.4 & 1 & i105.1 & 19.2 & 104.9 & 19.3 & 97.0 & Pass & N/A \\
\hline SL-014 & $|10 / 31 / 93|$ & 44121.2 & 78385.6 & 1 & 102.6 & 20.1 & 103.5 & 19.1 & 95.7 & Pass & $H / A$ \\
\hline SL-015 & $|10 / 31 / 93|$ & 44067.1 & 78479.3 & 1 & $i 101.0$ & 20.1 & 101.9 & 19.1 & 94.2 & Pass & $\mathrm{N} / \mathrm{A}$ \\
\hline SL-016 & $|10 / 31 / 93|$ & 44224.3 & 78568.1 & 1 & |102.9 & 20.5 & 103.1 & 20.3 & 95.3 & Pass & N/A \\
\hline SL-016RB & $|10 / 31 / 93|$ & 44224.3 & $7856 B .1$ & 1 & {$[H / A$} & $\mathrm{H} / \mathrm{A}$ & 104.8 & 20.3 & 96.9 & Pass & $\mathrm{M} / \mathrm{A}$ \\
\hline SL-017 & $|10 / 31 / 93|$ & 44103.3 & 78658.1 & 1 & 102.5 & 20.2 & 103.0 & 19.6 & 95.2 & Pass & N/A \\
\hline SL-018 & $|10 / 31 / 93|$ & 44088.1 & 78621.9 & 1 & 102.5 & 19.4 & 102.3 & 19.6 & 94.6 & Pass & $N / A$ \\
\hline SL-019 & $|10 / 31 / 93|$ & 44123.2 & 78787.9 & 1 & | 98.7 & 20.7 & 99.0 & 20.3 & 91.5 & Pass & $M / A$ \\
\hline$S L-020$ & $|10 / 31 / 93|$ & 44280.7 & 78757.6 & 1 & | 98.3 & 23.4 & 100.4 & 20.8 & 92.8 & Pass & $\mathrm{H} / \mathrm{A}$ \\
\hline$S L-021$ & $|11 / 01 / 93|$ & 44268.2 & 78801.5 & 1 & $i 100.9$ & 21.1 & 102.2 & 19.7 & 94.4 & Pass & N/A \\
\hline$S L-022$ & $|11 / 01 / 93|$ & 44047.4 & 78757.8 & 1 & 103.3 & 19.9 & 102.2 & 21.1 & 94.5 & Pass & $N / A$ \\
\hline$S L-023$ & $|11 / 01 / 93|$ & 44321.9 & 78750.9 & 2 & 106.4 & 19.7 & 106.5 & 19.6 & 98.4 & Pass & $\mathrm{N} / \mathrm{A}$ \\
\hline$S L-024$ & $|11 / 01 / 93|$ & 44294.9 & 78650.2 & 2 & 104.1 & 21.2 & 105.4 & 19.7 & 97.4 & Pass & $\mathrm{H} / \mathrm{A}$ \\
\hline SL-D25 & $|11 / 01 / 93|$ & 44260.8 & 78572.5 & 2 & 101.1 & 20.9 & 102.2 & 19.7 & 94.4 & Pass & N/A \\
\hline SL-026 & $|11 / 01 / 93|$ & 44292.6 & 78509.4 & 2 & 103.0 & 19.8 & 104.0 & 18.6 & 96.1 & Fail & H/A \\
\hline$S L=026 \mathrm{~A}$ & $|11 / 01 / 93|$ & 44292.6 & 78509.4 & 2 & |102.9 & 20.0 & 103.9 & 19.0 & 96.0 & Pass & N/A \\
\hline SL-027 & $|11 / 01 / 93|$ & 44297.1 & 78475.8 & 2 & 102.4 & 19.9 & 104.4 & 17.6 & 96.5 & Fail & N/A \\
\hline SL-027A & $|11 / 01 / 93|$ & 44277.0 & 78472.2 & 2 & 100.4 & 21.6 & 102.3 & 19.3 & 94.6 & Pass & N/A \\
\hline SL-02B & $|11 / 01 / 93|$ & 44314.1 & 78391.5 & 2 & 105.2 & 19.9 & 107.2 & 17.6 & 99.1 & Fall & H/A \\
\hline SL-028A & $|11 / 01 / 93|$ & 44314.1 & 78391.5 & 2 & 104.8 & 19.7 & 105.4 & 19.0 & 97.4 & Pass & $N / A$ \\
\hline SL-029 & $|11 / 01 / 93|$ & 44230.7 & 78364.8 & 2 & i103.9 & 20.4 & 103.8 & 20.5 & 95.9 & Pass & $H / A$ \\
\hline$S L-030$ & $|11 / 01 / 93|$ & 44149.9 & 78416.3 & 2 & 103.6 & 18.4 & 102.6 & 19.6 & 94.8 & Pass & N/A \\
\hline$S L-031$ & $|11 / 01 / 93|$ & 44116.8 & 78381.5 & 2 & |103.1 & 19.3 & 103.4 & 18.9 & 95.6 & Pass & N/A \\
\hline SL-031RB & $|11 / 01 / 93|$ & 44116.8 & 78381.5 & 2 & IN/A & N/A & $|106.6|$ & 18.9 & 98.5 & I Pass & N/A \\
\hline
\end{tabular}


WHC-SD-W025-RPT-001, Rev. 0

GOLDER COHSTRUCTIOH SERVICES INC.

SUMHARY OF SOIL LINER MOISTURE-DENSITY TESTS

DEPARTMENT OF EMERGY

NON-DRAG OFF LAKDFILL FACILITY

HAKFORD NUCLEAR RESERVATIOH SECONDARY SOIL LIMER

\begin{tabular}{|c|c|c|c|c|c|c|c|c|c|c|c|}
\hline \multirow{4}{*}{$\begin{array}{c}\text { TEST } \\
\text { HUMBER } \\
(1,2,3)\end{array}$} & \multirow{4}{*}{ DATE } & \multicolumn{3}{|c|}{ TEST LOCATIOH IHFORMATION } & \multicolumn{2}{|c|}{ TROXLER } & \multicolumn{3}{|c|}{$\begin{array}{l}\text { LABORATORY CALCULATED } \\
\text { TEST VALUES }\end{array}$} & \multicolumn{2}{|c|}{ 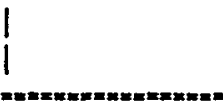 } \\
\hline & & \multicolumn{3}{|c|}{ I NORTH I WEST | LIFT } & \multicolumn{5}{|c|}{ | DRY | FIELL | ORY | LAB | PERCERT } & \multirow{2}{*}{ I PASS } & \multirow{3}{*}{$\begin{array}{l}\text { NOTES } \\
\text { (4) }\end{array}$} \\
\hline & & \multicolumn{2}{|c|}{ |COORDIKATE|COORDIKATE| } & \multirow[t]{2}{*}{ No. } & \multirow{2}{*}{$\begin{array}{l}\mid \text { DENS. } \mid \\
\mid \text { (pef) } \mid\end{array}$} & \multirow{2}{*}{$\begin{array}{l}\text { MOIST } \\
(x)\end{array}$} & \multirow{2}{*}{$\mid \begin{array}{l}\mid \text { DERSITY| } \\
\mid \text { (pef) } \mid\end{array}$} & \multirow{2}{*}{$\begin{array}{l}\text { MOIST } \\
(x)\end{array}$} & \multirow{2}{*}{$\mid$ COMPACTIOM } & & \\
\hline & & 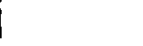 & & & & & & & & $\begin{array}{c}\text { or } 1 \\
\text { FAIL } 1\end{array}$ & \\
\hline SL-032 & $|21 / 09 / 93|$ & 44083.6 & 78805.8 & 2 & 99.2 & 22.5 & 100.5 & 20.9 & 92.7 & Pass & N/A \\
\hline$S L-033$ & $|11 / 09 / 93|$ & 44138.3 & 78752.9 & 2 & 103.9 & 20.4 & 104.3 & 19.8 & 96.3 & Pass & N/A \\
\hline$S L-034$ & $|11 / 09 / 93|$ & 44176.0 & 78725.1 & 2 & 103.7 & 19.8 & 104.4 & 19.0 & 96.3 & Pass & $K / A$ \\
\hline SL-035 & $|11 / 09 / 93|$ & 44219.0 & 78714.4 & 2 & |103.7 & 19.7 & 103.8 & 19.6 & 95.7 & Pass & N/A \\
\hline SL-036 & $|11 / 09 / 93|$ & 44337.1 & 78704.5 & 3 & 102.4 & 20.8 & 102.0 & 21.3 & 94.1 & Pass & $\mathrm{N} / \mathrm{A}$ \\
\hline SL-037 & $|11 / 09 / 93|$ & 44276.6 & 78647.8 & 3 & 104.7 & 19.4 & 105.2 & 18.9 & 97.1 & Pass & $N / A$ \\
\hline SL-038 & |11/09/93| & 44278.2 & 78551.9 & 3 & 102.0 & 19.9 & 103.0 & 18.7 & 95.0 & Pass & H/A \\
\hline SL-039 & $|11 / 09 / 93|$ & 44297.5 & 78494.3 & 3 & 101.2 & 21.3 & 103.2 & 19.0 & 95.2 & Pass & $\mathrm{H} / \mathrm{A}$ \\
\hline$S L-040$ & $|11 / 09 / 93|$ & 44176.3 & 78502.0 & 1 & |103.3 & 20.8 & 103.9 & 20.1 & 95.9 & Pass & $H / A$ \\
\hline$S L-041$ & $|11 / 09 / 93|$ & 44234.0 & 78448.4 & 2 & ¡101.0 & 21.8 & 102.4 & 21.3 & 93.5 & Pass & $H / A$ \\
\hline$S L=042$ & $|11 / 09 / 93|$ & 44167.9 & 78527.9 & 1 & | 99.9 & 19.0 & 98.3 & 20.8 & 90.7 & Pass & $H / A$ \\
\hline$S L-043$ & $|11 / 09 / 93|$ & 44162.0 & 78496.5 & 1 & |105.1 & 20.7 & 104.9 & 21.0 & 96.7 & Pass & H/A \\
\hline$S L-044$ & |11/09/93| & 44201.8 & 78520.5 & 1 & | 98.6 & 23.5 & 99.3 & 22.6 & 91.6 & Pass & H/A \\
\hline$S L-045$ & $|11 / 09 / 93|$ & 44194.0 & 78586.2 & 1 & 105.9 & 18.8 & 105.1 & 19.8 & 96.9 & Pass & H/A \\
\hline$S L-045 R B$ & $|11 / 09 / 93|$ & 44194.0 & 78586.2 & 1 & IN/A & $H / A$ & 106.3 & 19.8 & 98.1 & Pass & H/A \\
\hline$S L-046$ & |11/10/93| & 44166.7 & 78682.2 & 1 & |105.2 & 20.3 & 105.3 & 19.9 & 97.2 & Pass & $N / A$ \\
\hline SL-047 & $|11 / 10 / 93|$ & 44044.6 & 78778.8 & 1 & |101.6 & 19.4 & 100.7 & 20.6 & 92.9 & Pass & $N / A$ \\
\hline$S L-04 B$ & $|11 / 10 / 93|$ & 44170.0 & 78621.4 & 1 & 102.6 & 21.4 & 103.4 & 20.4 & 95.4 & Pass & N/A \\
\hline$S L-049$ & $|11 / 10 / 93|$ & 44151.6 & 78611.6 & 1 & | 99.3 & 22.2 & 99.3 & 22.2 & 91.6 & Pass & $N / \mathrm{A}$ \\
\hline$S L-050$ & $|11 / 10 / 93|$ & 44123.8 & 78516.4 & 1 & 100.7 & 22.3 & 100.9 & 22.0 & 93.1 & Pass & H/A \\
\hline SL-051 & $|11 / 10 / 93|$ & 44113.8 & 78405.1 & 3 & $|101.2|$ & 21.8 & 102.1 & 20.7 & 94.2 & Pass & $H / A$ \\
\hline SL-052 & $|11 / 10 / 83|$ & 44193.5 & 78433.9 & 3 & $|98.5|$ & 19.0 & 97.7 & 20.0 & 90.1 & Pass & $H / A$ \\
\hline$S L-053$ & $|11 / 10 / 93|$ & 44223.0 & 78392.6 & 3 & i102.5 & 21.3 & 104.4 & 19.1 & 96.3 & Pass & $H / A$ \\
\hline SL-054 & $|11 / 10 / 93|$ & 44064.7 & 78533.4 & 2 & 103.5 & 19.6 & 105.2 & 17.6 & 97.0 & Fall & H/A \\
\hline$S L-054 A$ & $|11 / 11 / 93|$ & 44064.7 & 78533.4 & 2 & 1100.0 & 21.0 & 101.7 & 19.0 & 93.9 & Pass & H/A \\
\hline SL-055 & $|11 / 10 / 93|$ & 44058.4 & 78596.6 & 3 & |204.7 & 20.3 & 105.8 & 19.0 & 97.6 & Pass & She lby \\
\hline SL-056 & $|11 / 10 / 93|$ & 44089.2 & 78620.7 & 2 & 102.9 & 22.6 & 103.9 & 20.5 & 95.8 & Pass & $\mathrm{N} / \mathrm{A}$ \\
\hline SL-057 & $|11 / 10 / 93|$ & 44100.8 & 78661.2 & 3 & 1202.3 & 19.6 & 102.9 & 18.9 & 95.0 & Pass & N/A \\
\hline SL-058 & $|11 / 10 / 93|$ & 44141.7 & 78667.5 & 3 & |103.0 & 20.4 & 103.3 & 20.0 & 95.2 & Pass & $\mathrm{H} / \mathrm{A}$ \\
\hline$S L-059$ & $|21 / 10 / 93|$ & 44223.4 & 78626.9 & 2 & 102.2 & 20.6 & 102.1 & 20.8 & 94.2 & Pass & $H / A$ \\
\hline$S L=060$ & $|11 / 10 / 93|$ & 44149.8 & 78794.6 & 3 & $|103.1|$ & 19.8 & 103.6 & 19.2 & 95.6 & Pass & H/A \\
\hline SL-061 & $|11 / 10 / 93|$ & 44186.5 & 78738.8 & 3 & $|102.2|$ & 19.1 & 101.2 & 20.4 & 93.3 & Pass & H/A \\
\hline SL-OS1RB & $|11 / 10 / 93|$ & 44186.5 & 78738.8 & 3 & IH/A & $N / A$ & 101.6 & 20.4 & 93.7 & Pass & $H / A$ \\
\hline$S L-062$ & $|21 / 10 / 93|$ & 44205.5 & 78753.1 & 3 & i203.7 & 19.0 & 103.7 & 19.0 & 95.7 & Pass & $H / A$ \\
\hline$S L-063$ & $|11 / 10 / 93|$ & 44233.3 & 78728.5 & 3 & 101.5 & 20.4 & 102.2 & 19.6 & 94.3 & Pass & $H / A$ \\
\hline$S L=064$ & $|11 / 10 / 93|$ & 44231.9 & 78692.7 & 2 & $|106.2|$ & 19.2 & 104.2 & 21.4 & 96.1 & Pass & H/A \\
\hline SL-065 & $|11 / 10 / 93|$ & 44278.6 & 78693.2 & 4 & $|105.2|$ & 19.2 & 104.6 & 19.9 & 96.5 & Pass & $\mathrm{H} / \mathrm{A}$ \\
\hline$S L-056$ & $|11 / 10 / 93|$ & 44225.2 & 78662.8 & 2 & 105.0 & 19.0 & 103.9 & 20.3 & 95.9 & Pass & $N / A$ \\
\hline SL-066RB & $|11 / 10 / 93|$ & 44225.2 & 78662.8 & 2 & IN/A & $\mathrm{H} / \mathrm{A}$ & $106.2 i$ & 20.3 & 97.9 & Pass & $\mathrm{H} / \mathrm{A}$ \\
\hline
\end{tabular}


GOLDER COHSTRUCTIOH SERVICES IHC.

SURHARY OF SOIL LIKER HOISTURE-DEHSITY TESTS 933-1354

DEPARTHEHT OF ENERGY

NON-DRAG OFF LANDFILL FACILITY

HAHFORO HUCLEAR RESERVATIOH

05/01/94

SECONDARY SOIL LIHER

\begin{tabular}{|c|c|c|c|c|c|c|c|c|c|c|c|}
\hline \multirow{6}{*}{ 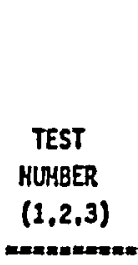 } & \multirow{5}{*}{ DATE } & \multirow{2}{*}{\multicolumn{3}{|c|}{ 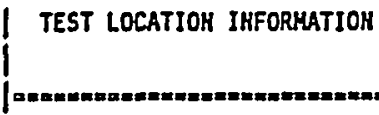 }} & \multirow{2}{*}{\multicolumn{2}{|c|}{ TROXLER }} & \multicolumn{3}{|c|}{$\begin{array}{l}\text { LABORATORY CALCULATED } \\
\text { TEST VALUES }\end{array}$} & \multirow{2}{*}{\multicolumn{2}{|c|}{ |x=" }} \\
\hline & & & & & & & & & & & \\
\hline & & \multirow{3}{*}{$\begin{array}{l}\text { I HORTH } \\
\text { |COOROIHATE }\end{array}$} & \multirow{3}{*}{\begin{tabular}{c|} 
WEST | \\
COORDIHATE|
\end{tabular}} & \multirow{3}{*}{$\begin{array}{l}\text { LIFT } \\
\text { Ho. }\end{array}$} & \multirow{3}{*}{\multicolumn{2}{|c|}{ 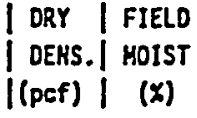 }} & \multirow{3}{*}{$\begin{array}{l}\mid \text { DRY } \\
\mid \text { DEKSITY| } \\
\mid \text { (pcf) } \mid\end{array}$} & \multirow{3}{*}{$\begin{array}{l}\text { LAB } \\
\text { HOIST } \\
(x)\end{array}$} & \multirow{3}{*}{$\begin{array}{l}\text { | PERCEHT | } \\
\text { |COHPACTIOH| }\end{array}$} & \multirow{3}{*}{\begin{tabular}{c|} 
PASS \\
or \\
FAIL
\end{tabular}} & \multirow{3}{*}{$\begin{array}{l}\text { NOTES } \\
(4)\end{array}$} \\
\hline & & & & & & & & & & & \\
\hline & & & & & & & & & & & \\
\hline & & & & & 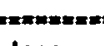 & $n=m=x=m$ & $y=E=z=x=$ & & & 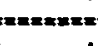 & \\
\hline$S L-067$ & $|11 / 10 / 93|$ & 44208.4 & 78590.2 & 2 & |103.8 & 19.8 & | 103.3 & 20.4 & 95.3 & Pass & $\mathrm{M} / \mathrm{A}$ \\
\hline SL-06B & $|11 / 10 / 93|$ & 44199.4 & 78552.5 & 2 & |102.3 & 19.4 & 101.1 & 20.8 & 93.2 & Pass & $H / A$ \\
\hline SL-069 & $|11 / 10 / 93|$ & 44128.6 & 78486.1 & 2 & |101.4 & 21.3 & 101.7 & 20.8 & 93.9 & Pass & H/A \\
\hline SL-070 & $\{11 / 10 / 93\}$ & 44135.1 & 78450.2 & 2 & | 99.8 & 22.5 & 101.5 & 20.4 & 93.6 & Pass & H/A \\
\hline SL-07L & $|11 / 11 / 93|$ & 44281.6 & 78578.2 & 4 & |102.1 & 19.3 & 102.8 & 19.7 & 94.0 & Pass & $H / A$ \\
\hline SL- 072 & $|111 / 11 / 93|$ & 44228.0 & 78573.8 & 3 & 98.0 & 21.2 & 98.3 & 20.9 & 90.8 & Pass & H/A \\
\hline SL-073 & $|11 / 11 / 93|$ & 44218.6 & 78546.9 & 3 & |102.8 & 21.0 & 103.2 & 20.5 & 95.3 & Pass & $M / A$ \\
\hline SL-074 & $|121 / 21 / 93|$ & 44266.8 & 78529.5 & 4 & |102.6 & 19.2 & 101.8 & 20.1 & 94.0 & Pass & $H / A$ \\
\hline SL-075 & $|11 / 11 / 93|$ & 44216.0 & 78504.2 & 3 & | 99.8 & 22.7 & 101.3 & 20.8 & 93.6 & Pass & H/A \\
\hline SL-076 & $|11 / 11 / 93|$ & 44217.1 & 78486.4 & 3 & |102.8 & 19.5 & 101.5 & 21.0 & 93.7 & Pass & H/A \\
\hline$S L-077$ & $|11 / 11 / 93|$ & 44253.0 & 78475.5 & 4 & 102.6 & 19.9 & 101.8 & 20.7 & 94.0 & Pass & H/A \\
\hline$S L-07 B$ & $|11 / 11 / 93|$ & 44182.8 & 78497.4 & 3 & 103.0 & 19.5 & 102.2 & 20.4 & 94.3 & Pass & $\mathrm{H} / \mathrm{A}$ \\
\hline SL-079 & $|11 / 11 / 93|$ & 44188.5 & 78564.2 & 3 & | 99.4 & 23.6 & 101.7 & 20.9 & 93.9 & Pass & H/A \\
\hline SL-080 & $|11 / 11 / 93|$ & 44164.4 & 78647.9 & 3 & |104.8 & 19.1 & 103.6 & 20.5 & 95.6 & Pass & H/A \\
\hline SL-081 & $|11 / 11 / 93|$ & 44142.3 & 78416.4 & 4 & 1102.0 & 19.6 & 101.5 & 20.2 & 93.7 & Pass & $H / A$ \\
\hline SL-082 & $|11 / 11 / 93|$ & 44209.9 & 78419.2 & 4 & | 99.2 & 22.6 & 101.0 & 20.5 & 93.3 & Pass & $\mathrm{H} / \mathrm{A}$ \\
\hline SL -083 & $|11 / 11 / 93|$ & 44221.3 & 78431.1 & 4 & 1101.8 & 19.9 & 101.8 & 19.9 & 94.0 & Pass & M/A \\
\hline SL-084 & $|11 / 11 / 93|$ & 44063.5 & 78432.3 & 1 & 104.8 & 19.6 & 104.5 & 20.0 & 96.5 & Pass & H/A \\
\hline SL-085 & $|11 / 11 / 93|$ & 44109.9 & 78551.2 & 1 & |103.4 & 19.5 & 103.3 & 19.7 & 95.3 & Pass & H/A \\
\hline SL-085RB & $|11 / 11 / 93|$ & 44109.9 & 78551.2 & 1 & |K/A & $H / A$ & 105.2 & 19.7 & 97.2 & Pass & $\boldsymbol{H} / \boldsymbol{A}$ \\
\hline$S L=086$ & |11/11/93| & 44130.2 & 78694.3 & 4 & |101.9 & 21.2 & 102.3 & 20.7 & 94.5 & Pass & Shelby \\
\hline SL-0B6A & |11/16/93| & 44130.2 & 78694.3 & 4 & |101.9 & 20.5 & 101.5 & 21.0 & 93.7 & Pass & Shelby \\
\hline SL-0B7 & $|12 / 11 / 93|$ & 44104.1 & 78691.3 & 4 & | 99.2 & 20.9 & 99.9 & 20.0 & 92.3 & Pass & $H / A$ \\
\hline$S L-0 B B$ & |11/13/93| & 44146.7 & 78554.7 & 6 & 100.7 & 20.1 & 100.8 & 19.8 & 93.1 & Pass & $H / A$ \\
\hline SL-089 & $|21 / 11 / 93|$ & 44079.5 & 78631.9 & 4 & 100.6 & 20.1 & 101.0 & 19.6 & 93.3 & Pass & $\mathrm{H} / \mathrm{A}$ \\
\hline$S L-090$ & |11/11/93| & 44065.4 & 78573.7 & 4 & 102.0 & 20.5 & 102.3 & 20.1 & 94.5 & Pass & $\mathrm{H} / \mathrm{A}$ \\
\hline SL-091 & $|11 / 11 / 93|$ & 44115.7 & 78754.9 & 4 & 101.0 & 22.0 & 103.1 & 19.5 & 95.2 & Pass & $\mathrm{H} / \mathrm{A}$ \\
\hline$S L-092$ & $|11 / 11 / 93|$ & 44184.4 & 78741.9 & 4 & |103.2 & 19.7 & 103.9 & 19.0 & 95.9 & Pass & $H / A$ \\
\hline SL-093 & |11/11/93| & 44236.2 & 78736.4 & 4 & 100.0 & 22.7 & 101.9 & 20.4 & 94.1 & Pass & $\mathrm{N} / \mathrm{A}$ \\
\hline SL-094 & |11/11/93| & 44239.2 & 78704.6 & 4 & 100.0 & 22.0 & 101.7 & 20.1 & 93.9 & Pass & M/A \\
\hline SL-095 & $|11 / 11 / 93|$ & 44242.7 & 78651.4 & 5 & |102.1 & 21.0 & 102.9 & 20.0 & 95.0 & Pass & M/A \\
\hline SL-096 & $|11 / 11 / 93|$ & 44280.3 & 78588.4 & 5 & 103.5 & 20.4 & 104.7 & 19.0 & 96.7 & Pass & H/A \\
\hline SL- 097 & $|11 / 11 / 93|$ & 44272.4 & 78528.7 & 5 & 104.2 & 19.4 & 103.3 & 20.4 & 95.4 & Pass & $\mathrm{H} / \mathrm{A}$ \\
\hline$S L-09 B$ & $|11 / 11 / 93|$ & 44248.0 & 78488.4 & 5 & |101.7 & 20.4 & 101.7 & 20.5 & 93.9 & Pass & $M / A$ \\
\hline SL-099 & |11/11/93| & 44259.7 & 78430.4 & 5 & 102.0 & 20.7 & 102.2 & 20.5 & 94.3 & Pass & $\mathrm{H} / \mathrm{A}$ \\
\hline$S L-100$ & $|11 / 11 / 93|$ & 44223.4 & 78414.0 & 5 & |101.1 & 22.2 & 102.5 & 20.6 & 94.6 & Pass & H/A \\
\hline $5 L-101$ & $|11 / 11 / 93|$ & 44137.4 & 78423.4 & 5 & 102.0 & 20.3 & 102.1 & 20.3 & 94.3 & Pass & N/A \\
\hline$S L-102$ & $|11 / 11 / 93|$ & 44251.7 & 78484.8 & 4 & 100.2 & 21.8 & 101.2 & 20.5 & 93.5 & Pass & H/A \\
\hline SL-103 & |11/11/93| & 44198.9 & 78495.7 & 4 & | 99.3 & 22.1 & $i 00.3$ & 20.8 & 92.6 & Pass & $H / A$ \\
\hline
\end{tabular}


GOLDER CONSTRUCTION SERVICES IMC.

SUMMARY OF SOIL LIMER HOISTURE-DENSITY TESTS

933-1354

DEPARTMEKT OF ENERGY

NOH-DRAG OFF LANDFILL FACILITY

$05 / 01 / 94$

HANFORD HUCLEAR RESERVATION

Page 4 SECONDARY SOIL LINER

\begin{tabular}{|c|c|c|c|c|c|c|c|c|c|c|c|}
\hline \multirow[b]{2}{*}{$\begin{array}{l}\text { TEST } \\
\text { NUMBER } \\
(1,2,3)\end{array}$} & \multirow[b]{2}{*}{ DATE } & \multicolumn{3}{|c|}{ 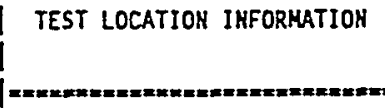 } & \multicolumn{2}{|c|}{ TROXLER } & \multicolumn{3}{|c|}{$\begin{array}{l}\text { LABORATORY CALCULATED } \\
\text { TEST VALUES }\end{array}$} & \multicolumn{2}{|c|}{$l_{1}^{1}$} \\
\hline & & MORTH & $\begin{array}{c}\text { VEST } \\
\text { COORDIMATE }\end{array}$ & $\begin{array}{l}\text { LIFT } \\
\text { No. }\end{array}$ & $\begin{array}{l}\mid \text { DRY } \mid \\
\mid \text { DEHS. } \\
\mid \text { (pcf) } \mid\end{array}$ & $\begin{array}{l}\text { FIELD } \\
\text { MOIST } \\
(x)\end{array}$ & $\begin{array}{l}\mid \text { ORY } \mid \\
\text { |DENSITY| } \\
\mid \text { (pof) } \mid\end{array}$ & $\begin{array}{l}\text { LAB } \\
\text { MOIST } \\
(x)\end{array}$ & $\begin{array}{l}\mid \text { PERCENT } \\
\text { |COMPACTIOH }\end{array}$ & $\left|\begin{array}{c}\text { PASS } \\
\text { or } \\
\text { FAIL }\end{array}\right|$ & $\begin{array}{l}\text { HOTES } \\
\text { (4) }\end{array}$ \\
\hline$S L-103 R B$ & $|11 / 11 / 93|$ & 44198.9 & 78515.3 & 4 & $\mid N / A$ & $\mathrm{H} / \mathrm{A}$ & 103.3 & 20.8 & 95.4 & Pass & $H / A$ \\
\hline$S L-104$ & |11/11/93| & 44202.3 & 78580.5 & 4 & 103.8 & 19.0 & 102.8 & 20.1 & 95.0 & Pass & $\mathrm{H} / \mathrm{A}$ \\
\hline$S L-105$ & |11/11/93| & 44142.5 & 78561.3 & 3 & 103.1 & 19.5 & 102.8 & 20.0 & 94.9 & Pass & H/A \\
\hline$S L-106$ & $|11 / 11 / 93|$ & 44120.6 & 78483.8 & 3 & |101.5 & 20.4 & 102.1 & 19.7 & 94.3 & Pass & H/A \\
\hline$S L-107$ & $|11 / 12 / 93|$ & 44197.2 & 78668.8 & 5 & 99.6 & 22.0 & 101.3 & 20.0 & 93.6 & Pass & M/A \\
\hline$S L-108$ & $|11 / 12 / 93|$ & 44207.1 & 78589.7 & 5 & 103.0 & 20.0 & 102.4 & 20.6 & 94.6 & Pass & H/A \\
\hline$S L-109$ & $|11 / 12 / 93|$ & 44183.9 & 78569.3 & 5 & 102.3 & 20.5 & 102.8 & 19.9 & 94.9 & Pass & $M / A$ \\
\hline$S L-110$ & $|11 / 12 / 93|$ & 44132.9 & 78441.0 & 5 & 103.0 & 20.1 & 102.5 & 20.6 & 94.6 & Pass & $M / A$ \\
\hline$S L-111$ & |11/12/93] & 44095.2 & 78431.0 & 5 & $\mid 102.9$ & 19.6 & 102.7 & 20.0 & 94.8 & Pass & $\mathrm{H} / \mathrm{A}$ \\
\hline$S L-112$ & $|11 / 12 / 93|$ & 44069.5 & 78628.0 & 5 & 197.8 & 22.8 & 99.6 & 20.6 & 92.0 & Pass & N/A \\
\hline$S L-113$ & $|11 / 12 / 93|$ & 44091.7 & 78642.9 & 5 & 1201.0 & 19.4 & 100.5 & 20.0 & 92.8 & Pass & H/A \\
\hline$S L-114$ & $|11 / 12 / 93|$ & 44188.0 & 78641.1 & 3 & | 97.8 & 21.4 & 99.1 & 19.8 & 91.5 & Pass & $\mathrm{N} / \mathrm{A}$ \\
\hline$S L-115$ & $|11 / 12 / 93|$ & 44078.1 & 78697.8 & 5 & 101.2 & 21.2 & 101.8 & 20.5 & 94.0 & Pass & $H / A$ \\
\hline$S L-116$ & $|11 / 12 / 93|$ & 44052.6 & 78681.6 & 5 & $\lceil 101.6$ & 21.3 & 103.1 & 19.5 & 95.2 & Pass & K/A \\
\hline$S L-117$ & $|11 / 12 / 93|$ & 44150.9 & 78452.1 & 4 & 99.7 & 22.2 & 200.4 & 21.3. & 92.7 & Pass & H/A \\
\hline$S L-118$ & $|11 / 12 / 93|$ & 44151.8 & 78599.4 & 4 & 102.7 & 20.5 & 103.5 & 19.5 & 95.6 & Pass & H/A \\
\hline$S L-119$ & $|11 / 12 / 93|$ & 44249.0 & 78781.2 & 5 & 103.4 & 19.9 & 103.3 & 20.0 & 95.4 & Pass & H/A \\
\hline$S L-120$ & $|11 / 12 / 93|$ & 44138.5 & 78759.9 & 5 & | 99.2 & 22.0 & 101.4 & 19.3 & 93.7 & Pass & $H / A$ \\
\hline$S L-121$ & $|11 / 12 / 93|$ & 44179.8 & 78724.2 & 5 & 1101.5 & 20.8 & 102.3 & 19.8 & 94.5 & Pass & $\mathrm{N} / \mathrm{A}$ \\
\hline$S L-122$ & $|11 / 12 / 93|$ & 44235.8 & 78720.8 & 5 & 100.6 & 22.3 & 102.5 & 20.1 & 94.6 & Pass & $M / A$ \\
\hline$S L-123$ & $|11 / 12 / 93|$ & 44267.8 & 78651.6 & 6 & |202.6 & 18.9 & 102.3 & 19.3 & 94.4 & Pass & H/A \\
\hline$S L-123 R B$ & $|11 / 12 / 93|$ & 44267.8 & 78651.6 & 6 & $\mathrm{IN} / \mathrm{A}$ & $N / A$ & 103.0 & 19.3 & 95.1 & Pass & $\mathrm{H} / \mathrm{A}$ \\
\hline$S L-124$ & $|11 / 12 / 93|$ & 44255.6 & 78612.2 & 6 & |101.4 & 20.3 & 101.8 & 19.9 & 94.0 & Pass & H/A \\
\hline$S L-125$ & $|11 / 12 / 93|$ & 44270.1 & 78534.8 & 6 & 103.4 & 19.2 & 103.0 & 19.7 & 95.1 & Pass & $\mathrm{H} / \mathrm{A}$ \\
\hline$S L-126$ & $|11 / 12 / 93|$ & 44284.0 & 78471.7 & 6 & 1102.8 & 20.7 & 103.6 & 19.7 & 95.7 & Pass & $H / A$ \\
\hline$S L-127$ & $|11 / 13 / 93|$ & 44259.8 & 78390.3 & 6 & | 99.8 & 22.1 & 102.0 & 19.5 & 94.2 & Pass & $N / A$ \\
\hline$S L-128$ & $|11 / 13 / 93|$ & 44189.9 & 78395.0 & 6 & 100.4 & 21.8 & 102.0 & 19.9 & 94.2 & Pass & $N / A$ \\
\hline$S L-129$ & $|11 / 13 / 93|$ & 44168.3 & 78443.6 & 6 & 1103.9 & 19.8 & 103.7 & 20.0 & 95.7 & Pass & Shelby \\
\hline$S L-130$ & $|11 / 13 / 93|$ & 44218.3 & 78401.8 & 6 & |103.6 & 19.6 & 103.6 & 19.6 & 95.7 & Pass & H/A \\
\hline$S L-131$ & $|11 / 13 / 93|$ & 44210.5 & 78588.6 & 6 & |102.2 & 21.4 & 103.5 & 19.9 & 95.6 & Pass & $\mathrm{K} / \mathrm{A}$ \\
\hline$S L-132$ & $|11 / 13 / 93|$ & 44166.6 & 78695.3 & 6 & i101.3 & 21.0 & 102.5 & 19.6 & 94.7 & Pass & $N / A$ \\
\hline$S L-133$ & |11/13/93! & 44220.5 & 78645.4 & 6 & 1103.2 & 19.8 & 103.3 & 19.7 & 95.4 & Pass & $K / A$ \\
\hline SL-134 & $|11 / 13 / 93|$ & 44096.0 & 78516.1 & 6 & 1103.8 & 19.2 & 102.9 & 20.2 & 95.0 & Pass & $N / A$ \\
\hline$S L-135$ & $|11 / 13 / 93|$ & 44163.7 & 78466.1 & 6 & 103.5 & 19.3 & 102.3 & 20.7 & 94.5 & Pass & $N / A$ \\
\hline$S L-136$ & $|11 / 13 / 93|$ & 44181.6 & 78517.1 & 6 & 100.9 & 22.1 & 102.7 & 20.0 & 94.8 & Pass & She lby \\
\hline$S L-137$ & $|11 / 23 / 93|$ & 44041.3 & 78458.8 & 6 & 104.5 & 20.2 & 105.1 & 19.5 & 97.0 & Pass & $K / A$ \\
\hline$S L-137 R B$ & $|11 / 13 / 93|$ & 44041.3 & 78458.8 & 6 & $\mid K / A$ & $H / A$ & 105.6 & 19.5 & 97.5 & Pass & $K / A$ \\
\hline SL-138 & $|11 / 13 / 93|$ & 44101.7 & 78597.6 & 6 & j102.1 & 20.6 & 103.41 & 19.0 & 95.4 & Pass & $N / A$ \\
\hline SL-139 & {$[11 / 13 / 93 \mid$} & 44076.2 & 78722.7 & 6 & | 99.1 & 19.2 & $98.9 i$ & 19.5 & 91.3 & Pass & $K / A$ \\
\hline
\end{tabular}


WHC-SD-W025-RPT-001, Rev. 0

GOLDER COHSTRUCTIOH SERVICES IHC.

SUAALRY OF SOIL LIKER HOISTURE-DEKSITY TESTS 933-1354 DEPARTHEKT OF ENERGY 05/01/94

HOK-DRAG OFF LAHDFILL. FACILITY Page 5

HANFORD RUCLEAR RESERVATIOH

SECOHDARY SOIL LINER

\begin{tabular}{|c|c|c|c|c|c|c|c|c|c|c|c|}
\hline \multirow[b]{2}{*}{$\begin{array}{c}\text { TEST } \\
\text { NUWBER } \\
(1,2,3)\end{array}$} & \multirow{2}{*}{$\begin{array}{l}1 \\
1 \\
\text { DATE }\end{array}$} & \multicolumn{3}{|c|}{ TEST LOCATIOH IHFORHATIOH } & \multicolumn{2}{|c|}{ TROXLER } & \multicolumn{3}{|c|}{$\begin{array}{l}\text { LABORATORY CALCULATED } \\
\text { TEST VALUES }\end{array}$} & \multicolumn{2}{|c|}{1} \\
\hline & & $\begin{array}{c}\text { HORTH } \\
\text { COOROIKATE }\end{array}$ & $\begin{array}{c}\text { WEST } \\
\text { COOROIHATE }\end{array}$ & $\begin{array}{l}\text { LIFT } \\
\text { Ho. }\end{array}$ & \begin{tabular}{|l|}
$\mid$ DRY \\
$\mid$ DEHS.| \\
$\mid$ (pef) |
\end{tabular} & $\begin{array}{l}\text { FIELD } \\
\text { MOIST } \\
(X)\end{array}$ & $\begin{array}{l}\text { DRY | } \\
\text { |DEKSITY| } \\
\mid \text { (pef) | }\end{array}$ & $\begin{array}{c}\text { LAB } \\
\text { MOIST } \\
(x)\end{array}$ & $\mid \begin{array}{l}\text { PERCEHT } \\
\text { |COMPACTIOH }\end{array}$ & $\left|\begin{array}{c}\text { PASS } \\
\text { or } \\
\text { faIL }\end{array}\right|$ & $\begin{array}{l}\text { HOTES } \\
\text { (4) }\end{array}$ \\
\hline$S L-140$ & $|11 / 13 / 93|$ & 44128.9 & 78751.1 & 6 & $|101.4|$ & 20.8 & 102.2 & 19.9 & 94.3 & Pass & N/A \\
\hline$S L-141$ & $|11 / 13 / 93|$ & 44166.6 & 78708.0 & 6 & $|99.0|$ & 22.1 & 100.8 & 19.9 & 93.1 & Pass & K/A \\
\hline$S L-142$ & $|11 / 13 / 93|$ & 44077.2 & 78373.2 & 6 & 103.0 & 18.6 & 101.9 & 19.8 & 94.1 & Pass & H/A \\
\hline$S L=143$ & $|11 / 13 / 93|$ & 44198.9 & 78481.6 & 1 & |102.9 & 20.4 & 104.2 & 18.9 & 96.2 & Pass & $K / A$ \\
\hline SL-144 & $|11 / 13 / 93|$ & 44179.1 & 78470.3 & 2 & |1201.3 & 20.9 & 102.9 & 18.9 & 95.1 & Pass & N/A \\
\hline SL-145 & $|11 / 13 / 93|$ & 44205.8 & 78502.7 & 3 & 1102.9 & 18.2 & 102.1 & 19.1 & 94.3 & Pass & M/A \\
\hline SL-146 & $|11 / 13 / 93|$ & 44178.6 & 78462.5 & 4 & 104.3 & 18.8 & 104.1 & 19.0 & 96.1 & Pass & $H / A$ \\
\hline$S L-146 R B$ & |11/13/93| & 44178.6 & 78462.5 & 4 & IH/A & $H / A$ & 106.3 & 19.0 & 98.2 & Pass & H/A \\
\hline$S L-147$ & $|11 / 14 / 93|$ & 44200.8 & 78509.3 & 5 & | 99.5 & 22.3 & 102.4 & 18.9 & 94.6 & Pass & H/A \\
\hline SL-148 & $|11 / 14 / 93|$ & 44187.1 & 78477.1 & 6 & |105.4 & 19.4 & 105.0 & 19.9 & 97.0 & Pass & $H / A$ \\
\hline$S L-148 R B$ & $|11 / 14 / 93|$ & 44187.1 & 78477.1 & 6 & |H/A & $\mathrm{H} / \mathrm{A}$ & 104.2 & 19.9 & 96.2 & Pass & H/A \\
\hline$S L-149$ & $|11 / 15 / 93|$ & 44072.6 & 78463.6 & 7 & |103.6 & 21.8 & $105.6 i$ & 19.5 & 97.5 & Pass & H/A \\
\hline$S L-150$ & $|11 / 15 / 93|$ & 44118.3 & 78553.3 & 8 & |102.3 & 19.8 & $102.8 \mid$ & 19.3 & 94.9 & Pass & $H / A$ \\
\hline$S L-151$ & $|11 / 15 / 93|$ & 44124.5 & 78593.6 & 9 & 100.0 & 21.5 & 102.1 & 18.9 & 94.3 & Pass & H/A \\
\hline$S L-152$ & $|11 / 15 / 93|$ & 44079.2 & 78513.0 & 10 & 1205.3 & 19.2 & 105.2 & 19.3 & 97.1 & Pass & H/A \\
\hline SL-15RRB & $|11 / 15 / 93|$ & 44079.2 & 78513.0 & 10 & H/A & $H / A$ & 206.1 & 19.3 & 98.0 & Pass & $H / A$ \\
\hline$S L-153$ & $|11 / 16 / 93|$ & 44230.8 & 78692.9 & 4 & 101.2 & 22.4 & 102.9 & 20.4 & 95.0 & f.Pass & She Iby \\
\hline$S L-154$ & $|11 / 16 / 93|$ & 44151.0 & 78702.0 & 4 & |104.1 | & 19.9 & 104.8 & 19.2 & 96.8 & Pass & She lby \\
\hline$S L-155$ & $|11 / 16 / 93|$ & 44120.7 & 78705.8 & 1 & |102.3 & 19.9 & 102.0 & 20.2 & 94.2 & Pass & $H / A$ \\
\hline$S L-156$ & $|11 / 16 / 93|$ & 44127.9 & 78695.0 & 2 & |102.1 & 20.7 & 102.2 & 20.6 & 94.4 & Pass & H/A \\
\hline SL-157 & $|11 / 16 / 93|$ & 44117.4 & 78711.2 & 3 & |102.7 & 18.9 & 101.4 & 20.4 & 93.6 & Pass & $M / A$ \\
\hline SL-158 & $|11 / 16 / 93|$ & 44130.5 & 78695.4 & 5 & |101.7 | & 19.8 & 101.0 & 20.6 & 93.3 & Pass & H/A \\
\hline$S L-159$ & $|11 / 16 / 93|$ & 44143.4 & 78688.5 & 6 & |103.7 & 19.2 & 103.1 & 20.0 & 95.2 & Pass & $N / A$ \\
\hline$S L-159 R B$ & $|11 / 16 / 93|$ & 44143.4 & 78688.5 & 6 & $\mid H / A$ & K/A & 105.6 & 20.0 & 97.5 & Pass & N/A \\
\hline$S L-160$ & $|12 / 01 / 93|$ & 44080.6 & 78501.9 & 6 & 108.0 & 17.4 & 106.6 & 18.9 & 98.4 & Pass & She lby \\
\hline$S L-161$ & $|12 / 01 / 93|$ & 44185.2 & 78600.9 & 6 & 106.1 & 18.4 & 105.4 & 19.2 & 97.2 & Pass & She lby \\
\hline SL-162 & $|12 / 01 / 93|$ & 44143.0 & 78435.3 & 6 & 1105.1 & 18.4 & 104.5 & 19.1 & 96.4 & Pass & She lby \\
\hline$S L-162 R B$ & $|12 / 01 / 93|$ & 44143.0 & 78435.3 & 6 & IN/A & $H / A$ & $103.9 i$ & 19.1 & 95.9 & Pass & $\mathrm{H} / \mathrm{A}$ \\
\hline
\end{tabular}

NOTES

(1) Test numbers with a suffix of " $A$ " indicate retests of failing motsture-density tests.

(2) Test numbers with suffixes of RB indicate rubber balloon tests.

(3) Test number SL-08B is not in chronological order due to an error in ordering sequence.

(4) The note "Shelby" indicates that a Shelby tube sample of the soll liner was obtained at this test location. 
WHC-SD-W025-RPT-001, Rev. 0

APPENDIX K-2

PRIMARY SOIL LINER

$k-2$ 
WHC-SD-W025-RPT-001, Rev. 0

GOLDER CONSTRUCTION SERVICES INC.

SUMMARY OF SOIL LIHER MOISTURE-DENSITY TESTS $\cdot$ 933-1354

DEPARTMEHT OF ENERGY 05/01/94

HON-DRAG OFF LANDFILLL FACILITY Page 1

HANFORD NUCLEAR RESERVATIOK

PRIMARY SOIL LIMER

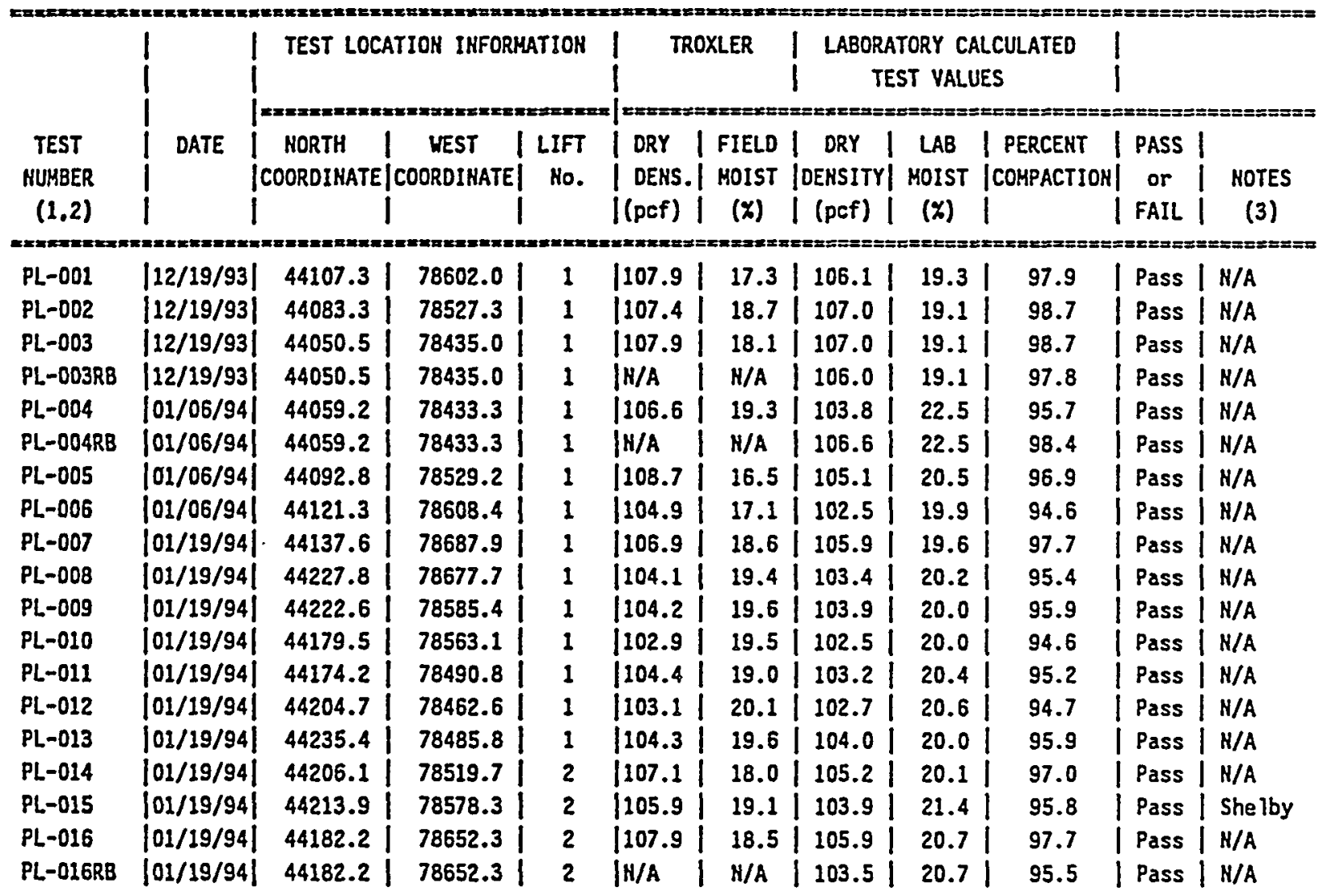

MOTES

(1) Test numbers with a suffix of "A" indicate retests of falling moisture-density tests.

(2) Test numbers with suffixes of RB indicate rubber balloon tests.

(3) The note "Shelby" indicates that a Shelby tube sample of the soil liner was obtained at this test location. 
WHC-SD-W025-RPT-001, Rev. 0

APPENDIX L

SUBGRADE ACCEPTANCE CERTIFICATES

$L-i$ 


\section{CERTTICATE OF ACCEPTANCE OF SOII SURFACE}

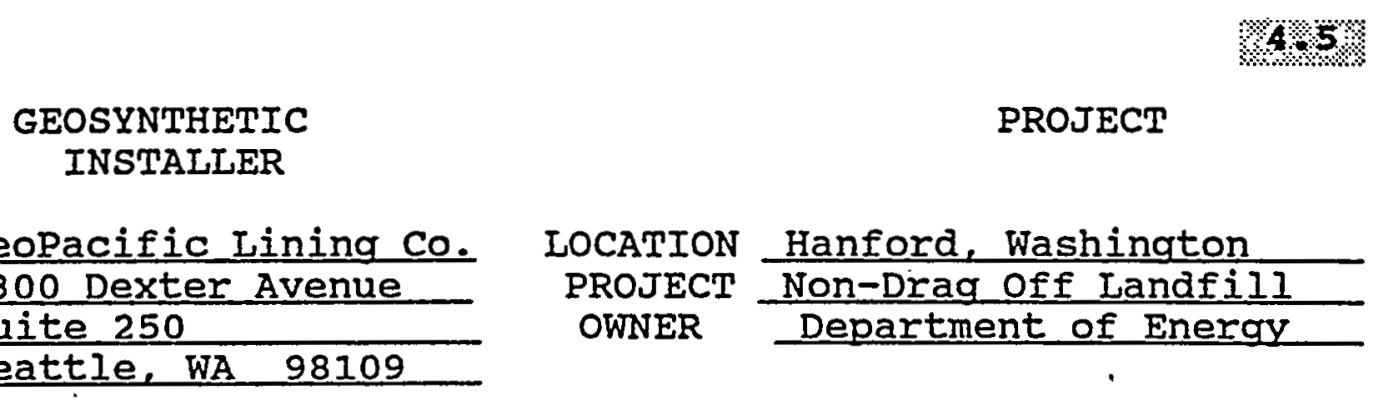

I, the Undersigned, the duly authorized representative of Geopacific Iining Inc. do hereby accept the area of soil surface bounded by:

The north slope and $20^{\prime}$ of the floor on the north end of the cell.

and shall be responsible for maintaining its integrity and suitability in accordance with project specifications from this date to the completion of the installation.

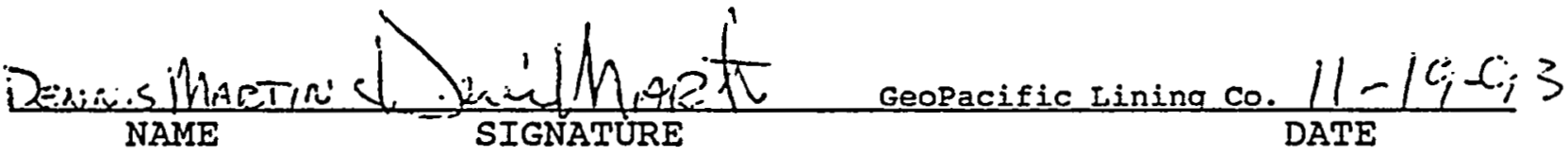

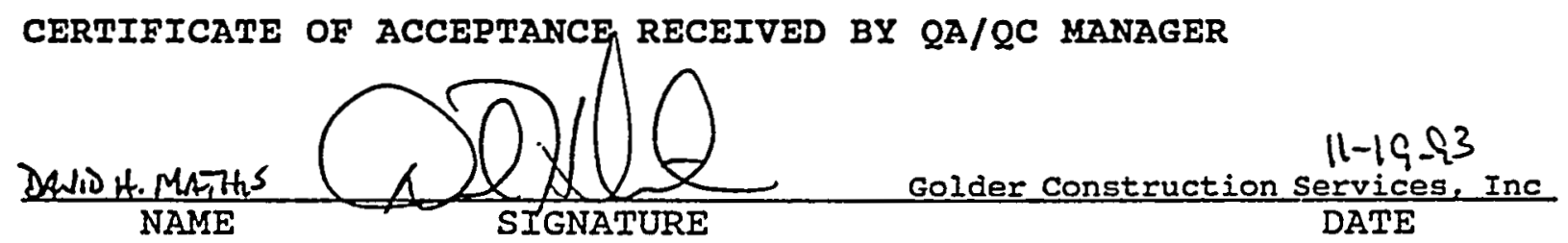

CERTIFICATE OF ACCEPTANCE RECEIVED BY GENERAL CONTRACTOR

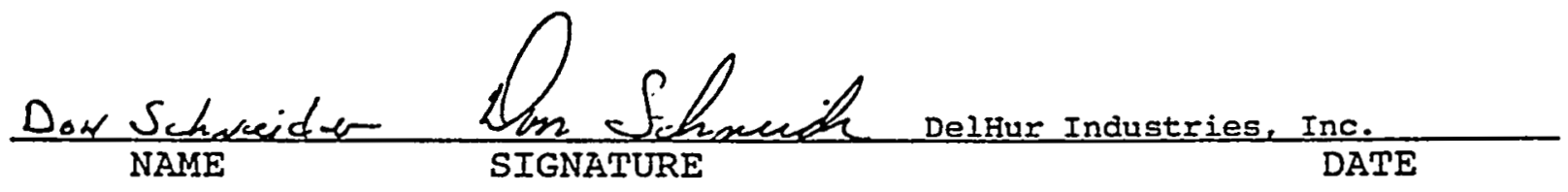
GCS FORM: G4 


\title{
CERTIFICATE OF ACCEPTANCE OF SOIL SURFACE
} \\ GEOSYNTHETIC \\ INSTALLER \\ PROJECT
}

COMPANY GeoPacific Lining Co. ADDRESS

$\frac{1300 \text { Dexter Avenue }}{\text { Suite } 250}$

LOCATION Hanford, Washington PROJECT Non-Drag Off Landfill OWNER Department of Energy

I, the Undersigned, the duly authorized representative of Geopacific Iining Inc. do hereby accept the area of soil surface bounded by:

The west and south slopes and $20^{\prime}$ of the floor on the west and souths ends of the cell.

and.shall be responsible for maintaining its integrity and suitability in accordance with project specifications from this date to the completion of the installation.

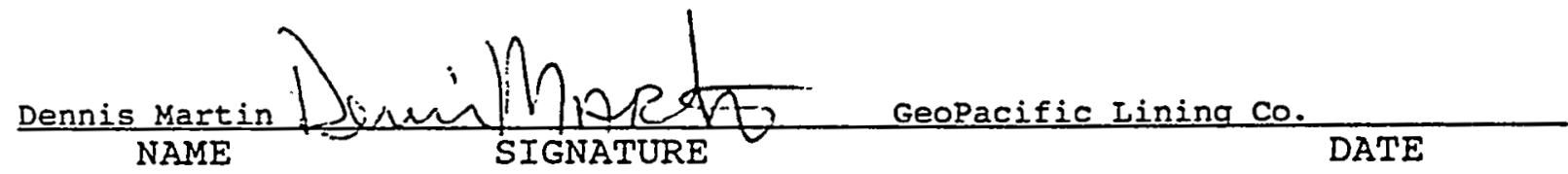

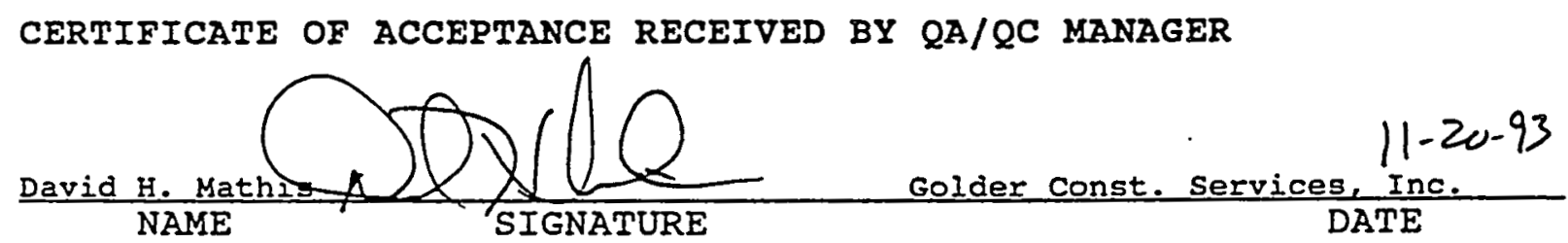

CERTIFICATE OF ACCEPTANCE RECEIVED BY GENERAL CONTRACTOR

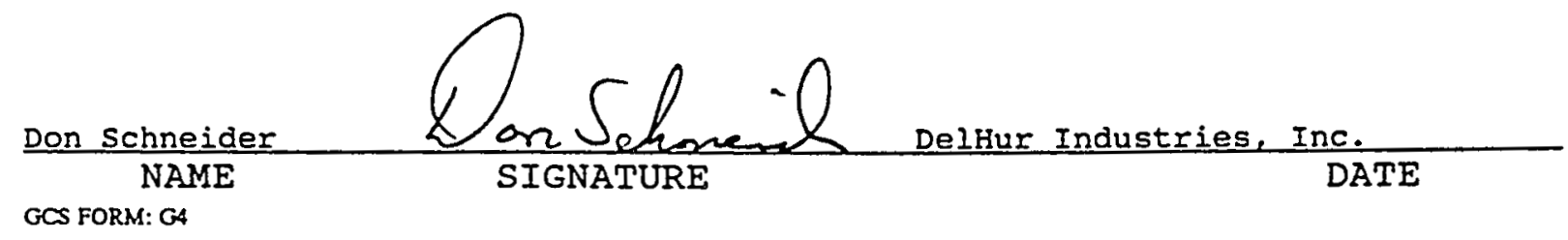




\section{CERTIFICATE OF ACCEPTANCE OF SOIL SURFACE}

\section{GEOSYNTHETIC INSTALLER}

COMPANY GeoPacific Lining Co. ADDRESS 1300 Dexter Avenue Suite 250 Seattle, WA 98109
LOCATION Hanford, Washington PROJECT Non-Drag off Landfill OWNER Department of Energy

I, the Undersigned, the duly authorized representative of Geopacific Iining Inc. do hereby accept the area of soil surface bounded by:

Panels $s-043$ through $s-049$ on the south slope and panels $s-050$ through $\mathrm{s}-052$ on the floor of the cell.

and shall be responsible for maintaining its integrity and suitability in accordance with project specifications from this date to the completion of the installation.

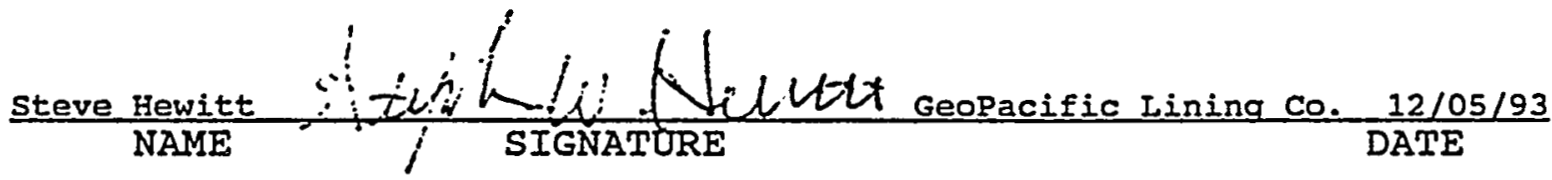

CERTIFICATE OF ACCEPTANCE RECEIVED BY QA/QC MANAGER

$\frac{\text { David H. Mathis }}{\text { NAME }} \frac{(102 \text { Golder const. Services, Inc. 12/05/93 }}{\text { DATE }}$

CERTIFICATE OF ACCEPTANCE RECEIVED BY GENERAL CONTRACTOR

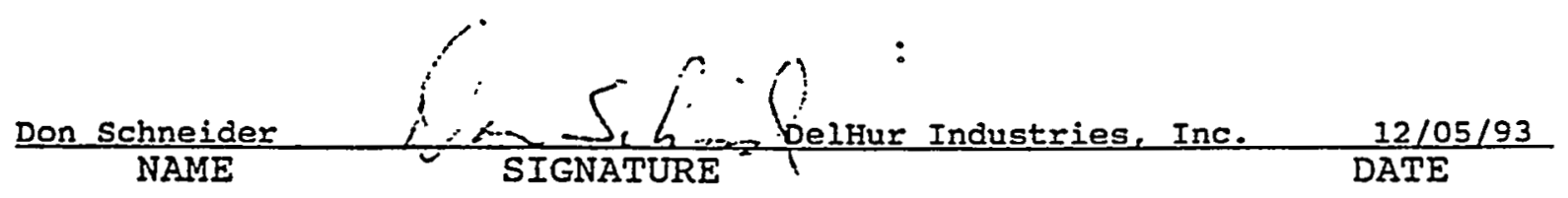
GCS FORM: CA 


\section{CERTIFICATE OF ACCEPTANCE OF SOL SURFACE}

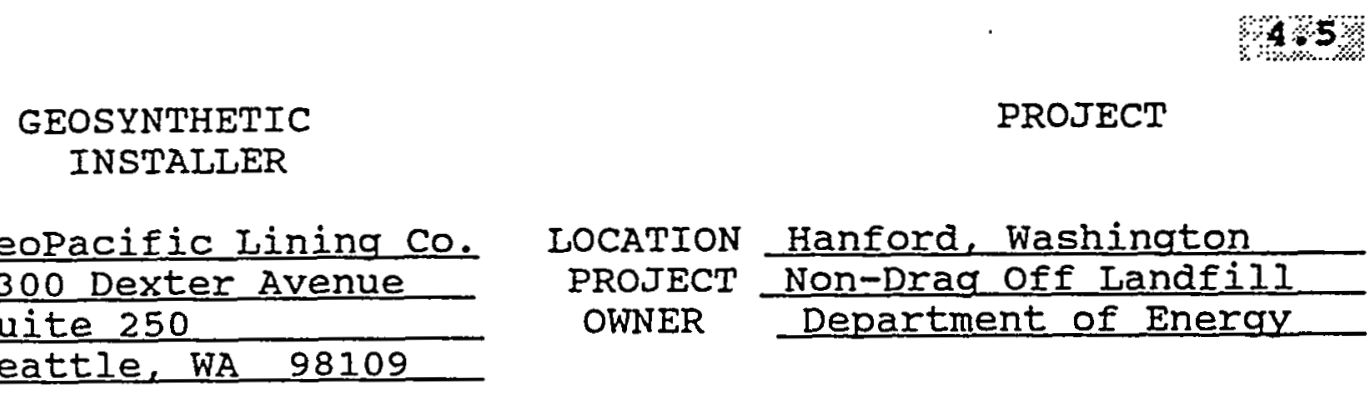

I, the Undersigned, the duly authorized representative of Geopacific Iining Inc. do hereby accept the area of soil surface bounded by:

Panels S-053 through S-055 on the south slope and panels $S-056$ through $s-065$ on the east slope of the cell.

and-shall be responsible for maintaining its integrity and suitability in accordance with project specifications from this date to the completion of the installation.

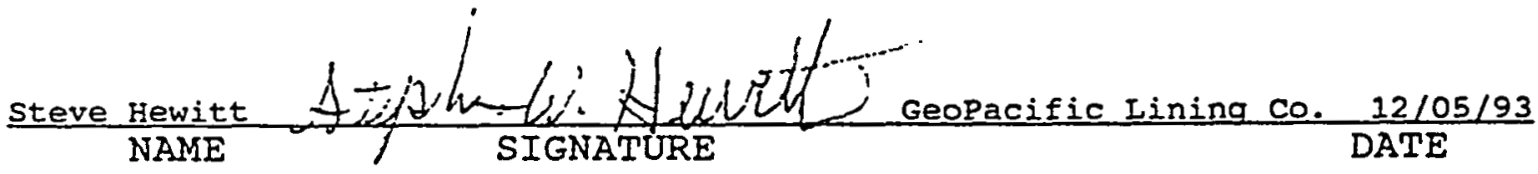

CERTIFICATE OF ACCEPTANCE RECEIVED BY QA/QC MANAGER

$\frac{\text { David H. Mathis }}{\text { NAME }} \frac{\text { Golder Const. Services, Inc. 12/05/93 }}{\text { DATE }}$

CERTIFICATE OF ACCEPTANCE RECEIVED BY GENERAL CONTRACTOR

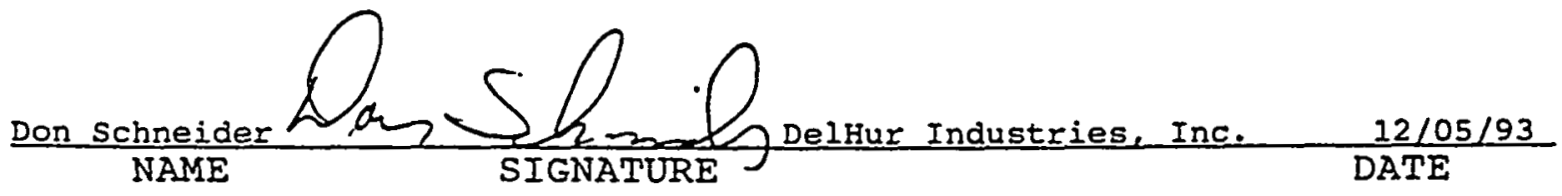

GCS FORM: C4 


\title{
CERTIFICATE OF ACCEPTANCE OF SOIL SURFACE
} \\ GEOSYNTHETIC \\ INSTALLER \\ PROJECT
}

COMPANY GeoPacific Lining Co. ADDRES

$\frac{1300 \text { Dexter Avenue }}{\text { Suite } 250}$

LOCATION Hanford, Washington PROJECT Non-Drag Off Landfill OWNER Department of Energy

I, the Undersigned, the duly authorized representative of Geopacific Lining Inc. do hereby accept the area of soil surface bounded by:

Panels S-066 through S-071 on the south slope and north slopes of the cell.

and shall be responsible for maintaining its integrity and suitability in accordance with project specifications from this date to the completion of the installation.

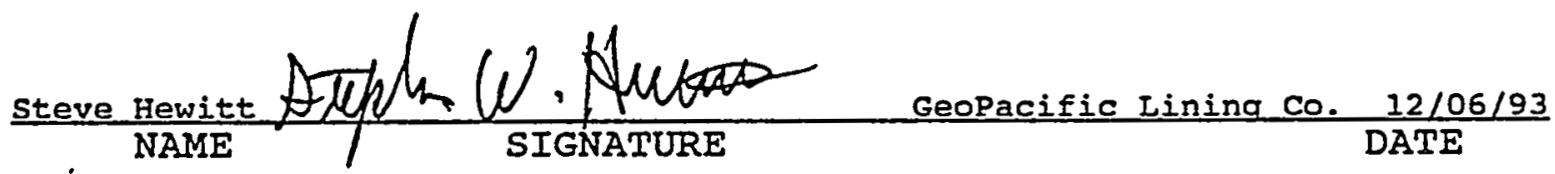

CERTIFICATE OF ACCEPTANCE RECEIVED BY QA/QC MANAGER

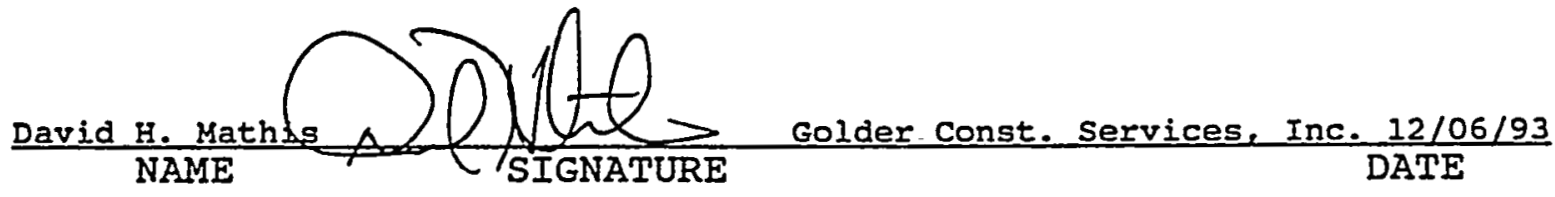

CERTIFICATE OF ACCEPTANCE RECEIVED BY GENERAL CONTRACTOR

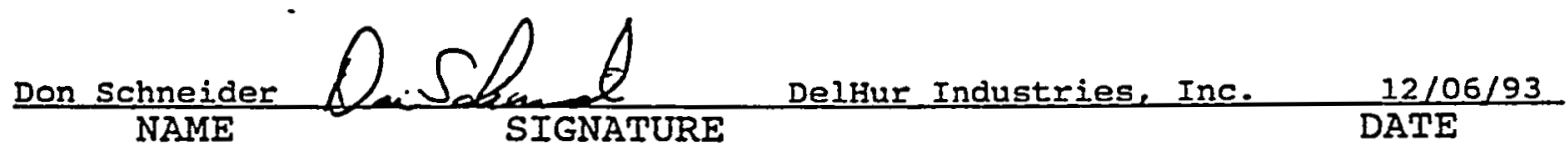
GCS FORM: G4 


\section{CERTIFICATE OF ACCEPTANCE \\ OF SOIL SURFACE}

\section{GEOSYNTHETIC \\ INSTALLER}
COMPANY GeoPacific Lining, Co. ADDRESS 1300 Dexter Avenue Suite 250 Seattle, WA 98109

PROJECT

LOCATION Hanford, Washington PROJECT Non-Drag off Landfill OWNER Department of Energy

I, the Undersigned, the duly authorized representative of Geopacific Lining Inc. do hereby accept the area of soil surface bounded by:

The entire surface of primary admix on the floor of the cell except the area at the end of the access ramp in the southwest corner of the cell.

and shall be responsible for maintaining its integrity and suitability in accordance with project specifications from this date to the completion of the installation.

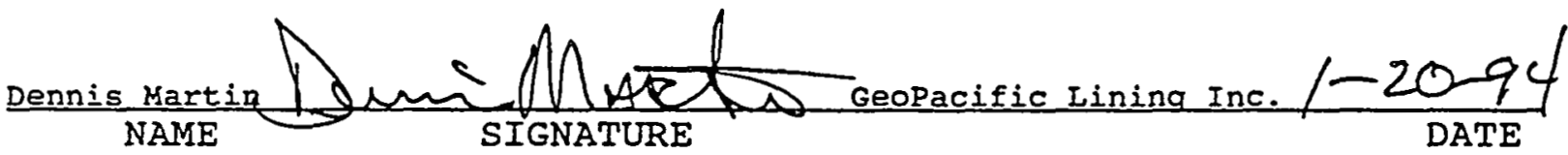

CERTIFICATE OF ACCEPTANCE RECEIVED BY QA/QC MANAGER

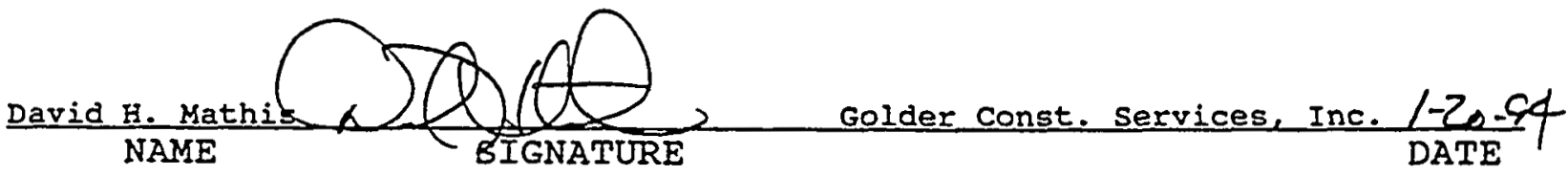

CERTIFICATE OF ACCEPTANCE RECEIVED BY GENERAL CONTRACTOR

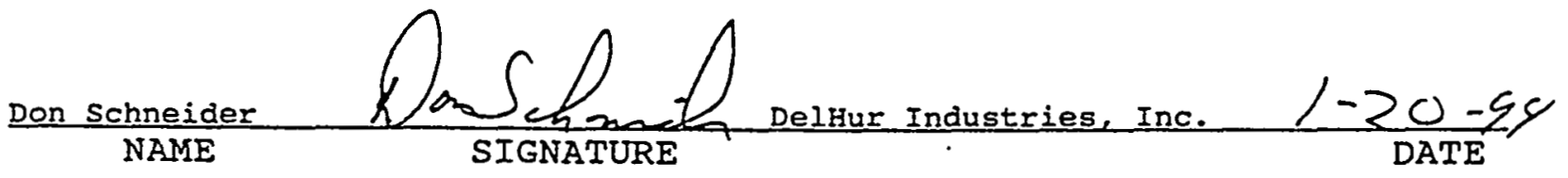

GCS FORM: G4 


\section{CERTIFICATE OF ACCEPTANCE OF SOL SURFACE}

GEOSYNTHETIC
INSTALLER
eOPacific Lining, CO.
300 Dexter Avenue
Suite 250 ,

\section{OSYNTHETIC \\ INSTALLER}

COMPANY GeoPacific Lining, Co. ADDRESS
PROJECT

LOCATION Hanford, Washington PROJECT Non-Drag Off Landfill OWNER Department of Energy

I, the Undersigned, the duly authorized representative of Geopacific Iining Inc. do hereby accept the area of soil surface bounded by:

The surface of primary admix on the access ramp into the cell.

and shall be responsible for maintaining its integrity and suitability in accordance with project specifications from this date to the completion of the installation.

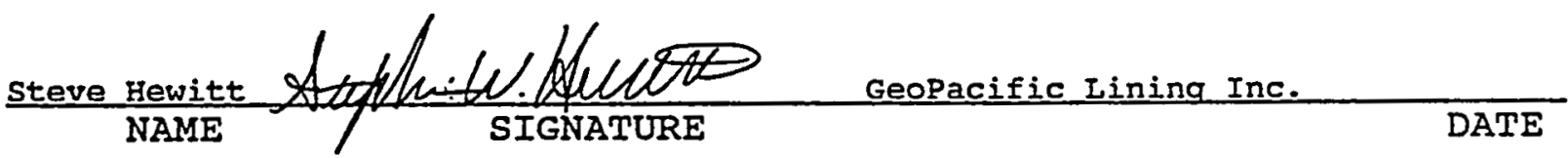

CERTIFICATE OF ACCEPTANCE RECEIVED BY QA/QC MANAGER

$\frac{\text { David H. Mathis }}{\text { NAME }} \frac{\text { Golder Const. Services, Inc. } 1 / 26 / 94}{\text { DATE }}$

CERTIFICATE OF ACCEPTANCE RECEIVED BY GENERAI CONTRACTOR-

$\frac{\text { Don schneider }}{\text { NAME }}$

GCS FORM: G4 


\section{CERTIFICATE OF ACCEPTANCE OF SOIL SURFACE}

\begin{tabular}{|c|c|c|}
\hline $\begin{array}{l}\text { GEOSYNTHETIC } \\
\text { INSTALLER }\end{array}$ & & PROJECT \\
\hline oPacific Lining, $\mathrm{Co}$. & \multirow{2}{*}{\multicolumn{2}{|c|}{$\begin{array}{l}\text { LOCATION Hanford, Washington } \\
\text { PROJECT Non-Drag Off Landfill }\end{array}$}} \\
\hline 00 Dexter Avenue & & \\
\hline ite 250 & OWNER & Department of Energy \\
\hline
\end{tabular}

I, the Undersigned, the duly authorized representative of Geopacific Iining Inc. do hereby accept the area of soil surface bounded by:

The entire surface of primary admix on the floor of the cell.

and shall be responsible for maintaining its integrity and suitability in accordance with project specifications from this date to the completion of the installation.

$\frac{\text { Dennis Martin }}{\text { NAME }} \frac{r \text { Noteth Geopacific Lining Inc. } 1-26-94}{\text { DATE }}$

CERTIFICATE OF ACCEPTANCE RECEIVED BY QA/QC WANAGER

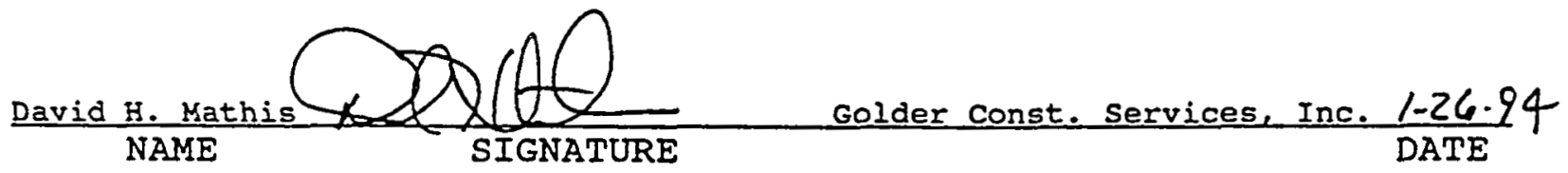

CERTIFICATE OF ACCEPTANCE RECEIVED BY GENERAL CONTRACTOR

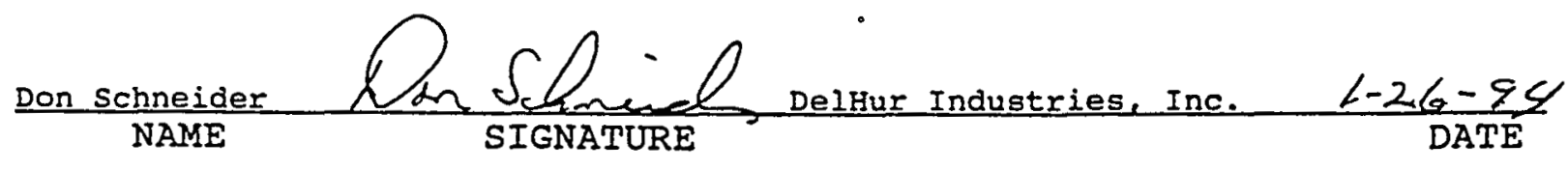

GCS FORM: $G 4$ 


\section{CERTIFICATE OF ACCEPTANCE \\ OF SOIL SURFACE}

\section{GEOSYNTHETIC \\ INSTAIIER}

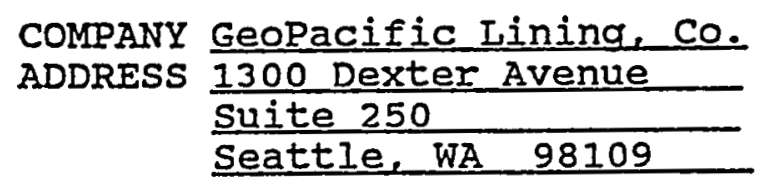

\author{
4.5 \\ PROJECT \\ IOCATION Hanford, Washington \\ PROJECT Non-Drag Off Landfill \\ OWNER
}

I, the Undersigned, the duly authorized representative of Geopacific Iining Inc. do hereby accept the area of soil surface bounded by:

The surface of subgrade in the truck turn around area where panels T-001 through T-007 were installed.

and shall be responsible for maintaining its integrity and suitability in accordance with project specifications from this date to the completion of the installation.
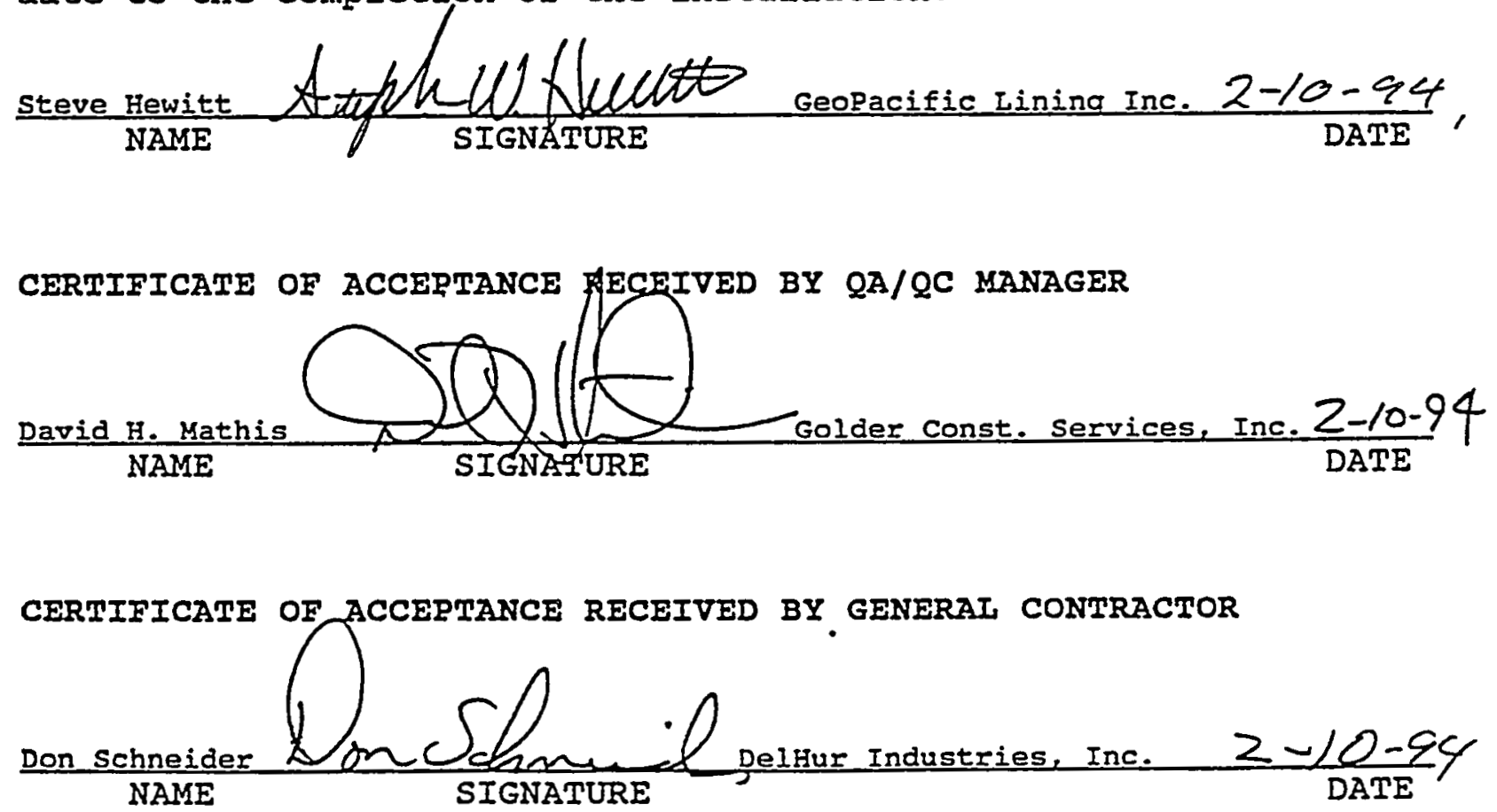

GCS FORM: G4 


\section{CERTIFICATE OF ACCEPTANCE OF SOIL SURFACE}

\begin{tabular}{|c|c|c|c|}
\hline & $\begin{array}{l}\text { GEOSYNTHETIC } \\
\text { INSTALIER }\end{array}$ & & PROJECT \\
\hline COMPANY & Geopacific Lining, $\mathrm{Co}$. & \multirow{2}{*}{\multicolumn{2}{|c|}{$\begin{array}{l}\text { LOCATION Hanford, Washington } \\
\text { PROJECT Non-Drag off Landfill }\end{array}$}} \\
\hline \multirow{3}{*}{ ADDRESS } & 1300 Dexter Avenue & & \\
\hline & Suite 250 & \multirow{2}{*}{ OWNER } & Department of Energy \\
\hline & Seattle, WA 98109 & & \\
\hline
\end{tabular}

I, the Undersigned, the duly authorized representative of Geopacific Iining Inc. do hereby accept the area of soil surface bounded by:

The surface of subgrade in the truck staging area where panels $T=$ 001 through T-007 were installed.

and shall be responsible for maintaining its integrity and suitability in accordance with project specifications from this date to the completion of the installation.

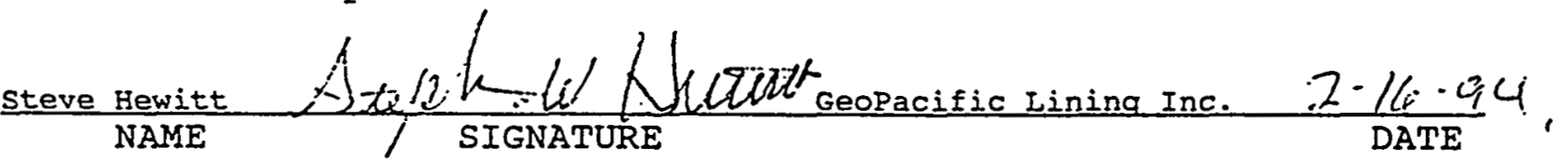

CERTIFICATE OF ACCEPTANCE RECEIVED BY QA/QC MANAGER

$\frac{\text { David H. Mathis }}{\text { NAME }}$

CERTIFICATE OF ACCEPTANCE RECEIVED BY GENERAL CONTRACTOR

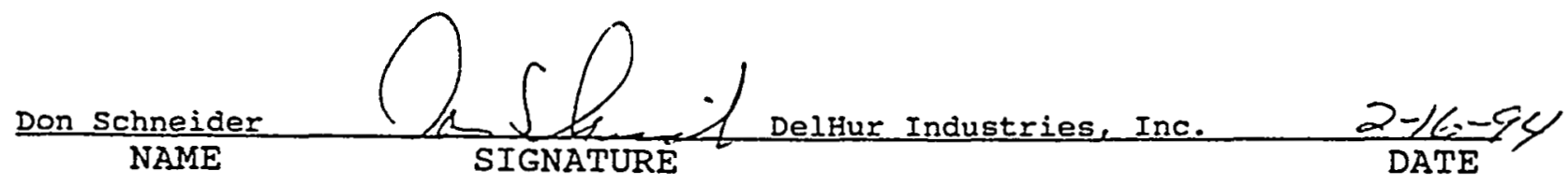

GCS FORM: GA 
WHC-SD-W025-RPT-001, Rev. 0

APPENDIX M

SECONDARY HDPE GEOMEMBRANE LINER INSTALLATION

$M-i$ 
WHC-SD-H025-RPT-001, Rev。0

APPENDIX M-1

GEOMEMBRANE DEPLOYMENT OBSERVATION SUMMARY

$M-1$ 
GOLOER CONSTRUCTION SERVICES, INC.

933-1354

SECONDARY GEOMEMBRANE LINER DEPLOYMENT OBSERVATION SUMMARY

05/11/94

DEPARTHENT OF ENERGY

Page 1

NON-DRAG OFF LANDFILL FACILITY

HANFORD NUCLEAR RESERVATION

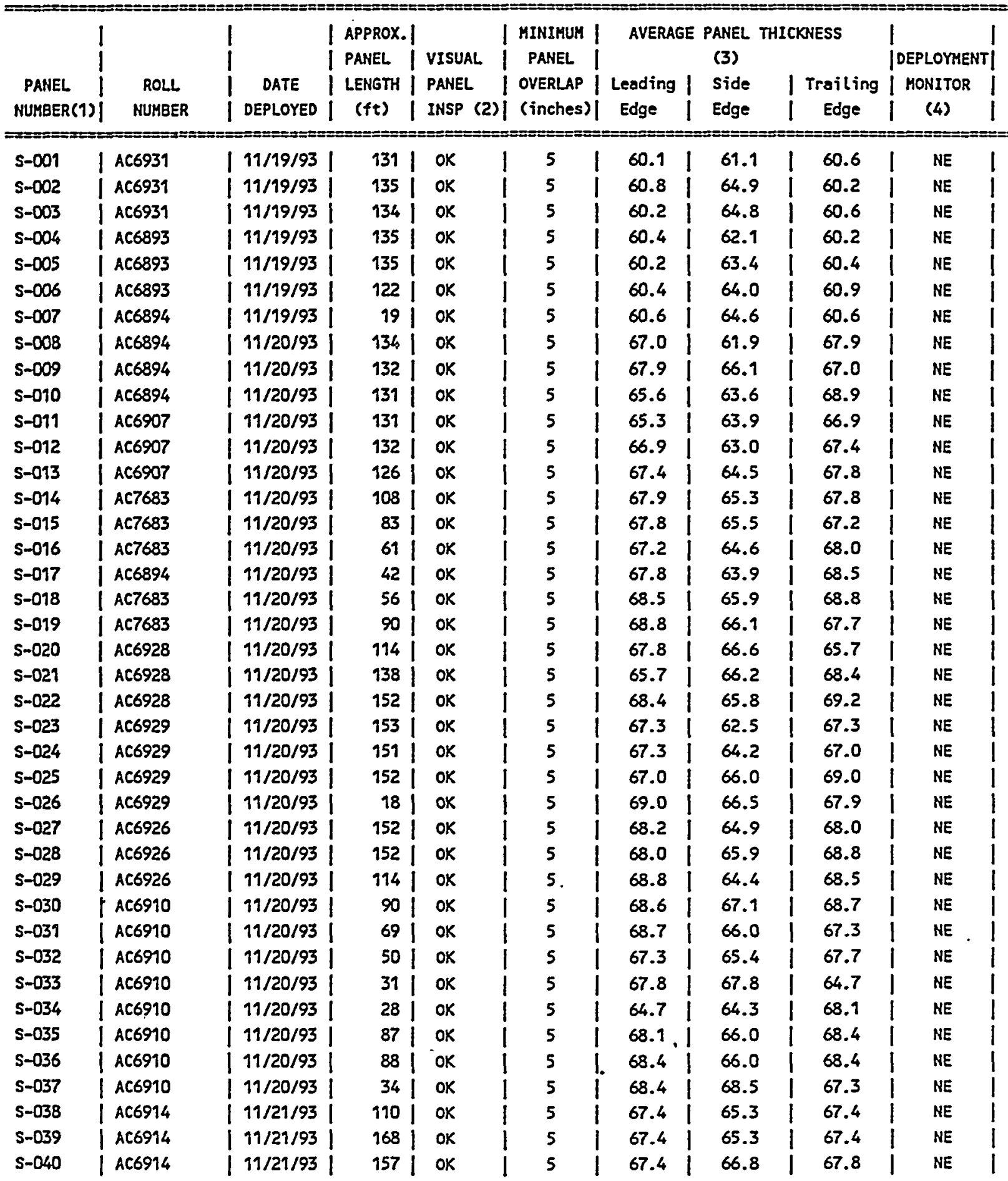


GOLDER CONSTRUCTION SERVICES, INC.

933-1354

SECONDARY GEOMEMBRANE LINER DEPLOYMENT OBSERVATION SUMMARY

$05 / 11 / 94$

DEPARTMENT OF ENERGY

Page 2

NON-DRAG OFF LANDFILL FACILITY

HANFORD NUCLEAR RESERVATION

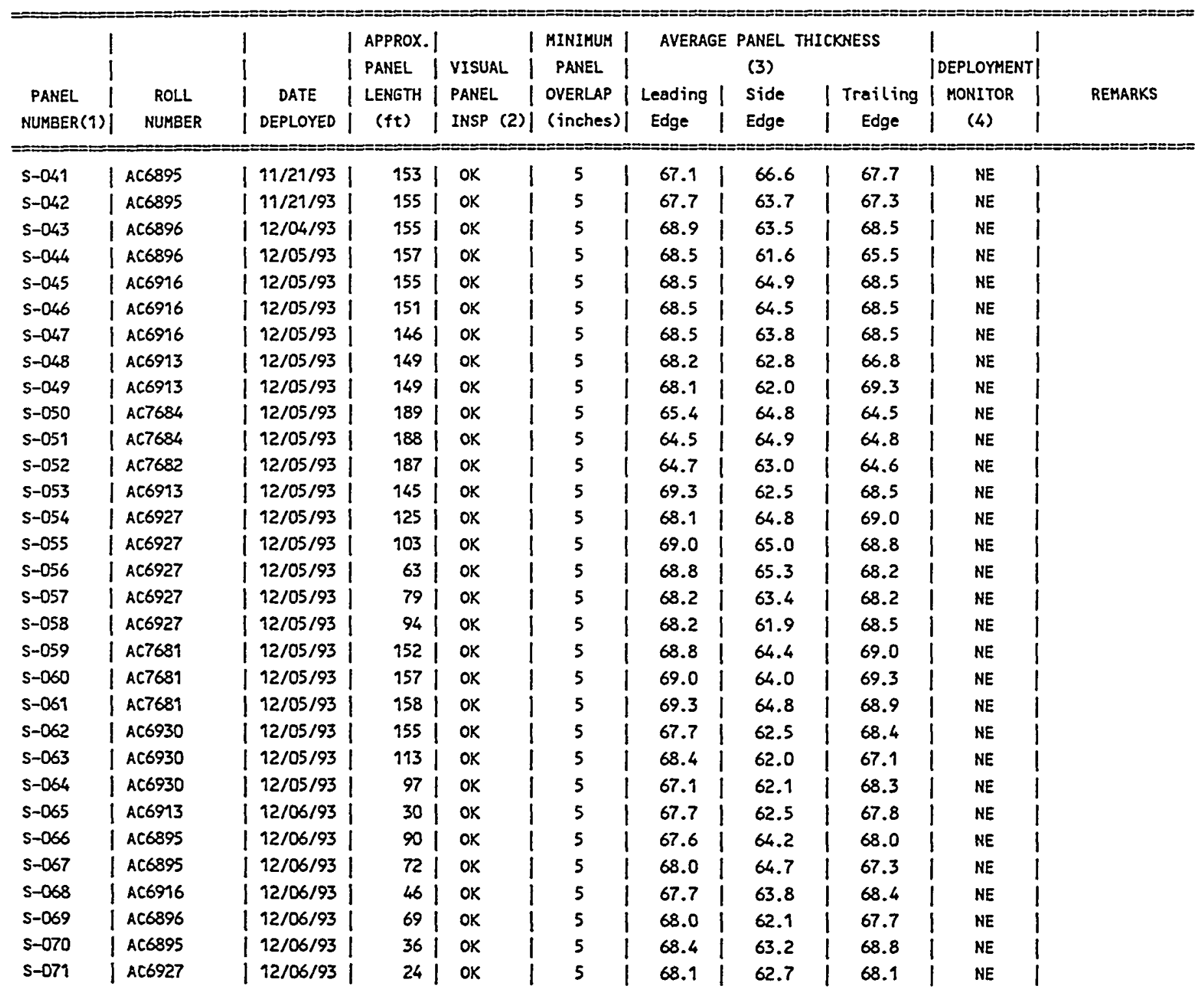




\section{WHC-SD-W025-RPT-001, Rev. 0}

NOTES

(1) A panel number with a prefix of $\mathbf{S}$ indicates a panel of the secondary HDPE geomembrane liner.

(2) OK indicates that the panel was free of visual defects when deployed in the landfill.

(3) Average sheet thickness based upon the average of five micrometer measurements made along each end and one side of the panel.

(4) See Held Observation Summary for monitor identification. 
WHC-SD-W025-RPT-001, Rev。0

APPENDIX M-2

TRIAL SEAM OBSERVATION SUMMARY

$M-2$ 
GOLDER CONSTRUCTION SERVICES, INC.

933-1354

SECONDARY GEOMEMBRANE LINER TRIAL SEAM OBERVATION SUMMARY

$05 / 11 / 94$

DEPARTHENT OF ENERGY

Page 1

NON-DRAG OFF LANDFILL FACILITY

HANFORD NUCLEAR RESERVATION

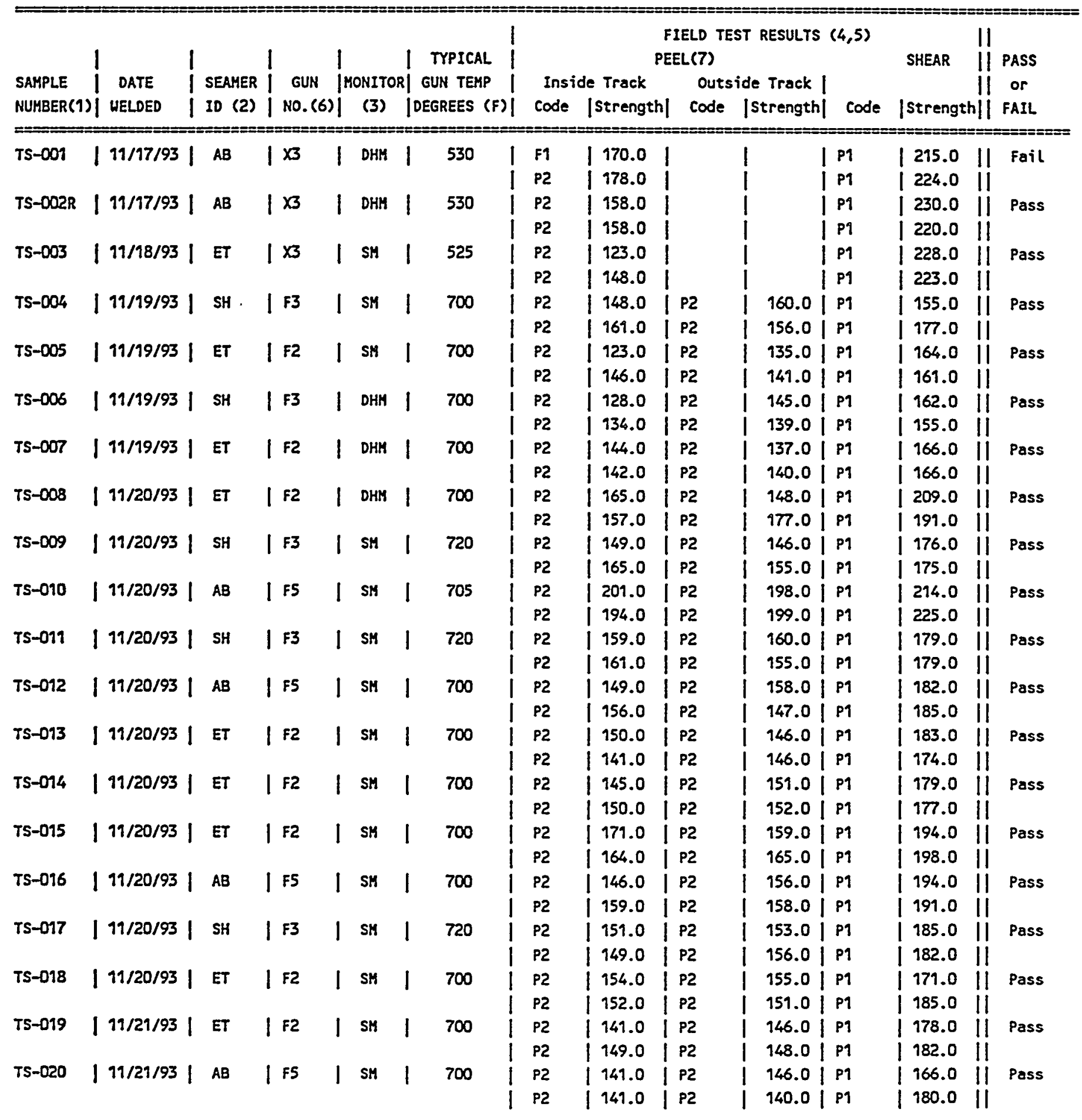


GOLDER CONSTRUCTION SERVICES, INC.

933-1354

SECONDARY GEOMEMBRANE LINER TRIAL SEAM OBERVATION SUMMARY

$05 / 11 / 94$

DEPARTMENT OF ENERGY

Page 2

NON-DRAG OFF LANDFILL FACILITY

HANFORD NUCLEAR RESERVATION

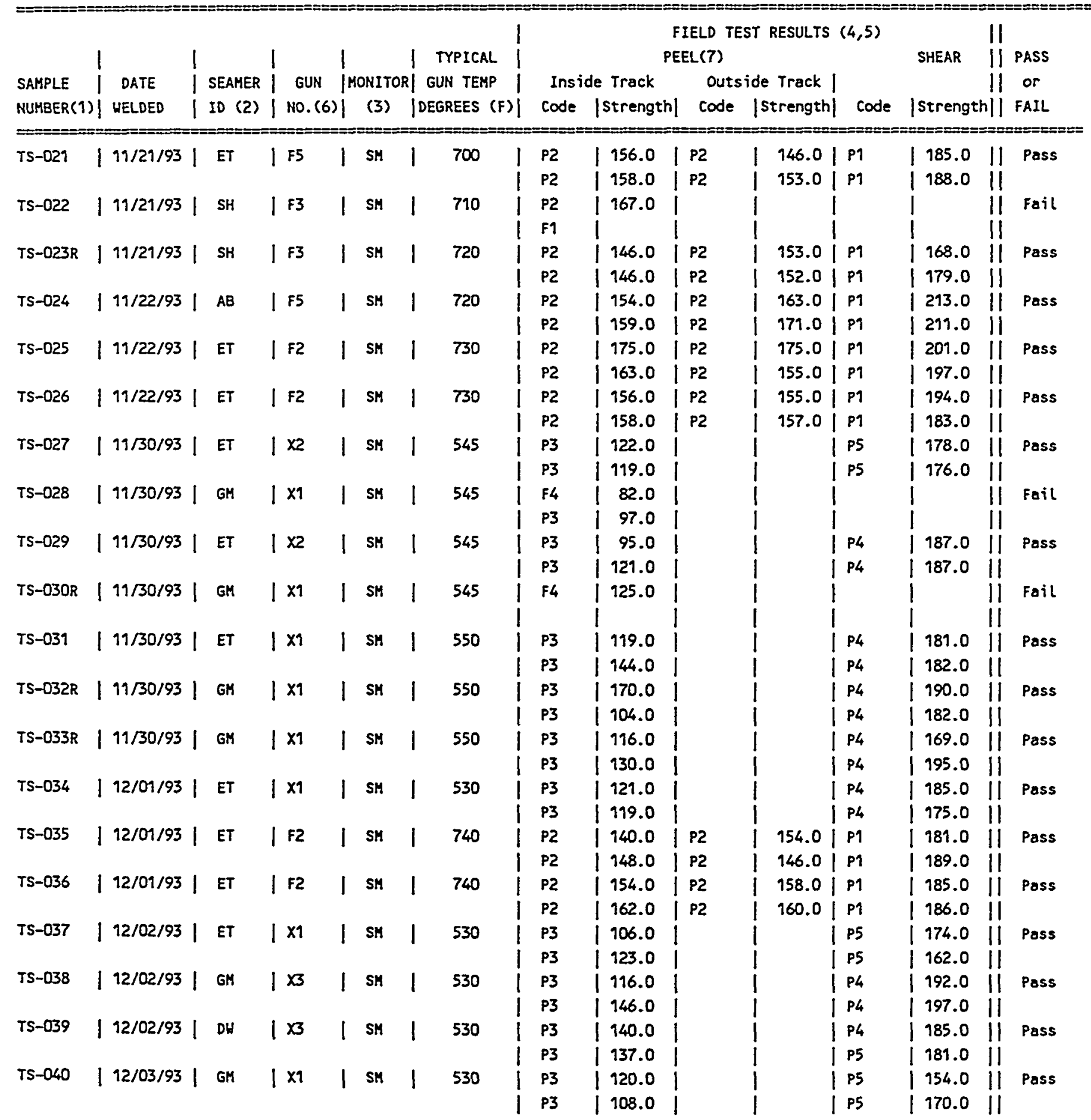


GOLDER CONSTRUCTION SERVICES, INC.

933-9354

SECONDARY GEOMEMBRANE LINER TRIAL SEAM OBERVATION SUMMARY

$05 / 19 / 94$

DEPARTHENT OF ENERGY

Page 3

NON-DRAG OFF LANDFILL FACILITY

HANFORD NUCLEAR RESERVATION

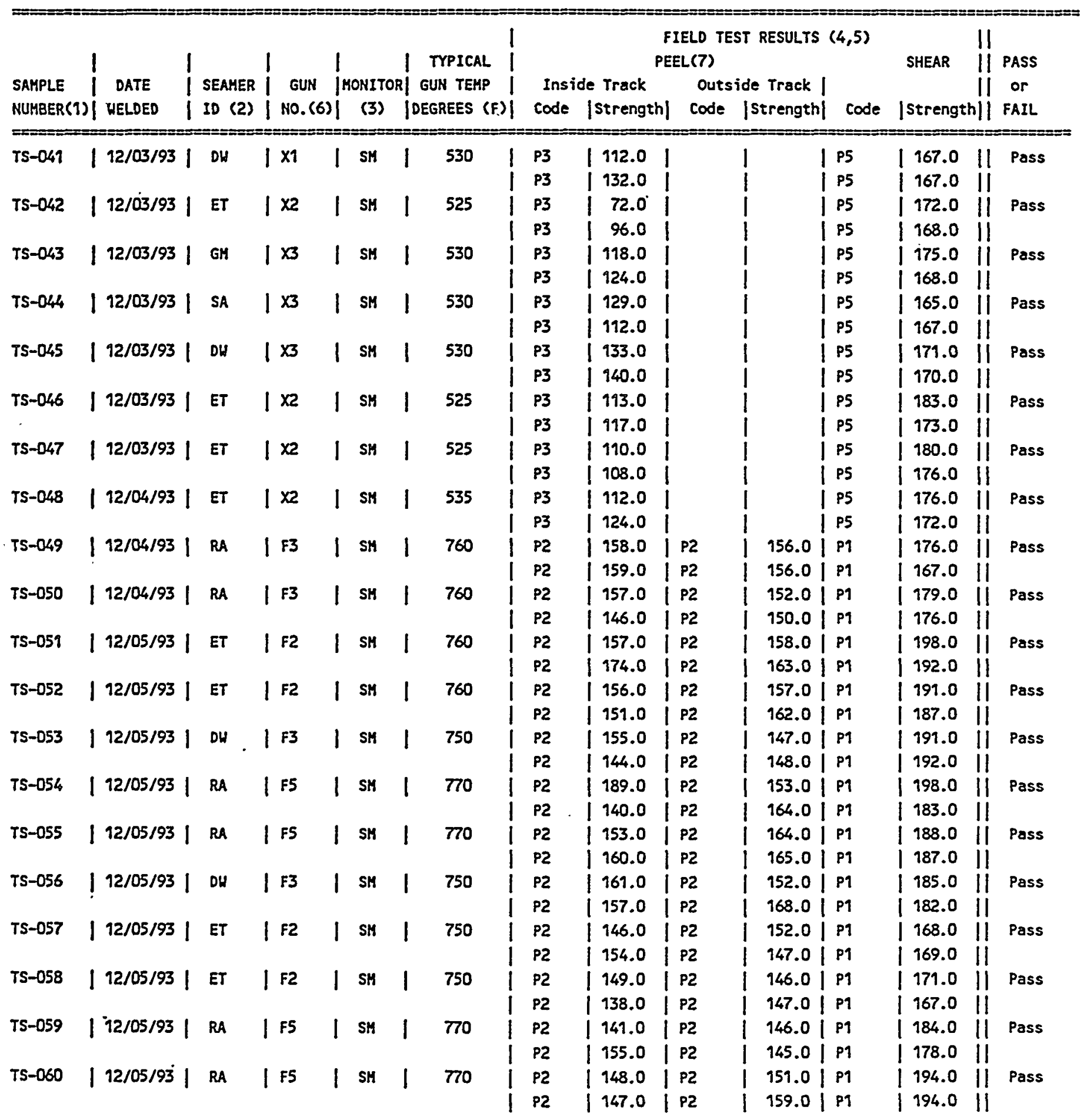


GOLDER CONSTRUCTION SERVICES, INC.

933-1354

SECONDARY GEOMEMBRANE LINER TRIAL SEAM OBERVATION SUMMARY

$05 / 11 / 94$

DEPARTMENT OF ENERGY

Page 4

NON-DRAG OFF LANDFILL FACILITY

HANFORD NUCLEAR RESERVATION

\begin{tabular}{|c|c|c|c|c|c|c|c|c|c|c|c|c|c|c|c|c|}
\hline $\begin{array}{l}\text { SAMPLE } \\
\text { NUMBERC1 }\end{array}$ & $\begin{array}{ll}1 & \\
1 & \text { DATE } \\
1 & \text { KELDED }\end{array}$ & $\begin{array}{l}\text { SEAMER } \\
\text { ID (2) }\end{array}$ & $\left\{\begin{array}{l}\text { GUN } \\
\text { No.(6) }\end{array}\right.$ & & $\begin{array}{l}\text { MONITO } \\
\text { (3) }\end{array}$ & & $\begin{array}{l}\text { TYPICAL } \\
\text { GUN TEMP } \\
\text { EGREES }\end{array}$ & & $\begin{array}{l}\text { Insi } \\
\text { code }\end{array}$ & $\begin{array}{l}\text { ide Track } \\
\text { |Strength }\end{array}$ & $\begin{array}{l}\text { FIELD TE } \\
\text { EEL( } 7 \text { ) } \\
\text { Outs } \\
\text { I cooie }\end{array}$ & $\begin{array}{l}\text { ide Track | } \\
\text { |Strength| }\end{array}$ & $(4,5)$ & $\begin{array}{l}\text { SHEAR } \\
\text { |Strength }\end{array}$ & $\begin{array}{l}11 \\
11 \\
11 \\
n 11\end{array}$ & $\begin{array}{l}\text { PASS } \\
\text { or } \\
\text { FAIL }\end{array}$ \\
\hline \multirow[t]{2}{*}{$T S-061$} & | 12/05/93 & DH & I F3 & 1 & SM & 1 & 750 & 1 & P2 & 1173.0 & P2 & 156.01 & PI & | 203.0 & II & Pass \\
\hline & & & & & & & & & P2 & | 153.0 & P2 & 174.0 & P1 & | 200.0 & 11 & \\
\hline \multirow[t]{2}{*}{$T S-062$} & | 12/05/93 & ET & I F2 & 1 & SM & 1 & 750 & ! & P2 & | 149.0 & P2 & 153.0 & Pq & | 195.0 & II & Pass \\
\hline & & & & & & & & & P2 & | 1954.0 & P2 & 151.01 & Pq & | 190.0 & 11 & \\
\hline \multirow[t]{2}{*}{ TS-063 } & | 12/05/93 & ET & I F2 & 1 & SM & 1 & 750 & 1 & P2 & | 154.0 & P2 & 168.0 & P1 & | 190.0 & 11 & Pass \\
\hline & & & & & & & & & P2 & | 159.0 & P2 & 162.0 & Pq & | 190.0 & 11 & \\
\hline \multirow[t]{2}{*}{ TS-064 } & | 12/06/93 & SH & $1 \times 3$ & 1 & SM & 1 & 525 & 1 & P3 & | 129.0 & | & 1 & P5 & | 188.0 & 11 & Pass \\
\hline & & & & & & & & & P3 & | 151.0 & & 1 & P5 & | 203.0 & 11 & \\
\hline \multirow[t]{2}{*}{ TS-065 } & | 12/06/93 & $\mathrm{DH}$ & $1 F 3$ & 1 & SM & 1 & 750 & 1 & P2 & | 142.0 & | P2 & 161.01 & $P 1$ & | 186.0 & 11 & Pass \\
\hline & & & & & & & & & P2 & | 169.0 & P2 & 161.0 & P9 & | 189.0 & II & \\
\hline \multirow[t]{2}{*}{ TS-066 } & | 12/06/93 & ET & $1 \times 2$ & 1 & SM & 1 & 535 & I & P3 & 1 97.0 & & 1 & P5 & 157.0 & 11 & Pass \\
\hline & & & & & & & & & P3 & | 122.0 & & 1 & P4 & 184.0 & II & \\
\hline \multirow[t]{2}{*}{ TS-057 } & | 12/06/93 & DH & I F3 & 1 & SM & 1 & 750 & & $\mathbf{P 2}$ & | 145.0 & | P2 & 148.01 & $P 1$ & | 181.0 & 11 & Pass \\
\hline & & & & & & & & & P2 & | 151.0 & I P2 & 152.01 & $P 1$ & 1168.0 & II & \\
\hline \multirow[t]{2}{*}{ TS-068 } & | 12/06/93 & ET & $1 \times 2$ & 1 & SM & 1 & 535 & 1 & P3 & | 121.0 & & 1 & P4 & | 171.0 & II & Pass \\
\hline & & & & & & & & & P3 & | 132.0 & & i & P5 & | 174.0 & 11 & \\
\hline \multirow[t]{2}{*}{ TS-069 } & | 12/06/93 & DH & $1 \times 3$ & 1 & SM & 1 & 530 & 1 & P3 & | 127.0 & & 1 & P4 & 173.0 & 11 & Pass \\
\hline & & & & & & & & 1 & P3 & | 128.0 & 1 & 1 & P4 & 177.0 & 11 & \\
\hline \multirow[t]{2}{*}{ TS-070 } & | 12/10/93 & ET & $1 \times 2$ & 1 & SM & 1 & 535 & 1 & P3 & 197.0 & j & i & P4 & 168.0 & II & Pass \\
\hline & & & & & & & & & P3 & | 129.0 & & 1 & $P 4$ & 156.0 & II & \\
\hline \multirow[t]{2}{*}{ TS-071 } & | 12/10/93 & ET & $1 \times 3$ & 1 & SM & 1 & 535 & I & p3 & $\mid 104.0$ & & 1 & PS & | 158.0 & II & Pass \\
\hline & & & & & & & & & P3 & $\mid 128.0$ & & 1 & P5 & | 142.0 & II & \\
\hline \multirow[t]{2}{*}{ TS-072 } & | 12/90/93 & DW & $1 \times 3$ & 1 & SM & 1 & 535 & & P3 & | 128.0 & 1 & 1 & P4 & | 158.0 & II & Pass \\
\hline & & & & & & & & & P3 & | 127.0 & & 1 & P4 & | 156.0 & 11 & \\
\hline \multirow[t]{2}{*}{ TS-073 } & | 12/11/93 & ET & $1 \times 2$ & 1 & SM & 1 & 535 & 1 & P3 & | 917.0 & I & 1 & $P 4$ & 1777.0 & II & Pass \\
\hline & & & & & & & & & P3 & | 103.0 & ! & i & P5 & 181.0 & II & \\
\hline \multirow[t]{2}{*}{ TS-074 } & | 12/11/93 & ET & $1 \times 2$ & 1 & SM & 1 & 535 & 1 & P3 & | 188.0 & 1 & 1 & P4 & | 162.0 & II & Pass \\
\hline & & & & & & & & & P3 & | 117.0 & & 1 & P4 & 163.0 & 11 & \\
\hline \multirow[t]{2}{*}{ TS-075 } & | 12/12/93 & ET & $1 \times 2$ & 1 & SM & 1 & 535 & 1 & P3 & | 126.0 & | & 1 & $P 4$ & 193.0 & II & Pass \\
\hline & & & & & & & & & P3 & 192.0 & & 1 & PS & 196.0 & II & \\
\hline \multirow[t]{2}{*}{ TS-076 } & | 12/12/93 & DH & I F2 & 1 & SM & 1 & 750 & 1 & P2 & | 164.0 & P2 & 159.0 & P1 & | 186.0 & 11 & Pass \\
\hline & & & & & & & & & $\mathbf{P 2}$ & | 159.0 & P2 & 167.0 & P1 & 184.0 & II & \\
\hline \multirow[t]{2}{*}{ TS-077 } & | 12/12/93 & $D H$ & I F3 & 1 & SM & 1 & 750 & & P2 & | 155.0 & P2 & 130.0 & P1 & 1990.0 & II & Pass \\
\hline & & & & & & & & & P2 & | 165.0 & P2 & 175.01 & P1 & | 196.0 & II & \\
\hline \multirow[t]{2}{*}{ TS-078 } & | 12/12/93 & DH & 183 & 1 & SM & 1 & 750 & 1 & $\mathrm{PZ}$ & | 161.0 & P2 & 158.0 & P1 & 176.0 & II & Pass \\
\hline & & & & & & & & & P2 & 1964.0 & P2 & I 150.0 & Pq & 176.0 & 11 & \\
\hline \multirow[t]{2}{*}{ TS-079 } & | 12/12/93 & SH & I F2 & 1 & SM & 1 & 750 & & P2 & | 156.0 & P2 & 161.0 & Pq & 179.0 & 11 & Pass \\
\hline & & & & & & & & & P2 & | 154.0 & P2 & 153.0 & P1 & | 184.0 & II & \\
\hline \multirow[t]{2}{*}{ TS-080 } & | 12/12/93 & ET & $1 \times 2$ & 1 & $S H$ & 1 & 535 & $t$ & P3 & | 120.0 & & 1 & P4 & | 168.0 & 11 & Pass \\
\hline & & & & & & & & & P3 & | 144.0 & & & P4 & | 169.0 & 11 & \\
\hline
\end{tabular}


GOLDER CONSTRUCTION SERVICES, INC.

SECONDARY GEOMEMBRANE LINER TRIAL SEAM OBERVATION SUMMARY DEPARTMENT OF ENERGY

NON-DRAG OFF LANDFILL FACILITY HANFORD NUCLEAR RESERVATION
933-1354

05/11/94

Page 5

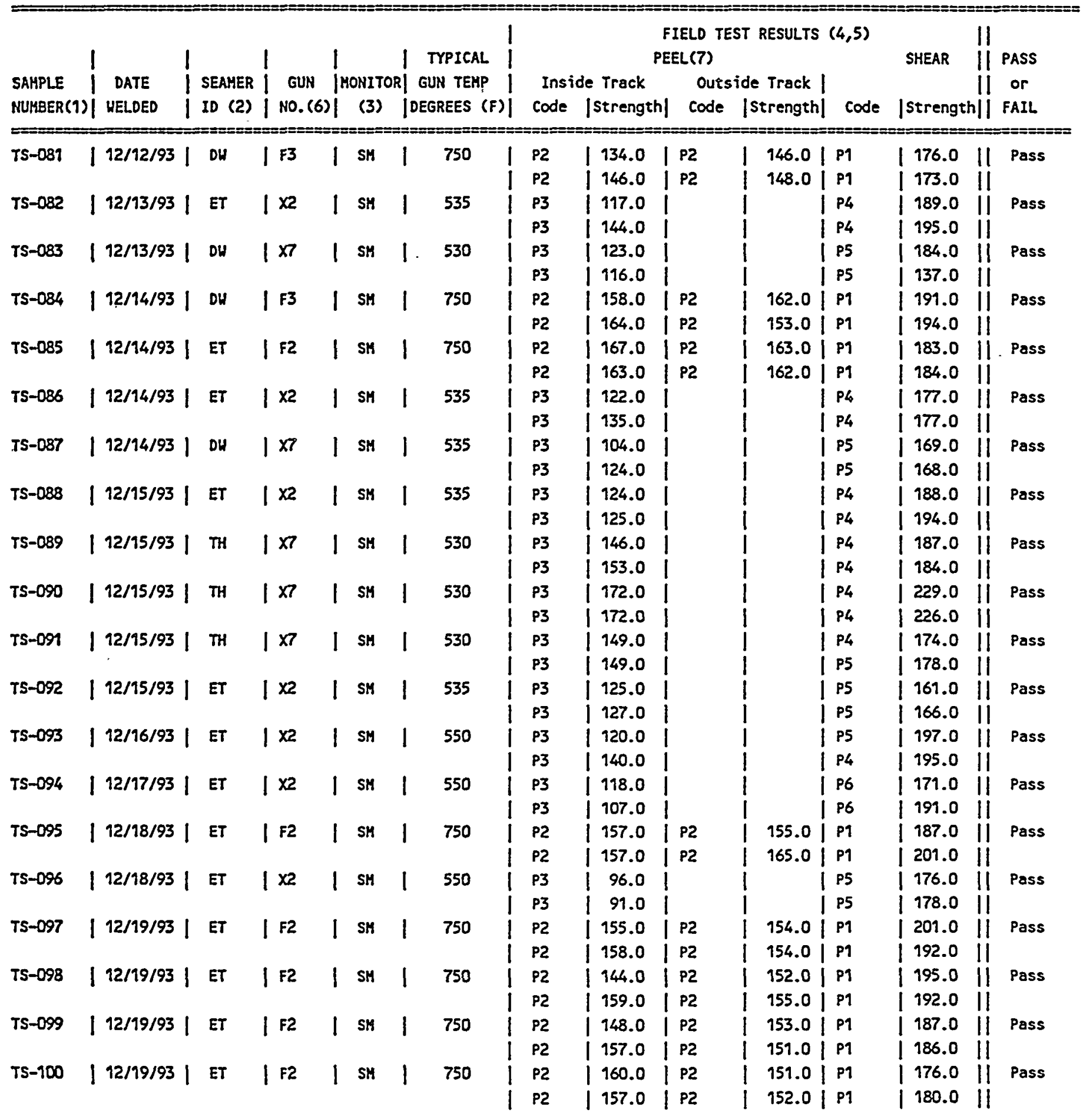


GOLDER CONSTRUCTION SERVICES, INC.

933-1354

SECONDARY GEOMEMBRANE LINER TRIAL SEAM OBERVATION SUMMARY

$05 / 11 / 94$

DEPARTMENT OF ENERGY

Page 6

NON-DRAG OFF LANDFILL FACILITY

HANFORD NUCLEAR RESERVATION

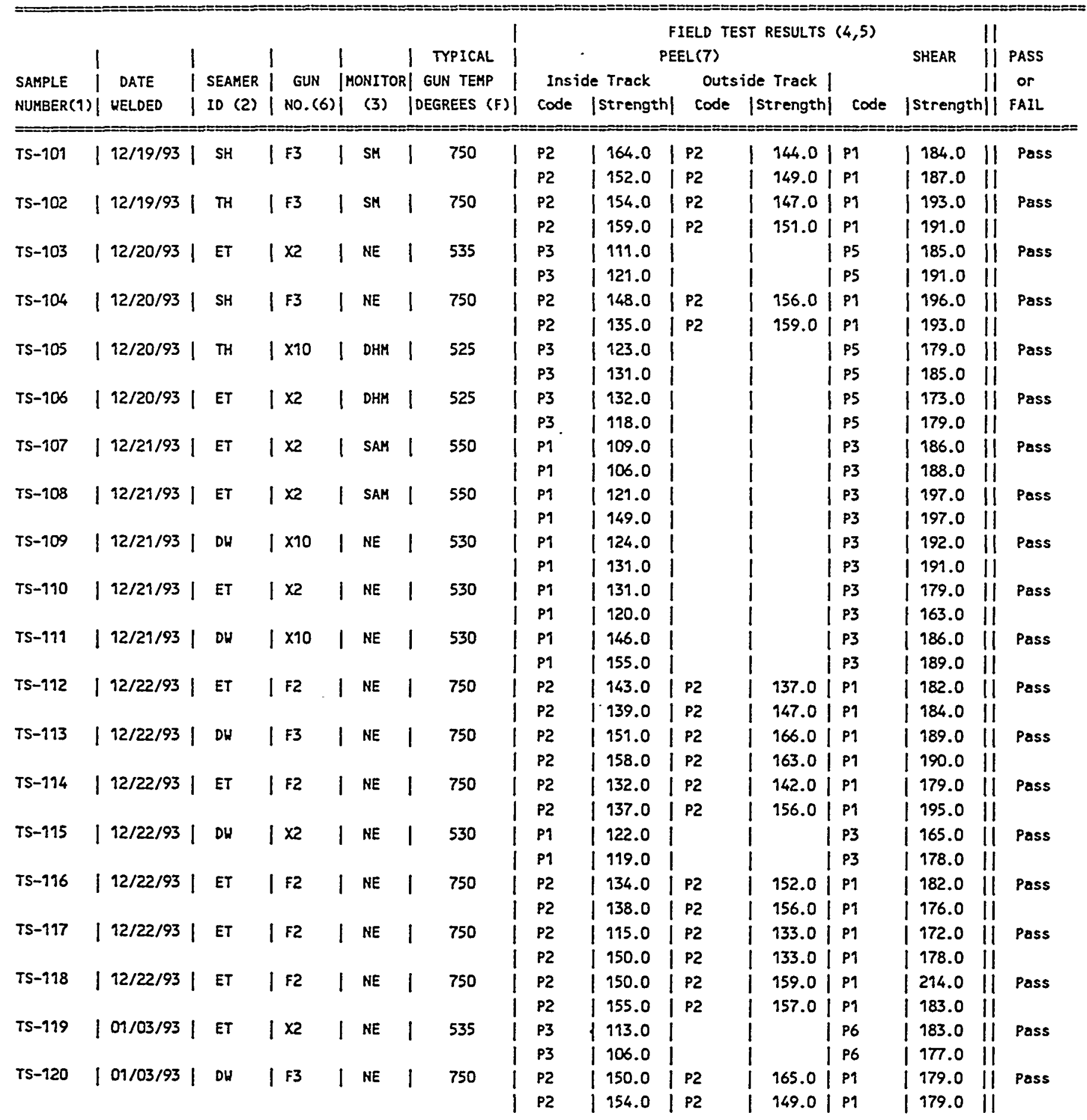


GOLDER CONSTTRUCTION SERVICES, INC.

SECONDARY GEOMEMBRANE LINER TRIAL SEAM OBERVATION SUMMARY

DEPARTMENT OF ENERGY

NON-DRAG OFF LANDFILL FACILITY

HANFORD NUCLEAR RESERVATION
933-1354

$05 / 11 / 94$

Page 7

\begin{tabular}{|c|c|c|c|c|c|c|c|c|c|c|c|c|c|c|}
\hline \multirow{4}{*}{$\begin{array}{l}\text { SAMPLE } \\
\text { NUMBER(1) }\end{array}$} & \multirow{4}{*}{$\begin{array}{l}\text { DATE } \\
\text { UELDED }\end{array}$} & \multirow{4}{*}{$\begin{array}{l}\text { SEAHER } \\
\text { ID (2) }\end{array}$} & \multirow{4}{*}{\multicolumn{2}{|c|}{$\left|\begin{array}{l}\mid \\
\mid \text { GUN } \\
\mid \text { NO.(6) }\end{array}\right|$}} & \multirow{3}{*}{$\begin{array}{l}\text { | } \\
\text { |MONITOR }\end{array}$} & \multirow[b]{2}{*}{ I TYPICAL } & \multirow{2}{*}{\multicolumn{5}{|c|}{$\begin{array}{l}\text { FIELD TEST RESULTS }(4,5) \\
\text { PEEL(7) }\end{array}$}} & \multirow{3}{*}{\multicolumn{2}{|c|}{11}} & \multirow{3}{*}{$\begin{array}{c}\text { PASS } \\
\text { or }\end{array}$} \\
\hline & & & & & & & & & & & & & & \\
\hline & & & & & & R| GUN TEHP & \multicolumn{2}{|c|}{ Inside Track } & \multicolumn{2}{|c|}{ Outside Track | } & \multirow[b]{2}{*}{ Code } & & & \\
\hline & & & & & (3) & |DEGREES (F) & code & |strength| & code & |strength| & & \multicolumn{2}{|c|}{ |Strength|| } & FAIL \\
\hline \multirow{3}{*}{$T S-121$} & $====$ & $==8=$ & 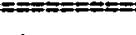 & sto & $===$ & 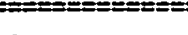 & & & & & $=====$ & $==\pi=====$ & & $====$ \\
\hline & $|01 / 04 / 94|$ & ET & $1 \times 2$ & 1 & NE & 535 & $\mathbf{P 2}$ & | 146.0 & & I & PI & | 173.0 & II & Pass \\
\hline & & & & & & & $\mathbf{P 2}$ & | 137.0 & & & P1 & 171.0 & 11 & \\
\hline \multirow[t]{2}{*}{$T S-122$} & $|01 / 04 / 94|$ & ET & $1 \times 2$ & l & NE & 535 & P2 & | 127.0 & & & P1 & 149.0 & il & Pass \\
\hline & & & & & & & P2 & $|115.0|$ & & & P1 & 158.0 & il & \\
\hline \multirow[t]{2}{*}{ TS-123 } & $|01 / 04 / 94|$ & ET & $1 \times 2$ & I & NE & 535 & P2 & $i 128.0$ & & i & Pq & 162.0 & II & Pass \\
\hline & & & & & & & P2 & 137.0 & & & P1 & 162.0 & il & \\
\hline \multirow[t]{2}{*}{ TS-124 } & |01/04/94 | & ET & $1 \times 2$ & I & NE & 535 & P2 & 138.0 & & & Pq & 148.0 & II & Pass \\
\hline & & & & & & & P2 & 122.0 & & & P1 & 152.0 & 11 & \\
\hline \multirow[t]{2}{*}{ TS-125 } & $|01 / 05 / 94|$ & ET & $1 \times 2$ & I & NE & 535 & $\mathbf{P 2}$ & 920.0 & & & P1 & 153.0 & II & Pass \\
\hline & & & & & & & P2 & 913.0 & & & P1 & | 155.0 & il & \\
\hline TS-126 & $01 / 05 / 94$ & ET & $1 \times 2$ & 1 & NE & 535 & P2 & 110.0 & & & $P 1$ & 148.0 & il & Pass \\
\hline & & & & & & & $P 2$ & 120.0 & & & P1 & | 148.0 & il & \\
\hline TS-127 & $01 / 06 / 94$ & ET & $1 \times 2$ & 1 & NE & 535 & $P 2$ & 112.0 & & & P1 & | 158.0 & II & Pass \\
\hline & & & & & & & P2 & 124.0 & & & P1 & 152.0 & 11 & \\
\hline TS-12B & $01 / 06 / 94$ & ET & $1 \times 2$ & 1 & NE & 535 & P2 & 103.0 & & & P1 & | 141.0 & II & Pass \\
\hline & & & & & & & P2 & | 104.0 & & & PI & 151.0 & II & \\
\hline TS-129 & $01 / 06 / 94$ & DH & I F2 & 1 & $\mathrm{NE}$ & 750 & $\mathbf{P 2}$ & 145.0 & P2 & $132.0 \mid$ & P1 & | 169.0 & II & Pass \\
\hline & & & & & & & P2 & 136.0 & P2 & 149.0 & P1 & | 162.0 & II & \\
\hline TS-130 & $01 / 06 / 94$ & DH & | F2 & 1 & NE & 750 & P2 & | 135.0 & $P 2$ & 128.0 & $\mathbf{P 1}$ & | 147.0 & II & Pass \\
\hline & & & & & & & $P 2$ & | 132.0 & P2 & 126.0 & P1 & 153.0 & 11 & \\
\hline TS-131 & $01 / 06 / 94 \mid$ & ET & $1 \times 2$ & 1 & NE & 535 & P2 & 125.0 & & & P1 & 146.0 & il & Pass \\
\hline & & & & & & & P2 & | 139.0 & & & P1 & | 148.0 & 11 & \\
\hline TS-132 & $01 / 06 / 94$ & ET & $1 \times 2$ & 1 & NE & 535 & $P 2$ & | 130.0 & & & P1 & | 147.0 & 11 & Pass \\
\hline & & & & & & & P2 & 118.0 & & & $P q$ & | 152.0 & 11 & \\
\hline TS-133 & $01 / 06 / 94$ & $\mathrm{TH}$ & | x10 & 1 & NE & 525 & P2 & | 130.0 & & & P1 & | 162.0 & 11 & Pass \\
\hline & & & & & & & P2 & | 124.0 & & & P1 & | 174.0 & 11 & \\
\hline TS-134 & $01 / 07 / 94$ & $\mathrm{DW}$ & $1 F 2$ & 1 & DHM & 750 & $\mathrm{P2}$ & i 129.0 & $P 2$ & $139.0 \mid$ & P1 & 176.0 & II & Pass \\
\hline & & & & & & & $P 2$ & 1 145.0 & $P 2$ & 153.0 & P1 & | 177.0 & 11 & \\
\hline TS-135 & $01 / 07 / 94$ & TH & $x 2$ & 1 & DHH & 535 & P1 & | 119.0 & & & $P 3$ & | 187.0 & II & Pass \\
\hline & & & & & & & Pq & 130.0 & & & P3 & | 186.0 & 11 & \\
\hline TS-136 & $01 / 07 / 94$ & DH & $F 2$ & I & DHM & 750 & $P 2$ & | 140.0 & P2 & 149.0 & P1 & | 172.0 & 11 & Pass \\
\hline & & & & & & & $P 2$ & 146.0 & $\mathrm{P2}$ & 153.0 & P1 & 171.0 & 11 & \\
\hline TS-137 & $01 / 07 / 94$ & DH & I F2 & 1 & DHM & 750 & P2 & | 147.0 & $P Z$ & 150.0 & Pq & 180.0 & 11 & Pass \\
\hline & & & & & & & P2 & | 149.0 & $P 2$ & 128.0 & P1 & | 181.0 & 11 & \\
\hline TS-138 & $01 / 07 / 94$ & SH & I F3 & 1 & DHH & 750 & $P 2$ & 146.0 & $P 2$ & 114.0 & & & 11 & Fail \\
\hline & & & & & & & F1 & | 101.0 & & & & & $\|$ & \\
\hline TS-139 & $01 / 07 / 94$ & TH & $x 2$ & I & DHM & 535 & P1 & .1129 .0 & & & P3 & 167.0 & 11 & Pass \\
\hline & & & & & , & & PI & | 109.0 & & & $P 3$ & | 187.0 & 11 & \\
\hline TS-140 & $01 / 07 / 94$ & SH & I F2 & 1 & DHM & 750 & $P 2$ & | 130.0 & P2 & 135.0 & $P 2$ & 176.0 & 11 & Pass \\
\hline & & & & & & & $P 2$ & | 126.0 & $P 2$ & 130.0 & $P 2$ & | 177.0 & 11 & \\
\hline
\end{tabular}


WHC-SD-W025-RPT-001, Rev. 0

GOLDER CONSTRUCTION SERVICES, INC.

933-1354

SECONDARY GEOMEMBRANE LINER TRIAL SEAM OBERVATION SUMMARY

$05 / 11 / 94$

DEPARTMENT OF ENERGY

Page 8

NON-DRAG OFF LANDFILLL FACILITY

HANFORD NUCLEAR RESERVATION

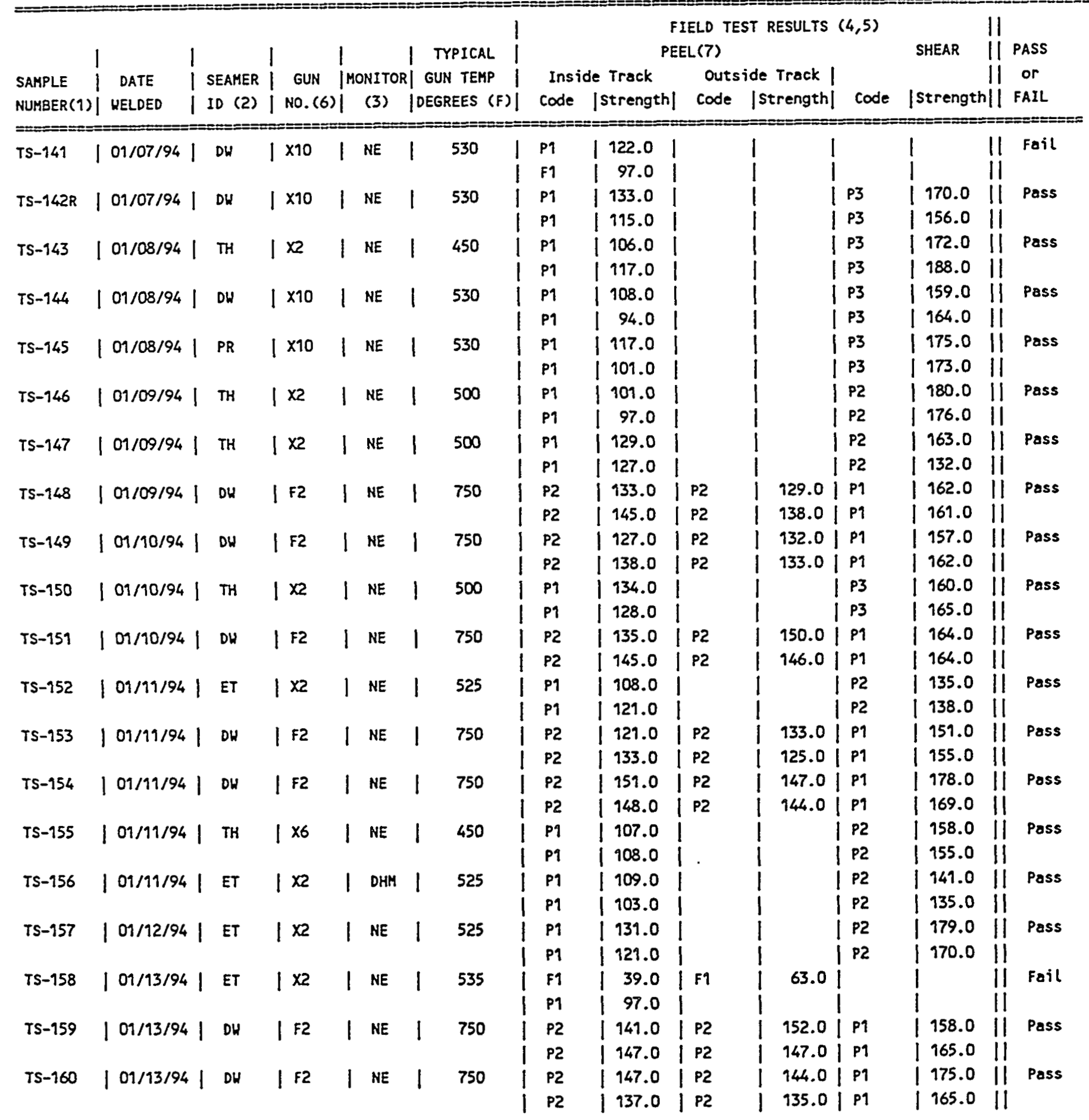


GOLDER CONSTRUCTION SERVICES, INC.

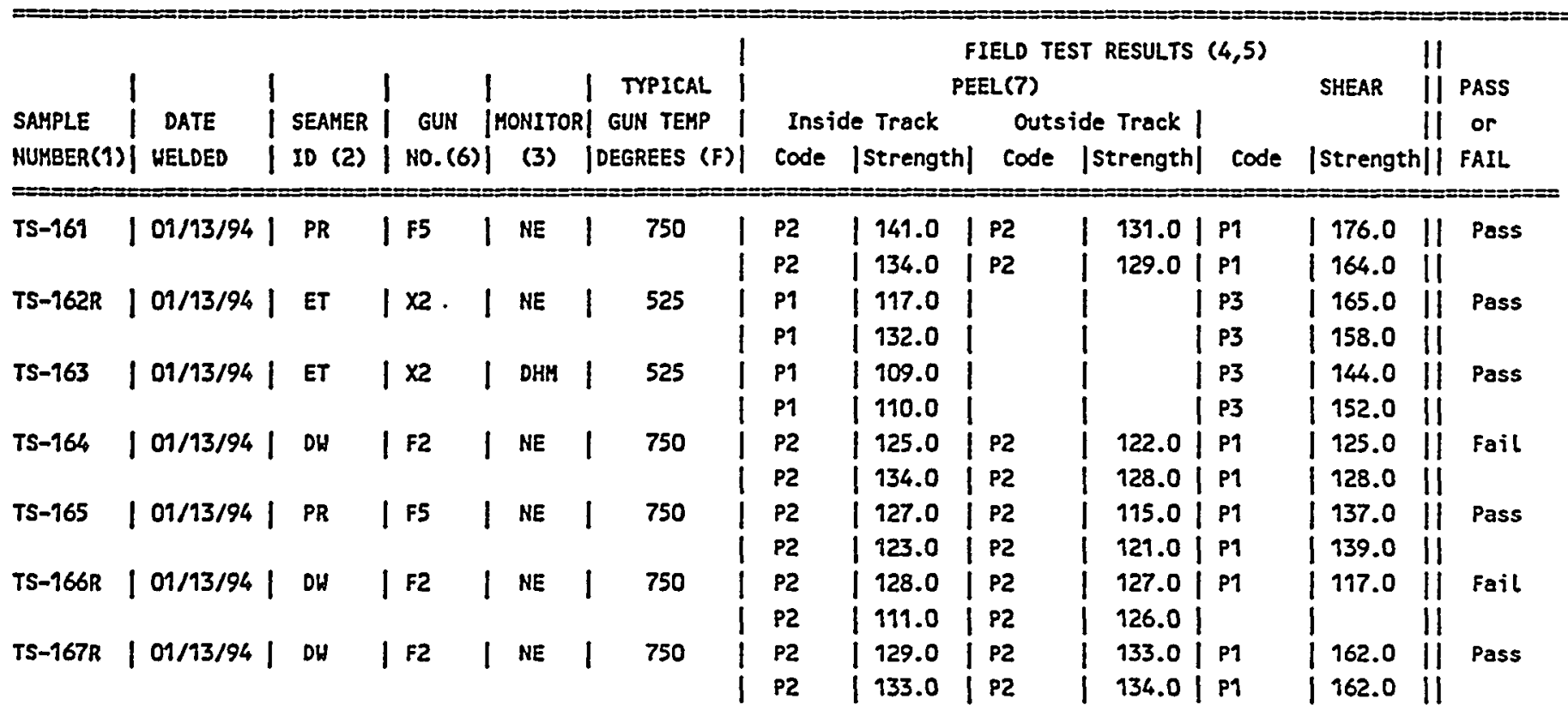

NOTES

(1) Trial Held Sample Numbers with an $R$ suffix are retest samples for a previously failed test performed by the same welder on the same date. e.g. TS-162R is a retest for failed test sample TS-158 welded by $x 2$.

(2) See Weld Inspection Summary for seamer identification.

(3) See Held Inspection Summary for monitor identifcation.

(4) See Figures for illustrations of modes of passing or failing test codes.

(5) Strengths are reported in pounds per inch width.

(6) Gun Numbers with an $X$ prefix are extrusion welders. Gun Numbers with an $F$ prefix are fusion welders.

(7) Passing Trial Seams without an outside track test result are for extrusion trial seams. 
WHC-SD-W025-RPT-001，Rev. 0

APPENDIX M-3

GEOMEMBRANE WELD OBSERVATION AND NON-DESTRUCTIVE TESTING SUMMARY

$M-3$ 
GOLDER CONSTRUCTION SERVICES, INC.

933-1354

SECONDARY GEOHEMBRANE LINER HELD OBSERVATION AND NON-DESTRUCTIVE TESTING SUMMARY

$05 / 11 / 94$

DEPARTMENT OF ENERGY

Page 1

NON-DRAG OFF LANDFILL FACILITY .

HANFORD NUCLEAR RESERVATION

\begin{tabular}{|c|c|c|c|c|c|c|c|c|c|c|c|}
\hline \multirow{3}{*}{$\begin{array}{l}\text { SEAM } \\
\text { NUMBER(1) }\end{array}$} & \multicolumn{2}{|c|}{$\mid$ PANEL NUMBERS } & \multirow{3}{*}{$=\begin{array}{l}1 \\
1 \text { DATE } \\
1 \text { HELDED }\end{array}$} & \multirow{3}{*}{$\begin{array}{l}\mid \\
\mid \\
\mid \text { SEAMER } \\
\mid \text { IDC(2)| }\end{array}$} & \multirow{3}{*}{$\begin{array}{l}\text { Gun } \\
\text { No. }\end{array}$} & \multirow{3}{*}{$\begin{array}{l}\mid \\
\text { TYPICAL } \\
\text { GUN TEMP } \\
\text { |DEGREES }(F) \mid\end{array}$} & \multirow{3}{*}{$\begin{array}{l}\text { DESTR. } \\
\text { TEST }\end{array}$} & \multirow{3}{*}{ 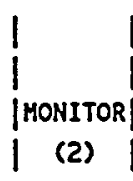 } & \multicolumn{3}{|c|}{$\mid \begin{array}{l}\text { NON-DESTRUCTIVE TEST } \\
\mid====\end{array}$} \\
\hline & I PANEL & I PANEL & & & & & & & & | HONITOR & R| DEFECTS \\
\hline & $\# 1$ & $\# 2$ & & & & & & & $\mid$ DATE (3) $\mid$ & i (2) & $i$ \\
\hline s-001/002 & | s-001 & s-002 & | 11/19/93 & SH & F3 & 700 & & DHM & | 11/20/93 & SH & 1 \\
\hline$s-001 / 063$ & $s-\infty$ & $s-063$ & | 12/05/93 & $\mathrm{DH}$ & F3 & 750 & & SH & | 12/11/93 & SM & i \\
\hline s-001/064 & $s-\infty 01$ & $s-064$ & | 12/05/93 & DH & F3 & 750 & & SM & $12 / 11 / 93$ & SH & i \\
\hline$s-001 / 066$ & $s-001$ & $5-066$ & | 12/06/93 & DH & F3 & 750 & & NE & | 12/08/93 & SH & 1 \\
\hline$s-002 / 003$ & $s-002$ & $s-003$ & | 91/19/93 & ET & F2 & 700 & & DHM & | 11/20/93 & SH & 1 \\
\hline$s-002 / 062$ & $s-002$ & $s-062$ & | 12/05/93 & DH & F3 & 750 & & SH & | 12/08/93 & SH & 1 \\
\hline$s-003 / 004$ & $s-003$ & $5-004$ & | 11/19/93 & SH & F3 & 700 & DS-001 & DHM & $11 / 20 / 93$ & SH & 1 \\
\hline$s-003 / 052$ & s-003 & $s-052$ & | 12/05/93 & $R A$ & F5 & 770 & & SH & $11 / 99 / 91$ & DHM & 13 \\
\hline$s-003 / 062$ & $s-003$ & $s-062$ & | 12/05/93 & $D H$ & F3 & 750 & & SH & $12 / 11 / 93$ & SM & \\
\hline$S-003 / 501 A$ & s-003 & SOIA & | 12/03/93 & GM & $x_{1}$ & 530 & DS-015 & SH & $12 / 03 / 93$ & SH & 1 \\
\hline S- $003 / S 01 A$ & $s-003$ & SOTA & | 12/03/93 & ET & $\mathrm{x} 2$ & 525 & & SM & $12 / 03 / 93$ & SH & 1 \\
\hline$s-003 / s 10 \mathrm{~g}$ & $s-003$ & $\$ 106$ & | 12/22/93 & ET & F2 & 750 & & NE & $01 / 07 / 94$ & NE & i \\
\hline$s-004 / 005$ & $s-004$ & is s-005 & i 11/19/93 & ET & $F 2$ & 700 & DS-002 & DHM & | 11/20/93 & SH & i \\
\hline$s-004 / 052$ & $s-004$ & s-052 & $12 / 05 / 93$ & RA & F5 & 770 & & SH & | 12/19/93 & SM & 1 \\
\hline$s-004 / 501 \lambda$ & $s-004$ & so1A & | $12 / 03 / 93$ & ET & $x 2$ & 525 & & SM & | 12/03/93 & SM & I \\
\hline$S-004 / 501 A$ & $s-004$ & is so1A & | $12 / 03 / 93$ & GN & $x 1$ & 530 & & SM & | 12/03/93 & SH & i \\
\hline$s-004 / s 106$ & $5-004$ & is10G & | 12/22/93 & ET & F2 & 750 & & NE & | 01/04/94 & NE & i \\
\hline s-005/006 & $s-00 s$ & $s-006$ & $11 / 19 / 93$ & SH & F3 & 700 & & DHM & $11 / 20 / 93$ & SM & i \\
\hline$s-005 / 007$ & $s-005$ & s-007 & | 11/19/93 & SH & F3 & 700 & & DHM & | 11/20/93 & $S M$ & i \\
\hline s-005/052 & $s-005$ & $s-052$ & 12/05/93 & RA & F5 & 770 & & SM & | 12/11/93 & SM & i \\
\hline$s-006 / 007$ & $s-006$ & s-007 & | 11/19/93 & ET & F2 & 700 & & DHM & | 11/20/93 & SM & I \\
\hline$s-006 / 008$ & $s-006$ & $s-008$ & | 11/20/93 & ET & F2 & 720 & & DHM & i 11/20/93 & $S M$ & i \\
\hline$s-007 / 008$ & s-007 & is-008 & | $11 / 20 / 93$ & ET & F2 & 720 & & DHM & i 11/20/93 & $S H$ & i \\
\hline$s-007 / 052$ & 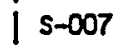 & is s-052 & | 12/05/93 & RA & F5 & 770 & & SM & | 12/11/93 & $S M$ & 1 \\
\hline s-008/009 & is s-008 & s-009 & | 11/20/93 & ET & F2 & 720 & & DHM & | 11/20/93 & SM & 1 \\
\hline s-008/052 & | s-008 & | s-052 & | $12 / 05 / 93$ & RA & F5 & 770 & & $1 . S H$ & | 12/11/93 & SM & | \\
\hline$s-009 / 010$ & | s-009 & | s-010 & | 11/20/93 & $A B$ & F5 & 705 & & SH & | 11/20/93 & SM & 1 \\
\hline$s-009 / 052$ & $s-009$ & | s-052 & i 12/05/93 & RA & F5 & 770 & & SM & | 12/06/93 & SH & i \\
\hline$s-010 / 011$ & $s-010$ & is s-011 & $11 / 20 / 93$ & SH & F3 & 720 & & SM & 11/20/93 & SM & i \\
\hline s-010/052 & $s-010$ & $s-052$ & 12/05/93 & RA & F5 & 770 & & SM & $12 / 06 / 93$ & SM & \\
\hline$s-011 / 012$ & s-019 & s-012 & | 11/20/93 & $A B$ & F5 & 705 & & SM & | 11/22/93 & SM & 1 \\
\hline s-019/052 & | s-019 & | s-052 & | 12/05/93 & RA & F5 & 770 & & SM & $12 / 06 / 93$ & SM & \\
\hline $5-011 / 503 x$ & $s-019$ & i so3x & | 12/04/93 & ET & $x 2$ & 535 & & SM & $12 / 04 / 93$ & SM & i \\
\hline s-012/013 & $s-012$ & | s-013 & i 11/20/93 & SH & F3 & 720 & DS-003 & SH & $11 / 22 / 93$ & SM & I \\
\hline$s-012 / 022$ & $s-012$ & $s-022$ & i 12/06/93 & ET & $x 2$ & 535 & & SM & $12 / 20 / 93$ & DHM & \\
\hline$s-012 / 022$ & $s-012$ & is-022 & | $11 / 20 / 93$ & ET & F2 & 700 & & $S M$ & | 12/20/93 & DHM & \\
\hline$s-012 / 052$ & is -012 & | s-052 & | $12 / 05 / 93$ & RA & F5 & 770 & DS -023 & SM & | 12/06/93 & SM & \\
\hline $5-012 / 503 x$ & is $s-012$ & i so3x & | 12/04/93 & ET & $x 2$ & 535 & & SM & $12 / 04 / 93$ & SM & \\
\hline s-013/014 & $s-013$ & $5-014$ & 11/20/93 & ET & F2 & 720 & DS-004 & DHM & $11 / 22 / 93$ & $S M$ & 1 \\
\hline $5-013 / 029$ & $s-013$ & $s-021$ & 11/20/93 & ET & $F 2$ & 700 & & i SM & $12 / 02 / 93$ & i SM & i \\
\hline
\end{tabular}


GOLDER CONSTRUCTION SERVICES, INC.

933-1354 SECONDARY GEOMEMBRANE LINER WELD OBSERVATION AND NON-DESTRUCTIVE TESTING SUMMARY $05 / 19 / 94$ DEPARTMENT OF ENERGY Page 2 NON-DRAG OFF LANDFILL FACILITY HANFORD NUCLEAR RESERVATION

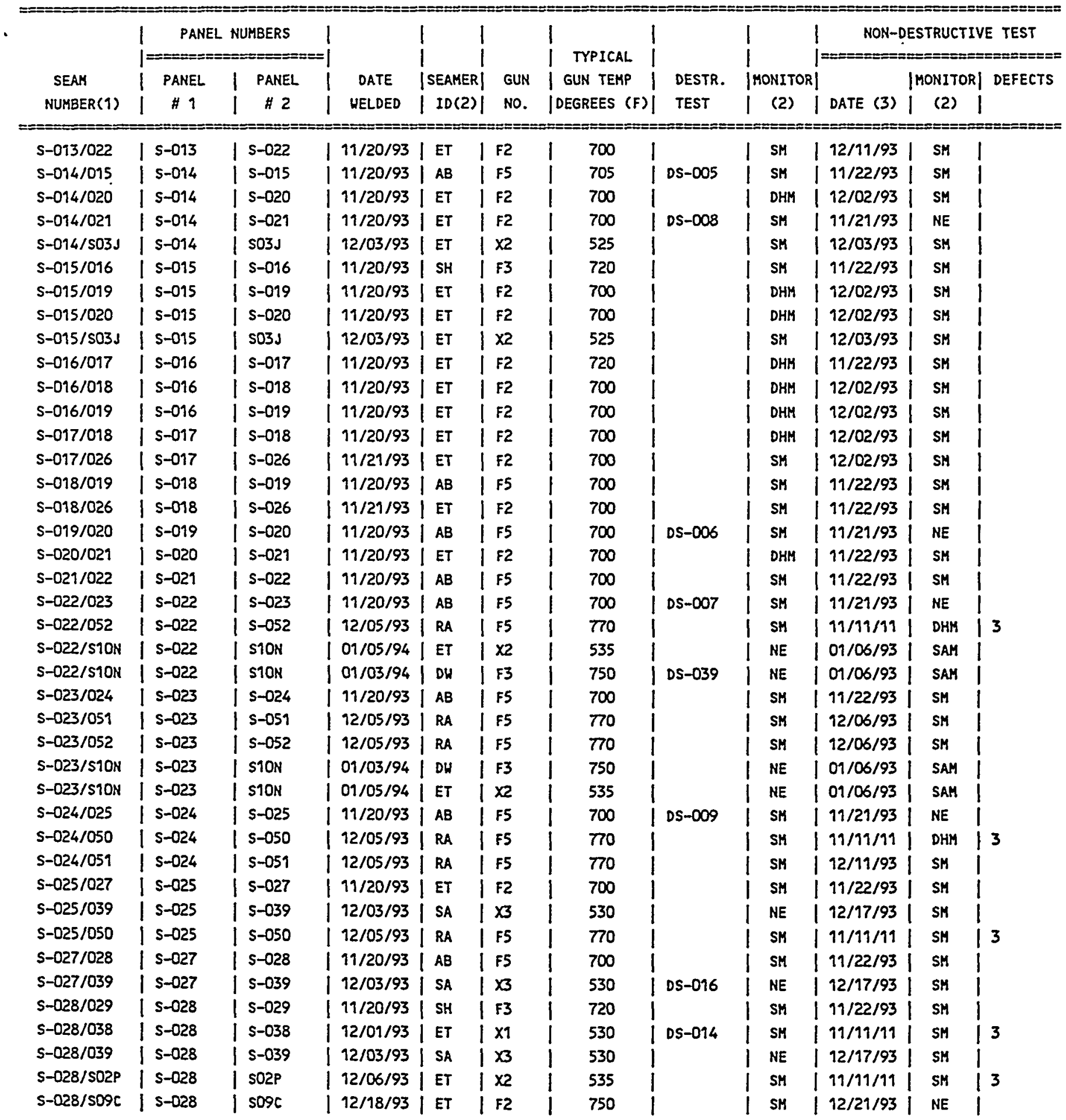


GOLDER CONSTRUCTION SERVICES, INC.

933-1354

SECONDARY GEOMEMBRANE LINER HELD OBSERVATION AND NON-DESTRUCTIVE TESTING SUMMARY

$05 / 91 / 94$

DEPARTMENT OF ENERGY

Page 3

NON-DRAG OFF LANDFILL FACILITY

HANFORD NUCLEAR RESERVATION

\begin{tabular}{|c|c|c|c|c|c|c|c|c|c|c|c|}
\hline \multirow{2}{*}{$\begin{array}{l}\text { SEAH } \\
\text { NUMBER(1) }\end{array}$} & \multicolumn{2}{|c|}{ I PANEL NUMBERS } & \multirow{2}{*}{$\begin{array}{c}\text { DATE } \\
\text { HELDED }\end{array}$} & \multirow{2}{*}{$\begin{array}{ll}\mid & \mid \\
1 & \mid \\
\text { |SEAMER } \\
\mid \text { ID(2) }\end{array}$} & \multirow{2}{*}{$\begin{array}{l}\text { GUN } \\
\text { No. }\end{array}$} & \multirow{2}{*}{$\mid$} & \multirow[b]{2}{*}{$\begin{array}{l}\text { DESTR. } \\
\text { TEST }\end{array}$} & \multirow{2}{*}{$\begin{array}{l}1 \\
1 \\
\text { HONITOR } \\
1 \text { (2) }\end{array}$} & \multicolumn{3}{|c|}{ NON-DESTRUCTIVE TEST } \\
\hline & PANEL & $\begin{array}{c}\text { PANEL } \\
\# 2\end{array}$ & & & & & & & DATE (3) & | MONITOR & DEFECTS \\
\hline s-029/030 & s-029 & $s-030$ & $11 / 20 / 93$ & $E T$ & F2 & 700 & & SM & $111 / 22 / 93$ & SM & \\
\hline$s-029 / 037$ & s-029 & $s-037$ & $11 / 20 / 93$ & ET & F2 & 700 & & SM & $11 / 11 / 11$ & SM & 3 \\
\hline$s-029 / S 02 P$ & $s-029$ & SO2P & $12 / 06 / 93$ & ET & $\times 2$ & 535 & & SM & $19 / 19 / 11$ & SM & 3 \\
\hline$s-029 / 5096$ & $s-029$ & sogc & $12 / 18 / 93$ & ET & F2 & 750 & & SM & $12 / 21 / 93$ & NE & \\
\hline s-030/031 & $s-030$ & $s-031$ & $11 / 20 / 93$ & SH & F3 & 720 & & SM & | 11/22/93 & SH & \\
\hline$s-030 / 035$ & s-030 & s-035 & | 11/20/93 & ET & F2 & 700 & & SM & | 11/91/91 & NE & 3 \\
\hline$S-030 / 036$ & s-030 & s-036 & | 11/20/93 & ET & F2 & 700 & & SH & | 11/111/11 & NE & 3 \\
\hline$S-030 / S 02 P$ & $s-030$ & SO2P & $12 / 06 / 93$ & ET & $x 2$ & 535 & DS -030 & SH & | 11/11/11 & NE & 3 \\
\hline$s-030 / S 09 C$ & $s-030$ & sogc & 12/18/93 & ET & F2 & 750 & & SH & | 12/21/93 & NE & \\
\hline s-031/032 & $s-039$ & $s-032$ & $11 / 20 / 93$ & $A B$ & F5 & 700 & & SM & | 11/22/93 & SM & \\
\hline$s-031 / 034$ & $s-039$ & $s-034$ & $11 / 20 / 93$ & ET & F2 & 700 & $D S-011$ & SM & | 11/21/93 & NE & \\
\hline$s-031 / 035$ & $s-031$ & s-035 & $11 / 20 / 93$ & ET & F2 & 700 & & SM & | 117/11/11 & NE & 3 \\
\hline s-039/s09c & $s-031$ & sogc & | 12/18/93 & ET & F2 & 750 & & SM & | $12 / 21 / 93$ & NE & \\
\hline $5-032 / 033$ & s-032 & s-033 & | 11/20/93 & ET & F2 & 700 & & SH & | 19/11/11 & NE & 3 \\
\hline $5-032 / 034$ & $s-032$ & $s-034$ & | 11/20/93 & ET & F2 & 700 & & SM & | 11/111/11 & NE & 3 \\
\hline $5-032 / 5096$ & 5-032 & so9c & | 12/18/93 & ET & F2 & 750 & & SH & | 12/21/93 & NE & \\
\hline $5-033 / 034$ & s-033 & s-034 & $11 / 20 / 93$ & ET & F2 & 700 & & SH & | 19/22/93 & SH & \\
\hline S-033/S09C & $s-033$ & so9c & $12 / 18 / 93$ & ET & F2 & 750 & & SM & | 12/18/93 & SH & \\
\hline S-034/035 & $s-034$ & s-035 & $11 / 20 / 93$ & $A B$ & F5 & 700 & $D S-010$ & SM & $11 / 21 / 93$ & NE & \\
\hline$S-034 / 5096$ & $s-034$ & so9c & $12 / 18 / 93$ & ET & F2 & 750 & DS- 033 & SM & $12 / 18 / 93$ & SM & \\
\hline S-035/036 & $s-035$ & $s-036$ & $11 / 21 / 93$ & $A B$ & F5 & 700 & & SM & $12 / 15 / 93$ & SM & \\
\hline S- $035 / \mathrm{s} 09 \mathrm{C}$ & $s-035$ & SO9C & 12/18/93 & ET & F2 & 750 & & SM & $12 / 18 / 93$ & SH & \\
\hline S-036/037 & s-036 & s-037 & $19 / 20 / 93$ & ET & F2 & 700 & & SH & $11 / 11 / 11$ & NE & 3 \\
\hline s-036/038 & $s-036$ & s-038 & $11 / 21 / 93$ & $A B$ & F5 & 700 & & SM & $11 / 22 / 93$ & SM & \\
\hline$s-036 / 5096$ & $s-036$ & SO9C & $12 / 18 / 93$ & ET & F2 & 750 & & SH & $12 / 18 / 93$ & SM & \\
\hline S-037/038 & s-037 & s-038 & $12 / 01 / 93$ & ET & $x 1$ & 530 & & SH & $11 / 11 / 11$ & NE & 3 \\
\hline$S-037 / S 02 P$ & $s-037$ & SO2P & $12 / 06 / 93$ & ET & $x 2$ & 535 & & SM & $11 / 91 / 11$ & NE & 3 \\
\hline S-037/S09C & s-037 & sogc & $12 / 18 / 93$ & ET & F2 & 750 & & SM & $12 / 18 / 93$ & SH & \\
\hline S-038/039 & $s-038$ & s-039 & $11 / 21 / 93$ & ET & F2 & 700 & & SH & $11 / 22 / 93$ & SH & \\
\hline$S-038 / S 02 P$ & s-038 & SOZP & | 12/06/93 & ET & $x 2$ & 535 & & SM & 11/11/11 & NE & 3 \\
\hline$S-038 / S 096$ & s-038 & so9c & | 12/18/93 & ET & F2 & 750 & & SM & $12 / 21 / 93$ & NE & \\
\hline s-039/040 & s-039 & $s-040$ & $11 / 21 / 93$ & $A B$ & F5 & 700 & & SM & 11/22/93 & SM & \\
\hline$s-039 / 050$ & s-039 & $s-050$ & $12 / 05 / 93$ & RA & F5 & 770 & & $S M$ & $12 / 11 / 93$ & SM & \\
\hline S-039/S02P & s-039 & SO2P & $12 / 06 / 93$ & ET & $x 2$ & 535 & & SM & $11 / 19 / 11$ & NE & 3 \\
\hline$s-040 / 049$ & $s-040$ & $s-041$ & $11 / 21 / 93$ & SH & F3 & 720 & DS -012 & SM & $11 / 22 / 93$ & SM & \\
\hline$s-040 / 050$ & $s-040$ & $5-050$ & $12 / 05 / 93$ & RA & F5 & 770 & & SH & 12/15/93 & NE & \\
\hline$s-041 / 042$ & $s-049$ & $s-0<2$ & $11 / 21 / 93$ & ET & F2 & 700 & DS-013 & SH & $11 / 22 / 93$ & SM & \\
\hline$s-041 / 050$ & $s-041$ & $s-050$ & $12 / 05 / 93$ & RA & F5 & 770 & & $S M$ & $12 / 11 / 93$ & SM & \\
\hline$s-042 / 043$ & $s-042$ & s-043 & $12 / 04 / 93$ & RA & F3 & 760 & DS -017 & SM & 11/11/19 & NE & \\
\hline$S-042 / 050$ & | s-042 & $s-050$ & $12 / 05 / 93$ & RA & I F5 & 770 & DS-024 & SM & | 12/15/93 & NE & \\
\hline$S-042 / S 06 R$ & | s-0.42 & SO6R & $12 / 12 / 93$ & DH & F3 & 750 & DS-039 & SM & | 12/13/93 & SM & \\
\hline
\end{tabular}


GOLDER CONSTRUCTION SERVICES, INC.

SECONDARY GEOMEMBRANE LINER WELD OBSERVATION AND NON-DESTRUCTIVE TESTING SUMMARY

933-1354 DEPARTMENT OF ENERGY

$05 / 11 / 94$

NON-DRAG OFF LANDFILL FACILITY

Page 4

HANFORD NUCLEAR RESERVATION

\begin{tabular}{|c|c|c|c|c|c|c|c|c|c|c|c|}
\hline \multirow{3}{*}{$\begin{array}{l}\text { SEAM } \\
\text { NUMBER(1) }\end{array}$} & \multicolumn{2}{|c|}{ | PANEL NUMBERS $\mid$} & \multirow{3}{*}{$\begin{array}{l}\text { DATE } \\
\text { WELDED }\end{array}$} & \multirow{3}{*}{$\begin{array}{l}\mid \\
\mid \\
\mid \text { SEAMER } \mid \\
\mid \text { ID(2)| }\end{array}$} & \multirow{3}{*}{$\begin{array}{l}\text { GUN } \\
\text { No. }\end{array}$} & \multirow{3}{*}{$\begin{array}{l}\mid \\
\mid \text { TYPICAL } \\
\mid \text { GUN TEMP } \\
\mid \text { DEGREES (F) } \mid\end{array}$} & \multirow{3}{*}{$\begin{array}{l}\text { DESTR. } \\
\text { TEST }\end{array}$} & \multirow{3}{*}{$\begin{array}{l}\mid \\
\mid \\
\mid \text { MONITOR } \\
\mid \text { (2) }\end{array}$} & \multicolumn{3}{|c|}{ 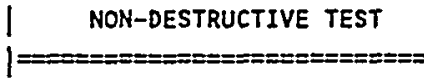 } \\
\hline & I PANEL & | PANEL & & & & & & & & |HONITOR| & DEFECTS \\
\hline & $\# 1$ & $\# 2$ & & & & & & & DATE (3) & $\mid$ (2) $\mid$ & \\
\hline$s-043 / 050$ & $s-043$ & $5-050$ & | 12/05/93 & RA & F5 & 770 & & SM & $12 / 15 / 93$ & NE & \\
\hline$S-043 / S O S R$ & $s-0,3$ & SO6R & | 12/12/93 & DH & F3 & 750 & & SH & | 12/15/93 & SM & \\
\hline s- $044 / 045$ & $s-044$ & $s-045$ & | 12/05/93 & ET & F2 & 760 & & SM & | 12/08/93 & SM & \\
\hline$s-044 / 045$ & $s-044$ & $s-045$ & | $12 / 05 / 93$ & DH & F3 & 750 & & SM & $192 / 08 / 93$ & SH & \\
\hline$s-044 / 050$ & $5-044$ & $s-050$ & | 12/05/93 & $R A$ & F5 & 770 & & SH & | 12/06/93 & SM & \\
\hline$s-045 / 046$ & $s-045$ & $s-0.46$ & | $12 / 05 / 93$ & ET & $F 2$ & 760 & DS -018 & SM & | 12/08/93 & SM & \\
\hline $5-045 / 050$ & $s-045$ & $5-050$ & | $12 / 05 / 93$ & RA & FS & 770 & & SM & $12 / 06 / 93$ & SH & \\
\hline$s-046 / 047$ & $s-046$ & $s-047$ & | $12 / 05 / 93$ & DW & F3 & 750 & DS-019 & SM & | 12/08/93 & SM & \\
\hline$s-046 / 050$ & $s-046$ & $5-050$ & | $12 / 05 / 93$ & RA & F5 & 770 & & SM & | 12/06/93 & SM & \\
\hline$S-046 / S 06 G$ & $s-046$ & SOSE & $12 / 13 / 93$ & ET & $x 2$ & 535 & & SM & | $12 / 15 / 93$ & SH & \\
\hline$s-047 / 048$ & $s-047$ & $5-048$ & | $12 / 05 / 93$ & ET & F2 & 760 & & SM & | $12 / 08 / 93$ & SM & \\
\hline$s-047 / 050$ & $s-047$ & $s-050$ & $112 / 05 / 93$ & RA & F5 & 770 & & SM & | 12/16/93 & SH & \\
\hline$s-047 / 050$ & $s-047$ & $s-050$ & $12 / 05 / 93$ & RA & F5 & 770 & & SH & | 12/16/93 & SH & \\
\hline$s-047 / 059$ & $s-047$ & $s-059$ & $12 / 05 / 93$ & DH & F3 & 750 & & SH & | 12/16/93 & SH & \\
\hline S-047/SO6G & $s-047$ & SO6G & $12 / 13 / 93$ & ET & $\times 2$ & 535 & & $S M$ & | 12/08/93 & SM & \\
\hline$s-048 / 049$ & $s-048$ & $5-049$ & | $12 / 05 / 93$ & ET & F2 & 760 & DS-020 & SM & | 12/08/93 & SH & \\
\hline $5-048 / 059$ & $5-048$ & $5-059$ & | 12/05/93 & DW & F3 & 750 & DS-026 & SM & | $12 / 16 / 93$ & SM & \\
\hline$s-049 / 053$ & $s-049$ & $s-053$ & | 12/05/93 & DW & F3 & 750 & & SM & | 12/11/93 & SM & \\
\hline$s-049 / 059$ & $s-049$ & $s-059$ & | 12/05/93 & OH & 83 & 750 & & SM & $12 / 12 / 93$ & SH & \\
\hline$s-050 / 051$ & $s-050$ & s-051 & | 12/05/93 & ET & $\mathbf{F 2}$ & 750 & & SM & | 12/06/93 & $S M$ & \\
\hline$s-050 / 059$ & $s-050$ & $5-059$ & | 12/05/93 & RA & F5 & 770 & & SM & 1 12/06/93 & SH & \\
\hline $5-050 / 060$ & $\mathrm{~s}-050$ & $s-060$ & $12 / 05 / 93$ & RA & F5 & 770 & & SM & | 12/11/93 & $S M$ & \\
\hline $5-051 / 052$ & $s-051$ & $s-052$ & | 12/05/93 & ET & F2 & 750 & DS-021 & SM & | 12/06/93 & SM & \\
\hline$s-051 / 060$ & $s-051$ & $s-060$ & | $12 / 05 / 93$ & RA & F5 & 770 & & SM & | 12/11/93 & $S M$ & \\
\hline$s-051 / 061$ & $s-051$ & $s-061$ & $12 / 05 / 93$ & RA & F5 & 770 & & SM & | 12/06/93 & SH & \\
\hline$s-052 / 069$ & $5-052$ & $S-061$ & | 12/05/93 & RA & F5 & 770 & & SM & 12/16/93 & SK & \\
\hline$s-052 / 062$ & $5-052$ & $S-062$ & | $12 / 05 / 93$ & RA & F5 & 770 & & SM & | 12/16/93 & $S H$ & \\
\hline $5-053 / 054$ & $5-053$ & $5-054$ & $12 / 05 / 93$ & $\mathrm{DH}$ & F3 & 750 & DS-022 & SM & | 12/11/93 & SH & \\
\hline s-053/058 & $s-053$ & $5-058$ & | 12/05/93 & $\mathrm{OH}$ & F3 & 750 & & SH & | $12 / 16 / 93$ & SM & \\
\hline s-053/059 & $s-053$ & $5-059$ & | $12 / 05 / 93$ & DH & F3 & 750 & & SH & | 12/16/93 & SM & . \\
\hline$S-054 / 055$ & $s-054$ & $s-055$ & | 12/05/93 & DW & F3 & 750 & & SH & | 12/11/93 & SM & \\
\hline $5-054 / 056$ & $s-054$ & $s-056$ & | 12/05/93 & OW & F3 & 750 & & SM & $12 / 12 / 93$ & SH & \\
\hline s-054/057 & $s-054$ & $s-057$ & | 12/05/93 & DH & F3 & 750 & & $S M$ & | 12/12/93 & SH & \\
\hline$s-054 / 058$ & $s-054$ & $5-058$ & | 12/05/93 & DW & F3 & 750 & & $S M$ & | $12 / 16 / 93$ & SH & \\
\hline s-055/056 & $5-055$ & $5-056$ & | $12 / 05 / 93$ & DH & F3 & 750 & & SM & | 12/12/93 & SH & \\
\hline$s-055 / 079$ & $s-055$ & s-071 & | 12/06/93 & DH & F3 & 750 & & $S M$ & | $12 / 16 / 93$ & SH & \\
\hline$s-056 / 057$ & $s-056$ & $5-057$ & | $12 / 05 / 93$ & ET & F2 & $\cdot 750$ & & SM & | 12/11/93 & SH & \\
\hline$s-056 / 065$ & $s-056$ & $s-065$ & | 12/06/93 & DH & F3 & 750 & & $S M$ & $12 / 11 / 93$ & SH & \\
\hline S-057/058 & $s-057$ & $s-058$ & | $12 / 05 / 93$ & ET & F2 & 750 & & $S M$ & | 12/16/93 & SH & \\
\hline $5-058 / 059$ & $s-058$ & $5-059$ & | 12/05/93 & ET & F2 & 750 & DS-025 & SM & | $12 / 06 / 93$ & SH & \\
\hline $5-059 / 060$ & s-059 & $s-060$ & | 12/05/93 & ET & $\mathbf{F 2}$ & 750 & & SM & | $12 / 06 / 93$ & SM & \\
\hline
\end{tabular}


GOLDER CONSTRUCTION SERVICES, INC.

933-1354

SECONDARY GEOMEMBRANE LINER HELD OBSERVATION AND NON-DESTRUCTIVE TESTING SUMMARY

$05 / 11 / 94$

DEPARTMENT OF ENERGY

Page 5

NON-DRAG OFF LANDFILL FACILITY

HANFORD NUCLEAR RESERVATION

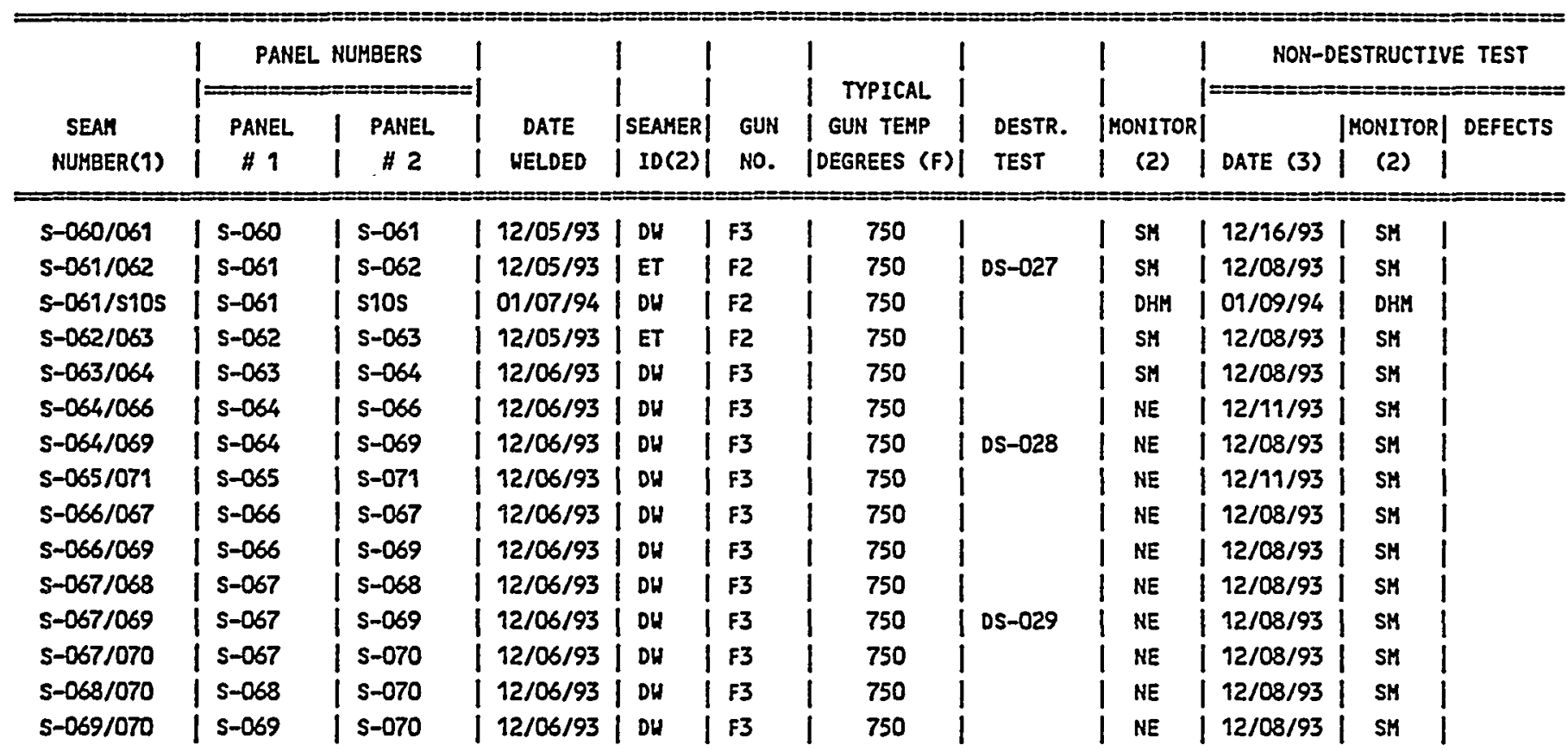

NOTES

$=$

(7) A prefix of $S$ indicates a seam between panels of secondary liner.

(2) Inspector Identification:

DHM - David H. Mathis

SH - Steve Milka

SAM - Scott A. Matthees

NE - Nancy Evans
Seamer Identification:

$\begin{array}{ll}\text { ET - Erwin Tsosie } & \text { DH - Dwight Wilson } \\ \text { AB - Arnold Barker } & \text { TH - Tim Harding } \\ \text { SH - Steve Hewitt } & \text { RA - Raymond Ashley } \\ \text { GH - Grant Martin } & \text { SA - Simon Ashley } \\ \text { PR - Paul Rhodes } & \text { SP - Scott Prydatko }\end{array}$

(3) Seams with a Non-Destructive Test Date of 11/11/11 indicates that the seam was capped, so non-destructive testing of seam was not required. The cap that covered the seam was non-destructively tested. 
WHC-SD-W025-RPT-001, Rev. 0

\section{APPENDIX M-4}

\section{DESTRUCTIVE TEST OBSERVATION SUMMARY}

$M-4$ 
GOLDER CONSTRUCTION SERVICES, INC.

933-1354

SECONDARY GEOMEMBRANE LINER DESTRUCTIVE TEST OBSERVATION SUMMARY

$05 / 11 / 94$

DEPARTMENT OF ENERGY

Page 1

NON-DRAG OFF LANDFILL FACILITY

HANFORD NUCLEAR RESERVATION

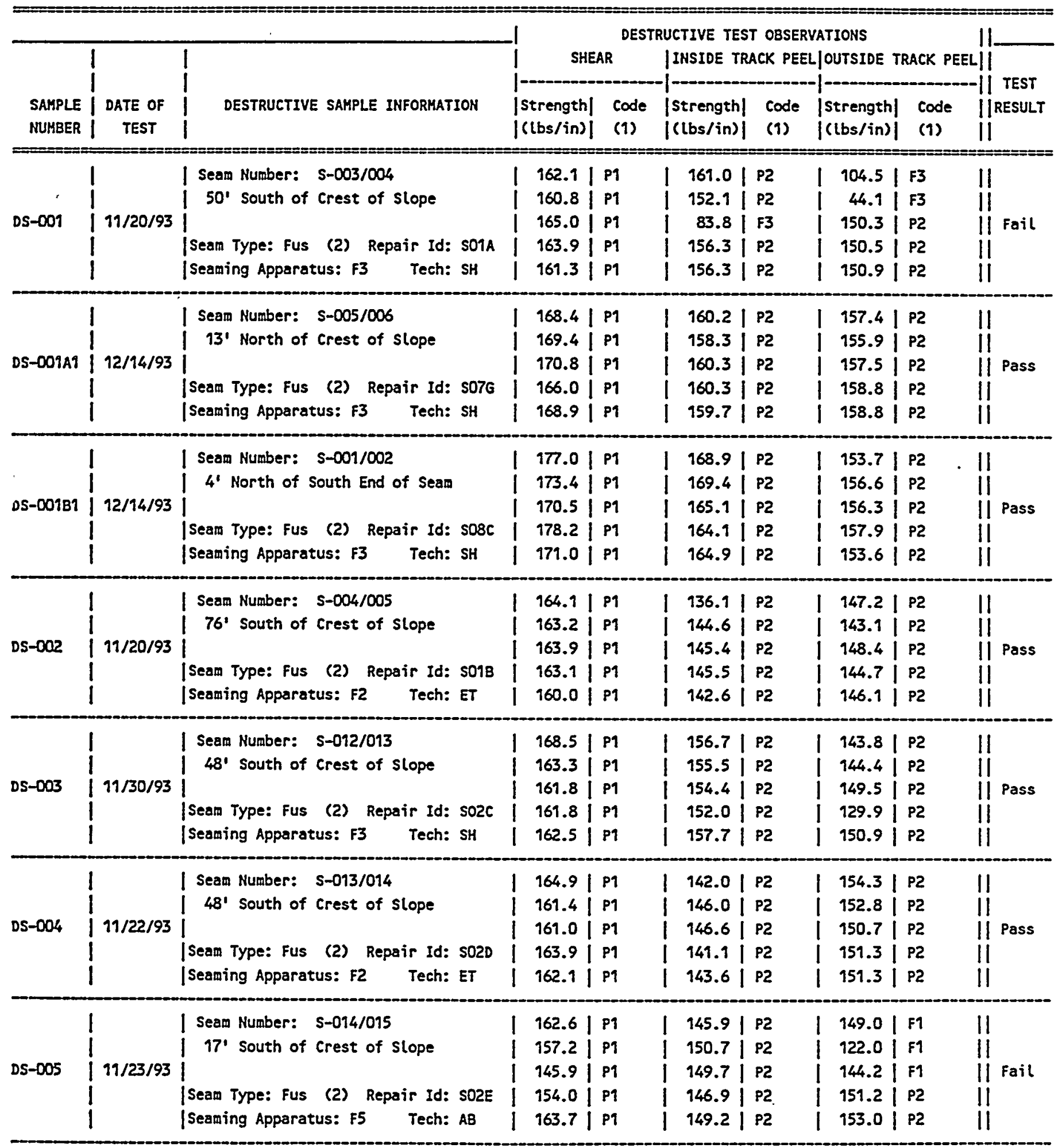


GOLDER CONSTRUCTION SERVICES, INC.

933-1354

SECONDARY GEOMEMBRANE LINER DESTRUCTIVE TEST OBSERVATION SUMMARY

$05 / 11 / 94$

DEPARTMENT OF ENERGY

Page 2

NON-DRAG OFF LANDFILL FACILITY

HANFORO NUCLEAR RESERVATION

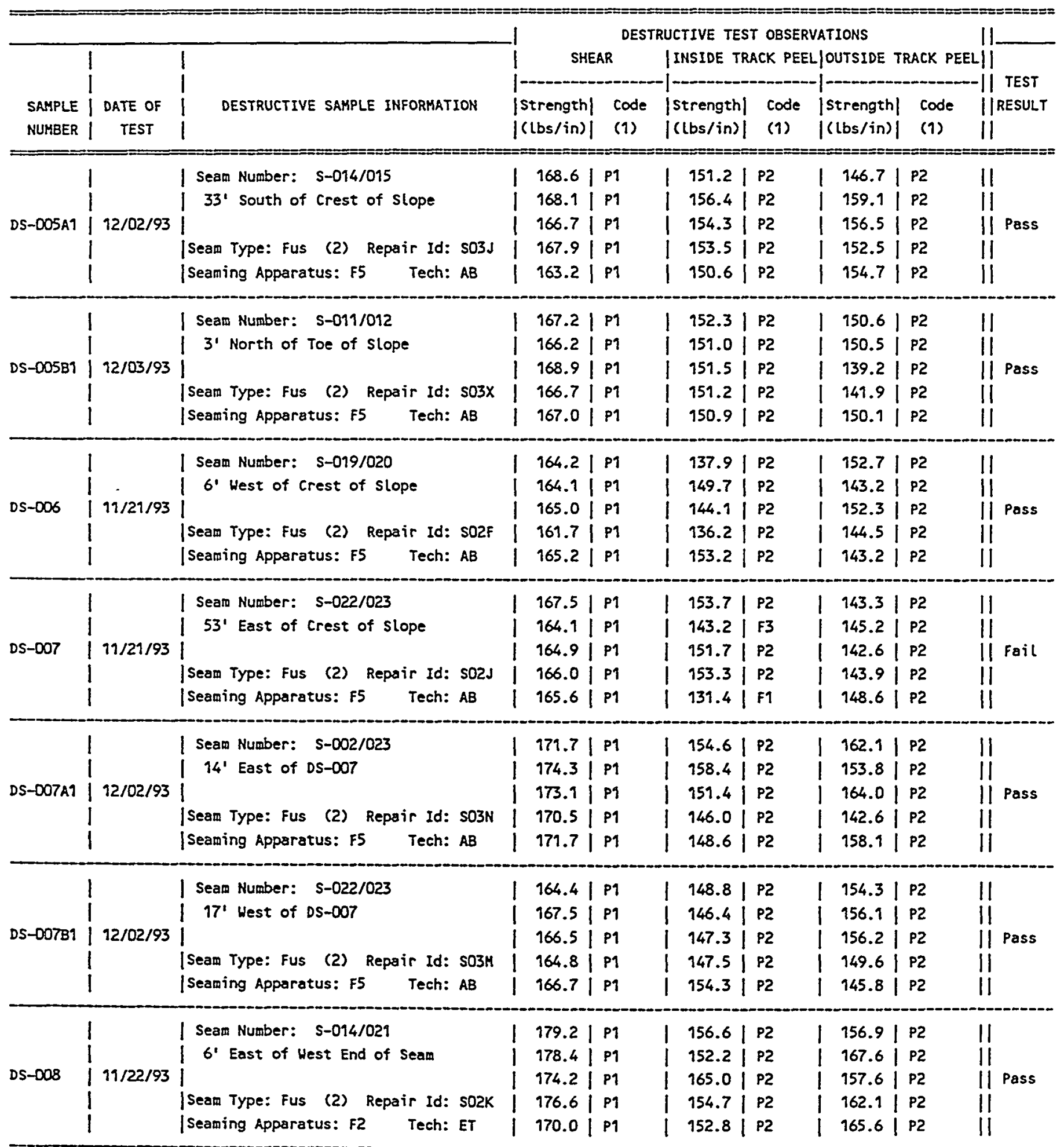


GOLDER CONSTRUCTION SERVICES, INC.

933-1354

SECONDARY GEOMEMBRANE LINER DESTRUCTIVE TEST OBSERVATION SUMMARY

$05 / 11 / 94$

DEPARTHENT OF ENERGY

Page 3

NON-DRAG OFF LANDFILL FACILITY

HANFORD NUCLEAR RESERVATION

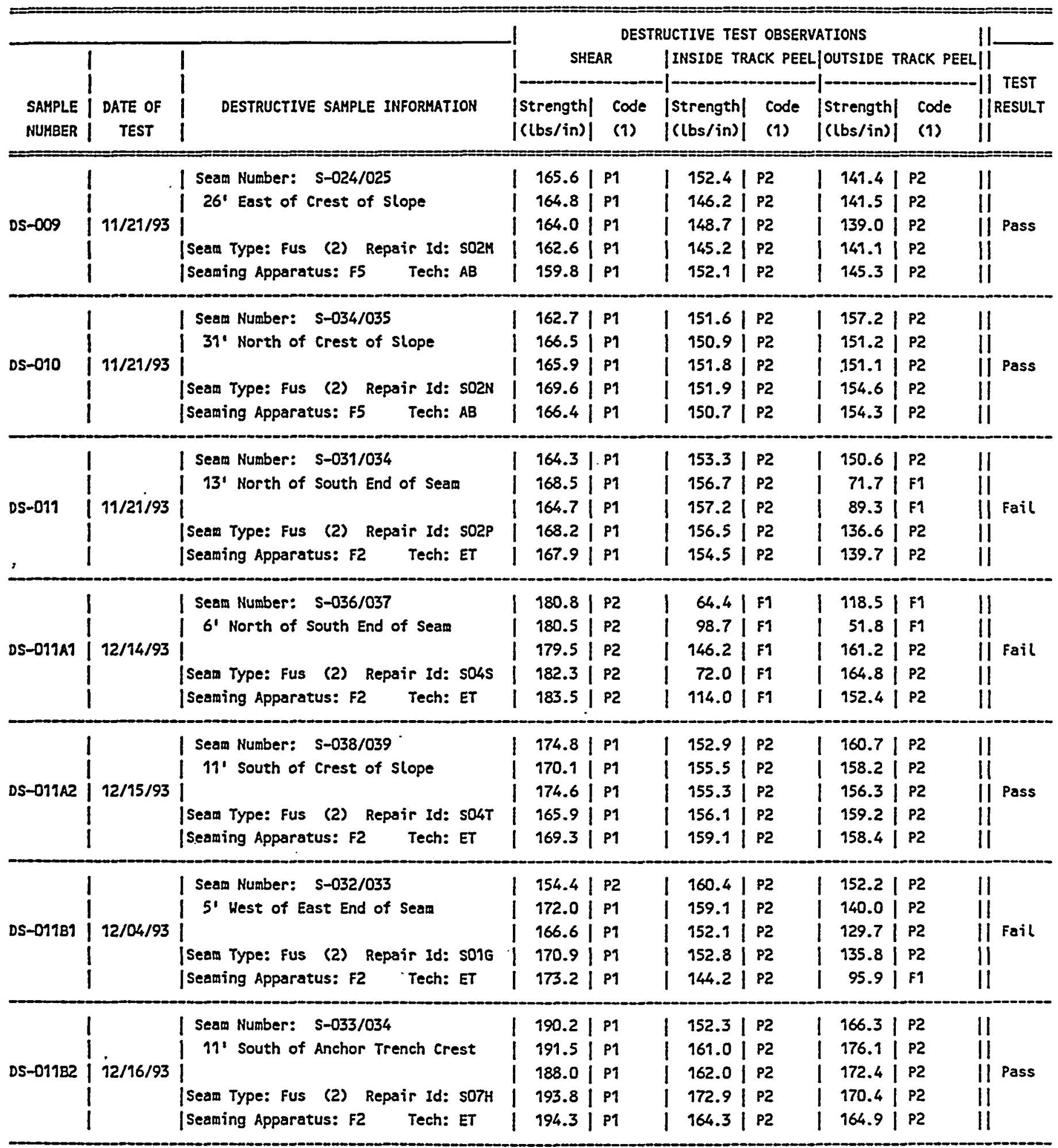


GOLDER CONSTRUCTION SERVICES, INC.

933-1354

SECONDARY GEOMEMBRANE LINER DESTRUCTIVE TEST OBSERVATION SUMMARY

$05 / 11 / 94$

DEPARTMENT OF ENERGY

Page 4

NON-DRAG OFF LANDFILL FACILITY

HANFORD NUCLEAR RESERVATION

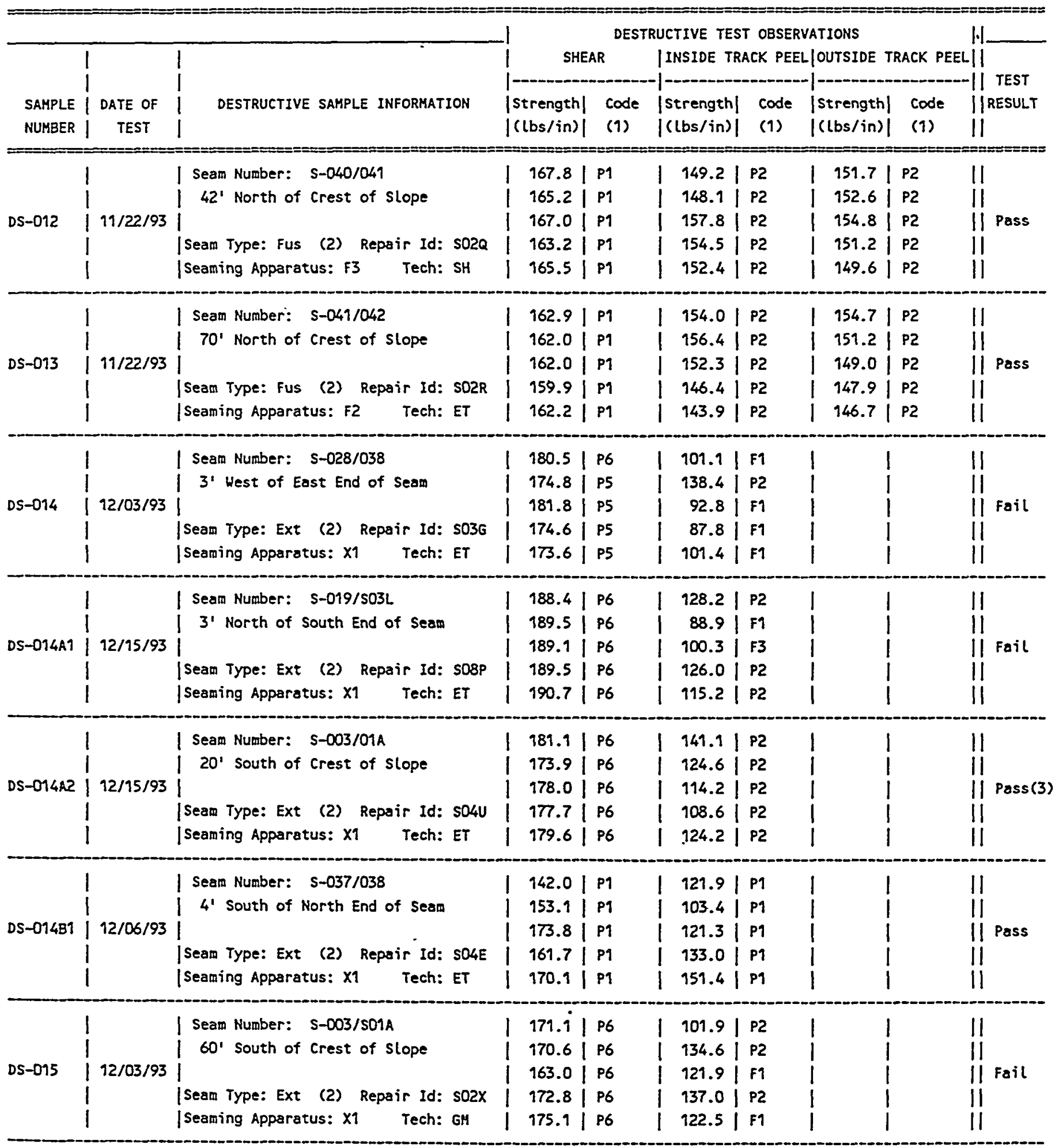


GOLDER CONSTRUCTION SERVICES, INC.

933-1354

SECONDARY GEOMEMBRANE LINER DESTRUCTIVE TEST OBSERVATION SUMMARY

05/11/94

DEPARTMENT OF ENERGY

Page 5

NON-DRAG OFF LANDFILL FACILITY

HANFORD NUCLEAR RESERVATION

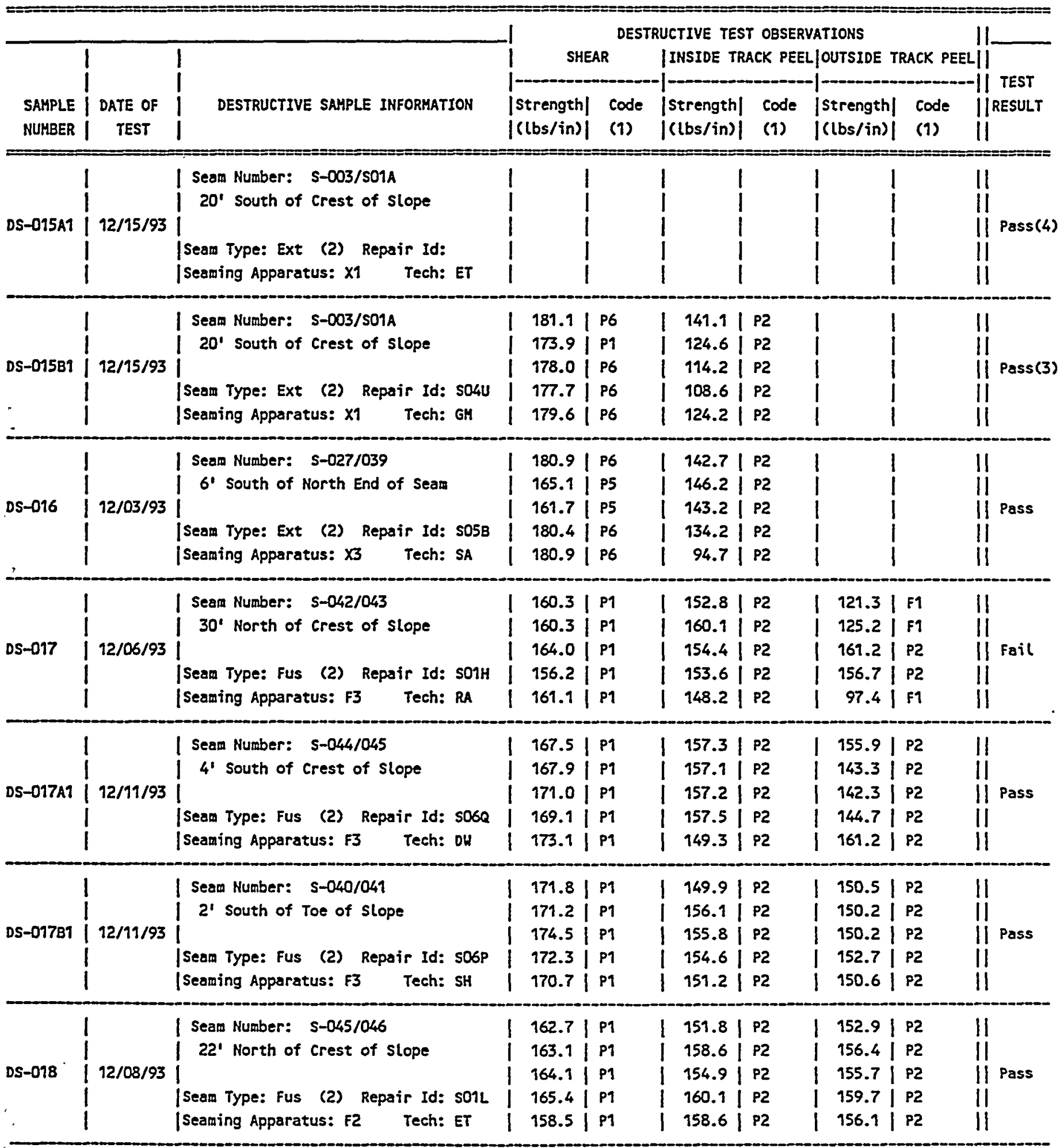


GOLDER CONSTRUCTION SERVICES, INC.

933-1354

SECONDARY GEOMEMBRANE LINER DESTRUCTIVE TEST OBSERVATION SUMMARY

$05 / 11 / 94$

DEPARTMENT OF ENERGY

Page 6

NON-DRAG OFF LANDFILL FACILITY

HANFORO NUCLEAR RESERVATION

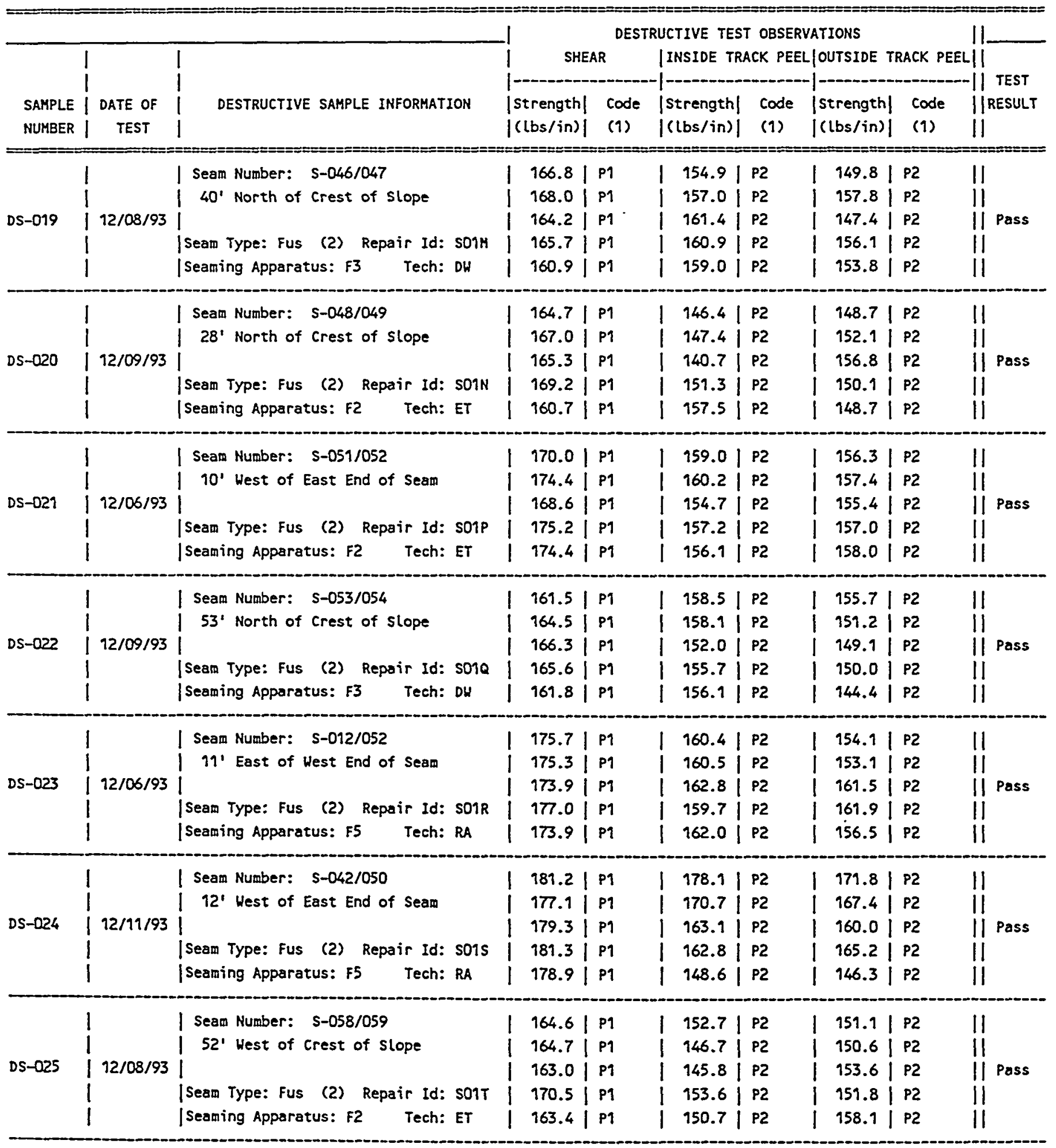


GOLDER CONSTRUCTION SERVICES, INC.

933-1354

SECONDARY GEOMEMBRANE LINER DESTRUCTIVE TEST OBSERVATION SUMMARY

05/11/94

DEPARTMENT OF ENERGY

Page 7

NON-DRAG OFF LANDFILL FACILITY

HANFORD NUCLEAR RESERVATION

\begin{tabular}{|c|c|c|c|c|c|c|c|c|c|}
\hline & & & & DEST & RUCTIVE TEST & T OBSER & VATIONS & & 11 \\
\hline & & 1 & SHEA & & |INSIDE TRA & ACK PEE & LOOUTSIDE TI & RACK PEEL & LII \\
\hline $\begin{array}{l}\text { SAMPLE } \\
\text { NUMBER }\end{array}$ & $\begin{array}{l}\text { DATE OF } \\
\text { TEST }\end{array}$ & DESTRUCTIVE SAMPLE INFORMATION & $\begin{array}{l}\text { |strength| } \\
\mid \text { (lbs/in)| }\end{array}$ & $\begin{array}{l}\text { code } \\
\text { (1) }\end{array}$ & $\begin{array}{l}\text { |strength } \mid \\
\mid \text { (lbs/in) } \mid\end{array}$ & $\begin{array}{l}\text { code } \\
\text { (1) }\end{array}$ & $\begin{array}{l}\text { |strength| } \\
\mid \text { (lbs/in)| }\end{array}$ & $\begin{array}{l}\text { code } \\
\text { (1) }\end{array}$ & $\|$ RESULT \\
\hline & & | Seam Number: s-048/059 & 192.21 & P1 & | 177.3 & P2 & | 169.5 | & P2 & 11 \\
\hline & & | 13' East of West End of Seam & $182.0 i$ & P1 & 175.0 & P2 & 164.4 & P2 & il \\
\hline DS-026 & $12 / 11 / 93$ & & 188.1 & Pq & 174.7 & P2 & 161.4 & P2 & II Pass \\
\hline & & |seam Type: fus (2) Repair Id: so1U & 184.1 & P1 & 155.2 & P2 & 157.1 & P2 & 11 \\
\hline & & | Seaming Apparatus: F3 Tech: DH & $|182.5|$ & P1 & 179.4 & P2 & $163.0 \mid$ & P2 & II \\
\hline & & | Seam Number: S-061/062 & 167.11 & P1 & $153.3 \mid$ & P2 & | 144.0 | & P2 & 11 \\
\hline & & | $39^{\prime}$ Hest of Crest of Slope & $164.9 i$ & Pq & 950.6 & P2 & $145.2 i$ & P2 & ii \\
\hline DS-027 & 12/08/93 & & 164.8 & P1 & 957.5 & P2 & $148.3 i$ & P2 & II Pass \\
\hline & & |Seam Type: fus (2) Repair Id: s014 & 164.4 & P1 & 954.7 & P2 & 144.8 & P2 & iI \\
\hline$\because$ & & |Seaming Apparatus: F2 Tech: ET & $|168.4|$ & P1 & 954.7 & P2 & $|148.5|$ & $\mathbf{P 2}$ & 11 \\
\hline & & | Seam Number: s-064/069 & | $165.1 \mid$ & P1 & | 152.4 | & P2 & $|153.2|$ & P2 & 11 \\
\hline & & I g' West of Crest of slope & $173.8 i$ & P1 & 155.6 & P2 & $150.4 i$ & P2 & ii \\
\hline DS-028 & $92 / 08 / 93$ & & $963.2 i$ & P1 & 152.4 & P2 & 949.8 & P2 & ii Pass \\
\hline & & |Seam Type: fus (2) Repair Id: S05C & 168.9 & P1 & 154.2 & P2 & 149.5 & P2 & ii \\
\hline & & |Seaming Apparatus: F3 Tech: DW & 169.8 & P1 & 152.1 & $\mathbf{P 2}$ & 145.71 & P2. & II \\
\hline & & I Seam Number: s-067/069 & $178.8 \mid$ & P1 & 151.3 & P2 & 156.71 & P2 & 11 \\
\hline & & i $5^{\prime}$ North of South End of Seam & $174.2 i$ & P1 & 144.1 & P2 & $156.7 i$ & P2 & ii \\
\hline DS-029 & 12/08/93 & & 176.5 & Pq & 151.1 & P2 & 161.9 & P2 & II Pass \\
\hline & & |Sean Type: Fus (2) Repair Id: SO5F & 170.9 & Pq & 154.6 & P2 & 162.3 & P2 & ii \\
\hline & & |Seaming Apporatus: F3 Tech: oH & 172.21 & P1 & $i 146.0 i$ & P2 & $|768.0|$ & P2 & II \\
\hline & & | Seam Number: s-030/s02p & 189.81 & P6 & 108.7 & Pq & 1 & & 11 \\
\hline & & i $4^{\prime}$ Hest of East End of Sean & 186.2 & P6 & 72.5 & F1 & i & & 11 \\
\hline DS-030 & $12 / 08 / 93$ & & $190.3 i$ & P6 & 73.8 & Fq & i & & il Fail \\
\hline & & |Seam Type: Ext (2) Repair Id: SO4J & 184.8 & P6 & 99.2 & Pq & $i$ & & il \\
\hline & & |Seaming Apparatus: $X 2 \quad$ Tech: ET & i $189.4 i$ & P6 & i 111.2 i & F1 & i & & 11 \\
\hline & & | Seam Number: S-030/S02P & $187.2 \mid$ & P6 & 140.0 & P1 & 1 & & 11 \\
\hline & & I 10' North of South End of Sean & 186.9 & P6 & 162.0 & P1 & j & & ii \\
\hline DS-030A1 & $12 / 14 / 93$ & & $187.3 \mid$ & P6 & 144.7 & Pq & I & & II Pass \\
\hline & & |seam Type: Ext (2) Repair Id: s04R & $\mid 186.2$ & P6 & 147.6 & Pq & i & & iI \\
\hline & & [Seaming Apparatus: X2 Tech: ET & i $183.3 i$ & PG & $125.8 i$ & P1 & i & & 11 \\
\hline & & I Seam Number: S-038/SO4E & 172.71 & .86 & | 170.9 | & P2 & 1 & & II \\
\hline & & I South of North End of Seam & $174.5 i$ & P6 & 155.7 & P2 & $i$ & & 11 \\
\hline DS-03089 & $12 / 95 / 93$ & & 176.8 & PG & 160.2 & P2 & $\mathrm{i}$ & & II Pass \\
\hline & & |Seam Type: Ext (2) Repair Id: SOsQ & 178.8 & P6 & 156.9 & P2 & i & & II \\
\hline & & |Seaming Apparatus: $X 2$ Tech: $E T$ & $177.6 i$ & P6 & i $160.3 i$ & P2 & i & & il \\
\hline
\end{tabular}


GOLDER CONSTRUCTION SERVICES, INC.

933-1354

SECONDARY GEOMEMBRANE LINER DESTRUCTIVE TEST OBSERVATION SUMMARY

$05 / 11 / 94$

DEPARTMENT OF ENERGY

Page 8

NON-DRAG OFF LANDFILL FACILITY

HANFORD NUCLEAR RESERVATION

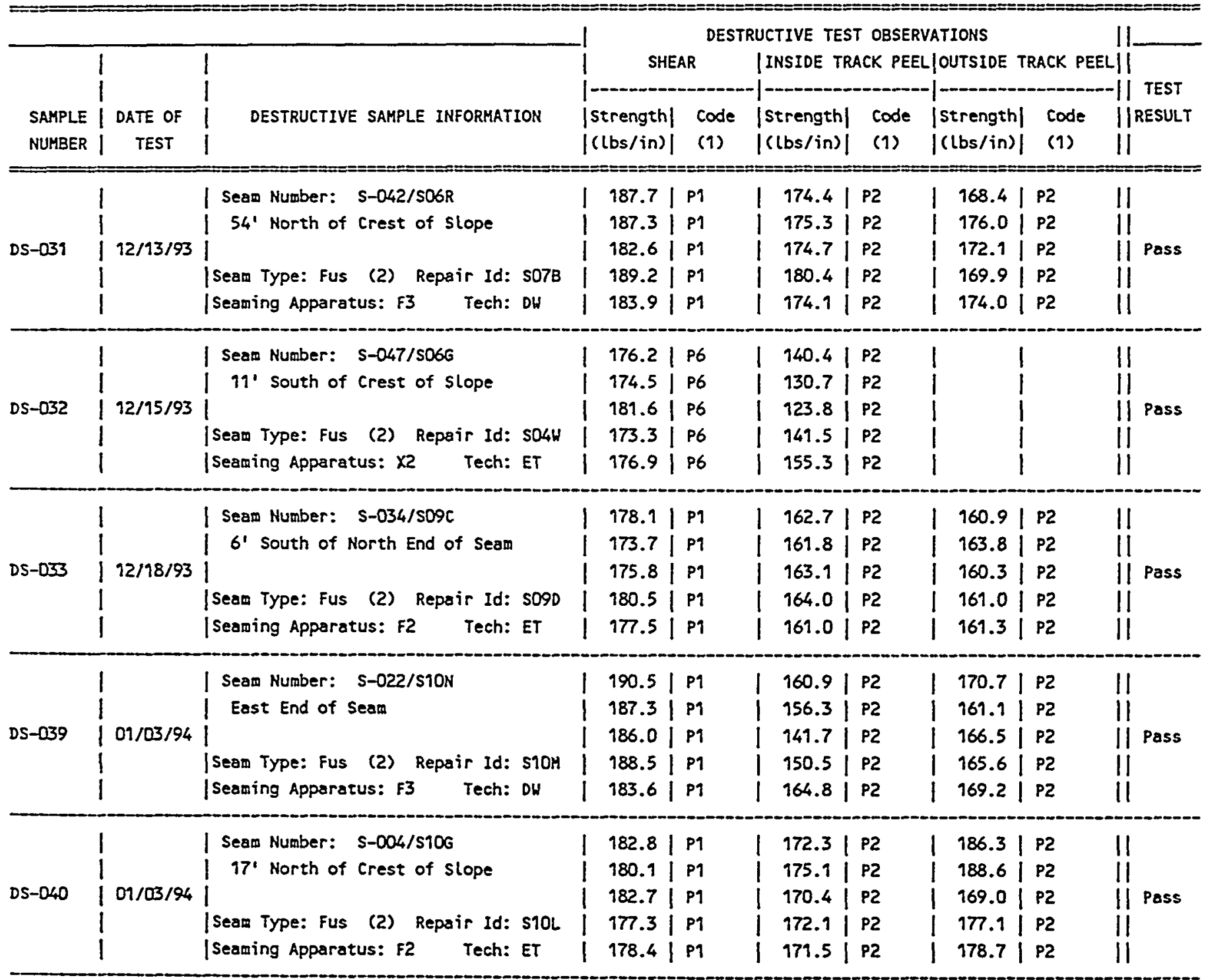


WHC-SD-W025-RPT-001, Rev. 0

NOTES

1) See Figures for illustration of mode of failure codes.

2) Fus - Fusion Seam

Ext - Extrusion Sean

3) Destructive Test Sample DS-014A2 is the same sample as DS-015B1.

Tracking failed destructive sample DS-014 in the after direction ended at the same location as tracking failed destructive test sample DS-015 in the before direction.

4) All deficient seams and repairs welded by extrusion welder $X 1$ were capped. $X 1$ was removed from the project after welding the seam from which sample DS-015 was obtained. 
WHC-SD-W025-RPT-001, Rev。 0

APPENDIX M-5

GEOMEMBRANE REPAIR SUMMARY

$M-5$ 
GOLDER CONSTRUCTION SERVICES, INC.

933-1354

SECONDARY GEOMEMBRANE LINER REPAIR SUMMARY

05/11/94

DEPARTMENT OF ENERGY

Page 1

NON-DRAG OFF LANDFILL FACILITY

HANFORD NUCLEAR RESERVATION

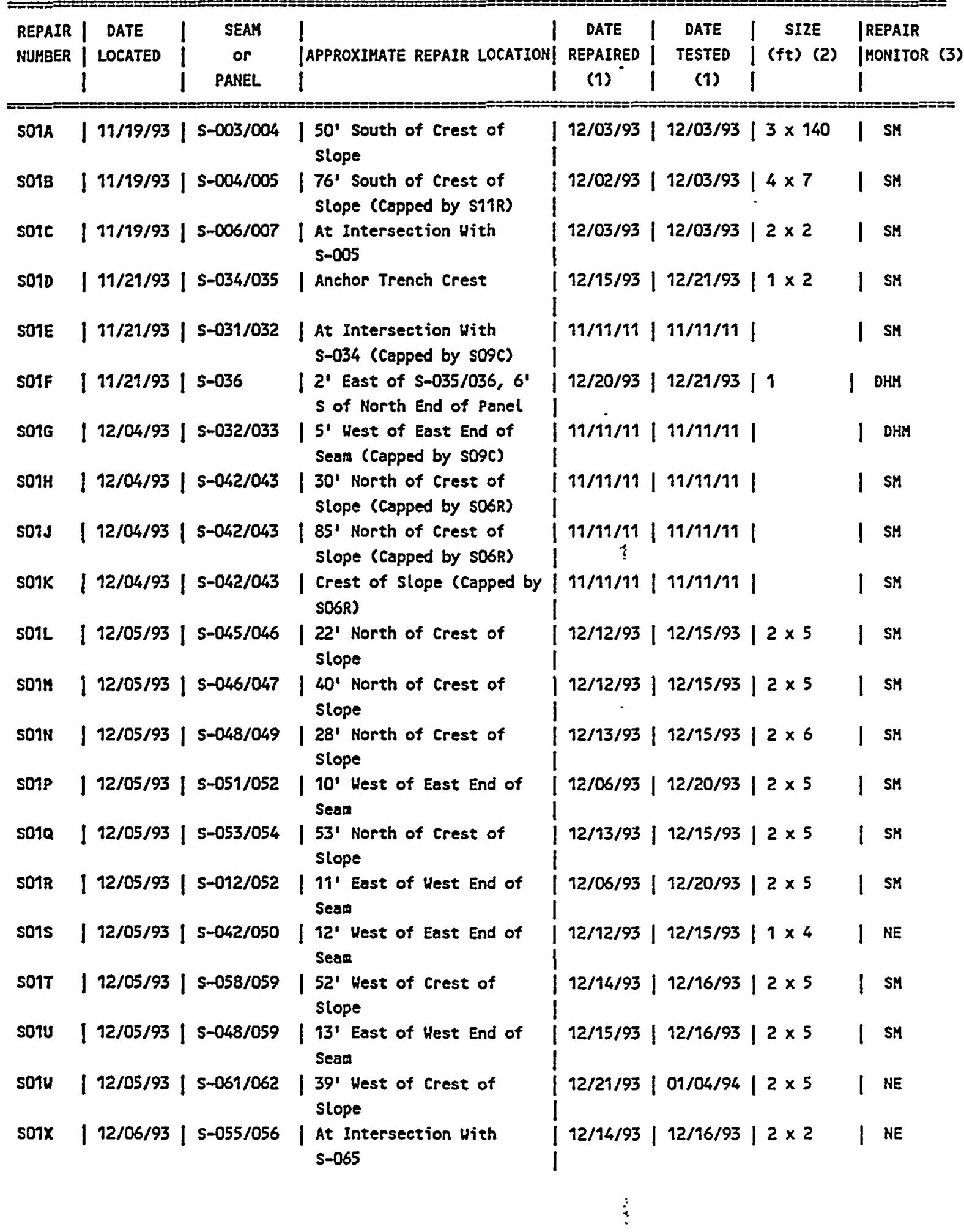


GOLDER CONSTRUCTION SERVICES, INC.

\begin{tabular}{|c|c|c|c|c|c|c|}
\hline $\begin{array}{l}\text { REPAIR } \\
\text { MUMBER }\end{array}$ & $\left\{\begin{array}{l}\text { DATE } \\
\mid \text { LOCATED }\end{array}\right.$ & $\begin{array}{l}\text { SEAM } \\
\text { or } \\
\text { PANEL }\end{array}$ & $\mid \begin{array}{l}\mid \\
\mid \text { APPROXIMATE REPAIR LOCATION| }\end{array}$ & \begin{tabular}{c|c|c} 
DATE & DATE & SIZE \\
REPAIRED & TESTED & $(f t)(2)$ \\
$(1)$ & (1) &
\end{tabular} & $\begin{array}{l}R \\
\text { | }\end{array}$ & $\begin{array}{l}\text { REPAIR } \\
\text { YONITOR (3) }\end{array}$ \\
\hline sads & | 11/20/93 & $s-011 / 012$ & $\begin{array}{l}14 \text { North of South End of } \\
\text { Seam (Capped by } 503 x \text { ) }\end{array}$ & $12 / 02 / 93|11 / 19 / 19| 2 \times 2$ & & SH \\
\hline 5023 & | 11/20/93 | & s-019 & $\begin{array}{l}\mid 1 \text { North, } 11 \text { ' of S End } \\
\text { of Panel (Copped by SO46) }\end{array}$ & $12 / 02 / 93|11 / 19 / 11| 1 \times 1$ & 1 & SK \\
\hline 5026 & $|11 / 20 / 93|$ & $s-012 / 013$ & $\begin{array}{l}\text { I } 48^{\prime} \text { south of Crest of } \\
\text { slope }\end{array}$ & $19 / 30 / 93|12 / 02 / 93| 2 \times 6$ & 1 & SK \\
\hline$S O 2 D$ & $|11 / 20 / 93|$ & s-013/014 & $\begin{array}{l}\text { | 48' South of Crest of } \\
\text { slope (capped by s11p) }\end{array}$ & $12 / 02 / 93|12 / 02 / 93| 2 \times 5$ & & SM \\
\hline SOZE & $|11 / 20 / 93|$ & s-014/015 & $\begin{array}{l}\text { I 17' South of Crest of } \\
\text { slope (Capped by So3J) }\end{array}$ & $12 / 06 / 93|11 / 11 / 11| 2 \times 5$ & 1 & SH \\
\hline sozF & $|11 / 20 / 93|$ & $s-019 / 020$ & 1 6' vest of Crest of Slope & $12 / 02 / 93|01 / 06 / 94| 2 \times 5$ & 1 & SH \\
\hline sQ26 & $|11 / 20 / 93|$ & $5-006 / 008$ & $\begin{array}{l}\text { I Anchor Trench crest } \\
\text { (Capped by } 519 x \text { ) }\end{array}$ & $12 / 02 / 93|12 / 02 / 93| 2 \times 2$ & 1 & SM \\
\hline SO2H & $|19 / 20 / 93|$ & s-006/007 & $\begin{array}{l}\text { I At Intersection With } \\
\text { S-008 }\end{array}$ & $12 / 03 / 93|12 / 03 / 93| 1 \times 3$ & $t$ & SH \\
\hline 5023 & $|11 / 20 / 93|$ & $s-022 / 023$ & $\begin{array}{l}\text { I 53. East of Crest of } \\
\text { slope (capped by SO3M) }\end{array}$ & $11 / 11 / 19|11 / 11 / 19|$ & & SH \\
\hline sazk & $|11 / 20 / 93|$ & $5-014 / 021$ & $\begin{array}{l}\text { I 6' East of West End of } \\
\text { Sean (Capped by S1OE) }\end{array}$ & $12 / 02 / 93|12 / 02 / 93| 3 \times 6$ & 1 & SH \\
\hline sor2. & $|11 / 20 / 93|$ & $5-012 / 013$ & $\begin{array}{l}\text { I At Intersection With } \\
\text { S-022 }\end{array}$ & $12 / 06 / 93|12 / 20 / 93| 2 \times 2$ & 1 & SH \\
\hline saza & $|11 / 20 / 93|$ & $5-024 / 025$ & $\begin{array}{l}\text { | 26' East of Crest of } \\
\text { Slope }\end{array}$ & $12 / 02 / 93|12 / 02 / 93| 2 \times 5$ & 1 & SH \\
\hline soar & $|11 / 21 / 93|$ & Is-034/035 & $\begin{array}{l}\text { I 10' North of Crest of } \\
\text { Slope }\end{array}$ & $12 / 15 / 93|12 / 21 / 93| 2 \times 5$ & 1 & SH \\
\hline$S D O P$ & | $91 / 21 / 93 \mid$ & $5-031 / 034$ & $\begin{array}{l}\text { I 13' N. of South End of } \\
\text { Sean (Cop by } 5096 \text { \& SO9T) }\end{array}$ & $11 / 11 / 11|19 / 11 / 19|$ & & SH \\
\hline 5020 & $|11 / 21 / 93|$ & $s-040 / 049$ & I 42' North of slope Crest & $12 / 06 / 93|12 / 15 / 93| 2 \times 4$ & I & SH \\
\hline SQRR & $|11 / 21 / 93|$ & $s-049 / 042$ & I $70^{\prime}$ Horth of Slope Crest & $92 / 08 / 93|12 / 15 / 93| 2 \times 5$ & & SH \\
\hline sees & $|11 / 21 / 93|$ & $s-017 / 018$ & $\begin{array}{l}\text { I At Intersection With } \\
\text { S-026 }\end{array}$ & $12 / 02 / 93|01 / 06 / 94| 2 \times 3$ & & SM \\
\hline SOLT & $|11 / 22 / 93|$ & $5-040 / 041$ & I 15' North of Toe of Slope & $12 / 03 / 93|12 / 15 / 93| 3 \times 4$ & & ME \\
\hline S020 & $|11 / 22 / 93|$ & s-035/036 & $\begin{array}{l}\text { 16' South of Crest of } \\
\text { slope }\end{array}$ & $12 / 15 / 93|12 / 21 / 93| 1 \times 1$ & & Sn \\
\hline sogu & $|01 / 03 / 94|$ & $5-028$ & $\begin{array}{l}\text { I T' N. of S-028/038, } 97^{\circ} \\
\text { E. of Crest of slope }\end{array}$ & $01 / 03 / 94|01 / 03 / 94| 4 \times 5$ & & HE \\
\hline $\sec x$ & $|12 / 03 / 93|$ & $5-003 / s 01 A$ & $\begin{array}{l}160 \text { ' South of Crest of } \\
\text { slope }\end{array}$ & $12 / 03 / 93|12 / 03 / 93| 3 \times 5$ & & SM \\
\hline $503 x$ & | 12/01/93 & $5-036 / 037$ & $\begin{array}{l}\text { I At intersection With } \\
\text { s-038 (Copped by s09s) }\end{array}$ & $12 / 01 / 93|11 / 11 / 19| 2 \times 2$ & & SH \\
\hline
\end{tabular}


GOLDER CONSTRUCTION SERVICES, INC. SECONDARY GEOHEMBRANE LINER REPAIR SUMHARY

933-1354 $05 / 11 / 94$ DEPARTMENT OF ENERGY NON-DRAG OFF LANDFILL FACILITY HANFORD NUCLEAR RESERVATION

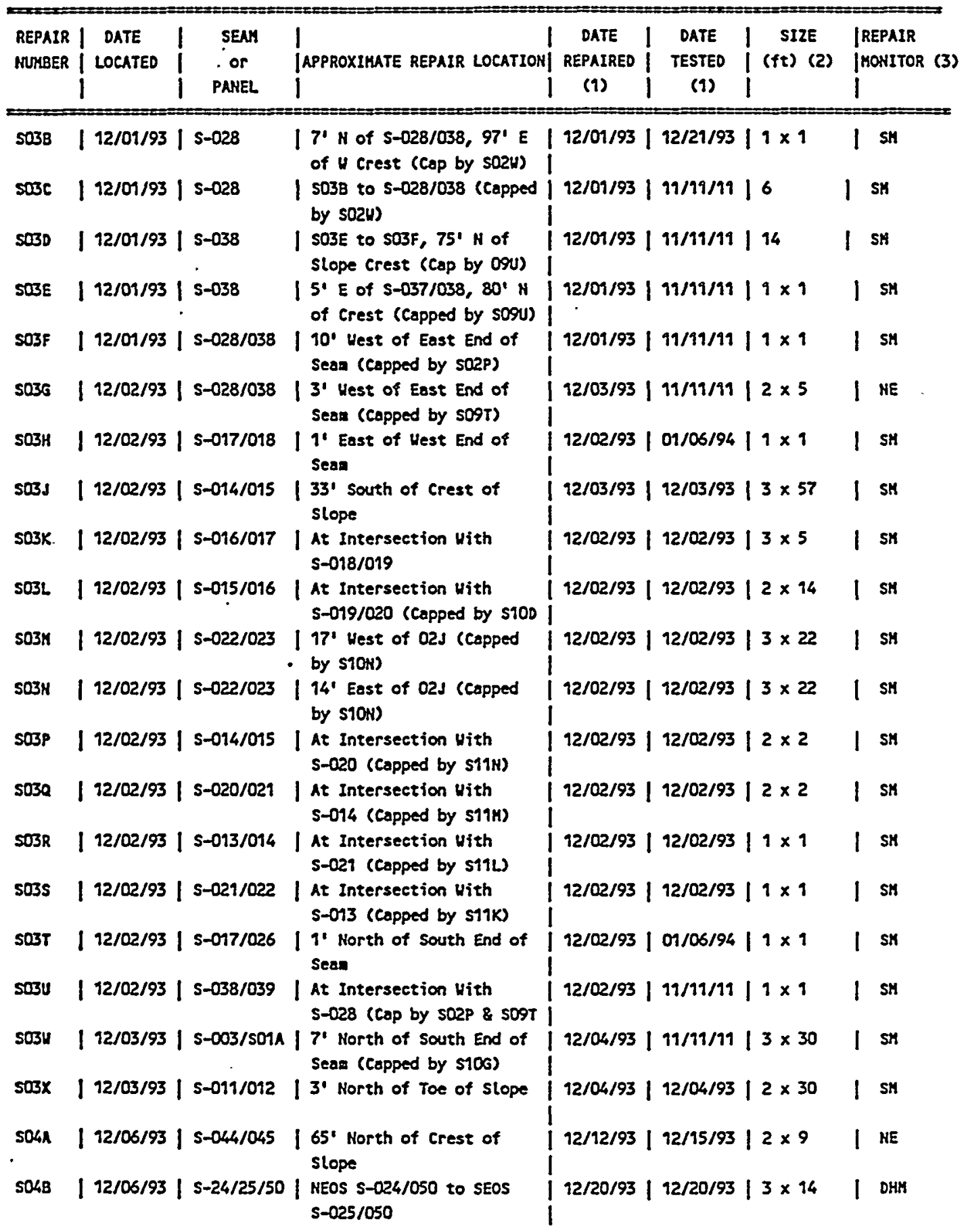


GOLDER CONSTRUCTION SERVICES, INC. DEPARTMENT OF ENERGY

\begin{tabular}{|c|c|c|c|c|c|c|c|c|}
\hline $\begin{array}{l}\text { REPAIR } \\
\text { NUMBER }\end{array}$ & $\left\{\begin{array}{l}\text { OATE } \\
1 \text { LOCATED }\end{array}\right.$ & $\begin{array}{l}\text { SEAM } \\
\text { or } \\
\text { PANEL }\end{array}$ & |APPROXIMATE REPAIR LOCATION| & \begin{tabular}{|c|} 
DATE \\
$\mid$ REPAIRED \\
$\mid$ \\
(1)
\end{tabular} & $\begin{array}{l}\text { DATE } \\
\text { TESTED } \\
\text { (1) }\end{array}$ & $\begin{array}{lc}1 & \text { SIZE } \\
1 & (f t)(2)\end{array}$ & $\begin{array}{l}\text { la } \\
\text { ln }\end{array}$ & $\begin{array}{l}\text { EEPAIR } \\
\text { ONITOR (3) }\end{array}$ \\
\hline sosc & | 12/06/93 & / s-051/052 & $\begin{array}{l}\text { I At Intersection With } \\
\text { s-023 }\end{array}$ & $|12 / 20 / 93|$ & $12 / 20 / 93$ & $13 \times 9$ & 1 & DHM \\
\hline 5040 & | $12 / 06 / 93$ & | s-022/052 & $\begin{array}{l}\text { I North End of Sean to } \\
\text { South End of Seam }\end{array}$ & | $12 / 06 / 93 \mid$ & $12 / 20 / 93$ & $11 \times 7$ & 1 & SM \\
\hline SO4E & $12 / 06 / 93$ & | s-037/038 & $\begin{array}{l}\text { I 4' South of North End of } \\
\text { seam (Capped by SO9S) }\end{array}$ & $|12 / 06 / 93|$ & $11 / 11 / 11$ & $12 \times 5$ & 1 & $S M$ \\
\hline SOLF & | 12/06/93 & | s-011/012 & $\begin{array}{l}\text { I At Intersection with } \\
s-052\end{array}$ & $|12 / 06 / 93|$ & $12 / 20 / 93$ & $12 \times 5$ & 1 & SK \\
\hline 5046 & | 12/0s/93 & | s-011/052 & $\begin{array}{l}\text { I 12' East of West End of } \\
\text { Seam }\end{array}$ & $|12 / 06 / 93|$ & $12 / 20 / 93$ & $12 \times 4$ & 1 & SH \\
\hline $\mathrm{SO} \angle \mathrm{H}$ & | $12 / 06 / 93$ & | s-010/019 & $\begin{array}{l}\text { I At Intersection with } \\
\text { S-052 }\end{array}$ & $12 / 06 / 93 \mid$ & $12 / 20 / 93$ & $12 \times 4$ & 1 & SM \\
\hline so64 & | 12/06/93 & | s-030/S02P & $\begin{array}{l}\text { 1 } 4 \text { West of East End of } \\
\text { Seam (Capped by SOSC) }\end{array}$ & $|11 / 11 / 11|$ & $11 / 11 / 11$ & 1 & 1 & SM \\
\hline so4x & $12 / 06 / 93$ & | s-047/059 & $\begin{array}{l}\text { At Intersection with } \\
\text { s-0so }\end{array}$ & $|12 / 15 / 93|$ & $12 / 96 / 93$ & $12 \times 2$ & 1 & SH \\
\hline so4. & | 12/06/93 & | s-050/0so & $\begin{array}{l}10^{\prime}-6^{2} \text { North of South } \\
\text { End of Sean }\end{array}$ & $|12 / 15 / 93|$ & $12 / 15 / 93$ & $13 \times 5$ & 1 & $S H$ \\
\hline so4x & $112 / 05 / 93$ & | s-061 & $\begin{array}{l}\text { I 45' West of Crest of } \\
\text { Slope }\end{array}$ & $|12 / 21 / 93|$ & $01 / 04 / 94$ & $12 \times 14$ & 1 & ME \\
\hline so4h & | $12 / 06 / 93$ & | s-060/081 & $\begin{array}{l}120^{\circ} \text { East of Hest End of } \\
\text { Sean }\end{array}$ & $|12 / 11 / 93|$ & $01 / 04 / 94$ & $11 \times 1$ & 1 & SH \\
\hline sokp & | 12/06/93 & $s-060 / 061$ & $\begin{array}{l}\text { I } 28^{\prime} \text { East of Vest End of } \\
\text { Seam }\end{array}$ & $\mid 12 / 19 / 93$ & $01 / 04 / 94$ & $12 \times 2$ & 1 & SH \\
\hline 5040 & | 12/06/93 & | s-009/010 & $\begin{array}{l}\text { I At Intersection with } \\
\text { S-052 }\end{array}$ & | 12/20/93 | & $12 / 20 / 93$ & $12 \times 4$ & 1 & $\mathrm{DHM}$ \\
\hline SO4R & $192 / 14 / 93$ & I $\mathrm{s}-030 / \mathrm{s} 02 \mathrm{p}$ & $\begin{array}{l}\text { I } 90^{\circ} \text { South of } 504 \mathrm{~J} \text { (Capped } \\
\text { by SO9C) }\end{array}$ & $|99 / 11 / 19|$ & 19/11/19 & & 1 & $S M$ \\
\hline 5045 & | 12/14/93 & $s-036 / 037$ & $\begin{array}{l}\text { 16' North of South End of } \\
\text { Seam (Capped by SOSR) }\end{array}$ & 11/11/11 & $11 / 11 / 11$ & | & 1 & DHK \\
\hline SOLT & | 12/15/93 & | s-038/039 & $\begin{array}{l}\text { I } 11 \text { ' South of Crest of } \\
\text { slope }\end{array}$ & | 12/21/93 | & 12/21/93 & $12 \times 5$ & 1 & DHM \\
\hline 5044 & | 12/15/93 & Is-003/s01A & $\begin{array}{l}\text { | } 20^{\prime} \text { s. of Crest of slope } \\
\text { (capped by } \$ 106 \text { ) }\end{array}$ & 99/าบ/า1 | & $11 / 11 / 11$ & 1 & 1 & DHM \\
\hline so44 & | 12/15/93 & Is-047/5066 & $\begin{array}{l}\text { I } 191 \text { south of Crest of } \\
\text { slope }\end{array}$ & $12 / 15 / 93$ & $12 / 16 / 93$ & $12 \times 4$ & 1 & SK \\
\hline $\operatorname{sos} x$ & | $12 / 15 / 93$ & $1 s-053 / 054$ & $\begin{array}{l}\text { I } 10^{\prime} \text { South of Crest of } \\
\text { slope }\end{array}$ & $|12 / 15 / 93|$ & $12 / 16 / 93$ & $12 \times 7$ & 1 & SH \\
\hline $\operatorname{sos} \lambda$ & | 12/03/93 & | s-028/039 & $\begin{array}{l}\text { I 4' North of South End of } \\
\text { Sean }\end{array}$ & $12 / 03 / 93$ & $12 / 17 / 93$ & $12 \times 2$ & & HE \\
\hline SOSB & | $12 / 03 / 93$ & |s-027/039 & $\begin{array}{l}16 \text { South of North End of } \\
\text { Sean }\end{array}$ & $\left\{\begin{array}{c}12 / 03 / 93 \\
0\end{array}\right.$ & $12 / 17 / 93$ & $12 \times 4$ & & $\mathrm{HE}$ \\
\hline sose & $112 / 06 / 93$ & $5-064 / 069$ & I g1 Nest of Crest of Slope & $|12 / \pi / 93|$ & $12 / 16 / 93$ & $12 \times 4$ & & NE \\
\hline
\end{tabular}


GOLDER CONSTRUCTION SERVICES, INC. DEPARTMENT OF ENERGY NON-DRAG OFF LANDFILL FACILITY HAHFORD NUCLEAR RESERVATION

\begin{tabular}{|c|c|c|c|}
\hline $\begin{array}{l}\text { REPAIR } \\
\text { NUMBER }\end{array}$ & $\mid \begin{array}{l}\text { DATE } \\
\mid \text { LOCATED }\end{array}$ & $\begin{array}{l}\text { SEAH } \\
\text { or } \\
\text { PANEL }\end{array}$ & $\begin{array}{l}\text { I } \\
\text { |APPROXIMATE REPAIR LOCATI }\end{array}$ \\
\hline Soso & | 12/06/93 & $s-064 / 069$ & I 3' East of Crest of Slo \\
\hline SOSE & | 12/06/93 & $s-001 / 063$ & $\begin{array}{l}\text { I } 14 \text { ' North of South End } 0 \\
\text { sean }\end{array}$ \\
\hline SOSF & $|12 / 06 / 93|$ & $s-0,05 / 1069$ & $\begin{array}{l}\text { I } 5 \text { North of South End of } \\
\text { Seam }\end{array}$ \\
\hline 5056 & $|12 / 06 / 93|$ & $s-055 / 071$ & $\begin{array}{l}\text { I 3' South of North End of } \\
\text { Sean }\end{array}$ \\
\hline SOSH & $|12 / 06 / 93|$ & $5-054 / 055$ & $\begin{array}{l}\text { I At Intersection With } \\
\text { s-056. }\end{array}$ \\
\hline sos 3 & $|12 / 06 / 93|$ & $s-056 / 057$ & $\begin{array}{l}\text { I At intersection with } \\
\text { S-054 }\end{array}$ \\
\hline sosk & $|12 / 06 / 93|$ & $s-057 / 058$ & $\begin{array}{l}\text { I At Intersection With } \\
\text { s-053/054 }\end{array}$ \\
\hline SDSL & $|12 / 06 / 93|$ & s-058/059 & $\begin{array}{l}\text { I At Intersection With } \\
\text { S-053 }\end{array}$ \\
\hline $\operatorname{sos} n$ & $|12 / 05 / 93|$ & $s-049 / 053$ & $\begin{array}{l}\text { I AE Intersection with } \\
\text { S-059 }\end{array}$ \\
\hline SOSH & $|12 / 06 / 93|$ & $5-048 / 049$ & $\begin{array}{l}\text { I At Intersection With } \\
\text { S-059 }\end{array}$ \\
\hline SOSP & | 12/06/93 & $s-046 / 047$ & $\begin{array}{l}\text { | At Intersection with } \\
\text { s-050 }\end{array}$ \\
\hline sosa & $|12 / 06 / 93|$ & $s-045 / 046$ & $\begin{array}{l}\text { I At Intersection With } \\
\text { s-050 }\end{array}$ \\
\hline SOSR & $|12 / 06 / 93|$ & $s-044 / 045$ & $\begin{array}{l}\text { I At Intersetion With } \\
\text { s-050 }\end{array}$ \\
\hline soss & $|12 / 06 / 93|$ & $s-0431044$ & $\begin{array}{l}\text { I At Intersection With } \\
\text { s-050 }\end{array}$ \\
\hline SOST & $|12 / 06 / 93|$ & $s-042 / 043$ & $\begin{array}{l}\text { I At Intersection with } \\
\text { s-050 }\end{array}$ \\
\hline SOSU & $|12 / 06 / 93|$ & $s-049 / 042$ & $\begin{array}{l}\text { At Intersection With } \\
\text { s-050 }\end{array}$ \\
\hline sosu & $|12 / 06 / 93|$ & $5-040 / 049$ & $\begin{array}{l}\text { I At Intersection With } \\
\text { s-050 }\end{array}$ \\
\hline $\operatorname{sos} x$ & $|12 / 06 / 93|$ & $s-039 / 040$ & $\begin{array}{l}\text { At Intersection With } \\
\text { s-0so }\end{array}$ \\
\hline SOSA & $|12 / 08 / 93|$ & $s-067 / 069$ & $\begin{array}{l}\text { I At Intersection With } \\
\text { S-068/070 }\end{array}$ \\
\hline 5068 & $|12 / 08 / 93|$ & $s-084 / 069$ & $\begin{array}{l}\text { I At Intersection With } \\
S-066\end{array}$ \\
\hline SO6E & $|12 / 08 / 93|$ & $s-001 / 066$ & $\begin{array}{l}\text { I At Intersection Uith } \\
\mathrm{S}-064\end{array}$ \\
\hline SOSD & | 12/08/93 & $5-063 / 064$ & 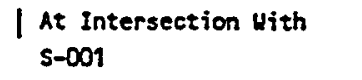 \\
\hline
\end{tabular}


GOLOER CONSTRUCTION SERVICES, INC.

933-1354 SECONDARY GEOMEMBRANE LINER REPAIR SUMHARY DEPARTMENT OF ENERGY

NON-DRAG OFF LANDFILL FACILITY

HANFORD NUCLEAR RESERVATIOH

\begin{tabular}{|c|c|c|c|c|c|c|c|c|}
\hline $\begin{array}{l}\text { REPAIR } \\
\text { NUMBER }\end{array}$ & $\mid \begin{array}{c}\text { DATE } \\
\mid \text { LOCATED } \\
\mid\end{array}$ & $\begin{array}{l}\text { SEAM } \\
\text { or } \\
\text { PANEL }\end{array}$ & $\mid$ & $\begin{array}{c}\text { DATE } \\
\text { REPAIRED } \\
\text { (1) }\end{array}$ & $\left\{\begin{array}{c}\text { OATE } \\
1 \text { TESTED } \\
1\end{array}\right.$ & $\left\{\begin{array}{c}\text { SILE } \\
1(f t)(2)\end{array}\right.$ & $\begin{array}{l}\mid \mathrm{RE} \\
\text { lKC } \\
1\end{array}$ & $\begin{array}{l}\text { EPAIR } \\
\text { ONITOR }\end{array}$ \\
\hline SOSE & | 12/08/93 & $s-062 / 063$ & 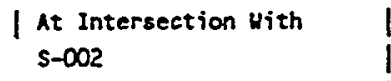 & $12 / 20 / 93$ & | 12/20/93 & $2 \times 4$ & 1 & OHS \\
\hline SOSF & $|12 / 08 / 93|$ & I $5-057 / 058$ & I 3' Vest of Crest of Slope & $12 / 16 / 93$ & | 12/16/93 & $12 \times 2$ & 1 & SM \\
\hline SOSG & $12 / 08 / 93$ & I s-046/047 & $\begin{array}{l}\text { I 13' North of Crest of } \\
\text { slope }\end{array}$ & $12 / 12 / 93$ & | 12/15/93 & $13 \times 29$ & 1 & SM \\
\hline SOSH & $\mid 12 / 08 / 93$ & Is-048/049 & $\begin{array}{l}\text { I 50. North of Crest of } \\
\text { slope }\end{array}$ & $12 / 13 / 93$ & | 12/15/93 & $9 \times 9$ & 1 & SM \\
\hline $506 \mathrm{~J}$ & | 12/08/93 | & $5-0.48 / 049$ & $\begin{array}{l}\text { I } 5 \text { ' South of Anchor Trench } \\
\text { Crest }\end{array}$ & $12 / 15 / 93$ & | $12 / 15 / 93$ & $11 \times 2$ & 1 & SM \\
\hline $506 x$ & | 12/08/93 & | s-049/053 & $\begin{array}{l}130^{\circ} \text { North of Crest of } \\
\text { slope }\end{array}$ & $12 / 13 / 93$ & | $12 / 15 / 93$ & & I & SH \\
\hline SO6L & $\mid 12 / 09 / 93$ & | s-049/053 & $\begin{array}{l}\text { I } 5 \text { South of Anchor Trench } \\
\text { Crest }\end{array}$ & $12 / 16 / 93$ & | $12 / 16 / 93$ & $12 \times 6$ & I & SM \\
\hline SOSK & | 12/09/93 | & 1 s-049/053 & $\begin{array}{l}\text { I } 24^{\prime} N \text { of Crest of Slope } \\
\text { (Covered by SOSK) }\end{array}$ & $12 / 13 / 93$ & | 12/15/93 & $12 \times 11$ & 1 & SM \\
\hline SOSN & | 12/11/93 | & $15-0471050$ & $\begin{array}{l}\text { I 5' South of North End of } \\
\text { Sear }\end{array}$ & $12 / 11 / 93$ & | 12/15/93 & $12 \times 4$ & I & SH \\
\hline SOSP. & | 12/11/93 & $5-040 / 041$ & I 3' South of Toe of slope & $12 / 12 / 93$ & | 12/15/93 & $12 \times 7$ & 1 & NE \\
\hline $506 a$ & | 12/19/93 & |s-044/045 & $\begin{array}{l}14 \text { South of Anchor Trench } \\
\text { Crest }\end{array}$ & $12 / 15 / 93$ & | $12 / 15 / 93$ & $12 \times 4$ & 1 & SH \\
\hline$S O S R$ & | 12/12/93 & $5-042 / 043$ & $\begin{array}{l}\text { I South End of Seam to } \\
\text { North End of Sean }\end{array}$ & $12 / 12 / 93$ & | 12/15/93 & $13 \times 150$ & 1 & SH \\
\hline soss & | 12/12/93 & I $5-043 / 506 R$ & I 3' North of Toe of Slope & $12 / 15 / 93$ & | $12 / 15 / 93$ & $14 \times 5$ & 1 & SM \\
\hline SOGT & | 12/12/93 & I $5-043 / S O S R$ & 1 73' North of slope Crest & $12 / 94 / 93$ & | 12/15/93 & $12 \times 3$ & I & SH \\
\hline SOSU & | 12/12/93 & I $s-0<3 / s 0 S R$ & $\begin{array}{l}\text { I 61' North of slope Crest } \\
\text { (Same Patch as SOSW) }\end{array}$ & $12 / 14 / 93$ & $12 / 15 / 93$ & $12 \times 12$ & & SH \\
\hline S064 & | 12/92/93 & I $5-0<3 / 506 R$ & $\begin{array}{l}\text { I 53. North of Slope Crest } \\
\text { (Same Patch as SOSU) }\end{array}$ & $12 / 14 / 93$ & | 12/95/93 & $19 \times 1$ & & SH \\
\hline SDSX & | 12/12/93 & I $s-0,33 / 506 R$ & | 22 ' North of Slope Crest & $12 / 14 / 93$ & | 12/15/93 & $11 \times 2$ & & NE \\
\hline SO7A & | 12/12/93 & I $s-0.43 / 50 S R$ & I Crest of slope & $12 / 14 / 93$ & $12 / 15 / 93$ & $11 \times 1$ & & KE \\
\hline 5078 & | 12/12/93 & I $s-042 / 505 R$ & $\begin{array}{l}154^{\prime} \text { North of Crest of } \\
\text { slope }\end{array}$ & $12 / 14 / 93$ & $12 / 15 / 93$ & $12 \times 5$ & & Sn \\
\hline SO7C & | 12/13/93 & I $5-042 / 5068$ & $\begin{array}{l}\text { | 101: North of Crest of } \\
\text { slope }\end{array}$ & $12 / 14 / 93$ & | 12/15/93 & $12 \times 7$ & & Sh \\
\hline 5070 & $\mid 12 / 13 / 93$ & $5-042 / 506 R$ & $\begin{array}{l}\text { I } 75^{\prime} \mathrm{N} \text { of Crest of Slope } \\
\text { (Same Patch as SO7E) }\end{array}$ & $12 / 14 / 93$ & $12 / 15 / 93$ & $12 \times 9$ & & SH \\
\hline SO7E & | 12/13/93 & $S-0.42 / S 06 R$ & $\begin{array}{l}\text { | 68' } N \text { of Crest of Slope } \\
\text { (Same Patch as s07D) }\end{array}$ & $12 / 14 / 93$ & | 12/15/93 & $12 \times 9$ & & SH \\
\hline
\end{tabular}


GOLOER CONSTRUCTION SERVICES, INC.

933-1354

SECONDARY GEOHEMBRUNE LINER REPAIR SUMMARY

$05 / 11 / 94$ DEPARTMENT OF ENERGY

Page 7

NON-DRAG OFF LANDFILL FACILITY

HANFORD NUCLEAR RESERVATION

\begin{tabular}{|c|c|c|c|c|c|c|c|c|}
\hline $\begin{array}{l}\text { REPAIR } \\
\text { HUMBER }\end{array}$ & $\mid \begin{array}{l}\mid \text { OATE } \\
\mid \text { LOCATED }\end{array}$ & $\begin{array}{l}\text { SEAM } \\
\text { or } \\
\text { PANEL }\end{array}$ & $\begin{array}{l}\text { |APPROXIMATE REPAIR LOCATION| } \\
\mid\end{array}$ & $\begin{array}{l}\text { DATE } \\
\text { REPAIRED } \\
\text { (1) }\end{array}$ & $\begin{array}{l}\text { DATE } \\
\text { TESTED } \\
\text { (1) }\end{array}$ & $\left\{\begin{array}{c}\text { SIZE } \\
(f t)(2) \\
1\end{array}\right.$ & $\begin{array}{l}\mid R \\
1 M \\
1\end{array}$ & $\begin{array}{l}\text { EPAIR } \\
\text { ONITOR (3) }\end{array}$ \\
\hline so7f & | 12/13/93 & $s-043 / 506 R$ & $\begin{array}{l}\text { | } 4 \text { ' South of Anchor Trench | } \\
\text { Crest }\end{array}$ & $12 / 15 / 93$ & $12 / 15 / 93$ & $11 \times 1$ & 1 & SH \\
\hline 5076 & $|12 / 14 / 93|$ & $s-005 / 006$ & $\begin{array}{l}\text { I 13' North of Crest of } \\
\text { slope }\end{array}$ & $01 / 09 / 94$ & $01 / 09 / 94$ & $12 \times 3$ & 1 & NE \\
\hline SOTH & | 12/16/93 & $s-033 / 034$ & $\begin{array}{l}\text { I 11' South of Crest of } \\
\text { slope }\end{array}$ & $12 / 21 / 93$ & $12 / 21 / 93$ & $12 \times 4$ & 1 & NE \\
\hline s07s & $|12 / 15 / 93|$ & $s-044 / 045$ & $\begin{array}{l}\text { I 11' South of Crest of } \\
\text { slope }\end{array}$ & $12 / 15 / 93$ & $12 / 15 / 93$ & $11 \times 1$ & I & SH \\
\hline so7k & | 12/15/93 & $s-046 / 047$ & $\begin{array}{l}\text { I Toe of Anchor Trench } \\
\text { (Capped by S0sG) }\end{array}$ & $12 / 15 / 93$ & $12 / 15 / 93$ & $12 \times 5$ & 1 & SH \\
\hline SOTL & | 12/15/93 | & $s-050 / 051$ & $\begin{array}{l}\text { I At intersection with } \\
\text { S-060 }\end{array}$ & $12 / 20 / 93$ & $12 / 20 / 93$ & $13 \times 4$ & I & SH \\
\hline SO7n & $|12 / 15 / 93|$ & $s-047 / 048$ & $\begin{array}{l}\text { I At Intersection With } \\
\text { S-059 }\end{array}$ & $12 / 15 / 93$ & $12 / 16 / 93$ & $12 \times 2$ & 1 & $S M$ \\
\hline SORH & | 12/15/93 | & $5-040$ & $\begin{array}{l}\text { I 8. E. of S-039/040, 30' } \\
\text { S. of Toe of Stope }\end{array}$ & $12 / 15 / 93$ & $12 / 16 / 93$ & $1 \times 1$ & I & SM \\
\hline SO7P & I 07/04/94 & $s-004 / s 906$ & $\begin{array}{l}161 \text { North of South End of } \\
\text { Sean }\end{array}$ & $01 / 04 / 94$ & $01 / 05 / 94$ & $12 \times 7$ & 1 & NE \\
\hline so7Q & | 01/04/94 | & $s-055$ & $\begin{array}{l}\mid 7^{\prime} \text { South, 4' E of Crest } \\
\text { of Slope to } 222^{\prime} \text { southeast }\end{array}$ & $12 / 96 / 93$ & $12 / 16 / 93$ & $13 \times 22$ & 1 & SH \\
\hline SOTR & 1 01/04/94 & $s-054$ & $\begin{array}{l}13^{\prime} \text { South of Acess Ramp, } \\
8^{\prime} \text { West of } 5-054 / 055\end{array}$ & $92 / 15 / 93$ & 12/16/93 & $13 \times 6$ & 1 & SM \\
\hline so7s & | 01/04/94 | & $5-054$ & $\begin{array}{l}\text { 15' South of Recess Ramp, } \\
\text { 4' Nest of S-054/055 }\end{array}$ & $12 / 15 / 93$ & $12 / 16 / 93$ & $12 \times 3$ & 1 & SM \\
\hline sort & $102 / 04 / 94$ & $s-054$ & $\begin{array}{l}\text { 16' South of Access Ramp, } \\
\text { 5' East of S-053/054 }\end{array}$ & $12 / 15 / 93$ & $12 / 16 / 93$ & $3 \times 7$ & 1 & SH \\
\hline sorn & | 01/04/94 | & $s-054$ & $\begin{array}{l}\text { I 5' South of Access Rasp, } \\
5^{\prime} \text { East of } 5-053 / 054\end{array}$ & $12 / 15 / 93$ & $12 / 16 / 93$ & $11 \times 2$ & 1 & SM \\
\hline SOT & | 01/04/94 & $s-054 / 055$ & I 2' South of Access Ramp & $12 / 16 / 93$ & $12 / 16 / 93$ & $11 \times 2$ & 1 & SH \\
\hline so7x & 1 01/04/94 & s-071 & \begin{tabular}{|}
$\mid 7^{\prime} S$ of Crest of Slope, \\
$2^{\prime}$ to $12^{\prime}$ SE of S-055/071 |
\end{tabular} & $12 / 16 / 93$ & $12 / 16 / 93$ & $3 \times 12$ & 1 & SH \\
\hline sose & | 12/13/93 & $s-024 / 051$ & $\begin{array}{l}13^{2} \text { North of South End of } \\
\text { Sean }\end{array}$ & $12 / 20 / 93$ & $12 / 20 / 93$ & $12 \times 2$ & 1 & DHM \\
\hline sosB & | 12/13/93 & $s-024 / 051$ & $\begin{array}{l}\text { I 9' Sourth of North End of } \\
\text { sean }\end{array}$ & $12 / 20 / 93$ & $12 / 20 / 93$ & $12 \times 2$ & l & DHH \\
\hline SOBC & | 12/94/93 & $s-001 / 002$ & $\begin{array}{l}14{ }^{\prime} \text { North of South End of } \\
\text { Sean }\end{array}$ & $12 / 20 / 93$ & $12 / 20 / 93$ & $12 \times 4$ & 1 & DHK \\
\hline SOBD & | 12/13/93 & $s-008 / 009$ & $\begin{array}{l}\text { I At Intersection with } \\
\text { S-052 }\end{array}$ & $12 / 20 / 93$ & $12 / 20 / 93$ & $12 \times 4$ & & DHM \\
\hline SOBE & | 12/13/93 & $s-007 / 008$ & $\begin{array}{l}\text { I At Intersection With } \\
\text { s-052 }\end{array}$ & $12 / 20 / 93$ & $12 / 20 / 93$ & $2 \times 2$ & 1 & DHM \\
\hline SOBF & | 12/13/93 & $s-005 / 006$ & $\begin{array}{l}\text { I At Intersection With } \\
\text { s-052 }\end{array}$ & $12 / 20 / 93$ & $12 / 20 / 93$ & $2 \times 2$ & & DHM \\
\hline
\end{tabular}


GOLDER CONSTRUCTION SERVICES, INC.

933-1354

SECONDARY GEOMEMBRANE LINER REPAIR SUMHARY 05/19/94

DEPARTHENT OF ENERGY

Page 8

NON-DRAG OFF LANDFILL FACILITY

HANFORD NUCLEAR RESERVATION

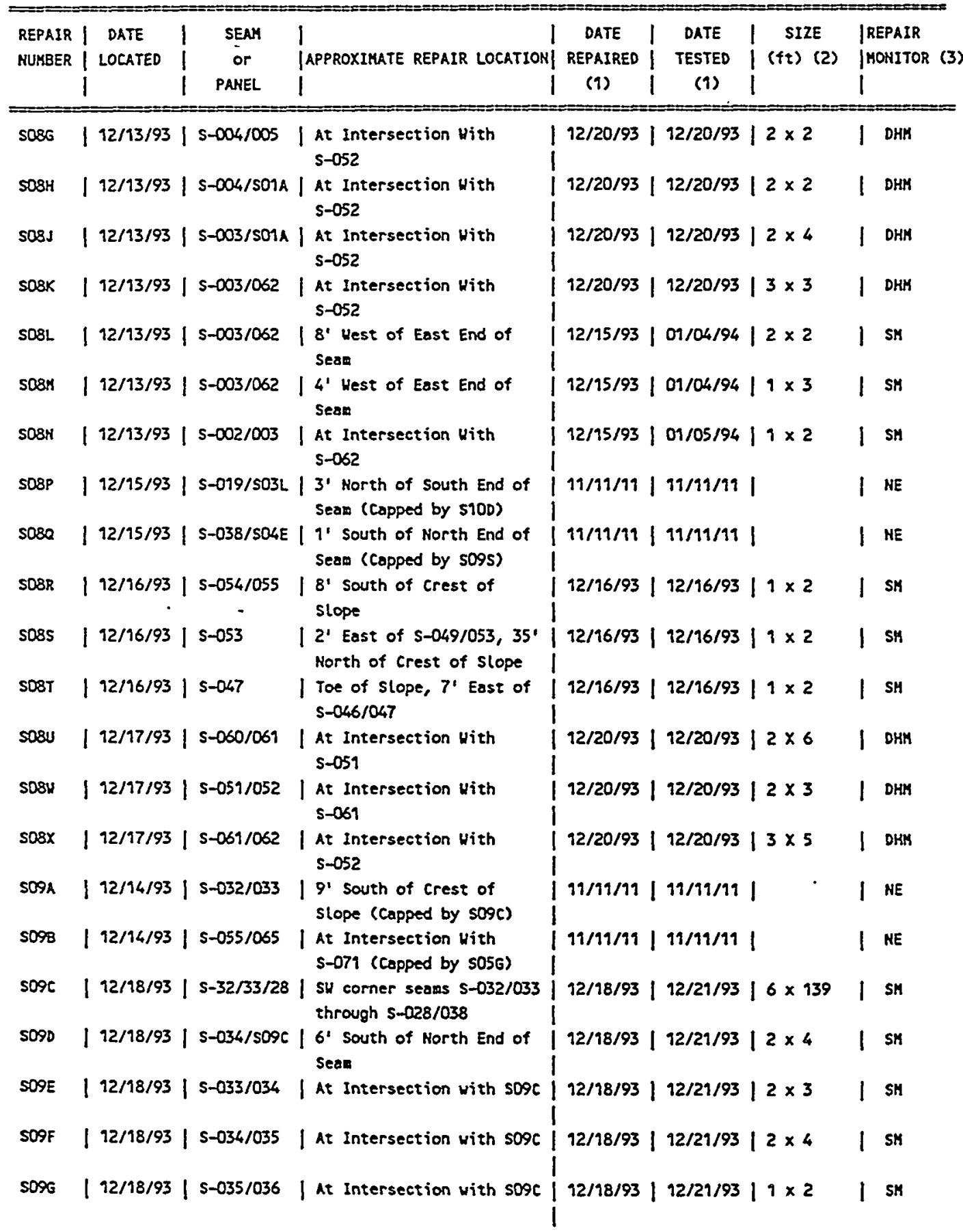


GOLDER CONSTRUCTION SERVICES, INC.

933-1354 SECONDARY GEOMEMBRANE LIMER REPAIR SUMHARY 05/19/94 DEPARTHENT OF ENERGY Page 9 NOW-DRAG OFF LANDFILL FACILITY HANFORD NUCLEAR RESERVATION

\begin{tabular}{|c|c|c|c|c|c|c|}
\hline $\begin{array}{l}\text { REPAIR } \\
\text { NUHBERR }\end{array}$ & $\mid \begin{array}{l}\text { DATE } \\
\text { LOCATEO }\end{array}$ & $\begin{array}{l}\text { SEAH } \\
\text { or } \\
\text { PANEL }\end{array}$ & |APPROXIMATE REPAIR LOCATION| & $\begin{array}{l}\text { DATE } \\
\text { REPAIRED } \\
\text { (1) }\end{array}$ & \begin{tabular}{cc|c}
$\mid$ & DATE & SIZE \\
$\mid$ & TESTED & (ft) $(2)$ \\
$\mid$ & $(1)$ &
\end{tabular} & $\begin{array}{l}\text { |REPAIR } \\
\text { |MONITOR (3) } \\
\end{array}$ \\
\hline SOSH & $112 / 18 / 93$ & $s-036 / 037$ & $\begin{array}{l}\text { I At Intersection with } 509 C \\
\text { (Copped by } S O 9 R \text { ) }\end{array}$ & $11 / 19 / 11$ & $|11 / 11 / 11|$ & SH \\
\hline sogs & $|12 / 18 / 93|$ & s-037/038 & $\begin{array}{l}\text { I At Intersection with s09C } \\
\text { (capped by SOgS) }\end{array}$ & 11/11/11 & | 11/11/11 | & SH \\
\hline sogk & $|12 / 18 / 93|$ & $5-029 / 509 C$ & $\begin{array}{l}\text { I 16' Horth of South End of } \\
\text { Sean }\end{array}$ & $12 / 21 / 93$ & $|12 / 21 / 93| 2 \times 2$ & ME \\
\hline so91. & $|12 / 18 / 93|$ & $s-028 / 029$ & I At Intersection With s09C & $12 / 21 / 93$ & $|12 / 21 / 93| 1 \times 3$ & HE \\
\hline SOSA & | 12/18/93 & $s-029 / 030$ & I At Intersection With s09C & 12/21/93 & | 12/21/93 | $2 \times 3$ & NE \\
\hline SOSH & $|12 / 18 / 93|$ & $s-030 / 031$ & I At Intersection vith s09C & $12 / 21 / 93$ & $|12 / 21 / 93| 2 \times 3$ & NE \\
\hline SO9P & | 12/18/93 | & $s-031 / 032$ & I At Intersection With sosc & $12 / 21 / 93$ & $|12 / 21 / 93| 1 \times 2$ & NE \\
\hline soga & $|12 / 18 / 93|$ & $\mathrm{s}-028 / \mathrm{s} 09 \mathrm{C}$ & $\begin{array}{l}\text { | } 10^{\prime} \text { North of South End of } \\
\text { Sean (Capped by sozw) }\end{array}$ & $12 / 21 / 93$ & $|12 / 21 / 93| 1 \times 1$ & ME \\
\hline SOPR & $|12 / 21 / 93|$ & $s-036 / 037$ & $\begin{array}{l}\text { I North End of Seas to } \\
\text { South End of Sean }\end{array}$ & $12 / 21 / 93$ & | $12 / 21 / 93 \mid 3 \times 16$ & NE \\
\hline sogs & $|12 / 21 / 93|$ & $s-037 / 038$ & $\begin{array}{l}\text { I North End of Sean to } \\
\text { South End of Seas }\end{array}$ & $12 / 21 / 93$ & $|12 / 21 / 93| 4 \times 14$ & NE \\
\hline SO9T & $|12 / 21 / 93|$ & s-028/038 & $\begin{array}{l}\text { I Hest End of Sean to East } \\
\text { End of Seam }\end{array}$ & $12 / 21 / 93$ & $|12 / 21 / 93| 6 \times 97$ & NE \\
\hline sogv & $|12 / 21 / 93|$ & $5-038$ & $\begin{array}{l}\text { I Caps repairs S030 and } \\
\text { SO3E }\end{array}$ & $12 / 21 / 93$ & $|12 / 21 / 93| 3 \times 12$ & NE \\
\hline sogu & | 12/21/93 & $s-033 / s 09 C$ & $\begin{array}{l}\text { I } 199 \text { South of Crest of } \\
\text { slope }\end{array}$ & $12 / 21 / 93$ & $|07 / 03 / 94| 6 \times 7$ & NE \\
\hline s09x & $|12 / 21 / 93|$ & $s-029 / s 09 C$ & $\begin{array}{l}\text { I } 14^{\circ} \text { North of South End of } \\
\text { Sean }\end{array}$ & $12 / 21 / 93$ & $12 / 21 / 93 \mid 1 \times 1$ & ME \\
\hline STOR & $|12 / 21 / 93|$ & S-028/S09T & | East End of SO9T & $12 / 21 / 93$ & | 12/21/93 | $2 \times 4$ & NE \\
\hline$\$ 108$ & $|12 / 21 / 93|$ & $\mathrm{S}-022 / \mathrm{SO} \mathrm{H}$ & $\begin{array}{l}\text { I 8' West of East End of } \\
\text { Sean (Copped by S10w) }\end{array}$ & $12 / 21 / 93$ & | 11/11/11 | $1 \times 1$ & NE \\
\hline $510 c$ & $\mid 12 / 21 / 93$ & $S-014 / 5020$ & $\begin{array}{l}\text { I Northwest Corner of } 5020 \\
\text { (Copped by S11p) }\end{array}$ & $12 / 21 / 93$ & | 11/11/11 | $1 \times 1$ & NE \\
\hline $\mathbf{S 9 0 0}$ & $|12 / 21 / 93|$ & $s-015 / 016$ & $\begin{array}{l}\text { I At Intersection with } \\
\text { S-019/020 }\end{array}$ & $12 / 21 / 93$ & $|01 / 06 / 94| 4 \times 15$ & NE \\
\hline STOE & | 12/21/93 & $5-014 / 021$ & $\begin{array}{l}\text { 16' East of Hest End of } \\
\text { Sean }\end{array}$ & $12 / 21 / 93$ & $|01 / 06 / 94| 4 \times 9$ & NE \\
\hline S10F & $|12 / 21 / 93|$ & $s-051$ & I sump & $12 / 21 / 93$ & $|01 / 05 / 94| 6 \times 15$ & I NE \\
\hline 5106 & $|12 / 22 / 93|$ & $5-003 / 004$ & $\begin{array}{l}1 \text { South End of Sean to } \\
\text { Horth End of Sean }\end{array}$ & $12 / 22 / 93$ & $|01 / 07 / 94| 5 \times 130$ & NE \\
\hline S1DH & | 01/03/94 & $s-028$ & $\begin{array}{l}\text { I } 11 \text { Hest of Slope Crest, } \\
6^{\prime} \text { south of S-027/028 }\end{array}$ & $01 / 05 / 94$ & $|01 / 05 / 94| 1 \times 2$ & I $\mathrm{NE}$ \\
\hline
\end{tabular}


GOLDER CONSTRUCTION SERVICES, INC.

\begin{tabular}{|c|c|c|c|c|c|c|}
\hline $\begin{array}{l}\text { REPAIR | } \\
\text { NLXBER ! }\end{array}$ & $\begin{array}{lll}1 & \text { DATE } & 1 \\
1 \text { LOCATED } & 1 \\
1 & & 1\end{array}$ & $\begin{array}{l}\text { SEAY } \\
\text { or } \\
\text { PANEL }\end{array}$ & |aPPROXIMATE REPAIR LOCATION] & $\left\{\begin{array}{c}\text { DATE } \\
\text { REPAIRED } \\
\text { (1) }\end{array}\right.$ & \begin{tabular}{c|c} 
DATE & SIZE \\
TESTED & $(f t)(2)$ \\
$(1)$ &
\end{tabular} & $\begin{array}{l}\text { |REPAIR } \\
\text { |MONITOR Q }\end{array}$ \\
\hline sios & $|09 / 03 / 94|$ & $5-024 / 025$ & I 14' West of Slope Crest & $01 / 05 / 941$ & $01 / 05 / 94 \mid 2 \times 3$ & KE \\
\hline s10K & | 01/03/94 | & s-021/022 & I 15' West of slope Crest & $09 / 06 / 94$ & $01 / 06 / 94 \mid 2 \times 3$ & I NE \\
\hline S1OL & $|01 / 03 / 94|$ & | s-004/s10s & I 17 North of Slope Crest & $01 / 07 / 94$ & $01 / 07 / 94 \mid 3 \times 3$ & I DHк \\
\hline sion & $\mid 01 / 03 / 94$ & s-oz2/s10N & I East End of Seam & $|01 / 05 / 94|$ & $01 / 06 / 94 \mid 3 \times 5$ & I $N E$ \\
\hline sion & $|01 / 05 / 94|$ & $s-022 / 023$ & $\begin{array}{l}\text { I } 35^{\prime}-78^{\prime} \text { East of Crest of } \\
\text { slope }\end{array}$ & $01 / 05 / 94 \mid$ & $01 / 06 / 94 \mid 7 \times 42$ & I NE \\
\hline S10P & $|02 / 04 / 94|$ & $5-057 / 058$ & $\begin{array}{l}\text { I } 12 \text { East of Crest of } \\
\text { Slope }\end{array}$ & | 01/10/94 | & $01 / 11 / 94 \mid 2 \times 3$ & I ME \\
\hline$s 100$ & | $01 / 04 / 94 \mid$ & $5-059$ & I At East End of Panel & $01 / 09 / 94$ & $01 / 11 / 94 \mid 2 \times 23$ & NE \\
\hline S1OR & $|01 / 04 / 94|$ & $5-060 / 069$ & $\begin{array}{l}\text { I } 10 \text { ' East of Crest of } \\
\text { slope }\end{array}$ & | 01/09/94 | & $01 / 09 / 94 \mid 1 \times 1$ & I NE \\
\hline s10s & | 01/04/94 | & $5-061$ & I At East End of Panel & $01 / 09 / 94$ & $01 / 09 / 94 \mid 3 \times 23$ & I HE \\
\hline sior & $|01 / 04 / 94|$ & $s-0633 / 064$ & $\begin{array}{l}\text { I 1' West of East End of } \\
\text { Sean }\end{array}$ & | 01/07/94 | & $01 / 09 / 94$ | $2 \times 2$ & DHY \\
\hline sios & $101 / 04 / 94$ & $5-064 / 069$ & $\begin{array}{l}\text { I 1' West of East End of } \\
\text { Sean }\end{array}$ & $\mid \begin{array}{l}01 / 07 / 94 \mid \\
\mid\end{array}$ & $01 / 09 / 94 \mid 3 \times 3$ & DHM \\
\hline s10n & $|01 / 04 / 94|$ & $5-015 / 016$ & | North End of Sean & $01 / 09 / 94$ & $01 / 09 / 94 \mid 2 \times 2$ & I OHM \\
\hline siox & $|01 / 04 / 94|$ & $s-016 / 017$ & I North End of Seas & $01 / 09 / 94$ & $01 / 09 / 94 \mid 2 \times 2$ & ME \\
\hline $\operatorname{si1a}$ & $|12 / 22 / 93|$ & s-004/s106 & | At South End of Sean & $12 / 22 / 93$ & $01 / 04 / 94 \mid 1 \times 1$ & HE \\
\hline S11B & $|12 / 22 / 93|$ & $s-003 / s 106$ & I At South End of Sears & $12 / 22 / 93$ & $01 / 04 / 94 \mid 1 \times 1$ & NE \\
\hline s11e & $|01 / 04 / 04|$ & $s-\infty, s 106$ & $\begin{array}{l}\text { | } 53^{\prime} \text { North of South End of } \\
\text { Sean }\end{array}$ & $01 / 04 / 94$ & $01 / 11 / 94 \mid 2 \times 6$ & ME \\
\hline 5110 & $109 / 04 / 04 \mid$ & $5-003 / 5100$ & $\begin{array}{l}131 \text { North of South End of } \\
\text { Sean }\end{array}$ & $01 / 04 / 94 \mid$ & $01 / 05 / 94 \mid 2 \times 2$ & NE \\
\hline SIIE & $|04 / 04 / 04|$ & $s-003 / s 106$ & $\begin{array}{l}\text { I } 21 \text { south of Crest of } \\
\text { slope }\end{array}$ & $09107 / 941$ & $01 / 07 / 94 \mid 4$ & DHM \\
\hline S7TF & $101 / 04 / 04 \mid$ & $5-003 / 5100$ & $\begin{array}{l}12^{\prime} \text { North of Crest of } \\
\text { Slope }\end{array}$ & $01 / 07 / 94 \mid$ & $01 / 07 / 45 \mid 2 \times 2$ & DHM \\
\hline S11G & $|01 / 05 / 94|$ & s-023/s10 & | East End of Sean & $04 / 05 / 94$ & $01 / 06 / 94 \mid 2 \times 2$ & I HE \\
\hline S19H & | 01/05/94 & $s-023 / s 10 \mathrm{~N}$ & | West End of Sear & $01 / 05 / 94$ I & $01 / 06 / 94 \mid 1 \times 2$ & I NE \\
\hline s11s & $109 / 05 / 94$ & s-022/s10N & | West End of Sean & $01 / 05 / 94$ & $01 / 05 / 94 \mid 9 \times 2$ & NE \\
\hline
\end{tabular}


GOLDER CONSTRUCTION SERVICES, INC.

933-1356

SECONDARY GEOMEMBRUNE LINER REPAIR SUMMARY

05/11/94

DEPARTHENT OF ENERGY

Page 11

NON-DRAG OFF LANDFILL FACILITY

HAHFORD NUCLEAR RESERVATION

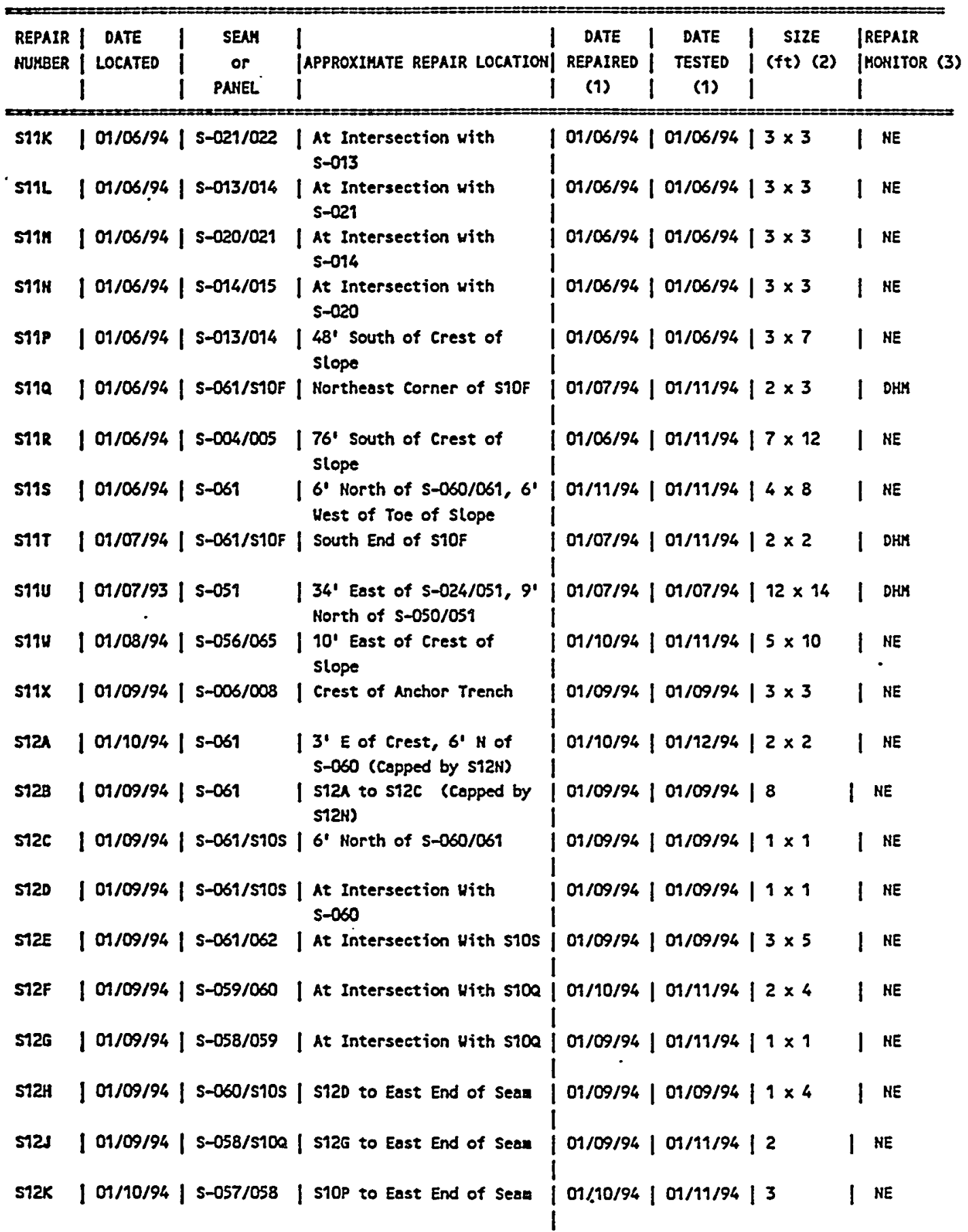


WHC-SD-W025-RPT-001, Rev. 0

GOLOER CONSTRUCTION SERVICES, INC.

933-1354

SECONDARY GEOKEMBRANE LINER REPAIR SUMHARY

$05 / 11 / 94$

DEPARTMENT OF ENERGY

Page 12

NON-DRAG OFF LANDFILL FACILITY

HANFORD NUCLEAR RESERVATION

\begin{tabular}{|c|c|c|c|c|c|c|c|}
\hline $\begin{array}{l}\text { REPAIR } \\
\text { NUMBER }\end{array}$ & $\begin{array}{l}\text { DATE } \\
1 \text { LOCATED }\end{array}$ & $\begin{array}{l}\text { SEAY } \\
\text { or } \\
\text { PANEL }\end{array}$ & $\mid \begin{array}{l}\mid \\
\mid \text { APPROXIHATE REPAIR LOCATION| }\end{array}$ & $\begin{array}{l}\text { DATE } \\
\text { REPAIRED } \\
\text { (1) }\end{array}$ & $\begin{array}{l}\text { DATE } \\
\text { TESTED } \\
\text { (१) }\end{array}$ & $\left\{\begin{array}{c}\text { SIZE } \\
\mid(f t)(2)\end{array}\right.$ & $\begin{array}{l}\text { |REPAIR } \\
\text { | MOHITOR (3) } \\
1\end{array}$ \\
\hline s12L & | 01/12/94 & | s-061 & $\begin{array}{l}\text { I I' North of } 5-060 / 061 \text {, } \\
\text { Toe to Crest of slope }\end{array}$ & $01 / 13 / 94$ & $07 / 13194$ & $16 \times 95$ & HE \\
\hline s12n & | 11/22/93 & | s-027/028 & I Intersection with s-039 & $12 / 20 / 93$ & $12 / 20 / 93$ & $11 \times 1$ & SNM \\
\hline SI2N & | 01/99/94 & $s-0,1$ & $\begin{array}{l}\text { I 3' } N \text { of S-060/061, 3' } \mathrm{K} \\
\text { of } E \text { End of Panel }\end{array}$ & $01 / 27 / 94$ & $101 / 27 / 94$ & $14 \times 6$ & 1 онM \\
\hline
\end{tabular}

NOTES:

(1) A repair date and/or test date of 19/19/19 indicates a repair which was capped.

(2) A repair with no size indicates a repair which was capped.

A repair with only one size dimension indicates a grind and weld repair.

(3) See Veld Observation Sumary for monitor identification. 
WHC-SD-W025-RPT-001, Rev. 0

APPENDIX N

PRIMARY HDPE GEOMEMBRANE LINER INSTALLATION

$N-i$ 
WHC-SD-H025-RPT-001, Rev. 0

\section{APPENDIX N-1}

GEOMEMBRANE DEPLOYMENT OBSERVATION SUMMARY 
GOLDER CONSTRUCTION SERVICES, INC.

933-1354

PRIMARY GEOMEMBRANE LINER DEPLOYMENT OBSERVATION SUMMARY

$05 / 11 / 94$ DEPARTHENT OF ENERGY

Page 1

NON-DRAG OFF LANDFILL FACILITY

HANFORD NUCLEAR RESERVATION

\begin{tabular}{|c|c|c|c|c|c|c|c|c|c|c|}
\hline \multirow{4}{*}{$\begin{array}{l}\text { PANEL } \\
\text { NUMBER(1) }\end{array}$} & \multirow{4}{*}{$\begin{array}{c}\text { ROLL } \\
\text { NUMBER }\end{array}$} & \multirow{4}{*}{$\begin{array}{l}1 \\
1 \\
\text { DATE } \\
\text { DEPLOYED }\end{array}$} & \multirow{4}{*}{$\begin{array}{c}\text { APPROX.| } \\
\text { PANEL } \\
\text { LENGTH ! } \\
(f T) !\end{array}$} & \multirow{4}{*}{$\begin{array}{l}\text { VISUAL } \\
\text { PANEL } \\
\text { INSP (2) }\end{array}$} & \multirow{4}{*}{$\begin{array}{l}\text { MINIHUM | } \\
\text { PANEL | } \\
\text { OVERLAP } \\
\text { (inches) }\end{array}$} & \multirow{2}{*}{\multicolumn{3}{|c|}{$\begin{array}{c}\text { AVERAGE PANEL THICKNESS } \\
\text { (3) }\end{array}$}} & \multirow{2}{*}{$\mid$} & \\
\hline & & & & & & & & & & \\
\hline & & & & & & Leading & side & I Trailing & | MONITOR | & REMARKS \\
\hline & & & & & & Edge & Edge & Edge & I (4) & \\
\hline$L-\infty 01$ & $A C 8309$ & | 11/177/93 & 501 & OK & & 82.5 & 80.5 & 82.1 & DHM & \\
\hline$P-\infty 01$ & AC6924 & 12/19/93 & $100 i$ & OK & 5 & 67.8 & 65.1 & 68.1 & $N E$ & \\
\hline$P-002$ & AC6924 & $12 / 19 / 93$ & 137 & OK & 5 & 68.1 & 64.6 & 68.1 & NE & \\
\hline$P-003$ & AC6924 & $12 / 19 / 93$ & 153 & ox & 5 & 68.1 & 64.4 & 68.0 & NE & \\
\hline$P-004$ & AC6909 & $12 / 19 / 93$ & 148 & OK & 5 & 68.1 & 63.1 & 67.8 & $\mathrm{NE}$ & \\
\hline$P-005$ & AC6909 & $12 / 19 / 93$ & 138 & OK & 5 & 67.8 & 63.5 & 68.3 & NE & \\
\hline P-D06 & AC6909 & $12 / 19 / 93$ & 136 & ox & 5 & 68.3 & 63.6 & 67.8 & NE & \\
\hline P-007 & AC6923 & $12 / 19 / 93$ & 138 & OK & 5 & 67.1 & 61.7 & 68.1 & NE & \\
\hline P-008 & AC6923 & $12 / 19 / 93$ & 141 & OK & 5 & 68.1 & 61.9 & 68.0 & NE & \\
\hline P-009 & AC6923 & $12 / 19 / 93$ & 139 & OK & 5 & 68.0 & 69.4 & 68.3 & NE & \\
\hline$P-010$ & AC6911 & $12 / 19 / 93$ & 139 & OK & 5 & 68.3 & 62.5 & 68.0 & NE & \\
\hline P-011 & AC6911 & $12 / 19 / 93$ & 146 & OK & 5 & 68.0 & 62.3 & 68.4 & NE & \\
\hline P-012 & AC6906 & 12/19/93 & 146 & OK & 5 & 68.9 & 63.3 & 68.6 & NE & \\
\hline$P-013$ & AC6906 & $12 / 22 / 93$ & 150 & OK & 5 & 65.1 & 61.2 & 66.1 & NE & \\
\hline$P-014$ & AC6906 & $12 / 22 / 93$ & 118 & OK & 5 & 66.1 & 61.9 & 67.2 & NE & \\
\hline P-D15 & AC6925 & $12 / 22 / 93$ & 118 & OK & 5 & 65.8 & 64.7 & 66.3 & NE & \\
\hline$P-016$ & AC6925 & $12 / 22 / 93$ & 96 & OK & 5 & 66.3 & 63.3 & 66.0 & NE & \\
\hline$P-017$ & AC6925 & $12 / 22 / 93$ & 83 & OK & 5 & 66.0 & 63.8 & 67.2 & NE & \\
\hline$P-018$ & AC6906 & $12 / 22 / 93$ & 48 & OK & 5 & 67.2 & 62.5 & 67.1 & NE & \\
\hline P-019 & AC6898 & 1 01/06/94 & 59 & ox & 5 & 67.7 & 63.9 & 67.8 & NE & \\
\hline$P-020$ & AC6898 & 1 01/06/94 & 78 & OK & 5 & 67.8 & 64.2 & 68.0 & NE & \\
\hline P-021 & AC6898 & 1 01/06/94 & 103 & ox & 5 & 68.0 & 64.2 & 68.5 & $N E$ & \\
\hline P-022 & AC6898 & 1 01/06/94 & 128 & OK & 5 & 68.5 & 63.9 & 67.7 & $N E$ & \\
\hline$P-023$ & AC6917 & $01 / 06 / 94$ & 149 & OK & 5 & 67.4 & 62.0 & 67.6 & $N E$ & \\
\hline$P-024$ & AC6917 & $01 / 06 / 94$ & 150 & OK & 5 & 67.6 & 62.7 & 67.1 & NE & \\
\hline$P-025$ & AC6932 & $01 / 06 / 94$ & 153 & OK & 5 & 67.6 & 61.3 & 67.9 & NE & \\
\hline P-026 & AC6932 & $101 / 06 / 94$ & 153 & $\mathrm{OK}$ & 5 & 67.9 & 61.0 & 67.1 & NE & \\
\hline$P-027$ & AC6932 & | 01/07/94 & 39 & OK & 5 & 67.5 & 64.0 & 67.6 & DHM & \\
\hline P-028 & AC6904 & $01 / 10 / 94$ & 156 & ox & 5 & 67.7 & 64.1 & 68.1 & NE & \\
\hline P-029 & AC6904 & $01 / 10 / 94$ & 158 & OK & 5 & 68.1 & 63.9 & 68.1 & NE & \\
\hline$P=030$ & AC6904 & $01 / 10 / 94$ & 120 & OK & 5 & 68.1 & 62.2 & 68.0 & NE & \\
\hline P-031 & AC6905 & | 01/10/94 & 100 & oK & 5 & 67.6 & 62.5 & 67.8 & NE & \\
\hline$P-032$ & AC6932 & $101 / 10 / 94$ & 77 & OK & 5 & 67.2 & 62.1 & 67.2 & NE & \\
\hline$P-033$ & AC6904 & $01 / 13 / 94$ & 39 & ox & 5 & 70.8 & 65.6 & 67.7 & NE & \\
\hline$P=034$ & AC6904 & 1 01/13/94 & 8 & OK & 5 & 67.7 & 65.5 & 67.8 & NE & \\
\hline$P-035$ & AC6917 & $01 / 13 / 94$ & 59 & ox & 5 & 67.5 & 64.2 & 68.4 & NE & \\
\hline$P-036$ & AC6905 & $01 / 13 / 94$ & 82 & OK & 5 & 68.0 & 62.8 & 68.3 & NE & \\
\hline$P-037$ & AC6905 & $01 / 13 / 94$ & 921 & OK & 5 & 68.3 & 62.6 & 68.5 & NE & \\
\hline$p-038$ & AC6905 & | 01/13/94 & $110 \mid$ & OK & 5 & 68.5 & 61.7 & 68.5 & NE & \\
\hline$P-039$ & AC6915 & | 01/13/94 & 119 & ox & 5 & 67.9 & 64.3 & 68.0 & NE & \\
\hline
\end{tabular}


GOLDER CONSTRUCTION SERVICES, INC.

PRIMARY GEOMEMBRANE LINER DEPLOMMENT OBSERVATION SUMMARY

NON-DRAG OFF LANDFILL FACILITY

HANFORD NUCLEAR RESERVATION

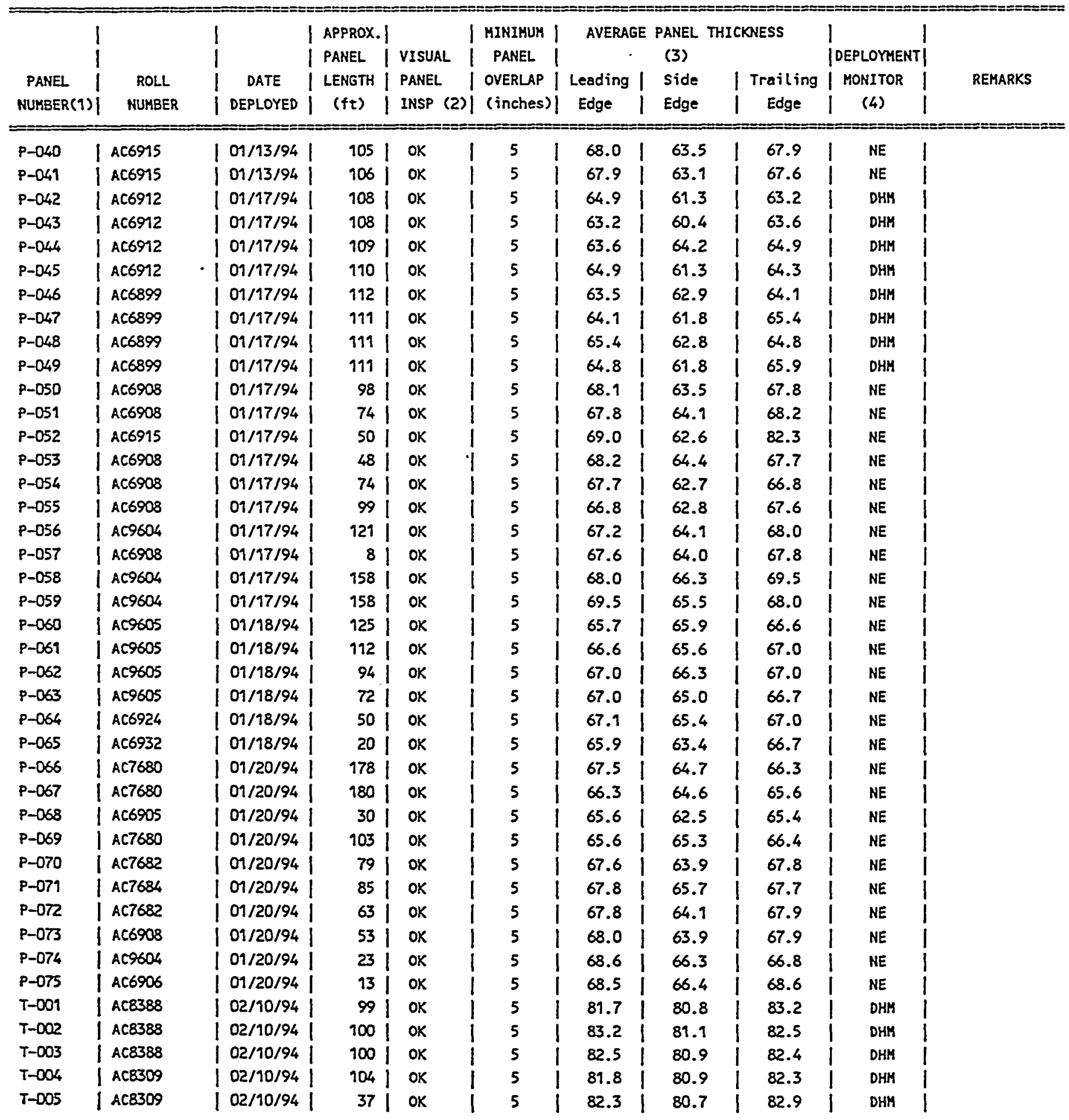


WHC-SD-W025-RPT-001, Rev. 0

GOLDER CONSTRUCTION SERVICES, INC.

933-1354

PRIMARY GEOMEMBRANE LINER DEPLOYMENT OBSERVATION SUMMARY

$05 / 11 / 94$

DEPARTMENT OF ENERGY

Page 3

NON-DRAG OFF LANDFILL FACILITY

HANFORD NUCLEAR RESERVATION

\begin{tabular}{|c|c|c|c|c|c|c|c|c|c|c|}
\hline & & & $\begin{array}{l}\text { APPROX. } \\
\text { PANEL }\end{array}$ & YISUAL & MINIMUM & AVERAGE & $\begin{array}{l}\text { PANEL T } \\
\text { (3) }\end{array}$ & :KNESS & $I_{\text {IOEPIOYMENT }}$ & \\
\hline $\begin{array}{c}\text { PANEL } \\
\text { MUMBER(1) }\end{array}$ & $\begin{array}{c}\text { ROLL } \\
\text { NUHBER }\end{array}$ & $\begin{array}{c}\text { DATE } \\
\text { DEPLOYED }\end{array}$ & $\begin{array}{l}\text { LENGTH } \\
\text { (ft) }\end{array}$ & $\begin{array}{l}\text { PANEL } \\
\text { INSP (2) }\end{array}$ & $\begin{array}{l}\text { OVERLAP | } \\
\text { (inches) }\end{array}$ & $\begin{array}{c}\text { Leading | } \\
\text { Edge }\end{array}$ & $\begin{array}{l}\text { Side } \\
\text { Edge }\end{array}$ & $\begin{array}{c}\text { Trailing } \\
\text { Edge }\end{array}$ & $\left\{\begin{array}{c}\text { MONITOR } \\
\text { (4) }\end{array}\right.$ & REMARKS \\
\hline$T-006$ & AC8388 & $02 / 10 / 94$ & 48 & OK & 5 & 82.7 & 81.2 & 82.6 & DHH & \\
\hline$T-\infty 07$ & AC8388 & $02 / 10 / 94$ & 19 & OK & 5 & 82.8 & 81.0 & 83.3 & DHM & \\
\hline
\end{tabular}

NOTES

(7) A panel number with a prefix of $L$ indicates a panel of HDPE geomembrane liner installed in the containment basin for leachate temporary storage tank.

A panel number with a prefix of $P$ indicates a panel of the primary HDPE geomembrane liner in the landfill.

A panel number with a prefix of $T$ indicates a panel of HOPE geomembrane liner installed in the sruck unloading area.

(2) OK indicates that the panel was free of visual defects when deployed in the landfill.

(3) Average sheet thickness based upon the average of five micrometer measurements made along each end and one side of the panel.

(4) See Held Observation Summary for monitor identification. 
WHC-SD-W025-RPT-001, Rev. 0

APPENDIX N-2

TRIAL SEAM OBSERVATION SUMMARY

$N-2$ 
GOLDER CONSTPUCTIOH SERVICES, INC.

PRIMARY GEOMEMBRANE LINER TRIAL SEAM OBERVATION SUMMARY DEPARTHENT OF ENERGY

NON-DRAG OFF LANDFILL FACILITY HANFORD NUCLEAR RESERVATION
933-1354

05/11/94

Page 1

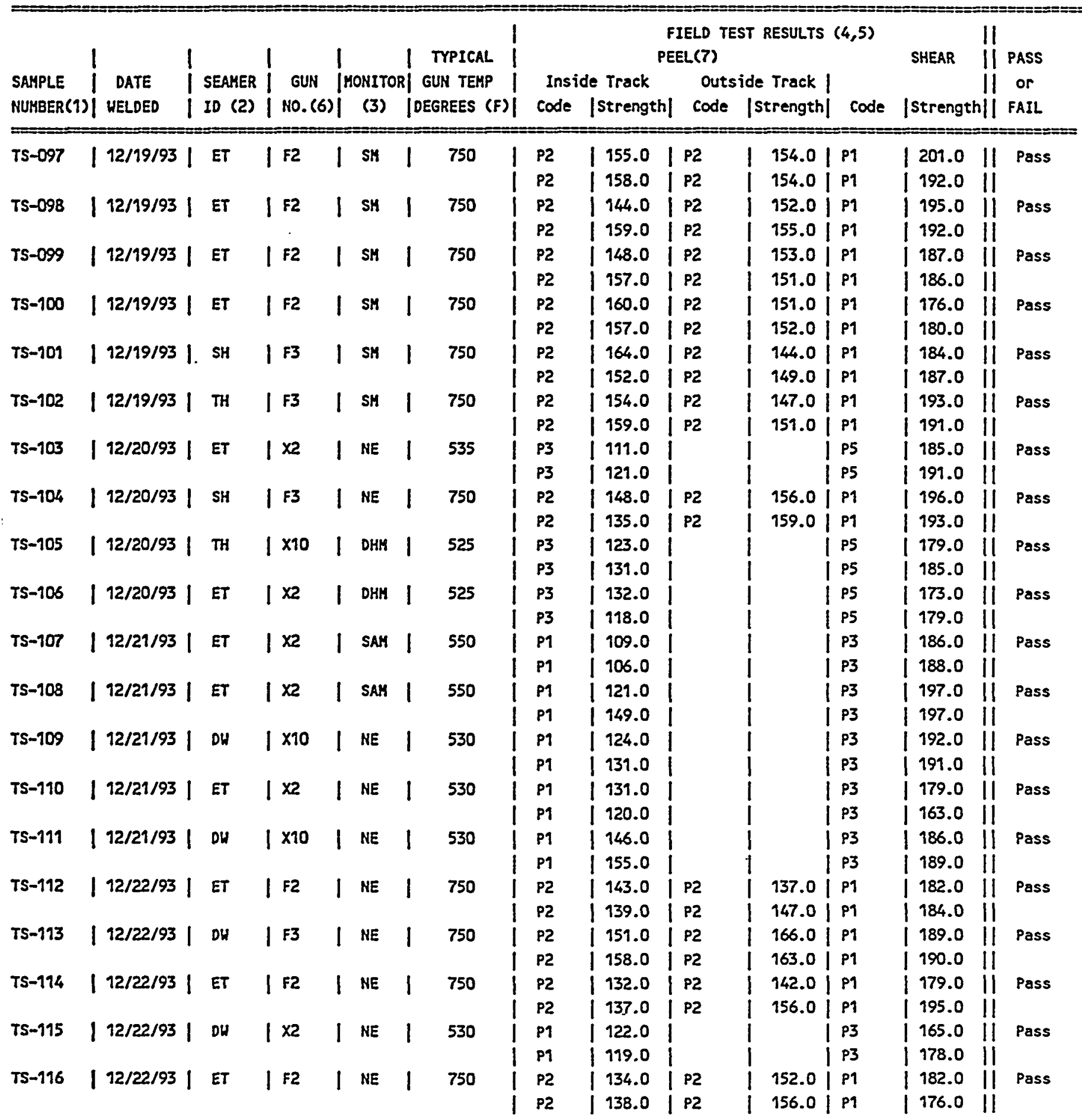


GOLDER CONSTRUCTION SERVICES, INC.

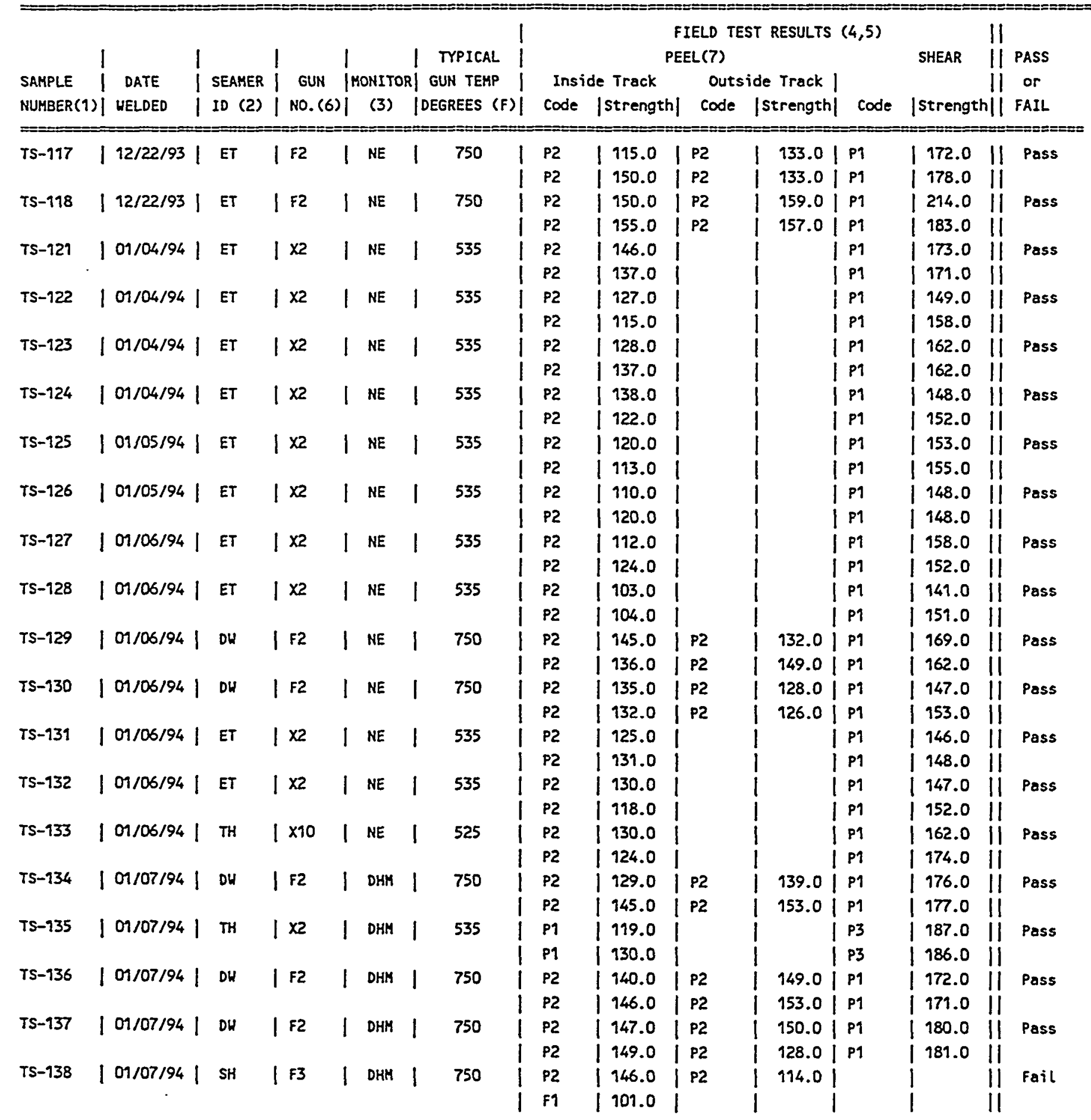


WHC-SD-W025-RPT-001, Rev. 0

GOLDER CONSTRUCTION SERVICES, INC.

933-1354

PRIMARY GEOMEMBRANE LINER TRIAL SEAM OBERVATION SUMMARY

$05 / 11 / 94$

DEPARTMENT OF ENERGY

NON-DRAG OFF LANDFILL FACILITY

Page 3

HANFORD NUCLEAR RESERVATION

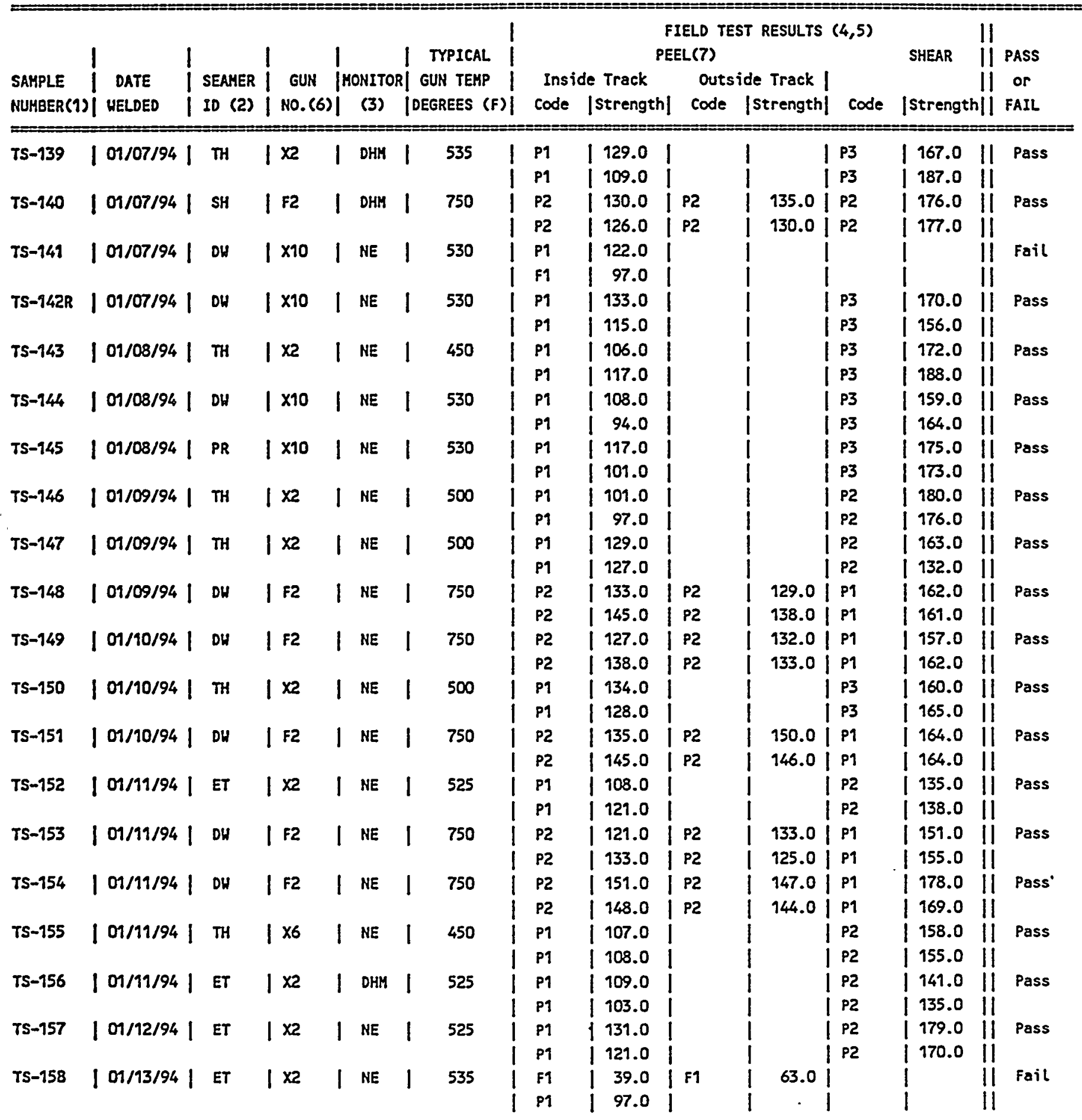


GOLDER CONSTRUCTION SERVICES, INC.

933-1354

PRIMARY GEOMEMBRANE LINER TRIAL SEAM OBERVATION SUMMARY

$05 / 11 / 94$

DEPARTMENT OF ENERGY

Page 4

NON-DRAG OFF LANDFILL FACILITY

HANFORD NUCLEAR RESERVATION

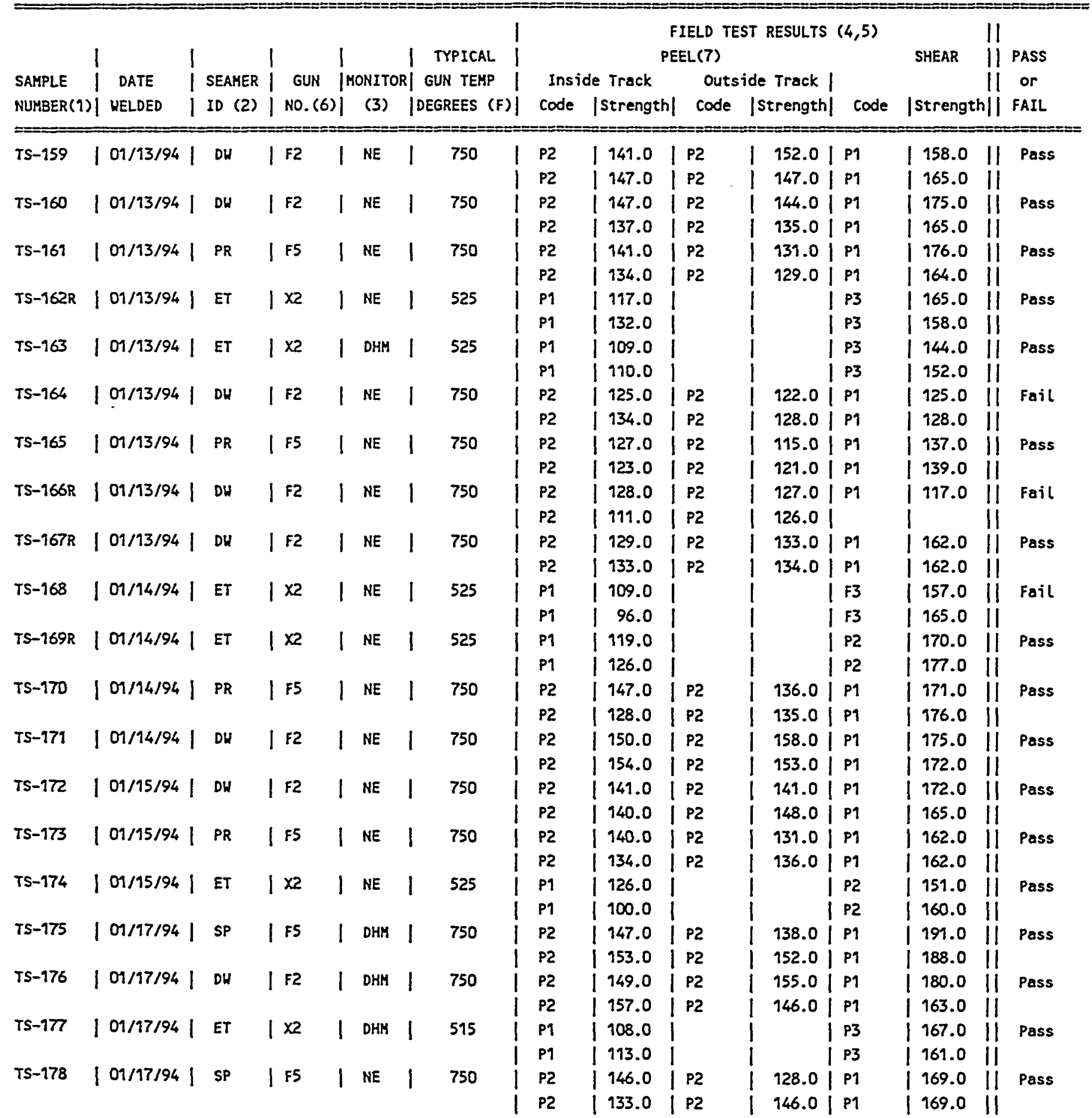


GOLDER CONSTRUCTION SERVICES, INC.

933-1354

PRIMARY GEOMEMBRANE LINER TRIAL SEAM OBERVATION SUMMARY

$05 / 11 / 94$

DEPARTHENT OF ENERGY

NON-DRAG OFF LANDFILL FACILITY

Page 5

HANFORD NUCLEAR RESERVATION

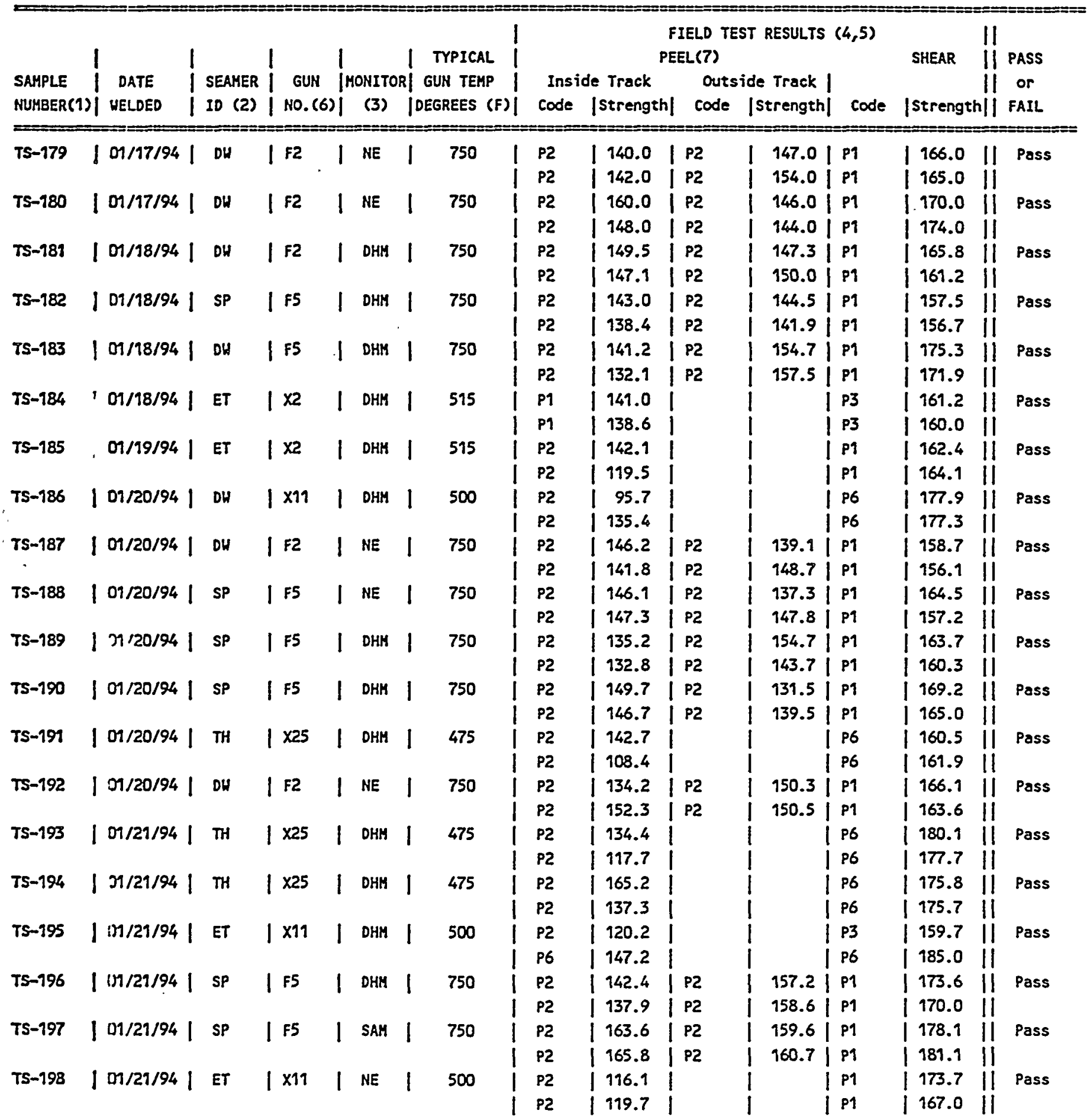


GOLDER CONSTRUCTION SERVICES, INC.

933- 1354

PRIMARY GEOMEMBRANE LINER TRIAL SEAM OBERVATION SUMMARY

$05 / 11 / 94$

DEPARTMENT OF ENERGY

Page 6

NON-DRAG OFF LANDFILL FACILITY

HANFORD NUCLEAR RESERVATION

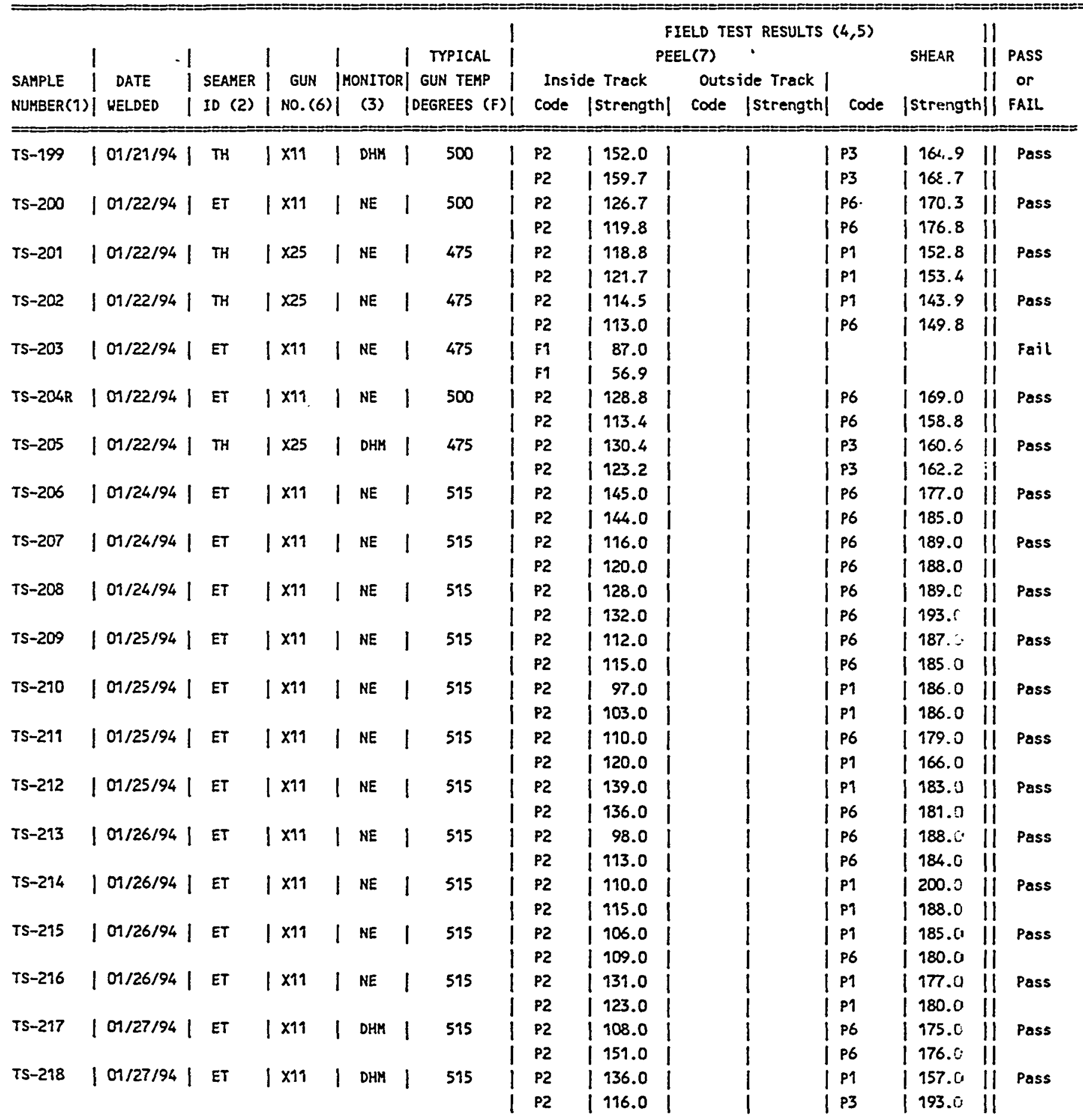


GOLDER CONSTRUCTION SERVICES, INC.

PRIHARY GEOMEMBRANE LINER TRIAL SEAM OBERVATION SUMMARY

DEPARTHENT OF ENERGY

NON-DRAG OFF LANDFILLL FACILITY

HANFORD NUCLEAR RESERVATION
933-1354

$05 / 11 / 94$

Page 7

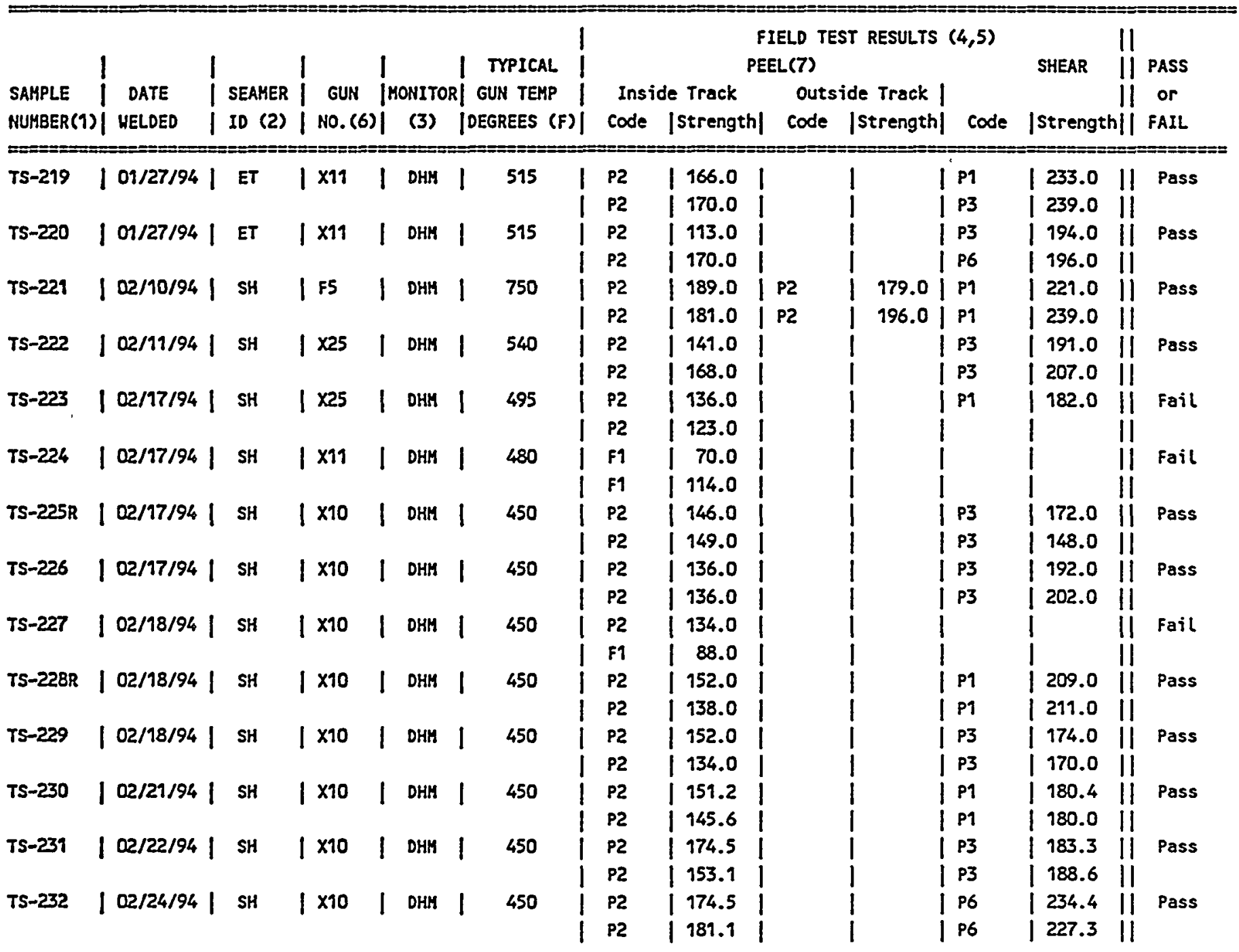


(1) Trial Weld Sample Numbers with an $R$ suffix are retest samples for a previously failed test performed by the same welder on the same date. e.g. TS- $162 R$ is a retest for failed test sample TS-158 weided by $\times 2$.

(2) See Weld inspection Summary for seamer identification.

(3) See Weld Inspection Summary for monitor identifcation.

(4) See Figures for illustrations of modes of passing or failing test codes.

(5) Strengths are reported in pounds per inch width.

(6) Gun Numbers with an $X$ prefix are extrusion welders. Gun Numbers with an $F$ prefix are fusion welders.

(7) Passing Trial Seams without an outside track test result are for extrusion trial seams. 
WHC-SD-W025-RPT-001, Rev. 0

\begin{abstract}
APPENDIX N-3
GEOMEMBRANE WELD OBSERVATION AND NON-DESTRUCTIVE TESTING SUMMARY
\end{abstract}

$N-3$ 
GOLDER CONSTRUCTION SERVICES, INC.

PRIMARY GEOMEMBRANE LINER UELD OBSERVATION AND NON-DESTRUCTIVE TESTING SUMMARY

933-1354

DEPARTMENT OF ENERGY

$05 / 11 / 94$

NON-DRAG OFF LANDFILLL FACILITY

Page 1 HANFORD NUCLEAR RESERVATION

\begin{tabular}{|c|c|c|c|c|c|c|c|c|c|c|c|}
\hline \multirow{3}{*}{$\begin{array}{c}\text { SEAH } \\
\text { NUMBER( } 1)\end{array}$} & \multicolumn{2}{|c|}{ PANEL NUMBERS } & \multirow{3}{*}{$\begin{array}{ll}1 & \\
1 & \\
1 & \text { DATE } \\
1 & \text { HELDED }\end{array}$} & \multirow{3}{*}{$\begin{array}{l}\mid \\
\mid \\
\mid \text { SEAMER } \\
\mid \text { ID(2) }\end{array}$} & \multirow{3}{*}{$\begin{array}{l}\text { GUN } \\
\text { No. }\end{array}$} & \multirow{3}{*}{$\mid \begin{array}{c}\text { TYPICAL } \\
\mid \text { GUN TEMP } \\
\text { DEEREES (F) }\end{array}$} & \multirow{3}{*}{$\begin{array}{l}\text {. DESTR. } \\
\text { TEST }\end{array}$} & \multirow{3}{*}{$\begin{array}{ll}\mid & \\
1 & \\
\text { |MONITOR) } \\
\mid & \text { (2) }\end{array}$} & \multicolumn{3}{|c|}{ NON-DESTRUCTIVE TEST } \\
\hline & I PANeL & 1 PANEL & & & & & & & & |MONITOR| & DEFECTS \\
\hline & $\# 1$ & $\not 2$ & & & & & & & DATE (3) & | (2) $\mid$ & \\
\hline$p-001 / 002$ & $P-001$ & | P-002 & | 12/19/93 & ET & $F 2$ & 750 & & SM & $12 / 20 / 93$ & DHM & \\
\hline$P-001 / 063$ & $P-001$ & | P-063 & $01 / 18 / 94$ & DH & F5 & 750 & & NE & $01 / 18 / 94$ & NE & \\
\hline$P-001 / 064$ & P-001 & | P-064 & | 01/18/94 & DH & F5 & 750 & & NE & $01 / 18 / 94$ & NE & \\
\hline$P-001 / 065$ & $P-001$ & | P-0.55 & | 01/18/94 & DH & F5 & 750 & & NE & $01 / 18 / 94$ & NE & \\
\hline$P-002 / 003$ & $P-002$ & | P-003 & | 12/19/93 & ET & F2 & 750 & DS-034 & SM & $12 / 20 / 93$ & DHM & \\
\hline$P-002 / 069$ & $P-002$ & $p-061$ & $01 / 20 / 94$ & DH & F2 & 750 & & DHM & $01 / 21 / 94$ & NE & \\
\hline$P-002 / 062$ & $p-002$ & $p-062$ & | $01 / 18 / 94$ & DH & F5 & 750 & & NE & $01 / 18 / 94$ & $\mathrm{NE}$ & \\
\hline$P-003 / 004$ & $P=003$ & | $p-\infty$ & | 12/19/93 & ET & F2 & 750 & & SH & $12 / 20 / 93$ & DHM & \\
\hline$P-003 / 061$ & $P-003$ & P-051 & $01 / 20 / 94$ & DH & $F 2$ & 750 & & DHM & $01 / 21 / 94$ & NE & \\
\hline$P-003 / P 06 A$ & $P-003$ & POSA & | 01/20/94 & DH & F2 & 750 & & DHA & $01 / 21 / 94$ & NE & \\
\hline P-004/005 & P-004 & $P-005$ & | 12/19/93 & ET & F2 & 750 & DS-035 & SM & $12 / 20 / 93$ & DHM & \\
\hline$P-004 / 073$ & $P-004$ & $P=073$ & | 01/21/94 & SP & F5 & 750 & & SAM & $11 / 11 / 11$ & SAM & 3 \\
\hline$P-004 / P 06 A$ & $P-004$ & PO\&A & $01 / 20 / 94$ & DH & F2 & 750 & & DHM & $01 / 25 / 94$ & $\mathrm{NE}$ & \\
\hline$P-005 / 006$ & $P-005$ & $P=006$ & | 12/19/93 & ET & F2 & 750 & & SH & $12 / 20 / 93$ & DHM & \\
\hline$P-005 / 073$ & $P-005$ & $P-073$ & | 01/21/94 & SP & F5 & 750 & & SAM & $01 / 25 / 94$ & NE & \\
\hline$P-006 / 007$ & $P-006$ & | $P=-\infty 7$ & | 12/19/93 & ET & F2 & 750 & & SH & $12 / 20 / 93$ & DHM & \\
\hline$P-006 / 073$ & $P-006$ & $P-073$ & | 01/21/94 & $\mathbf{S P}$ & F5 & 750 & & SAM & $01 / 25 / 94$ & NE & \\
\hline$P-007 / 008$ & $P-007$ & $P-008$ & | 12/19/93 & ET & E2 & 750 & DS-036 & SM & $01 / 25 / 94$ & DHM & \\
\hline$P-007 / 008$ & $P-\infty 07$ & $P-008$ & | 01/20/94 & DH & F2 & 750 & & DHM & $01 / 25 / 94$ & DHM & \\
\hline P-007/072 & $P-007$ & $P-072$ & | 01/21/94 & $\mathbf{S P}$ & F5 & 750 & & SAM & $01 / 25 / 94$ & NE & \\
\hline$P-007 / 073$ & $P-\infty 07$ & P -073 & | 01/21/94 & SP & F5 & 750 & & SAM & $01 / 25 / 94$ & $\mathrm{NE}$ & \\
\hline$P-008 / 009$ & $P-008$ & | p- -009 & | 12/19/93 & SH & F3 & 750 & & SM & $12 / 20 / 93$ & DHM & \\
\hline P-008/072 & $P-008$ & $P-072$ & | 01/21/94 & SP & F5 & 750 & DS-056 & SAM & $01 / 24 / 94$ & $N E$ & \\
\hline P-009/010 & $P-009$ & $P-010$ & i 12/19/93 & ET & F2 & 750 & & $S M$ & $12 / 20 / 93$ & DHM & \\
\hline$P-009 / 072$ & P-009 & $p-072$ & $01 / 21 / 94$ & SP & F5 & 750 & & SAM & $01 / 24 / 94$ & NE & \\
\hline P-010/011 & $P-010$ & p-011 & $01 / 20 / 94$ & DH & F2 & 750 & & DHY & $01 / 25 / 94$ & DHM & \\
\hline$P-010 / 011$ & $P=010$ & $P-011$ & $12 / 19 / 93$ & SH & 83 & 750 & DS-037 & SH & $01 / 25 / 94$ & DHM & \\
\hline P-010/079 & $P-010$ & $P-071$ & | 01/21/94 & SP & F5 & 750 & & SAM & $01 / 24 / 94$ & NE & \\
\hline$P-010 / 072$ & $P=010$ & $P-072$ & | 01/21/94 & SP & F5 & 750 & & SAM & $19 / 11 / 19$ & NE & 3 \\
\hline$P-011 / 012$ & P-011 & $P-012$ & | 12/20/93 & SH & F3 & 750 & & DHM & $12 / 20 / 93$ & DHM & \\
\hline P-011/071 & P-019 & $P-071$ & | 01/21/94 & sp & F5 & 750 & & SAM & $01 / 24 / 94$ & NE & \\
\hline$P-012 / 013$ & P-012 & $P-013$ & 12/22/93 & ET & F2 & 750 & 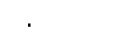 & NE & $12 / 22 / 93$ & NE & \\
\hline$P-012 / 071$ & P-012 & $P-071$ & $01 / 21 / 94$ & $\mathbf{S P}$ & F5 & 750 & & SAM & $01 / 24 / 94$ & $\mathrm{NE}$ & \\
\hline P-012/P08S & $P=012$ & P08s & $01 / 21 / 94$ & $\mathbf{S P}$ & F5 & 750 & & SAM & $01 / 25 / 94$ & NE & \\
\hline P-013/014 & $P-013$ & $P-014$ & $12 / 22 / 93$ & DH & 83 & 750 & & NE & $12 / 22 / 93$ & NE & \\
\hline$P-013 / 023$ & $P-013$ & $p-023$ & $01 / 21 / 94$ & SP & F5 & 750 & & SAH & $01 / 24 / 94$ & KEE & \\
\hline$P-013 / 024$ & $P=013$ & $P-024$ & | 01/21/94 & SP & F5 & 750 & & SAY & $01 / 24 / 94$ & NE & \\
\hline$P=013 / 071$ & $P-013$ & $P=071$ & | 01/21/94 & SP & F5 & 750 & & SAM & $01 / 21 / 94$ & NE & \\
\hline$P-014 / 015$ & P-074 & $P-015$ & | 12/22/93 & ET & $F 2$ & 750 & & NE & $12 / 22 / 93$ & NE & \\
\hline$P-014 / 022$ & P-014 & | P-022 & | 01/21/94 & I SP & F5 & 750 & & SAM & | 01/25/94 & I NE & \\
\hline
\end{tabular}


GOLDER CONSTRUCTION SERVICES, INC.

933-1354

PRIMARY GEOMEMBRANE LINER HELD OBSERVATION AND NON-DESTRUCTIVE TESTING SUMMARY

05/11/94

DEPARTMENT OF ENERGY

Page 2

NON-DRAG OFF LANDFILL FACILITY

HANFORD NUCLEAR RESERVATION

\begin{tabular}{|c|c|c|c|c|c|c|c|c|c|c|c|}
\hline \multirow{4}{*}{$\begin{array}{l}\text { SEAR } \\
\text { NUMBER(१) }\end{array}$} & \multicolumn{2}{|c|}{ PANEL NUMBERS } & 1 & \multirow{2}{*}{1} & & \multirow{2}{*}{ TYPICAL } & \multirow[b]{3}{*}{ DESTR. } & \multirow{4}{*}{$\begin{array}{l}1 \\
\mid \\
\mid \text { MONITOR| } \\
\mid \text { (2) }\end{array}$} & \multicolumn{3}{|c|}{ NON-DESTRUCTIVE TEST } \\
\hline & & $===$ & & & & & & & & $=$ & \\
\hline & PANEL & PANEL & DATE & |SEAMER| & GUN & I GUN TEMP & & & & [MONITOR] & DEFECTS \\
\hline & $\# 1$ & $\# 2$ & WELDED & $|I D(2)|$ & No. & |DEGREES (F)| & TEST & & DATE (3) & | (2) & \\
\hline$P-014 / 023$ & P-014 & $P-023$ & | 01/21/94 & SP & F5 & 750 & & SAM & $11 / 11 / 11$ & NE & 3 \\
\hline$P-015 / 016$ & $P-015$ & $p-016$ & | 12/22/93 & DH & F3 & 750 & & NE & $12 / 22 / 93$ & NE & \\
\hline$p-015 / 021$ & $P-015$ & $\mathrm{P}-021$ & | 01/07/94 & DH & F2 & 750 & & DHH & $11 / 11 / 11$ & NE & 3 \\
\hline$P-015 / 022$ & $P-015$ & P-022 & | 01/21/94 & SP & F5 & 750 & & SAM & $01 / 24 / 94$ & NE & \\
\hline$P-016 / 017$ & $P-016$ & $P-017$ & 1 12/22/93 & ET & F2 & 750 & DS-038 & NE & $11 / 11 / 11$ & NE & \\
\hline$p-016 / 029$ & P-016 & $p-021$ & | 01/07/94 & DH & F2 & 750 & & DHM & $01 / 15 / 94$ & DHM & \\
\hline P-016/P014 & $P-016$ & P01W & | 12/22/93 & ET & F2 & 750 & & NE & 01/05/94 & $\mathrm{NE}$ & \\
\hline P-016/P01W & $P-016$ & POIW & 01/05/94 & ET & $\times 2$ & 535 & & NE & $01 / 05 / 94$ & NE & \\
\hline$P-017 / 018$ & $P-017$ & $P-018$ & 1 12/22/93 & DH & F3 & 750 & & NE & $12 / 22 / 93$ & NE & \\
\hline$P-017 / 019$ & $P-017$ & P-019 & | 01/07/94 & DW & F2 & 750 & & DHM & $11 / 11 / 11$ & DHM & 3 \\
\hline$P-017 / 020$ & $P-017$ & $P=020$ & | 01/07/94 & DH & F2 & 750 & & DHM & $01 / 07 / 94$ & NE & \\
\hline P-017/P014 & P-017 & Po1w & $12 / 22 / 93$ & ET & F2 & 750 & & $\mathrm{NE}$ & $01 / 05 / 94$ & NE & \\
\hline P-018/019 & $P-018$ & P-019 & | 01/07/94 & DU & F2 & 750 & & DHM & $01 / 07 / 94$ & NE & \\
\hline$P-018 / 027$ & $P-018$ & $p-027$ & | 01/07/94 & DW & F2 & 750 & & DHM & $01 / 07 / 94$ & DHH & \\
\hline$P-019 / 020$ & P-019 & $P-020$ & | 01/06/94 & DW & F2 & 750 & & NE & $01 / 06 / 94$ & NE & \\
\hline$P-019 / 027$ & P-019 & $P=027$ & | 01/07/94 & DH & F2 & 750 & & DHM & 101/07/94 & DHM & \\
\hline P-020/029 & $P-020$ & $P-021$ & $01 / 06 / 94$ & DH & F2 & 750 & & NE & $01 / 06 / 94$ & $\mathrm{NE}$ & \\
\hline P-020/P01Y & $P-020$ & POIH & $01 / 07 / 94$ & DH & F2 & 750 & & DHM & $19 / 11 / 11$ & DHM & 3 \\
\hline P-021/022 & $P-021$ & P-022 & | 09/06/94 & DW & F2 & 750 & $D S-0<1$ & NE & $01 / 06 / 94$ & $\mathrm{NE}$ & \\
\hline$P-022 / 023$ & $P-022$ & $P=023$ & $01 / 06 / 94$ & DW & F2 & 750 & & NE & $01 / 06 / 94$ & NE & \\
\hline$p-023 / 024$ & $P-023$ & $P-024$ & $01 / 06 / 94$ & DH & F2 & 750 & & NE & | 01/06/94 & NE & \\
\hline$P-024 / 025$ & $\mathrm{P}-024$ & $P-025$ & $01 / 06 / 94$ & DW & F2 & 750 & DS-042 & NE & 01/24/94 & NE & \\
\hline$P-024 / 079$ & $P-024$ & $P-071$ & $01 / 20 / 94$ & SP & F5 & 750 & & DHM & $01 / 24 / 94$ & NE & \\
\hline$P-025 / 026$ & $P-025$ & $P-026$ & $01 / 06 / 94$ & DW & F2 & 750 & & NE & $01 / 06 / 94$ & NE & \\
\hline$p-025 / 070$ & $P-025$ & $P-070$ & $01 / 20 / 94$ & SP & F5 & 750 & & DHK & $01 / 24 / 94$ & NE & \\
\hline p-025/071 & $P-025$ & $P-071$ & $01 / 20 / 94$ & SP & F5 & 750 & & DHM & $01 / 24 / 94$ & NE & \\
\hline$p-026 / 028$ & $P-026$ & $P-028$ & $01 / 20 / 94$ & SP & F5 & 750 & & DHM & $01 / 20 / 94$ & NE & \\
\hline P-026/028 & $P-026$ & $P-028$ & $01 / 10 / 94$ & DH & F2 & 750 & DS- 043 & NE & $01 / 20 / 94$ & NE & \\
\hline P-026/070 & $P-026$ & P-OT0 & $01 / 20 / 94$ & $\mathbf{S P}$ & F5 & 750 & & DHM & $01 / 24 / 94$ & NE & \\
\hline P-026/P05B & $P-026$ & POSB & $07 / 20 / 94$ & SP & F5 & 750 & & DHM & $01 / 24 / 94$ & NE & \\
\hline P-028/029 & P-028 & $P-029$ & $01 / 10 / 94$ & DW & $F 2$ & 750 & & NE & $01 / 20 / 94$ & NE & \\
\hline P-028/066 & $P-028$ & P-066 & $01 / 20 / 94$ & $\mathbf{S P}$ & F5 & 750 & & DHM & $01 / 24 / 94$ & NE & \\
\hline$P-028 / 067$ & $P-028$ & $p-067$ & $01 / 20 / 94$ & SP & F5 & 750 & & DHM & $01 / 24 / 94$ & NE & \\
\hline P-028/P05B & $P-028$ & P05B & $01 / 20 / 94$ & SP & F5 & 750 & & DHM & $01 / 26 / 94$ & NE & \\
\hline$P-029 / 030$ & $p-029$ & $P-030$ & $01 / 10 / 94$ & DW & F2 & 750 & & $\mathrm{NE}$ & $01 / 10 / 94$ & NE & \\
\hline$P-029 / 037$ & $P-029$ & $P-037$ & $01 / 20 / 94$ & SP & F5 & 750 & & DHM & $11 / 11 / 11$ & NE & 3 \\
\hline$p-029 / 038$ & $P-029$ & $p-038$ & $01 / 20 / 94$ & SP & F5 & 750 & & DHM & $01 / 20 / 94$ & NE & \\
\hline$p-029 / 039$ & P-029 & $P-039$ & $01 / 20 / 94$ & $\mathbf{S P}$ & F5 & 750 & & DHM & $01 / 20 / 94$ & NE & \\
\hline$P-030 / 031$ & $P-030$ & $P-031$ & | 01/10/94 & DW & F2 & 750 & & NE & $01 / 10 / 94$ & NE & \\
\hline$P-030 / 036$ & $P-030$ & $P-036$ & | 01/13/94 & DH & F2 & 750 & & NE & $01 / 13 / 94$ & OHM & \\
\hline$P-030 / 037$ & $P-030$ & $P-037$ & $01 / 13 / 94$ & $\mathrm{OH}$ & if & 750 & & NE & $01 / 13 / 94$ & DHM & \\
\hline
\end{tabular}


GOLDER CONSTRUCTION SERVICES, INC.

PRIMARY GEOMEMBRANE LINER WELD OBSERVATION AND NON-DESTRUCTIVE TESTING SUMMARY

933-1354

DEPARTMENT OF ENERGY

$05 / 11 / 94$

NON-DRAG OFF LANDFILL FACILITY

Page 3

HANFORD NUCLEAR RESERYATION

\begin{tabular}{|c|c|c|c|c|c|c|c|c|c|c|c|}
\hline \multirow{2}{*}{$\begin{array}{l}\text { SEAN } \\
\text { NUMBER(1) }\end{array}$} & \multicolumn{2}{|c|}{ PANEL NUMBERS } & \multirow{2}{*}{$\begin{array}{l}\text { DATE } \\
\text { HELDED }\end{array}$} & \multirow{2}{*}{$\begin{array}{l}\mid \\
\mid \text { SEAKER } \mid \\
\mid \text { ID(2)| }\end{array}$} & \multirow{2}{*}{$\begin{array}{l}\text { GUN } \\
\text { NO. }\end{array}$} & \multirow{2}{*}{$\mid \begin{array}{l}\mid \\
\mid \text { TYPICAL } \\
\mid \text { GUN TEMP } \\
\text { |DEGREES (F)| }\end{array}$} & \multirow{2}{*}{$\begin{array}{l}\text { DESTR. } \\
\text { TEST }\end{array}$} & \multirow{2}{*}{$\begin{array}{l}\mid \\
\mid \text { MONITOR } \\
\mid \text { (2) }\end{array}$} & \multicolumn{3}{|c|}{ 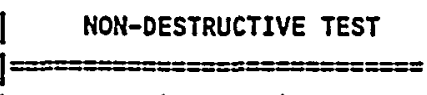 } \\
\hline & PANEL & PANEL & & & & & & & & |MONITOR| & DEFECTS \\
\hline$P-031 / 032$ & $P-039$ & P-032 & $01 / 10 / 94$ & DH & F2 & 750 & & NE & $01 / 10 / 94$ & NE & \\
\hline$P-031 / 036$ & $P-031$ & $P=036$ & $01 / 13 / 94$ & DH & F2 & 750 & & NE & $01 / 13 / 94$ & DHM & \\
\hline P-032/033 & $P-032$ & $P=033$ & $01 / 13 / 94$ & DH & F2 & 750 & & NE & $11 / 11 / 11$ & DHM & 3 \\
\hline$p-032 / 034$ & $P-032$ & $P-036$ & $01 / 13 / 94$ & DH & $\mathbf{F 2}$ & 750 & & NE & $01 / 13 / 94$ & DHM & \\
\hline$P=032 / P 02 X$ & $P-032$ & P02X & $01 / 13 / 94$ & DH & $\mathbf{F 2}$ & 750 & & NE & $01 / 13 / 94$ & DHM & \\
\hline$P-033 / 034$ & $P-033$ & $P-034$ & $01 / 13 / 94$ & DH & $\mathbf{F 2}$ & 750 & & NE & $01 / 13 / 94$ & NE & \\
\hline$P-033 / 035$ & $P-033$ & $P-035$ & $01 / 13 / 94$ & DH & $\mathbf{F 2}$ & 750 & DS-044 & NE & $01 / 13 / 94$ & NE & \\
\hline$P-037 / 038$ & $P-037$ & $P-038$ & $01 / 13 / 94$ & PR & F5 & 750 & & NE & $01 / 13 / 94$ & NE & \\
\hline P-038/039 & $P-038$ & $p-039$ & $01 / 13 / 94$ & PR & F5 & 750 & & NE & $01 / 14 / 94$ & NE & \\
\hline$P-039 / 040$ & $P-039$ & $P-040$ & $01 / 13 / 94$ & PR & F5 & 750 & DS-045 & NE & $01 / 14 / 94$ & NE & \\
\hline$p-039 / 066$ & $P-039$ & $p=066$ & $01 / 20 / 94$ & SP & F5 & 750 & & DHM & $01 / 24 / 94$ & NE & \\
\hline$P-040 / 041$ & $P=040$ & $P-041$ & $01 / 13 / 94$ & PR & F5 & 750 & & NE & $01 / 14 / 94$ & NE & \\
\hline$P-040 / 066$ & $P-040$ & $P-066$ & $01 / 20 / 94$ & $\mathbf{S P}$ & F5 & 750 & & DHM & $01 / 24 / 94$ & NE & \\
\hline$P-041 / 042$ & $P-041$ & $P-042$ & $01 / 17 / 94$ & SP & F5 & 750 & & DHM & $01 / 17 / 94$ & DHM & \\
\hline$p-041 / 066$ & $p-041$ & $P-066$ & $01 / 20 / 94$ & SP & f5 & 750 & & OHM & $01 / 24 / 94$ & NE & \\
\hline$P-042 / 043$ & $p-042$ & $P-043$ & $01 / 17 / 94$ & DH & F2 & 750 & & DHM & $01 / 25 / 94$ & DHM & \\
\hline$P-042 / 043$ & $P-042$ & $P-043$ & $01 / 20 / 94$ & SP & F5 & 750 & & DHM & $01 / 25 / 94$ & DHM & \\
\hline$p-042 / 066$ & $P-042$ & $P-066$ & $01 / 20 / 94$ & SP & F5 & 750 & DS -054 & DHM & $01 / 20 / 94$ & DHM & \\
\hline P-046/047 & $P-046$ & $P-047$ & $01 / 17 / 94$ & DH & F2 & 750 & & DHM & $01 / 20 / 94$ & OHM & \\
\hline$P-046 / 066$ & $P-046$ & $p-066$ & $01 / 20 / 94$ & SP & F5 & 750 & & DHM & $01 / 20 / 94$ & DHM & \\
\hline$p-047 / 048$ & $P-047$ & $P-048$ & $01 / 17 / 94$ & DH & F2 & 750 & · & DHM & $01 / 17 / 94$ & DHM & \\
\hline$P-047 / 066$ & $P-047$ & $p-066$ & $01 / 20 / 94$ & $\mathbf{S P}$ & F5 & 750 & & DHM & $01 / 20 / 94$ & DHM & \\
\hline$P-047 / 068$ & $P-047$ & $P-068$ & $01 / 20 / 94$ & SP & F5 & 750 & & DHH & $01 / 21 / 94$ & $\mathrm{NE}$ & \\
\hline$P-047 / P 068$ & $P=047$ & PO6B & $01 / 20 / 94$ & SP & F5 & 750 & & $\mathrm{DHM}$ & $01 / 21 / 94$ & NE & \\
\hline$P-048 / 049$ & $P=048$ & P-049 & $01 / 17 / 94$ & SP & F5 & 750 & & DHM & $01 / 17 / 94$ & DHM & \\
\hline$P=048 / 068$ & $P-048$ & $p-068$ & $01 / 20 / 94$ & $\mathbf{S P}$ & F5 & 750 & & DHM & $01 / 21 / 94$ & NE & \\
\hline$P=049 / 050$ & $P=049$ & $P=050$ & $01 / 17 / 94$ & SP & F5 & 750 & & $\mathrm{OHM}$ & $01 / 17 / 94$ & NE & \\
\hline$P-049 / 055$ & $P-049$ & P-055 & $01 / 20 / 94$ & SP & F5 & 750 & & DHM & $01 / 21 / 94$ & NE & \\
\hline P-049/056 & $P=049$ & $8-056$ & $01 / 20 / 94$ & I SP & F5 & 750 & & I DHм & | 01/21/94 & I NE & \\
\hline
\end{tabular}


GOLDER CONSTRUCTION SERVICES, INC.

933-1354

PRIMARY GEOMEMBRANE LINER HELD OBSERVATION AND NON-DESTRUCTIVE TESTING SUMMARY

05/11/94 DEPARTMENT OF ENERGY

NON-DRAG OFF LANDFILL FACILITY

Page 4 HANFORD NUCLEAR RESERVATION

\begin{tabular}{|c|c|c|c|c|c|c|c|c|c|c|c|}
\hline \multirow{3}{*}{$\begin{array}{l}\text { SEAM } \\
\text { NUKBER( }(1)\end{array}$} & \multicolumn{2}{|c|}{ PANEL NUMBERS } & \multirow{3}{*}{$\begin{array}{c}\text { DATE } \\
\text { WELDED }\end{array}$} & \multirow{3}{*}{$\mid \begin{array}{l}\mid \\
\mid \text { SEAMER } \mid \\
\mid \text { ID(2) }\end{array}$} & \multirow{3}{*}{$\begin{array}{l}\text { GUN } \\
\text { No. }\end{array}$} & \multirow{3}{*}{$\begin{array}{l}\mid \\
1 \text { TYPICAL } \\
\mid \text { GUH TEMP } \\
\text { |DEGREES (F) }\end{array}$} & \multirow{3}{*}{$\begin{array}{l}\text { DESTR. } \\
\text { TEST }\end{array}$} & \multirow{3}{*}{$\begin{array}{l}1 \\
j_{\text {MONITOR }} \\
1 \text { (2) }\end{array}$} & \multicolumn{3}{|c|}{ I NON-DESTRUCTIVE TEST } \\
\hline & PANEL & I PANEL & & & & & & & & MONITOF & DR| DEFECTS \\
\hline & $\# 1$ & $\# 2$ & & & & & & & $\mid$ DATE (3) $\mid$ & (2) & i \\
\hline$P-050 / 051$ & I P-050 & $\mid P-051$ & | 01/17/94 & DH & 52 & 750 & & DHM & | 09/17/94 | & NE & 1 \\
\hline$P-050 / 054$ & $P-050$ & | $P-054$ & | 01/17/94 & DH & F2 & 750 & & DHM & | 11/11/11 | & NE & 13 \\
\hline$P-050 / 055$ & P $P-050$ & P $P-055$ & | 01/17/94 & DH & F2 & 750 & . & DHM & | 01/21/94 | & NE & 1 \\
\hline$P-050 / 055$ & $P-050$ & p $p-055$ & | $01 / 20 / 94$ & SP & F5 & 750 & & DHM & $01 / 29 / 94$ & NE & i \\
\hline$P-051 / 052$ & | $P-051$ & $P-052$ & | 01/17/94 & DW & F2 & 750 & & DHM & | $01 / 17 / 94$ & NE & \\
\hline$P-051 / 053$ & $P-059$ & $P-053$ & | 01/17/94 & DH & F2 & 750 & & DHM & | 11/11/11 | & NE & 13 \\
\hline$P-051 / 054$ & $P-051$ & $1 P-054$ & | 01/17/94 & DW & F2 & 750 & & DHM & | 01/18/94 | & ME & \\
\hline$P-052 / 053$ & | P-052 & | P-053 & | 01/17/94 & DH & F2 & 750 & DS-050 & ОНM & | 01/18/94 | & NE & 1 \\
\hline$P-052 / 057$ & | P-052 & | $P-057$ & | 01/17/94 & $\mathrm{DH}$ & F2 & 750 & & DHM & | 01/18/94 | & NE & \\
\hline$P-053 / 054$ & | P-053 & I $P-054$ & | 01/17/94 & SP & F5 & 750 & & DHM & | 01/17/94 | & DHM & \\
\hline$P-053 / 057$ & | P-053 & | P-057 & $01 / 17 / 94$ & DH & F2 & 750 & & DHM & | 01/18/94 i & NE & \\
\hline$P-054 / 055$ & $P-054$ & | P-055 & | 01/17/94 & SP & F5 & 750 & DS-049 & DHM & $01 / 17 / 94$ & DHM & \\
\hline$P-055 / 056$ & $P-055$ & | P-056 & | 01/17/94 & DH & F2 & 750 & & DHM & $01 / 20 / 94$ & DHM & \\
\hline P-056/058 & $P-056$ & | $P-058$ & | 01/17/94 & SP & F5 & 750 & & $\mathrm{NE}$ & $01 / 20 / 94$ & NE & i \\
\hline$P-056 / 058$ & | P -056 & $1 \mathrm{P}-058$ & | 01/20/94 & DH & F2 & 750 & & NE & | 01/20/94 | & NE & 1 \\
\hline$P-056 / 068$ & | P-056 & | $p-068$ & 1 01/20/94 & SP & F5 & 750 & & ОНн & | 01/21/94 | & NE & i \\
\hline$P-058 / 059$ & | $P-058$ & | P-059 & | 01/20/94 & SP & F5 & 750 & & DHK & | 01/20/94 | & NE & $i$ \\
\hline P-058/059 & $p-058$ & | p-059 & | 01/17/94 & $S P$ & F5 & 750 & & DHM & $01 / 20 / 94$ & ME & i \\
\hline$P-058 / 066$ & $P-058$ & P-OS6 & | 01/20/94 & SP & F5 & 750 & & DHM & $01 / 25 / 94 \mid$ & NE & 1 \\
\hline$P-058 / 067$ & $P-058$ & $P-057$ & | 01/20/94 & SP & F5 & 750 & & DHM & 1 01/25/94 & NE & 1 \\
\hline$P-058 / 068$ & $P-058$ & P-068 & | 01/20/94 & $\mathbf{S P}$ & F5 & 750 & & DHM & 01/21/94 & NE & 1 \\
\hline$P-058 / P 06 B$ & P-058 & | PO6B & | 01/20/94 & SP & F5 & 750 & & DHH & $01 / 26 / 94$ & NE & 1 \\
\hline P-059/060 & P-059 & | $P-060$ & | 01/20/94 & DW & F2 & 750 & & DHM & $|01 / 21 / 94|$ & NE & 1 \\
\hline$P-059 / 060$ & $P-059$ & | P-060 & | 01/18/94 & DH & F2 & 750 & & NE & | 01/21/94 | & NE & 1 \\
\hline$P-059 / 057$ & $P-059$ & $\mid P-067$ & | 01/20/94 & $\mathbf{S P}$ & F5 & 750 & & DHM & | 09/25/94 | & NE & 1 \\
\hline$P-059 / 069$ & $P-059$ & | P-069 & | 01/20/94 & SP & F5 & 750 & & DHH & $|01 / 25 / 94|$ & NE & 1 \\
\hline P-059/074 & $P-059$ & | $P-074$ & | 01/20/94 & DH & F2 & 750 & & DHM & $|01 / 21 / 94|$ & ME & 1 \\
\hline P-059/075 & | P-059 & | P-075 & | $01 / 20 / 94$ & SP & F5 & 750 & & DHM & | 01/25/94 | & NE & i \\
\hline$P-060 / 051$ & | $P-0 \leq 0$ & | P-069 & $01 / 18 / 94$ & $\mathbf{S P}$ & F5 & 750 & & NE & | 01/24/94 i & NE & i \\
\hline$P-060 / 074$ & $p-060$ & | $p-074$ & 1 01/20/94 & $\mathbf{S P}$ & F5 & 750 & & DHM & | 01/21/94 | & NE & ! \\
\hline$P-060 / P 04 G$ & $P-050$ & | PO46 & | 01/20/94 & DH & F2 & 750 & & DHM & | $01 / 21 / 94$ & NE & 1 \\
\hline$P-060 / P 06 A$ & $P-060$ & I PO6A & | 01/20/94 & SP & F5 & 750 & DS-055 & DHM & 01/21/94 & ME & i \\
\hline$P-061 / 062$ & $\mid P-069$ & | P-062 & | 01/18/94 & SP & F5 & 750 & & NE & | 01/18/94 & NE & 1 \\
\hline$P-061 / P 04 G$ & | P-069 & | PO4G & | 01/20/94 & DW & F2 & 750 & & DHM & | 01/21/94 | & NE & 1 \\
\hline$P-062 / 063$ & | P-062 & $\mid P-063$ & | 01/18/94 & DW & F2 & 750 & & NE & | 01/18/94 & ME & 1 \\
\hline$P-063 / 064$ & $P-063$ & | P-064 & | 01/18/94 & $\mathbf{S P}$ & F5 & 750 & DS-051 & NE & | 01/18/94 & NE & 1 \\
\hline$P-064 / 065$ & P-OS4 & | $p-065$ & | $01 / 18 / 94$ & DH & F2 & .750 & & NE & | 01/18/94 | & ME & i \\
\hline$P-064 / P 04 \mathrm{~s}$ & $P-064$ & i PO4J & 1 01/18/94 & DH & F2 & 750 & & $\mathrm{NE}$ & $|07 / 18 / 94|$ & ME & 1 \\
\hline$P-065 / P 04 \mathrm{~J}$ & $P-065$ & I PO4J & $01 / 18 / 94$ & DW & F2 & 750 & & $\mathrm{NE}$ & | 01/18/94 & NE & \\
\hline$P-066 / 067$ & $18-066$ & $\mid P-067$ & 101/20/94 & DH & F2 & 750 & & DHM & $01 / 20 / 94 \mid$ & NE & 8 \\
\hline$P-066 / P 06 B$ & $1 P-066$ & | PO6B & | 01/20/94 & SP & F5 & 750 & & OHM & $|01 / 25 / 94|$ & NE & i \\
\hline
\end{tabular}


GOLDER CONSTRUCTION SERVICES, INC.

$933-1354$

PRIMARY GEOMEMBRANE LINER UELD OBSERVATION AND NON-DESTRUCTIVE TESTING SUMMARY

05/11/94

DEPARTMENT OF ENERGY

NON-DRAG OFF LANDFILL FACILITY

Page 5

HANFORD NUCLEAR RESERVATION

\begin{tabular}{|c|c|c|c|c|c|c|c|c|c|c|c|}
\hline \multirow[b]{2}{*}{$\begin{array}{l}\text { SEAH } \\
\text { NUMBER(1) }\end{array}$} & \multicolumn{2}{|c|}{ PANEL NUMBERS } & \multirow{2}{*}{$=\begin{array}{ll}1 & \\
1 & \\
1 & \text { DATE } \\
1 & \text { HELDED }\end{array}$} & \multirow{2}{*}{$\mid \begin{array}{l}1 \\
\mid \text { SEAMER } \\
\mid \text { IDC(2) }\end{array}$} & \multirow{2}{*}{$\begin{array}{l}\text { GUN } \\
\text { no. }\end{array}$} & \multirow{2}{*}{$\begin{array}{l}\mid \\
\mid \text { TYPICAL } \\
\mid \text { GUN TEMP } \\
\text { |DEGREES (F) }\end{array}$} & \multirow[b]{2}{*}{$\begin{array}{l}\text { DESTR. } \\
\text { TEST }\end{array}$} & \multirow{2}{*}{$\begin{array}{l}1 \\
1 \\
\mid \text { MONITOR } \\
\mid \text { (2) }\end{array}$} & \multicolumn{3}{|c|}{ NON-DESTRUCTIVE TEST } \\
\hline & {$\left[\begin{array}{c}\text { PANEL } \\
\# 1\end{array}\right.$} & $\begin{array}{c}\text { PANEL } \\
\# 2\end{array}$ & & & & & & & DATE (3) & 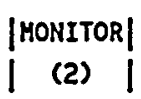 & DEFECTS \\
\hline P-067/069 & | P-067 & $P-069$ & | 01/20/94 & I OH & F2 & 750 & DS-053 & DHM & $01 / 20 / 94$ & NE & \\
\hline$P-067 / 070$ & | P-067 & $P-070$ & $01 / 20 / 94$ & OH & F2 & 750 & & DHM & $01 / 24 / 94$ & NE & \\
\hline$P-067 / P 058$ & $P-067$ & POSB & | 01/20/94 & SP & F5 & 750 & & DHM & $01 / 24 / 94$ & NE & \\
\hline$P-068 / P 068$ & P-068 & PO6B & $01 / 20 / 94$ & $\mathbf{S P}$ & F5 & 750 & & DHM & $01 / 21 / 94$ & NE & \\
\hline P-069/070 & | P-069 & $P-070$ & $01 / 20 / 94$ & DH & F2 & 750 & & DHM & $01 / 20 / 94$ & DHM & \\
\hline$P=069 / 071$ & | P-069 & P -071 & | 01/20/94 & DW & F2 & 750 & & DHM & $01 / 25 / 94$ & NE & \\
\hline$P=069 / 072$ & | P-069 & $P=072$ & $101 / 20 / 94$ & OH & F2 & 750 & & DHM & $01 / 25 / 94$ & NE & \\
\hline$P-069 / 073$ & P P-069 & $P-073$ & $01 / 20 / 94$ & OH & F2 & 750 & & DHM & $01 / 25 / 94$ & NE & \\
\hline P-069/075 & | P-069 & $P-075$ & $01 / 20 / 94$ & SP & F5 & 750 & & DHM & $01 / 25 / 94$ & NE & \\
\hline P-070/079 & | P-070 & $P=071$ & $01 / 20 / 94$ & OH & F2 & 750 & & DHM & $01 / 20 / 94$ & NE & \\
\hline$P-070 / P 05 B$ & $P-070$ & PO5B & $01 / 22 / 94$ & $T H$ & $x 25$ & 475 & & NE & $01 / 26 / 94$ & NE & \\
\hline P-071/072 & p-079 & P-072 & $01 / 20 / 94$ & DH & F2 & 750 & & DHM & $01 / 24 / 94$ & NE & \\
\hline$P-072 / 073$ & P-072 & $P-073$ & $01 / 20 / 94$ & SP & F5 & 750 & & DHM & $01 / 25 / 94$ & NE & \\
\hline$P-073 / 074$ & p-073 & P-074 & $01 / 20 / 94$ & SP & F5 & 750 & & DHM & $01 / 21 / 94$ & NE & \\
\hline P-073/075 & $P-073$ & $P-075$ & $01 / 20 / 94$ & SP & F5 & 750 & & DHM & $01 / 21 / 94$ & NE & \\
\hline$P-073 / P 06 A$ & $P-073$ & POSA & $01 / 20 / 94$ & SP & F5 & 750 & & DHM & $01 / 21 / 94$ & NE & \\
\hline P-074/075 & P-074 & $P-075$ & $01 / 20 / 94$ & SP & F5 & 750 & & DHM & $01 / 21 / 94$ & NE & \\
\hline P-074/PDSA & P-074 & POSA & $01 / 20 / 94$ & SP & F5 & 750 & & DHM & $01 / 21 / 94$ & NE & \\
\hline$T-001 / 002$ & | T-001 & $T-002$ & $02 / 10 / 94$ & SH & F5 & 750 & & DHM & $02 / 10 / 94$ & DHM & \\
\hline$T-002 / 003$ & | T-002 & $T-\infty 03$ & $02 / 10 / 94$ & SH & F5 & 750 & & DHM & $02 / 10 / 94$ & DHM & \\
\hline$T-003 / 004$ & | T-003 & $T=004$ & 102/90/94 & SH & F5 & 750 & & DHM & $02 / 10 / 94$ & DHM & \\
\hline$T-004 / 005$ & | T-004 & $T-005$ & | 02/10/94 & SH & F5 & 750 & DS-060 & DHM & $02 / 10 / 94$ & DHM & \\
\hline$T-004 / 006$ & | $T-004$ & $T-006$ & | 02/10/94 & SH & F5 & 750 & & DHM & $02 / 10 / 94$ & DHM & \\
\hline$T-004 / 007$ & I T-004 & $T-\infty 07$ & | 02/10/94 & SH & F5 & 750 & & DHM & $02 / 10 / 94$ & DHM & \\
\hline$T-005 / 006$ & | T-005 & $T-006$ & | 02/10/94 & SH & F5 & 750 & & DHM & $02 / 10 / 94$ & DHM & \\
\hline$T-006 / 007$ & T-006 & $T-007$ & $02 / 10 / 94$ & SH & F5 & 750 & & DHM & $02 / 10 / 94$ & DHM & \\
\hline$T P-001 / 002$ & | T-001 & P-002 & | 02/18/94 & $\mathrm{SH}$ & $\times 10$ & 450 & & I DHM & $02 / 21 / 94$ & DHM & \\
\hline$T P-001 / 002$ & | T-001 & $P-002$ & | 02/22/94 & I SH & $\times 10$ & 450 & & I DHM I & | 02/21/94 & I DHM I & \\
\hline
\end{tabular}


(1) A seam number with a prefix of $P$ indicates a seam between panels of the primary HDPE liner.

A seam number with a prefix of $T$ indicates a seam between panels of the HDPE Liner in the truck unloading area.

A seam number with a prefix of TP indicates a tie-in seam between truck unloading area HDPE liner panels and and Primary HDPE liner panels.

(2) Inspector Identification:

DHM - David H. Mathis

SM - Steve Milka

SAM - Scott A. Matthees

NE - Nancy Evans
Seamer Identification:

$\begin{array}{ll}\text { ET - Erwin Tsosie } & \text { DW - Dwight Wilson } \\ \text { AB - Arnold Barker } & \text { TH - Tim Harding } \\ \text { SH - Steve Hevitt } & \text { RA - Raymond Ashley } \\ \text { GH - Grant Martin } & \text { SA - Simon Ashley } \\ \text { PR - Paul Rhodes } & \text { SP - Scott Prydatko }\end{array}$

(3) Seams with a Non-Destructive Test Date of $11 / 11 / 11$ indicates that the seam was capped, so non-destructive testing of seam was not required. The cap that covered the seam was non-destructively tested. 
WHC-SD-W025-RPT-001, Rev. 0

\section{APPENDIX N-4 \\ DESTRUCTIVE TEST OBSERVATION SUMMARY}

$\mathrm{N}-4$ 
GOLDER CONSTRUCTION SERVICES, INC.

933-1354

PRIHARY GEOMEHBRANE LINER DESTRUCTIVE TEST OBSERVATION SUMMARY

05/11/94

DEPARTMENT OF ENERGY

Page 1

NON-DRAG OFF LANDFILL FACILITY

HANFORD NUCLEAR RESERVATION

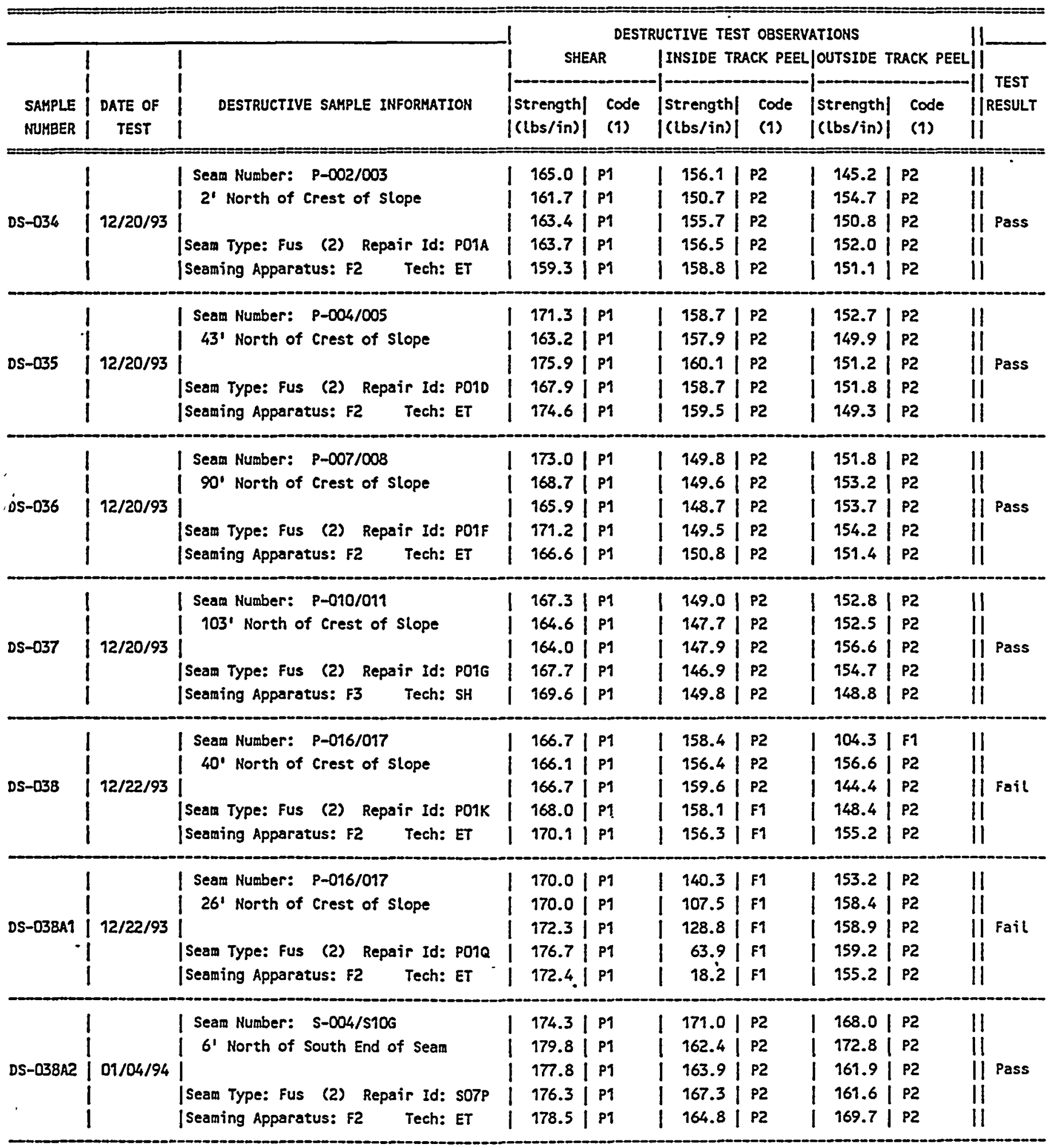


GOLDER CONSTRUCTION SERVICES, INC.

933-1354

PRIMARY GEOMEMBRANE LINER DESTRUCTIVE TEST OBSERVATION SUMMARY

$05 / 19 / 94$

DEPARTMENT OF ENERGY

Page 2

NON-DRAG OFF LANDFILL FACILITY

HANFORD NUCLEAR RESERVATION

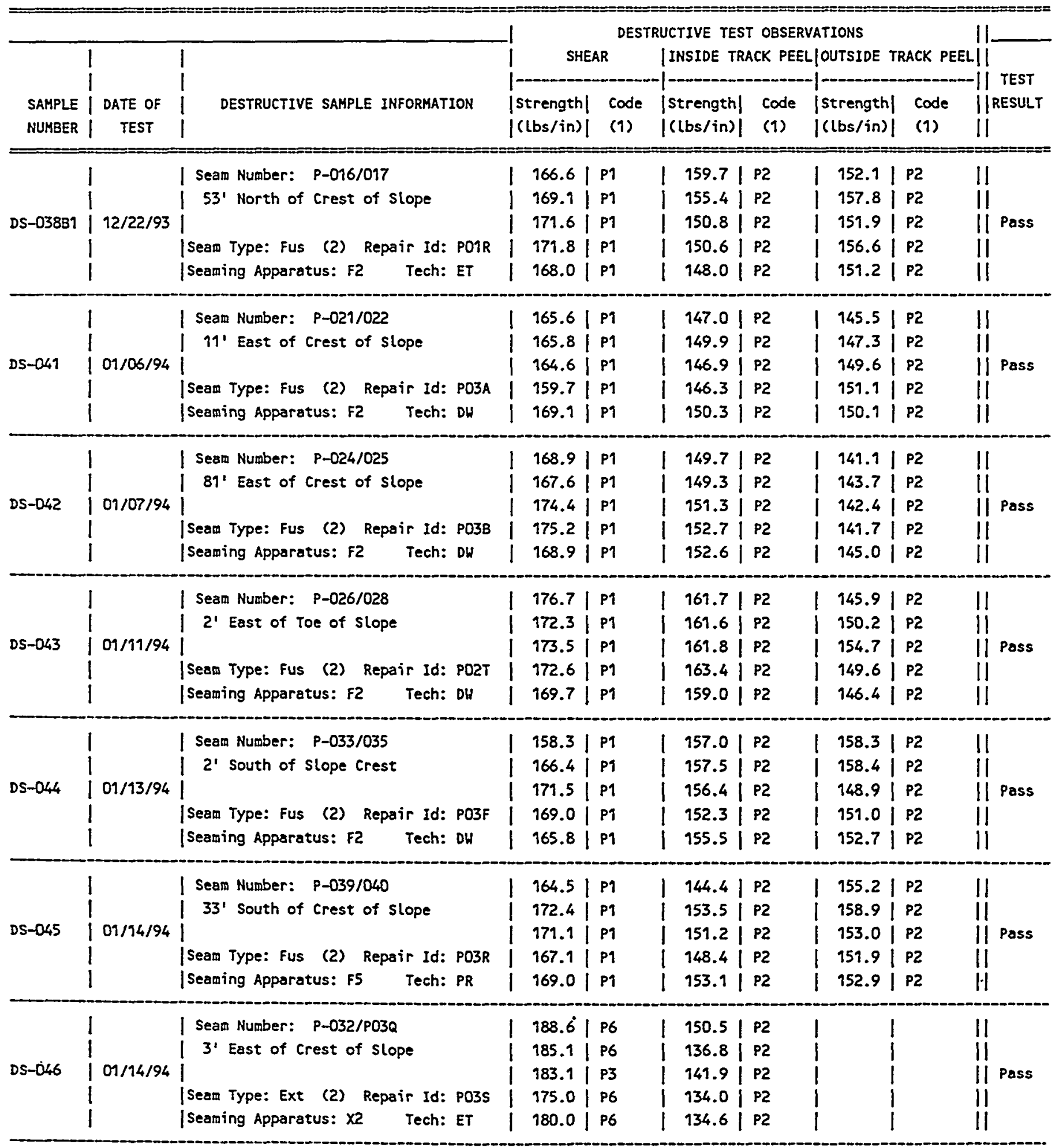


GOLDER CONSTRUCTION SERVICES, INC.

933-1354

PRIMARY GEOMEMBRANE LINER DESTRUCTIVE TEST OBSERVATION SUMMARY

$05 / 11 / 94$

DEPARTMENT OF ENERGY

NON-DRAG OFF LANDFILL FACILITY

Page 3

HANFORD NUCLEAR RESERVATION

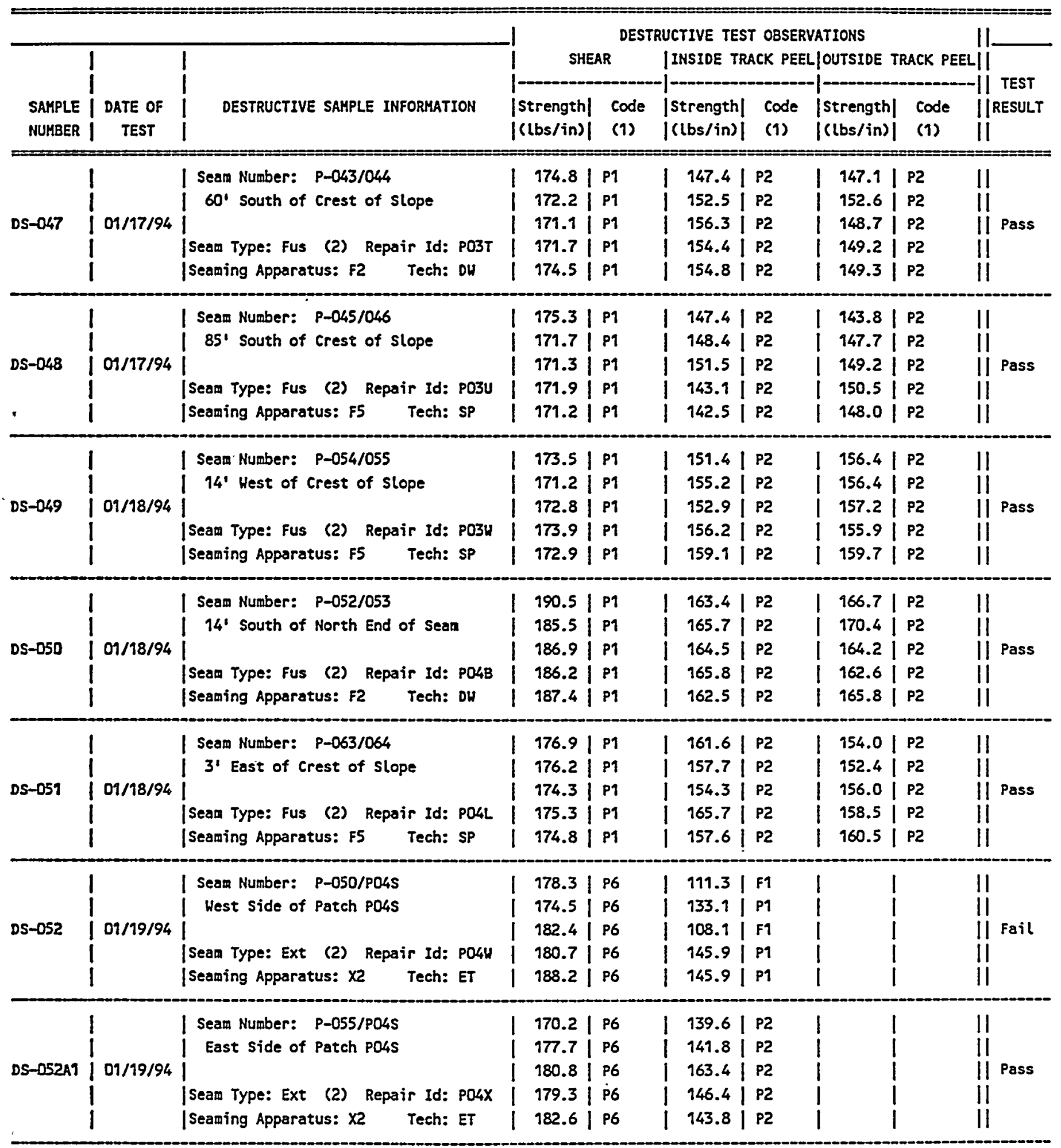


GOLDER CONSTRUCTION SERVICES, INC.

933-1354

PRIMARY GEOMEMBRANE LINER DESTRUCTIVE TEST OBSERVATION SUMMARY

05/11/94

DEPARTMENT OF ENERGY

Page 4

NON-DRAG OFF LANDFILL FACILITY

HANFORD NUCLEAR RESERVATION

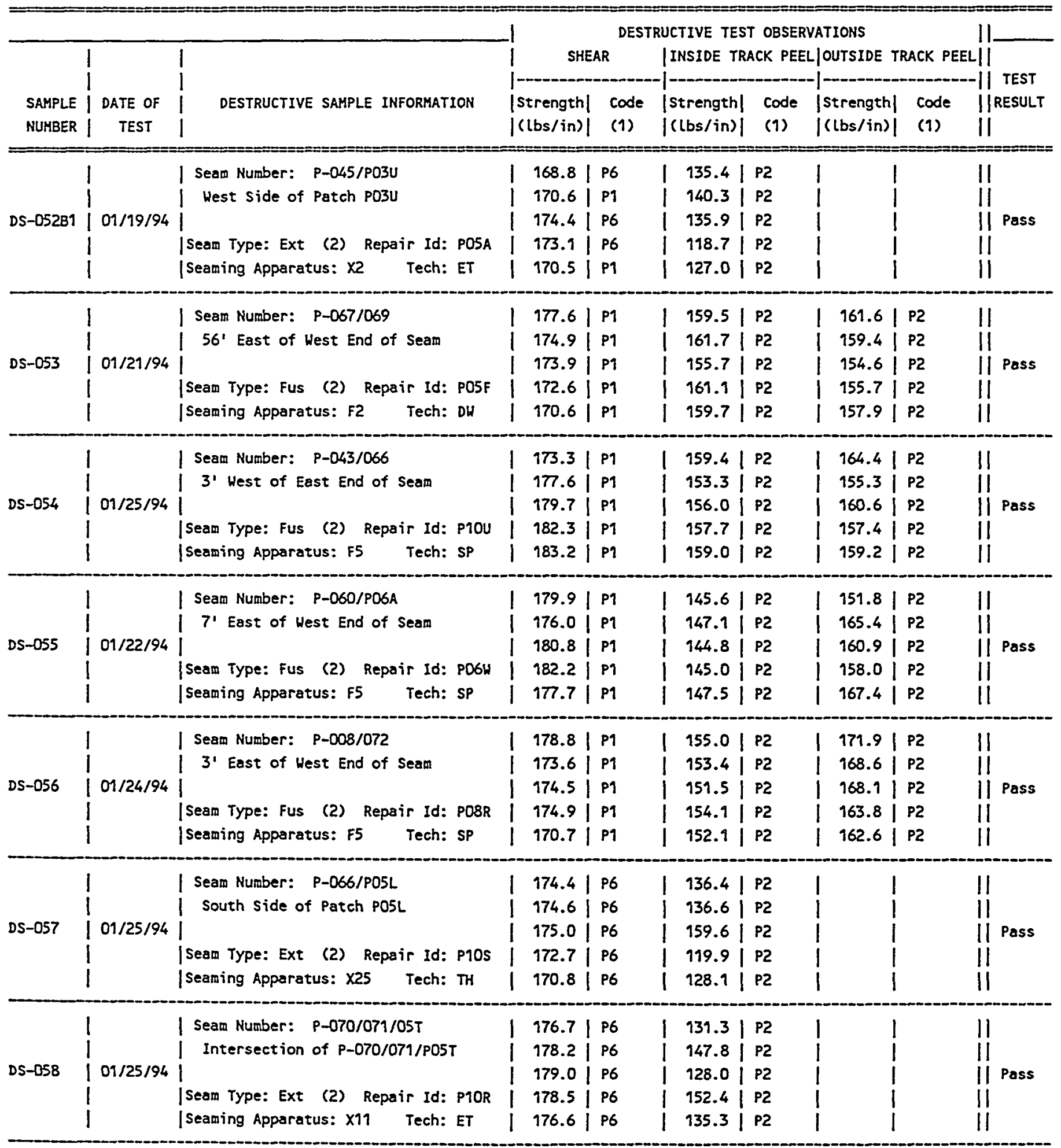


GOLDER CONSTRUCTION SERVICES, INC. PRIMARY GEOMEMBRANE LINER OESTRUCTIVE TEST OBSERVATION SUMMARY DEPARTMENT OF ENERGY NON-DRAG OFF LANDFILL FACILITY

933-1354 $05 / 11 / 94$ Page 5 HANFORD NUCLEAR RESERVATION

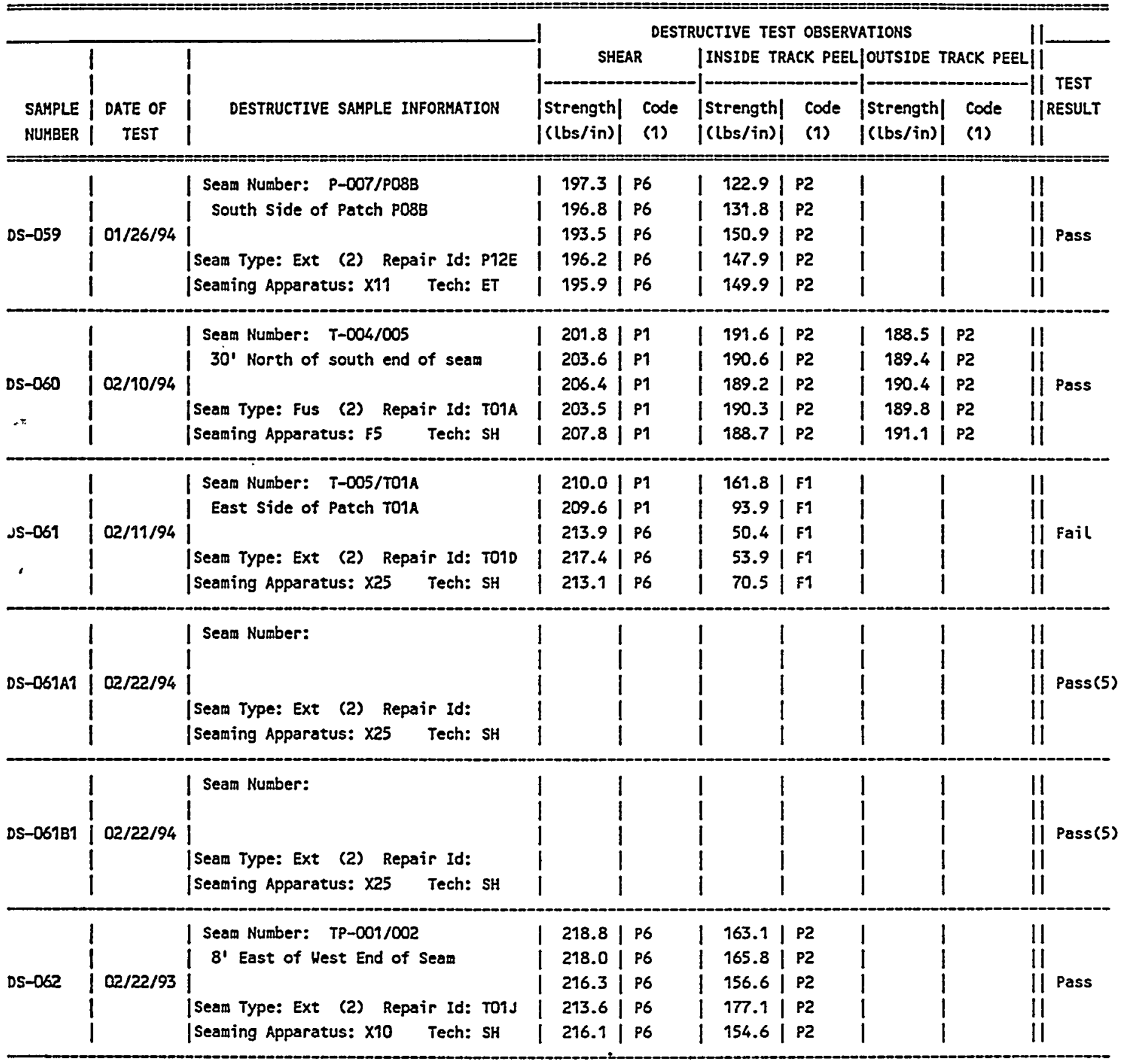




\section{WHC-SD-W025-RPT-001, Rev. 0}

NOTES

1) See Figures for illustration of mode of failure codes.

2) Fus - Fusion Seam

Ext - Extrusion Seam

3) All deficient repairs welded by extrusion welder X25 in the truck unloading area were capped. Destructive test DS-061 was performed on a repair welded by $X 25$, and DS-061 failed. Because all velding completed by $X 25$ in the truck unloading area was capped, "dummy" destructive test numbers DS-OS1A1 and DS-0S1B1 were designated to indicate that all deficient welding performed by $X_{25}$ was capped, and DS-061 was bracketed by the capping. 
WHC-SD-W025-RPT-001, Rev. 0

APPENDIX N-5

GEOMEMBRANE REPAIR SUMMARY

$N-5$ 
GOLDER CONSTRUCTION SERVICES, INC.

933-1356

PRIMARY GEOHEMBRANE LINER REPAIR SUMMARY

05/11/94

DEPARTMENT OF ENERGY

Page 1

NON-DRAG OFF LANDFILL FACILITY

HANFORD NUCLEAR RESERVATION

\begin{tabular}{|c|c|c|c|c|c|c|c|c|}
\hline $\begin{array}{l}\text { REPAIR } \\
\text { NUMBER } \\
\text { (1) }\end{array}$ & $\begin{array}{l}\text { DATE } \\
\text { LOCATED }\end{array}$ & $\begin{array}{l}\text { SEAH } \\
\text { or } \\
\text { PANEL }\end{array}$ & $\begin{array}{l}\mid \\
\text { |APPROXIMATE REPAIR LOCATION| }\end{array}$ & $\begin{array}{l}\text { DATE } \\
\text { REPAIRED } \\
\text { (2) }\end{array}$ & $\begin{array}{l}\text { DATE } \\
\text { TESTED } \\
\text { (2) }\end{array}$ & $\left\{\begin{array}{r}\text { SIZE } \\
(f t) \\
(3)\end{array}\right.$ & $\begin{array}{l}\text { IRE } \\
\text { IMC } \\
1\end{array}$ & $\begin{array}{l}\text { EPAIR } \\
\text { DNITOR } \\
(4)\end{array}$ \\
\hline PO1A & | 12/19/93 & $P-002 / 003$ & $\begin{array}{l}\text { I } 2 \text { North of Crest of } \\
\text { slope }\end{array}$ & $12 / 20 / 93$ & $12 / 20 / 93$ & $12 \times 5$ & 1 & DHM \\
\hline PDIB & | 12/19/93 & $P-003 / 004$ & $\begin{array}{l}\text { I 40' North of Crest of } \\
\text { slope }\end{array}$ & $12 / 20 / 93$ & $12 / 20 / 93$ & $12 \times 4$ & l & NE \\
\hline POIC & | 12/19/93 & $P-003 / 004$ & $\begin{array}{l}\text { I South End of Seam to 7' } \\
\text { North of Crest of Slope }\end{array}$ & $12 / 20 / 93$ & $12 / 20 / 93$ & $13 \times 22$ & 1 & NE \\
\hline PO10 & | 12/19/93 & $P-004 / 005$ & $\begin{array}{l}\text { I } 43^{*} \text { North of Crest of } \\
\text { Slope }\end{array}$ & $12 / 20 / 93$ & $12 / 20 / 93$ & $12 \times 5$ & 1 & NE \\
\hline PD1E & | 12/19/93 & $P-005 / 006$ & $\begin{array}{l}\text { I South End of Seam to } \\
\text { Anchor Trench Crest }\end{array}$ & $12 / 20 / 93$ & $01 / 04 / 94$ & $12 \times 12$ & 1 & NE \\
\hline POIF & | 12/19/93 & $p-007 / 008$ & $\begin{array}{l}\text { I 90' North of Crest of } \\
\text { slope }\end{array}$ & $12 / 20 / 93$ & $12 / 20 / 93$ & $12 \times 5$ & l & NE \\
\hline PO1G & | 12/19/93 & $p-010 / 011$ & $\begin{array}{l}\text { I 103' North of Crest of } \\
\text { slope }\end{array}$ & $12 / 20 / 93$ & $12 / 20 / 93$ & $12 \times 5$ & 1 & DHM \\
\hline POIH & | 12/20/93 & $P-007 / 008$ & $\begin{array}{l}\text { I 63' North of Crest of } \\
\text { slope }\end{array}$ & $12 / 20 / 93$ & $12 / 20 / 93$ & $12 \times 3$ & 1 & OHM \\
\hline PoIs & | 12/20/93 & $P-005 / 006$ & I Crest of Anchor Trench & $12 / 20 / 93$ & $01 / 04 / 94$ & $11 \times 1$ & l & ME \\
\hline POIK & | 12/22/93 & $P=016 / 017$ & $\begin{array}{l}\text { I 40' North of Crest of } \\
\text { Slope (Capped by Po1u) }\end{array}$ & $12 / 22 / 93$ & $19 / 11 / 11$ & $12 \times 5$ & | & NE \\
\hline POIL & | 12/22/93 & $P-018$ & $\begin{array}{l}\text { I11' South of Crest of } \\
\text { slope }\end{array}$ & $12 / 22 / 93$ & $01 / 07 / 94$ & $15 \times 24$ & I & NE \\
\hline POIM & | 12/22/93 & $P-017 / 018$ & $\begin{array}{l}\text { I 11' South of crest of } \\
\text { slope to crest of slope }\end{array}$ & $12 / 22 / 93$ & $01 / 05 / 94$ & $12 \times 14$ & 1 & NE \\
\hline PDIM & $101 / 07 / 93$ & P-018/019 & $\begin{array}{l}\text { I At Intersection with } \\
P-027\end{array}$ & $01 / 07 / 94$ & $01 / 12 / 94$ & $12 \times 2$ & 1 & ME \\
\hline POIP & | 12/22/93 & $P-016 / 017$ & $\begin{array}{l}\text { I Crest of slope (Capped by } \\
\text { poIw) }\end{array}$ & 11/19/11 & $11 / 11 / 11$ & | & 1 & NE \\
\hline Pola & | 12/22/93 & $P-016 / 017$ & $\begin{array}{l}\text { I 26'North of Crest of } \\
\text { slope (Capped by Po1w) }\end{array}$ & $11 / 11 / 11$ & $11 / 11 / 11$ & & 1 & NE \\
\hline POIR & | 12/22/93 & $P-016 / 017$ & $\begin{array}{l}\text { I 53' North of Crest of } \\
\text { slope (Capped by P01W) }\end{array}$ & $11 / 11 / 11$ & $11 / 11 / 11$ & & | & NE \\
\hline P015 & | 12/22/93 & $P-016 / 017$ & $\begin{array}{l}\text { I } 13^{\prime} \text { North of Crest of } \\
\text { slope (Capped by P01W) }\end{array}$ & $11 / 11 / 11$ & $11 / 11 / 11$ & & I & NE \\
\hline PO1T & $12 / 22 / 93$ & $p-016 / 017$ & $\begin{array}{l}\text { I } 16^{\circ} \text { south of crest of } \\
\text { stope (Capped by po1w) }\end{array}$ & $11 / 11 / 11$ & $11 / 11 / 11$ & & 1 & NE \\
\hline PO1U & | 12/22/93. & $P-017 / 018$ & $\begin{array}{l}\text { I } 2^{t} \text { North of Crest of } \\
\text { Slope }\end{array}$ & $12 / 22 / 93$ & $01 / 05 / 94$ & $1 \times 1$ & 1 & NE \\
\hline POTu & | 12/22/93 & P-016/017 & $\begin{array}{l}\text { I South End of Seam to } \\
\text { North End of Seam }\end{array}$ & $01 / 05 / 94$ & $01 / 05 / 94$ & $3 \times 83$ & 1 & NE \\
\hline POIX & | 12/22/93 & P-016/P01W & $\begin{array}{l}\text { I } 40^{\prime} \text { North of Crest of } \\
\text { Slope }\end{array}$ & $01 / 05 / 94$ & $01 / 05 / 94$ & $12 \times 8$ & 1 & NE \\
\hline
\end{tabular}


GOLDER CONSTRUCTION SERVICES, INC.

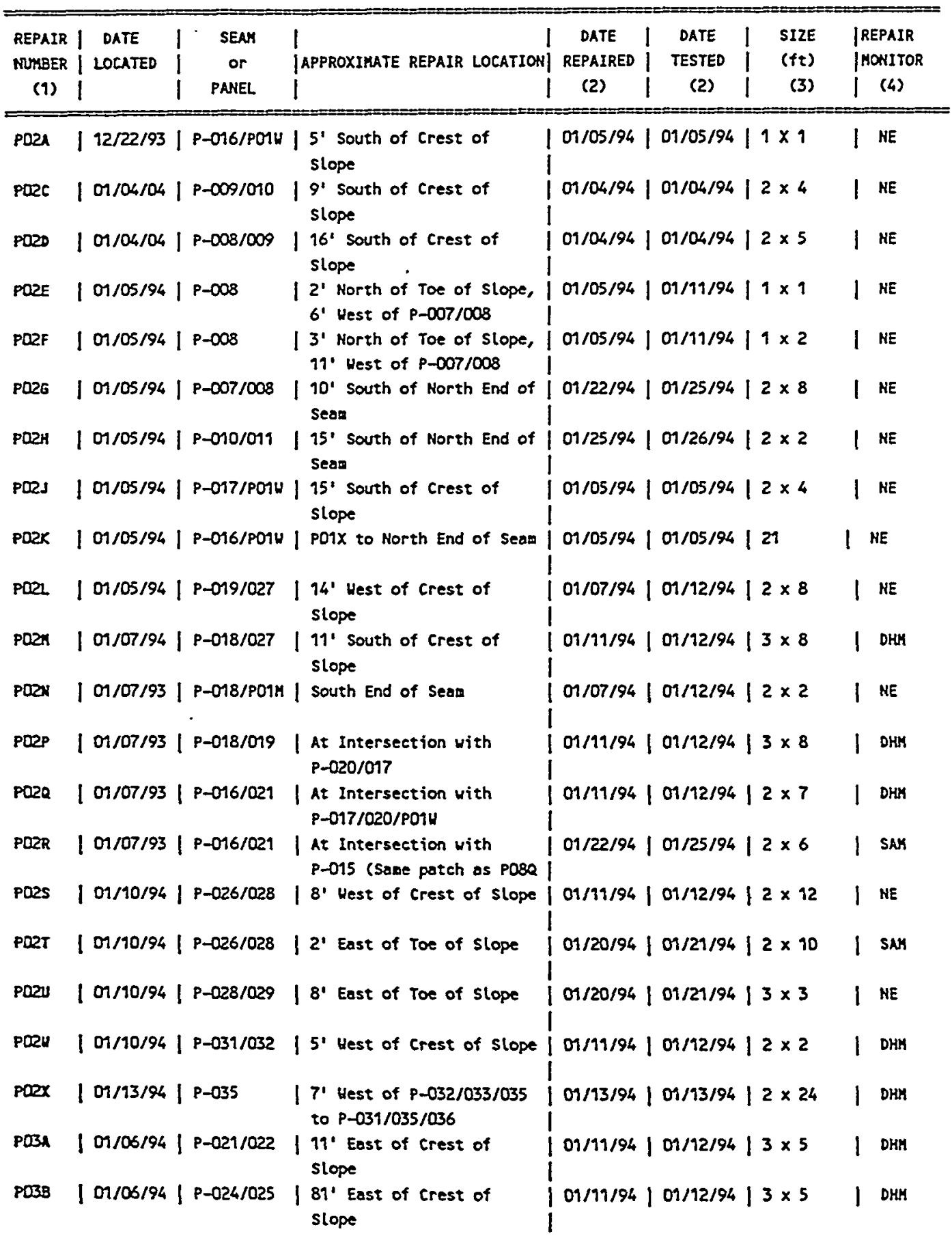


GOLDER CONSTRUCTION SERVICES, INC.

933-1354

PRIHARY GEOMEMBRANE LINER REPAIR SUMMARY

05/19/94

DEPARTHENT OF ENERGY

Page 3

NON-DRAG OFF LANDFILL FACILITY

HANFORO NUCLEAR RESERVATION

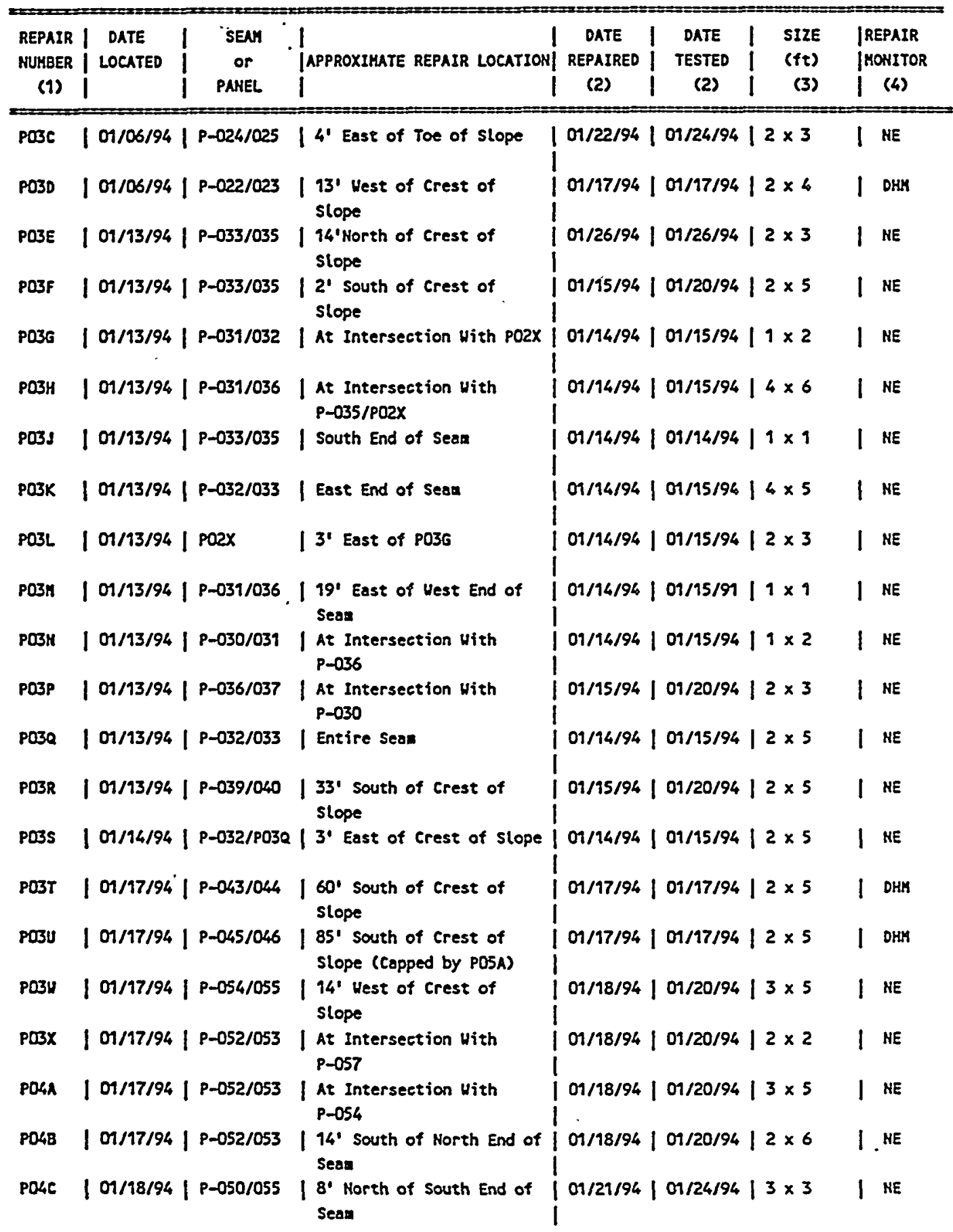


GOLDER CONSTRUCTION SERVICES, INC.

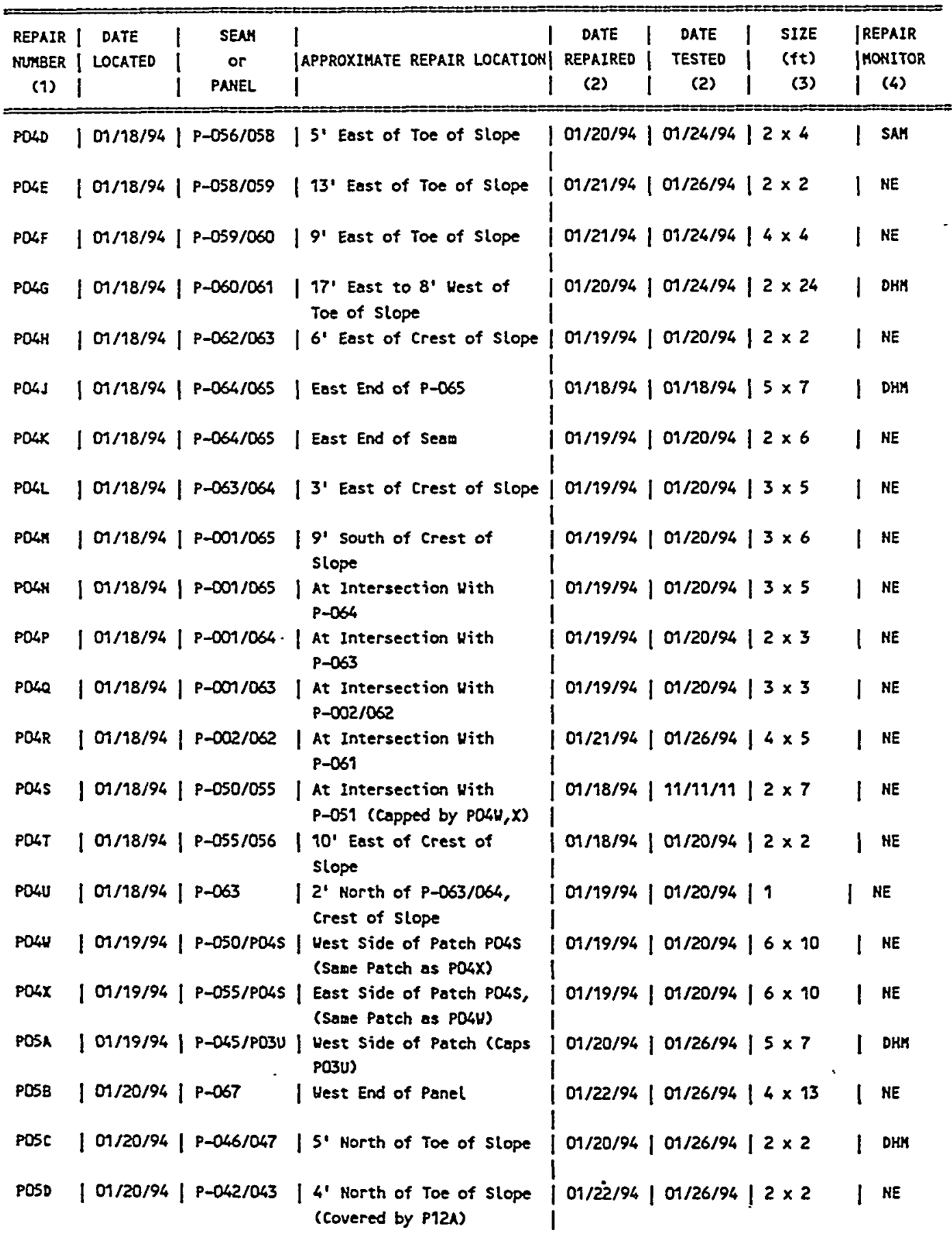


GOLDER CONSTRUCTION SERVICES, INC.

\begin{tabular}{|c|c|c|c|c|c|c|c|c|}
\hline $\begin{array}{l}\text { REPAIR } \\
\text { MUHBER } \\
\text { (1) }\end{array}$ & $\begin{array}{l}1 \text { DATE } \\
1 \\
1\end{array}$ & $\mid \begin{array}{c}\text { SEAH } \\
\text { or } \\
\text { PANEL }\end{array}$ & $\mid$ & $\left\{\begin{array}{l}\text { DATE } \\
\text { REPAIRED } \\
\text { (2) }\end{array}\right.$ & $\begin{array}{l}\text { DATE } \\
\text { TESTED } \\
\text { (2) }\end{array}$ & $\left\{\begin{array}{r}\text { SIZE } \\
1 \\
1 \\
(f t) \\
(3)\end{array}\right.$ & & $\begin{array}{l}\text { EPAIR } \\
\text { ONITOR } \\
\text { (4) }\end{array}$ \\
\hline POSE & $101 / 20 / 94$ & | P-0, $17 / 069$ & $\begin{array}{l}\text { I At Intersection With } \\
\text { P-OTO }\end{array}$ & | 01/22/94 & $01 / 26 / 94$ & $12 \times 2$ & 1 & ME \\
\hline POSF & $101 / 20 / 94$ & | P-067/069 & $\begin{array}{l}\text { I 56' East of West End of } \\
\text { Seam }\end{array}$ & $01 / 21 / 94$ & $01 / 26 / 94$ & $12 \times 4$ & 1 & ME \\
\hline PO5G & $101 / 20 / 94$ & | $P-046 / 047$ & $\begin{array}{l}\text { I At Intersection With } \\
\text { P-066 }\end{array}$ & $01 / 20 / 94$ & $01 / 25 / 94$ & $12 \times 2$ & 1 & OHY \\
\hline POSH & $101 / 20 / 94$ & | P-045/046 & $\begin{array}{l}\text { I At Intersection With } \\
\text { P-066 }\end{array}$ & $01 / 20 / 94$ & $01 / 25 / 94$ & $12 \times 2$ & 1 & DHY \\
\hline POSJ & | 01/20/94 & | P-044/045 & $\begin{array}{l}\text { | At Intersection With } \\
\text { P-066 }\end{array}$ & $01 / 20 / 94$ & $01 / 25 / 94$ & $12 \times 2$ & 1 & DHM \\
\hline POSK & | 01/20/94 & | P-043/044 & $\begin{array}{l}\text { I At Intersection With } \\
\text { P-OS6 }\end{array}$ & $01 / 20 / 94$ & $01 / 25 / 94$ & $12 \times 2$ & 1 & DHM \\
\hline POSL & $101 / 20 / 94$ & | P-042/066 & $\begin{array}{l}\text { I } 51 \text { Hest of East End of } \\
\text { Sean }\end{array}$ & $01 / 22 / 94$ & $01 / 25 / 94$ & $13 \times 7$ & 1 & ME \\
\hline POSM & $101 / 20 / 94$ & | P-04i/042 & $\begin{array}{l}\text { I At Intersection Uith } \\
P-066\end{array}$ & $01 / 22 / 94$ & $01 / 25 / 94$ & $12 \times 2$ & 1 & ME \\
\hline POSM & $107 / 20 / 94$ & | P-040/041 & $\begin{array}{l}\text { I At Intersection Vith } \\
\text { P-DS6 }\end{array}$ & $01 / 22 / 94$ & $01 / 25 / 94$ & $12 \times 2$ & 1 & NE \\
\hline PO5P & $101 / 20 / 94$ & | P-039/040 & I East End of Seam to P106 & $01 / 22 / 94$ & $01 / 25 / 94$ & $15 \times 6$ & 1 & NE \\
\hline PO5a & | 01/20/94 & | P-028/029 & $\begin{array}{l}\text { I At Intersection with } \\
\text { P-0.56 }\end{array}$ & $01 / 22 / 94$ & $01 / 25 / 94$ & $13 \times 5$ & 1 & ME \\
\hline POSR & $109 / 20 / 94$ & | P-037/038 & $\begin{array}{l}\text { I At Intersection vith } \\
\text { P-029 }\end{array}$ & $01 / 20 / 94$ & $01 / 21 / 94$ & $12 \times 2$ & I & NE \\
\hline PO5S & $101 / 20 / 94$ & | P-071/072 & $\begin{array}{l}\text { I At Intersection With } \\
\text { Pm-069 }\end{array}$ & $01 / 22 / 94$ & $01 / 25 / 94$ & $19 \times 1$ & 1 & NE \\
\hline POST & $101 / 20 / 94$ & | P-069/070 & $\begin{array}{l}\text { I At Intersection Hith } \\
\text { P-071 (Capped by P1OR) }\end{array}$ & $01 / 22 / 94$ & $19 / 19 / 91$ & $12 \times 3$ & 1 & NE \\
\hline POSU & $107 / 20 / 94$ & | P-029/030 & $\begin{array}{l}\text { | At Intersection With } \\
\text { P-037 }\end{array}$ & $01 / 20 / 94$ & $01 / 21 / 94$ & $19 \times 9$ & 1 & SAM \\
\hline POSH & | 01/20/94 & | P-047/066 & I At Intersection With POSB & $01 / 20 / 94$ & $01 / 24 / 94$ & $13 \times 3$ & 1 & NE \\
\hline POSA & $101 / 20 / 94$ & | P-003 & $\begin{array}{l}1 \text { North End of Panels P-003 } \\
\text { and P-004 }\end{array}$ & $01 / 20 / 94$ & | 01/21/94 & $14 \times 30$ & 1 & SAM \\
\hline PO6B & $101 / 20 / 94$ & | P-058 & I Hest End of Panel & $01 / 26 / 94$ & | 01/26/94 & $15 \times 16$ & I & HE \\
\hline POSC & | 01/20/94 & | P-026/028 & $\begin{array}{l}\text { I } 94 \text { West of East End of } \\
\text { Sean }\end{array}$ & $01 / 20 / 94$ & | 01/21/94 & $12 \times 2$ & & DHM \\
\hline POSD & | $01 / 20 / 94$ & | P-072/073 & $\begin{array}{l}\text { I At Intersection With } \\
\text { P-069 }\end{array}$ & $01 / 22 / 94$ & | 01/24/94 & $12 \times 2$ & t & ME \\
\hline PO6E & $101 / 20 / 94$ & $1 P-047 / 048$ & $\begin{array}{l}\text { 1 At Intersection with } \\
\text { P-068 }\end{array}$ & $01 / 21 / 94$ & | 01/24/94 & $12 \times 2$ & & ME \\
\hline POSF & | 01/20/94 & | P-048/049 & $\begin{array}{l}\text { | At Intersection With } \\
\text { P-O68 }\end{array}$ & 01/21/94 & | 01/24/9 & $13 \times 5$ & & NE \\
\hline
\end{tabular}


GOLDER CONSTRUCTION SERVICES, INC.

\begin{tabular}{|c|c|c|c|c|c|c|c|c|}
\hline $\begin{array}{l}\text { REPAIR } \\
\text { NUMBER } \\
\text { (1) }\end{array}$ & $\begin{array}{ll}\mid \text { DATE } & \mid \\
\mid & \text { LOCATED } \\
1 & \end{array}$ & $\begin{array}{l}\text { SEAM } \\
\text { or } \\
\text { PAKEL }\end{array}$ & $\mid$ & $\begin{array}{l}\text { DATE } \\
\text { REPAIRED } \\
\text { (2) }\end{array}$ & $\begin{array}{c}\text { DATE } \\
\text { TESTED } \\
\text { (2) }\end{array}$ & $\left\{\begin{array}{r}\text { SIZE } \\
\mid(f t) \\
1 \quad(3)\end{array}\right.$ & $\begin{array}{l}\mid R \\
\mid M\end{array}$ & $\begin{array}{l}\text { REPAIR } \\
\text { IONITOR } \\
\text { (4) }\end{array}$ \\
\hline POSG & $|01 / 20 / 94|$ & $P-055 / 056$ & $\begin{array}{l}\text { I At Intersection With } \\
P-049\end{array}$ & 01/21/94 & | $01 / 24 / 94$ & $13 \times 3$ & 1 & ME \\
\hline POSH & | 01/20/94 | & $P-056 / 058$ & $\begin{array}{l}\text { I At Intersection With } \\
P-068\end{array}$ & $01 / 21 / 94$ & | 01/24/94 & $13 \times 6$ & 1 & ME \\
\hline POSJ & | 01/20/94 | & $P-058 / 068$ & | At Intersection With POSB & $01 / 21 / 94$ & | 01/24/94 & $12 \times 2$ & 1 & HE \\
\hline POSK & | 01/20/94 | & $P-058 / 066$ & | At Intersection With POSB & $01 / 21 / 94$ & | 01/24/94 & $12 \times 2$ & 1 & ME \\
\hline POSL & | 01/20/94 | & $P-066 / 067$ & $\begin{array}{l}\text { I At Intersection With } \\
\text { P-058 }\end{array}$ & $01 / 21 / 94$ & $01 / 24 / 94$ & $12 \times 2$ & 1 & NE \\
\hline POSM & $|01 / 20 / 94|$ & $P-058 / 059$ & $\begin{array}{l}\text { At Intersection With } \\
P-067\end{array}$ & $01 / 21 / 94$ & | 01/24/94 & $12 \times 2$ & 1 & NE \\
\hline POSN & | 01/20/94 | & $P-057 / 069$ & $\begin{array}{l}\text { I At Intersection With } \\
\text { p-059 }\end{array}$ & $01 / 21 / 94$ & | 01/24/94 & $12 \times 2$ & I & NE \\
\hline POSP & $|01 / 20 / 94|$ & $P-059 / 075$ & $\begin{array}{l}\text { I At Intersection With } \\
\text { P-069 }\end{array}$ & $01 / 22 / 94$ & | 01/24/94 & $13 \times 6$ & 1 & ME \\
\hline POSO & | 01/20/94 | & P-073/075 & $\begin{array}{l}\text { I At Intersection With } \\
\text { P-069 (Some patch as po7u }\end{array}$ & $01 / 22 / 94$ & 1 01/24/94 & $13 \times 6$ & I & NE \\
\hline POSR & | 01/20/94 | & $P-073 / 074$ & $\begin{array}{l}\text { At Intersection With } \\
\text { P-075 }\end{array}$ & $01 / 21 / 94$ & 101/24/94 & $13 \times 3$ & 1 & NE \\
\hline POSS & | 01/20/94 | & $P-059 / 074$ & $\begin{array}{l}\text { I At Intersection With } \\
P-075\end{array}$ & $01 / 21 / 94$ & $01 / 21 / 94$ & $3 \times 4$ & 1 & NE \\
\hline POST & | 01/20/94 | & $P-059 / 060$ & $\begin{array}{l}\text { I At Intersection With } \\
\text { P-074 }\end{array}$ & $01 / 21 / 94$ & 1 01/24/94 & $13 \times 4$ & 1 & NE \\
\hline POSU & $|01 / 20 / 94|$ & $P-047 / 068$ & $\begin{array}{l}\text { At Intersection with } \\
P-O S B\end{array}$ & $01 / 21 / 94$ & 01/24/94 & $12 \times 2$ & 1 & NE \\
\hline POSW & | 01/20/94 | & $P-060 / P 06 A$ & $\begin{array}{l}\text { I 7' East of West End of } \\
\text { Sear }\end{array}$ & $01 / 21 / 94$ & | 01/24/94 & $13 \times 5$ & 1 & NE \\
\hline$P 06 x$ & $101 / 20 / 94$ & $P-073 / 074$ & I At Intersection With POSA & $01 / 21 / 94$ & | 01/24/94 & $2 \times 2$ & 1 & NE \\
\hline POTA & | 01/20/94 | & $P-066 / 067$ & | At Intersection With P028 & $01 / 22 / 94$ & $101 / 25 / 94$ & $12 \times 2$ & 1 & NE \\
\hline PO7B & $|01 / 20 / 94|$ & $P-028 / 067$ & I At Intersection With POSB & $01 / 22 / 94$ & $101 / 26 / 94$ & $12 \times 2$ & I & ME \\
\hline POTC & $|07 / 20 / 94|$ & $P-067 / 070$ & I At Intersection With POSB & $01 / 22 / 94$ & 01/25/94 & $13 \times 3$ & l & NE \\
\hline POTD & $|01 / 20 / 94|$ & $P-026 / 070$ & I At Intersection With POSB & $01 / 22 / 94$ & 1 01/25/94 & $12 \times 2$ & & NE \\
\hline POTE & $|07 / 20 / 94|$ & $P-025 / 026$ & $\begin{array}{l}\text { I At Intersection With } \\
\text { p-070 }\end{array}$ & $09 / 22 / 94$ & $01 / 25 / 94$ & $12 \times 2$ & & HE \\
\hline PO7F & $101 / 20 / 94$ & $P=070 / 071$ & $\begin{array}{l}\text { I At intersection with } \\
P-025\end{array}$ & $01 / 22 / 94$ & $01 / 25 / 94$ & $12 \times 2$ & & ME \\
\hline PO7G & $\mid 01 / 20 / 94$ & $P-024 / 025$ & $\begin{array}{l}\text { I At intersection with } \\
\text { P } 079\end{array}$ & $01 / 22 / 94$ & 101/25/94 & & & NE \\
\hline
\end{tabular}


GOLDER CONSTRUCTION SERUICES, INC.

\begin{tabular}{|c|c|c|c|c|c|c|c|}
\hline $\begin{array}{l}\text { REPAIR } \\
\text { NUMBER } \\
\text { (1) }\end{array}$ & $\left\{\begin{array}{l}\mid \begin{array}{l}\text { DATE } \\
\text { LOCATED } \\
1\end{array} \\
\end{array}\right.$ & $\begin{array}{c}\text { SEAM } \\
\text { or } \\
\text { PAMEL }\end{array}$ & $\mid$ & $\left|\begin{array}{c}\text { DATE } \\
\text { REPAIRED } \\
\text { (2) }\end{array}\right|$ & $\mid \begin{array}{c}\text { DATE } \\
\text { TESTED } \\
\text { (2) }\end{array}$ & $\left\{\begin{array}{l}\text { SIZE } \\
(f t) \\
1 \\
(3)\end{array}\right.$ & $\begin{array}{l}\text { |REPAIR } \\
\text { | HOWITOR } \\
\mid \text { (4) }\end{array}$ \\
\hline POTH & | 01/21/94 | & $P-012$ & $\begin{array}{l}\mid \begin{array}{l}51 \\
\text { East of } P-012 / 013,51\end{array} \\
\text { South of } P-012 / 079\end{array}$ & $|01 / 21 / 94|$ & 01/25/94 & $12 \times 4$ & I NE \\
\hline PO7J & $|01 / 21 / 94|$ & P-060/074 & | At Intersection With POSA | & $|01 / 21 / 94|$ & | 01/24/94 & $12 \times 2$ & I NE \\
\hline POTK & $|01 / 21 / 94|$ & P-003/004 & | At Intersection With POSA | & $01 / 21 / 94$ & 107/24/94 & $12 \times 2$ & I $\mathrm{NE}$ \\
\hline PORL & $|01 / 21 / 94|$ & P $-002 / 003$ & $\begin{array}{l}\text { | At Intersection Hith } \\
\text { P-OS1 }\end{array}$ & |01/21/94 & 109/24/94 & $12 \times 2$ & I HE \\
\hline POTn & $|01 / 21 / 96|$ & | P-060/061 & $\begin{array}{l}\text { | At Intersection With } \\
\text { P-003/P06//P04G }\end{array}$ & $\left.\right|^{01 / 21 / 94}$ & 1 01/24/94 & $13 \times 6$ & I NE \\
\hline PO7N & $|01 / 21 / 94|$ & | P-074 & $\begin{array}{l}141 \text { Horth of } P-074 / P 06 A \text {, } \\
51 \text { Hest of } P-060 / 074\end{array}$ & $|01 / 21 / 94|$ & 101/24/94 & $12 \times 2$ & I NE \\
\hline PO7P & $|04 / 21 / 94|$ & P $-004 / 005$ & $\begin{array}{l}\text { I At Intersection with } \\
\text { P-OT3/POSA }\end{array}$ & $|01 / 29 / 94|$ & 1 01/24/94 & $12 \times 5$ & I NE \\
\hline POTa & $|09 / 21 / 94|$ & P-005/006 & $\begin{array}{l}\text { I At Intersection with } \\
\text { P-OT3, NEOS to } 10^{\prime} \text { South }\end{array}$ & $01 / 22 / 94 \mid$ & $101 / 24 / 94$ & $13 \times 12$ & I ME \\
\hline POTR & $|01 / 21 / 94|$ & | P-049/050 & \begin{tabular}{|}
$\mid$ At Intersection With \\
$p-055$
\end{tabular} & $01 / 21 / 94$ & | 01/24/94 & $13 \times 3$ & I NE \\
\hline PO75 & $|01 / 22 / 94|$ & | P-026/028 & I At Intersection With POSB & $01 / 22 / 94$ & 101/25/94 & $14 \times 4$ & I NE \\
\hline POTT & $|01 / 22 / 94|$ & | P-010/079 & $\mid$\begin{tabular}{|} 
At Intersection Hith \\
$p \rightarrow 072$
\end{tabular} & $07 / 22 / 94$ & 1 01/25/94 & $12 \times 4$ & I NE \\
\hline POTS & $|09 / 22 / 94|$ & | P-069/075 & $\begin{array}{l}\mid \text { At Intersection With } \\
\text { P-OT3 (sase patch as POSQ }\end{array}$ & $|09 / 22 / 94|$ & $109 / 24 / 94$ & $13 \times 6$ & I NE \\
\hline POTN & | 01/24/94 | & P $p-007 / 008$ & I 2' West of $P-007 / 008 / 072$ & $09 / 25 / 94$ & 01/26/94 & $12 \times 3$ & I NE \\
\hline PO7x & $|01 / 24 / 94|$ & | P-010/079 & I 3' West of $P-010 / 071 / 072$ & $01 / 24 / 94$ & $101 / 25 / 94$ & $12 \times 2$ & I NE \\
\hline pose & $|01 / 21 / 94|$ & | P-006/007 & $\begin{array}{l}\text { I At Intersection With } \\
P \rightarrow 073\end{array}$ & $\left.\right|^{01 / 25 / 94}$ & | 09/26/94 & $12 \times 3$ & I NE \\
\hline P083 & $|01 / 21 / 94|$ & | $P-072 / 073$ & $\begin{array}{l}\text { I At Intersection with } \\
p \rightarrow-\infty 07\end{array}$ & $\left.\right|^{01 / 25 / 94}$ & $101 / 26 / 94$ & $14 \times 4$ & I NE \\
\hline Pose & $|01 / 21 / 94|$ & | P-007/008 & $\begin{array}{l}\text { I At Intersection With } \\
\text { P-o72 }\end{array}$ & $01 / 22 / 94$ & 1 01/26/94 & $12 \times 2$ & I NE \\
\hline PDBD & $|07 / 21 / 94|$ & | $P-008 / 009$ & $\begin{array}{l}\text { I At Intersection With } \\
p=072\end{array}$ & $\mid 01 / 22 / 94$ & 1 01/25/94 & & I NE \\
\hline POBE & $|01 / 21 / 94|$ & | $P-009 / 010$ & $\begin{array}{l}\text { I NEOS to } 13 \text { ' Sourth of } \\
\text { North End of Sean }\end{array}$ & $01 / 22 / 94$ & | 01/25/94 & $13 \times 13$ & I $\mathrm{ME}$ \\
\hline POBF & $|01 / 21 / 94|$ & | P-010/011 & $\begin{array}{l}\text { I At Intersection With } \\
\text { P-071 }\end{array}$ & $|01 / 22 / 94|$ & $101 / 25 / 94$ & $19 \times 1$ & I NE \\
\hline 8085 & $|01 / 21 / 94|$ & | P-011/012 & $\begin{array}{l}\text { I At Intersection With } \\
\text { P } 071\end{array}$ & $01 / 22 / 94$ & | 01/25/94 & $13 \times 4$ & I NE \\
\hline POSH & $|01 / 21 / 94|$ & | P-012 & $\begin{array}{l}\mid 2^{2} \text { South of } P-012 / 071,8^{8} \\
\text { West of } P-011 / 012\end{array}$ & $07 / 22 / 94$ & | 01/25/94 & $12 \times 2$ & I NE \\
\hline
\end{tabular}


GOLDER CONSTRUCTION SERVICES, INC.

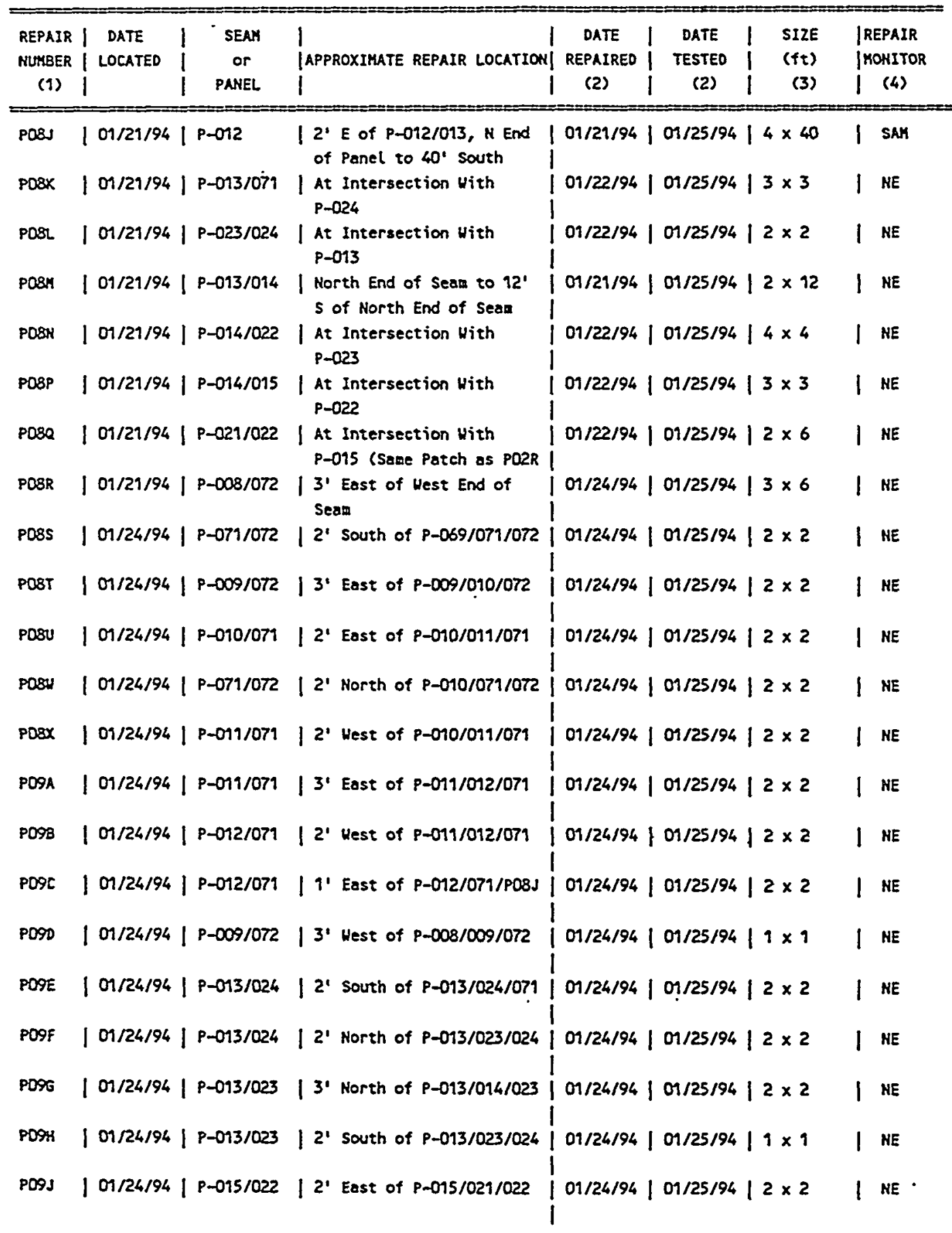


GOLDER CONSTRUCTION SERVICES, IHC.

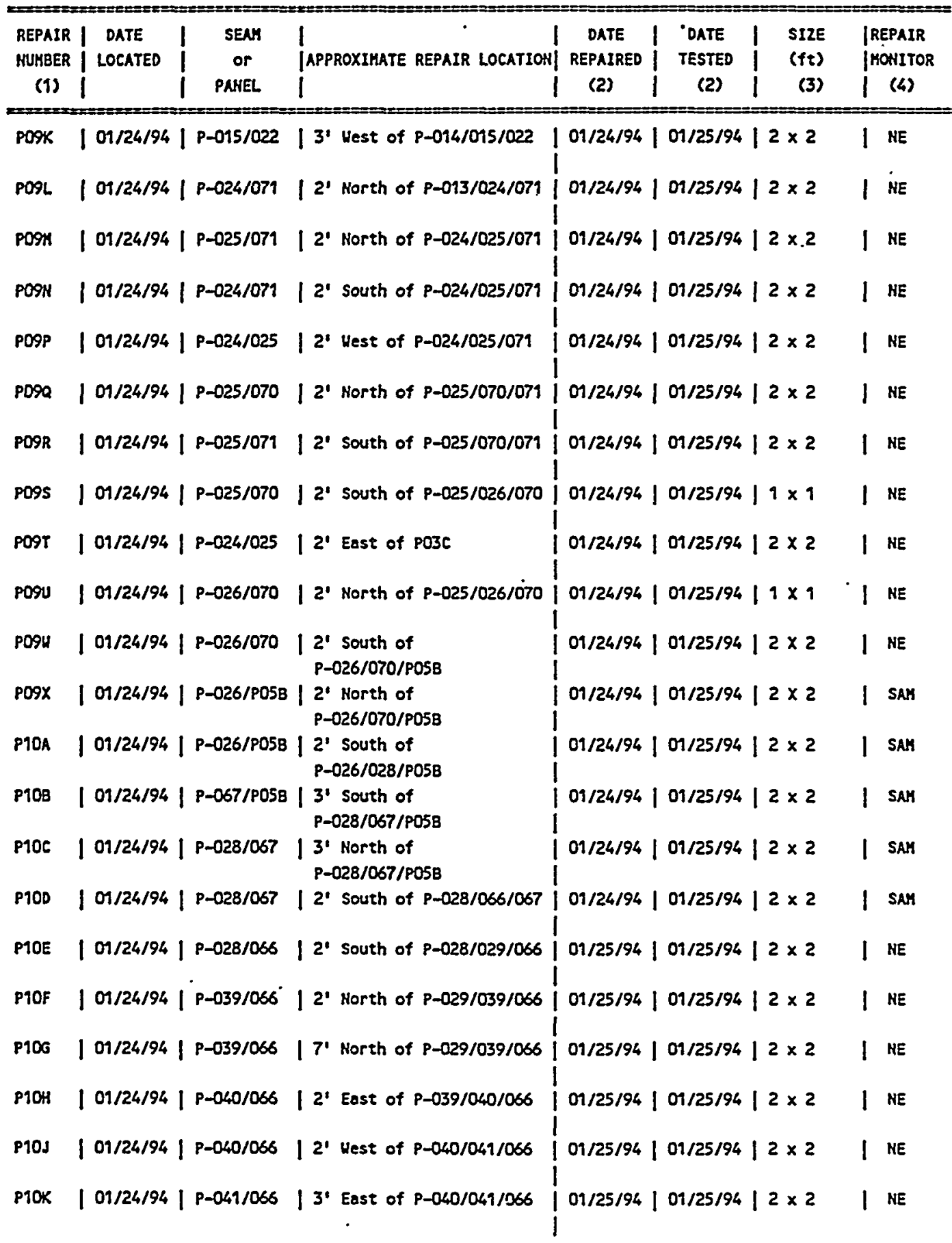


GOLDER CONSTRUCTION SERVICES, INC.

933-1354

PRIMARY GEOHEMBRANE LINER REPAIR SUMMARY

$05 / 91 / 94$

DEPARTMENT OF ENERGY

Page 10

NON-DRAG OFF LANDFILL FACILITY

HANFORD NUCLEAR RESERVATION

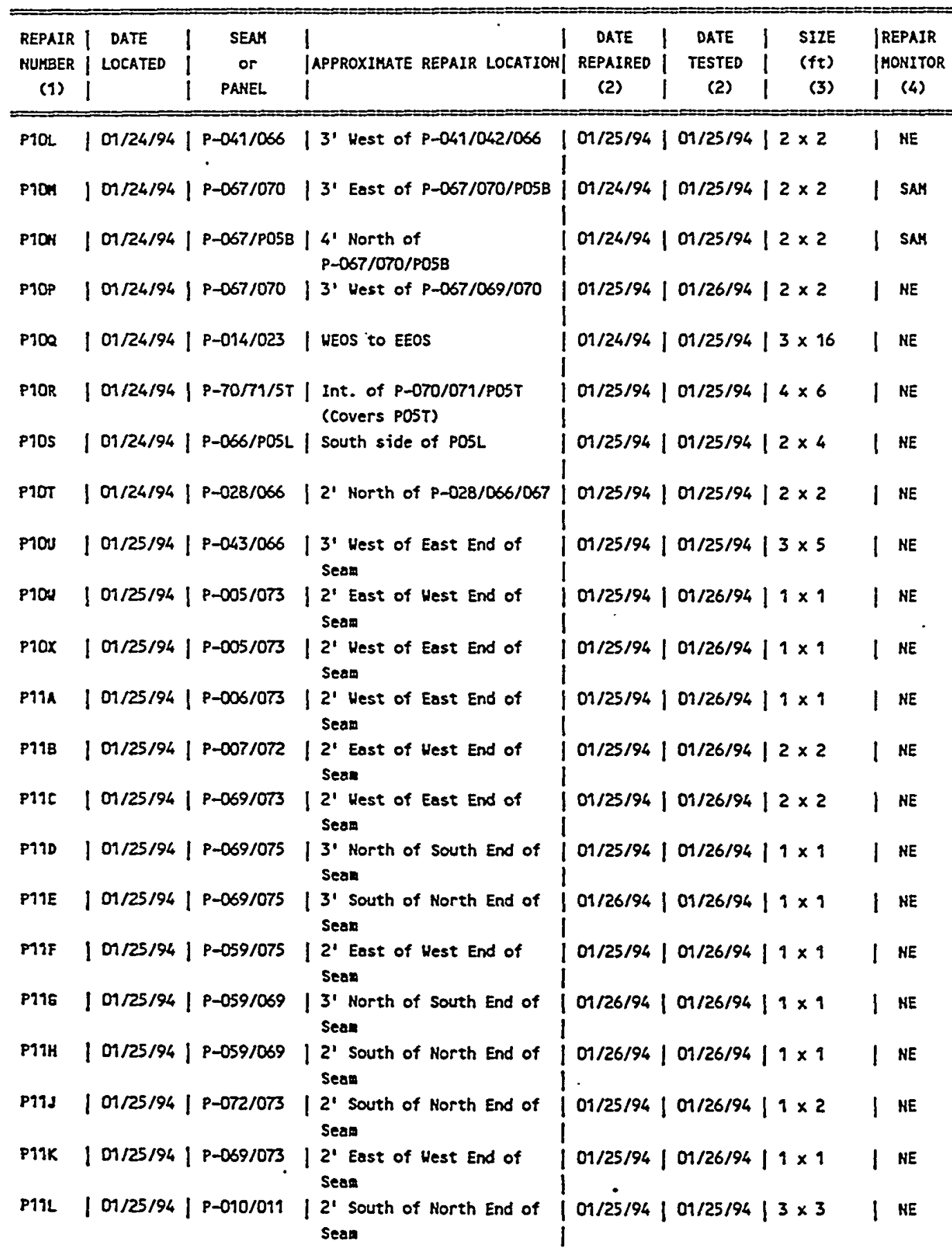


GOLDER CONSTRUCTION SERVICES, INC.

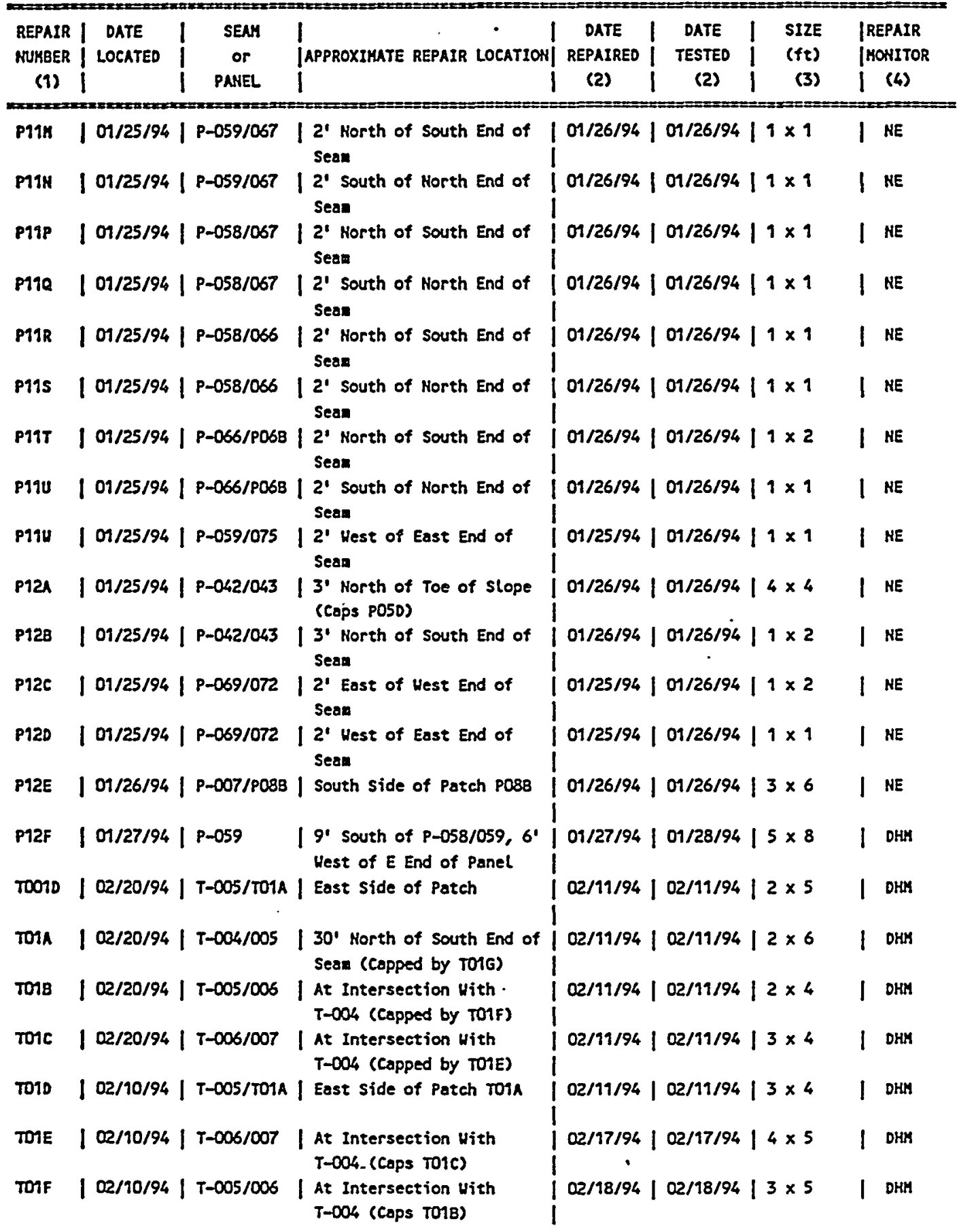


GOLDER CONSTRUCTION SERVICES, IHC.

NOW-DRAG OFF LANDFILL FACILITY HANFORD NUCLEAR RESERVATION

\begin{tabular}{|c|c|c|c|c|c|c|c|}
\hline $\begin{array}{l}\text { REPRIR | } \\
\text { NUMBER ! } \\
\text { (1) । }\end{array}$ & $\begin{array}{l}\text { DATE } \\
\text { LOCATED } \\
1\end{array}$ & $\begin{array}{l}\text { SEAH } \\
\text { or } \\
\text { PANEL }\end{array}$ & $\begin{array}{l}\mid \\
\text { |APPROXIHATE REPAIR LOCATION! }\end{array}$ & $\begin{array}{l}\text { DATE } \\
\text { REPAIRED } \\
\text { (2) }\end{array}$ & $\begin{array}{l}\text { DATE } \\
\text { TESTED } \\
(2)\end{array}$ & $\begin{array}{l}1 \\
\text { SIZE } \\
1 \\
(f t) \\
(3)\end{array}$ & $\begin{array}{l}\text { |REPAIR } \\
\text { | MONITOR } \\
1 \text { (4) }\end{array}$ \\
\hline TOIG & 1 02/10/94 & | T-004/005 & $\begin{array}{l}\text { I At Intersection Vith } \\
\text { T-004 (Cops To1A) }\end{array}$ & $02 / 18 / 94$ & $02 / 18 / 94$ & $13 \times 7$ & DHH \\
\hline TOIH & 1 02/21/94 & I $T P-\infty 01 / 002$ & $\begin{array}{l}\text { At Intersection With } \\
\text { P-Dot/002 }\end{array}$ & $02 / 21 / 94$ & $02 / 21 / 94$ & $14 \times 34$ & DHM \\
\hline TO1 & $102 / 22 / 94$ & I T-001/TO1K & $\begin{array}{l}\text { I } 8^{\prime} \text { West of East End of } \\
\text { Sean }\end{array}$ & $02 / 22 / 94$ & $02 / 22 / 94$ & $12 \times 5$ & DHM \\
\hline TOIK & 1 02/22/94 & | TP-001/002 & $\begin{array}{l}\mid \text { Vest End of Sean to } 24^{\circ} \\
\text { South }\end{array}$ & $02 / 22 / 94$ & $02 / 22 / 94$ & $12 \times 24$ & DHM \\
\hline
\end{tabular}

NOTES:

(9) A repair number beginning with the letter "pn indicates a repair on the Primary HDPE liner. A repair number beginning with the letter "T" indicates a repair on the HDPE liner in the truck unloading area.

(2) A repair date and/or test date of 11/91/99 indicates a repair which was capped.

(3) A repair with no size indicates a repair which was capped.

A repsir vith only one size dimension indicates a grind and weld repair.

(4) See veld observation Summery for monitor identification. 
WHC-SD-W025-RPT-001, Rev. 0

APPENDIX 0

TOP COURSE MOISTURE-DENSITY TESTS 
GOLDER CONSTRUCTION SERVICES INC.

SUMMARY OF TOP COURSE MOISTURE-DENSITY TESTS 933-1354

DEPARTMENT OF ENERGY

05/01/94

NON-DRAG OFF LANDFILL FACILITY

HANFORD NUCLEAR RESERVATION

Page 1

TOP COURSE

\begin{tabular}{|c|c|c|c|c|c|c|c|c|c|c|c|}
\hline \multirow[b]{3}{*}{ TEST } & \multirow{4}{*}{ DATE } & \multicolumn{3}{|c|}{ TEST LOCATION INFORMATION } & \multicolumn{2}{|c|}{ TROXLER } & \multicolumn{3}{|c|}{$\begin{array}{l}\text { LABORATORY CALCULATED } \\
\text { TEST YALUES }\end{array}$} & \multirow{2}{*}{\multicolumn{2}{|c|}{ 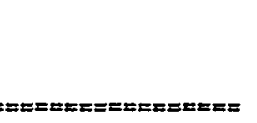 }} \\
\hline & & \multicolumn{8}{|c|}{ | } & & \\
\hline & & | NORTH I & WEST | & LIFT & | DRY | & FIELD & | DRY I & LAB & | PERCENT | & PASS & \\
\hline $\begin{array}{r}\text { NUMBER } \\
(1,2)\end{array}$ & & $\mid$ COORDIHATE $\mid$ & COORDINATE | & Ho. & $\begin{array}{l}\mid \text { DENS. } \\
\mid \text { (pcf) } \mid\end{array}$ & $\begin{array}{l}\text { MOIST } \\
(x)\end{array}$ & $\begin{array}{l}\text { |DENSITY } \mid \\
\mid \text { (pcF) } \mid\end{array}$ & $\begin{array}{l}\text { MOIST } \\
(X)\end{array}$ & [COMPACTION] & $\begin{array}{l}\text { or } \\
\text { FAIL }\end{array}$ & NOTES \\
\hline & 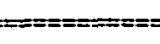 & & & $=$ & $z==x==s=$ & $x=s==8$ & $s=x=x=x$ & $s=x==x$ & & $=0$ & \\
\hline TC-006 & $|03 / 02 / 94|$ & 43991.51 & 78607.9 & 1 & |134.2 & 4.2 & 132.9 & 5.2 & 95.1 & Pass & $N / A$ \\
\hline TC-007 & $|03 / 02 / 94|$ & 43986.2 & 78683.2 & 1 & 1234.0 & 4.5 & 133.5 & 4.9 & 95.5 & Pass & $\mathrm{H} / \mathrm{A}$ \\
\hline$T C-008$ & $|03 / 02 / 94|$ & 43986.1 & 78861.4 & 1 & 134.3 & 4.2 & 133.6 & 4.7 & 95.6 & Pass & $N / A$ \\
\hline TC-009 & $|03 / 02 / 94|$ & 44182.9 & 78282.6 & 1 & 137.7 & 3.3 & 136.7 & 4.0 & 97.8 & Pass & $N / A$ \\
\hline$T C-010$ & $|03 / 02 / 94|$ & 44357.9 & 78312.3 & 1 & 135.4 & 2.8 & 133.7 & 4.1 & 95.6 & Pass & $N / A$ \\
\hline TC-011 & $|03 / 02 / 94|$ & 44389.6 & 78424.7 & 1 & 135.3 & 3.4 & 133.5 & 4.8 & 95.5 & Pass & $N / A$ \\
\hline TC-012 & $|03 / 02 / 94|$ & 44388.5 & 78572.8 & 1 & 134.5 & 3.4 & 133.7 & 4.0 & 95.6 & Pass & $N / A$ \\
\hline$T C-013$ & $|03 / 02 / 94|$ & 44379.8 & 78732.3 & 1 & 134.9 & 3.3 & 133.7 & 4.2 & 95.6 & Pass & $H / A$ \\
\hline$T C-014$ & $|03 / 02 / 94|$ & 44376.5 & 78846.5 & 1 & |135.7 & 4.2 & 135.9 & 4.0 & 97.2 & Pass & $N / A$ \\
\hline TC-015 & $|03 / 02 / 94|$ & 44171.7 & 78867.5 & 1 & |135.7 & 4.2 & 135.9 & 4.0 & 97.2 & Pass & $N / A$ \\
\hline TC-016 & $|03 / 07 / 94|$ & 43950.5 & 78249.5 & 1 & $\mid 135.3$ & 6.8 & 144.5 & $N / A$ & 103.4 & Pass & K/A \\
\hline TC-017 & $|03 / 07 / 94|$ & 43944.2 & 78358.9 & 1 & |133.2 & 6.1 & 141.4 & $N / A$ & 101.1 & Pass & N/A \\
\hline TC-018 & $|03 / 07 / 94|$ & 43948.0 & 78175.7 & 1 & 1137.2 & 7.2 & 147.1 & $N / A$ & 105.2 & Pass & N/A \\
\hline TC-D19 & $|03 / 11 / 94|$ & 44050.9 & 78400.5 & 1 & |134.9 & 5.3 & 133.1 & 6.7 & 95.2 & Pass & $N / A$ \\
\hline$T C-020$ & $|03 / 11 / 94|$ & 43983.1 & 78356.2 & 1 & |135.8 & 4.9 & 133.6 & 6.1 & 95.6 & Pass & N/A \\
\hline$T C-021$ & $|03 / 11 / 94|$ & 44057.5 & 78295.1 & 1 & |134.8 & 7.4 & $135.1 \mid$ & 7.2 & 96.6 & Pass & $N / A$ \\
\hline TC-022 & $|03 / 23 / 94|$ & 44111.6 & 78598.1 & 1 & 135.7 & 3.0 & 133.11 & 5.0 & 95.2 & Pass & N/A \\
\hline TC-023 & $|03 / 23 / 94|$ & 44083.5 & 78529.0 & 1 & 135.8 & 4.3 & $133.5 i$ & 6.1 & 95.5 & Pass & $N / A$ \\
\hline$T C-024$ & $|03 / 23 / 94|$ & 44083.7 & 78500.3 & 1 & |137.8 & 4.6 & 135.9 & 6.0 & 97.2 & Pass & $N / A$ \\
\hline
\end{tabular}

NOTES

$=x==$

(1) Test numbers TC-001 through TC-005 were assigned to laboratory tested samples of top course material.

(2) See General Fill test no. GF-055RB for the rubber balloon test performed on 03/02/94. See General Fill test no. GF-056RB for the rubber balloon test performed on 03/07/94. See General Fill test no. GF-057RB for the rubber balloon test performed on 03/11/94. See General Fill test no. GF-058RB for the rubber balloon test performed on 03/23/94. 
WHC-SD-W025-RPT-001, Rev. 0

APPENDIX P

ASPHALT PAVEMENT

$p-i$ 
WHC-SD-W025-RPT-001, Rev. 0

APPENDIX P-1

LABORATORY TESTS AND FIELD DENSITY TESTS 
WHC-SD-W025-RPT-001, Rev. 0

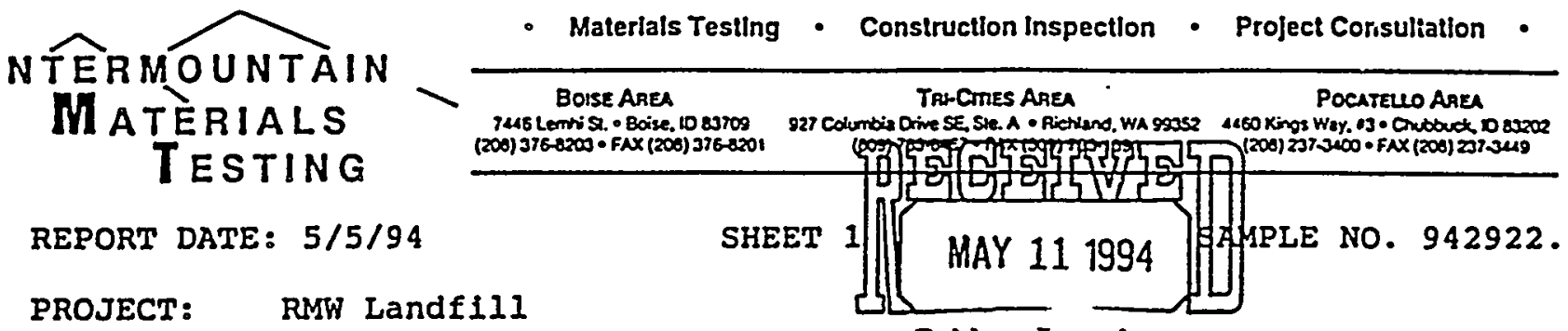

REPORT TO: Golder Associates

Galúker Assaciales

4104148 th Avenue NE

Redmond, Wa. 98052. Invoice No.: 2-940322

Attn: Cliff Knitter File No.: 93-167

\begin{tabular}{ll}
\hline Material Source: & Acme Materials \& Construction \\
Date sampled: & $3 / 16 / 94$ \\
Sampled by: & Wiley Yeakey (IMT) \\
Date received: & $3 / 16 / 94$
\end{tabular}

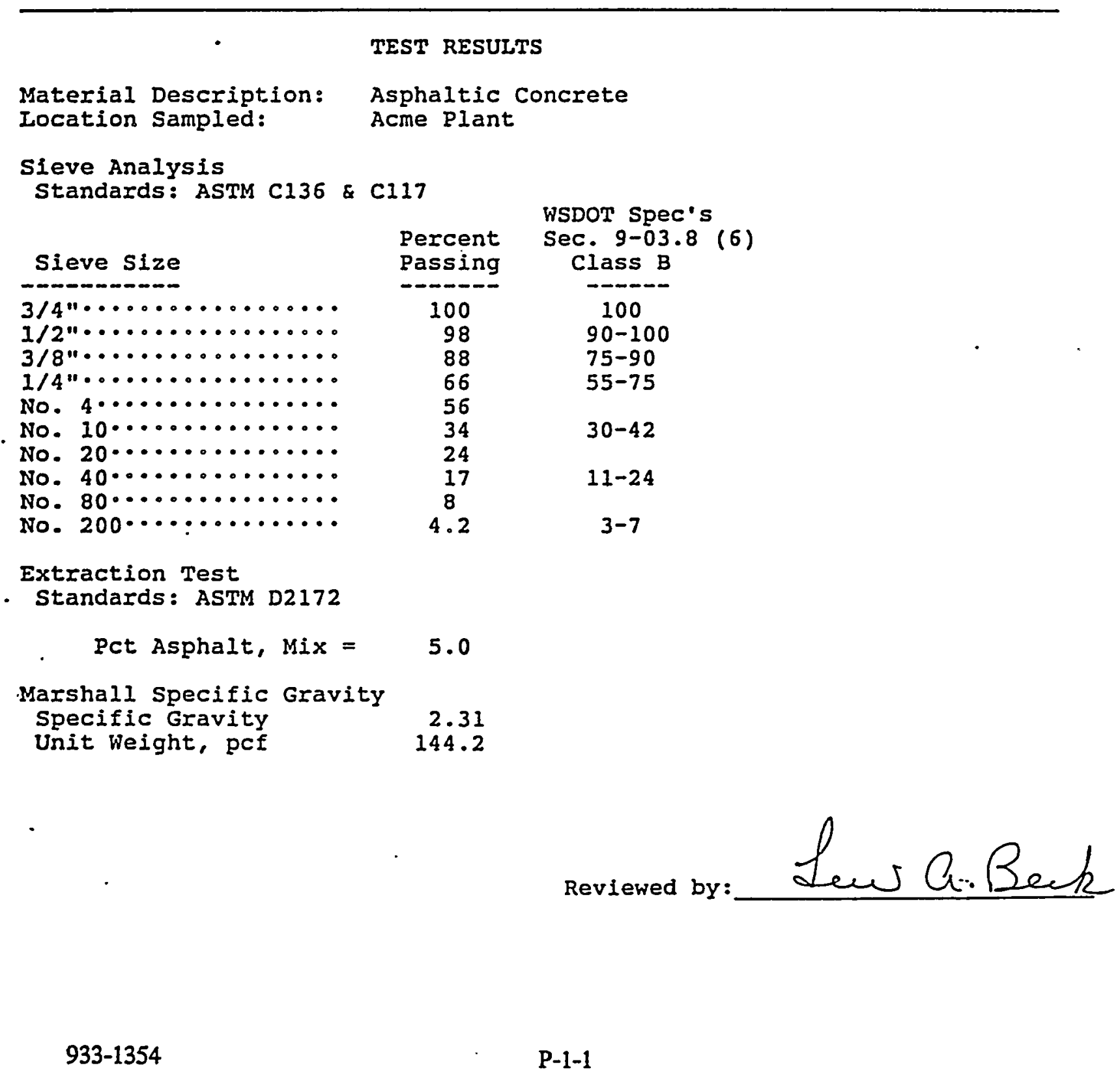


WHC-SD-W025-RPT-001, Rev. 0

INTERMOUNTAIN
$\begin{array}{r}\text { MI ATERIALS } \\ \text { TESTING }\end{array}$

REPORT TO: Golder Associates 4104148 th Ave, NE Redmond, Wa. 98052 ATTN: Cliff Knitter
DATE: $\quad 3 / 16 / 94$

FILE NUMBER: $93-167$

SHEET: 1 of 1

INVOICE: 2-940322

PROJECT: RMW Landfill, Richland, Wa.

\section{SAMPLE IDENTIFICATION}

Summarized below are the results of Asphaltic Concrete density tests performed on the above referenced project at the location shown below.

Unless otherwise note, our personnel utilized the nuclear densometer method of testing in accordance with ASTM D2922.

The project specifications require a minimum in-place density of 93

percent of the Marshall density as determined by ASTM D1559.

The test results are as follows:

\section{TEST RESULTS}

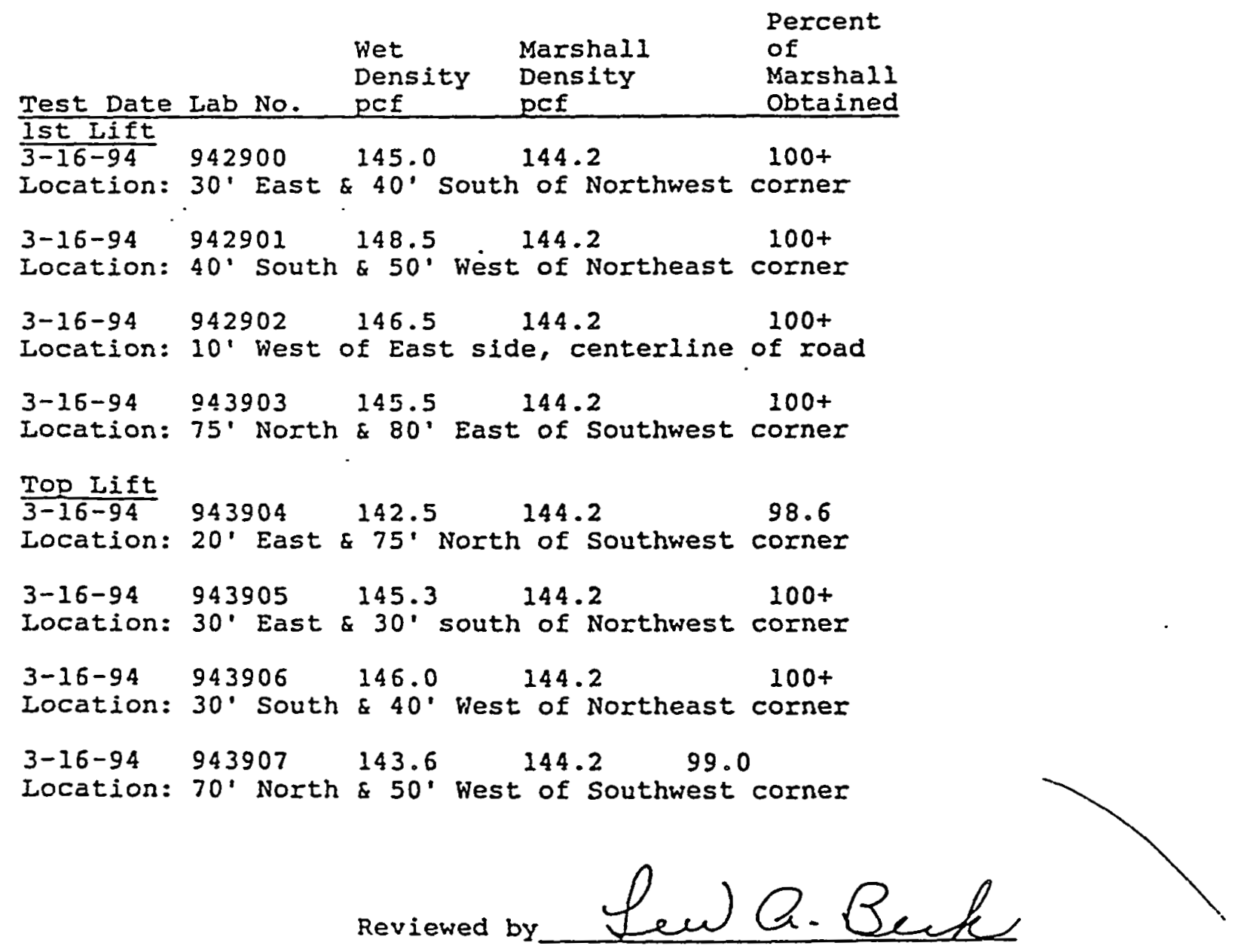


WHC-SD-W025-RPT-001, Rev. 0

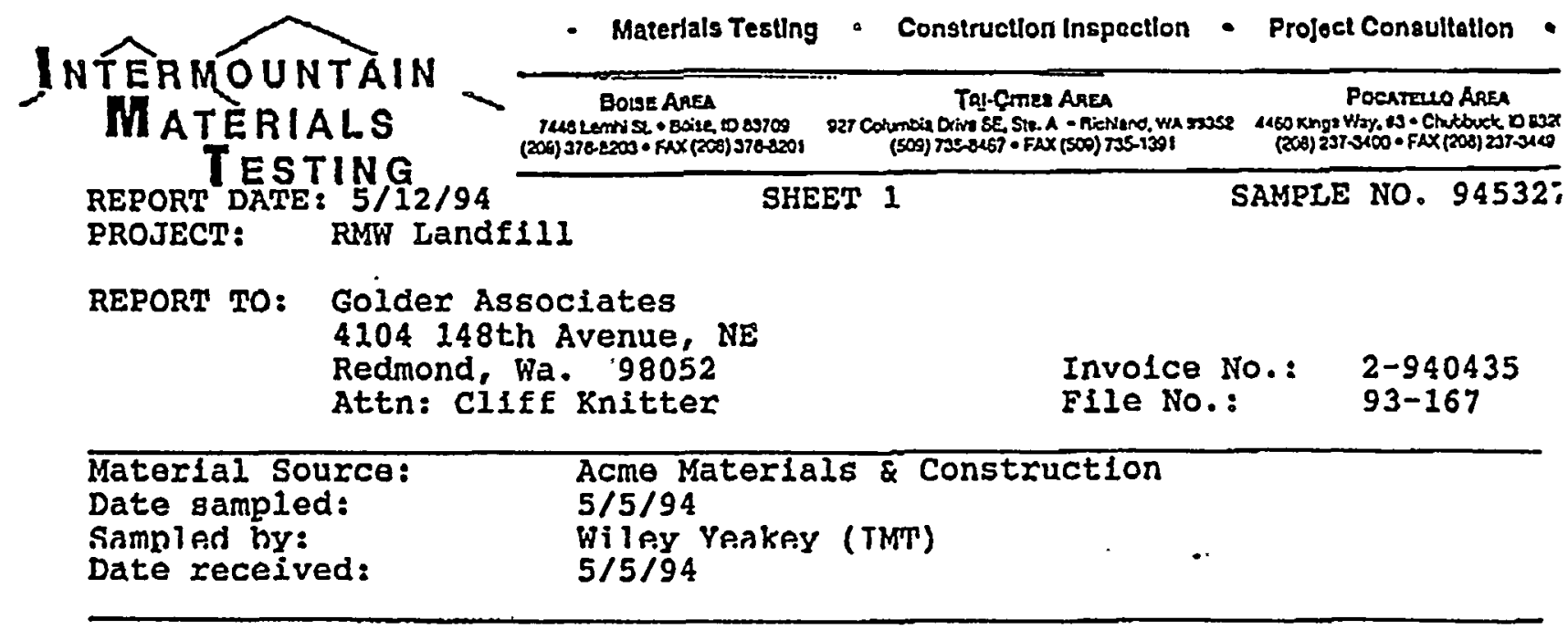

\section{TEST RESULTS}

Material Description:

Asphaltic Concrete Location Sampled:

From truck asphalt plant

\section{Sleve Analysis \\ Standards: ASTM C136 $7 \mathrm{C117}$}

Sleve Size

$3 / 411 \ldots \ldots \ldots \ldots$

$1 / 21 \ldots \ldots \ldots \ldots . . . \ldots$

$3 / 81 \ldots \ldots \ldots \ldots \ldots$

$1 / 411 \ldots \ldots \ldots \ldots \ldots \ldots$

No. $4 \ldots \ldots \ldots \ldots \ldots \ldots$

No. $10 \ldots \ldots \ldots \ldots \ldots . . . .$.

No. $20 \ldots \ldots \ldots \ldots$

No. $40 \ldots \ldots \ldots \ldots \ldots \ldots$

No. $80 \ldots \ldots \ldots \ldots \ldots . . . .$.

No. $200 \ldots \ldots \ldots . . . .$.

Extraçtion Test

Standards: ASTM D2172

Pct Asphalt, M1X =

Marshall Specific Gravity Specific Gravity Unit Weight, pof

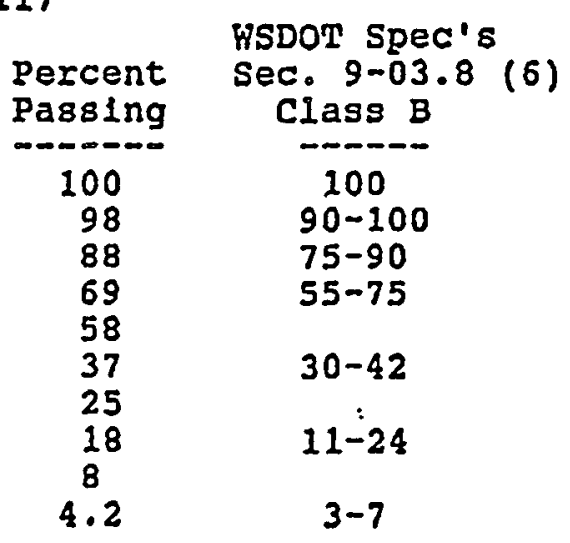

5.5

2.34

146.0

Reviewed by: 
WHC-SD-W025-RPT-001, Rev. 0

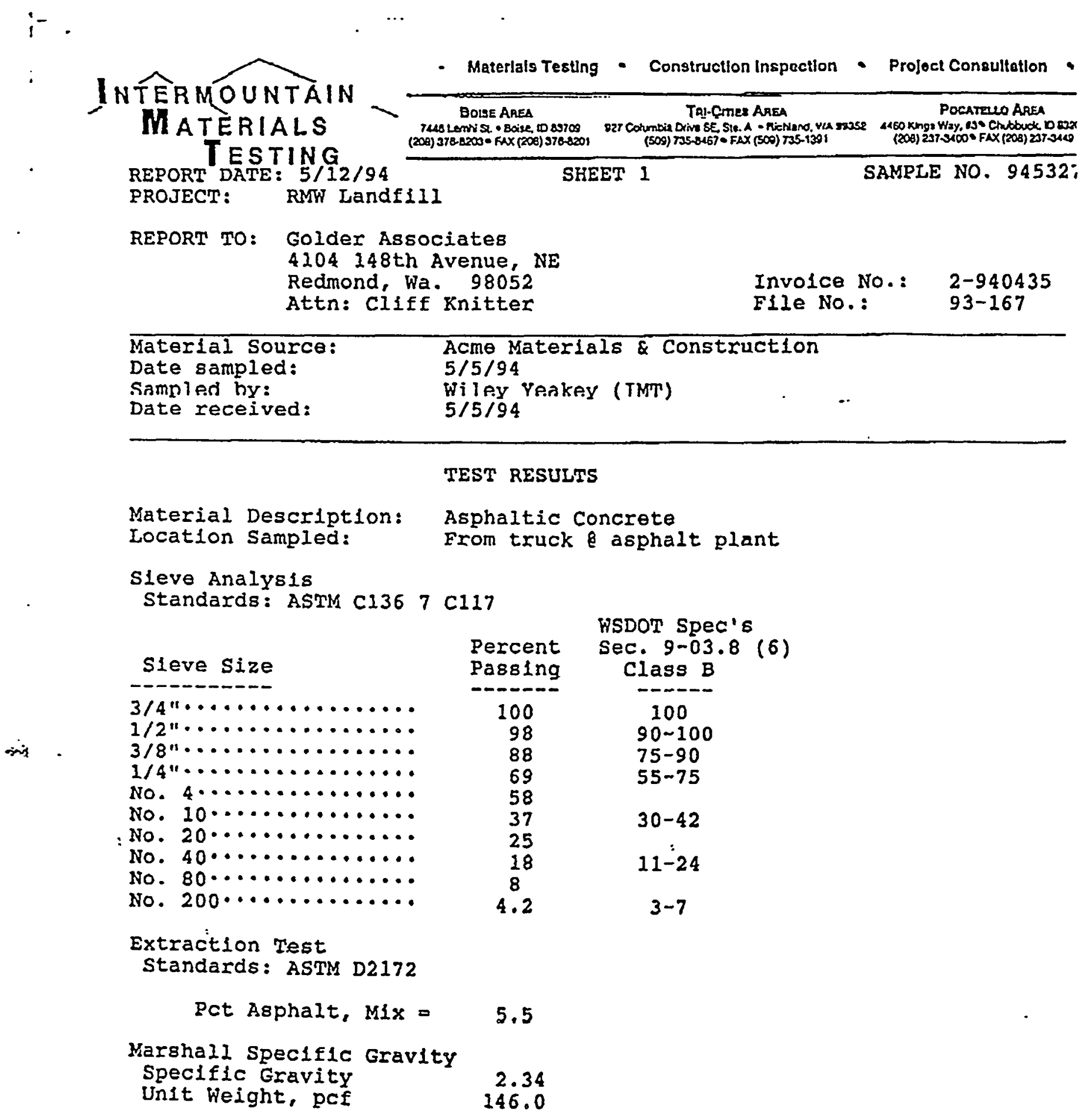

Revlewed by: 


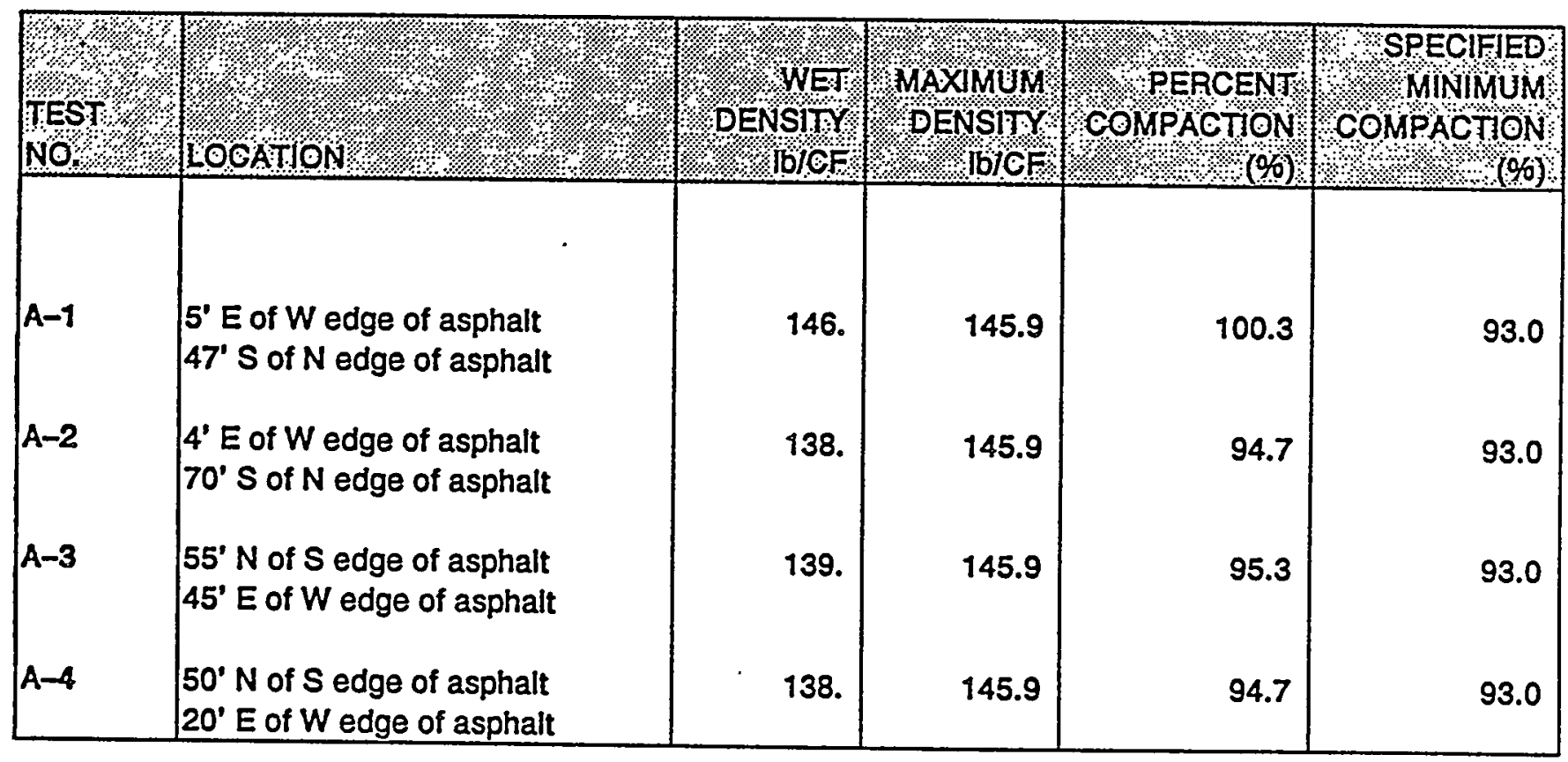




\section{FIELD MONITORING REPORT}

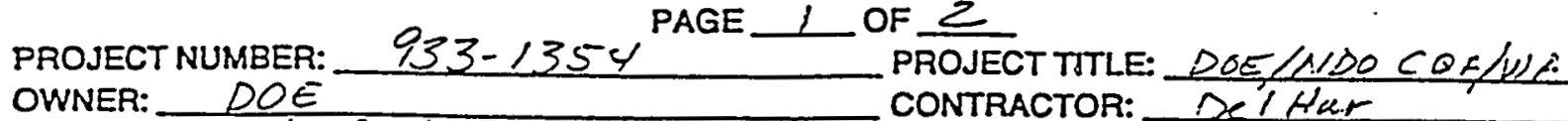

LOCATION: Hanford Reseriation

$$
\text { DATE } 5-5-94 \text { S M T W } P=s
$$

THE FOLLOWING WAS NOTED:

On site at 8:30 am. Met with Kaiser, Puton Racklen ind firnie persennel. Payina antivity was aoreed upon

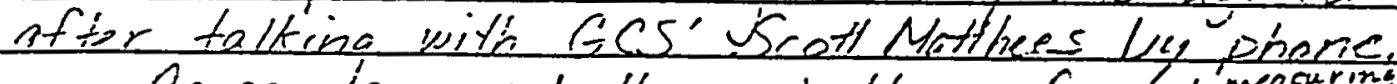
Annie jorepored the aspinalt curfecebyimsurinispine the

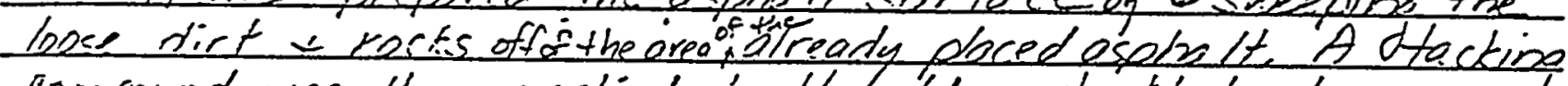

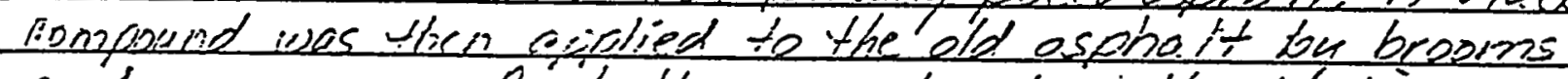

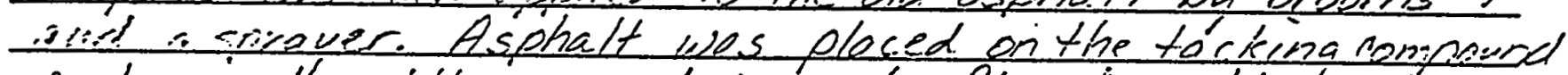
and sreed' with reaard to water flow directions. A

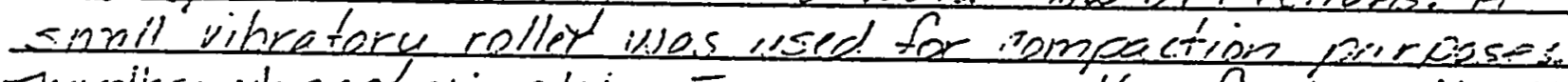

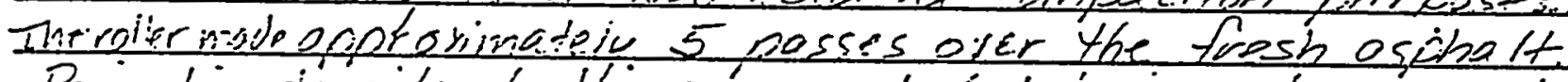

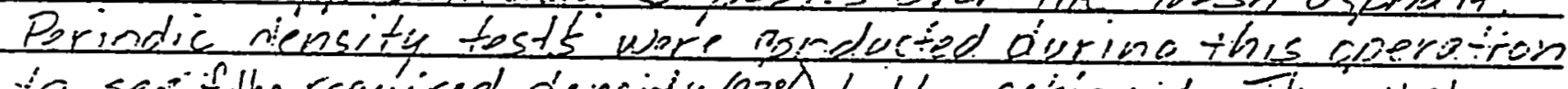
in se. if the rearied rensity $193 \%$ ) hed been achicued. The western

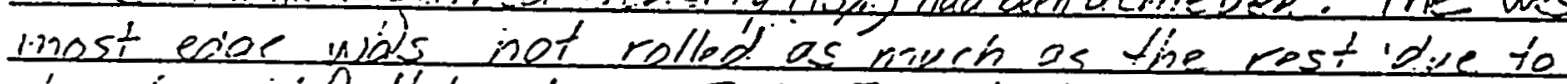

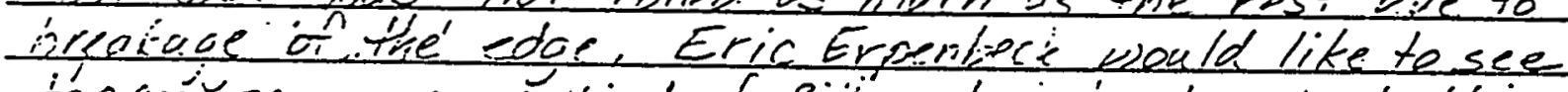

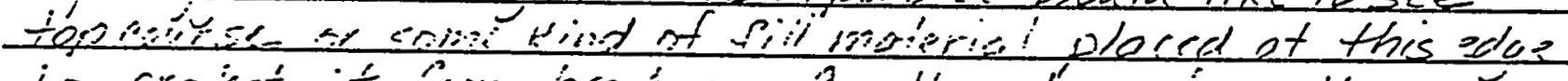

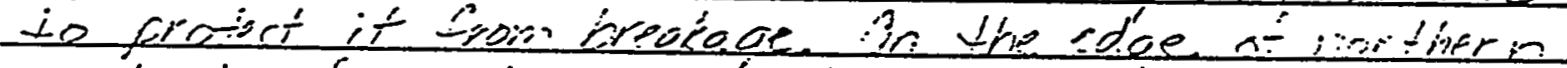

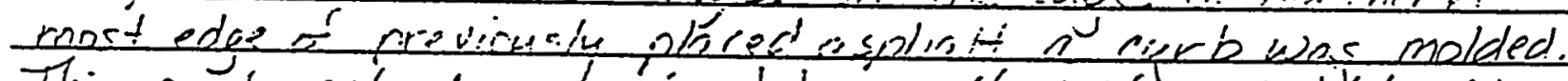
This neivh oxtond ardroximatelu 100 ' E tow and $11 / 2$ ' wids ond 4" high. A hand soreaned was ised to manipalete tire esphelt winile a flat tupe hond compartor was ucen. - .

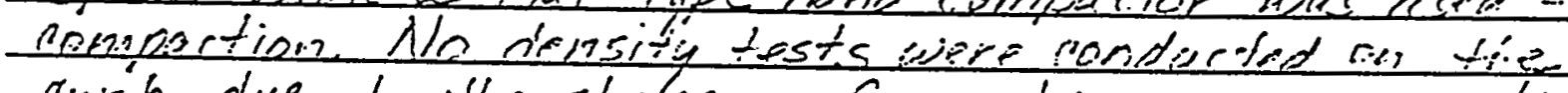
Murh due to the shepe. See diagram on next paoe. Afier Acme completed comportino aivinlts a water arainoce fest wes run. Drainece dif the under inent

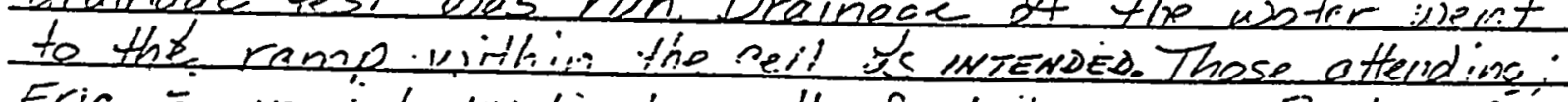

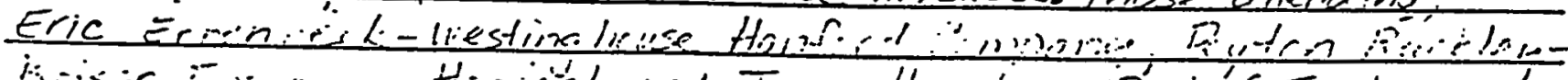

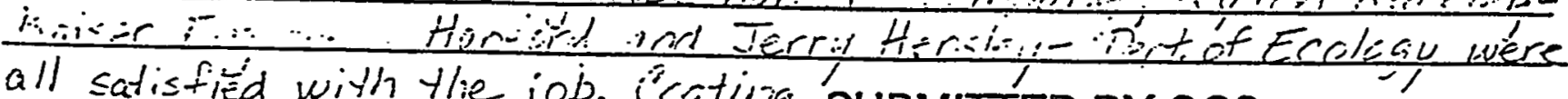
all satisfied with the job. icatiog 'sUBMITTED BY GCS
stili roceis to be afplied to surface.

ande rosos

$$
\begin{gathered}
\text { COMT. } \\
\text { NANC YONITOR } \\
\text { GOLIOER CONSTRUCTION SERVICES, INC. }
\end{gathered}
$$




\section{FIELD MONITORING REPORT}

PROJECT NUMBER:

OWNER: DOE

$933-1354$ PAGE 2
PROJECT TITLE: DOE/NOD SOA/UA CONTRACTOR: Del Har LOCATION: Han Hord Reservot line

\section{DATE $5-5-94$ S $T$ WCTF $S$}

$\because$

THE FOLLOWING WAS NOTED:

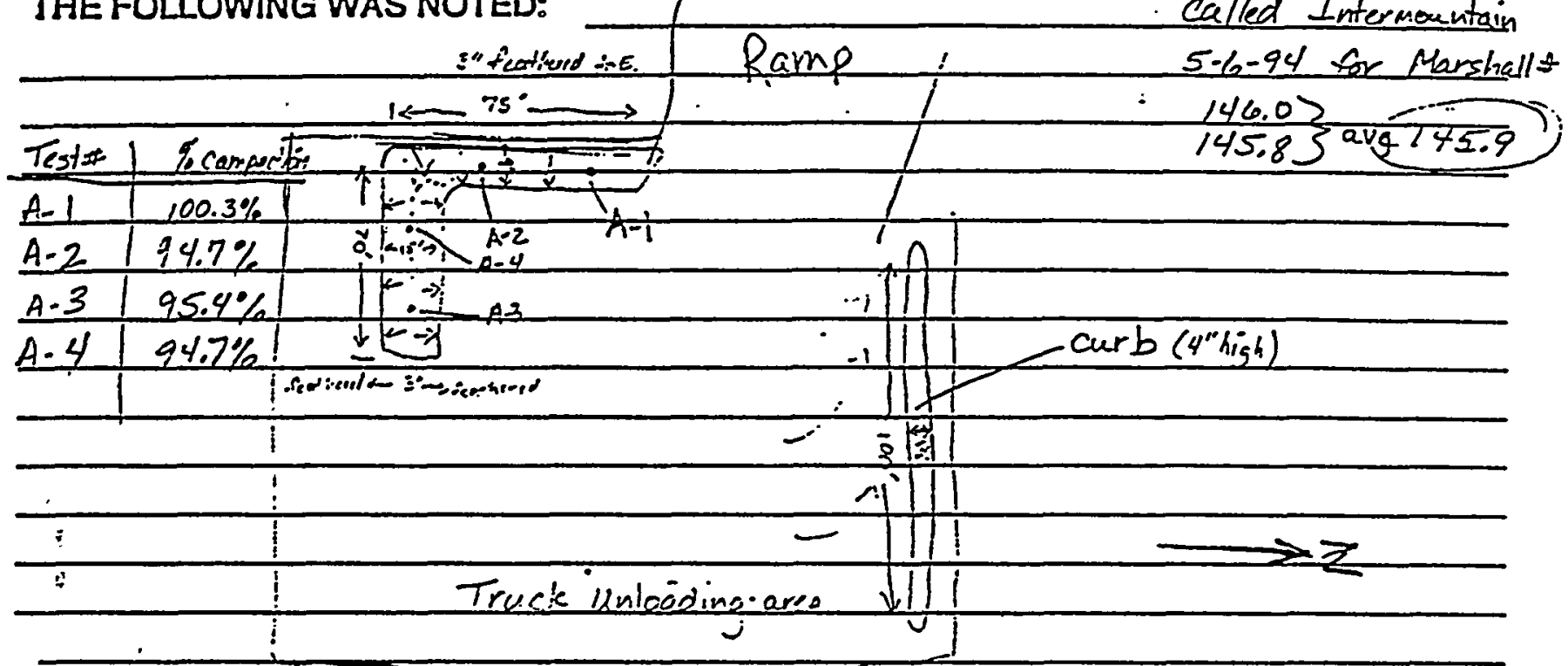

- Nucier zauce uses recalibroted after foilino stordard count.

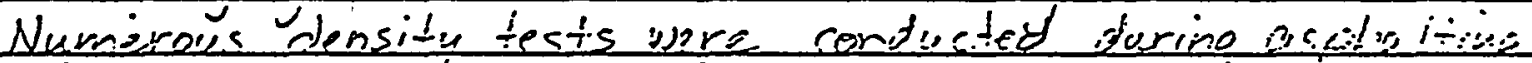

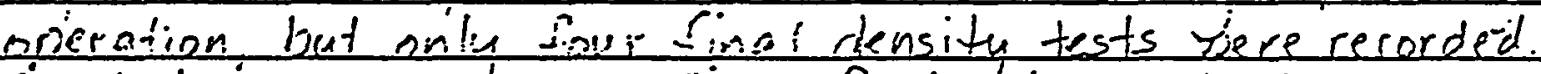
Aill tests were $\geq 93 \%$ compertion of Morshall 145.9 . Phecked the insulation se time tonk. Every thino looked correct civept for laheck value did not have indsulation covprina it Eric was also aliohtly concern about insulation residue on edae of slider on woter meter. Eric wnots the flow meter problem resolued. He also wants to know why there is increasing auncants of water in serondary liner Most likelu beroine spueced from odwix. He will momitor ste. depth. 
WHC-SD-W025-RPT-001, Rev. 0

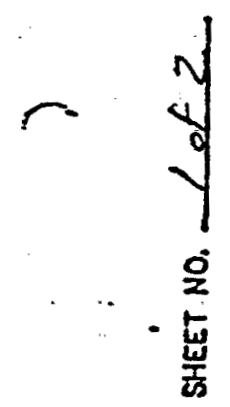

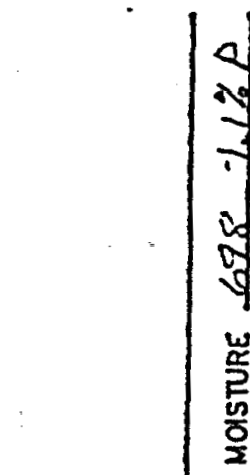

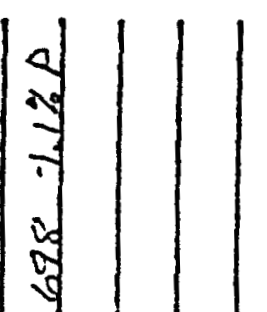

宸

|

|

|

.

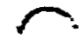

933-1354

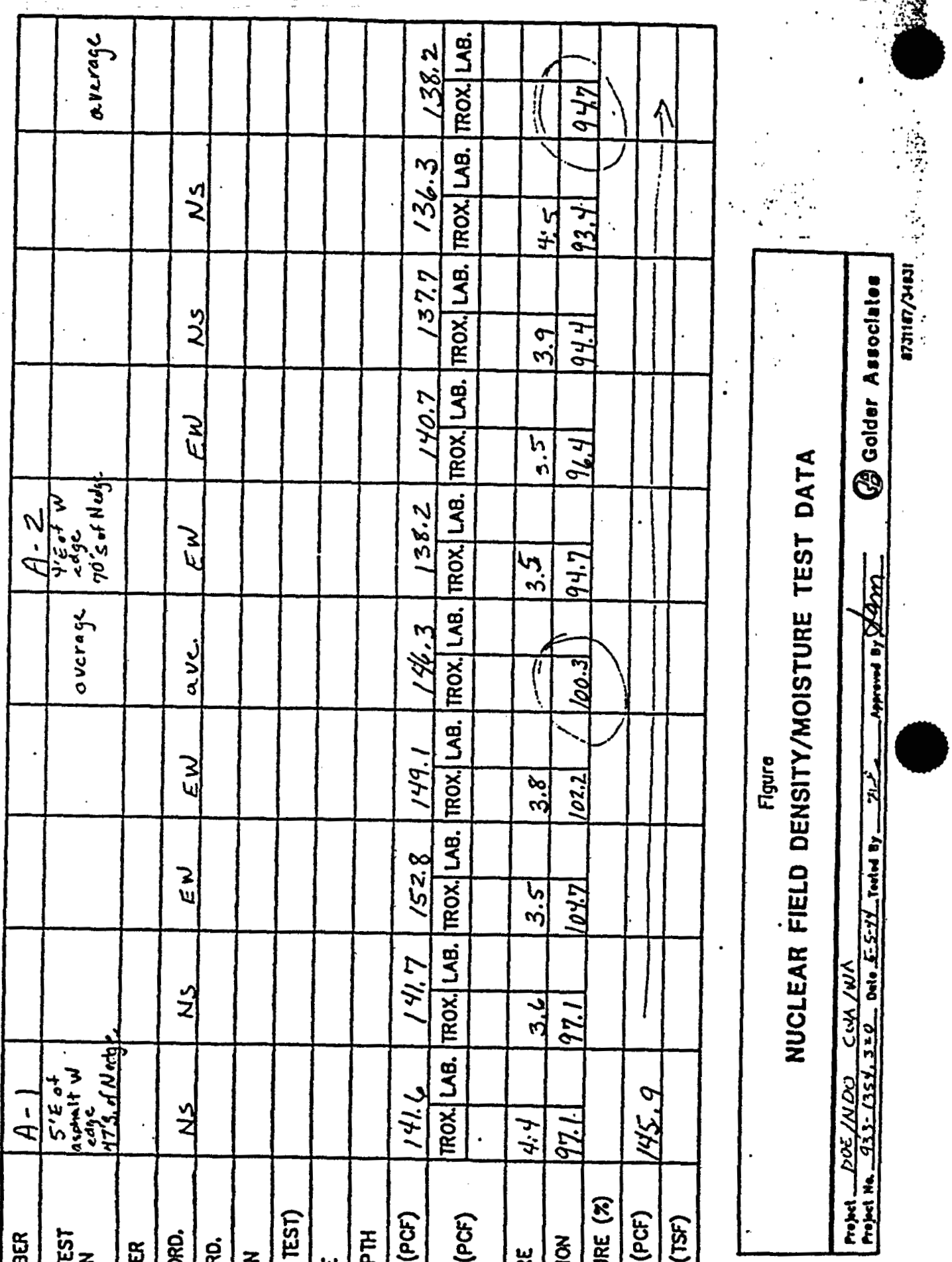

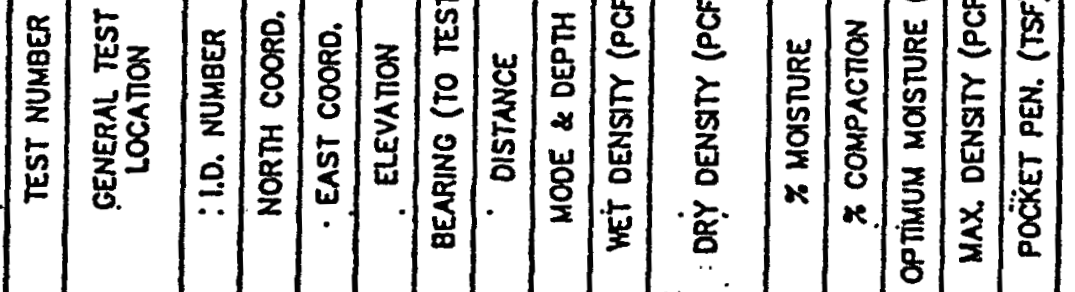

3XV15 30N383538

P-1-8 
WHC-SD-W025-RPT-001, Rev. 0

$y$
$y$
$y$
$\frac{0}{2}$
$-\frac{5}{5}$
$\frac{1}{5}$

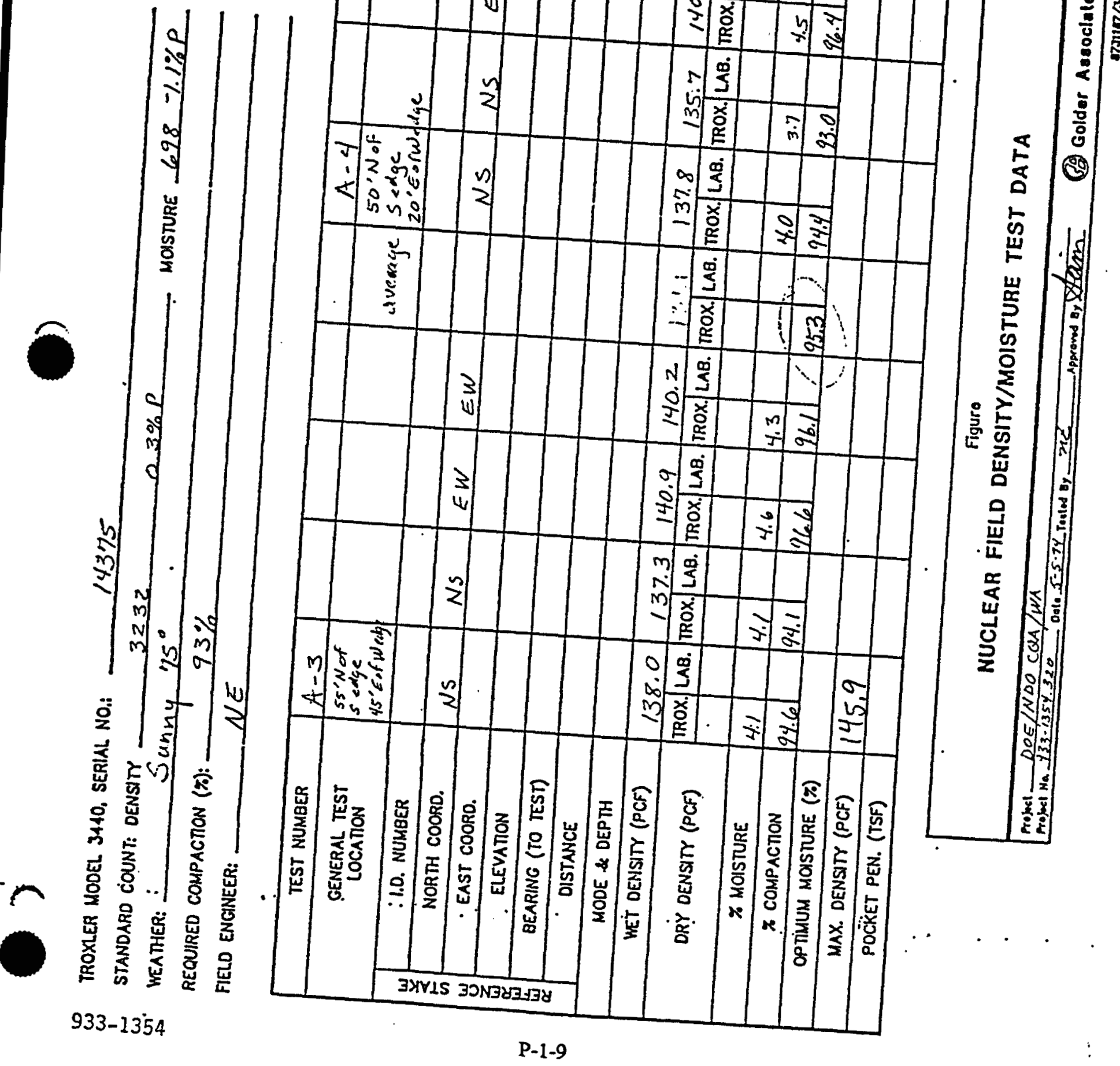


WHC-SD-W025-RPT-001, Rev. 0

APPENDIX P-2

DESIGN ENGINEER'S MEMO REGARDING TOLERANCES

$\mathrm{P}-2$ 
WHC-SD-W025-RPT-001, Rev. 0

MEMORANDUM

TO: Scott Matthees, GCS Redmond

April 17, 1994

FR: Frank Shuri, GCS Redmond Fes

RE: PROJECT W-025 LANDFILL, TRUCK UNLOADING AREA PAVEMENT ELEVATION, Job No. $933-1360$

As we discussed at the project site on April 14, 1994, the surface of the asphalt pavement in the truck unloading area is generally high. The difference between the actual and specified elevations exceeds the allowable tolerance of 0.5 inches in many cases. This is not a problem in itself, because if the base course was at the correct elevation, as indicated by the contractor's survey, the thickness of the asphalt is greater than required.

The problem occurs where the elevation of the asphalt surface would cause water to drain to areas other than the landfill. This could potentially allow contaminated material to enter the environment. Reviewing the as-built data, uncontrolled drainage could occur on the northeast and west sides of the truck unloading area. Acceptable remedies include raising the elevation of the asphait, installing a continuous curb, or other methods that might be proposed by the contractor. Any remedy must ensure that all water falling on the truck unloading area drains into the landfill. 
WHC-SD-H025-RPT-001, Rev. 0

APPENDIX Q

CONCRETE

$Q-i$ 
September 2, 1993

Certificate of conformance MB-VR standard

Master Builders Air-Entraining Admixture for Concrete

Iot: : 20770883

TO WHOY IT MAY CONCERN:

I, Brian E. Caine, Manager, Chemistry Laboratory for Master Builders, Inc., Cleveland, onio, certify:

That $M B-V R$ Standard (Master Builders Neutralized Vinsol Resin Solution) is an aqueous solution of vinsol resin that has been neutralized with sodium hydroxide, the ratio of sodiur hydroxide to vinsol resin is one part to six parts, with a nominal 13 percent by weight residue when dried at . $105^{\circ} \mathrm{C}$; and

That no calcium chloride or chloride based ingredient is used in the manufacture of $\mathrm{HB}-V R$-standard; and.

That MB-VR Standard, based on the chlorides originating from all the ingredients used in its manufacture, contributes less than 0.000068 percent $(0.68 \mathrm{ppm})$ chloride ions by weight of the cement when used at the rate of one (1) fluid ounce per 100 pounds of cement; ana

That MB-VR Standard meets the requirements of ASTM C 260-86 and Corps of Engineers' CRD C 13-86, the Standard Specifications for Air-Entraining Adrixtures for concrete, and AASHTO M154-B7I, the Interim Specification for Air-? Entraining Admixtures for Concrete.

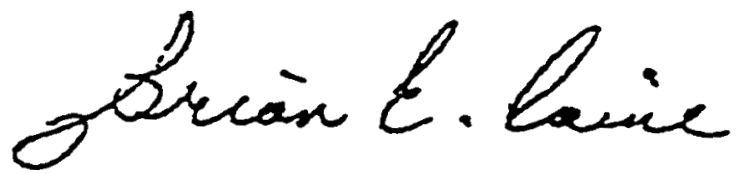

Brian $\mathrm{E}$, Caine

Ifanager, Chemistry Laboratory

Admixture Research \& Development 


\section{National Ready Mixed Concrete Association}

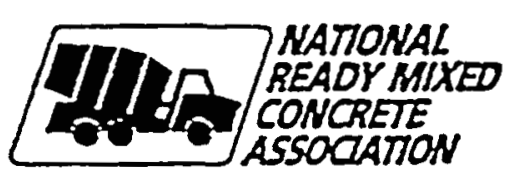

\author{
Certificate of Conformance \\ for
}

Concrete Production Facilities

THIS IS TO CERTIFY THAT

\section{Plant No. 2, Richland, Washington Acme Materials and Construction}

has boen inspected by the undersigned. registered professional. engineer for conformance with requirements of the "Check List for Ready Mixed Concrete.Production Facilities.". As of the inspection date, the facilities met requirements for production by,

\section{Truck Mixing with Automatic Batching}
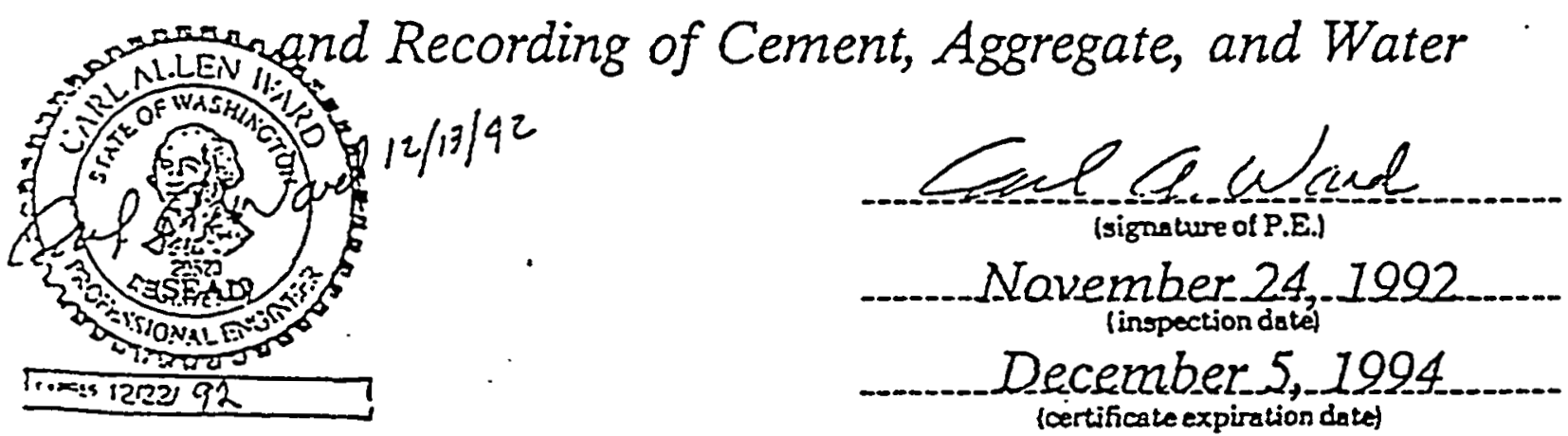

This company will maintain these facilities in compliance with the Check List requirements and will correct promptly any deficiencies which develop.

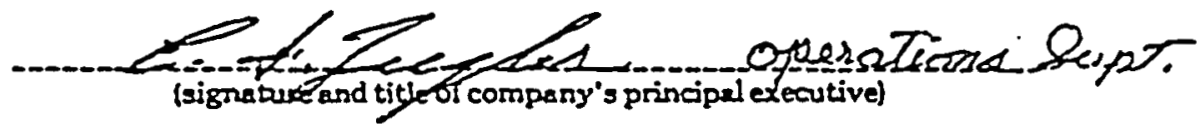

(sigrabue and titj- ff company's principal executive)

NOTICE: The Check List indicates only that plant facilities are satisiaclory for the production of concrete when properly operaled. Conformance of the concrete itself with specification requirements must be verified by usual inspection methods in accordance with sales agreements.

(- 1955.1992 
Report Lo:

Bolder Construction sor Redmond, Ha. 98052 Atin: clift knitter

Project: RMU Land OLsposal Faciifit

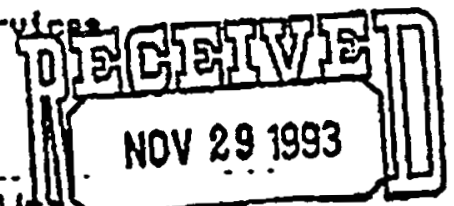

NOV 291993
Report No...

Date.........10/26/93

FIle $10 \ldots . .93-167$

Involce No.. T930979

\section{Contractor: Delhur Industries Goldar Lasodata}

Structure: Slab

Loeat10 T: Leachats Tank Pad

HATERIALS ANO MIX DATA

Reported by Acme Haterlals \& Const

$\mathrm{MLX}$ No. ....6111

Sacks/CY....5

Cement.......Ashgrove I\&II

Admlxtures. . AEA

Speclificallons
Slunp, In...
Alr Cont, $x$

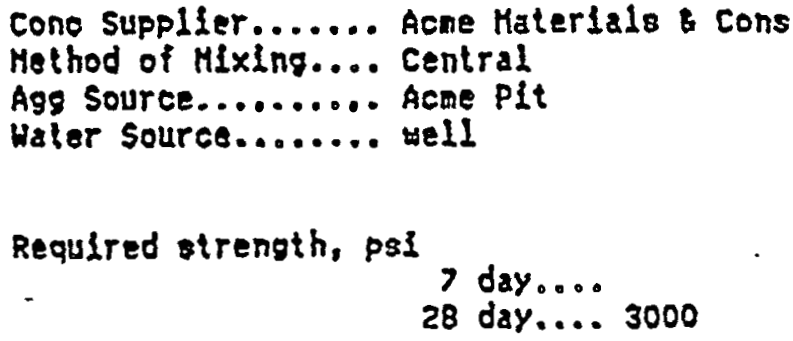

FIELD TEST DATA

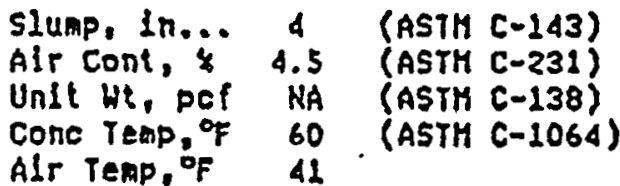

Concrete Samplied (ASTH C-172),

lested, Cylinders Cast (ASTH C-31)

Date Cast.. 10/27/93

Tlne .. 4:50 PH

By ... Dustin Back (IHT)
Truak No. .........5116

Tlekel No. ..........309189

Tline Batchad..........2:15 PH

Ho. CY represented...6.0

Hater added, ga1.....0

Cylinder Curing (ASTH C-31)

Fleld Cure, days...... I

- Temp Range, of.....

Lab cure Iron:

Date Received.........10/28/93
Sample No... ID

$-\infty-\infty$

$93792-1$

93792-2

93792-3

$93792-4$.

$\begin{array}{cc}\begin{array}{c}\text { Cyllnder } \\ \text { Dlas }\end{array} & \begin{array}{c}\text { X-5ec } \\ \text { Area }\end{array} \\ \text { (Inches) } & \text { (sq In) } \\ & 0.00 \\ & 0.00 \\ 6.01 & 28.37 \\ 6.01 & 28.37 \\ 6.01 & 28.37\end{array}$

LABORATORY TEST DATA

(ASTH C-39)

\begin{tabular}{lc} 
Total & Fracture \\
Load & Type \\
(16a) & \\
\hline & \\
65000 & cone \\
112500 & cone \\
112000 & cone
\end{tabular}

Date
Tested
$11 / 3 / 93$
$11 / 28 / 93$
$11 / 24 / 93$

Test

Test

(days)

Comp

Slr

(paI)

0

7

28

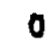

2290

3970

3950 ce: Acme Materlads \& Const.
Revieued by: Dess) R.Bsek 


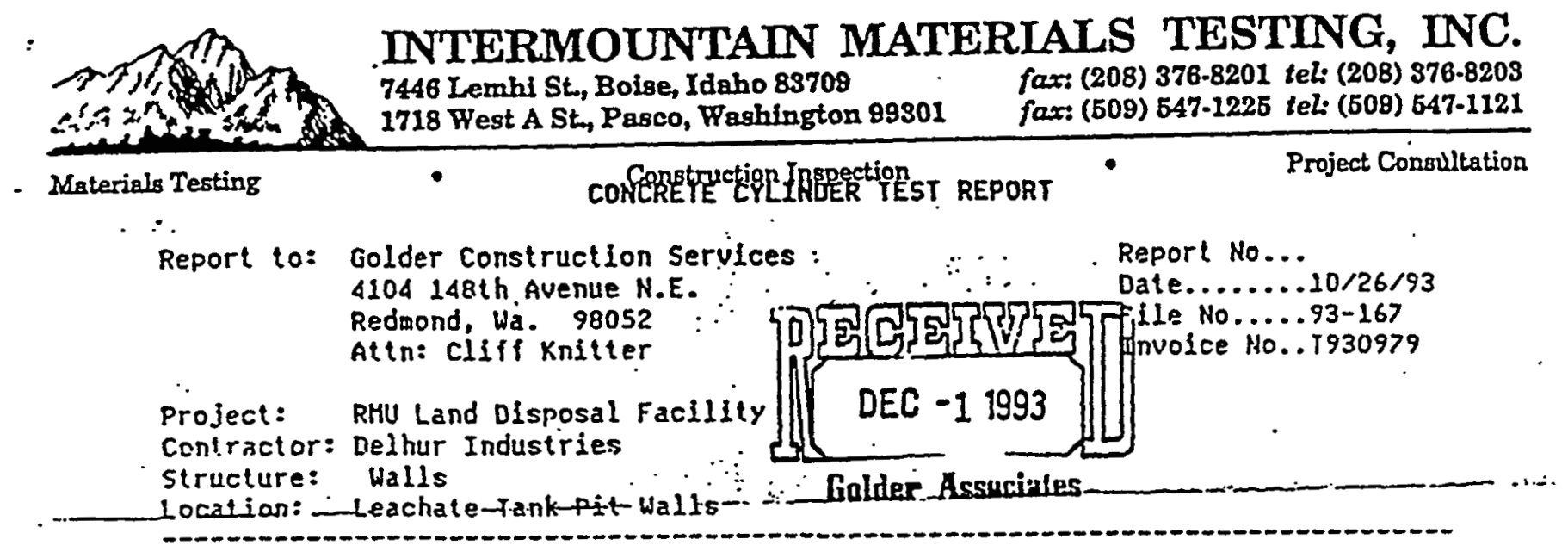

MATERIALS AND MIX DATA

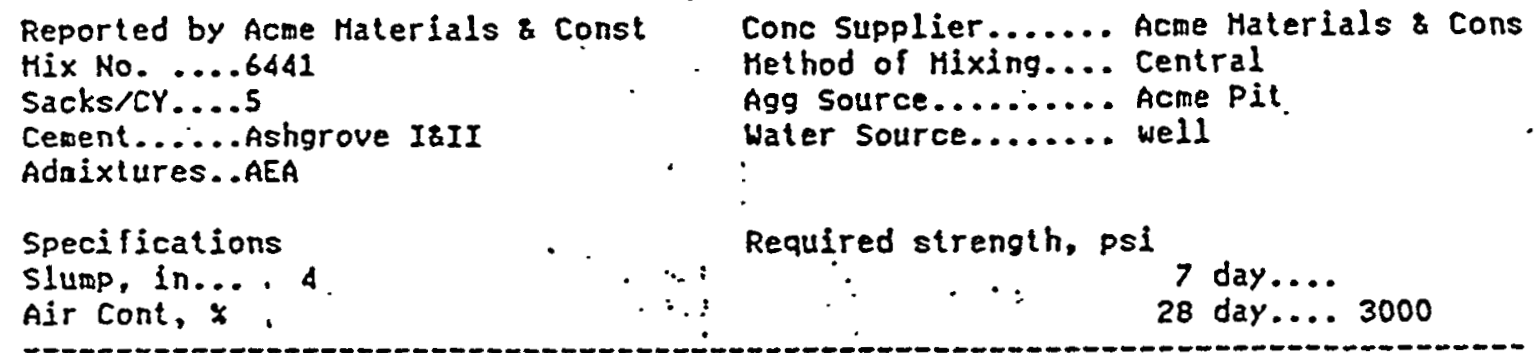

FIELD TEST DATA

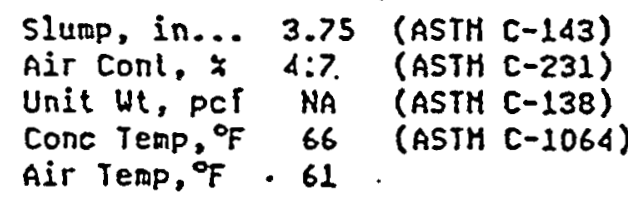

Concrete Sampled (ASTH C-172),

tested, \& Cylinders Cast (ASTM C-31)

Date Cast.. 10/29/93

Tine .. 2:05 PH

By .. Dustin Reck (IHT) $\because$

Fruck No. .........171

Tlcket No. .........309339

Time Batched.........12:18 PH

No. CY represented...8.0

Water added, gal.....2x

Cylinder Curing (ASTH C-31)

Field Cure, days......

e Temp Range, of.....

Lab cure from:

Dale Received........10/30/93
Sample Ho...

LABORATORY TEST DATA

$\underset{\text { ID }}{\text { Cylinder }}$

93793-1

$93793-2$

93793-3

93793-4
Cylinder

(inches)

(sques) in)

6.01

6.01

6.01
(ASTH C-39)

\begin{tabular}{|c|c|}
\hline $\begin{array}{c}x-\text { sec } \\
\text { area } \\
\text { (sq in) }\end{array}$ & $\begin{array}{l}\text { Total } \\
\text { Load } \\
\text { (lbs) }\end{array}$ \\
\hline & \\
\hline $\begin{array}{l}28.37 \\
28.37 \\
28.37\end{array}$ & $\begin{array}{r}89500 \\
144000 \\
145500\end{array}$ \\
\hline
\end{tabular}

Fracture
Type

\begin{tabular}{c} 
Dale \\
Tested \\
\hline 1 \\
$11 / 5 / 93$ \\
$11 / 29 / 93$ \\
$11 / 29 / 93$
\end{tabular}

\begin{tabular}{cc}
$\begin{array}{c}\text { Age } \\
\text { Tested } \\
\text { (days) }\end{array}$ & $\begin{array}{c}\text { Comp } \\
\text { Str } \\
\text { (psi) }\end{array}$ \\
\hline 0 & 0 \\
7 & 3150 \\
31 & 5080 \\
31 & 5130
\end{tabular}

xwater was added after test was taken

cc: Acme Materials \& Const.

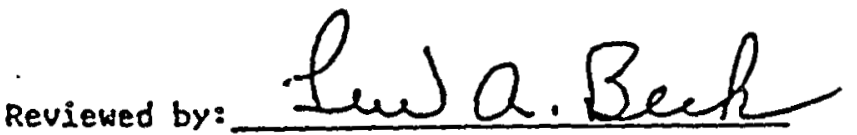




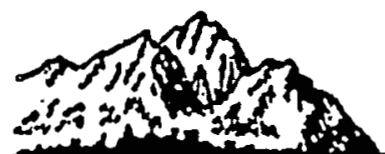

INTERMOUNTAIN MATERIALS TESTING, INC. 7446 Lombi St, Bolse, Idaho 83708 fars (208) 376.8201 tel: (208) 376.8203 1718 Weat A St., Pasco, Washlagton 99801

fars (508) 847-1225 tel: (509) 847-1121

Materials Testing

- Constraction Inspection CONCRETE CYLIHDER TEST REPORT

Report to: Goldor Construotion Serulees 4104148 th Avenue N.E. Redaonid, Hz. 98052 Attn: Cllff Knitter.

Project Consultation

Project: Riv Land Disposal Fasility

Contractor: Delhur Induatries

Structure: Stalis \& Floor slab

Location: - Tank pad stalrs \& control bidg. slab

MATERIALS AND MIX DATA

Report Ho...

Date.........12/1/93.

File No.....93-167

Invoice No..T931098

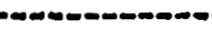

Reported by Acme Materlals Conat
HIX No. ....6452

H1X No. ....6452

Sacks/CY....5.5

Cenent.......ABhgrove IEII.

Adm2xtures. .AEA

Cone Suppller....... Acae Materials \& Cons

Method of Mixing..... Central

Agg Source........... Achs Pit

Water source......... wedl

Specilications Requlred strength, pei

Slump, in...

7 day....

Air Cont, $x$

28 day.... 4000

FIELD TEST DATA

Sluap, In... 4.5 (ASTM C-143)

Alr Cont, $*$ 7.0 (ASTH C-231)

UnIt Ut, pEF NA (ASTH C-138)

CONE TENP, ${ }^{\circ}$ 61 (ASTH C-1064)

Alr TEMP, ${ }^{\circ} \mathrm{F} \quad 46$

(ASTH $[-1064$ )

Concrete Sampled (ASTH C-172),

tested, a Cylinder Cast (ASTH C-31)

Date Cast.. 12/1/93

T1: .. 12:55 PH

By .. Durtin Beck (IHT)

Truek No. ..........2198

Tlaket No. ...........310808

Tine satched..........11:23 AH

No. CY represented...5.0

Nator added, sal.....3

Cylinder Curing (ASTM C-31)

Fleld Curs, days......l

Q Teap Range, "F....66-80

Lab cure fron:

Date Recelved.........12/2/93

\begin{tabular}{|c|c|c|c|c|c|c|c|}
\hline \multicolumn{2}{|c|}{ Sample No... } & \multicolumn{3}{|c|}{$\begin{array}{c}\text { LABORATORY TEST DATA } \\
\text { (ASTH }(-39)\end{array}$} & \multirow[b]{2}{*}{$\begin{array}{l}\text { Date } \\
\text { Teated }\end{array}$} & \multirow[b]{2}{*}{$\begin{array}{l}\text { Age } \\
\text { Tested } \\
\text { (dxys) }\end{array}$} & \multirow[b]{2}{*}{$\begin{array}{c}\text { Conp } \\
\text { Str } \\
(p \otimes I)\end{array}$} \\
\hline $\begin{array}{l}\text { Cylinder } \\
\text { I0 }\end{array}$ & $\begin{array}{l}\text { Cyllnder } \\
\text { olan } \\
\text { (Inches) }\end{array}$ & $\begin{array}{c}x-5 e c \\
\text { Area } \\
\text { (sa in) }\end{array}$ & $\begin{array}{l}\text { Total } \\
\text { Load } \\
\text { (1bs) }\end{array}$ & $\begin{array}{c}\text { Fracture } \\
\text { Type }\end{array}$ & & & \\
\hline $\begin{array}{l}93892-1 \\
93892-2 \\
93892-3 \\
93892-4\end{array}$ & $\begin{array}{l}6.01 \\
6.01 \\
6.01 \\
6.01\end{array}$ & $\begin{array}{l}28.37 \\
28.37 \\
28.37 \\
28.37\end{array}$ & $\begin{array}{l}113500 \\
118500 \\
171500 \\
173000\end{array}$ & $\begin{array}{l}\text { cone } \\
\text { cone } \\
\text { cone } \\
\text { cone }\end{array}$ & $\begin{array}{l}12 / 8 / 93 \\
12 / 8 / 93 \\
12 / 29 / 93 \\
12 / 29 / 93\end{array}$ & $\begin{array}{l}7 \\
7 \\
28 \\
28\end{array}$ & $\begin{array}{l}4000 \\
4180 \\
6050 \\
6100\end{array}$ \\
\hline
\end{tabular}

cc: Acme Haterlals \& Construction

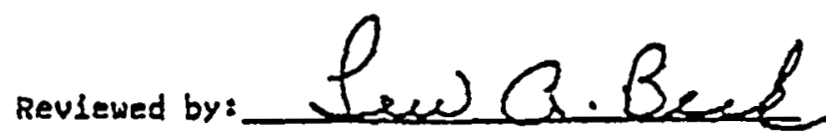

933-1354

- Q-5 


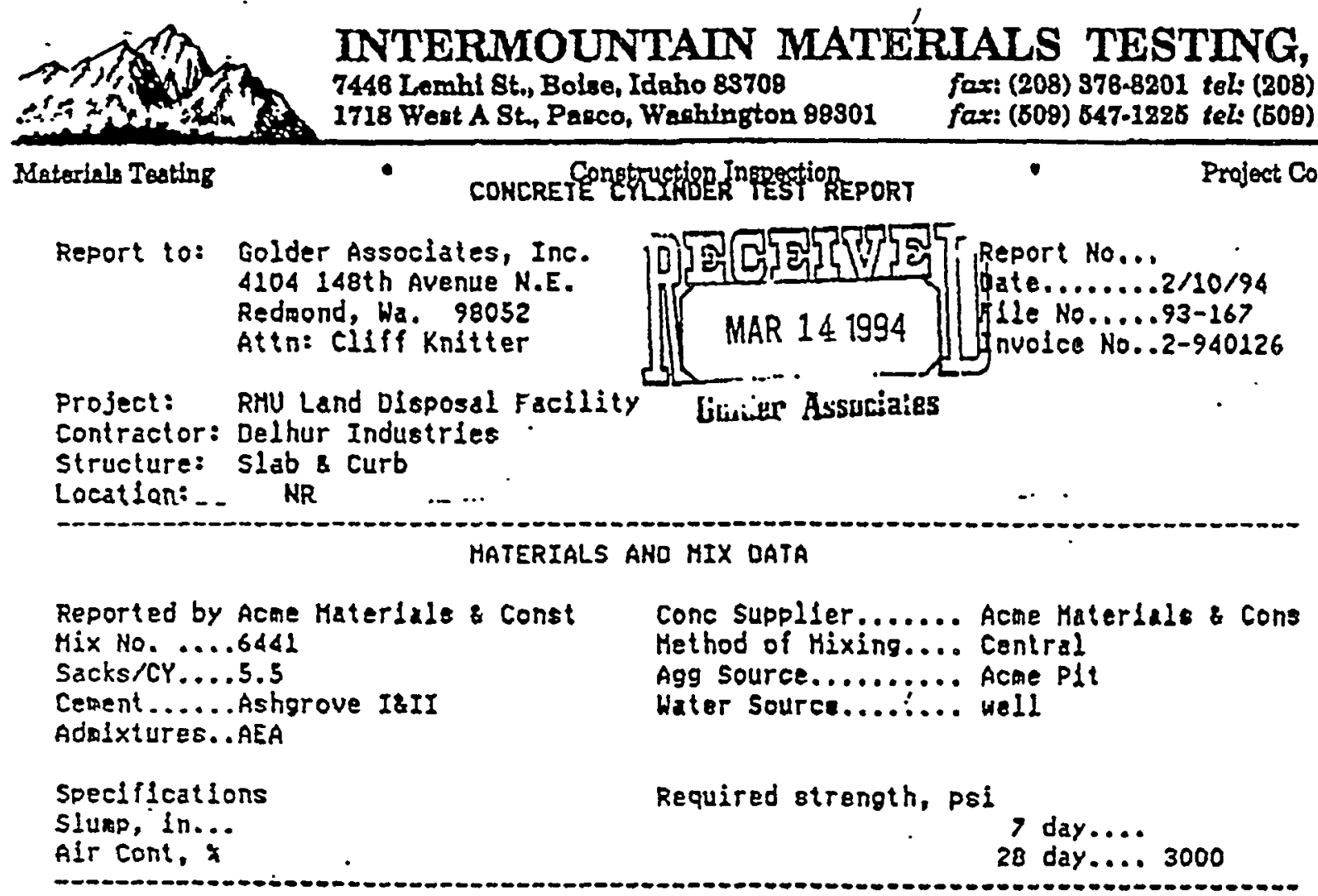

FIELD IEST DATA

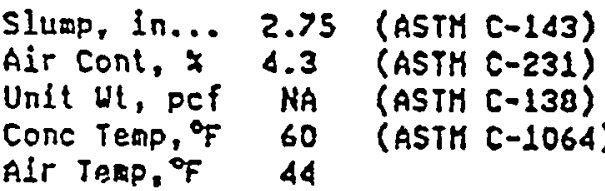

Concrete Samoled (ASTM C-172). tested, \& Cylinders Cast (ASIM $C_{2}=31$ ). Date Cast.. 2/10/94 Time .. 12:15 AM By .. Dustin Beck (IHT)
Truck No. ..........5116

Tleket No: .........323791

Time Batched........9:24 AH

No. CY represented...5.5

Hater added, gal......d

Cylinder Curing (ASTH C-31)

Fleld cure, days......

a Temp Range, of....60-80

Lab cure fron:

Date Recelved........2/11/94

\begin{tabular}{|c|c|c|c|c|c|c|c|}
\hline ample No.. & & & $\begin{array}{l}\text { ORATORY T } \\
\text { (ASTH C }\end{array}$ & ST OATA & & & \\
\hline $\begin{array}{c}\text { Eylinder } \\
\text { ID }\end{array}$ & $\begin{array}{l}\text { Cylinder } \\
\text { Olan } \\
\text { (Inches) }\end{array}$ & $\begin{array}{c}x-\text { Sec } \\
\text { Ares } \\
(s q \text { in) }\end{array}$ & $\begin{array}{l}\text { Total } \\
\text { Load } \\
\text { (1bs) }\end{array}$ & $\begin{array}{c}\text { Fracture } \\
\text { Type }\end{array}$ & $\begin{array}{l}\text { Date } \\
\text { Tested }\end{array}$ & $\begin{array}{l}\text { Age } \\
\text { Tested } \\
\text { (days) }\end{array}$ & $\begin{array}{r}\text { Conp } \\
\text { Str } \\
(p 81)\end{array}$ \\
\hline $\begin{array}{l}94001-1 \\
94001-2 \\
94081-3 \\
94081-4\end{array}$ & $\begin{array}{l}6.01 \\
6.01 \\
6.01 \\
6.01\end{array}$ & $\begin{array}{l}28.37 \\
28.37 \\
28.37 \\
28.37\end{array}$ & $\begin{array}{r}89000 \\
.113500 \\
137000 \\
139000\end{array}$ & $\begin{array}{c}\text { - cone } \\
\text { cone } \\
\text { cone } \\
\text { cone }\end{array}$ & $\begin{array}{l}2 / 17 / 94 \\
2 / 24 / 94 \\
3 / 10 / 94 \\
3 / 10 / 94\end{array}$ & $\begin{array}{l}7 \\
14 \\
28 \\
28\end{array}$ & $\begin{array}{l}3140 \\
1000 \\
1830 \\
4900\end{array}$ \\
\hline
\end{tabular}

cc: Acwe Materials : Construction

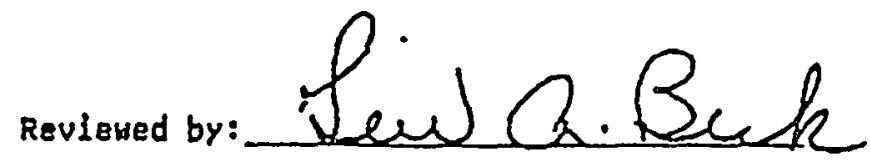




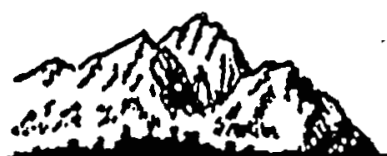

INTERMOUNTAIN MATERIALS TESTING, FNC. 7446 Lamhl St, Bolse, Ideho 88709 for: (208) 376-8201 tel: (208) 376.820s 1718 West A St., Pasoo, Waahington 98301 fart (509) 647.1228 tel: (808) 847-1121

Materials Tasting

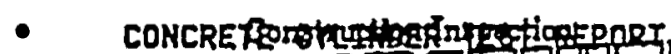

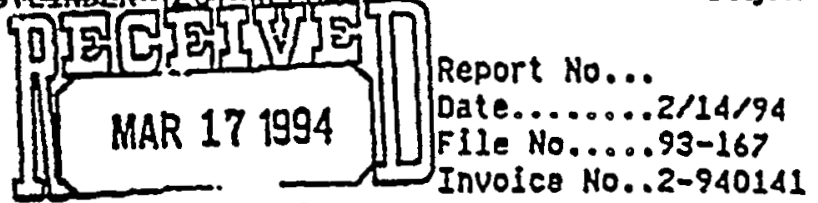

Report to: Golder Associales, Inc. \$104 \$4Bth Avenue K.E. Redrond. Ha. 98052 Attn: Cllft Knitter

Project: RHW Landfill Disposal FaciLity

Contractor: Delhur Industries

structure: Wall

Location: Slope Riser Crest wall

MATERIALS ANB MIX DATA

Reported by Acine Materlals Const Mix No. ...668d1

Sacks $/ C Y \ldots . .5,5$

Cement.......Ashgrove I\&II

Adoixtures. . AEA

Specifications

slump, in...

Air Cont, $x: \cdots$.
Conc supplier....... Acne Materlals \& Cons Hethod of Mixing..... Central

Agg Source.......... Acne PIt

Hater Source......... well

Required sirength, poi

7 day....

28 day.... 3000

FIELD TEST DATA

$\begin{array}{lll}\text { Slump, in... } & 4.25 & \text { (ASTH C-143) } \\ \text { Alr Cont, \% } & 5.2 & \text { (ASTH C-231) } \\ \text { Unit wt, pcf } & \text { NA } & \text { (ASTH C-138) } \\ \text { Conc Temp: of } & 59 & \text { (ASTH C-1064) } \\ \text { Alr Teap, } \mathrm{F} & 48 & \end{array}$

Concrete Sampled (ASTH C-172).

tested, cyllnders Cást (AsTr C-31)

Date Cast.. 2/14/94

iine .. 4:35 PM

By ... Dustin Beck (IMT)
Truck No..........5116

Ticket No: .........323965

TIme 8atched.........3:10 PH

No. CY represented...1.0

Water added, gal.....

Cylinder curing (ASTM C-3I)

Field cure, days......1

- Témp Range, of....60-80

Lab cure fron:

Oate Received........2/15/94
Sample No...

Cylinder

$94087-$

$940 B 7-2$

94087-3

$94087-4$

\begin{tabular}{cc}
$\begin{array}{c}\text { Cylinder } \\
\text { Oian }\end{array}$ & $\begin{array}{c}\text { X-Sec } \\
\text { Area }\end{array}$ \\
(Inches) & (89 in) \\
\hline 6.01 & 28.37 \\
6.01 & 28.37 \\
6.01 & 28.37 \\
6.01 & 28.37
\end{tabular}

\section{LABORATORY TEST DATA}

(ASTH C-39)

Total
Load
$(165)$
-60000
77500
105000
105500

Fracture
Type
cone
cone
cone
cone

\begin{tabular}{ccc}
$\begin{array}{c}\text { Date } \\
\text { Tésted }\end{array}$ & $\begin{array}{c}\text { Age } \\
\text { Tested } \\
\text { (days) }\end{array}$ & $\begin{array}{c}\text { Comp } \\
\text { Str } \\
\text { (p8i) }\end{array}$ \\
\hdashline $2121 / 94:$ & 7 & 2110 \\
$2 / 28 / 94$ & 14 & 2730 \\
$3 / 14 / 94$ & 28 & 3700 \\
$3 / 14 / 94$ & 28 & 3720
\end{tabular}

cc: Acne Haterlals \& Construction

$933-1354$

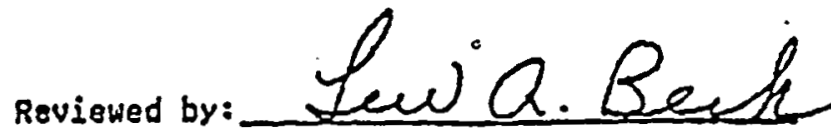


WHC-SD-W025-RPT-001, Rev. 0

\section{APPENDIX R}

LEACHATE TEMPORARY STORAGE TANK

$$
R-i
$$


WHC-SD-W025-RPT-001, Rev. 0

APPENDIX R-1

MANUFACTURER'S QC DOCUMENTATION

$R-1$ 
FORM U.1A MANUFACTURERS' DATA REPORT FOR PRESSURE VESSELS (Altemate Form for Singlo Chamber, Completely Shop.Fabricated Vessels Only) is requirod by the provistonis of the ASHE Code rules, Section VIII. DIrislon 1

7. Manufacturod and certitiod by PULLITY TLKK \& coxSTRUCTIOK CC. 16!11 SE. 362XO OR., SAKOY, OR ITCS5

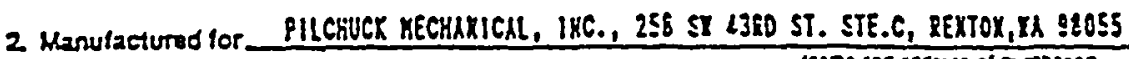

3 Logtion of Installation HAXFORO XUCLELR RESERYLTIOR, IL

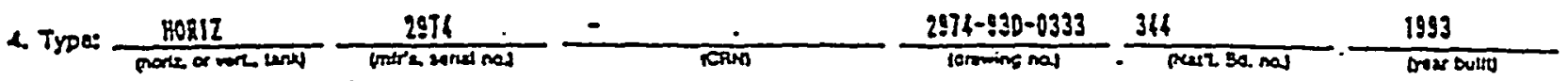

5. The chemleal and physleal propertles of all parts meet the requirements of material specifieations of the ASME BOILER AND PRESSURE VESSEL CODE The desigr, construction and workmanship conform 10 ASME Code. Section VII, Division 1: 1992

\begin{tabular}{|c|c|c|c|c|c|c|c|c|c|c|}
\hline & $19: 2$ & & & & & & & & & rean \\
\hline & Heoonat buted & $r_{\infty}$ & 6 cesentes & & & & t200ecies 200 & 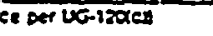 & & \\
\hline She & Alt $5 h-36$ & & 0.3125 & & 0 & & & $-11.375^{\circ}$ & $35^{\prime} \cdot 0$ & \\
\hline & $\tan 32120$ & $\propto m a$ grsel & inom the & $\operatorname{ces} \cos x$ & $\mathrm{ked}$ & $10=\sin \theta$ & & 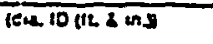 & $\operatorname{sens}$ & in toveretg (1) 6 ind \\
\hline 7. Sez & ms DBL BUTT & & roks & 10 & $\div$ & 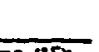 & & DEL \&UTा & DORE & 1 \\
\hline 8. Hea & 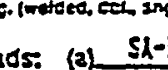 & $\begin{array}{l}6 \text { hen oulo } \\
15-70\end{array}$ & GAT lados of lurrs & 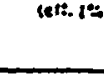 & & $m=r^{\circ}$ & $S h-515-T E$ & 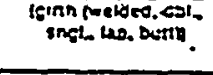 & 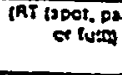 & tha ct coursest \\
\hline & & & mal7. 12000, nn & croets & & & & Imat. (SODOC & no sriesed & \\
\hline & $\begin{array}{l}\text { Lecallon rop } \\
\text { Eerom enotil. }\end{array}$ & $\begin{array}{l}\text { Mininum } \\
\text { indelums }\end{array}$ & $\begin{array}{l}\text { Corrosion } \\
\text { Nlowence }\end{array}$ & $\begin{array}{l}\text { Crown } \\
\text { ruevis }\end{array}$ & $\begin{array}{l}\text { Koncile } \\
\text { Pyours }\end{array}$ & Sthoseds & 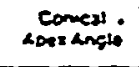 & $\begin{array}{c}\text { Mermis=naneal } \\
\text { Raews }\end{array}$ & Esimeter & 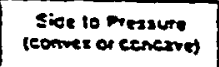 \\
\hline (a) & EXO & 0.2736 & 0 & $99^{2}$ & 81 & & & & & COXCAYE \\
\hline (b) & EXD & 0.2734 & 0 & $90^{2}$ & 58 & & & & & GXCAYE \\
\hline
\end{tabular}

If removable. belts usec (deseribe oiner lasteningst:

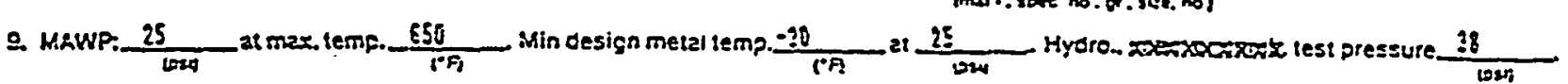

10. Nozles. Inspection and safery valve openings:

\begin{tabular}{|c|c|c|c|c|c|c|c|c|}
\hline Inis: culportasingeles & 100 & cas. & Tree & sent? & Sincom & $\begin{array}{c}\text { Aemigoepment } \\
\text { Malt }\end{array}$ & A:senen & becerion \\
\hline IXIET & 1 & 4 & $1: 98550$ & $515: 05$ & .519 & I. YOKE & IE!O & SMELI! \\
\hline YEXI & 1 & 4 & IHDSTME & $S h 59 E$ & 510 & TXORE & $I E L O$ & ShE!l \\
\hline LEYEL GREE & 3 & 1.5 & SETEFIE & Shlo: & 36008 & XClE & IEID & SHELI \\
\hline LEYEL STliCH & 1 & 5.75 & SECPLC & 54105 & 30008 & IXOXE & $I E: D$ & SWELL \\
\hline
\end{tabular}

12. Femarks: Manufaclurers" Panial Eata Fesons Dropeny isentulied ane signec by Commissioned Inseec:ors nave been furnishee lor the lollewirg items of the seoort:

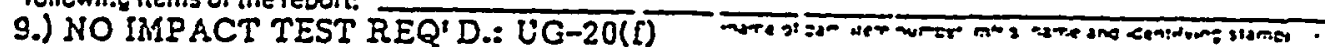

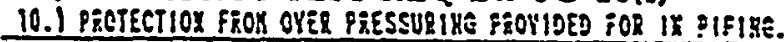

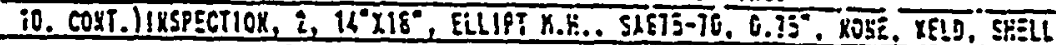

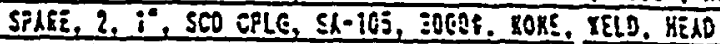

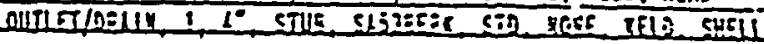

CERTIFICATE OF SHOP CCMPLIANCE

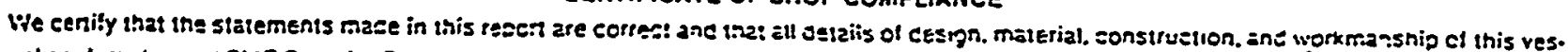

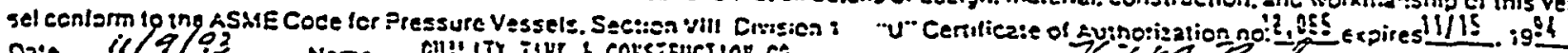

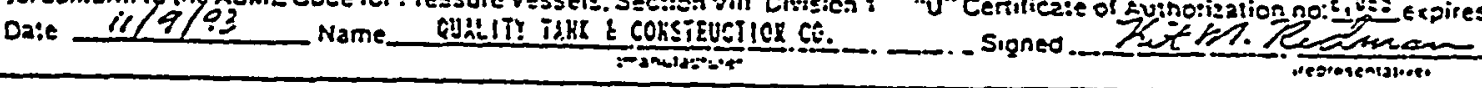

CERTIFICATE OF SHOP INSPECTION

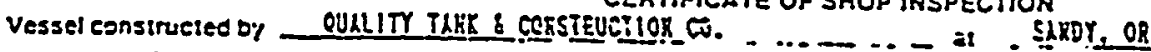

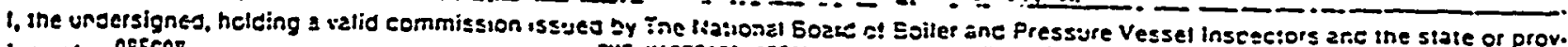

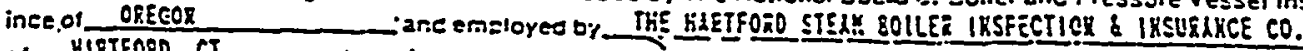

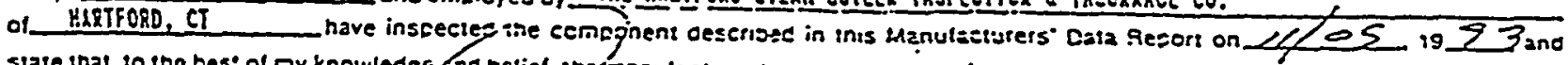

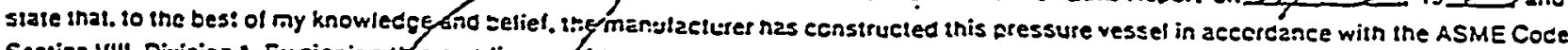
Sestion VII!. Division 1. Ey signing yhis certlicate peither the inspector nor nis exployer makes ariy watranty. expressed or implice. concern.

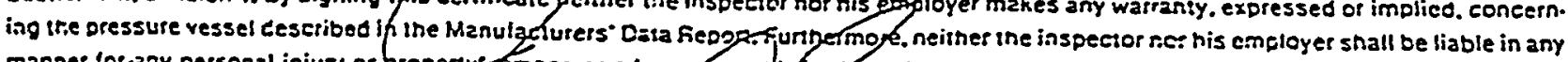

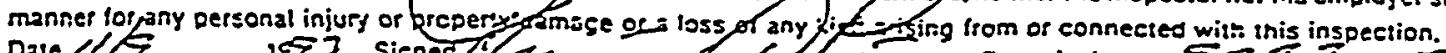

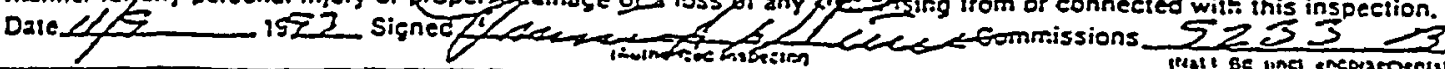

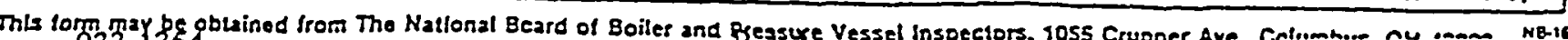
$933-1354$ 
WHC-SD-W025-RPT-001, Rev. 0

APPENDIX R-2

REGISTERED PROFESSIONAL ENGINEER'S STATEMENT

$\mathrm{R}-2$ 
HHC-SD-W025-RPT-001, ReV.0

APPENDIX R-2

ENGINEERS STATEMENT ABOUT LEACHATE STORAGE TANK

This Appendix presents the results of our review of the design of the Leachate Storage Tank. The review was performed in general accordance with the requirements of WAC 173-303-640 3(a). The review consisted of a review of the tank and foundation design, discussions with the Tank designers Quality Tank \& Construction (QTC), and an assessment of the suitability of the design. The overall design and function of the Leachate Storage Tank was reviewed as depicted on Golder Associates, Inc. (GAI) Design Drawing H-2-131585, dated 4/2/90 and Drawing H-2-131585 by QTC dated $8 / 26 / 93$. The GAI drawing showed the overall design concept while the QTC drawing showed the detailed design of the tank structure.

Our comments on the design include:

- The tank foundation is supported on naturally occurring eolian sand which is more than adequate to support the light bearing loads of the tank. The tank foundation has an appropriate allowable bearing pressure and an adequate depth for frost penetration.

- The tank was pressure tested to 25 psi without leaking and will not be pressurized in actual service.

- QTC has a certification from the American Society of Mechanical Engineers (ASME) to design and construct pressure vessels. The structural design was completed by QTC in accordance with the ASME Code, Section VIII, Division 1. Based on our review, their design meets the intent of the GAI design and the requirements of the ASME Code for these types of tanks.

- An HDPE lined concrete collection basin is located under the tank such than the entire volume of the tank could be stored in the very unlikely case of a tank failure or the need to drain the tank.

- The tank is above ground and will not be in contact with any soil. It will contain liquid leachate from the adjacent landfill and has been epoxy coated. Based on 9090 testing of a simulated leachate, the leachate will contain primarily metallic salts. We contacted the 
epoxy supplier and confirmed that the epoxy used to coat the tank is highly resistant to the type of leachate anticipated.

- The construction of the tank foundations and tank installation was subjected to full CQA monitoring by GCS.

Based on this review the tank design meets the design requirements of the project and is suitable for storing the anticipated leachate. The review and the Appendix section was prepared by:
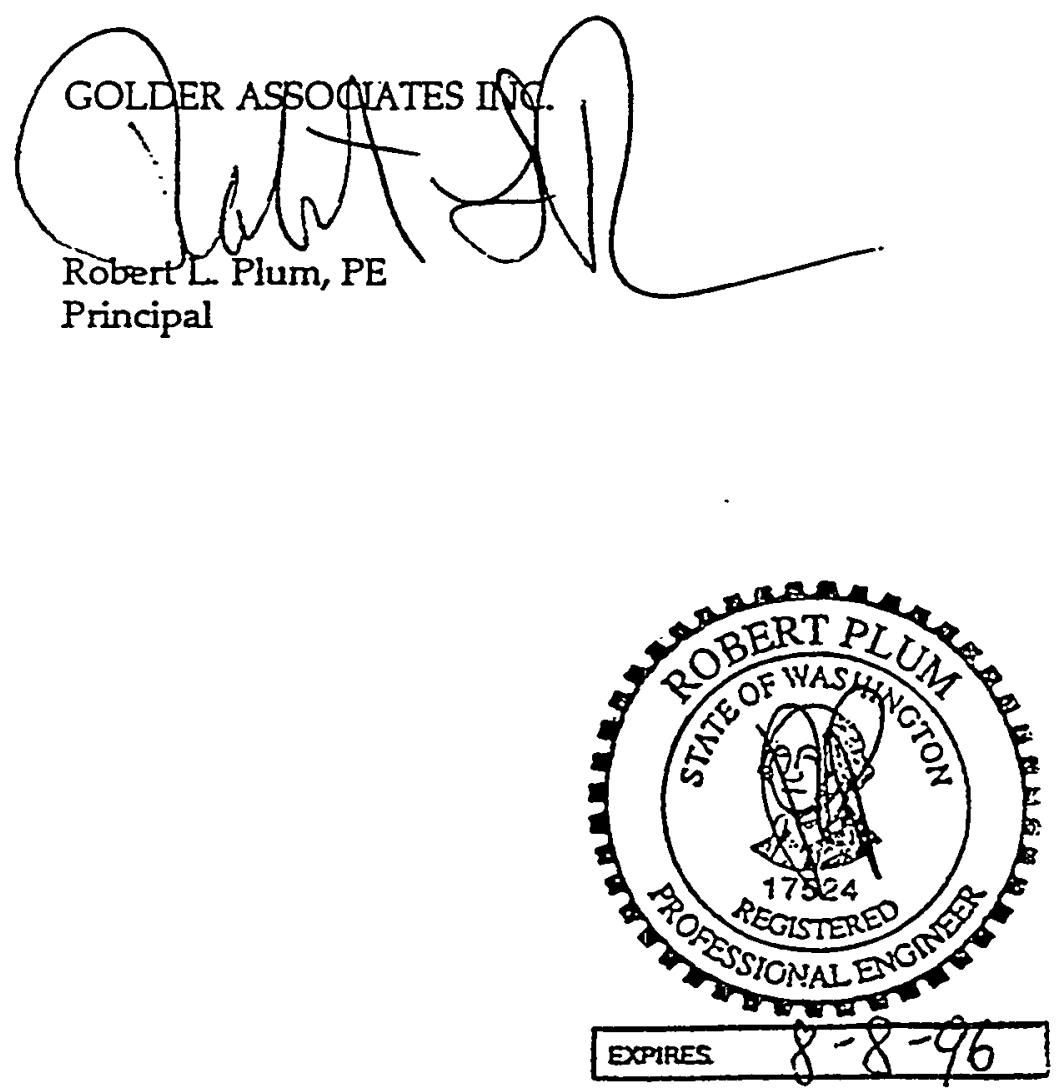
WHC-SD-W025-RPT-001, Rev。O

APPENDIX R-3

SELF PRIMING PUMP TESTING

$R-3$ 


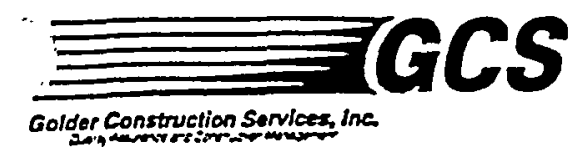

Golder Construetion Services, inc
PRESSWRE TEST-DSTHARGE SUET PIPE FOR SELE PRIMUNG PUMP Joo No. 933-1354| Made Dy $\left.\right|_{\text {and }}$ Date 3-15Rel.
Sheet of

TESTING NNCLUDES 3 " PRIMNNG PUNP DSSCHARGE PIPE) FROM SELF PRIMINE PUMP TO VALVE .ON. TANK SIDE OF CREST WALL.

\section{TME}

1100

1130

1300

\section{EVENT}

PILCHUCK MECHANHCAL INSTALLED DLANKS ON EACH END OE PIPE TO BE TESTED. PIPE WAS FILLED W/ HZO.

PILCHULK PRESSURIZED PIPE TO 5\% 5 PSI. VERY MINOR LEAK@ BLANK END NEPR CREST WALL, SO PILCHUCK TIGHTENES CONNECTION.' PRESSURE DID NOT DROP DELDW 50 PSI, SO TEST CONTINUED. 
WHC-SD-W025-RPT-001, Rev. 0

\section{APPENDIX R-4}

\section{LEACHATE PIPING PRESSURE TESTING}

$\mathrm{R}-4$ 
WHC-SD-W025-RPT-001, Rev. 0

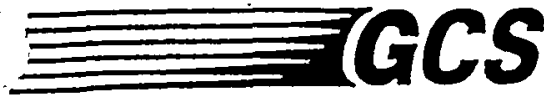

Goldor Construcflon Services, inc.
SUBUET PIPINE SHSTEIn PRESSWRE TEST

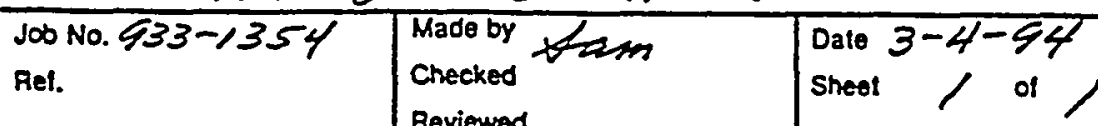

TESTING INCLUDES PINING \& VALVES FROM VALVES Q.CREST WALL TO VALVES ON TOP OF TANK

TO TRANSFER PUMP TO ENO OF 4 "O STANAESS STEEL DNCAARGE PNPE T NNCLUDES STANALESS SUCTION PIPE BETWEEN TANK $\rightarrow$ TPANSFER PUMP. (CHECKS REMOVED FRON VALVES ON TOP OF TANK). (BLANKS INSTALLED IN LANDFILL SIDE OF VALVESE CREST WALL, - BLANK INSTALLED BENEATH VALVE ON TOP OF TANK. - BLANKS INSTALCED TO ISOLATE PIPNNG SISTEM. BLANK ALSO INSTALLED BETWEEN TANK - PIPE ON BOTOM OF TANK).

TIME EVENT

1400 PILCHUCK DECH. FILED P,DES W/ HZO. 14/5 PIPHG SYSTEM PRESSUPIZED TO 35.5 PSI. SOME MINOR LEAKS Q YALVES. HALYES TIGHTENED \& LEAKS STOPPED-

1430 PE-PRESSWRIEE PPNNG TO $395 \mathrm{PSI}$ CHECKED FOR LEAKS DURNG TEST NO $\angle E A K S$ ORSERVED.

1545 MANHALLY CLOSED O OPENED YALVE NEAR CREST WALC, THEN ENDED TEST

SUMMARY - PAN TEST Q 395 PS/ FOR / HR 15 MN. NO LEAKS OBSERVED.. TEST D.K. 
WHC-SD-W025-RPT-001, Rev. 0

APPENDIX R-5

CONTAINMENT BASIN LEAK TEST

$R-5$ 
WHC-SD-W025-RPT-001, Rev. 0

$12-4-93$

CALCULATED DEPTH OF H2O PEQUIRED IN LEACHATE TEMPORARY STORAGE TANK CONTANMENT BASIN. H=O QuANTITY SHOULD BE $D$ CAPACITH OF FuLL TANK. TANX CAPACITH = 10.000 GAL.

CONTANNMENT BASNA 'DIMENSIONS $=12^{\prime}$ WIDE $\times 35^{\circ}$ LONG

$$
\begin{aligned}
12^{\circ} \times 35^{\prime} \times X & =10,000 \mathrm{GAC} / 7.48 \mathrm{Gac} / \mathrm{FT}^{3}=1,336.9 \mathrm{FT}^{3} \\
12^{\prime} \times 35^{\prime} \times X & =1336.9 \mathrm{FT}^{3} \\
X & =3.18 \mathrm{FT}
\end{aligned}
$$

HzO IN BASIN MUST DE $\geq 3.18^{\circ}$ DEEP

ADVISED BELHUR TO FILL BASIN $\geq 3$ FT $4 \mathrm{HW}$ DEEP

DELHUR FNLLED BASIN $\sim 3^{\prime} 5^{\prime \prime}$ DEED

$12-5-93$

USED SPI MODEL 31-414 CALIPER

CALIPER MOUNTED ON STEEL POD.

FILED A PONT ON THE END OF A NAIL, NAIL TO END OF POD TO CREATE A PONT GAMGE.

CALIPET READS IN INCREMENTS OF O.I $\mathrm{mm}$ ON DIAL GAWGE. $0.1 \mathrm{~mm} \times \frac{1 \mathrm{NCH}}{25.4 \mathrm{~mm}}=0.003937 \mathrm{1NCHES}$

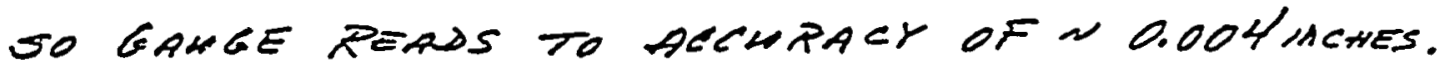

ESTABLISHED A PEFERENCE POINT ON THE TANK SADDLE SUPPORT, Y MEASWRED DISTANCE $\therefore$ FROM REFERENLE' POINT TO WATER SWRIACE.

MEASWREMENTS

DATE TIME DISTANCE FREM REF MARK TO tho SURFACE

$12-5-54 \quad 1300$

$27.4 \mathrm{~mm}$

$12-6-94 \quad 0700$

$27.4 \mathrm{~mm}$

$12-7-54 \quad 1230-1300$

$27.4 \mathrm{~mm}$

No.CHANGE IN HZO LEVEL DURING 48 HR TEST. DELIMR PENOVED H2O FROM CONTANMENT BASIN. MATHEES + MATHIS (BOTH GCS) WALKED ON HDPE LNER .... IN BASIN cONLD FEEL NO SIGNS OF PUDDLED HOO

..' BENEATH LINER - No LEAKS DETECTED. 
WHC-SD-W025-RPT-001, Rev. 0

APPENDIX S

ELECTRICAL DESIGN ENGINEERS OBSERVATIONS DURING ATPS 


\begin{tabular}{ll}
\hline Project & $\begin{array}{l}\text { Non Drag Off Facility } \\
\text { Richland, Washington }\end{array}$ \\
\hline & \\
\hline Locoton: & Hanford Reservation \\
\hline Contradar & \\
\hline Chest: & Frank Shuri, Golder Assoclates \\
\hline
\end{tabular}

\begin{tabular}{ll}
\hline Date: & April 18, 1994 \\
\hline Date Obcaved: & April 14, 1994 \\
\hline Spuring Represertertive & Rowe Moyle \\
\hline Bas: & 1 of 2 \\
\hline
\end{tabular}

This report presents opinlons formed as i result of our observation of the Controctor's activitles Contractor lo comply with the Contract Documents throughout the durallon of the Project Irespective of the presence of the Sparting Representative. The presence of our field repreaentalive will be for the purpose of providing observalton. Our senvkes do not indude wupervision or direction of the actual wark of tive Contrator, his employess $\alpha$ igents. Nelther the grtesence of our representative soz the obsurvation by our firm shall excuse the Contractor in any way for defects dlsocvered in his work. This report dost not athorize any work which will increase constriction cost.

Project Site was visited by Rowe Moyle on April 14, 1994. Acceptance testing procedures and project electrical work were observed as noted below.

People Present: Scott Matthees, Golder Construction Services

Eric Erpenbeck, Westinghouse Hanford

Frank Shuri, Golder Associates

Mike Brown, Golder Asssocjates

Rowe Moyle, Sparling

Joe Gonzalez, Electrical Contractor

Jack McGee, Westinghouse Hanford

Larry Ply, Westinghouse Hanford

Kathy Busching Westinghouse Hanford

Cliff Knitter, Golder Associates

1. The question whether of normally open and normally closed contacts should be used for contacts CR2, CR4 and CR6 was reviewed. It was determined that the primary transducer and secondary transducer produce a 4 to 20 milliamp signal which is processed into on/off signals for coils to CR2, CR4 and CR6. It was described by the Electrical Contractor that the outputs from the process controller for the 4 to 20 milliamp transducers energize the CR2 and CR6 relay coils. The coils are not energized when a low sump condition exists. This would indicate that a normally closed contact would be appropriate. The low level switch, Item FS2, is a normally closed toat switch. This would indicate that a normally closed contact in the control circuit for the motor is appropriate.

2. Frank Shuri indicated that the control wiring allowed contacts CR7A at position 17,21 and 25 have been rewired so that in the manual position the wiring goes through the high tank relay rather than bypassing it. A second mechanical tank float switch has been wired in parallel with a storage tank float high level switch No. FS3, equipment schedule item No. 32.

3. The ATP testing procedure was observed and all items functioned in acoordance with the testing requirements. 
Non Drag Off Facility - Richland, Washington

Page 2 of 2 April 15, 1994

a. Heat trace thermostat operation was verified and line currents were measured.

b. Control cabinet relay positions were observed to operate in expected mode.

c. Water level sensors were calibrated.

d. GFI receptacle was tested.

e. Lighting fixtures were tested and operated properly.

E. Pump motors were tested and operated properly. All the over-load relays were observed to be set in accordance with the nameplate ratings of the motors.

Following work was observed to be incomplete or not in compliance with the project contract drawings:

A. Control panel MS-3 has a hub conduit fitting opening in the top of the enclosure that is not sealed off. Remove hub fitting and provide knockout seal fitting.

B. Enclosure for motor starter MS2 has an opening at the top of the panel sealed with duct tape. Remove duct tape and provide metal sealing fitting.

C. Heat trace at vicinity of upstream water meter at the head end valve manifold has been damaged. The outer jacket has been abraded and shall be repaired or replaced.

D. Control panel $C_{i}$ Provide nameplate on panel indicating all sources of power ger specification 16100, paragraph 2.5. Label shall indicate source of control power and space heater power.

E. Most electrical enclosure interiors were dusty. Vacuum, seal all openings and keep enclosure doors closed tightly to prevent entry of more dust.

Eric Erpenbeck indicated that as-built drawings of the control schematios will be provided to the $A / E$ team for review and comment. Eric is looking for the A/E team to verify that the record drawings do Indicate the as-built condition as best we can determine.

Copta la Mike Brown, Golder Associates

Scott Mathees, Golder Construction Services

tosthen

Gorsonderpor 
WHC-SD-W025-RPT-001, Rev. 0

APPENDIX T

CQA VERIFICATION SURVEYS

$T-i$ 
WHC-SD-W025-RPT-001, Rev. 0

APPENDIX T-1

SUBGRADE 


\section{WHC-SD-Wr025-RPT-001, Rev. 0}

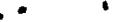

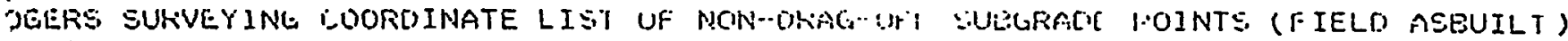
$.0-18-93$

Fage 1

M.ATCH STRING :

SLLECT ENTIFE FILE LIET COORDINATES

\begin{tabular}{|c|c|c|c|c|c|c|}
\hline & $\frac{\text { GOIDER }}{\text { POINT }}$ & & $\frac{\text { R.S.I. }}{\text { Hil }}$ & NORTH & EAST & ELEV \\
\hline COINTROL PO & NTS $\angle W$ & $\sqrt{214}$ & 414 & 44501.090 & -78194.990 & 685.71 \\
\hline & & EAAR & 417 & 44347.803 & -78582.660 & 701.75 \\
\hline & SHLD & 602 & 500 & 44346.323 & -78752.975 & 707.99 \\
\hline . & SHLD & 603 & 501 & 14343.875 & -78727.580 & 706.60 \\
\hline & SHLD & $\in 10$ & 502 & 44339.351 & -713677.200 & 703.132 \\
\hline & SHLD & .618 & 503 & 44334.230 & -78642.602 & 701.86 \\
\hline & EHLD & 014 & 504 & $4433 \% .568$ & -78602.011 & 701.33 \\
\hline & SHLD & $6 I I$ & sos & $44 \div 39.594$ & -78551.902 & 200.11 \\
\hline & SHLO & 607 & 505 & 44341.392 & -78501.080 & 700.13 \\
\hline & SHILD & 6053 & 507 & 44342.443 & -78470.815 & $6 \%$ \\
\hline & SAHLO & 615 & 508 & 14333.80 & -7ة426.473 & 397.35 \\
\hline & EHILO & $1.1 \%$ & $50 \%$ & $44337.1=5$ & $-7 \overline{0091.460}$ & 605.93 \\
\hline & EILO & 520 & 310 & $4132.1 \%$ & .78068 .299 & 6.98. \\
\hline & SHHLD & $6 \because 3$ & $\therefore 1 \mathrm{i}$ & 4.1307 .158 & $-7 \Omega 35 \%-431$ & $\therefore 55.78$ \\
\hline & StiLo & $6: 6$ & 512 & 44:24.963 & $-7505 \%=20$ & $\therefore 5.08$ \\
\hline & SHILD & 613 & 513 & $4 i=0 ; .173$ & . .3559 .2 sis & $\because 0.01$ \\
\hline & SHLD & 61 & $5: 3$ & $4.4: 3.08 \%$ & -70350.955 & 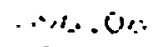 \\
\hline & EHHLO & \&Et & 515 & 44121.246 & 70059.050 & $\therefore 0$ \\
\hline & EHILO & $71 \div 704$ & 512 & $400 \div 324$ & $\cdots 9550.07$ & $\cdots-1, \ldots<$ \\
\hline & 51160 & 716 & 517 & 1.9070 .783 & 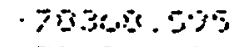 & $\therefore * . \% \geq$ \\
\hline & SIILO & 7.43 & 518 & 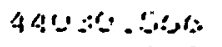 & -73433.5800 & $\because \because \ldots$ \\
\hline & $51+L 0$ & 729 & $\xi_{1} 1 \div$ & 490.819 & .73480 .101 & $\therefore$ \\
\hline & SHLO & ra & 50 & 4.4030 .58 & -78.05 .526 & 607.97 \\
\hline & SFILD & 7255 & 521 & $490 \$ 7.797$ & $.7050 \% .96$ & $6 \div 7.90$ \\
\hline & SHALD & 731 & $5:=$ & 11032.760 & 7:51 . & 700.50 \\
\hline & SHLO & 733 & $5 ะ 3$ & $110=7.799$ & $-7 e 631.542$ & 703.79 \\
\hline & SHLO & 7306 & 524 & $1402=.601$ & -78082.153 & 708.30 \\
\hline & $\because H L D$ & 739 & $5 \approx 5$ & 44019.127 & .78792 .500 & 705.20 \\
\hline & SHILO & 740 & 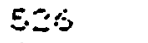 & 44017.037 & -7897.704 & $\because 10 . \div 8$ \\
\hline & $\because 4 L 0$ & 741 & 527 & $\therefore 4016.315$ & $-78790.15,4$ & צים \\
\hline & SHLO & 724 & 525 & 44045.381 & 778517.975 & $73.1 . \div 9$ \\
\hline & $\because$ HILD & 701 & $5 \div$ & $14100.3=1$ & $9 \cos 17.23$ & 711.94 \\
\hline & $\therefore 4$ LLO & BEt & 50 & 44150.845 & $-78817.6 \%$ & 711.35 \\
\hline & SHLO & 649 & 531 & $14=00.914$ & $-78017: 609$ & 711.95 \\
\hline & SHILD & 527 & 5.32 & 14250.1591 & -78817.552 & 711.87 \\
\hline & SHILD & 624 & シミ3 & 44301.050 & -781317.573 & 711.39 \\
\hline & SHLD & 622 & 534 & $4.4: 18.029$ & -70817.635 .5 & $7+\because 3$ \\
\hline & SHLD & 604 & $.5 \Xi こ$ & 49342.571 & $-75306.0 \geq 1$ & נ10. \\
\hline & SHLD & 601 & 5340 & 44348.352 & -78783.040 .3 & $7(10.20)$ \\
\hline IUE & R:AMP & 720 & 537 & 44050.808 & .73474 .441 & $6 \% 8.37$ \\
\hline $10 E$ & RAMP & 714 & $5=8$ & 44072.589 & $-7850 \% .794$ & 60.94 \\
\hline TOE & RAMP & 746 & 539 & 44080.125 & -73533.128 & 604.00 \\
\hline TOE & RAMP & 697. & 540 & 44107.543 & .786 .16 .218 & 6.75 .90 \\
\hline TOE & PAMFO & $6 \% 1$ & $541^{\circ}$ & 44118.626 & -73049.317 & $6 \%+.=0$ \\
\hline TOE & EOPE & 748 & .542 & 44124.308 & 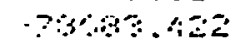 & $\therefore 7 \div .44$ \\
\hline
\end{tabular}


.

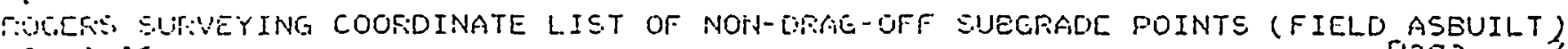
$1020 \%$

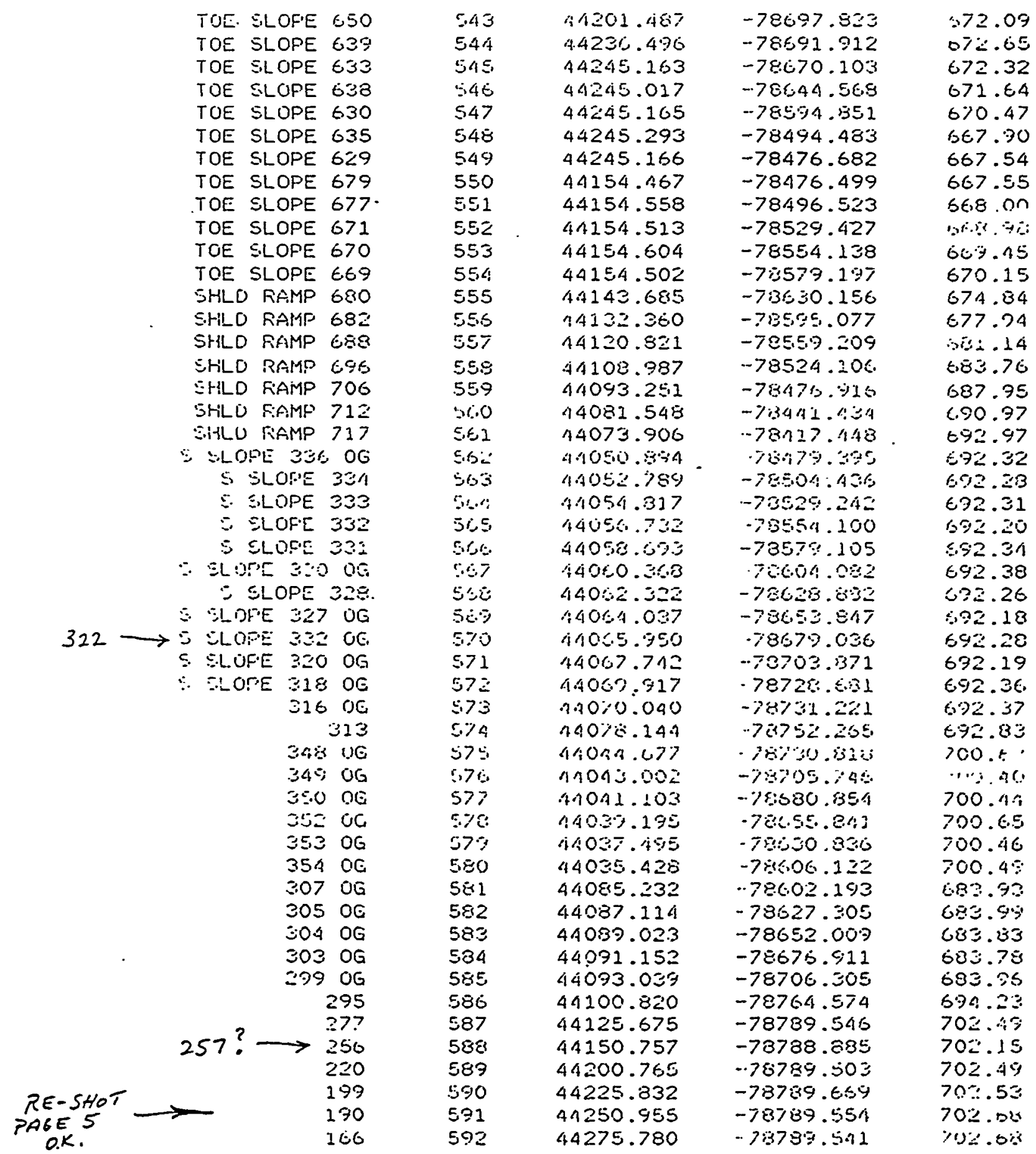


WHC-SD-W025-RPT-001, Rev. 0

.

OGERS SURVEYING CODROINATE LIST OF NON-URAG-OFF SUBGRADE POINTS (FIELO ASEUILT) $-0-18-93$

Page 3

\begin{tabular}{|c|c|c|c|c|c|c|c|}
\hline & $W$ & SLOPE & 144 & $5,9.3$ & 44209.532 & $-7878 \% .54$ & 702.57 \\
\hline & $N$ & SLOFE & 127 & .594 & 44319.026 & -78750.793 & 692.89 \\
\hline & N & SLOPE & 127 & 595 & 4.4318 .851 & -78750.748 & 698.67 \\
\hline & $N$ & SLOPE & $1 \approx 5$ & 596 & 44320.917 & -78725.941 & 633.87 \\
\hline & & & 124 & 597 & 44322.675 & -78700.976 & 698.89 \\
\hline & $\cdot N$ & SLOPE & 121 & 598 & 44326.476 & -78651.141 & 698.71 \\
\hline & $N$ & SLOPE & 118 & 599 & 44330.225 & -78601.338 & 698.90 \\
\hline & + & & 114 & 600 & 44335.835 & -78526.382 & 698.91 \\
\hline - & $N$ & SLOPE & 132 & 601 & 44319.644 & -78407.406 & 690.46 \\
\hline & & & 130 & 602 & 44316.498 & -78449.784 & 690.55 \\
\hline 136 & $N$ & SLOPE & 134 & 603 & 44312.754 & -78499.770 & 690.52 \\
\hline 138 & & & $3 / 2$ & 604 & 44309.064 & -78549.577 & 690.54 \\
\hline 138 & & & $\begin{array}{l}38 \\
41\end{array}$ & $\begin{array}{l}605 \\
60 \%\end{array}$ & 44307.152 & $\begin{array}{l}-73.574 .411 \\
-785.71\end{array}$ & 690.57 \\
\hline 145 & & $\rightarrow$ & 45 & 507 & $\begin{array}{l}44303.527 \\
.44299 .779\end{array}$ & $\begin{array}{l}-78 \leqslant 34.312 \\
-7867.4 .126\end{array}$ & $\begin{array}{l}690.58 \\
690.57\end{array}$ \\
\hline & $N$ & SI OFE & 149 & 505 & 44205.771 & -73725.911 & 690.58 \\
\hline & $W$ & SLOFE & 168 & $50 \%$ & 44276.158 & - ҰE7૯A. . 500 & $\therefore \div$. IE. \\
\hline & & & 210 & $\leqslant 10$ & if $=00.67=$ & - & 694.01 \\
\hline & & & $\because \because 3$ & 611 & 14175.861 & -75.744. .27 & $=54.20$ \\
\hline & $\omega$ & SLOTEE & 284 & 612 & $4410 \% 331$ & $73751 . \div 24$ & O00 \\
\hline & - & & 255 & $\because 12$ & $4+151.002$ & .73.75.45\% & 685.90 \\
\hline & & & 237 & $\therefore 14$ & 41175.8055 & ーフターシ\% .559 & 635.37 \\
\hline & & & 218 & 0.15 & 44200.6035 & 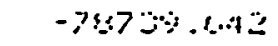 & 825.91 \\
\hline & & & 201 & SIt. & 44225.598 & -78373.259 & Biss . . 2 2 \\
\hline & $\omega$ & SLOPE & $1 \% 3$ & 617 & $4425 \div .744$ & - & 285.65 \\
\hline & in & SLOPE & 173 & 618 & $1423 \div .135$ & -737こ7.201 & $634.1=$ \\
\hline & in & SLOFE & 163 & $52 \%$ & 14284.103 & $.7: 371 \therefore .4 .84$ & 686.40 \\
\hline 71 & $N$ & SLOPE & 177 & $\therefore=0$ & 44272. ㄷ․ & -739.7 .241 & 632.15 \\
\hline & $n$ & SLOPE & 170 & $6 \geq 1$ & 44274.560 & -7:30:2. & SBE. $=0$ \\
\hline & $M$ & SLOPE & 16.4 & 522 & 44278.570 & 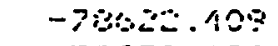 & $6.3=.20$ \\
\hline & & & 160 & $\therefore 23$ & $44 \because 3=.201$ & -755.72. & $68 \div .13$ \\
\hline & $N$ & SLOPE & 1.55 & $6=1$ & $44=35.987$ & ᄀフВ5ニニ.S1C & 6.82 .19 \\
\hline & $N$ & SLOFE & 151 & $\therefore 25$ & $44=89.692$ & 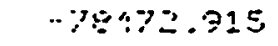 & $68: .08$ \\
\hline & rl & 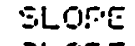 & 1403 & 520 & $4429=.824$ & -7i:1:ić. 5シ6 & $6: 2:-2: 1$ \\
\hline & iv & $\because L O F E$ & 152 & $5: 27$ & $44=36.18 \%$ & $-78400.34=$ & $\therefore=03$ \\
\hline & & & $16 \%$ & 628 & 14271.630 & $-7839 \div .110$ & 6.3 .1 .95 \\
\hline & & & 194 & $\leq \approx 9$ & 4.1249 .848 & $\because+5: 0=019$ & 634.97 \\
\hline & & & 214 & 530 & $1422.9 .5 \% 4$ & -78392.246 & 6.34 .53 \\
\hline & & & 250 & 6.31 & 44175.095 & $-78: 92.275$ & 684.92 \\
\hline & & & $25 \%$ & 632 & 44149.956 & $-7339 \because .205$ & 631.51 \\
\hline & $E$ & SLOPE & $\geq 80$ & 633 & 44123.233 & -78392.123 & 631.4 .70 \\
\hline & & & 290 & 634 & 44097.195 & -78423.038 & 605.25 \\
\hline & & & 264 & 635 & 44123.95 .4 & -73448.031 & 576.98 \\
\hline & & & $27 \cdot 3$ & 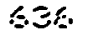 & 44120.096 & -7ロイフ: . & $57 \% .00$ \\
\hline & RAMP & SLOPE & 274 & 537 & $441=7.792$ & -7E158.75.1 & $67.0 \%$ \\
\hline & RAMF & SLOPE & 270 & 538 & 44131.704 & -785.16 .424 & $67 \therefore 8$ \\
\hline & & SUMP & 656 & 539 & 44123.676 & 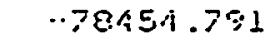 & $6.4 .0=$ \\
\hline & & SUMP & 547 & 640 & 44207.507 & -7.345.4.855 & 664. 08 \\
\hline & ri & SLOPE & 134 & 641 & $44205.36: 3$ & .78457 .540 & 673.87 \\
\hline & $H$ & SLOPE & 178 & 642 & 44264.851 & -7シ4:1.0ะ5 & בוּ. \\
\hline
\end{tabular}


..,$\cdot$ ROGERS SURVEYIHG LDORDINATE LIST OF MON-OFAG-OFF SUEGRADE POINTS (FIELD ASBUILT)
$20-18 \cdots ?$

\begin{tabular}{|c|c|c|c|}
\hline & N ELOPE & 181 & 54 \\
\hline & rL SLOCE & 182 & 64 \\
\hline & N SLOPE & 183 & $6.1:$ \\
\hline & N SLOPE & 185 & 54 \\
\hline & & 188 & 647 \\
\hline & BOTTOM & 222 & 648 \\
\hline & EOTTOM & 221 & 64 \\
\hline & BOTTOM & 211 & 55 \\
\hline . & BOTTOM & 223 & 65 \\
\hline & BOT TOM & 246 & 65 \\
\hline & BOTTOM & 244 & 65 \\
\hline & BOT TOM & 225 & 65 \\
\hline & BOTTOM & 208 & 65 \\
\hline & BOT TOM & 207 & 65 \\
\hline & EOTTOM & 226 & 65 \\
\hline & EOITOM & 2.73 & $E s$ \\
\hline & SUMP & 750 & 65 \\
\hline & surir & $\therefore \because$ & נ \\
\hline & EUMR & 749 & st \\
\hline & & $\because 2:$ & $6 i$ \\
\hline & & $=81$ & $\therefore$ \\
\hline & SUMF & 233 & 68 \\
\hline & & ز5 & $\therefore$ \\
\hline & & 347 & $c$ \\
\hline & & $\$ 15$ & 6 \\
\hline & & $=11$ & $\leq x$ \\
\hline & & $3=2$ & رrs \\
\hline & & 345 & $\therefore 7$ \\
\hline & & 33.5 & בה \\
\hline & & 923 & $\Leftrightarrow$ \\
\hline & & : & $\Leftrightarrow 7$ \\
\hline & & 284 & 37 \\
\hline & & $=00$ & $\$ 27$ \\
\hline & & 306 & $6 ?$ \\
\hline & & $\because 94$ & 57 \\
\hline 288 & 1 & ะ゚ะ & $6 . ?$ \\
\hline & & $=69$ & 6 \\
\hline & & 273 & 6.5 \\
\hline & & 291 & eis \\
\hline 683 & 7 & 600 & 8.3 \\
\hline & & $E 44$ & 68 \\
\hline & & 254 & 68 \\
\hline & & $18 ! 7$ & 68 \\
\hline & $\cdot$ & 173 & 68 \\
\hline & & 154 & 68 \\
\hline & & 158 & 68 \\
\hline & & 159 & 6.8 \\
\hline & & 142 & 605 \\
\hline & IS SLOPE & 113 & 69 \\
\hline & & $12 i$ & $6 \%$ \\
\hline
\end{tabular}

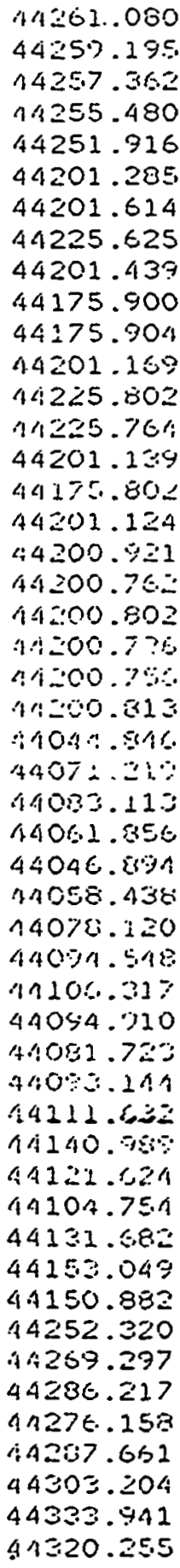

.735 .00 .8012

$-7.545 .790$

$-78570.80 \%$

$-78595.627$

$-78645.471$

$-78679.270$

$-78654.342$

$-78654.127$

$-78629.538$

$-786.29 .498$

$-78579.358$

$-78579.342$

$-78579.37 \%$

.78554 .311

.78554 .354

-78:50. 31

-75505 ..53

.78479.276.

ن

$-7841.555$

.7:115. .

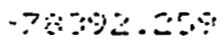

-7:35. 25.4

- 주요

$737 \%$.64

$.9377 \% .140$

$-78770.111$

$-73.55 .044$

- .

-7375: . 208

$-737 \div 4.952$

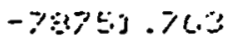

$.70 .391 .15 \%$

.7:?10.540

- 7а706. .

$\because 816.321$

$\because \because 314.898$

$-73705.505$

$.796 \% 0.502$

-7815.9: .

$-75701.719$

$.78714 .5=0$

$-78706.96 .5$

$-78.727 .230$

$-78745.6 .84$

$-7876.499$

$-70777.074$

-78704.014

$-78766.918$

.78\%:35. 008
.73 .89

673.89

$6>3.88$

673.90

673.98

671.43

670.74

$5 \%: .53$

670.22

670.23

66.9 .62

$668: .96$

$66 \% .5 \%$

669.97

608.34

.4. 9 \%

66.7 .12

564.05

EC: .04

$\therefore 6, .04$

$\therefore 75.04$

:

हण4. 36

$70 \% .75$

$\because 02.50$

. 39

ヒジ.૯\%

7or. Er.

:01.

$20 \div .80$

$0 \div 4.27$

$3.59 .9 \%$

50.80

205.02

60.93

650.73

$6, \cdots .55$

6.77 .17

679.73

E.?. 5 ;

$6.7 \Xi .1 \%$

677.55

$6 \% 8$.

684.40

七\%0.

6\% . 15

6\% .

$6 \%$. 55

704.25

702.75 
WHC-SD-W025-RPT-001, Rev. 0

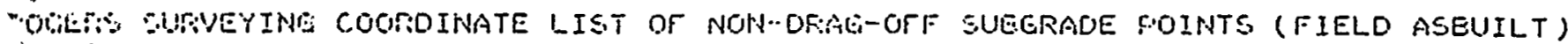
$.0-18.93$

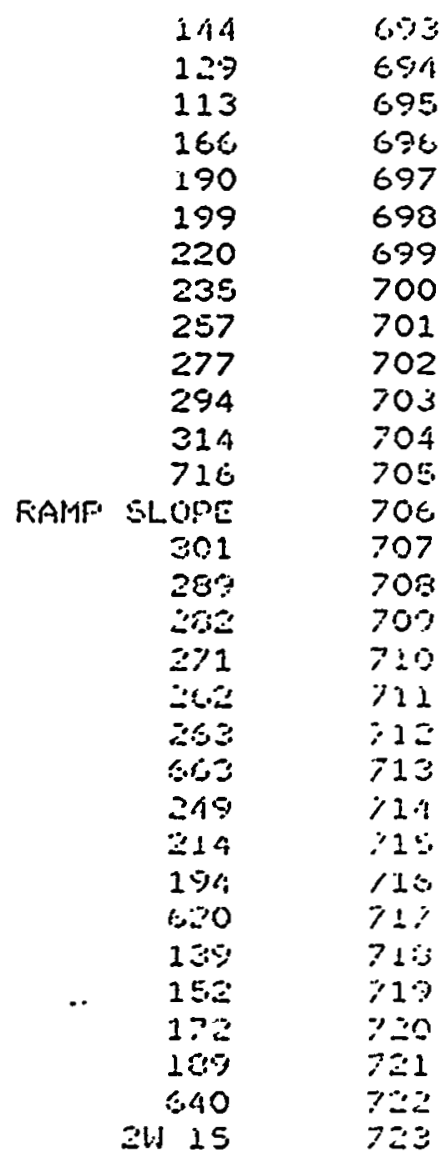

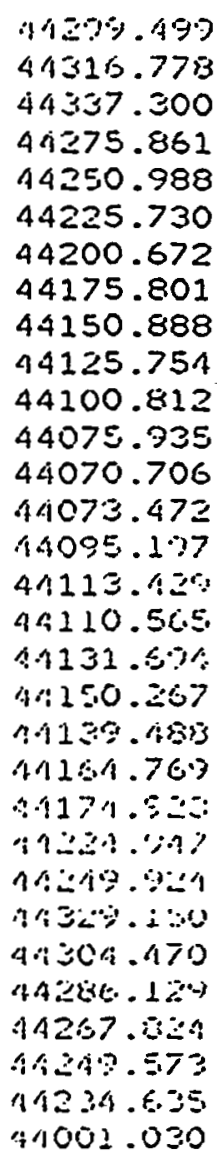

.0 .9789 .484

$-7880 \mathrm{~s} .5=0$

$-78800.68 ?$

$-78739.569$

$-79789.492$

$-78789.655$

$-78789.511$

$-78789.615$

$-78789.564$

$-78789.586$

$-73789.670$

.78789 .506

$-73368.641$

$-78400.33 \pi$

-73591 . 279

-.7310?. .33

-73455.50

-7E.

-7844:․ㅗㄴ

$.7345=20 \%$

. 73454.

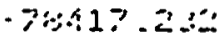

- 390

- ?ㅜ. 300

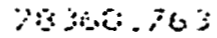

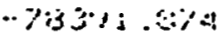

- 78 80ri. $\$ 12$

$\because 3195$

$7944: 5$

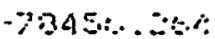

. $291 \% .002$
Page 5

702.55

708.73

703.80

702.69

702.52

702.53

702.51

702.41

702.44

702.47

702.35

$70 \div .51$

695.94

692.74

653.34

$68=.67$

Sis 1.04

677.02

671.34

9.72 .84

56.

670.63

מํ.

अ13.

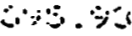

stis. 27

602.58

$67 \% .63$

$67 .=5$

6.75

Sith 
WHC-SD-W025-RPT-001, Rev. 0

APPENDIX T-2

TOP OF SECONDARY SOIL LINER 
ROGERS SURVEYING COORDINATE LIST OF NON-DRAG-OFF SECONDARY ADMIX POINTS $11-18-93$

SELECT POINTS BY NUMBER

POINT \# [,THRU POINT \#] : 1015,1320

POINT \# [,THRU POINT \#] :

LIST COORDINATES

\begin{tabular}{|c|c|c|c|c|c|}
\hline \multicolumn{2}{|c|}{ GOLDER } & $\begin{array}{l}\text { RSI } \\
\text { PT\#\# }\end{array}$ & NORTH & & \\
\hline $\begin{array}{l}\text { PTH } \\
\text { OG }\end{array}$ & 1126 & 1015 & 44320.28 & $\begin{array}{r}\text { EAST } \\
-78782.07\end{array}$ & $\begin{array}{r}\text { ELEV } \\
706.20\end{array}$ \\
\hline$O G$ & 1125 & 1016 & 44320.91 & -78725.91 & 702.18 \\
\hline OG & 2124 & 1017 & 44322.99 & -78700.84 & 702.24 \\
\hline OG & 1122 & 1018 & 44324.85 & -78675.97 & 702.27 \\
\hline OG & 1121 & 1019 & 44326.60 & -78651.23 & 702.23 \\
\hline OG & 1142 & 1020 & 44303.39 & -78764.01 & 700.11 \\
\hline OG & 1154 & 1021 & 44286.31 & -78745.65 & 693.94 \\
\hline OG & 1146 & 1022 & 44297.76 & -78698.97 & 693.93 \\
\hline OG & 1145 & 1023 & 44299.85 & -78674.12 & 693.92 \\
\hline OG & 1143 & 1024 & 44301.60 & -78649.06 & 693.79 \\
\hline OG & 1141 & 1025 & 44303.58 & -78624.30 & $693^{\circ} .87$ \\
\hline OG & 1140 & 1026 & 44305.30 & -78599.37 & 693.90 \\
\hline OG & 1138 & 1027 & 44307.26 & -78574.41 & 693.88 \\
\hline OG & 1136 & 1028 & 44309.22 & -78549.51 & 693.92 \\
\hline OG & 1135 & 1029 & 44310.99 & -78524.55 & 693.88 \\
\hline$O G$ & 1134 & 1030 & 44312.91 & -78499.57 & 693.91 \\
\hline OG & 1131 & 1031 & 44314.73 & -78474.77 & 693.94 \\
\hline OG & 1130 & 1032 & 44316.57 & -78449.91 & 693.90 \\
\hline$O G$ & 1128 & 1033 & 44318.09 & -78425.06 & 693.92 \\
\hline$O G$ & 1132 & 1034 & 44319.58 & -78407.48 & 693.86 \\
\hline OG & 1139 & 1035 & 44304.31 & -78391.77 & 691.62 \\
\hline OG & 1152 & 1036 & 44286.04 & -78408.79 & 685.99 \\
\hline OG & 1137 & 1037 & 44306.28 & -78419.86 & 689.69 \\
\hline OG & 1148 & 1038 & 44292.75 & -78432.62 & 685.59 \\
\hline OG & 1150 & 1039 & 44291.70 & -78447.91 & 685.52 \\
\hline$O G$ & 1151 & 1040 & 44289.84 & -78472.72 & 685.59 \\
\hline OG & 1153 & 1041 & 44288.01 & -78497.66 & 685.57 \\
\hline$O G$ & 1155 & 1042 & 44286.08 & -78522.58 & 685.59 \\
\hline OG & 1157 & 1043 & 44284.29 & -78547.52 & 685.59 \\
\hline OG & 1160 & 1044 & 44282.41 & -78572.56 & 685.59 \\
\hline OG & 1164 & 1045 & 44278.65 & -78622.34 & 685.53 \\
\hline OG & 1165 & 1046 & 44276.73 & -78647.24 & 685.55 \\
\hline OG & 1173 & 1047 & 44269.39 & -78727.25 & 687.83 \\
\hline OG & 1187 & 1048 & 44252.25 & -78709.01 & 681.70 \\
\hline OG & 1197 & 1049 & 44229.69 & -78714.73 & 681.01 \\
\hline$O G$ & 1202 & 1050 & 44225.88 & -78714.74 & 680.97 \\
\hline OG & 1217 & 1051 & 44200.73 & -78714.83 & 680.97 \\
\hline$O G$ & 1238 & 1052 & 44175.71 & -78714.75 & 680.93 \\
\hline 06 & 1254 & 1053 & 44150.83 & -78714.86 & 680.90 \\
\hline OG & 1269 & 1054 & 44140.92 & -78714.69 & 680.87 \\
\hline$O G$ & 1278 & 1055 & 44117.80 & -78739.68 & 689.30 \\
\hline OG & 1275 & 1056 & 44125.85 & -78739.74 & 689.36 \\
\hline OG & 1255 & 1057 & 44150.86 & -78739.69 & 689.38 \\
\hline OG & 1237 & 1058 & 44175.88 & -78739.79 & 689.27 \\
\hline
\end{tabular}


WHC-SD-W025-RPT-001, Rev. 0

ROGERS SURVEYING COORDINATE LIST OF NON-DRAG-OFF SECONDARY ADMIX POINTS 11-18-93

\begin{tabular}{|c|c|c|c|c|c|}
\hline $\begin{array}{l}\text { OG } \\
\text { OG }\end{array}$ & $\begin{array}{l}1218 \\
1201\end{array}$ & $\begin{array}{l}1059 \\
1060\end{array}$ & $\begin{array}{l}44200.96 \\
44225.76\end{array}$ & $\begin{array}{l}-78739.55 \\
-78739.75\end{array}$ & $\begin{array}{l}689.18 \\
689.27\end{array}$ \\
\hline OG & 1192 & 1061 & 44250.80 & -78739.84 & 689.37 \\
\hline OG & 1193 & 1062 & 44252.90 & -78739.68 & 689.32 \\
\hline OG & 1176 & 1063 & 44264.48 & -78752.31 & 693.39 \\
\hline OG & 1168 & 1064 & 44276.13 & -78764.72 & 697.63 \\
\hline OG & 1191 & 1065 & 44250.81 & -78764.75 & 697.52 \\
\hline OG & 1200 & 1066 & 44225.80 & -78764.70 & 697.54 \\
\hline OG & 1219 & 1067 & 44200.78 & -78764.76 & 697.64 \\
\hline OG & 1236 & 1068 & 44175.76 & -78764.71 & 697.64 \\
\hline$O G$ & 1256 & 1069 & 44150.79 & -78764.80 & 697.68 \\
\hline OG & 1276 & 1070 & 44125.93 & -78764.71 & 697.69 \\
\hline OG & 1295 & 1071 & 44100.85 & -78764.69 & 697.67 \\
\hline OG & 1296 & 1072 & 44094.58 & -78764.55 & 697.66 \\
\hline$O G$ & 1311 & 1073 & 44082.94 & -78777.19 & 701.80 \\
\hline$O G$ & 1315 & 1074 & 44071.45 & -78789.60 & 706.00 \\
\hline OG & 1314 & 1075 & 44075.81 & -78789.52 & 705.99 \\
\hline$O G$ & 1294 & 1076 & 44100.87 & -78789.64 & 706.04 \\
\hline OG & 1277 & 1077 & 44125.69 & -78789.82 & 705.98 \\
\hline$O G$ & 1257 & 1078 & 44150.83 & -78789.67 & 706.05 \\
\hline OG & 1235 & 1079 & 44175.92 & -78789.75 & 706.01 \\
\hline OG & 1220 & 1080 & 44200.76 & -78789.66 & 705.97 \\
\hline OG & 1199 & 1081 & 44226.01 & -78789.75 & 705.92 \\
\hline$O G$ & 1190 & 1082 & 44250.76 & -78789.81 & 705.94 \\
\hline OG & 1166 & 1083 & 44276.04 & -78789.88 & 705.93 \\
\hline OG & 1144 & 1084 & 44299.38 & -78789.71 & 705.94 \\
\hline$O G$ & 1345 & 1085 & 44046.73 & -78756.13 & 704.02 \\
\hline OG & 1348 & 1086 & 44044.83 & -78730.55 & 704.02 \\
\hline OG & 1349 & 1087 & 44042.95 & -78705.45 & 704.09 \\
\hline OG & 1350 & 1088 & 44041.03 & -78680.85 & 704.10 \\
\hline OG & 1352 & 1089 & 44039.11 & -78655.84 & 704.10 \\
\hline OG & 1353 & 1090 & 44037.14 & -78630.85 & 704.05 \\
\hline OG & 1336 & 1091 & 44050.79 & -78479.49 & 695.78 \\
\hline OG & 1334 & 1092 & 44052.88 & -78504.21 & 695.74 \\
\hline OG & 1333 & 1093 & 44054.70 & -78529.29 & 695.70 \\
\hline OG & 1332 & 1094 & 44056.54 & -78554.18 & 695.78 \\
\hline OG & 1331 & 1095 & 44058.37 & -78579.12 & 695.77 \\
\hline OG & 1330 & 1096 & 44060.35 & -78603.93 & 695.65 \\
\hline OG & 1328 & 1097 & 44062.14 & -78628.98 & 695.72 \\
\hline OG & 1327 & 1098 & 44064.00 & -78653.89 & 695.80 \\
\hline OG & 1322 & 1099 & 44065.92 & -78678.83 & 695.72 \\
\hline OG & 1320 & 1100 & 44067.72 & -78704.02 & 695.71 \\
\hline OG & 1318 & 1101 & 44069.64 & -78728.86 & 695.80 \\
\hline OG & 1316 & 1102 & 44069.98 & -78731.15 & 695.67 \\
\hline OG & 1299 & 1103 & 44093.14 & -78706.33 & 687.36 \\
\hline OG & 1302 & 1104 & 44092.79 & -78701.93 & 687.37 \\
\hline OG & 1303 & 1105 & 44090.95 & -78677.11 & 687.39 \\
\hline OG & 1304 & 1106 & 44088.93 & -78652.14 & 687.36 \\
\hline OG & 1305 & 1107 & 44087.12 & -78627.16 & 687.43 \\
\hline OG & 1307 & 1108 & 44085.31 & -78602.26 & 687.37 \\
\hline
\end{tabular}


WHC-SD-W025-RPT-001, Rev. 0

ROGERS SURVEYING COORDINATE LIST OF NON-DRAG-OFF SECONDARY ADMIX POINTS $11-18-93$

\begin{tabular}{|c|c|c|}
\hline $\begin{array}{l}O G \\
O G\end{array}$ & $\begin{array}{l}1309 \\
1283\end{array}$ & $\begin{array}{l}1109 \\
1110\end{array}$ \\
\hline$O G$ & 1261 & 1111 \\
\hline OG & 1180 & 1112 \\
\hline$O G$ & 1188 & 1113 \\
\hline OG & 1186 & 1114 \\
\hline $\begin{array}{l}\text { OG } \\
. O G\end{array}$ & $\begin{array}{l}1185 \\
1183\end{array}$ & $\begin{array}{l}1115 \\
1116\end{array}$ \\
\hline$O G$ & 1182 & 1117 \\
\hline OG & 1178 & 1118 \\
\hline $\begin{array}{l}O G \\
O G\end{array}$ & $\begin{array}{l}1161 \\
1150\end{array}$ & $\begin{array}{l}1119 \\
1120\end{array}$ \\
\hline OG & 1152 & 1121 \\
\hline$O G$ & 1156 & 1122 \\
\hline OG & 1169 & 1123 \\
\hline$O G$ & Iat $/ 194$ & 1124 \\
\hline OG & $1214^{\circ}$ & 1125 \\
\hline OG & 1233 & 1126 \\
\hline$O G$ & 1250 & 1127 \\
\hline OG & 1259 & 1128 \\
\hline$O G$ & 1280 & 1129 \\
\hline OG & 1286 & 1130 \\
\hline OG & 1267 & 1131 \\
\hline $\begin{array}{l}O G \\
O G\end{array}$ & 1260 & 1132 \\
\hline OG & 1252 & 1133 \\
\hline OG & 1249 & 1134 \\
\hline OG & 1231 & 1135 \\
\hline OG & 1213 & 1136 \\
\hline OG & $1295-1195$ & 1137 \\
\hline OG & 1175 & 1138 \\
\hline OG & 1172 & 1139 \\
\hline$O G$ & 1189 & 1140 \\
\hline OG & 1215 & 1141 \\
\hline BOT & 1212 & 1142 \\
\hline BOT & 1211 & 1143 \\
\hline BOT & 1210 & 1144 \\
\hline BOT & 1209 & 1145 \\
\hline BOT & 1208 & 1146 \\
\hline BOT & 1207 & 1147 \\
\hline BOT & 1206 & 1148 \\
\hline BOT & 1205 & 1149 \\
\hline BOT & 1204 & 1150 \\
\hline $80^{\circ}$ & 1229 & 1151 \\
\hline 3 & 1750 & 1152 \\
\hline BOT & 1227 & 1153 \\
\hline BOT & 1226 & 1154 \\
\hline 30 & 1225 & 1155 \\
\hline 80 & 1224 & 1156 \\
\hline 80 & 1223 & 1157 \\
\hline 3 & 1221 & 1158 \\
\hline
\end{tabular}

$\begin{array}{ll}44083.32 & -78577.23 \\ 44115.86 & -78675.22 \\ 44116.34 & -78681.28 \\ 44261.31 & -78688.58 \\ 44252.00 & -78645.38 \\ 44253.71 & -78620.39 \\ 44255.45 & -78595.63 \\ 44257.37 & -78570.76 \\ 44259.24 & -78545.70 \\ 44264.77 & -78471.02 \\ 44279.36 & -78444.89 \\ 44291.46 & -78447.82 \\ 44286.00 & -78408.79 \\ 44287.86 & -78376.88 \\ 44271.64 & -78392.12 \\ 44249.92 & -78392.14 \\ 44225.01 & -78392.13 \\ 44200.71 & -78392.21 \\ 44175.09 & -78392.13 \\ 44149.82 & -78392.13 \\ 44128.27 & -78392.17 \\ 44111.94 & -78377.12 \\ 44141.79 & -78404.61 \\ 44155.21 & -78417.25 \\ 44168.72 & -78429.57 \\ 44174.97 & -78417.16 \\ 44200.75 & -78417.11 \\ -44224.93 & -78416.90 \\ 44244.58 & -78417.18 \\ 44258.20 & -78404.67 \\ 44267.87 & -78425.76 \\ 44249.33 & -78442.46 \\ 44217.63 & -78442.21 \\ 44225.85 & -78679.21 \\ 44225.85 & -78654.20 \\ 44225.76 & -78629.39 \\ 44225.78 & -78604.36 \\ 44225.73 & -78579.22 \\ 44225.74 & -78554.38 \\ 44225.70 & -78529.30 \\ 44225.76 & -78504.32 \\ 44225.74 & -78479.27 \\ 44.200 .86 & -78479.32 \\ 44200.86 & -78504.65 \\ 44201.10 & -78529.08 \\ 44201.16 & -78554.30 \\ 44201.25 & -78579.30 \\ 44201.33 & -78604.35 \\ 44201.41 & -78629.28 \\ 44201.43 & -78654.27\end{array}$

687.34

679.06

679.12

681.54

677.33

677.25

677.18

677.19

677.23

677.18

681.43

685.57

685.99

693.24

688.21

688.29

688.31

688.31

688.39

688.36

688.33

693.36

684.23

680.03

675.93

680.03

679.96

679.98

679.92

684.10

680.23

674.49

671.59

675.33

674.63

673.96

673.36

672.89

672.12

671.60

670.90

670.29

668.20

670.39

671.03

671.50

672.17

672.85

673.44

674.17 
ROGERS SURVEYING COORDINATE LIST OF NON-DRAG-OFF SECONDARY ADMIX POINTS $11-18-93$

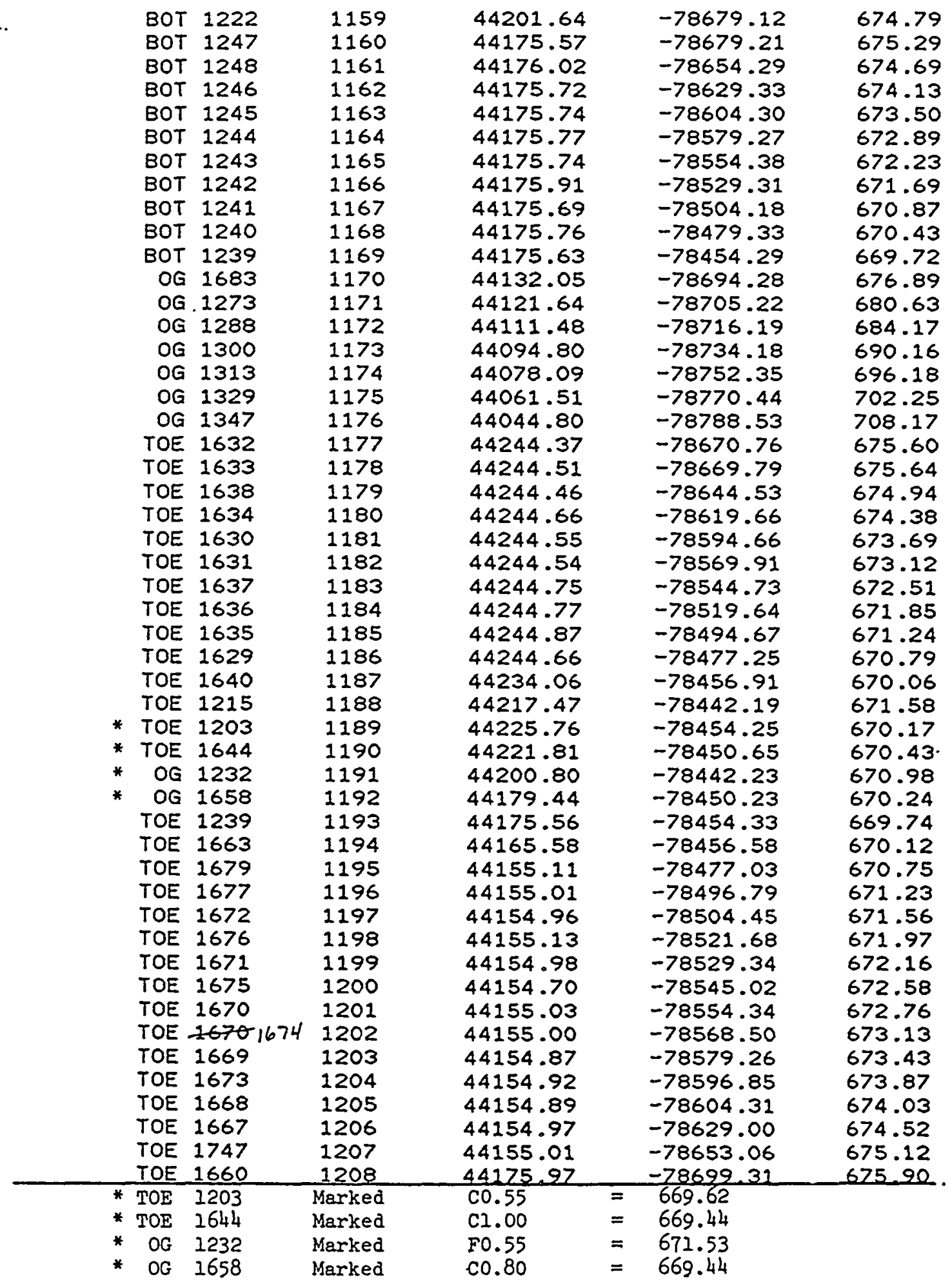


BOGERS SURVEYING COORDINATE LIST OF NON-DRAG-OFF SECONDARY ADMIX POINTS $1-18-93$

\begin{tabular}{|c|c|c|}
\hline $\begin{array}{l}\text { TOE } \\
\text { OG }\end{array}$ & $\begin{array}{l}1650 \\
1265\end{array}$ & $\begin{array}{l}1209 \\
1210\end{array}$ \\
\hline $\begin{array}{l}O G \\
O G\end{array}$ & $\begin{array}{l}1268 \\
1270\end{array}$ & $\begin{array}{l}1211 \\
1212\end{array}$ \\
\hline $\begin{array}{l}O G \\
O G\end{array}$ & $\begin{array}{l}1272 \\
1274\end{array}$ & $\begin{array}{l}1213 \\
1214\end{array}$ \\
\hline$O G$ & 1279 & 1215 \\
\hline${ }_{2}$ & $\begin{array}{r}1264 \\
W-15\end{array}$ & $\begin{array}{l}1216 \\
1217\end{array}$ \\
\hline OG & 1290 & 1218 \\
\hline $\begin{array}{l}\text { OG } \\
\text { OG }\end{array}$ & $\begin{array}{l}1298 \\
1293\end{array}$ & $\begin{array}{l}1219 \\
1220\end{array}$ \\
\hline OG & 1292 & 1221 \\
\hline TOE & 13471346 & 1222 \\
\hline TOE & 1723 & 1223 \\
\hline TOE & 1722 & 1224 \\
\hline $\begin{array}{l}\text { TOE } \\
\text { TOE }\end{array}$ & $\begin{array}{l}1721 \\
1720\end{array}$ & $\begin{array}{l}1225 \\
1226\end{array}$ \\
\hline $\begin{array}{l}\text { TOE } \\
\text { TOE }\end{array}$ & $\begin{array}{l}1719 \\
1718\end{array}$ & $\begin{array}{l}1227 \\
1228\end{array}$ \\
\hline $\begin{array}{l}\text { TOE } \\
\text { TOE }\end{array}$ & $\begin{array}{l}1714 \\
1713\end{array}$ & $\begin{array}{l}1229 \\
1230\end{array}$ \\
\hline $\begin{array}{l}\text { TOE } \\
\text { TOE }\end{array}$ & $\begin{array}{l}1746 \\
1711\end{array}$ & $\begin{array}{l}1231 \\
1232\end{array}$ \\
\hline $\begin{array}{l}\text { TOE } \\
\text { TOE }\end{array}$ & $\begin{array}{l}1709 \\
1707\end{array}$ & $\begin{array}{l}1233 \\
1234\end{array}$ \\
\hline $\begin{array}{l}\text { TOE } \\
\text { TOE }\end{array}$ & $\begin{array}{l}1705 \\
1702\end{array}$ & $\begin{array}{l}1235 \\
1236\end{array}$ \\
\hline $\begin{array}{l}\text { TOE } \\
\text { TOE }\end{array}$ & $\begin{array}{l}1699 \\
1697\end{array}$ & $\begin{array}{l}1237 \\
1238\end{array}$ \\
\hline $\begin{array}{l}\text { TOE } \\
\text { TOE }\end{array}$ & $\begin{array}{l}1695 \\
1693\end{array}$ & $\begin{array}{l}1239 \\
1240\end{array}$ \\
\hline $\begin{array}{l}\text { TOE } \\
\text { TOE }\end{array}$ & $\begin{array}{l}1690 \\
1689\end{array}$ & $\begin{array}{l}1241 \\
1242\end{array}$ \\
\hline $\begin{array}{l}\text { OG } \\
\text { OG }\end{array}$ & $\begin{array}{l}1301 \\
1289\end{array}$ & $\begin{array}{l}1243 \\
1244\end{array}$ \\
\hline OG & 1271 & 1245 \\
\hline OG & 1262 & 1246 \\
\hline $\begin{array}{l}\text { LDR } \\
\text { LDR }\end{array}$ & $\begin{array}{l}1725 \\
1727\end{array}$ & $\begin{array}{l}1247 \\
1248\end{array}$ \\
\hline $\begin{array}{l}\text { LDR } \\
\text { LDR }\end{array}$ & $\begin{array}{l}1729 \\
1743\end{array}$ & $\begin{array}{l}1249 \\
1250\end{array}$ \\
\hline $\begin{array}{l}\text { LDR } \\
\text { LDR }\end{array}$ & $\begin{array}{l}1321 \\
1716\end{array}$ & $\begin{array}{l}1250 \\
1251 \\
1252\end{array}$ \\
\hline ILDR & 1704 & 1253 \\
\hline ILDR & 1686 & 1254 \\
\hline LLOR & 1678 & 1255 \\
\hline ILDR & 1661 & 1256 \\
\hline ILOR & 1653 & 1257 \\
\hline ILDR & 1643 & 1258 \\
\hline
\end{tabular}

44200.87
44135.07
44132.85
44131.29
44129.47
44127.42
44125.69
44123.68
44001.00
44096.92
44098.82
44100.80
44102.55
44045.23
44043.61
44047.49
44051.38
44055.22
44059.21
44063.09
44067.05
44071.18
44074.69
44078.61
44082.28
44086.30
44090.18
44094.13
44098.09
44102.03
44105.87
44109.70
44115.76
44121.12
44095.25
44113.53
44131.86
44150.16
44044.28
44042.69
44040.79
44036.98
44066.73
44076.13
44099.54
44124.85
44150.07
44174.95
44200.68
44224.98

$-78697.32$

$-78598.27$

$-78570.02$

$-78546.96$

$-78523.63$

$-78498.64$

$-78473.86$

$-78448.06$

$-78195.02$

$-78423.13$

$-78450.74$

$-78475.75$

$-78500.64$

$-78426.95$

$-78440.49$

$-78452.54$

$-78464.32$

$-78476.22$

$-78488.17$

$-78500.05$

$-78511.85$

$-78523.68$

$-78534.91$

$-78546.88$

$-78558.73$

$-78570.66$

$-78582.44$

$-78594.45$

$-78606.13$

$-78617.96$

$-78629.97$

$-78641.69$

$-78660.29$

$-78676.07$

$-78391.71$

$-78408.46$

$-78425.39$

$-78442.34$

$-78529.81$

$-78504.97$

$-78480.13$

$-78431.21$

$-78395.01$

$-78373.59$

$-78365.54$

$-78365.55$

$-78365.51$

$-78365.61$

$-78365.70$

$-78365.52$
675.17 680.28 680.46 680.34 680.35 680.38 680.39 680.39 696.65 688.76 688.70 688.75 688.69 698.19 697.18 696.26 695.26 694.24 693.11 692.23 691.23 690.13 689.32 688.30 687.22 686.26 685.17 684.21 683.21 682.18 681.24 680.23 678.76 677.32 691.69 686.13 680.45 674.75 699.22 699.13 699.07 699.22 698.20 697.68 697.16 697.11 697.14 697.18 697.21 697.07 
ROGERS SURVEYING COORDINATE LIST OF NON-DRAG-OFF SECONDARY AOMIX POINTS $11-18-93$

SHLDR 1628

SHILDR 1626

SHLDR 1623

SHLDR 1620

SHLDR 1619

SHLDR 1615

SHLDR 1612

SHLDR 1608

SHLDR 1606

SHLDR 1607

SHLDR 1609

SHLDR 1611

SHLDR 1613

SHLDR 1614

SHLDR 1617

SHLDR 1618

SHLDR 1610

SHLDR 1605

SHLDR 1603

SHLDR 1602

SHLDR 1601

SHLDR 1604

SHLDR 1621

SHLDR 1624

SHLDR 1625

SHLDR 1627

SHLOR 1641

SHLDR 1649

SHLDR 1659

SHLDR 1666

SHLDR 1685

SHLDR 1701

SHLDR 1715

SHLDR 1724

SHLDR 1735

SHLDR 1742

SHLDR 1740

SHLDR 1739

SHLDR 1738

SHLDR 1736

SHLDR 1734

SHLDR . 1733

SHLDR 1732

SHLDR 1731

SHLDR 1728

SHLDR RAMR 1710

SHLDR RAMP 1708

SHLDR RAMP 1706

SHLDR RAMP 1703

SHLDR RAMP 1700

1259
1260
1261
1262
1263
1264
1265
1266
1267
1268
1269
1270
1271
1272
1273
1274
1275
1276
1277
1278
1279
1280
1281
1282
1283
1284
1285
1286
1287
1288
1289
1290
1291
1292
1293
1294
1295
1296
1297
1298
1299
1300
1301
1302
1303
1304
1305
1306
1308

1259

1261

1262

263

1265

1266

1267

1268

269
270

1271

1272

1273

1274

1276

1277

1278

1280

1281

1282

1284

1285

1286

1287

1289

1290

1291

1292

1293

1295

1296

1297

1290

1300

1301

302

1304

1305

1307

1308
44249.84

44274.82

44300.12

44324.02

44330.82

44332.47

44333.66

44334.92

44335.59

44334.83

44334.12

44332.96

44332.13

44331.24

44330.32

44329.50

44332.80

44335.12

44337.32

44339.57

44341.58

44336.71

44319.23

44301.01

44276.00

44250.97

44225.87

44200.86

44175.85

44150.97

44126.10

44100.95

44075.91

44051.55

44032.04

44023.08

44024.40

44025.73

44027.01

44029.14

44031.88

44034.36

44036.90

44039.52

44041.87

44079.76

44083.81

44087.60

44091.66

44095.39
$-78365.59$

$-78365.64$

$-78365.72$

$-78373.62$

$-78397.17$

$-78425.64$

$-78450.94$

$-78476.24$

$-78487.11$

$-78501.11$

$-78526.23$

$-78551.22$

$-78576.36$

$-78601.36$

$-78626.37$

$-78642.67$

$-78676.80$

$-78701.93$

$-78727.13$

$-78752.39$

$-78775.11$

$-78799.98$

$-78811.09$

$-78811.26$

$-78811.27$

$-78811.15$

$-78811.39$

$-78811.31$

$-78811.30$

$-78811.31$

$-78811.47$

$-78811.20$

$-78811.37$

$-78811.23$

$-78802.43$

$-78781.59$

$-78757.28$

$-78732.12$

$-78707.06$

$-78681.64$

$-78656.34$

$-78631.23$

$-78606.13$

$-78580.59$

$-78555.44$

$-78455.06$

$-78466.82$

$-78478.83$

$-78490.64$

$-78502.47$
697.05

697.12

697.12

697.70

697.42

698.58

699.63

700.60

701.17

701.33

701.72

702.02

702.35

702.68

702.98

703.15

705.03

706.39

707.78

709.18

710.44

711.99

713.05

713.09

713.13

713.03

713.07

713.11

713.07

713.10

713.00

713.03

713.06

713.17

712.87

712.35

711.47

710.39

709.34

708.01

706.45

705.13

703.56

701.99

700.72

695.20

694.22

693.24

692.24

691.25 
BOGERS SURVEYING COORDINATE LIST OF NON-DRAG-OFF SECONDARY ADMIX POINTS 11-18-93

$\begin{array}{lll}\text { SHLDR RAMP } & 1698 & 1309 \\ \text { SHLDR RAMP } & 1696 & 1310 \\ \text { SHLDR RAMP } & 1694 & 1311 \\ \text { SHLDR RAMP } & 1692 & 1312 \\ \text { SHLDR RAMP } & 1688 & 1313 \\ \text { SHLDR RAMP } & 1687 & 1314 \\ \text { SHLDR RAMP } & 1684 & 1315 \\ \text { SHLDR RAMP } & 1682 & 1316 \\ \text { SHLDR RAMP } & 1681 & 1317 \\ \text { SHLDR RAMP } & 1745 & 1318 \\ \text { SHLDR RAMP } & 1680 & 1319 \\ \text { SHLDR RAMP } & 1665 & 1320\end{array}$

44099.49
44103.03
44106.93
44110.93
44114.73
44118.74
44122.76
44126.60
44130.33
44134.44
44138.18
44139.60

$-78514.33$

$-78525.55$

$-78537.41$

$-78549.31$

$-78561.31$

$-78573.10$

$-78584.83$

$-78596.74$

$-78608.96$

$-78620.83$

$-78632.59$

$-78636.19$

690.22

689.32

688.27

687.28

686.24

685.21

684.32

683.31

682.27

681.29

680.24

679.95 
WHC-SD-W025-RPT-001, Rev。0

APPENDIX T-3

TOP OF SECONDARY GRAVEL 
WHC-SD-W025-RPT-001, Rev. 0

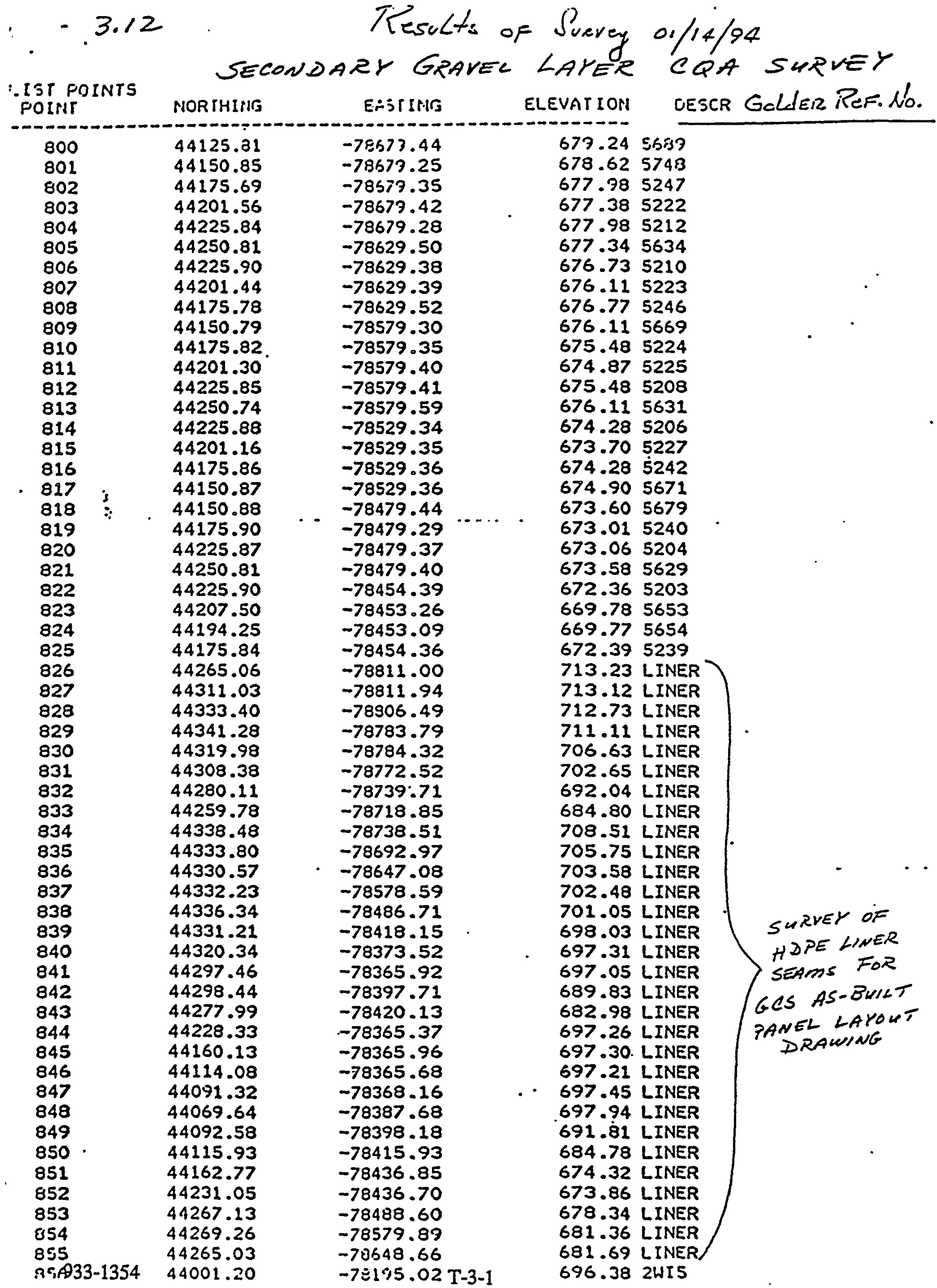


WHC-SD-H025-RPT-001, Rev. 0

APPENDIX T-4

TOP OF PRIMARY SOIL LINER 
CQA SURVEY OF PRIMARY ADMIX SURFACE PERFORMED BY ROGERS SURVEYING, INC. ON 01-20-94

\begin{tabular}{|c|c|c|c|c|}
\hline $\begin{array}{r}\text { Rogers } \\
\text { Point } \\
\text { No. } \\
\end{array}$ & Easting & Northing & Elevation & $\begin{array}{r}\text { Grid } \\
\text { Point } \\
\text { No. }\end{array}$ \\
\hline 1 & 1 & 1 & 1 & 1 \\
\hline 417 & -78582.6 & 44347.80 & 701.75 & 2 \\
\hline 414 & -78194.9 & 44501.09 & 685.71 & 2 \\
\hline 500 & -78679.4 & 44125.81 & 679.235 & 5689 \\
\hline 501 & -78679.2 & 44150.85 & 678.624 & 5748 \\
\hline 502 & -78679.3 & 44175.69 & 677.979 & 5247 \\
\hline 503 & -78679.4 & 44201.56 & 677.384 & 5222 \\
\hline 504 & -78679.2 & 44225.84 & 677.982 & 5212 \\
\hline 505 & -78629.4 & 44250.80 & 677.338 & 5634 \\
\hline 506 & -78629.3 & 44225.89 & 676.73 & 5210 \\
\hline 507 & -78629.3 & 44201.43 & $676: 108$ & 5223 \\
\hline 508 & -78629.5 & 44175.78 & 676.77 & 5246 \\
\hline 509 & -78579.2 & 44150.78 & 676.106 & 5669 \\
\hline 510 & -78579.3 & 44175.81 & 675.479 & 5224 \\
\hline 511 & -78579.4 & 44201.29 & 674.865 & 5225 \\
\hline 512 & -78579.4 & 44225.85 & 675.483 & 5208 \\
\hline 513 & -78579.5 & 44250.73 & 676.113 & 5631 \\
\hline 514 & -78529.3 & 44225.87 & 674.281 & 5206 \\
\hline 515 & -78529.3 & 44201.16 & 673.703 & 5227 \\
\hline 516 & -78529.3 & 44175.85 & 674.282 & 5242 \\
\hline 517 & -78529.3 & 44150.86 & 674.895 & 5671 \\
\hline 518 & -78479.4 & 44150.88 & 673.596 & 5679 \\
\hline 519 & -78479.2 & 44175.89 & 673.009 & 5240 \\
\hline 520 & -78479.3 & 44225.87 & 673.057 & 5204 \\
\hline 521 & -78479.4 & 44250.81 & 673.576 & 5629 \\
\hline 522 & -78454.3 & 44225.9 & 672.361 & 5203 \\
\hline 523 & -78453.2 & 44207.50 & 669.776 & 5653 \\
\hline 524 & -78453.0 & 44194.24 & 669.765 & 5654 \\
\hline 525 & -78454.3 & 44175.83 & 672.388 & 5239 \\
\hline
\end{tabular}


WHC-SD-H025-RPT-001, Rev. 0

APPENDIX T-5

TOP OF PRIMARY GRAVEL 
$\therefore$ ROGERS SURVEYING INC. $6272934 \quad P .02$

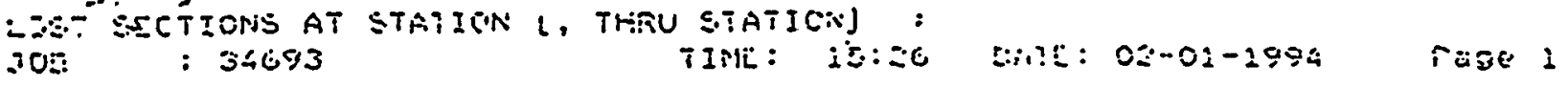

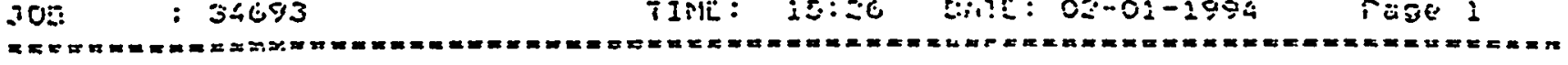

I. $5000.000000,5000.000000, \quad 100.000000$, STAFT

415, 44001.200000,-78195.02000C, 690.400000, FOS KI 0415

$4: 6,44022.346000,-73643.533000, \quad 707.140000$, STN TP 0416

$417,49002.200742,-78195.035733$, 695.3926:4, FOS KI 04is

$4: 8,44225.956448,-78454.376982,673.43673:, 3203$

$429,44225.838345,-75474.539619, \quad 674.005 * 6,3204$

$420,44226.029956,-78504.419063,674.682239,320 \equiv$

$421,44225.939067,-78529.281186,675.282673,3206$

$422 ; 44225.775989,-78554.378653, \quad 675.905875,3207$

$423,44225.691925,-78579.286507, \quad 676.565987,3208$

$424,44225.82105 \%,-78604.430132$; 677.\$70955, 3203

$425,44225.842297 .-78629.420570,577.799621,3210$

$426,44225.809373,-78654.362688,678.4305 .82,3211$

$427,44225.693550,-78679.288490, \quad 679.017406,3212$

$428,44201.022923,-78454.439585, \quad 672.803929,3228$

$429,44201.112143,-73479.362431, \quad 673.417299,3225$

$430,44200.982148,-78504.494211,674.046061,3750$

$431,44201.154649,-78529.364997, \quad 674.692455,3227$

$432,44201.128861,-78554.393475,675.353295,3226$

$433,44201.138484,-78579.324721,675.945520,3225$

$434,44201.344635,-78604.105219, \quad 675.5 .384=3.3224$

$435,44201.288336,-786.29 .445960, \quad 677.1986 .50,3.223$

$425,44201.487075,-70554.309587, \quad 877.407500,3221$

$437,64201.761552,-786.79 .395026, \quad 675.4155 \%, 322$

$438,44175.794521,-7 E 575.378423, \quad 6 \% .086523, \quad 3247$

$439,44175.790701,-78654.355188, \quad 678.450352,3248$

$440, \angle 4: 75.5 i 5406,-78629.455573, \quad 577.85320 \%, 3246$

$44 i, 44175.760035,-78604.486426,677.21513:, 3245$

$442,44175.730339,-78579.339876, \quad 676.5925: 7,3264$

$443,44175.919948,-78554.291761, \quad 675.953137,3243$

444, iA $175.828587,-78529.377486, \quad 675.338240,3242$

$445,44175.925910,-78504.426828,574.687404 .5241$

$446,44175.940216,-78477.29883 \%, 674.059857,3240$

$447,44: 75.859345,-78454.422255,673.456538,5239$

$448:=4001.303933,-78195.052142, \quad 596.406852,415$ 
WHC-SD-W025-RPT-001, Rev. 0

\section{APPENDIX T-6}

TOP OF OPERATIONS LAYER 
WHC-SD-W025-RPT-001, Rev. 0

ROGERS SURVEYING INC.

6272994

P.02

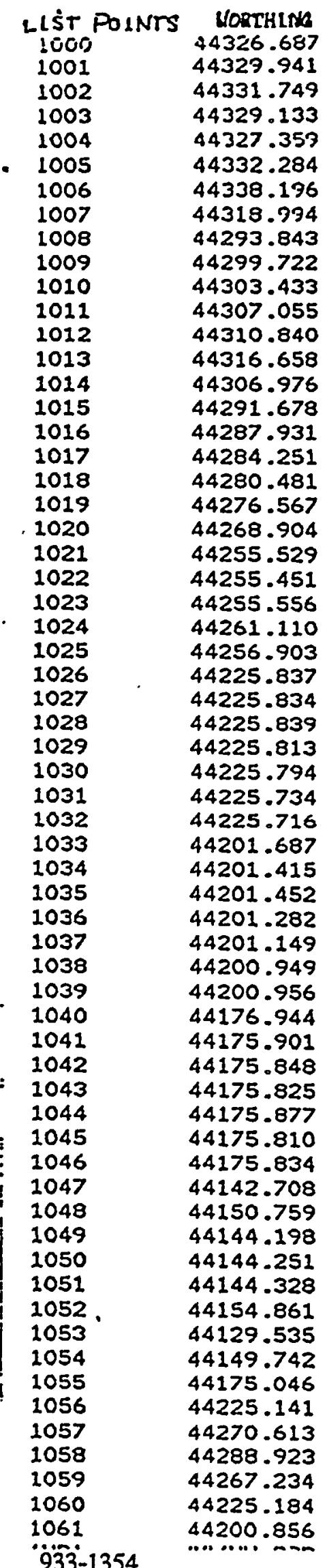

EASTING

$.7 .001 .30 \%$

$-73450.3 .57$

-78501.201

$-78576.293$

$-73626.421$

$-713701.734$

$-78771.666$

$-78750.915$

$-78724.054$

$-78674.394$

$-78624.489$

$-78574.598$

$-78524.756$

$-784.49 .956$

$-78419.579$

$-78448.056$

$-78497.983$

$-78547.851$

$-78597.786$

$-78647.639$

$-78697.053$

$-78682.628$

$-78620.846$

$-78570.759$

$-78520.943$

$-78470.535$

$-78454.413$

$-78504.427$

$-78554.393$

$-78604.347$

$-78654.401$

$-78710.015$

$-78714.692$

$-78708.079$

$-78679.256$

$-78629.449$

$-78579.412$

$-78529.470$

$-78479.420$

$-78437.300$

$-78437.276$

$-78479.450$

$-78529.402$

$-78579.429$

$-78629.388$

$-78679.344$

$-78710.112$

$-78712.673$

$-78679.377$

$-78597.924$

$-78545.928$

$-78497.629$

$-78446.587$

$-78423.072$

$-78411.974$

$-78417.196$

$-78417.233$

$-78423.356$

$-78406.322$

$-78396.605$

$-78392.288$

$-78392.169$

$\cdots$ T-6-1
EREYATIGKS GOLDER REF,*

$3 \% .32 \%$ :3:5

101.544 4611)

703.5074605

704.5574611

705.2734615

708.13154604

712.5034600

705.5774127

636.603 .4149

$697.260^{\circ} 4145$

$697.328 \quad 4141$

697.2304138

697.3154135

697.2934130

$693.451 \quad 4137$

688.9614150

688.9784153

688.9764157

688.9814162

688.9374165

687.6254174

682.9814623

681.3954626

$680.154 \quad 4628$

680.6564181

678.0824184

676.7854203

677.7714205

679.0184207

680.2574209

681.5094211

682.9054638

684.2884202

682.2394642

681.4914222

680.2614223

$679.006 \quad 4225$

$677.766 \quad 4227$

$676.800 \quad 4229$

676.8044232

676.7864251

677.1314240

$678.399 \quad 4242$

679.6474244

680.9244246

682.1454247

682.9254646

683.8064650

682.7654258

680.8964662

679.6084654

678.3244664

676.8384648

684.5064271

685.0454260

683.2754249

683.3104213

684.5114172

690.1684152

690.2454175

691.5684214

691.6654233 
WHC-SD-W025-RPT-001, Rev. 0

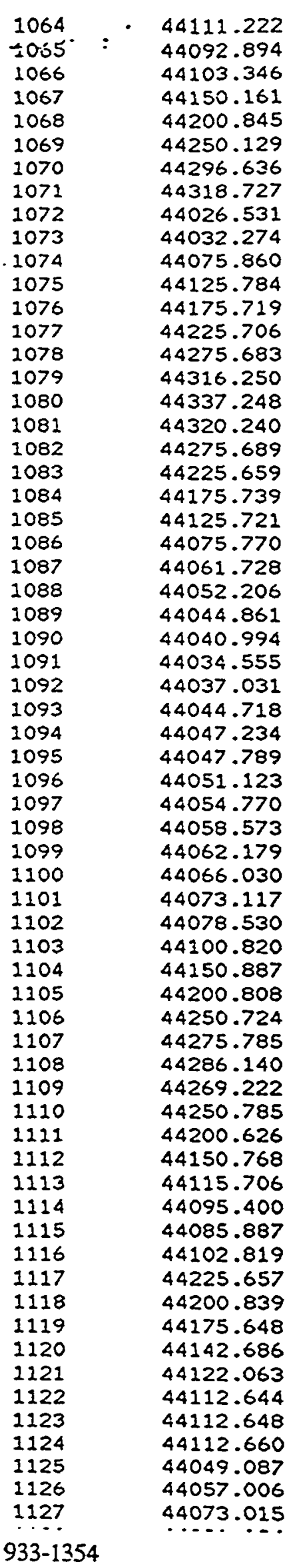

ROGERS SURVEYING INC. 6272994 P.OS

$-78406.036 \quad 690.2144289$ -

$-78388.946 \quad 695.8644301$ 人

-78368.922 . 699.3404683 \%

$-78368.938 \quad 699.3704651$

$-78369.104 \quad 699.3954643$ '

$-78369.071$

$-78369.223$

$-78378.692$

$-78778.022$

$-78801.789$

$-78808.061$

$-78808.180$

$-78808.155$

$-78808.084$

$-78808.186$

$-78808.089$

$-78800.752$

$-78782.454$

$-78789.677$

$-78789.565$

$-78789.683$

$-78789.604$

$-78785.503$

$-78770.564$

$-78750.743$

$-78730.671$

$-78680.831$

$-78656.220$

$-78630.948$

$-78555.147$

$-78504.825$

$-78450.996$

$-78479.439$

$-78529.298$

$-78579.190$

$-78629.020$

$-78678.883$

$-78728.564$

$-78752.672$

$-78759.051$

$-78764.579$

$-78764.802$

$-78764.719$

699.3744634 \%

699.3804620 -

$699.3624617^{\prime}$

714.6164725

715.3654720

715.4094152 wRond 4700

715.4164669

715.3834645

$715.4004637 \checkmark$

715.4064621

715.3474618 -

$715.4404113^{\prime}$

709.3284126

$709.2954166 \alpha$

709.2704199 -

$709.2524235^{\prime}$

$709.2744277^{-}$

707.8794315

705.3984329

$705.2714335^{2}$

$707.2624348^{2}$

707.2354350

$708.8404719^{\prime}$

707.4184718

702.9354715

700.9184712

$699.9964710 \checkmark$

698.953 4336

$699.0094333-$

698.9174331

$698.9464328^{\prime}$

698.9504322

.698.058 4316

699.3854313

$699.0234296 \alpha$

$700.9464256 \%$

$700.9764219^{\prime}$

700.8734191 -

$-78764.376$

$-78745.745$

$-78727.161$

$-78737.372$

$-78739.616$

$-78739.676$

$-78743.311$

$-78734.754$

$-78715.011$

$-78697.341$

$-78714.740$

$-78714.769$

$-78714.664$

$-78712.699$

$-78706.640$

$-78685.308$

$-78675.474$

$-78648.626$

$-78454.455$

$-78479.037$

$-78527.875$

700.8524168 -

697.1004154

$690.9744173^{\prime}$

691.8124193

$692.6014218^{\prime}$

692.6064255

693.7474284

$693.3814300 /$

$693.1924306^{\prime}$

687.1504291

684.3004202

$684.3054217^{\prime}$

$684.2604238^{\prime}$

$683.8294650^{-}$

$684.1384672^{\circ}$

683.8554677

$683.6204675^{\circ}$

682.9514676

699.05147090

$696.9394706^{\prime}$

$692.8474702^{\prime}$

T-6-2 
WHC-SD-W025-RPT-001, Rev. 0

ROGERS SURVEYING INC.

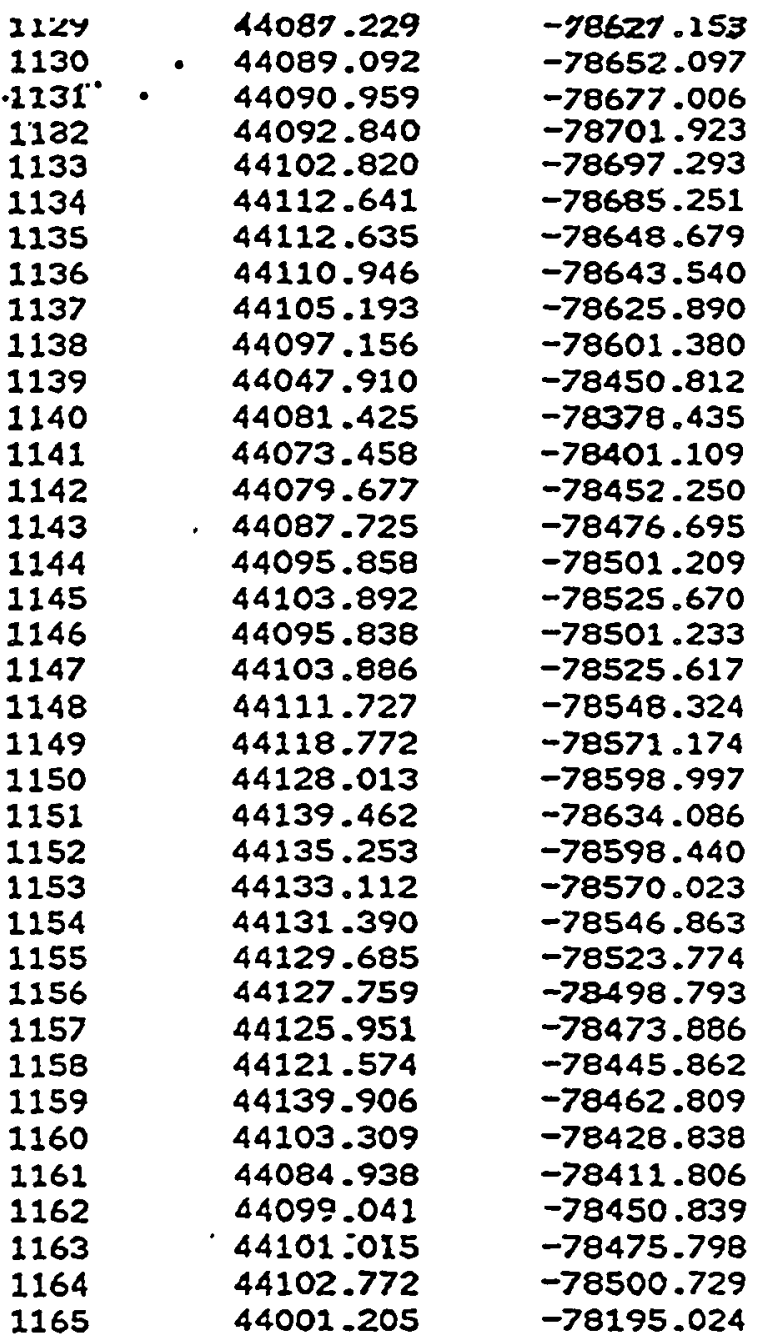

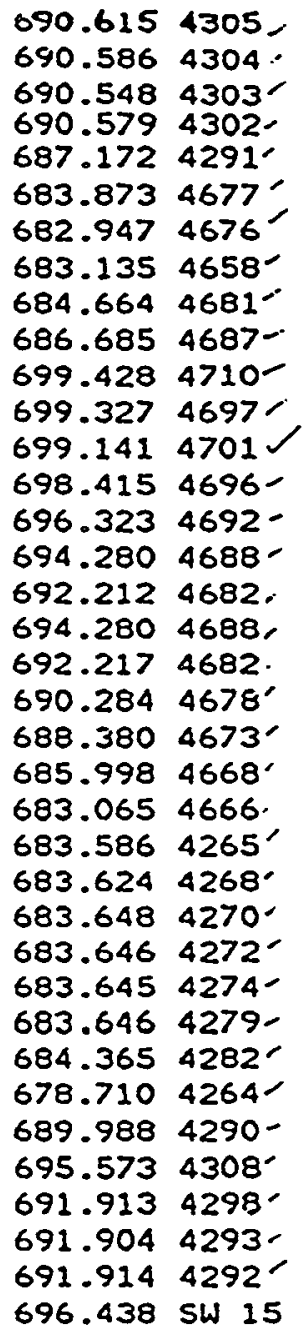


HHC-SD-H025-RPT-001, Rev。 0

APPENDIX U

REPORT: SOIL LINER TEST FILL AND SDRI TEST

$U-i$ 
WHC-SD-W025-RPT-001, Rev. 0

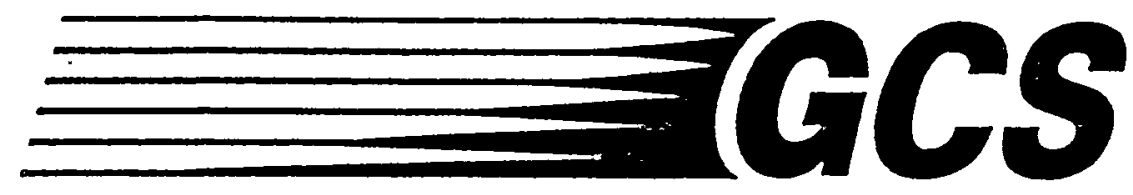

Golder Construction Services, Inc.

Quality Assurance and Construction Management

REPORT TO

UNITED STATES DEPARTMENT

OF ENERGY

\section{ON}

\section{CONSTRUCTION QUALITY ASSURANCE OBSERVATION AND}

TESTING, SOIL LINER TEST FILL AND SDRI TEST

RADIOACTIVE MIXED WASTE LAND DISPOSAL FACILITY

NON-DRAG-OFF, PROJECT W-025

Prepared by:

GOLDER CONSTRUCTION SERVICES, INC.

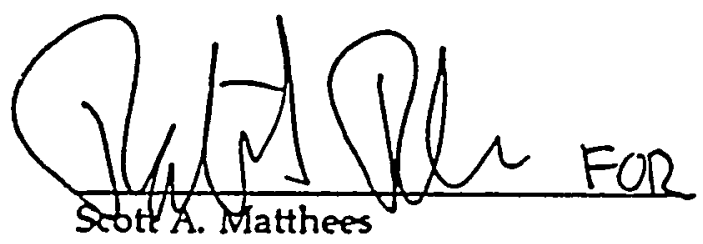

Construction Quality Assurance Engineer

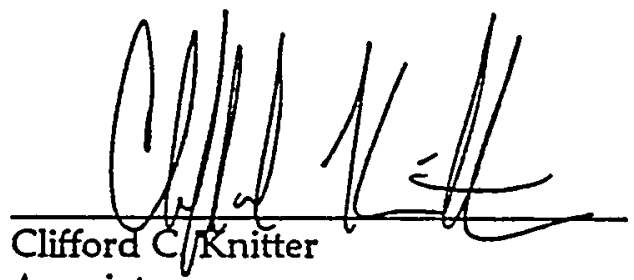

Associate

Fraitas S. Shuir

Frank S. Shuri, P.E.

Associate

May 13, 1994 


\section{TABLE OF CONTENTS}

Page No.

1. INTRODUCTION

1.1 Purpose

1.2 Scope of Services

1.3 Project Specifications

1

1

1

2. TEST FILL CONSTRUCTION PROCEDURES

2.1 Admix Preparation and Testing

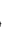

2.2 Test Fill Construction and Testing

3. INFILTROMETER INSTALLATION AND TEST PROCEDURES 5

3.1 Infiltrometer Installation Procedure $\quad 5$

3.2. Infiltrometer Test Procedures 6

4. TEST FILL PERMEABILITY RESULTS $\quad 7$

4.1 Laboratory Test Results $\quad 7$

4.2 SDRI Test Results $\quad 8$

5. CONCLUSION 10

\section{LIST OF TABLES}

U-1. Test Fill - SDRI Equipment Summary

U-2. Soil-Bentonite Admix Laboratory Testing Summary

U-3. Test Fill Moisture-Density Test Summary

U-4. Test Fill Shelby Tube Sample Test Summary

\section{LIST OF FIGURES}

U-1. Infiltrometer Schematic

U-2. Test Fill Moisture-Density Test Locations

U-3. Test Fill No. 2 Moisture-Density Test Locations

U-4. Test Fill Shelby Tube Sample Locations

\section{LIST OF APPENDICES}

Appendix U-1 Summary of Moisture-Density Testing Appendix U-2 SDRI Test Information 


\section{INTRODUCTION}

Golder Construction Services, Inc. (GCS) has performed quality assurance observation and testing for the construction of a soil liner test fill and the installation of a sealed double-ring infiltrometer (SDRD) at DOE's Radioactive Mixed Waste Land Disposal Facility, Non Drag Off, located in the 200 West area of the Hanford Reservation, near Richland, Washington. This report provides a summary of the observation and testing results associated with test fill construction and the SDRI installation.

\subsection{Purpose}

A soil liner test pad was built prior to the construction of the landfill to demonstrate that the contractor's equipment and construction methods could install a soil liner that meets the project specifications. Therefore, the contractor used the same soil liner materials, equipment, and methods to construct the test fill as were proposed for construction of the landfill.

Evaluation of the test fill permeability included testing of relatively undisturbed samples of the compacted soil liner and a large-scale permeability test using a sealed double-ring infiltrometer (SDRI).

\subsection{Scope of Services}

'The scope of GCS's services consisted of the observation and testing of the test fill, observation of the infiltrometer installation, and evaluation of the infiltrometer data. GCS started providing full-time observation services for the construction of the test fill on October 5, 1993. Monitoring of the SDRI took place from October 8, 1993 to February 9, 1994.

\subsection{Project Specifications}

The work was performed in accordance with the following documents:

- Material Specifications and Construction Requirements for the Radioactive Mixed Waste Land Disposal Facility, Non-Drag-Off, Project W-025, Revision 2, prepared for the U.S. Department of Energy-Richland Operations Office, - Contract No. DE-AC06-89RL11615 by Golder Associates Inc., dated May 26, 1993.

- Construction Quality Assurance Plan for Project W-025, Radioactive Mixed Waste (RMW) Land Disposal Facility - Non-Drag-Off, Revision 3, prepared for the U.S. Department of Energy-Richland Operations Office, Contract No. DE-AC06-89RL11615 by Golder Associates Inc., dated May 21, 1993. 


\section{TEST FILL CONSTRUCTION PROCEDURES}

The test fill for the Non-Drag-Off Land Disposal Facility was constructed by Delhur Industries, of Port Angeles, Washington, using the same equipment, procedures, and key personnel that they proposed to use during the placement and compaction of the soil liner for Non-Drag-Off. The test fill was constructed approximately 300 feet south of the Non-Drag-Off truck staging area.

\subsection{Admix Preparation and Testing}

The test fill was constructed using onsite eolian sand admixed with approximately twelve percent by dry weight of powdered bentonite. The bentonite was obtained from American Colloid Company, of Upton, Wyoming. Eolian sand, bentonite, and water were mixed in Delhur's pugmill. Small batches of the admixture were produced to calibrate the feed rate of the water, with a target moisture content of 19.5 percent.

Prior to production of the admix for the test fill, a sample of a small batch of the admix material produced by Delhur was obtained for laboratory testing, as was a sample of the eolian sand which was used in the admixture. The bentonite used in the admixture had previously been tested by GCS personnel. The results of the sieve and hydrometer tests (ASTM D 422) performed on the admix and sand samples, combined with the previous information gained from tests on the bentonite, indicated that the sample of admix contained a bentonite content of 12.9 percent, meeting the specification's requirement of a bentonite content of 11 percent to 14 percent. A Standard Proctor moisture-density test (ASTM D 698) was also performed on the sample of admix. The results of this test indicated a maximum dry density of $108.2 \mathrm{lbs} / \mathrm{ft}^{3}$, and an optimum moisture content of 17.5 percent. A summary of these laboratory tests is presented in Table U-2.

\subsection{Test Fill Construction and Testing}

On October 5, 1993, Delhur stripped, scarified, moisture conditioned, and compacted the subgrade for the test fill. GCS personnel performed 5 nuclear moisture-density tests on the compacted subgrade. The results of these tests indicated that the subgrade at each test location had been compacted to at least 90 percent of the maximum dry density as determined by ASTM D 1557. See Appendix U-1 for a summary of test results.

Delhur produced the admix for the test fill on October 5, 1993. The admix was stockpiled near the test fill location, and was allowed to hydrate until placement and compaction activities began on the following day.

On October 6, 1993, Delhur constructed a test fill with the admixture. GCS personnel performed CQA observation and testing during the construction of the test fill. The soil liner material was placed in the test fill using a Caterpillar 988 Front-End Loader and spread to approximately 8- to 10-inch thick loose lifts using a Caterpillar D-5 dozer 
equipped with low ground pressure (LGP) tracks. The loose lifts were then compacted to approximately 6-inch thick lifts using a Caterpillar $825-\mathrm{C}$ padfoot compactor. The final lift was rolled with a RayGo Rascal 410 A smooth-drum vibratory compactor. See Table U-1 for an equipment summary.

During the placement and compaction of the admix, GCS personnel performed density and moisture tests using a Troxler Model 3440 nuclear density gauge (ASTM D 2922 and ASTM D 3017, respectively). See Appendix U-1 for a summary of moisture-density test results.

Oven dried moisture tests were performed on a sample of material from each test location. Both a microwave oven (ASTM D 4643) and a conventional oven (ASTM D 2216) were used to determine the moisture content of each sample. The conventional oven moisture test result was used as the actual moisture content for each test sample, but the moisture content data determined by the two ovens was used to develop a correlation between the two methods in order to allow the use of the microwave oven during construction of the landfill. The microwave oven allows a relatively quick moisture content determination as opposed to the overnight turn-around time required for the conventional oven.

In addition to the moisture-density tests performed using the nuclear gauge, one side-byside nuclear density test (ASTM D 2922) and rubber balloon density test (ASTM D 2167) were performed on each lift of material placed in the test fill. The rubber balloon density tests were performed to confirm the accuracy of the nuclear gauge. There was good agreement between the test results determined using the rubber balloon and nuclear test methods.

During the placement and compaction of the first two lifts of admix in the test fill, at least six density tests were performed for every two, one-way passes of the compactor. The compactor made a total of six, one-way passes on each of the first two lifts of material. Nineteen moisture-density tests were performed on the first lift, and eighteen moisture-density tests were performed on the second lift. The high frequency of testing was performed on the first two lifts of the test fill to determine the optimum number of passes by the compactor to achieve the required compaction and kneading of the soil liner material.

The results of the observation and testing on the first two lifts indicated that two, one-way passes with the compactor could achieve the compaction required by the specifications, but the material did not appear to be adequately kneaded, an important consideration for layer bonding and permeability. Four, one-way passes by the compactor increased the density of the material, yielded more consistent test results, and appeared to adequately knead the material. Six, one-way passes with the compactor increased the density slightly, but did not appear to be significant enough to warrant the use of this many passes by the compactor. Therefore, four, one-way passes by the compactor was determined to provide the desired end product. 
Six moisture-density tests were performed after four, one-way passes of the compactor on the third lift of material placed, and three moisture-density tests were performed after four one-way passes of the compactor on the 4th, 5th, and 6th lifts of material placed for the test fill. A rubber balloon moisture-density test was performed on each lift of the test fill as previously noted.

Following the completion of the test fill construction, two test pits were excavated through the full thickness of the test fill to allow examination of the lifts for layer bonding. GCS personnel observed the walls of the test pits to determine the adequacy of the layer bonding between the compacted lifts of material. The lifts of compacted material were not distinguishable in the test pits. Close examination of the walls of the test pits indicated that the lifts of material had been adequately kneaded together, and the three foot thick test fill appeared to be monolithic.

In the southwest corner of the test fill, Delhur removed, then replaced with the same soil and recompacted the top three lifts of the test fill to demonstrate their method of removing and replacing soil liner material, should the event arise that "out-of-spec" material is placed during the construction of the landfill. GCS personnel performed a moisture-density test on each lift of the replaced material, and the test results indicated that the material had been adequately compacted. Following the completion of construction and testing activities, the surface of the test fill was back-dragged by the D-5 dozer, and then statically rolled with a Ray Go $410 \mathrm{~A}$ smooth-drum vibratory roller.

The results of the moisture-density tests indicated that the soil liner had been compacted to at least 90 percent of the maximum dry density as determined by ASTM D 698 and that most of the soil liner had been placed and compacted at a moisture content of one to five percent above the optimum moisture content as determined by ASTM D 698. A few of the moisture tests on material sampled at the density test locations indicated moisture contents slightly below the specified moisture content (the specifications require one to five percent above optimum moisture content). The moisture-density results are summarized in Table U-3, and the moisture-density test data are presented in Appendix U-1. The approximate moisture-density test locations are shown in Figures U-2 and U-3.

Shelby tube samples of the compacted soil liner were obtained from each lift of the test fill Flexible-wall permeability tests (ASTM D 5084) were performed on these samples in Golder Associates soils laboratory, located in Redmond, Washington. Holes remaining in the soil liner (after the removal of the Shelby tube samples) were backfilled and recompacted by hand. A Shelby tube sample was obtained from one of these repaired areas and a permeability test was performed to determine whether the soil had been adequately recompacted to achieve the permeability required by the specifications. In addition to the permeability tests performed on Shelby tube samples from the test fill, a permeability test was also performed on a laboratory-recompacted sample of the soil liner. A summary of the permeability test results is presented in Table U-4. The approximate Shelby tube sample locations are shown in Figure U-4. 


\section{INFILTROMETER INSTALLATION AND TEST PROCEDURES}

A sealed double-ring infiltrometer (SDRI) test program was performed to evaluate the insitu permeability of the twelve percent bentonite/sand admix over a relatively large area. The specific objectives of the SDRI test program included:

- An evaluation of the permeability of the placed soil;

- A comparison of the large-scale in-situ permeability test results with the laboratory permeability test results performed on undisturbed Shelby tube samples of the placed and compacted admix.

\subsection{Infiltrometer Installation Procedure}

The SDRI was assembled and installed by Delhur and GCS personnel upon completion of the test fill construction. The SDRI components were supplied by Trautwein Soil Testing Equipment of Houston, Texas. The SDRI consisted of a 12 foot by 12 foot, square, outer ring, a 5 foot by 5 foot, square, inner ring, and nine soil tensiometers. A schematic of the SDRI is shown in Figure U-1. The SDRI was installed in accordance with ASTM D 5093 using the following sequence:

- The surface the test fill was graded using a Caterpillar D-5 dozer and statically rolled using a Ray Go $410 \mathrm{~A}$ vibratory, smooth-drum roller;

- A four-inch wide by eighteen-inch deep trench for the outer ring was excavated using a Ditch-witch excavator;

- A two-inch wide by four-inch deep trench for the inner ring was excavated using a chain saw and hand labor;

- The outer ring was assembled and sealed in-place with Volclay bentonite grout;

- The inner ring was sealed in-place with Volclay bentonite grout;

- The nine soil tensiometers were installed to depths of six, twelve, and eighteen inches between the inner and outer rings;

- The inner ring was leak tested for 60 minutes by siphoning approximately 15 gallons of water into the inner ring;

- The outer ring was slowly filled with water to depth of one foot and examined for leaks; and

- The fittings, hoses, and water bags were then installed and the infiltration test started. 
The SDRI was installed on October 7, 1993. Water leaked out of the outer ring of the SDRI in the southwest comer of the trench, where a fairly dry batch of Volclay grout had been placed. The dry grout was removed and replaced, the outer ring of the SDRI was refilled with water, and the SDRI permeability test was started on October 8, 1993.

\subsection{Infiltrometer Test Procedures}

The SDRI test was completed in accordance with ASTM D 5093. Monitoring of the SDRI consisted of the following tasks:

- Weighing and Changing the Flexible Bag - The amount of water infiltration in the inner ring is determined by the water loss in the flexible bag which is connected to the inner ring. Initially the volume loss from the bag was measured twice a day. During the later stages of the test, the bag was measured at less frequent intervals of up to 7 days. Bag weights were recorded before attachment to and after detachment from the inner ring of the SDRI. The difference between these two weights was the infiltration for that time period.

- Monitoring the Tensiometers - The tensiometers are used to monitor the progression of the wetting front. Readings of the tensiometers were recorded each time the flexible bags were changed.

- $\quad$ Recording Water Temperature - Water temperature was recorded each time the flexible bags were changed.

- Swell Survey - The SDRI was checked for vertical swell of the inner ring due to the potential swelling of the soil liner. This was accomplished by surveying the elevation of each corner of the inner ring relative to a benchmark. No swelling of the soil liner was recorded. 
WHC-SD-W025-RPT-001, Rev. 0

May 13, 1994

7

$933-1354$

\section{TEST FILL PERMEABILITY RESULTS}

\subsection{Laboratory Test Results}

The results of the permeability tests on the Shelby tube samples from the test fill indicated an average permeability of $5 \times 10^{-8} \mathrm{~cm} / \mathrm{s}$, and have demonstrated that the twelve percent bentonite/sand admix will achieve a permeability of less than or equal to 1 $\times 10^{-7} \mathrm{~cm} / \mathrm{s}$ when the soil liner is placed and compacted in accordance with the project specifications for moisture content and compaction.

The permeability test on the Shelby tube sample from the first lift of the test fill indicated a hydraulic conductivity slightly greater than $1 \times 10^{-7} \mathrm{~cm} / \mathrm{s}$. The apparent reason for the difference in permeabilities between this sample and samples from subsequent lifts of the test fill is that the first lift was contaminated by subgrade material. The contamination likely occurred due to a combination of the weight of the Caterpillar $825-\mathrm{C}$ compactor, the six- to seven-inch length of the pegs for the padfoot compactor, and the thickness of the first lift of material ( 10 inches loose, 6 inches compacted). The pegs of the compactor likely forced admix into the subgrade, displacing the subgrade sand, and pushing the subgrade sand upward between the pegs of the compactor.

Golder's laboratory manager indicated that the Shelby tube sample from the first lift did not appear to have the cohesive quality that the rest of the samples displayed. Atterberg limits tests performed on this sample of material and a sample from the Shelby tube taken in the second lift of the test fill showed that the sample from the first lift was significantly less plastic, suggesting a higher sand content.

To demonstrate that a first lift of material could be placed and compacted to achieve the required permeability, and to demonstrate that contamination of this lift could be minimized or eliminated, Delhur constructed a second test fill. The second test fill was only one lift thick, because Delhur had already demonstrated that subsequent lifts could be placed and compacted to achieve the required permeability. Delhur increased the first lift loose thickness from ten inches to twelve inches for the second test fill, and used the same equipment to compact the material as was used on the first test fill. Four, one-way passes with the compactor were used to compact the soil. The compacted thickness of the second test fill was eight to nine inches. GCS personnel performed two moisturedensity tests on the subgrade prior to the construction of the second test fill, and performed four moisture-density tests and one side-by-side rubber balloon moisturedensity test on the admix in the second test fill. A Shelby tube sample was obtained from the second test fill. The permeability test result on this sample was $5 \times 10^{-8} \mathrm{~cm} / \mathrm{s}$, meeting the specification's permeability requirement and is consistent with the results of the permeability tests performed on lifts 2 through 6 from the first test fill.

In addition to the permeability tests performed on the Shelby tube samples, a permeability test was performed on a laboratory-recompacted sample of the soil liner material. The results of this test are indicated a permeability comparable to that indicated 
by the tests on samples from lifts two through six in the first test fill. The results of the permeability tests on the Shelby tube samples and the recompacted sample are presented in Table U-4.

\subsection{SDRI Test Results}

The SDRI was monitored on a daily basis when it was initially installed. Daily readings include the water level in the outer ring, the amount of water infiltration in the inner ring, the water temperature, and the tensiometer readings. A swell survey was incorporated to check the inner ring of SDRI for vertical swell due to the potential swelling of the admix. No swelling of the admix was detected. The SDRI test was run for 124 days.

The depth of the wetting front was not detected by the shallowest of the tensiometers which were installed at a six-inch depth, as evidenced by the soil suction indicated by the vacuum readings on the gauges, so the depth of the wetting front was less than six inches. In order to determine the depth of the wetting front, samples of the soil liner material were collected at one-inch intervals to a depth of six-inches. The oven-dried moisture contents of these samples were determined and compared to moisture contents measured during construction. The moisture contents of the samples from the 0 - to 1inch interval, 1 - to 2 -inch interval, and the $2-$ to 3 -inch interval were significantly greater than those measured during construction (greater than three standard deviations above the mean value). The moisture content of the sample from the 3- to 4-inch interval was slightly higher than the range of moisture content measured during construction.

Therefore, the depth to the wetting front was conservatively determined to be 3.5 inches. The final moisture contents of the test fill are shown in Appendix U-2.

The permeability of the test fill based on the data from the SDRI test was determined based on the following relationship:

$$
\begin{array}{ll}
\mathrm{K}=\mathrm{V} \mathrm{i} \quad \text { where, } \quad & \mathrm{K}=\text { Permeability } \\
& \mathrm{I}=\text { Infiltration Rate } \\
& \mathrm{i}=\text { Hydraulic Gradient }
\end{array}
$$

The SDRI test results are summarized in Appendix U-2. Infiltration rates of $1 \times 10^{-6}$ to $2 \times$ $10^{-8} \mathrm{~cm} / \mathrm{s}$ were measured during the duration of the test. The highest infiltration rates were measured during the first week of the test. The cumulative infiltration rate for the 124 day duration of the test was $6 \times 10^{-8} \mathrm{~cm} / \mathrm{s}$.

The hydraulic gradient was determined by the following relationship:

$$
\begin{array}{ll}
i=(D+h) / D \text { where }, \quad & i=\text { Hydraulic Gradient } \\
& D=\text { Depth to the Wetting Front } \\
& h=\text { Depth of Ponded Water }
\end{array}
$$


The depth of ponded water throughout the SDRI test was twelve inches. The depth of the wetting front was measured directly from the test fill based on the soil moisture contents and was determined to be 3.5 inches.

Based on the data from the SDRI test, the calculated permeability of the test fill, using the cumulative infiltration rate over the 124 -day test period, was $1.4 \times 10^{-8} \mathrm{~cm} / \mathrm{s}$. This represents a conservative estimate of the test fill permeability because, the cumulative infiltration rate and not the final infiltration rate $\left(3 \times 10^{-8} \mathrm{~cm} / \mathrm{s}\right)$ was used and we did not include soil suction, as measured by the tensiometers, in the determination of the hydraulic gradient. 


\section{CONCLUSION}

The proposed material and placement and compaction procedures should result in a uniform soil liner that meets the performance specifications for Non-Drag-Off. The first lift of the admix should be increased to a twelve-inch loose lift thickness, and should be compacted by four passes of the 825-C compactor. The construction of the second test fill demonstrated that this procedure could achieve the required compaction and permeability of the soil liner. The placement and compaction of the first lift should be monitored closely, especially on the slopes of the excavation so that the soil liner material is not contaminated by incorporation of the subgrade material. The contractor is planning on placing the material in a downhill direction. In the event that contamination of the first lift occurs, the contractor will be required to remove and replace the contaminated material, and will be required to change his procedures, possibly placing the material in an uphill direction.

Subsequent lifts of the soil liner should be placed in maximum loose-lift thicknesses of ten inches or less, and the compacted thickness of these lifts should not exceed six inches. If the compacted lift thicknesses exceed six inches, the contractor will be required to decrease the loose-lift thicknesses of these lifts from ten inches to eight inches. A total of six lifts must be placed and compacted. Four passes of the 825-C compactor should be used to compact each lift.

The contractor should adjust the water feed rate for the pug mill as necessary to compensate for drying of the admix material. The target moisture content of the first test fill was approximately 19.5 percent, and drying of the admix was apparent as the construction of the test fill proceeded. 
WHC-SD-W025-RPT-001, Rev. 0

TABLES

Golder Construction Services, Inc. 


\section{TEST FILL - SDRI EQUIPMENT SUMMARY}

\begin{tabular}{|c|c|}
\hline EQUIPMENT & FUNCTION \\
\hline Pug Mill (Owner Fabricated) & Admixing of natural soils with bentonite \\
\hline Caterpillar 988 Front-End Loader & Hauling admix from stockpile to test fill \\
\hline Caterpillar D-5 LPG Dozer & Spreading admix on test fill lifts \\
\hline Caterpillar 825-C Tamping Foot Compactor & Compacting admix lifts of test fill \\
\hline $\begin{array}{l}\text { RayGo Rascal 410A Smooth Drum } \\
\text { Vibratory Compactor }\end{array}$ & Final surface prep for final lift of test fill \\
\hline Water Truck & Moisture conditioning of subgrade \\
\hline $\begin{array}{l}\text { Troxler } 3440 \text { Nuclear Moisture-Density } \\
\text { Gauge }\end{array}$ & $\begin{array}{l}\text { Moisture-density testing of the compactive } \\
\text { effort applied to the subgrade and each lift } \\
\text { of the test fill }\end{array}$ \\
\hline Rubber Balloon Density Apparatus & $\begin{array}{l}\text { Correlation of the nuclear moisture-density } \\
\text { gauge }\end{array}$ \\
\hline $\begin{array}{l}\text { Travtwein Sealed Double-Ring Infiltrometer } \\
\text { (SDRI) (12'x12' outer ring, 5'x5' inner ring) }\end{array}$ & $\begin{array}{l}\text { In-situ permeability testing of the test fill } \\
\text { compacted admix }\end{array}$ \\
\hline
\end{tabular}


WHC-SD-W025-RPT-001, Rev. 0

May 13, 1994

TABLE U-2

SOIL-BENTONITE ADMIX LABORATORY TESTING SUMMARY

(Admix Produced By Pug Mill)

\begin{tabular}{|c|c|c|c|c|c|}
\hline \multicolumn{6}{|c|}{ BENTONITE PROPERTIES } \\
\hline Property & Lab Result & Specification & \multicolumn{3}{|c|}{ API Specification } \\
\hline Free Swell & $25 \mathrm{ml}$ & $25 \mathrm{ml}$ & \multicolumn{3}{|c|}{$2 A \mathrm{ml}$ minimum } \\
\hline Wet Screen & . $2.6 \%$ & N/A & \multicolumn{3}{|c|}{$4 \%$ maximum } \\
\hline Moisture & $7.3 \%$ & N/A & \multicolumn{3}{|c|}{$N / A$} \\
\hline Filtrate & $15 \mathrm{ml}$ & N/A & \multicolumn{3}{|c|}{$15 \mathrm{ml}$ maximum } \\
\hline Marsh Funnel & $69 \mathrm{~s}$ & & & & \\
\hline \multicolumn{6}{|c|}{ API Spec = American Petroleum Institute Specification for 91 Barrel yield Bentonite: } \\
\hline \multicolumn{6}{|c|}{ ADMIX PROPERTIES } \\
\hline Sample & Soil Description & Maximum Dry & Optimum & Percent & Percent \\
\hline TXS-1 & Sand for Admix & N/A & N/A & $20.2 \%$ & $2.0 \%$ \\
\hline $\mathrm{TX}-1$ & Pug Mill & 108.2 pcf & $17.5 \%$ & $30.5 \%$ & $10.4 \%$ \\
\hline
\end{tabular}

\section{CALCULATIONS:}

Given: 30.5 percent of the Admix (A) passes the \#200 Sieve.

202 percent of the sand $(S)$ passes the \#200 sieve.

Assume: 100 percent of the bentonite (B) passes the \#200 Sieve.

Find: The percentage of the sand and bentonite components of the admixture based on the dry weights of the materials.

Solution: Based on the percentages of the components (A, S, and B) passing the \#200 Sieve, the percentages of sand and bentonite in the admix can be determined as follows:

$(B \times \%$ passing \#200) $+(S \times \%$ passing \#200) $=(A \times \%$ passing \#200)

$(B \times 100)+(S \times 20.2)=(A \times 30.5)=100 B+20.2 S=30.5 A$

$A=100$ percent of the Admix; and

Bentonite $(B)+$ Sand $(S)=100$ percent of the Admix

So $B+S=100 ;$ and $S=100 \cdot B$

Therefore:

$$
\begin{aligned}
& 100 B+(202 \times(100-B)=100 \times 30.5 \\
& 100 B+2020-20.2 B=3050 \\
& B=12.91
\end{aligned}
$$

Answer: The bentonite comprises 12.91 percent of the admix. 
TEST FILL MOISTURE-DENSITY TEST SUMMARY

\begin{tabular}{|c|c|c|c|c||}
\hline $\begin{array}{c}\text { Lift } \\
\text { Number }\end{array}$ & $\begin{array}{c}\text { Number of Tests } \\
\text { Performed }\end{array}$ & $\begin{array}{c}\text { Number of } \\
\text { Passes } \\
\text { with Compactor }\end{array}$ & $\begin{array}{c}\text { Average Percent } \\
\text { Compaction } \\
(1)\end{array}$ & $\begin{array}{c}\text { Average } \\
\text { Moisture } \\
\text { Content } \\
(\%)(2)\end{array}$ \\
\hline 1 & 7 & 2 & 93.7 & 19.4 \\
\hline 1 & 6 & 4 & 94.7 & 19.1 \\
\hline 1 & 6 & 6 & 96.6 & 19.1 \\
\hline 2 & 6 & 2 & 95.1 & 19.2 \\
\hline 2 & 6 & 4 & 96.7 & 19.1 \\
\hline 2 & 6 & 6 & 97.0 & 18.9 \\
\hline 3 & 6 & 4 & 95.2 & 19.2 \\
\hline 4 & 3 & 4 & 97.0 & 18.5 \\
\hline 5 & 3 & 4 & 96.3 & 18.9 \\
\hline 6 & 3 & 4 & 96.9 & 18.5 \\
\hline \multicolumn{5}{|c|}{ Second Test Fill Constructed on October 20, 1993 } \\
\hline 1 & (One Lift, 12 Inch Loose Lift Thickness) & \\
\hline
\end{tabular}

Notes: 1. Percent of maximum dry density as determined by ASTM D 698.

2. Percent moisture content as determined by ASTM D 2216. 
TEST FILL SHELBY TUBE SAMPLE TEST RESULTS

\begin{tabular}{|c|c|c|c|c|c|c|c|c|c|c|}
\hline $\begin{array}{c}\text { Sample } \\
\text { No. }\end{array}$ & Lift & $\begin{array}{c}\text { No. of } \\
\text { Compactor } \\
\text { Passes }\end{array}$ & $\begin{array}{c}\text { Moisture } \\
\text { Content } \\
(\%)\end{array}$ & $\begin{array}{l}\text { Percent } \\
\text { Over } \\
\text { Optimum } \\
\text { Moisture } \\
\text { Content }\end{array}$ & $\begin{array}{c}\text { Dry } \\
\text { Density } \\
\text { (pef) }\end{array}$ & $\begin{array}{c}\text { Percent } \\
\text { Compaction }\end{array}$ & $\begin{array}{l}\text { Perm } \\
(\mathrm{cm} / \mathrm{s})\end{array}$ & LL & PL & PI \\
\hline$T X-1$ & $N / A$ & $N / A$ & 20.0 & 25 & 1029 & 95.1 & $6 \times 10^{-}$ & $N / A$ & N/A & $N / A$ \\
\hline TF-18 & 1 & 6 & 19.0 & 15 & 103.4 & 955 & $1 \times 10^{\prime}$ & 25 & 24 & 1 \\
\hline$T F-33$ & 2 & 6 & 19.0 & 1.5 & 103.4 & 95.6 & $6 \times 10^{4}$ & 45 & 21 & 24 \\
\hline TF38 & 3 & 4 & 19.0 & 15 & 1028 & 95.0 & $5 \times 10^{4}$ & $N / A$ & N/A & N/A \\
\hline TF- 46 & 4 & 4 & 18.7 & 1.2 & 105.4 & 97.4 & $4 \times 10^{-4}$ & N/A & N/A & N/A \\
\hline TF-49 & 5 & 4 & 19.2 & 1.7 & 1024 & 95.9 & $5 \times 10^{0}$ & $N / A$ & $N / A$ & $\mathrm{~N} / \mathrm{A}$ \\
\hline TF-49A & 5 & 4 & 19.3 & 1.8 & 1045 & 96.6 & $4 \times 10^{0}$ & N/A & N/A & N/A \\
\hline TF.50 & 6 & 4 & 18.8 & 1.3 & 105.1 & 97.6 & $3 \times 10^{4}$ & $N / A$ & N/A & N/A \\
\hline$T F 2-2$ & 1 & 4 & 22.0 & 4.5 & 100.7 & 93.1 & $5 \times 10^{-4}$ & N/A & $N / A$ & $N / A$ \\
\hline
\end{tabular}

Notes: 1. Sample TX-1 is a recompacted laboratory sample.

2. Sample TF2-2 shelby tube taken from second test fill, one lift thick. 
WHC-SD-W025-RPT-001, Rev. 0

s.

FIGURES

933-1354

$\mathrm{U}-18$

Golder Construction Services, Inc. 


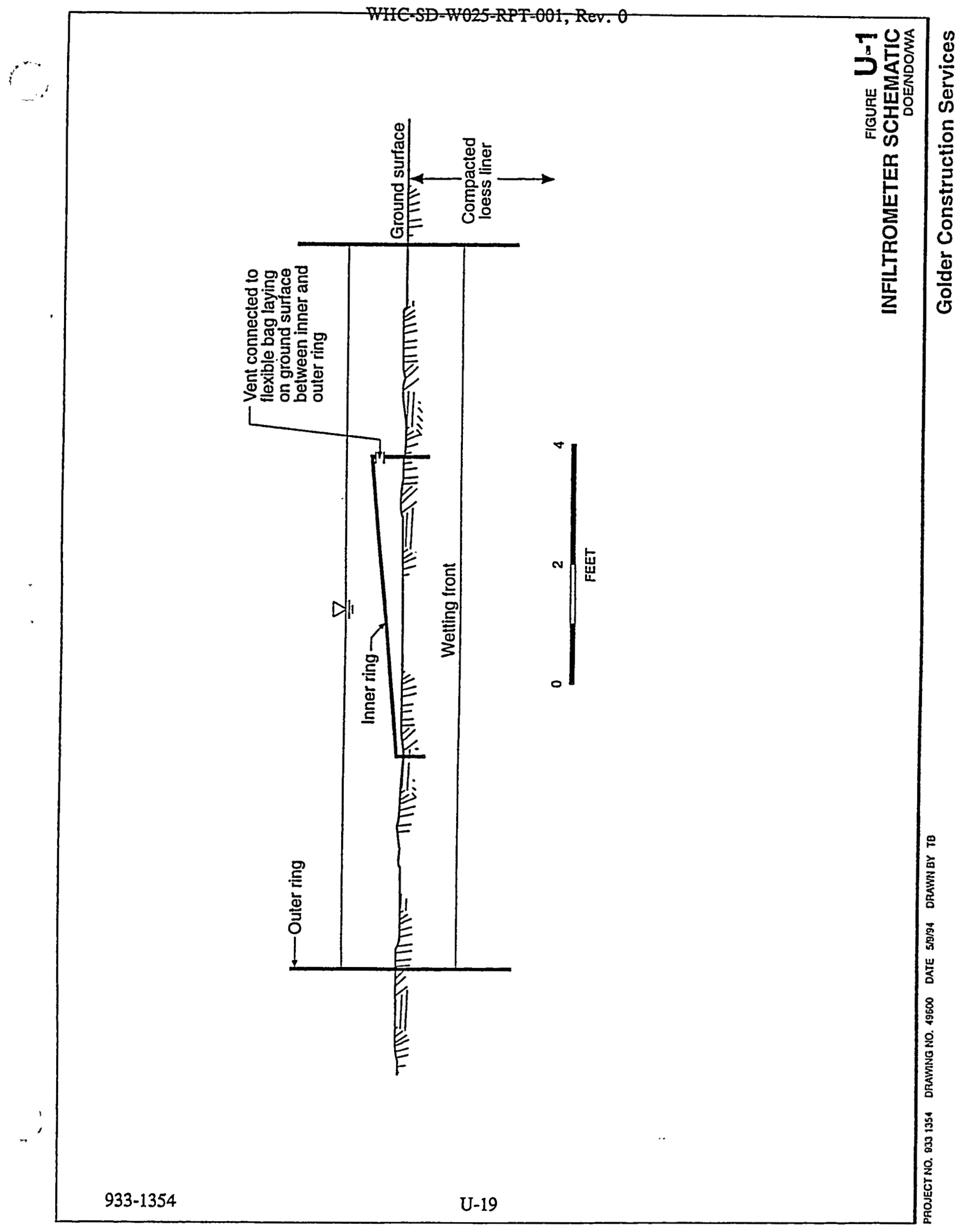



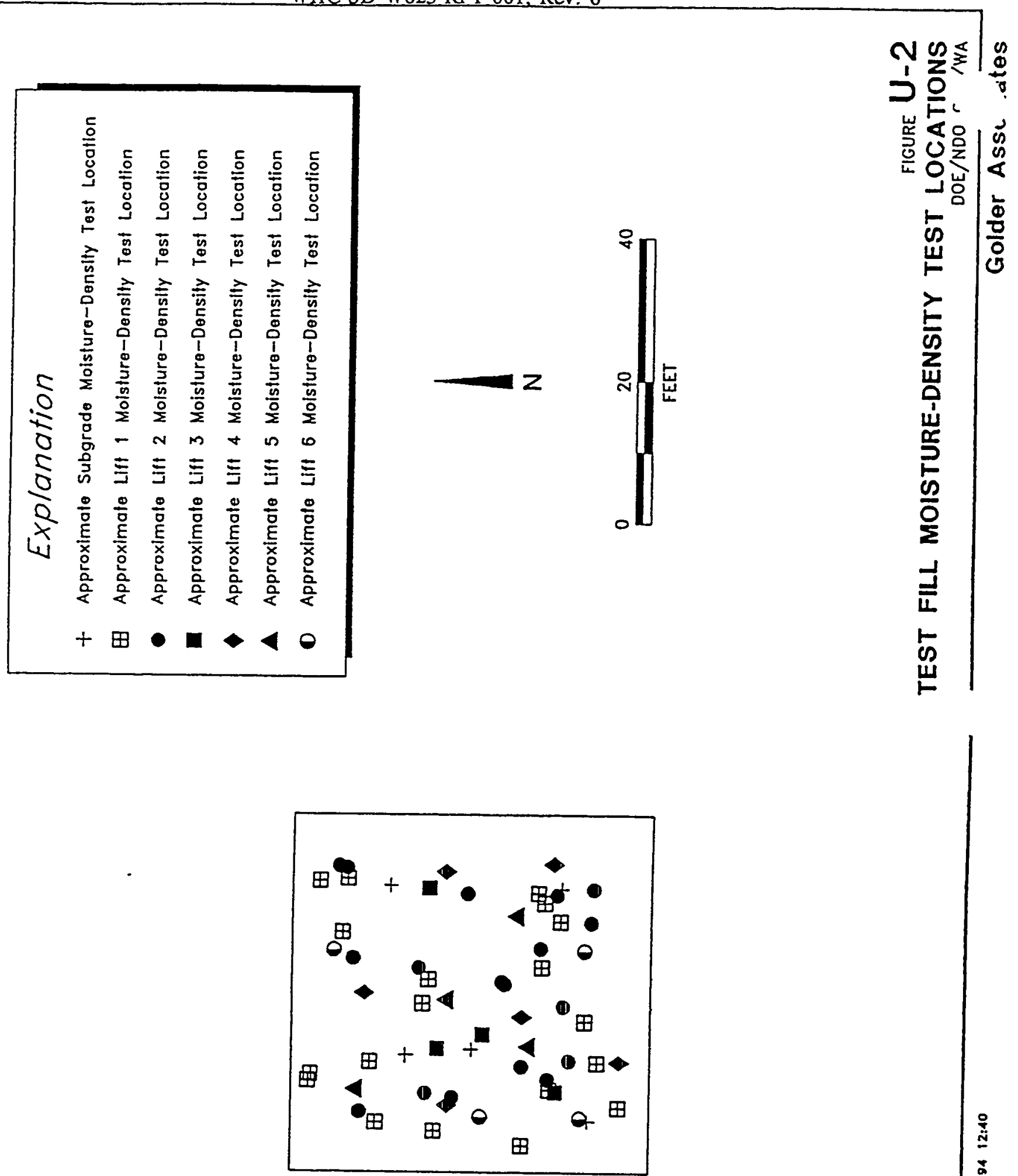

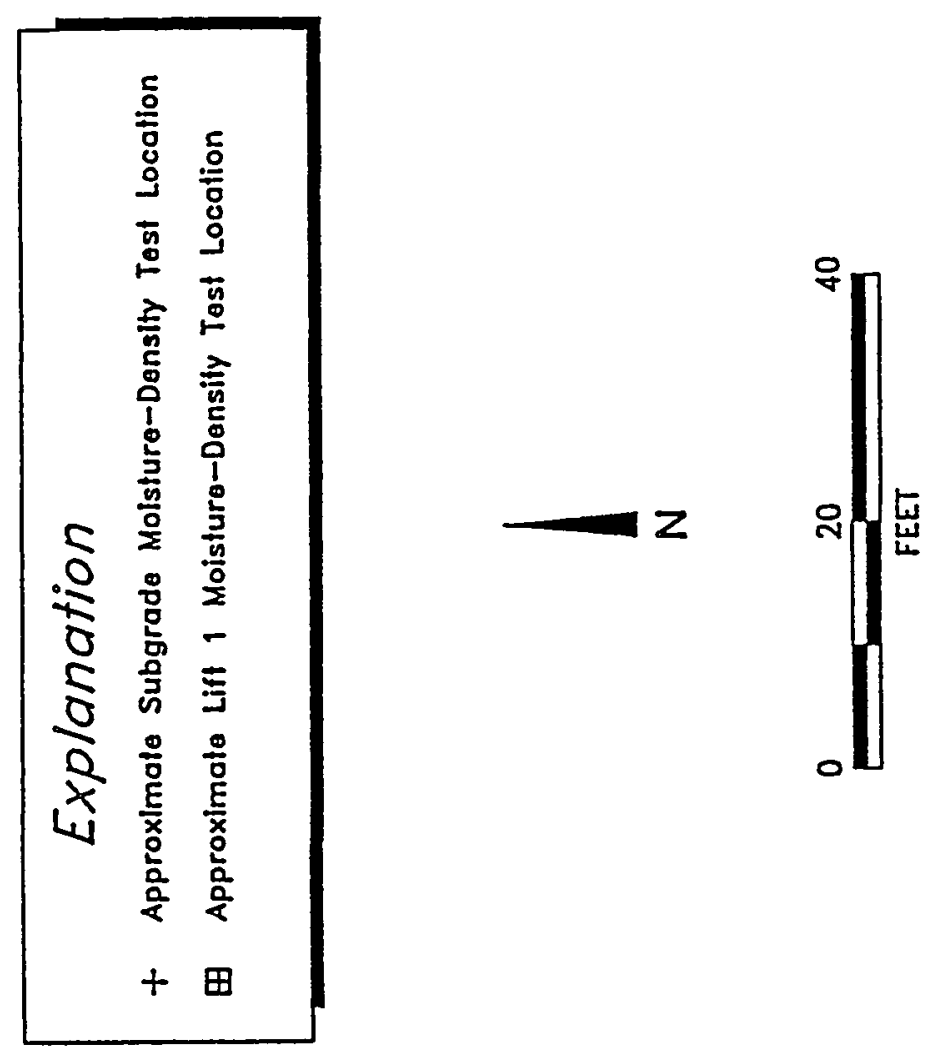

es

닌

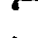

E

$\sum_{11}^{\infty}$

9

u

$\stackrel{\infty}{5}$

es

올

N

$\dot{8}$

$\frac{1}{21}$

占

$\underset{5}{\rightleftarrows}$

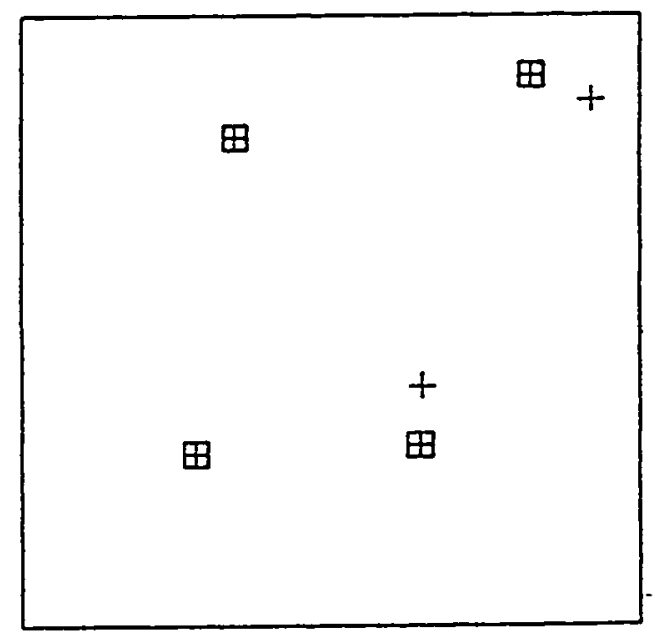




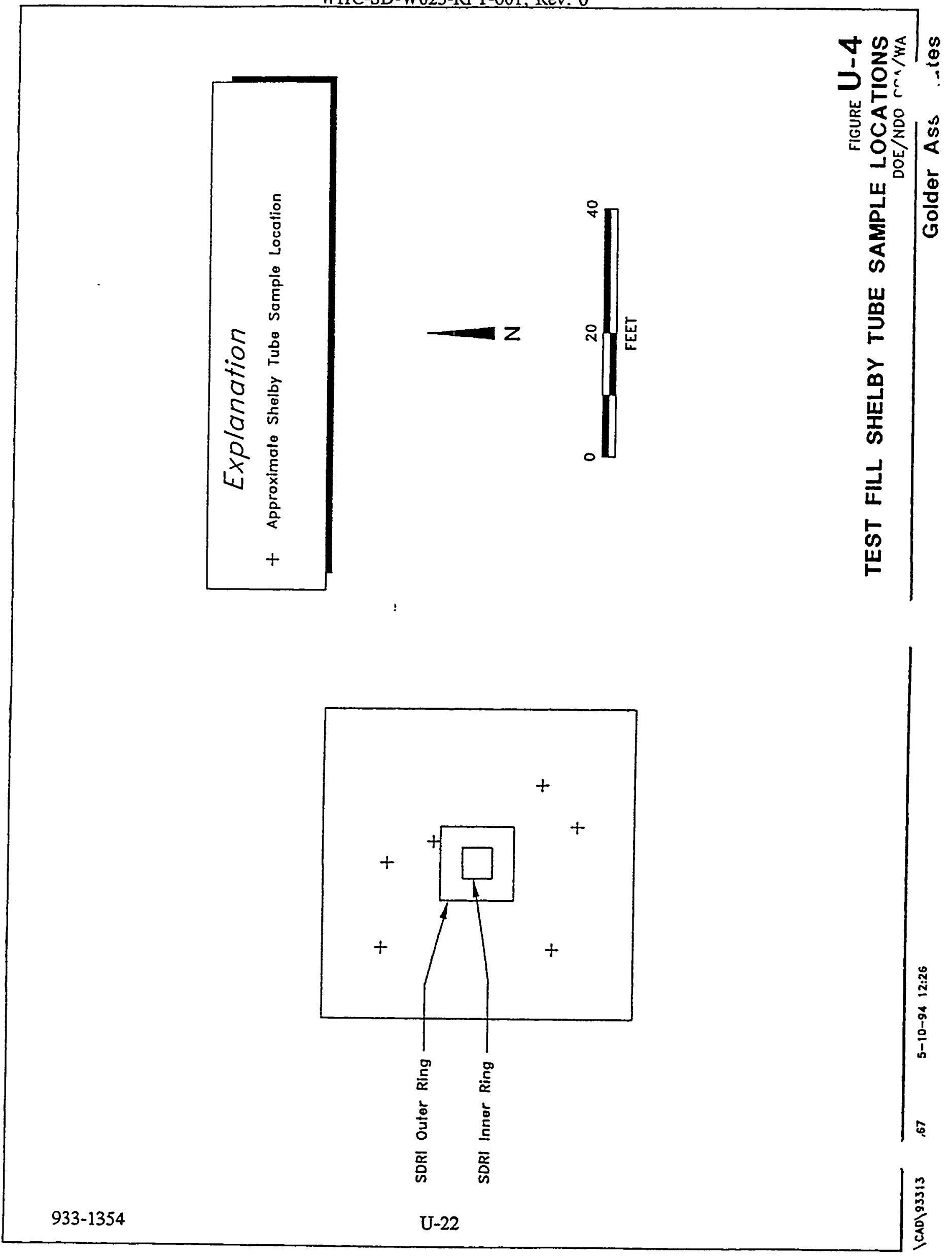


WHC-SD-W025-RPT-001, Rev. 0

\author{
APPENDIX U-1 \\ SUMMARY OF MOISTURE-DENSITY TESTING
}

$U-1 . j$ 
GOLOER CONSTRUCTION SERVICES INC.

SUMMARY OF SOIL LINER MOISTURE-DENSITY TESTS

DEPARTHENT OF ENERGY 05/12/94

NON-DRAG OFF LANDFILL FACILITY

HANFORD NUCLEAR RESERVATION

Page 1

SECONDARY SOIL LINER

TEST FILL

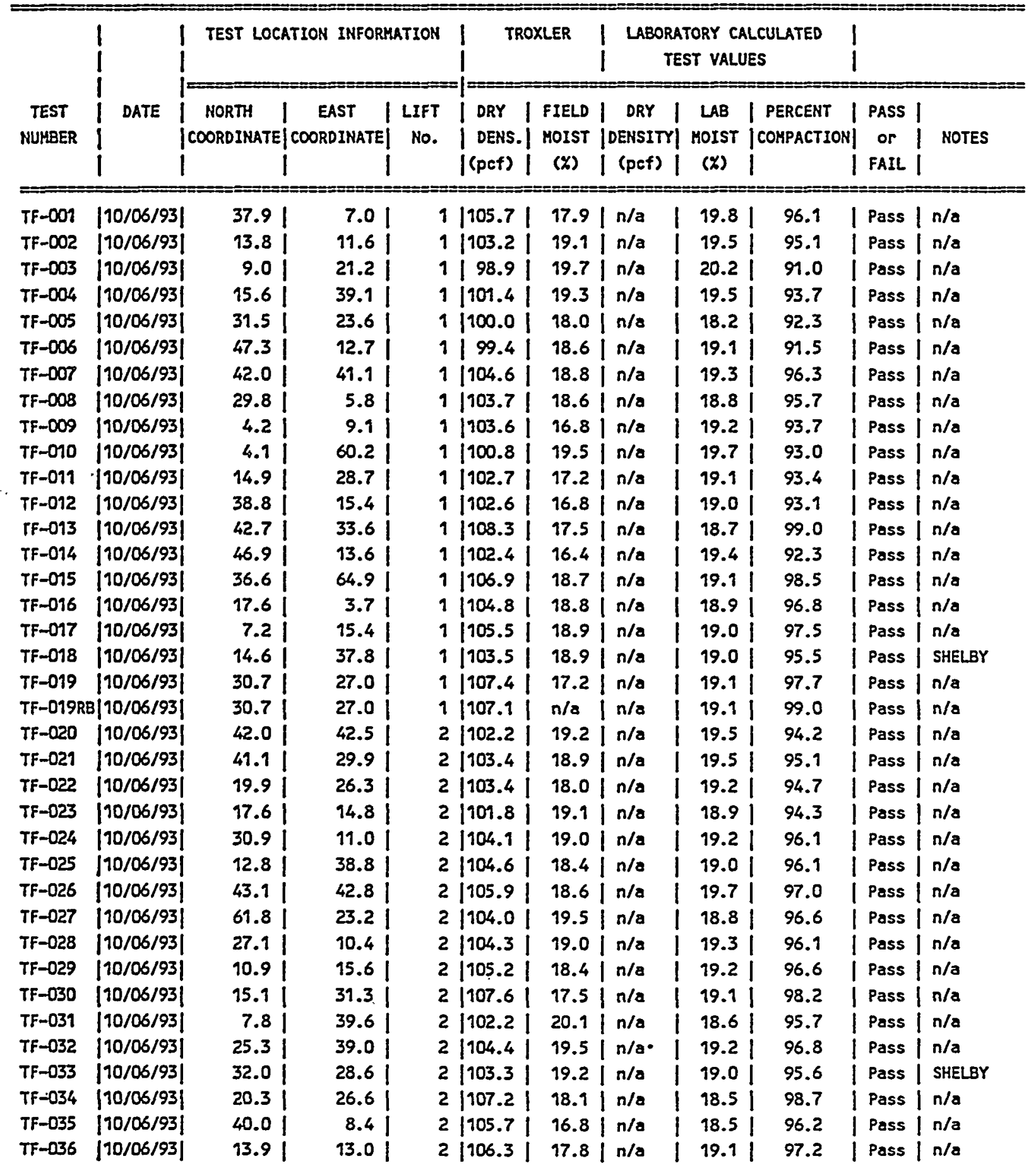


WHC-SD-W025-RPT-001, Rev. 0

GOLDER CONSTRUCTION SERVICES INC.

SUMMARY OF SOIL LINER MOISTURE-DENSITY TESTS 933-1354

DEPARTMENT OF ENERGY 05/12/94

NON-DRAG OFF LANDFILL FACILITY $\quad$ Page 2

HANFORD NUCLEAR RESERVATION

SECONDARY SOIL LINER

TEST FILL

\begin{tabular}{|c|c|c|c|c|c|c|c|c|c|c|c|c|c|}
\hline \multirow[b]{2}{*}{$\begin{array}{l}\text { TEST } \\
\text { NUMBER }\end{array}$} & \multirow[b]{2}{*}{ DATE } & \multirow{2}{*}{$\begin{array}{l}\text { TEST LOCA } \\
\mid= \\
\mid \text { NORTH }\end{array}$} & \multicolumn{3}{|c|}{ ITION INFORHATION } & \multicolumn{2}{|c|}{ TROXLER } & \multicolumn{4}{|c|}{$\begin{array}{l}\text { LABORATORY CALCULATED } \\
\text { TEST VALUES }\end{array}$} & & \multirow[b]{2}{*}{ NOTES } \\
\hline & & & $\begin{array}{c}\text { EAST } \\
\text { COORDINATE! }\end{array}$ & $\begin{array}{l}\text { LIFT } \\
\text { No. }\end{array}$ & & \begin{tabular}{|l|}
$\mid$ DRY \\
$\mid$ DENS. \\
$\mid$ (pcf) $\mid$
\end{tabular} & $\begin{array}{c}\text { FIELD } \\
\text { MOIST } \\
(\%)\end{array}$ & $\begin{array}{l}\text { DRY } \\
\text { |DENSITY } \\
\mid \text { (pcf) }\end{array}$ & & $\begin{array}{l}\text { LAB } \\
\text { MOIST } \\
(x)\end{array}$ & $\begin{array}{l}\text { I PERCENT } \\
\text { |COMPACTION } \\
\mid\end{array}$ & $\left|\begin{array}{c}\text { PASS } \\
\text { or } \\
\text { FAIL }\end{array}\right|$ & \\
\hline$T F-037$ & $|10 / 06 / 93|$ & 8.01 & 35.01 & & 2 & |106.2 & 18.1 & $\mathrm{n} / \mathrm{a}$ & & 19.0 & 97.4 & Pass & $n / a$ \\
\hline$T F-037 \mathrm{RB}$ & $3|10 / 06 / 93|$ & 8.0 i & 35.01 & & 2 & 105.0 & $\mathrm{n} / \mathrm{a}$ & $\mathrm{n} / \mathrm{a}$ & & 19.0 & 97.0 & Pass & $\mathrm{n} / \mathrm{a}$ \\
\hline$T F-038$ & $|10 / 06 / 93|$ & $39.5 i$ & $25.1 i$ & & 3 & |103.7 & 18.0 & $\mathrm{n} / \mathrm{a}$ & i & 19.0 & 95.0 & Pass & SHELBY \\
\hline$T F-039$ & $|10 / 06 / 93|$ & $27.9 i$ & 9.41 & & 3 & |103.8 & 18.8 & $n / a$ & & 19.9 & 95.1 & Pass & $\mathrm{n} / \mathrm{a}$ \\
\hline$T F-040$ & $|10 / 05 / 93|$ & 4.1 & $15.5 i$ & & 3 & i204.6 & 18.3 & $n / a$ & & 19.3 & 95.8 & Pass & $n / a$ \\
\hline$T F-D \& 1$ & $|10 / 06 / 93|$ & $97.6 \mid$ & $21.8 i$ & & 3 & 104.5 & 18.7 & $n / a$ & i & 19.9 & 95.6 & Pass & $\mathrm{n} / \mathrm{a}$ \\
\hline$T F-042$ & $|10 / 06 / 93|$ & $13.4 i$ & 43.21 & & 3 & 1704.2 & 18.8 & $n / a$ & i & 19.3 & 95.8 & Pass & $n / a$ \\
\hline$T F-043$ & $|10 / 06 / 93|$ & 28.4 & 42.11 & & 3 & 104.2 & 17.6 & $\mathrm{n} / \mathrm{a}$ & 1 & 18.0 & 95.9 & Pass & $\mathrm{n} / \mathrm{a}$ \\
\hline$T F-043 R B$ & $3|10 / 06 / 93|$ & $28.4 \mid$ & 42.11 & & 3 & |101.4 & $n / a$ & $n / a$ & 1 & 18.0 & 93.8 & Pass & $n / a$ \\
\hline$T F-044$ & $|10 / 06 / 93|$ & 30.6 & 39.71 & & 4 & 105.5 & 17.0 & $n / a$ & $i$ & 18.8 & 96.1 & Pass & $\mathrm{n} / \mathrm{a}$ \\
\hline$T F-045$ & $|10 / 06 / 93|$ & $23.0 i$ & $19.3 \mid$ & & 4 & i 106.1 & 17.5 & $n / a$ & $i$ & 18.1 & 97.5 & Pass & $\mathrm{n} / \mathrm{a}$ \\
\hline$T F-046$ & $|10 / 06 / 93|$ & $12.8 i$ & $11.2 \mid$ & & 4 & 104.8 & 18.5 & $n / a$ & 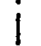 & 18.7 & 96.7 & Pass & SHELBY \\
\hline$T F-046 R B$ & 3| $10 / 06 / 93 \mid$ & $12.8 \mid$ & 11.21 & & 4 & 105.4 & $n / a$ & $n / a$ & i & 18.7 & 97.4 & Pass & $\mathrm{n} / \mathrm{a}$ \\
\hline$T F-047$ & $|10 / 06 / 93|$ & $18.1 \mid$ & $35.8 i$ & & 5 & 106.4 & 17.6 & $n / a$ & & 18.8 & 97.4 & Pass & $\mathrm{n} / \mathrm{a}$ \\
\hline$T F-048$ & $|10 / 06 / 93|$ & 16.4 & $17.6 i$ & & 5 & 105.6 & 17.6 & $n / a$ & & 18.6 & 96.8 & Pass & $n / a$ \\
\hline$T F-049$ & $|10 / 06 / 93|$ & $40.4 i$ & 11.5 & & 5 & 104.4 & 18.5 & $\mathrm{n} / \mathrm{a}$ & 1 & 19.2 & 95.9 & Pass & SHELBY \\
\hline$T F-049 R$ & $|10 / 06 / 93|$ & $40.4 I$ & $11.5 \mid$ & & 5 & 105.3 & 18.4 & $n / a$ & & 19.3 & 96.6 & Pass & SHELBY \\
\hline TF-049RB & $8|10 / 06 / 93|$ & $40.4 i$ & $11.5 i$ & & 5 & 102.4 & $\mathrm{n} / \mathrm{a}$ & n/a & & 19.2 & 94.7 & Pass & $\mathrm{n} / \mathrm{a}$ \\
\hline$T F-05 D$ & $|10 / 06 / 93|$ & $8.9 \mid$ & 31.01 & & 6 & i 105.2 & 18.6 & $n / a$ & ] & 18.8 & 97.1 & Pass & SHELBY \\
\hline$T F-059$ & $|10 / 06 / 93|$ & 9.31 & 7.61 & & 6 & |104.8 & 18.4 & $n / a$ & & 19.0 & 96.4 & Pass & $\mathrm{n} / \mathrm{a}$ \\
\hline TF-DS2 & $|10 / 06 / 93|$ & $56.2 i$ & 19.01 & & 6 & 105.5 & 17.9 & n/a & & 17.7 & 97.6 & Pass & $\mathrm{n} / \mathrm{a}$ \\
\hline$T F-052 R B$ & $8|10 / 06 / 93|$ & 56.21 & 19.0 .1 & & 6 & i 105.1 & $n / a$ & $\mathrm{n} / \mathrm{a}$ & 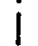 & 17.7 & 97.1 & Pass & $n / a$ \\
\hline$T F-053$ & $|10 / 07 / 93|$ & $29.4 \mid$ & 17.31 & & 4 & |105.9 & 17.9 & $\mathrm{n} / \mathrm{a}$ & & 19.0 & 96.9 & Pass & $n / a$ \\
\hline$T F-054$ & $|10 / 07 / 93|$ & 27.8 & 24.1 & & 5 & |105.6 & 18.4 & $n / a$ & & 18.6 & 97.5 & Pass & $\mathrm{n} / \mathrm{a}$ \\
\hline$T F-055$ & |10/07/93| & 23.11 & 7.7 & & 6 & |106.2 & 18.2 & $\mathrm{n} / \mathrm{a}$ & 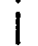 & 19.2 & 97.3 & Pass & $n / a$ \\
\hline$T F-055 R B$ & $3|10 / 07 / 93|$ & 23.11 & 7.7 & & 6 & 1905.5 & $n / 8$ & $n / a$ & & 19.2 & 97.5 & Pass & $n / a$ \\
\hline TF2-1 & $|70 / 20 / 93|$ & $535.9 i$ & 514.1 & & 1 & 1700.5 & 21.9 & $n / a$ & & 22.3 & 91.9 & Pass & $n / a$ \\
\hline TF2-2 & $|10 / 20 / 93|$ & 517.6 & 514.8 & & 9 & |101.8 & 20.8 & $n / a$ & & 22.0 & 92.4 & Pass & $\mathrm{n} / \mathrm{a}$ \\
\hline TF2-3 & $|10 / 20 / 93|$ & 508.7 & 545.0 & & 1 & |701.1 & 20.7 & $\mathrm{n} / \mathrm{a}$ & & 22.2 & 91.7 & Pass & $\mathrm{n} / \mathrm{a}$ \\
\hline TFZ-3RB & |10/20/93| & 508.7 & 545.0 & & 1 & | 98.8 & $n / a$ & $\mathrm{n} / \mathrm{a}$ & & 22.2 & 90.6 & Pass & $n / a$ \\
\hline$T F 2-4$ & $|10 / 20 / 93|$ & 532.7 & 540.0 & & 1 & 101.6 & 21.8 & $\mathrm{n} / \mathrm{a}$ & & 22.4 & 92.8 & Pass & $\mathrm{n} / \mathrm{a}$ \\
\hline TF2SG-1 & [10/20/93] & $504.0 i$ & 543.1 & $n / a$ & & |103.5 & 5.2 & $n / a$ & & 7.0 & 93.4 & Pass & $\mathrm{n} / \mathrm{a}$ \\
\hline TF2SG-2 & $|10 / 20 / 93|$ & 517.5 & $519.7 i$ & $n / a$ & & $\mid 102.2$ & 4.4 & $n / a$ & & 8.4 & 90.4 & Pass & $n / a$ \\
\hline TSG-01 & $|10 / 04 / 93|$ & 33.7 & 16.3 & $n / a$ & & $\mid 108.3$ & 12.5 & $\mathrm{n} / \mathrm{s}$ & & 13.9 & 98.1 & Pass & $\mathrm{n} / \mathrm{a}$ \\
\hline TSG-02 & $|10 / 04 / 93|$ & 36.1 & 40.2 & $n / a$ & & $\mid 109.8$ & 13.7 & $\mathrm{n} / \mathrm{a}$ & & 16.8 & 98.1 & Pass & $n / a$ \\
\hline TSG-0B & |70/04/93| & $12.3 i$ & 39.7 & $n / a$ & & 103.3 & 11.5 & $\mathrm{n} / \mathrm{a}$ & & 13.4 & 93.2 & Pass & $n / a$ \\
\hline TSE-04 & $|30 / 04 / 93|$ & 8.31 & 7.2 & $n / a$ & & 105.5 & 10.4 & $n / a$ & & 10.1 & 97.1 & Pass & $n / a$ \\
\hline TSG-OS & $|10 / 04 / 93|$ & 24.6 & 17.2 & $n / a$ & & $|106.1|$ & 11.7 & $n / a$ & & 13.1 & 96.2 & Pass & $n / a$ \\
\hline TSG-DSRB & $|10 / 04 / 93|$ & $24.6 i$ & 17.2 & $n / a$ & & $|105.6|$ & $n / a$ & $n / a$ & & 13.1 & 96.9 & Pass & $n / a$ \\
\hline
\end{tabular}


(1) Test numbers with prefixes of TSG indicate density tests on the subgrade of the first test fill.

(2) Test numbers with prefixes of TF2SG indicate density tests on the subgrade of the second test fill.

(3) Test numbers with prefixes of TF indicate density tests on the soil liner in the first test fill.

(4) Test numbers with prefixes of TF2 indicate density tests on the soil liner in the second test fill.

(5) Test numbers with suffixes of RB indicate rubber balloon density tests.

(3) Test numbers with suffuxes of $R$ indicate a recompacted perm test location. 
WHC-SD-W025-RPT-001, Rev, 0

APPENDIX U-2

SDRI TEST INFORMATION

$U-2 . i$ 
WHC-SD-W025-RPT-001, Rev. 0

Page 1

NON-DRAG-OFF SDRI TEST RESULTS

Project W-025

GCS Project No. 933-1354

\begin{tabular}{|c|c|c|c|c|c|c|}
\hline मै। & $\begin{array}{l}\text { कि? } \\
\text { INTERVAL } \\
\text { TIME (s) }\end{array}$ & $\begin{array}{l}\text { की } \\
\text { CUMULATIVE } \\
\text { TIME(S) }\end{array}$ & $\begin{array}{l}\text { CUMULATIVE } \\
\text { DAYS } \\
\text { DAY }\end{array}$ & $\begin{array}{r}\text { INTERVAL } \\
\text { FLOW } \\
\text { (mi) }\end{array}$ & $\begin{array}{l}\text { CUMULATIVE } \\
\text { FLCOW } \\
\text { (mi) }\end{array}$ & $\begin{array}{l}\text { INTERVAL } \\
\text { INFILTRATION } \\
\text { (cm/s) }\end{array}$ \\
\hline 08 Oct 93 & 6360 & 6360 & 0.07 & 187 & 187 & 1.27E-06 \\
\hline 08 Oct 93 & 11640 & 18000 & 0.21 & 4 & 191 & $1.48 E-08$ \\
\hline 09 Oct 93 & 58800 & 76800 & 0.89 & 786 & 977 & 5.76E-07 \\
\hline 090 ct 93 & 27900 & 104700 & 1.21 & 77 & 1054 & 1.19E-07 \\
\hline 10 Oct 93 & 98220 & 202920 & 2.35 & 247 & 1301 & $1.08 E-07$ \\
\hline $110 \mathrm{ct} 93$ & 52260 & 255180 & 2.95 & 158 & 1459 & 1.30E-07 \\
\hline $120 \mathrm{ct} 93$ & 87300 & 342480 & 3.96 & 335 & 1794 & $1.65 E-07$ \\
\hline 130 ct 93 & 100380 & 442860 & 5.13 & 275 & 2069 & $1.18 E-07$ \\
\hline 140 ct 93 & 71100 & 513960 & 5.95 & 295 & 2364 & $1.79 E-07$ \\
\hline 15 Oct 93 & 85920 & 599880 & 6.94 & 166 & 2530 & $8.32 E-08$ \\
\hline $17 \cot 93$ & 169800 & 769680 & 8.91 & 269 & 2799 & $6.82 E-08$ \\
\hline 18 Oct 93 & 86040 & 855720 & 9.90 & 130 & 2929 & $6.51 E-08$ \\
\hline 19 oct 93 & 97020 & 952740 & 19.03 & 361 & 3290 & $1.60 \mathrm{E}-07$ \\
\hline 200 ct 93 & 84060 & 1036800 & 12.00 & 140 & 3430 & 7.17E-08 \\
\hline $210 c t 93$ & 79560 & 1116360 & 12.92 & 261 & 3691 & $1.41 E-07$ \\
\hline 22 Oct 93 & 91680 & 1208040 & 13.98 & 180 & 3871 & $8.45 E-08$ \\
\hline 22 Oct 93 & 16740 & 1224780 & 14.18 & 36 & 3907 & $9.26 E-08$ \\
\hline 250 ct 93 & 249720 & 1474500 & 17.07 & 544 & 4451 & $9.38 E-08$ \\
\hline 26 Oct 93 & 82020 & 1556520 & 18.02 & 356 & 4807 & $1.87 E-07$ \\
\hline 27 Oct 93 & 77100 & 1633620 & 18.91 & 101 & 4908 & $5.64 E-08$ \\
\hline 280 ct 93 & 92580 & 1726200 & 19.98 & 93 & 5001 & 4.33E-08 \\
\hline 29 Oct 93 & 80820 & 1807020 & 20.91 & 160 & 5161 & $8.52 E-08$ \\
\hline 30 Oct 93 & 95820 & 1902840 & 22.02 & 132 & 5293 & $5.93 E-08$ \\
\hline 31 Oct 93 & 86280 & 1989120 & 23.02 & 107 & 5400 & $5.34 E-08$ \\
\hline O1 Nov 93 & 92580 & 2081700 & 24.09 & 568 & 5968 & $2.64 E-07$ \\
\hline 02 Nov 93 & 81060 & 2162760 & 25.03 & 107 & 6075 & $5.68 E-08$ \\
\hline 03 Nov 93 & 89160 & 2251920 & 26.06 & 126 & 6201 & $6.08 \mathrm{E}-08$ \\
\hline 04 Nov 93 & 77400 & 2329320 & 26.96 & 226 & 6427 & $1.26 \mathrm{E}-07$ \\
\hline 05 Nov 93 & 94380 & 2423700 & 28.05 & 157 & 6584 & $7.16 E-08$ \\
\hline 08 Nov 93 & 256140 & 2679840 & 31.02 & 538 & 7122 & $9.04 E-08$ \\
\hline 09 Nov 93 & 86340 & 2766180 & 32.02 & 100 & 7222 & 4.99E-08 \\
\hline 10 Nov 93 & 96180 & 2862360 & 33.13 & 110 & 7332 & $4.92 E-08$ \\
\hline 13 Nov 93 & 244680 & 3107040 & 35.96 & 245 & 7577 & 4.31E-08 \\
\hline 16 Nov 93 & 278820 & 3385860 & 39.19 & 552 & 8129 & $8.52 E-08$ \\
\hline 19 Now 93 & 244140 & 3630000 & 42.01 & 329 & 8458 & $5.80 E-08$ \\
\hline 22 Nov 93 & 265260 & 3895260 & 45.08 & 177 & 8635 & 2.87E-08 \\
\hline 24 Nov 93 & 174600 & 4069860 & 47.10 & 215 & 8850 & $5.30 E-08$ \\
\hline 29 Nov 93 & 420360 & 4490220 & 51.97 & 361 & 9211 & $3.70 E-08$ \\
\hline 93334658 & 369000 & 4859220 & 3724 & 430 & 9641 & $5.02 E-08$ \\
\hline
\end{tabular}


WHC-SD-W025-RPT-001, Rev. 0

Page 2

NON-DRAG-OFF SDRI TEST RESULTS

Project W-025

GCS Project No. 933-1354

\begin{tabular}{|c|c|c|c|c|c|c|}
\hline \begin{tabular}{c}
$\cdots$ \\
DATE \\
\hdashline
\end{tabular} & $\begin{array}{r}\text { INTERVAL } \\
\text { TIME (s) }\end{array}$ & $\begin{array}{r}\text { CUMULATIVE } \\
\text { TIME (s) }\end{array}$ & $\begin{array}{l}\because \\
\text { CUMULATIVE } \\
\because \quad \text { DAYS } \\
\end{array}$ & $\begin{array}{r}\text { INTERVAL } \\
\because \text { FLOW } \\
\quad \text { (mi) } \\
\end{array}$ & $\begin{array}{l}\text { CUMULATIVE } \\
\text { FLOW } \\
\vdots \\
(\mathrm{ml}) \\
\end{array}$ & $\begin{array}{r}\text { INTERVAL } \\
\text { INFILTRATION } \\
(\because \mathrm{cm} / \mathrm{s}) \\
\end{array}$ \\
\hline 07 Dec 93 & 329700 & 5188920 & 60.06 & 421 & 10062 & $5.50 \mathrm{E}-08$ \\
\hline 11 Dec 93 & 344760 & 5533680 & 64.05 & 440 & 10502 & $5.49 E-08$ \\
\hline 15 Dec 93 & 353040 & 5886720 & 68.13 & 313 & 10815 & $3.82 E-08$ \\
\hline $19 \operatorname{Dec} 93$ & 350100 & 6236820 & 72.19 & 576 & 11391 & $7.08 E-08$ \\
\hline $22 \operatorname{Dec} 93$ & 249300 & 6486120 & 75.07 & 157 & 11548 & 2.71E-08 \\
\hline 29 Dec 93 & 591300 & 7077420 & 81.91 & 429 & 11977 & $3.12 E-08$ \\
\hline 04 Jan 94 & 532200 & 7609620 & 88.07 & 191 & 12168 & $1.55 E-08$ \\
\hline 11 Jan 94 & 612600 & 8222220 & 95.16 & 546 & 12714 & $3.84 E-08$ \\
\hline 19 Jan 94 & 684900 & 8907120 & 103.09 & 717 & 13431 & $4.51 E-08$ \\
\hline $27 \operatorname{Jan} 94$ & 689400 & 9596520 & 111.07 & 721 & 14152 & $4.50 E-08$ \\
\hline 04 Feb 94 & 682200 & 10278720 & 118.97 & 523 & 14675 & $3.30 E-08$ \\
\hline 09 Feb 94 & 451800 & 10730520 & 124.20 & 299 & 14974 & $2.85 \mathrm{E}-08$ \\
\hline
\end{tabular}


WHC-SD-W025-RPT-001, Rev. 0

NONDRAG-OFF SDAI TEBT

CUMULATIVE INFILTRATION VS TIME
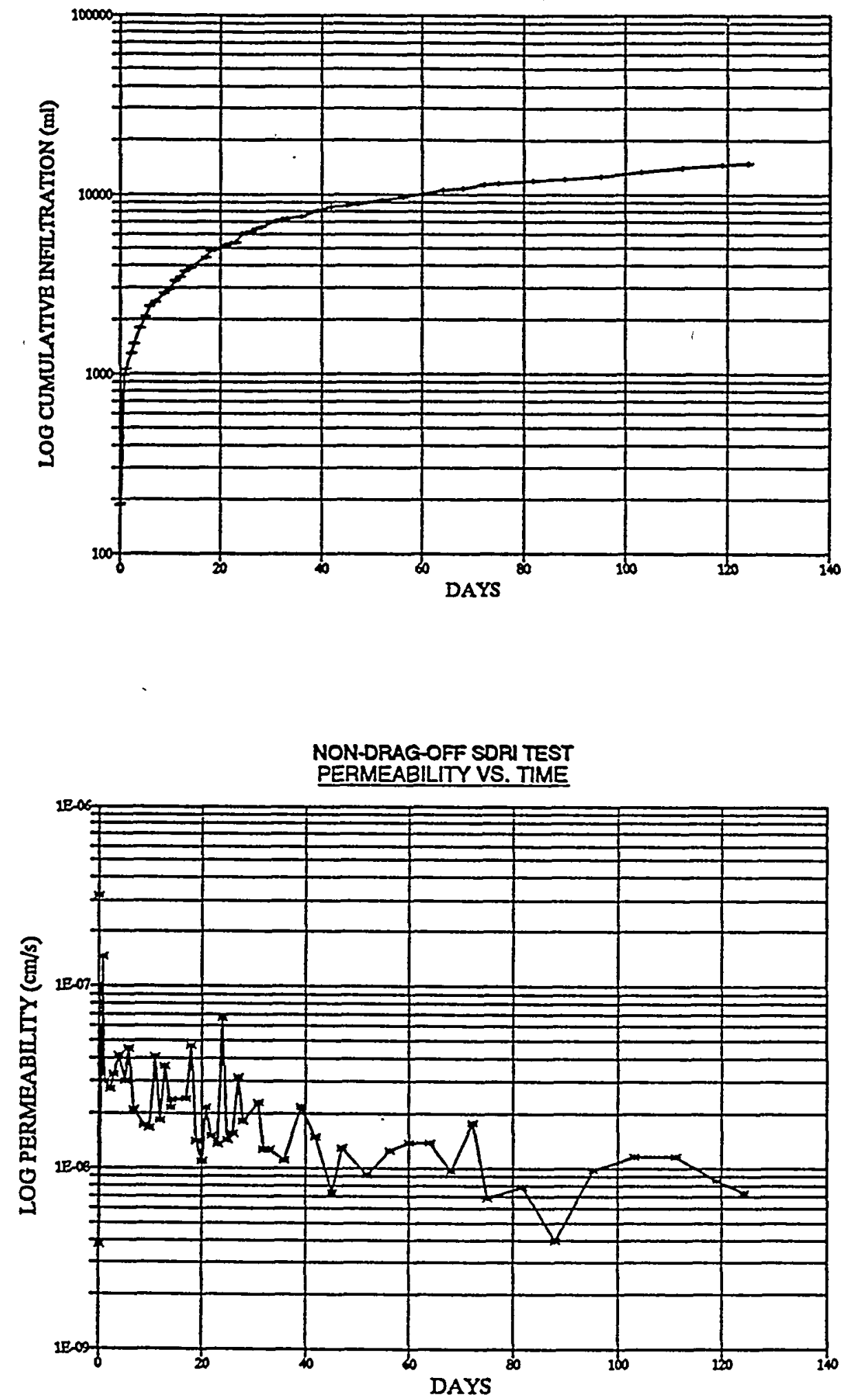
WHC-SD-W025-RPT-001, Rev. 0

WETTING FRONT DETERMINATION

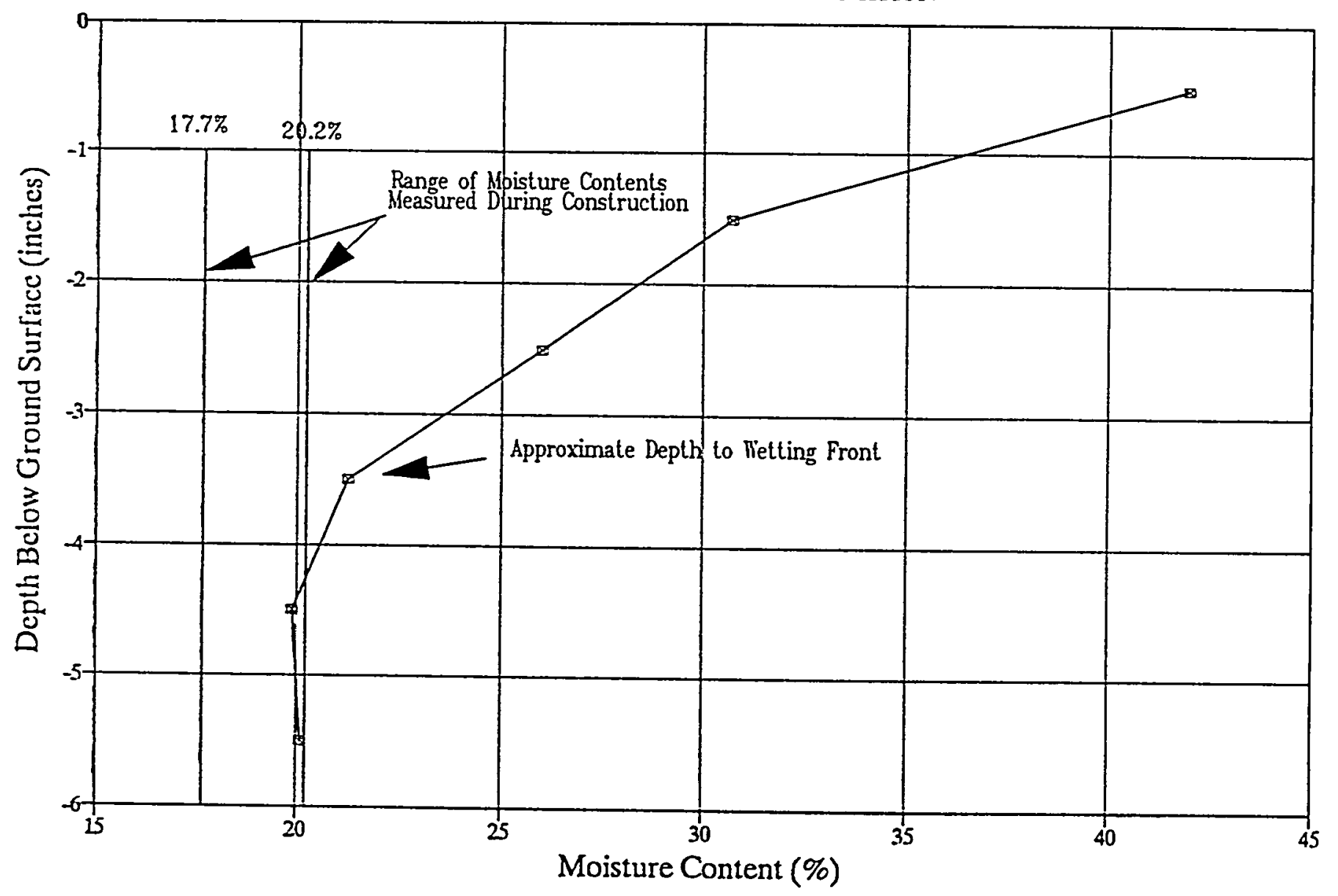

Data Points 
WHC-SD-W025-RPT-001, Rev. 0

DRAWINGS

$v-i$ 
WHC-SD-H025-RP.T-001, Rev. 0

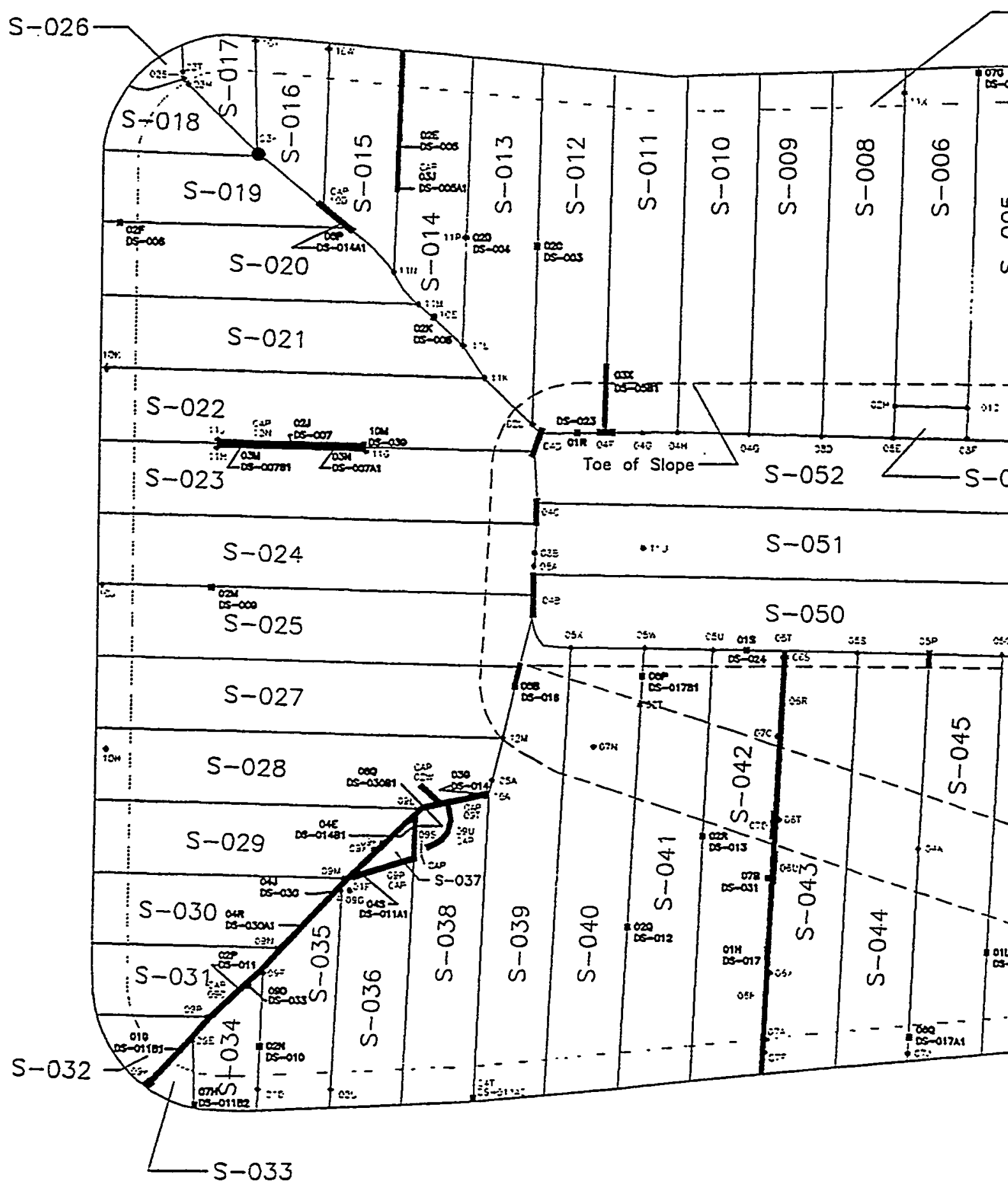




\section{Explanation}

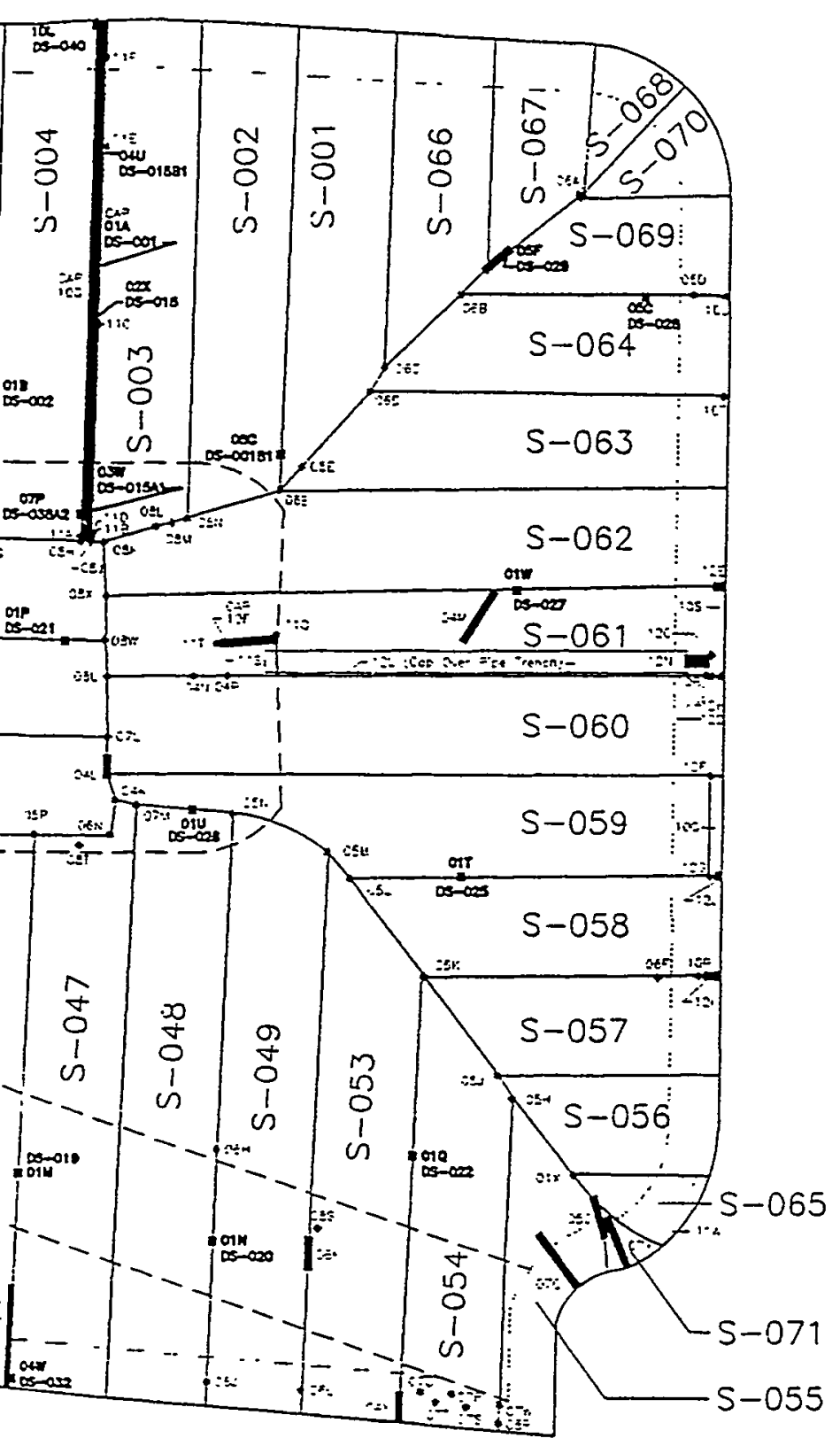

Repoir Number and Approximete Repoir Locotion (ropairs shown os dots are not to rede: the length of the ropeirs sthown os bold tines are to seote)

- ors Repeir Number Oentructive Somole Number and

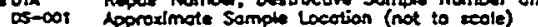
S-001 Secondory Pand Number and Approximete

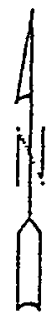

50

100

FEET

SHEET $S 1$

SECONDARY HDPE GEOMEMBRANE LINER AS-BUILT DOE/NDO/WA 


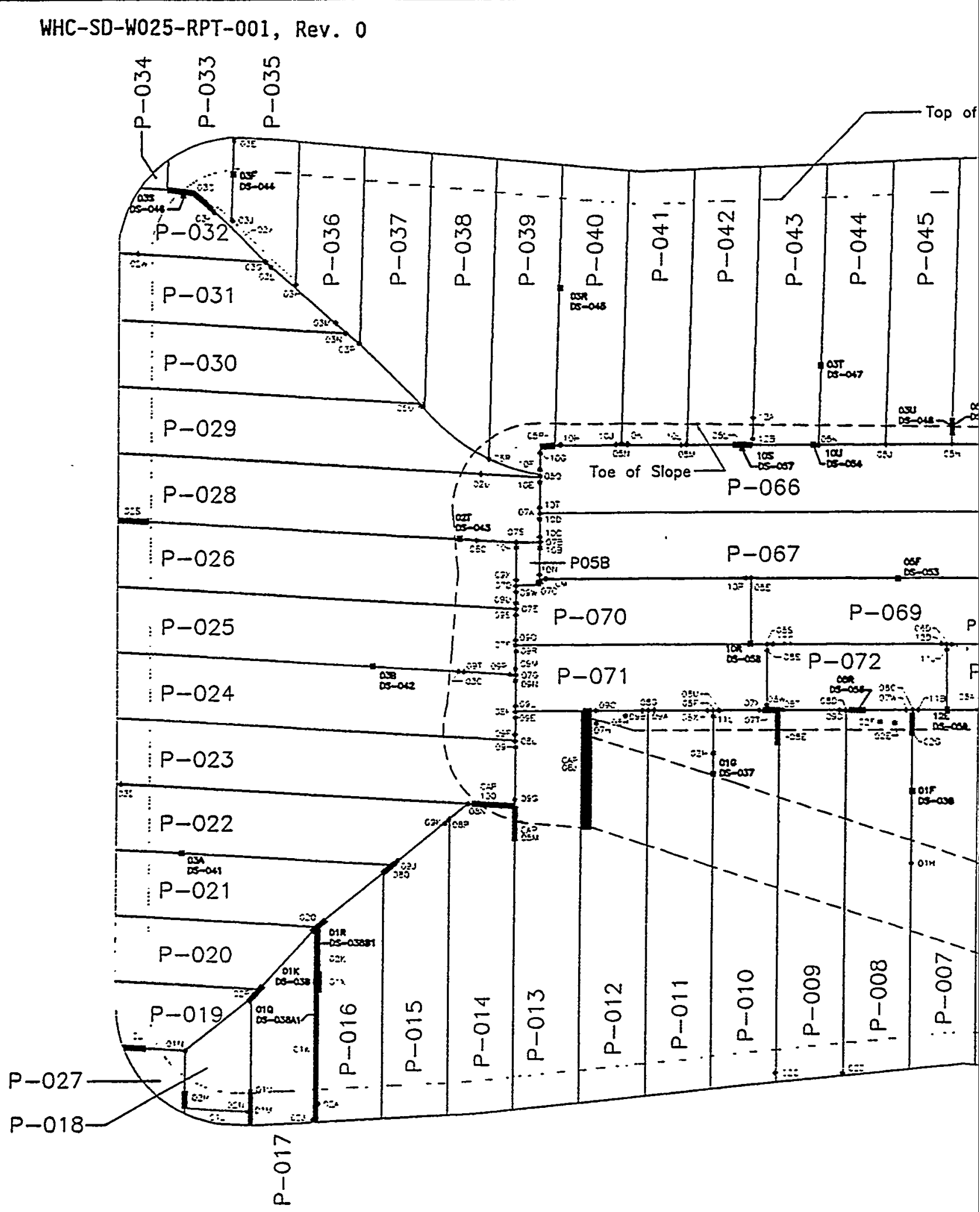


WHC-SD-W025-RPT-001, Rev. 0

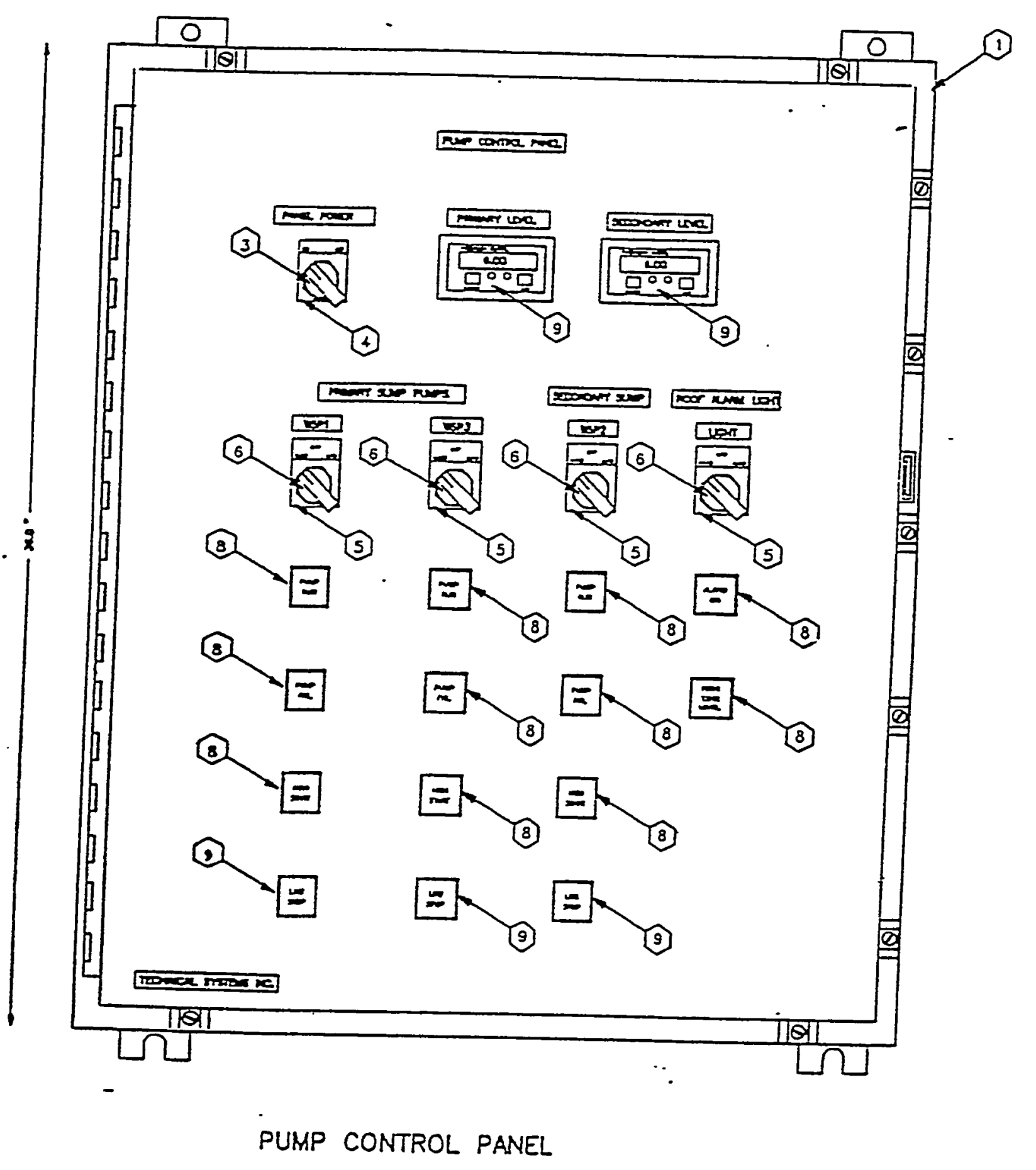

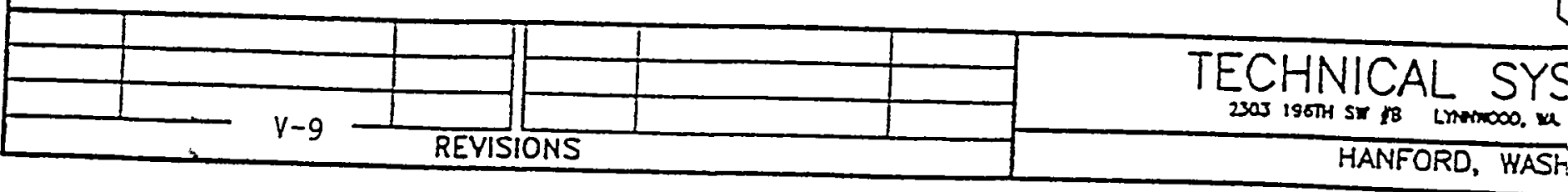


WHC-SD-H025-RPT-001, Rev. 0

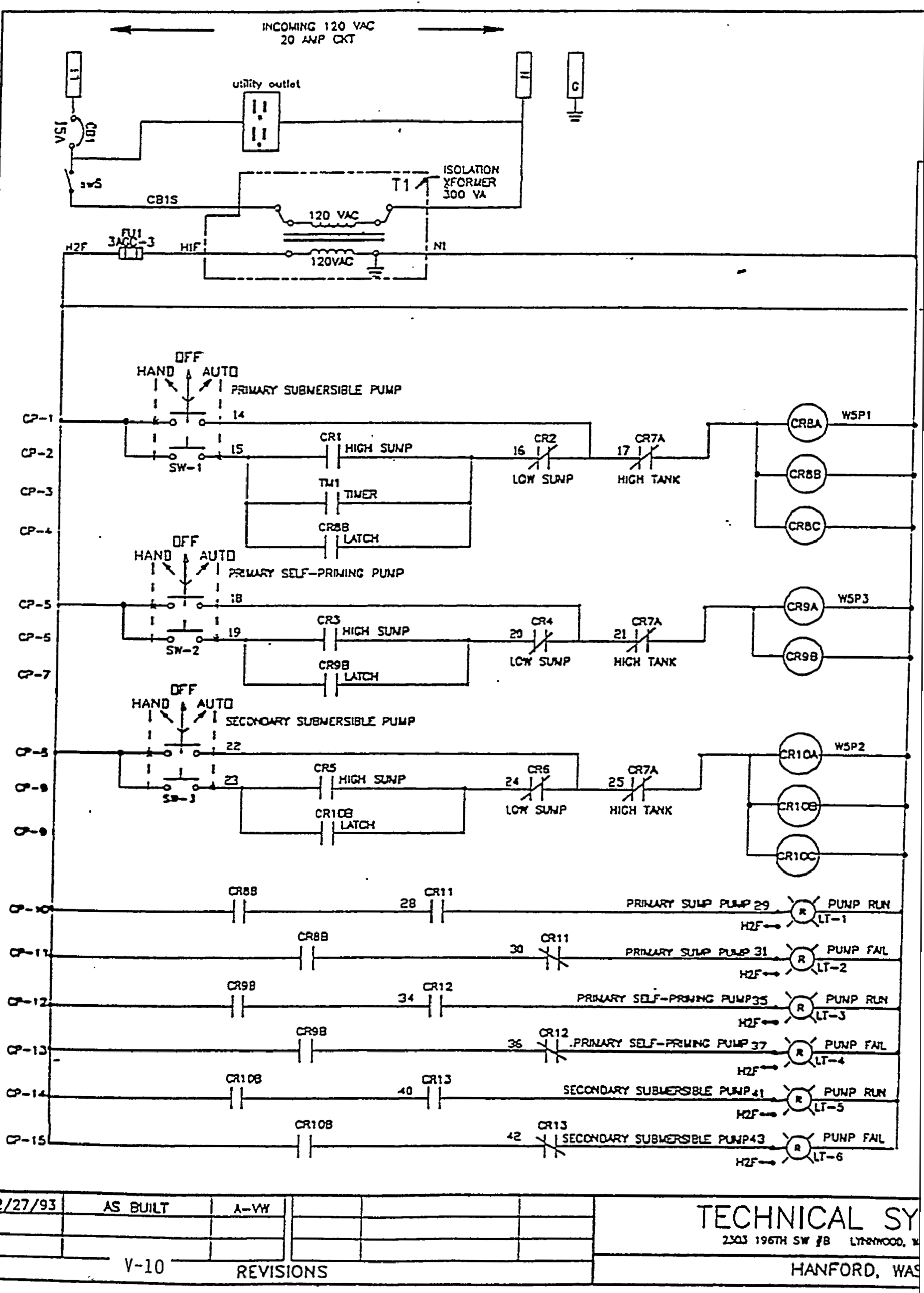




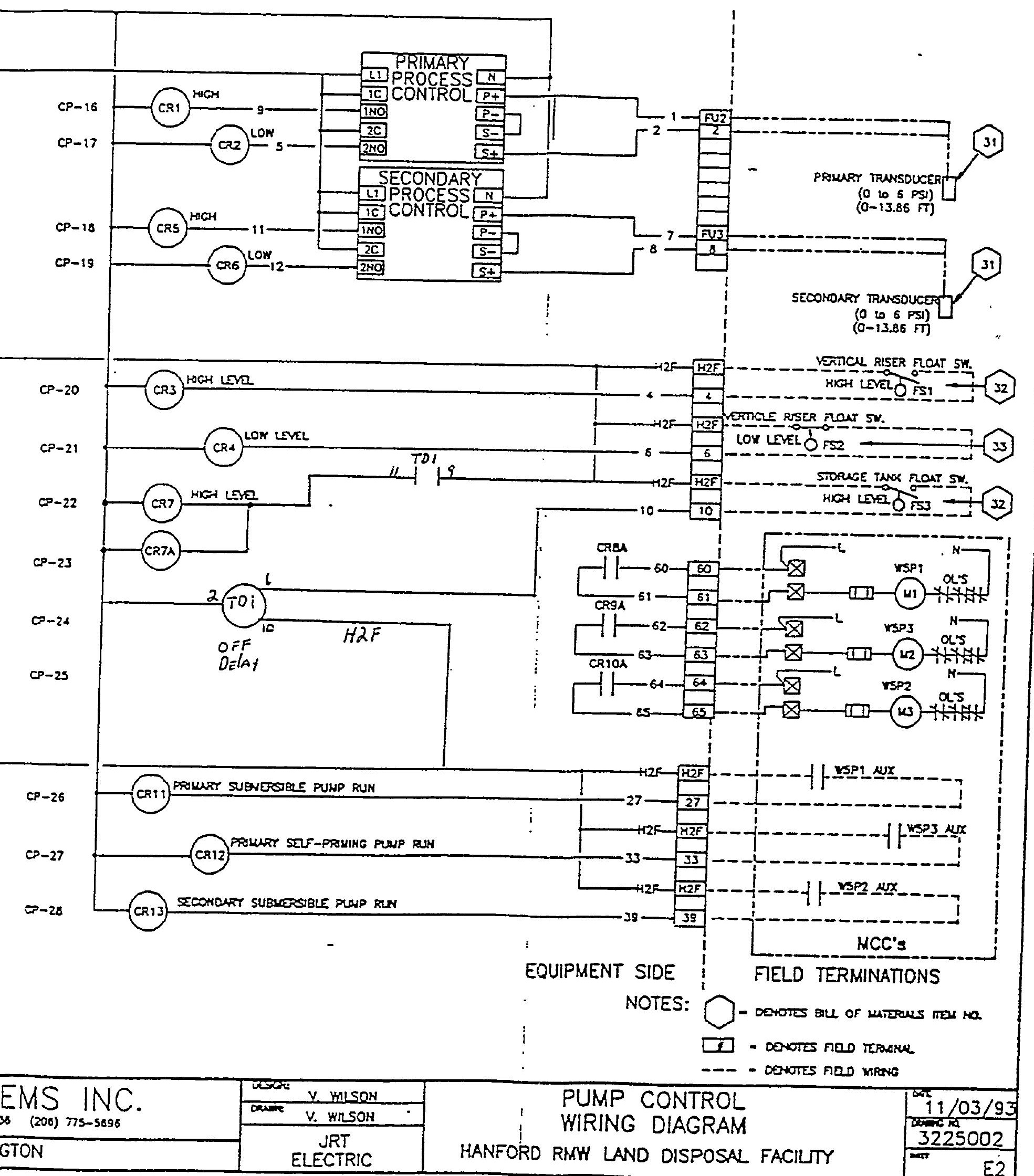


WHC-SD-H025-RPT-001, Rev. 0

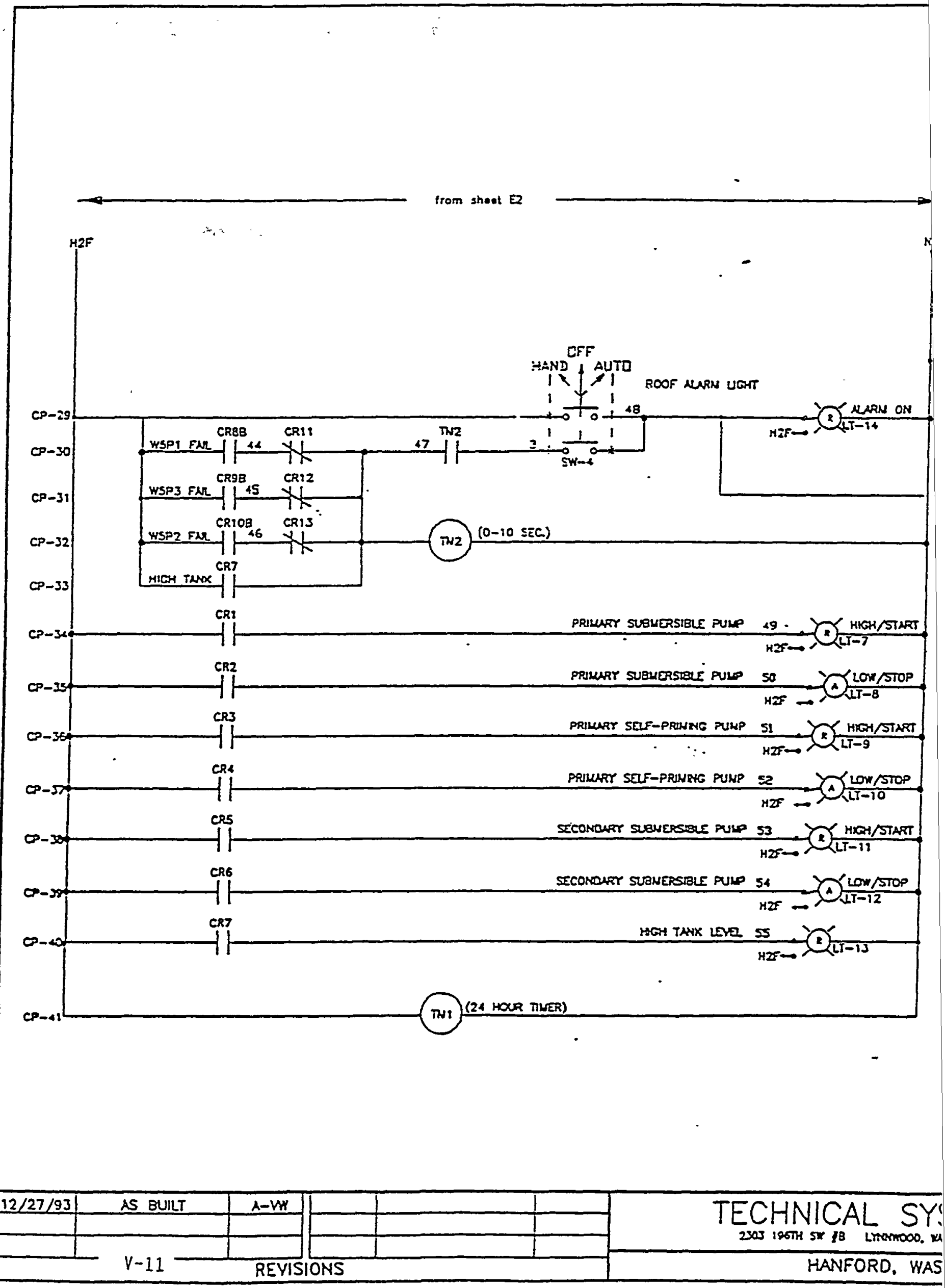




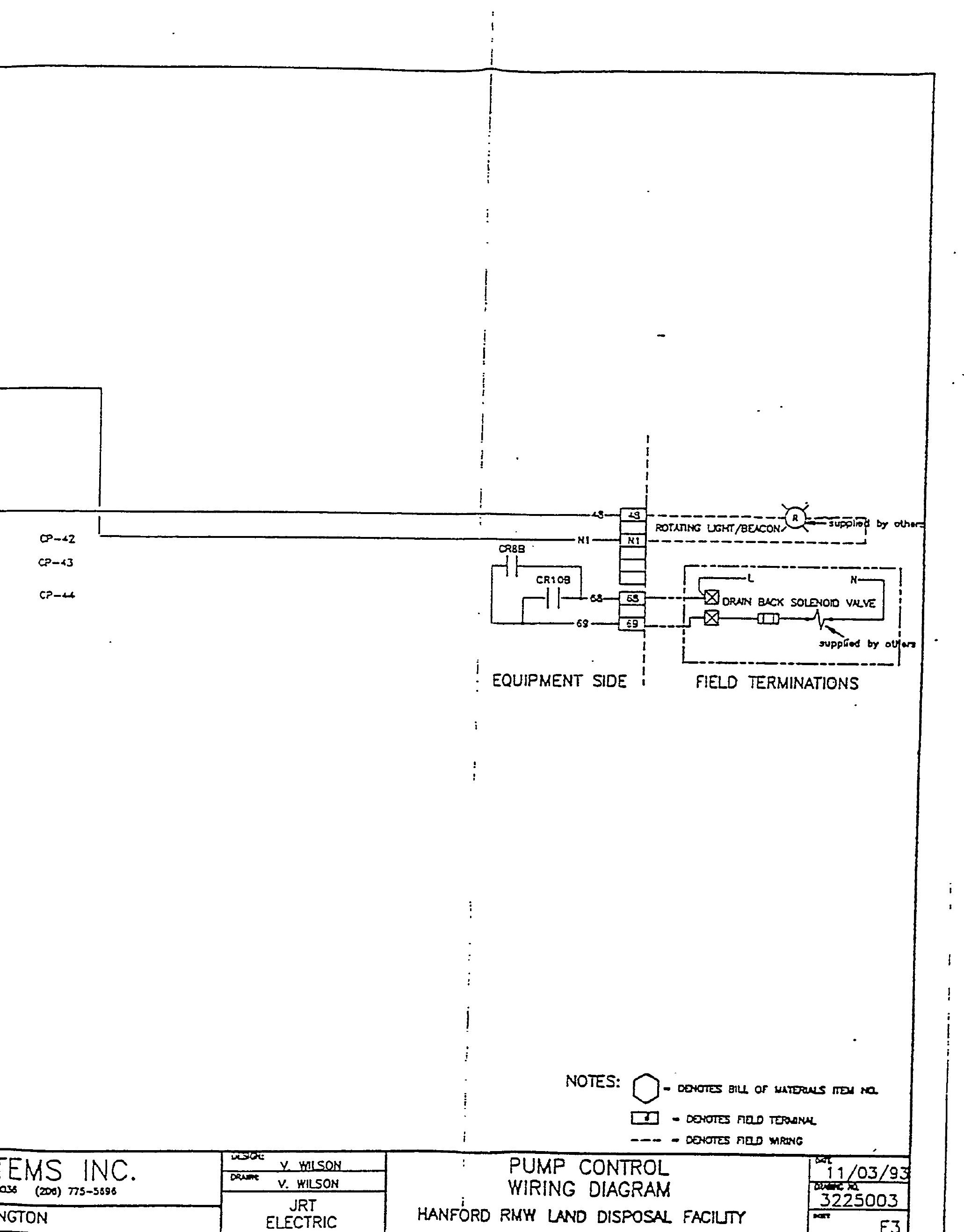


WHC-SD-H025-RPT-001, Rev. 0

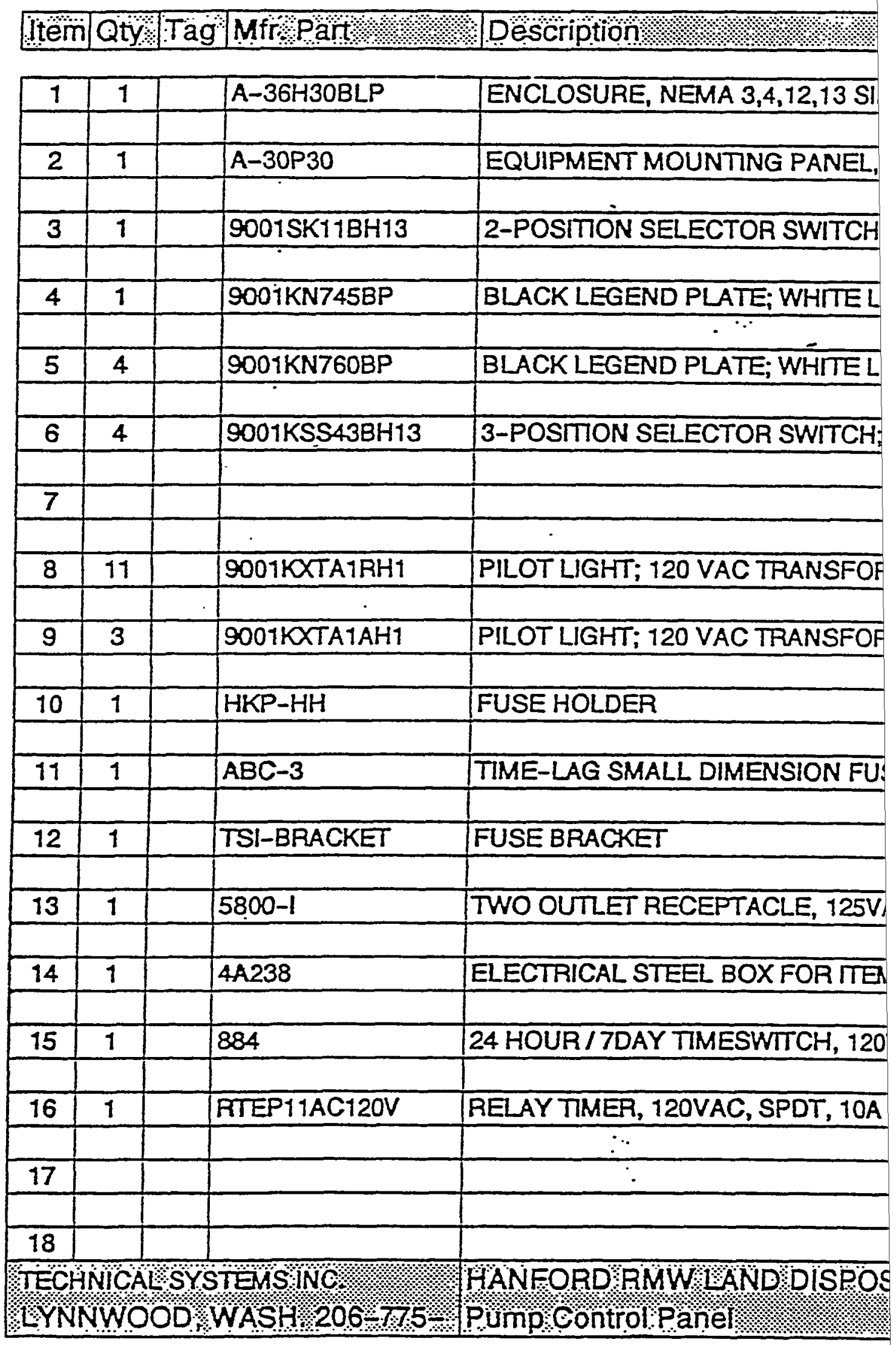




\begin{tabular}{|c|c|c|c|}
\hline ( & $\mathrm{Mff}=2$ & Veno & POH \\
\hline 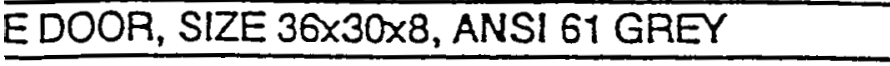 & HOFFMAN & STUSSER & 25795 \\
\hline ERIOR OF ITEM 1, PAINTED GLOSS WHITE & $4 O$ & STICSED & \\
\hline & & & \\
\hline INTAINED; GLOVE HAND KNOB; 2-NC, 2-NO & SQUARED & STUSSER & 25795 \\
\hline RONG (ON/OFF) & SQUARE D & STUSSER & 25795 \\
\hline & & & \\
\hline RONG (HAND/OFF/AUTO) & SQUARED & STUSSER & 25795 \\
\hline \multirow{4}{*}{ INTAINED; GLOVE HAND KNOB; 2-NC, 2-NO } & SQUARED & STUSSER & 25795 \\
\hline & 1 & & \\
\hline & & & \\
\hline & 1 & & \\
\hline RTYPE; PUSH TO TEST; (2) RED & SQUARE D & STUSSER & 25795 \\
\hline \multirow[t]{3}{*}{ RTYPE; PUSH TO TEST; (2) AMBER } & SQLARE D & STUSSER & 25795 \\
\hline & & & \\
\hline & BUSSMAN & ICC & STOCK \\
\hline \multirow[t]{3}{*}{$3 \mathrm{~A}$} & BUSSMAN & ICC & STOCK \\
\hline & & & \\
\hline & TSI & TSI & STOCK \\
\hline $15 \mathrm{~A}$ & LEVTTON & GRAINGER & STOCK \\
\hline 3 & RACO & GRAINGER & STOCK \\
\hline \multirow[t]{2}{*}{ SPDT, 10 AMPS } & DIEHL & ICC & 25797 \\
\hline & IDEC & ICC & STOCK \\
\hline \\
\hline & & & \\
\hline \multicolumn{4}{|l|}{$\frac{-}{15 n^{2}}$} \\
\hline FACILITYY & JOB 3225 & & \\
\hline
\end{tabular}


WHC-SD-W025-RPT-001, Rev. 0

Itêm|Qty/Tag Mifreart: Description

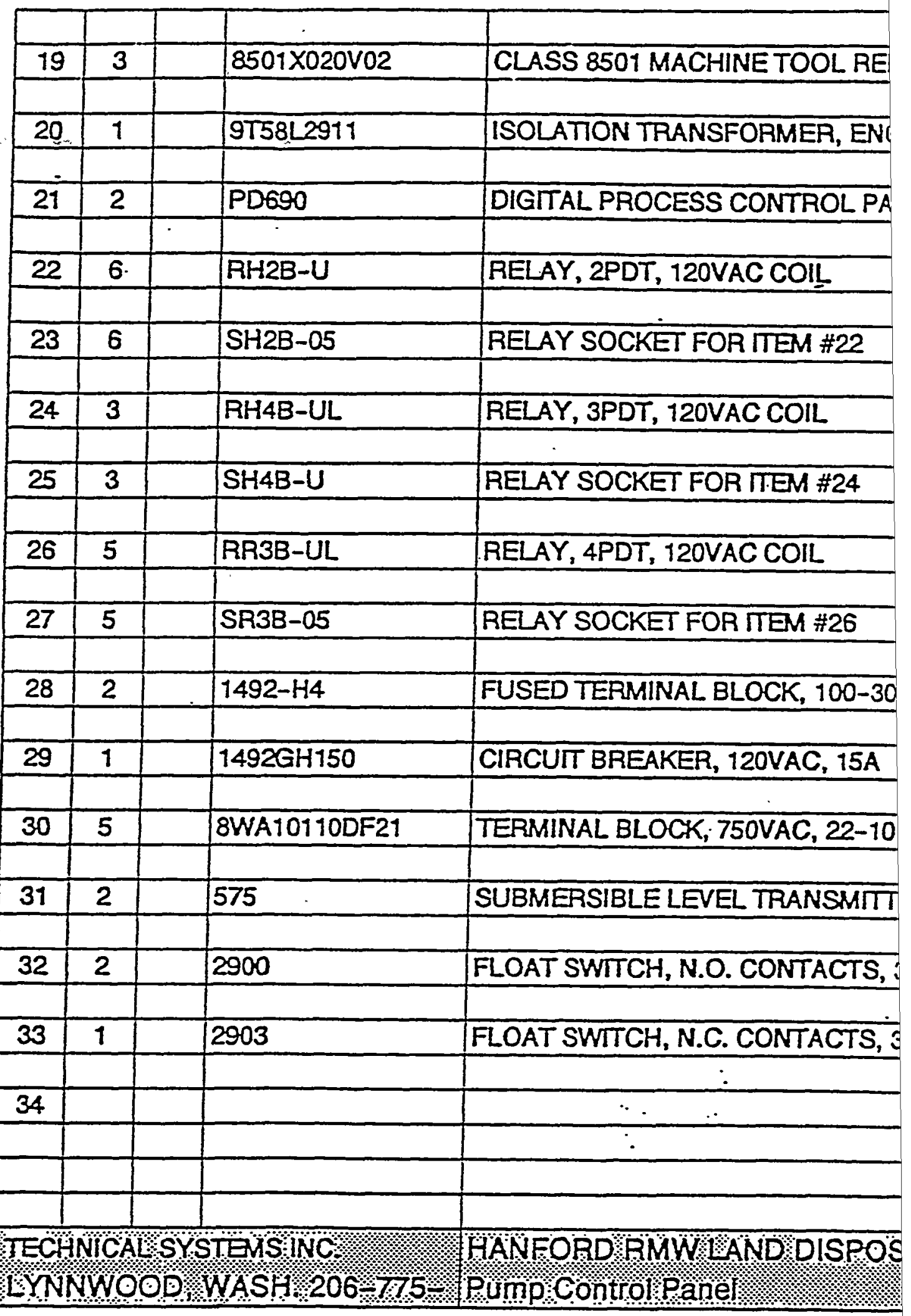




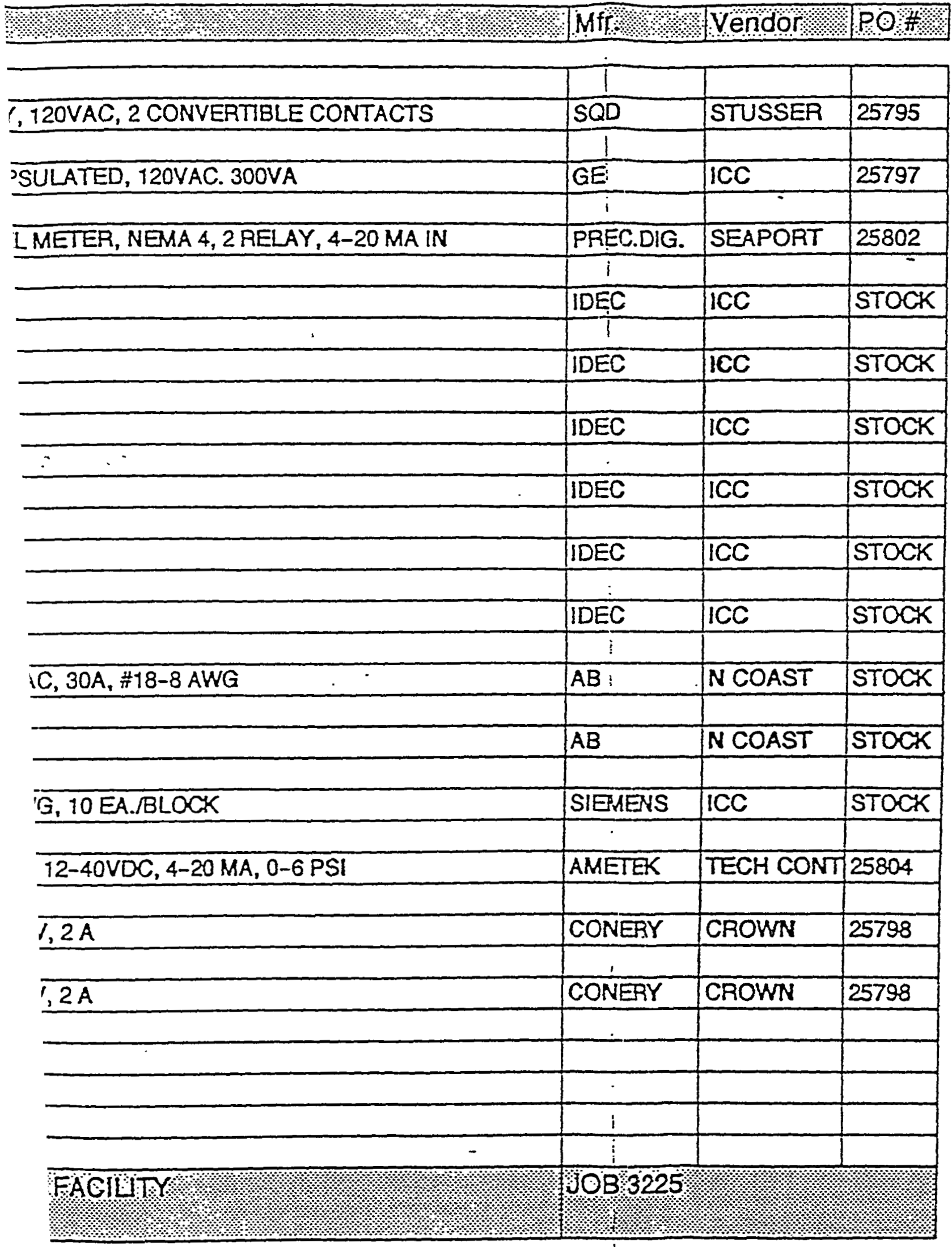




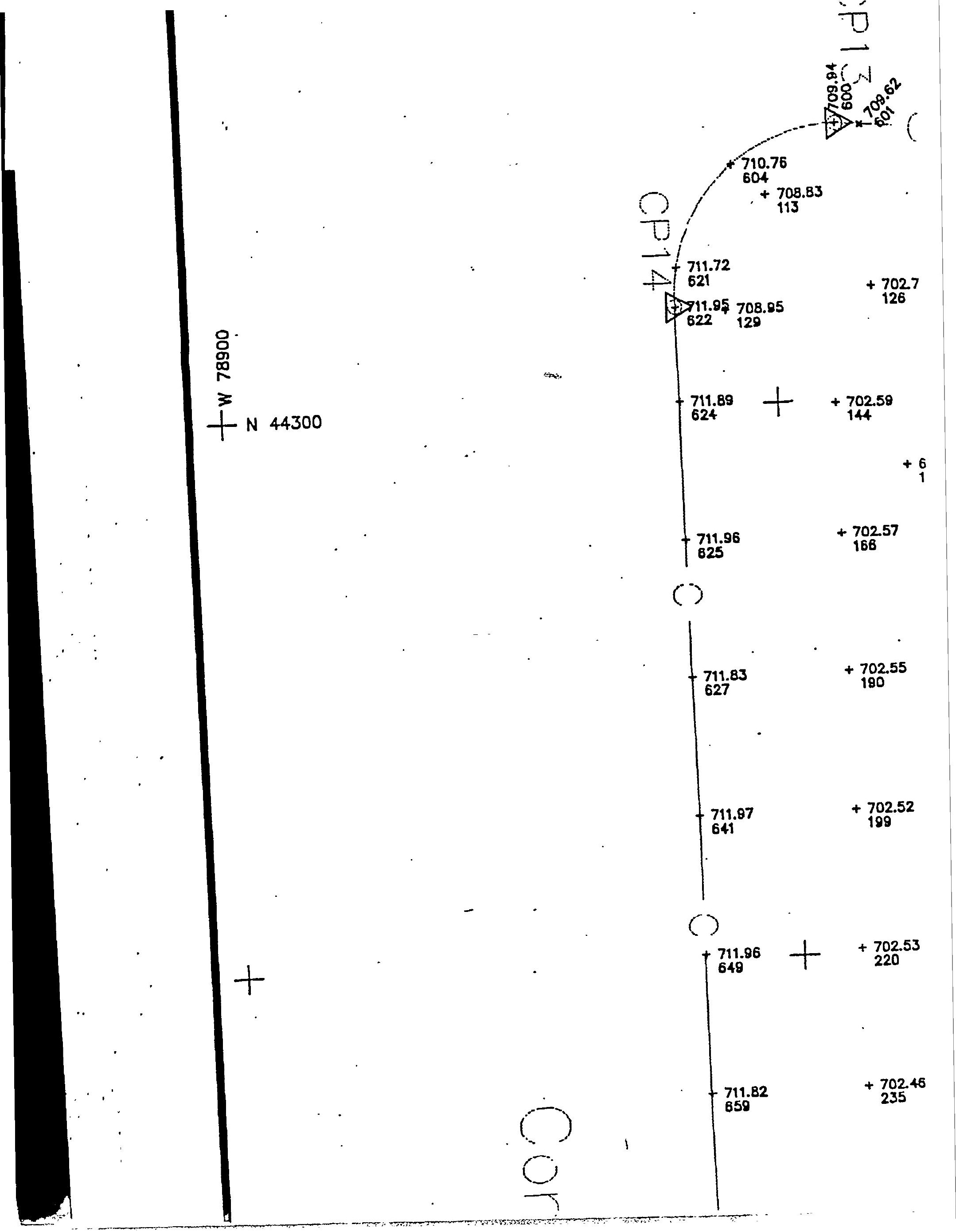




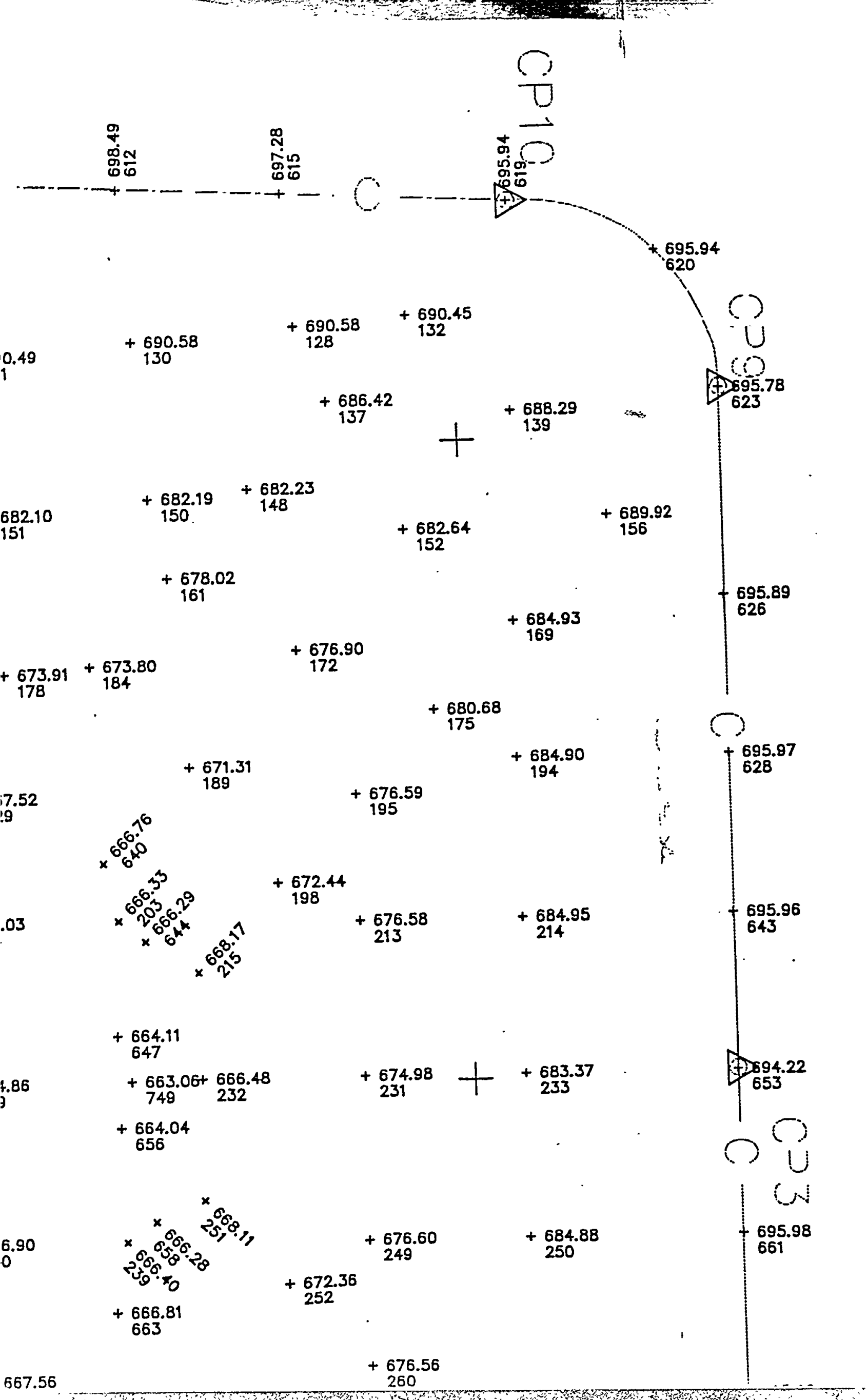


1

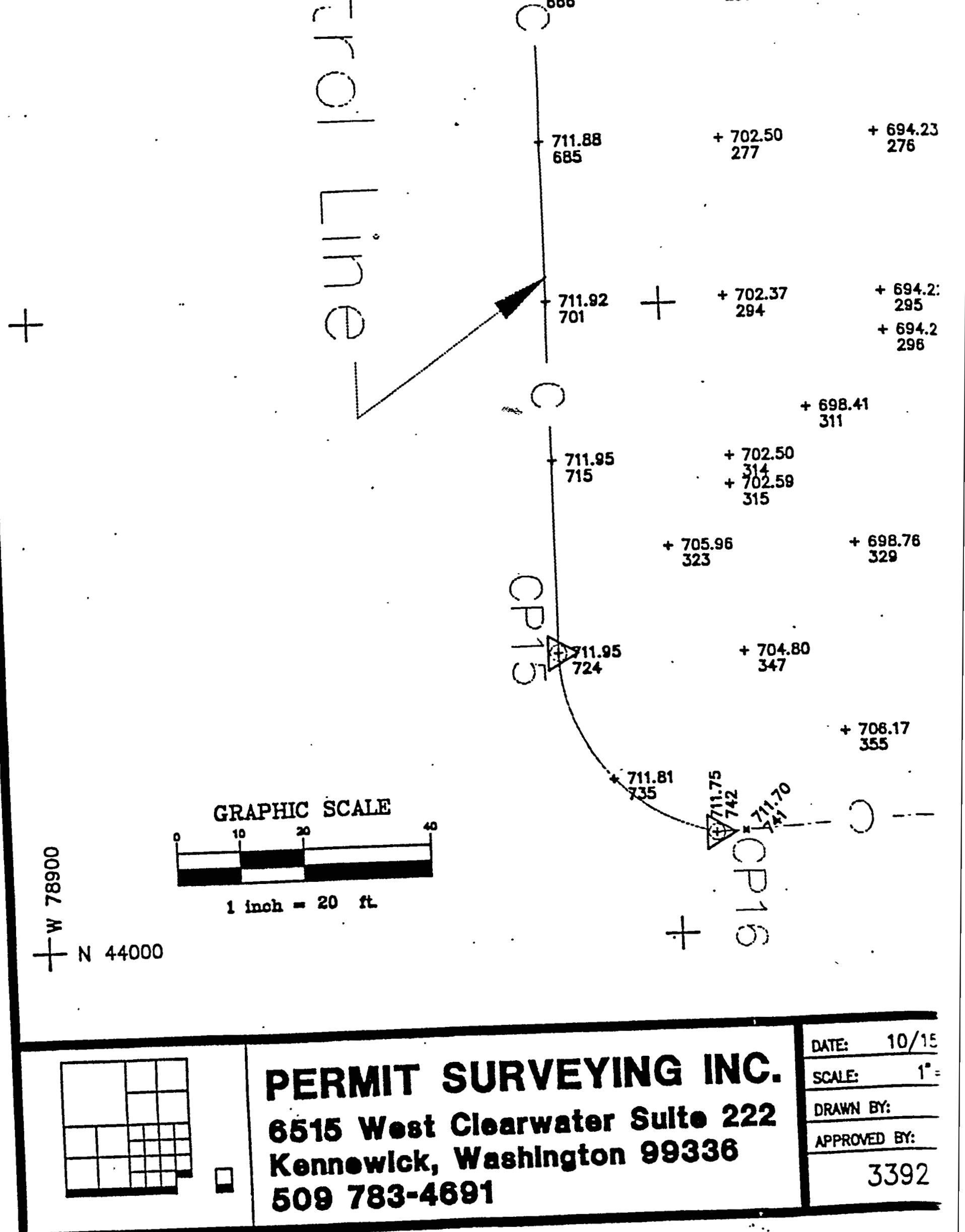




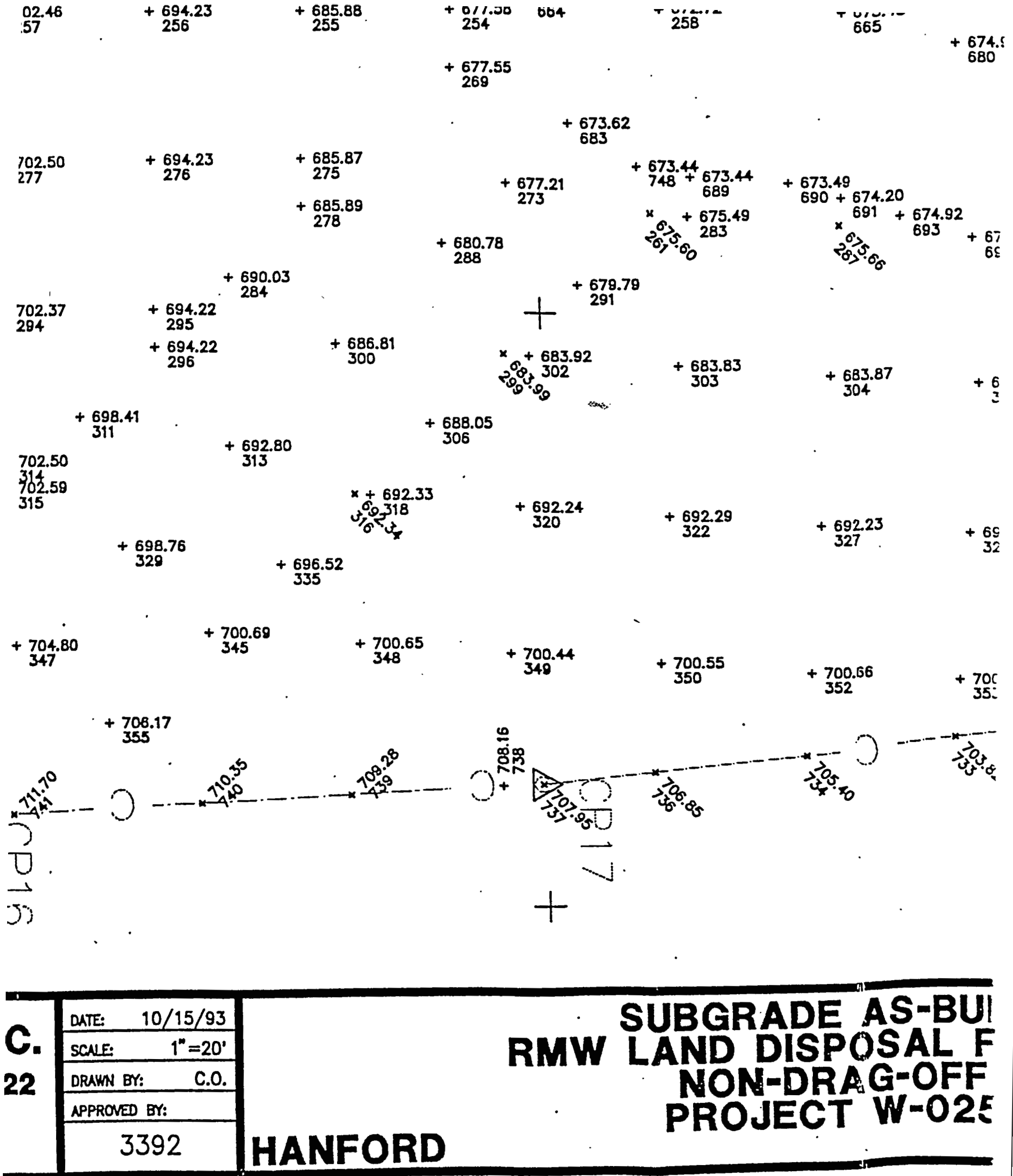




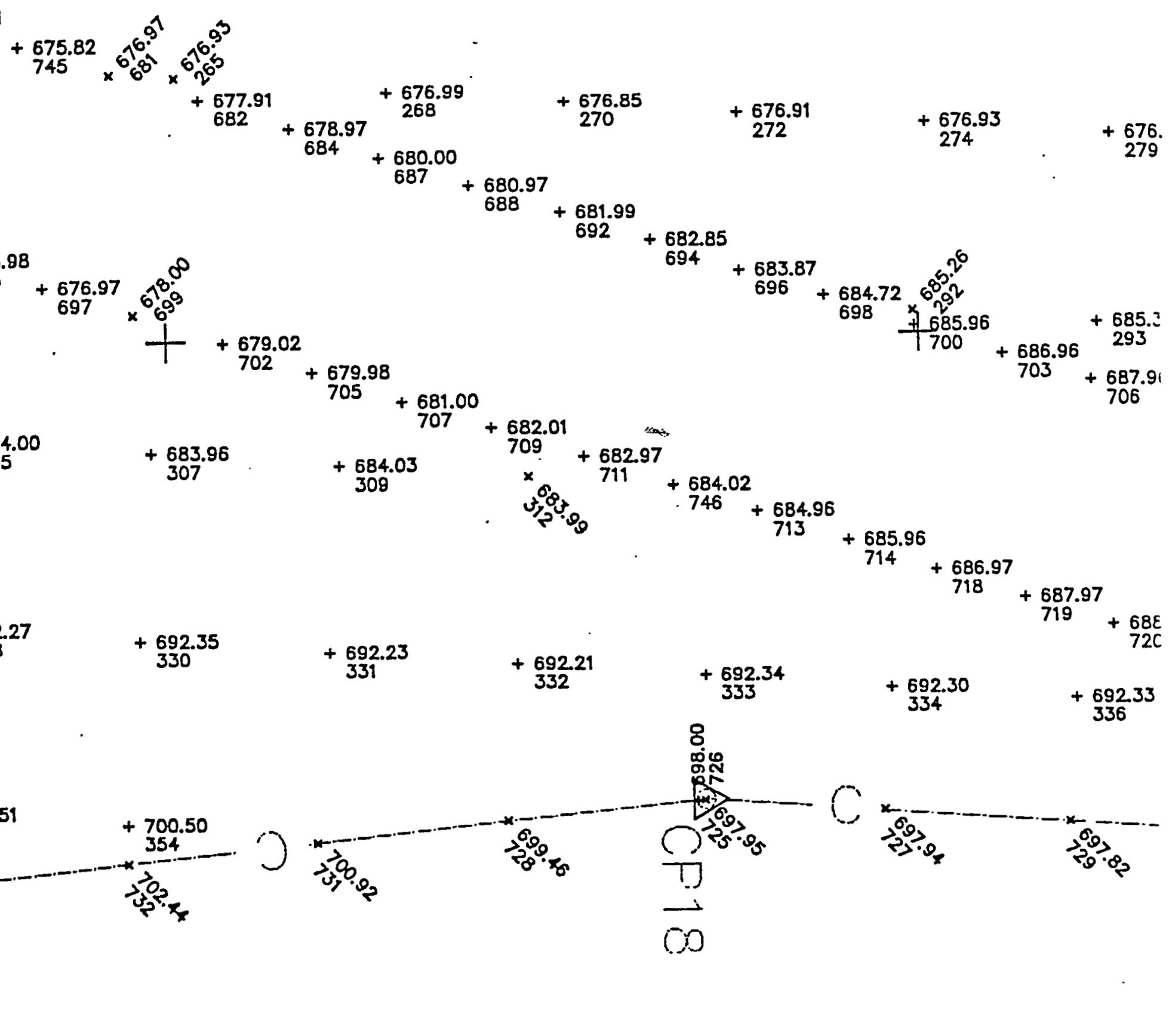

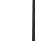

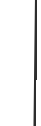


+680.74
267

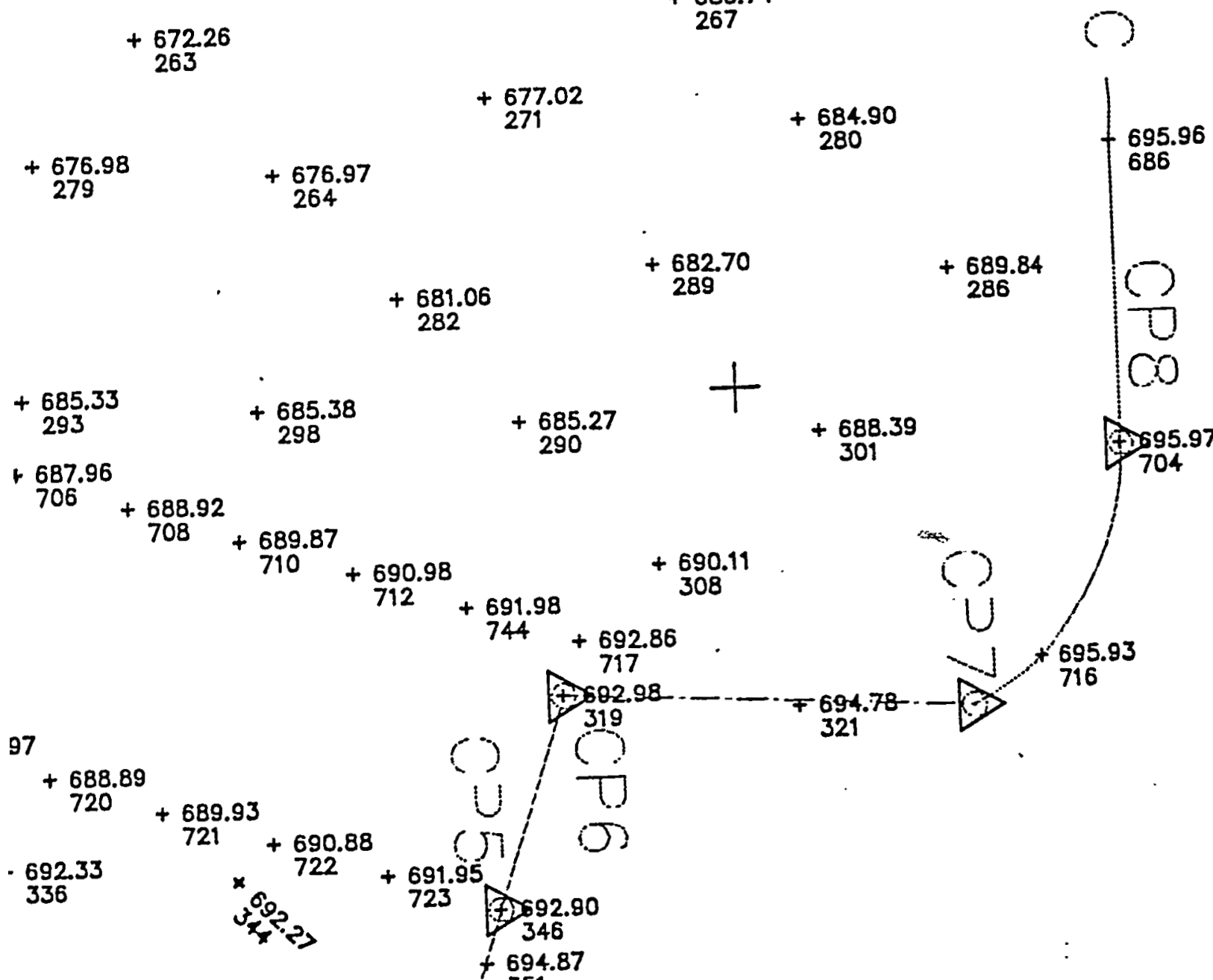

$+672.26$

263
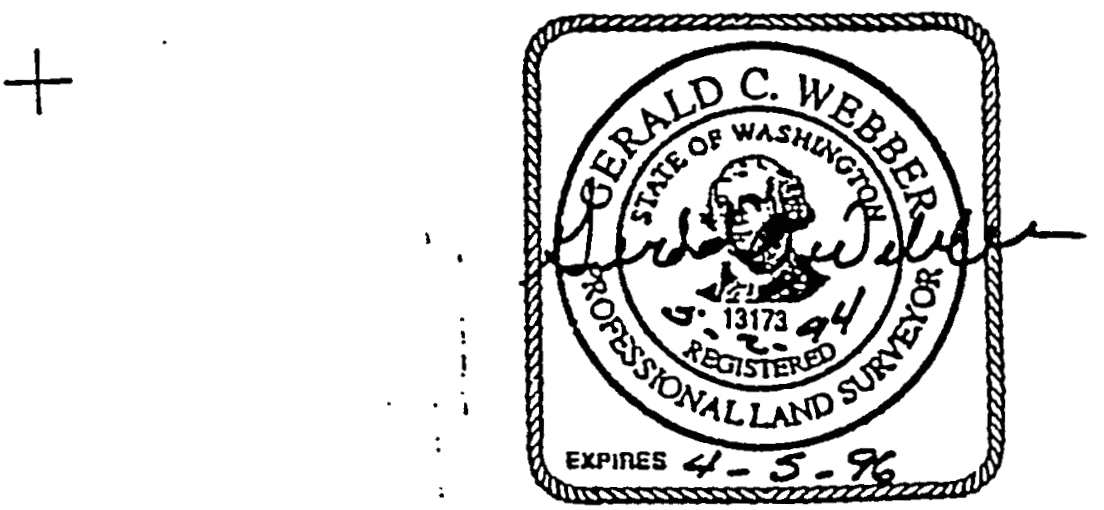

WHC-SD-WO 

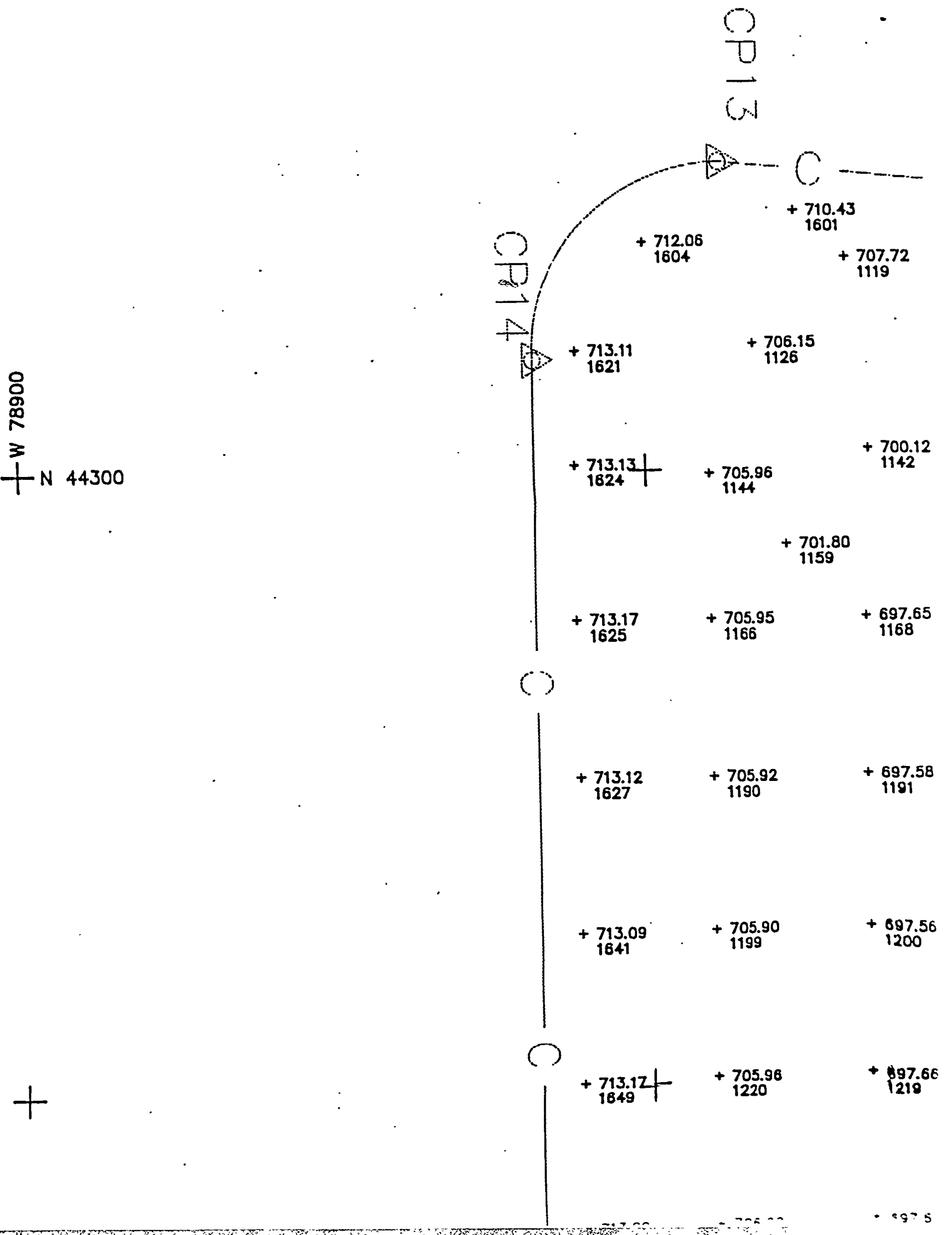


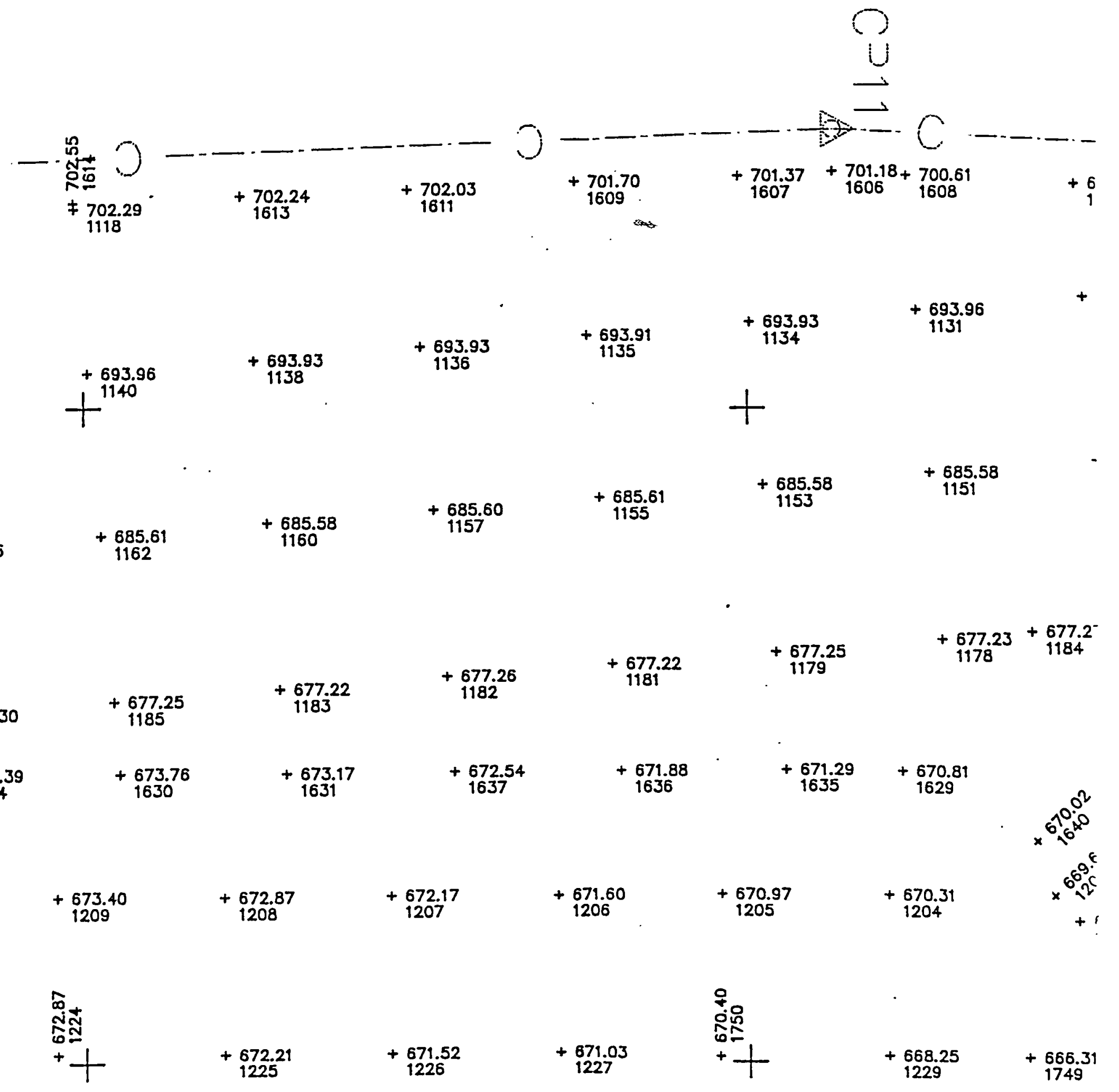




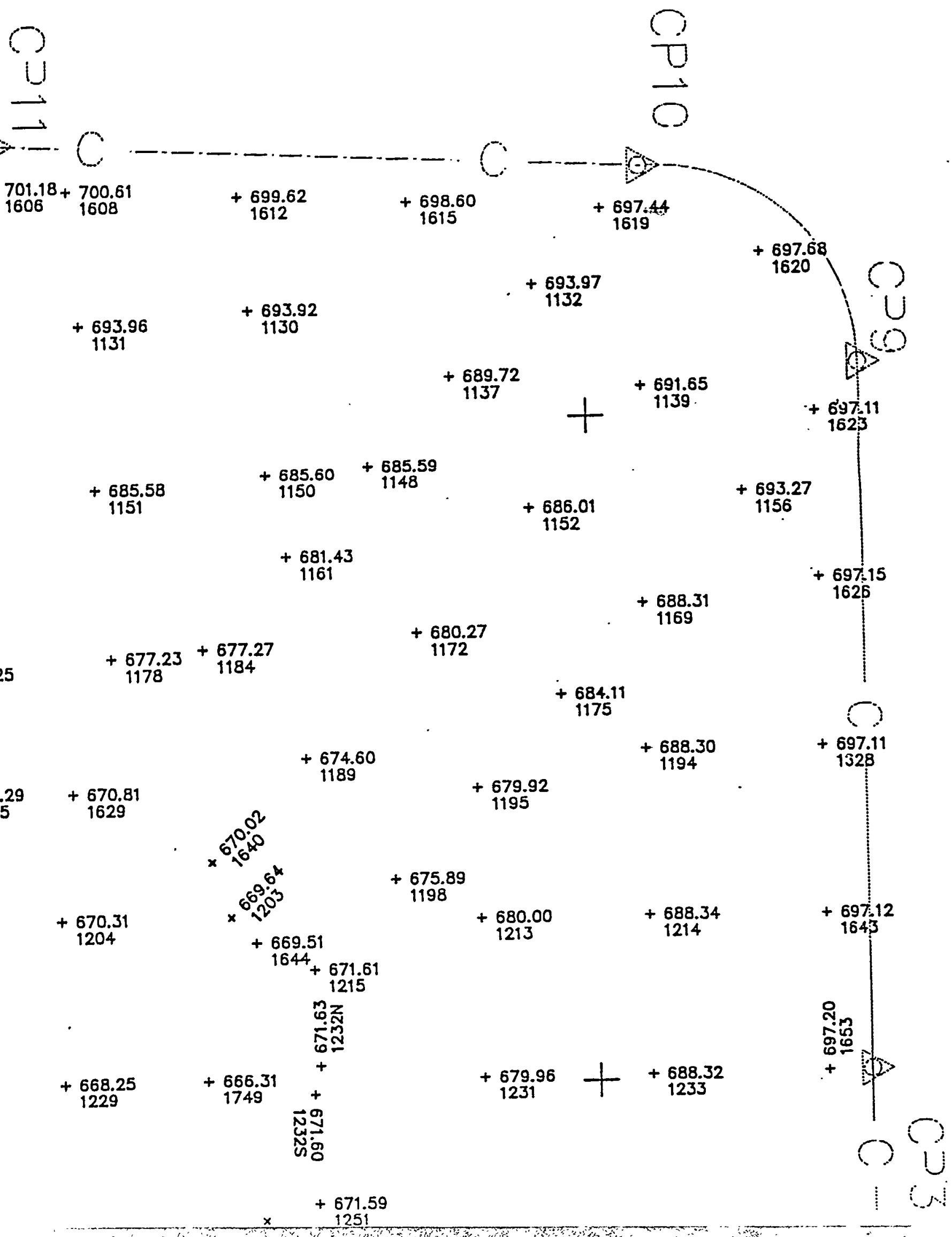




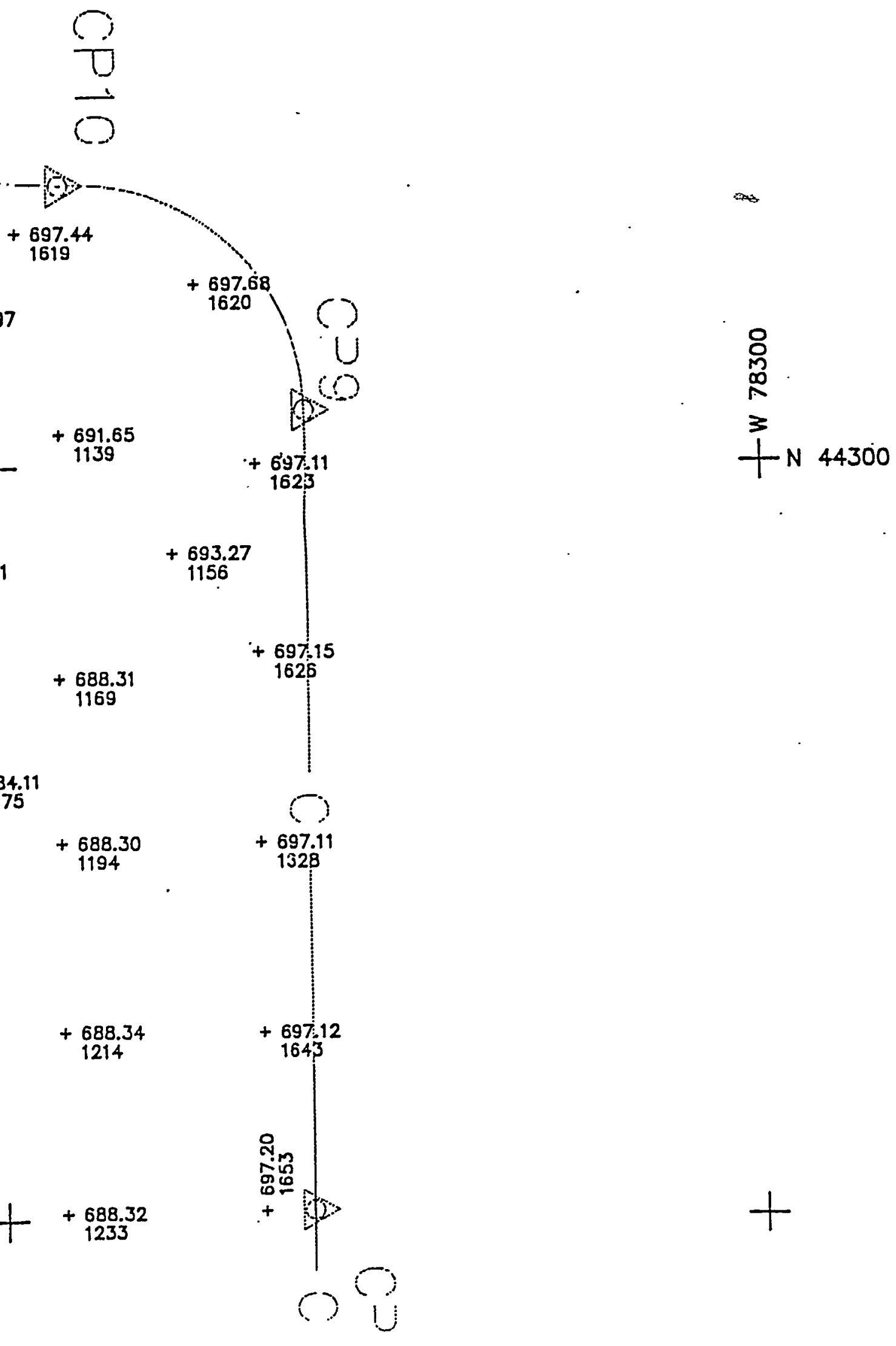




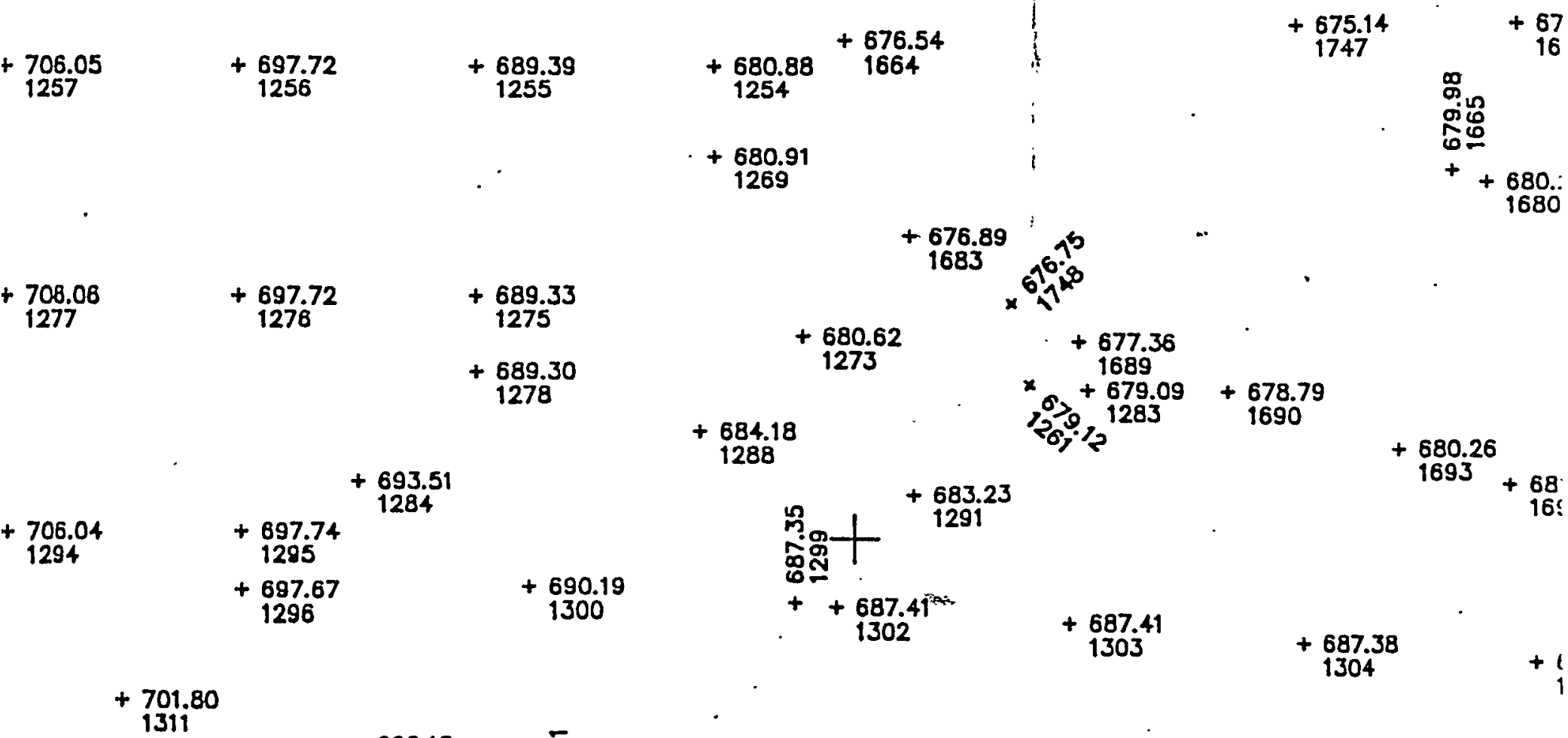

1284

$+680.26$

$1693+68$

$$
+687.41
$$

$+687.38$

1304

\section{$+701.80$}

1311

705.95

1314.04

696.18
1313

1315

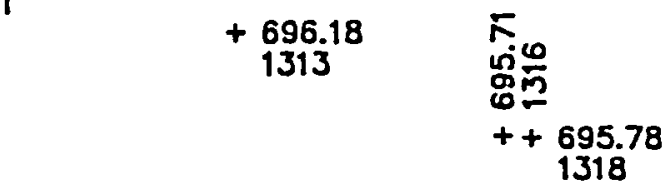

708.17
1347

$+702.20$
$+699.93$ 1335
$+704.10$ 1345
$+704.05$ 1348

$$
\begin{gathered}
704.05 \\
1349
\end{gathered}
$$

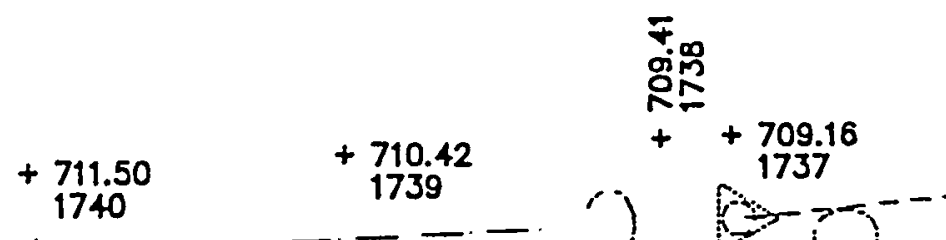

$R_{N}+709.53$ Nำ 1355

$++712.25$ 1741

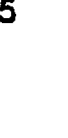

$$
\begin{aligned}
& +695.74 \\
& 1322 \\
& +
\end{aligned}
$$$$
+695.77
$$$$
1327
$$$$
\begin{array}{r}
65 \\
13
\end{array}
$$

$$
1
$$

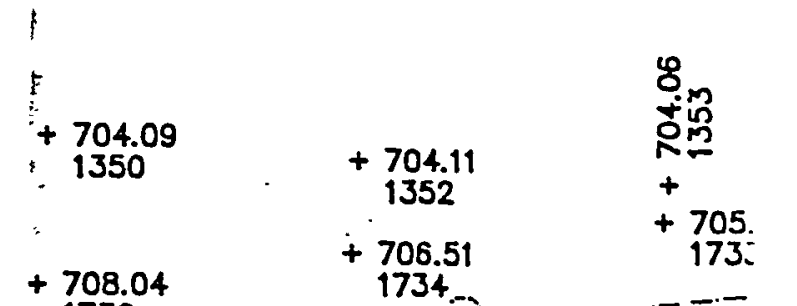




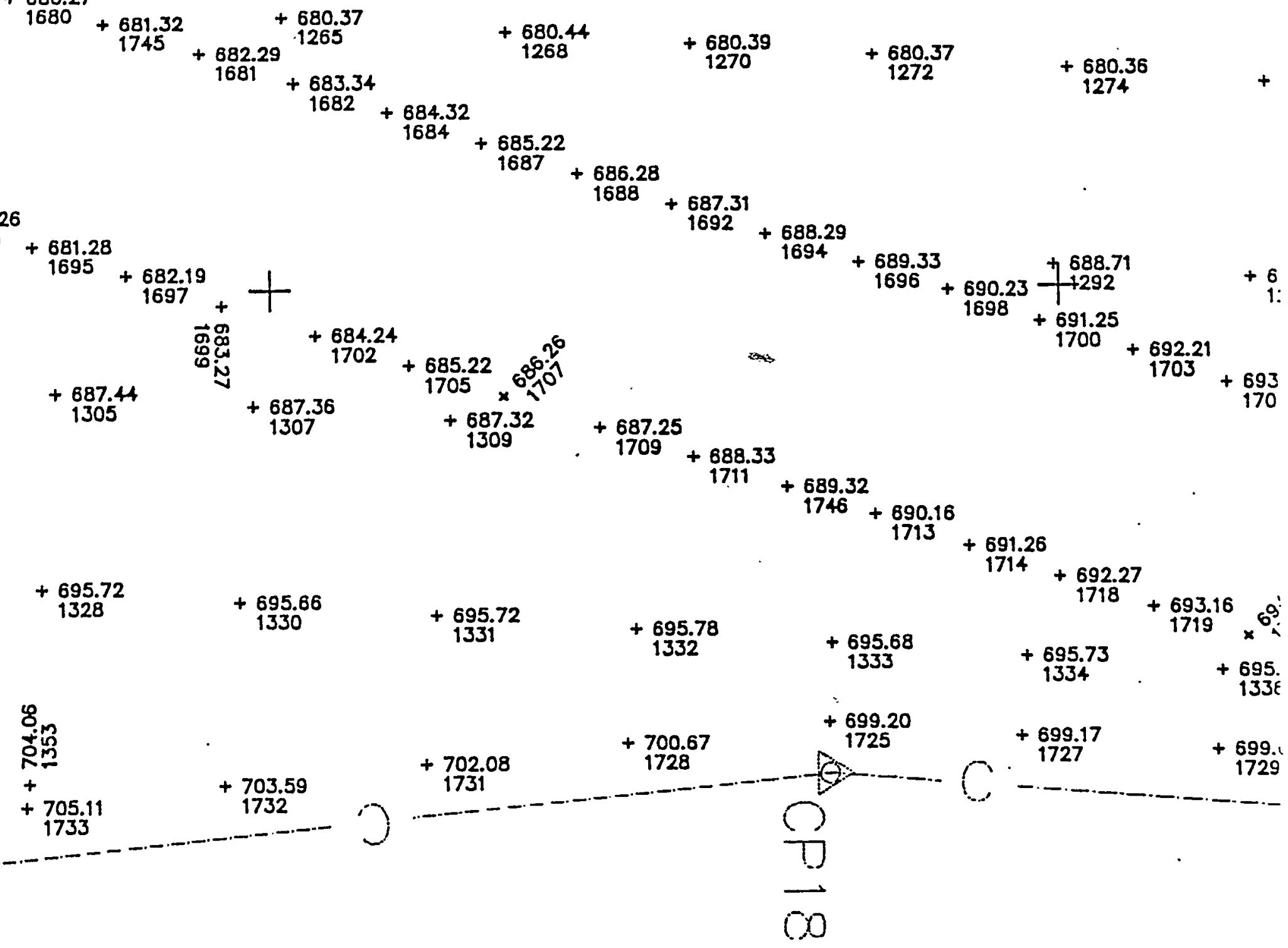

$+699.17$

1727

$+680.36$

1274

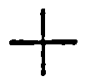

NOTE:

SURVEY PERFORMED $11 / 15$ THRU $11 / 17$ AND 


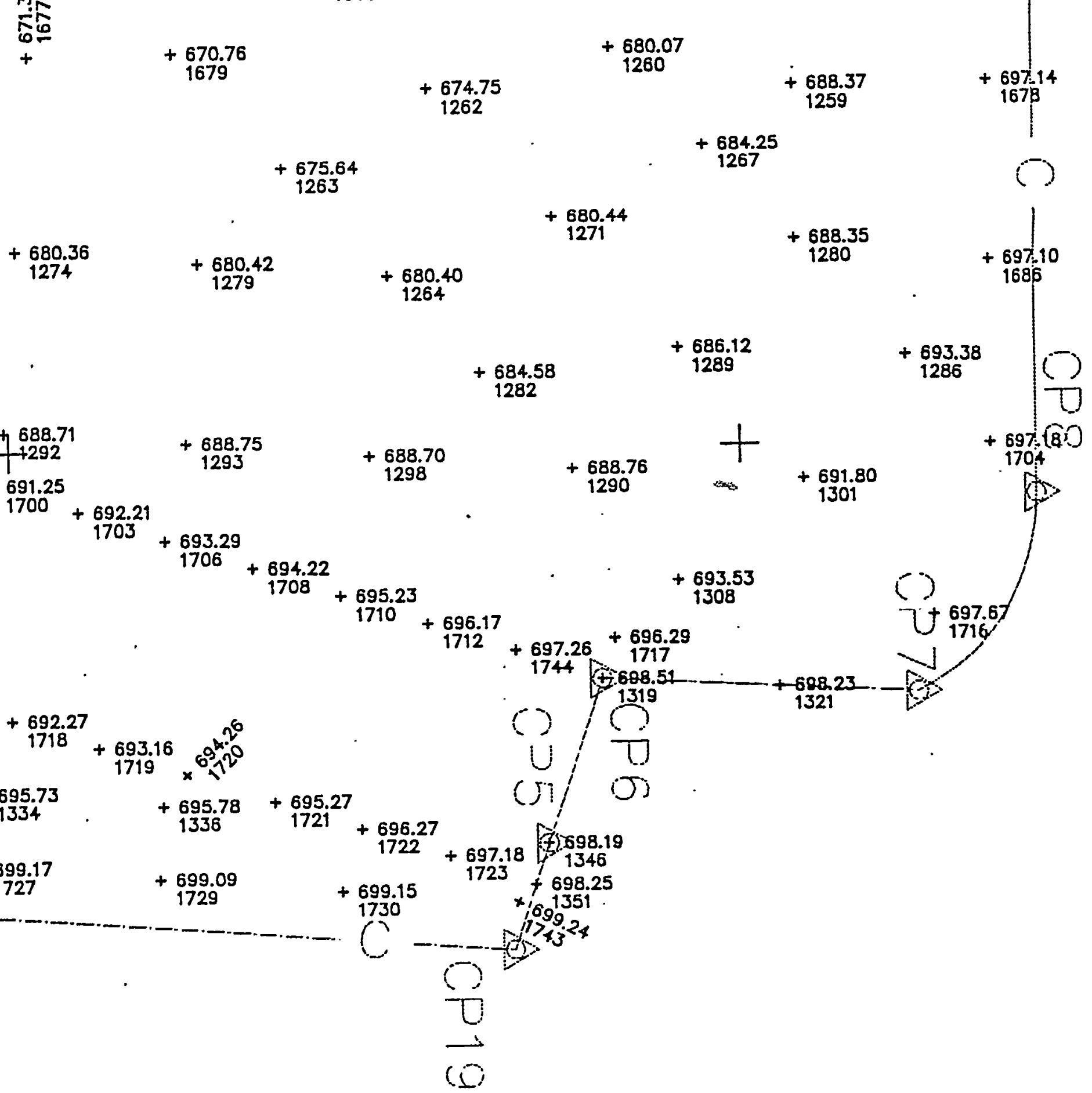
1290$$
\rightarrow
$$

THRU 11/17 AND 11/19 1993 

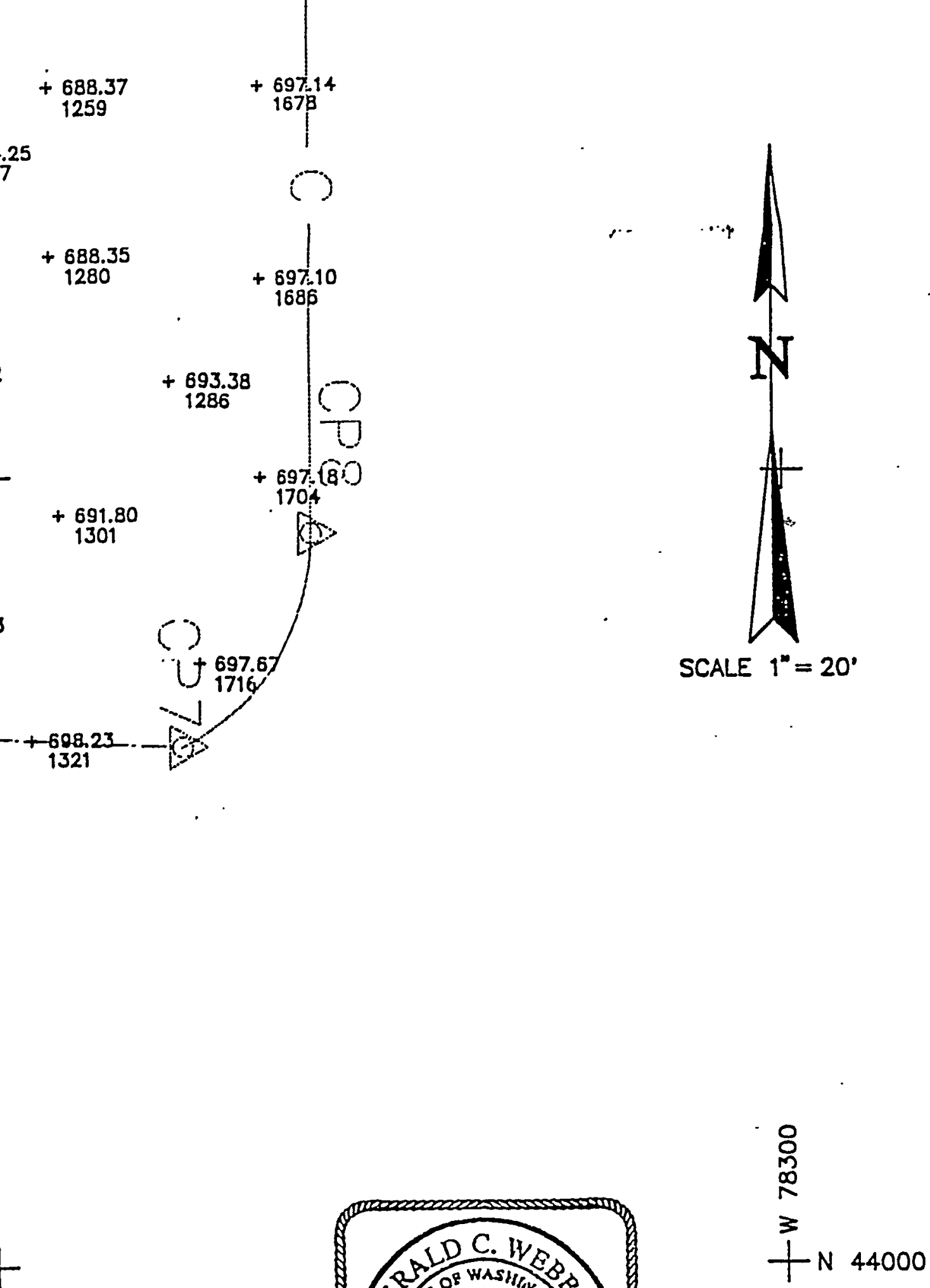


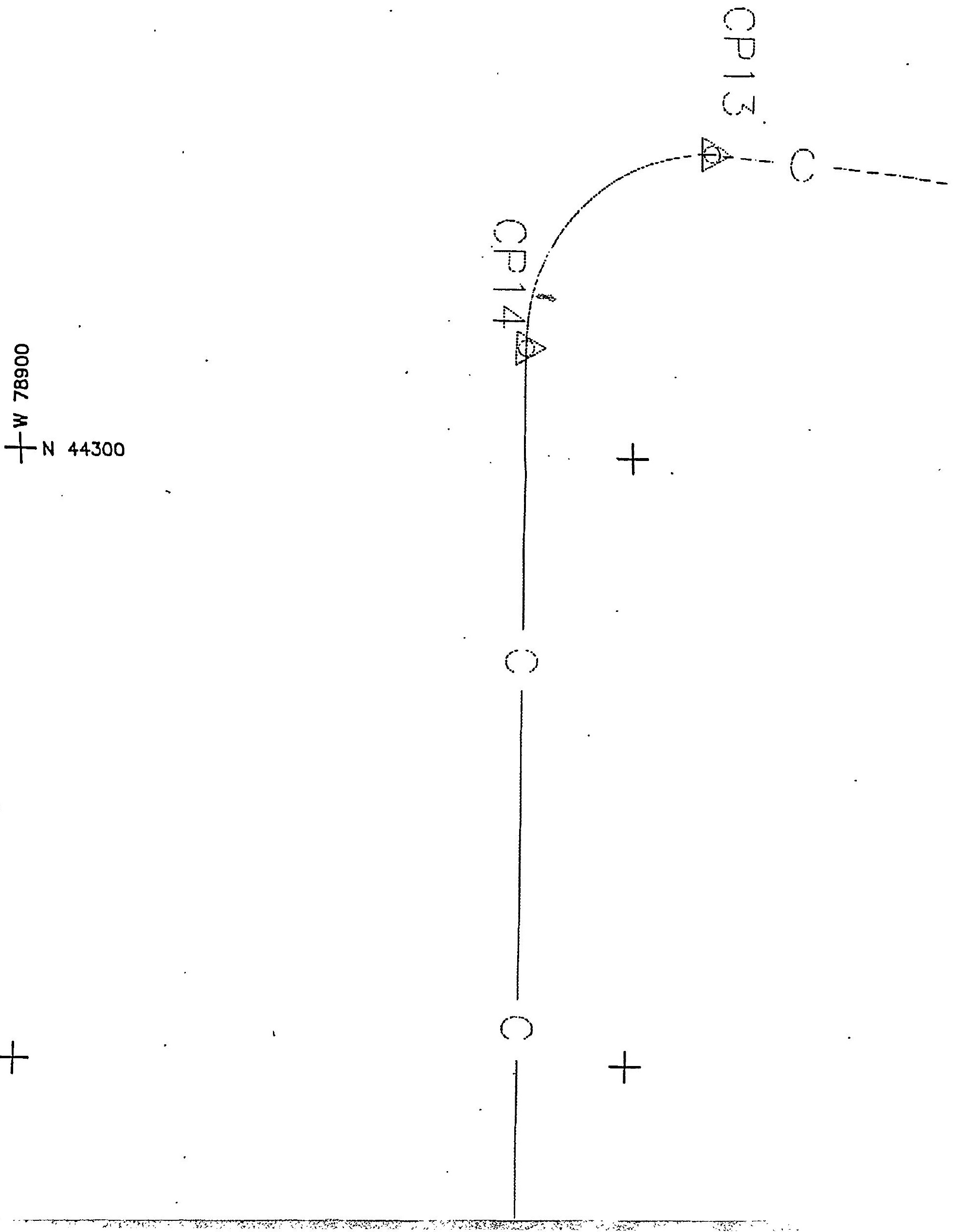



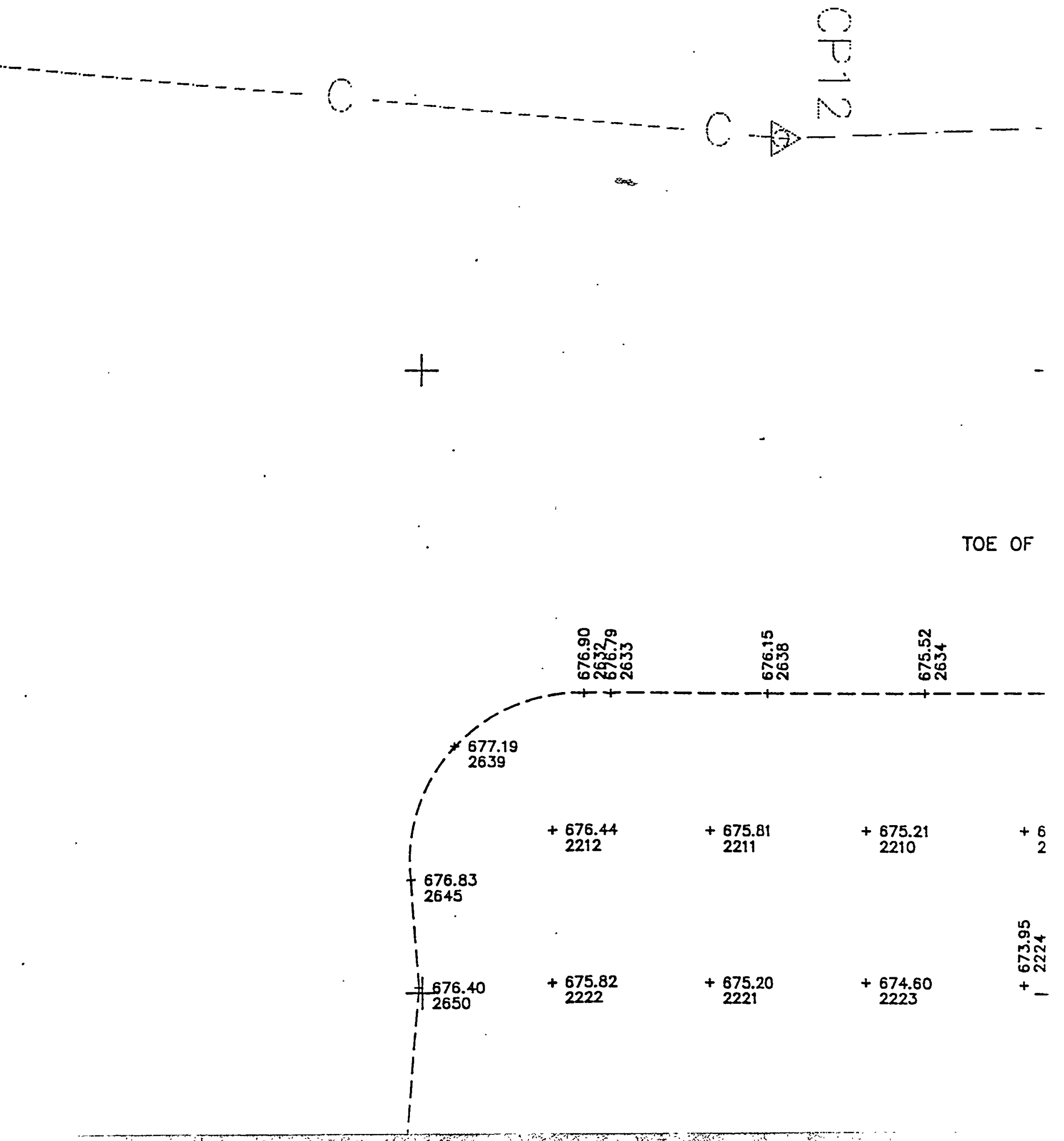

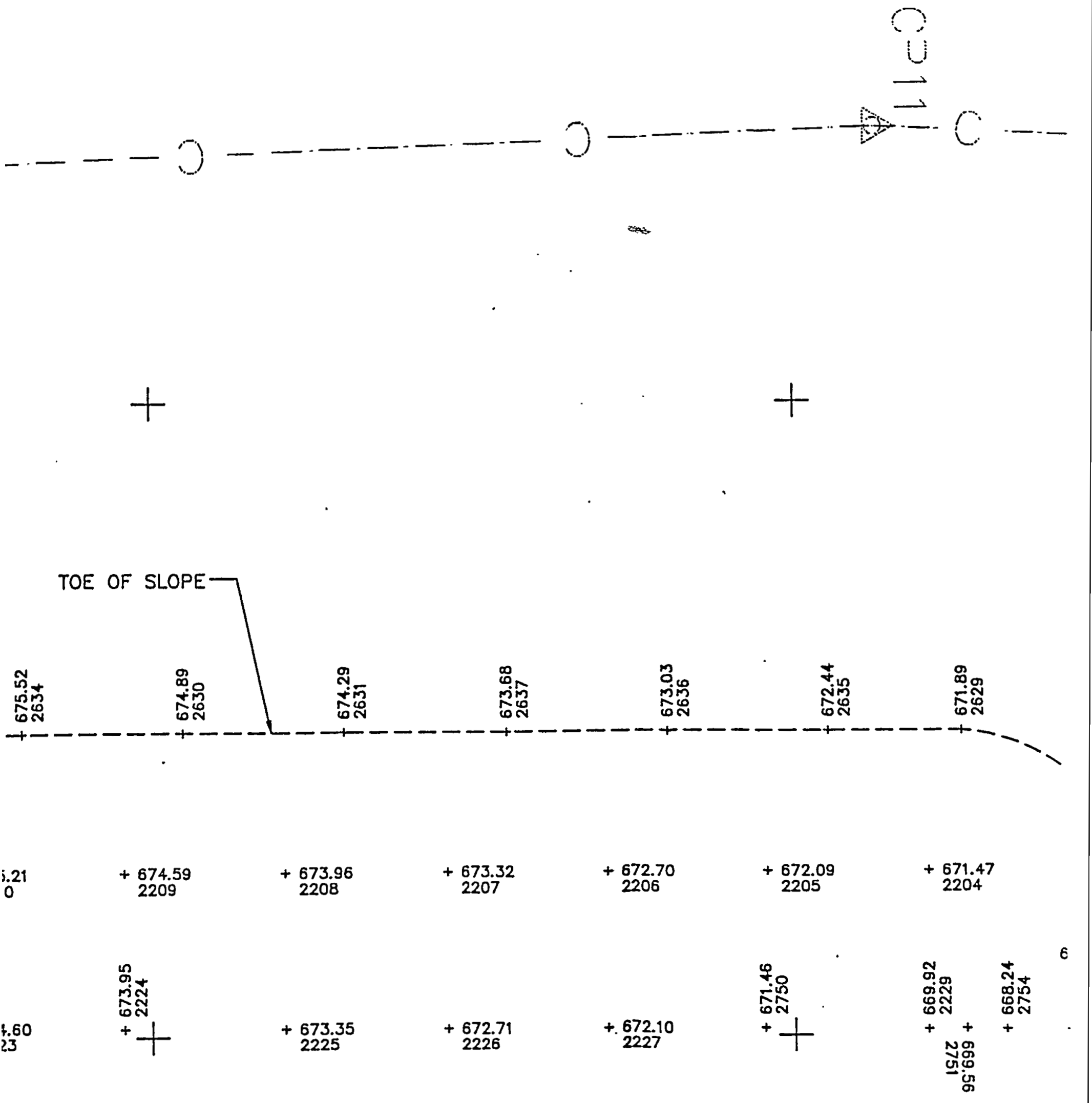


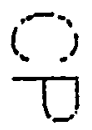

$\overrightarrow{0}$

-

$\infty$

مُ

$\frac{3}{T N} 44300$

6

RY SLOPE RISER

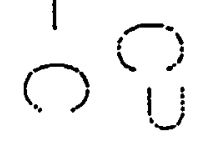


sos

NOTE:

SURVEY PERFORMED $1 / 141994$ 


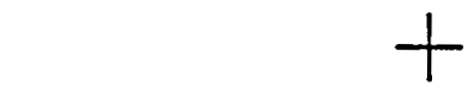

$$
+
$$

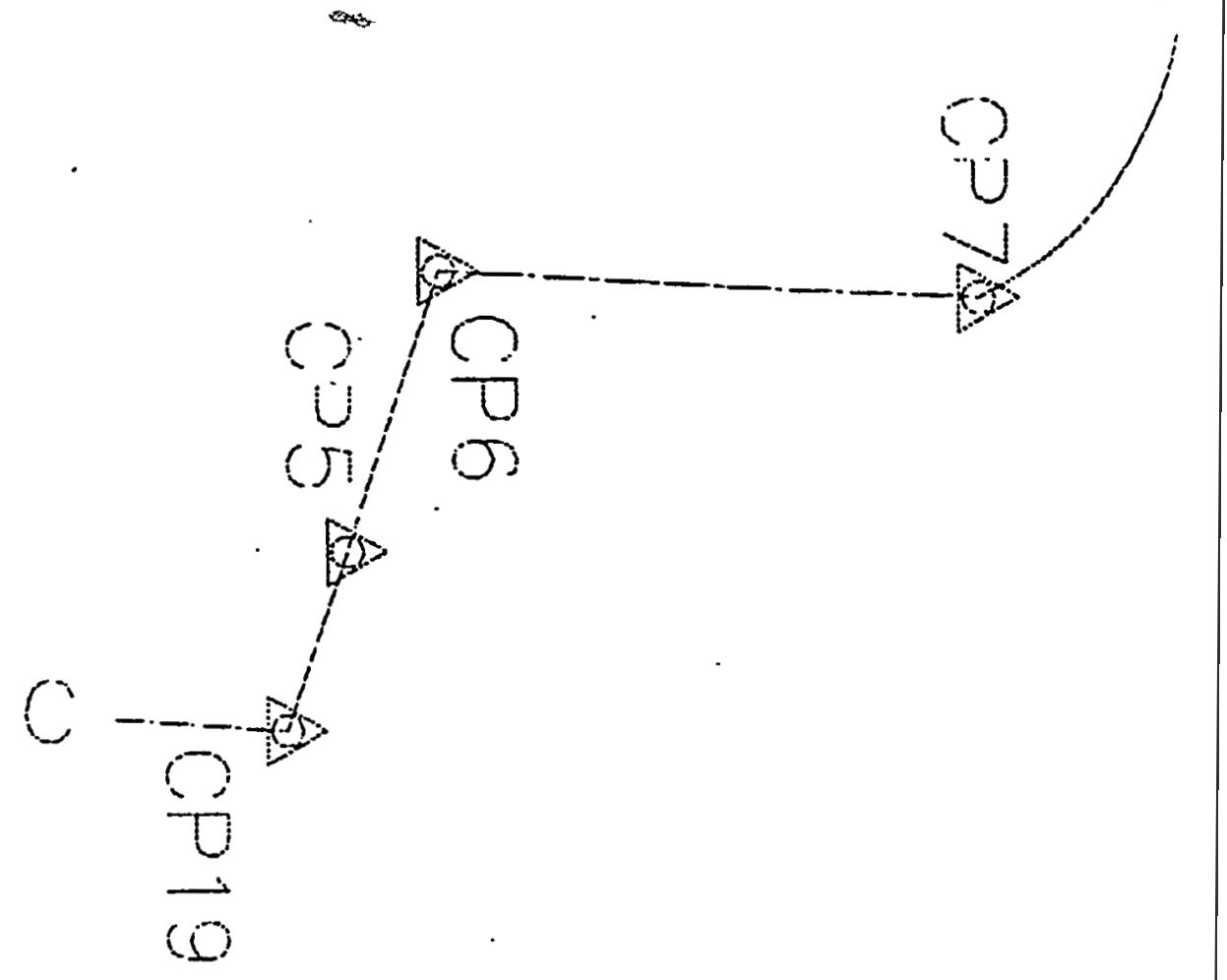

1

1 

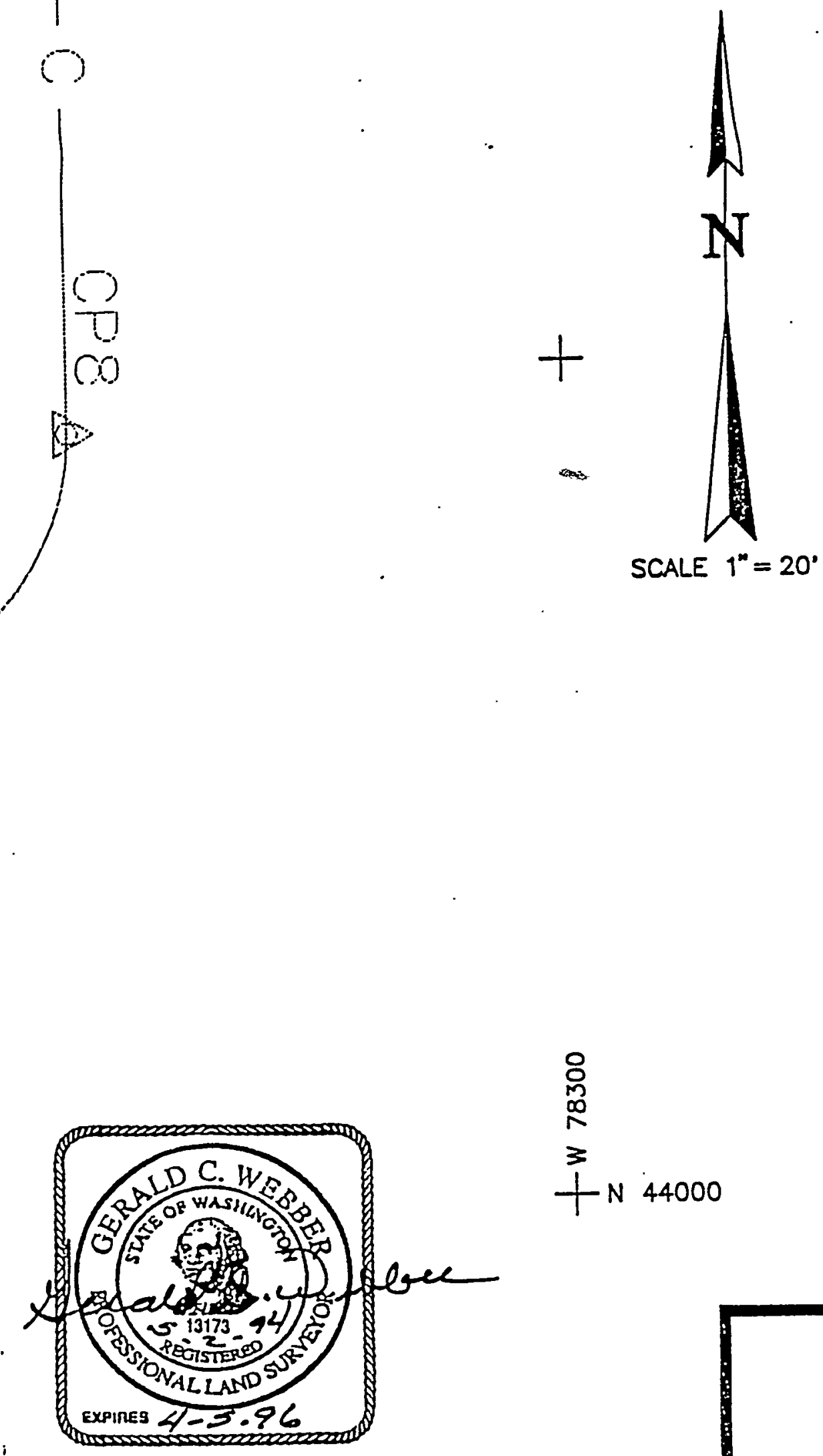
8
品
9
3

TN 44300

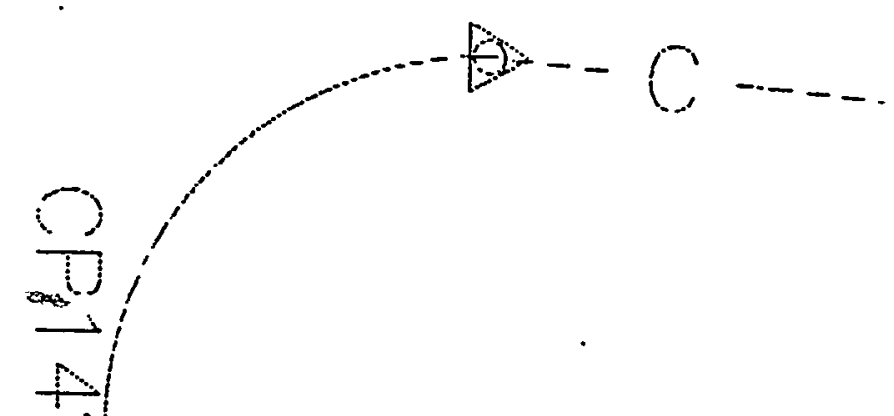

()

$\mid$

0

$+$

$+$ 


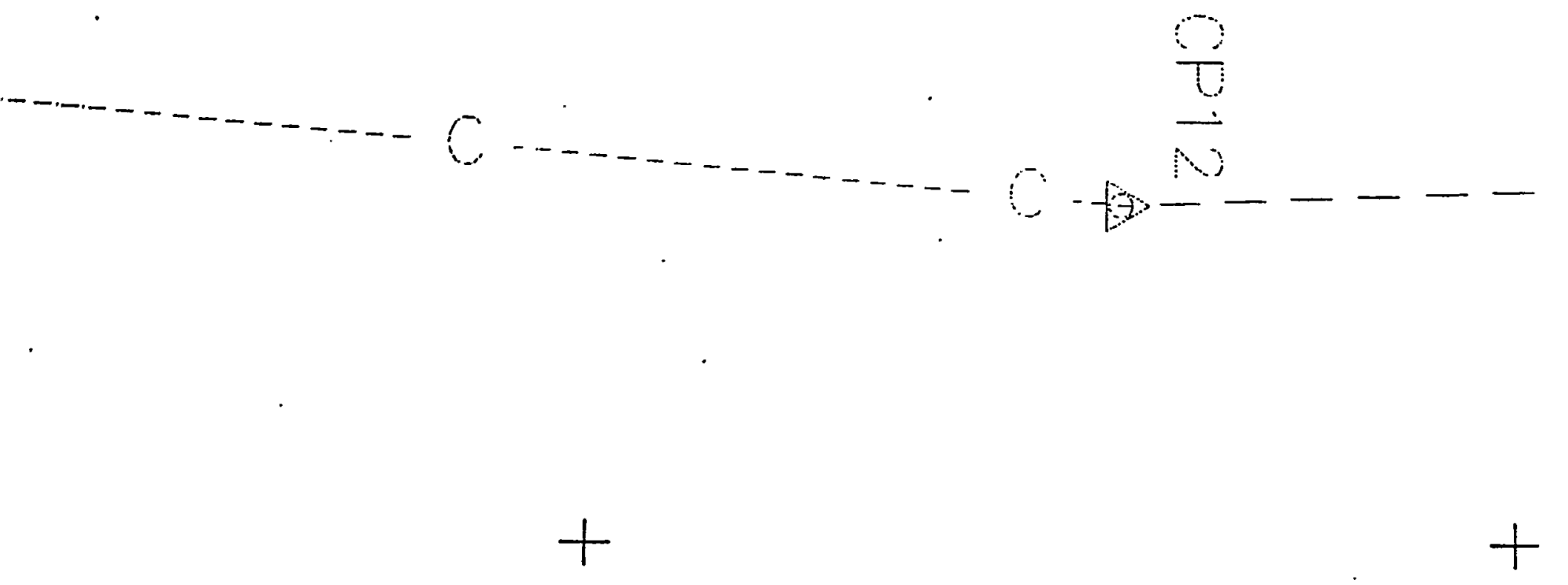


$\underset{+\infty}{\longrightarrow}$

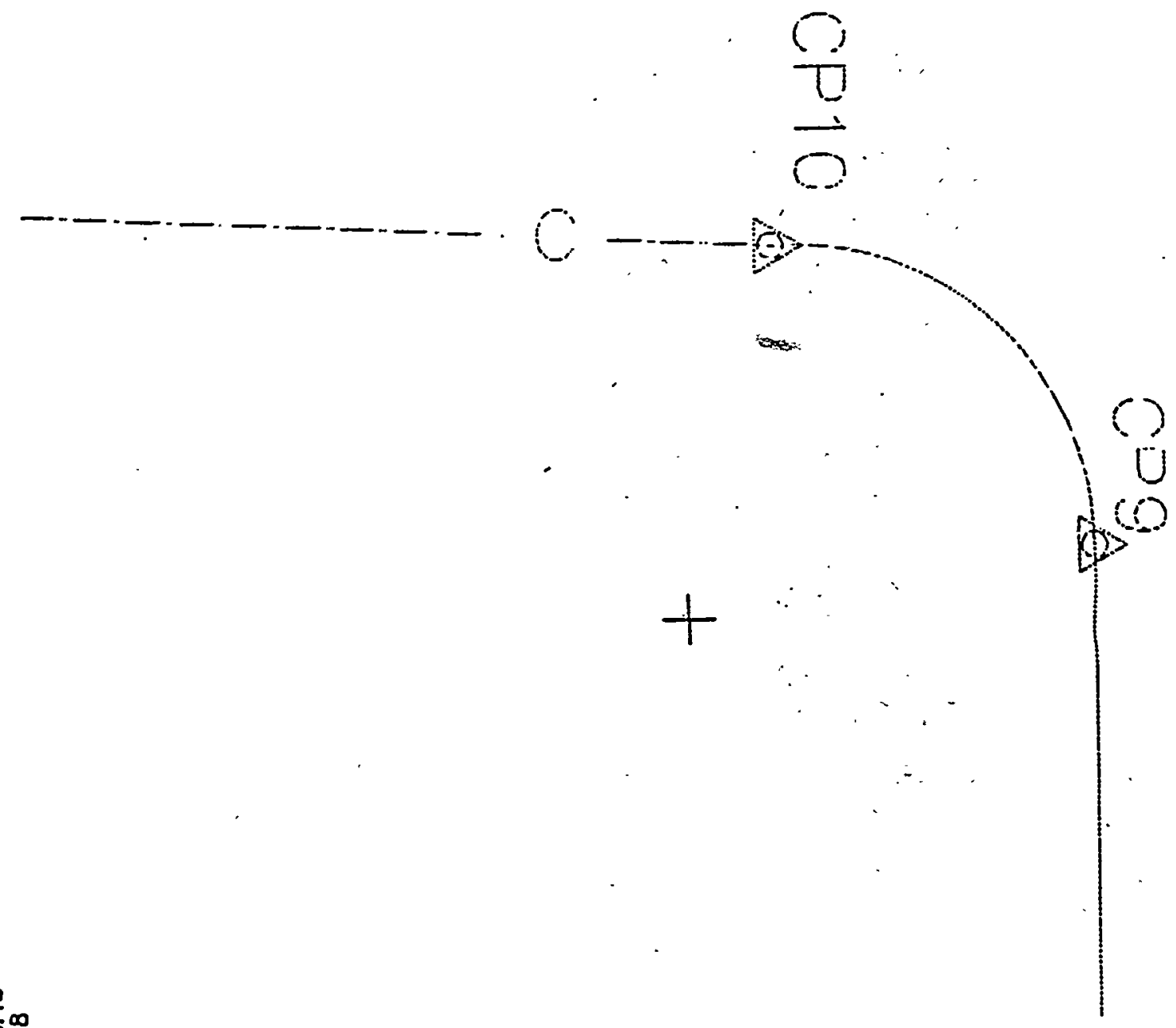

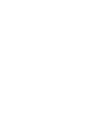

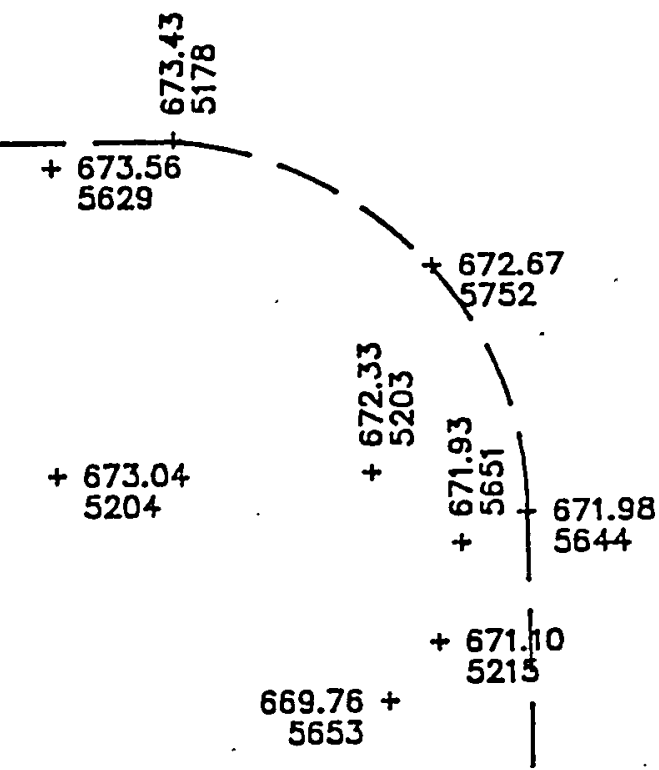

$+671.44$

5229
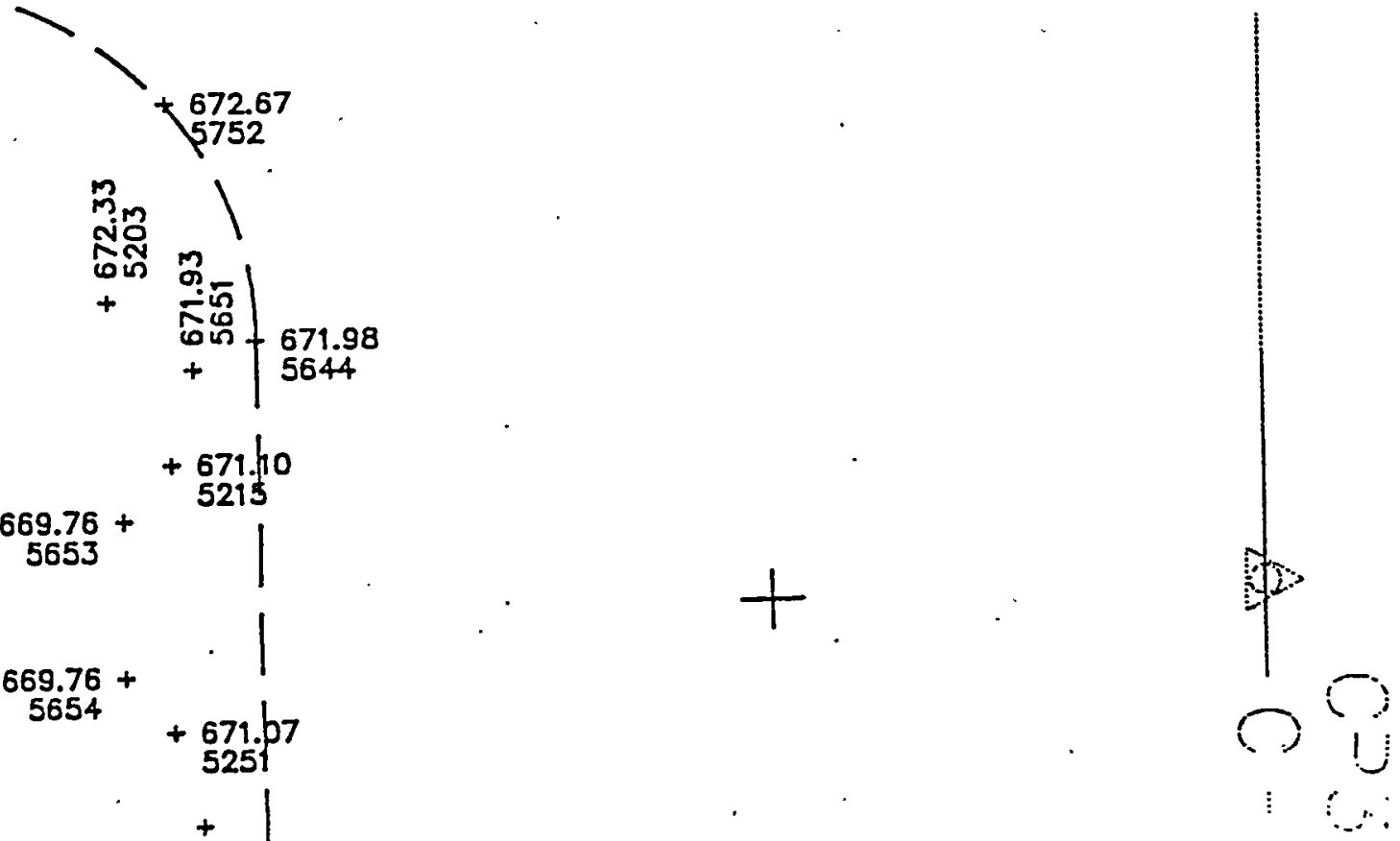

$+57200$ 
$+$
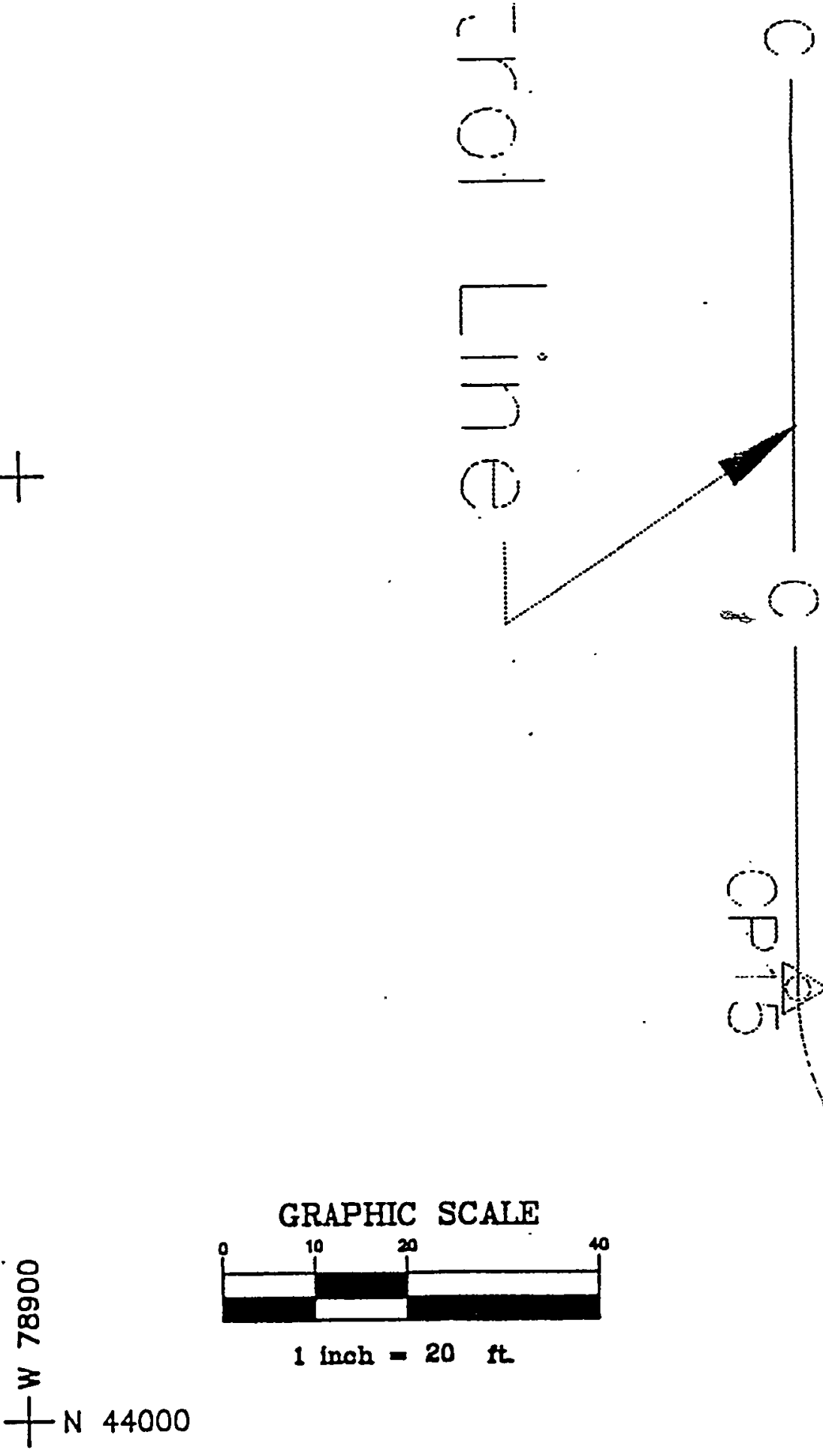

0

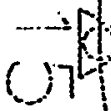

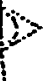

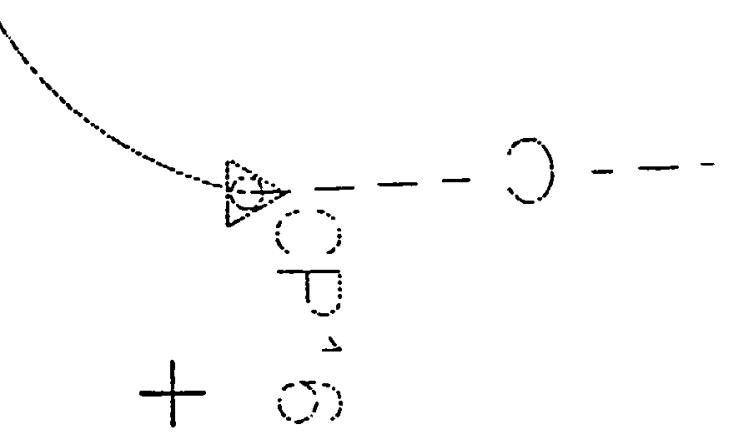

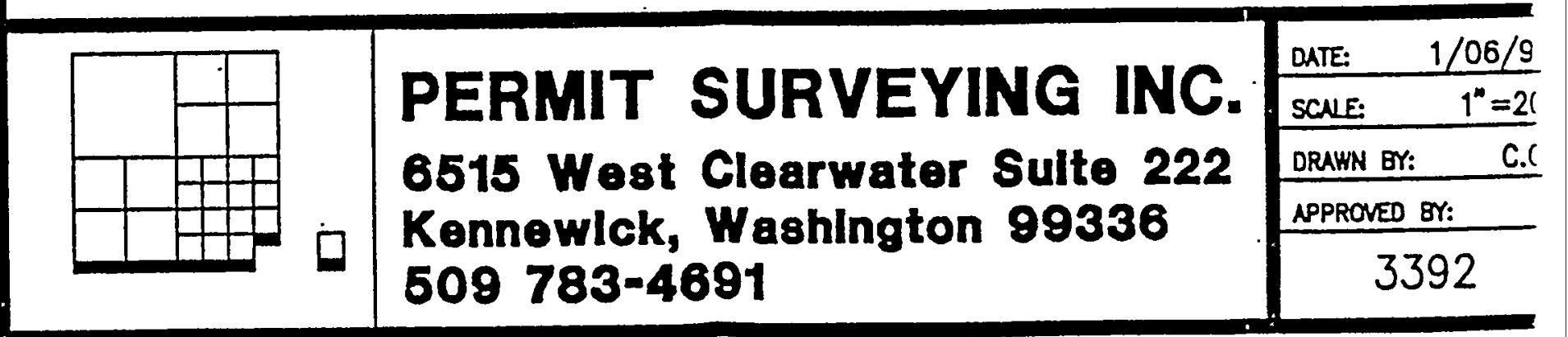



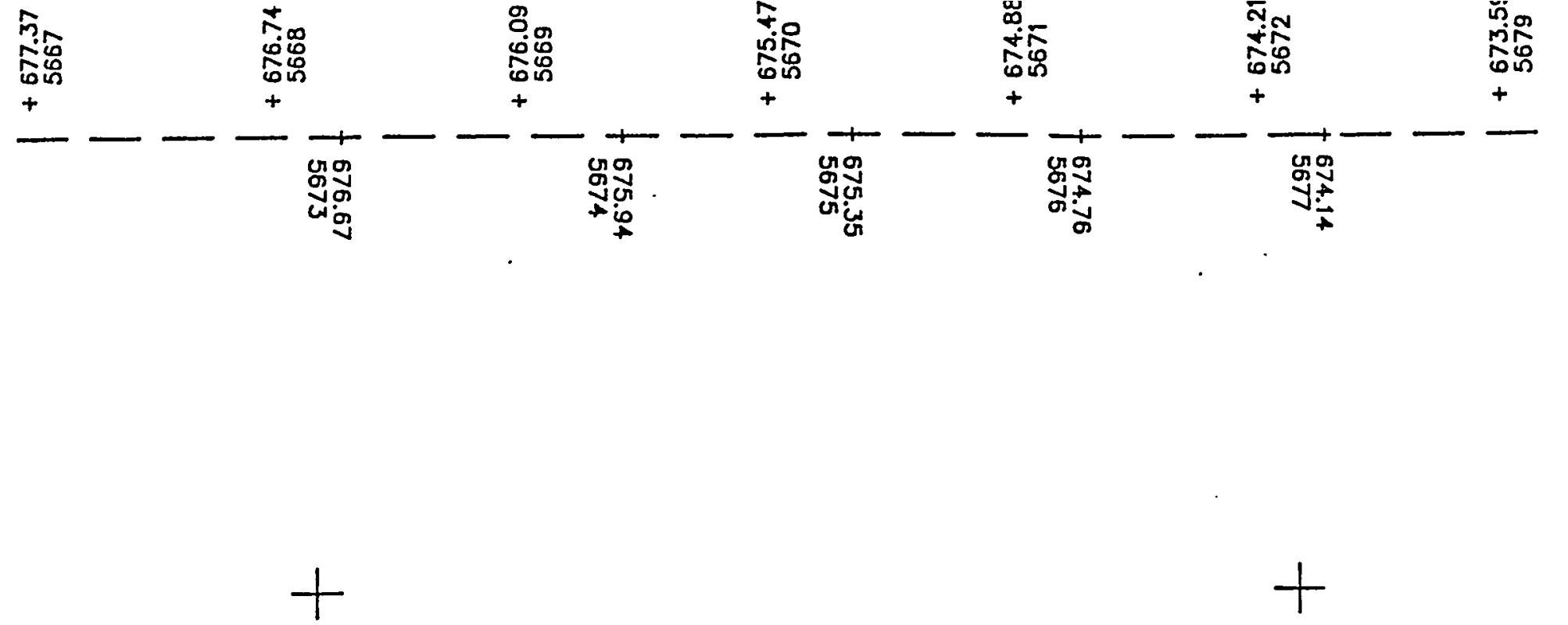

1

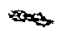

MIX AS-BUILT

AL FACILITY

$+$

NOTE:

SURVEY PERFORMED $1 / 19$ THRU $1 / 20 / 1$ s 



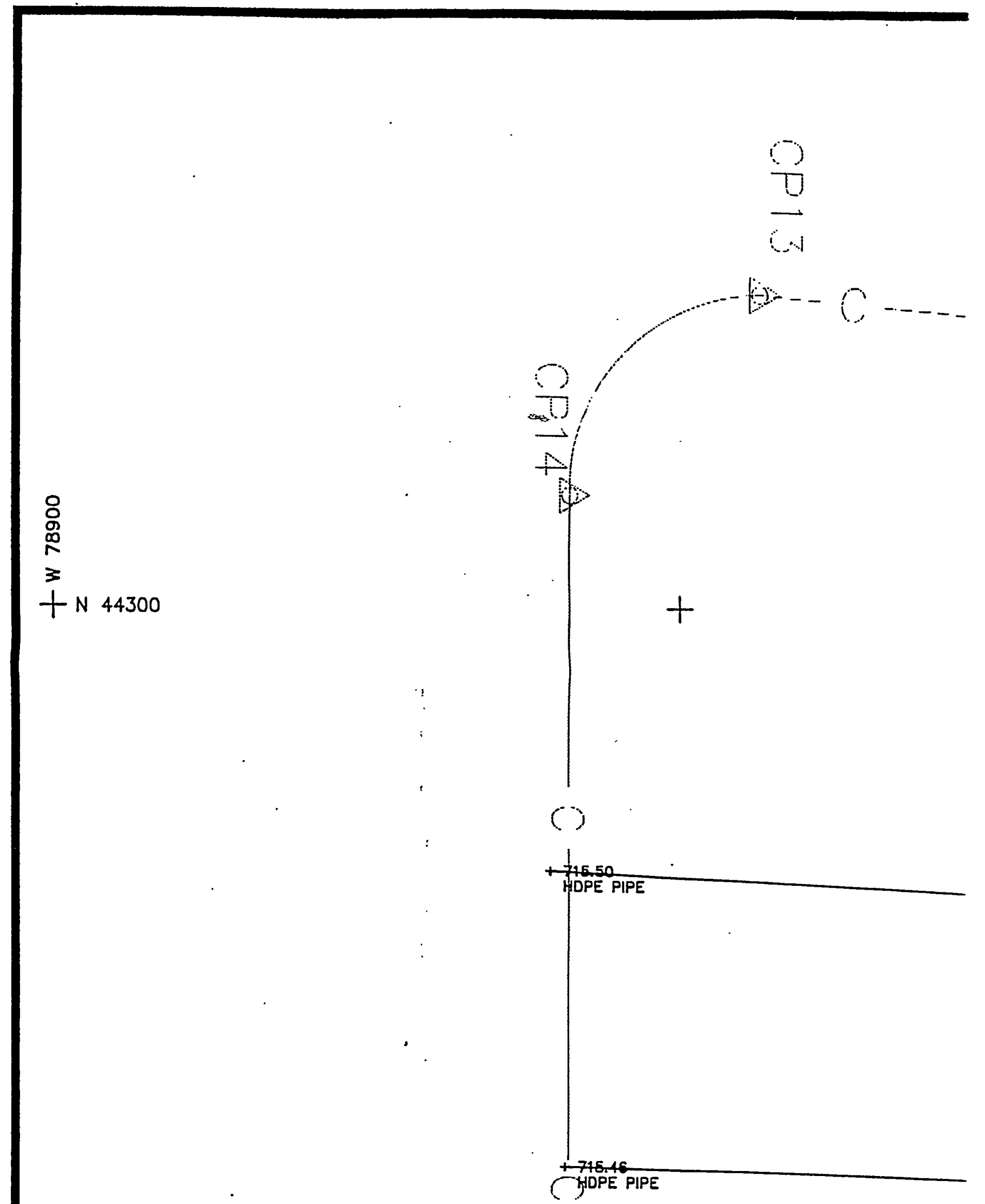

$+$

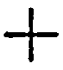




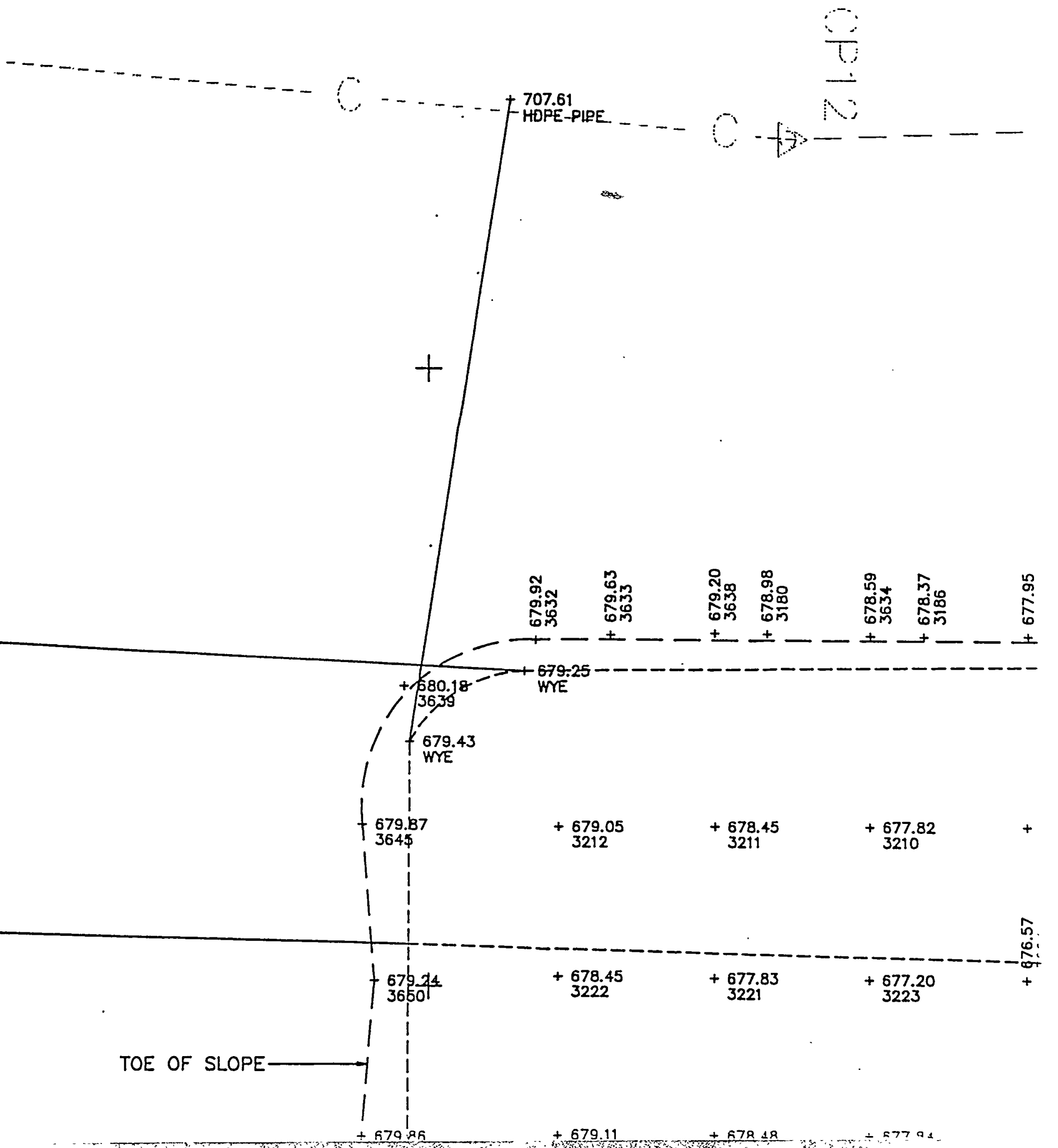




\section{LEGEND}

\section{$-----4^{N}$ HDPE PERFORATED PIPE}

4* HDPE NON-PERFORATED PIPE

6

$\frac{1}{6}$

$+$<smiles></smiles>

怘

3

$+N 44300$

ats

AARY SLOPE RISER PIPE

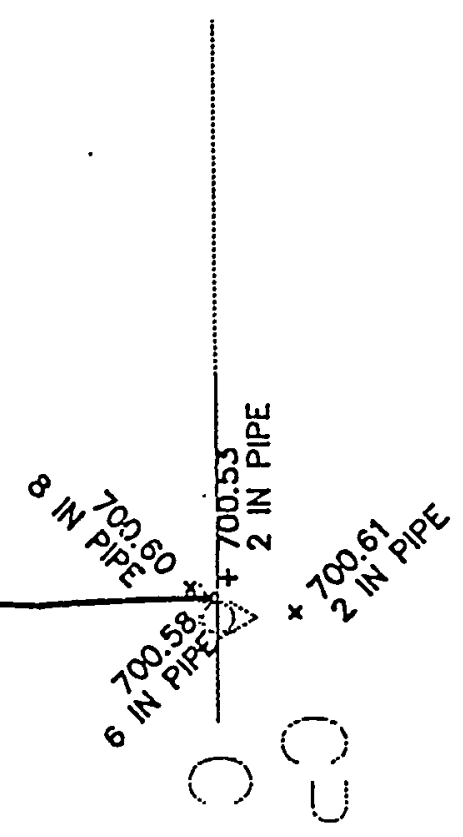




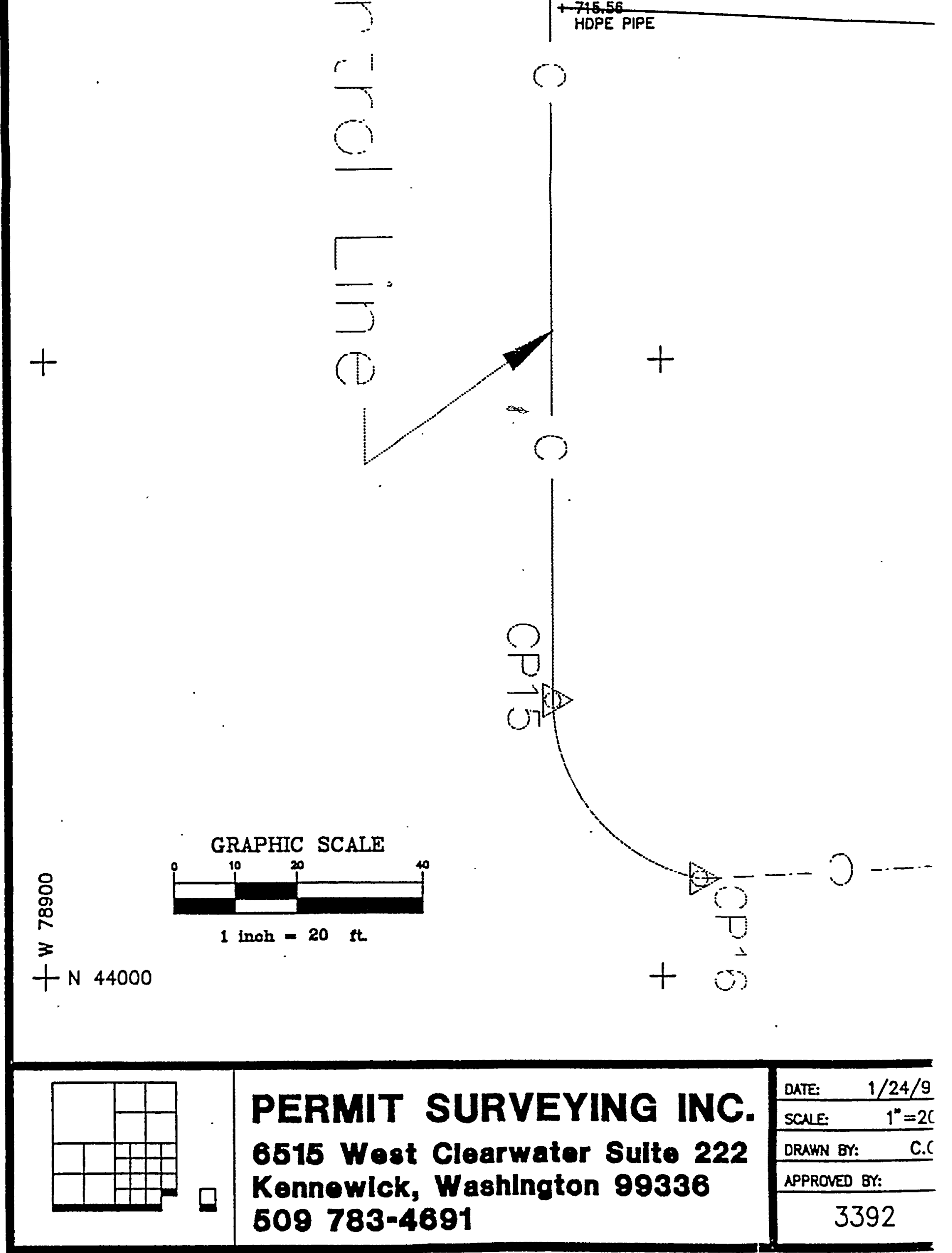




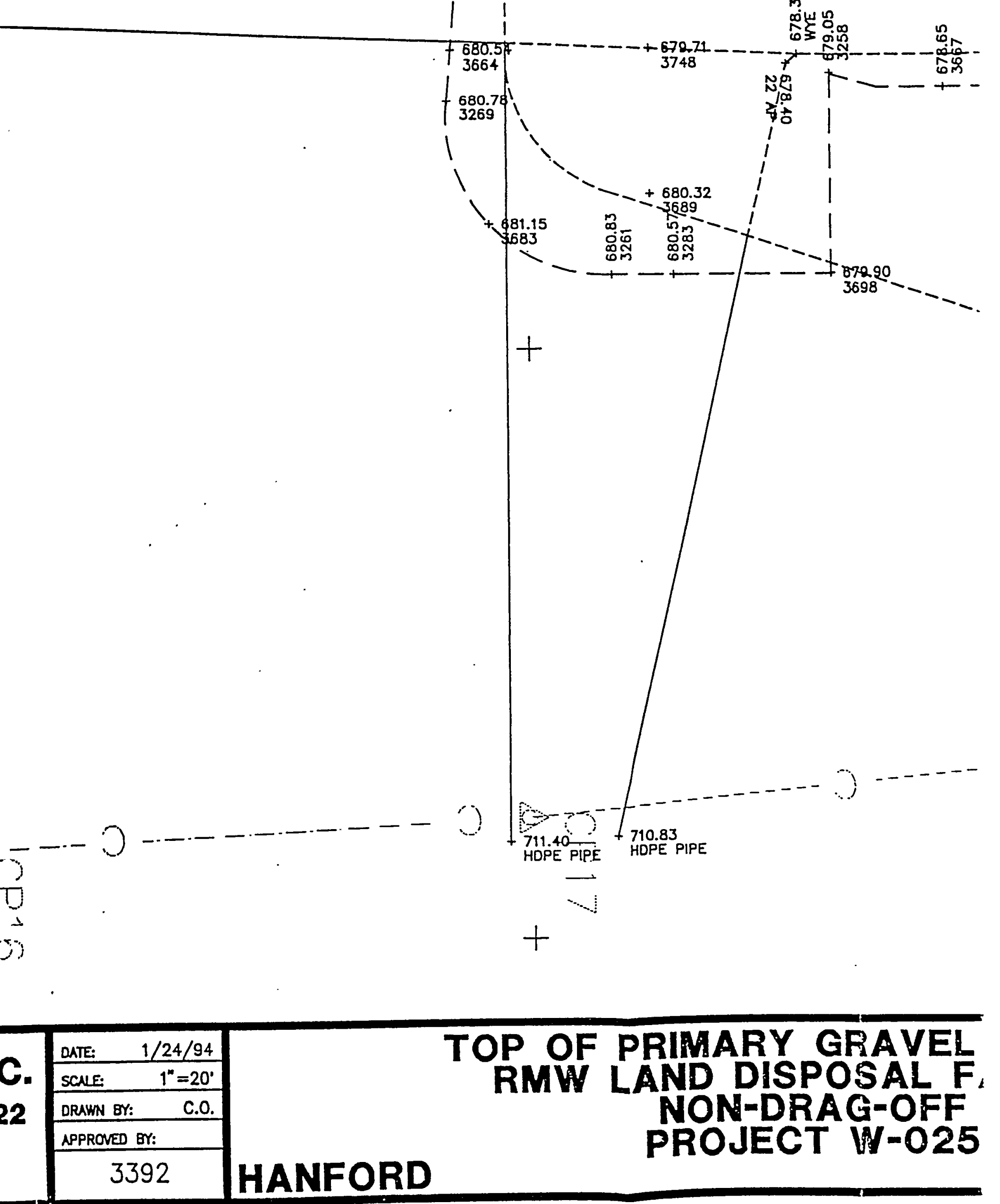



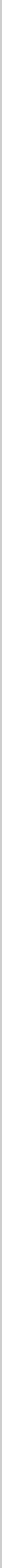


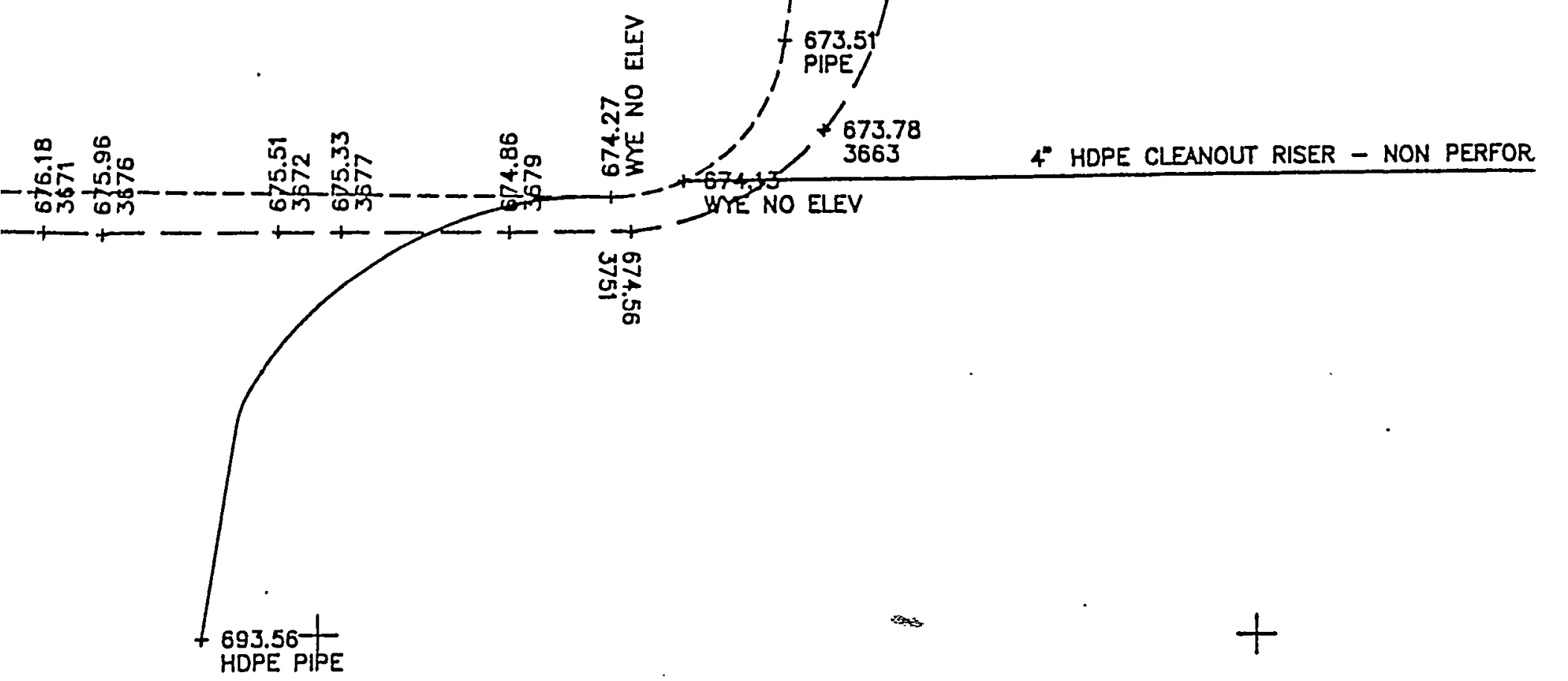




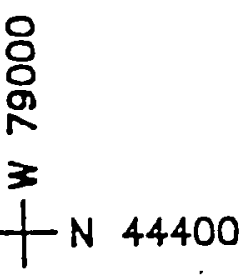

$\rightarrow$

1
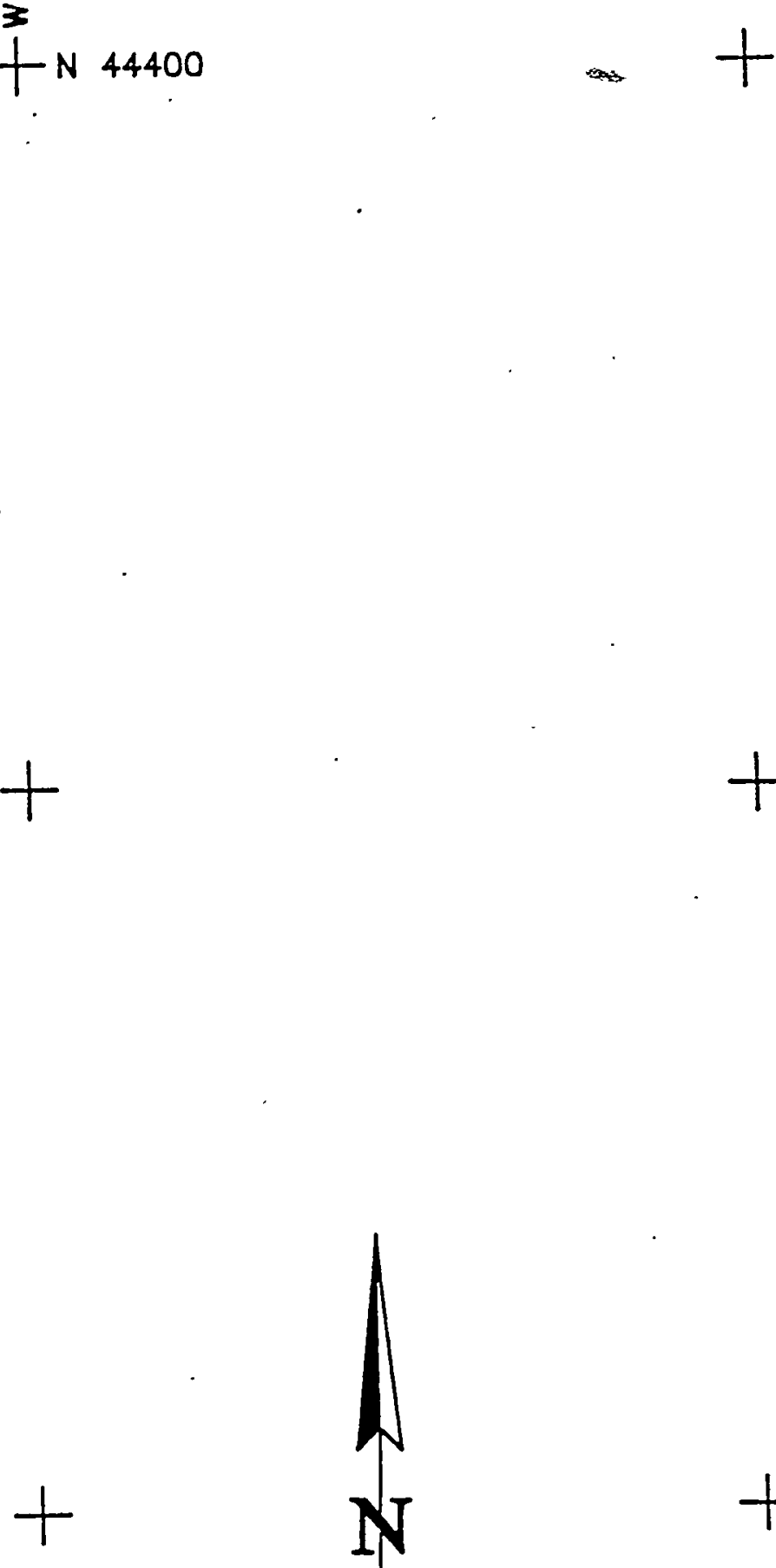

1

$N$
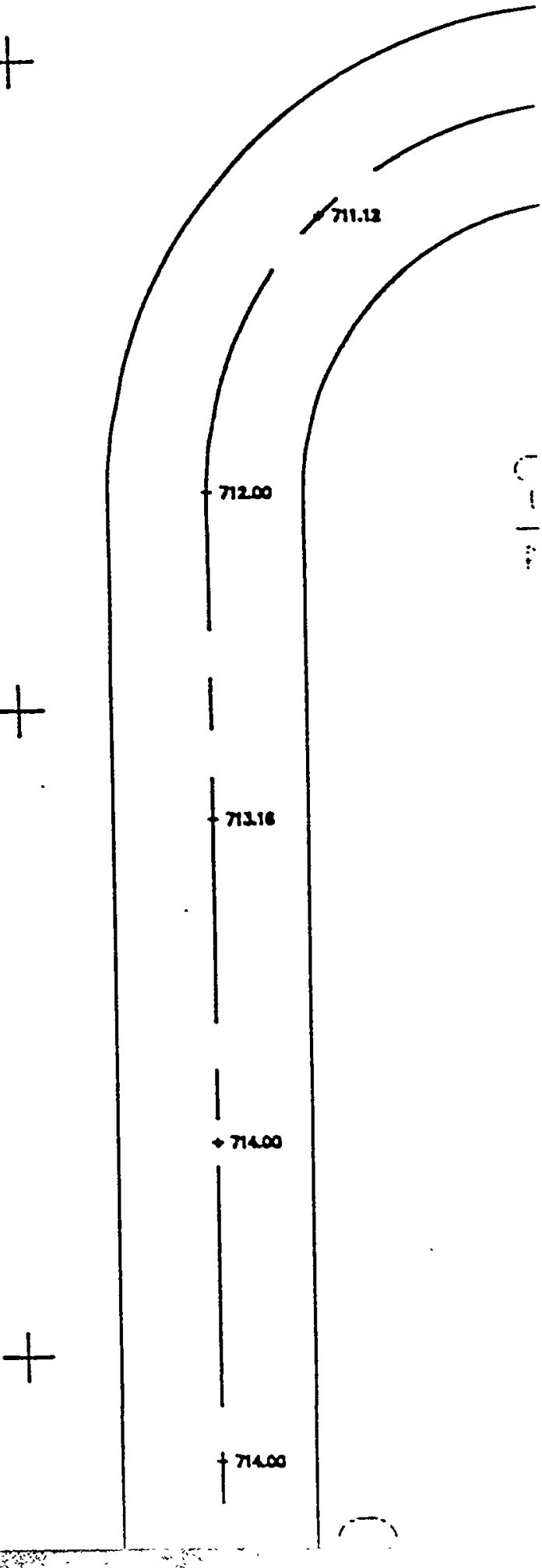


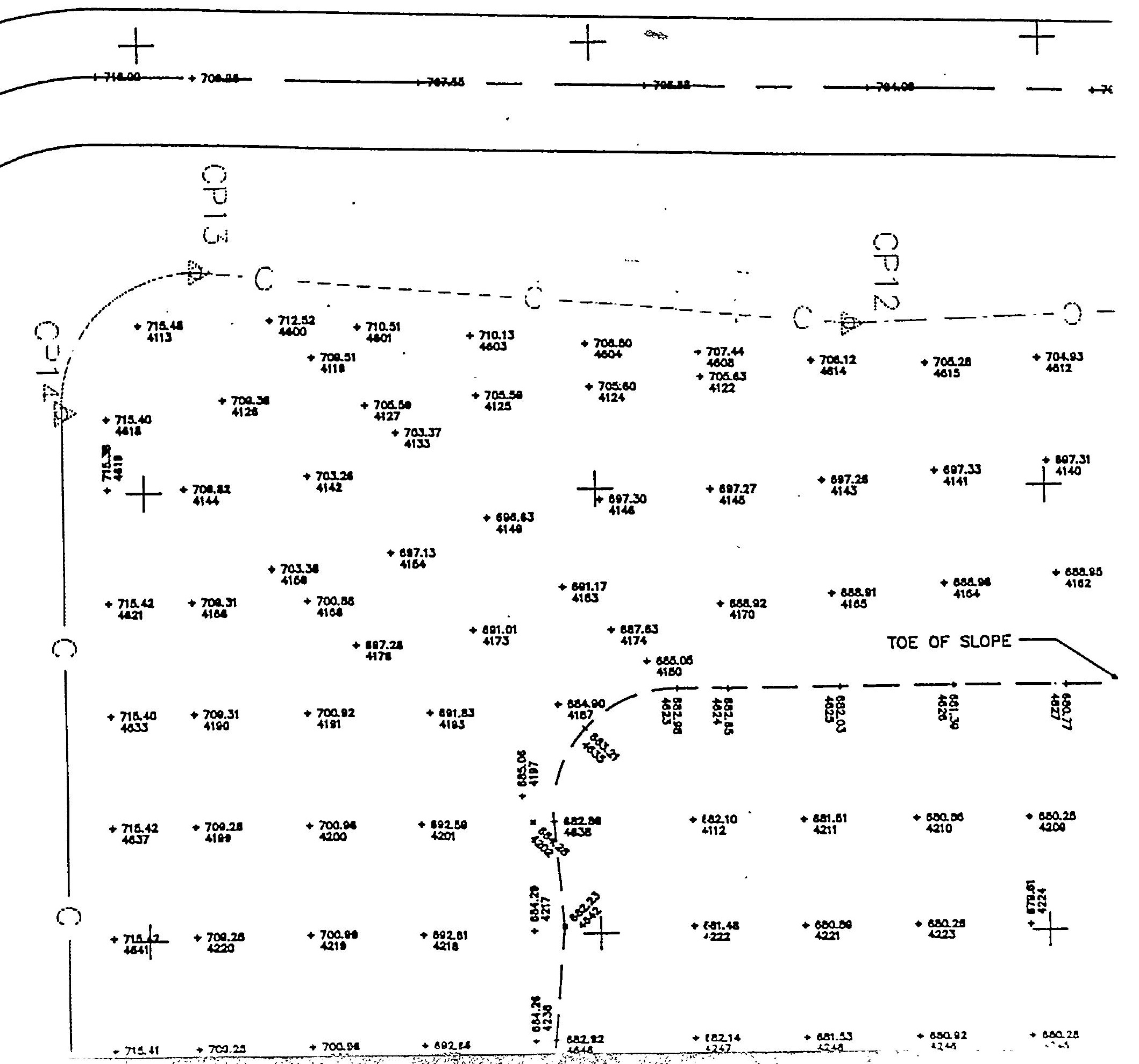




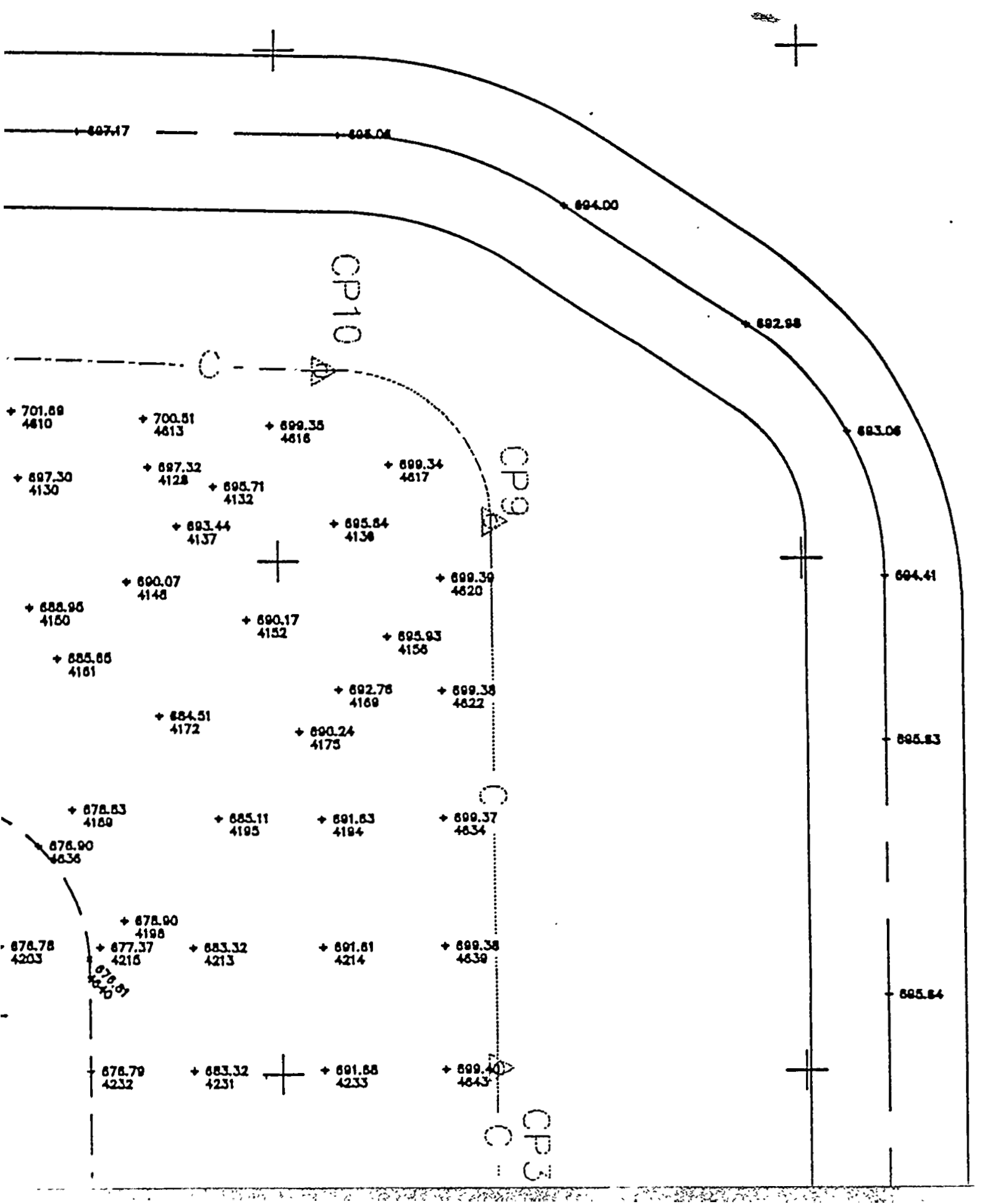




\section{ABBREVATIONS}

$\begin{array}{ll}\text { AP } & =\text { ANGLE POINT } \\ \text { EP } & =\text { EDGE PAVENENT } \\ \text { EB } & =\text { EDGE ASPHALT BERM } \\ \text { LOH PT. } & =\text { LOW POINT } \\ \text { MID } & =\text { MIODLE POINT } \\ \text { PC } & =\text { POINT OF CURVE } \\ \text { POC } & =\text { POINT ON CURVE } \\ \text { PT } & =\text { POINT OF TANGENT } \\ \text { TB } & =\text { TOP OF ASPHALT BERM }\end{array}$
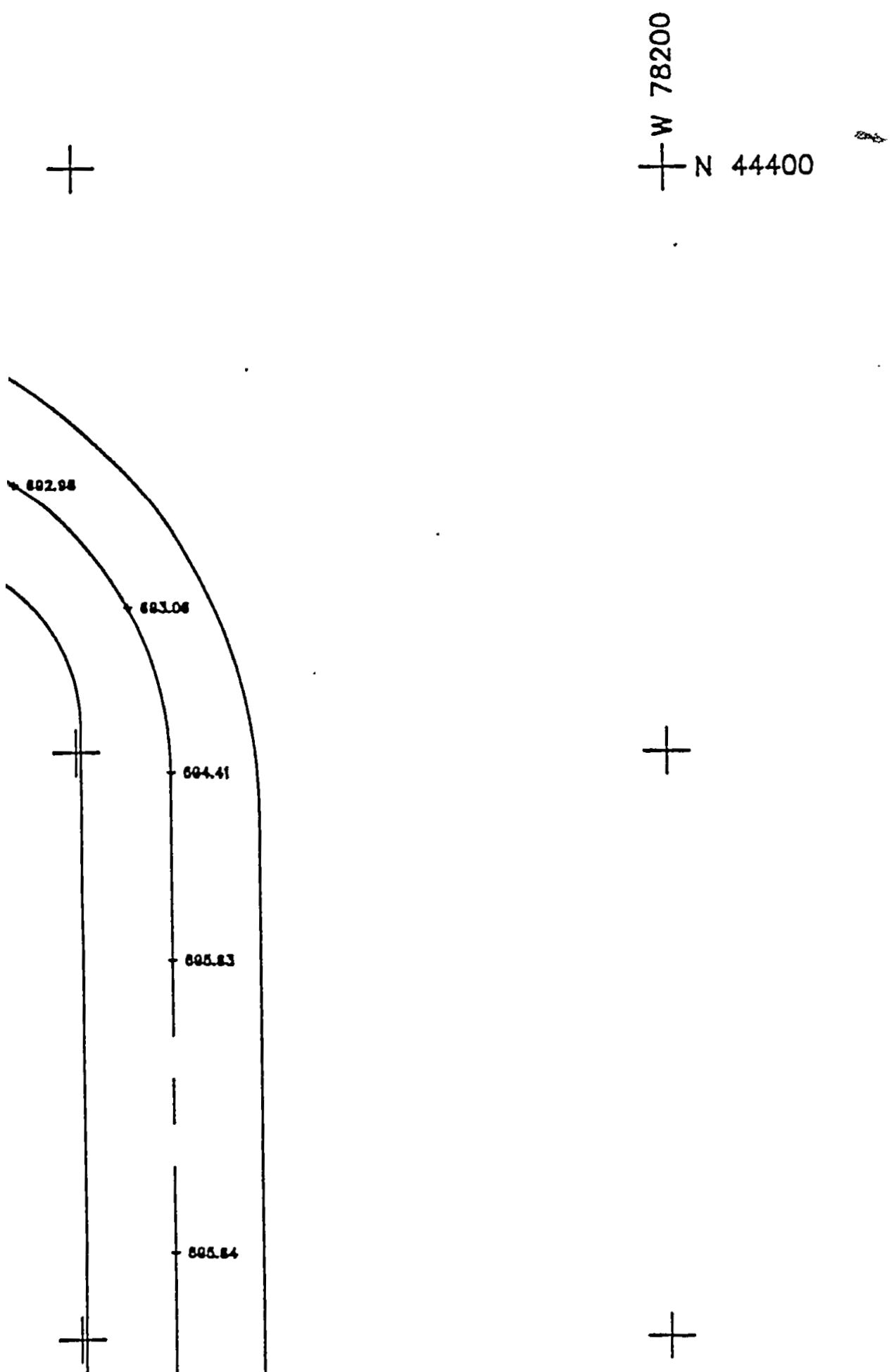


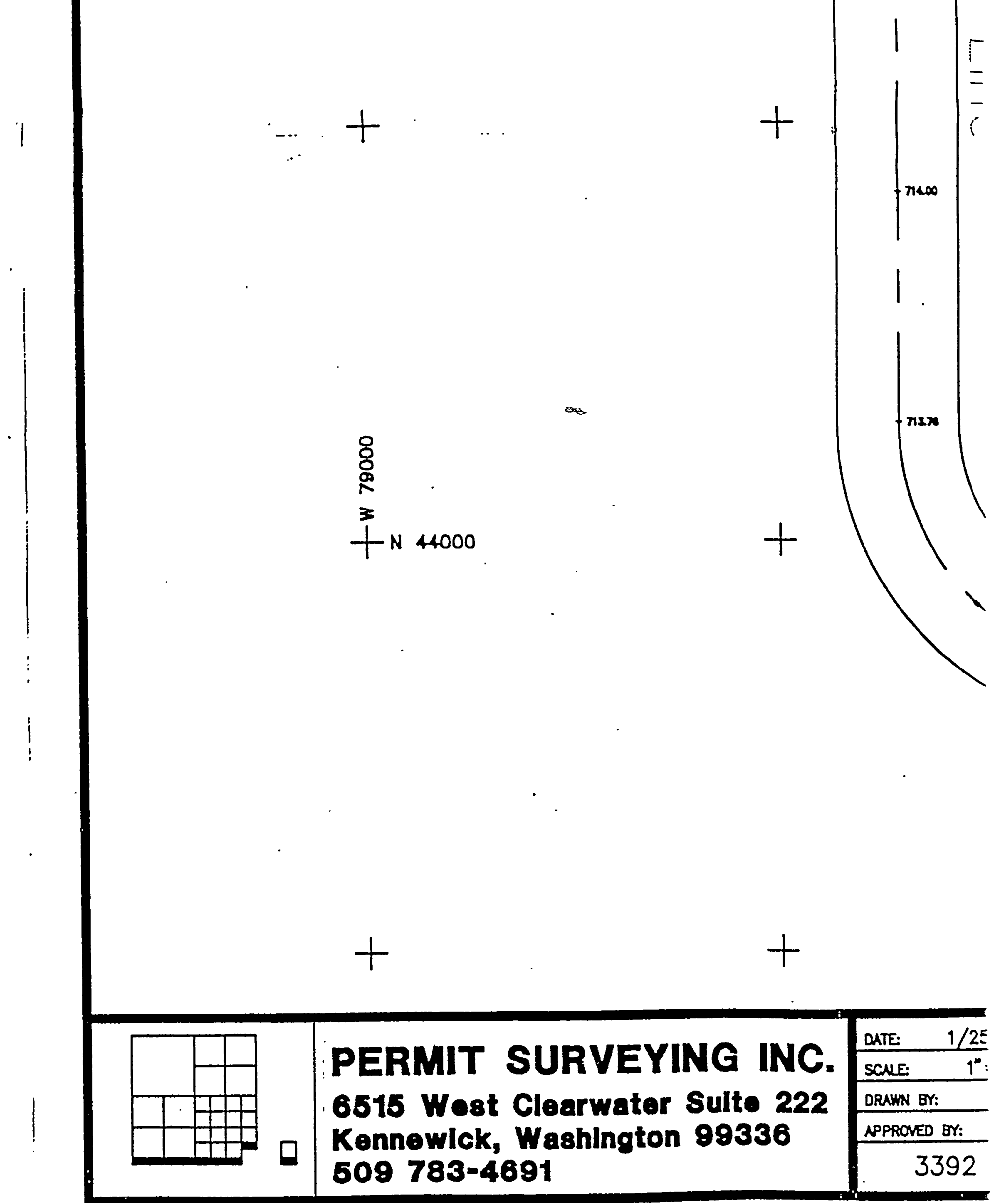




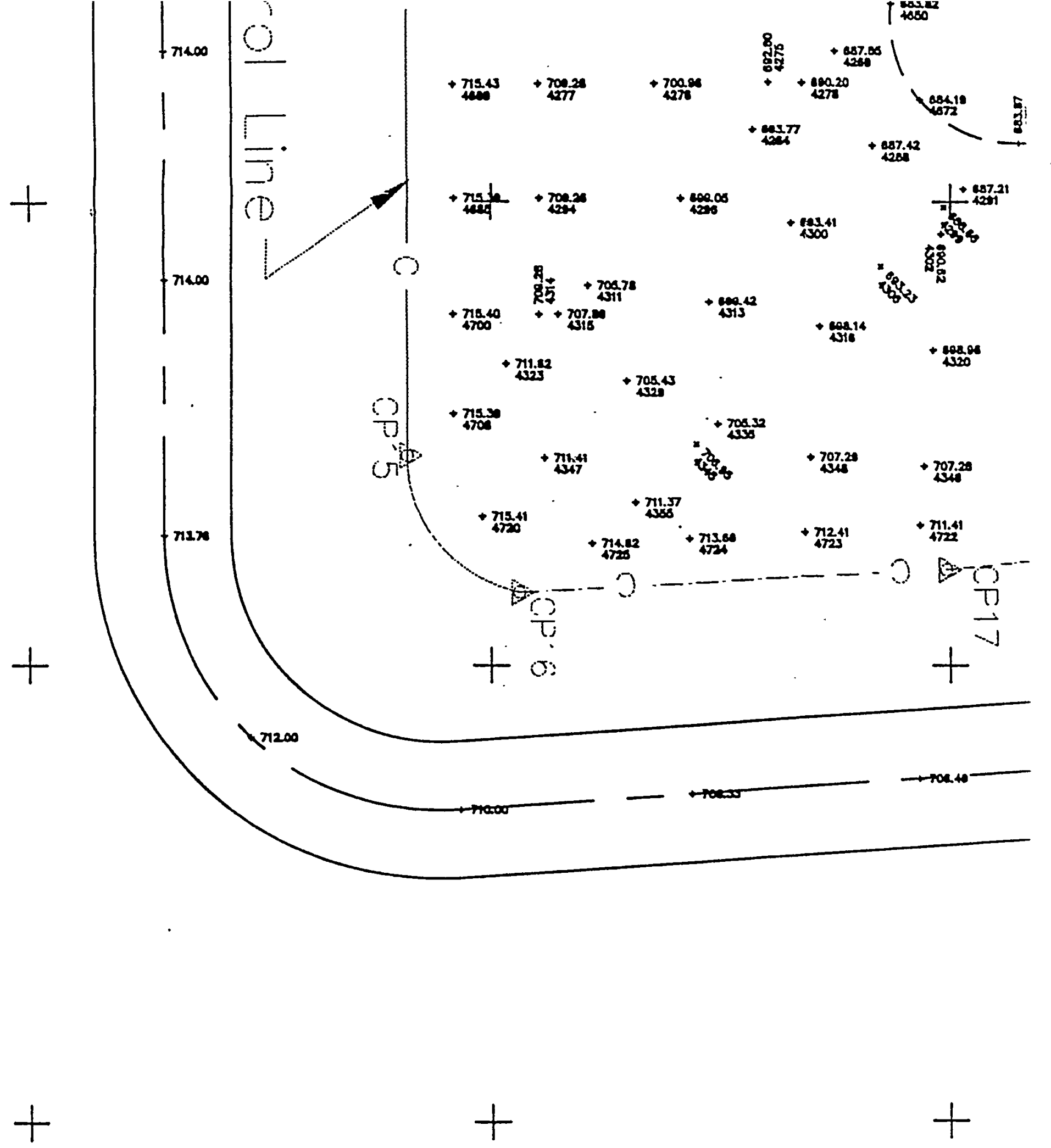

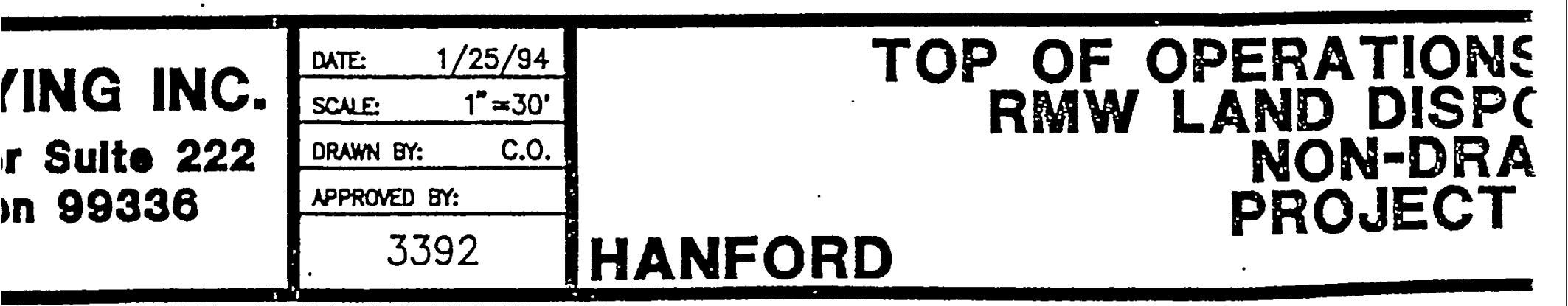




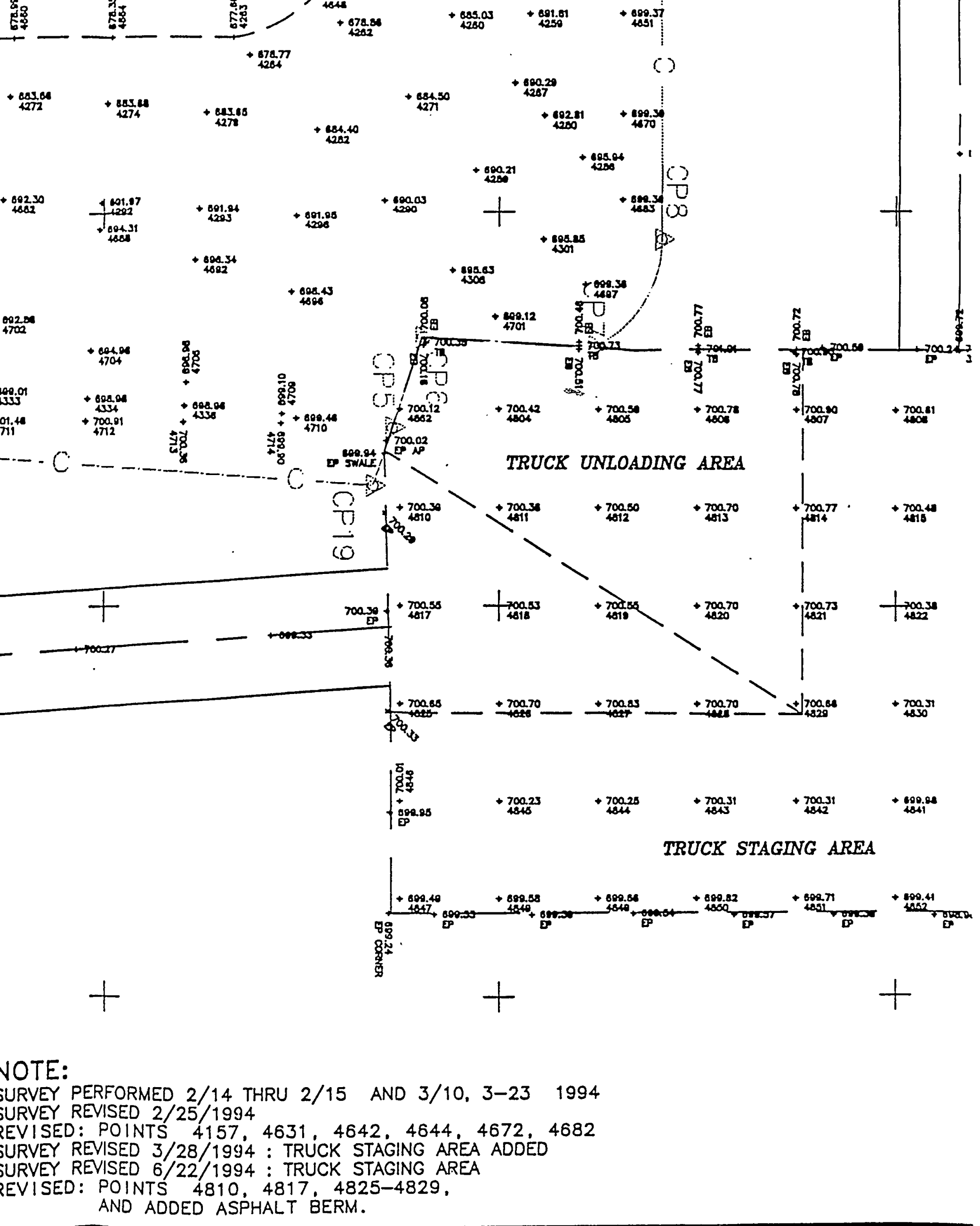




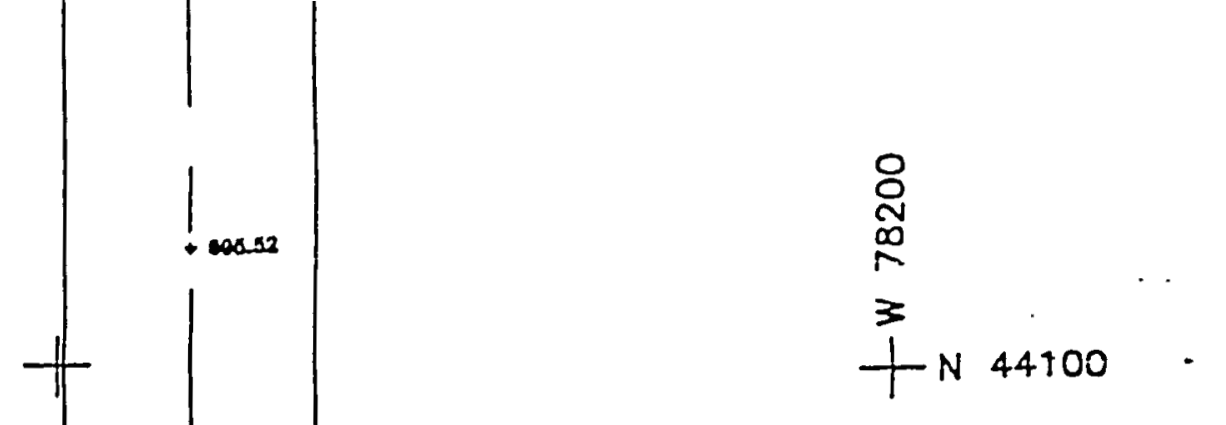

1002 is coseres
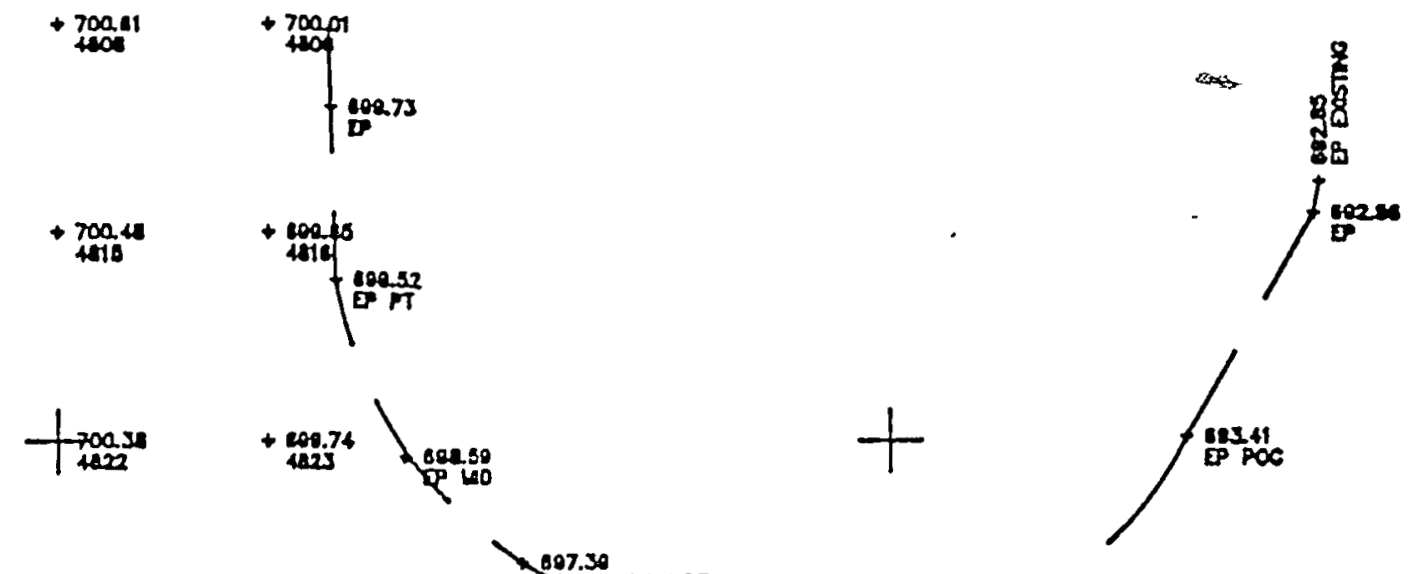

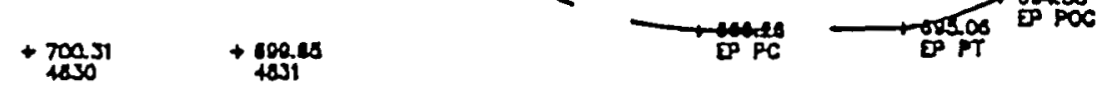
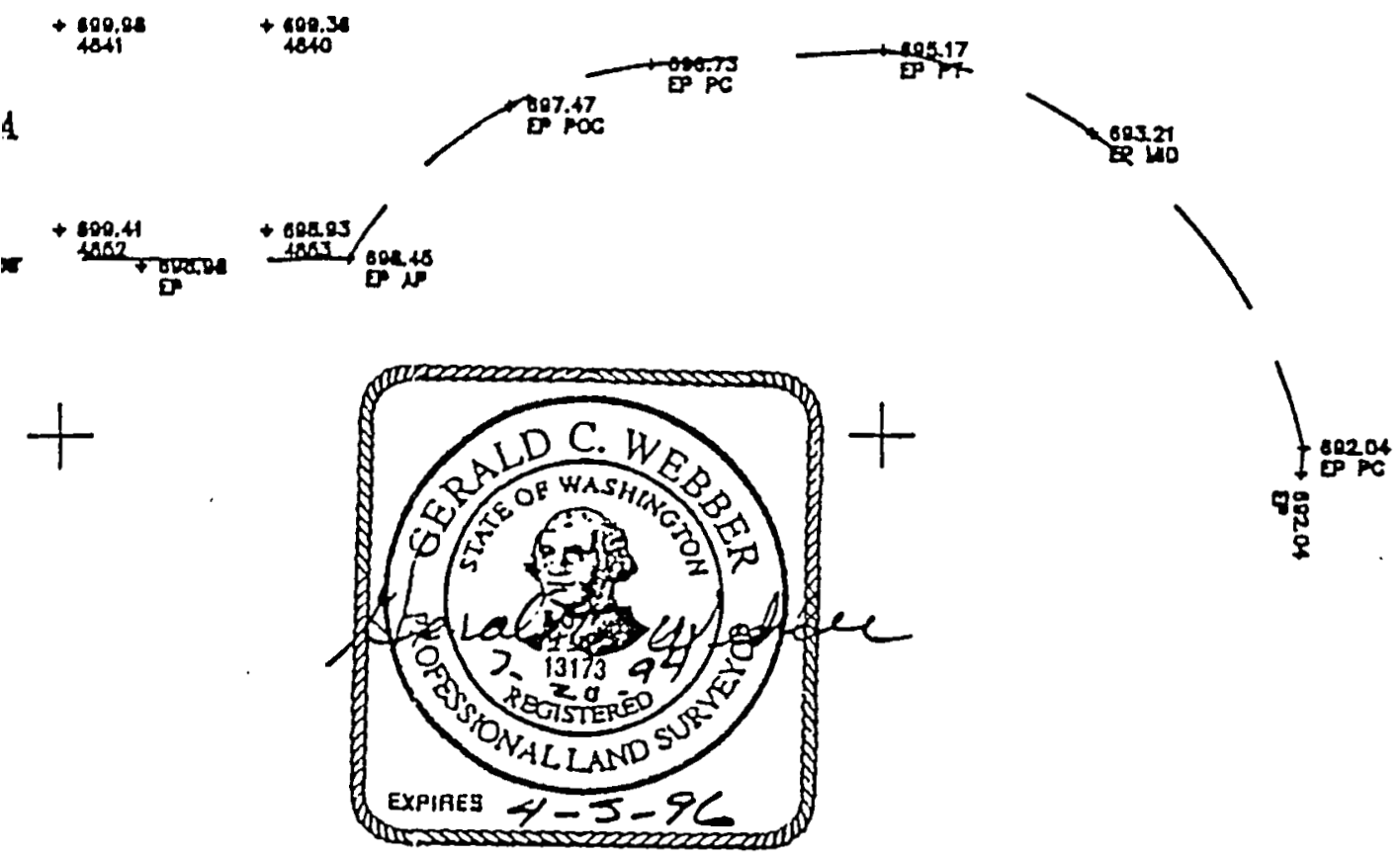

WHC-SD-W025-RPT-001, Rev. 0 